\title{
Evaluation of Metals Release from Oxidation of Fly Ash during Dredging of the Emory River, TN
}

Jeffery A. Steevens, Anthony J. Bednar, Mark A. Chappell, Alan J. Kennedy, Jennifer M. Seiter, Jacob K. Stanley, and Daniel E. Averett

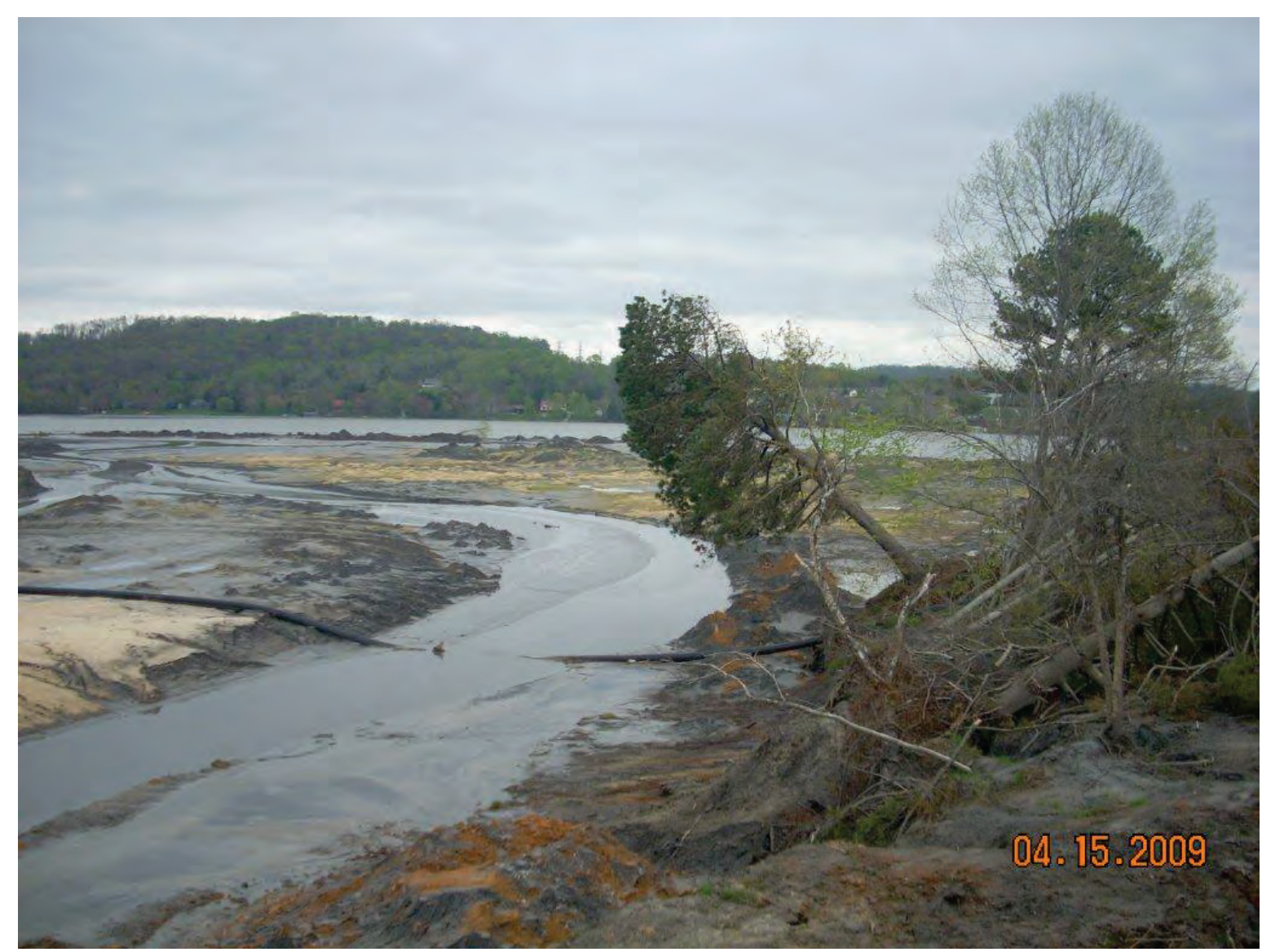




\section{Evaluation of Metals Release from Oxidation of Fly Ash during Dredging of the Emory River, TN}

Jeffery A. Steevens, Anthony J. Bednar, Mark A. Chappell, Alan J. Kennedy, Jennifer M. Seiter, Jacob K. Stanley, and Daniel E. Averett

Environmental Laboratory

U.S. Army Engineer Research and Development Center 3909 Halls Ferry Road

Vicksburg, MS 39180-6199

Final report

Approved for public release; distribution is unlimited. 


\begin{abstract}
This report describes a study examining the effects of Kingston Fossil Plant fly ash on geochemistry, water quality, and aquatic biota relevant to the Emory River in Tennessee. It focuses on the processes specific to the changes in the fly ash chemistry and potential releases as a result of a spill that occurred on December 22, 2008 and the subsequent dredging operations to remove the fly ash from the Emory River. Fly ash from three sites including the original pile, fly ash spilled in the Emory River, and dredged fly ash from the sluice channel were evaluated to assess the potential for metal releases. Fly ash from the Emory River and sluice channel were used to prepare relevant suspensions (e.g., elutriates) to represent extreme conditions for the release, dissolution, and metal speciation changes that might occur during dredging. Advanced chemical assessment techniques were used to quantify the chemical concentrations and speciation in this system. Because multiple metals were present, biological studies were also conducted to assess the potential for toxic effects and uptake in aquatic organisms. Chemistry results from this study were compared to measurements from ongoing monitoring at the site.
\end{abstract}

DISCLAIMER: The contents of this report are not to be used for advertising, publication, or promotional purposes. Citation of trade names does not constitute an official endorsement or approval of the use of such commercial products. All product names and trademarks cited are the property of their respective owners. The findings of this report are not to be construed as an official Department of the Army position unless so designated by other authorized documents. 


\section{Contents}

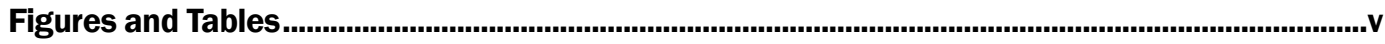

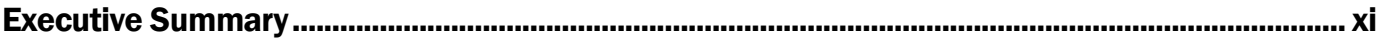

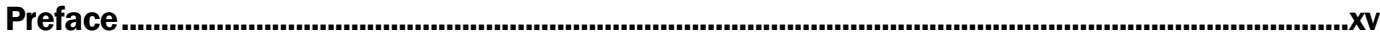

Unit Conversion Factors ..................................................................................................................xvi

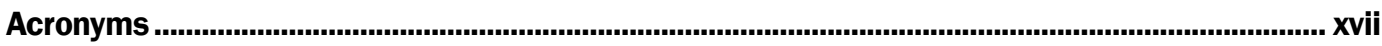

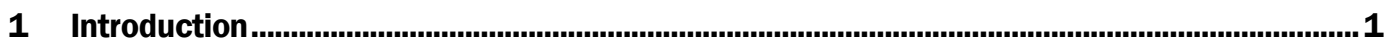

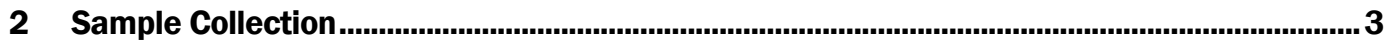

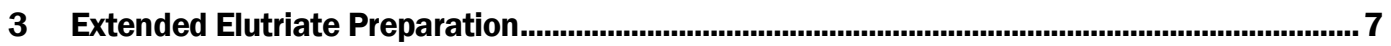

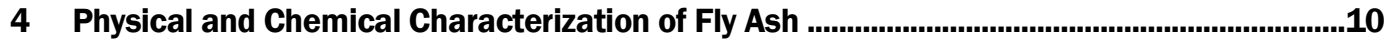

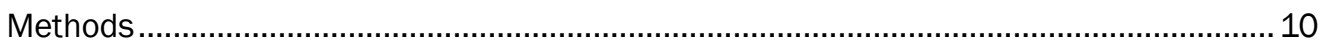

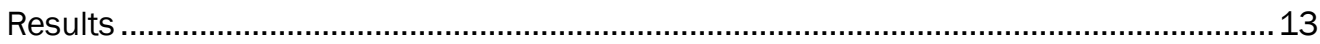

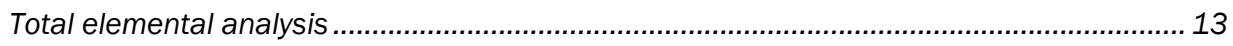

Ash metal speciation: Sequential extraction analysis...................................................... 19

Ash elemental speciation: X-ray absorption spectroscopy analysis................................... 21

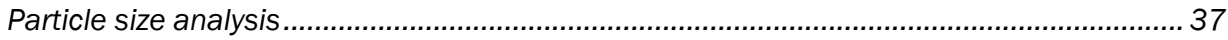

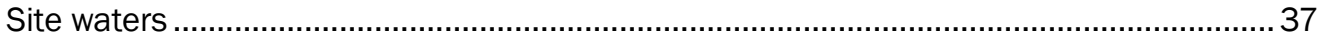

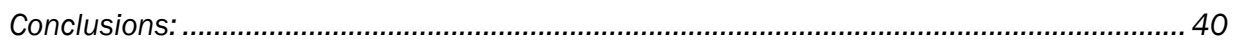

5 Physical and Chemical Characterization of Elutriate ........................................................

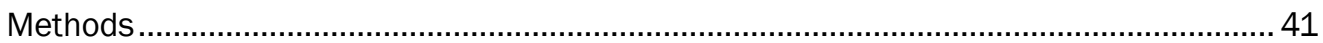

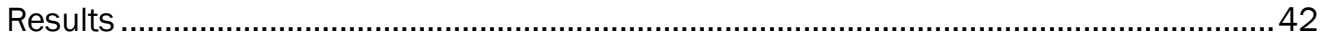

Solid-phase metal speciation .................................................................................... 51

Sediment particle size distributions during the EET .......................................................... 56

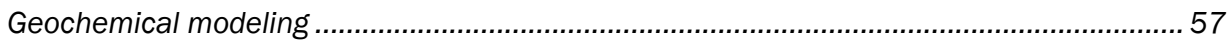

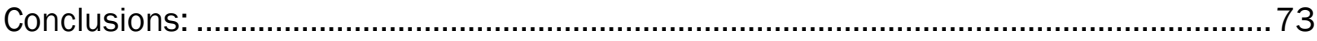

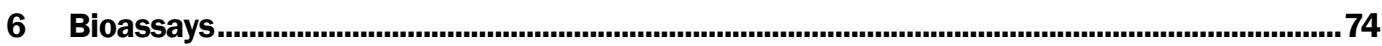

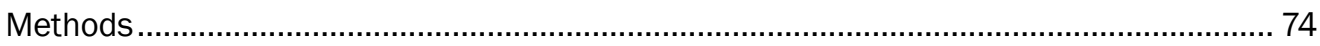

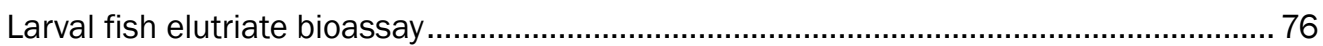

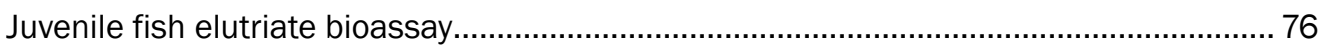

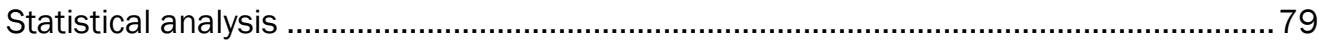

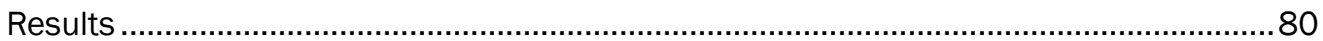

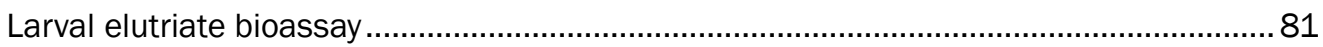

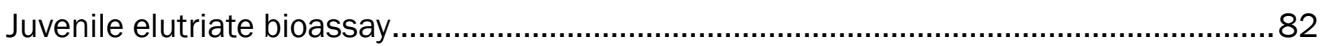

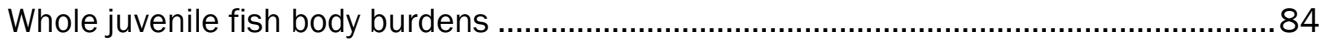

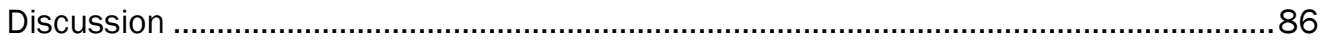




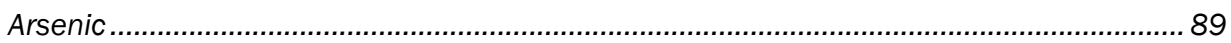

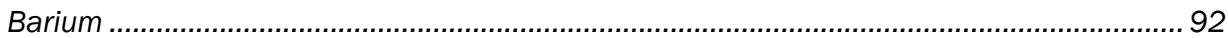

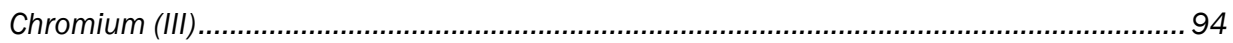

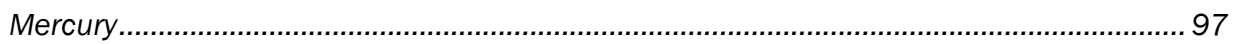

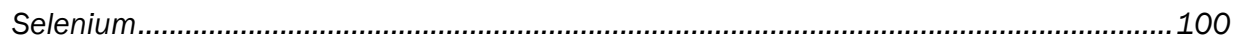

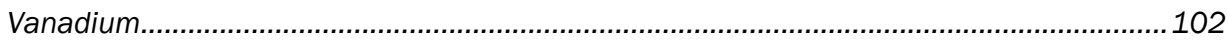

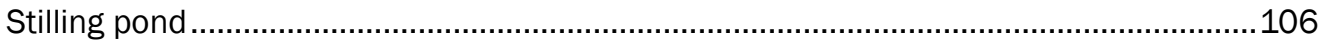

Gill histology and mussel bioassay.................................................................. 107

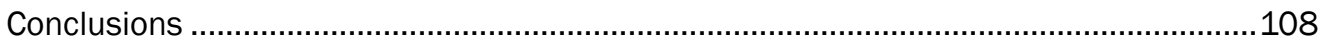

7 Comparison of Laboratory Elutriate Results to Field Observations.....................................110

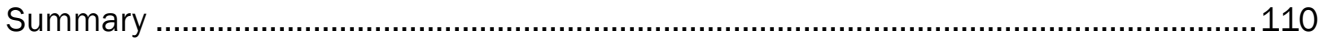

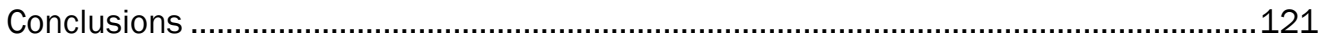

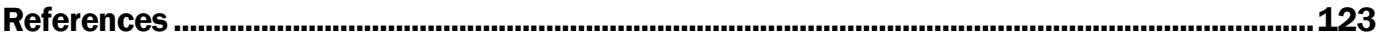

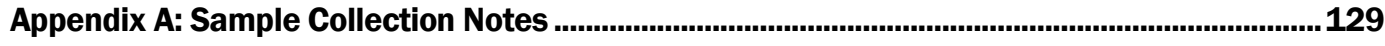

Appendix B: Elutriate Laboratory Data Sheets ..............................................................................142

Appendix C: Particle Size Distribution ..................................................................................149

Appendix D: Chemistry Data Reports .......................................................................................... 170

Appendix E: TOC data, Grain Size Analysis, and Speciation Data Sheets ...................................513

Appendix F: Elutriate Bioassay Laboratory Data Sheets ................................................................. 524

Appendix G: Elutriate Bioassay Water Quality Parameters............................................................. 579

Appendix H: Histological Examination of Fish Gills ................................................................582

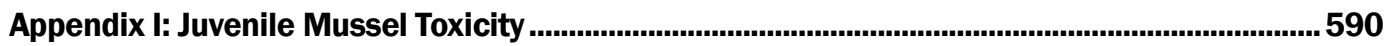

Report Documentation Page 


\section{Figures and Tables}

\section{Figures}

Figure 1. Sampling locations.

Figure 2. Factor loading plot demonstrating interdependent structure in the two factors from principal factor analysis of the solid-phase constituents from the ash samples collected at the original source pile (Pile), the Emory River (EMR), and the sluice channel (SLC).

Figure 3. Plots showing the distribution of case-wise factor scores for reference soil and sediment (RF1 - 6) samples relative to the ash samples with respect to factor loading.......

Figure 4. Carbon NEXAFS spectra for ash collected from the source pile, the Emory River, and sluice channel sediments and elutriates.

Figure 5. Nitrogen NEXAFS spectra for ash collected from the source pile, the Emory River, and sluice channel sediments and elutriates.

Figure 6. XANES data collected at the manganese K-edge energy for bulk ash and elutriate samples, where 0 means oxic regime elutriate samples..

Figure 7. XANES data collected at the iron K-edge for bulk ash and elutriate samples.

Figure 8. XANES spectra collected at the arsenic K-edge for bulk ash and elutriate samples.

Figure 9. Linear combination results for arsenic XANES data (Figure 8) on bulk ash and elutriate samples.

Figure 10. XANES spectra collected at the selenium K-edge for bulk ash and elutriate samples.

Figure 11. XANES spectra collected at the selenium K-edge for use as reference samples, including the selenium-bound thiol compounds: Se-methyl-selenocysteine and Semethionine.

Figure 12. Linear combination results for selenium XANES data (Figure 10) on bulk ash and elutriate samples.

Figure 13. Synchrotron X-ray fluorescence maps depicting elemental distribution in source pile fly ash.

Figure 14. Synchrotron X-ray fluorescence maps depicting elemental distribution in fly ash collected from the Emory River.

Figure 15. Spot $\mu$-XANES analysis for determining localized arsenic speciation in fly ash and elutriate samples..

Figure 16. Linear combination results of spot $\mu$-XANES data (Figure 15) for localized arsenic speciation of ash and elutriate samples.

Figure 17. Localized selenium speciation determined by differential energy $\mu$-XRF mapping.

Figure 18. Selenium speciation of the spots defined in (Figure 17) for the original source pile fly ash material using $\mu$-XANES.

Figure 19. Linear combination results of selenium $\mu$-XANES (Figure 18) data from spots defined on the XRF maps (Figure 17). 
Figure 20. $\mu$-XANES analysis of selenium in the fly ash collected from the Emory River and the sluice channel sediments and elutriates. $\mu$-XANES spectra for $\mathrm{Se}(0)$, $\mathrm{Se}(\mathrm{IV})$, and $\mathrm{Se}(\mathrm{VI})$ are included for reference.

Figure 21. Linear combination results for selenium $\mu$-XANES data for spots on the XRF maps..

Figure 22. Textural triangle showing the percentages of sand-, silt-, and clay-sized particle in the ash materials collected from the source pile, Emory River, and the sluice channel.

Figure 23. Dissolved metals concentrations in the Emory River oxic regime (i.e., bubbled with air) elutriate as a function of time.

Figure 24. Dissolved metals concentrations in the Emory River anoxic regime (i.e., bubbled with nitrogen gas) elutriate as a function of time.

Figure 25. Dissolved metals concentrations in the sluice channel oxic regime elutriate as a function of time.

Figure 26. Dissolved metals concentrations in the sluice channel anoxic regime elutriate as a function of time.

Figure 27. Expanded view of dissolved metals concentrations in the Emory River elutriates as a function of time.

Figure 28. Expanded view of dissolved metals concentrations in the sluice channel elutriates as a function of time.

Figure 29. TOC concentrations in elutriate waters over the 10-day preparation period.

Error bars represent the standard deviation of triplicate elutriate preparations.

Figure 30. Arsenic speciation in the oxic and anoxic regime Emory River and sluice channel elutriate experiments. Error bars represent the standard deviation of triplicate elutriate preparations. Non-detect concentrations of arsenic species $(<2 \mu \mathrm{g} / \mathrm{L})$ are plotted as one-half the detection limit (i.e. $1 \mu \mathrm{g} / \mathrm{L}$ )

Figure 31. Selenium speciation in the oxic and anoxic regime Emory River and sluice channel elutriate experiments.

Figure 32. Eh values measured over the course of the 10-day elutriate experiment........................50

Figure 33. pH values measured over the course of the 10-day elutriate experiment. .......................50

Figure 34. Pourbaix $(\mathrm{Eh}-\mathrm{pH})$ stability diagram for iron oxides........................................................51

Figure 35. Pourbaix (Eh - pH) stability diagrams for manganese oxides............................................52

Figure 36. Results from linear combination fits of arsenic XANES data on bulk ash and elutriate samples.

Figure 37. Results from linear combination fitting of selenium XANES on bulk Emory River and sluice channel ash and elutriate samples.

Figure 38. Results from linear combination fittings of $\mu$-XANES data collected at the arsenic K-edge on discrete locations in the ash and treated with oxic regime elutriate samples.

Figure 39. Results from linear combination fittings of $\mu$-XANES data collected at the selenium K-edge on discrete locations in the Emory River and sluice channel ash and oxic regime Emory River and sluice channel elutriate samples.

Figure 40. Particle size data for the Emory River elutriate experiments. .57

Figure 41. Particle size data for the sluice channel elutriate experiments. 58

Figure 42. Results from geochemical modeling predicting manganese pairs and complexes in the Emory River elutriate $(\mathrm{t}=0 \mathrm{hr})$, based on total Mn determined in 
solution. Calculations were performed for a pH sweep of 4 to 10 , and redox sweep of 300 to $+300 \mathrm{mV}$...

Figure 43. Results from geochemical modeling predicting manganese pairs and complexes in the Emory River elutriate $(t=240 \mathrm{hr}$ ), based on total Mn determined in solution. Calculations were performed for a pH sweep of 4 to 10 , and redox sweep of 300 to $+300 \mathrm{mV}$..

Figure 44. Results from geochemical modeling predicting manganese pairs and complexes in the sluice channel elutriate $(t=0 \mathrm{hr})$, based on total Mn determined in solution. Calculations were performed for a pH sweep of 4 to 10 , and redox sweep of 300 to $+300 \mathrm{mV}$.

Figure 45. Results from geochemical modeling predicting manganese pairs and complexes in the sluice channel elutriate $(t=240 \mathrm{hr}$ ), based on total Mn determined in solution. Calculations were performed for a pH sweep of 4 to 10 , and redox sweep of 300 to $+300 \mathrm{mV}$.

Figure 46. Results from geochemical modeling predicting chromium pairs and complexes in the Emory River elutriate $(\mathrm{t}=240 \mathrm{hr}$ ), based on total $\mathrm{Mn}$ determined in solution. Calculations were performed for a pH sweep of 4 to 10 , and redox sweep of -300 to $+300 \mathrm{mV}$.

Figure 47. Results from geochemical modeling predicting chromium pairs and complexes in the sluice channel elutriate $(t=240 \mathrm{~h}$ ), based on total Mn determined in solution. Calculations were performed for a pH sweep of 4 to 10 , and redox sweep of -300 to $+300 \mathrm{mV}$.

Figure 48. Results from geochemical modeling predicting arsenic pairs and complexes in the Emory River elutriate ( $\mathrm{t}=0 \mathrm{hr}$ ), based on total and speciated arsenic determined in solution. Calculations were performed for a pH sweep of 4 to 10 , and redox sweep of 300 to $+300 \mathrm{mV}$.

Figure 49. Results from geochemical modeling predicting arsenic pairs and complexes in the Emory River elutriate ( $\mathrm{t}=240 \mathrm{hr}$ ), based on total and speciated arsenic determined in solution. Calculations were performed for a pH sweep of 4 to 10, and redox sweep of 300 to $+300 \mathrm{mV}$...

Figure 50. Results from geochemical modeling predicting arsenic pairs and complexes in the sluice channel elutriate ( $\mathrm{t}=0 \mathrm{hr}$ ), based on total and speciated arsenic determined in solution. Calculations were performed for a pH sweep of 4 to 10 , and redox sweep of 300 to $+300 \mathrm{mV}$.

Figure 51. Results from geochemical modeling predicting arsenic pairs and complexes in the sluice channel elutriate $(t=0 \mathrm{hr})$, based on total and speciated arsenic determined in solution. Calculations were performed for a pH sweep of 4 to 10 , and redox sweep of 300 to $+300 \mathrm{mV}$..

Figure 52. Results from geochemical modeling predicting selenium pairs and complexes in the Emory River elutriate $(\mathrm{t}=240 \mathrm{hr}$ ), based on total and speciated Se determined in solution. Calculations were performed for a pH sweep of 4 to 10 , and redox sweep of 300 to $+300 \mathrm{mV}$.

Figure 53. Results from geochemical modeling predicting selenium pairs and complexes in the sluice channel $(\mathrm{t}=0 \mathrm{hr}$ ), based on total and speciated Se determined in solution. Calculations were performed for a $\mathrm{pH}$ sweep of 4 to 10 , and redox sweep of -300 to $+300 \mathrm{mV}$.

Figure 54. Results from geochemical modeling predicting selenium pairs and complexes in the sluice channel $(\mathrm{t}=240 \mathrm{hr})$, based on total and speciated Se determined in solution. Calculations were performed for a pH sweep of 4 to 10 , and redox sweep of -300 to $+300 \mathrm{mV}$. 
Figure 55. Results from geochemical modeling predicting precipitation of barium compounds (in mol/L) at $\mathrm{t}=\mathrm{O} \mathrm{h}$ in the (left) Emory River and (right) sluice channel elutriates. Predictions are based on total Ba and total and speciated As determined in solution. Calculations were performed for a pH sweep of 4 to 10 , and redox sweep of 300 to $+300 \mathrm{mV}$.

Figure 56. Images of (a) larval and (b) juvenile Pimephales promelas............................................ 74

Figure 57. Image of exposure systems for (a) larval and (b) juvenile Pimephales promelas. ............78

Figure 58. Gills dissected from an unexposed juvenile Pimephales promelas...................................80

Figure 59. Daily survival of larval Pimephales promelas assessed over the 10-day exposure duration for (a) SLC-EL-LF, and (b) EMR-EL-LF.

Figure 60. Dissolved arsenic concentrations in Emory River Reference water (EMR-WA), Sluice water (SLC-WA), Emory River Fly Ash Elutriate (EMR-EL), sluice fly ash elutriate (SLC$\mathrm{EL})$, and the U.S. Environmental Protection Agency chronic water quality criterion (Criterion Continuous Concentration or $\mathrm{CCC}$ ).

Figure 61. Arsenic concentrations (as wet weight, $\mathrm{mg} / \mathrm{kg}$ ) in whole Pimephales promelas exposed to EMR-EL-AF for 10 days.

Figure 62. Dissolved barium concentrations in Emory River reference water (EMR-WA), sluice water (SLC-WA), Emory River fly ash elutriate (EMR-EL) and sluice fly ash elutriate (SLC-EL).

Figure 63. Dissolved chromium (III) concentrations in Emory River reference water (EMRWA), sluice water (SLC-WA), Emory River fly ash elutriate (EMR-EL), sluice fly ash elutriate (SLC-EL), and the standard and hardness adjusted USEPA chronic water quality criteria (criterion continuous concentration or CCC).

Figure 64. Chromium concentrations (as wet weight, $\mathrm{mg} / \mathrm{kg}$ ) in whole Pimephales promelas exposed to EMR-EL-AF for 10 days.

Figure 65. Dissolved mercury concentrations in Emory River reference water (EMR-WA), sluice water (SLC-WA), Emory River fly ash elutriate (EMR-EL), sluice fly ash elutriate (SLC$\mathrm{EL}$ ) and the USEPA chronic water quality criterion (criterion continuous concentration or CCC)

Figure 66. Mercury concentrations (as wet weight, $\mathrm{mg} / \mathrm{kg}$ ) in whole Pimephales promelas exposed to EMR-EL-AF for 10 days.

Figure 67. Dissolved selenium (predominately selenite) concentrations in Emory River reference water (EMR-WA), sluice water (SLC-WA), Emory River fly ash elutriate (EMR-EL), sluice fly ash elutriate (SLC-EL), and the USEPA chronic water quality criterion (criterion continuous concentration or (CC).

Figure 68. Selenium concentrations (converted to dry weight, $\mathrm{mg} / \mathrm{kg}$ ) in whole Pimephales promelas exposed to EMR-EL-AF for 10 days.

Figure 69. Dissolved vanadium (V) concentrations in Emory River reference water (EMRWA), sluice water (SLC-WA), Emory River fly ash elutriate (EMR-EL), and sluice fly ash elutriate (SLC-EL).

Figure 70. Vanadium concentrations (as wet weight, $\mathrm{mg} / \mathrm{kg}$ ) in whole Pimephales promelas exposed to EMR-EL-AF for 10 days.

Figure 71. Elutriate supernatant TSS concentrations compared to field observations (verified, not validated) by TVA.

Figure 72. Emory River elutriate supernatant arsenic compared to TVA's field observations (verified, not validated) of the dredge plume.

Figure 73. Emory River elutriate supernatant barium compared to TVA's field observations (verified, not validated) of the dredge plume. 
Figure 74. Emory River elutriate supernatant selenium compared to TVA's field observations (verified, not validated) of the dredge plume.

Figure 75. Emory River elutriate supernatant vanadium compared to TVA's field observations (verified, not validated) of the dredge plume.

Figure 76. Arsenic in sluice channel elutriate supernatant arsenic compared to TVA's field observations (verified, not validated) of stilling pond effluent (AP_IMP001).

Figure 77. Selenium in sluice channel elutriate supernatant selenium compared to TVA's field observations (verified, not validated) of stilling pond effluent (AP_IMP001).......................... 116

Figure 78. Particulate arsenic concentrations (verified, not validated)...........................................117

Figure 79. Particulate barium concentrations (verified, not validated)..........................................117

Figure 80. Particulate selenium concentrations (verified, not validated). ...................................... 118

Figure 81. Particulate vanadium concentrations (verified, not validated).................................... 118

Figure 82. TVA field observations (verified, not validated) of TSS in receiving waters................... 119

Figure 83. TVA field observations (verified, not validated) of arsenic in receiving waters.............. 120

Figure 84. TVA field observations of barium in receiving waters.................................................... 120

Figure 85. TVA field (verified, not validated) observations of the percent dissolved/total arsenic and barium compared to TSS in receiving waters.

\section{Tables}

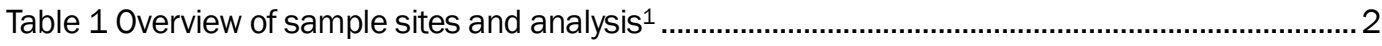

Table 2. Sampling station coordinates.................................................................................. 4

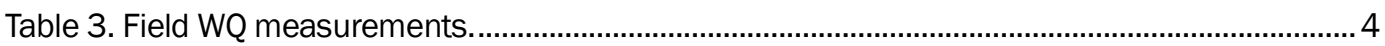

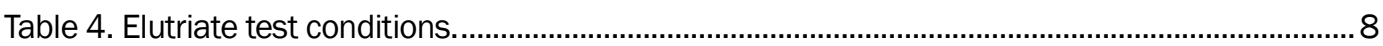

Table 5. Concentrations $(\mathrm{mg} / \mathrm{kg}$ ) of metals in fly ash samples from TVA Kingston Fossil

Plant.

Table 6. Concentrations ( $\mathrm{mg} / \mathrm{kg}$ ) of metals in reference samples upstream from TVA

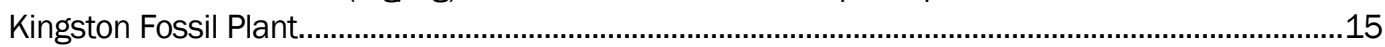

Table 7. Varimax-rotated factor loadings for elemental quantities from Tables 5 and 6 ...................16

Table 8. Partitioning of Emory River fly ash metals into six geochemical phases.............................19

Table 9. Partitioning of storage pile fly ash metals into six geochemical phases. .............................20

Table 10. Partitioning of Sluice Channel fly ash metals into six geochemical phases. ......................20

Table 11. Linear combination results for Fe XANES data on bulk ash and elutriate samples.

Table 12. Concentrations of metals ( $\mathrm{mg} \mathrm{L}^{-1}$ ) in site waters (one grab sample each). …...................39

Table 13. Concentration $(\mu \mathrm{g} / \mathrm{L})$ of arsenic and selenium species measured in sitecollected waters.

Table 14. Summary of conditions for fly ash elutriate toxicity tests conducted by ERDC.

Table 15. Confirmation of elutriate homogenization consistency by total suspended solids (TSS) concentrations prior to water renewals (days 0,3 , and 7). NA = data not available, sample not taken.

Table 16. Summary of larval Pimephales promelas survival and biomass after 10 days of exposure. 
Table 17. Summary of mean juvenile Pimephales promelas survival and biomass (and one standard deviation from the mean) after 10 days of exposure to EMR-EL-AF.

Table 18. Whole Pimephales promelas body burdens ( $\mathrm{mg} / \mathrm{kg}$ wet weight) for arsenic (As), barium (Ba), chromium ( $\mathrm{Cr}$ ), mercury ( $\mathrm{Hg}$ ), and vanadium $(\mathrm{V})$ and other metals (below the double line).

Table 19. Comparison of dissolved metals concentrations $(\mathrm{mg} / \mathrm{L})$ in the stilling pond, sluice channel water, and sluice channel elutriate. 106 


\section{Executive Summary}

This report describes a study examining the effects of Kingston Fossil Plant fly ash on geochemistry, water quality, and aquatic biota relevant to the Emory River in Tennessee. It focuses on the processes specific to the changes in the fly ash chemistry and potential releases as a result of a spill that occurred on December 22, 2008 and the subsequent dredging operations to remove the fly ash from the Emory River. Fly ash from three sites including the original pile, fly ash spilled in the Emory River, and dredged fly ash from the sluice channel were evaluated to assess the potential for metal releases. Fly ash from the Emory River and sluice channel were used to prepare relevant suspensions (e.g., elutriates) to represent extreme conditions for the release, dissolution, and metal speciation changes that might occur during dredging. Advanced chemical assessment techniques were used to quantify the chemical concentrations and speciation in this system. Because multiple metals were present, biological studies were also conducted to assess the potential for toxic effects and uptake in aquatic organisms. Chemistry results from this study were compared to measurements from ongoing monitoring at the site. The following conclusions were made based on the results of the study:

\section{Chemical characterization of elutriate}

- Fly ash collected from three locations (original source pile, the Emory River, and sluice channel) contain elevated concentrations of several metals relative to local soils and sediments, including arsenic, selenium, and barium.

- Fly ash has been modified by its residence in the Emory River in the following ways:

- Net carbon and nitrogen profiles changed to represent accumulated natural organic matter present in the river.

o Manganese in the source pile transformed from an oxidized to reduced state. Iron species were stable as Fe(III) oxides, and did not change.

- Approximately 30 to $50 \%$ of total selenium transformed from selenite (Se(IV)) to more reduced Se species after the ash was deposited in the river. Naturally forming organoselenium, present 
as Se(II), may be among these reduced forms. No evidence of selenate was found in the Emory River ash or the sluice channel.

o Fly ash arsenic remained predominantly as As(V), with evidence of some slight shift to reduced arsenic-thiol species.

- Selenate was only found in the waters collected from the stilling pond. Given that selenate was not detected in any of the systems preceding the stilling pond, it can be hypothesized that selenium is photo-oxidized due to the exposure of surface waters to direct sunlight. More investigations are warranted to resolve this mechanism.

\section{Chemical characterization of fly ash}

- Increased dissolution of several elements (e.g., As, Ba, V, Se) was observed over the 10-day effluent elutriate test, while dissolved Mn concentrations decreased. Even though system redox remained relatively stable, it was demonstrated that fly ash Mn was partially oxidized by extensive oxic regime treatment. Iron phases were unaffected. The one order of magnitude increase in dissolved organic carbon over the effluent elutriate test suggests the experimental conditions promoted microbial activity.

- With the exception of Mn, bulk metal(loid) speciation remained relatively constant over the 10-day period. The major exception to this observation came from $\mu$-XANES (micro-X-ray near edge structure) spectroscopic analysis showing a domain of selenate in Emory River elutriate solid after the 10-day air bubbling period, even though selenate was not detected in solution. It is unclear whether this domain was caused by the elutriate conditions or existed before the experiments were performed. However, the results do open up the possibility that extensive oxidation of the system can produce selenate. Geochemical modeling confirms that the Emory River conditions represent a lower boundary where selenate is thermodynamically stable in this system, although a relatively minor species relative to selenite.

- Given that both the anoxic and oxic regime (nitrogen- and air-bubbled) systems displayed nearly identical behavior, it is hypothesized that the trend in the dissolution of metals over the 10-day period was due to the extended agitation of the fly ash. The corresponding spike in microbial activities supports the view that constantly dispersing the fly ash enhances the microbial bioavailability to surface-adsorbed nutrients. 


\section{Biological characterization}

- No statistically significant adverse effects were observed for larval or juvenile fish exposed to the oxic regime elutriates prepared with fly ash from the Emory River. Histological analysis of the gills was inconclusive, and no effect was found on juvenile mussel survival.

- Dissolved As, Ba, $\mathrm{Cr}, \mathrm{Hg}, \mathrm{V}$ concentrations in the oxic regime elutriate were below the chronic water quality criteria and/ or relevant literature or screening values.

o Dissolved concentrations of As, $\mathrm{Cr}$, and $\mathrm{Hg}$ were below the criterion continuous concentration (CCC).

o Ba and $\mathrm{V}$ concentrations were lower than literature toxicity reference values.

o While the dissolved concentrations of Se measured in the Emory River elutriate $(5.4 \mu \mathrm{g} / \mathrm{L})$ and the sluice elutriate $(12.7 \mu \mathrm{g} / \mathrm{L})$ exceeded the CCC $=5.0 \mu \mathrm{g} / \mathrm{L}$ for total Se, it is generally understood that tissue residues are more predictive of Se toxicity than are water concentrations (U.S. Environmental Protection Agency (USEPA) 2004).

- In general, concentration of metals in tissues increased in a dosedependent manner. However, this increase is likely due to metals present in the gut as ingested fly ash, not metals integrated into the tissue.

o Arsenic (As) was near reference values reported in the literature at which effects are observed. However, this was a conservative assessment because the less toxic arsenate $(\mathrm{As}(\mathrm{V}))$ dominated in the oxic regime elutriates, but the levels in water were compared to screening values derived for the more toxic arsenite (As(III)).

o There was very little literature data and no screening values to compare barium (Ba) concentrations in tissues.

- Chromium (Cr) was present as $\mathrm{Cr}$ (III) and at concentrations near the no observable effects residue (NOER) from literature studies.

o Mercury (Hg) and selenium (Se) concentrations in tissues were below relevant screening values.

- Vanadium (V) exceeded literature-based screening values, but the elevated concentrations are likely the result of fly ash in the gut. 
- In a weight of evidence approach where chemistry, toxicity, and bioaccumulation data are integrated, these results suggest little potential for toxicity to related fish species given the extreme conditions represented by oxidized elutriates in this study.

- Several areas of uncertainty could be addressed through additional data collection including longer-term exposures of fish to suspended or bedded fly ash, assessing food web exposure or trophic transfer, and evaluation of sensitive species not assessed in this study.

\section{Comparison to field conditions}

- The elutriate experiments performed in this study used mixing times well beyond established elutriate procedures for the purpose of assessing potential shifts in metal speciation when fly ash contaminants are released to the water environment. The exaggerated terminal mixing times (10 days) produced elutriates that overestimate total dissolved metal concentrations for the dredge plume and the ash settling/ stilling pond effluent, compared to those observed by TVA's field monitoring program for the plume and stilling pond.

- Suspended solids and metal concentrations decline significantly at the monitoring station immediately downstream of the dredging operation.

- Dissolved metals account for much of the total metal concentrations observed in the pond effluent, plume, and river monitoring stations, largely because of the relative low suspended solids concentration. This suggests that the small suspended particulate source limits further dissolved metal desorption in the water column, but that most of the metal constituents present in the water column are mobile and subject to downstream transport.

- Results of the field monitoring program should be closely observed for deviations from the trends observed to date. Increased dredge production rates may increase contaminant releases at the dredge, as well as increase flow rates through the settling ponds, reducing retention time and impacting the effectiveness of the settling pond system in retaining suspended solids and contaminants. Cumulative effects of dredging operations over time may also affect water quality through deposits of erodible solids downstream of the dredge and solids buildup in the settling ponds. 


\section{Preface}

This report was prepared through a research effort funded by the Tennessee Valley Authority. It describes the results of research focusing on the geochemistry, water quality, and aquatic biota following the Kingston Fossil Plant spill on December 22, 2008. The research effort was conducted and the report prepared by Dr. J effery Steevens, Dr. Anthony J . Bednar, Dr. Mark A. Chappell, Alan J . Kennedy, Dr. J ennifer M. Seiter, Dr. J acob K. Stanley, Daniel E. Averett of the Environmental Laboratory (EL), U.S. Army Engineer Research and Development Center (ERDC). The authors acknowledge support provided by J onathan Burr and Paul Schmierbach from the State of Tennessee, Department of Environment and Conservation, with sampling design and collection. The authors would also like to thank reviewers from the U.S. Fish and Wildlife Service, U.S. Environmental Protection Agency, Tennessee Valley Authority, and State of Tennessee for providing constructive review of this report.

This report was prepared under the U.S. Army Corps of Engineers' Dredging Operations and Environmental Research (DOER) Program and Dredging Operations Technical Support (DOTS) Program, for which Dr. Todd S. Bridges serves as Program Manager. Dr. Beth Fleming was Director, EL. COL Kevin J. Wilson was Commander and Dr. J effery P. Holland was Director. 


\section{Unit Conversion Factors}

\begin{tabular}{|l|c|l|}
\hline Multiply & By & To Obtain \\
\hline cubic yards & 0.7645549 & cubic meters \\
\hline degrees Fahrenheit & $(\mathrm{F}-32) / 1.8$ & degrees Celsius \\
\hline feet & 0.3048 & meters \\
\hline miles (nautical) & 1,852 & meters \\
\hline miles (U.S. statute) & $1,609.347$ & meters \\
\hline ounces (mass) & 0.02834952 & kilograms \\
\hline pints (U.S. liquid) & 0.473176 & liters \\
\hline
\end{tabular}




\section{Acronyms}

ACR - acute-to-chronic toxicity ratio, used to derive chronic no-effect concentrations

AF - application factor, used to derive chronic no-effect concentrations; or, adult fish (when used in identifying exposure regimes)

APHA - American Public Health Association

CAMD - the J. Bennett J ohnston Sr. Center for Advanced Microstructures and Devices in Baton Rouge, LA

CCC - Criterion Continuous Concentration - chronic water quality criterion (WQC) for the protection of aquatic life

CDF - confined disposal facility - a structure designed to receive sediments dredged from a navigation channel and safely contain the contaminants

d - duration, in days, of the toxicity exposure

DCM - double-crystal monochromator - an optical device that can be used in spectrophotometry

DRET - dredging elutriate test

EC50 - the median effects concentration - represents a modeled concentration that has a biological effect (e.g., growth, reproduction, etc.) on $50 \%$ of the test subjects

EET - effluent elutriate test

EFL - effluent from settling pond (used to identify samples or exposure regimes)

EL - elutriate (used to identify samples or exposure regimes) 
EMR - Emory River (used to identify samples or exposure regimes)

ER - effects residue (see ER50)

ERED - the Environmental Residue Effects Database provided by the U.S. Army Corps of Engineers (http://el.erdc.usace.army.mil/ered/)

ER50 - the median effects residue - represents a modeled tissue residue at which $50 \%$ of the test subjects show a biological effect (e.g., growth, reproduction, etc.)

ERDC - U.S. Army Engineer Research and Development Center

FA - fly ash (used to identify samples or exposure regimes)

GPS - global positioning system

$\mathbf{h}$ - duration, in hours, of the treatment

HDPE - high-density polyethylene, a type of plastic that is hard and opaque

HPLC - high-performance liquid chromatography - analytical method for detecting and quantifying compounds of interest

ICP-AES - inductively coupled plasma atomic emission spectroscopy analytical method for detecting trace metals

ICP-MS - inductively coupled plasma mass spectrometry - analytical method for detecting and quantifying a range of compounds of interest

$\mathbf{k g}$ - kilogram, a unit of mass equal to one thousand grams

L - liter, a unit of volume

LC50 - the lethal median effects concentration - represents a modeled concentration that kills $50 \%$ of the test subjects

LF - larval fish (used to identify exposure regimes) 
LOEC - lowest observable effect concentration - represents the lowest concentration at which an adverse biological effect was observed

LOER- lowest observable effect residue - represents the lowest tissue residue at which an adverse biological effect was observed

LR50 - the lethal median effects residue - represents a modeled tissue residue at which $50 \%$ of the test subjects die

MATC - maximum allowable toxicant concentration - a concentration assumed not to be toxic. This is usually derived as the geometric mean of the NOEC and LOEC and is better suited for application to chronic toxicity test data.

MCL - maximum concentration level - usually applies to drinking water standards

mg - milligram, a unit of mass equal to one thousandth of a gram

MPC - maximum permissible concentration (see MATC)

MS - mass spectrometry, a general analytical method for detecting and quantifying compounds; or, mussel (when used in identifying exposure regimes)

$\mathbf{m V}$ - millivolt, a unit of voltage or electric potential difference equal to one thousandth of a volt

NA - not available

ND - not detected by analytical methods

NEXAFS, C-NEXAFS - near-edge X-ray absorption fine structure, carbon near-edge X-ray fine structure - specific spectroscopic methods for studying compounds of interest

NOEC - no observable effect concentration - represents the highest concentration at which no adverse biological effect was observed 
NOER - no observable effect residue - represents the highest tissue residue at which no adverse biological effect was observed

NSLS - National Synchrotron Light Source at the Brookhaven National Laboratory in Upton, NY

PIL - fly ash pile (used to identify samples or exposure regimes)

QAPP - quality assurance project plan

RF1, RF2, ... RF6 - References 1 through 6, respectively (used to identify samples)

SD - sediment (used to identify samples or exposure regimes)

SET - standard elutriate test

SLC - sluice channel (used to identify samples or exposure regimes)

SSRL - Stanford Synchrotron Radiation Lightsource at the SLAC National Accelerator Laboratory in Menlo Park, CA

TDEC - Tennessee Department of Environment \& Conservation

TEY - total electron yield, response measurement recorded in NEXAFS technique

TIC - total inorganic carbon - the sum of all inorganic carbon species in a solution

TOC - total organic carbon - the sum of all organic carbon species in a solution

TRV - toxicity reference value - a reference exposure level or reference dose used to determine risk to a wildlife receptor, defined for a specific contaminant and species

TSS - total suspended solids, the amount of suspended particulates in a water sample and a measure of water quality 
t-TEL - tissue threshold effect level - a tissue concentration, above which adverse biological effects are seen, but below which no adverse effects are seen

TVA - Tennessee Valley Authority

USACE - U.S. Army Corps of Engineers

USCS - united soil classification system

USEPA - U.S. Environmental Protection Agency

UV - ultraviolet, as in ultraviolet radiation - the section of the electromagnetic spectrum between visible and $\mathrm{X}$-ray radiation

VLSPGM - varied-line-space plane-grating-monochromator - an x-ray focusing element used in NEXAFS speciation

WA - water (used to identify samples or exposure regimes)

WOE - weight of evidence - an approach to inference or decision making based upon the plausibility or believability of the available evidence on all sides of a case

WQ - water quality

WQC - Water quality criterion for the protection of aquatic life as defined by the U.S. Environmental Protection Agency or state water quality agency

XANES, $\boldsymbol{\mu}$-XANES - X-ray absorption near edge structure, micro-X-ray near edge structure - a type of spectroscopy for determining metal species in solids

$\mathbf{X R F}, \boldsymbol{\mu}$-XRF - X-ray fluorescence, micro-X-ray fluorescence - analytical techniques for mapping elements of interest

$\boldsymbol{\mu g}$ - microgram, a unit of mass equal to one millionth of a gram

$\boldsymbol{\mu S} \mathbf{~ c m}^{-1}$ - microsiemens, units of electrical conductivity 


\section{Introduction}

On December 22, 2008 over 5,000,000 yd $\mathrm{yd}^{3}$ of fly ash were released to the Emory River, TN from the Tennessee Valley Authority Kingston Fossil Plant (U.S. Environmental Protection Agency (USEPA) 2009). Remediation and recovery efforts for this event are currently underway. The impact of the release on the river and surrounding area is currently under investigation.

The following results describe the potential effects of fly ash on the geochemistry, water quality, and aquatic biota of the Emory River. The analysis is intended to provide baseline information regarding the potential geochemical and biological impacts from exposure to the fly ash in the Emory River as well as potential impacts of fly ash removal. To address this goal, an elutriate that uses fly ash and waters taken directly from the site was characterized over a 10-day period. The approach used in this study provides significant control over the system and the greatest potential for accurately simulating conditions for contaminant release.

Fly ash was collected from three representative sites: material in its original conditions (Pile), material that has been released into the Emory River, and material that has been dredged and settled in the ash recovery ditch (herein referred to as the Sluice Channel). The sites selected represent the three conditions that may affect the release of metals from the system. In addition, sediment and soils from six sites at least 12 miles upstream of the spill area were used to develop a reference envelope for metals concentrations. Geochemical, physical, and biological properties of the elutriate preparations from the Emory River and sluice channel were determined. The most important physical and geochemical parameters include physical fly-ash characteristics, system oxidation shifts associated with suspension in water, and metal speciation and dissolution into water. These data were used for geochemical modeling of the system. Bioassays were conducted using elutriates prepared from the Emory River and sluice channel fly ash. Two life stages of the fathead minnow, Pimephales promelas, and the early life stage of freshwater mussel, Villosa iris, were used in the elutriate bioassay. 
Table 1. Overview of sample sites and analysis ${ }^{1}$.

\begin{tabular}{|l|l|l|l|l|}
\hline Sample Collection Site & $\begin{array}{l}\text { Chemical and } \\
\text { Physical }\end{array}$ & $\begin{array}{l}\text { Larval Fish } \\
\text { Bioassay }\end{array}$ & $\begin{array}{l}\text { Adult Fish } \\
\text { Bioassay }\end{array}$ & $\begin{array}{l}\text { Mussel } \\
\text { Bioassay }\end{array}$ \\
\hline Original Storage Pile Material & X & & & \\
\hline Emory River Material & X & X & X & X \\
\hline Sluice Channel Material & X & X & & X \\
\hline Emory River Reference & X & & \\
\hline $\begin{array}{l}1 \text { Note that fish histology and mussel bioassay results were conducted by separate laboratories and are } \\
\text { provided as supplemental reports in appendices. }\end{array}$
\end{tabular}

The goals of this analysis, using the data described, are to determine the following:

- Concentration and species of metals released during the suspension and potential oxidation of fly ash. Concentrations will be directly compared to relevant standards as a conservative screening assessment.

- Biological responses to contaminants, in order to evaluate potential toxic effects.

- Time-dependent release of metals to predict the potential for significant metals release from fly ash particles.

- Physical and chemical parameters to estimate contaminant and particle releases to the Emory River. 


\section{Sample Collection}

All field samples were collected on J une 12, 2009 and immediately packed on ice and transported overnight by truck to the US Army Engineer Research and Development Center (ERDC) in Vicksburg, MS. Sample collection data sheets are provided in Appendix A. Sample locations are shown in Figure 1 and coordinates are listed in Table 2. A handheld water quality probe was used to measure $\mathrm{pH}$ and redox potential (Beckman Coulter, [phi] 225, Fullerton, CA, USA) as well as conductivity (EcTestr ${ }^{\mathrm{TM}}$ 11 Plus, OAKTON Instruments, Vernon Hills, IL, USA) at the sampling sites given in Table 3 at the time of water, fly ash, or sediment sampling to provide information on ambient water quality conditions. Fly ash from the Emory River was collected by boat using a polypropylene (U.S. Food and Drug Administration-approved) shovel in areas where the fly ash was submerged by the river at depths less than $1 \mathrm{~m}$. An initial attempt was made to use a Ponar grab sampler for fly ash collection; however, because the submerged fly ash had developed a crust, an acceptable grab sample using this method was not possible. Therefore, samples were collected

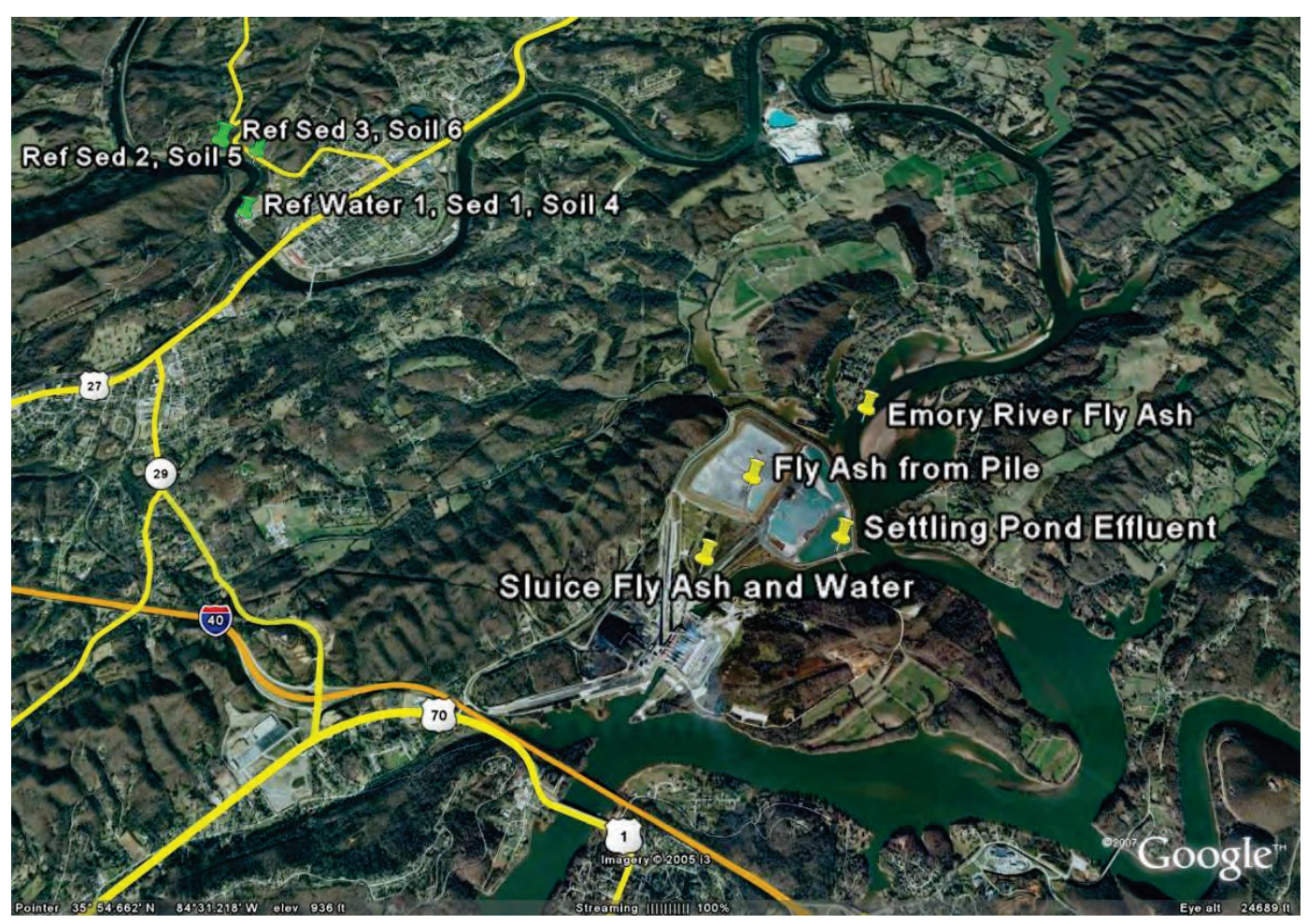

Figure 1. Sampling locations. Ref $=$ Reference water, sediment or soil. Sed $=$ Sediment. Number value is respective reference sample. Map created using Google ${ }^{\mathrm{TM}}$ Earth Enterprise Client (CorpsGlobe). 
Table 2. Sampling station coordinates.

\begin{tabular}{|c|c|c|}
\hline Sample Description & Latitu & / Longitude \\
\hline Emory River Fly Ash - Composited Sample \# 1 & N $35^{\circ} 46.987^{\prime *}$ & W $084^{\circ} 31.511^{\prime *}$ \\
\hline Emory River Fly Ash - Composited Sample \# 2 & N $35^{\circ} 54.877^{\prime}$ & W $084^{\circ} 30.163^{\prime}$ \\
\hline Emory River Fly Ash - Composited Sample \# 3 & 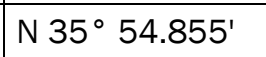 & W $084^{\circ} 30.165^{\prime}$ \\
\hline Emory River Fly Ash - Composited Sample \# 4 & N $35^{\circ} 54.858^{\prime}$ & W $084^{\circ} 30.155^{\prime}$ \\
\hline Emory River Fly Ash - Composited Sample \# 5 & N $35^{\circ} 54.845^{\prime}$ & W $084^{\circ} 30.145^{\prime}$ \\
\hline Reference Water, Reference Sediment 1 & N $35^{\circ} 55.995^{\prime}$ & W $084^{\circ} 33.554^{\prime}$ \\
\hline Reference Sediment 2 & N $35^{\circ} 56.394^{\prime}$ & W $084^{\circ} 33.566^{\prime}$ \\
\hline Reference Sediment 3 & N $35^{\circ} 56.448^{\prime}$ & W $084^{\circ} 33.793^{\prime}$ \\
\hline Reference Soil 4 - Composited Sample \# 1 & N 35 56.009' & W $084^{\circ} 33.560^{\prime}$ \\
\hline Reference Soil 4 - Composited Sample \# 2 & N 35 $56.012^{\prime}$ & W $084^{\circ} 33.571^{\prime}$ \\
\hline Reference Soil 4 - Composited Sample \# 3 & N 35 $56.007^{\prime}$ & W $084^{\circ} 33.565^{\prime}$ \\
\hline Reference Soil \# 5 & N $35^{\circ} 56.394^{\prime}$ & W $084^{\circ} 33.566^{\prime}$ \\
\hline Reference Soil \# 6 & N $35^{\circ} 56.448^{\prime}$ & W $084^{\circ} 33.793^{\prime}$ \\
\hline Sluice Channel Fly Ash & N $35^{\circ} 54.192^{\prime}$ & W $084^{\circ} 31.020^{\prime}$ \\
\hline Sluice Channel Water & N $35^{\circ} 54.192^{\prime}$ & W $084^{\circ} 31.016^{\prime}$ \\
\hline Stilling Pond Effluent (Water) & N $35^{\circ} 54.279^{\prime}$ & W $084^{\circ} 30.363^{\prime}$ \\
\hline Fly Ash from Pile - Composited Sample \# 1 & N 35 54.550' & W $084^{\circ} 30.770^{\prime}$ \\
\hline Fly Ash from Pile - Composited Sample \# 2 & N $35^{\circ} 54.586^{\prime}$ & W $084^{\circ} 30.769^{\prime}$ \\
\hline Fly Ash from Pile - Composited Sample \# 3 & N $35^{\circ} 54.604^{\prime}$ & W $084^{\circ} 30.811^{\prime}$ \\
\hline
\end{tabular}

Table 3. Field WQ measurements.

\begin{tabular}{|l|l|l|l|}
\hline Sampling Site & $\mathbf{p H}$ & Conductivity $\left(\boldsymbol{\mu \mathbf { S ~ c m } ^ { - 1 } )}\right.$ & Redox Potential $(\mathrm{mV})$ \\
\hline Emory River & 6.96 & 120 & 296.0 \\
\hline Reference Site 1 & 6.81 & 90 & 267.8 \\
\hline Reference Site 3 & 6.70 & 90 & 109.8 \\
\hline Sluice Channel & 7.95 & Not Measured & 135.0 \\
\hline Stilling Pond Effluent & 8.59 & 300 & 12.9 \\
\hline
\end{tabular}

using the polypropylene shovel to a depth of at least $15 \mathrm{~cm}$. Samples were taken from five discrete locations (Table 2) approximately 2.2 miles upstream of the confluence of the Emory and Clinch Rivers near N $35^{\circ}$ $54.877^{\prime}, \mathrm{W} 084^{\circ} 30.163^{\prime}$ in an area where ash removal by hydraulic dredging was occurring. These five samples were composited, homogenized by mixing with a polypropylene shovel until visually 
homogenous, and passed through a 1.25-cm sieve to remove any large debris from the samples. The homogenized samples were placed into two 18.9-L plastic buckets.

Reference water for use in preparing elutriates and in toxicity studies was collected from the Emory River at least 12 miles upstream of the Kingston Fossil Plant site (N 35 55.995', W 084 33.554'). This water was collected using an electric pump at approximately $0.75 \mathrm{~m}$ depth at a 1.5-m-deep site located approximately $7.6 \mathrm{~m}$ from the shoreline. The pump and associated hoses were flushed with site water for 15 minutes prior to sampling. Water was held in 208-L high-density polyethylene (HDPE) drums. Bags of ice were packed around the exterior of the drums for transportation to ERDC.

Reference sediment and soil were also collected within $50 \mathrm{~m}$ of the site at which the reference water was collected by HDPE shovel (four discrete samples that were composited) and handheld coring device (three discrete samples that were composited), respectively. Composites were collected using three or four locations rather than the five originally described in the Quality Assurance Project Plan (QAPP) (U.S. Engineer Research and Development Center (ERDC) 2009) due to time constraints. Additional reference sediment and soil composite samples were similarly collected further upstream at the following coordinates: N $35^{\circ} 56.394^{\prime}, \mathrm{W} 084^{\circ}$ $33.566^{\prime}$ and $\mathrm{N} 35^{\circ} 56.448^{\prime}$, W $084^{\circ} 33.793^{\prime}$, for a total of three reference sediment samples and three reference soil samples, all placed in 1-L HDPE bottles.

Fly ash and water samples were taken from the sluice channel (N $35^{\circ}$ 54.192', W $\left.084^{\circ} 31.020^{\prime}\right)$. In previous TVA reports, this part of the channel has been described as the ash ditch. The water was sampled using the same electric pump as used for the reference site water, which was flushed with sluice channel water prior to sample collection. Sluice channel water was collected in 16-L cubitainers and packed in coolers with ice for transportation. Fly ash was sampled from the bottom of the sluice channel at a depth of approximately $3 \mathrm{~m}$, using an excavator, and then placed in an 18.9-L plastic bucket for transportation to ERDC.

Samples of fly ash were also collected from the top of the fly ash pile. Three composite samples were taken using a hand sediment corer at $\mathrm{N} 35^{\circ}$ $54.550^{\prime}$, W $084^{\circ} 30.770^{\prime}, \mathrm{N} 35^{\circ} 54.586^{\prime}$, W $084^{\circ} 30.769^{\prime}$, and N $35^{\circ}$ $54.604^{\prime}, \mathrm{W} 084^{\circ} 30.811^{\prime}$. Three composite samples were taken instead of 
the five listed in the QAPP due to difficulty in penetrating the pile's surface with the sediment corer and time limitations. At each sampling site, the upper $5 \mathrm{~cm}$ of substrate was scraped away prior to sampling to remove the stabilizer added to the pile, and sediment cores were taken at a depth of $0.5 \mathrm{~m}$. These samples were composited, homogenized, and placed into three 1-L HDPE bottles. Water samples of the effluent of the stilling pond were taken just prior to entry into the weir leading to the Emory River ( N $35^{\circ} 54.279^{\prime}, \mathrm{W} 084^{\circ} 30.363^{\prime}$ ). These were grab samples, taken just under the water's surface. Two replicate grab samples were taken in 1-L HDPE bottles. 


\section{Extended Elutriate Preparation}

Environmental concerns for dredging projects are often directed toward releases of contaminants from the sediment to the receiving waters at the point of dredging and at the point of disposal. Compliance with water quality standards and protection of aquatic organisms are frequently issues for dredging projects, particularly when the sediments are contaminated. Dredging releases are related to the dredge head's mechanical movement and cutting action, which propel sediment particles and interstitial water into the water column and generate a plume of suspended sediment and associated contaminants. Contaminant releases may occur during discharge of sediment from a dredge pipeline into the water column, emptying a barge or hopper of sediment into the water column, or effluent from a confined placement site following sedimentation to remove most of the solids.

The US Army Corps of Engineers has developed laboratory elutriate tests to estimate contaminant releases from each of these pathways. The Upland Testing Manual (USACE 2003) defines elutriate as "A sample generated by washing contaminants from a sediment sample using water, usually by mixing water with the sediment, allowing the sediment to settle, and extracting the sample from the overlying water." Elutriate procedures have been developed to simulate the point of dredging, open water disposal, and confined placement or disposal facility effluent. Sediment elutriates may also be prepared for biological effects testing. The primary differences in the tests are associated with mixing time, settling time, and solids-to-water ratio or solids concentration as shown in Table 4 . The tests are listed in chronological order of their development.

The two pathways of concern for the Kingston Dredging Operations are releases at the dredging site and effluent from the sluice and stilling pond system. Consequently, the EET and the DRET procedures should most closely replicate the dredging operations currently associated with cleanup operations. However, due to specific information available on total solids loads in the sluice channel, the laboratory protocols were modified for this study, as well as to investigate the effects of longer-term aeration and oxidation on metal speciation and dissolved metal releases to the water column. One of the specific concerns raised for the dredging of 
Table 4. Elutriate Test Conditions.

\begin{tabular}{|c|c|c|c|c|c|}
\hline Test Name & Purpose & Solids:Water & Mixing Time & Settling Time & Reference \\
\hline $\begin{array}{l}\text { Standard } \\
\text { Elutriate Test } \\
\text { (SET) }\end{array}$ & $\begin{array}{l}\text { Open water disposal } \\
\text { (dissolved } \\
\text { contaminants) }\end{array}$ & $\begin{array}{l}1 \text { part sediment to } \\
4 \text { parts water by } \\
\text { volume }\end{array}$ & $0.5 \mathrm{hr}$ & $1.0 \mathrm{hr}$ & $\begin{array}{l}\text { USEPA/ USACE } \\
\text { (1998) }\end{array}$ \\
\hline $\begin{array}{l}\text { Effluent } \\
\text { Elutriate Test } \\
\text { (EET) } \\
\text { (formerly } \\
\text { named } \\
\text { Modified } \\
\text { Elutriate Test) }\end{array}$ & \begin{tabular}{l|} 
Confined disposal \\
facility (CDF) effluent \\
(particulate and \\
dissolved contaminants)
\end{tabular} & $\begin{array}{l}\text { CDF influent } \\
\text { concentration } \\
\text { Default: } 150 \text { g dry } \\
\text { sediment solids per } \\
\text { liter elutriate }\end{array}$ & $1.0 \mathrm{hr}$ & $\begin{array}{l}\text { CDF retention } \\
\text { time } \\
\text { Default: } 24 \mathrm{hr}\end{array}$ & USACE (2003) \\
\hline $\begin{array}{l}\text { Dredging } \\
\text { Elutriate Test } \\
\text { (DRET) }\end{array}$ & $\begin{array}{l}\text { Point of dredging } \\
\text { (particulate and } \\
\text { dissolved contaminants) }\end{array}$ & $\begin{array}{l}1 \text { to } 10 \mathrm{~g} \text { dry } \\
\text { sediment solids per } \\
\text { liter elutriate }\end{array}$ & $1.0 \mathrm{hr}$ & $1.0 \mathrm{hr}$ & $\begin{array}{l}\text { Palermo et al. } \\
\text { (2008) }\end{array}$ \\
\hline
\end{tabular}

fly ash is whether or not introduction of oxygenated water into the fly ash slurry will result in oxidation and release of metals, particularly selenium. Therefore, the elutriate procedure developed for the current work uses an extended aeration time, far in excess of aeration times occurring in the dredging operation to answer the question of metals release once exposed to oxic conditions.

For the current investigations, the elutriate tests were created following slight modifications of the DRET procedure outlined above. Fly ash samples were homogenized in the laboratory prior to subsampling from sample containers. Specifically, $10 \mathrm{~g}$ (wet weight) of fly ash material was added per liter of Emory River or sluice channel water to create the elutriate mixture. The Emory River fly ash sample was 53\% solid material, and therefore, the elutriate sediment load is calculated to be approximately $5.3 \mathrm{~g} / \mathrm{L}$. The sluice channel fly ash sample was $73 \%$ solid material, and therefore the elutriate sediment load is calculated to be approximately $8 \mathrm{~g} / \mathrm{L}$ (accounting for the total solids of the sluice channel water of $\sim 0.7 \mathrm{~g} / \mathrm{L}$ ). These loading values are in line with historical solids loads measured during dredging operations at the site. Monitoring data available from TVA indicates TSS at this location ranged from 69 to 17,100 $\mathrm{mg} / \mathrm{L}$ with an average value of $5,000 \mathrm{mg} / \mathrm{L}$, and median value of $606 \mathrm{mg} / \mathrm{L} .{ }^{1}$ Additionally, all elutriate experiments were performed in triplicate. Furthermore, to test the effect of redox potential on the release and speciation of metals from the fly ash, two redox systems were tested, one oxic, created by bubbling the elutriate mixture with humidified air, and one anoxic, created by bubbling the

1 Personal Communication. July 23, 2009. M. Houck, Engineer, Jacobs Engineering, Oak Ridge, TN. 
elutriate mixture with humidified nitrogen, for 10-day experiments. Increased water vapor in the air and nitrogen used for bubbling the elutriates was needed to prevent evaporative concentration of the elutriates over the 10- day experiment. The nitrogen bubbling treatment did not result in completely anoxic conditions. The two redox systems will therefore be referred to as the anoxic regime (nitrogen-bubbled) and the oxic regime (air-bubbled).

Elutriates for the sluice channel oxic and anoxic regime experiments were prepared in 19-L HDPE containers. Emory River anoxic regime elutriates were also prepared in 19-L HDPE containers. However, due to the volume of water needed for the biological tests, Emory River oxic regime elutriates were prepared in 200-L HDPE drums. All elutriate preparations were performed in a $20^{\circ} \mathrm{C}$ secured environmental chamber.

Aliquot samples (1-L) were collected from the elutriate preparation containers at 1-, 24-, 48-, 96-, and 240-hr timepoints, allowed to settle for $1 \mathrm{hr}$ following the standard DRET procedure, and the supernatant analyzed for dissolved and total metals, arsenic and selenium speciation, and total organic carbon (USEPA 2007, Bednar et al. 2004, 2009). Redox potential (Eh) and pH were also measured at each timepoint in the bulk elutriate containers prior to collection of the 1-L aliquot. Data sheets for the elutriate preparations with associated water quality data are found in Appendix B. 


\section{Physical and Chemical Characterization of Fly Ash}

Different techniques were used to determine the physical and chemical properties of the fly ash. The purpose was to gain detailed information about the composition and potential changes in the physical and chemical properties of the ash from the original source pile, to the Emory River, and eventually to the sluice channel following dredging. In addition, reference sediment and soil samples were collected at various points upstream of the spill and similarly intensely characterized for comparison.

\section{Methods}

Solid phase concentrations of metals in the fly ash and reference materials were determined by Inductively Coupled Plasma Atomic Emission Spectroscopy and Inductively Coupled Plasma Mass Spectrometry following USEPA methods 6010C (ICP-AES) and 6020A (ICP-MS), using a Perkin Elmer Optima 5300DV and Elan DRC-II, respectively. The solids were first dried, ground to pass a \#40 ASTM sieve, and acid digested following USEPA method 3050B. The grinding step, though not specified in method 3050B, is a commonly used procedure that has been found to increase analytical precision (Felt et al. 2008). The digestate was filtered through a \#41 Whatman filter and diluted to $50 \mathrm{~mL}$ with $1 \%$ nitric acid. This digestate was further diluted with $1 \%$ nitric acid prior to ICP-AES and ICP-MS analysis as needed, such that the analyte concentrations were within the instrument calibration range. Mercury was determined using cold vapor atomic fluorescence using a PS Analytical Atomic Fluorescence Instrument following USEPA method 7474. Dissolved organic and inorganic carbon was determined using a Shidmadzu TOC-V analyzer using a catalytic combustion technique following USEPA method 9060. (For all methods, see USEPA (2007).)

Factor analysis was conducted using the total elemental data and the StatisiXL statistical software package, as described by Mathangwane et al. (2008). Analysis was performed using a Varimax rotation to avoid data order biases. Number of factors was determined using a Scree test criterion, in which factors with eigenvalues $<1$ were ignored. 
Known arsenic, selenium, and chromium species were analyzed as standards to aid in the identification of unknown species within the Kingston site test samples. These standards were analyzed at beamlines 11-2 at the Stanford Synchrotron Radiation Lightsource (SSRL) at the SLAC National Accelerator Laboratory in Menlo Park, CA and at the Double Crystal Monochromator line at J . Bennett J ohnston, Sr. Center for Advanced Microstructures and Devices (CAMD) in Baton Rouge, LA. Standards were also tested alongside the experimental spectra at beamlines 2-3 at the SSRL and X27A at the National Synchrotron Light Source (NSLS) at the Brookhaven National Laboratory in Upton, NewYork. Arsenic was calibrated to $11874 \mathrm{eV}$ using an inline arsenate [As(V)] standard, $\mathrm{Ca} 3 \mathrm{As}\left(\mathrm{O}_{4}\right)_{2}$. Selenium was calibrated using an $\mathrm{Se}(0)$ foil, selenite [Se(IV)], and selenate [Se(VI)] standards were analyzed periodically to ensure beamline calibration. Chromium calibration was tested using $\mathrm{Cr}$ (III) and $\mathrm{Cr}(\mathrm{VI})$ salt standards.

Experimental X-ray absorption near edge structure (XANES) spectroscopy was conducted at the microprobe beamlines X27A at the NSLS and 2-3 at the SSRL, and bulk experimental XANES were collected at beamline 11-2 at the SSRL. The fly ash was desiccated under oxygen (source pile; Emory River and sluice elutriates for $240 \mathrm{hr}$ treated with oxic regime) or nitrogen conditions (sluice and Emory River sediments; Emory River and sluice elutriates for $240 \mathrm{hr}$ treated with anoxic regime) and ground to establish a homogeneous sample. Samples were dried on Mylar film in order to establish a single/ thin layer on the film. The Mylar film was analyzed for total metal(loid) content to minimize contamination of the arsenic, chromium, and selenium signals. Samples were prepared directly before arrival at the synchrotron facilities. The Mylar film was used to minimize the effects of beam-induced species reduction.

After collection on beamline X27A, the XANES data and X-ray fluorescence (XRF) maps were analyzed using the X27A Plot program. The $\mathrm{X} 27 \mathrm{~A}$ Plot was also used in the formation of XRF maps and correlation plots. The XRF maps collected on beamline 2-3 were analyzed using the SMAK microtoolkit. All XANES analysis and metalloid speciation was determined using WinXAS 3.1 and Athena 0.8.051 (Newville 2001, Ressler 1998). Arsenic and selenium speciation was determined by comparing the whiteline and derivative values of the experimental and standard spectra. Where possible, linear combination fitting (LCF) was performed using Athena 0.8.051 (Newville 2001) and Six Pack's Linear Least Squares 
Fitting (Webb 2005). An error of about 5-10\% is associated with LCF results (Manceau et al. 2002).

Iron and manganese XANES analysis was conducted on samples at the double-crystal monochromater (DCM) beamline at CAMD. Iron and Mn analysis was calibrated using their respective metallic foils. Samples were analyzed in fluorescence mode using a 13-element solid-state detector. XANES data were processed and analyzed as described previously.

Composition of the carbon, nitrogen, and oxygen functional group was investigated by near-edge X-ray absorption spectroscopy (NEXAFS). Measurements were carried out at the varied-line-space plane-gratingmonochromator (VLSPGM) beamline at CAMD. Drops of concentrated suspensions (100 mg in $1 \mathrm{~mL}$ water) of fly ash samples were deposited on $\mathrm{Au}$-coated silicon wafers, and dried in a dessicator at room temperature to make a thin film of sample. These samples were mounted to a stainlesssteel plate and placed into the beamline sample chamber. The pressure at the sample chamber during the measurements was $\sim 3 \times 10^{-9}$ torr, which was obtained after baking (at $\sim 100^{\circ} \mathrm{C}$ ) the chamber overnight. NEXAFS measurements were performed by acquiring the sample current (total electron yield detection). The total electron yield (TEY) NEXAFS spectroscopy experiments were carried out on the VLSPGM beamline with resolution better than $0.2 \mathrm{eV}$ at the Cls edge. The photon energy scale was calibrated for the $\mathrm{C} 1 \mathrm{~s}-\pi^{*}$ resonance peak using a polystyrene sample (Sigma-Aldrich), which was fixed at $285.4 \mathrm{eV}$. Sample spectra were $\mathrm{I}_{0}$ normalized using the total yield of clean gold mesh placed in the incident beam before sample. The incident beam intensity was monitored concurrently by a gold mesh and used to correct the measured spectra. C-NEXAFS spectra were processed using the program Athena from the IFEFFIT software package (Newville 2001). Linear combination fits of the C-NEXAFS spectra were compared to carbon reference standards also analyzed at VLSPGM beamline.

A sequential extraction procedure was also performed on the fly ash materials as a companion analysis to the synchrotron-based speciation analysis. The sequential extraction targets specific geochemical phases (soluble, exchangeable, carbonate, metal oxide, organic matter/ sulfide, and residual) to determine what metals are present in each operationally defined phase. This analysis is useful for predicting under what type of geochemical environment metals likely will become mobile (e.g. acid 
environments dissolving carbonate-bound elements). The method used was modified from published procedures (Tessier et al. 1979).

Total and dissolved metals in the site waters were determined using Inductively Coupled Plasma Atomic Emission Spectroscopy and Mass Spectrometry (ICP-AES and ICP-MS), following EPA Methods 6010C and 6020A, using a Perkin Elmer Optima 5300DV and Elan DRC-II, respectively (USEPA 2007). Scandium was used as the internal standard during ICP-AES analysis, and rhodium, terbium, and holmium were used as internal standard during ICP-MS analysis to correct for instrumental drift. Oxygen and methane were used as the reaction cell gases for certain elements and isotopes to improve accuracy through removal of polyatomic interferences using the Dynamic Reaction Cell ICP-MS. Arsenic (III) and (V) and selenium (IV) and (VI) were determined using recently developed HPLC-ICP-MS techniques, which use anion exchange to separate the species of interest with elemental specific detection at the $\mu \mathrm{g} / \mathrm{L}$ level using the ICP-MS (Bednar et al. 2004, 2009).

\section{Results}

\section{Total elemental analysis}

Table 5 below lists the concentrations of metals and total organic and inorganic carbon measured in the fly ash samples collected from the Kingston TVA sites. The concentrations of elements measured in the three fly ash samples are elevated compared to the six reference soils and sediments collected from the Emory River area, upstream of the Kingston Fossil Plant. The concentrations of metals measured in the reference materials are listed in Table 6. In general, metals concentrations in the fly ash samples are elevated compared to the six reference soils and sediments, in some cases by an order of magnitude or more (e.g. arsenic, barium, copper, molybdenum, selenium, thallium, and vanadium). Arsenic, barium, selenium, molybdenum and vanadium are noted to be in soluble forms of the fly ash material as described below in the geochemical description of the fly ash, as well as observed to dissolve into the elutriate samples, as well as being measured in the field-collected waters. 
Table 5. Concentrations $(\mathrm{mg} / \mathrm{kg}$ ) of metals in fly ash samples from TVA Kingston Fossil Plant.

\begin{tabular}{|c|c|c|c|}
\hline \multirow[b]{3}{*}{ Analyte } & \multicolumn{3}{|c|}{ Fly Ash Sampling Location } \\
\hline & Emory River & Sluice Channel & Storage Pile \\
\hline & \multicolumn{3}{|c|}{ Concentration $(\mathrm{mg} / \mathrm{kg})$} \\
\hline TOC 1 & 44930 & 16780 & 51840 \\
\hline $\mathrm{TIC}^{2}$ & 170 & 370 & 120 \\
\hline Aluminum & 30600 & 29900 & 18600 \\
\hline Antimony & 0.233 & 0.212 & 0.145 \\
\hline Arsenic & 65.8 & 76.2 & 46.9 \\
\hline Barium & 655 & 515 & 510 \\
\hline Beryllium & 7.15 & 6.03 & 4.88 \\
\hline Cadmium & 0.576 & 0.631 & 0.272 \\
\hline Calcium & 9090 & 6510 & 6780 \\
\hline Chromium & 48.7 & 49.2 & 33.5 \\
\hline Cobalt & 25.6 & 23.1 & 16.9 \\
\hline Copper & 84.1 & 76.4 & 50 \\
\hline Iron & 17700 & 22200 & 17500 \\
\hline Lead & 36.4 & 33.1 & 20.2 \\
\hline Magnesium & 2780 & 2380 & 1940 \\
\hline Manganese & 153 & 187 & 88.7 \\
\hline Mercury & 0.0732 & 0.0294 & 0.0279 \\
\hline Molybdenum & 4.04 & 4.23 & 3.2 \\
\hline Potassium & 4690 & 4890 & 2760 \\
\hline Nickel & 52.6 & 49.9 & 34 \\
\hline Selenium & 6.97 & 6.08 & 5.44 \\
\hline Sodium & 709 & 608 & 482 \\
\hline Silver & 0.426 & 0.335 & 0.22 \\
\hline Thallium & 2.19 & 2.04 & 1.35 \\
\hline Vanadium & 133 & 124 & 86.2 \\
\hline Zinc & 77.4 & 72.1 & 41.5 \\
\hline $\begin{array}{l}1 \text { Total organic } \\
{ }^{2} \text { Total inorgar }\end{array}$ & & & \\
\hline
\end{tabular}


Table 6. Concentrations $(\mathrm{mg} / \mathrm{kg})$ of metals in reference samples upstream from TVA Kingston Fossil Plant.

\begin{tabular}{|c|c|c|c|c|c|c|}
\hline Sample & RF-1 (Sed) & RF-2 (Sed) & RF-3 (Sed) & RF-4 (Soil) & RF-5 (Soil) & RF-6 (Soil) \\
\hline Analyte & \multicolumn{6}{|c|}{ Concentration (mg/kg) } \\
\hline TOC & 18920 & 19370 & 3490 & 27400 & 69560 & 41850 \\
\hline TIC & $<0.1$ & $<0.1$ & 260 & 760 & 4930 & 1480 \\
\hline Aluminum & 4650 & 4950 & 4040 & 8560 & 15100 & 6070 \\
\hline Antimony & 0.0262 & 0.292 & 0.0162 & 0.0201 & 0.05 & 0.0236 \\
\hline Arsenic & 1.71 & 2.69 & 1.91 & 2.42 & 10.9 & 3.15 \\
\hline Barium & 48 & 73.5 & 33.6 & 100 & 118 & 56 \\
\hline Beryllium & 0.584 & 0.528 & 0.496 & 0.87 & 1.51 & 0.707 \\
\hline Cadmium & 0.0991 & 0.111 & 0.0971 & 0.248 & 0.208 & 0.117 \\
\hline Calcium & 1800 & 2440 & 6150 & 6860 & 14200 & 7320 \\
\hline Chromium & 8.25 & 8.33 & 9.11 & 13 & 27.9 & 11.1 \\
\hline Cobalt & 7.01 & 6.16 & 6.27 & 8.76 & 9.92 & 8.41 \\
\hline Copper & 5.58 & 5.33 & 5.49 & 10 & 24 & 7.59 \\
\hline Iron & 7990 & 10600 & 8670 & 10900 & 39600 & 10900 \\
\hline Lead & 6.68 & 9.06 & 9.33 & 16.8 & 45.3 & 12.9 \\
\hline Magnesium & 674 & 566 & 1130 & 1700 & 2070 & 1610 \\
\hline Manganese & 313 & 233 & 209 & 724 & 518 & 453 \\
\hline Mercury & 0.0211 & 0.0239 & 0.0110 & 0.0395 & 0.0875 & 0.0221 \\
\hline Molybdenum & 0.255 & 0.273 & 0.224 & 0.28 & 1.03 & 0.284 \\
\hline Potassium & 760 & 633 & 782 & 1080 & 1910 & 1060 \\
\hline Nickel & 11.5 & 9.96 & 12.4 & 15 & 16.9 & 14.8 \\
\hline Selenium & 0.247 & 0.271 & 0.114 & 0.757 & 1.48 & 0.473 \\
\hline Sodium & 52 & 66.1 & 48.3 & 49.9 & 182 & 54.1 \\
\hline Silver & 0.0593 & 0.078 & 0.0805 & 0.104 & 0.172 & 0.0667 \\
\hline Thallium & 0.0776 & 0.103 & 0.0595 & 0.131 & 0.297 & 0.0947 \\
\hline Vanadium & 9.16 & 9.95 & 8.33 & 15.6 & 33.8 & 12.8 \\
\hline Zinc & 35 & 31.9 & 61.2 & 63.1 & 64.8 & 50.5 \\
\hline
\end{tabular}

Multi-linear regression analysis was limited in showing relationships among ash constituents, so multivariate factor analysis (using the Scree test criterion) was employed to show the basic structure of the data through interdependent relationships of the ash constituents (Mathangwane et al. 2008). The factor analysis of the ash was limited by the size of the data set, 
but still allowed for some structural determination in the ash data between two different factors (Table 7). In general, hard cations were highly loaded with Factor 1 (except potassium, but significant as loading $>0.5$ ), while remaining soft cations (i.e., metals) were highly loaded in Factor 2, with the exception of silver and selenium. Some metals were highly loaded in both factors; differences would be discriminated with a larger data set. Note that arsenic and manganese are both highly loaded on Factor 2 while selenium and vanadium are significantly loaded in Factor 1. Note also that TOC is highly loaded in Factor 1 while TIC is loaded in Factor 2.

Table 7. Varimax-rotated factor loadings for elemental quantities from Tables 5 and 6 . The number of factors was determined using the Scree test criterion.

\begin{tabular}{|c|c|c|c|c|c|}
\hline \multicolumn{3}{|c|}{ Ash samples } & \multicolumn{3}{|c|}{ Ash samples and reference soils/sediments } \\
\hline Variable & Factor 1 & Factor 2 & Factor 1 & Factor 2 & Factor 3 \\
\hline TOC & -0.938 & -0.347 & 0.111 & -0.824 & -0.081 \\
\hline TIC & 0.207 & -0.978 & -0.289 & -0.950 & 0.008 \\
\hline $\mathrm{Al}$ & 0.848 & -0.529 & 0.955 & -0.281 & -0.029 \\
\hline $\mathrm{Ca}$ & -0.077 & -0.997 & 0.190 & -0.930 & 0.124 \\
\hline $\mathrm{Fe}$ & 0.876 & 0.483 & 0.231 & -0.907 & -0.138 \\
\hline Mg & 0.539 & -0.842 & 0.768 & -0.513 & 0.223 \\
\hline $\mathrm{K}$ & 0.913 & -0.408 & 0.971 & -0.195 & -0.059 \\
\hline $\mathrm{Na}$ & 0.569 & -0.823 & -0.003 & 0.049 & 0.980 \\
\hline Sn & 0.741 & -0.671 & -0.136 & 0.072 & 0.965 \\
\hline $\mathrm{Ba}$ & 0.048 & -0.999 & 0.970 & -0.064 & -0.102 \\
\hline $\mathrm{Be}$ & 0.522 & -0.853 & 0.984 & -0.098 & -0.107 \\
\hline $\mathrm{Cd}$ & 0.935 & -0.355 & 0.957 & -0.127 & 0.139 \\
\hline $\mathrm{Mn}$ & 0.988 & -0.158 & -0.518 & -0.361 & 0.737 \\
\hline $\mathrm{Hg}$ & 0.047 & -0.999 & 0.282 & -0.862 & 0.116 \\
\hline $\mathrm{Cr}$ & 0.860 & -0.510 & 0.939 & -0.326 & -0.081 \\
\hline Co & 0.705 & -0.709 & 0.986 & -0.129 & -0.047 \\
\hline $\mathrm{Cu}$ & 0.750 & -0.661 & 0.982 & -0.164 & -0.091 \\
\hline $\mathrm{Pb}$ & 0.765 & -0.644 & 0.560 & -0.811 & 0.019 \\
\hline As & 0.992 & -0.128 & 0.982 & -0.052 & -0.130 \\
\hline Mo & 0.804 & -0.595 & 0.980 & -0.108 & -0.145 \\
\hline $\mathrm{Ni}$ & 0.802 & -0.598 & 0.992 & -0.087 & -0.051 \\
\hline $\mathrm{Ag}$ & 0.572 & -0.820 & 0.946 & -0.223 & -0.034 \\
\hline Th & 0.781 & -0.624 & 0.992 & -0.058 & -0.108 \\
\hline $\mathrm{V}$ & 0.773 & -0.635 & 0.986 & -0.128 & -0.101 \\
\hline $\mathrm{Zn}$ & 0.800 & -0.599 & 0.584 & -0.441 & 0.396 \\
\hline Se & 0.433 & -0.902 & 0.974 & -0.112 & -0.108 \\
\hline
\end{tabular}


The interplay between these two factors describing ash constituents is shown in Figure 2. Most of the ash constituents consistently showed up in the fourth quadrant of the plot (determined by dotted lines) suggesting similar interdependencies in the materials. Note that TOC is positioned nearly opposite of iron, manganese, and arsenic, suggesting a negative correspondence among these components. In addition, Figure 2 shows the relationship of the case-wise factor scores for the different ash samples. The fact that samples are positioned in different quadrants suggests that the ash composition differs to some extent as ash is transported from the source pile, to the Emory River, and finally to the sluice channel. Figure 3 shows the combined factor analysis for the ash samples to the reference soils and sediments. Compared to the reference materials, the Emory River and sluice channel ash samples factor out similarly, while the source pile ash shows similar compositional interrelationships to reference river sediment, RF2.

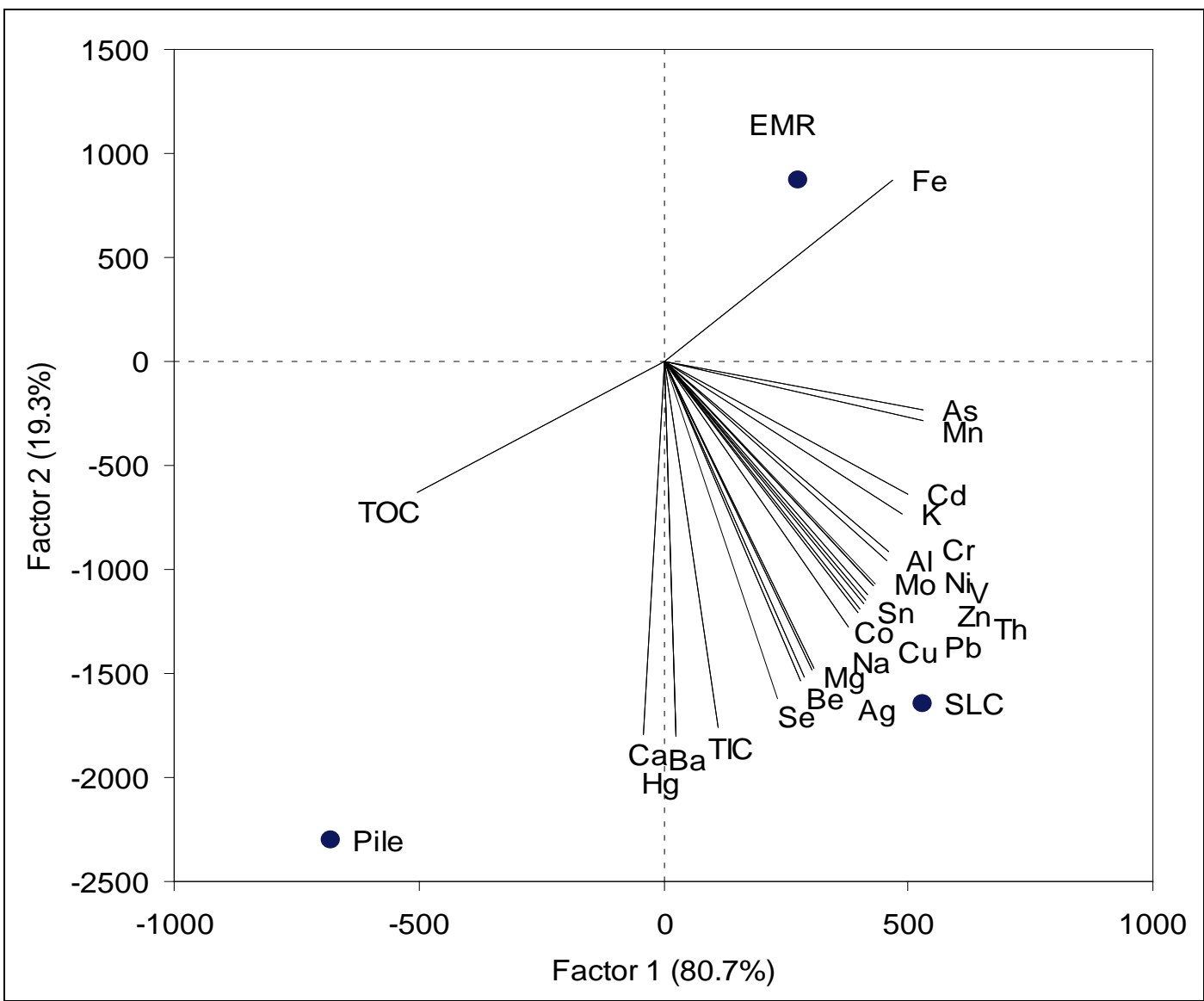

Figure 2. Factor loading plot demonstrating interdependent structure in the two factors from principal factor analysis of the solid-phase constituents from the ash samples collected at the original source pile (Pile), the Emory River (EMR), and the sluice channel (SLC). Data points are case-wise factor scores while solid lines represent the element eigenvectors. Values in parentheses represent the percent variance explained by each factor. 


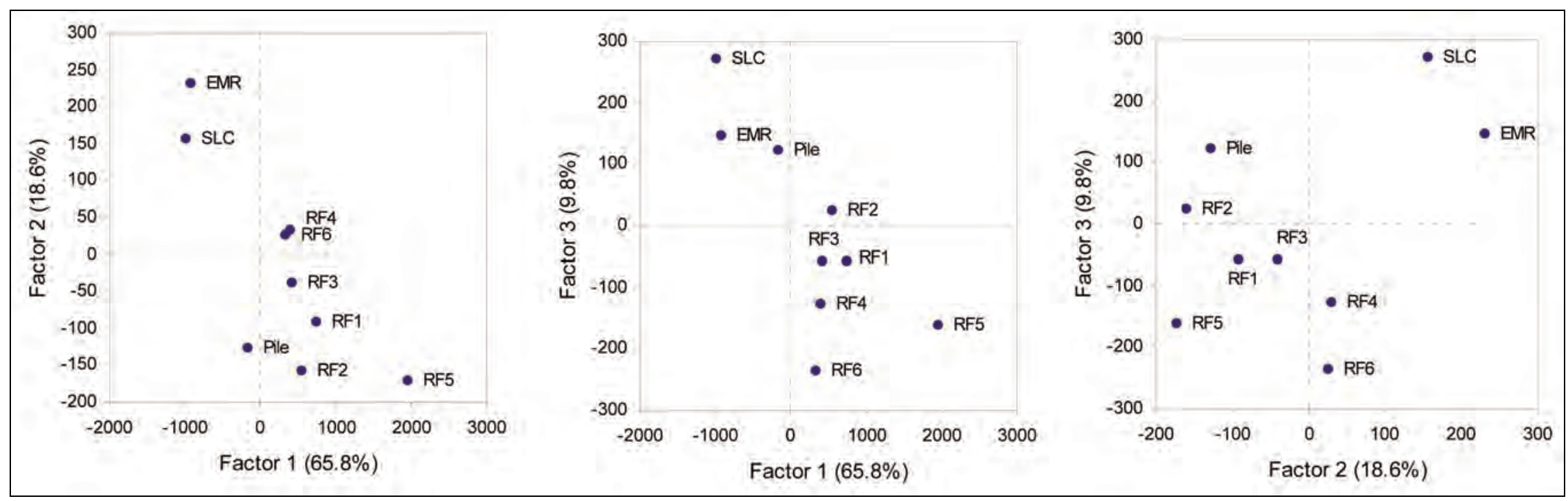

Figure 3. Plots showing the distribution of case-wise factor scores for reference soil and sediment (RF1 - 6) samples relative to the ash samples with respect to factor loading. Details of the plot are described in the caption of Figure 2. 


\section{Ash metal speciation: Sequential extraction analysis}

The sequential extraction procedure is designed to represent the potential for removing the contaminant with increasingly more "aggressive" solvents (going from the left to the right sides of Tables 8-10). Contaminants removed in the early steps of the sequential extraction suggest the contaminants (soluble and exchangeable phases) are readily dissolved from the surface. Contaminants removed in the later stages represent the more residual phases, or nonextractable phases, and are recalcitrant and difficult to remove from the system.

The concentrations of metals in each of the geochemical phases obtained from the sequential extraction procedure are listed in Tables 8-10 for the three fly ash samples tested. Results of the sequential extraction analysis suggest several elemental associations present in the fly ash materials. Specifically, the extraction simulating metal oxide phase association was enriched in arsenic, suggesting a possible association with iron and manganese oxide phases in the ash. High amounts of arsenic and iron

Table 8. Partitioning of Emory River fly ash metals into six geochemical phases.

\begin{tabular}{|l|l|l|l|l|l|l|l|}
\hline Phase & Soluble & Exchangeable & Carbonate & Oxide & Organic/Sulfide & Residual \\
\hline \multicolumn{7}{|c|}{ Metal Concentration (mg/kg) } \\
\hline Arsenic & 2.4 & 1.8 & 11.8 & 32.7 & 4.8 & 42.6 \\
\hline Barium & 0.3 & 35.5 & 68.2 & 110.5 & 44.0 & 204.3 \\
\hline Beryllium & $<0.1$ & $<1$ & 0.3 & 1.0 & 0.3 & 2.8 \\
\hline Cobalt & $<0.1$ & $<1$ & 0.3 & 1.8 & 0.5 & 9.8 \\
\hline Chromium & $<0.1$ & $<1$ & 3.3 & 11.9 & 3.1 & 17.8 \\
\hline Copper & $<0.1$ & $<1$ & 6.1 & 2.1 & 13.3 & 31.7 \\
\hline Iron & 0.1 & 1.1 & 110.0 & 1086.5 & 63.3 & 5457.8 \\
\hline Manganese & $<0.1$ & 3.4 & 19.6 & 24.9 & 3.4 & 34.8 \\
\hline Mercury & $<0.008$ & $<0.008$ & 0.016 & 0.018 & $<0.008$ & 0.022 \\
\hline Molybdenum & 0.2 & $<1$ & 0.2 & 1.4 & 0.5 & 1.6 \\
\hline Nickel & $<0.1$ & $<1$ & 0.7 & 3.1 & 1.1 & 19.9 \\
\hline Lead & $<0.1$ & $<1$ & 0.4 & 1.4 & 0.6 & 16.2 \\
\hline Antimony & $<0.1$ & $<1$ & 0.3 & 0.4 & 0.3 & 0.1 \\
\hline Selenium & 0.4 & $<1$ & 0.7 & 1.2 & 4.0 & 2.5 \\
\hline Thallium & $<0.1$ & $<1$ & 0.2 & 0.6 & 0.3 & 0.6 \\
\hline Vanadium & 1.2 & $<1$ & 4.0 & 38.1 & 8.2 & 41.1 \\
\hline Zinc & 0.2 & 1.7 & 2.5 & 7.3 & 2.6 & 30.2 \\
\hline
\end{tabular}


Table 9. Partitioning of storage pile fly ash metals into six geochemical phases.

\begin{tabular}{|c|c|c|c|c|c|c|}
\hline Phase & Soluble & Exchangeable & Carbonate & Oxide & Organic/Sulfide & Residual \\
\hline \multicolumn{7}{|c|}{ Metal Concentration $(\mathrm{mg} / \mathrm{kg})$} \\
\hline Arsenic & $<0.1$ & $<1$ & 4.9 & 9.4 & 1.9 & 33.7 \\
\hline Barium & 1.4 & 67.1 & 77.2 & 76.8 & 14.2 & 190.2 \\
\hline Beryllium & $<0.1$ & $<1$ & 0.4 & 0.9 & 0.3 & 1.5 \\
\hline Cobalt & $<0.1$ & $<1$ & 0.3 & 1.9 & 0.6 & 5.4 \\
\hline Chromium & $<0.1$ & $<1$ & 3.1 & 9.0 & 2.2 & 11.3 \\
\hline Copper & $<0.1$ & $<1$ & 3.8 & 0.4 & 14.5 & 19.0 \\
\hline Iron & $<0.1$ & 1.4 & 165.1 & 1212.8 & 152.3 & 5225.3 \\
\hline Manganese & $<0.1$ & $<1$ & 5.7 & 24.4 & 3.7 & 25.9 \\
\hline Mercury & $<0.008$ & $<0.008$ & 0.008 & 0.010 & $<0.008$ & 0.014 \\
\hline Molybdenum & 0.1 & $<1$ & $<0.2$ & 1.0 & $<0.2$ & 1.4 \\
\hline Nickel & $<0.1$ & $<1$ & 0.7 & 2.9 & 1.3 & 10.7 \\
\hline Lead & $<0.1$ & $<1$ & 0.3 & 0.8 & 0.2 & 10.2 \\
\hline Antimony & $<0.1$ & $<1$ & 0.2 & 0.2 & $<0.2$ & 0.1 \\
\hline Selenium & 0.1 & $<1$ & 0.7 & 1.4 & 2.4 & 2.1 \\
\hline Thallium & $<0.1$ & $<1$ & 0.2 & 0.4 & $<0.2$ & 0.4 \\
\hline Vanadium & $<0.1$ & $<1$ & 2.1 & 32.9 & 3.1 & 29.0 \\
\hline Zinc & 0.2 & 1.9 & 2.2 & 5.7 & 2.7 & 15.1 \\
\hline
\end{tabular}

Table 10. Partitioning of Sluice Channel fly ash metals into six geochemical phases.

\begin{tabular}{|l|l|l|l|l|l|l|l|}
\hline Phase & Soluble & Exchangeable & Carbonate & Oxide & Organic/Sulfide & Residual \\
\hline \multicolumn{7}{|l|}{ Metal Concentration $(\mathrm{mg} / \mathrm{kg})$} \\
\hline Arsenic & 2.2 & 1.2 & 11.7 & 38.9 & 3.2 & 41.5 \\
\hline Barium & 0.3 & 24.8 & 50.4 & 79.9 & 19.3 & 140.9 \\
\hline Beryllium & $<0.1$ & $<1$ & 0.2 & 0.7 & 0.3 & 1.9 \\
\hline Cobalt & $<0.1$ & $<1$ & 0.4 & 1.7 & 0.7 & 7.1 \\
\hline Chromium & $<0.1$ & $<1$ & 2.5 & 11.1 & 2.7 & 15.5 \\
\hline Copper & $<0.1$ & $<1$ & 6.7 & 1.1 & 13.3 & 23.5 \\
\hline Iron & 0.4 & 1.5 & 111.3 & 1107.6 & 134.1 & 5374.3 \\
\hline Manganese & $<0.1$ & 3.8 & 23.6 & 37.4 & 4.7 & 31.4 \\
\hline Mercury & $<0.008$ & $<0.008$ & $<0.008$ & $<0.008$ & $<0.008$ & 0.016 \\
\hline Molybdenum & 0.2 & $<1$ & $<0.2$ & 1.1 & $<0.2$ & 1.7 \\
\hline Nickel & $<0.1$ & $<1$ & 0.6 & 3.1 & 1.5 & 15.3 \\
\hline Lead & $<0.1$ & $<1$ & 0.4 & 1.6 & 0.5 & 12.5 \\
\hline Antimony & $<0.1$ & $<1$ & $<0.2$ & 0.3 & $<0.2$ & $<0.1$ \\
\hline Selenium & 0.4 & $<1$ & 0.4 & 1.3 & 3.4 & 1.9 \\
\hline Thallium & $<0.1$ & $<1$ & $<0.2$ & 0.5 & 0.3 & 0.5 \\
\hline Vanadium & 1.1 & $<1$ & 3.9 & 31.4 & 3.5 & 36.7 \\
\hline Zinc & 0.1 & 2.0 & 3.1 & 6.7 & 3.0 & 23.9 \\
\hline
\end{tabular}


were also observed in the residual phase, suggesting limited aqueous mobility. Although much lower in total concentration, selenium association with the oxide and residual phases is also noted. In general, a relatively large amount of the metals observed in the fly ash materials are present in the residual phase. Therefore, it would be expected that these metals would not easily become mobile in normal aqueous environments. There are, however, measureable ( $<1 \mathrm{mg} / \mathrm{kg}$ ) amounts of several elements, including arsenic, selenium, barium, zinc, and vanadium, that are in the soluble and exchangeable phases, and therefore represent potentially mobile compounds.

Iron and manganese were primarily present in the residual phase, suggesting that somewhat crystalline oxide phases are present, although approximately $20 \%$ of the iron measured was in the reducible oxide phase, suggesting at least some amorphous oxyhydroxides were present. The oxyhydroxides are well known to sorb other elements, and therefore explain the association of arsenic, selenium, chromium, and vanadium. Furthermore, significant amounts of iron and manganese are observed in the carbonate phase, indicating precipitation of carbonates may also have occurred. Also, the sulfide and organic matter phase is notably elevated in copper, which would be expected to precipitate as an insoluble sulfide. The presence of arsenic in this phase would also suggest that realgar or orpiment type phases may be present as well. However, of particular interest is the presence of selenium in the organic matter-sulfide phase.

\section{Ash elemental speciation: X-ray absorption spectroscopy analysis}

Bulk carbon and nitrogen speciation. Carbon-edge NEXAFS data shows a peak at $\sim 290 \mathrm{eV}$ for the ash collected from the source pile (Figure 4) representing $\mathrm{C}(1 \mathrm{~s}) \rightarrow \pi^{*}$ transition for $\mathrm{C}=\mathrm{O}$ carbonyl $\mathrm{C}$, a $\sim 285 \mathrm{eV}$ for aromatic $\mathrm{C}$, and a secondary oxygen peak at $\sim 271 \mathrm{eV}$. The ash pulled from the Emory River shows an additional peak at $\sim 287 \mathrm{eV}$, possibly representing phenolic $\mathrm{C}$, while the secondary oxygen peak is barely visible. The carbon groups observed from the ash in the sluice channel are similar to the source pile. Nitrogen-edge NEXAFS (Figure 5) demonstrates a low $\mathrm{N}$ content in the source pile - with very limited information observable for source pile nitrogen functional groups. Ash pulled from the Emory River and sluice channel, however, showed evidence of nitrated and amino groups. The data suggest that the ash accumulated natural organic matter while residing in the Emory River. It should be noted that TOC levels in the source pile are higher than those measured for the Emory River and 
sluice channel samples (Table 5). This may be a result of the fact that the source pile samples were collected at the top of the remaining ash pile, which was sprayed with a polysaccharide-based binder material to stabilize the particles. Thus, source pile $\mathrm{N}$ was barely detectable, but clearly visible in the Emory River and sluice channel samples. $\mathrm{N}$ and $\mathrm{C}$ profiles were observed to be somewhat different in the sluice channel samples, probably due to losses of humic material from the turbulent dredging process. However, the data suggest that the ash does remain permanently modified by its residence in the river.

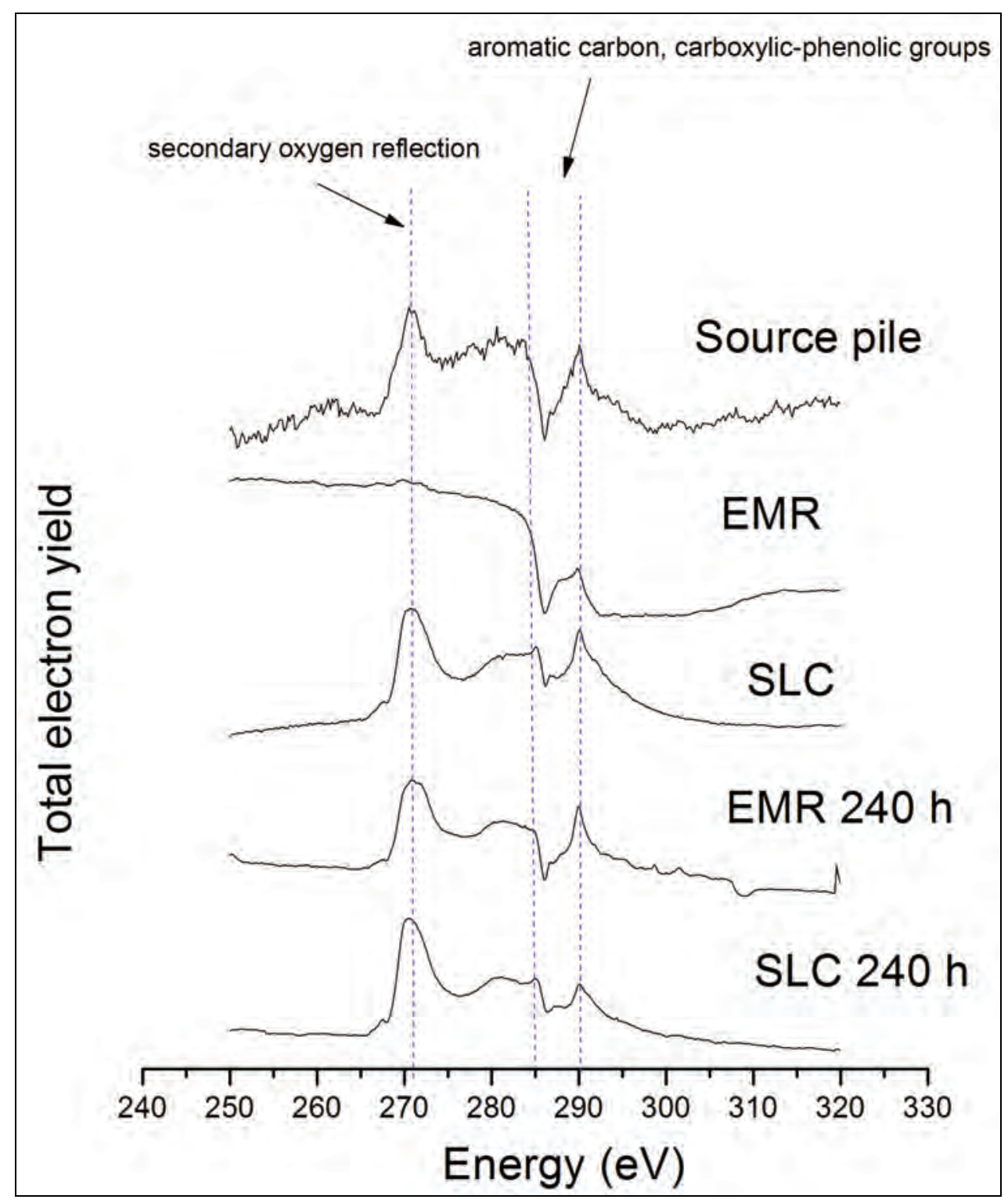

Figure 4. Carbon NEXAFS spectra for ash collected from the source pile, the Emory River, and sluice channel sediments and elutriates. 


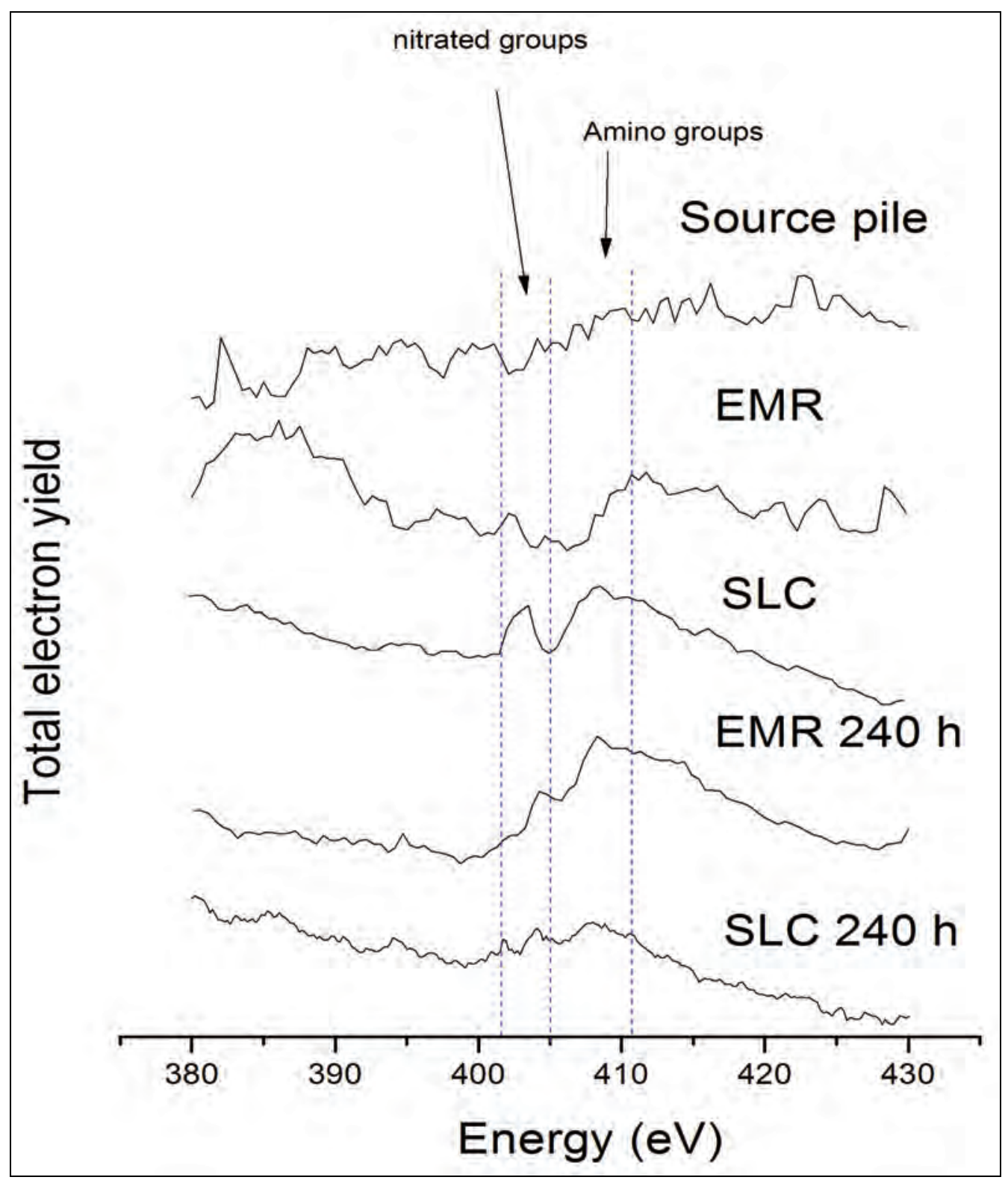

Figure 5. Nitrogen NEXAFS spectra for ash collected from the source pile, the Emory River, and sluice channel sediments and elutriates." $240 \mathrm{~h}$ " denotes the 10-day elutriate preparation period.

Bulk manganese speciation. Bulk Mn XANES data are of relatively poor quality and required considerable smoothing of the data. However, the data (Figure 6) showed the source pile ash contained oxidized Mn (e.g., $\mathrm{Mn}(\mathrm{IV})$ ) with a minor proportion of reduced Mn (e.g., Mn(II)). Once deposited into the river, the $\mathrm{Mn}$ in the ash appears to reduce to predominantly $\mathrm{Mn}$ (II). Ash collected in the sluice channel shows similarly reduced Mn, suggesting dredging activities did not affect the net redox of the ash. The XANES data for Mn were not sufficiently detailed to estimate the Mn compounds contained within the ash. 


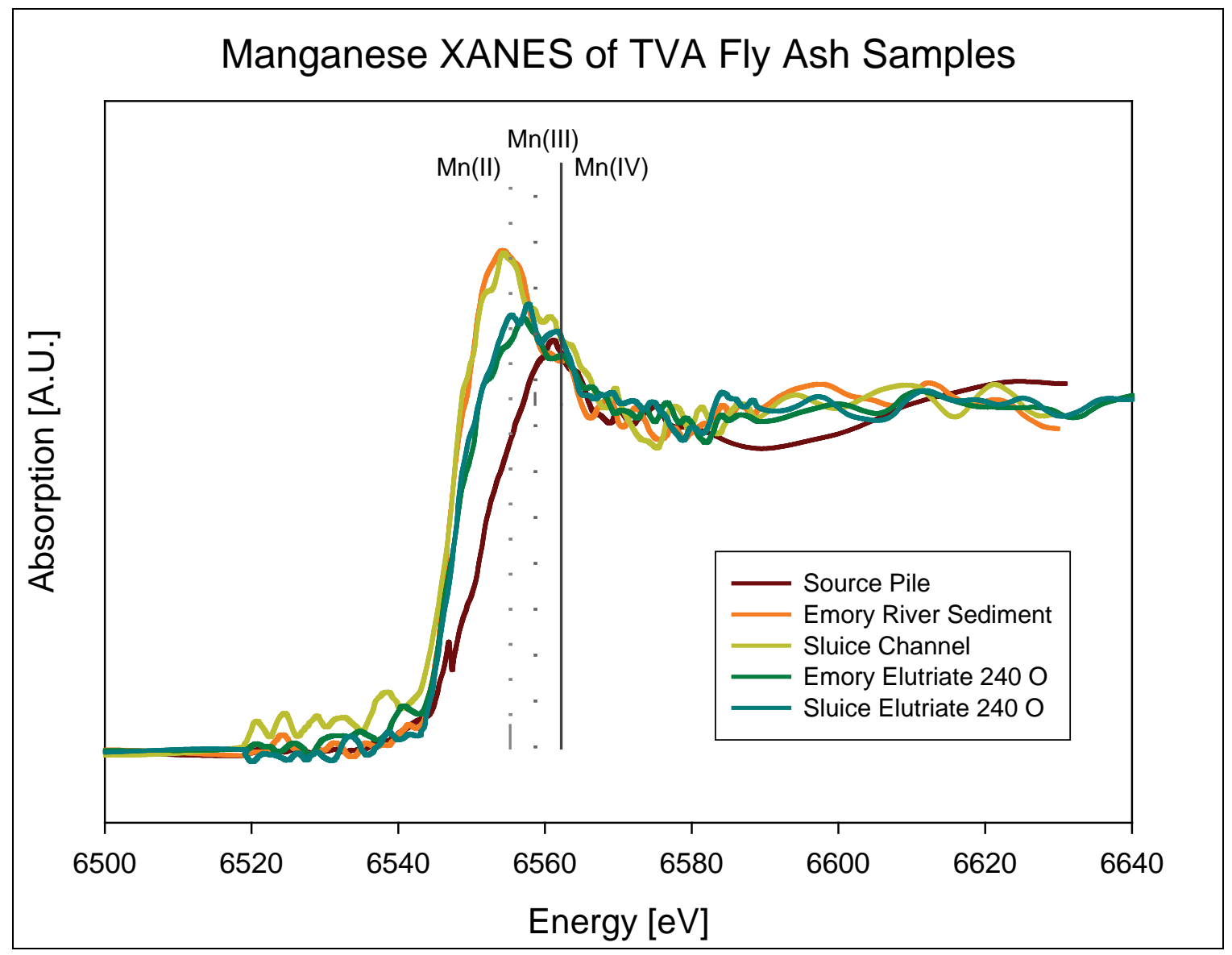

Figure 6. XANES data collected at the manganese K-edge energy for bulk ash and elutriate samples, where 0 means oxic regime elutriate samples. " $240 \mathrm{~h}$ " denotes the 10-day elutriate preparation period.

Bulk iron speciation. LCF analysis of Fe K-edge XANES (Figure 7) showed the ash contained approximately $70 \%$ oxidized Fe(III) and $30 \%$ reduced $\mathrm{Fe}(\mathrm{II})$ (Table 11). No change was observed in the proportion of Fe(II) to Fe(III) with residence of the ash in the river or following dredging activities. This means that the Fe phases in the source pile ash did not reduce to secondary Fe-oxide minerals, but remained stable while residing in the river. LCF results suggest that the Fe forms are a mixture of ferrihydrite and maghemite minerals.

Bulk chromium speciation. Bulk chromium speciation was difficult due to the low concentrations and limitations of the experimental setup. XANES analysis (data not shown) suggests the ash contains predominantly $\mathrm{Cr}$ (III), with the possibility of trace levels of $\mathrm{Cr}(\mathrm{VI})$, yet the data are insufficiently clear to verify this distinction. 


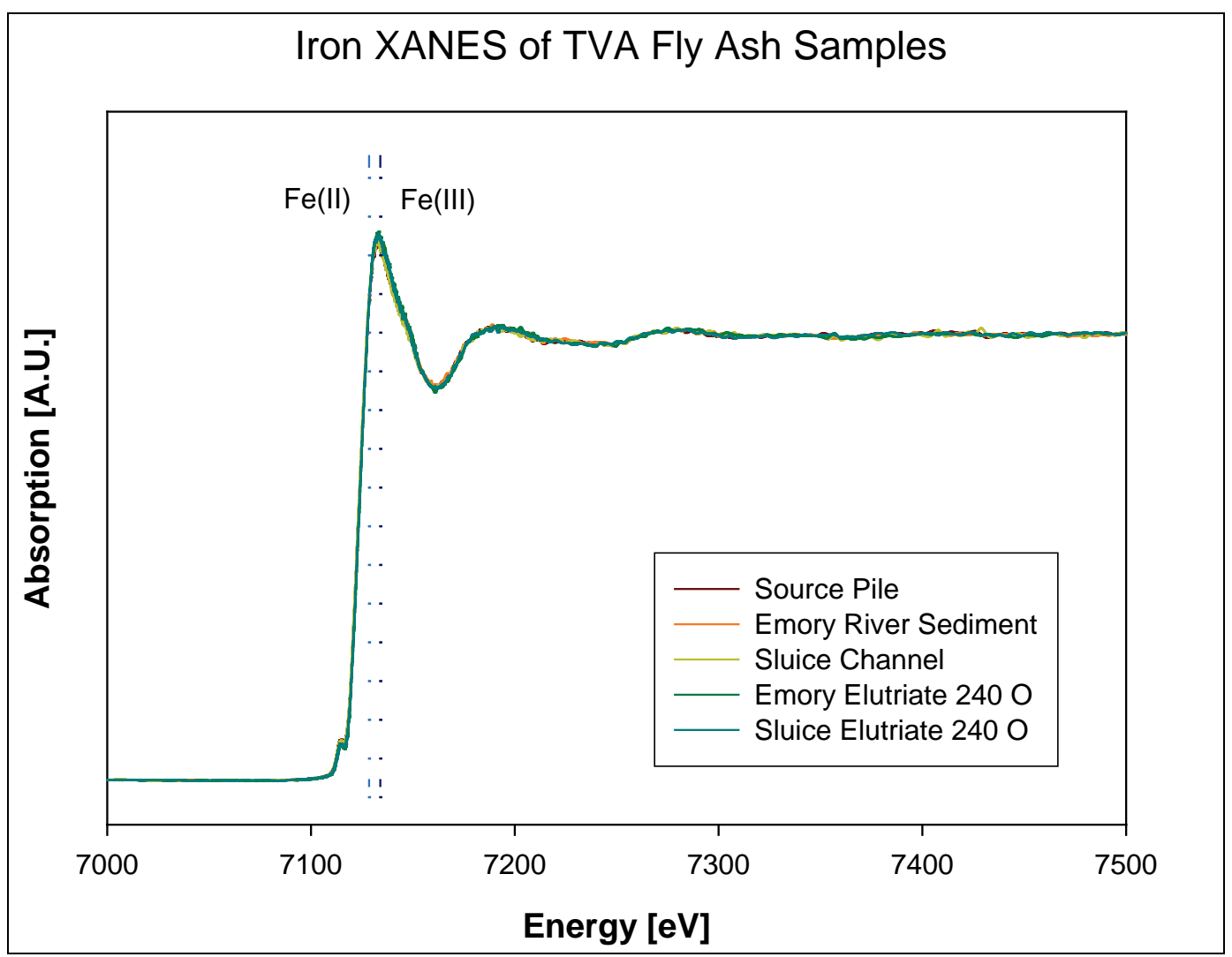

Figure 7. XANES data collected at the iron K-edge for bulk ash and elutriate samples where

" 0 " denotes elutriates treated with oxic regime. " $240 \mathrm{~h}$ " denotes the 10-day elutriate preparation period.

Table 11. Linear combination results for Fe XANES data on bulk ash and elutriate samples.

\begin{tabular}{|l|l|l|}
\hline Samples & $\begin{array}{l}\text { Fe(II) } \\
\%\end{array}$ & $\begin{array}{l}\text { Fe(III) } \\
\%\end{array}$ \\
\hline \multicolumn{3}{|c|}{ Ash Samples } \\
\hline Source Pile Elutriates \\
\hline Emory River Sediments & 30.0 & 70.0 \\
\hline Sluice Channel & 30.3 & 71.7 \\
\hline \multicolumn{3}{|c|}{} \\
\hline Emory EL 240 h (air) & 21.0 & 69.3 \\
\hline Sluice EL 240 h (air) & 23.3 & 79.0 \\
\hline
\end{tabular}

Bulk arsenic speciation. XANES data (Figure 8) show arsenic existed primarily as $\mathrm{As}(\mathrm{V})$ in the source pile, with trace proportions of $\mathrm{As}(\mathrm{III})$ and sulfide-bound arsenic (Figure 9). The bulk analysis shows little change in arsenic speciation in the ash collected from the Emory River and the sluice channel from the source pile. 


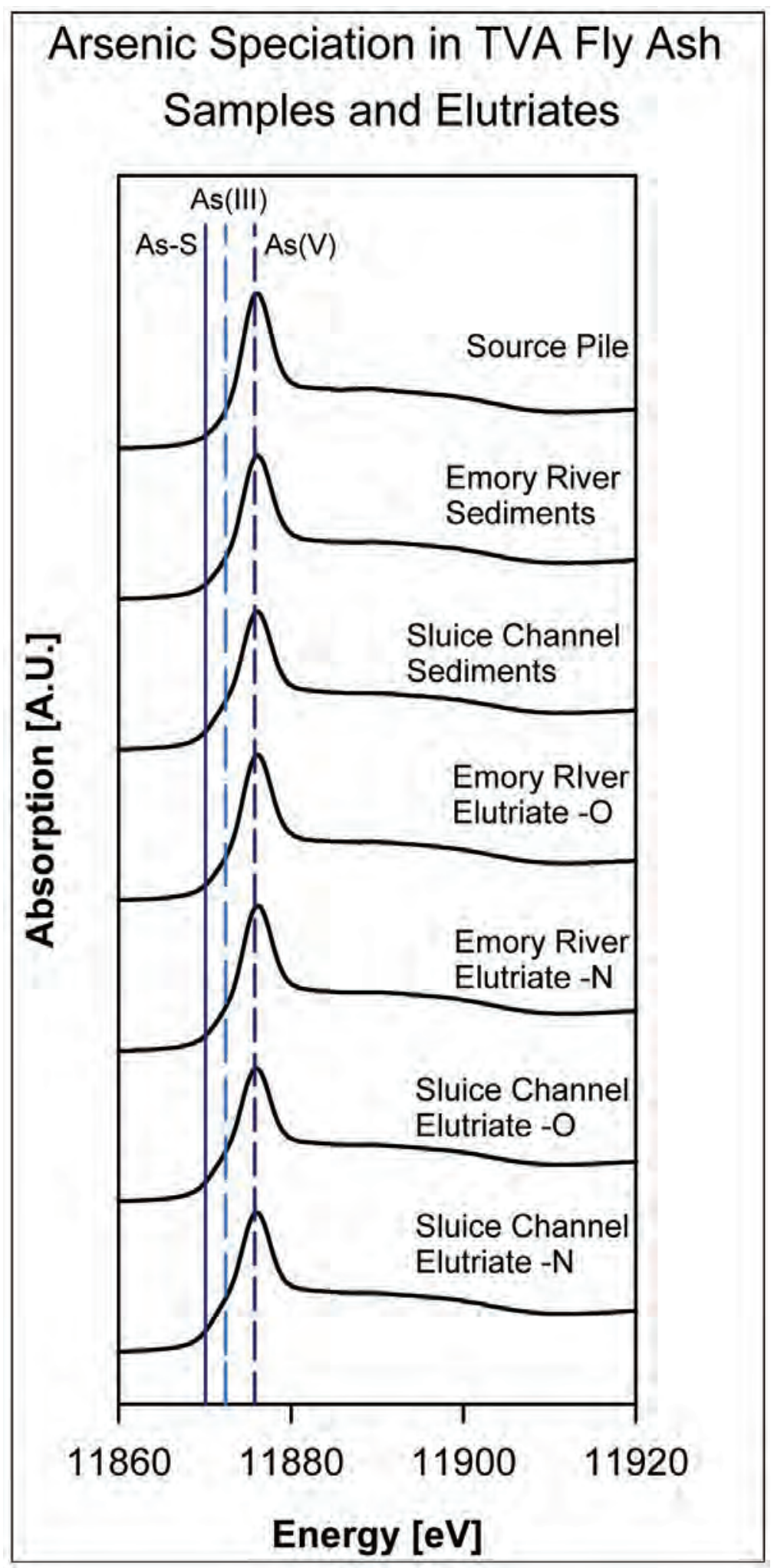

Figure 8. XANES spectra collected at the arsenic K-edge for bulk ash and elutriate samples. 


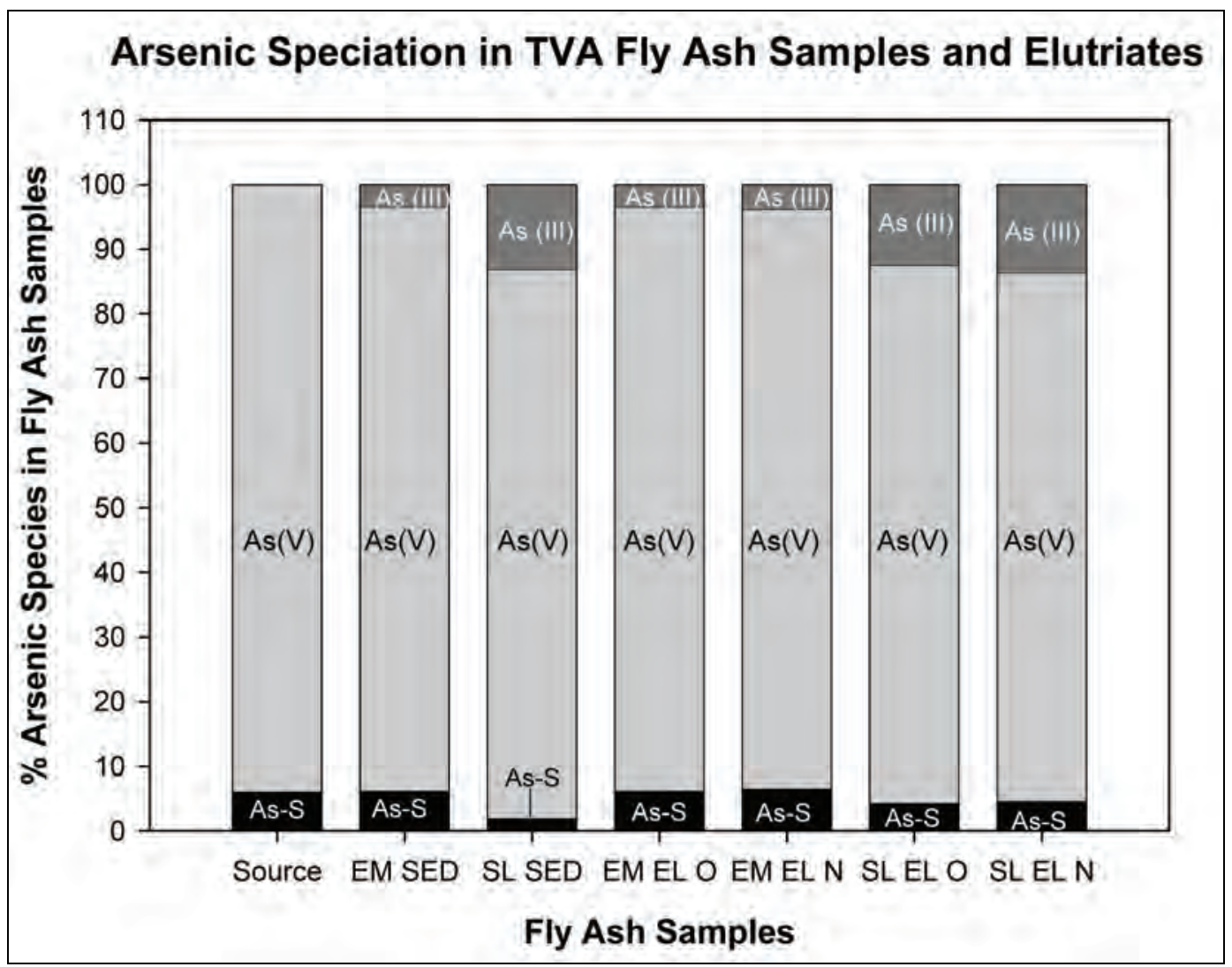

Figure 9. Linear combination results for arsenic XANES data (Figure 8) on bulk ash and elutriate samples, where: EM = Emory River, SL = Sluice Channel, SED = sediment, and EL = elutriate samples. The " $O$ " and" N" represent whether the elutriates were bubbled with air (oxic regime) or nitrogen (anoxic regime), respectively.

Bulk selenium speciation. Selenium K-edge XANES (Figures 10-12) analysis shows a notable change in form of selenium after the ash was introduced into the river. The analysis shows that the selenium form changed from predominantly selenite in the source pile to a mixture of species in the Emory River and sluice channel sediments. Linear combination fittings for the Emory River and sluice channel sediments estimated that 30 to $50 \%$ of the Se exhibited relatively low absorption edge energies. A number of more reduced selenium species have absorption edge energies in this range including: elemental Se, selenium sulfide ( $\left.\mathrm{SeS}_{2}\right)$, and selenium bound to thiol bearing C-compounds (e.g. cysteines and methionines), see Figure 11. Closer investigation of these samples suggests that a portion of these reduced selenium species may be composed of selenium species covalently bound to thiol and organic carbon-bearing materials, as the Se(II) species. As demonstrated in the NEXAFS data, natural humic materials, either dissolved or suspended in the Emory River water, accumulated on the surface of the ash particles after the spill. Once introduced to natural humic material, the data show that selenium 


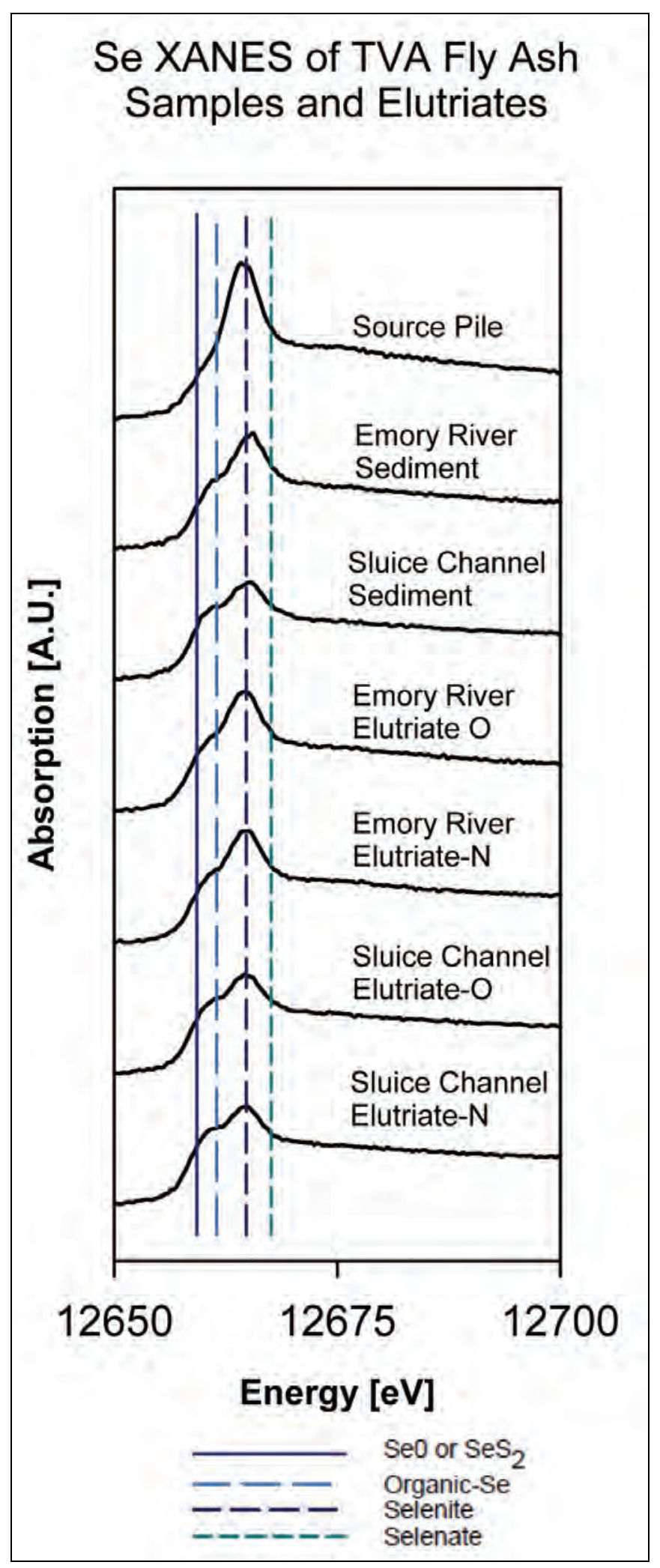

Figure 10. XANES spectra collected at the selenium K-edge for bulk ash and elutriate samples. Elutriate data are discussed in the next chapter. 


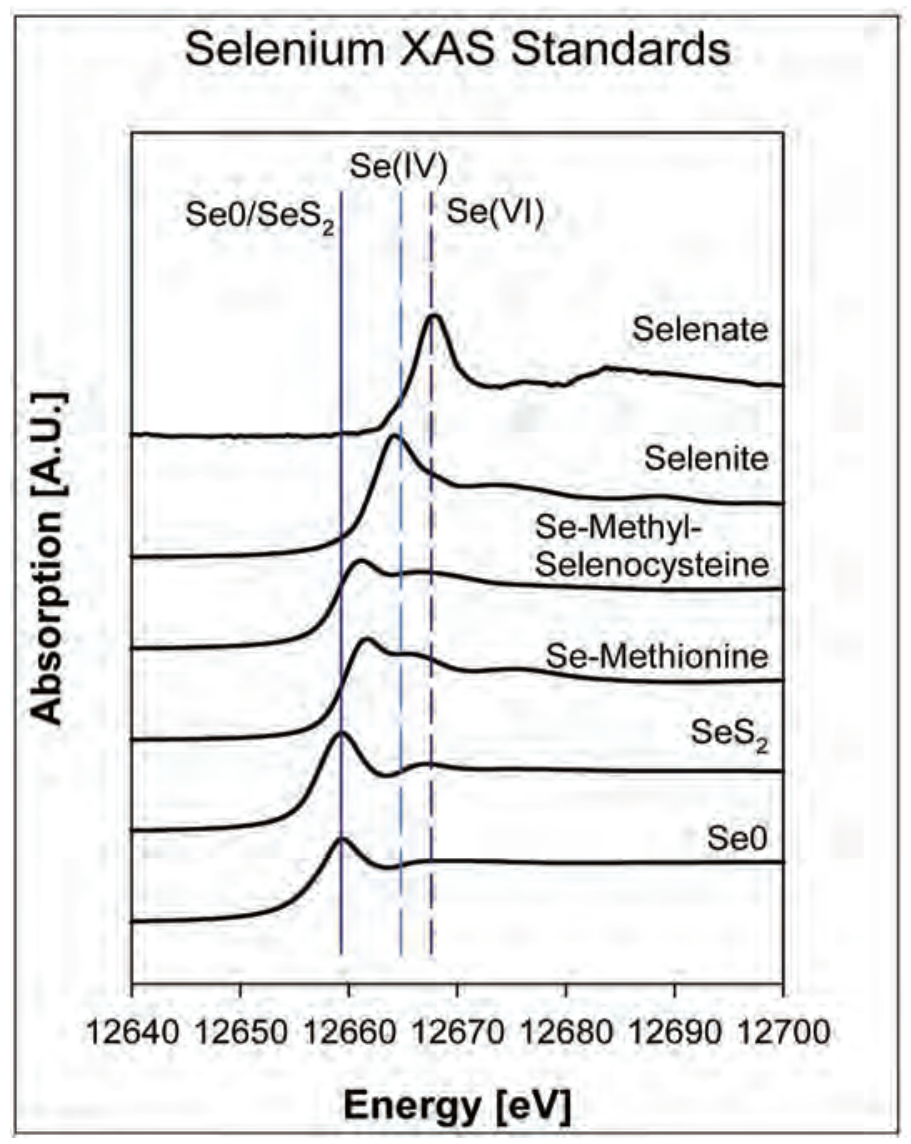

Figure 11. XANES spectra collected at the selenium K-edge for use as reference samples, including the seleniumbound thiol compounds: Se-methyl-selenocysteine and Semethionine.

somehow reacted to form what appears to be covalently bound complexes with the organic carbon and thiol-rich material - commonly referred to as organoselenium. This conclusion is consistent with the scientific literature (Chaissaigne et al. 2004, Haratake et al. 2009, Tarze et al. 2007), showing that selenium forms very strong, covalent-nature complexes with organic thiol groups, and in the process is reduced to Se(II). For example, Huggins et al. (2007) used XANES to determine Se speciation in fly ash and found an organoselenium component. A few studies dispute the direct association of organoselenium with natural humics, citing incompatibilities with sequential extraction experiments and competitive sorption processes (Kamei-Ishikawa et al. 2007, Ponce de Leon et al. 2003, Séby et al. 1997) while others claim organoselenium can be extracted with soil fulvic acids (Rael and Frankenberger 1995) under alkaline extracting conditions. Further investigations are justified in elucidating the nature of the Se-humic complex, as this represents the dominant form of selenium in the spilled ash materials. 


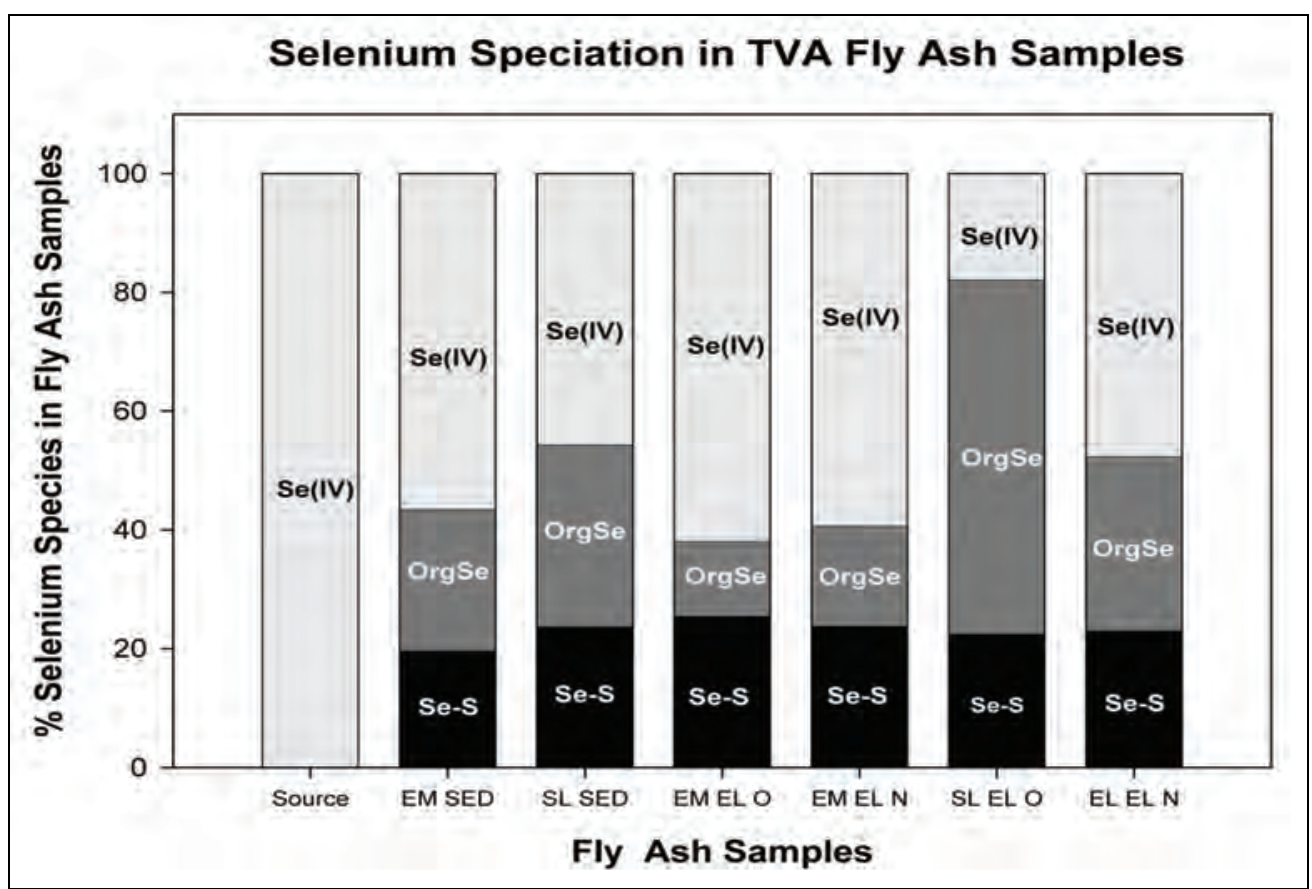

Figure 12. Linear combination results for selenium XANES data (Figure 10) on bulk ash and elutriate samples.

Micro-metal distribution and speciation. Realizing the large degree of heterogeneity that exists in natural systems, micro-mapping analysis was performed to assess the accuracy of the bulk representations of ash metal species on a microscopic scale. Using the $\mu$-XRF technique (Figure 13), analysis found that metals were non-uniformly distributed, exhibiting $\mu \mathrm{m}$ range heterogeneity throughout the fly ash material collected from the original source pile and the Emory River. In some cases, the data showed overlapping domains of selenium, manganese, iron, chromium, calcium, mercury, and arsenic (and sulfur), such as the large particle visible in Figure 13 near the upper right-hand corner of the maps. The occurrence of the metals in this particular arrangement is important as overlapping elemental domains typically represent sites where metals are loaded by a similar chemical mechanism, such as sorption/ accumulation on Fe-oxide phases. Such accumulations are commonly observed in natural systems, e.g., soils, sediments. A map for the Emory River fly ash (Figure 14) showed similarly distributed selenium "hotspots" as observed in the source pile (small white spots in the figure). In general, arsenic, selenium, and chromium seem to track the distribution of iron domains as well. According to the $\mu$-XANES analysis (Figure 15), the source pile arsenic is predominantly composed of As(V) with a minor proportion of As(III). The Emory River and sluice channel ash only showed evidence of $\mathrm{As}(\mathrm{V})$, with little As(III) detected. 


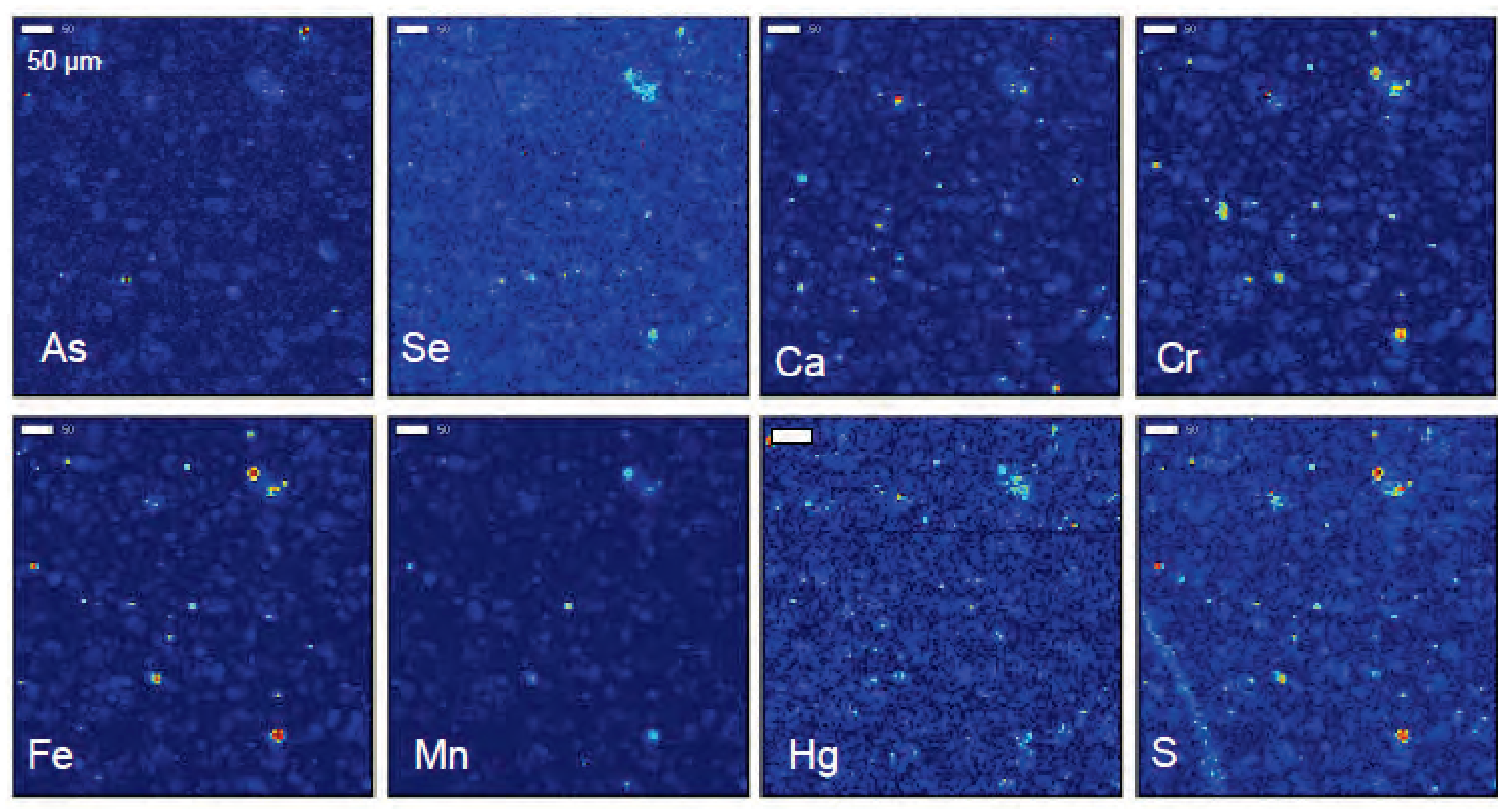

Figure 13. Synchrotron X-ray fluorescence maps depicting elemental distribution in source pile fly ash. Maps were collected at $13000 \mathrm{eV}$. Areas of warmer colors (white, yellows, and reds) indicate areas of higher concentration, whereas cooler colors like blues indicate areas of lower concentration. Solid white bar represents a $50-\mu \mathrm{m}$ scale. 

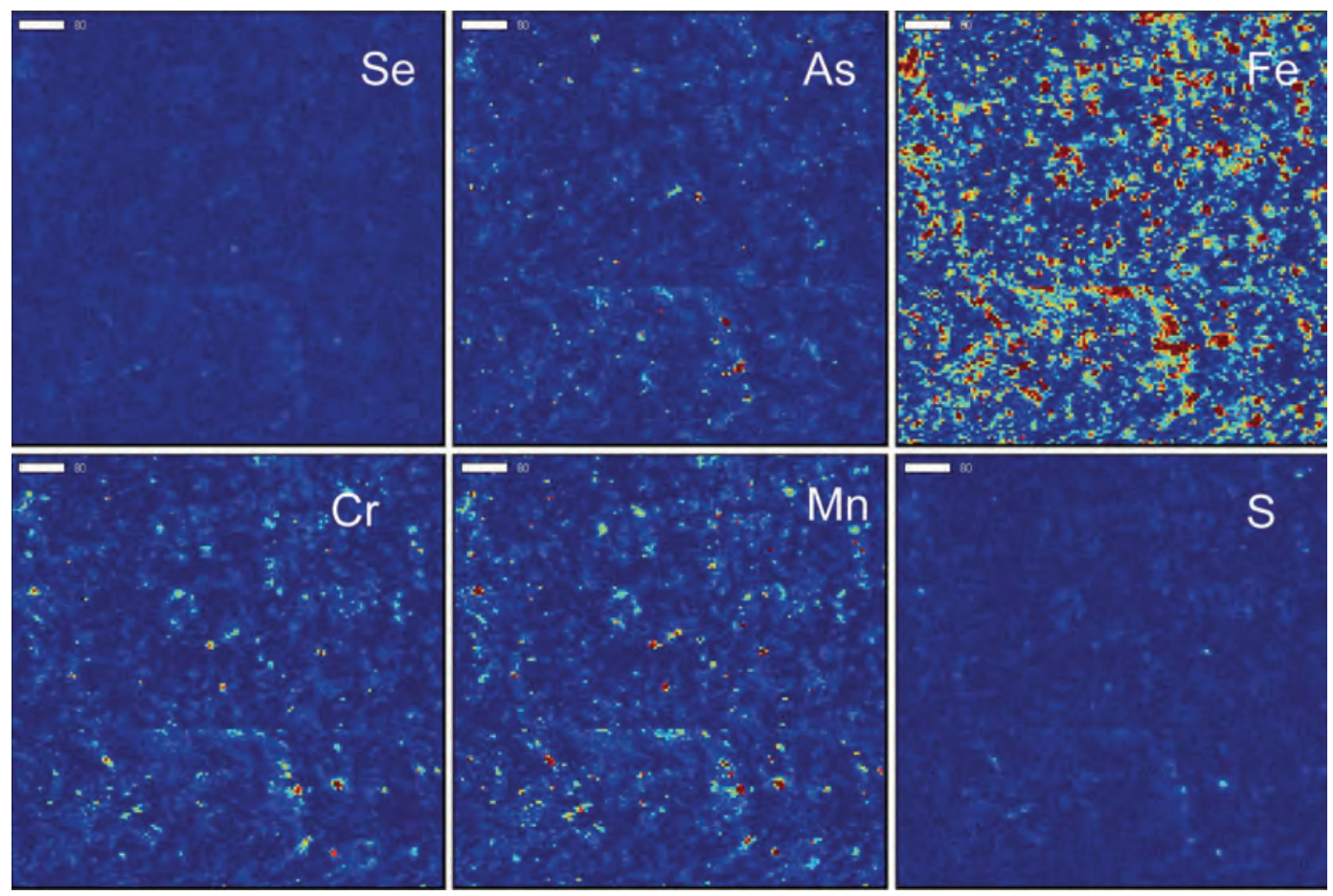

Figure 14. Synchrotron X-ray fluorescence maps depicting elemental distribution in fly ash collected from the Emory River. Maps were collected at $13000 \mathrm{eV}$. Areas of warmer colors (white, yellows, and reds) indicate areas of higher concentration, whereas cooler colors like blues indicate areas of lower concentration. Solid white bar represents a80- $\mu \mathrm{m}$ scale.

Differential $\mu$-XRF mapping was performed to determine the distribution of the selenium species present in the fly ash (Figure 17). The maps were collected by scanning the sample at the energies associated with the different species of selenium, e.g., Se(0, IV, VI). (Figure 18). The maps show significant domains where selenium was detected at the "lower" energies for Se, suggesting either "reduced" (elemental selenium or selenium sulfide, $\mathrm{SeS}_{2}$ ) or covalently bound Se(IV) forms (Figure 19). Se(IV) was also evident in the maps, but is much less abundant than "reduced" Se, while Se(VI) was not detected at all in the source pile material.

Further evidence for selenium speciation was obtained by focusing the $\mathrm{X}$-ray beam on particular spots on the source pile sample, and then conducting $\mu$-XANES. Figures 18 and 19 show that spot 1 is largely composed of selenite, while spot 2 contains predominantly "reduced" selenium (probably organoselenium) in the source pile. Similar species of selenium were found in $\mu$-XANES analysis of the Emory River and sluice channel ash samples, typically as mixtures of "reduced" or covalently bound selenium and selenite (Figures 20 and 21). No evidence of localized selenate domains was found in the source pile or the Emory River and sluice channel sediments. 


\section{Arsenic XANES in Fly Ash Samples}

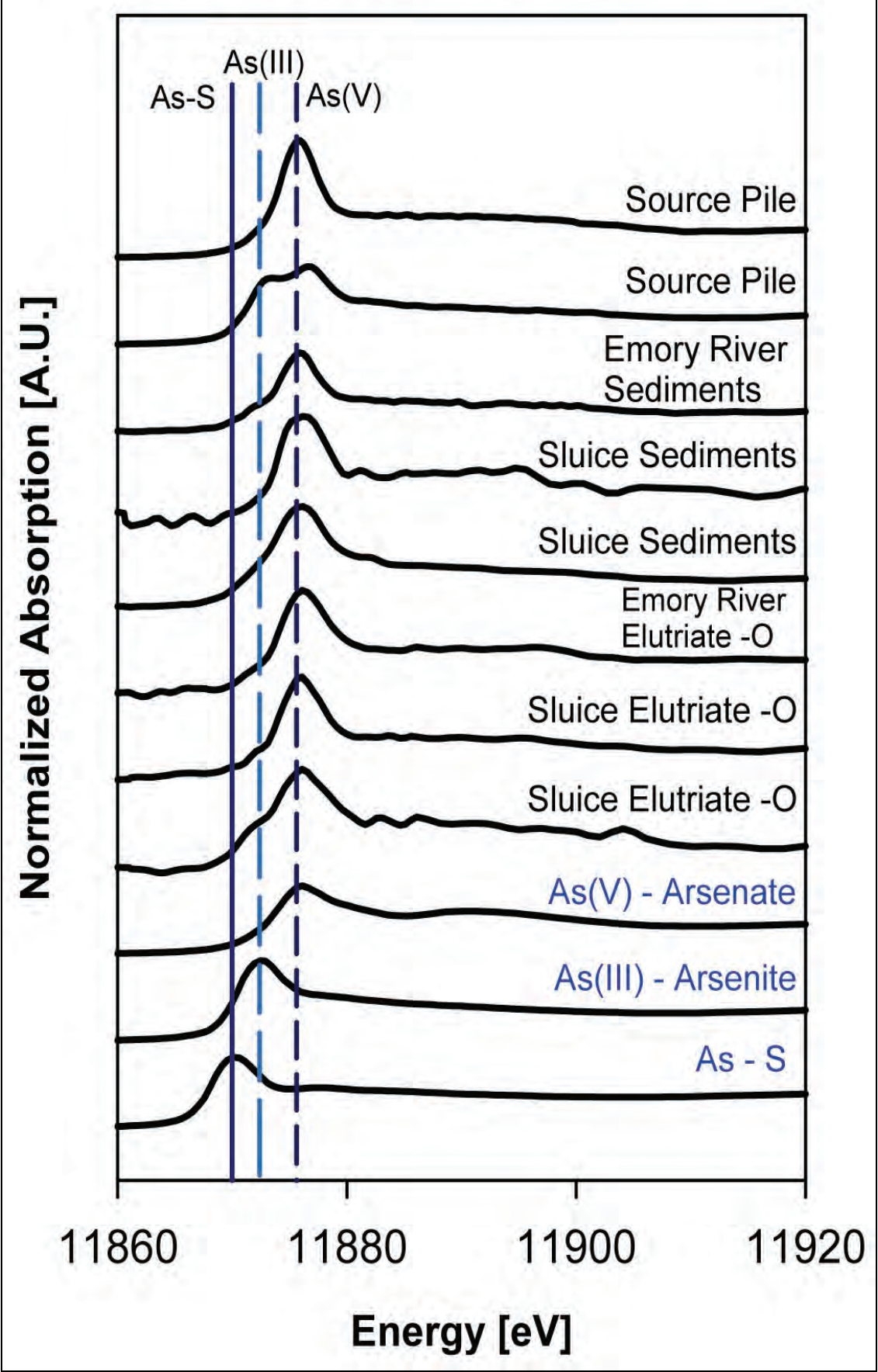

Figure 15. Spot $\mu$-XANES analysis for determining localized arsenic speciation in fly ash and elutriate samples. $\mu$-XANES spectra for arsenate, arsenite, and arsenic bound to $S$ are included for reference. 


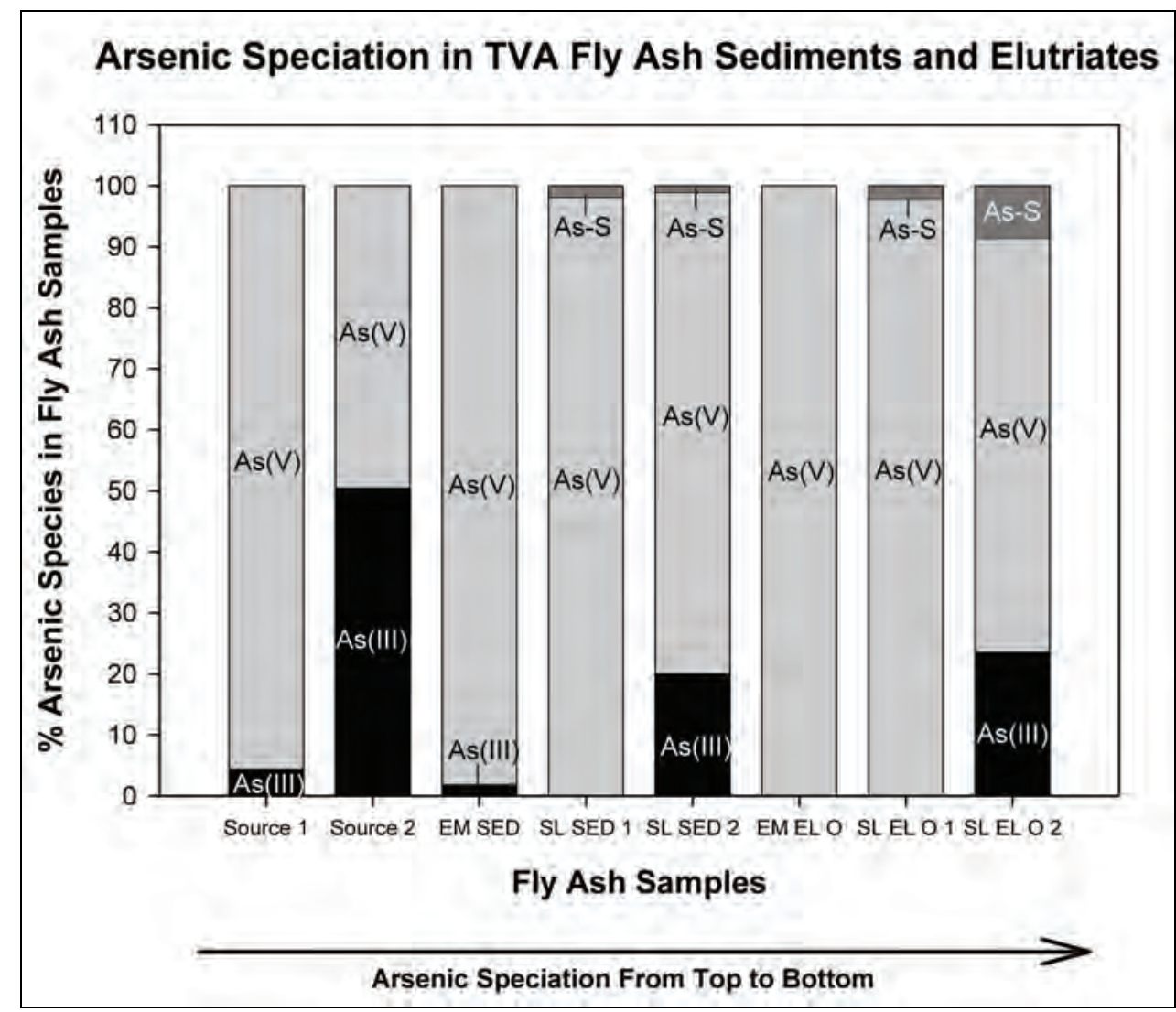

Figure 16. Linear combination results of spot $\mu$-XANES data (Figure 15) for localized arsenic speciation of ash and elutriate samples.
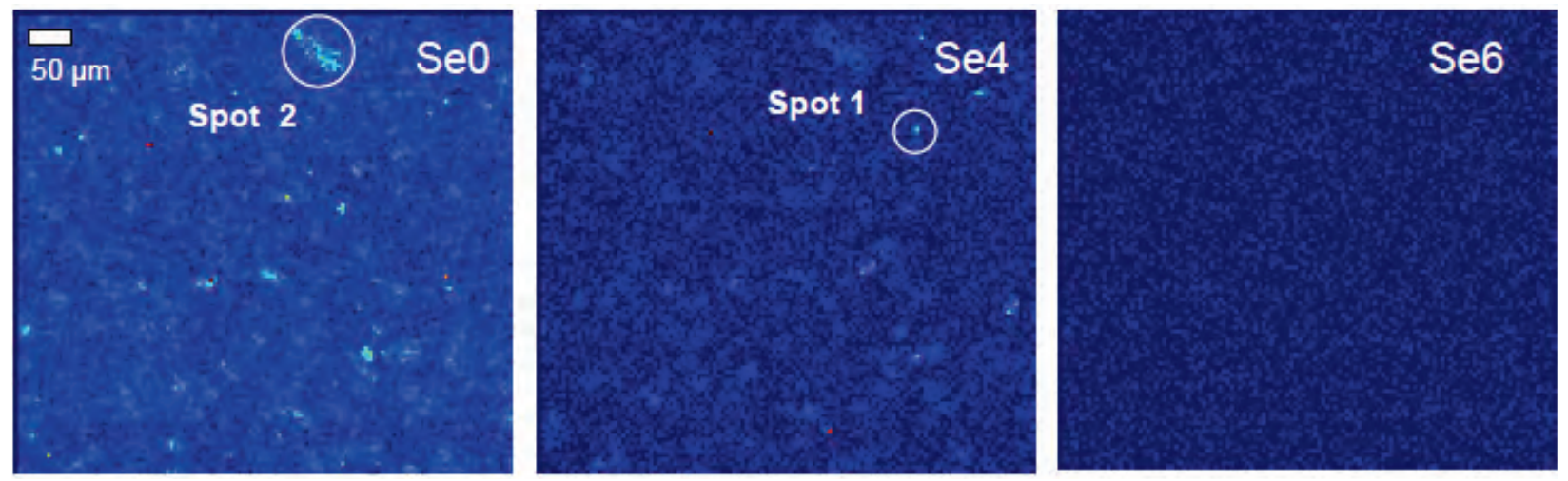

Figure 17. Localized selenium speciation determined by differential energy $\mu$-XRF mapping. This technique allows for selenium speciation of selected areas in the elemental maps. The particular area mapped in the source pile sample shows selenium existing as predominantly "reduced" forms - indicative of either elemental or covalently bound selenium, such as on organic thiol-bearing compounds. 


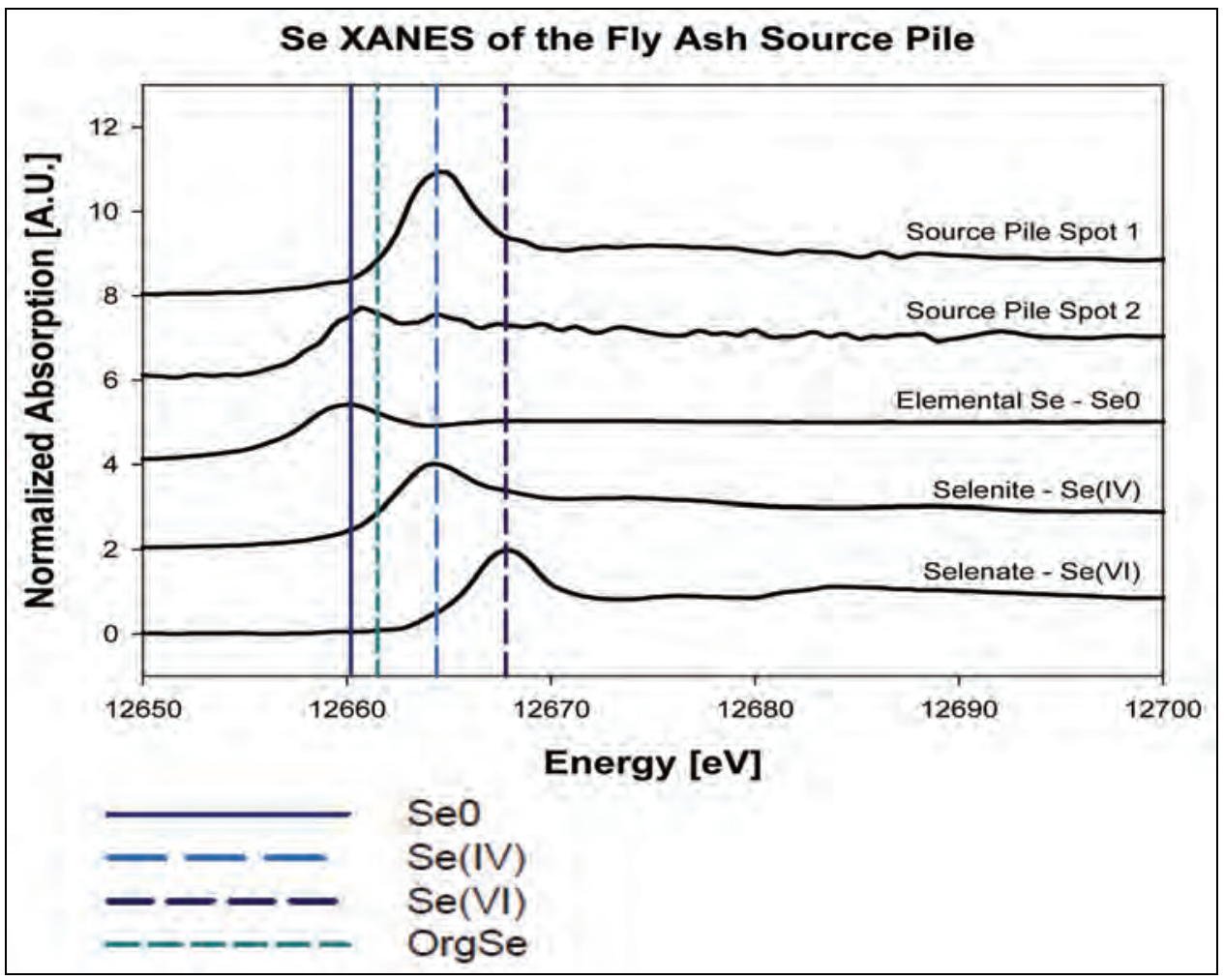

Figure 18. Selenium speciation of the spots defined in Figure 17 for the original source pile fly ash material using $\mu$-XANES. Spectra for $\mathrm{Se}(0, \mathrm{IV}$, and VI) are shown for comparison.

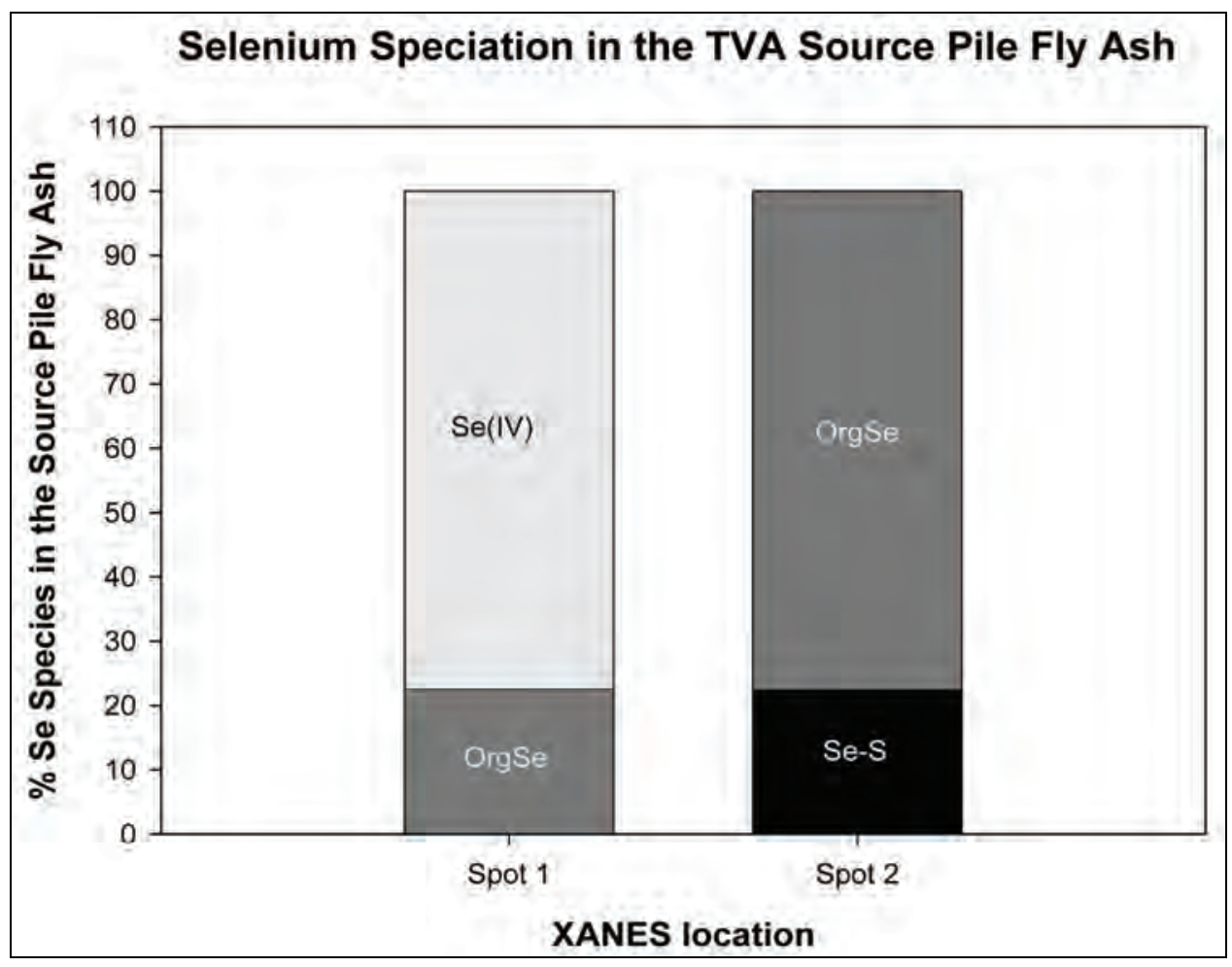

Figure 19. Linear combination results of selenium $\mu$-XANES (Figure 18) data from spots defined on the XRF maps (Figure 17). 


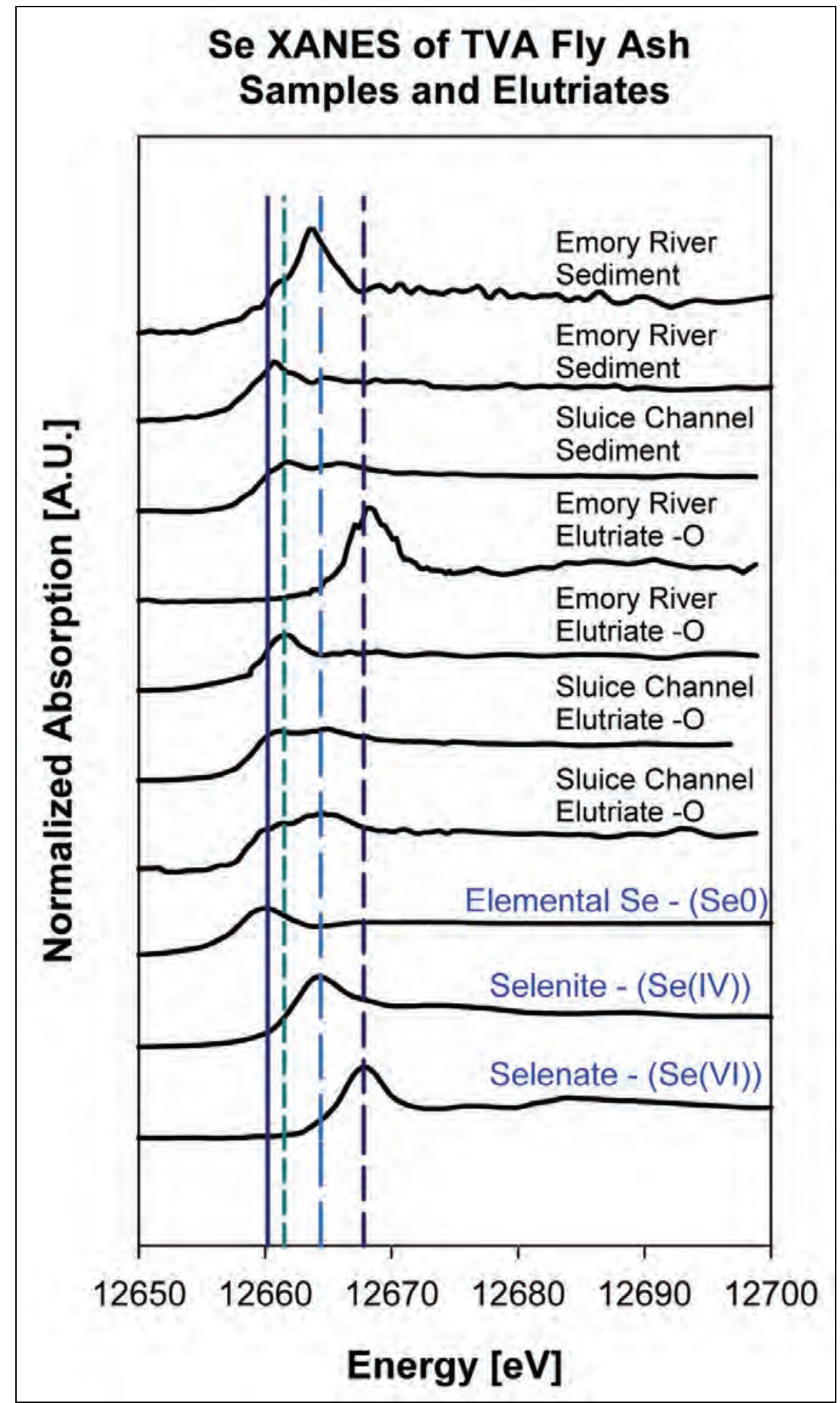

Figure 20. $\mu$-XANES analysis of selenium in the fly ash collected from the Emory River and the sluice channel sediments and elutriates. $\mu$-XANES spectra for $\mathrm{Se}(0), \mathrm{Se}(\mathrm{IV})$, and $\mathrm{Se}(\mathrm{VI})$ are included for reference. Elutriate data are discussed in the next section. 


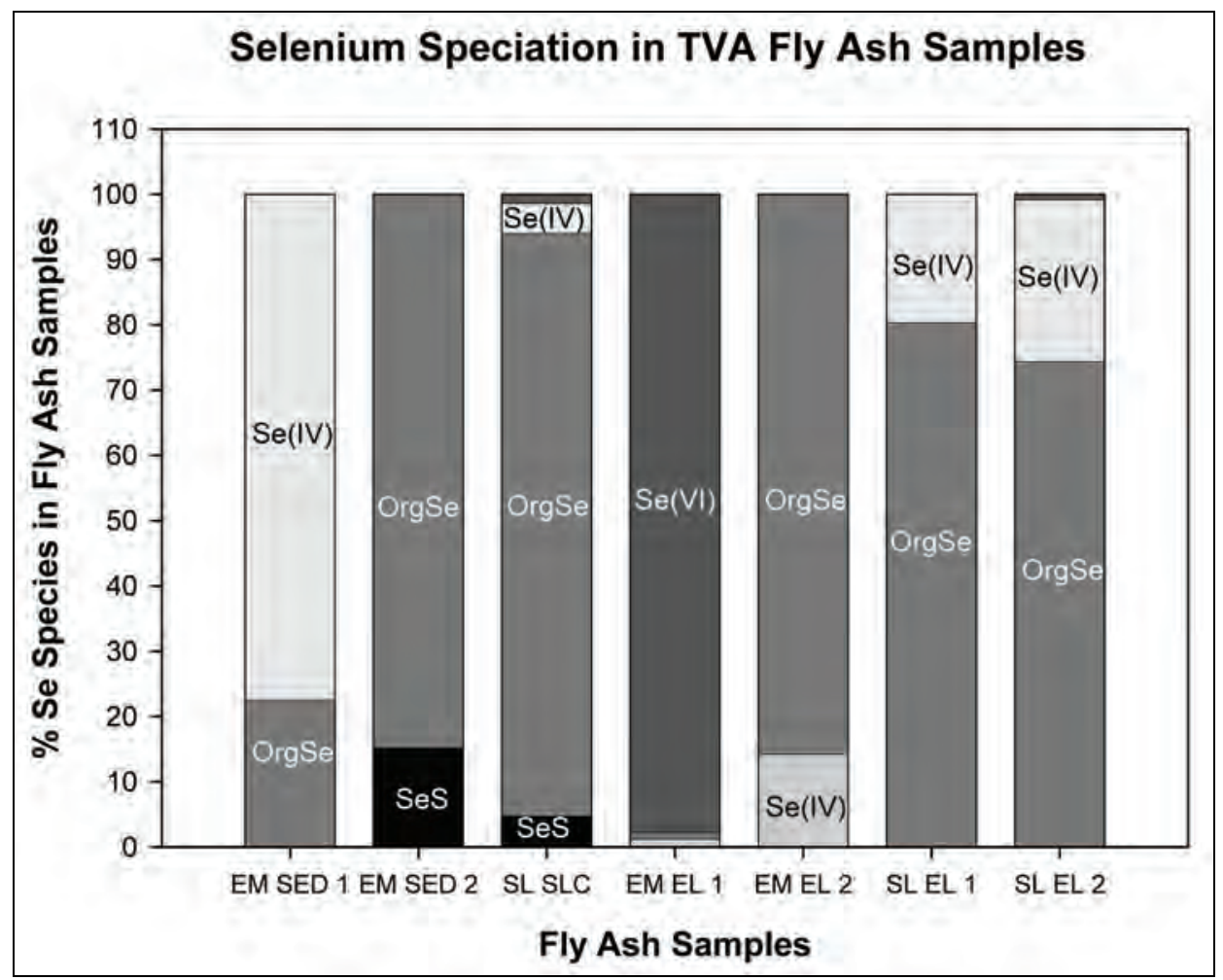

Figure 21. Linear combination results for selenium $\mu$-XANES data for spots on the XRF maps. Elutriate data are discussed in the next section.

\section{Particle size analysis}

Particle size distributions (Figure 22) of the fly ash collected from the source pile, the Emory River, and the sluice channel were determined as predominantly composed of silty-sized material (USCS Classification Silt (Silt(ML, Gray). Specific gravity of the particles was determined as 2.37, 2.41, and 2.35 for the Emory River, sluice channel, and pile samples, respectively. As expected, deposition and dredging activity had little effect on the particle size distribution of the ash.

\section{Site waters}

Three site water samples (Emory River, sluice channel, and stilling ponds) were collected from the field and analyzed for dissolved metals (Table 12) and concentrations of dissolved arsenic and selenium species (Table 13). Combined, these data represent the influence of the ash materials on the equilibrium concentration of dissolved metals. In addition, a sample was collected from the stilling pond outflow, prior to release of the dredged waters back to the Emory River after the removal of solids. 


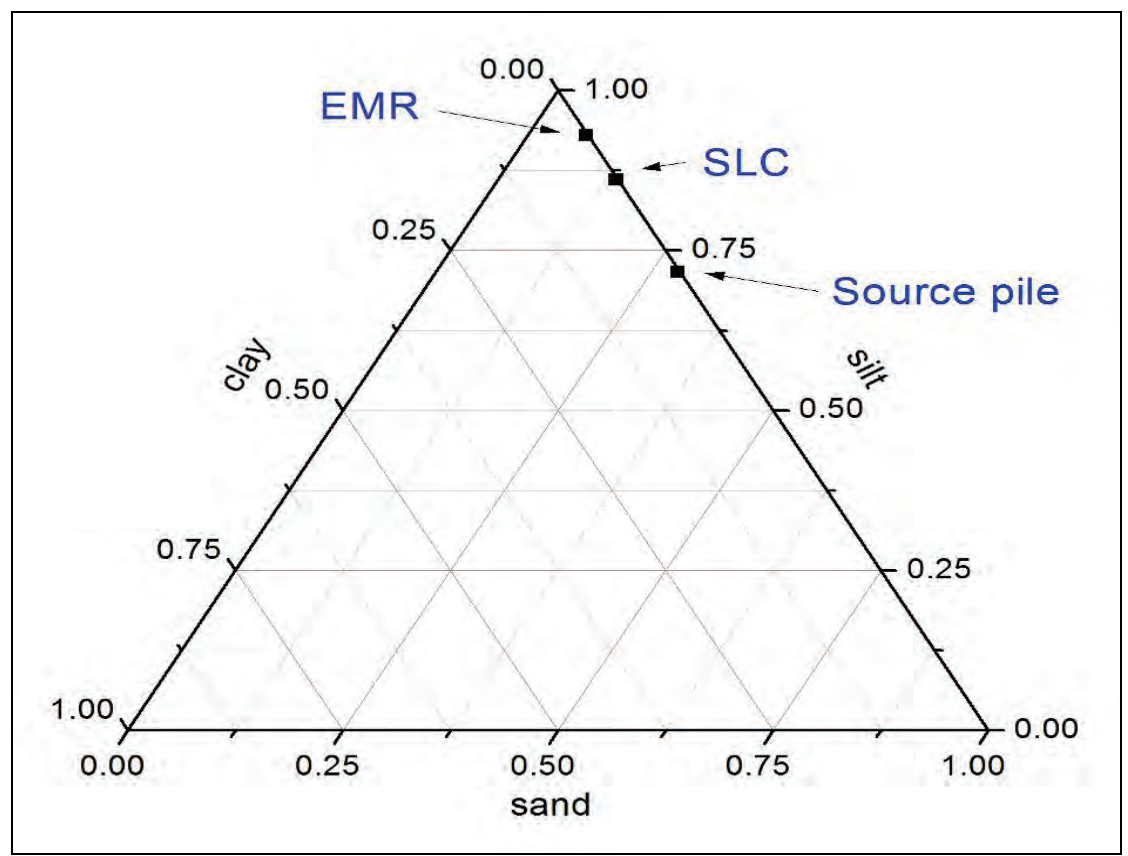

Figure 22. Textural triangle showing the percentages of sand-, silt-, and claysized particle in the ash materials collected from the source pile, Emory River, and the sluice channel.

The results of the field water analysis indicate that the dredging operation increases the concentration of the major elements (calcium, magnesium, sodium, and potassium) - a result consistent with the increased electroconductivity (EC) measurement shown in Chapter 2. In addition, several trace elements are also elevated above ambient levels, including arsenic, barium, molybdenum, selenium, and vanadium. Arsenic and selenium are of particular interest, as the concentrations in the sluice channel water reach 78 and $6 \mu \mathrm{g} / \mathrm{L}$, respectively, which are close to the values obtained in the elutriate experiments. The concentration of arsenic falls by a factor of 4 in the stilling pond effluent compared to the sluice channel water. The concentration of selenium in the stilling pond effluent is approximately the same as the sluice channel water ( $5.4 \mathrm{vs} 5.9 \mathrm{\mu g} / \mathrm{L}$ ).

The presence of selenium (VI) in the stilling pond may be a result of photooxidation of selenium, resulting from the residence time of the waters in the settling system, similar to photooxidation previously reported for arsenic (Bednar et al. 2002, 2003). This is potentially an important area of research as the photooxidation of selenium may be related to the presence of dissolved selenite after the ash has settled. There are many different pathways in which selenium may be oxidized, such as photoreduction of suspended iron oxide particles or radicalization of dissolved organic matter by absorbed UV radiation (Voelker et al. 1997). 
Table 12. Concentrations of metals ( $\mathrm{mg} \mathrm{L}^{-1}$ ) in site waters (one grab sample each).

\begin{tabular}{|c|c|c|c|c|c|c|}
\hline \multirow[b]{2}{*}{ Analyte } & Emory River & $\begin{array}{l}\text { Sluice } \\
\text { Channel }\end{array}$ & $\begin{array}{l}\text { Stilling Pond } \\
\text { Effluent }\end{array}$ & Emory River & $\begin{array}{l}\text { Sluice } \\
\text { Channel }\end{array}$ & $\begin{array}{l}\text { Stilling Pond } \\
\text { Effluent }\end{array}$ \\
\hline & \multicolumn{3}{|c|}{ Dissolved Concentration (mg/L) } & \multicolumn{3}{|c|}{ Total Concentration (mg/L) } \\
\hline Aluminum & $<0.0500$ & 0.252 & 0.281 & 0.228 & 5.58 & 1.14 \\
\hline Antimony & $<0.0002$ & 0.0081 & 0.0023 & 0.0088 & 0.0411 & 0.0088 \\
\hline Arsenic & 0.0008 & 0.0779 & 0.0208 & 0.0011 & 0.0767 & 0.0192 \\
\hline Barium & 0.0307 & 0.107 & 0.175 & 0.0340 & 0.167 & 0.182 \\
\hline Beryllium & $<0.0002$ & $<0.0002$ & $<0.0002$ & $<0.0004$ & 0.0009 & $<0.0004$ \\
\hline Cadmium & $<0.0002$ & 0.0003 & $<0.0002$ & $<0.0004$ & $<0.0004$ & $<0.0004$ \\
\hline Calcium & 8.55 & 30.7 & 34.7 & 8.93 & 31.0 & 34.4 \\
\hline Chromium & $<0.0002$ & $<0.0002$ & 0.0041 & 0.00095 & 0.0063 & 0.0081 \\
\hline Cobalt & $<0.0002$ & $<0.0002$ & $<0.0002$ & $<0.0004$ & 0.0034 & 0.0012 \\
\hline Copper & 0.0005 & 0.0005 & 0.0012 & 0.0040 & 0.0190 & 0.0090 \\
\hline Iron & 0.0525 & $<0.0500$ & $<0.0500$ & 0.253 & 2.00 & 0.333 \\
\hline Lead & $<0.0002$ & $<0.0002$ & $<0.0002$ & 0.0007 & 0.0065 & 0.0017 \\
\hline Magnesium & 2.1 & 5.15 & 7.34 & 2.17 & 5.47 & 7.38 \\
\hline Manganese & 0.0005 & 0.0296 & $<0.0002$ & 0.0351 & 0.0482 & 0.0235 \\
\hline Mercury & $<0.000005$ & $<0.000005$ & 0.000014 & 0.000148 & 0.000306 & 0.000431 \\
\hline Molybdenum & $<0.0002$ & 0.0733 & 0.0236 & $<0.0004$ & 0.0738 & 0.0236 \\
\hline Nickel & 0.0009 & 0.0005 & 0.0013 & 0.0027 & 0.0115 & 0.0055 \\
\hline Potassium & 1.29 & 1.88 & 2.3 & 1.71 & 4.09 & 2.49 \\
\hline Selenium & $<0.0002$ & 0.0059 & 0.0054 & $<0.0004$ & 0.0046 & 0.0043 \\
\hline Silver & 0.0005 & 0.0004 & 0.001 & 0.0016 & 0.0010 & 0.0023 \\
\hline Sodium & 2.44 & 4.05 & 7.79 & 3.01 & 4.79 & 8.23 \\
\hline Strontium & 0.0424 & 0.544 & 0.353 & 0.0429 & 0.571 & 0.352 \\
\hline Thallium & $<0.0002$ & 0.0005 & 0.00029 & $<0.0004$ & 0.0008 & $<0.0004$ \\
\hline Vanadium & $<0.0002$ & 0.0509 & 0.0317 & 0.0005 & 0.0769 & 0.0390 \\
\hline Zinc & 0.0057 & 0.0058 & 0.0064 & 0.0210 & 0.0334 & 0.0285 \\
\hline
\end{tabular}

Table 13. Concentration $(\mu \mathrm{g} / \mathrm{L})$ of arsenic and selenium species measured in site-collected waters.

\begin{tabular}{|l|l|l|l|l|}
\hline \multirow{2}{*}{ Sample } & As(III) & As(V) & Se(IV) & Se(VI) \\
\cline { 2 - 6 } & \multicolumn{5}{|c|}{ Concentration $(\mu \mathrm{g} / \mathrm{L})$} \\
\hline Emory River & $<2$ & $<2$ & $<2$ & $<2$ \\
\hline Sluice Channel & $<2$ & 60.3 & 4 & $<2$ \\
\hline Stilling Pond Effluent & $<2$ & 14.8 & 4.7 & 3.7 \\
\hline
\end{tabular}




\section{Conclusions:}

- Fly ash collected from three locations (original source pile, the Emory River, and sluice channel) contained elevated concentrations of several metals relative to local soils and sediments, including arsenic, selenium, and barium.

- Fly ash has been modified by its residence in the Emory River in the following ways:

o Net carbon and nitrogen profiles changed to represent accumulated natural organic matter present in the river.

o Manganese in the source pile transformed from an oxidized to reduced state. Iron species were stable as Fe(III) oxides, and did not change.

o Approximately 30 to $50 \%$ of total selenium transformed from selenite (Se(IV)) to more reduced Se species after the ash was deposited in the river. Naturally forming organoselenium, present as Se(II), may be among these reduced Se forms. No evidence of selenate was found in the ash pulled from the Emory River or the sluice channel.

o Fly ash arsenic remained predominantly as $\mathrm{As}(\mathrm{V})$, with evidence of some slight shift to reduced arsenic-thiol species.

- Selenate was only found in the waters collected from the stilling pond. Given that selenate was not detected in any of the systems preceding the stilling pond, it is hypothesized that selenium is photooxidized due to the exposure of surface waters to direct sunlight. More investigations are warranted to resolve this mechanism. 


\section{Physical and Chemical Characterization of Elutriate}

This chapter describes the experimental results of the extended effluent elutriate tests (EET) performed on ash materials collected from the Emory River and the sluice channel in J une 2009, as described in Chapter 2. EET experiments were constructed as described in Chapter 3.

\section{Methods}

Total and dissolved metals were determined as described in Chapter 4. Arsenic (III) and (V) and selenium (IV) and (VI) were also determined as described in Chapter 4. Dissolved organic and inorganic carbon was determined using a Shidmadzu TOC-V analyzer with a catalytic combustion technique. Solid phase speciation of As and Se in elutriate sediments was conducted using $\mu$-XANES as described previously in Chapter 4. Solid phase speciation of bulk metals was conducted using XANES, also described in Chapter 4. Elutriate particle size distributions were determined using a Beckman Coulter LS Series 100Q laser particlesizer generally in accordance with the procedure described by Poppe et al. (2000). The LS100Q measures particles from $0.4 \mu \mathrm{m}$ to $900 \mu \mathrm{m}$ in size.

Geochemical modeling was conducted using the software Visual Minteq (v. 2.61; Gustafsson 2009) to calculate the speciation of dissolved constituents in solution based on their thermodynamic solubility and complexation constants. These calculations focused on Se, As, Mn, and Cr. Elemental constituents were input into the program using the analytically determined concentrations as redox couples: Se was evaluated using the redox couple $\mathrm{HSeO}^{3-} / \mathrm{SeO}_{4}{ }^{2-}$; As using the $\mathrm{HAsO}_{3} / \mathrm{AsO}_{4}$ couple; $\mathrm{Mn}$ using $\mathrm{Mn}(\mathrm{II}), \mathrm{Mn}(\mathrm{III})$, and $\mathrm{Mn}(\mathrm{IV})$; and $\mathrm{Cr}$ using the $\mathrm{Cr}^{2+} \mathrm{Cr}(\mathrm{OH})_{2}{ }^{2+}$ couple, $\mathrm{Cr}(\mathrm{VI})$ was not found to be significant $\mathrm{Cr}$ species. An appropriate redox couple for Hg was not defined in the program, and thus was not calculated. For selenium and arsenic metals, the concentrations of species were defined based on the analytical determinations - Se (IV) and As(V) were input based on the HPLC-ICP-MS analysis. The difference in selenium or arsenic concentration (total metals subtracted by concentrations of metal species) was assigned to Se(VI) and As(III), respectively, representing a "worse-case" scenario calculation, even though neither of the latter species were detected in solution. The Cr input was the divalent cation species. 
Speciation calculations were performed by allowing the $\mathrm{pH}$ of the system to sweep from $\mathrm{pH} 4$ to 10. For each set of calculations, system Eh was fixed at either $-300,-100,100$, or $300 \mathrm{mV}$. The calculations were conducted to test both the $\mathrm{pH}$ and redox boundary conditions in which selenium and arsenic may undergo redox changes to their more toxic species in solution.

Software constraints did not allow for the simultaneous input of all dissolved constituents in the elutriate systems. Therefore, As, Se, Mn, and Cr were tested in separate calculations in the presence of one additional constituent, added at the concentration detected in solution, to determine if the added constituent underwent a specific reaction (e.g., formed new pairs or precipitate solids). For each one of these calculations, and all subsequent calculations described in this chapter, solution anions were inputted based on the analytically determined concentrations (TIC concentrations were input as carbonate anions). For all calculations, computed species were allowed to over-saturate and produce secondary precipitates if thermodynamically appropriate. In addition, these secondary precipitates were allowed to further re-dissolve based on thermodynamic solubility.

Preliminary calculations showed new species produced when Se was modeled in the presence of either $\mathrm{Zn}, \mathrm{Ni}$, or $\mathrm{Cu}$, yet the predicted activities of these species were so low as to have virtually no effect on the activity of the major species predicted.

\section{Results}

The purpose of the elutriate tests described above was to simulate the extent of metals in the fly ash samples (Table 5) that might be released during dredging activities under extreme conditions. Dissolved metals concentrations in the aerated and nitrogen bubbled (oxic and anoxic regime) experiments for the elutriate tests created with Emory River and sluice channel waters and fly ash are shown in Figures 23-28.

The solution data (Figures 23-26) show the concentration profiles of dissolved elements over the 240 -hr elutriate test. The dissolved element concentrations span about 5 orders of magnitude with the major cations sodium, magnesium, potassium, and calcium remaining relatively constant over the $240-\mathrm{hr}$ period at 1 - $10 \mathrm{mg} / \mathrm{L}$ or above. Some elements (e.g., beryllium, thallium, cadmium, chromium, nickel, and silver) were never detected above the $1 \mu \mathrm{g} / \mathrm{L}$ reporting limit, while others like barium, arsenic, manganese, selenium, and vanadium exhibit trends in 
concentration over the EET experiment. For clarity, plots containing expanded scales of these latter elements are shown in Figures 27 and 28. These data show that arsenic concentrations in the Emory River elutriate reached about $50 \mu \mathrm{g} / \mathrm{L}$, while arsenic concentrations in the sluice channel elutriates exceeded $80 \mu \mathrm{g} / \mathrm{L}$ by $240 \mathrm{hr}$ of gas bubbling. Vanadium concentrations remained approximately constant in the sluice channel elutriates over the 10-day experiment, while increasing in the Emory River elutriates from about 10 to over $30 \mu \mathrm{g} / \mathrm{L}$. Selenium concentrations increased in all four elutriate systems over the 10-day experiment, from $<1$ to $5 \mu \mathrm{g} / \mathrm{L}$ in the Emory River systems and from 5 to $13 \mu \mathrm{g} / \mathrm{L}$ in the sluice channel elutriates.

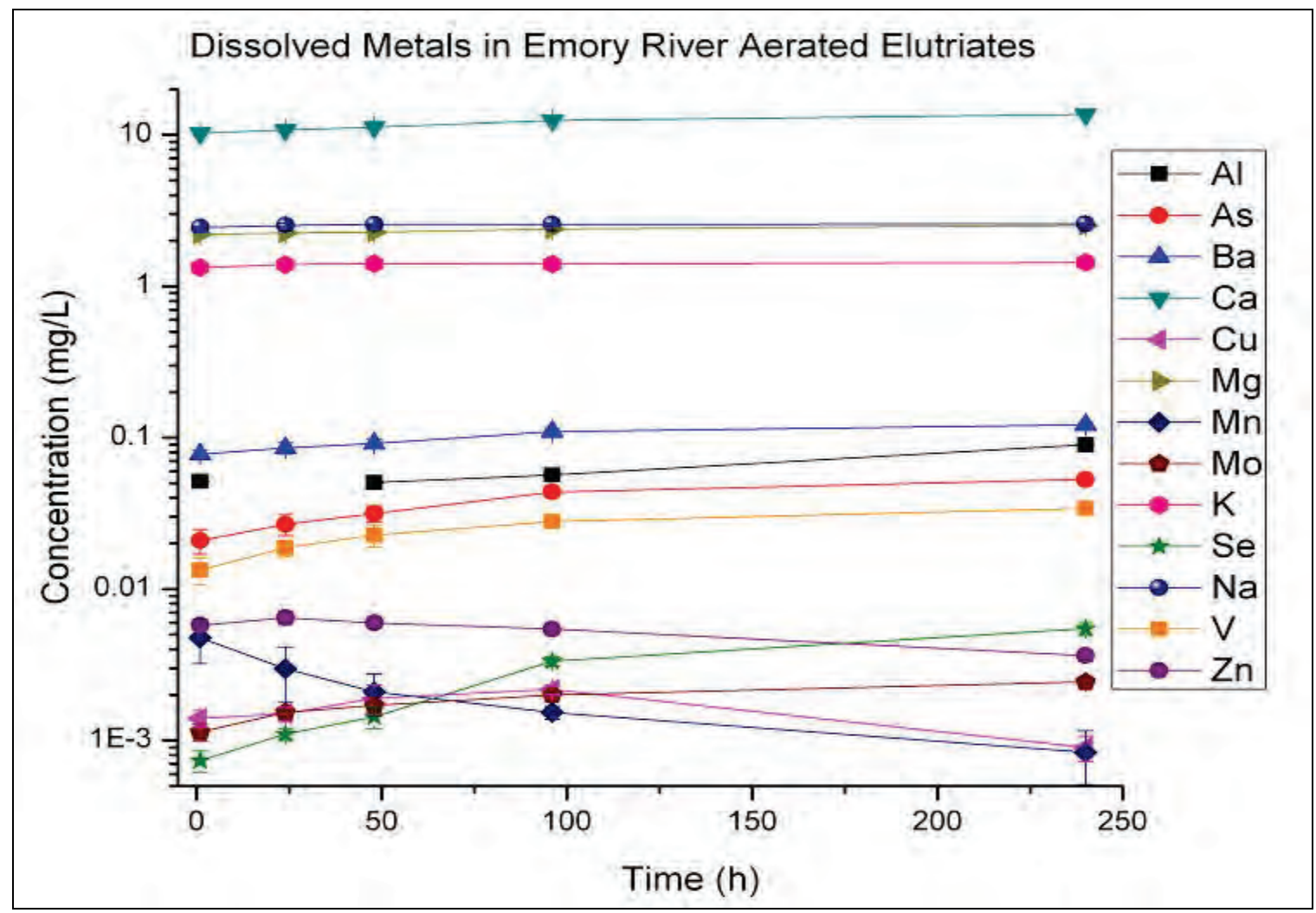

Figure 23. Dissolved metals concentrations in the Emory River oxic regime (i.e., bubbled with air) elutriate as a function of time. Error bars represent the standard deviation of triplicate elutriate preparations. 


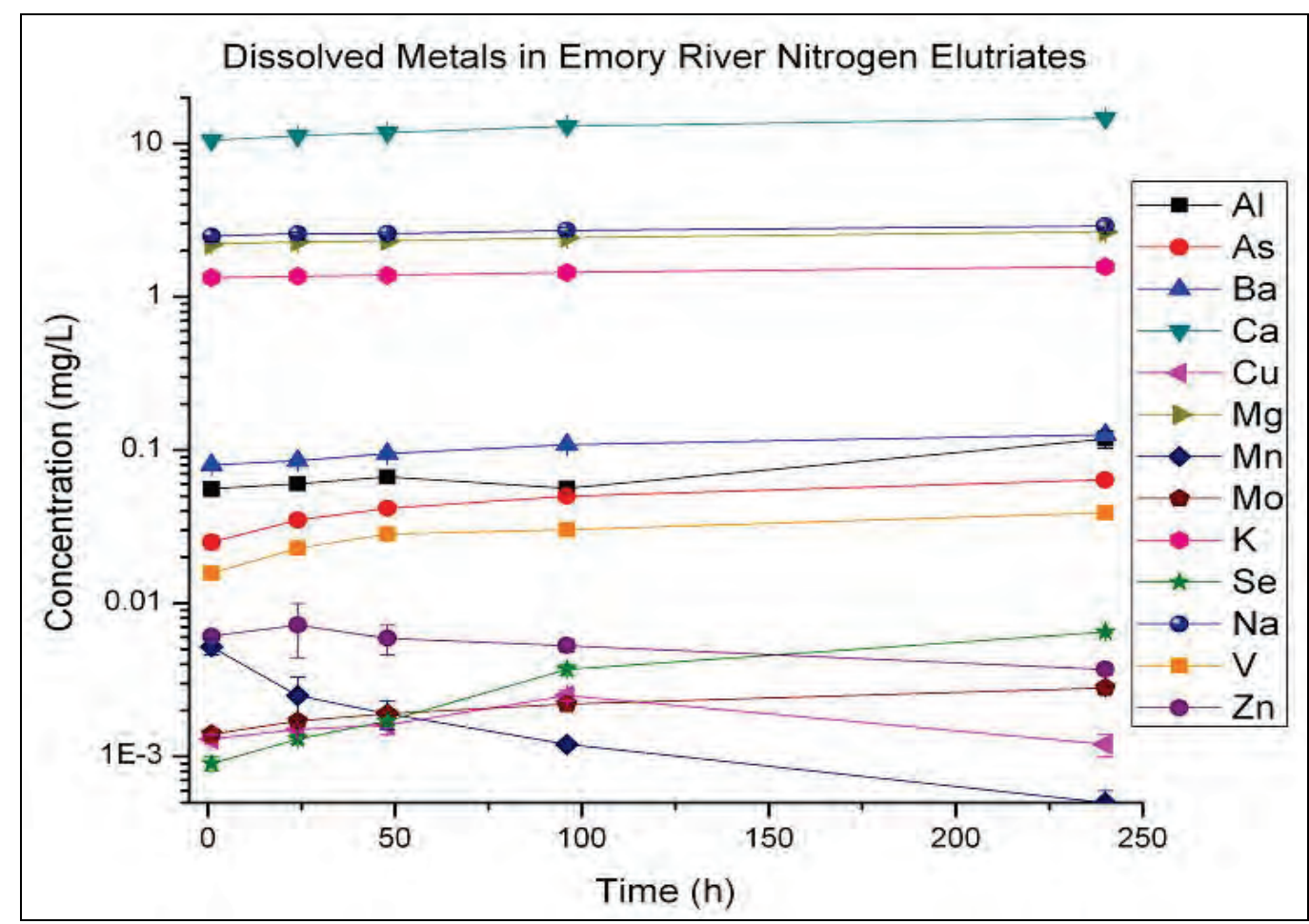

Figure 24. Dissolved metals concentrations in the Emory River anoxic regime (i.e., bubbled with nitrogen gas) elutriate as a function of time. Error bars represent the standard deviation of triplicate elutriate preparations.

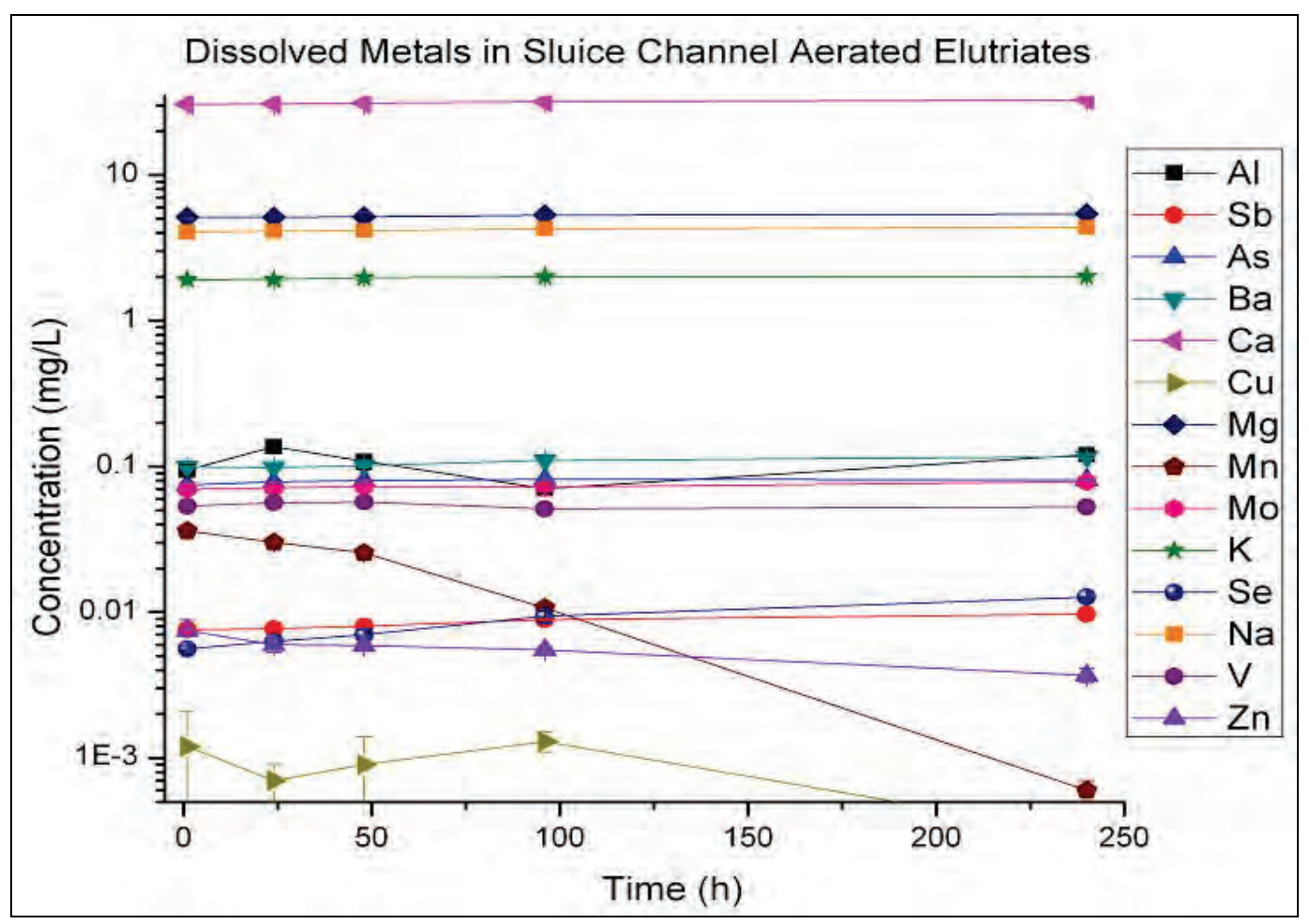

Figure 25. Dissolved metals concentrations in the sluice channel oxic regime elutriate as a function of time. Error bars represent the standard deviation of triplicate elutriate preparations. 


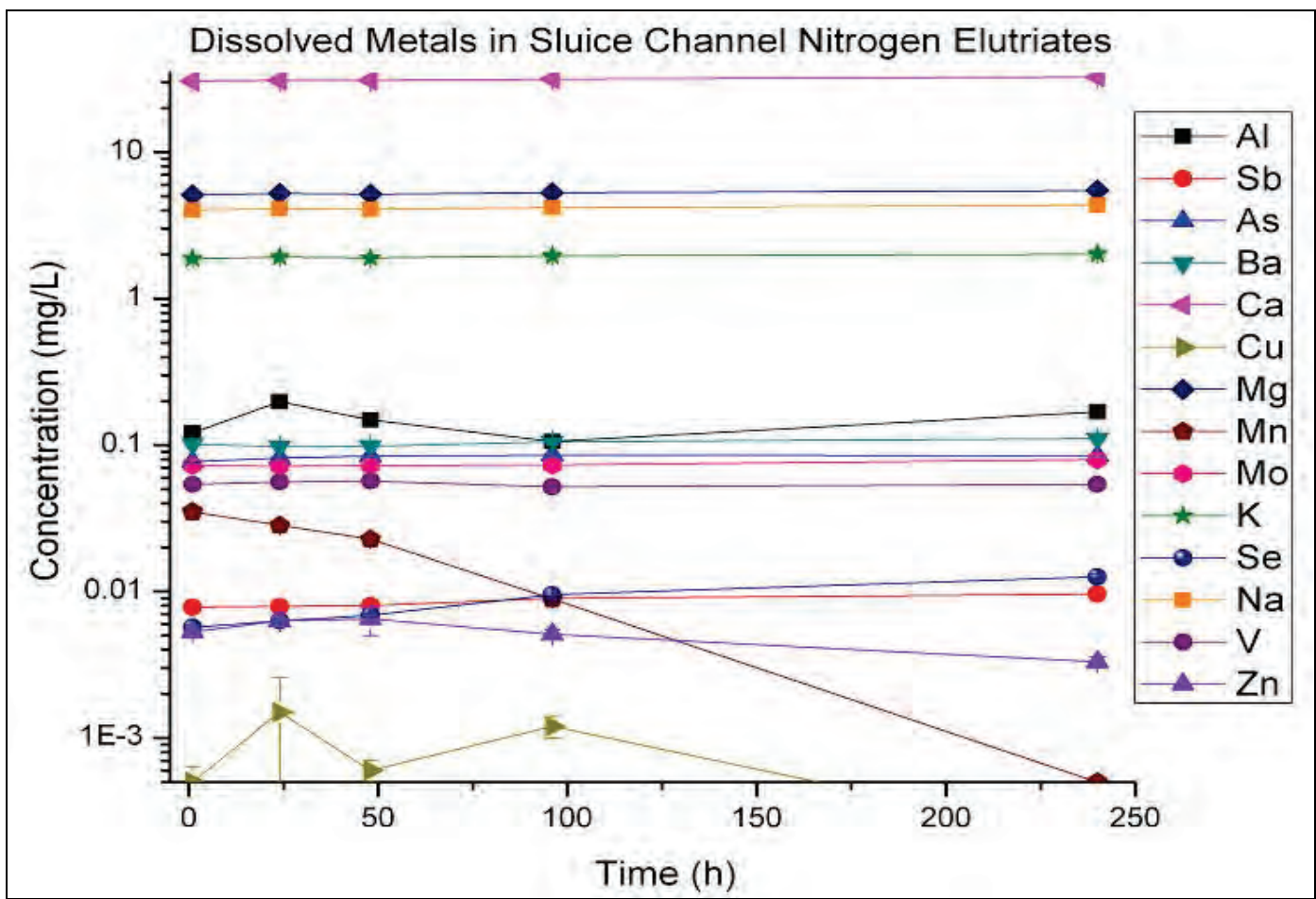

Figure 26. Dissolved metals concentrations in the sluice channel anoxic regime elutriate as a function of time. Error bars represent the standard deviation of triplicate elutriate preparations.

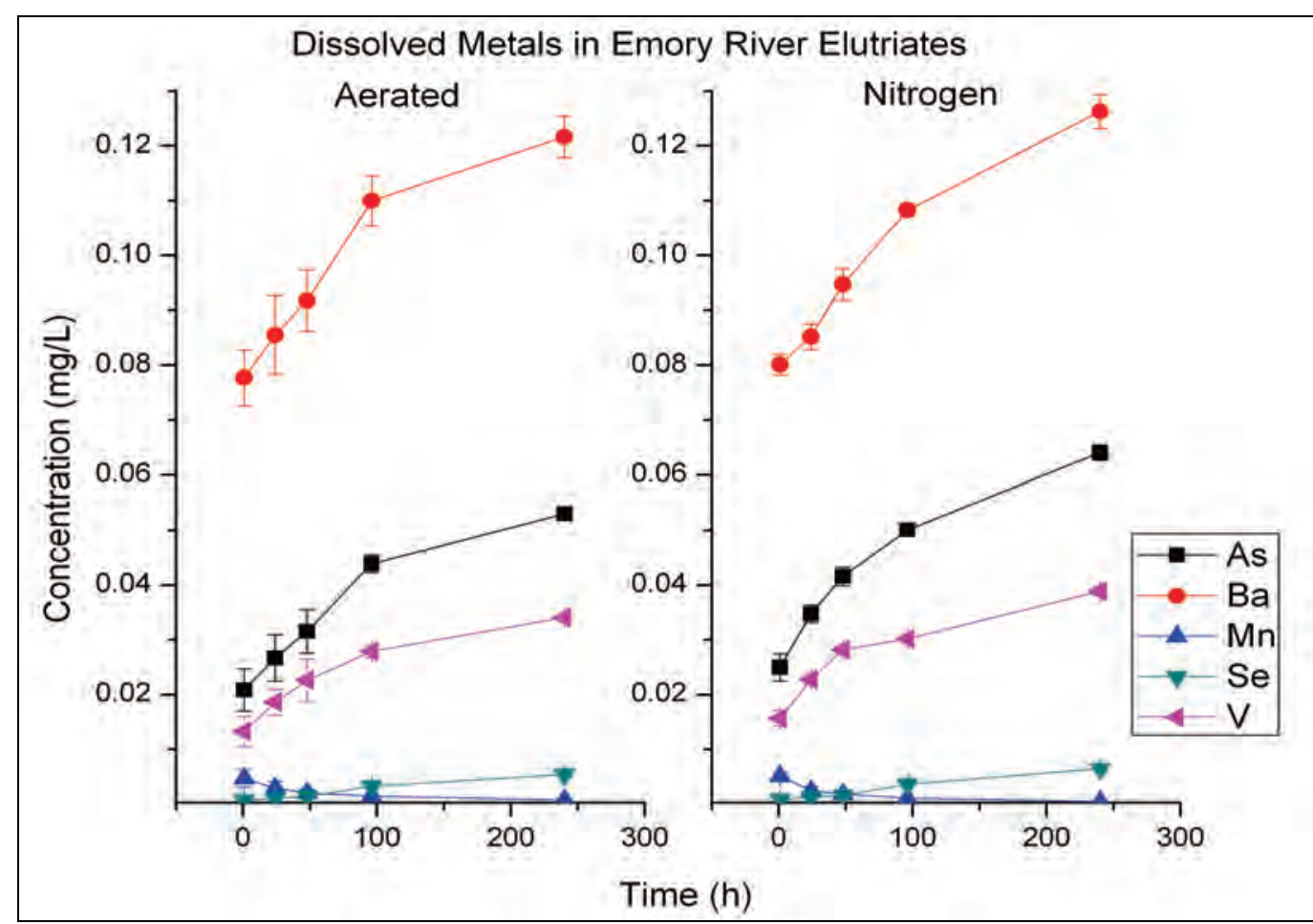

Figure 27. Expanded view of dissolved metals concentrations in the Emory River elutriates as a function of time. Error bars represent the standard deviation of triplicate elutriate preparations. 


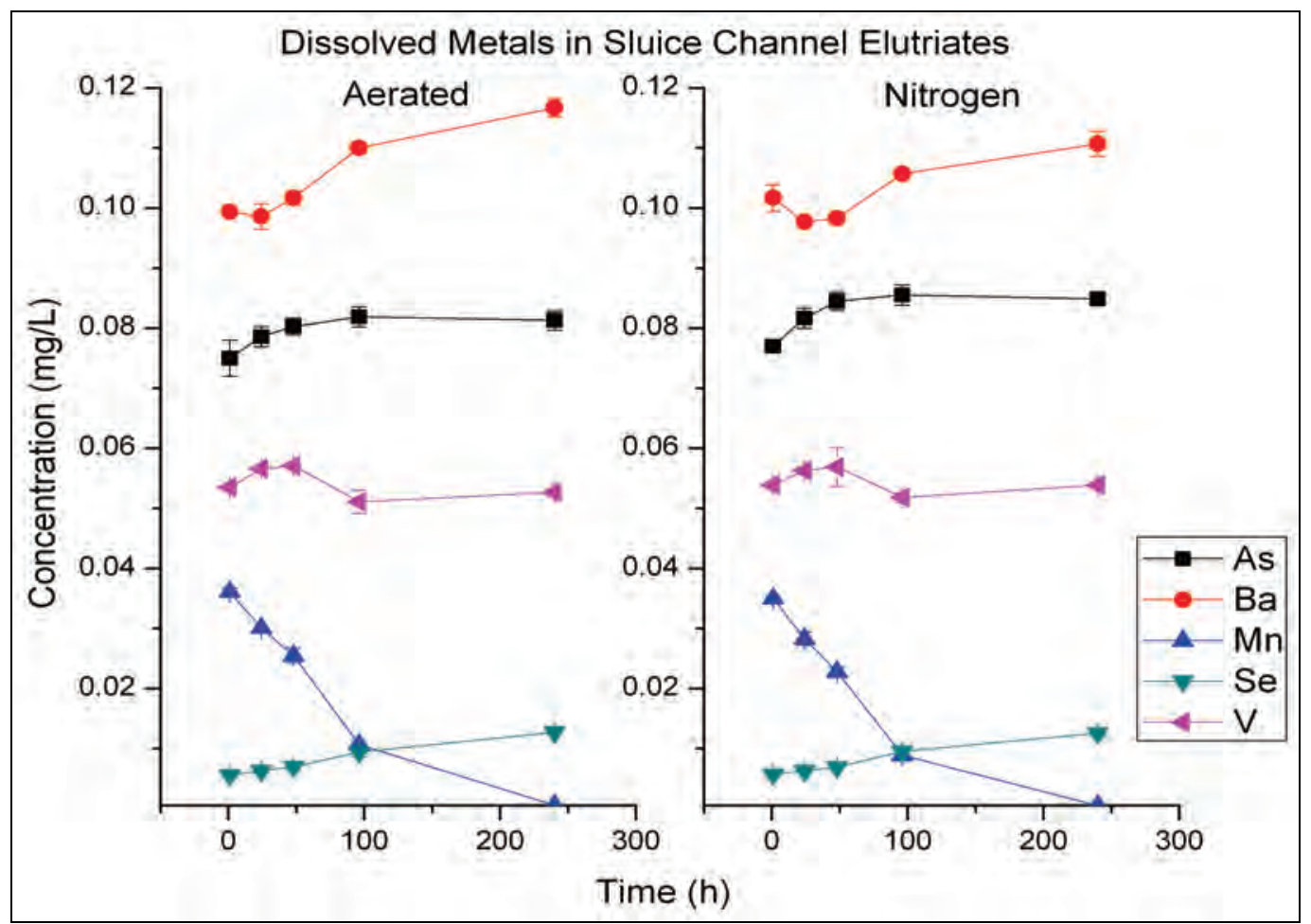

Figure 28. Expanded view of dissolved metals concentrations in the sluice channel elutriates as a function of time. Error bars represent the standard deviation of triplicate elutriate preparations.

The total concentration of dissolved organic carbon (Figure 29) remained relatively constant at $3 \mathrm{mg} / \mathrm{L}$ over the first $96 \mathrm{hr}$ of the EET, followed by a substantial increase by $240 \mathrm{hr}$ in both the Emory River and sluice channel elutriates. The increase in total organic carbon between 96 and $240 \mathrm{hr}$ suggests that the efforts to keep the ash materials suspended in solution over the EET stimulated microbial activity, which after an initial lag phase, may have exhibited an effect on the system. Note that this behavior is equally apparent in both the oxic and anoxic regime (air- and nitrogenbubbled) systems. Expected metal behaviors based on geochemical speciation modeling are discussed later in this section.

In addition to the total dissolved concentrations, HPLC-ICP-MS was utilized to determine the concentrations of As(III) and As(V) (Figure 30) and Se(IV) and Se(VI) (Figure 31) in solution over the course of the 10-day elutriate experiment. These data show possible shifts in metal speciation with time, particularly as affected by the oxic and anoxic regime treatments on the systems. The data demonstrate the persistent redox stability of the elutriate solution over the course of the 10-day study. For example, no $\mathrm{Se}(\mathrm{VI})$, e.g., selenate, was detected in solution in any of the elutriates, even after 10 days of oxic regime treatment. The speciation data 
show the measured increase in dissolved selenium concentration was attributed solely to the increase in Se(IV), e.g., selenite. This suggests that even with aeration, the redox potential of the system is poised such that selenite is the stable species. Similarly, speciation data showed that the measured increase in total arsenic was attributed to an increase in arsenic (V), e.g., arsenate. No As(III) was observed in these systems, even in the anoxic regime experiments.

Figures 32 and 33 show the measured Eh and $\mathrm{pH}$ values for the 10-day elutriate experiments. Little variability is observed between the various oxic and anoxic regime elutriates. Variability in Eh drops approximately $50 \mathrm{mV}$ in all the systems from 1 to $24 \mathrm{hr}$, followed by a slow increase in Eh to near $275 \mathrm{mV}$ by $240 \mathrm{hr}$. The $\mathrm{pH}$ of all the elutriates held between 7.25 and 7.75 until $48 \mathrm{hr}$, after which the $\mathrm{pH}$ increases approximately 1.5 units. Due to the low concentrations of redox-active elements (e.g. Fe, $\mathrm{Mn}$ ) in these systems, it is hypothesized that the redox potential may be strongly influenced by organic matter and oxygen produced by biological activity. Furthermore, the increase in $\mathrm{pH}$ could be due to the biological production of alkalinity. Biological production of oxygen could also explain the similarities observed between the oxic and anoxic regime elutriate.

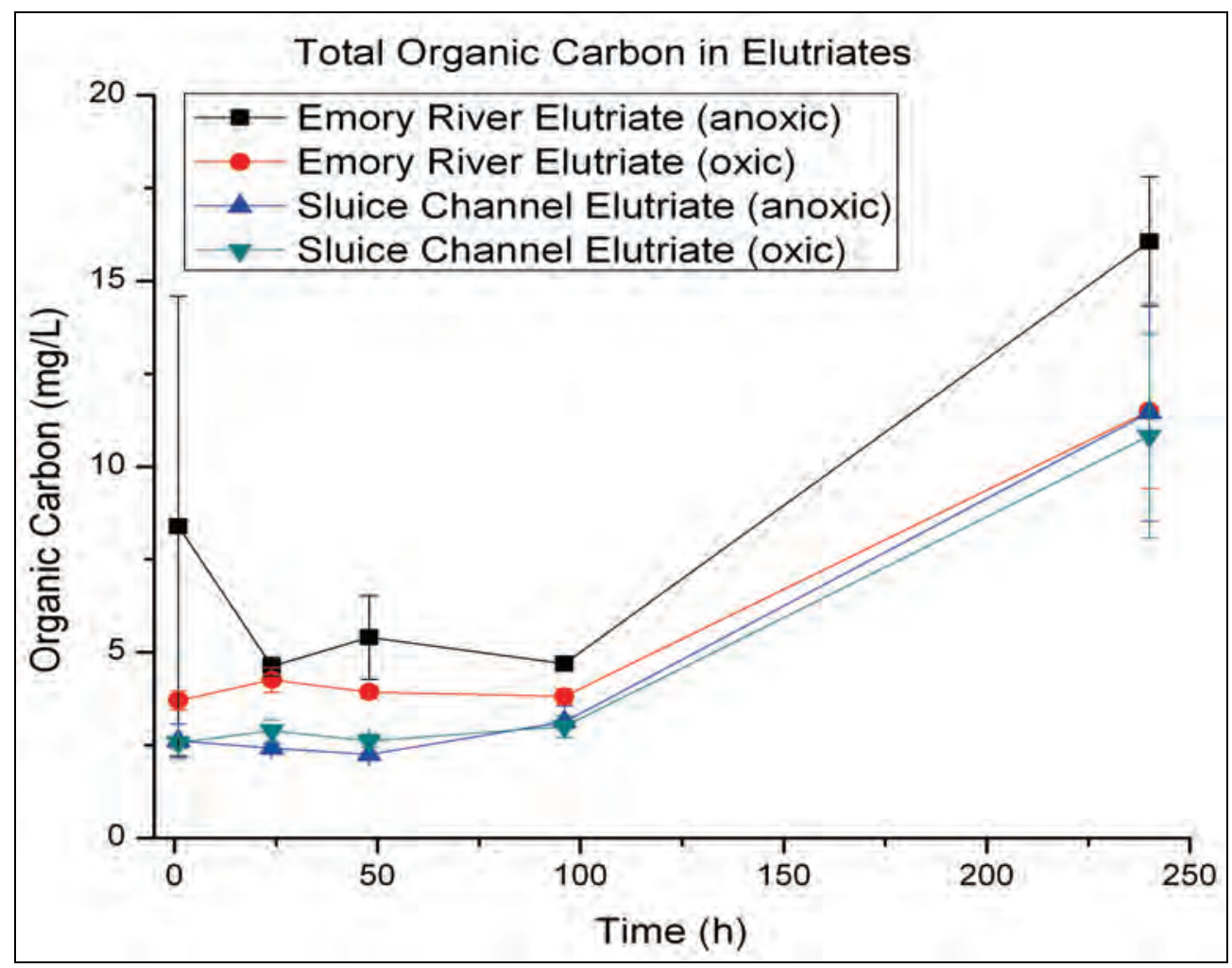

Figure 29. TOC concentrations in elutriate waters over the 10-day preparation period. Error bars represent the standard deviation of triplicate elutriate preparations. 


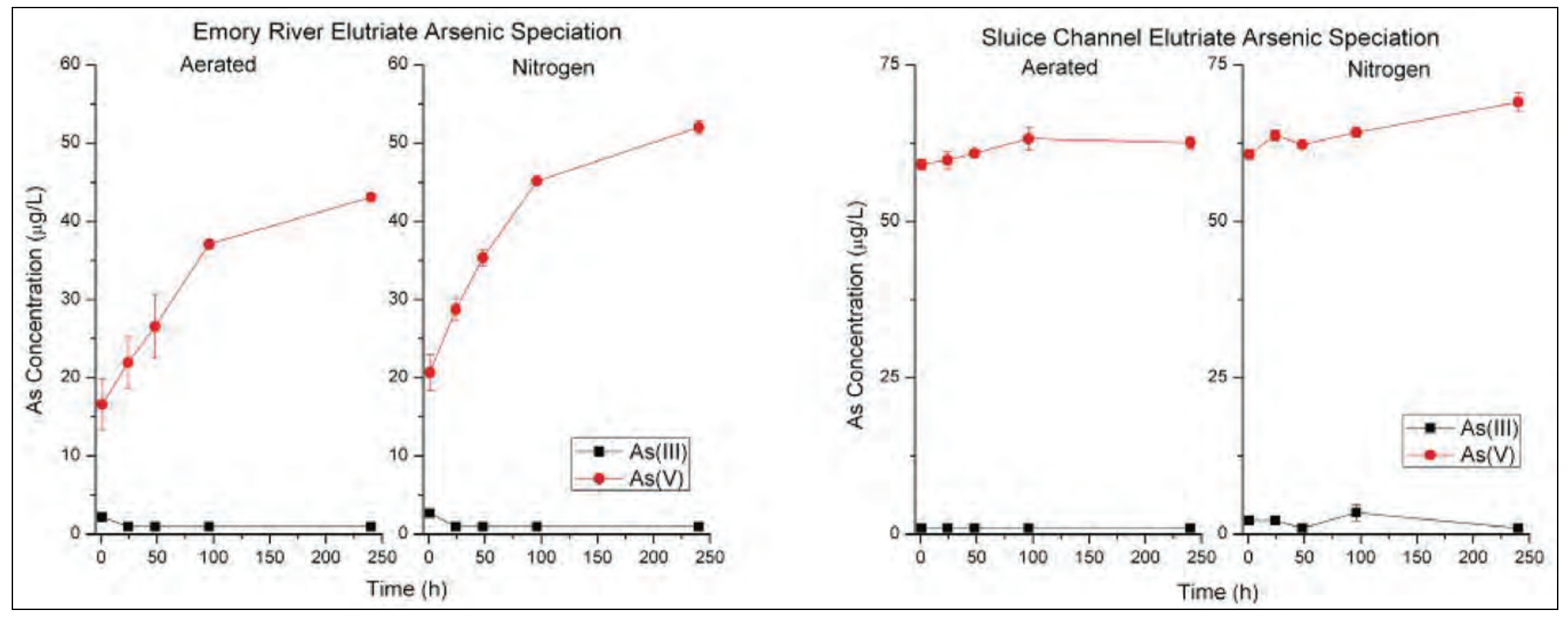

Figure 30. Arsenic speciation in the oxic and anoxic regime Emory River and sluice channel elutriate experiments. Error bars represent the standard deviation of triplicate elutriate preparations. Non-detect concentrations of arsenic species $(<2 \mu \mathrm{g} / \mathrm{L}$ ) are plotted as one-half the detection limit (i.e. $1 \mu \mathrm{g} / \mathrm{L}$ ). 


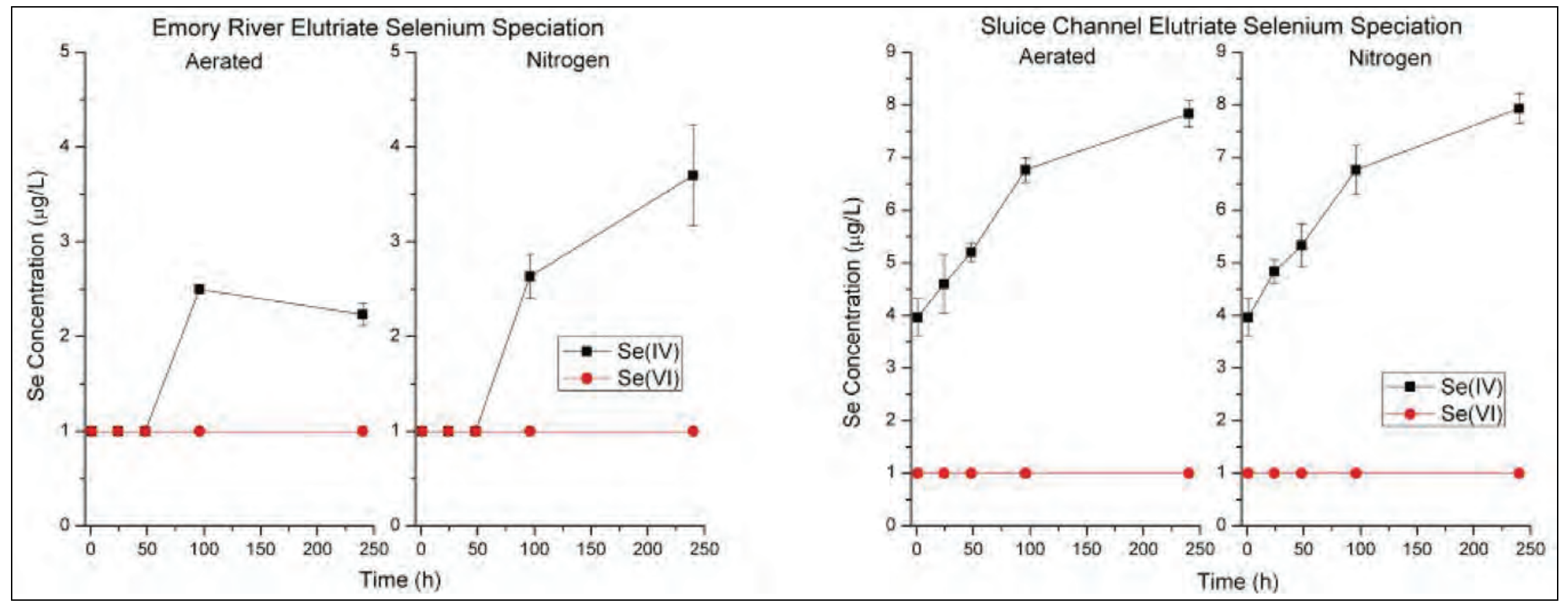

Figure 31. Selenium speciation in the oxic and anoxic regime Emory River and sluice channel elutriate experiments. Error bars represent the standard deviation of triplicate elutriate preparations. Non-detect concentrations of selenium species ( $<2 \mu \mathrm{g} / \mathrm{L}$ ) are plotted as one-half the detection limit (i.e. 1 $\mu \mathrm{g} / \mathrm{L})$. 


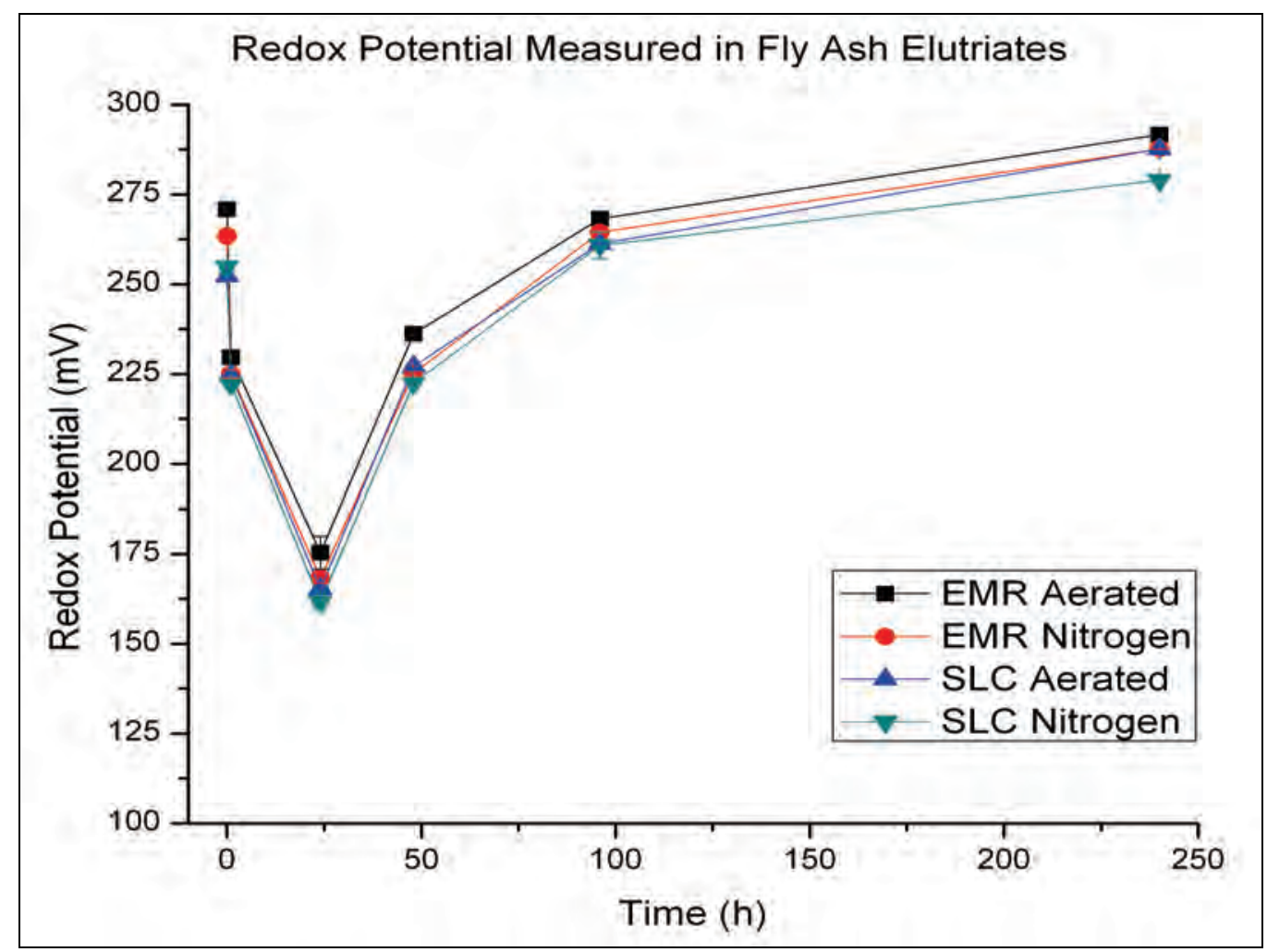

Figure 32. Eh values measured over the course of the 10-day elutriate experiment. Measured Eh values have been adjusted to compensate for the platinum electrode having a silver:silver chloride reference electrode ( $200 \mathrm{mV}$ added).

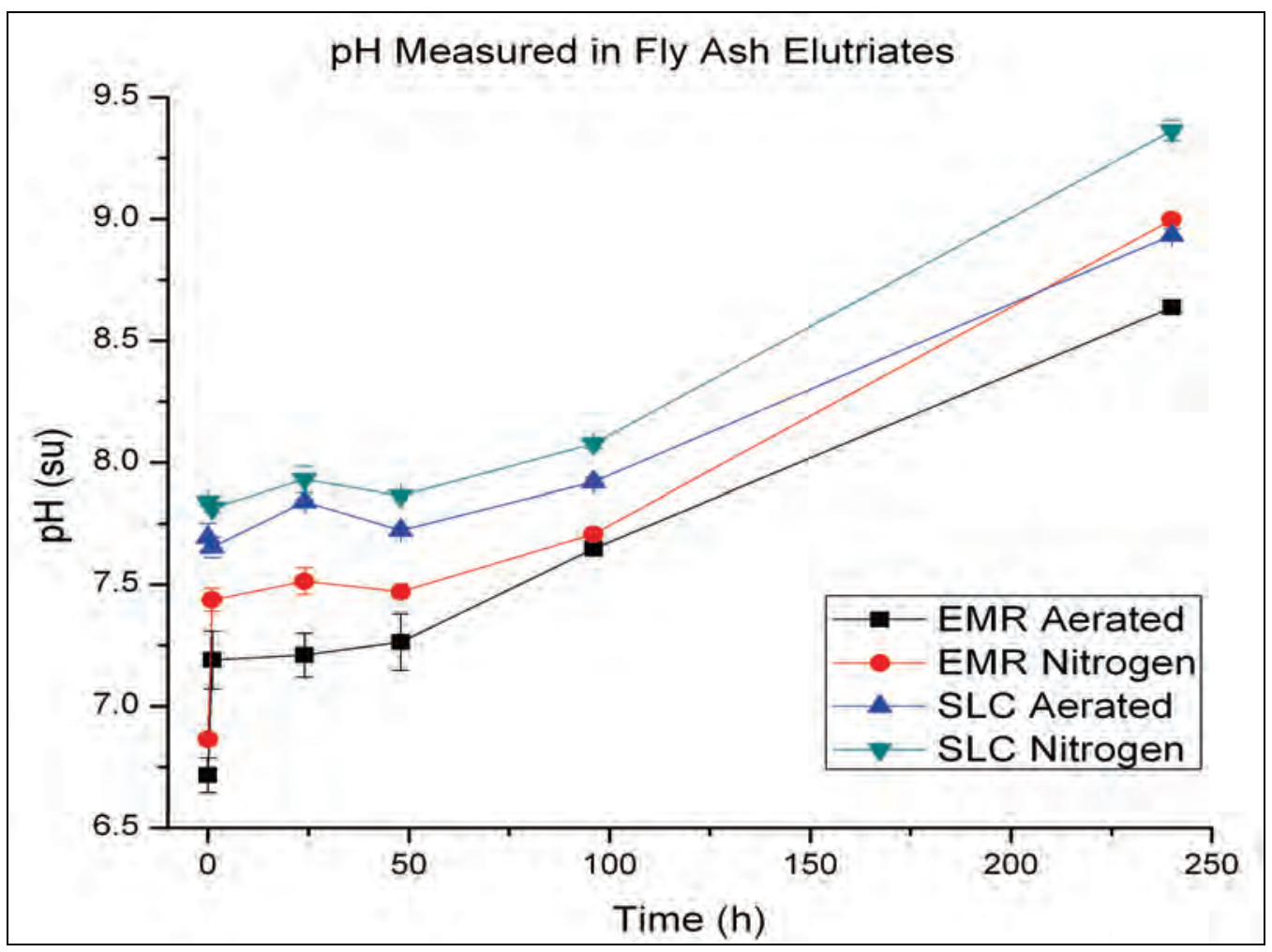

Figure 33. $\mathrm{pH}$ values measured over the course of the 10-day elutriate experiment. 


\section{Solid-phase metal speciation}

BulkXANES analysis for Mn in the ash samples (see Figure 6) shows the effect of the extensive air bubbling (oxic regime treatment) on the ash. The data show a noticeable shift in the Mn XANES spectra, from containing predominantly reduced $\mathrm{Mn}$ species to a partially oxidized $\mathrm{Mn}$ species in both the Emory River and sluice channel systems. Therefore, this provides evidence that the system did undergo oxidation to some extent, even though the solution redox remained relatively constant. This result also explains the reduction in dissolved Mn over the 240-hr period as shown in Figures 27 and 28.

Interestingly, bulk FeXANES (see Figure 7) showed relatively minor change in the Fe redox state over the $240-\mathrm{hr}$ oxic regime period. The elutriate Fe species (see Table 11) remained relatively stable, similar to that of the source pile. Thus, the data indicate that the $\mathrm{Mn}$ in the ash was responsive to the treatment conditions, not the Fe phases. Figures 34 and 35 show the stability diagrams for Fe and Mn oxides, respectively. Based on the $\mathrm{pH}$ and redox measurements (Figures 32 and 33), Fe(III) was expected to remain stable while EET conditions put the system close to the redox barrier between $\mathrm{Mn}$ (II) and Mn(IV). Similar observations have been

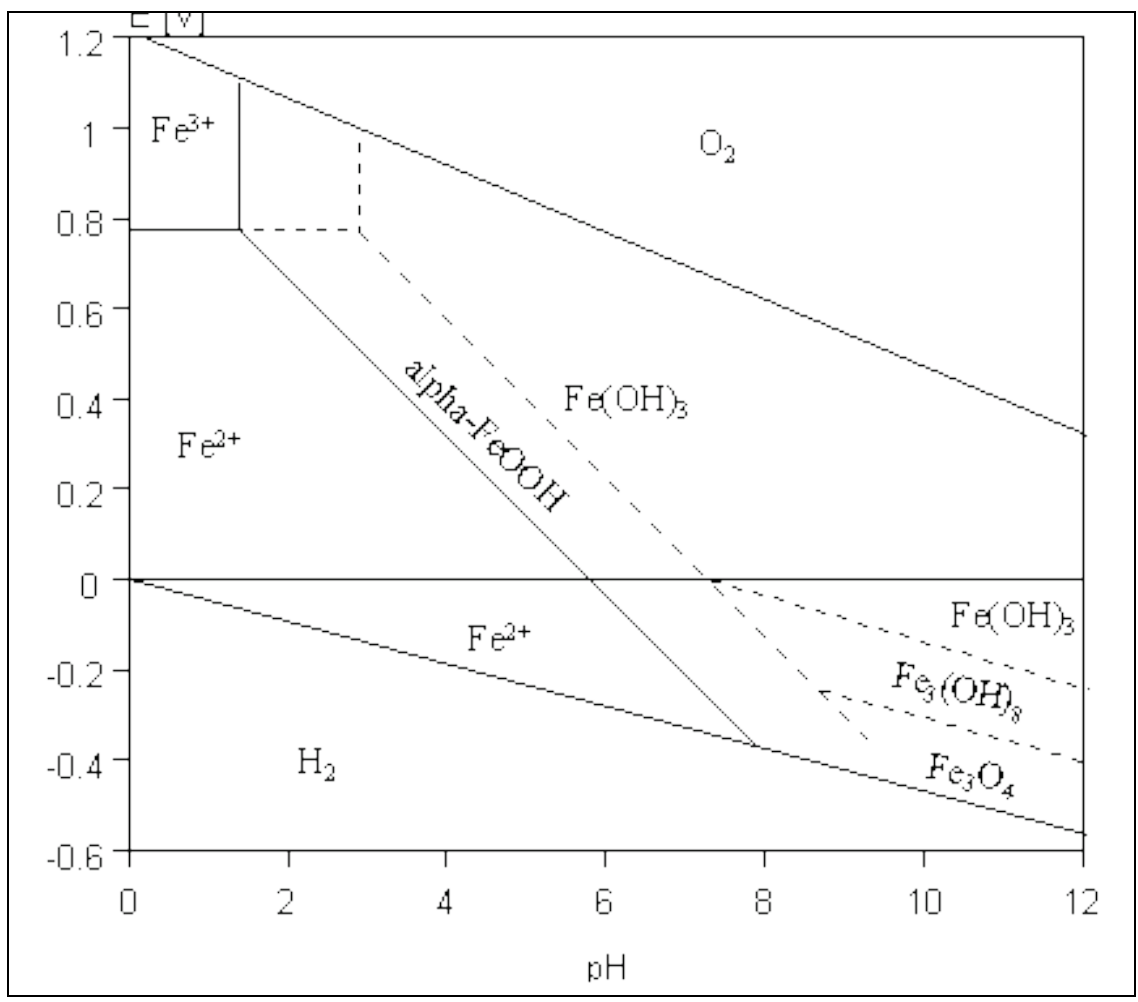

Figure 34. Pourbaix $(\mathrm{Eh}-\mathrm{pH})$ stability diagram for iron oxides. 


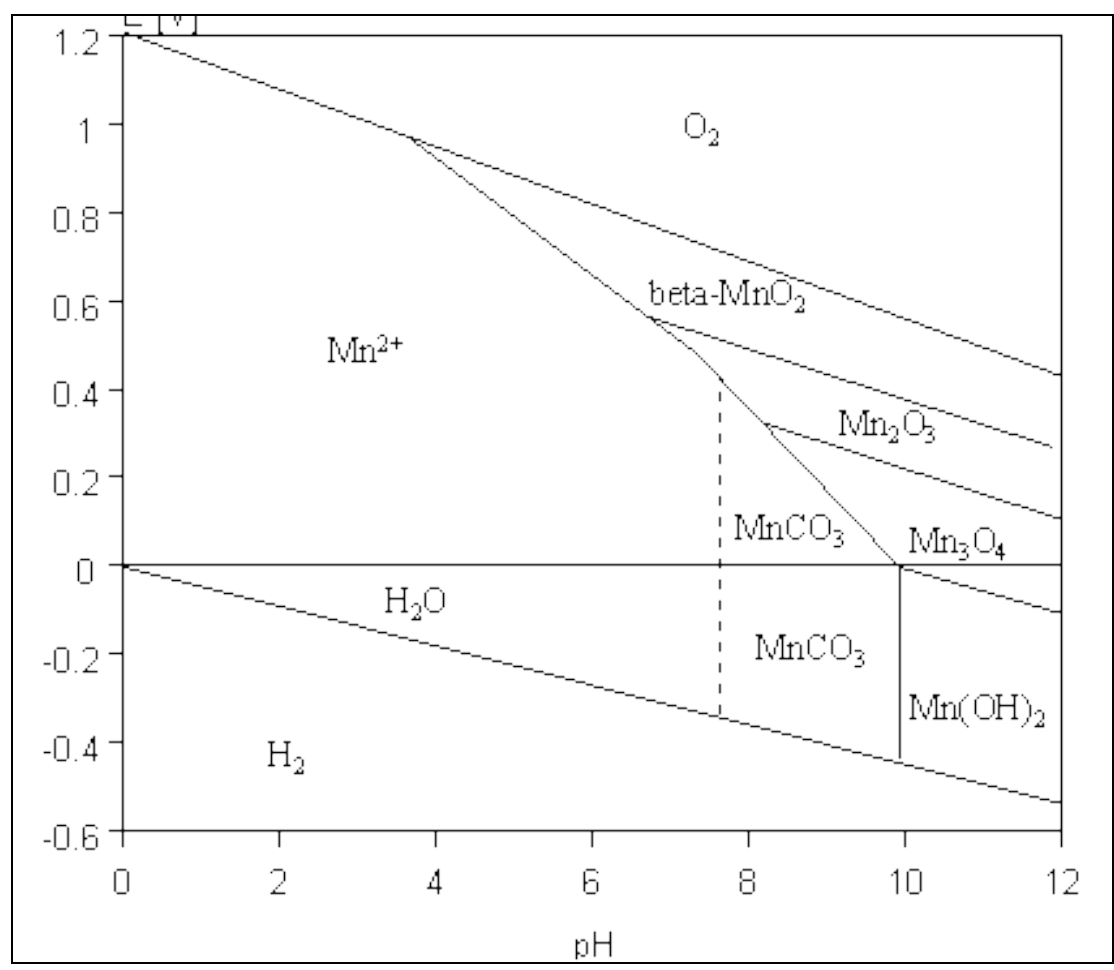

Figure 35. Pourbaix $(\mathrm{Eh}-\mathrm{pH})$ stability diagrams for manganese oxides.

previously reported for dissolved iron and arsenic species in poised redox systems (Bednar et al. 2005). Thus, in the Emory River and sluice channel systems, Mn serves as a more responsive indicator of potential shifts in the river conditions that may be associated with redox changes in Se and As. The formation of oxidized $\mathrm{Mn}$ in the elutriate solid samples is in agreement with the decrease in the amount of total dissolved Mn in the elutriate waters.

Bulk XANES analysis shows arsenic (Figure 36) in the EET systems mainly as the $\mathrm{As}(\mathrm{V})$ species after $240 \mathrm{hr}$ of oxic regime treatment. Evidence was also observed for 3 to $12 \%$ arsenite in the Emory River and sluice channel elutriates, respectively, whether treated with an oxic or anoxic regime. The data suggest increasing levels of arsenite as the ash moves from the river to the sluice channel through dredging, although the mechanism for this transformation is unclear.

Bulk XANES data (Figure 37) determined selenium speciation in the EET systems as approximately 40-80\% "reduced" selenium (probably organoselenium) and $20-60 \%$ selenite. This proportion of covalently bound Se is similar to that observed in Chapter 4, suggesting that this form of Se may be chemically stable in the river system. 
$\mu$-XANES analysis was conducted on sediments collected from the extended elutriate experiments after bubbling with gas for $240 \mathrm{hr}$. Given time constraints associated with this work, investigations focused on the sediments treated with the oxic regime (bubbled with air), to understand the potential for Se oxidation and $\mathrm{Se}(\mathrm{VI})$ release from the elutriate systems. The data showed that $\mathrm{As}(\mathrm{V})$ made up the predominant arsenic species in both the Emory River and sluice channel elutriates (Figure 38). Evidence was also found for As(III) in the $\mu$-XANES spectrum for one site on the sluice channel elutriate sediments, yet $\mathrm{As}(\mathrm{V})$ remained the major arsenic species in the ash.

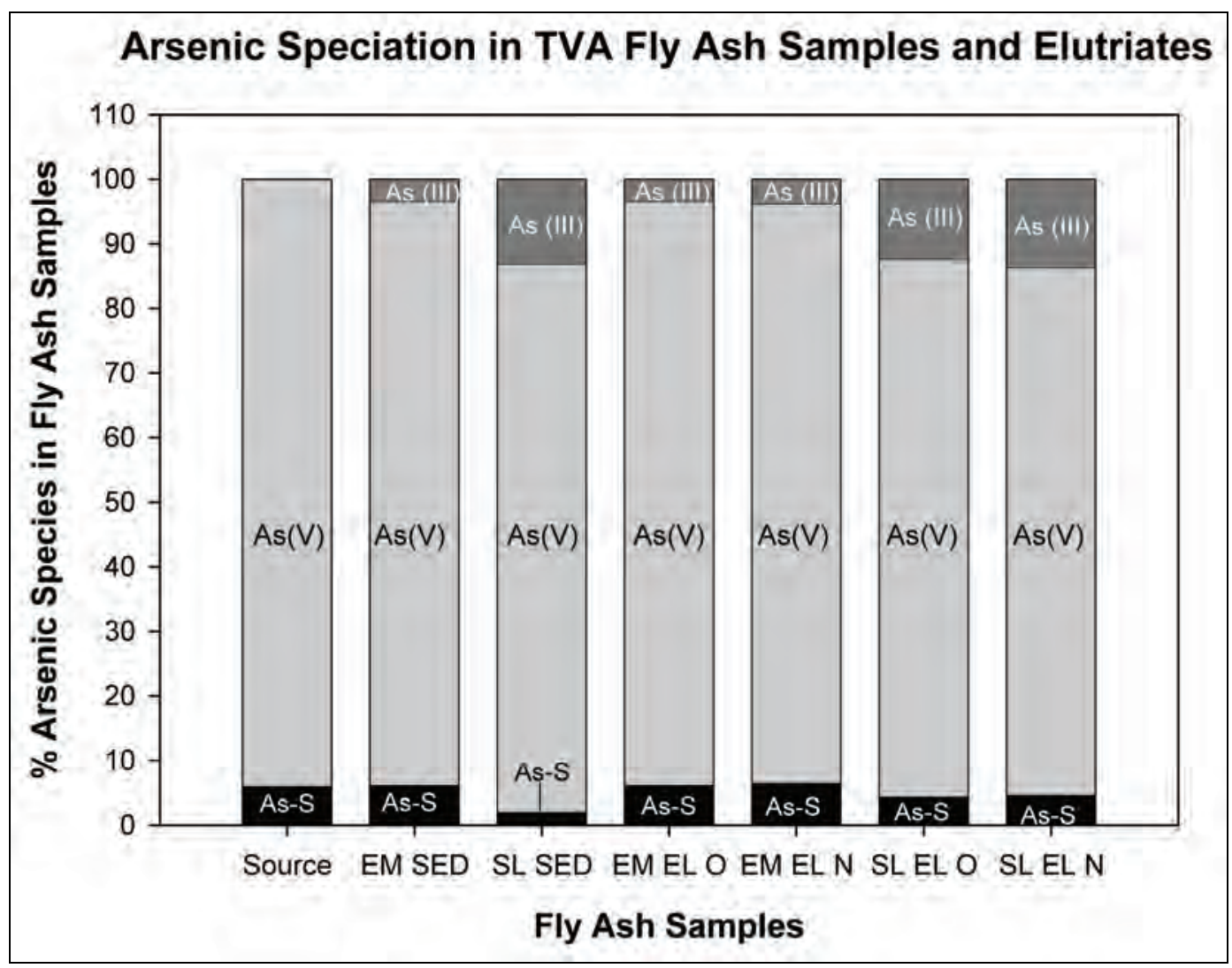

Figure 36. Results from linear combination fits of arsenic XANES data on bulk ash and elutriate samples. Source = source pile, EM SED = Emory River sediment, SL SED = sluice channel sediment, EM EL $\mathrm{O}=$ Emory River elutriate treated with oxic regime, EM EL N = Emory River elutriate treated with anoxic regime, SL EL $O=$ sluice channel elutriate treated with oxic regime, and SL EL N = Sluice Channel Elutriate treated with anoxic regime. As(V) = arsenate, $\mathrm{As}(\mathrm{III})=$ arsenite and As-S is arsenic bound to sulfur. 


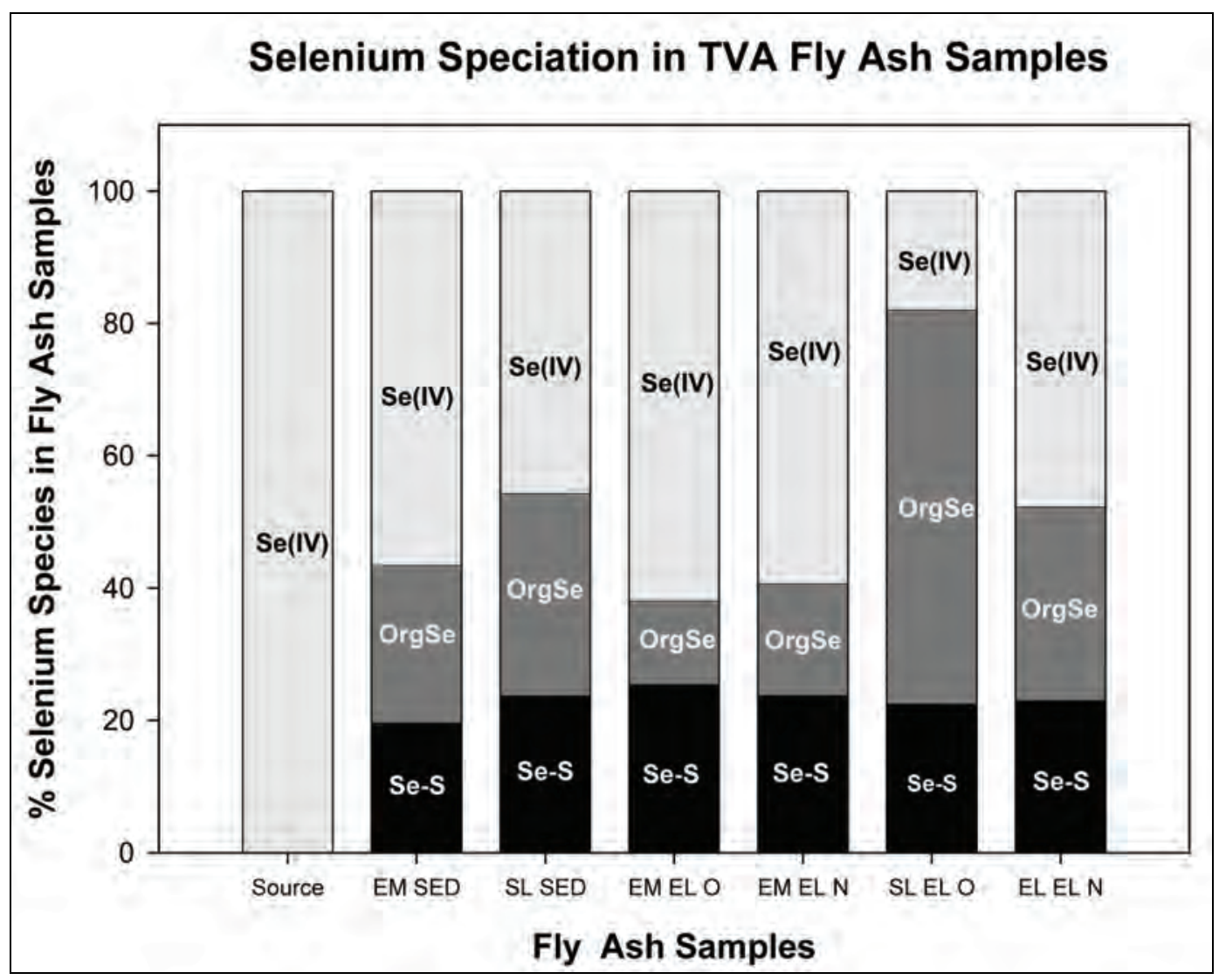

Figure 37. Results from linear combination fitting of selenium XANES on bulk Emory River and sluice channel ash and elutriate samples. Source = source pile, EM SED = Emory River sediment, SL SED = sluice channel sediment, EM EL O = Emory River elutriate treated with oxic regime, EM EL N = Emory River elutriate treated with anoxic regime, SL EL $0=$ sluice channel elutriate treated with oxic regime, and SL EL N = sluice channel elutriate treated with anoxic regime. $\mathrm{Se}(\mathrm{IV})=$ selenite, OrgSe $=\mathrm{Se}$ bound to thiol bearing $\mathrm{C}$-compounds, and $\mathrm{Se}-\mathrm{S}$ is selenium bound to sulfur. 


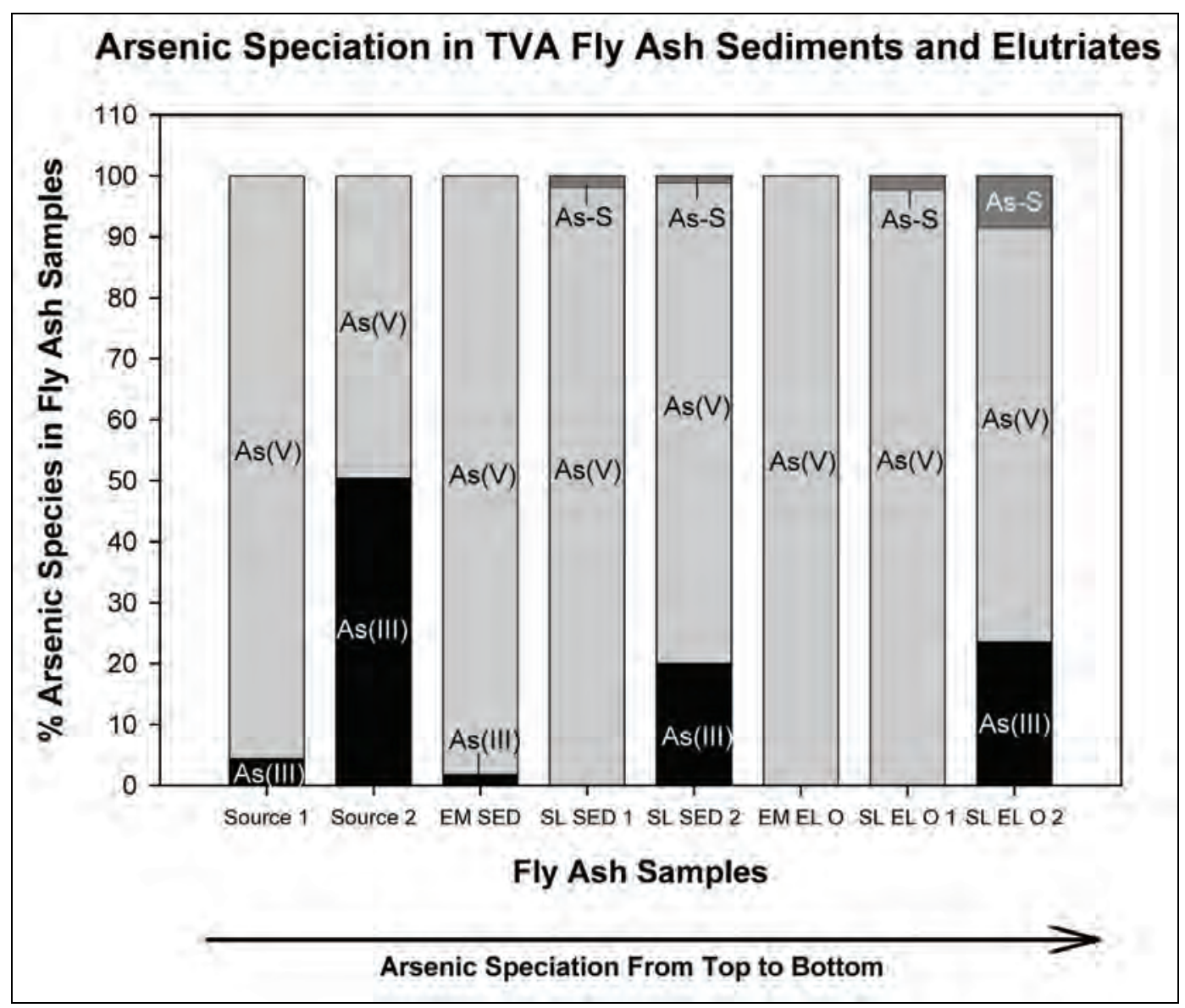

Figure 38. Results from linear combination fittings of $\mu$-XANES data collected at the arsenic $\mathrm{K}$ edge on discrete locations in the ash and treated with oxic regime elutriate samples. Fittings refer back to Figure 15.

Se $\mu$-XANES analysis of the Emory River elutriate sediments (Figure 39) showed evidence for both Se(IV) and "reduced" Se species (organoselenium as discussed previously). However, the presence of a micro-domain containing Se(VI) in the Emory River elutriate sediments confirms the possibility of obtaining Se(VI) through extended oxidation, although no $\mathrm{Se}(\mathrm{VI})$ was detected in solution during any of the elutriates. Thus, if Se(VI) was released from the elutriate sediments, it was released at concentrations undetectable at the 2-ppb limit. For the sluice channel elutriates, no evidence of Se(VI) was found, only mixtures of "reduced" Se and Se(IV). 


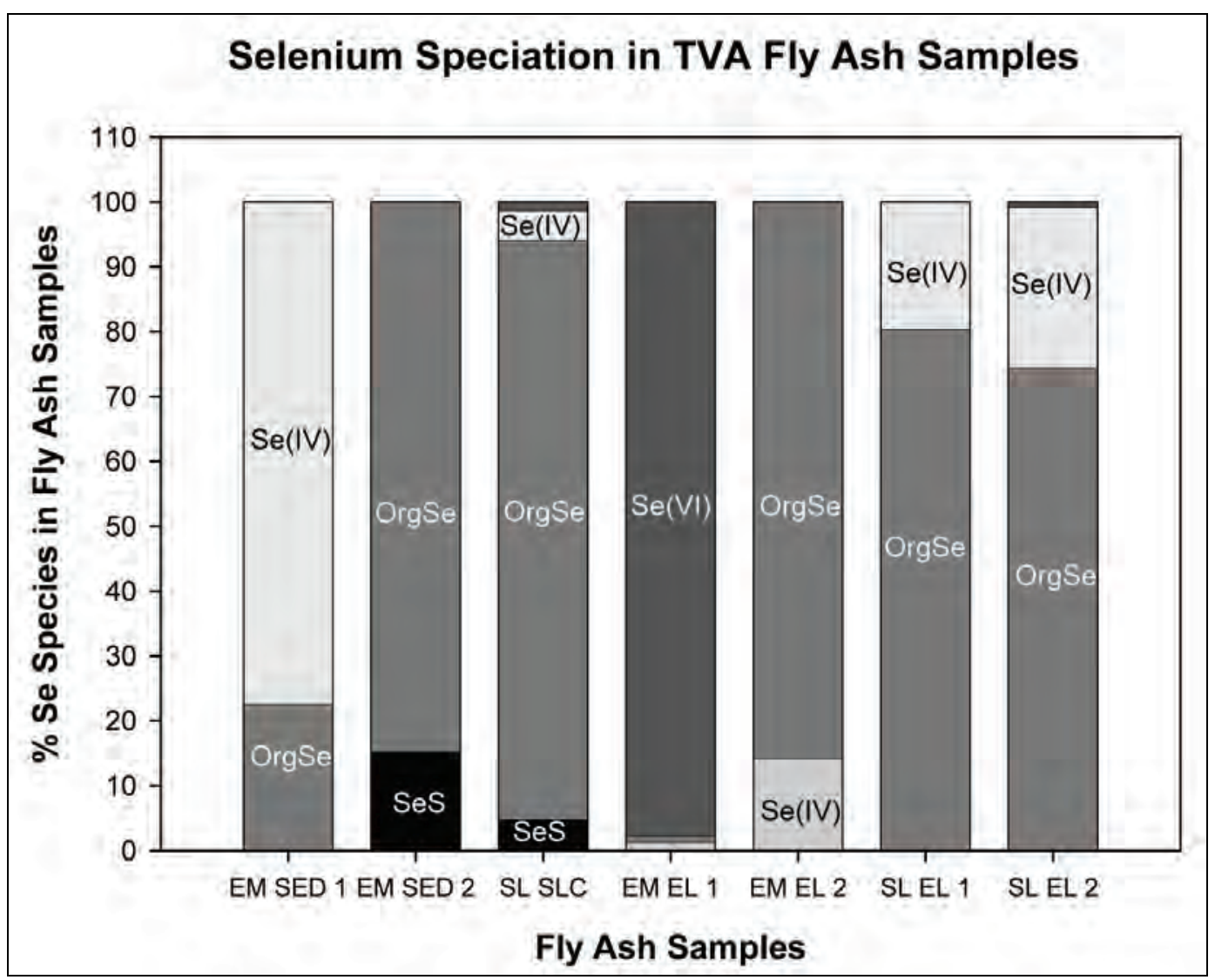

Figure 39. Results from linear combination fittings of $\mu$-XANES data collected at the selenium K-edge on discrete locations in the Emory River and sluice channel ash and oxic regime Emory River and sluice channel elutriate samples. Source = source pile, EM SED = Emory River sediment, SL SED = sluice channel sediment, EM EL = Emory River elutriate, and SL EL= sluice channel elutriate. The numbers refer to the number of XANES scans per sample. Se(IV) $=$ selenite, OrgSe $=\mathrm{Se}$ bound to thiol bearing $\mathrm{C}$-compounds, and Se-S is selenium bound to sulfur.

\section{Sediment particle size distributions during the EET}

Figures 40 and 41 show particle size distribution data obtained during the 240-hr bubbling period for the Emory River and sluice channel elutriates, respectively. The data show a slight shift in the particle size distribution curves toward smaller particle sizes with prolonged gas bubbling. This shift was more pronounced in the sluice EET than the Emory River EET, particularly for the larger particle sizes. Such shifts may be indicative of the effect of prolonged agitation of the sediment materials, and successful disaggregation of larger particles. Also, this effect may be related to the difficulty of fully suspending the ash material during the EET experiments. With time, ash material accumulating on the bottom of the EET vessels tended to "concrete" or form a crust so that it was necessary to periodically break up by mechanical stirring. Thus, particle size distributions would be 


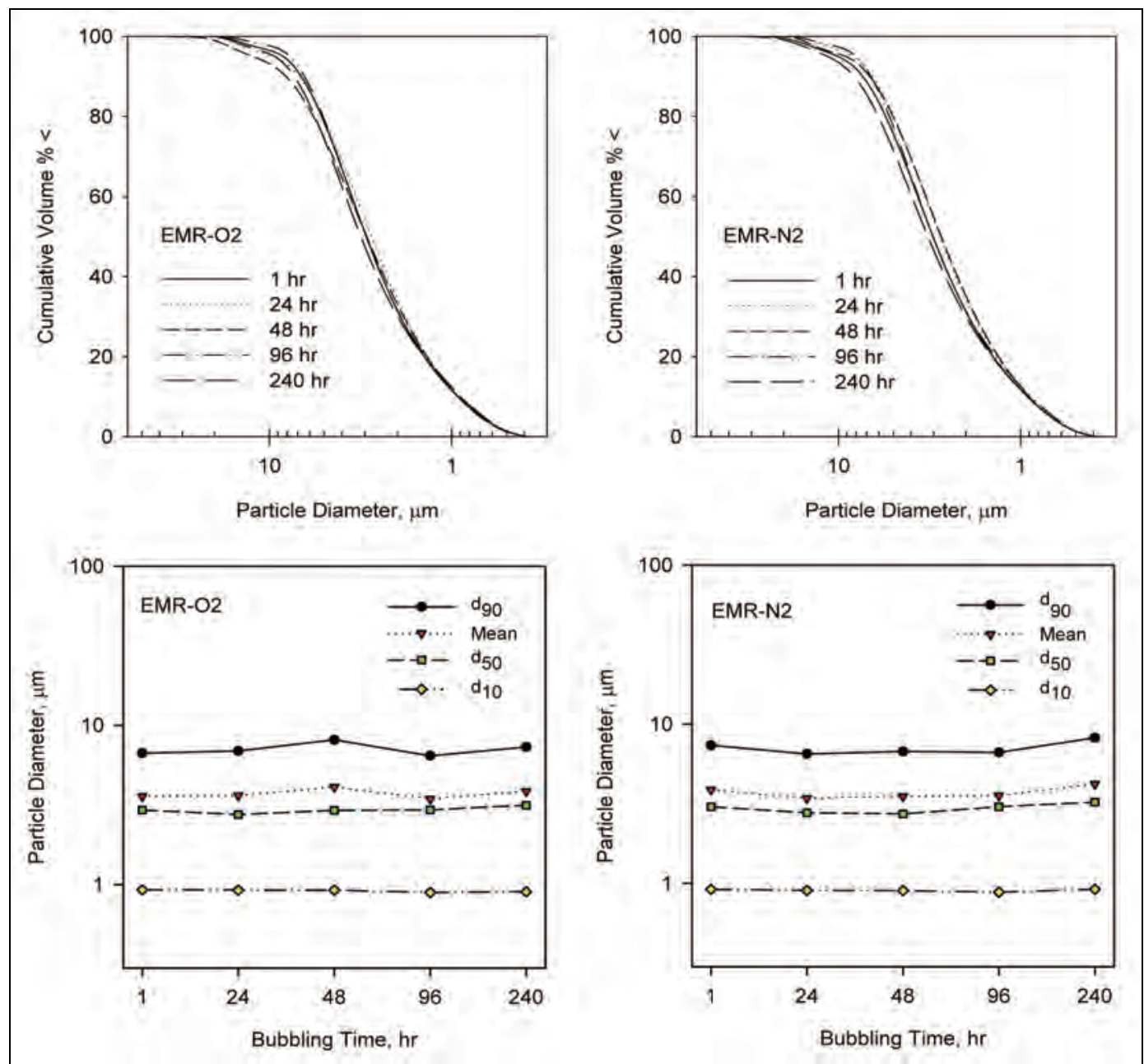

Figure 40. Particle size data for the Emory River elutriate experiments.

expected to have shifted slightly due to the removal of larger aggregates in the crust, which became difficult to resuspend. In any case, the data show that the prolonged bubbling did have a dramatic effect on the particle size distribution (better defined as a one to two order of magnitude difference). It is unclear to what degree such a shift in particle size distribution may have impacted the dissolution of Se and As.

\section{Geochemical modeling}

While the above studies provide information relevant to the species of arsenic and selenium, the actual chemistry of these metals is controlled by the existence of pairs and complexes made up from these particular species. Given the almost impossible task of analytically determining these pairs and complexes, geochemical modeling was used to predict the constituents based on reported literature values describing solubility and pairing complexation constants. 


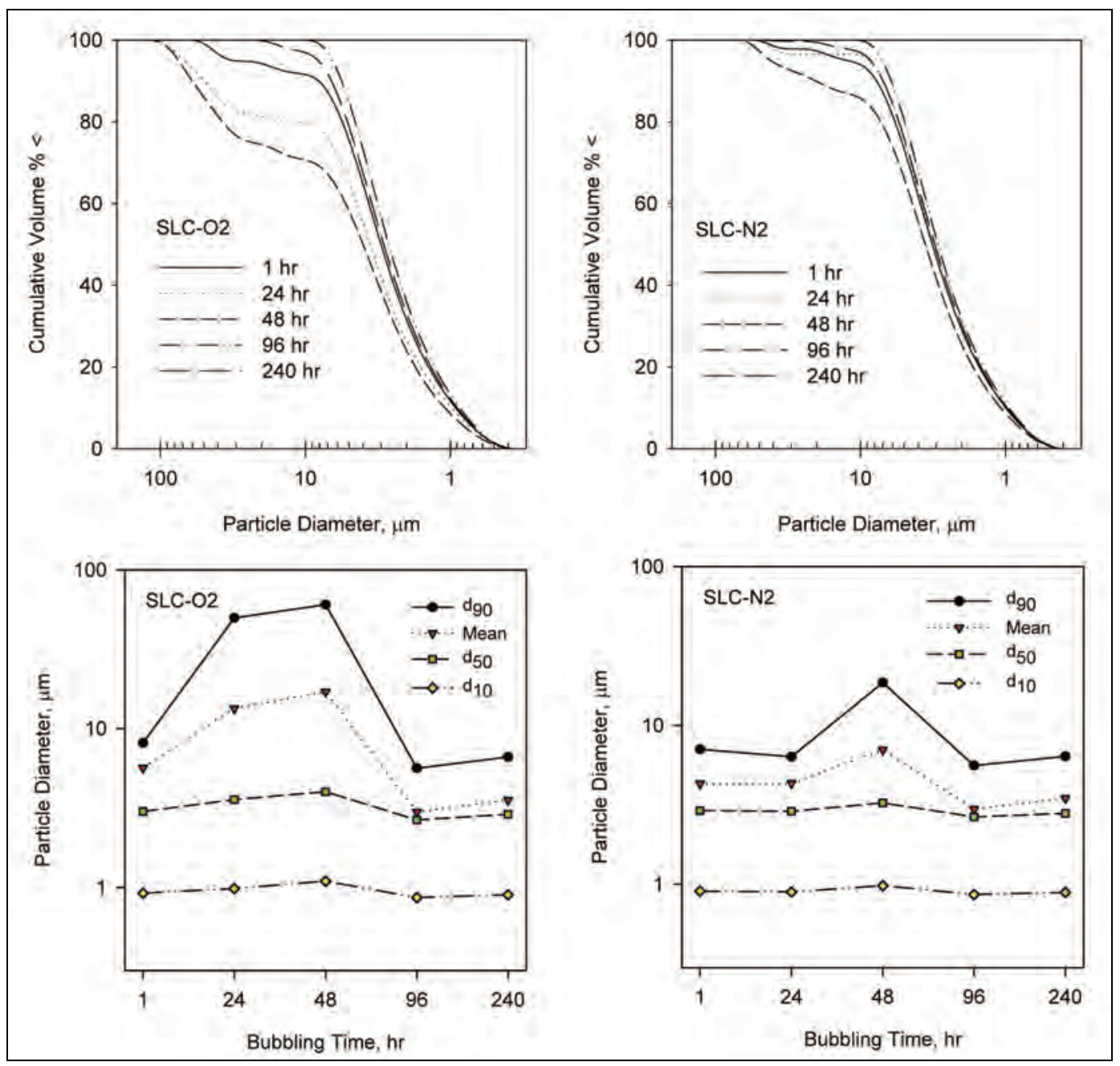

Figure 41. Particle size data for the sluice channel elutriate experiments.

Manganese. Equilibrium modeling of $\mathrm{Mn}$ in elutriate systems (Figures 42-45) shows the main $\mathrm{Mn}$ species as $\mathrm{Mn}^{2+}$, with $\mathrm{MnCO}_{3}{ }^{0}$ aqueous pair becoming important at $\mathrm{pH}>8$ at all redox ranges tested. According to this calculation, $\mathrm{Mn}$ (III) and $\mathrm{Mn}$ (IV) were not predicted to be stable in the measured elutriate conditions, in spite of evidence of oxidation under the oxic regime systems. The evidence suggests that the redox exhibited during the elutriate system actually represents a $\mathrm{pE}$ buffering capacity of this system, around the quantity of $\mathrm{Mn}$ in the system. If the EET was extended to complete Mn oxidation, the bulk Eh would have increased accordingly. 


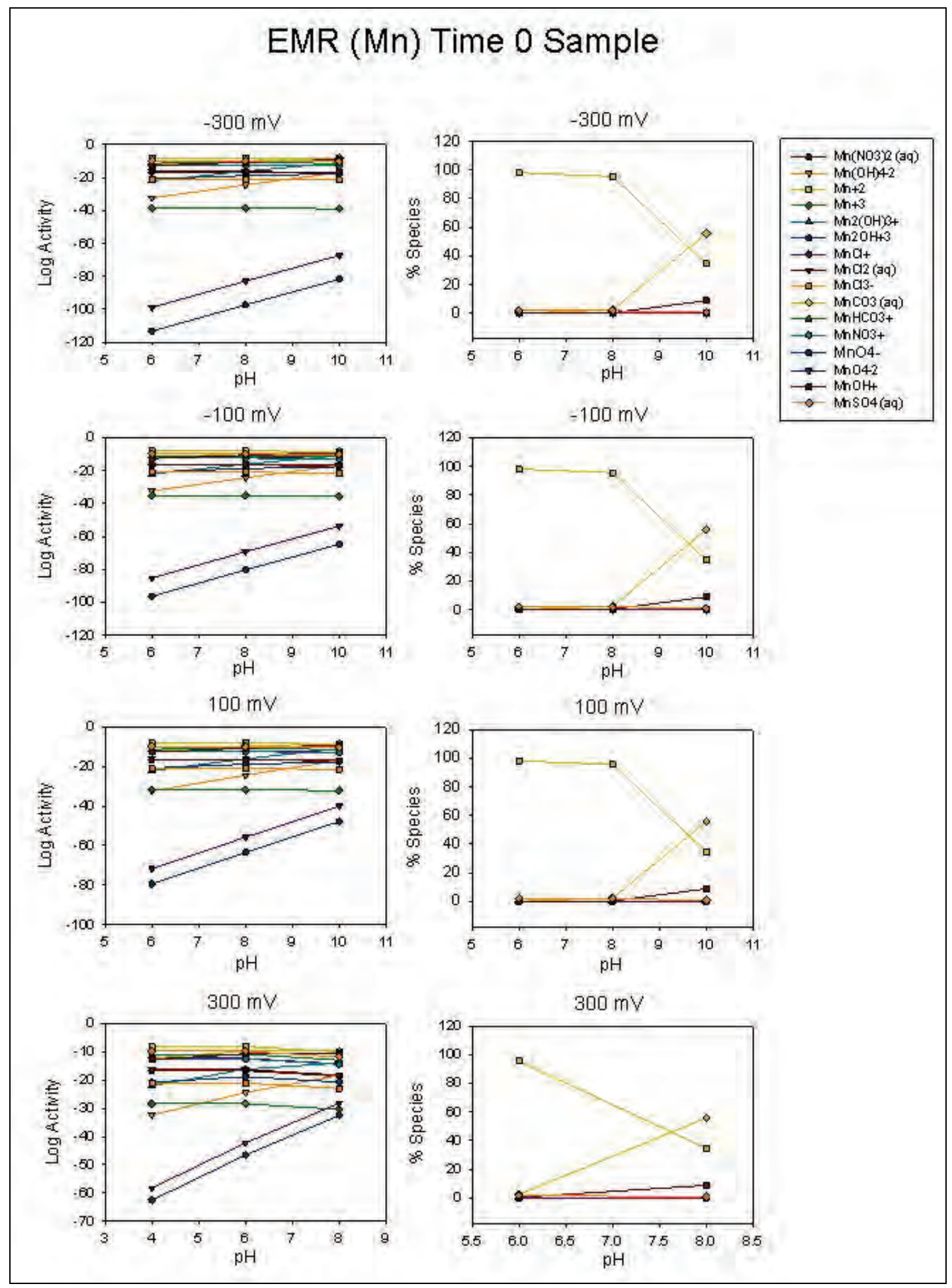

Figure 42. Results from geochemical modeling predicting manganese pairs and complexes in the Emory River elutriate ( $t=0 \mathrm{hr}$ ), based on total $\mathrm{Mn}$ determined in solution. Calculations were performed for a pH sweep of 4 to 10 , and redox sweep of -300 to $+300 \mathrm{mV}$. 


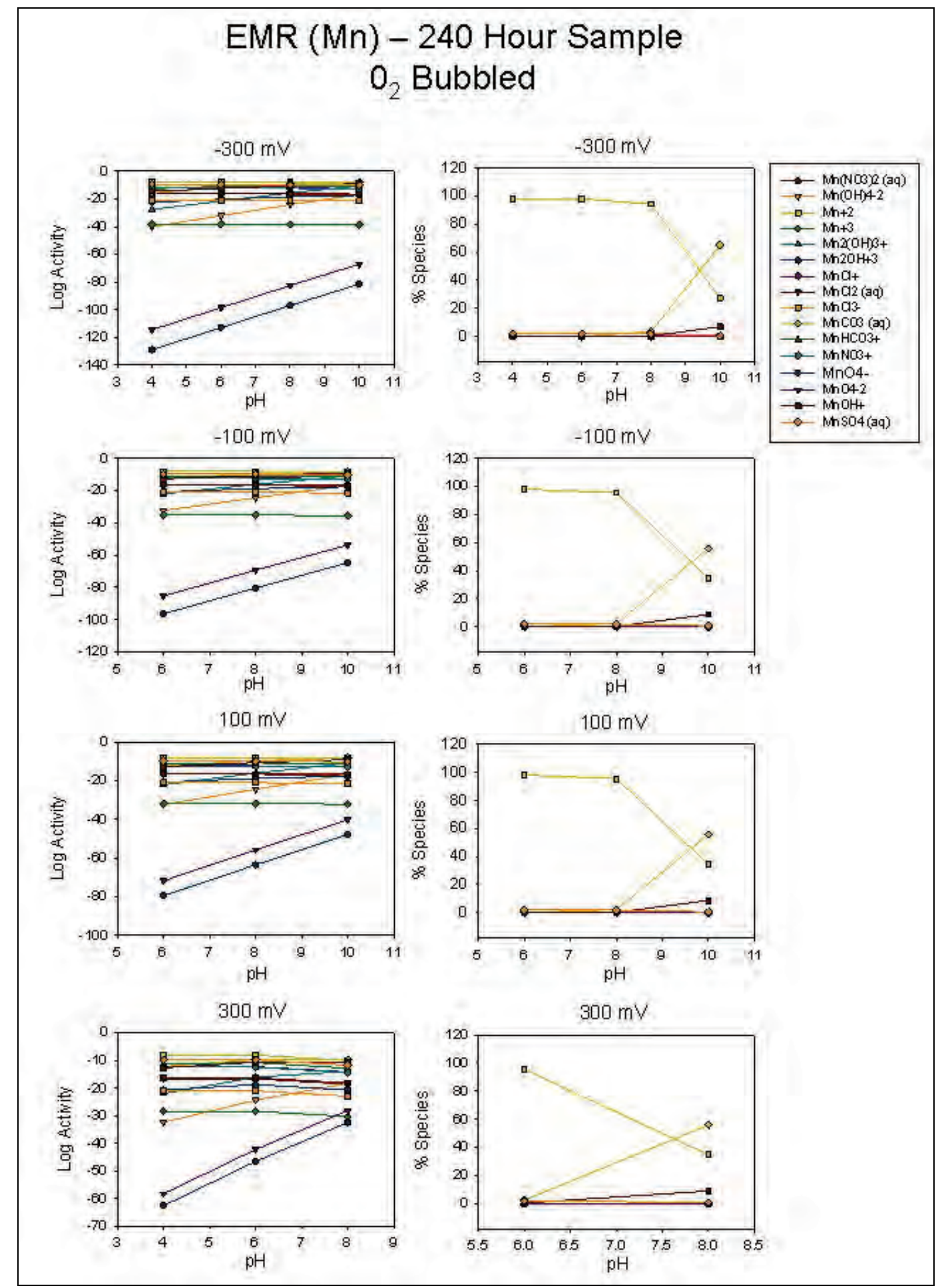

Figure 43. Results from geochemical modeling predicting manganese pairs and complexes in the Emory River elutriate $(\mathrm{t}=\mathbf{2 4 0} \mathrm{hr}$ ), based on total $\mathrm{Mn}$ determined in solution. Calculations were performed for a pH sweep of 4 to 10 , and redox sweep of -300 to $+300 \mathrm{mV}$. 


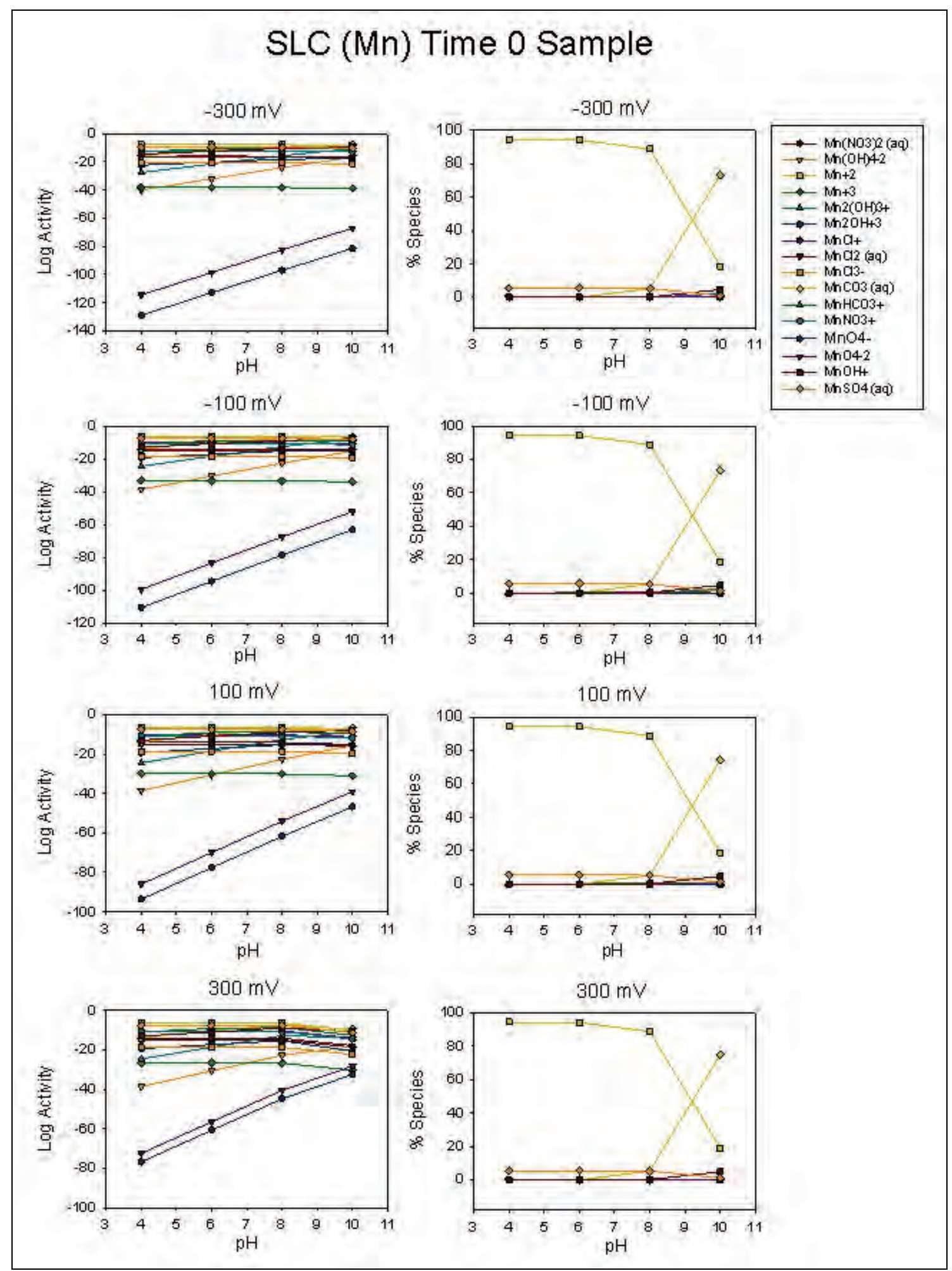

Figure 44. Results from geochemical modeling predicting manganese pairs and complexes in the sluice channel elutriate $(\mathrm{t}=0 \mathrm{hr})$, based on total $\mathrm{Mn}$ determined in solution. Calculations were performed for a pH sweep of 4 to 10 , and redox sweep of -300 to $+300 \mathrm{mV}$. 


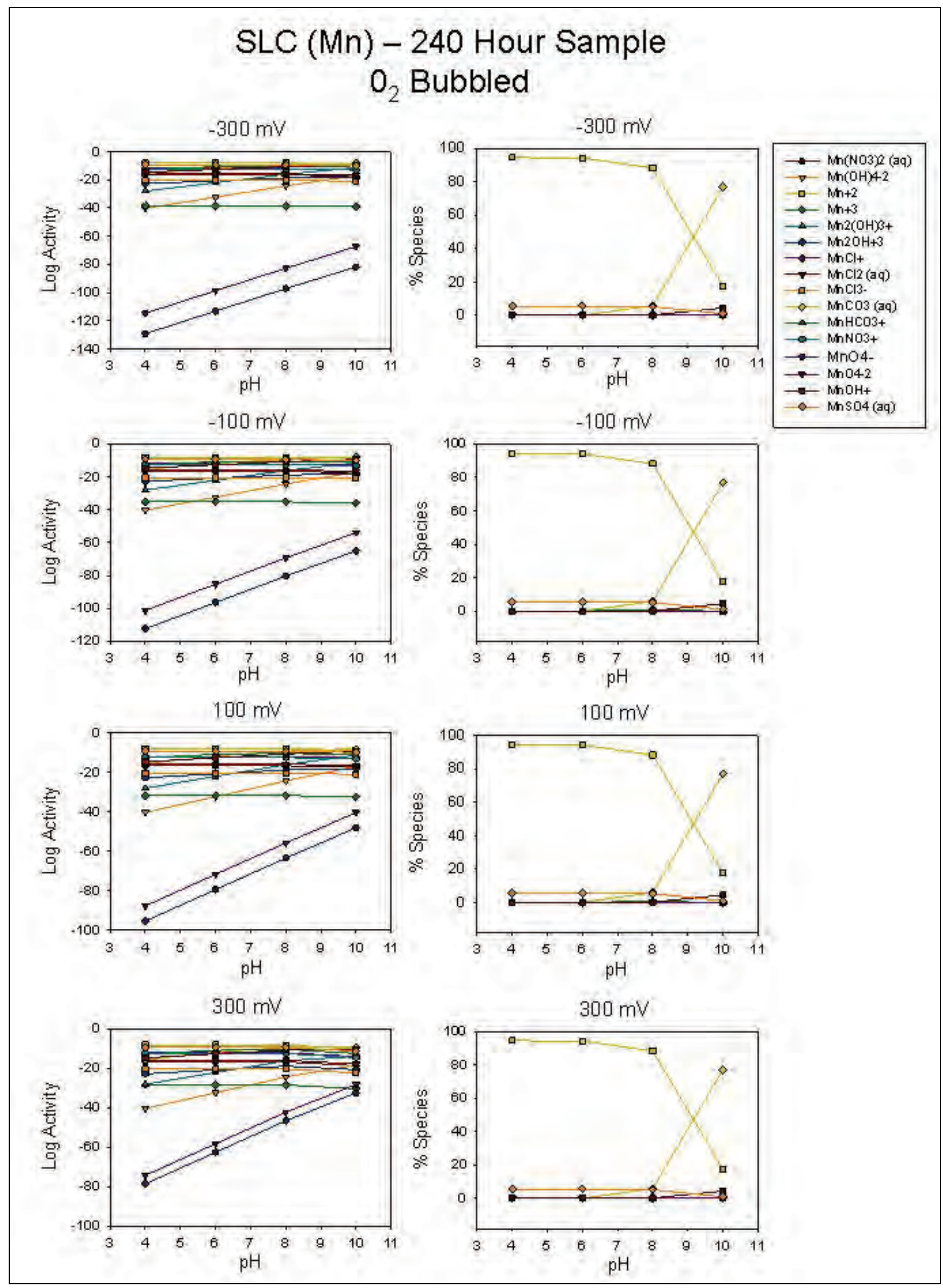

Figure 45. Results from geochemical modeling predicting manganese pairs and complexes in the sluice channel elutriate $(\mathrm{t}=240 \mathrm{hr})$, based on total $\mathrm{Mn}$ determined in solution.

Calculations were performed for a pH sweep of 4 to 10 , and redox sweep of -300 to +300 $\mathrm{mV}$. 
Chromium. Equilibrium modeling of chromium (Figures 46 and 47) was limited to detection of the metal at $\mathrm{t}=240 \mathrm{hr}$. In general, the modeling predicted dominance of $\mathrm{Cr}$ (II) complexes at acid $\mathrm{pH}$ and $\mathrm{Cr}$ (III) complexes at basic $\mathrm{pH}$ values for all redox conditions tested.

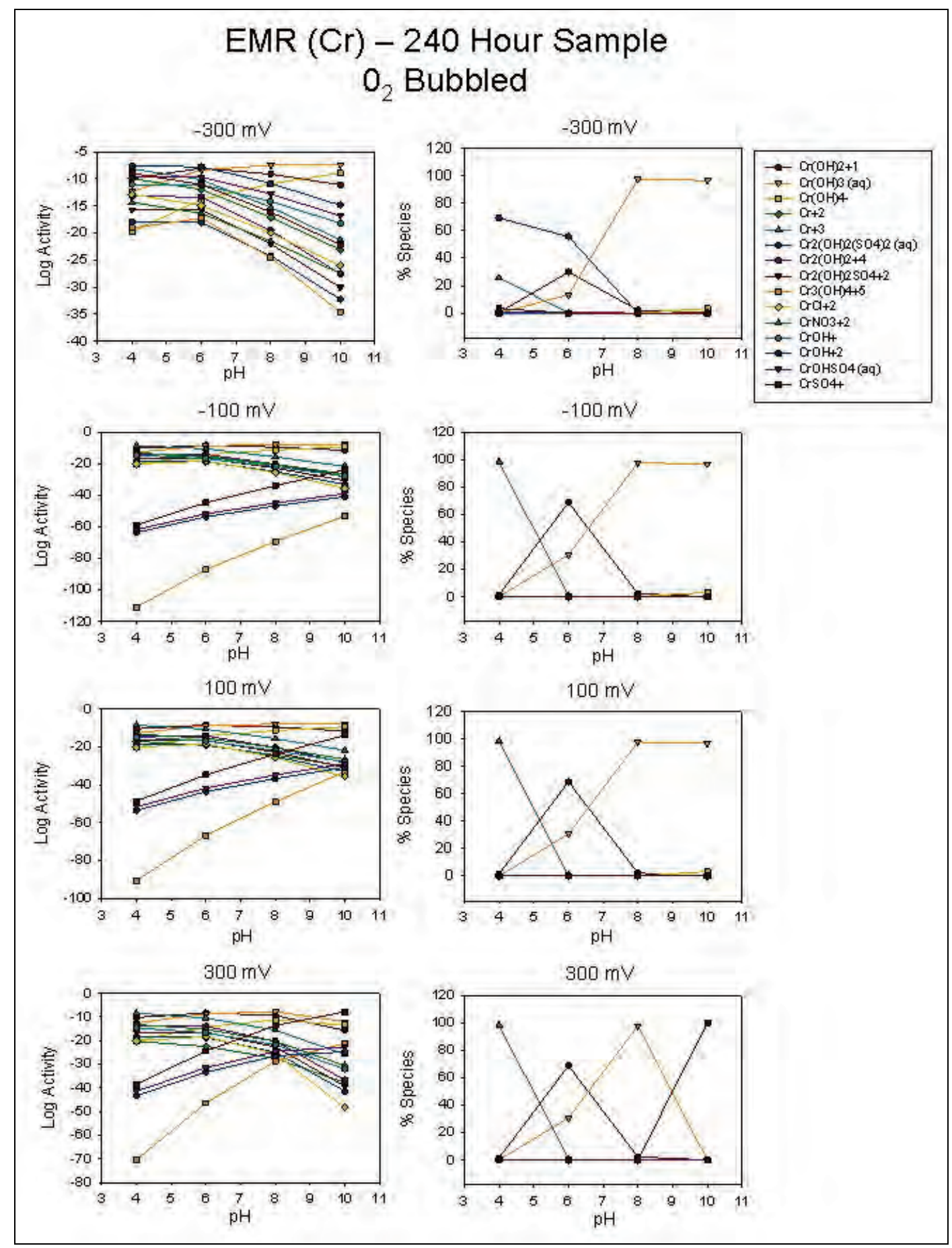

Figure 46. Results from geochemical modeling predicting chromium pairs and complexes in the Emory River elutriate $(t=240 \mathrm{hr}$ ), based on total Mn determined in solution. Calculations were performed for a $\mathrm{pH}$ sweep of 4 to 10 , and redox sweep of -300 to $+300 \mathrm{mV}$. 


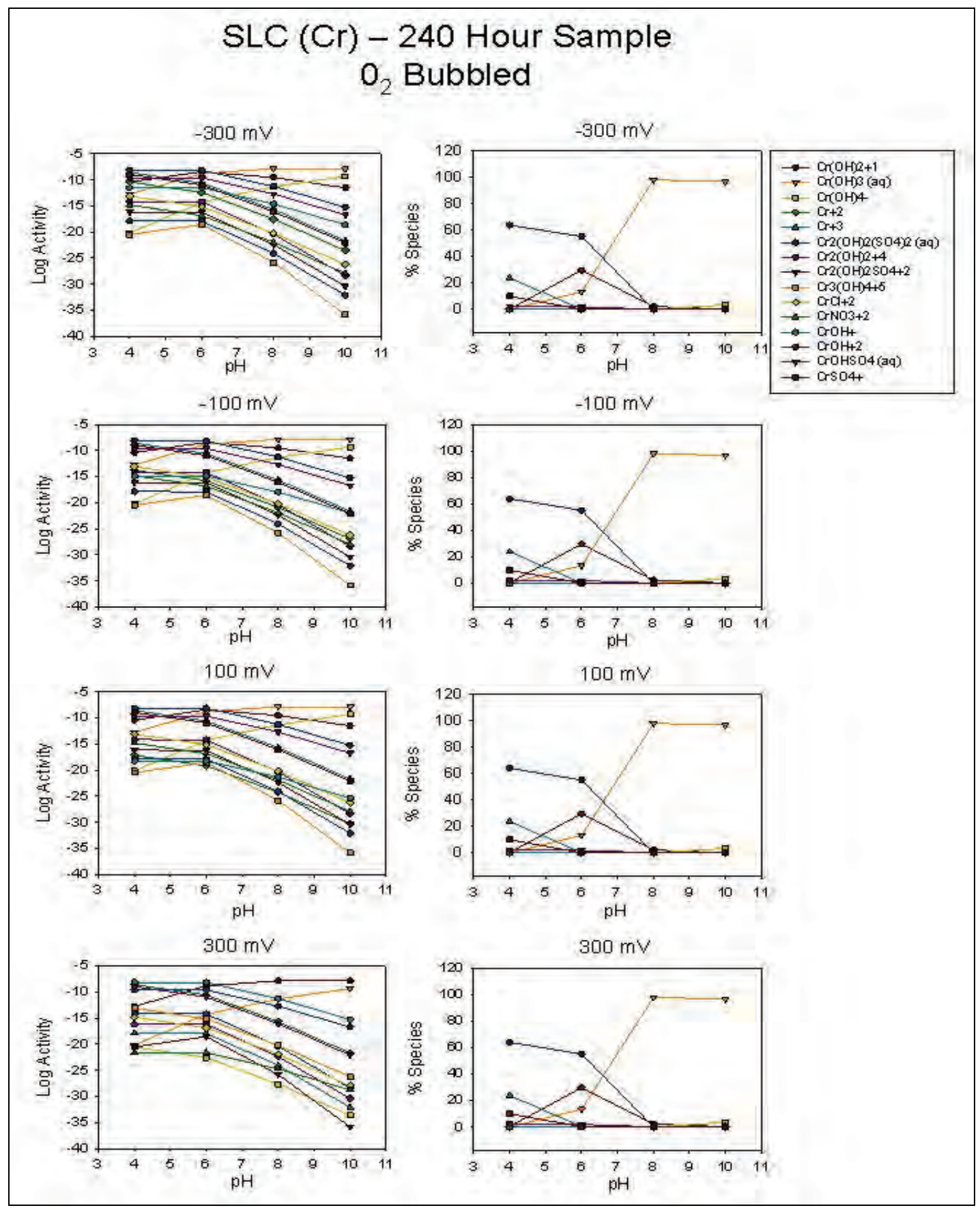

Figure 47. Results from geochemical modeling predicting chromium pairs and complexes in the sluice channel elutriate $(t=240 \mathrm{~h})$, based on total $\mathrm{Mn}$ determined in solution. Calculations were performed for a pH sweep of 4 to 10 , and redox sweep of -300 to $+300 \mathrm{mV}$.

Arsenic. Figures 48-51 show the geochemical speciation calculations for arsenic. Log activity plots show a wide variety of predicted arsenic pairs and complexes, based on the arsenite/ arsenate redox couple. For the Emory River sediment, speciation calculations predicted the main arsenic species as $\mathrm{As}(\mathrm{V})$, in the forms of $\mathrm{H}_{2} \mathrm{AsO}_{4}{ }^{-}$and $\mathrm{HAsO}_{4}{ }^{2-}$ ( $\mathrm{pKa}$ for these two species 

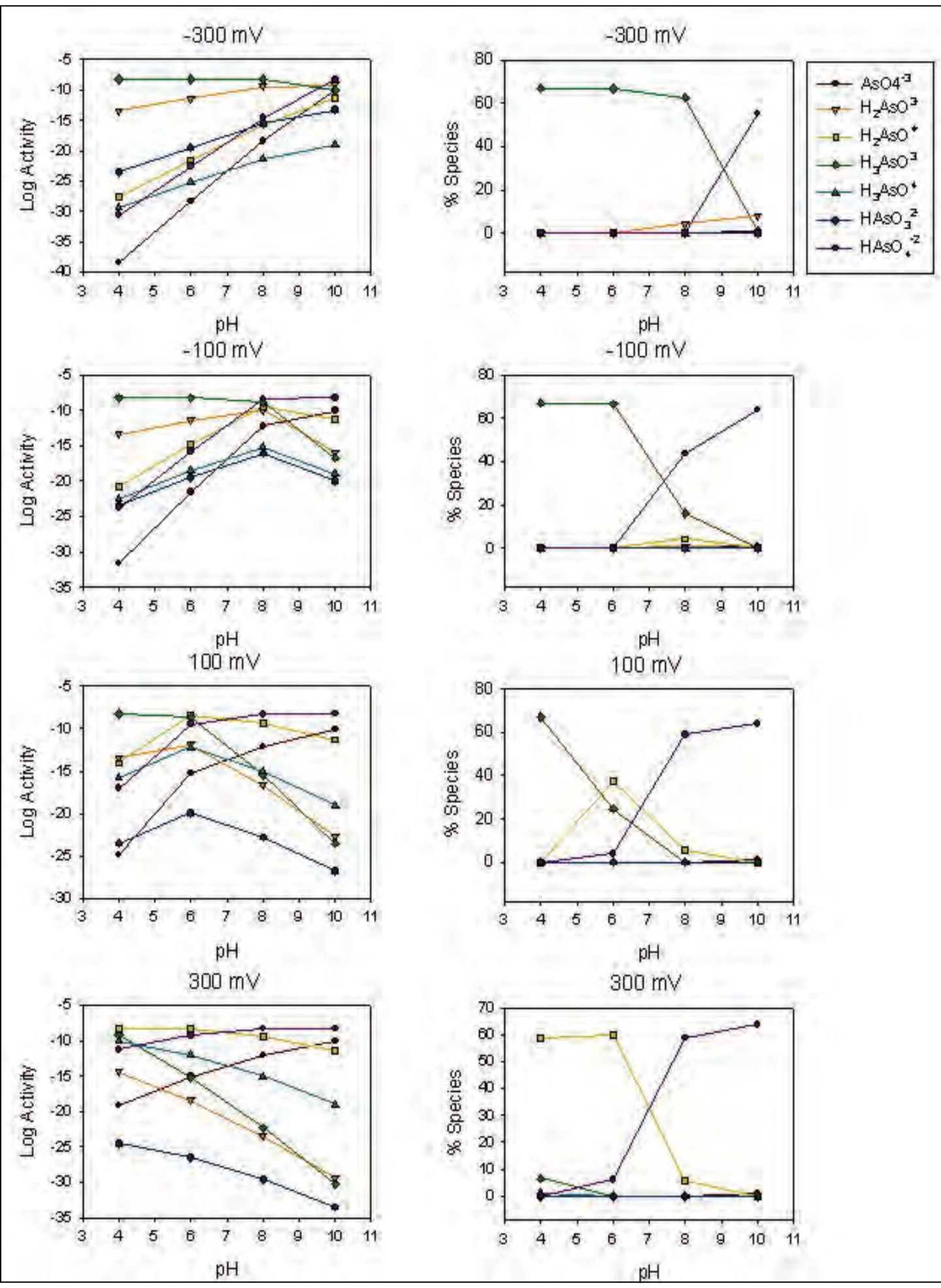

Figure 48. Results from geochemical modeling predicting arsenic pairs and complexes in the Emory River elutriate $(\mathrm{t}=0 \mathrm{hr})$, based on total and speciated arsenic determined in solution. Calculations were performed for a pH sweep of 4 to 10 , and redox sweep of -300 to $+300 \mathrm{mV}$. 


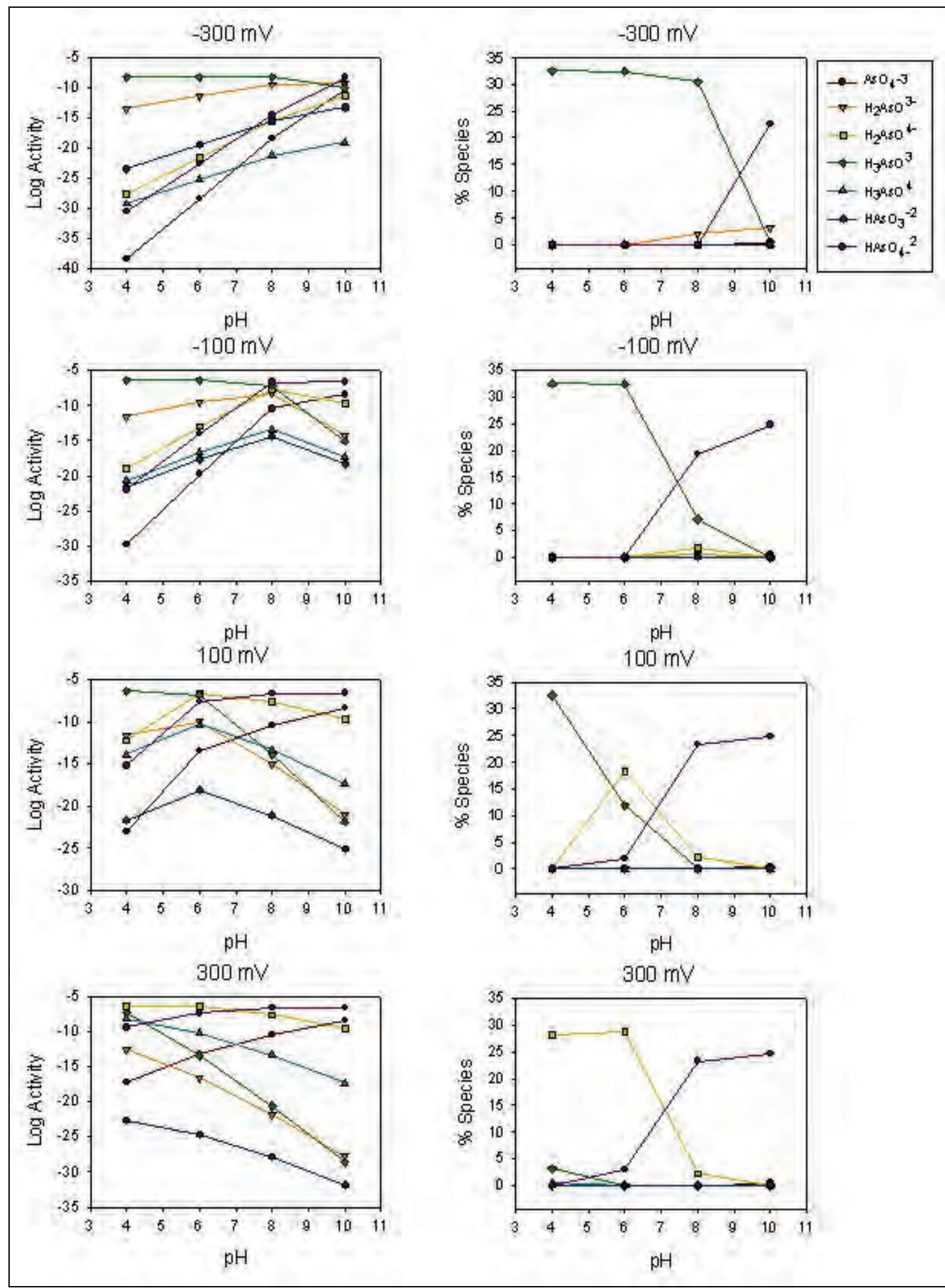

Figure 49. Results from geochemical modeling predicting arsenic pairs and complexes in the Emory River elutriate $(t=240 \mathrm{hr}$ ), based on total and speciated arsenic determined in solution. Calculations were performed for a pH sweep of 4 to 10 , and redox sweep of -300 to $+300 \mathrm{mV}$. 


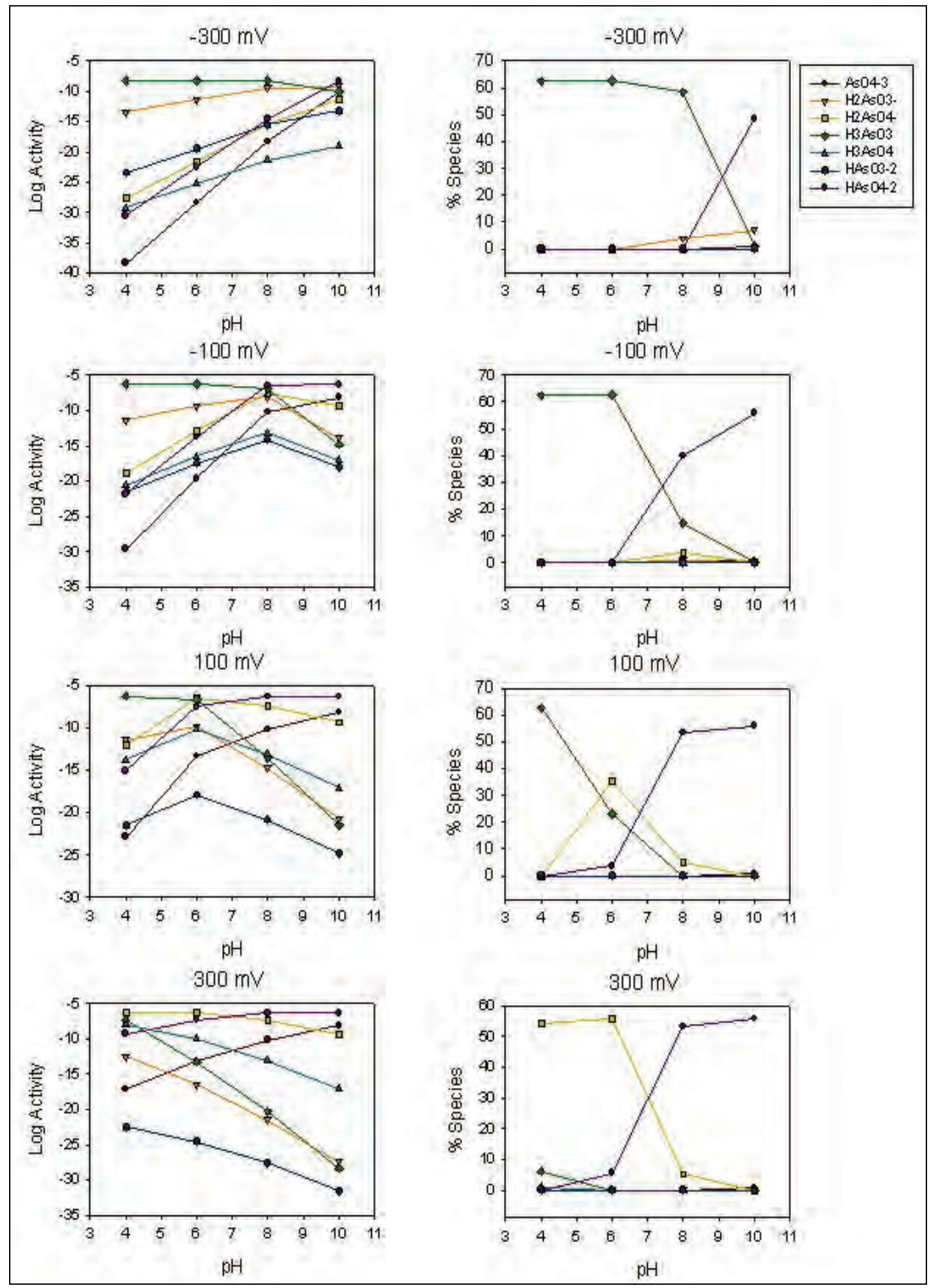

Figure 50. Results from geochemical modeling predicting arsenic pairs and complexes in the sluice channel elutriate $(t=0 \mathrm{hr}$ ), based on total and speciated arsenic determined in solution. Calculations were performed for a pH sweep of 4 to 10 , and redox sweep of -300 to $+300 \mathrm{mV}$. 

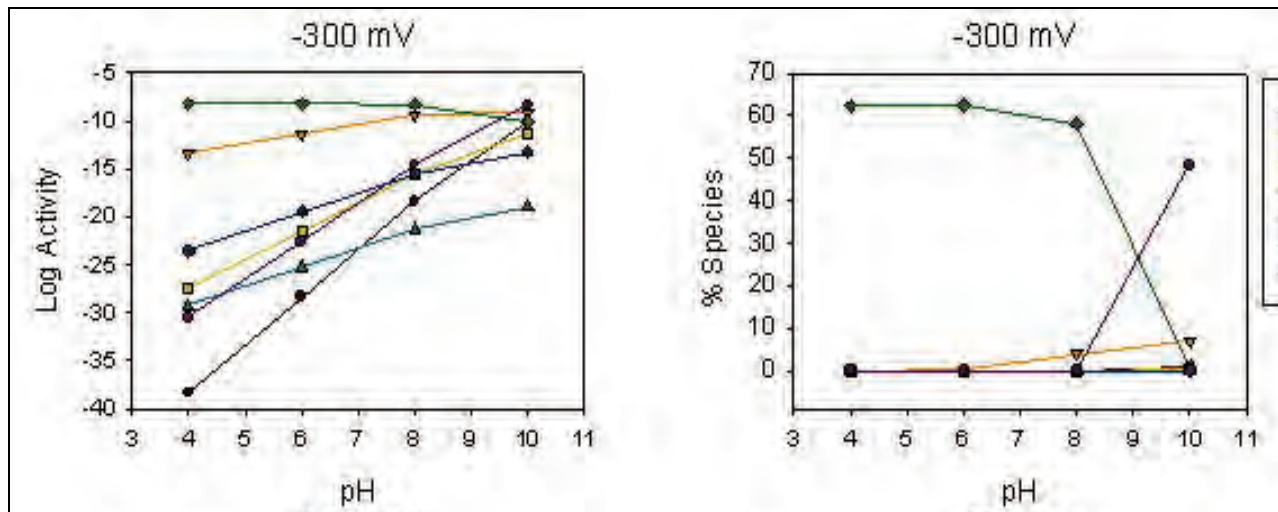

$\rightarrow-A s 043$ Y. $\mathrm{H} 2 \mathrm{As} 03-$ - $\mathrm{H}_{2} \mathrm{As} \mathrm{SO}$ $\rightarrow \mathrm{H} 3 \mathrm{AsO} 04$

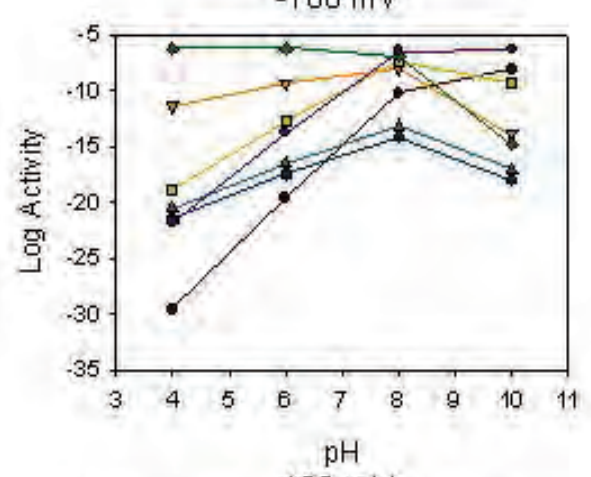

$-100 \mathrm{mV}$

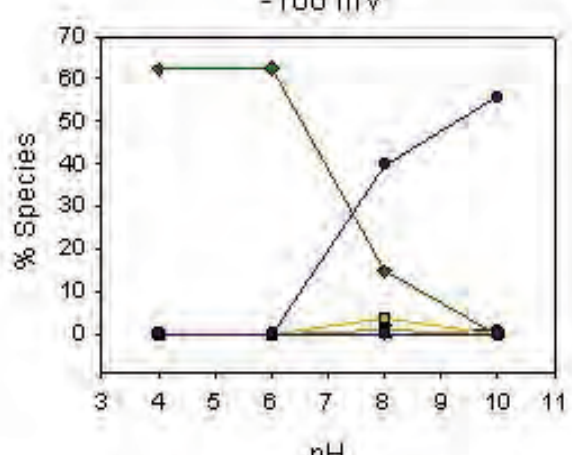

$100 \mathrm{mV}$

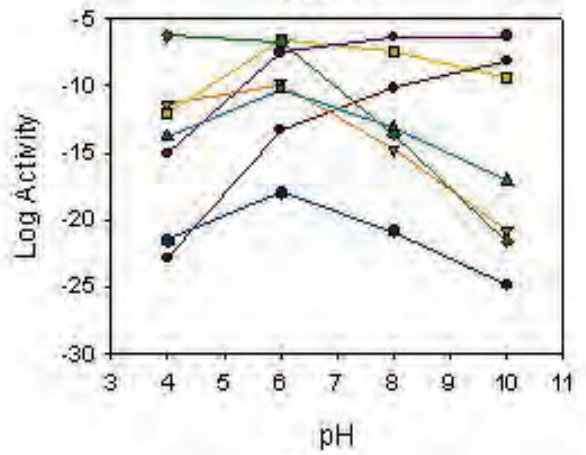

$100 \mathrm{mV}$
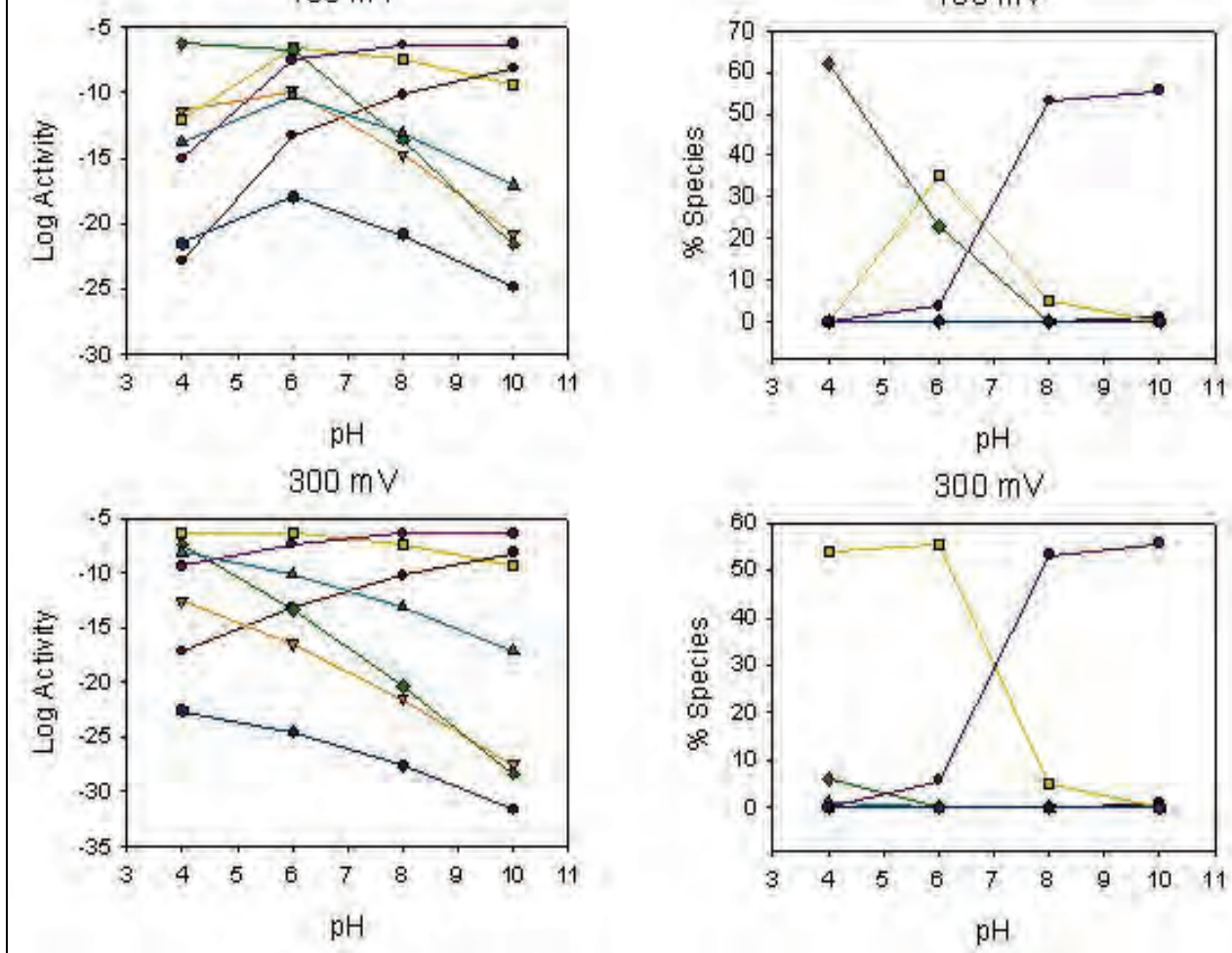

Figure 51. Results from geochemical modeling predicting arsenic pairs and complexes in the sluice channel elutriate $(t=240 \mathrm{hr}$ ), based on total and speciated arsenic determined in solution. Calculations were performed for a $\mathrm{pH}$ sweep of 4 to 10 , and redox sweep of -300 to $+300 \mathrm{mV}$. 
is 6.94). At $\mathrm{Eh}=300 \mathrm{mV}$, $\mathrm{As}(\mathrm{V})$ represented the dominant species, with the percent distribution of the $\mathrm{H}_{2} \mathrm{AsO}_{4}{ }^{-}$and $\mathrm{HAsO}_{4}{ }^{2-}$ pairs well described by simple Henderson-Hasselbauch relationships. With decreasing Eh, the calculations predicted an increase in As(III) species, in the form of $\mathrm{H}_{3} \mathrm{AsO}_{3}$ (arsenious acid) and $\mathrm{H}_{2} \mathrm{AsO}_{3}-(\mathrm{pKa}=9.2)$. Thus, the predictions show that the thermodynamically favored arsenic species in the Emory River and sluice channel systems as As(V) - evidence supporting both the liquid and solid phase data.

Figure 49 shows the arsenic speciation calculations for the Emory River EET after $240 \mathrm{hr}$ of oxic regime treatment. Thermodynamically, the $\mathrm{t}=0$ and $240 \mathrm{hr}$ systems are very similar with respect to the arsenic pairs predicted. However, it was observed that the log activity values for the various arsenic pairs were shifted somewhat due to the higher level of barium in the elutriate system. Thus, the model predicted the precipitation of $\mathrm{BaHAsO}_{4} \bullet 2 \mathrm{H}_{2} \mathrm{O}$ (Figure 55). Redox-associated shifts in $\mathrm{BaHAsO}_{4} \bullet$ $2 \mathrm{H}_{2} \mathrm{O}$ are shown in Figure 55. In the SLC, $\mathrm{t}=0$ and 240-hr systems, the proportion of arsenic pairs is also predicted to be similar to Emory River sediments, except the higher concentration of sulfate is predicted to result in the precipitation of barite $\left(\mathrm{BaSO}_{4}\right)$ instead of $\mathrm{BaHAsO}_{4} \bullet 2 \mathrm{H}_{2} \mathrm{O}$ (Figure 55).

Selenium. Se speciation calculations were not performed on the Emory River ( $\mathrm{t}=0 \mathrm{hr}$ ) elutriates as no Se was detected in solution. However, $\mathrm{Se}(\mathrm{IV})$ was detected in the oxic regime elutriates. Calculations predicted $\mathrm{Se}(\mathrm{IV})$ as the dominant species in this system, in the form of $\mathrm{HSeO}_{3}$ - and $\mathrm{SeO}_{3}{ }^{2-}$ (Figure 52), even after assigning "excess" selenium concentration (total selenium - Se(IV)) to Se(VI). Similar predictions were obtained for the sluice channel systems (Figures 53 and 54). Predictions show that $\mathrm{SeO}_{4}{ }^{2-}$ only emerges as a major selenium species under oxidation conditions ( $\mathrm{Eh}=300 \mathrm{mV}$ ) much higher than those generally observed in the Emory River or sluice channel. 


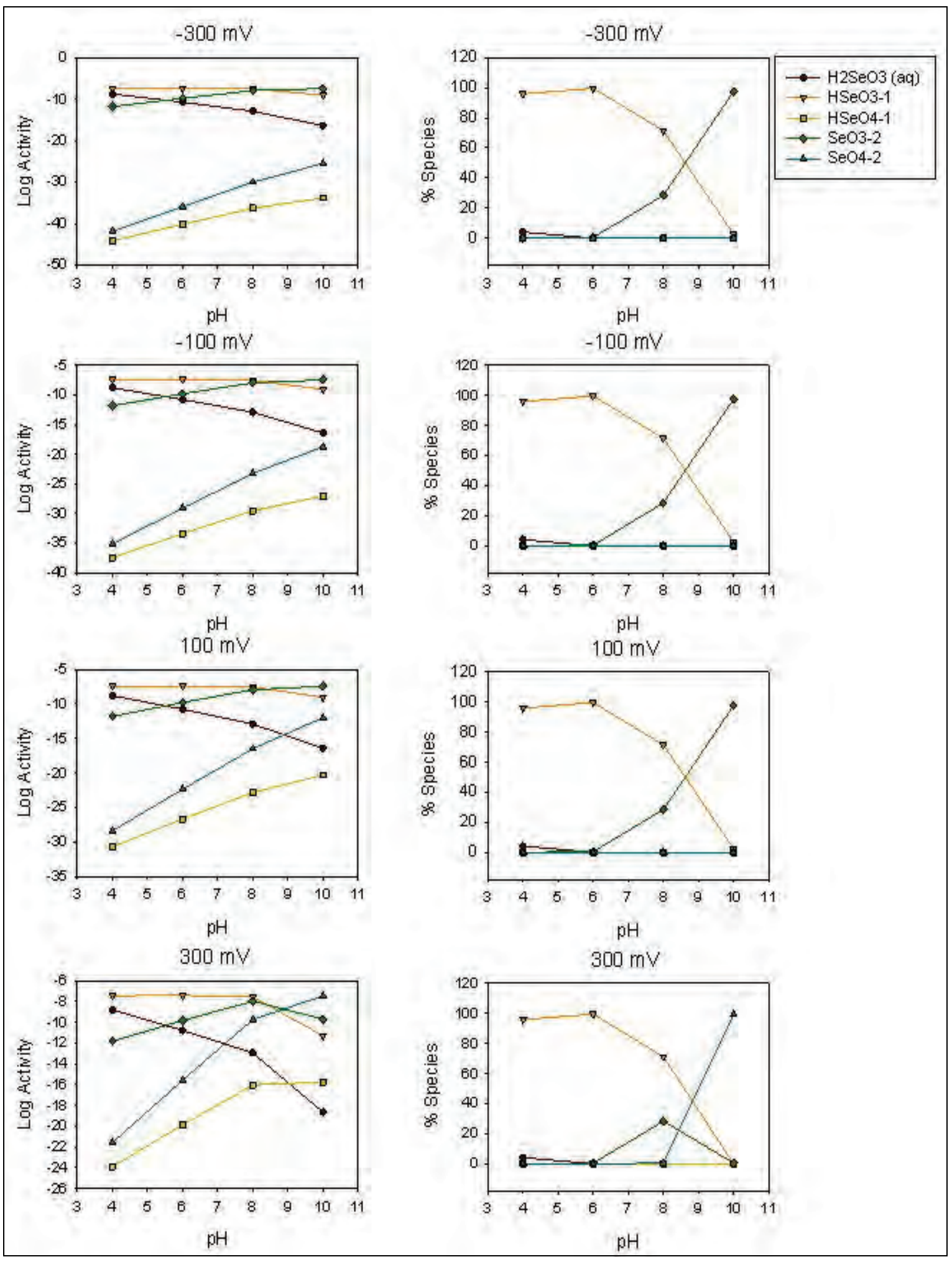

Figure 52. Results from geochemical modeling predicting selenium pairs and complexes in the Emory River elutriate ( $t=240 \mathrm{hr}$ ), based on total and speciated Se determined in solution. Calculations were performed for a pH sweep of 4 to 10 , and redox sweep of -300 to $+300 \mathrm{mV}$. 


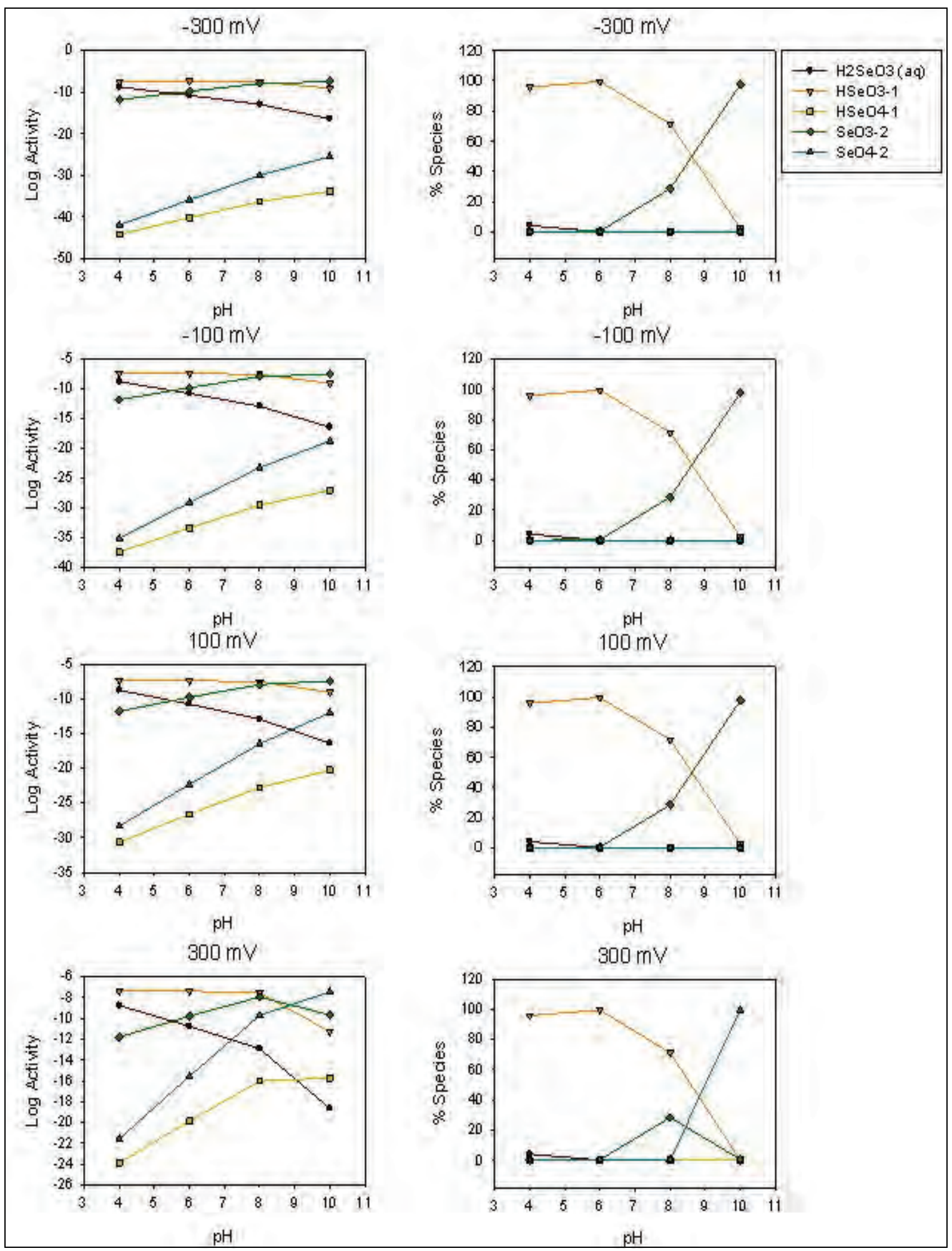

Figure 53. Results from geochemical modeling predicting selenium pairs and complexes in the sluice channel $(t=0 \mathrm{hr}$ ), based on total and speciated Se determined in solution.

Calculations were performed for a pH sweep of 4 to 10 , and redox sweep of -300 to +300 $\mathrm{mV}$. 


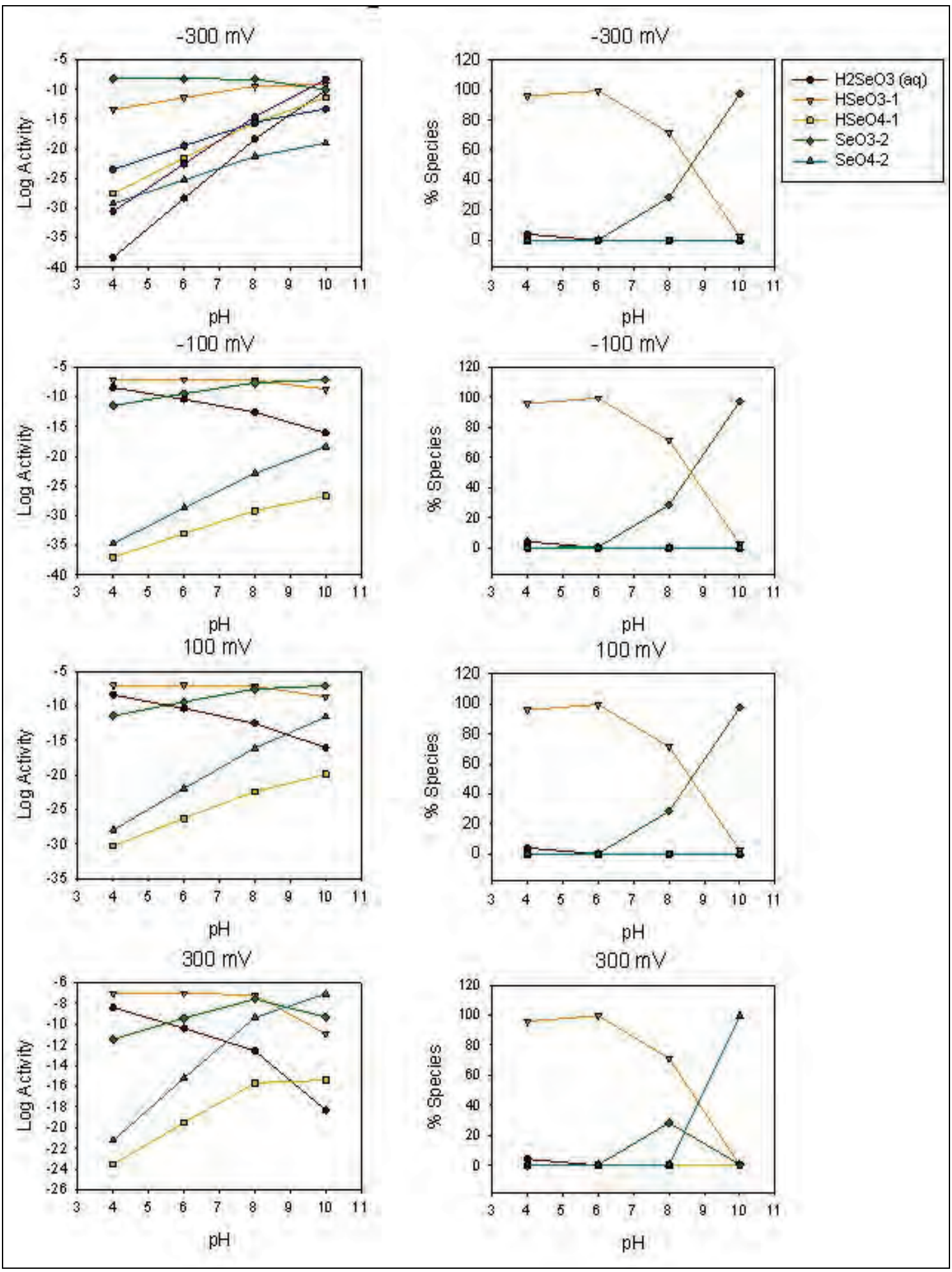

Figure 54. Results from geochemical modeling predicting selenium pairs and complexes in the sluice channel $(t=240 \mathrm{hr}$ ), based on total and speciated Se determined in solution. Calculations were performed for a pH sweep of 4 to 10, and redox sweep of -300 to +300 $\mathrm{mV}$. 


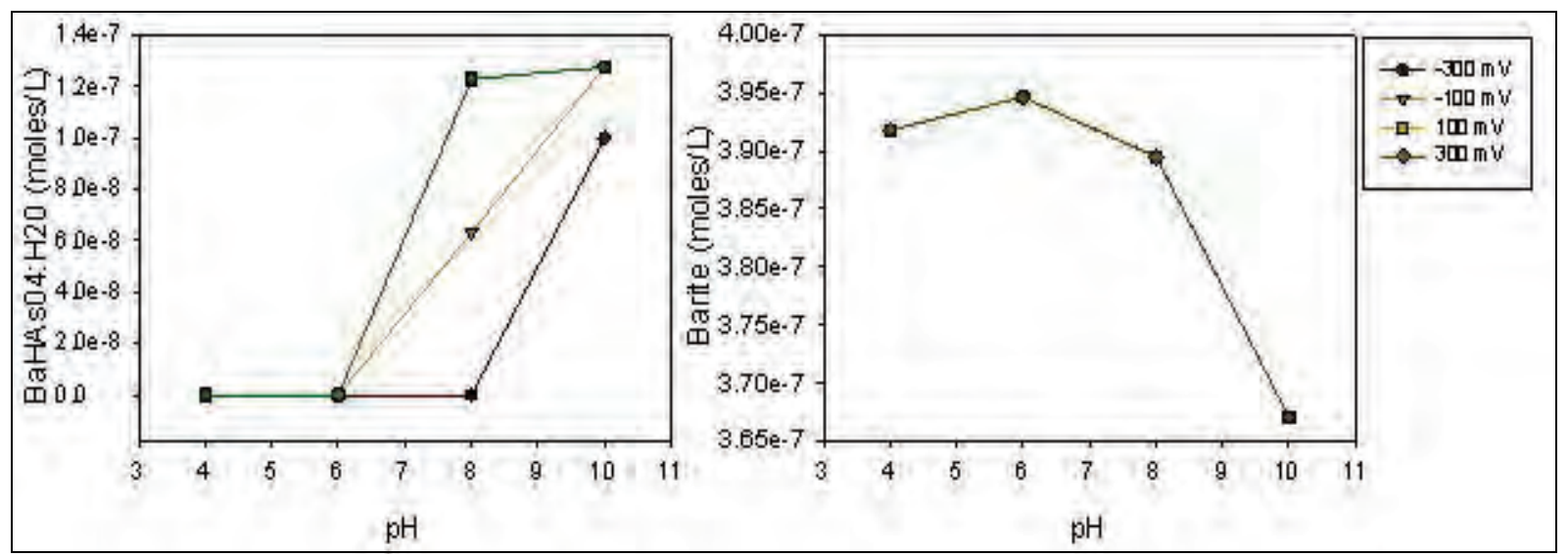

Figure 55. Results from geochemical modeling predicting precipitation of barium compounds (in mol/L) at $\mathrm{t}=\mathrm{O} \mathrm{h}$ in the (left) Emory River and (right) sluice channel elutriates. Predictions are based on total $\mathrm{Ba}$ and total and speciated As determined in solution. Calculations were performed for a pH sweep of 4 to 10 , and redox sweep of -300 to $+300 \mathrm{mV}$.

\section{Conclusions:}

- Increased dissolution of several elements (e.g., As, Ba, V, Se) was observed over the 10-day EET, while dissolved Mn concentrations decreased. Even though system redox remained relatively stable, fly ash $\mathrm{Mn}$ was demonstrated to partially oxidize by extensive oxic regime treatment. Iron phases were unaffected. The one order of magnitude increase in dissolved organic carbon over the EET suggests the experimental conditions promoted microbial activity.

- With the exception of $\mathrm{Mn}$, bulk metal (loid) speciation remained relatively constant over the 10-day period. The major exception to this observation came from $\mu$-XANES analysis showing a domain of selenate in Emory River elutriate solid after the 10-day oxic regime period, even though selenate was not detected in solution. It is unclear whether this domain was caused by the elutriate conditions or existed before the experiments were performed. However, the results do open up the possibility that extensive oxidation of the system can produce selenate. Geochemical modeling confirms that the Emory River conditions represent a lower boundary where selenate is thermodynamically stable in this system, although a relatively minor species relative to selenite.

- Given that both the anoxic and oxic regime (nitrogen- and air-bubbled) systems displayed nearly identical behavior, it is hypothesized that the trend in the dissolution of metals over the 10-day period was due to the extended agitation of the fly ash. The corresponding spike in microbial activities supports the view that constantly dispersing the fly ash enhances the microbial bioavailability to surface-adsorbed nutrients. 


\section{Bioassays}

\section{Methods}

The potential for sediment elutriate effects was assessed by 10-day exposures of two different life stages of the fathead minnow Pimephales promelas. This fish is a recommended freshwater test species for elutriate exposures in USEPA and USACE guidance for testing dredged material (USEPA / U.S. Army Corps of Engineers (USACE) 1991, 1998). The P. promelas (Figure 56) is laboratory-cultured and was obtained from a commercial source (Aquatic Biosystems, Fort Collins, CO, USA). Larval fish were selected to assess the potential toxicity of a more sensitive life stage while juvenile fish were tested to gain information on whole body burdens and the potential for gill damage. The larval fish were exposed to

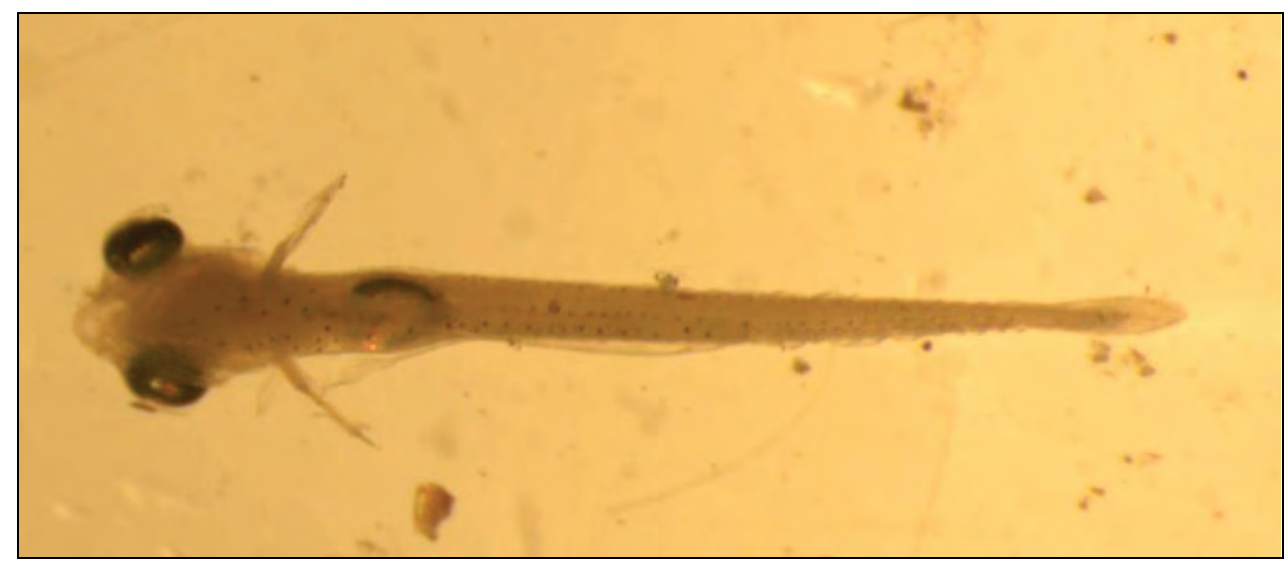

(a)

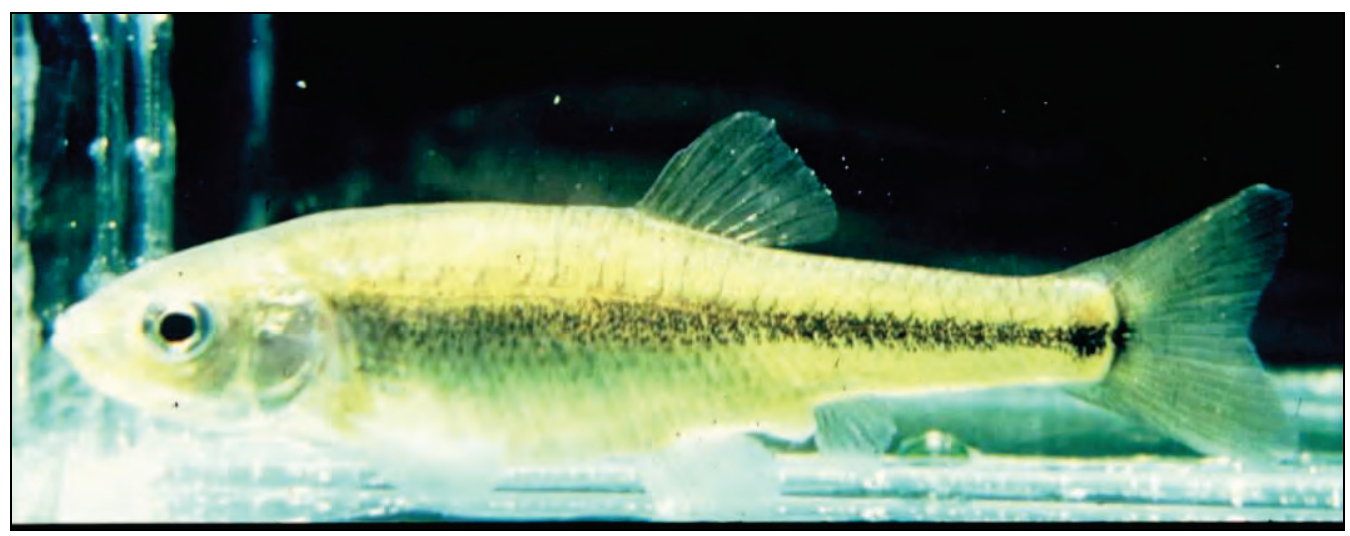

(b)

Figure 56. Images of (a) larval and (b) juvenile Pimephales promelas. 
both elutriates (EMR-EL and SLC-EL), while the juvenile fish were exposed only to EMR-EL-AF. For all exposures, the oxic regime 10-day extended elutriate preparation described above was considered the $100 \%$ elutriate treatment. In addition, this elutriate was diluted with Emory River site water (river mile 12; RF1-WA) to prepare $50 \%$ and $10 \%$ elutriate treatments. Comparative treatments included the reference site water (RF1-WA, 0\% treatment) and a performance control (dechlorinated tap water, Vicksburg, MS, USA, municipal source). Each treatment involved five experimental replicates and exposures were conducted at $20 \pm 1^{\circ} \mathrm{C}$ to represent conditions at the dredging site.

To maintain water quality during the exposures, water exchanges ( $90 \%$ of total volume) using freshly diluted treatments (control, 0, 10, 50, 100\%) were conducted on test days three and seven. Daily water exchanges were not practical due to 1) the amount of water that would be required for both bioassays (>800 L), and 2) the settling of the solid phase particles in the larval bioassay that necessitated transfer of larvae to new exposure chambers at each water renewal to avoid temporally increasing test concentrations. Prior to preparation of test concentrations, the stored $100 \%$ elutriates were thoroughly homogenized by mechanical mixers (Lightnin DuraMix, E78R2558N-RR, Mixing Equipment Inc., Avon, NY, USA for EMR-EL and IKA, Model RW 20 DS1, Wilmington, NC, USA for SLC-EL) for 5 minutes and elutriate consistency at each renewal was confirmed by total suspended solids (TSS) measurements (conducted according to American Public Health Association (APHA) 1999). During testing, larval and juvenile $P$. promelas received feeding rations of Artemia nauplii and fish flakes (Zeigler ${ }^{\circledR}$ AquaTox Feed, Aquatic EcoSystems, Inc., Apopka, FL, USA), respectively. In compensation for the forgoing daily water renewals, this ration was supplied every other day to maintain water quality. This procedure is in basic accordance with the 96-hr P. promelas test method in USEPA/ USACE (1998). Survival was assessed daily and deceased individuals were removed promptly.

Water quality parameters (temperature, $\mathrm{pH}$, conductivity, dissolved oxygen) were recorded for all replicates at test initiation and termination and for one replicate per treatment during test days 1-9. Ammonia was measured in one replicate per treatment over the duration of the exposure and alkalinity and hardness were measured at test initiation. A model 315i meter (WTW; Weilheim, Germany) was used for $\mathrm{pH}$ and temperature, a model Oxi 330 meter (WTW; Weilheim, Germany) was used for D.O. and 
an ECTestr low instrument (Oakton Instruments, Vernon Hills, IL, USA) was used for conductivity. Meters were calibrated daily and slopes were logged (Appendix F). Ammonia (Model PAN Code 4795), alkalinity (Model WAT-DR Code 4491) and hardness (Model PHT-DR-L1 Code 4482) were measured using LeMotte (Chestertown, MD, USA) titration kits.

\section{Larval fish elutriate bioassay}

Fish larvae were obtained and held for $72 \mathrm{hr}$ to allow acclimation to laboratory conditions. During each day of acclimation, a feeding ration of Artemia nauplii was supplied and water quality was monitored and recorded (Appendix F). The age of the larval fish at test initiation was 9 days post-hatching. This age was selected to be more sensitive than the juvenile life stage but more tolerant to the reported confounding factors inherent to the site (i.e., fungal infections) and to a low feeding ration. Tests were conducted in general accordance with guidance (USEPA/USACE 1998; USEPA 2002a, 2002b). Each of the five experimental replicates per treatment received 10 larvae. Each replicate was gently aerated from an oil-free source (trickle flow, 2-5 bubbles per second) to provide some turbulent flow and to maintain dissolved oxygen levels. More rigorous aeration was not implemented (to attempt to keep the solid phase material in suspension) to avoid energetic stress to the larval fish. At test termination, the endpoints assessed were survival, biomass, and abnormalities. Additionally, a reference toxicity test using $\mathrm{KCl}$ (positive control) was conducted to assess the larval fish health relative to historic control charts. Additional details on the general conduct of the bioassays are provided in Table 14. The exposure system is shown in Figure 57 (a).

\section{Juvenile fish elutriate bioassay}

J uvenile fish were also obtained and held for $72 \mathrm{hr}$ to allow acclimation to laboratory conditions. The acclimating fish received a daily feeding ration of AquaTox fish flakes and water quality parameters were recorded daily (Appendix F). J uvenile fish ( $4.2 \pm 0.3 \mathrm{~cm}$ ) were selected to supply adequate tissue mass for chemical analysis and gill histopathology. Larger adult fish were not used since they may reduce the water quality within the test chambers more rapidly. While a specific testing protocol was not available for this juvenile fish exposure, it was conducted in general accordance with bioassay guidance (USACE/ USEPA 1998; USEPA 2002a, 2002b). Each of the five experimental replicates per treatment received 
Table 14. Summary of conditions for fly ash elutriate toxicity tests conducted by ERDC.

\begin{tabular}{|c|c|c|}
\hline Description & Larval Pimephales promelas & Juvenile Pimephales promelas \\
\hline Test type & Static renewal on days 3 and 7 & Static renewal on days 3 and 7 \\
\hline Test duration & $240 \mathrm{hr}$ & 240 hours \\
\hline Temperature & $\begin{array}{l}\text { Mean: } 20.0 \pm 1.0^{\circ} \mathrm{C} \\
\text { Instantaneous: } 20.0 \pm 3.0^{\circ} \mathrm{C}\end{array}$ & $\begin{array}{l}\text { Mean: } 20.0 \pm 1.0^{\circ} \mathrm{C} \\
\text { Instantaneous: } 20.0 \pm 3.0^{\circ} \mathrm{C}\end{array}$ \\
\hline Salinity / conductivity & Site specific & Site specific \\
\hline $\mathrm{pH}$ & 7-9 SU & 7-9 SU \\
\hline Light quality & Ambient laboratory & Ambient laboratory \\
\hline Light intensity & $10-20 \mathrm{uE} / \mathrm{m} 2 / \mathrm{s}$ & $10-20 \mathrm{uE} / \mathrm{m} 2 / \mathrm{s}$ \\
\hline Photoperiod & 16L:8D & 16L:8D \\
\hline Test chamber size & 300-ml beakers & $4 \mathrm{~L}$ aquarium \\
\hline Test solution volume & $200 \mathrm{~mL}$ & $3.5 \mathrm{~L}$ \\
\hline Renewal of overlying water & $90 \%$ renewal on Days 3 and 7 & $90 \%$ renewal on Days 3 and 7 \\
\hline Age / size of test organisms & $\begin{array}{l}\text { Approximately } 9 \text { days old, } 24-\mathrm{hr} \\
\text { range }\end{array}$ & $3-5 \mathrm{~cm}$ \\
\hline No. organisms per chamber & 10 & 5 \\
\hline No. replicates & 5 & 5 \\
\hline No. organisms per concentration & 50 & 25 \\
\hline Feeding regime & $\begin{array}{l}0.2 \mathrm{ml} \text { concentrate of Artemia } \\
\text { nauplii every other day }\end{array}$ & Fish flakes ad libitum every other day \\
\hline Test chamber cleaning & $\begin{array}{l}\text { Settled elutriate rinsed away at } \\
\text { water exchanges }\end{array}$ & $\begin{array}{l}\text { Settled elutriate rinsed away at water } \\
\text { exchanges }\end{array}$ \\
\hline Test solution aeration & Trickle flow, 3-5 bubbles per second & Moderate to heavy \\
\hline Dilution water & Site water & Site water \\
\hline Test concentrations & $\begin{array}{l}\text { Three dilutions of elutriate, } \\
\text { reference, and control (lab) water }\end{array}$ & $\begin{array}{l}\text { Three dilutions of elutriate, reference, } \\
\text { and control (lab) water }\end{array}$ \\
\hline Dilution series & $100 \%, 50 \%, 10 \%, 0 \%$ & $100 \%, 50 \%, 10 \%, 0 \%$ \\
\hline Endpoint & $\begin{array}{l}\text { Survival, growth, } \\
\text { abnormalities }\end{array}$ & $\begin{array}{l}\text { Survival, organ mass, abnormalities, } \\
\text { histology, tissue residues }\end{array}$ \\
\hline Sampling and hold time & $\begin{array}{l}<8 \text { weeks for sediment } \\
\text { ASAP following elutriate preparation }\end{array}$ & $\begin{array}{l}<8 \text { weeks for sediment } \\
\text { ASAP following elutriate preparation }\end{array}$ \\
\hline Sample volume required & $7.2 \mathrm{~L}$ per site minimum & $126 \mathrm{~L}$ per site minimum \\
\hline Test acceptability criterion & $\begin{array}{l}\text { At least } 80 \% \text { survival in the } \\
\text { negative control }\end{array}$ & $\begin{array}{l}\text { At least } 80 \% \text { survival in the negative } \\
\text { control }\end{array}$ \\
\hline
\end{tabular}




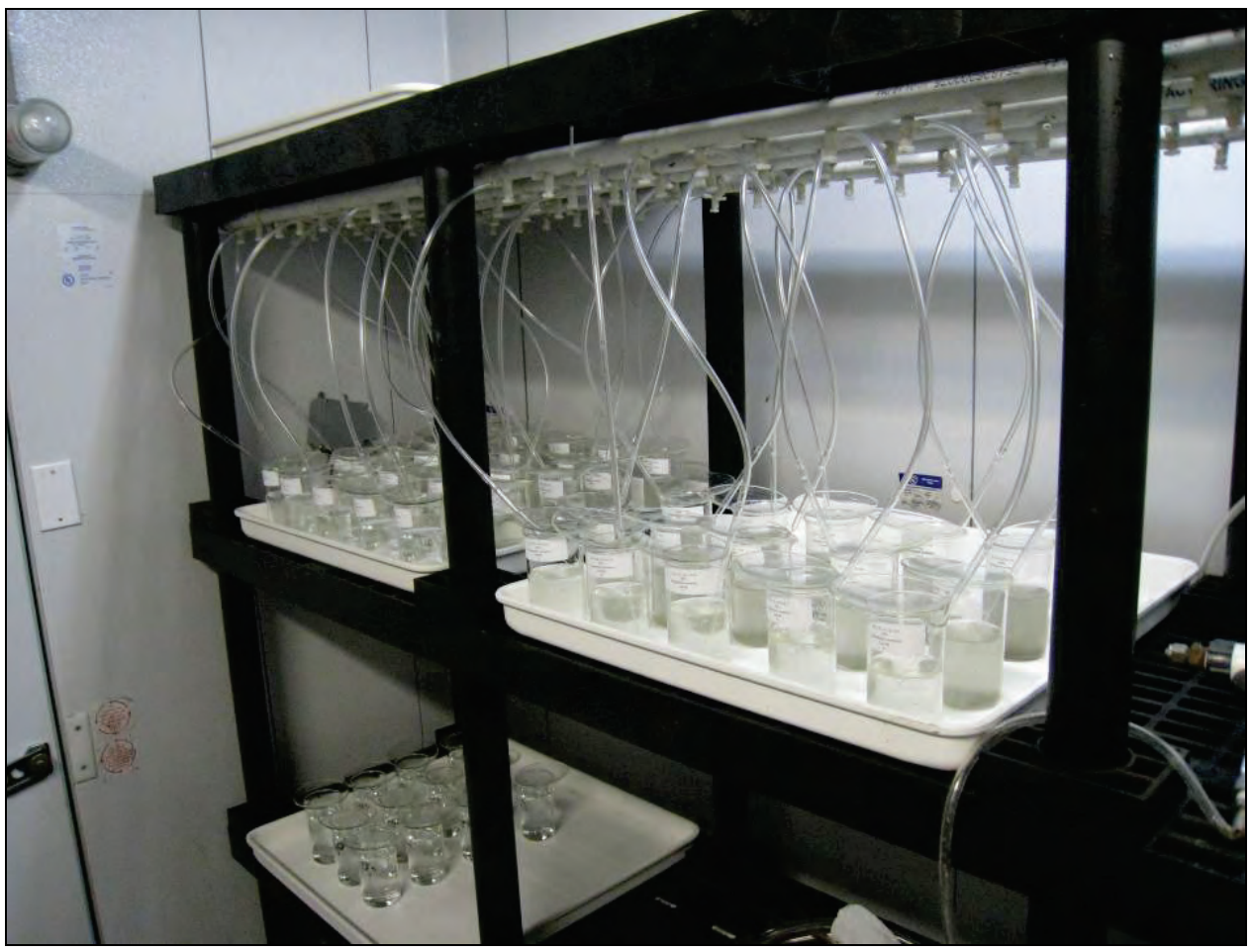

(a)

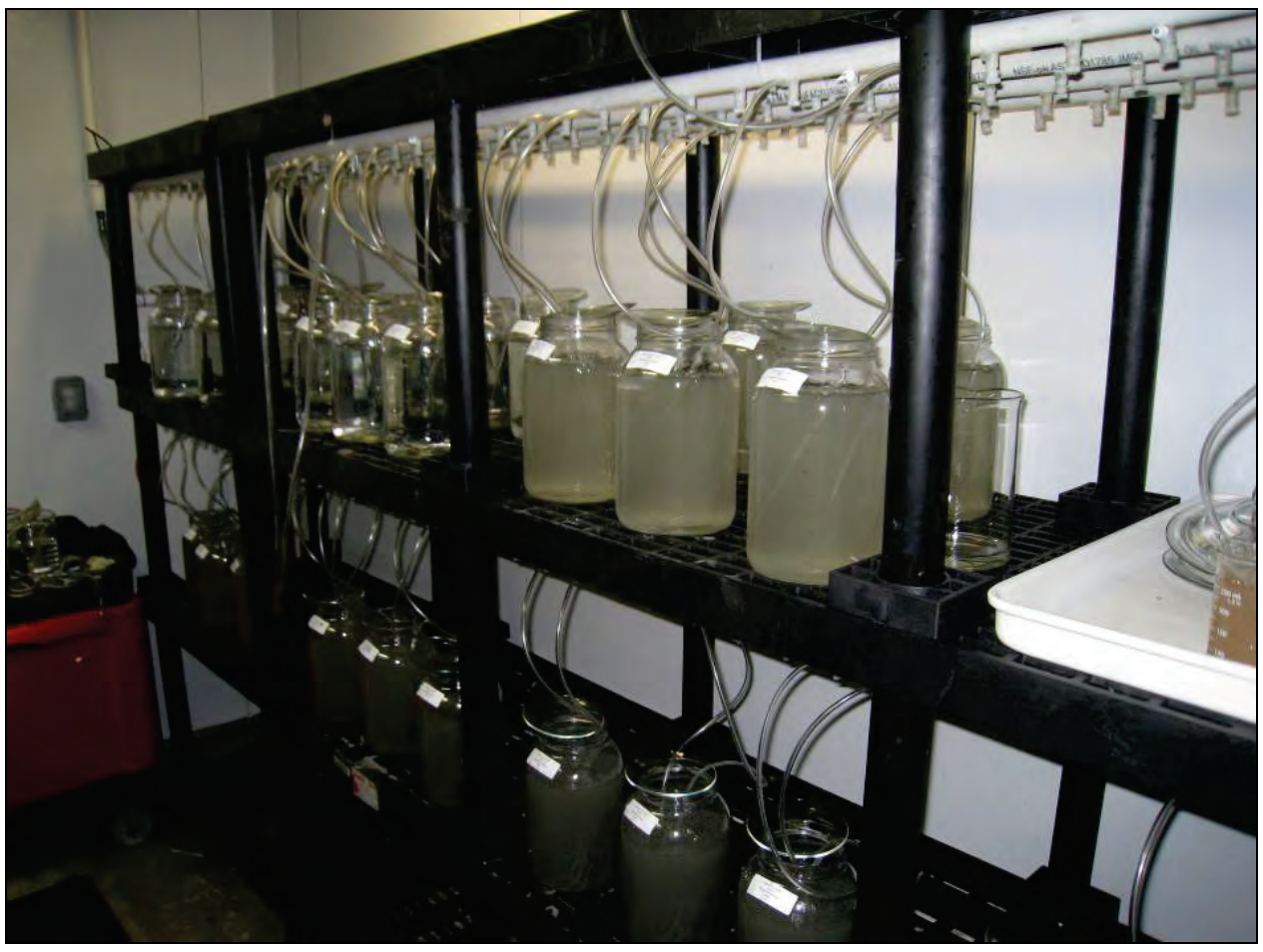

(b)

Figure 57. Image of exposure systems for (a) larval and (b) juvenile Pimephales promelas. 
five juvenile fish and was rigorously aerated from an oil-free source to represent turbulent lotic conditions, and to provide adequate agitation to keep the solid phase material suspended and maintain dissolved oxygen levels. This was in contrast with the larval exposure and was implemented in this case to assess extreme effects of the solid phase material on the gills. The exposure system is shown in Figure 57 (b).

At test termination, the endpoints assessed were survival, whole fish biomass, liver mass (to determine a liver to body mass index), gill histopathology, and abnormalities. At termination, all fish were blotted dry and three of the five fish per replicate were immediately frozen for analysis of chemical residues in whole fish residues. These fish were later composited and homogenized into a fine powder using a mortar and pestle over a liquid nitrogen bath and then submitted for chemical analysis.

Fish homogenates were digested by a modified USEPA method 3050B, explicitly, using additional hydrogen peroxide as needed to destroy lipids that may interfere with ICP-MS elemental analysis. The digestates were filtered and diluted as described above with the fly ash and reference sediment materials. Due to the generally low levels of metals present in tissue, ICP-AES was not used for analysis. Mercury was determined by cold vapor atomic fluorescence using a PS Analytical atomic fluorescence instrument following USEPA method 7474 (USEPA 2007).

Dissections were conducted at ERDC on the remaining two fish per replicate immediately following test termination. Fish were anesthetized (Tricaine methanesulfonate, TMS, MS-222) and livers and gills were carefully removed using Fisherbrand micro-dissection scissors (08-9531B, Thermo Fisher Scientific, Waltham, MD, USA) assisted by dissecting microscopes. Gills were imaged (Figure 58) and immediately placed into 10-ml Bouin's Solution (Sigma Chemical, HT10132-1L, Saint Louis, MO, USA) contained in 20-ml glass scintillation vials. Specimens were shipped overnight to the Department Pharmacology at the University of Mississippi (Oxford, MS, USA) for gill histopathology.

\section{Statistical analysis}

All statistical comparisons were conducted using SigmaStat software (SPSS, Chicago, IL). Data normality and homogeneity were assessed by the Kolmogorov-Smirnov and Levene's Tests, respectively. All survival data were arc-sine transformed. A one-way ANOVA was conducted to assess 


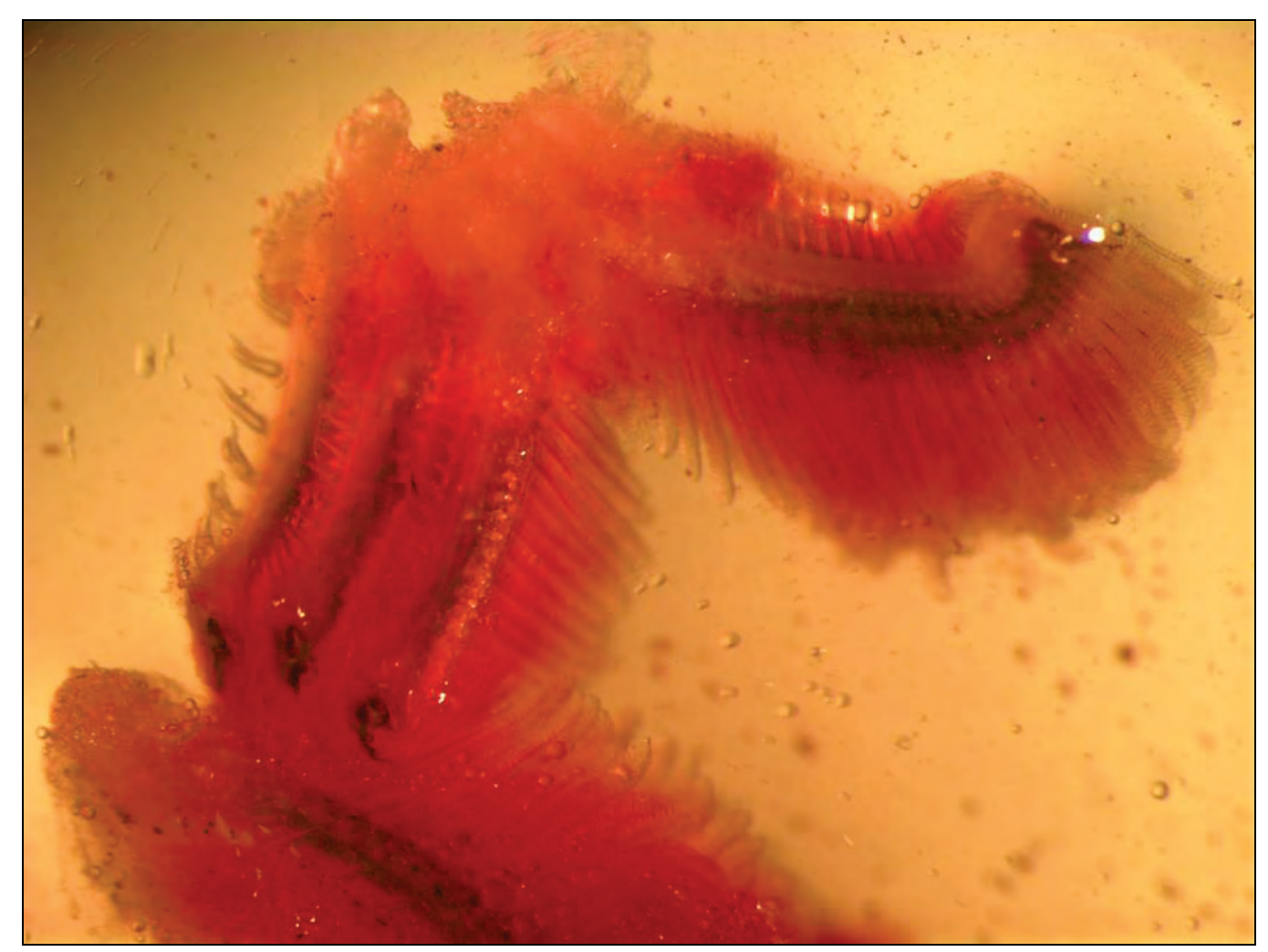

Figure 58. Gills dissected from an unexposed juvenile Pimephales promelas.

statistical significance between treatment levels $(\alpha=0.05)$, and individual differences were elucidated using Dunnett's test. When necessary, data were transformed (square root, $\log _{10}$ ) to accommodate the assumptions of normality. When data distributions did not allow parametric statistics, the Kruskal-Wallis ANOVA on ranks was applied. Statistical significance of endpoints for the various treatment levels (10, 50, 100\%) was compared relative to the RF1-WA site water ( $0 \%$ treatment). A paired t-test ( $0 \%$ vs. treatment) was used to determine the individual $p$-values. Tissue residues of juvenile fish were compared by one-way ANOVA and the Dunnett's method as described above.

\section{Results}

The survival in both life stages of $\mathrm{P}$. promelas in the negative control met acceptability criteria (4-day larval survival $=100 \%$, 10-day larval survival $=86 \%, 10$-day juvenile survival $=100 \%$ ) as defined in Table 14 . Additionally, all water parameters measured during the exposures (Appendix F) were within the ranges specified by test guidance. The 48-hr and 96-hr LC50 values for larvae exposed to $\mathrm{KCl}$ (positive control) were both $0.69(0.60-0.80) \mathrm{g} \mathrm{KCl} / \mathrm{L}$ (Appendix G), which falls within the 
required two standard deviations from the mean LC50 derived from control charts $(0.61-1.13 \mathrm{~g} / \mathrm{L})$. The TSS levels were determined to be comparable for the resuspended $100 \%$ treatment and freshly diluted 50 and $10 \%$ treatments on each of the water renewal days (Table 15).

Table 15. Confirmation of elutriate homogenization consistency by total suspended solids (TSS) concentrations prior to water renewals (days 0,3 , and 7 ). NA = data not available, sample not taken.

\begin{tabular}{|c|c|c|c|c|}
\hline \multirow[b]{2}{*}{ Site ID } & \multirow[b]{2}{*}{ Concentration } & \multicolumn{3}{|c|}{ Mean total suspended solids (mg/L) } \\
\hline & & Day 0 & Day 3 & Day 7 \\
\hline \multirow{3}{*}{ EMR-EL } & $10 \%$ & $36.92 \pm 10.63$ & $51.5 \pm 15.2$ & $50.00 \pm 2.38$ \\
\hline & $50 \%$ & $185.33 \pm 16.63$ & $215.5 \pm 33.81$ & $270.08 \pm 18.00$ \\
\hline & $100 \%$ & $500.42 \pm 32.17$ & $453.08 \pm 48.57$ & $527.58 \pm 17.32$ \\
\hline \multirow{3}{*}{ SLC-EL } & $10 \%$ & $45.08 \pm 14.68$ & $46.50 \pm 2.84$ & $46.17 \pm 39.81$ \\
\hline & $50 \%$ & NA & $157.58 \pm 12.13$ & $213.83 \pm 31.04$ \\
\hline & $100 \%$ & $435.17 \pm 12.79$ & $461.92 \pm 425.07$ & $468.75 \pm 14.34$ \\
\hline
\end{tabular}

\section{Larval elutriate bioassay}

In the larval fish exposure, daily survival counts were estimated in the 50 and $100 \%$ treatments due to the opaque nature of the media and settled solid phase material. However, survival counts were fully assessed on water exchange days (days 3 and 7) and at test termination (day 10). Normal feeding behavior was observed during the exposure. While some spinal curvature was observed in individual fish (denoted by asterisks in the raw data sheets, Appendix F), these abnormalities were not observed monotonically. Fungal growths were not noted. Relative to the site water treatment $(0 \%)$, there were no significant decreases in 4-day or 10-day larval survival (Table 16). However, 10-day survival was significantly greater in the EMR-EL-LF-100\% treatment relative to site water (0\% treatment). While slight decreases in survival were observed for the two highest SLC treatments (SLC-EL-LF-100\% and 50\%) as the exposure approached 10 days (Figure 59(a)), they were not statistically significant. This trend was not observed in the EMR-EL-LF treatments. Overall, larval growth did not occur relative to the initial weights of the fish, potentially due to the reduced feeding ration (once every other day vs. twice daily), which was employed to maintain water quality with fewer water exchanges. The reduced feeding ration was a deviation from the standard bioassay method. Data are consequently reported as biomass. Only one 
statistically significant decrease in 10-day biomass relative to the site water was observed for EMR-EL-LF-10\%. However, significant reductions were not observed in the higher treatments (EMR-EL-LF-100\% and 50\%).

Table 16. Summary of larval Pimephales promelas survival and biomass after 10 days of exposure. All statistical comparisons were to the site water $(0 \%)$. Asterisks indicate a statistically significant decrease in the endpoint while number signs indicate a statistically significant increase in the endpoint. Individual $p$ values were obtained through paired t-tests in comparison to the site water (0\%). One-way ANOVA and paired tests provided equivalent results. $N A=$ data not available.

\begin{tabular}{|c|c|c|c|c|c|c|c|}
\hline \multirow[b]{2}{*}{ Site ID } & \multirow[b]{2}{*}{ Concentration } & \multicolumn{2}{|c|}{$\begin{array}{l}\text { 4-day } \\
\text { Survival }\end{array}$} & \multicolumn{2}{|c|}{$\begin{array}{l}\text { 10-day } \\
\text { Survival }\end{array}$} & \multicolumn{2}{|c|}{$\begin{array}{c}\text { 10-day } \\
\text { Individual Fish } \\
\text { Biomass }\end{array}$} \\
\hline & & Mean (\%) & $P$ value & Mean (\%) & $P$ value & $\begin{array}{l}\text { Mean } \\
(\mathrm{mg})\end{array}$ & $P$ value \\
\hline \multirow{5}{*}{ EMR-EL-LF } & Control & $100 \pm 0$ & NA & $86 \pm 5$ & NA & $0.20 \pm 0.01$ & NA \\
\hline & $0 \%$ & $94 \pm 5$ & NA & $74 \pm 15$ & NA & $0.24 \pm 0.05$ & NA \\
\hline & $10 \%$ & $96 \pm 5$ & 0.690 & $74 \pm 15$ & 0.800 & $0.18 \pm 0.02 *$ & 0.043 \\
\hline & $50 \%$ & $100 \pm 0$ & 0.151 & $76 \pm 19$ & 0.667 & $0.20 \pm 0.02$ & 0.810 \\
\hline & $100 \%$ & $98 \pm 4$ & 0.242 & $94 \pm 5 \#$ & 0.017 & $0.20 \pm 0.01$ & 0.092 \\
\hline \multirow{5}{*}{ SLC-EL-LF } & Control & $100 \pm 0$ & NA & $86 \pm 5$ & NA & $0.20 \pm 0.01$ & NA \\
\hline & $0 \%$ & $94 \pm 5$ & NA & $74 \pm 15$ & NA & $0.24 \pm 0.05$ & NA \\
\hline & $10 \%$ & $88 \pm 4$ & 0.098 & $74 \pm 11$ & 0.976 & $0.21 \pm 0.03$ & 0.229 \\
\hline & $50 \%$ & $78 \pm 28$ & 0.244 & $64 \pm 31$ & 0.690 & $0.20 \pm 0.08$ & 0.306 \\
\hline & $100 \%$ & $90 \pm 12$ & 0.714 & $68 \pm 13$ & 0.487 & $0.21 \pm 0.04$ & 0.302 \\
\hline
\end{tabular}

\section{Juvenile elutriate bioassay}

No mortality or abnormality was observed during the 10-day exposure of juvenile fish. Fish length $(p=0.194)$, whole fish biomass $(p=0.188)$, liver mass $(p=0.506)$, and the liver somatic index $(p=0.789)$ were not significantly different between treatments relative to the site water $(0 \%$ treatment) (Table 17). The combination of the rigorous aeration and the current created by fish swimming kept the solid phase material in suspension (see photographs in Figure 57). The juvenile fish could not be quantified in the 50 and 100\% treatments during non-water exchange days due to the opaque nature of the suspension. Normal feeding behavior was observed in all treatments throughout the exposure. 


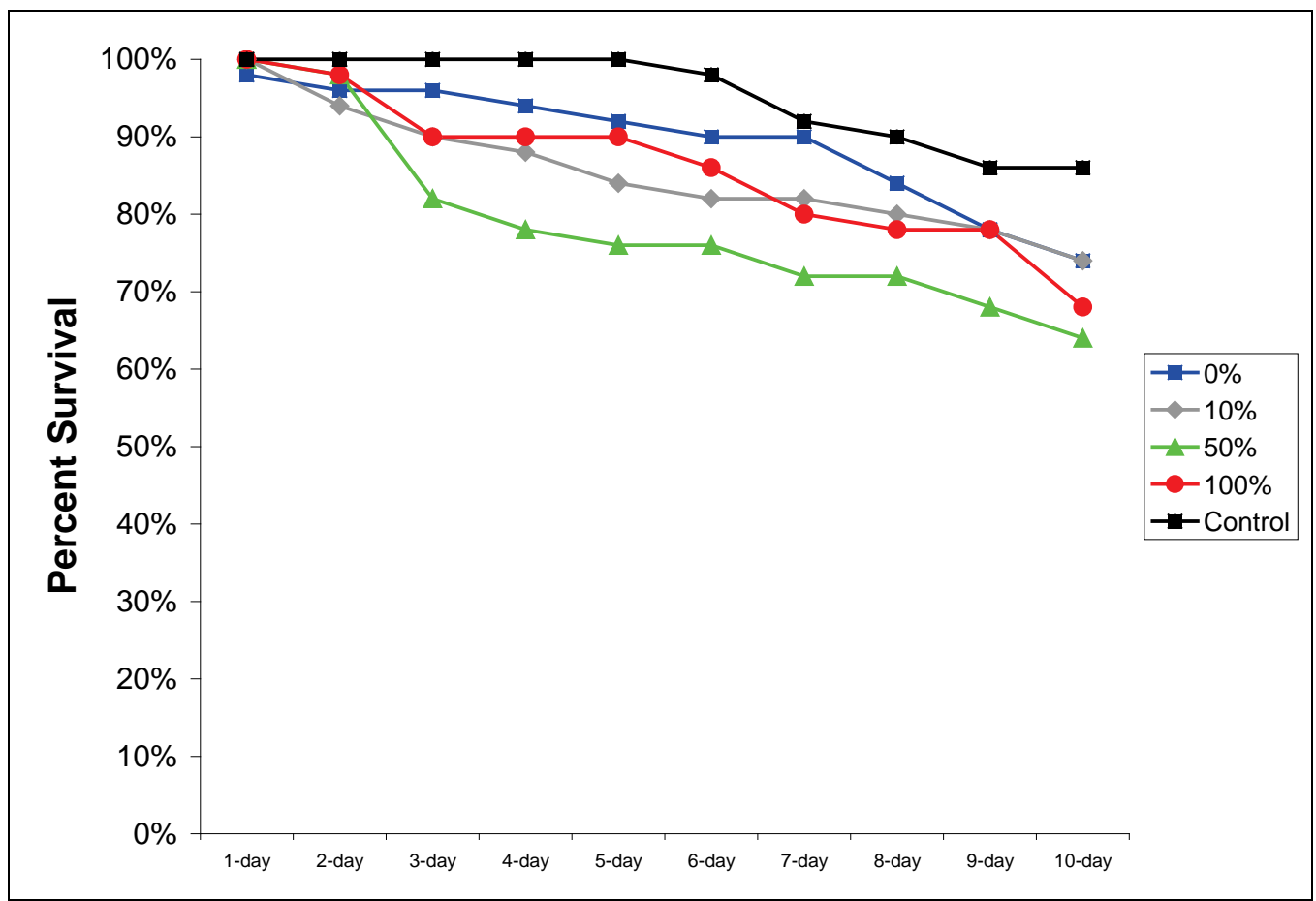

(a)

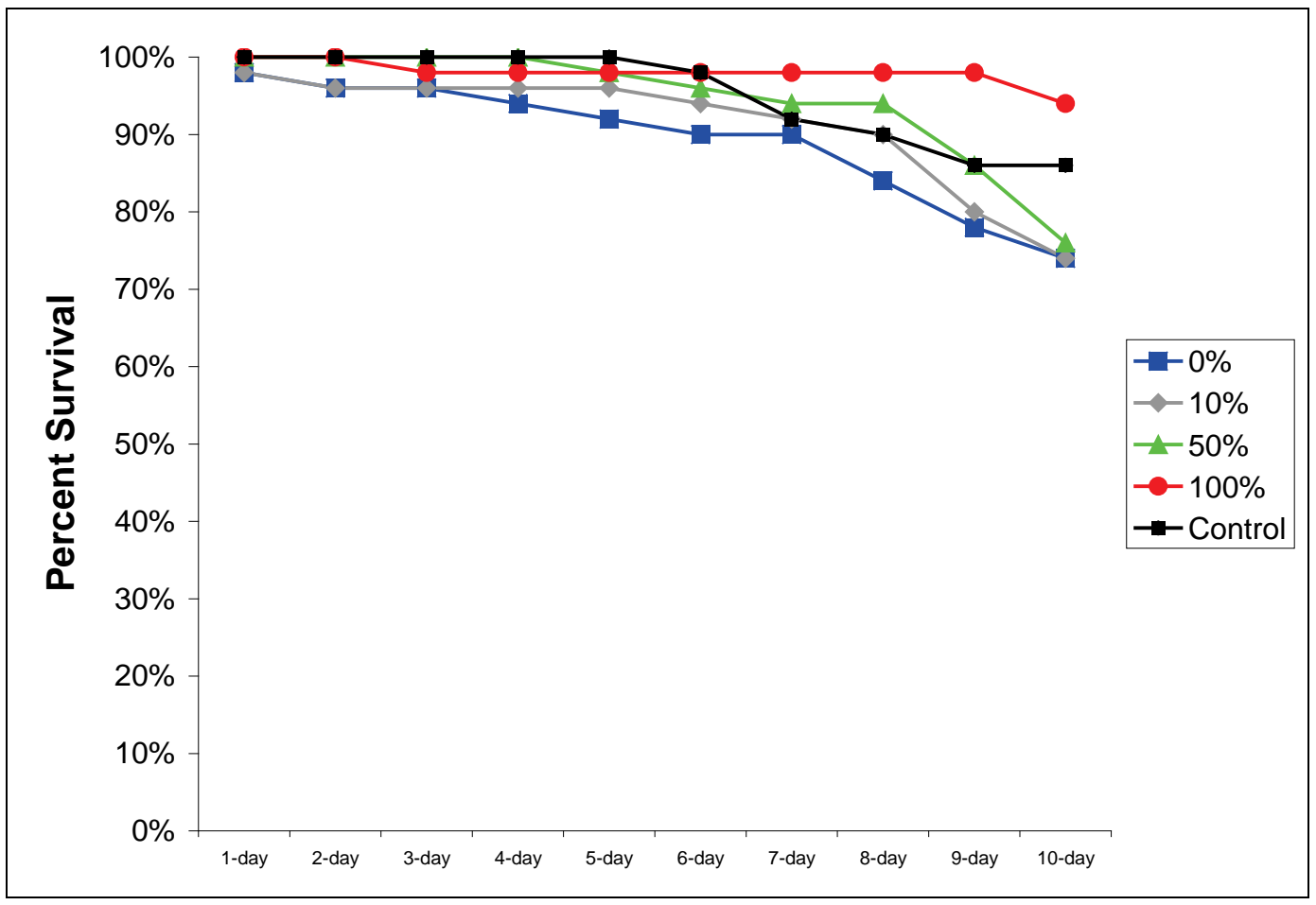

(b)

Figure 59. Daily survival of larval Pimephales promelas assessed over the 10-day exposure duration for (a) SLC-EL-LF, and (b) EMR-EL-LF. Estimates of the data distribution were omitted for clarity but are available in Tables 14 and 15. 
Table 17. Summary of mean juvenile Pimephales promelas survival and biomass (and one standard deviation from the mean) after 10 days of exposure to EMR-EL-AF. Numbers in parentheses represent the coefficient of variation. NA = data not available.

\begin{tabular}{|l|l|l|l|l|l|}
\hline Concentration & $\begin{array}{l}\text { Percent } \\
\text { Survival }\end{array}$ & $\begin{array}{l}\text { Length } \\
(\mathbf{c m})\end{array}$ & $\begin{array}{l}\text { Biomass } \\
(\mathrm{mg})\end{array}$ & $\begin{array}{l}\text { Liver mass } \\
(\mathrm{mg})\end{array}$ & $\begin{array}{l}\text { Liver Somatic } \\
\text { Index }\end{array}$ \\
\hline Control & $100 \pm 0$ & NA & NA & NA & NA \\
\hline $0 \%$ & $100 \pm 0$ & $\begin{array}{l}4.2 \pm 0.3 \\
(7 \%)\end{array}$ & $\begin{array}{l}888 \pm 188 \\
(21 \%)\end{array}$ & $\begin{array}{l}16.83 \pm 4.64 \\
(28 \%)\end{array}$ & $1.9 \pm 0.6(30 \%)$ \\
\hline $10 \%$ & $100 \pm 0$ & $\begin{array}{l}4.0 \pm 0.4 \\
(10 \%)\end{array}$ & $\begin{array}{l}796 \pm 173 \\
(22 \%)\end{array}$ & $\begin{array}{l}16.04 \pm 4.63 \\
(29 \%)\end{array}$ & $\begin{array}{l}2.0 \pm 0.3 \\
(16 \%)\end{array}$ \\
\hline $50 \%$ & $100 \pm 0$ & $\begin{array}{l}4.3 \pm 0.2 \\
(5 \%)\end{array}$ & $\begin{array}{l}959 \pm 138 \\
(14 \%)\end{array}$ & $\begin{array}{l}18.46 \pm 5.06 \\
(27 \%)\end{array}$ & $\begin{array}{l}1.9 \pm 0.5 \\
(24 \%)\end{array}$ \\
\hline $100 \%$ & $100 \pm 0$ & $\begin{array}{l}4.2 \pm 0.3 \\
(6 \%)\end{array}$ & $\begin{array}{l}943 \pm 167 \\
(18 \%)\end{array}$ & $\begin{array}{l}20.04 \pm 8.09 \\
(40 \%)\end{array}$ & $\begin{array}{l}2.2 \pm 0.8 \\
(37 \%)\end{array}$ \\
\hline
\end{tabular}

\section{Whole juvenile fish body burdens}

Analysis of the whole bodies of the juvenile Pimephales promelas revealed a dose-dependent response in the mean concentrations of the six metals of interest (Table 18). The increases in As, $\mathrm{Ba}$, Se, and $\mathrm{V}$ were significantly greater in all EMR-EL-AF treatments (10\%, 50\%, 100\%) relative to fish exposed to the Emory River reference water ( $0 \%)$. For Hg, the two highest treatments (50\%, 100\%) showed significantly higher concentrations in the fish. While Cr did increase monotonically, no statistical significance was found in body burdens relative to the $0 \%$ treatment. Significant increases in whole body burdens were observed for an additional 11 metals, 10 of which showed monotonic increases ( $\mathrm{Al}, \mathrm{Cd}, \mathrm{Co}, \mathrm{Cu}, \mathrm{Fe}, \mathrm{K}, \mathrm{Mg}, \mathrm{Mo}, \mathrm{Pb}, \mathrm{Tl}$ ). While metals were detected in the AquaTox fish food that was provided every 48 hr (As: 1.7, Ba: 3.17, Cr: 5.5, Hg, 0.002, Se: 1.3, V: 0.36 mg/ kg), the feeding ration was kept equal in all exposure chambers. Thus, the consistent and statistically significant dose-dependent relationship in the whole body burdens of the fish suggests that the source of the metal burdens in fish was the test material. However, provided that this assessment was a whole fish analysis in an exposure to metal-laden particles in suspension, the measured concentrations include the sum of metals accumulated in tissues and material that resided in the gut.

In order to assess the portion of metals in tissue measured during the juvenile P. promelas 10-day bioassay that were attributable to metal associated with ingested particles present in the gut, an additional 10-day juvenile P. promelas study was conducted. Determining what fraction of 
metals present in the gut vs. the fraction present in the rest of the body is important in that a significant portion of the metals in the gut may be passed out of the body and may not be bioavailable to the fish (Vijver et al. 2004).

Table 18. Whole Pimephales promelas body burdens (mg/kg wet weight) for arsenic (As), barium (Ba), chromium ( $\mathrm{Cr}$ ), mercury $(\mathrm{Hg})$, and vanadium $(\mathrm{V})$ and other metals (below the double line). Selenium is reported as a dry weight using an internally derived 0.238 conversion factor to compare to a USEPA (2004) tissue benchmark value. Asterisks denote a significant increase relative to tissue concentrations measured in the $0 \%$ (Emory River Reference) treatment. The detection limit was $0.10 \mathrm{mg} / \mathrm{kg}$ (detection limit for mercury was $0.005 \mathrm{mg} / \mathrm{kg}$ ).

\begin{tabular}{|c|c|c|c|c|c|}
\hline Metal & Control & $0 \%$ & $10 \%$ & $50 \%$ & $100 \%$ \\
\hline As & $0.12 \pm 0.02$ & $0.37 \pm 0.04$ & $1.21 \pm 0.22$ * & $2.95 \pm 0.76$ * & $2.84 \pm 0.99 *$ \\
\hline $\mathrm{Ba}$ & $1.83 \pm 0.17$ & $2.40 \pm 0.52$ & $8.20 \pm 1.82$ * & $18.54 \pm 4.99$ * & $20.24 \pm 7.90$ * \\
\hline $\mathrm{Cr}$ & $1.86 \pm 1.12$ & $3.53 \pm 3.20$ & $5.50 \pm 3.00$ & $5.45 \pm 2.14$ & $8.12 \pm 5.13$ \\
\hline $\mathrm{Hg}$ & $0.015 \pm 0.001$ & $0.017 \pm 0.003$ & $0.017 \pm 0.002$ & $0.023 \pm 0.004$ * & $0.023 \pm 0.005$ * \\
\hline $\mathrm{Se}$ & $1.02 \pm 0.06$ & $1.12 \pm 0.12$ & $1.46 \pm 0.11$ * & $2.14 \pm 0.21 *$ & $2.45 \pm 0.42 *$ \\
\hline V & $0.06 \pm 0.03$ & $0.10 \pm 0.05$ & $1.54 \pm 0.40$ * & $4.06 \pm 0.93$ * & $3.88 \pm 1.28$ * \\
\hline $\mathrm{Ag}$ & $<0.10$ & $0.17 \pm 0.27$ & $<0.10$ & $<0.10$ & $0.13 \pm 0.18$ \\
\hline $\mathrm{Al}$ & $11.10 \pm 3.58$ & $25.56 \pm 9.06$ & $400 \pm 112.17$ * & $955.80 \pm 253.59 *$ & $942.20 \pm 352.93$ * \\
\hline $\mathrm{Be}$ & $<0.10$ & $<0.10$ & $<0.10$ & $0.16 \pm 0.04$ & $0.16 \pm 0.04$ \\
\hline $\mathrm{Ca}$ & $5810 \pm 141$ & $5544 \pm 850$ & $5766 \pm 435$ & $5566 \pm 380$ & $5106 \pm 514$ \\
\hline $\mathrm{Cd}$ & $<0.10$ & $<0.10$ & $0.13 \pm 0.01$ * & $0.37 \pm 0.07$ * & $0.39 \pm 0.09$ * \\
\hline Co & $<0.10$ & $<0.10$ & $0.37 \pm 0.07$ * & $0.81 \pm 0.19$ * & $0.83 \pm 0.30$ * \\
\hline $\mathrm{Cu}$ & $1.07 \pm 0.14$ & $1.30 \pm 0.29$ & $2.24 \pm 0.37 *$ & $4.09 \pm 0.89 *$ & $4.07 \pm 1.03$ * \\
\hline $\mathrm{Fe}$ & $35.42 \pm 3.09$ & $71.10 \pm 24.41$ * & $248.60 \pm 51.26$ * & $517.60 \pm 127.75 *$ & $497.00 \pm 175.74 *$ \\
\hline K & $2202 \pm 141$ & $2304 \pm 48$ & $2374 \pm 156$ & $2498 \pm 170$ & $2586 \pm 159$ * \\
\hline $\mathrm{Mg}$ & $260 \pm 16$ & $266 \pm 19$ & $293 \pm 16$ & $338 \pm 29$ * & $344 \pm 36$ * \\
\hline $\mathrm{Mn}$ & $1.20 \pm 0.27$ & $3.41 \pm 1.25$ & $3.41 \pm 0.51$ & $5.62 \pm 1.33$ * & $5.22 \pm 1.72$ \\
\hline Mo & $<0.10$ & $<0.10$ & $0.26 \pm 0.19 *$ & $0.28 \pm 0.05 *$ & $0.31 \pm 0.12 *$ \\
\hline $\mathrm{Na}$ & $931 \pm 56$ & $917 \pm 48$ & $945 \pm 47$ & $972 \pm 24$ & $994 \pm 57$ \\
\hline $\mathrm{Ni}$ & $0.86 \pm 0.57$ & $1.71 \pm 1.46$ & $3.07 \pm 1.15$ & $3.83 \pm 0.84$ & $5.08 \pm 2.78$ \\
\hline $\mathrm{Pb}$ & $0.21 \pm 0.10 *$ & $0.36 \pm 0.20 *$ & $0.81 \pm 0.30$ * & $1.47 \pm 0.41$ * & $1.57 \pm 0.56$ * \\
\hline $\mathrm{Sb}$ & $0.20 \pm 0.34$ & $<0.10$ & $0.15 \pm 0.22$ & $<0.10$ & $0.45 \pm 0.36$ \\
\hline $\mathrm{Tl}$ & $<0.10$ & $<0.10$ & $<0.10$ & $0.15 \pm 0.03$ & $0.21 \pm 0.04$ * \\
\hline $\mathrm{Zn}$ & $24.94 \pm 2.04$ & $27.98 \pm 3.42$ & $27.12 \pm 1.08$ & $27.82 \pm 3.87$ & $26.96 \pm 3.87$ \\
\hline
\end{tabular}


Treatments in this study included EMR-WA, EMR-EL, and a dechlorinated tap water control. This bioassay was performed using the same methods as the original 10-day bioassay, except that in the second study, each treatment consisted of one exposure chamber with five fish in each, and water was renewed once on day 5 .

At the end of the 10-day exposure, there was $100 \%$ survival in each treatment. Fish were sacrificed using MS-222, and their digestive tracts were removed (the liver was not removed). The remainder of the fish carcass was homogenized into a fine powder using a mortar and pestle over a liquid nitrogen bath. The digestive tracts were dried overnight in a $60{ }^{\circ} \mathrm{C}$ oven, then ashed in a muffle furnace at $550{ }^{\circ} \mathrm{C}$ for $3 \mathrm{hr}$. Metal content in the ashed digestive tracts and the homogenized fish carcasses was then submitted for analytical determination using the same methods described for the original 10-day bioassay.

For all of the treatments described above, the total concentration of As, Ba, $\mathrm{Cr}$, Se, and $\mathrm{V}$ were much higher in the gut removed from the fish than from the whole fish with the gut removed. Mercury was not analyzed in the gut due to low mass of the recovered material. However, dissolved concentrations of $\mathrm{Hg}$ were not detected in the extended elutriate at the $10^{\text {th }}$ day of preparation. This effect was most dramatic when fish were exposed to fly ash in the elutriate preparation from the Emory River where concentrations in the gut were 50 to 2800 times higher than in the whole fish with the gut removed. This evidence would suggest the exposuredependent elevations in metal concentration in fish is more likely from gut contents than actual available fraction of metals in fish tissue. Further analysis of these data by normalization of metal concentration to aluminum concentration in fish (an indication of sediment present in gut) showed significant correlations ( $\mathrm{p}<0.05$ ) between aluminum concentration and levels of As, Ba, $\mathrm{Hg}, \mathrm{Se}$, and $\mathrm{V}$ in fish tissue. As a predominant element in the fly ash particles, aluminum concentrations can be considered a tracer for the particles that resided in the gut.

\section{Discussion}

Elutriate bioassay results may be interpreted through a weight of evidence (WOE) evaluation that considers multiple lines of evidence (e.g., chemistry, toxicity, accumulation) to assess the potential for the suspended fly ash to adversely affect aquatic life. A weight of evidence approach provides a more comprehensive evaluation using all available 
data in contrast to reliance on a single measurement endpoint (Adams et al. 2004). In this assessment, elutriate bioassay results are complemented by additional lines of evidence including dissolved water concentration in oxidized elutriate and tissue residues in fish from the elutriate bioassay. This section describes the interpretation of water and tissue residue chemistry results as compared to appropriate screening and literature values. Box and whisker plots were used to show the median, $25^{\text {th }}$ and $75^{\text {th }}$ percentiles, and $10^{\text {th }}$ and $90^{\text {th }}$ percentiles where adequate data were available.

To evaluate the most extreme scenario for exposure of fish to metals in the elutriate preparation, the highest dissolved concentration of each metal during the 10-day elutriate preparation was used. Most often this was the 10-day value (exception: $\mathrm{Hg}$ ). The Criterion Continuous Concentration (CCC) from the USEPA was selected as the preferred screening value protective of aquatic life. State water quality standards are not shown in these comparisons because the state values are the same as USEPA values. Generally, chemical concentrations were not compared against human health screening values because this is outside the scope of this study and not relevant to the other measures of fish toxicity and metal concentrations in whole fish. However, an MCL for Ba in drinking water was discussed in the text below for comparative purposes since USEPA water quality criteria were unavailable. In addition to the screening values, literature values were used for the comparison. A search of all relevant peer-reviewed publications was conducted using the U.S. Environmental Protection Agency's ECOTOX Database (http://cfpub.epa.gov/ecotox/) and Science Direct

(http://www.sciencedirect.com/).

Metal concentrations in whole fish were evaluated through interpretation of tissue residue values (TRVs). While metal TRVs are generally poor estimators of an effect, they can be useful in a weight of evidence assessment to determine the role of a metal in potential toxicity. Some metals, such as selenium, are best evaluated through the use of tissue-based TRVs. Relevant TRVs and literature-effect concentrations (e.g., lowest observed effect residues and no observed effect residues) for whole fish were obtained through literature searches and the USACE Environmental Residue Effects Database (ERED; http://el.erdc.usace.army.mil/ered/). This assessment was considered conservative as tissue TRVs were compared to the whole body burdens of metals in the fish exposed in this study that included metal-laden particles associated with the gut contents. 
Relevant literature studies used to compare water and tissue concentrations were screened following methods described by Steevens et al. (2005). Where relevant screening criteria were not available, literature values were sought to relate metal concentrations in water and tissues to effects observed in elutriate toxicity bioassays. Because there is a wide variety of laboratory and field-based studies reporting toxicity of metals, the available literature must be screened to obtain the most relevant comparable results to interpret the water and tissue concentrations. For this assessment, studies were limited to those reporting laboratory-only experiments with fish. Preference was given to those examining the effects in fathead minnow (Pimephales promelas) or another warm freshwater fish. For quality control, all studies must have had reported measured concentrations and included a range of concentrations. Exposures of longer duration were preferred. Responses included in the screening included decreases in survival, growth, or reproduction. No observed effect concentration (NOEC) or residue (NOER) and lowest observed effect concentration (LOEC) or residue (LOER) values were used from screened studies for comparison to measured concentrations. In some cases in which few TRVs were found in the available literature, median lethal concentration (LC50) and effects residue (ER) values were reported.

While the weight of evidence approach for evaluation of toxicity, chemistry, and bioaccumulation is one of the most rigorous methods for integration of data, there is significant uncertainty in its application to field conditions as well as other aquatic species. Uncertainty in the evaluation of water chemistry comes from the variability in water quality (i.e., hardness, $\mathrm{pH}$ ) for published studies as well as this laboratory study compared to field conditions. Furthermore, the conditions represented in the elutriate bioassay were extreme; dilution and settling at the site are expected to result in metal concentrations lower than this study.

Uncertainty in the evaluation of metal in tissues is from the short duration of exposure in the elutriate bioassay and assumption regarding the species of metal. Concentration of metals in tissue of fish in relationship to toxicity is poorly understood (Society of Environmental Toxicology and Chemistry (SETAC) 2008). In an organism, metals have the capacity to partition in freely dissolved, protein-bound, or metal-rich granule fractions. Except for organo metals (e.g., methyl mercury) or selenium, it is difficult to relate tissue concentrations to biological effects (Luoma and Rainbow 2005, Phillips and Rainbow 1989). 


\section{Arsenic}

Water

The measured dissolved As concentration in the EMR-WA was $0.8 \mu \mathrm{g} / \mathrm{L}$. Measured dissolved As concentration in the SLC-WA was $77.9 \mathrm{\mu g} / \mathrm{L}$. Mean dissolved As concentrations in the EMR-EL increased over the 240-hr elutriate preparation time from a concentration of $20.9( \pm 3.9) \mu \mathrm{g} / \mathrm{L}$ at the 1-hr time point to $53.0( \pm 1.1) \mathrm{\mu g} / \mathrm{L}$ at $240 \mathrm{hr}$. Dissolved As concentrations were higher in the SLC-EL than in the EMR-EL; the measured dissolved As concentration increased from $75.0( \pm 3.0) \mu \mathrm{g} / \mathrm{L}$ at the 1-hr time point to a high of $81.9( \pm 1.8) \mathrm{\mu g} / \mathrm{L}$ at the $96-\mathrm{hr}$ time point. The measured dissolved As concentration at the terminal 240-hr time point was $81.3( \pm 1.6) \mathrm{\mu g} / \mathrm{L}$. $\mathrm{As}(\mathrm{V})$ was by far the predominant As species in both elutriate preparations (Figure 30).

All measured aqueous dissolved concentrations of As were below the Criterion Continuous Concentration (CCC) of $150 \mu \mathrm{g} / \mathrm{L}$ recommended by USEPA (2002c). The highest mean concentration of dissolved As measured in the either elutriate preparation, $81.9( \pm 1.8) \mathrm{\mu g} / \mathrm{L}$, is 1.8-fold less than the CCC. Also, literature toxicity reference values (TRVs) for P. promelas exposure to As were much higher than the conservative CCC value (Figure 60). Arsenic measured in the oxidized elutriates was present as $\mathrm{As}(\mathrm{V})$ and not the more toxic As(III). However, for the purpose of providing a conservative screening level comparison, these concentrations of As(V) in the elutriate are compared to TRVs for the more toxic As(III). The 30-day P. promelas growth no observable effect concentration (NOEC) and lowest observable effect concentration (LOEC) were 2,130 and 4,300 $\mu \mathrm{g} / \mathrm{L}$, respectively, for As(III) in a P. promelas early life stage exposure in which the test organisms are exposed as eggs and then for 30-day post fertilization (about 24-day post hatch) (Call et al. 1983). The concentrations of As in water were below all screening thresholds and unlikely to result in adverse effects to aquatic biota. 


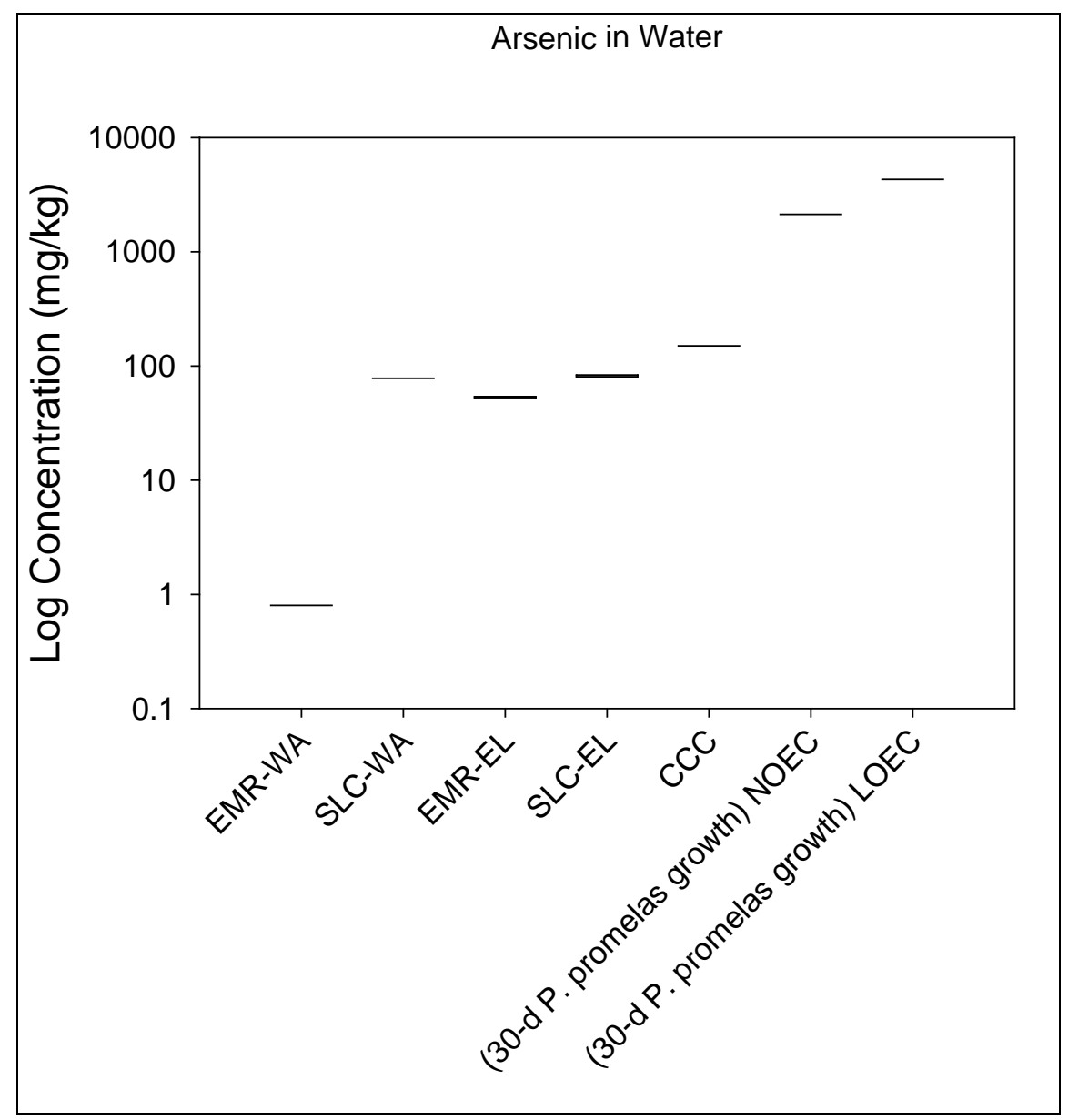

Figure 60. Dissolved arsenic concentrations in Emory River Reference water (EMR-WA), sluice water (SLC-WA), Emory River fly ash Elutriate (EMR-EL), sluice fly ash elutriate (SLC-EL), and the U.S. Environmental Protection Agency chronic water quality criterion (Criterion Continuous Concentration or CCC). Available toxicity reference values (TRVs) from the literature are summarized and defined in the definitions section. The exposure durations for the TRVs are indicated on the $x$-axis. Stand-alone horizontal lines indicate single points or tight distributions. ND $=$ not detected (reporting limits $=1 \mu \mathrm{g} / \mathrm{L}$ ).

Tissue

The whole body burden of As ranged from $0.37( \pm 0.04) \mathrm{mg} / \mathrm{kg}$ wet weight in the EMR-WA to $2.95 \pm 0.76 \mathrm{mg} / \mathrm{kg}$ wet weight in fish exposed to the $50 \%$ EMR-EL dilution. The $100 \%$ dilution yielded a mean As body burden of 2.84 ( \pm 0.99$) \mathrm{mg} / \mathrm{kg}$ wet weight.

The concentrations of As measured in the tissue of fish exposed to the EMR-WA are well below the literature TRVs for As in tissue; however, the highest amount of As in the whole fish body burden in the EMR-EL exposure, $2.95 \pm 0.76 \mathrm{mg} / \mathrm{kg}$ wet weight, was above or approximately equal to various TRVs for As in tissue for Lepomis macrochirus (bluegill) 
and Oncorhynchus mykiss (rainbow trout) (Figure 61) (Gilderhus 1966, McGeachy and Dixon 1990, Dixon and Sprague 1981). The elevated level of As in tissues is likely due to contributions of As from gut contents during analysis and unlikely to indicate toxicity to fish due to As in the test system.

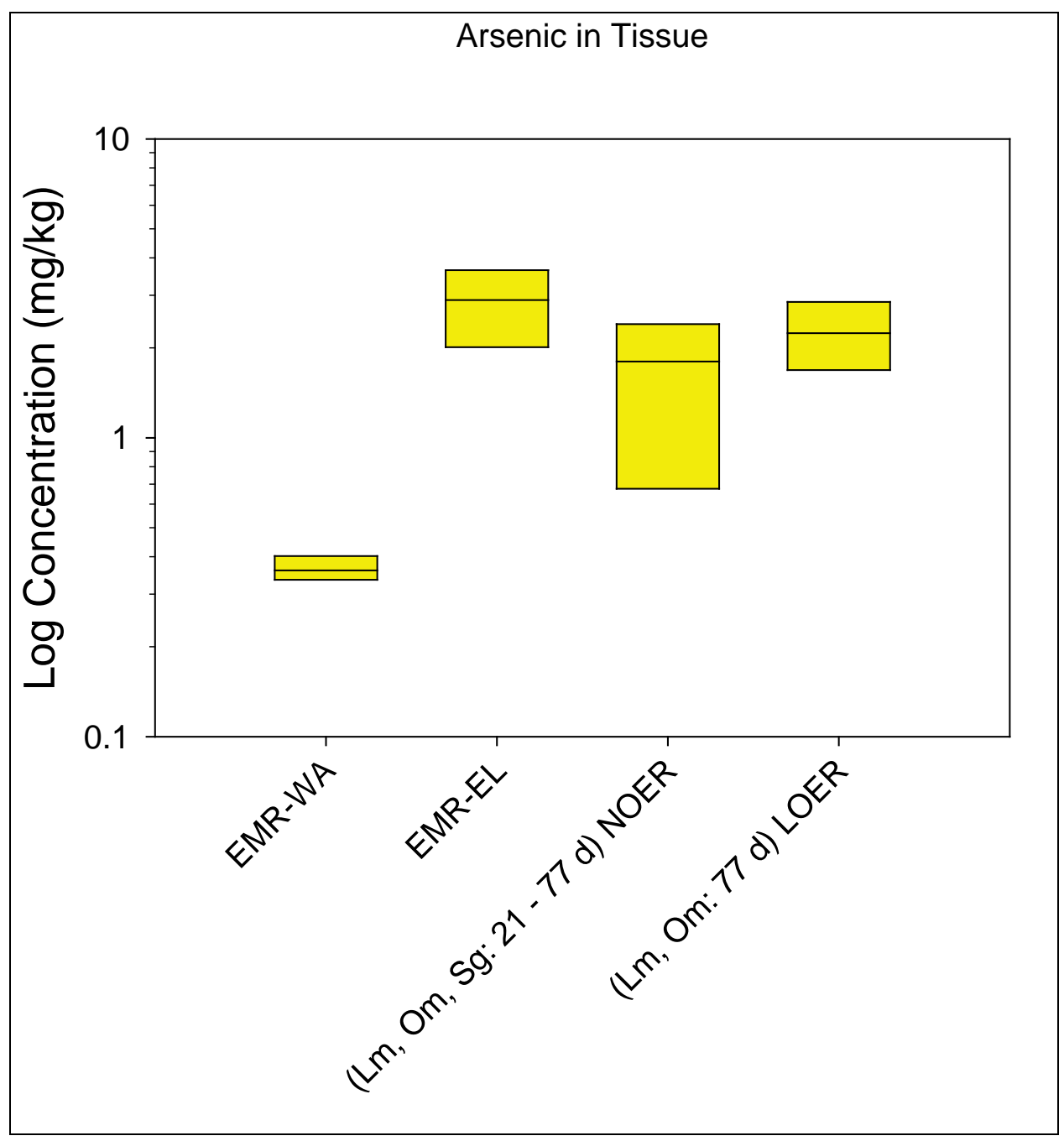

Figure 61. Arsenic concentrations (as wet weight, $\mathrm{mg} / \mathrm{kg}$ ) in whole Pimephales promelas exposed to EMR-EL-AF for 10 days. Available toxicity reference values (TRVs) from the literature are summarized and defined in the definitions section. The exposure durations for the TRVs are indicated on the x-axis. The ends of the boxes represent the $25^{\text {th }}$ and $75^{\text {th }}$ percentiles of the data distribution and the horizontal line indicates the median represented value. Stand-alone horizontal lines indicate single points or tight distributions. $\mathrm{Lm}=$ Lepomis macrochirus, $\mathrm{Om}=$ Oncorhynchus mykiss, $\mathrm{Sg}=$ survival and growth. Reporting limits $=0.5 \mathrm{mg} / \mathrm{kg}$. 


\section{Barium}

Water

Dissolved Ba was detected in the EMR-WA and sluice channel water (SLCWA) at concentrations of 30.7 and $107.0 \mu \mathrm{g} / \mathrm{L}$, respectively.

In the Emory River fly ash elutriate (EMR-EL), mean ( \pm SD) dissolved Ba concentrations increased over the 240 -hr elutriate preparation period from a concentration of $77.7( \pm 5.1)$ at the $1-h r$ time point to $121.7( \pm 3.8)$ $\mathrm{\mu g} / \mathrm{L}$ after $240 \mathrm{hr}$ of extended elutriate mixing in oxic conditions. Dissolved Ba levels were similar in the sluice fly ash elutriate (SLC-EL) and also increased over time, ranging from a concentration of $99.4( \pm 0.5)$ to 116.7 ( \pm 1.5$) \mu \mathrm{g} / \mathrm{L}$ at the 1-and 240 -hr time points, respectively.

There are no USEPA recommended water quality criteria for the protection of aquatic life for Ba. The measured concentrations in the 100\% EMR-EL and SLC-EL elutriate waters were considerably lower than acute NOECs (Figure 62) for the invertebrate Daphnia magna (68,000 $\mathrm{gg} / \mathrm{L}$ ), a marine larval fish Cyprinondon variegatus $(500,000 \mathrm{\mu g} / \mathrm{L}$ ) that is likely more tolerant to metals than the freshwater P. promelas (Leung et al. 2001, Wheeler et al. 2002), and a maximum permissible concentration (MPC) that was modeled from ecotoxicology data and is used in the Netherlands (Crommentuijn et al. 2000). Few additional no-effect toxicity reference values for Ba were found in the literature. Effect levels (48-hr LC50 and EC50 values ranging from 32,000 to 410,000 $\mu \mathrm{g} / \mathrm{L}$ ) were found for D. magna (LeBlanc 1980; Khangarot and Ray 1989). These values are likely nominal (measurement techniques not reported) and the acute duration is unlikely to be protective under chronic exposure durations. Safety multipliers (e.g., 0.01, 0.05, 0.10) have been employed in the past to generate more protective values from acute LC50 values, but this approach has been criticized (National Academy of Sciences/ National Academy of Engineering 1973; Mount 1977). However, acute to chronic ratios (acute LC50 divided by a chronic NOEC) do suggest some merit to AFs (Mount 1977, Verma et al. 1981, Giesy and Graney 1989, Heger et al. 1995, Lange et al. 1998, Ahlers et al. 2006), but it is clear that the magnitude of the application factor is chemical class specific (Roex et al. 2000). Application of the lowest of these factors (0.01) still results in toxicity reference values that are at least 2.6 fold higher than measured water concentrations. The USEPA's maximum contaminant level (MCL) for drinking water (2,000 pg/ L; USEPA 2009) and human consumption of organisms and 
water (1,000 $\mu \mathrm{g} / \mathrm{L}$; USEPA 2002c) guidelines are more than 16 and 8 times higher, respectively, than the Ba concentrations measured in the elutriate water. Overall, it is not expected that the concentrations of $\mathrm{Ba}$ in the investigated water samples present substantial toxicological implications.

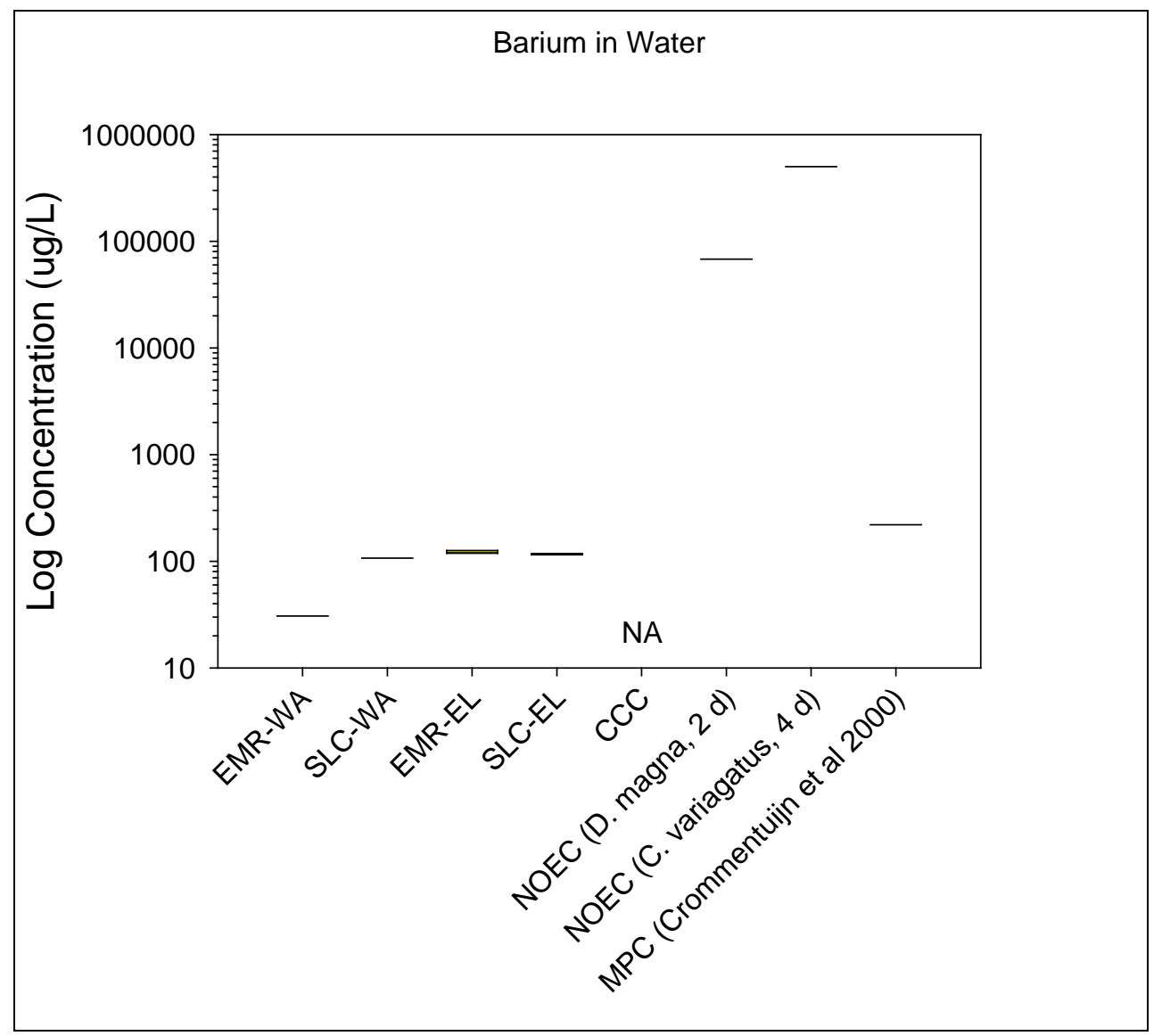

Pimephales promelas (freshwater fish), Daphnia magna (freshwater invertebrate), Cyprinodon variegatus (salt water fish).

Figure 62. Dissolved barium concentrations in Emory River reference water (EMR-WA), sluice water (SLC-WA), Emory River fly ash elutriate (EMR-EL) and sluice fly ash elutriate

(SLC-EL). Note that WQC are not available for barium but the USEPA drinking water quality criterion (MCL) is provided in the text. Available toxicity reference values (TRVs) from the literature are summarized and defined in the definitions section. The exposure durations for the TRVs are indicated on the X-axis. Stand-alone horizontal lines indicate single points or tight distributions. ND $=$ not detected (reporting limits $=1 \mu \mathrm{g} / \mathrm{L}$ ).

\section{Tissue}

In the EMR-EL bioaccumulation exposure of juvenile P. promelas, mean measured Ba body burdens ranged from 2.40 ( \pm 0.52$)$ to 20.24 ( \pm 7.90$)$ $\mathrm{mg} / \mathrm{kg}$ wet weight in the lowest ( $0 \%$ elutriate) and highest (100\% elutriate) treatments, respectively. No useful tissue residue benchmarks for Ba in fish were found in the available literature, except for one very low level 
NOER of $0.15 \mathrm{mg} / \mathrm{kg}$ (Dube et al. 2005). This value represents an arbitrarily measured body burden that induced no reported effect. Since effects level body burden benchmarks were not found, a figure comparing Ba body burdens in the current study is not included in this report.

\section{Chromium (III)}

\section{Water}

The only chromium species found in this study was $\mathrm{Cr}$ (III), which is widely reported to be less toxic than the more heavily studied $\mathrm{Cr}(\mathrm{VI})$ (Baral et al. 2006, Farag et al. 2006). Dissolved Cr(III) $(<0.2 \mu \mathrm{g} / \mathrm{L})$ was not detected above detection limits in the Emory River reference water (EMR-WA) or sluice channel water (SLC-WA).

In the Emory River fly ash elutriate (EMR-EL), mean ( \pm SD) dissolved $\mathrm{Cr}$ (III) concentrations increased over the 240-hr elutriate preparation period from a concentration that was less than DL $(<0.2 \mu \mathrm{g} / \mathrm{L})$ at the $1-\mathrm{hr}$ time point to $1.8( \pm 0.1) \mu \mathrm{g} / \mathrm{L}$ after $240 \mathrm{hr}$ of extended elutriate mixing under oxic conditions. Dissolved Cr(III) levels were slightly lower in the sluice fly ash elutriate (SLC-EL) and also increased over time, ranging from less than DL $(<0.2 \mu \mathrm{g} / \mathrm{L})$ to $0.7( \pm 0.0) \mu \mathrm{g} / \mathrm{L}$ at the 1 -and- $240 \mathrm{hr}$ time points, respectively.

These values are less than the USEPA's (2002c) continuous criterion concentration (CCC) for dissolved Cr(III) of 74 and $21 \mu \mathrm{g} / \mathrm{L}$ at water hardness of $100^{1}$ and $22^{2} \mathrm{mg} / \mathrm{L}$ as $\mathrm{CaCO}_{3}$, respectively. Figure 63 compares the measured $\mathrm{Cr}$ (III) concentrations in the water to the CCC and toxicity reference values found in the literature. All measured dissolved concentrations are well below a 48-hr NOEC (Baral et al. 2006) and a 30-day MATC (Spehar and Fiandt 1986) reported for P. promelas. Gendusa and Beitinger (1992) reported a 96-hr NOEC for Lepomis macrochirus of $5030 \mu \mathrm{g} / \mathrm{L}$ that was excluded from the graph since data were found for P. promelas. Overall, Cr(III) would not be expected to be of toxicological concern since the measured concentrations in the $100 \%$ elutriates were more than 11 times lower than the CCC and 1000 times lower than a literature-reported MATC.

\footnotetext{
1 Standard water hardness used in the water quality criterion document (USEPA 2002c).

2 Lowest water hardness $\left(22 \mathrm{mg} / \mathrm{L}\right.$ as $\mathrm{CaCO}_{3}$ ) measured by titration in a site water. This value was recalculated to be $30 \mathrm{mg} / \mathrm{L}$ as $\mathrm{CaCO}_{3}$ using the measured concentrations of $\mathrm{Ca}$ and $\mathrm{Mg}$.
} 


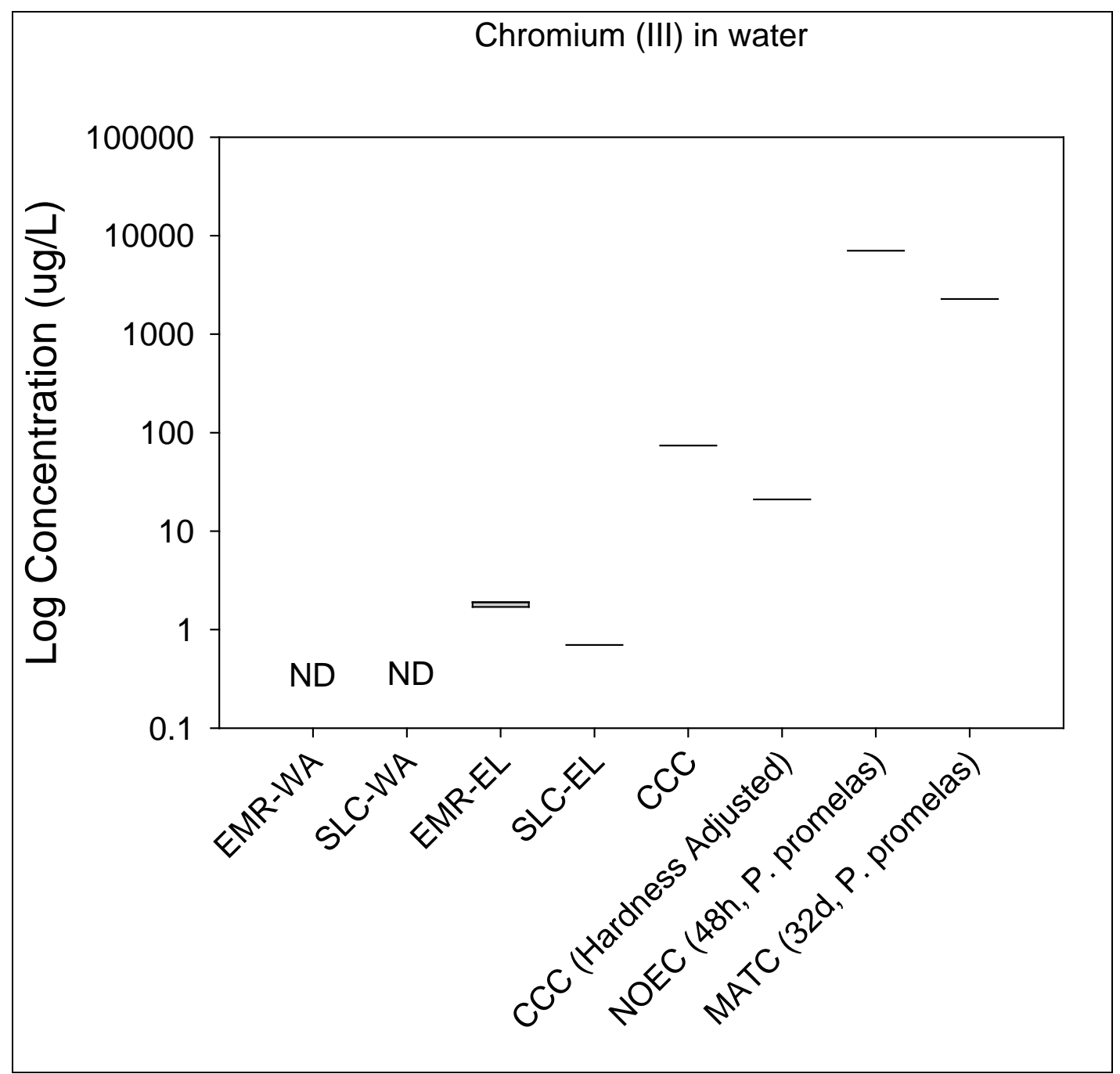

Figure 63. Dissolved chromium (III) concentrations in Emory River reference water (EMR-WA), sluice water (SLC-WA), Emory River fly ash elutriate (EMR-EL), sluice fly ash elutriate (SLC-EL), and the standard and hardness adjusted USEPA chronic water quality criteria (criterion continuous concentration or CCC). Available toxicity reference values (TRVs) from the literature are summarized and defined in the definitions section. The exposure durations for the TRVs are indicated on the X-axis. Stand-alone horizontal lines indicate single points or tight distributions. ND = not detected (reporting limits $=1 \mu \mathrm{g} / \mathrm{L}$ ).

\section{Tissue}

In the EMR-EL bioaccumulation exposure of juvenile P. promelas, mean measured Cr whole body burdens (including gut contents) ranged from 3.53 $( \pm 3.20)$ to $8.12( \pm 5.13) \mathrm{mg} / \mathrm{kg}$ wet weight in the lowest ( $0 \%$ elutriate) and highest (100\% elutriate) treatments, respectively (Figure 64). The median Cr body burden in the $100 \%$ elutriate treatment was comparable to a 108-day NOER reported for Oncorhynchus tshawytscha (Farag et al. 2006). Additionally, Roling et al. (2006) generated an ER15 (a tissue residue benchmark at which $15 \%$ mortality was modeled) of $44 \mathrm{mg} / \mathrm{L}$ for 
Fundulus heteroclitus exposed to hexavalent $\mathrm{Cr}(\mathrm{VI})$. While this freshwater fish was exposed to the more toxic $\mathrm{Cr}(\mathrm{VI})$ species, this ER15 value is still five times higher than measured body burdens of the less toxic $\mathrm{Cr}$ (III). ${ }^{1}$ It is not expected that the whole body burdens of $\mathrm{Cr}$ are of toxicological significance in the current investigation. In addition, most of the Cr detected was associated with the fish gut contents.

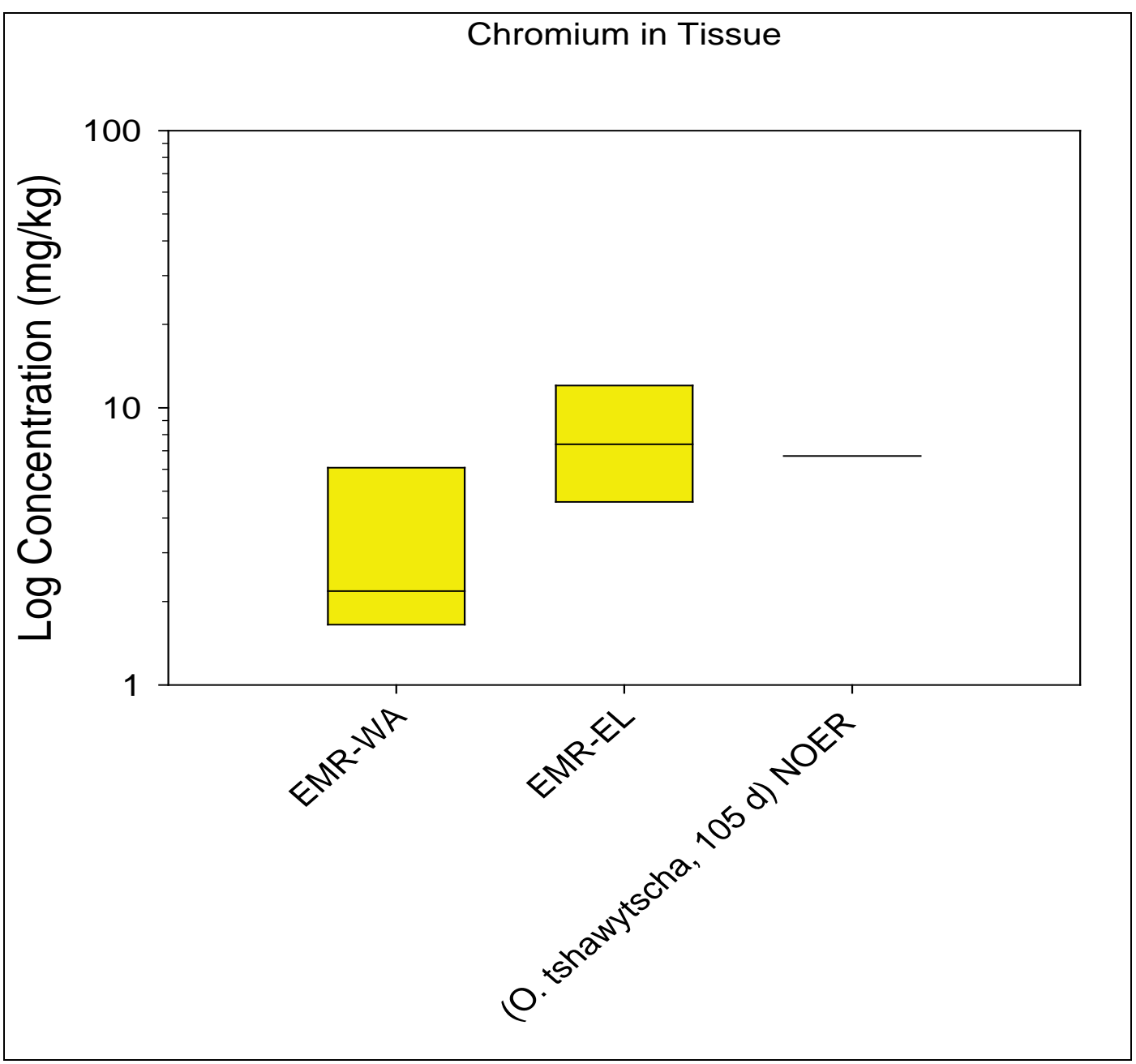

Figure 64. Chromium concentrations (as wet weight, $\mathrm{mg} / \mathrm{kg}$ ) in whole Pimephales promelas exposed to EMR-EL-AF for 10 days. Available toxicity reference values (TRVs) from the literature are summarized and defined in the definitions section. The exposure durations for the TRVs are indicated on the X-axis. The ends of the boxes represent the $25^{\text {th }}$ and $75^{\text {th }}$ percentiles of the data distribution and the horizontal line indicates the median represented value. Stand-alone horizontal lines indicate single points or tight distributions. Reporting limits $=0.5 \mathrm{mg} / \mathrm{kg}$.

${ }^{1}$ Chronic WQC suggest $\mathrm{Cr}(\mathrm{VI})$ is 6.7 times more toxic. 


\section{Mercury}

Water

The only inorganic $\mathrm{Hg}$ was found in this study, which is reported to be less toxic than methyl mercury (Friedmann et al. 1996). Dissolved Hg was below reporting limits ( $\mathrm{DL}<0.005 \mu \mathrm{g} / \mathrm{L}$ ) in the Emory River reference water (EMR-WA) and sluice channel water (SLC-WA).

In the Emory River fly ash elutriate (EMR-EL), mean $( \pm \mathrm{SD})$ dissolved Hg concentrations did not increase over time. In fact, the only detectable concentrations ( $\mathrm{RL}<0.005 \mu \mathrm{g} / \mathrm{L}$ ) in the Emory fly ash elutriate (EMR-EL) were for the $1-\mathrm{hr}(0.014 \pm 0.001 \mu \mathrm{g} / \mathrm{L})$ and $24-\mathrm{hr}(0.011 \pm 0.007 \mu \mathrm{g} / \mathrm{L})$ timepoints. Similarly, $\mathrm{Hg}$ was only detected in the sluice elutriate (SLCEL) in the 1-hr $(0.008 \pm 0.005 \mu \mathrm{g} / \mathrm{L})$ and $24-\mathrm{hr}(0.010 \pm 0.007 \mu \mathrm{g} / \mathrm{L})$ time points. These values are near reporting limits and should be interpreted cautiously. It is hypothesized that while the source of the $\mathrm{Hg}$ was likely the fly ash particles, the dissolved $\mathrm{Hg}$ either precipitated from solution or volatilized in the oxic conditions during the 10-day elutriate preparation.

The highest measured values of Hg are much less than the USEPA's (2002c) continuous criterion concentration (CCC) for dissolved $\mathrm{Hg}$ of $0.77 \mu \mathrm{g} / \mathrm{L}$. Figure 65 compares the measured Hg concentrations in the water to the CCC and toxicity reference values found in the literature. The measured dissolved concentrations are well below the median, long-term NOECs, and LOECs generated for P. promelas (Call et al. 1983; Snarski and Olson 1982). The large variability in the NOEC and LOEC data presented in Figure 65 are due in part to the inclusion of measurement endpoints that are inherently different in sensitivity (i.e., survival, growth, hatching success). Spehar and Fiandt (1986) reported a 32-day MATC for P. promelas growth of $0.89 \mu \mathrm{g} / \mathrm{L}$, a value 64 times higher than the $\mathrm{Hg}$ concentrations in the EMR-EL and SLC-EL elutriates. However, MATCs as low as $0.26 \mu \mathrm{g} / \mathrm{L}$ have been reported by others (Snarski and Olson 1982), a value that is still 19 times higher than the dissolved Hg concentrations in the elutriates. The MATC in Figure 65 is the median of the two values reported above. Overall, the dissolved $\mathrm{Hg}$ concentrations detected in the test elutriates are well below WQC ( 55 times lower) and toxicity reference values and are not likely to be toxicologically significant. 


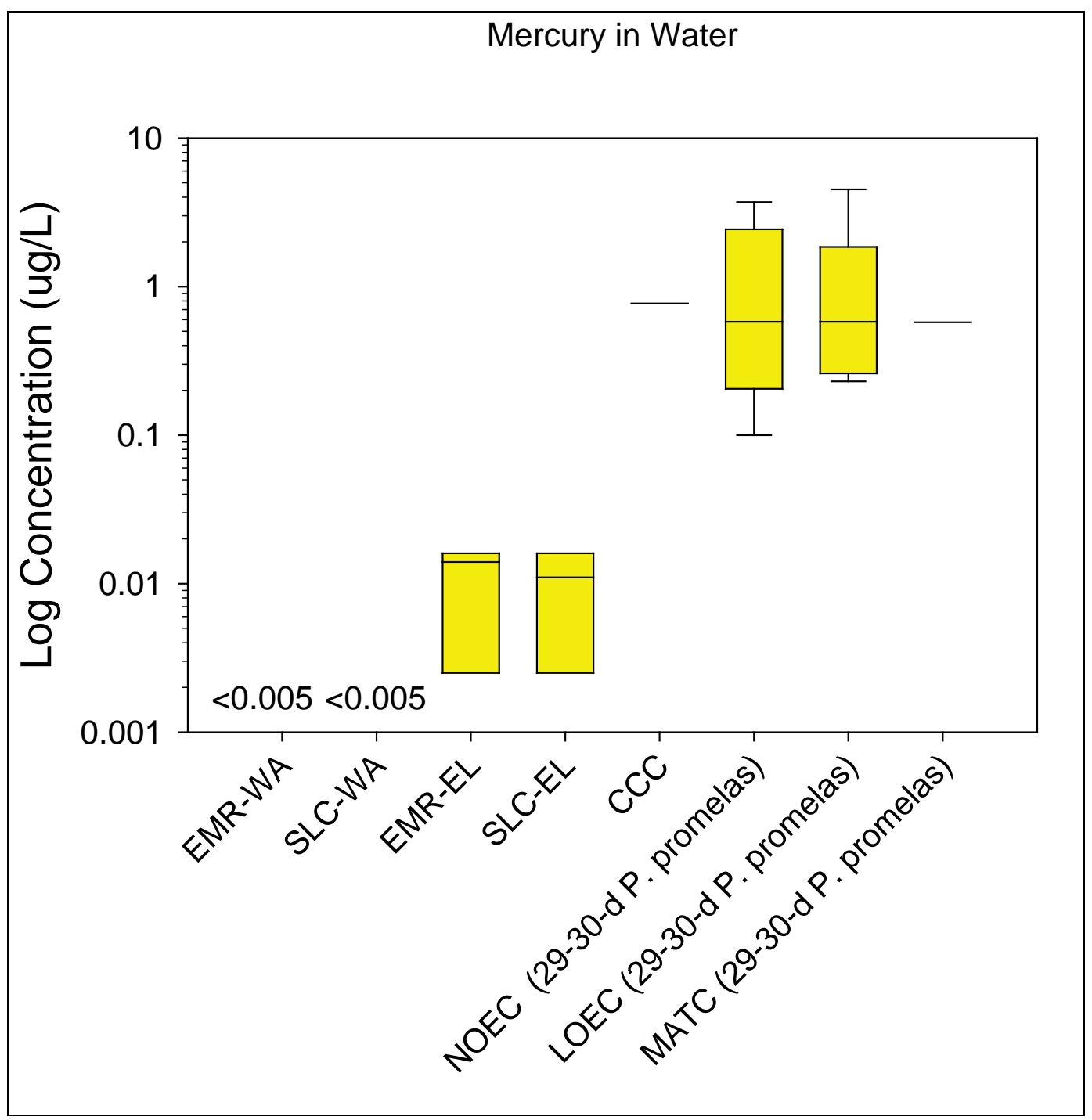

Figure 65. Dissolved mercury concentrations in Emory River reference water (EMR-WA), sluice water (SLC-WA), Emory River fly ash elutriate (EMR-EL), sluice fly ash elutriate (SLC-EL) and the USEPA chronic water quality criterion (criterion continuous concentration or CCC). Available toxicity reference values (TRVs) from the literature are summarized and defined in the definitions section. The exposure durations for the TRVs are indicated on the X-axis. The ends of the boxes represent the $25^{\text {th }}$ and $75^{\text {th }}$ percentiles of the data distribution, the horizontal line indicates the median represented value, and whiskers represent the $10^{\text {th }}$ and $90^{\text {th }}$ percentiles. Stand-alone horizontal lines indicate single points or tight distributions. ND = not detected (reporting limits $=0.005 \mu \mathrm{g} / \mathrm{L}$ ).

Tissue

In the EMR-EL bioaccumulation exposure of juvenile P. promelas, mean measured Hg body burdens ranged from 0.017 ( \pm 0.003 ) to 0.023 $( \pm 0.005) \mathrm{mg} / \mathrm{kg}$ wet weight in the lowest ( $0 \%$ elutriate) and highest (100\% elutriate) treatments, respectively (Figure 66). These body burdens are 87-and 130-fold lower than median NOERs and LOERs reported in the 
literature (Snarski and Olson 1982; Friedmann et al. 1996) and more than eight times lower than the tissue threshold effect level (t-TEL) modeled by Beckvar et al. (2005). These data suggested the body burdens of $\mathrm{Hg}$ in the juvenile P. promelas are not high enough to be of toxicological significance. Further, metals burdens in this study were primarily associated with the gut contents.

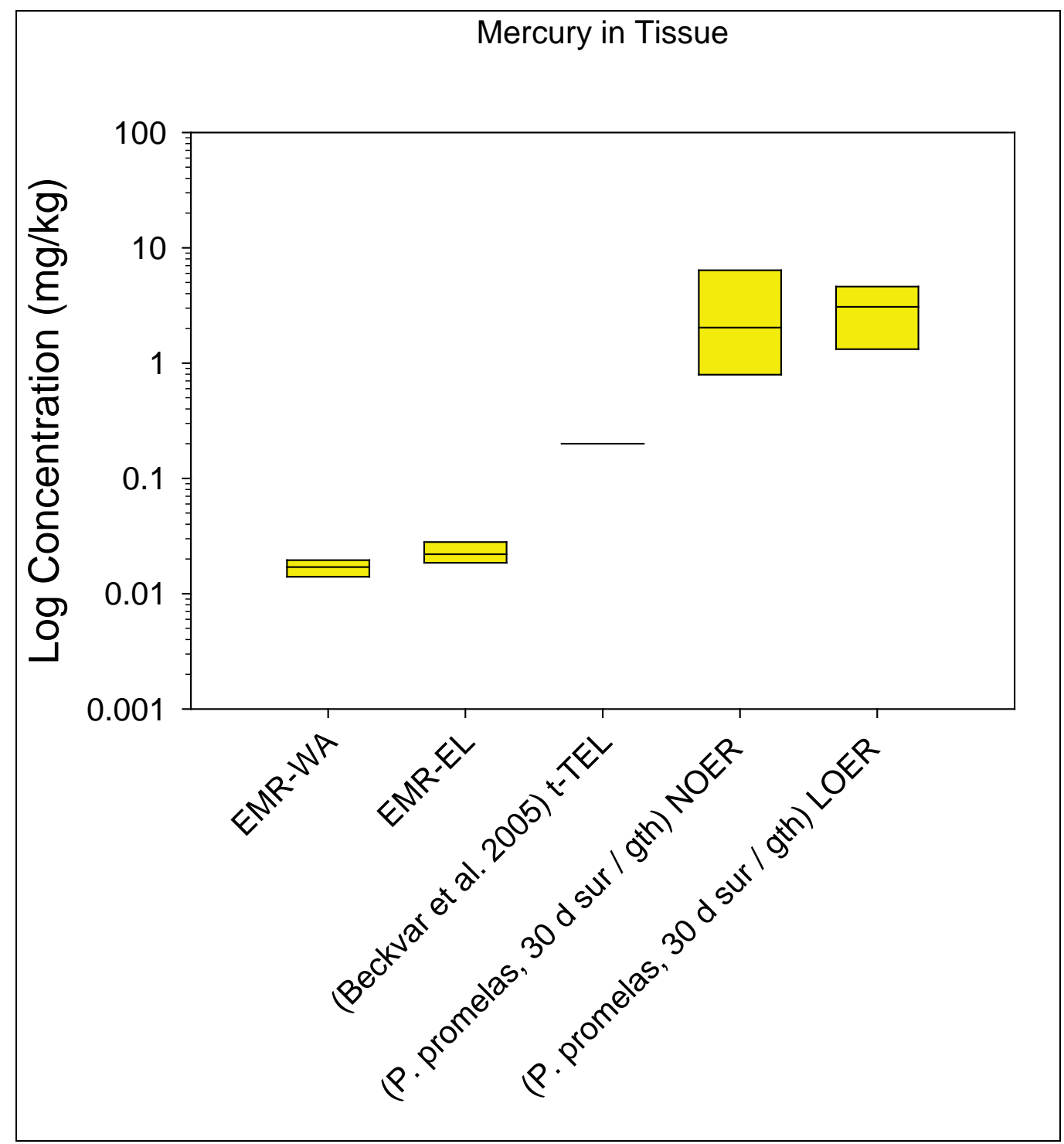

Figure 66. Mercury concentrations (as wet weight, $\mathrm{mg} / \mathrm{kg}$ ) in whole Pimephales promelas exposed to EMR-EL-AF for 10 days. Available toxicity reference values (TRVs) from the literature are summarized and defined in the definitions section. The exposure durations for the TRVs are indicated on the X-axis. The ends of the boxes represent the $25^{\text {th }}$ and $75^{\text {th }}$ percentiles of the data distribution and the horizontal line indicates the median represented. Stand-alone horizontal lines indicate single points or tight distributions. Reporting limits = $0.001-0.005 \mathrm{mg} / \mathrm{kg}$. 


\section{Selenium}

Water

Dissolved Se $(<0.2 \mu \mathrm{g} / \mathrm{L})$ was not detected above detection limits in the EMR-WA. The measured dissolved Se concentration in the SLC-WA was $5.9 \mu \mathrm{g} / \mathrm{L}$.

In the EMR-EL, mean dissolved Se concentrations increased over the 240-hr elutriate preparation time from a concentration of $0.7( \pm 0.1) \mathrm{\mu g} / \mathrm{L}$ at the $1-\mathrm{hr}$ time point to $5.5( \pm 0.5) \mathrm{\mu g} / \mathrm{L}$ at $240 \mathrm{hr}$. Dissolved Se levels were higher in the SLC-EL and also increased over time, ranging from 5.6 $( \pm 0.3)$ to $12.7( \pm 0.1) \mathrm{\mu g} / \mathrm{L}$ at the 1-and 240-hr time points, respectively. Se(IV) was the only species of Se observed in both elutriate preparations (Figure 31).

The concentrations of Se in the SLC-WA as well as in both the EMR-EL and the SLC-EL, at least at some time point(s) over the 240-hr elutriate preparation period, exceed the USEPA's and the State of Tennessee's continuous criterion concentration (CCC) of $5 \mu \mathrm{g} / \mathrm{L}$ for Se in water (Figure 67). Thus, some concern for the potential of Se to contribute to chronic toxicity exists. However, as outlined in USEPA's (2004) Draft Aquatic Life Water Quality Criteria for Selenium, it is technically more valid to use a whole-body tissue concentration of Se as the chronic criterion because diet is the primary route of Se exposure that controls chronic toxicity in fish. Thus, the Se body burdens measured at the conclusion of the 10-day juvenile P. promelas toxicity and bioaccumulation study (discussed below) should be used as the primary assessment of potential Se impacts to fish in this investigation.

Also, even though exceedances of water CCCs were observed, all measured dissolved Se concentrations are well below no observable effect concentrations (NOECs) reported by Norberg-King (1989) in 7-day studies with larval P. promelas that ranged from 377 to 1,450 $\mu \mathrm{g} / \mathrm{L}$ (Figure 67). Lowest observable effect concentrations (LOECs) for the same studies for the growth endpoint ranged from 836 to 2,920 $\mu \mathrm{g} / \mathrm{L}$ (Norberg-King 1989). Thus, the highest concentration of Se measured in the SLC-EL, 12.73( \pm 0.1$)$ $\mu \mathrm{g} / \mathrm{L}$, is at least 65-fold less than LOECs observed in this study. 


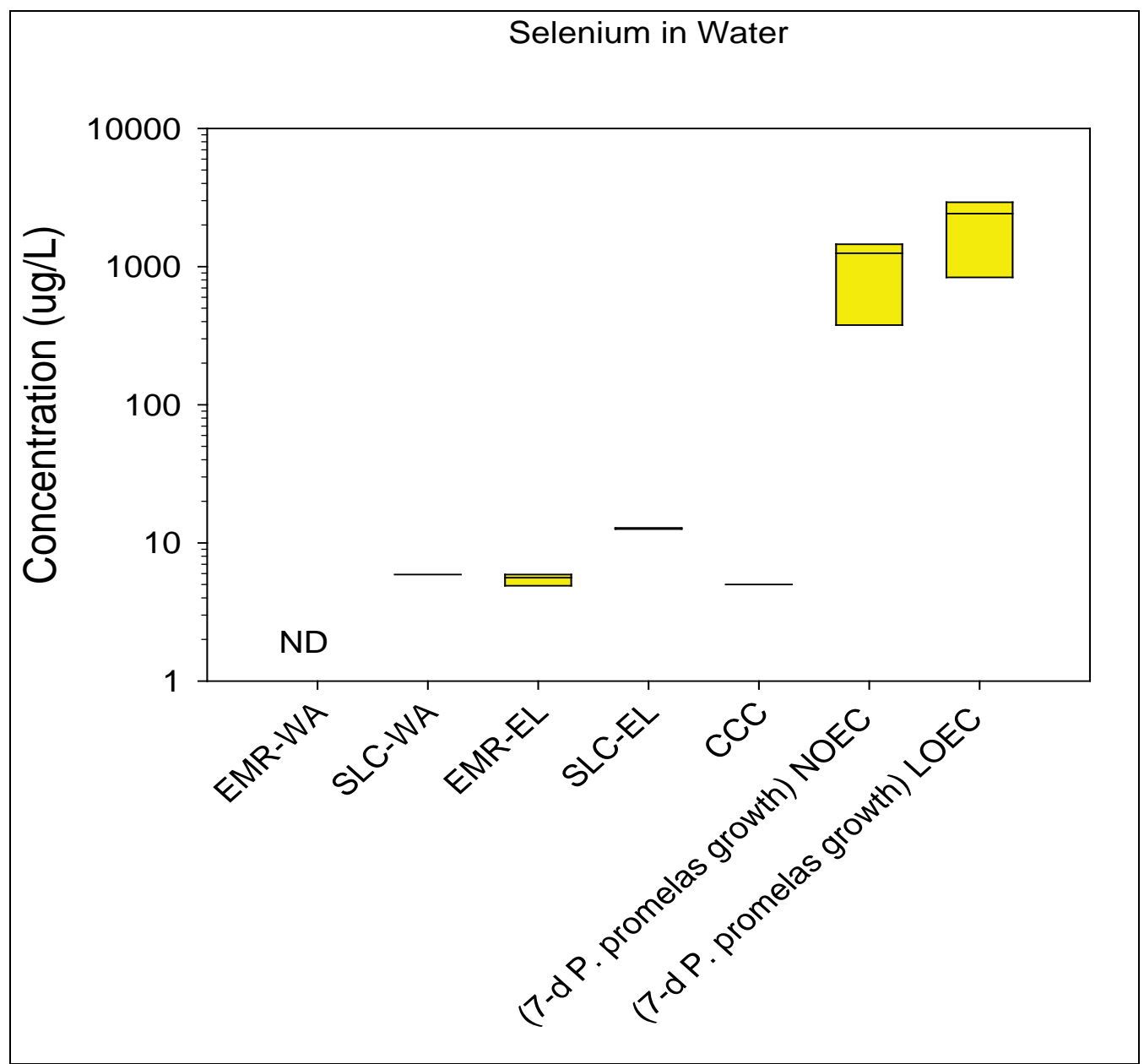

Figure 67. Dissolved selenium (predominately selenite) concentrations in Emory River reference water (EMR-WA), sluice water (SLC-WA), Emory River fly ash elutriate (EMR-EL), sluice fly ash elutriate (SLC-EL), and the USEPA chronic water quality criterion (criterion continuous concentration or CCC). Available toxicity reference values (TRVs) from the literature are summarized and defined in the definitions section. The exposure durations for the TRVs are indicated on the X-axis. The ends of the boxes represent the $25^{\text {th }}$ and $75^{\text {th }}$ percentiles of the data distribution and the horizontal line indicates the median represented. Stand-alone horizontal lines indicate single points or tight distributions. ND = not detected (reporting limits $=1 \mu \mathrm{g} / \mathrm{L}$ ).

\section{Tissue}

In the EMR-EL bioaccumulation study, the highest mean measured Se tissue concentration was 2.91 ( \pm 0.49$) \mathrm{mg} / \mathrm{kg}$ dry weight. Wet weights of P. promelas measured in the study were converted to dry weights using an experimentally determined factor of $23.8 \%$ in order to compare to the USEPA (2004) chronic exposure criterion for Se in tissue that is given in dry weight, $7.91 \mathrm{mg} / \mathrm{kg}$ dry weight. The highest mean measured Se tissue concentration in fish exposed to the EMR-WA was $1.33( \pm 0.14) \mathrm{mg} / \mathrm{kg}$ dry weight. 
The highest measured tissue Se concentrations in the EMR-EL of $2.91 \mathrm{mg} / \mathrm{kg}$ dry weight is 2.7 -fold less than the USEPA (2004) chronic exposure criterion for Se in tissue.

Also, all measured tissue Se values in this study are below literature LOER TRVs for P. promelas (Figure 68). TRVs used in this comparison are for larval and adult P. promelas obtained via water (Bertram and Brooks 1986, Schultz and Hermanutz 1990) and dietary exposure (Dobbs et al. 1996, Ogle and Knight 1989). This suggests the whole body burden of Se, which included gut contents, is unlikely to be of toxicological concern as measured in the current investigation. While some reported NOER TRVs are below tissue Se concentrations measured in fish exposed to the EMRWA and EMR-EL, no effect levels such as NOERs can be an artifact of spacing of treatments in experimental design, so exceedances of these values do not necessarily imply that a significant toxic response will occur.

\section{Vanadium}

Water

Dissolved V was not detected above detection limits $(<0.2 \mu \mathrm{g} / \mathrm{L})$ in the EMR-WA. Measured dissolved V concentration in the SLC-WA was $50.9 \mu \mathrm{g} / \mathrm{L}$.

In the EMR-EL, mean dissolved $\mathrm{V}$ concentrations increased over the 240-hr elutriate preparation time from a concentration of $13.3( \pm 2.7)$ $\mu \mathrm{g} / \mathrm{L}$ at the 1-hr time point to 34.1 ( \pm 0.1$) \mu \mathrm{g} / \mathrm{L}$ at $240 \mathrm{hr}$.

Dissolved V concentrations were higher in the SLC-EL than in the EMREL. In the SLC-EL, the measured dissolved V concentration remained approximately the same over the 240 - $h r$ period, ranging from $53.5( \pm 0.4)$ at the 1-hr time point to $52.7( \pm 0.6) \mathrm{\mu g} / \mathrm{L}$ at the $240-\mathrm{hr}$ time point, with a maximum concentration of $57.1( \pm 0.1) \mu g / L$ measured at the 48 -hr time point.

The measured dissolved V concentrations are lower than literature TRV values for $\mathrm{V}$ (Figure 69). The highest concentration of $\mathrm{V}$ observed in either elutriate preparation, $57.1( \pm 0.2) \mathrm{\mu g} / \mathrm{L}$, is 5.3-fold lower than the lethal concentration to 5\% (LC5) and 40.3-fold lower than the 7-day LC50 for Danio rerio (zebrafish) reported by Beusen and Neven (1987). The measured dissolved concentration of $\mathrm{V}$ is also almost 20-fold lower than a 


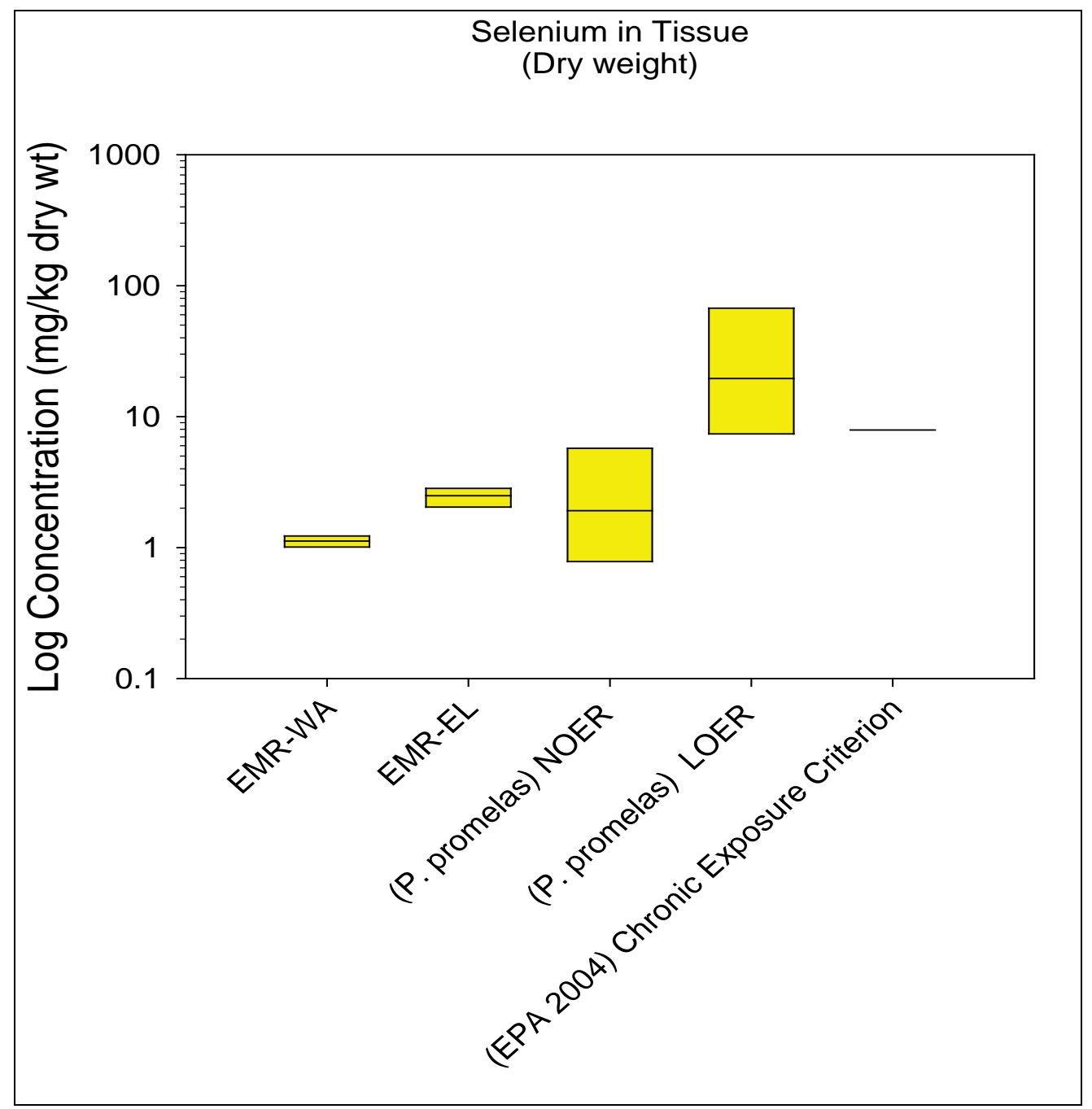

Figure 68. Selenium concentrations (converted to dry weight, $\mathrm{mg} / \mathrm{kg}$ ) in whole Pimephales promelas exposed to EMR-EL-AF for 10 days. Available toxicity reference values (TRVs) from the literature are summarized and defined in the definitions section. The exposure durations for the TRVs are indicated on the X-axis. The ends of the boxes represent the $25^{\text {th }}$ and $75^{\text {th }}$ percentiles of the data distribution and the horizontal line indicates the median represented. Stand-alone horizontal lines indicate single points or tight distributions. Reporting limits $=0.5 \mathrm{mg} / \mathrm{kg}$.

28-day LC50 for J ordanella floridae (American flagfish) reported by Holdway and Sprague (1979). Unfortunately endpoints suggesting no effect (e.g., NOECs, MATCs, LC50) or screening values were not available for V. Obviously the LC50 values in the available literature (Figure 69) inherently present concentrations that induced adverse biological effects. Investigators have derived application factors (AFs) but these values were derived to adjust acute (96-hr) LC50 values, not chronic 28-day LC50 values, and are therefore not applicable to the available toxicity reference values for $\mathrm{V}$. 


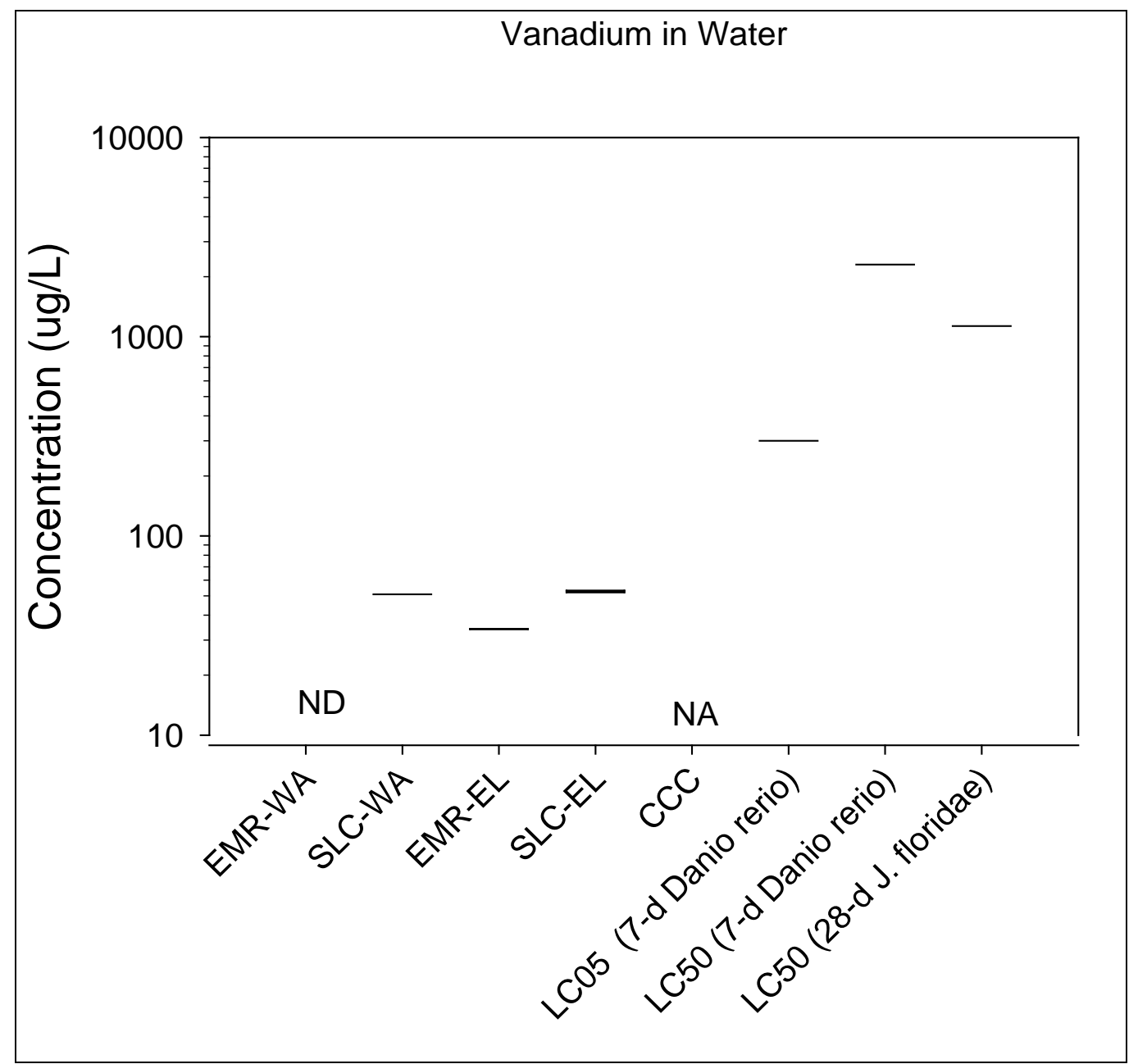

Figure 69. Dissolved vanadium (V) concentrations in Emory River reference water (EMR-WA), sluice water (SLC-WA), Emory River fly ash elutriate (EMR-EL), and sluice fly ash elutriate (SLCEL). Note that no WQC was available for V. Available toxicity reference values (TRVs) from the literature are summarized and defined in the definitions section. The exposure durations for the TRVs are indicated on the X-axis. Stand-alone horizontal lines indicate single points or tight distributions. ND $=$ not detected (reporting limits $=1 \mu \mathrm{g} / \mathrm{L}$ ). NA $=$ not available.

\section{Tissue}

In the EMR-EL bioaccumulation study, $\mathrm{V}$ body burden, including gut contents, ranged from $0.10( \pm 0.05) \mathrm{mg} / \mathrm{kg}$ wet weight in the Emory River reference water to $4.06( \pm 0.93) \mathrm{mg} / \mathrm{kg}$ wet weight at the $50 \%$ dilution. The $\mathrm{V}$ body burden in the fish exposed to the $100 \%$ dilution was similar at $3.88( \pm 1.28) \mathrm{mg} / \mathrm{kg}$ wet weight. While the concentration of V in $\mathrm{P}$. promelas exposed to EMR-WA was less than reported TRVs, the concentration of $\mathrm{V}$ in the P. promelas exposed to the EMR-EL exceeded NOER and LOERs reported by Holdway et al. (1983) for J . floridae (Figure 70). This indicates a potential for toxicity due to bioaccumulated $\mathrm{V}$ in the test system, although relative species-specific sensitivity to 
bioaccumulated V between P. promelas and J . floridae is unknown. Also, the limited amount of available literature data on $\mathrm{V}$ bioaccumulation and toxicity to freshwater fish suggests that care should be taken in the interpretation of the above comparison of measured values to these TRVs. Furthermore, as described earlier, the concentration of metals in whole fish may be high due to the presence of fly ash in the gut of the fish.

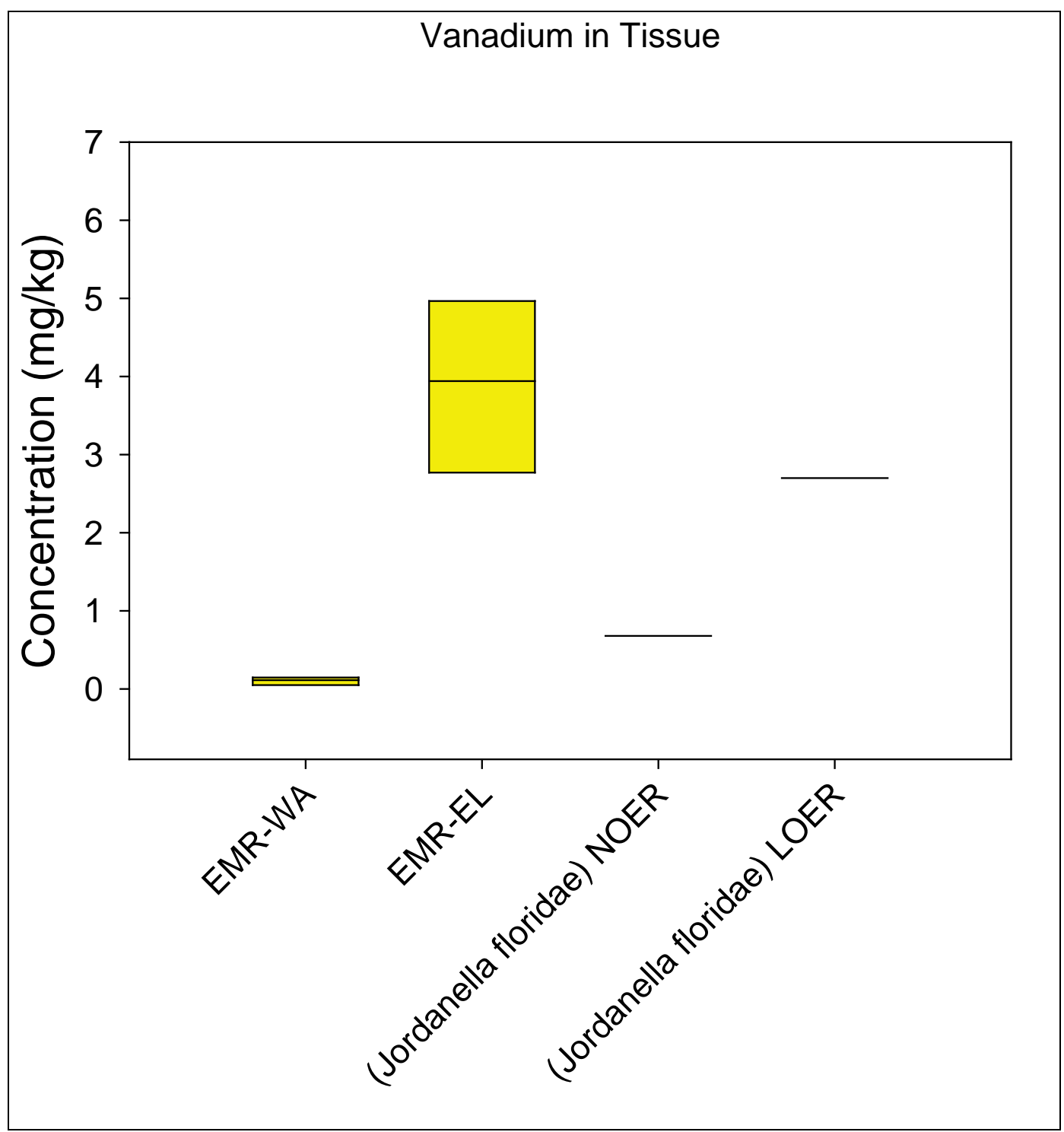

Figure 70. Vanadium concentrations (as wet weight, $\mathrm{mg} / \mathrm{kg}$ ) in whole Pimephales promelas exposed to EMR-EL-AF for 10 days. Available toxicity reference values (TRVs) from the literature are summarized and defined in the definitions section. The exposure durations for the TRVs are indicated on the X-axis. The ends of the boxes represent the $25^{\text {th }}$ and $75^{\text {th }}$ percentiles of the data distribution and the horizontal line indicates the median represented. Stand-alone horizontal lines indicate single points or tight distributions. Reporting limits $=0.5$ 


\section{Stilling pond}

The dredged fly ash initially enters the sluice channel (SLC) and proceeds to the stilling pond prior to discharge to the Emory River. Consequently, metals concentrations in the stilling pond may be more relevant to what the Emory River receives than metals concentrations in the field-collected sluice channel water or the sluice channel elutriate (SLC-EL, $10 \mathrm{~g} \mathrm{TSS} / \mathrm{L}$ ). Thus, a comparison of the dissolved concentrations of the six metals of interest (As, Ba, Cr, Hg, Se and V) was made between the sluice channel water and the stilling pond (Table 19). These concentrations were also compared to water quality criteria (WQC) or toxicity reference values (TRV), where needed. Metals concentrations were lower in the stilling pond for As (73\% lower) and V (38\% lower), while the concentration of Se was comparable ( $8 \%$ lower) to the sluice channel water. However, higher concentrations of the other three metals were found in the stilling pond relative to the sluice water. While it must be noted that statistical comparison of these trends was not possible provided only a single analytical measurement was made in the stilling pond, it is interesting that two metals that were not detected in the sluice channel water ( $\mathrm{Cr}$ and $\mathrm{Hg}$ ) increased to detectable levels in the stilling pond. The third metal, Ba, increased by $64 \%$. Furthermore, the concentrations of these three metals were higher in the stilling pond than in the laboratory prepared EMR-EL elutriate, which contained greater than $9 \mathrm{mg} / \mathrm{L}$ (i.e., the amount of additional fly ash added to the sluice water to prepare the extended elutriate) more fly ash than the sluice water that the stilling pond receives in the field.

Table 19. Comparison of dissolved metals concentrations $(\mathrm{mg} / \mathrm{L})$ in the stilling pond, sluice channel water, and sluice channel elutriate.

\begin{tabular}{|l|l|l|l|l|}
\hline Metal & $\begin{array}{l}\text { Stilling pond } \\
(\mu \mathrm{g} / \text { L dissolved })\end{array}$ & $\begin{array}{l}\text { Sluice Water } \\
(\mu \mathrm{g} / \text { L dissolved })\end{array}$ & $\begin{array}{l}\text { SLC-EL } \\
(\text { Mean })\end{array}$ & $\begin{array}{l}\text { Water Quality Criteria } \\
(\mathrm{CCC})\end{array}$ \\
\hline $\mathrm{As}$ & 20.8 & 77.9 & 81.9 & 150 \\
\hline $\mathrm{Ba}$ & 175 & 107 & 117 & NA \\
\hline $\mathrm{Cr}$ & 4.1 & 0.1 & 0.7 & $\begin{array}{l}74(21 \text { at } 22 \mathrm{mg} / \mathrm{L} \text { as } \\
\text { CaCO3 })\end{array}$ \\
\hline $\mathrm{Hg}$ & 0.014 & 0.0025 & 0.01 & 0.77 \\
\hline $\mathrm{Se}$ & 5.4 & 5.9 & 12.7 & 5.0 \\
\hline $\mathrm{V}$ & 31.7 & 50.9 & 52.7 & NA \\
\hline
\end{tabular}


For the metals that have USEPA-recommended WQC (As, Cr, Hg, Se), dissolved metals concentrations in the stilling pond (Table 19) were below the criterion continuous concentration (or CCC), with the exception of Se $(5.4 \mu \mathrm{g} / \mathrm{L}$ ), which was slightly above the dissolved CCC of $5 \mu \mathrm{g} / \mathrm{L}$ (USEPA 2002c). Also noteworthy is that the more toxic selenate species was detected at a higher fraction (Table 20) than the other field and laboratory waters that contained predominately selenite. However, the concentration of Se in the stilling pond $(5.4 \mu \mathrm{g} / \mathrm{L}$ ) was lower than TRVs (Figure 67). Further, a more recent publication than the USEPA (2002c) national recommended water quality criteria document suggests that the CCC value of $5.0 \mu \mathrm{g} / \mathrm{L}$ is not appropriate and Se toxicity is better predicted by tissue residues than by concentrations in the water column (USEPA 2004). For the metals that did not have USEPA-recommended WQC (Ba, V), concentrations were considerably less than TRVs (Figures 62 and 69), a Dutch screening value for Ba (Crommentuijn et al. 2000) and are not expected to be of toxicological significance in the stilling pond.

Complete characterization of the stilling pond was not a goal of this project. However, because of the suggestive results described above, the stilling pond may be an area of interest for future study.

\section{Gill histology and mussel bioassay}

Fish histology and mussel bioassay investigations were conducted by separate laboratories and are provided as supplemental reports in Appendices H and I, respectively. In the fish gill histopathology study, no statistically significant differences in indices such as gill filament width were observed for fish exposed to fly ash elutriate. However, difficulties were reported in sectioning and mounting that resulted in a limited number of available samples $(\mathrm{n}=10)$. Since there was less than desirable statistical power, it was not possible to form definitive conclusions. The 10-day mussel bioassay, performed with 5-month-old juvenile rainbow mussels (Villosa iris), showed no toxicity to the survival endpoint as determined by heartbeat in the $100 \%, 50 \%$, or $10 \%$ EMR-EL or SLC-EL. However, a significant reduction in foot movement was observed in the $100 \%$ SLC-EL, indicating the potential for effects to mussel behavior. See Appendices $\mathrm{H}$ and I for details. 


\section{Conclusions}

- No statistically significant adverse effects were observed for larval or juvenile fish exposed to the oxic regime elutriates prepared with fly ash from the Emory River. Histological analysis of the gills was inconclusive, and no effect was found on juvenile mussel survival.

- Dissolved As, Ba, Cr, Hg, V concentrations in the oxic regime elutriate were below the chronic water quality criteria and/ or relevant literature or screening values.

o Dissolved concentrations of As, $\mathrm{Cr}$ and $\mathrm{Hg}$ were below the CCC.

o Ba and $\mathrm{V}$ concentrations were lower than literature-reported toxicity reference values.

- While the dissolved concentrations of Se measured in the Emory River elutriate $(5.4 \mu \mathrm{g} / \mathrm{L})$ and the sluice elutriate $(12.7 \mu \mathrm{g} / \mathrm{L})$ exceeded the $\mathrm{CCC}=5.0 \mu \mathrm{g} / \mathrm{L}$ for total Se, it is generally understood that tissue residues are more predictive of Se toxicity than are water concentrations (USEPA 2004).

- In general, concentration of metals in tissues increased in a dosedependent manner. However, this increase is likely due to metals present in the gut as ingested fly ash, not metals integrated into the tissue.

- Arsenic (As) was near reference values reported in the literature at which effects are observed. However, this was a conservative assessment because the less toxic arsenate (As V) dominated in the oxic regime elutriates, but the levels in water were compared to screening values derived for the more toxic arsenite (As III).

o There was very little literature data and no screening values to compare barium (Ba) concentrations in tissues.

- Chromium (Cr) was present as $\mathrm{Cr}$ (III) and at concentrations near the NOER from literature studies.

o Mercury (Hg) and selenium (Se) concentrations in tissues were below relevant screening values.

- Vanadium (V) exceeded literature-based screening values, but the elevated concentrations are likely the result of fly ash in the gut.

- In a weight-of-evidence approach where chemistry, toxicity, and bioaccumulation data are integrated, these results suggest little 
potential for toxicity to related fish species given the extreme conditions represented by the oxidized elutriates in this study.

- Several areas of uncertainty could be addressed through additional data collection including longer-term exposures of fish to suspended or bedded fly ash, assessing food web exposure or trophic transfer, and evaluation of sensitive species not assessed in this study. Further characterization of stilling pond effluent may also be useful in future endeavors. 


\section{Comparison of Laboratory Elutriate Results to Field Observations}

\section{Summary}

Elutriate tests are generally used to predict contaminant releases to surface waters by dredging and disposal activities. These tests are performed during the planning or design stages of a project to provide an a priori estimate that can in turn lead to regulatory approval to proceed with the project or to defining a need for alternative disposal sites or operational controls to reduce contaminant releases. The laboratory test conditions for the standardized tests were selected to mimic field conditions and were then validated against field data. The elutriate test procedures used in this study deviated from standard procedures in order to investigate potential shifts in metal speciation for fly ash-water mixtures exposed to oxygen for an extended time period. Metal contaminants, dissolved and particulate, that escape the dredging and disposal area are exposed to oxygen-rich receiving waters for time periods exceeding the dredging and disposal times. Consequently, the terminal mixing times for the experiments used in this study were extended beyond the normal 1-hr mixing time.

Applying the elutriate concentrations for total metals measured by this study after $240 \mathrm{hr}$ of mixing to estimates of contaminant loadings from the project area to the receiving streams is not an appropriate use of the data because the experimental conditions differ from field operational parameters. Additionally, making contaminant loading estimates based on elutriate studies is not necessary for the Kingston site because at this point in time TVA and TDEC monitor the points of contaminant release providing direct field observations of contaminant concentrations for the stilling pond effluent and for the suspended solids plume produced by the dredge(s) operating in the Emory River. A USEPA Panel (2009) stated in its recent review of potential selenium issues for this site, "However, it should be noted that it is the Panel's opinion that the continued monitoring of unspeciated selenium in the water is the best current basis for assessing the potential hazards of any changes in selenium concentrations, whether through this proposed pathway, or through other means." 
This section of the report will compare the laboratory elutriate observations to TVA field observations, as well as illustrate the monitoring program results for various stations in the Emory, Clinch, and Tennessee Rivers. TVA provided ERDC the field monitoring data $^{1}$ for the dredging period (late March through early August) with the notation that all of the data have been verified, but many of the data have not been fully validated. TVA provided this statement of caution regarding use of the field monitoring data. ${ }^{2}$

The following data set has not been fully validated and should be used with some caution until validation is completed. TVA and TVA's data management contractor, Environmental Standards, Inc., believe the data to be of suitable quality for internal agency use, but data may be subject to recall and correction if errors are found during validation. Until validated, the data should not be released publicly.

The elutriate supernatant samples analyzed for the laboratory study were collected after $1 \mathrm{hr}$ of settling. As shown in Figure 71, the total suspended solids concentrations in the elutriate supernatants, which were analyzed for metals, ranged from 400 to $500 \mathrm{mg} / \mathrm{L}$. In comparison, the total suspended solids concentrations measured by the field monitoring program at the point of release to the river averages $32 \mathrm{mg} / \mathrm{L}$ in the dredge plume (approximately $200 \mathrm{yd}$ downstream of the dredge) ${ }^{3}$ and $13 \mathrm{mg} / \mathrm{L}$ in the settling pond effluent (Figure 71). Therefore, once the resuspended sediment from the dredge or the impoundment effluent is released to the river, the solids concentration and, hence, the mass of metals available for desorption from the solids to the water column, is reduced by more than an order of magnitude compared to the elutriate mixtures.

Figures 72-75 compare the dissolved concentrations of arsenic, barium, selenium, and vanadium measured in the Emory River elutriate supernatants after $1 \mathrm{hr}$ and after $240 \mathrm{hr}$ of mixing to field observations of the maximum concentration of these metals in the dredging plume. All of the data sets for the field monitoring program included a number of values below the reportable detection limits. Each of these values was assumed a

\footnotetext{
1 Personal Communication. August 21, 2009. Robert Crawford, Sampling and Monitoring Coordinator, Tennessee Valley Authority, Kingston, TN.

2 Personal Communication. August 24, 2009. William Rogers, Ph.D., Sampling and Monitoring Coordinator, Tennessee Valley Authority, Kingston, TN.

3 Personal Communication. July 23, 2009. Neil Carriker, Ph.D., Program Manager, Special Projects, Tennessee Valley Authority, Kingston, TN.
} 


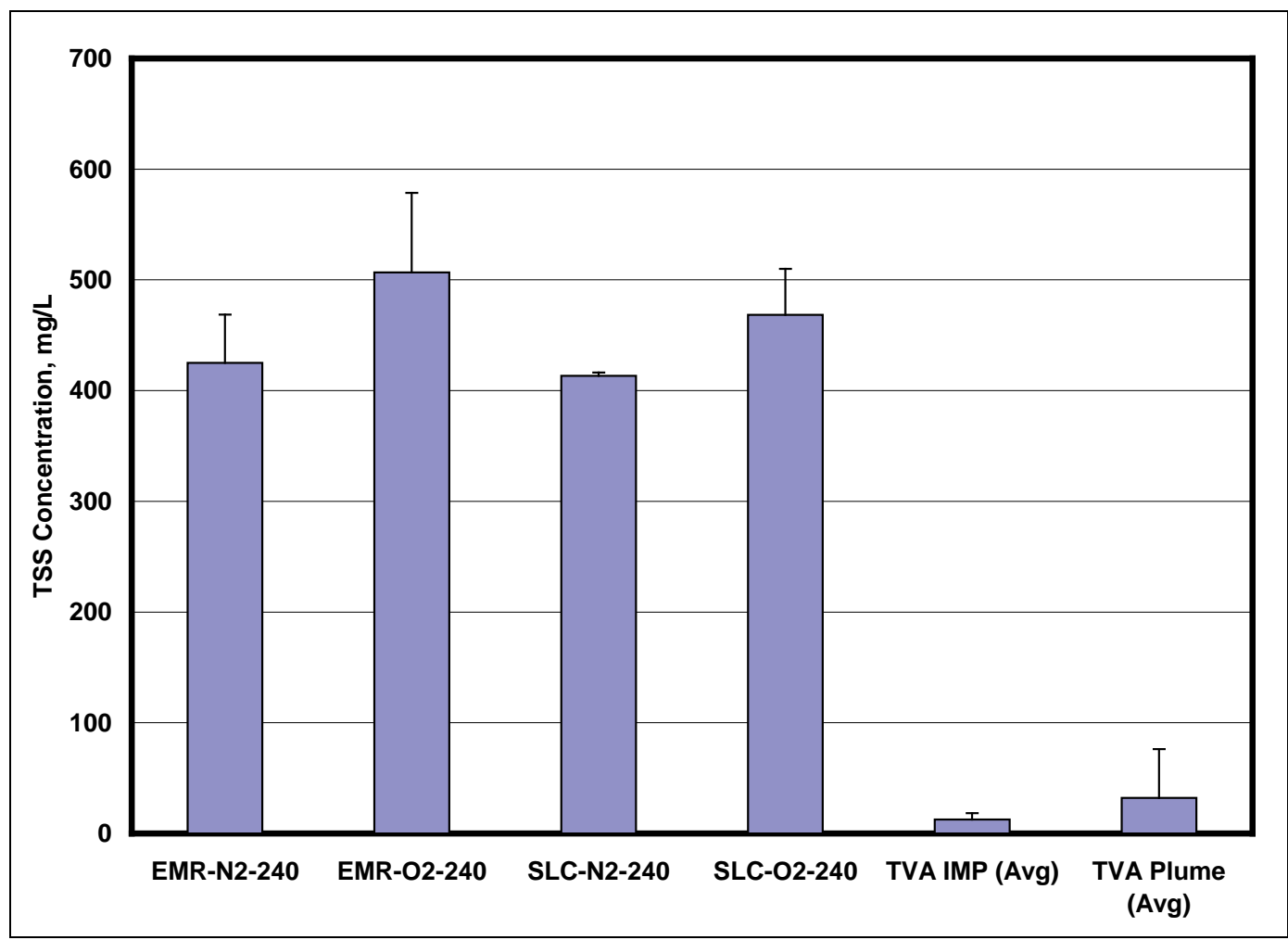

Figure 71. Elutriate supernatant TSS concentrations compared to field observations (verified, not validated) by TVA.

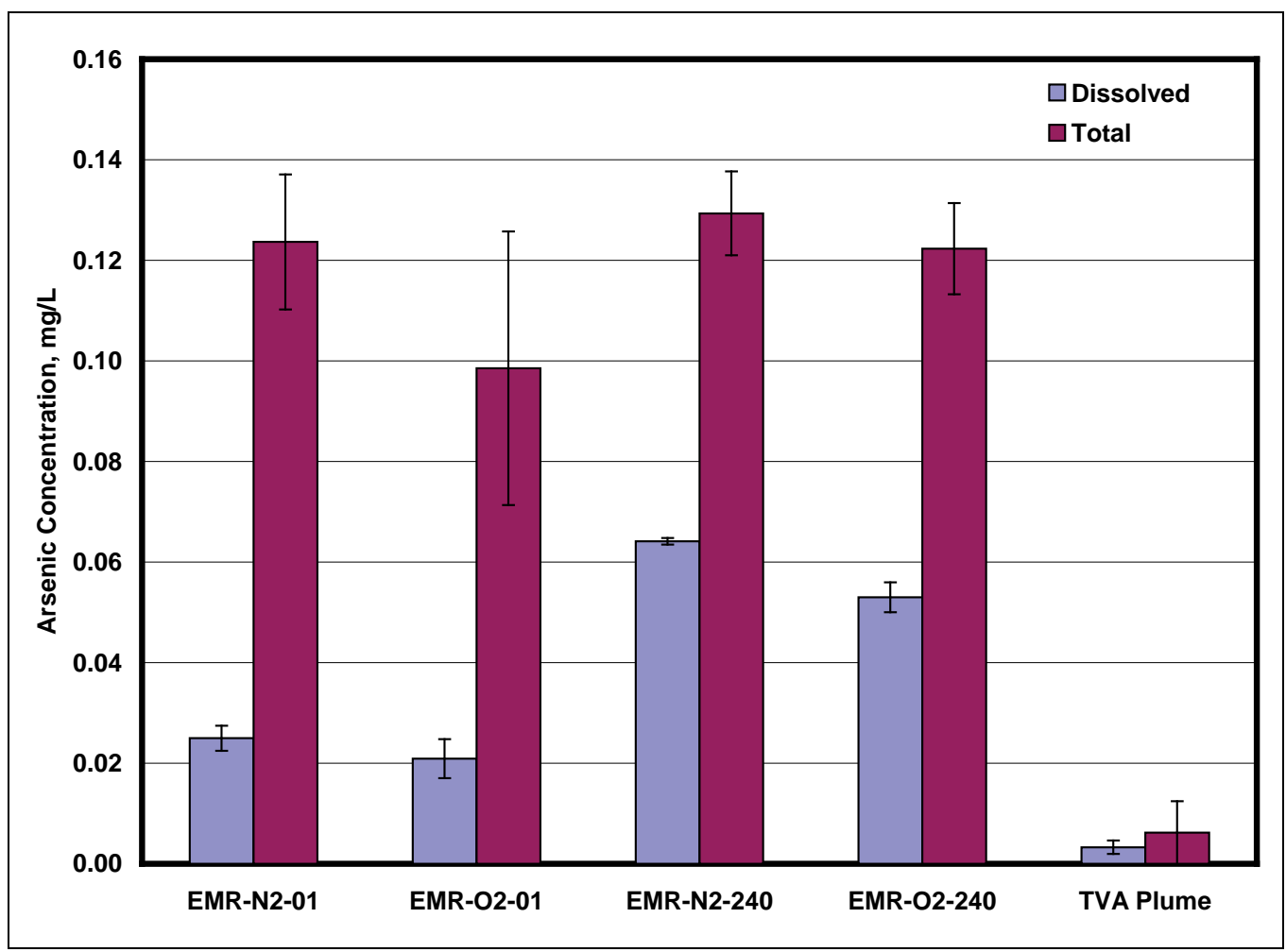

Figure 72. Emory River elutriate supernatant arsenic compared to TVA's field observations (verified, not validated) of the dredge plume. 


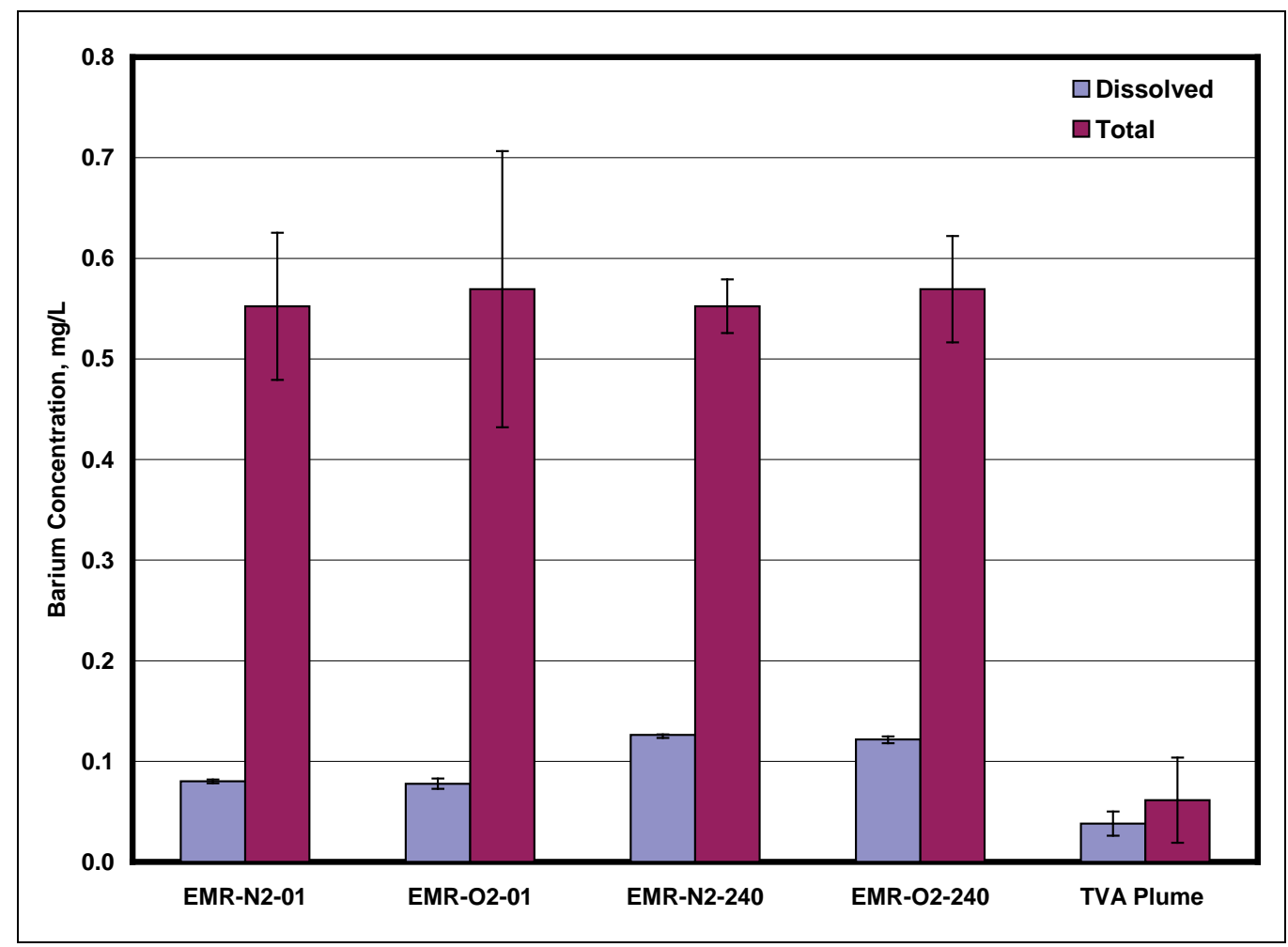

Figure 73. Emory River elutriate supernatant barium compared to TVA's field observations (verified, not validated) of the dredge plume.

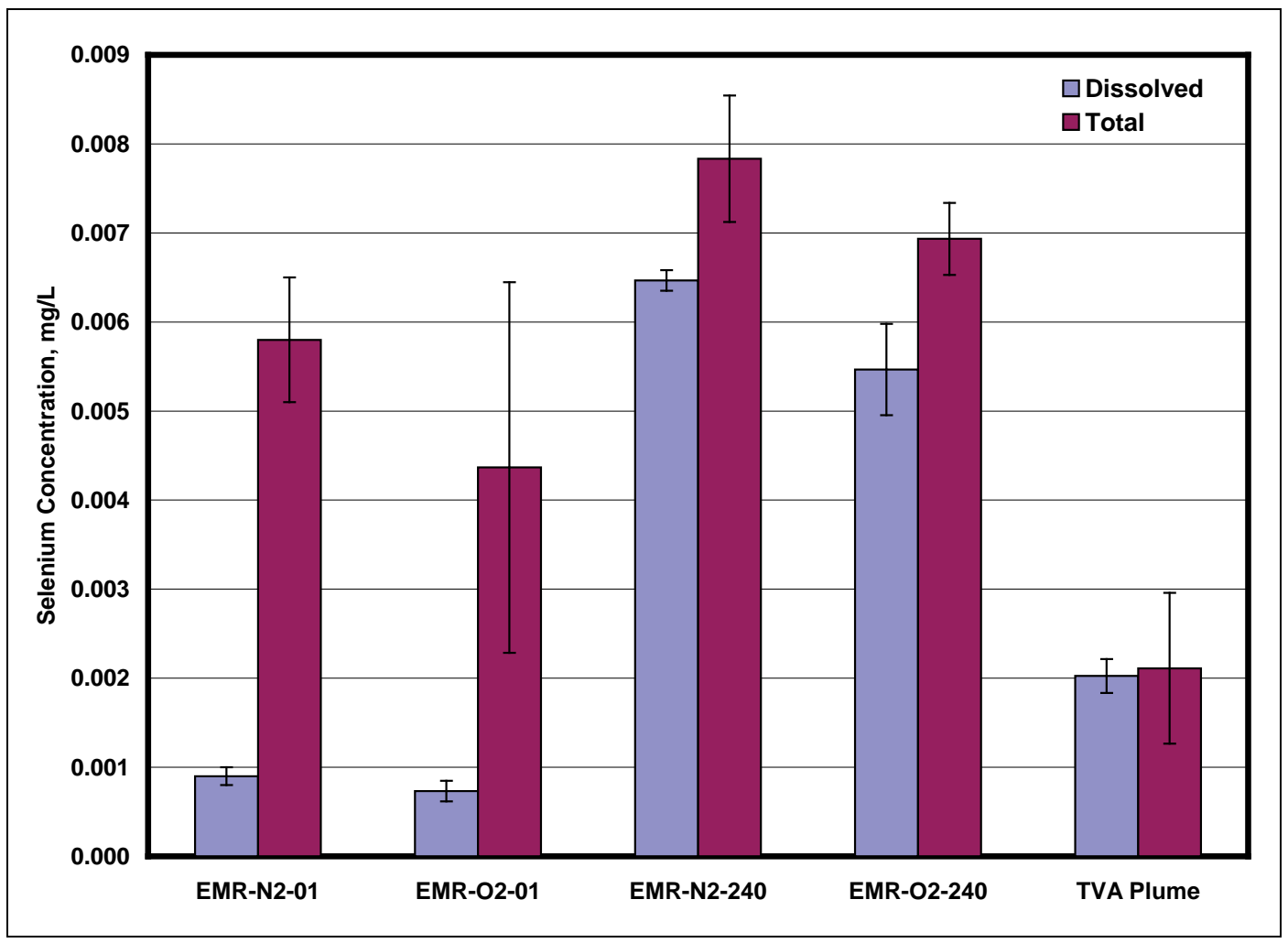

Figure 74. Emory River elutriate supernatant selenium compared to TVA's field observations (verified, not validated) of the dredge plume. 


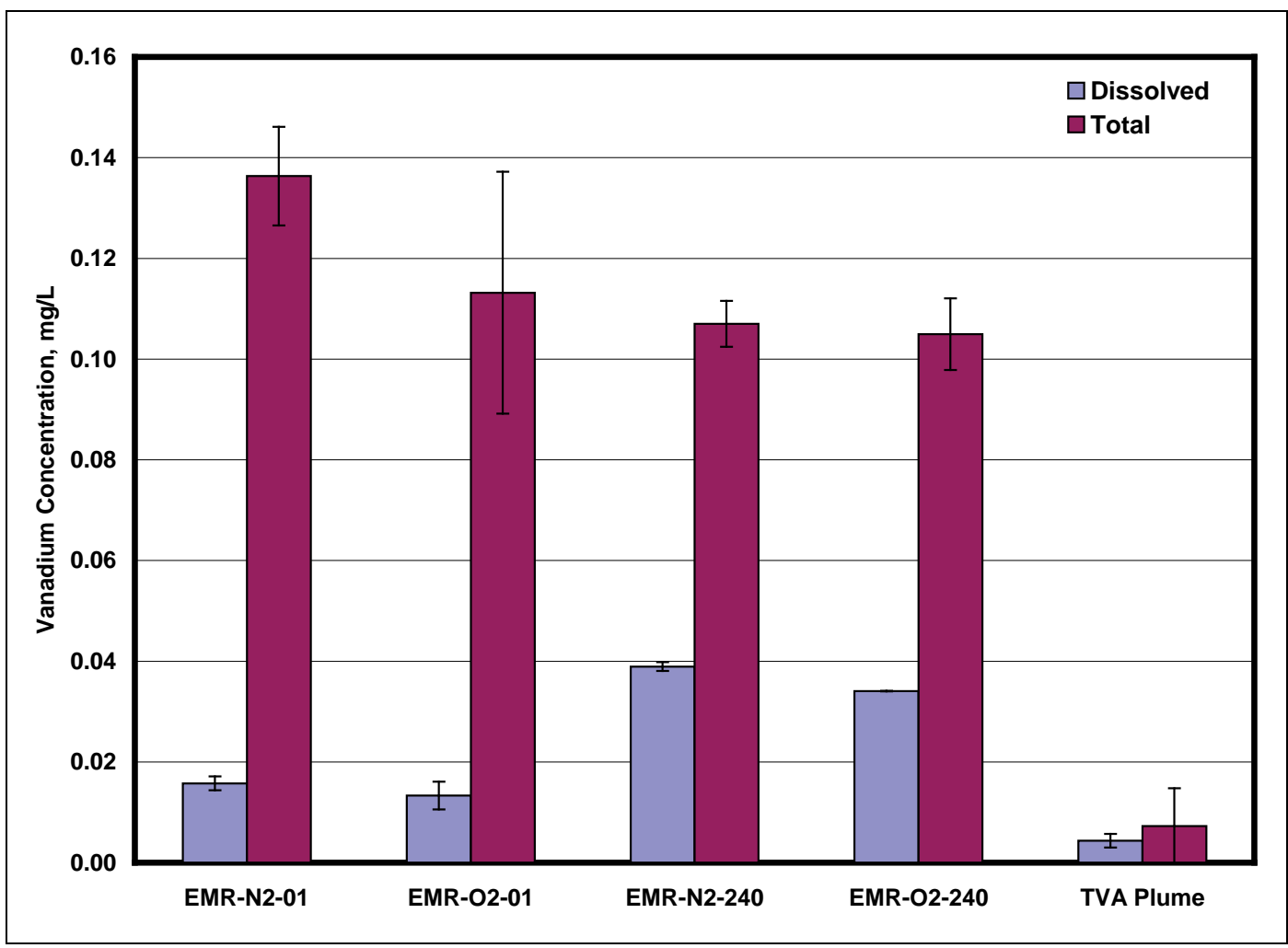

Figure 75. Emory River elutriate supernatant vanadium compared to TVA's field observations (verified, not validated) of the dredge plume.

value equal to the detection limit; therefore, the averages illustrated throughout this section of the report for field observations are greater than the true average. The arsenic, barium, and vanadium elutriate concentrations for both 1-hr and 240-hr mixing times exceeded the field monitoring observations, suggesting that the elutriate tests overestimate contaminant releases. The 1-hr elutriate dissolved concentrations are greater than the field values by a factor of 2 to 6 for the elutriates mixed with air; whereas, the 240-hr elutriates overestimate concentrations for these metals by a factor of 3 to 16 . Fifty percent of the plume dissolved arsenic values were below a detection limit of $0.002 \mathrm{mg} / \mathrm{L}$, and 80 percent of the plume dissolved vanadium values were less than $0.004 \mathrm{mg} / \mathrm{L}$.

Figure 74 indicates that selenium concentrations in the Emory River 1-hr elutriate underestimate plume values by a factor of 3, but the 240-hr elutriate overestimates the field observations by a factor of 3 . The plume selenium values are biased high because only 2 of 96 observations found selenium above the detection limit of $0.002 \mathrm{mg} / \mathrm{L}$. If one half the detection limit were assumed for the non-detects, the lab and field concentrations for the 1-hr elutriate would compare favorably. 
Figures 76 and 77 compare the dissolved concentrations of arsenic and selenium measured in the sluice channel elutriate supernatants after $1 \mathrm{hr}$ and after $240 \mathrm{hr}$ of mixing to field observations of the concentration of these metals in the stilling pond effluent (TVA discharge No. AP_IMP001). (Field observations for barium and vanadium were not available for comparison.) Complicating this comparison is the fact that the plant ash discharge mixes with and dilutes the dredge flow prior to entering the pond system. Arsenic and selenium contributions from the plant release, as well as relative flows from the plant and dredge streams would be needed to further assess the effects of the plant discharge. Figure 76 shows that both the 1-hr and the 240-hr elutriates overestimate the arsenic concentration in the pond effluent by a factor of 5. However, Figure 77 shows that the 1-hr elutriate approximates the pond selenium concentrations very well, and the $240-\mathrm{hr}$ selenium elutriate is greater than the monitored value by a factor of 3 . Figure 77 also suggests that essentially all of the elutriate selenium, as well as that in the pond, is in the dissolved or filterable fraction compared to the total (particulate plus dissolved) selenium.

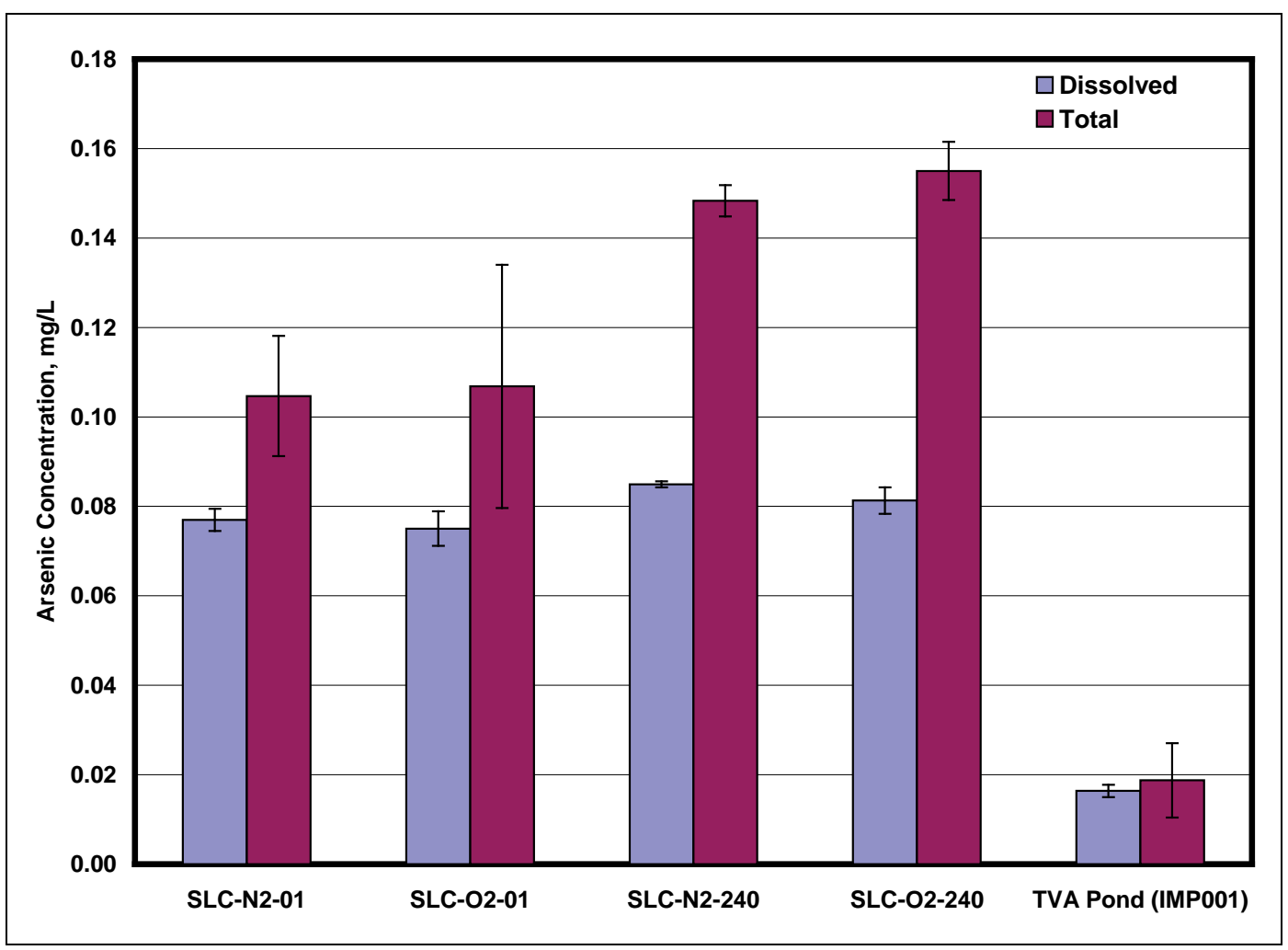

Figure 76. Arsenic in sluice channel elutriate supernatant arsenic compared to TVA's field observations (verified, not validated) of stilling pond effluent (AP_IMP001). 


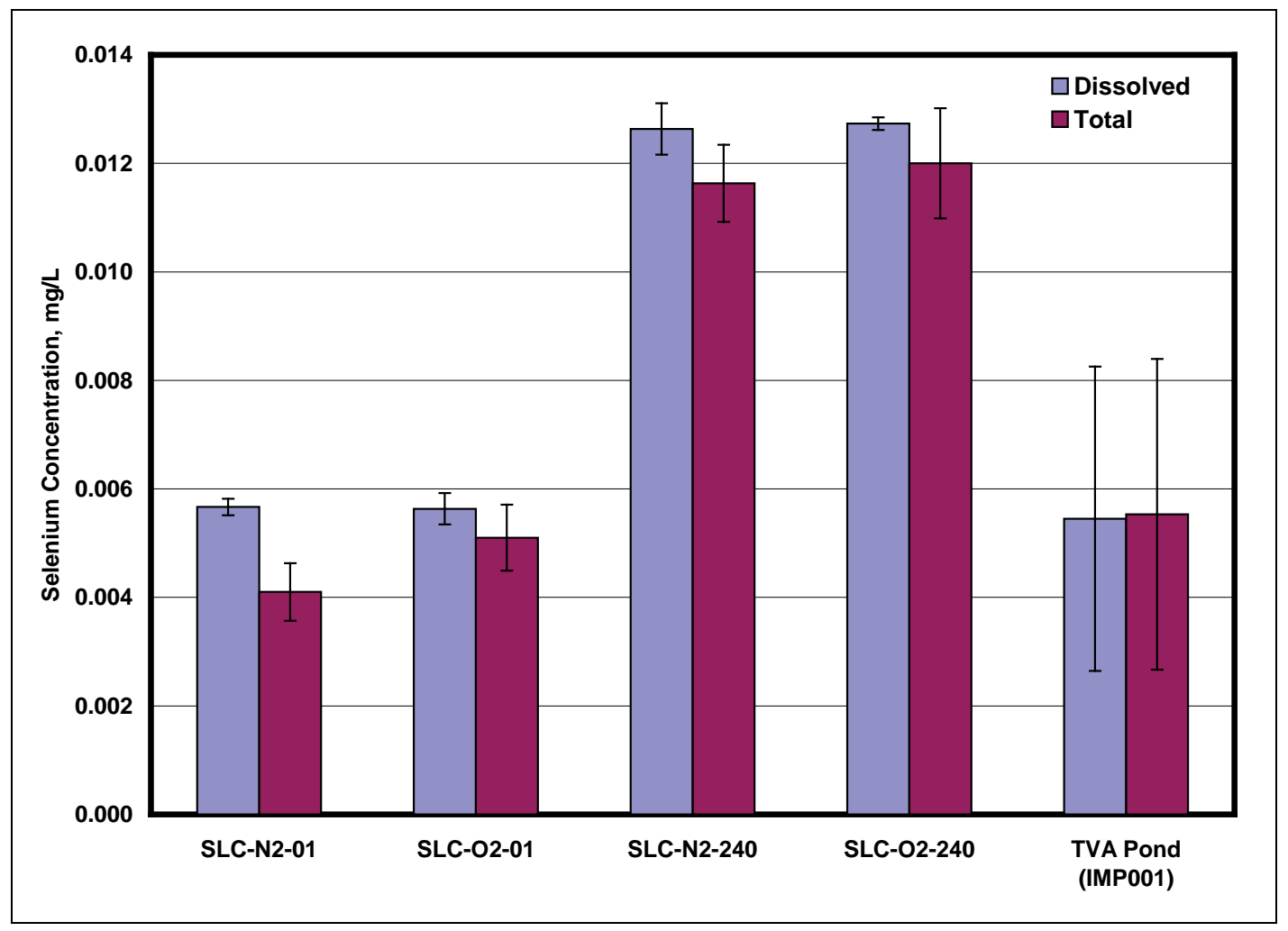

Figure 77. Selenium in sluice channel elutriate supernatant selenium compared to TVA's field observations (verified, not validated) of stilling pond effluent (AP_IMP001).

Figures 78-81 show the particulate concentration of arsenic, barium, selenium, and vanadium, respectively, for the elutriates and the field samples as well as the single analysis of the Emory River and sluice materials used as the basis for the elutriates. These values were calculated by the difference in the total and dissolved analyses divided by the suspended solids concentration. Generally, the values for arsenic, barium, and vanadium are all the same order of magnitude and with less than a factor of three from the highest to the lowest. The elutriates and field samples are slightly enriched with metals compared to the original ash samples. Because the selenium dissolved and total concentrations were very nearly the same, the calculated particulate values are near or below zero (analytical anomaly). The large variability in the field selenium observations are explained in part by the large number of total and dissolved samples reported as below the detection limit.

Figure 82 illustrates the trend in suspended solids concentrations upstream and downstream of the plume measurement. Where the upstream (background) suspended solids concentration averages $8 \mathrm{mg} / \mathrm{L}$ and plume concentration averages $31 \mathrm{mg} / \mathrm{L}$, the downstream stations drop 


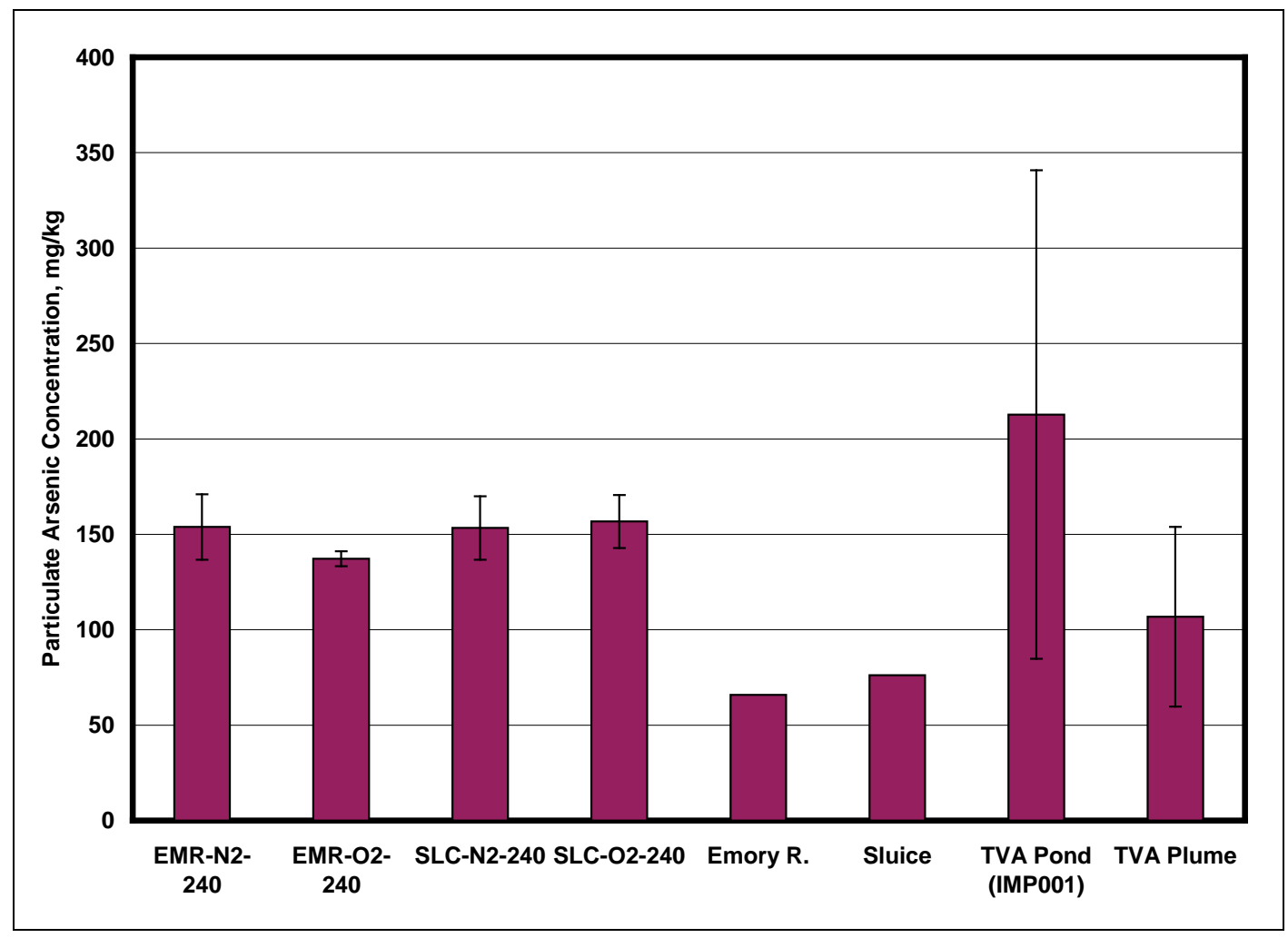

Figure 78. Particulate arsenic concentrations (verified, not validated).

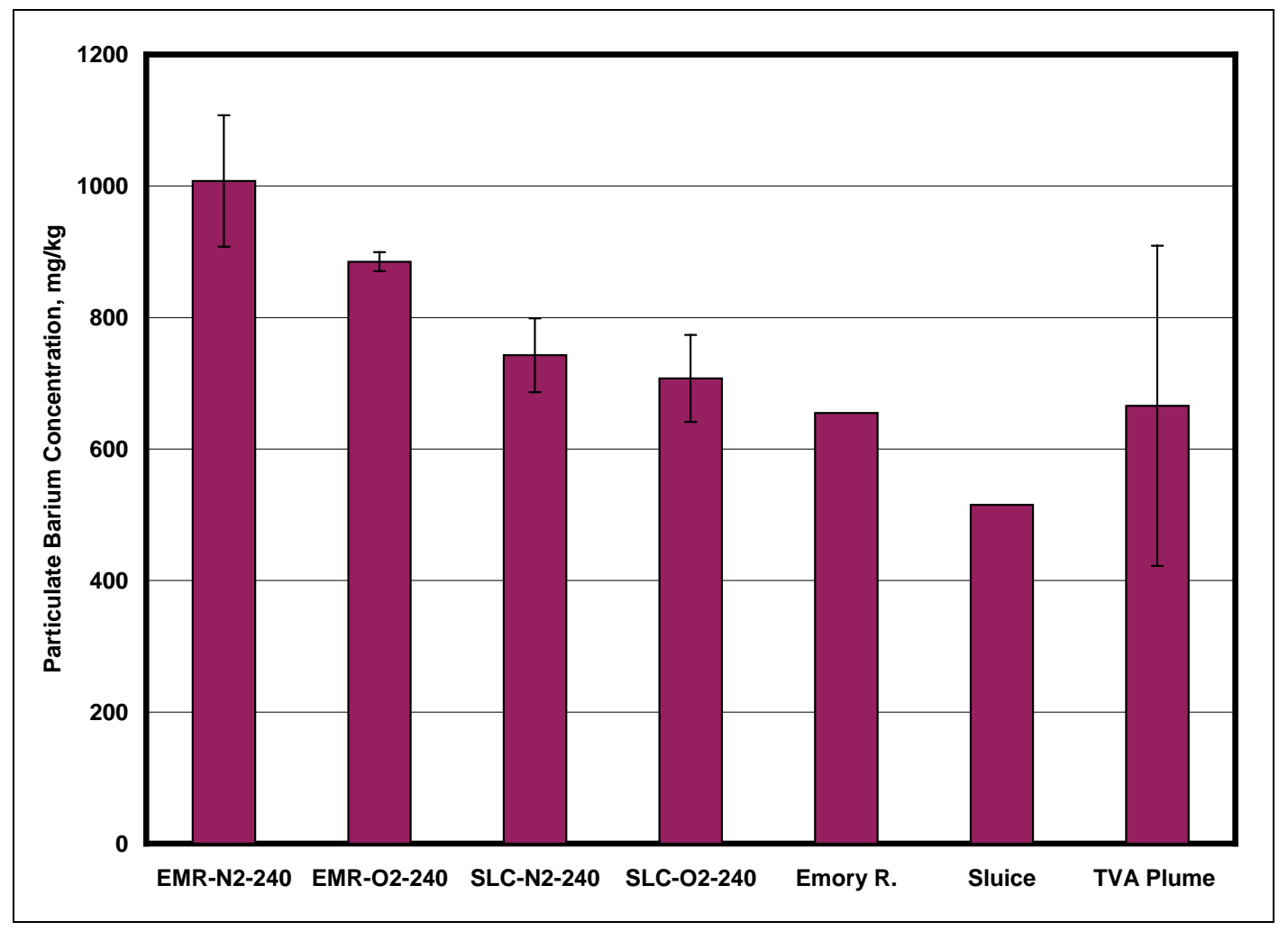

Figure 79. Particulate barium concentrations (verified, not validated). 


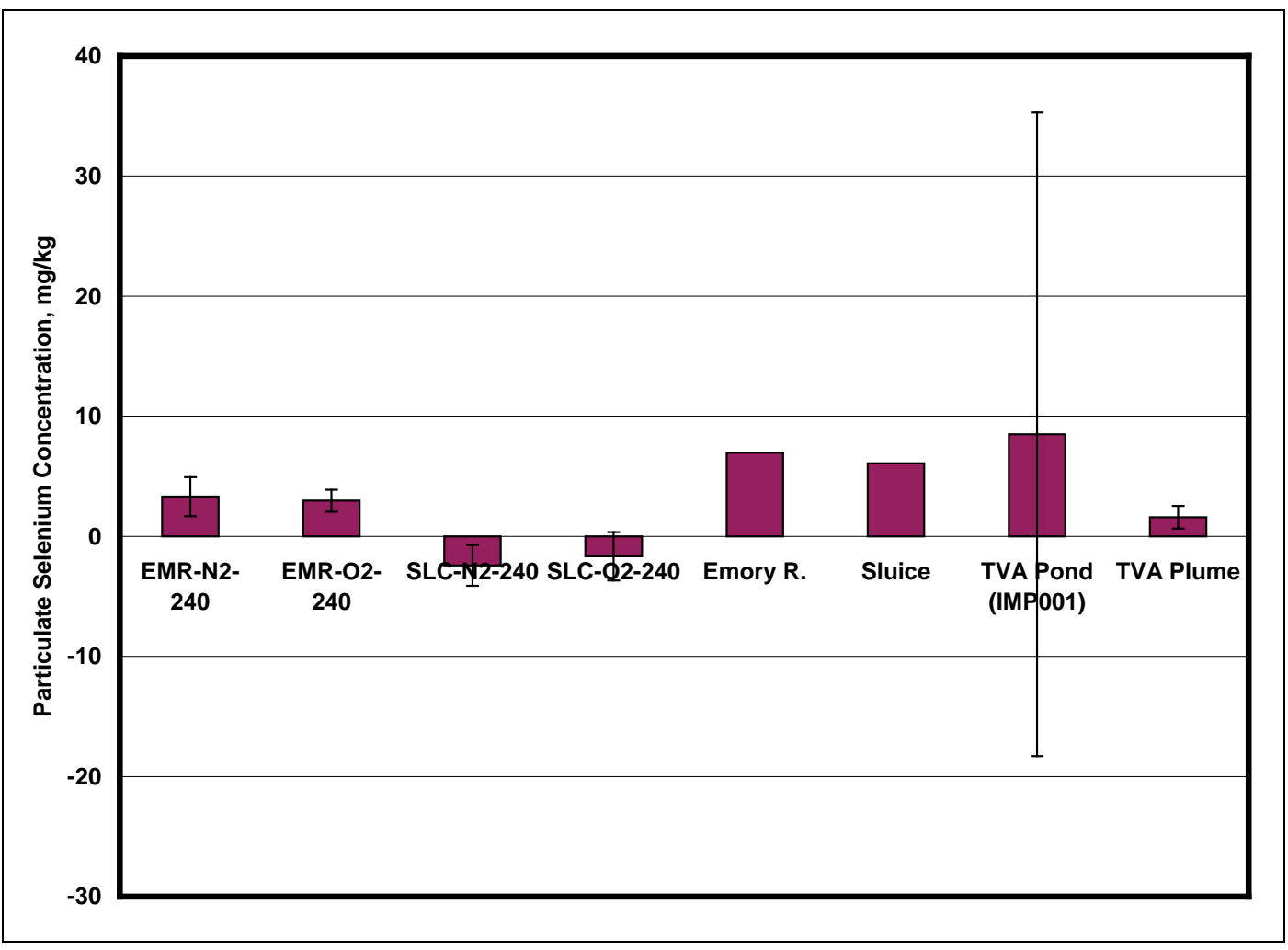

Figure 80. Particulate selenium concentrations (verified, not validated).

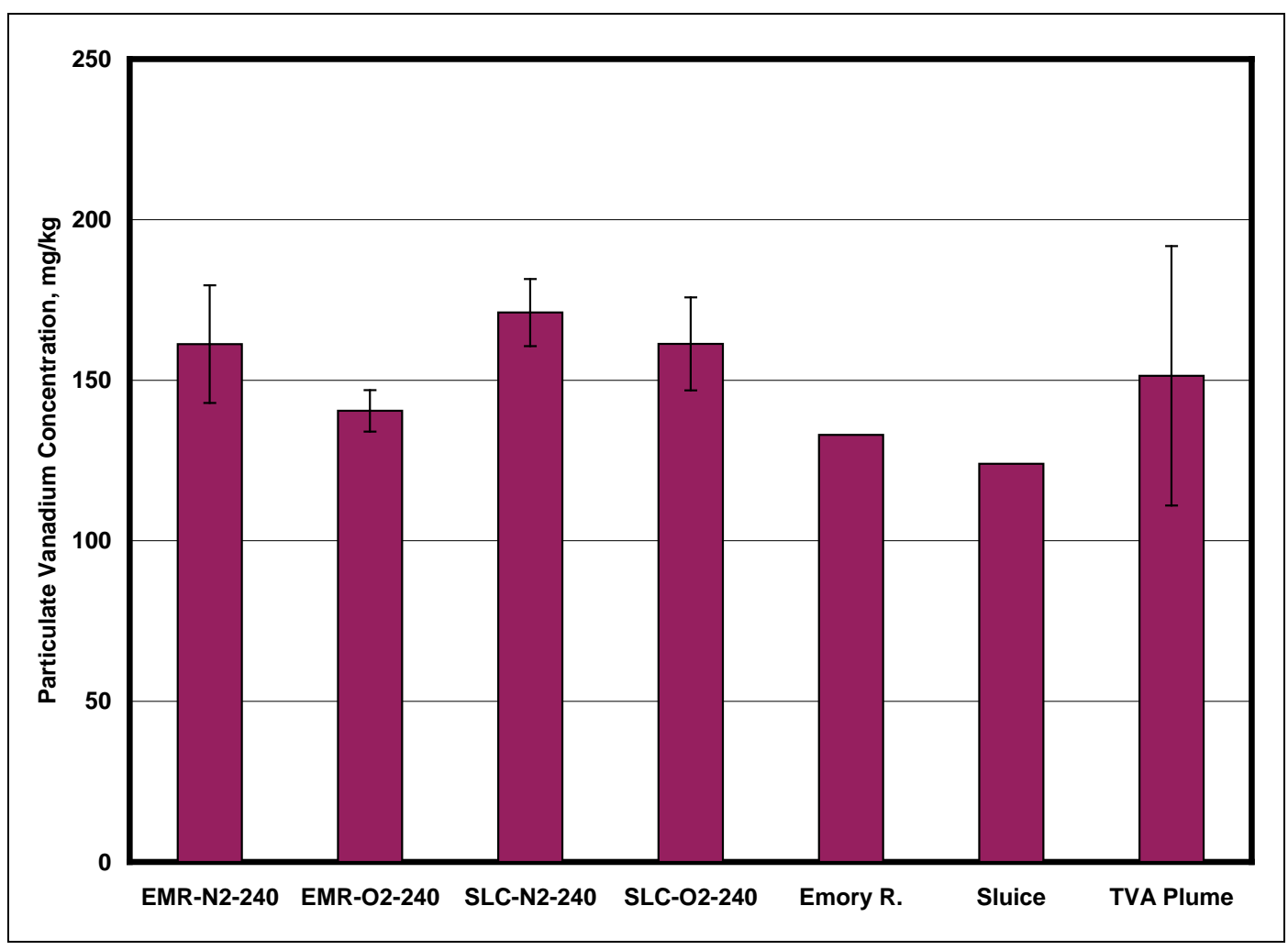

Figure 81. Particulate vanadium concentrations (verified, not validated). 


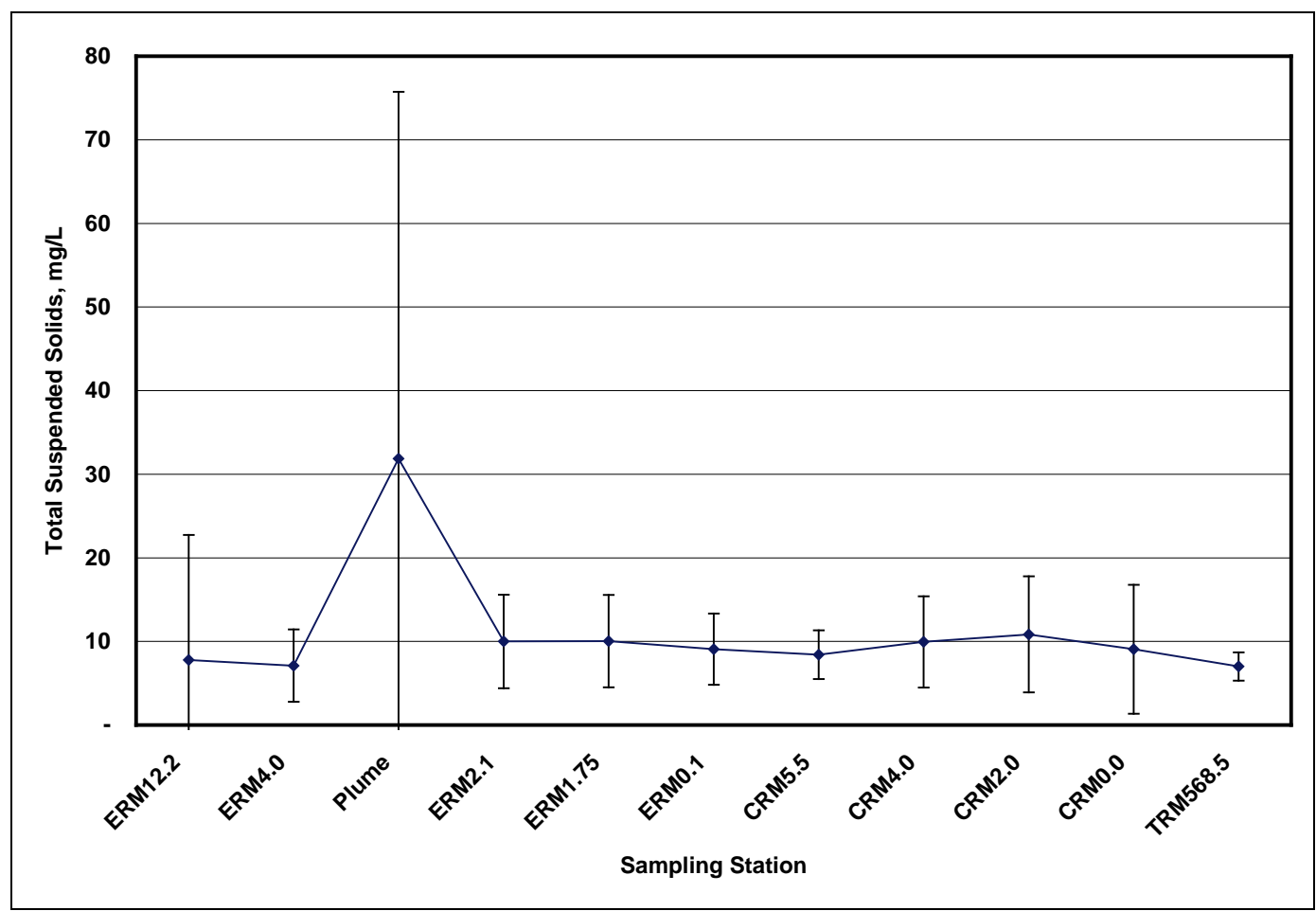

Figure 82. TVA field observations (verified, not validated) of TSS in receiving waters. Sampling stations on the $x$-axis are arranged upstream to downstream.

immediately to an average of 8 to $11 \mathrm{mg} / \mathrm{L}$ and remain in this range until mixing with the Tennessee River. The reduction is likely due to additional settling of the ash material resuspended by dredging, as well as by dispersion and dilution of the plume solids.

Figures 83 and 84 show the change in total and dissolved arsenic and barium in the river system upstream and downstream of the dredging activity. Selenium is not illustrated because all of the reported values downstream of the plume values are below TVA's detection limit of $0.002 \mathrm{mg} / \mathrm{L}$. Arsenic concentrations return to near the reporting limit of $0.002 \mathrm{mg} / \mathrm{L}$ before the confluence of the Emory and Clinch Rivers. The barium concentration decreases at the next station downstream from the plume and continues to be relatively steady until the confluence of the Clinch and Tennessee Rivers. The Kingston Plant's condenser cooling water discharge enters the Clinch River between river mile 4.0 and 2.0 and may contribute to the slight bump in arsenic and barium shown on the charts. Figure 85 shows the arsenic and barium in the dissolved fraction (as percent of the total), as well as for the river stations upstream and downstream of dredging. Downstream of dredging, the arsenic and barium is more than 85 percent in the dissolved phase, and the suspended solids concentration 


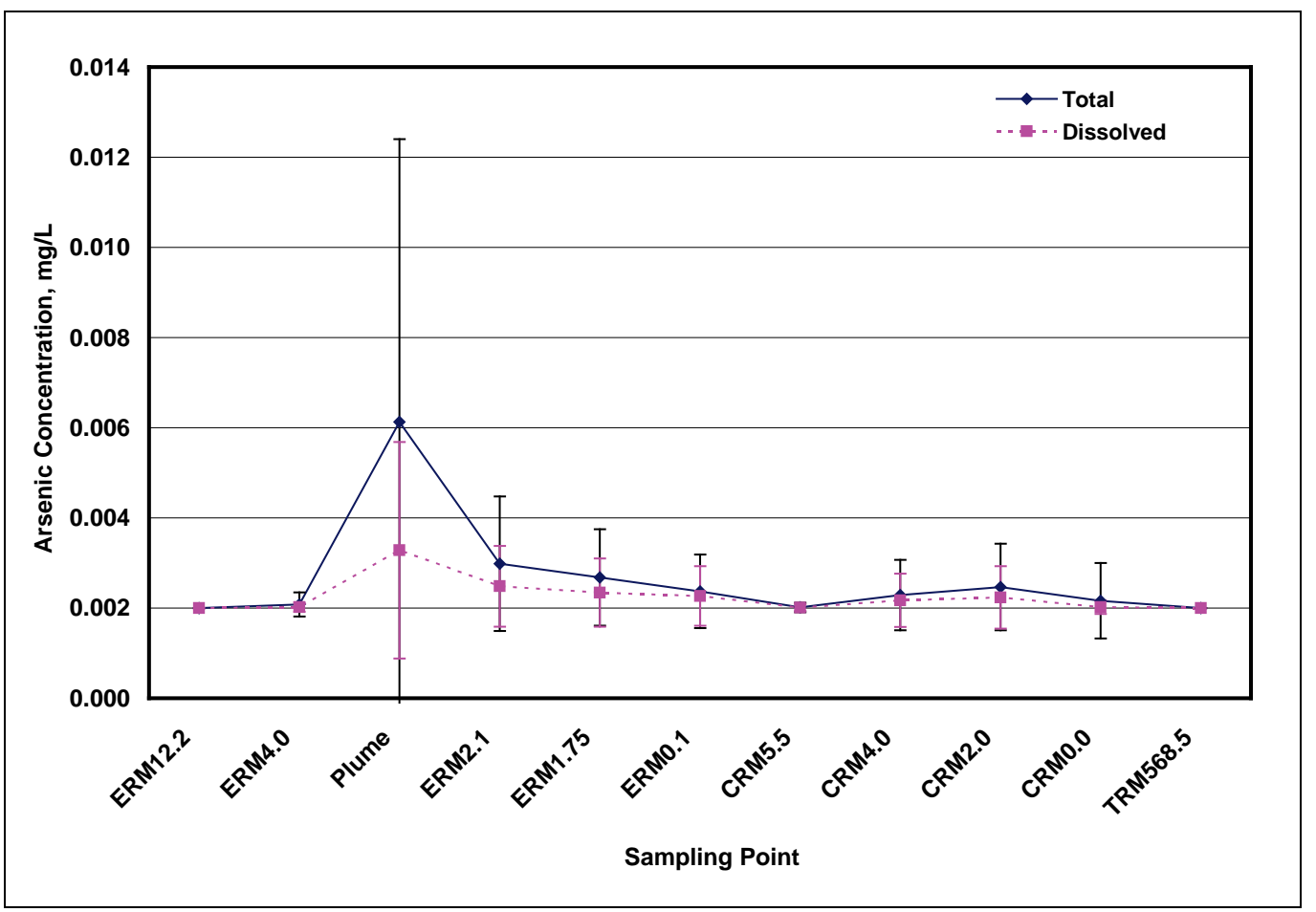

Figure 83. TVA field observations (verified, not validated) of arsenic in receiving waters. Sampling stations on the $\mathrm{x}$-axis are arranged upstream to downstream.

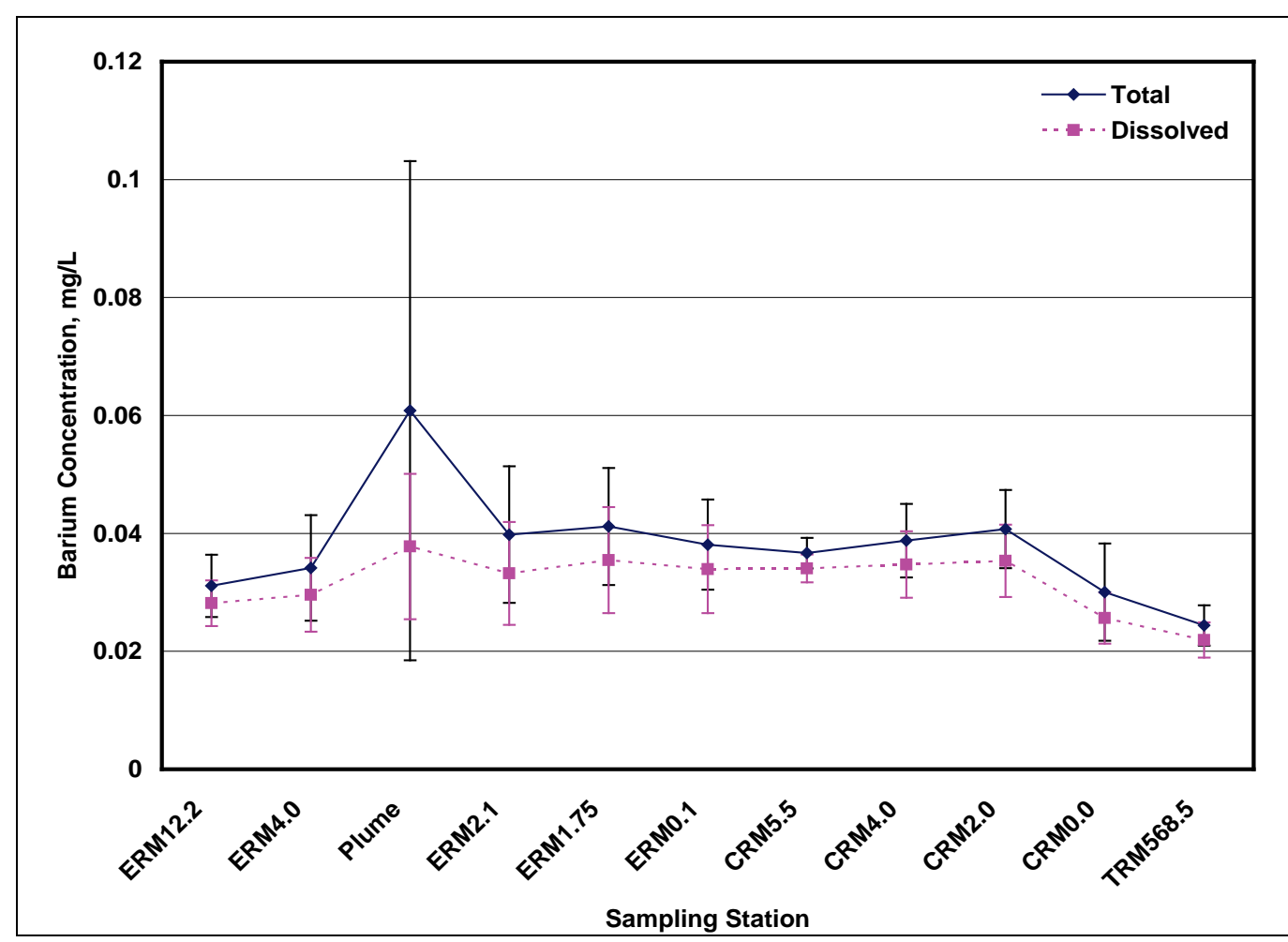

Figure 84. TVA field observations of barium in receiving waters. Sampling stations on the $x$ axis are arranged upstream to downstream. 


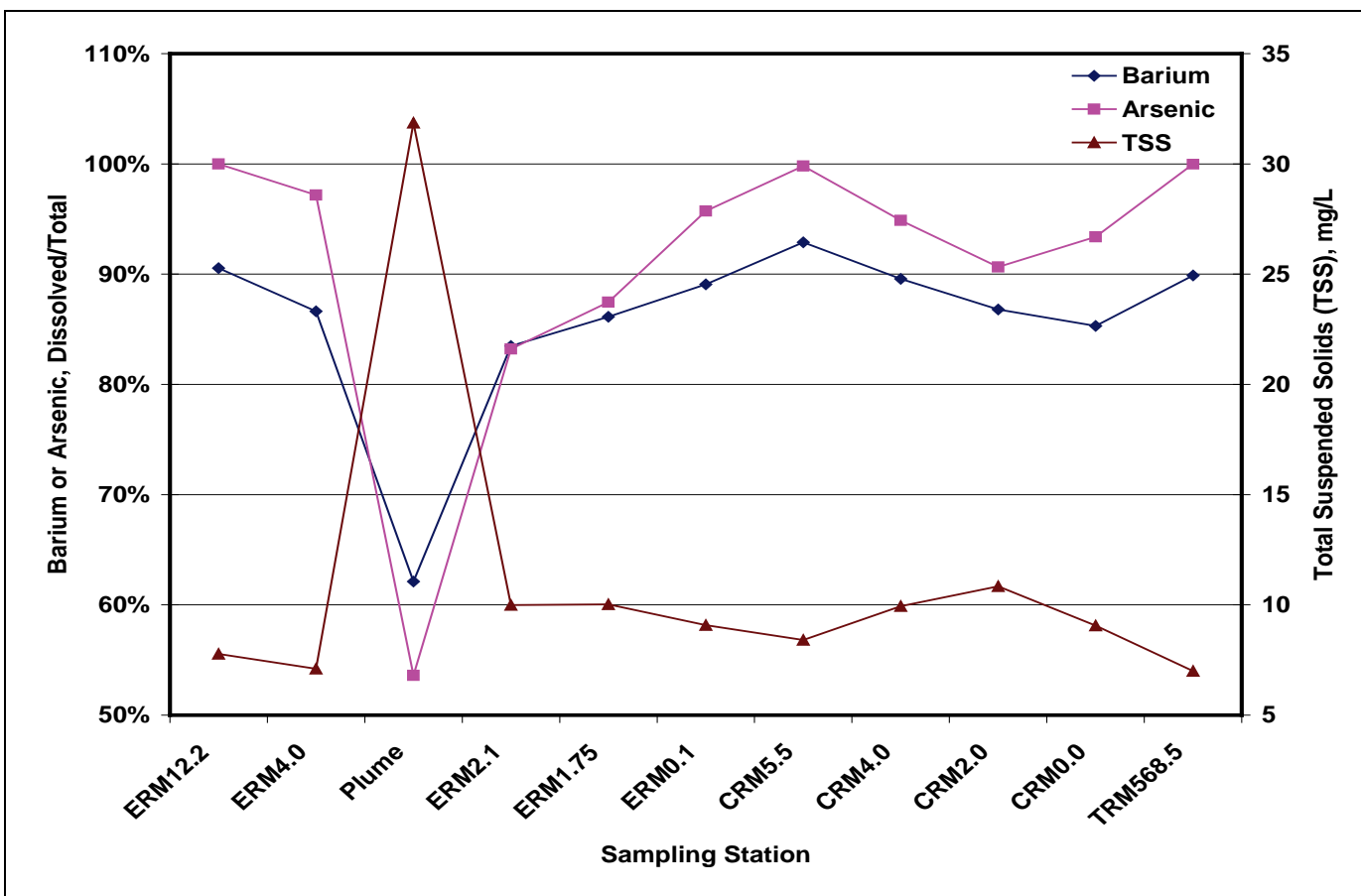

Figure 85. TVA field (verified, not validated) observations of the percent dissolved/total arsenic and barium compared to TSS in receiving waters. Sampling stations on the X-axis are arranged upstream to downstream.

is on the order of $10 \mathrm{mg} / \mathrm{L}$. (This analysis could not be performed for selenium because most of the samples had less than detectable concentrations.) The dissolved metals are readily transportable downstream without adsorption to suspended particulate matter or bed material. Metals sorbed to suspended particulates represent a smaller fraction of the load and are subject to settling, further decreasing their contribution to the water column, or to desorption increasing the dissolved concentration in the water column.

\section{Conclusions}

The following conclusions can be drawn on the basis of this study:

- The elutriate experiments performed in this study used mixing times well beyond established elutriate procedures for the purpose of assessing potential shifts in metal speciation when fly ash contaminants are released to the water environment. The exaggerated terminal mixing times ( 10 days) produced elutriates that overestimate total dissolved metal concentrations for the dredge plume and the ash settling/ stilling pond effluent based on comparison to those observed by TVA's field monitoring program for the plume and stilling pond. 
- Suspended solids and metal concentrations decline significantly at the monitoring station immediately downstream of the dredging operation.

- Dissolved metals account for much of the total metal concentrations observed in the pond effluent, plume, and river monitoring stations, largely because of the relative low suspended solids concentration. This suggests that the small suspended particulate source limits further dissolved metal desorption in the water column, but that most of the metal constituents present in the water column are mobile and subject to downstream transport.

- Results of the field monitoring program should be closely observed for deviations from the trends observed to date. Increased dredge production rates may increase contaminant releases at the dredge, as well as increase flow rates through the settling ponds, reducing retention time and impacting the effectiveness of the settling pond system in retaining suspended solids and contaminants. Cumulative effects of dredging operations with time may also affect water quality through deposits of erodible solids downstream of the dredge and solids buildup in the settling ponds. 


\section{References}

Adams, W. A. Green, W. Ahlf, A. Burton, S. Brown, B. Chadwick, M. Crane, R. Gouguet, K. Ho, T. Reynoldson, J . Savitz, P. Sibley, A. Ringwood, C. Hogstrand. 2004. Using sediment assessment tools and a weight of evidence approach. In Use of sediment quality guidelines and related tools for the assessment of contaminated sediments, ed. R. J. Wenning, G. E. Bately, G. C. Ingersoll, and D. W. Moore, 163-226. Pensacola, FL: Society of Environmental Toxicology and Chemistry.

Ahlers, J ., C. Riedhammer, M. Vogliano, R-U. Ebert, R. Kühne, G. Schüürmann. 2006. Acute to chronic ratios in aquatic toxicity: Variation across trophic levels and relationship with chemical structure. Environ Toxicol Chem. 25: 2937-2945.

American Public Health Association. 1999. Standard methods for the examination of water and wastewater, 20th edition. Alexandria, VA: Water Environmental Federation.

Baral, A., R. Engel, W. Stephens, J . Farris, R. Hannigan. 2006. Evaluation of aquatic toxicities of chromium and chromium-containing effluents in reference to chromium electroplating industries. Arch Environ Contam Toxicol. 50: 496-502.

Beckvar, N., T. M. Dillon, L. B. Read. 2005. Approaches for linking whole-body fish tissue residues of mercury or DDT to biological effects thresholds. Environ Toxicol Chem. 24: 2094-2105.

Bednar, A. J ., J . R. Garbarino, J . F. Ranville, T. R. Wildeman. 2002. Preservation of inorganic arsenic species in groundwater and acid mine drainage samples. Environ Scị Technol. 36: 2213-2216.

Bednar, A.J ., J . R. Garbarino, J . F. Ranville, T. R. Wildeman. 2003. Photodegradation of roxarsone in poultry litter leachates. Sci Total Environ. 302: 237-2.

Bednar, A.J ., J . R. Garbarino, J . F. Ranville, T. R. Wildeman. 2004. Methods of arsenic speciation and their application to natural water analysis. Water Res. 38(2): 355364 .

Bednar, A.J ., J . R. Garbarino, J . F. Ranville, T. R. Wildeman. 2005. Effects of iron on arsenic speciation and redox chemistry in acid mine water. J Geochem Explo. 85: 55-62.

Bednar, A.J ., R. A. Kirgan, W. T. J ones. 2009. Comparison of standard and reaction cell inductively coupled plasma mass spectrometry in the determination of chromium and selenium species by HPLC-ICP-MS. Anal Chim Acta. 632: 27-34.

Bertram, P.E., A. S. Brooks. 1986. Kinetics of accumulation of selenium from food and water by fathead minnows. Water Res. 20(7): 877-884.

Beusen, J . M., B. Neven. 1987. Toxicity of vanadium to different freshwater organisms. Bull Environ Contam Toxicol 39: 194-201. 
Call, D. J., L. T. Brooke, N. Ahmad, J . E. Richter. 1983. Toxicity and metabolism studies with EPA priority pollutants and related chemicals in freshwater organisms. EPA-600/3-83-095. Duluth, MN: US Environmental Protection Agency.

Chassiagne, H., C. C. Chery, G. Gordin, F. Vanhaecke, and A. Rodriguez. 2004. 2dimensional gel electrophoresis techique for yeast selenium-containing proteins sample preparation and MS approaches for processing 2-D gel protein spots. J Anal A Spectrom. 19: 85-95.

Crommentuijn, T., D. Sijm, J . de Bruijn, M. van den Hoop, K. van Leeuwen, E. van de Plassche. 2000. Maximum permissible and negligible concentrations for metals and metalloids in the Netherlands, taking into account background concentrations. J Environ Mgmt. 60: 121-143.

Dixon, D. G., and J . B. Sprague. 1981. Acclimation-induced changes in toxicity of arsenic and cyanide to rainbow trout, Salmo gairdneri Richardson. J Fish Biol. 18: 579589.

Dobbs, M. G., D. S. Cherry, J. Cairns. 1996. Toxicity and Bioaccumulation of Selenium to a Three-Trophic Level Food Chain. Environ Toxicol Chem. 15: 340-347.

Dube, M. G., L. D. MacLatchy, J . D. Kieffer, N. E. Glozier, J . M. Culp, and K. J . Cash. 2005. Effects of metal mining effluent on Atlantic salmon (Salmo salar) and slimy sculpin (Cottus cognatus): using artificial streams to assess existing effects and predict future consequences. Sci Total Environ. 343: 135- 154.

Farag, A. M., T. May, G. D. Marty, M. Easton, D. D. Harper, E. E. Little, and L. Cleveland. 2006. The effect of chronic exposure on the health of Chinook salmon (Oncorhynchus tshawtscha). Aquat Toxicol. 76: 246-257.

Felt, D. R., A. J . Bednar, and T. Georgian. 2008. The effects of grinding methods on metals concentrations in soils. Talanta 77: 380-387.

Friedmann, A. S., M. C. Watzin, T. Brinck-J ohnsen, and J . C. Leiter. 1996. Low levels of dietary methylmercury inhibit growth and gonadal development in juvenile walleye (Stizostedion vitreum). Aquat Toxicol. 35: 265-278.

Gendusa, T. C., and T. L. Beitinger. 1992. External biomarkers to assess chromium toxicity in adult Lepomis macrochirus. Bull Environ Contam Toxicol. 48: 237242.

Giesy, P., and R. L. Graney. 1989. Recent developments in and intercomparisons of acute and chronic bioassays and bioindicators. Hydrobiologia 188/ 189: 21-60.

Gilderhus, P. A. 1966. Some effects of sublethal concentrations of sodium arsenite on bluegills and the aquatic environment. Trans Am Fish Soc. 095: 289-296.

Gustafsson, J . P. 2009. Visual MINTEQ. Release 2.61.

Haratake, M., M. Hongoh, M. Ono, and M. Nakayama. 2009. Thiol-dependent membrane transport of selenium through an integral protein of the red blood cell membrane. Inorg Chem. 48: 7805-7811. 
Heger, W., S. J . J ung, S. Martin, and H. Peter. 1995. Acute and prolonged toxicity to aquatic organisms of new and existing chemicals and pesticides. Hydrobiologia 31: $2707-2726$.

Holdway, D. A., and J . B. Sprague. 1979. Chronic toxicity of vanadium to flagfish. Water Res. 13: 905-910.

Holdway, D. A., J . B. Sprague, and J . G. Dick. 1983. Bioconcentration of vanadium in American flagfish over one reproductive cycle. Water Res. 17: 937-941.

Huggins, F. E., C. L. Senior, P. Chu, K. Ladwig, and G. P. Huffman. 2007. Selenium and arsenic speciation in fly ash from full-scale coal-burning utility plants. Environ Sci Technol. 41: 3284-3289.

Kamei-Ishikawa, N., K. Tagami, and S. Uchida. 2007. Sorption kinetics of selenium on humic acid. J Radioanal Nuc Chem. 274: 555-561.

Khangarot, B. S., and P. K. Ray. 1989. Investigation of correlation between physicochemical properties of metals and their toxicity to the water flea Daphnia magna Straus. Ecotoxicol Environ. Safety 18: 109-120.

Lange, R., T. H. Hutchinson, N. Scholz, and J . Solbe. 1998. Analysis of the ECETOC Aquatic Toxicity (EAT) database II-comparison of acute to chronic ratios for various aquatic organisms and chemical substances. Chemosphere 36: 115- 127.

LeBlanc, G. A. 1980. Acute toxicity of priority pollutants to water flea (Daphnia magna). Bull Environ Contam Toxicol. 24: 384-691.

Leung, K. M. Y., D. Morritt, J. R. Wheeler, P. Whitehouse, N. Sorokin, R. Toy, M. Holt, and M. Crane. 2001. Can saltwater toxicity be predicted from freshwater data? Mar Pollut Bull. 42: 1007-1013.

Luoma, S. N., and P. S. Rainbow. 2005. Why is metal bioaccumulation so variable? Biodynamics as a unifying concept. Environ Sci Technol. 39(7): 1921-1931.

Manceau, A., M. A. Marcus, and N. Tamura. 2002. Quantitative speciation of heavy metals in soils and sediments by synchrotron X-ray techniques. In: Applications of Synchrotron Radiation In Low-Temperature Geochemistry and Environmental Sciences, 49(341-428).

Mathangwane, B. T., M. A. Chappell, J . R. V. Pils, L. S. Sonon, and V. P. Evangelou. 2008. Dispersion potential of selected Iowa lake sediments as influenced by dissolved and solid-phase constituents. Clean 36: 201-208.

MoGeachy, S. M., and D. G. Dixon. 1990. Effect of temperature on the chronic toxicity of arsenate to rainbow trout (Oncorhynchus mykiss). Can J Fish Aquat Sci. 47: 2228-2234.

Mount, D. I. 1977. An assessment of application factors in aquatic toxicology. In Recent advances in fish toxicology. A symposium held in Corvallis, Oregon on J anuary 13-14, 1977. Corvallis, OR: Office of Research and Development, USEPA.

National Academy of Sciences/ National Academy of Engineering. 1973. Water Quality Criteria 1972. EPA Ecological Res. Ser EPA R3-73-033, Washington, D.C. 
Newville, M. 2001. IFEFFIT: interactive XAFS analysis and FEFF fitting. J Synchrotr Radiat. 8: 322-324.

Norberg-King, T. 1989. An evaluation of the fathead minnow seven-day subchronic test for estimating chronic toxicity. Environ Toxicol Chem 8: 1075-1089.

Ogle, R. S., and A. W. Knight. 1989. Effects of elevated foodborne selenium on growth and reproduction of the fathead minnow (Pimephales promelas). Arch Environ Toxicol Chem. 18: 795-803.

Palermo, M. R., P. R. Schroeder, T. J . Estes, and N. R. Francingues. 2008. Technical guidelines for environmental dredging of contaminated sediments. ERDC/ EL TR-08-29. Vicksburg, MS: U.S. Army Engineer Research and Development Center.

Phillips, D. J . H., and P. S. Rainbow. 1989. Strategies of trace metal sequestration in aquatic organisms. Mar Environ Res. 28: 207-210.

Ponce de Leon, C. A., K. DeNicola, M. M. Bayon, and J . A. Caruso. 2003. Sequential extractions of selenium soils from Stewart Lake: Total selenium and speciation measurements with ICP-MS detection. J Environ Monit. 5: 435-440.

Poppe, L. J ., A. H. Eliason, J . J . Fredericks, R. R. Rendigs, D. Blackwood, and C. F. Polloni. 2000. U.S. Geological Survey Open File Report 00-358 (USGS 2000). Chapter 1: Grain-Size Analysis of Marine Sediments: Methodology and Data Processing. http://pubs.usgs.gov/of/2000/of00-358/text/chapter1.htm

Rael, R. M., and W. T. Frankenberger. 1995. Detection of selenmethionine in the fulvic fraction of seleiferous sediment. Soil Biol Biochem. 27: 241-242.

Ressler, T. 1998. WinXAS: A program for X-ray absorption spectroscopy data analysis under MS-windows. J Synchrotr Radiat. 5:118-122.

Roex, E. W. M., C. A. M. van Gestel, A. P. van Wezel, and N. M. van Straalen. 2000. Ratios between acute aquatic toxicity and effects on population growth rates in relation to toxicant mode of action. Environ Toxicol Chem. 19: 685 - 693.

Roling, J . A., L. J . Bain, J . Gardea-Torresdey, J . Bader, and W. S. Baldwin. 2006. Hexavalent chromium reduces larval growth and alters gene expression in mummichog (Fundulus heteroclitus). Environ Toxicol Chem. 25:2725-2733.

Schultz, R., and R. Hermautz. 1990. Transfer of toxic concentrations of selenium from parent to progeny in the fathead minnow (Pimephales promelas). Bull Environ Toxicol Chem 45: 568-573.

Séby, F., M. P. Gautier,G. Lespés,and M. Astruc. 1997. Selenium speciation in soils after alkaline extraction. Sci Total Environ. 207: 81-90.

Snarski, V. M., and G. F. Olson. 1982. Chronic toxicity and bioaccumulation of mercuric chloride in the fathead minnow (Pimephales promelas). Aquat Toxicol. 2: 143156. 
Society of Environmental Toxicology and Chemistry (SETAC). 2008. Tissue residue approach for toxicity assessment: Invertebrates and fish. In Proceedings Society of Environmental Toxicology and Chemistry, Leavenworth, WA, 7-12 J une 2007, published in 2009 by SETAC and CRC Press.

Spehar, R. L., and J. T. Fiandt. 1986. Acute and chronic effects of water quality-based metal mixtures on three aquatic species. Environ Toxicol Chem. 5: 917-931.

Steevens, J. A., M. Reiss, and A. V. Pawlisz. 2005. A methodology for deriving tissue residue benchmarks for fish exposed to 2,3,7,8-tetrachlorodienzo-p-dioxin. Integr Environ Assess Mgmt. 1(2): 142-151.

Tarze, A., M. Dauplais, I. Grigoras, M. Lazard, N-T. Ha-Duong, F. Barbier, S. Blanquet, and P. Plateau. 2007. Extracellular production of hydrogen selenide accounts for thiol-assisted toxicity of selenite against Saccharomyces cerevisiae. J Biol Chem. 282:8759-8767.

Tessier, A., P. G. C. Campbell, and M. Bisson. 1979. Sequential extraction procedure for the speciation of particulate trace metals. Anal Chem. 51:844-850.

U.S. Army Corps of Engineers (USACE). 2003. Evaluation of dredged material proposed for disposal at island, nearshore, or upland confined disposal facilities - Testing Manual." ERDC/ EL TR-03-1. Vicksburg, MS: U.S. Army Engineer Research and Development Center.

U.S. Army Engineer Research and Development Center (ERDC). 2009. Quality Assurance Project Plan. Evaluation of Metals Release from Oxidation of Fly Ash during Dredging of the Emory River, TN. J une 14, 2009.

U.S. Environmental Protection Agency (USEPA). 2002a. Methods for measuring the acute toxicity of effluents and receiving waters to freshwater and marine organisms. EPA/ 812/R/ 02/ 012. Washington, DC: U.S. Environmental Protection Agency, Office of Water.

U.S. Environmental Protection Agency (USEPA). 2002b. Short-term methods for estimating the chronic toxicity of effluents and receiving waters to freshwater organisms, $4^{\text {th }}$ edition. EPA/ 821/ R/ 02/ 013. Washington, DC: U.S. Environmental Protection Agency, Office of Water.

U.S. Environmental Protection Agency (USEPA). 2002c. National recommended water quality criteria. EPA/ 822/R/ 02/ 047. Washington, DC: U.S. Environmental Protection Agency, Office of Water.

U.S. Environmental Protection Agency (USEPA). 2004. Draft aquatic life water quality criteria for selenium - 2004. EPA-822-D-04-001. Washington, DC: U.S. Environmental Protection Agency, Office of Water, Office of Science and Technology.

U.S. Environmental Protection Agency (USEPA). 2007. SW-846 Test Methods for Evaluating Solid Waste, Method 3050B, Method 6010C, Method 6020A.

U.S. Environmental Protection Agency (USEPA). 2009. National Primary Drinking Water Regulations. EPA816/ F/ 09/ 004. Washington, DC: U.S. Environmental Protection Agency, Office of Water. 
U.S. Environmental Protection Agency (USEPA) Panel. 2009. Review of potential selenium issues following a coal ash spill at the Tennessee Valley Authority Kingston Fossil Plant--An EPA Science Panel review paper, prepared at the request of the Senate Committee on Environment and Public Works Staff, Washington, DC.

U.S. Environmental Protection Agency/ U.S. Army Corps of Engineers (USEPA/ USACE). 1991. Evaluation of dredged material proposed for ocean disposal. EPA-503-891-001, Washington, DC.

U.S. Environmental Protection Agency/ U.S. Army Corps of Engineers (USEPA/ USACE). 1998. Evaluation of dredged material proposed for discharge in waters of the U.S. - Testing Manual. EPA-823-B-98-004, Washington, DC.

Verma, S. R., I. P. Tonk, and R. C. Dalela. 1981. Determination of the maximum acceptable toxicant concentration and the safe concentration for certain aquatic pollutants. Acta Hydrochim Hydrobiol. 9(3): 247-254.

Vijver, M. G., C. A. M. Van Gestel, R. P. Lanno, N. M. Van Straalen, and W. J . G. M. Peijnenburg. 2004. Internal metal sequestation and its ecotoxicological relevance: a review. Environ Sci Tech. 38(18): 4705-4712.

Voelker, B. M., F. M. M. Morel, and B. Sulzberger. 1997. Iron redox cycling in surface waters: Effects of humic substances and light. Environ Sci Technol. 31: 10041011.

Webb, S. M. 2005. Sixpack: A graphical user interface for XAS analysis using IFEFFIT. Physica Scripta T115:1011-1014.

Wheeler, J . R., K. M. Y. Leung, D. Morritt, N. Sorokin, H. Rogers, R. Toy, M. Holt, P. Whitehouse, and M. Crane. 2002. Freshwater to saltwater toxicity extrapolation using species sensitivity distributions. Environ Toxicol Chem. 21: 2459-2467. 


\section{Appendix A: Sample Collection Notes}


Appendix A. Sample collection notes

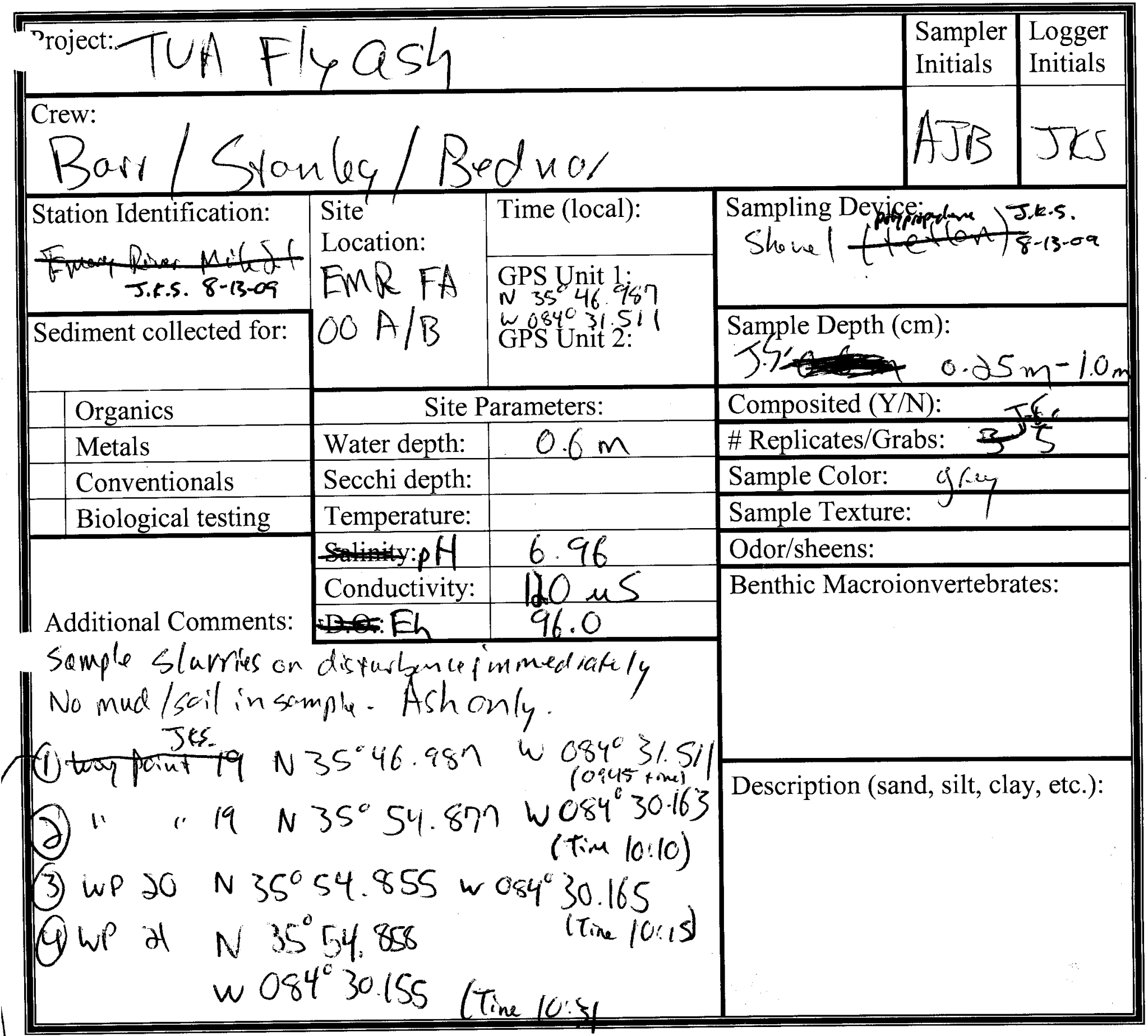

(5) WP N35' 54.845

$\omega 084^{\circ} 30.145^{\prime}$

I Time Instal 


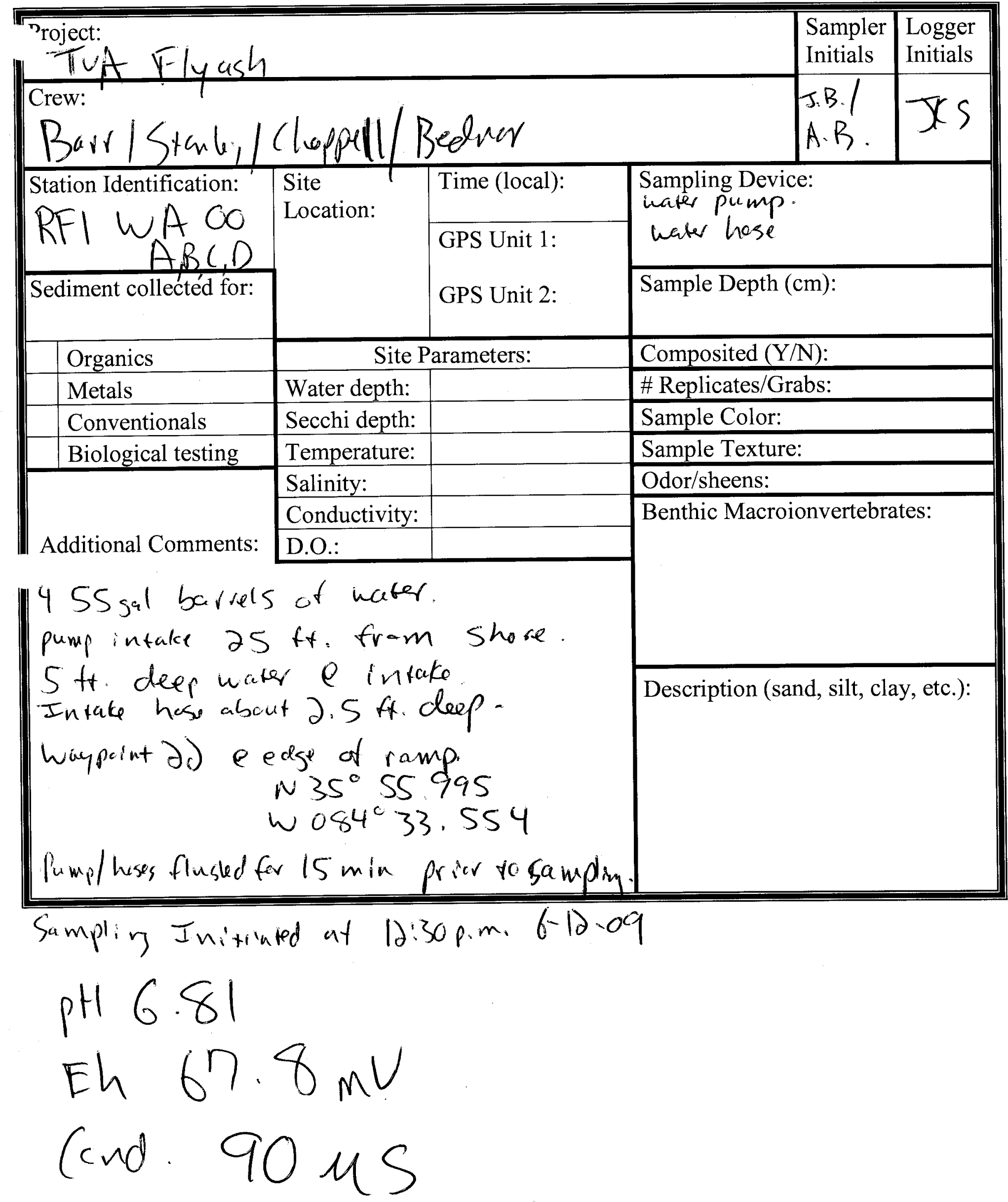




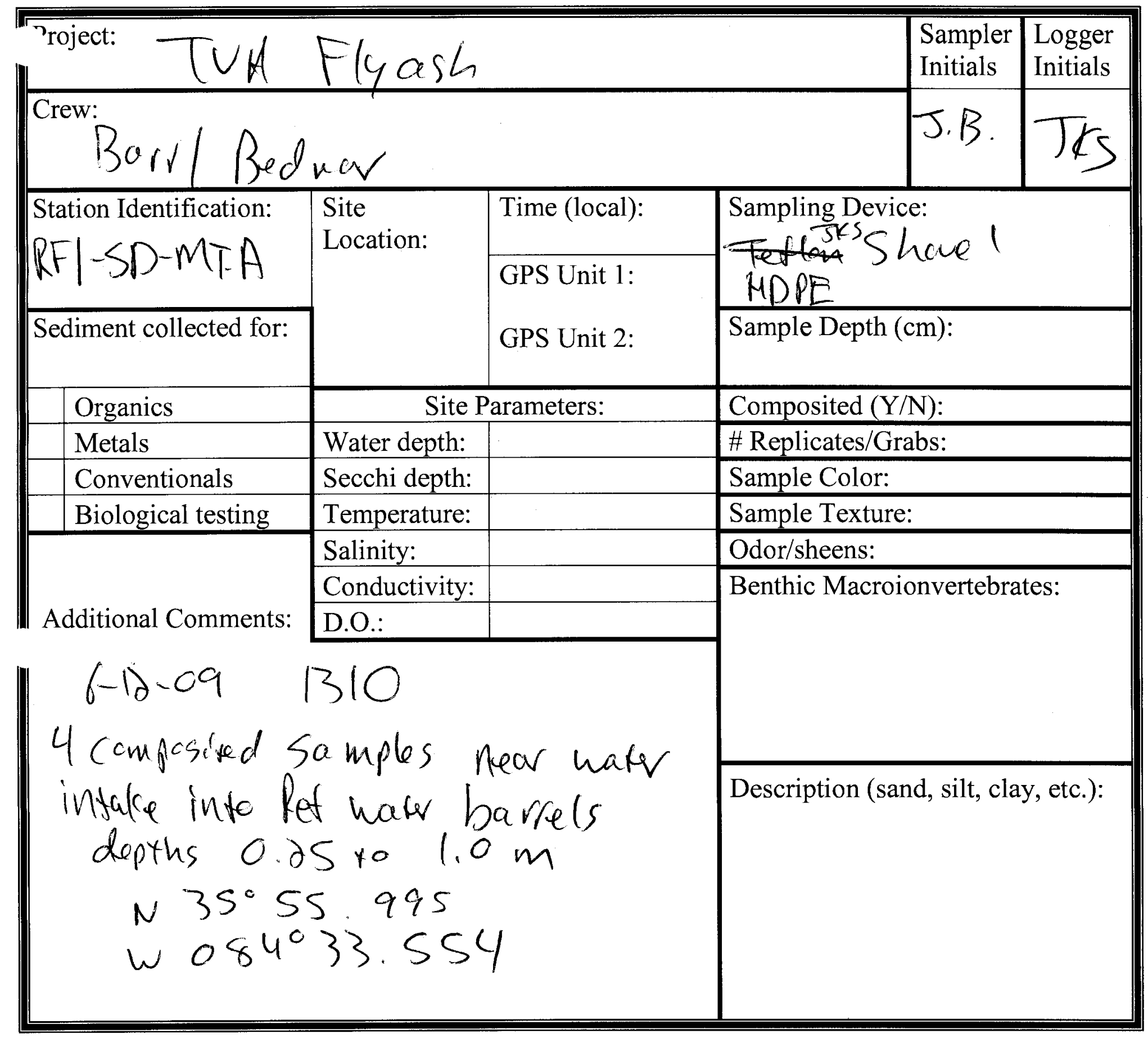




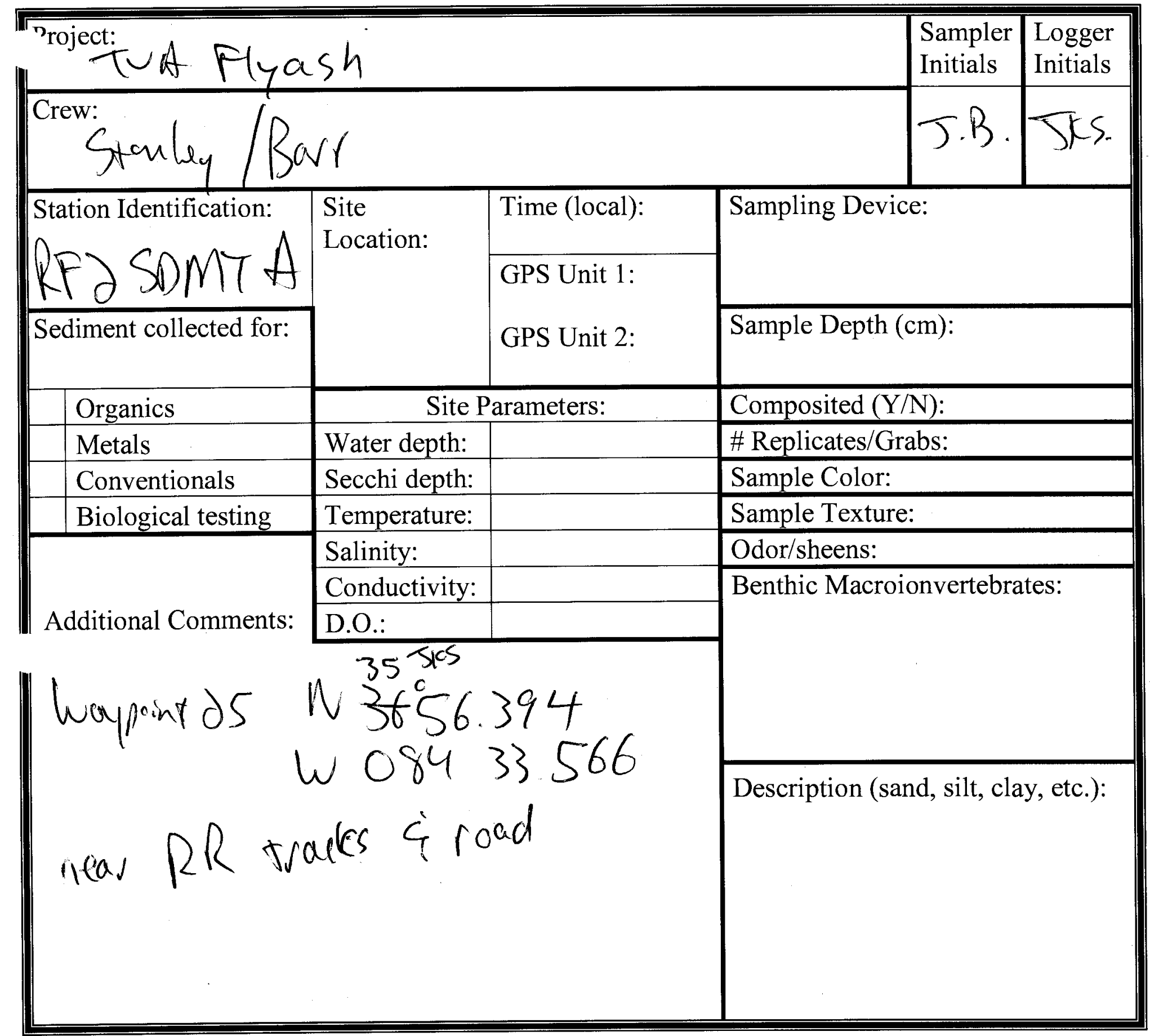




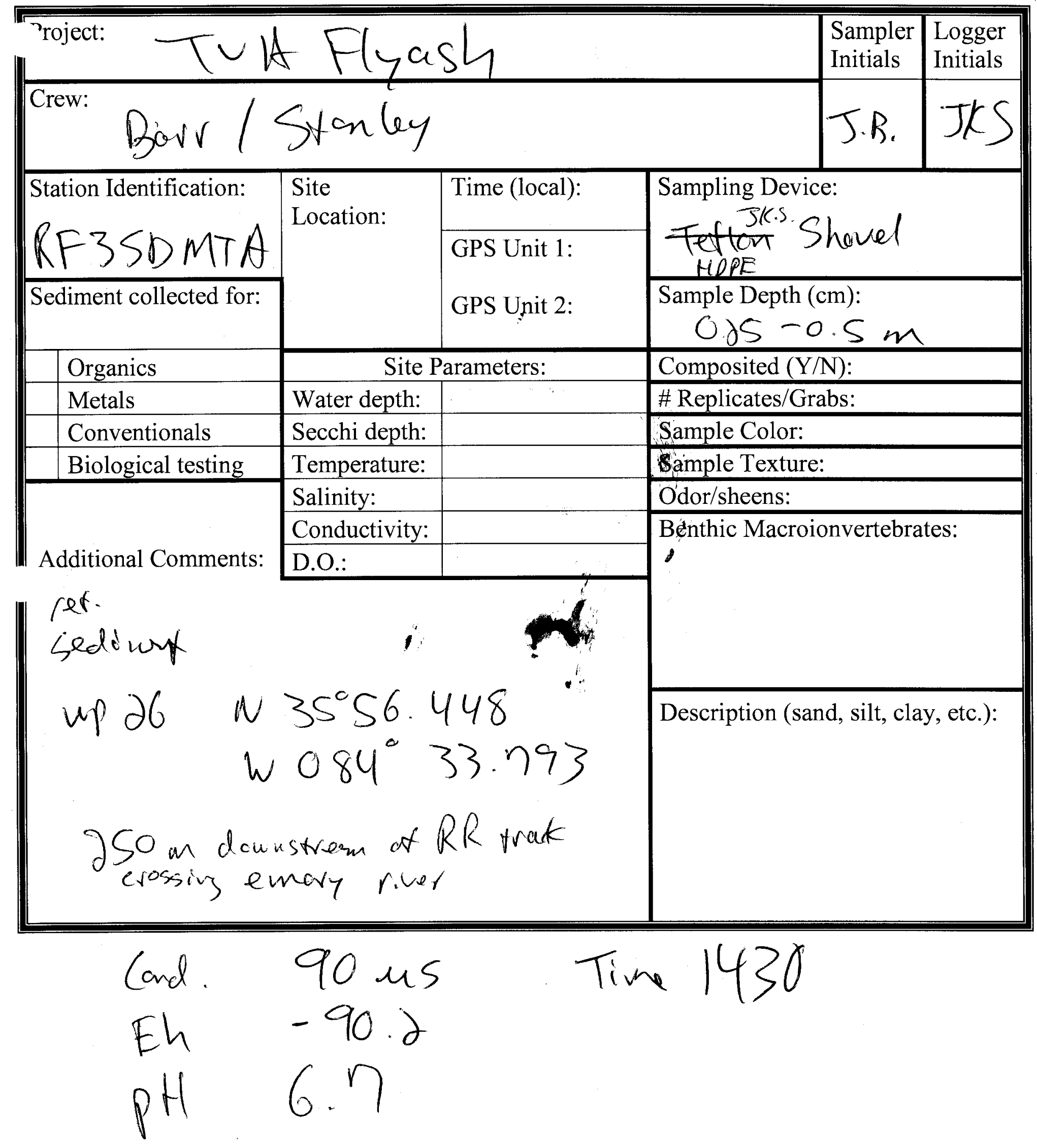




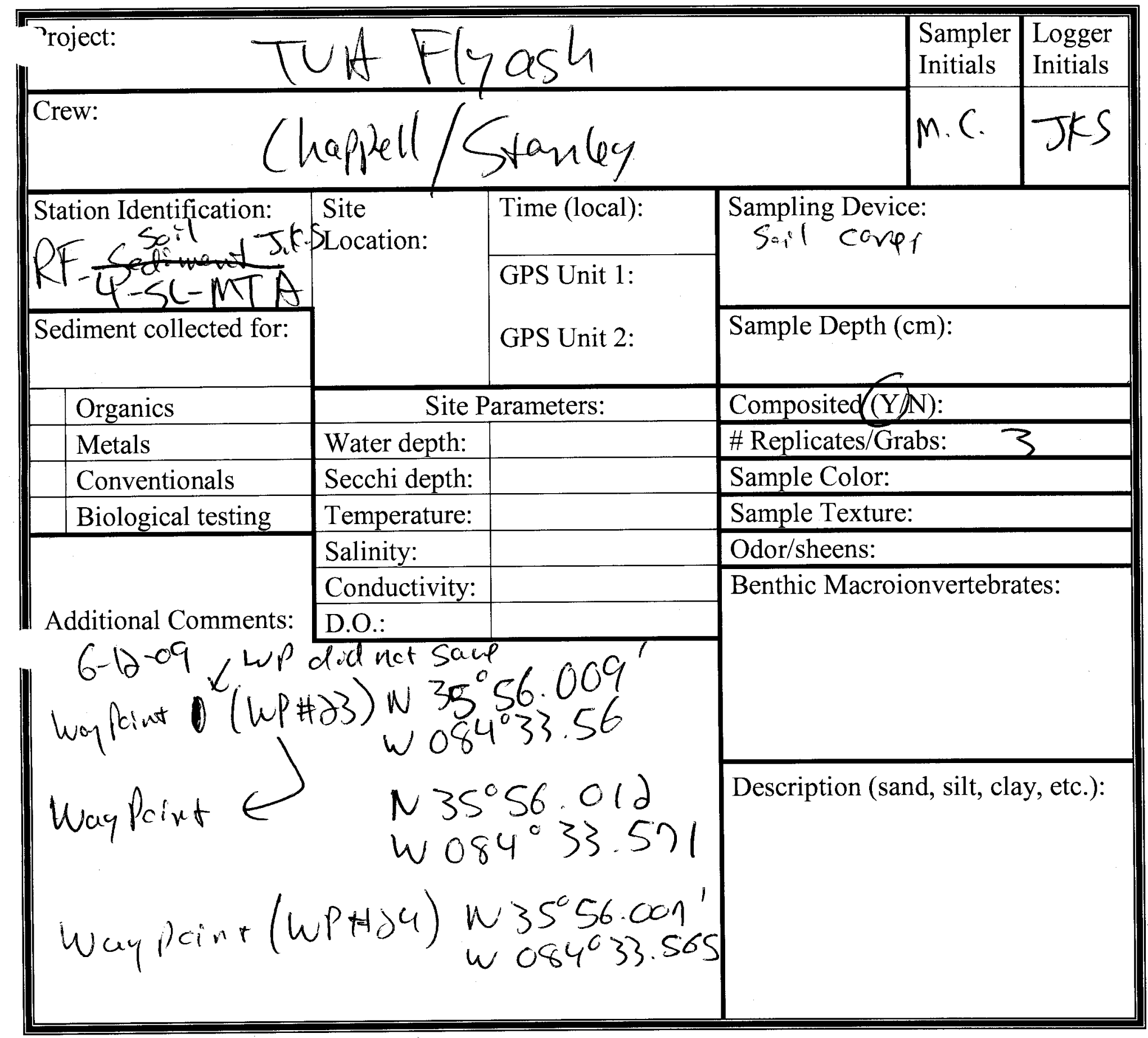

Wi $\mathrm{for} 3$ grabs that were composing on bank. Approx SO yod s upstream of batranp where drums of water tilled.

Rakes Sticks removed from sample. 


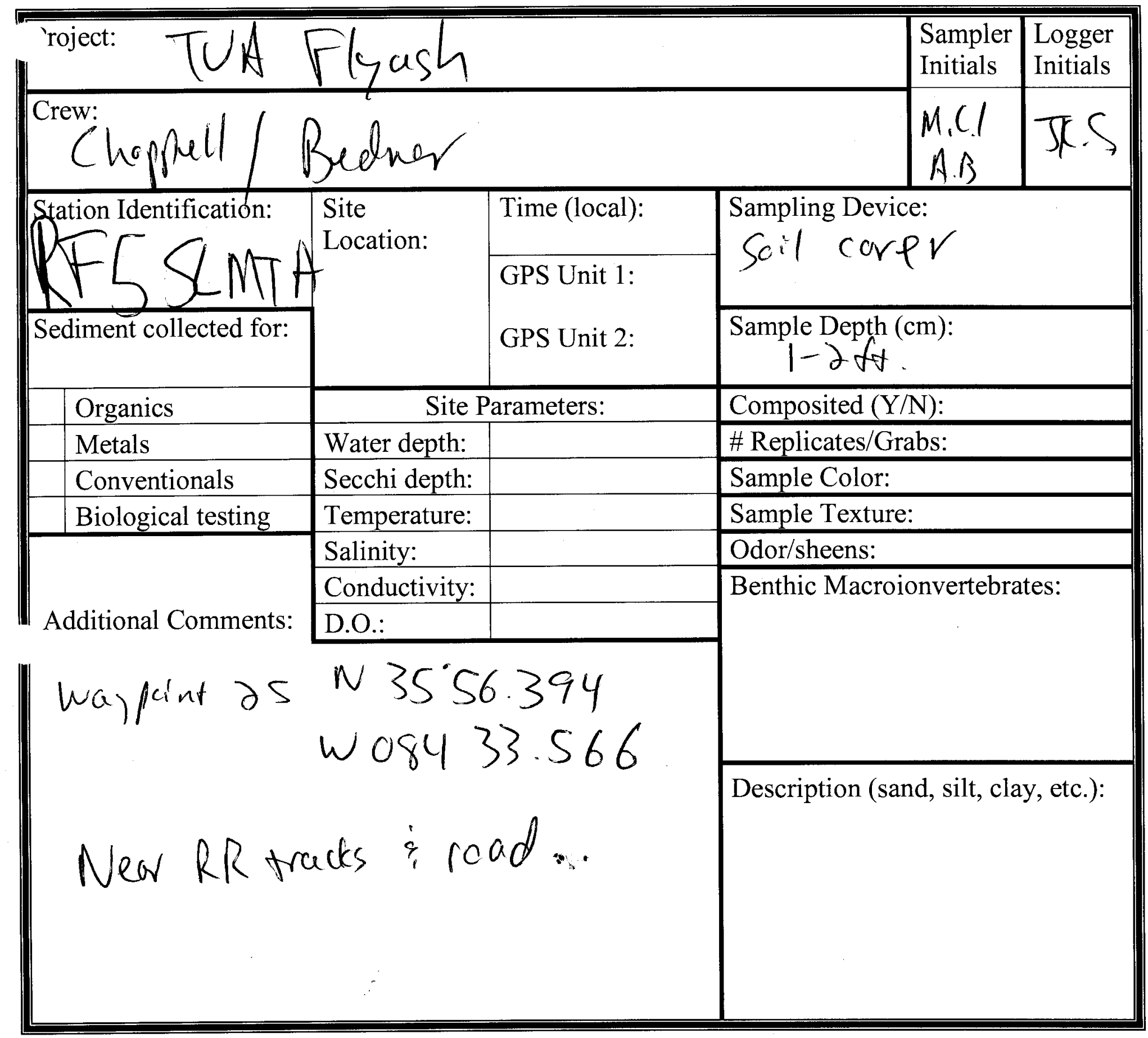




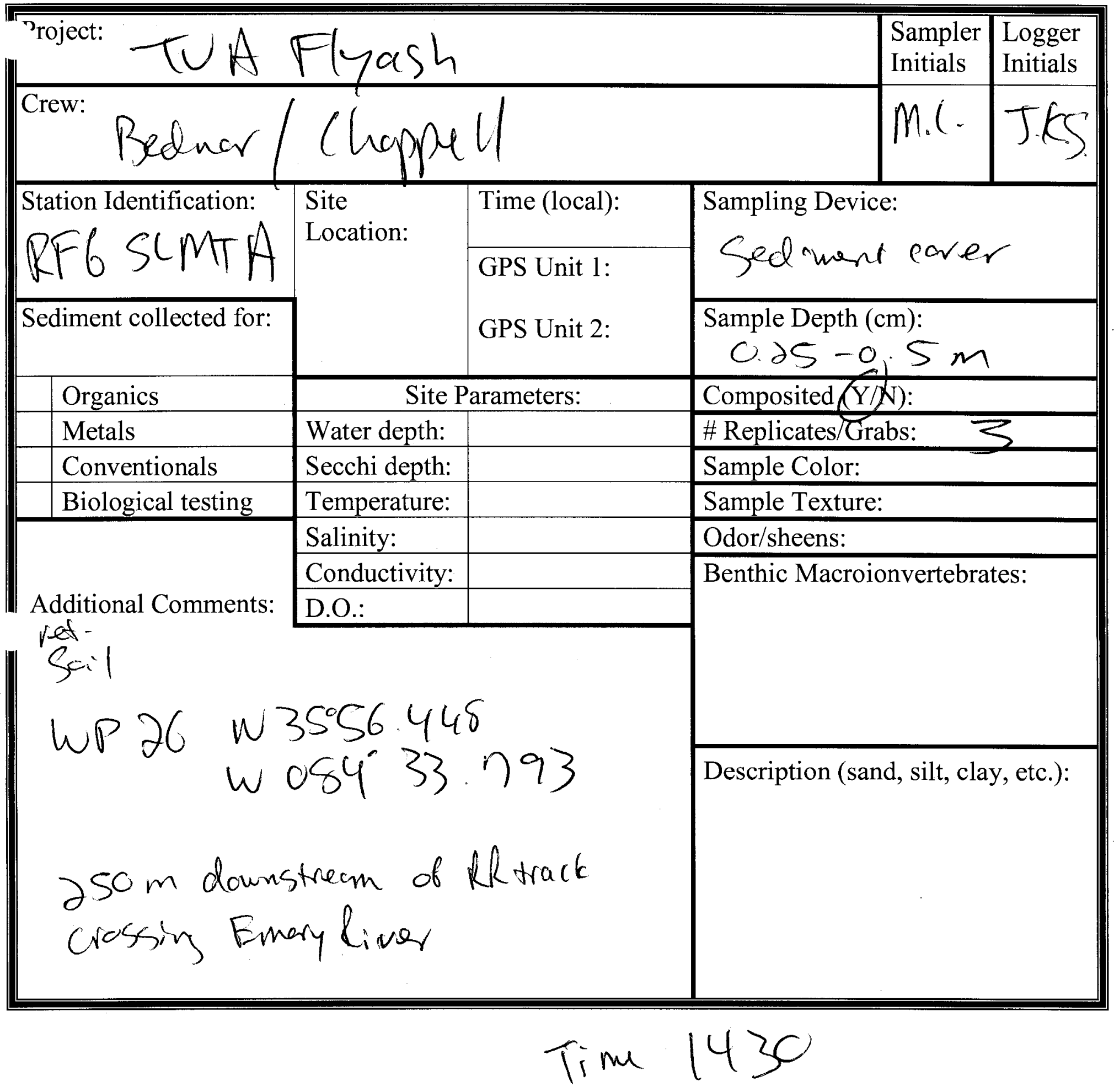




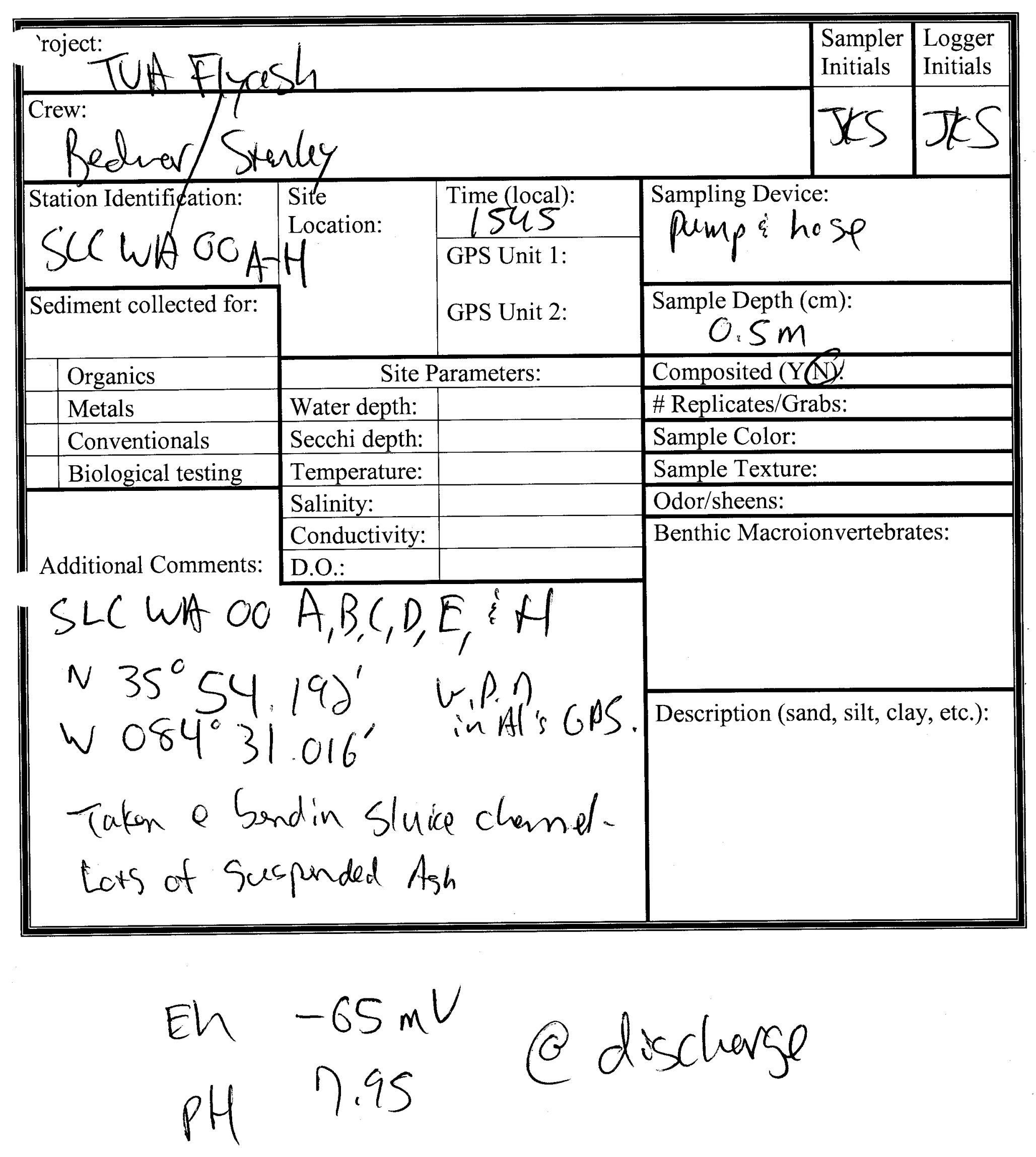




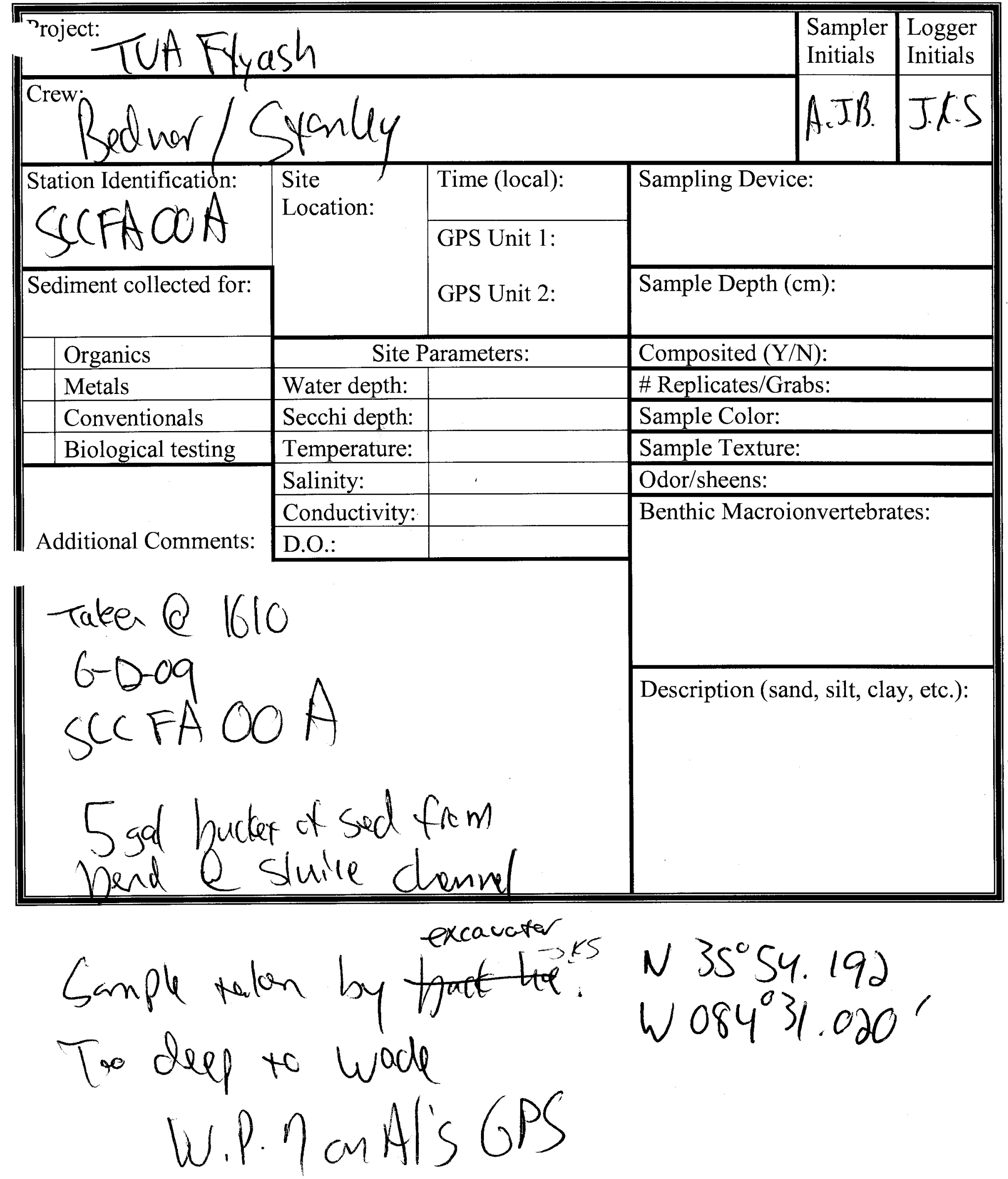




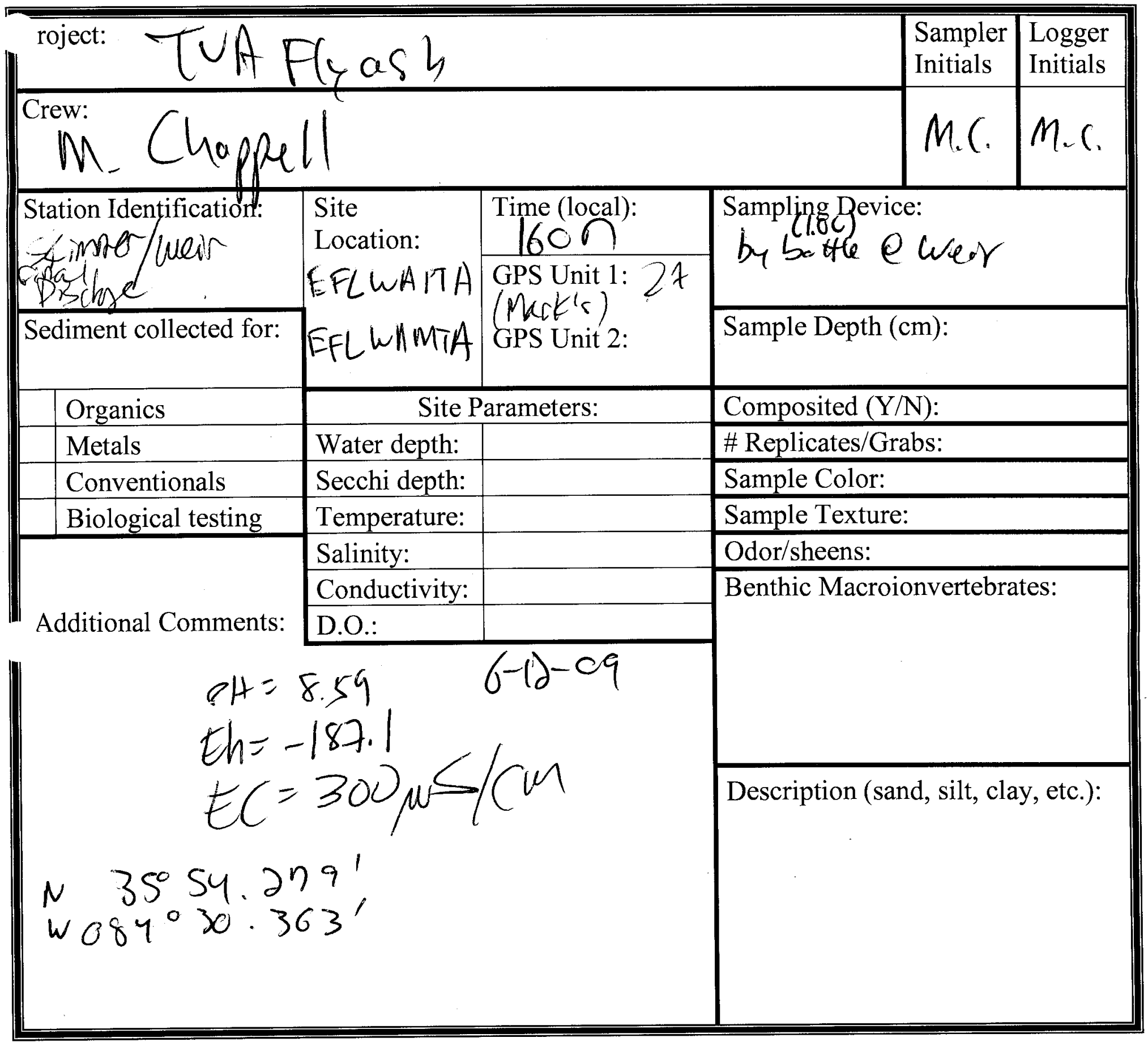




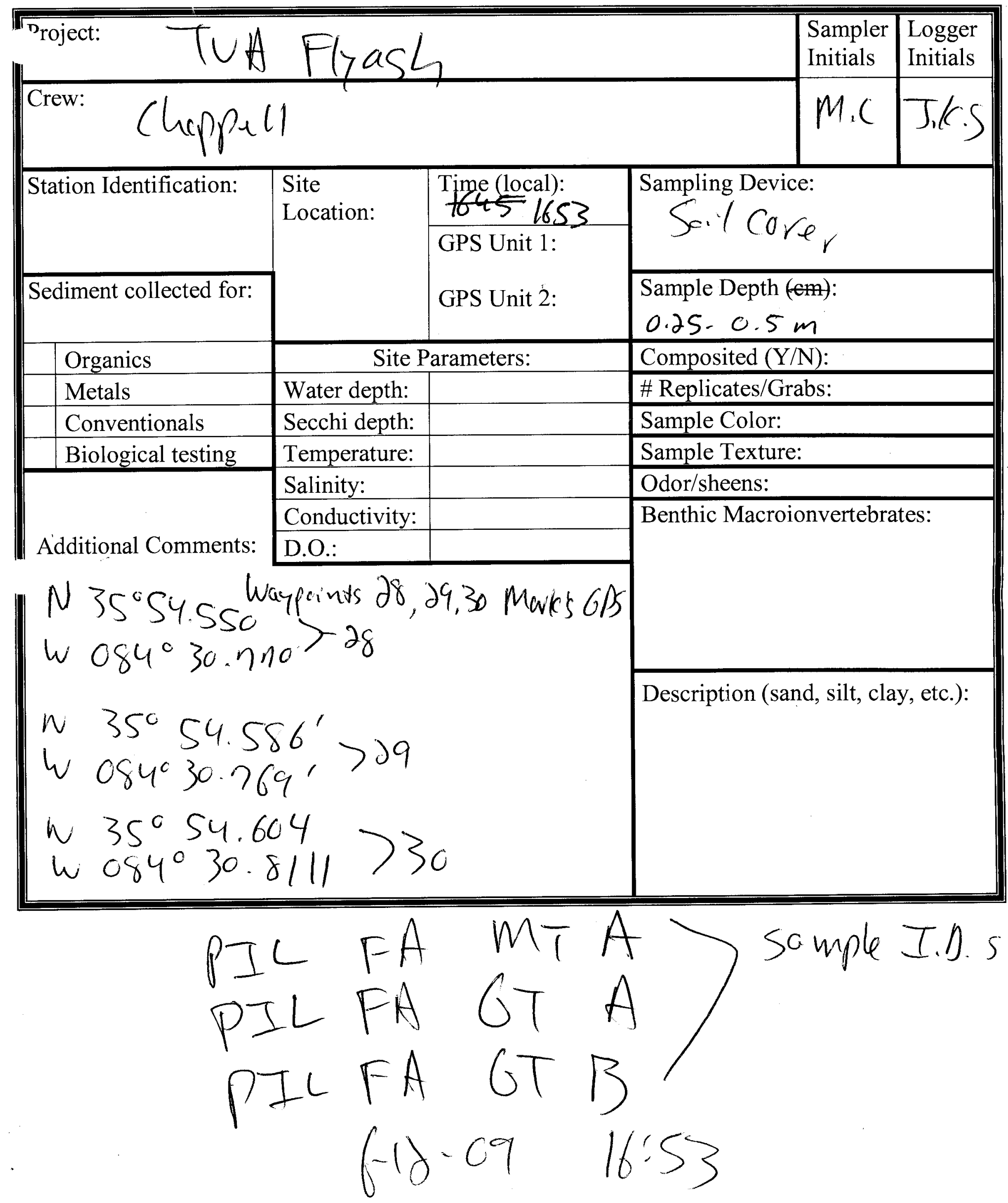




\section{Appendix B: Elutriate Laboratory Data Sheets}




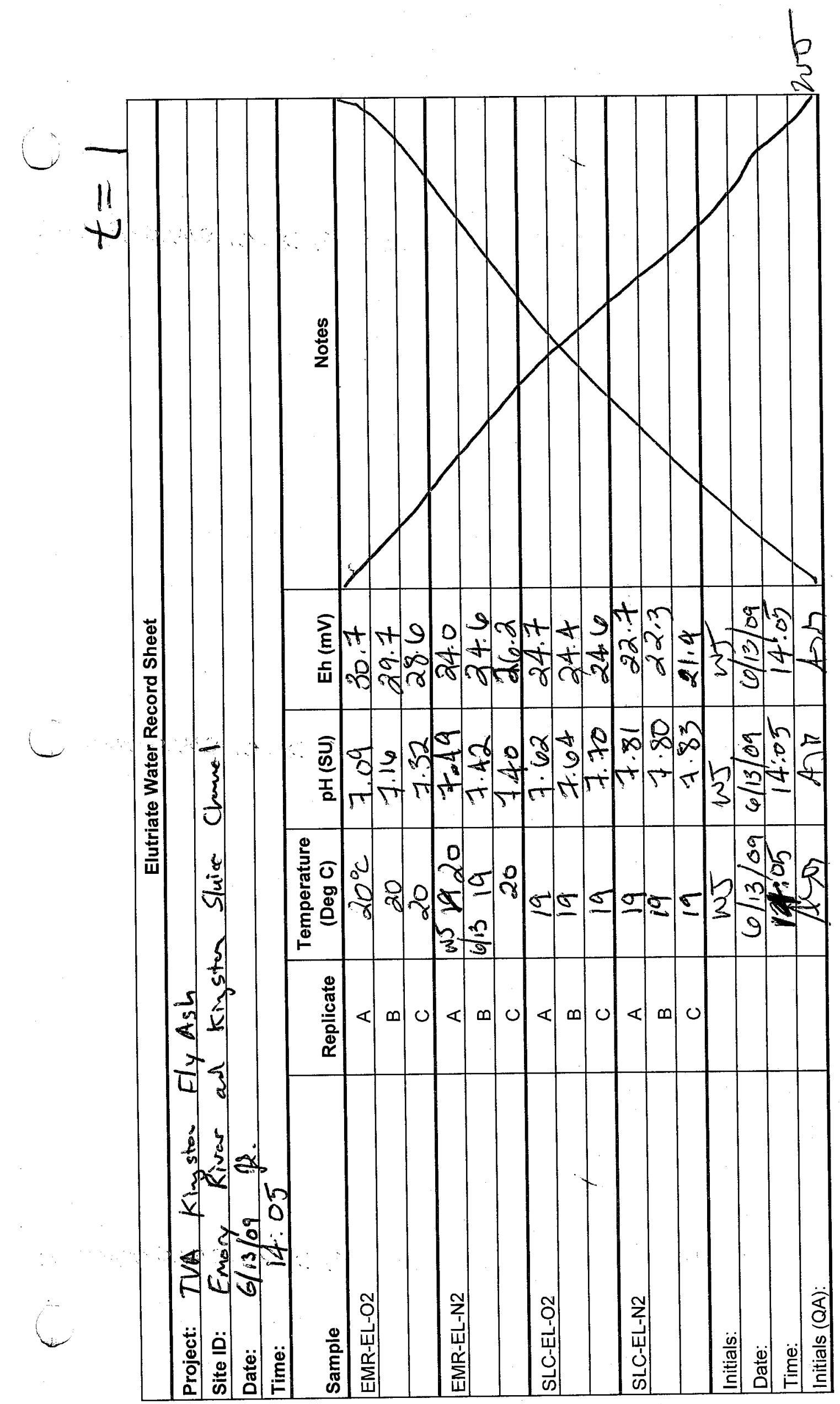




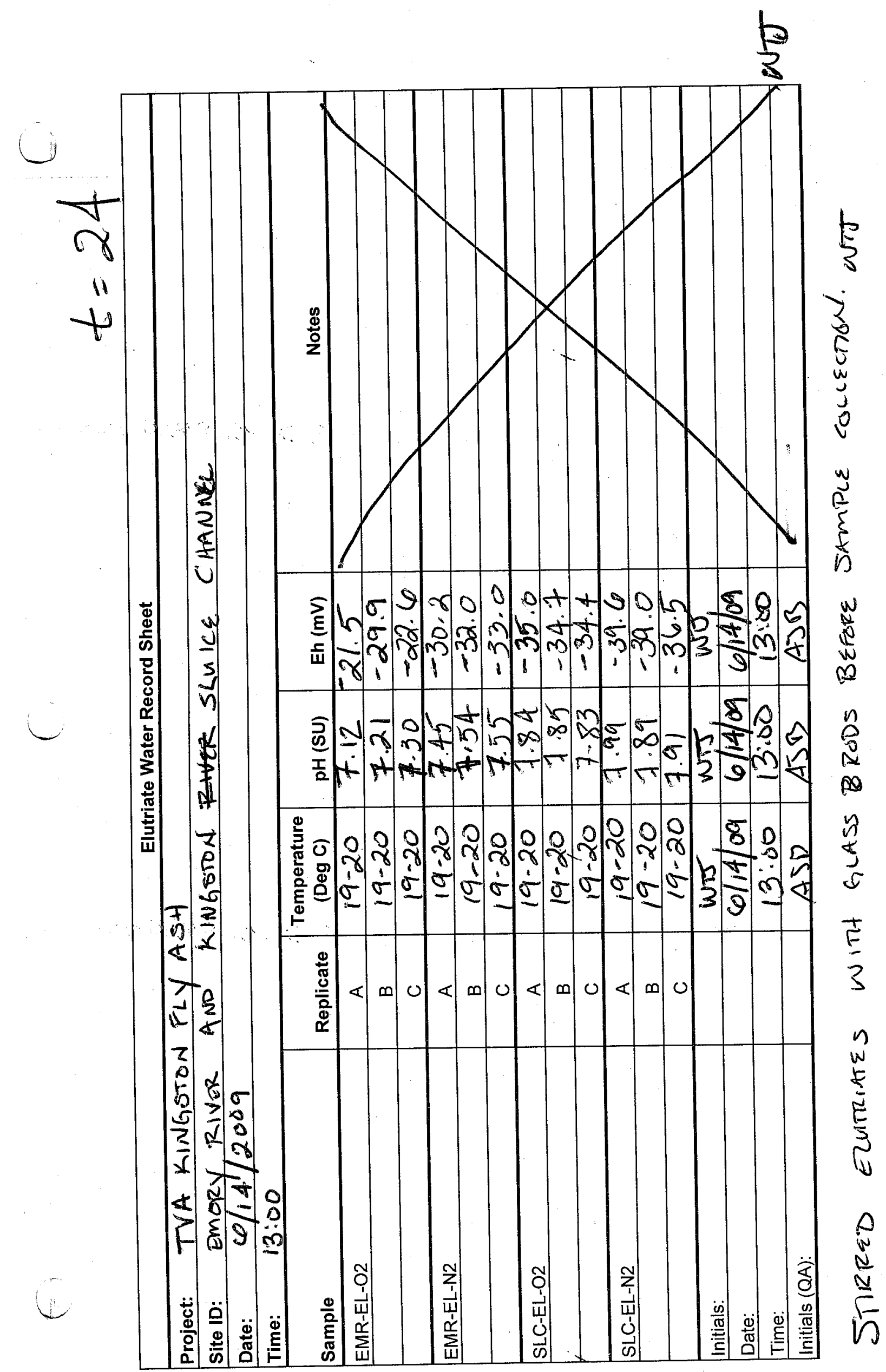




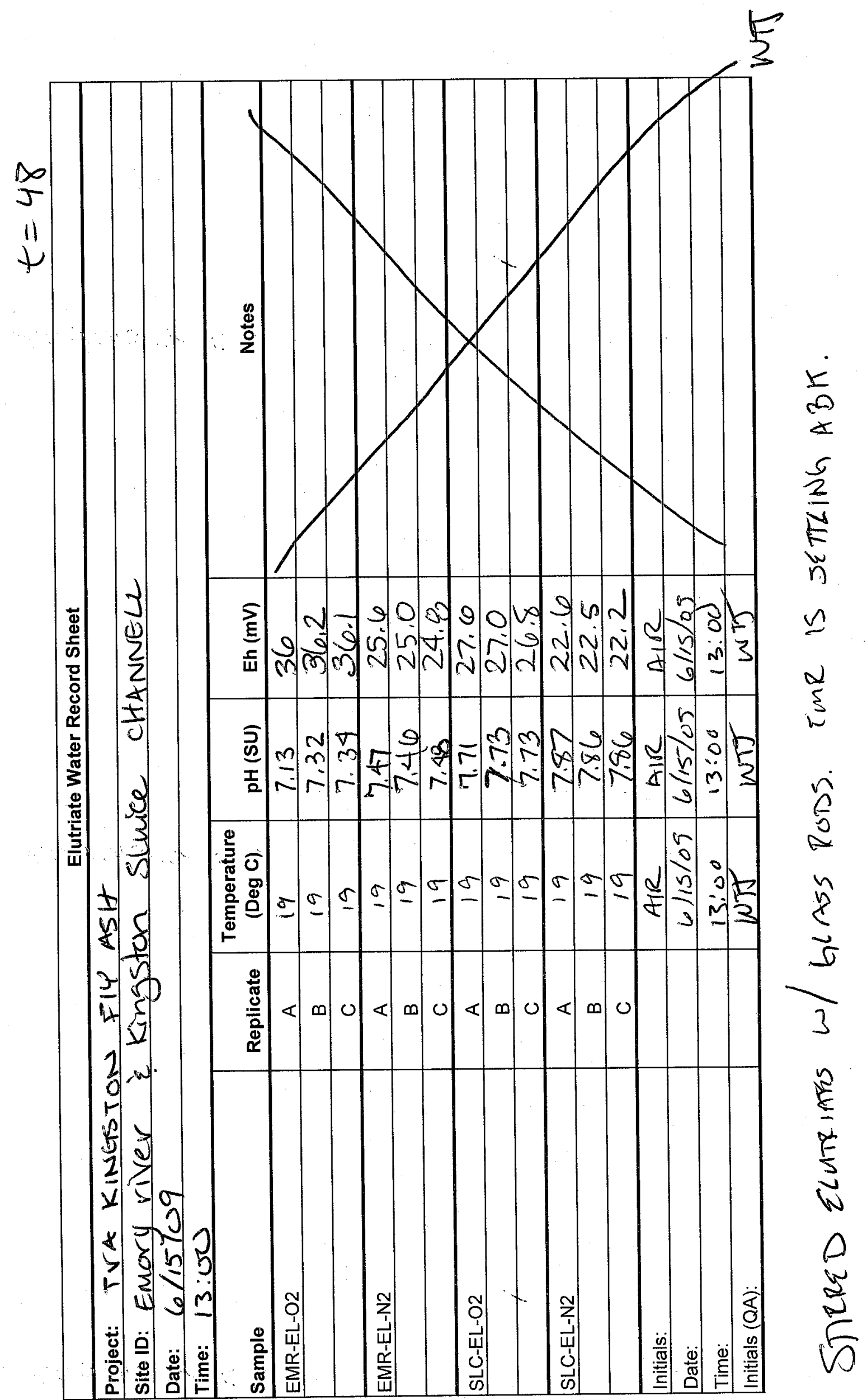




\section{Appendix C: Particle Size Distribution}


Appendix C

Elutriate Particle Size Analysis
TVA Kingston Elutriate Particle Size Distribution Averages

(Analysis by Coulter Counter)

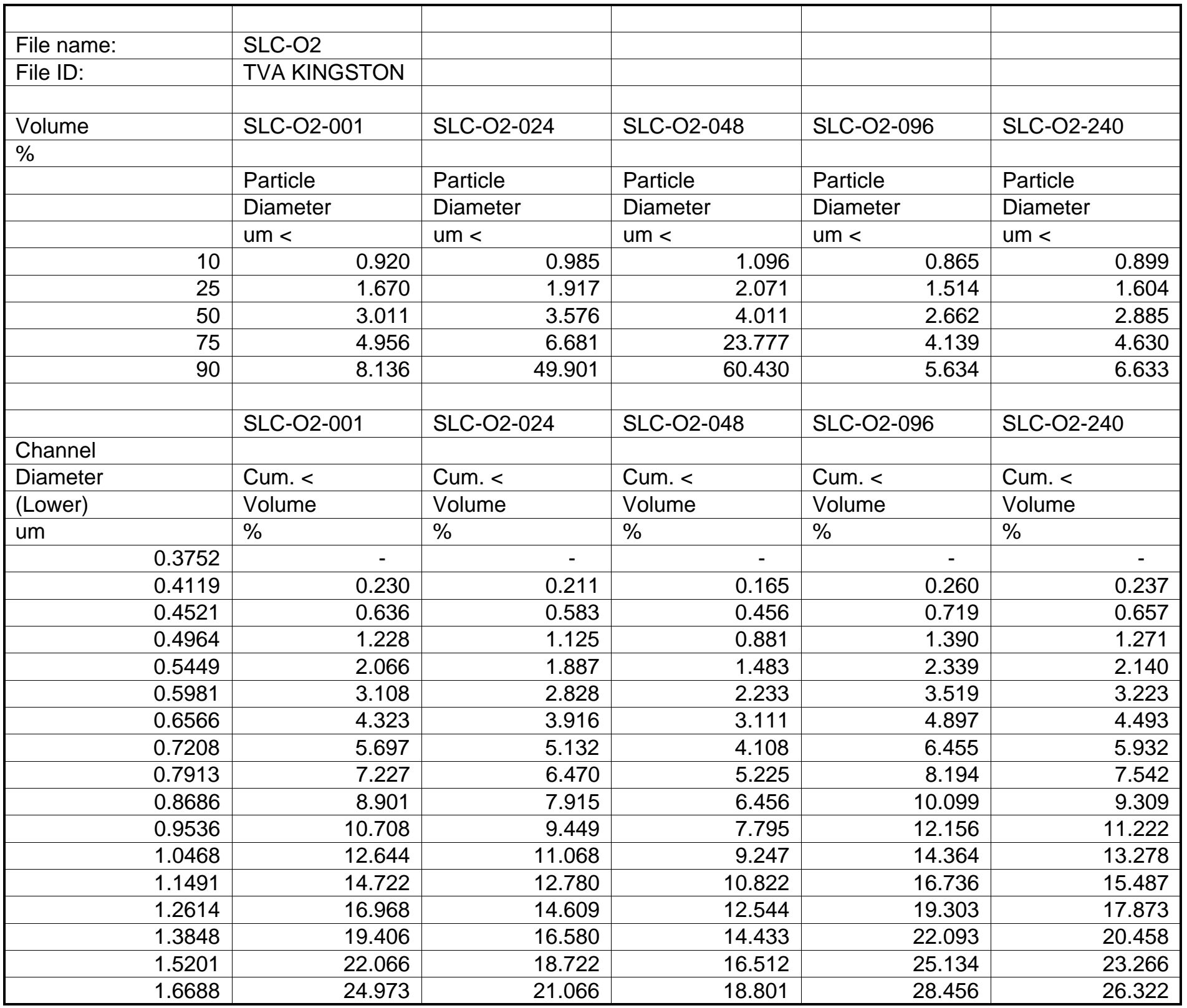


TVA Kingston Elutriate Particle Size Distribution Statistics

Elutriate Particle Size Analysis

(Analysis by Coulter Counter)

\begin{tabular}{|c|c|c|c|c|c|c|c|c|c|}
\hline \\
\hline \multicolumn{10}{|c|}{$\begin{array}{l}\text { Volume statistics (Aritnmetic) } \\
\text { Calculations from } 0.375 \mu \mathrm{m} \text { to } 948 \mu \mathrm{m}\end{array}$} \\
\hline & Mean & Median & $D(p, q)$ & S.D. & Mode & S.S.A. & d10 & d50 & d90 \\
\hline & $\mu \mathrm{m}$ & $\mu \mathrm{m}$ & $\mu \mathrm{m}$ & $\mu \mathrm{m}$ & $\mu \mathrm{m}$ & $\mathrm{cm}^{2} / \mathrm{mL}$ & $\mu \mathrm{m}$ & $\mu \mathrm{m}$ & $\mu \mathrm{m}$ \\
\hline SLC-O2-001.\$av & 5.66 & 3.01 & 0.682 & 9.49 & 3.36 & 28821 & 0.92 & 3.01 & 8.14 \\
\hline SLC-O2-024.\$av & 13.4 & 3.58 & 0.672 & 23.1 & 3.69 & 24985 & 0.985 & 3.58 & 49.9 \\
\hline SLC-O2-048.\$av & 17.1 & 4.01 & 0.691 & 25.1 & 3.36 & 22221 & 1.1 & 4.01 & 60.4 \\
\hline SLC-O2-096.\$av & 2.99 & 2.66 & 0.683 & 1.83 & 3.36 & 32134 & 0.865 & 2.66 & 5.63 \\
\hline SLC-O2-240.\$av & 3.55 & 2.88 & 0.684 & 2.88 & 3.69 & 30178 & 0.899 & 2.88 & 6.63 \\
\hline (Average) & 8.53 & 3.23 & 8 & 2.50 & 3.49 & 7,66 & 0.95 & 3.23 & 26.10 \\
\hline (C.V.) & $74.10 \%$ & $17.10 \%$ & $1.00 \%$ & $88.30 \%$ & $5.20 \%$ & $14.50 \%$ & $9.60 \%$ & $17.10 \%$ & $102.40 \%$ \\
\hline (Maximum) & 17.1 & 4.01 & 0.691 & 25.1 & 3.69 & 32134 & 1.1 & 4.01 & 60.4 \\
\hline (Minimum) & 2.99 & 2.66 & 0.672 & 1.83 & 3.36 & 22221 & 0.865 & 2.66 & 5.63 \\
\hline
\end{tabular}


Appendix C

Elutriate Particle Size Analysis
TVA Kingston Elutriate Particle Size Distribution Statistics

(Analysis by Coulter Counter)

\begin{tabular}{|c|c|c|c|c|c|c|c|c|c|}
\hline \multicolumn{10}{|c|}{ Volume Statistics (Arithmetic) } \\
\hline \multicolumn{10}{|c|}{ Calculations from $0.375 \mu \mathrm{m}$ to $948 \mu \mathrm{m}$} \\
\hline & Mean & Median & $D(p, q)$ & S.D. & Mode & S.S.A. & d10 & d50 & d90 \\
\hline & $\mu \mathrm{m}$ & $\mu \mathrm{m}$ & $\mu \mathrm{m}$ & $\mu \mathrm{m}$ & $\mu \mathrm{m}$ & $\mathrm{cm}^{2} / \mathrm{mL}$ & $\mu \mathrm{m}$ & $\mu \mathrm{m}$ & $\mu \mathrm{m}$ \\
\hline EMR-O2-C & 3.59 & 2.94 & 0.687 & 2.82 & 3.69 & 29480 & 0.926 & 2.94 & 6.7 \\
\hline EMR-O2-C & 3.63 & 2.76 & 0.705 & 3.23 & 3.06 & 30214 & 0.925 & 2.76 & 6.92 \\
\hline EMR-O2-C & 4.1 & 2.93 & 0.699 & 4.04 & 3.36 & 29284 & 0.925 & 2.93 & 8.11 \\
\hline EMR-O2-C & 3.46 & 2.95 & 0.674 & 2.52 & 3.69 & 30037 & 0.893 & 2.95 & 6.45 \\
\hline EMR-O2-¿ & 3.86 & 3.16 & 0.672 & 3.09 & 4.05 & 28902 & 0.904 & 3.16 & 7.31 \\
\hline (Average) & 3.73 & 2.95 & 0.687 & 3.14 & 3.57 & 29584 & 0.915 & 2.95 & 7.1 \\
\hline (C.V.) & $6.70 \%$ & $4.80 \%$ & $2.10 \%$ & $18.10 \%$ & $10.50 \%$ & $1.80 \%$ & $1.70 \%$ & $4.80 \%$ & $9.20 \%$ \\
\hline (Maximum & 4.1 & 3.16 & 0.705 & 4.04 & 4.05 & 30214 & 0.926 & 3.16 & 8.11 \\
\hline (Minimum) & 3.46 & 2.76 & 0.672 & 2.52 & 3.06 & 28902 & 0.893 & 2.76 & 6.45 \\
\hline
\end{tabular}


Appendix C

Elutriate Particle Size Analysis
TVA Kingston Elutriate Particle Size Distribution Statistics

(Analysis by Coulter Counter)

\begin{tabular}{|c|c|c|c|c|c|c|c|c|c|}
\hline \multicolumn{10}{|c|}{ Volume Statistics (Arithmetic) } \\
\hline \multicolumn{10}{|c|}{ Calculations from $0.375 \mu \mathrm{m}$ to $948 \mu \mathrm{m}$} \\
\hline & Mean & Median & $\mathrm{D}(p, q)$ & S.D. & Mode & S.S.A. & d10 & d50 & d90 \\
\hline & $\mu \mathrm{m}$ & $\mu \mathrm{m}$ & $\mu \mathrm{m}$ & $\mu \mathrm{m}$ & $\mu \mathrm{m}$ & $\mathrm{cm}^{2} / \mathrm{mL}$ & $\mu \mathrm{m}$ & $\mu \mathrm{m}$ & $\mu \mathrm{m}$ \\
\hline SLC-N2-0: & 4.31 & 2.91 & 0.685 & 5.96 & 3.36 & 29703 & 0.908 & 2.91 & 7.08 \\
\hline SLC-N2-0: & 4.33 & 2.89 & 0.68 & 6.75 & 3.69 & 30115 & 0.898 & 2.89 & 6.37 \\
\hline SLC-N2-0, & 7.03 & 3.26 & 0.689 & 11.1 & 3.36 & 26644 & 0.983 & 3.26 & 18.6 \\
\hline SLC-N2-O! & 2.98 & 2.66 & 0.681 & 1.81 & 3.36 & 32124 & 0.865 & 2.66 & 5.6 \\
\hline SLC-N2-2، & 3.49 & 2.8 & 0.684 & 3.27 & 3.36 & 30713 & 0.891 & 2.8 & 6.39 \\
\hline (Average) & 4.43 & 2.9 & 0.684 & 5.77 & 3.42 & 29860 & 0.909 & 2.9 & 8.81 \\
\hline (C.V.) & $35.30 \%$ & $7.60 \%$ & $0.50 \%$ & $62.00 \%$ & $4.30 \%$ & $6.80 \%$ & $4.90 \%$ & $7.60 \%$ & $62.50 \%$ \\
\hline (Maximum & 7.03 & 3.26 & 0.689 & 11.1 & 3.69 & 32124 & 0.983 & 3.26 & 18.6 \\
\hline (Minimum) & 2.98 & 2.66 & 0.68 & 1.81 & 3.36 & 26644 & 0.865 & 2.66 & 5.6 \\
\hline
\end{tabular}


TVA Kingston Elutriate Particle Size Distribution Statistics

Elutriate Particle Size Analysis

(Analysis by Coulter Counter)

\begin{tabular}{|c|c|c|c|c|c|c|c|c|c|}
\hline \multicolumn{10}{|c|}{ Volume Statistics (Arithmetic) } \\
\hline \multicolumn{10}{|c|}{ Calculations from $0.375 \mu \mathrm{m}$ to $948 \mu \mathrm{m}$} \\
\hline & Mean & Median & $D(p, q)$ & S.D. & Mode & S.S.A. & d10 & d50 & d90 \\
\hline & $\mu \mathrm{m}$ & $\mu \mathrm{m}$ & $\mu \mathrm{m}$ & $\mu \mathrm{m}$ & $\mu \mathrm{m}$ & $\mathrm{cm}^{2} / \mathrm{mL}$ & $\mu \mathrm{m}$ & $\mu \mathrm{m}$ & $\mu \mathrm{m}$ \\
\hline EMR-N2-001.\$av & 3.87 & 3.03 & 0.681 & 3.34 & 3.69 & 29080 & 0.919 & 3.03 & 7.36 \\
\hline EMR-N2-024.\$av & 3.43 & 2.77 & 0.692 & 2.68 & 3.36 & 30634 & 0.903 & 2.77 & 6.51 \\
\hline EMR-N2-048.\$av & 3.53 & 2.73 & 0.697 & 3 & 3.06 & 30738 & 0.902 & 2.73 & 6.74 \\
\hline EMR-N2-096.\$av & 3.56 & 3.03 & 0.668 & 2.63 & 4.05 & 29881 & 0.88 & 3.03 & 6.66 \\
\hline EMR-N2-240.\$av & 4.22 & 3.23 & 0.676 & 3.83 & 4.05 & 28239 & 0.92 & 3.23 & 8.22 \\
\hline (Average) & 3.72 & 2.96 & 0.683 & 3.1 & 3.64 & 29714 & 0.905 & 2.96 & 7.1 \\
\hline (C.V.) & $8.70 \%$ & $7.00 \%$ & $1.70 \%$ & $16.20 \%$ & $11.90 \%$ & $3.60 \%$ & $1.80 \%$ & $7.00 \%$ & $10.00 \%$ \\
\hline (Maximum) & 4.22 & 3.23 & 0.697 & 3.83 & 4.05 & 30738 & 0.92 & 3.23 & 8.22 \\
\hline (Minimum) & 3.43 & 2.73 & 0.668 & 2.63 & 3.06 & 28239 & 0.88 & 2.73 & 6.51 \\
\hline
\end{tabular}


Appendix C

Elutriate Particle Size Analysis
TVA Kingston Elutriate Particle Size Distribution Averages

(Analysis by Coulter Counter)

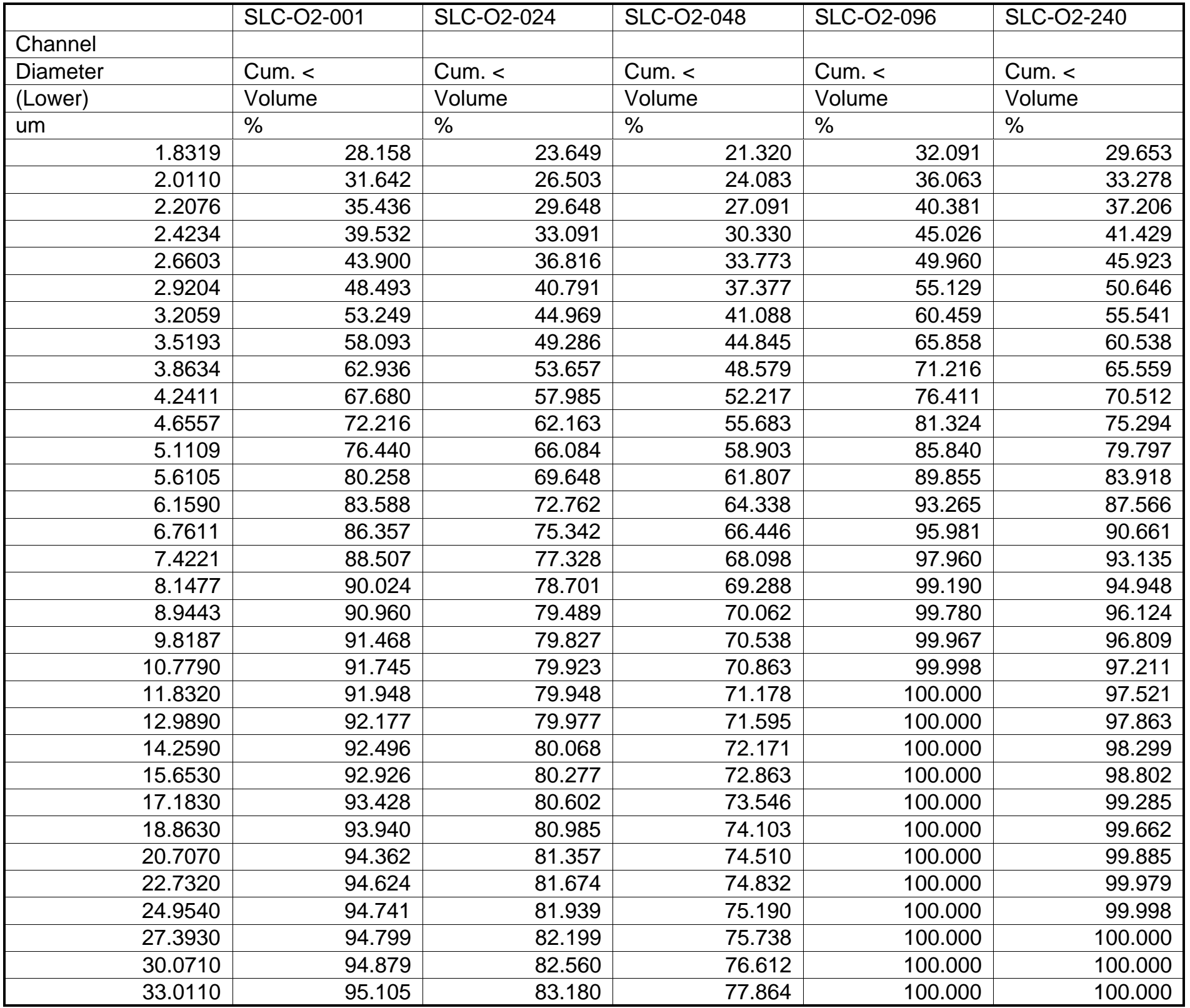


Appendix C

Elutriate Particle Size Analysis
TVA Kingston Elutriate Particle Size Distribution Averages

(Analysis by Coulter Counter)

\begin{tabular}{|c|c|c|c|c|c|}
\hline & SLC-O2-001 & SLC-O2-024 & SLC-O2-048 & SLC-O2-096 & SLC-O2-240 \\
\hline \multicolumn{6}{|l|}{ Channel } \\
\hline (Lower) & Volume & Volume & Volume & Volume & Volume \\
\hline 36.2390 & 95.639 & 84.213 & 79.445 & 100.000 & 100.000 \\
\hline 39.7810 & 96.524 & 85.679 & 81.241 & 100.000 & 100.000 \\
\hline 52.6260 & 99.466 & 91.023 & 87.010 & 100.000 & 100.000 \\
\hline 57.7710 & 99.887 & 92.617 & 89.013 & 100.000 & 100.000 \\
\hline 63.4190 & 99.989 & 94.016 & 91.109 & 100.000 & 100.000 \\
\hline 69.6190 & 100.000 & 95.291 & 93.277 & 100.000 & 100.000 \\
\hline 76.4250 & 100.000 & 96.458 & 95.404 & 100.000 & 100.000 \\
\hline 121.8400 & 100.000 & 99.864 & 99.989 & 100.000 & 100.000 \\
\hline 133.7500 & 100.000 & 99.971 & 100.000 & 100.000 & 100.000 \\
\hline 146.8200 & 100.000 & 99.997 & 100.000 & 100.000 & 100.000 \\
\hline 161.1800 & 100.000 & 100.000 & 100.000 & 100.000 & 100.000 \\
\hline 176.9300 & 100.000 & 100.000 & 100.000 & 100.000 & 100.000 \\
\hline 194.2300 & 100.000 & 100.000 & 100.000 & 100.000 & 100.000 \\
\hline 213.2200 & 100.000 & 100.000 & 100.000 & 100.000 & 100.000 \\
\hline 234.0700 & 100.000 & 100.000 & 100.000 & 100.000 & 100.000 \\
\hline 256.9500 & 100.000 & 100.000 & 100.000 & 100.000 & 100.000 \\
\hline 282.0700 & 100.000 & 100.000 & 100.000 & 100.000 & 100.000 \\
\hline 594.8700 & 100.000 & 100.000 & 100.000 & 100.000 & 100.000 \\
\hline 653.0200 & 100.000 & 100.000 & 100.000 & 100.000 & 100.000 \\
\hline
\end{tabular}


TVA Kingston Elutriate Particle Size Distribution Averages

Elutriate Particle Size Analysis

(Analysis by Coulter Counter)

\begin{tabular}{|c|c|c|c|c|c|}
\hline \multirow{2}{*}{\multicolumn{6}{|c|}{ SLC-O2-240 }} \\
\hline & & & & & \\
\hline Diameter & Cum. $<$ & Cum. $<$ & Cum. $<$ & Cum. $<$ & Cum. $<$ \\
\hline (Lower) & Volume & Volume & Volume & Volume & Volume \\
\hline um & $\%$ & $\%$ & $\%$ & $\%$ & $\%$ \\
\hline 716.8700 & 100.000 & 100.000 & 100.000 & 100.000 & 100.000 \\
\hline 786.9500 & 100.000 & 100.000 & 100.000 & 100.000 & 100.000 \\
\hline 863.8800 & 100.000 & 100.000 & 100.000 & 100.000 & 100.000 \\
\hline 948.3400 & 100.000 & 100.000 & 100.000 & 100.000 & 100.000 \\
\hline
\end{tabular}


Appendix C

Elutriate Particle Size Analysis
TVA Kingston Elutriate Particle Size Distribution Averages

(Analysis by Coulter Counter)

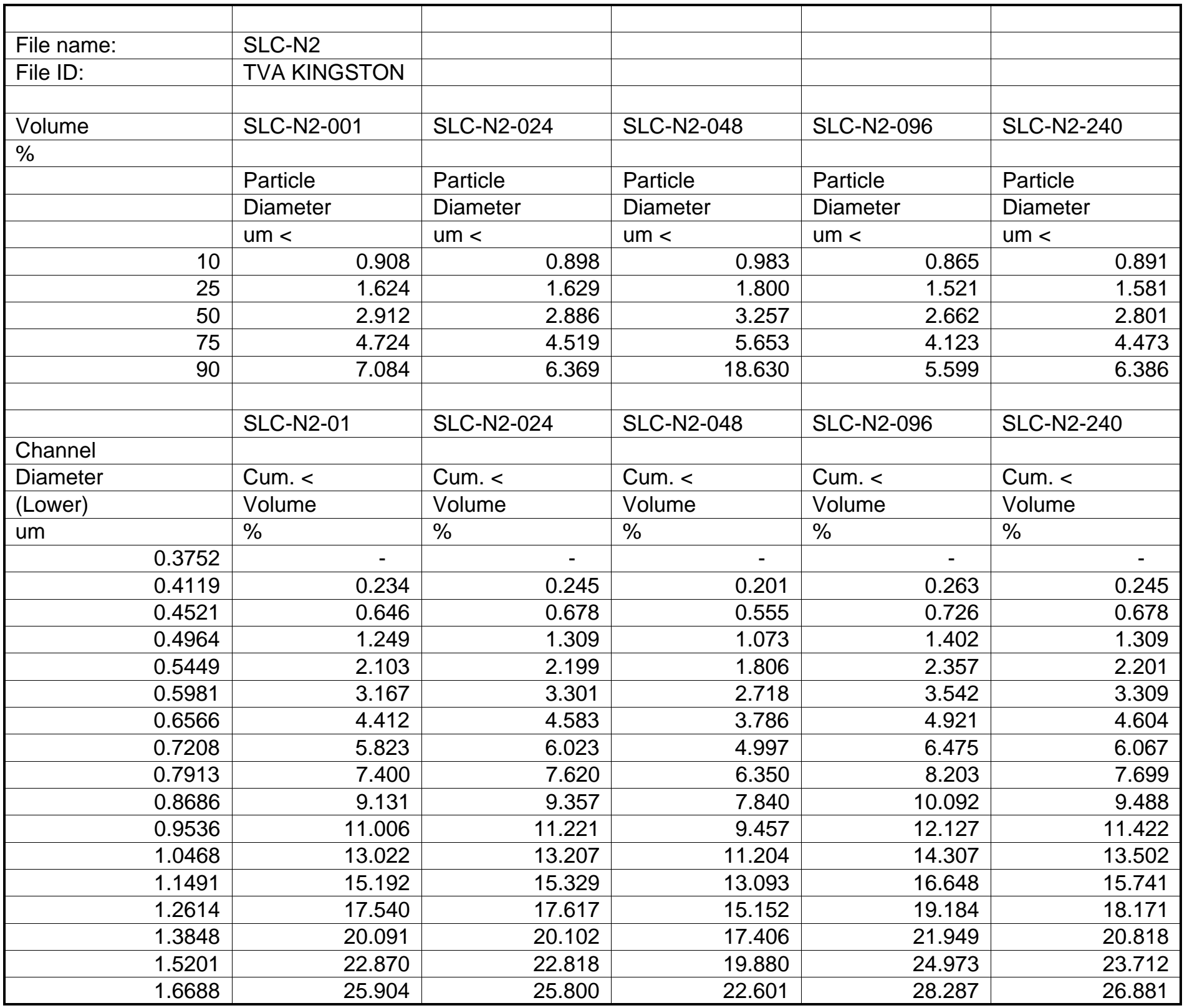


Appendix C

Elutriate Particle Size Analysis
TVA Kingston Elutriate Particle Size Distribution Averages

(Analysis by Coulter Counter)

\begin{tabular}{|c|c|c|c|c|c|}
\hline & SLC-N2-01 & SLC-N2-024 & SLC-N2-048 & SLC-N2-096 & SLC-N2-240 \\
\hline \multicolumn{6}{|l|}{ Channel } \\
\hline (Lower) & Volume & Volume & Volume & Volume & Volume \\
\hline 1.8319 & 29.219 & 29.087 & 25.594 & 31.928 & 30.351 \\
\hline 2.0110 & 32.832 & 32.712 & 28.878 & 35.921 & 34.142 \\
\hline 2.6603 & 45.454 & 45.702 & 40.445 & 49.957 & 47.362 \\
\hline 2.9204 & 50.156 & 50.654 & 44.775 & 55.182 & 52.256 \\
\hline 3.2059 & 55.012 & 55.821 & 49.255 & 60.564 & 57.287 \\
\hline 3.5193 & 59.950 & 61.119 & 53.812 & 66.012 & 62.375 \\
\hline 3.8634 & 64.885 & 66.440 & 58.367 & 71.410 & 67.433 \\
\hline 6.1590 & 86.020 & 88.992 & 77.963 & 93.495 & 88.901 \\
\hline 6.7611 & 88.897 & 91.888 & 80.674 & 96.190 & 91.816 \\
\hline 7.4221 & 91.157 & 94.050 & 82.840 & 98.125 & 94.102 \\
\hline 8.1477 & 92.781 & 95.465 & 84.449 & 99.290 & 95.733 \\
\hline 8.9443 & 93.815 & 96.185 & 85.549 & 99.822 & 96.763 \\
\hline 9.8187 & 94.434 & 96.437 & 86.260 & 99.976 & 97.347 \\
\hline 10.7790 & 94.827 & 96.482 & 86.740 & 99.999 & 97.683 \\
\hline 11.8320 & 95.167 & 96.485 & 87.133 & 100.000 & 97.937 \\
\hline 12.9890 & 95.546 & 96.485 & 87.551 & 100.000 & 98.202 \\
\hline 14.2590 & 96.001 & 96.485 & 88.060 & 100.000 & 98.520 \\
\hline 30.0710 & 97.882 & 96.706 & 93.103 & 100.000 & 99.720 \\
\hline 33.0110 & 98.091 & 97.128 & 93.738 & 100.000 & 99.751 \\
\hline
\end{tabular}


Appendix C

Elutriate Particle Size Analysis
TVA Kingston Elutriate Particle Size Distribution Averages (Analysis by Coulter Counter)

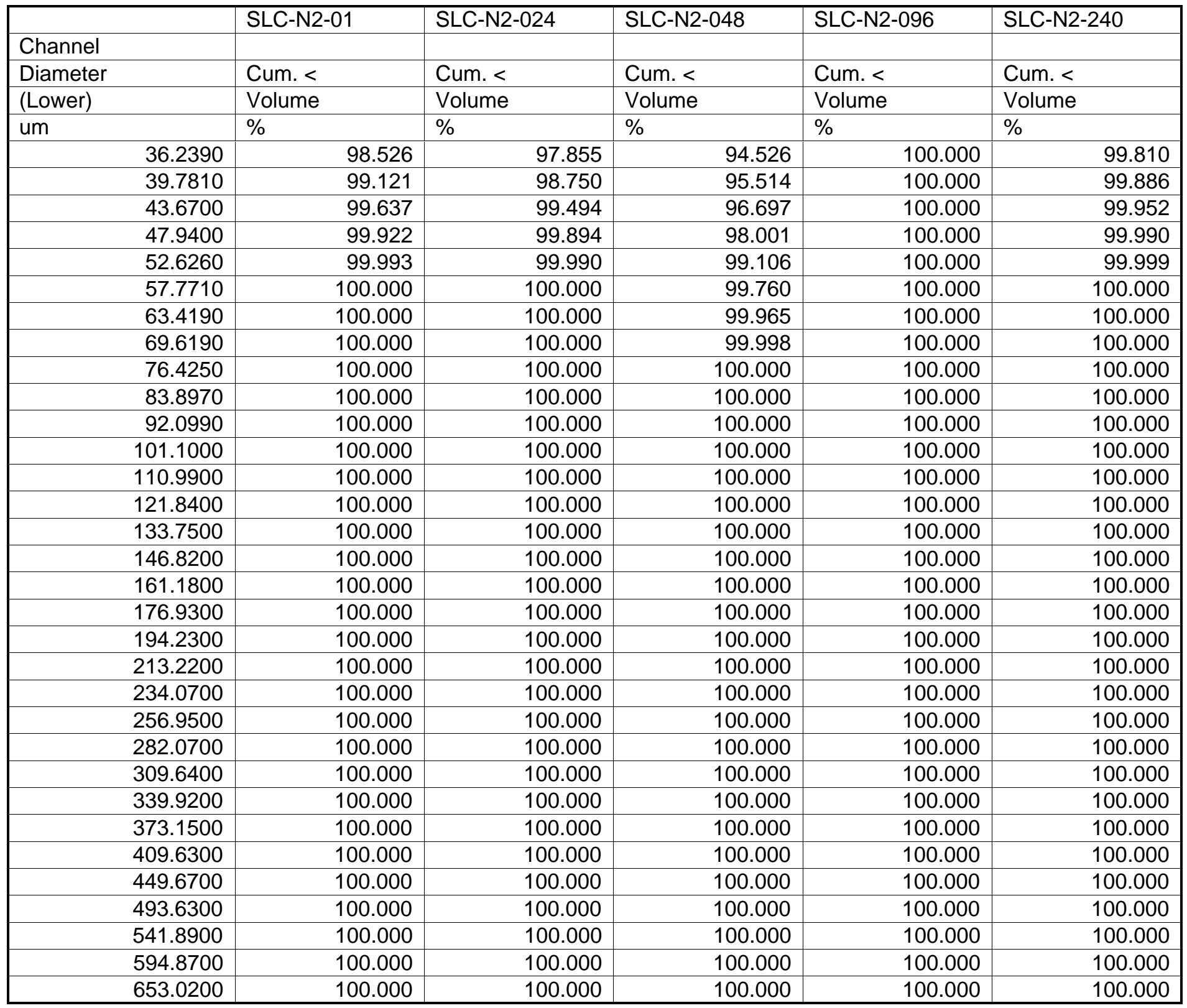


TVA Kingston Elutriate Particle Size Distribution Averages

Elutriate Particle Size Analysis

(Analysis by Coulter Counter)

\begin{tabular}{|c|c|c|c|c|c|}
\hline \multirow{2}{*}{\multicolumn{6}{|c|}{ SLC-N2-096 }} \\
\hline & & & & & \\
\hline Diameter & Cum. $<$ & Cum. $<$ & Cum. $<$ & Cum. $<$ & Cum. $<$ \\
\hline (Lower) & Volume & Volume & Volume & Volume & Volume \\
\hline um & $\%$ & $\%$ & $\%$ & $\%$ & $\%$ \\
\hline 716.8700 & 100.000 & 100.000 & 100.000 & 100.000 & 100.000 \\
\hline 786.9500 & 100.000 & 100.000 & 100.000 & 100.000 & 100.000 \\
\hline 863.8800 & 100.000 & 100.000 & 100.000 & 100.000 & 100.000 \\
\hline 948.3400 & 100.000 & 100.000 & 100.000 & 100.000 & 100.000 \\
\hline
\end{tabular}


Appendix C

Elutriate Particle Size Analysis
TVA Kingston Elutriate Particle Size Distribution Averages

(Analysis by Coulter Counter)

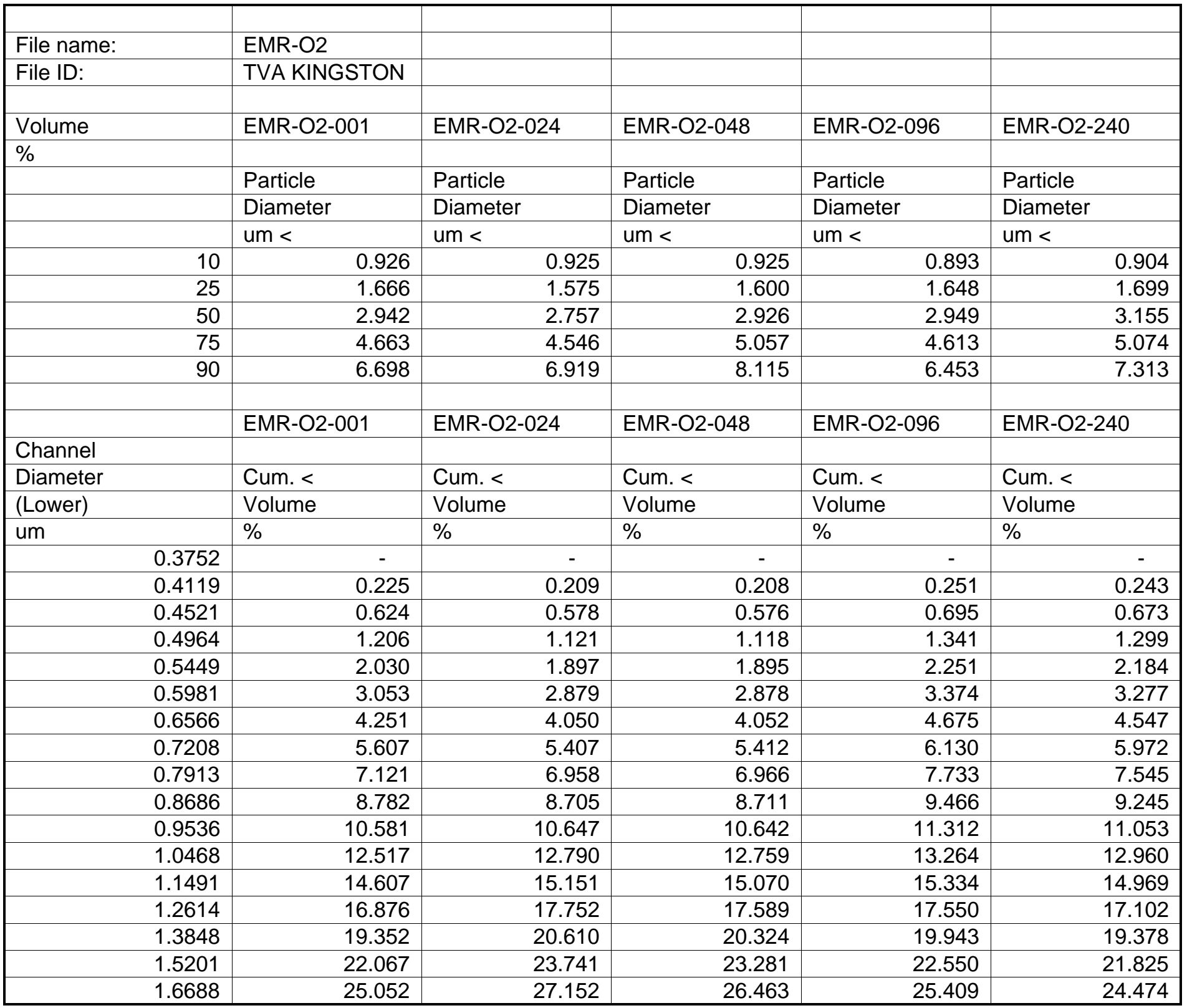


Appendix C

Elutriate Particle Size Analysis
TVA Kingston Elutriate Particle Size Distribution Averages

(Analysis by Coulter Counter)

\begin{tabular}{|c|c|c|c|c|c|}
\hline & EMR-O2-001 & EMR-O2-024 & EMR-O2-048 & EMR-O2-096 & EMR-O2-240 \\
\hline \multicolumn{6}{|l|}{ Channel } \\
\hline (Lower) & Volume & Volume & Volume & Volume & Volume \\
\hline 1.8319 & 28.339 & 30.852 & 29.875 & 28.561 & 27.362 \\
\hline 2.0110 & 31.951 & 34.835 & 33.512 & 32.044 & 30.521 \\
\hline 2.6603 & 44.766 & 48.217 & 45.595 & 44.633 & 41.824 \\
\hline 2.9204 & 49.611 & 52.994 & 49.908 & 49.484 & 46.195 \\
\hline 3.2059 & 54.647 & 57.820 & 54.293 & 54.577 & 50.821 \\
\hline 3.5193 & 59.794 & 62.622 & 58.702 & 59.835 & 55.650 \\
\hline 3.8634 & 64.961 & 67.325 & 63.081 & 65.162 & 60.615 \\
\hline 6.1590 & 87.264 & 86.826 & 82.505 & 88.450 & 83.953 \\
\hline 6.7611 & 90.322 & 89.488 & 85.473 & 91.628 & 87.521 \\
\hline 7.4221 & 92.750 & 91.634 & 88.011 & 94.102 & 90.490 \\
\hline 8.1477 & 94.521 & 93.259 & 90.094 & 95.842 & 92.793 \\
\hline 8.9443 & 95.688 & 94.405 & 91.727 & 96.929 & 94.421 \\
\hline 9.8187 & 96.412 & 95.182 & 92.960 & 97.562 & 95.486 \\
\hline 10.7790 & 96.905 & 95.746 & 93.894 & 97.975 & 96.190 \\
\hline 11.8320 & 97.352 & 96.248 & 94.656 & 98.339 & 96.752 \\
\hline 12.9890 & 97.871 & 96.806 & 95.369 & 98.744 & 97.334 \\
\hline 14.2590 & 98.485 & 97.471 & 96.122 & 99.190 & 98.004 \\
\hline 30.0710 & 100.000 & 100.000 & 99.842 & 100.000 & 100.000 \\
\hline 33.0110 & 100.000 & 100.000 & 99.876 & 100.000 & 100.000 \\
\hline
\end{tabular}


Appendix C

Elutriate Particle Size Analysis
TVA Kingston Elutriate Particle Size Distribution Averages

(Analysis by Coulter Counter)

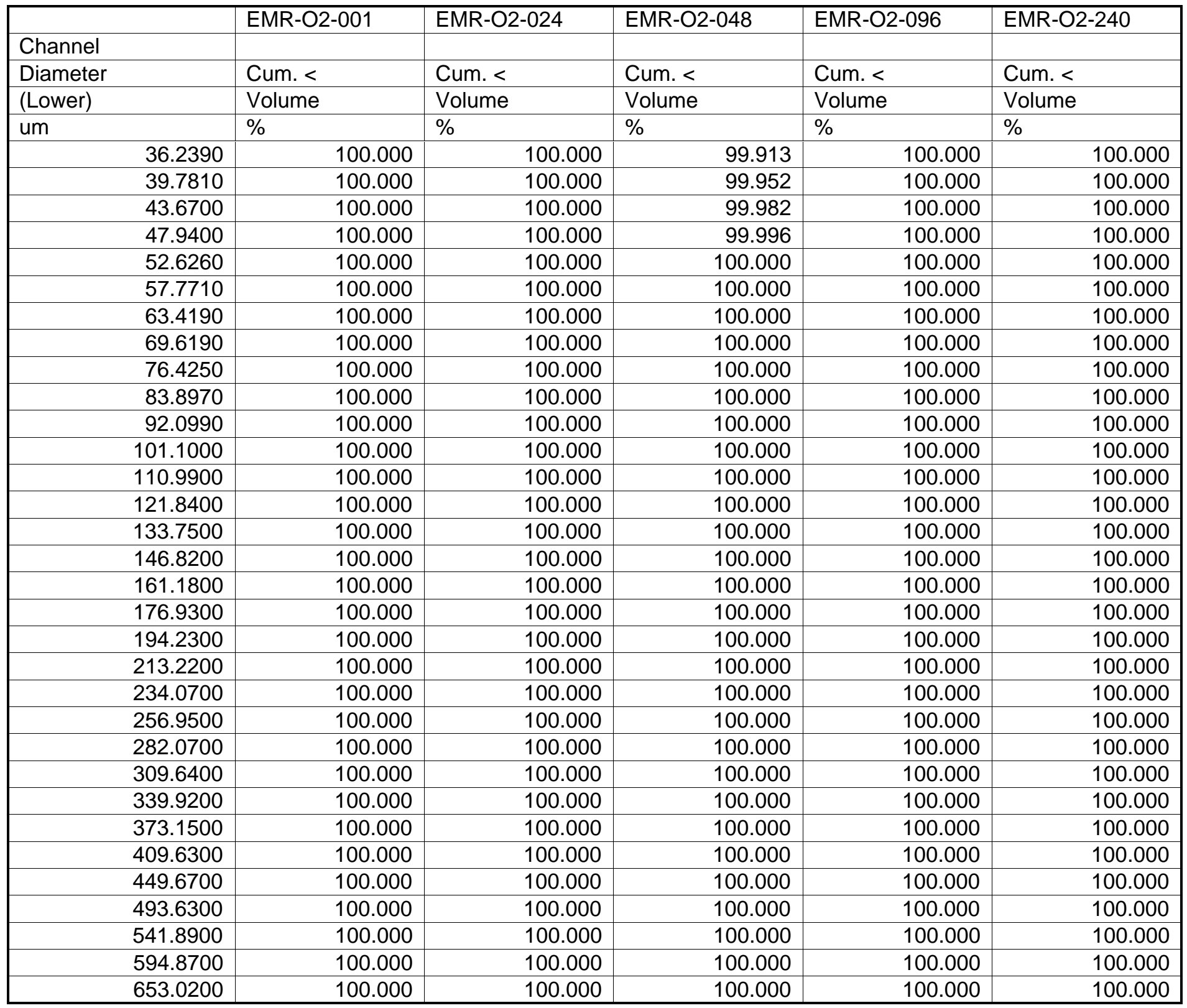


(Analysis by Coulter Counter)

\begin{tabular}{|c|c|c|c|c|c|}
\hline \multirow{2}{*}{\multicolumn{6}{|c|}{ EMR-02-096 }} \\
\hline & & & & & \\
\hline Diameter & Cum. $<$ & Cum. $<$ & Cum. $<$ & Cum. $<$ & Cum. $<$ \\
\hline (Lower) & Volume & Volume & Volume & Volume & Volume \\
\hline um & $\%$ & $\%$ & $\%$ & $\%$ & $\%$ \\
\hline 716.8700 & 100.000 & 100.000 & 100.000 & 100.000 & 100.000 \\
\hline 786.9500 & 100.000 & 100.000 & 100.000 & 100.000 & 100.000 \\
\hline 863.8800 & 100.000 & 100.000 & 100.000 & 100.000 & 100.000 \\
\hline 948.3400 & 100.000 & 100.000 & 100.000 & 100.000 & 100.000 \\
\hline
\end{tabular}


Appendix C

Elutriate Particle Size Analysis
TVA Kingston Elutriate Particle Size Distribution Averages

(Analysis by Coulter Counter)

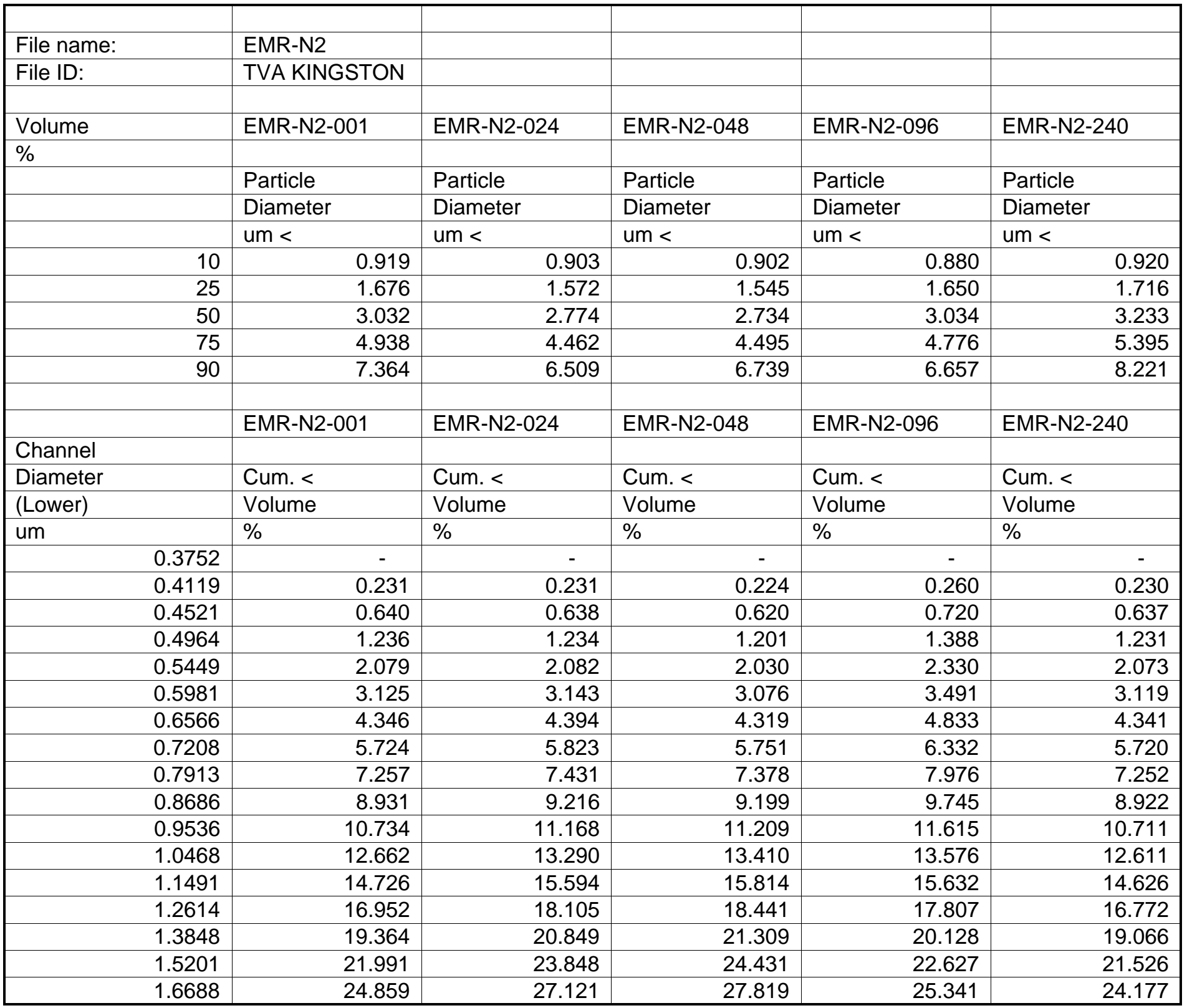


Appendix C

Elutriate Particle Size Analysis
TVA Kingston Elutriate Particle Size Distribution Averages

(Analysis by Coulter Counter)

\begin{tabular}{|c|c|c|c|c|c|}
\hline & EMR-N2-001 & EMR-N2-024 & EMR-N2-048 & EMR-N2-096 & EMR-N2-240 \\
\hline \multicolumn{6}{|l|}{ Channel } \\
\hline (Lower) & Volume & Volume & Volume & Volume & Volume \\
\hline 1.8319 & 27.999 & 30.687 & 31.482 & 28.313 & 27.048 \\
\hline 2.0110 & 31.436 & 34.555 & 35.417 & 31.582 & 30.163 \\
\hline 2.6603 & 43.566 & 47.878 & 48.660 & 43.429 & 41.089 \\
\hline 2.9204 & 48.137 & 52.753 & 53.412 & 48.045 & 45.241 \\
\hline 3.2059 & 52.894 & 57.740 & 58.228 & 52.937 & 49.605 \\
\hline 3.5193 & 57.768 & 62.763 & 63.038 & 58.043 & 54.136 \\
\hline 3.8634 & 62.680 & 67.737 & 67.771 & 63.282 & 58.775 \\
\hline 6.1590 & 84.457 & 88.413 & 87.449 & 87.166 & 80.764 \\
\hline 6.7611 & 87.626 & 91.143 & 90.099 & 90.590 & 84.282 \\
\hline 7.4221 & 90.228 & 93.259 & 92.204 & 93.308 & 87.317 \\
\hline 8.1477 & 92.237 & 94.763 & 93.747 & 95.261 & 89.819 \\
\hline 8.9443 & 93.704 & 95.768 & 94.791 & 96.508 & 91.778 \\
\hline 9.8187 & 94.759 & 96.471 & 95.487 & 97.240 & 93.254 \\
\hline 10.7790 & 95.561 & 97.068 & 96.033 & 97.706 & 94.368 \\
\hline 11.8320 & 96.243 & 97.691 & 96.602 & 98.103 & 95.261 \\
\hline 12.9890 & 96.905 & 98.378 & 97.297 & 98.540 & 96.056 \\
\hline 14.2590 & 97.581 & 99.052 & 98.104 & 99.031 & 96.826 \\
\hline 30.0710 & 99.999 & 100.000 & 100.000 & 100.000 & 99.903 \\
\hline 33.0110 & 100.000 & 100.000 & 100.000 & 100.000 & 99.948 \\
\hline
\end{tabular}


Appendix C

Elutriate Particle Size Analysis
TVA Kingston Elutriate Particle Size Distribution Averages

(Analysis by Coulter Counter)

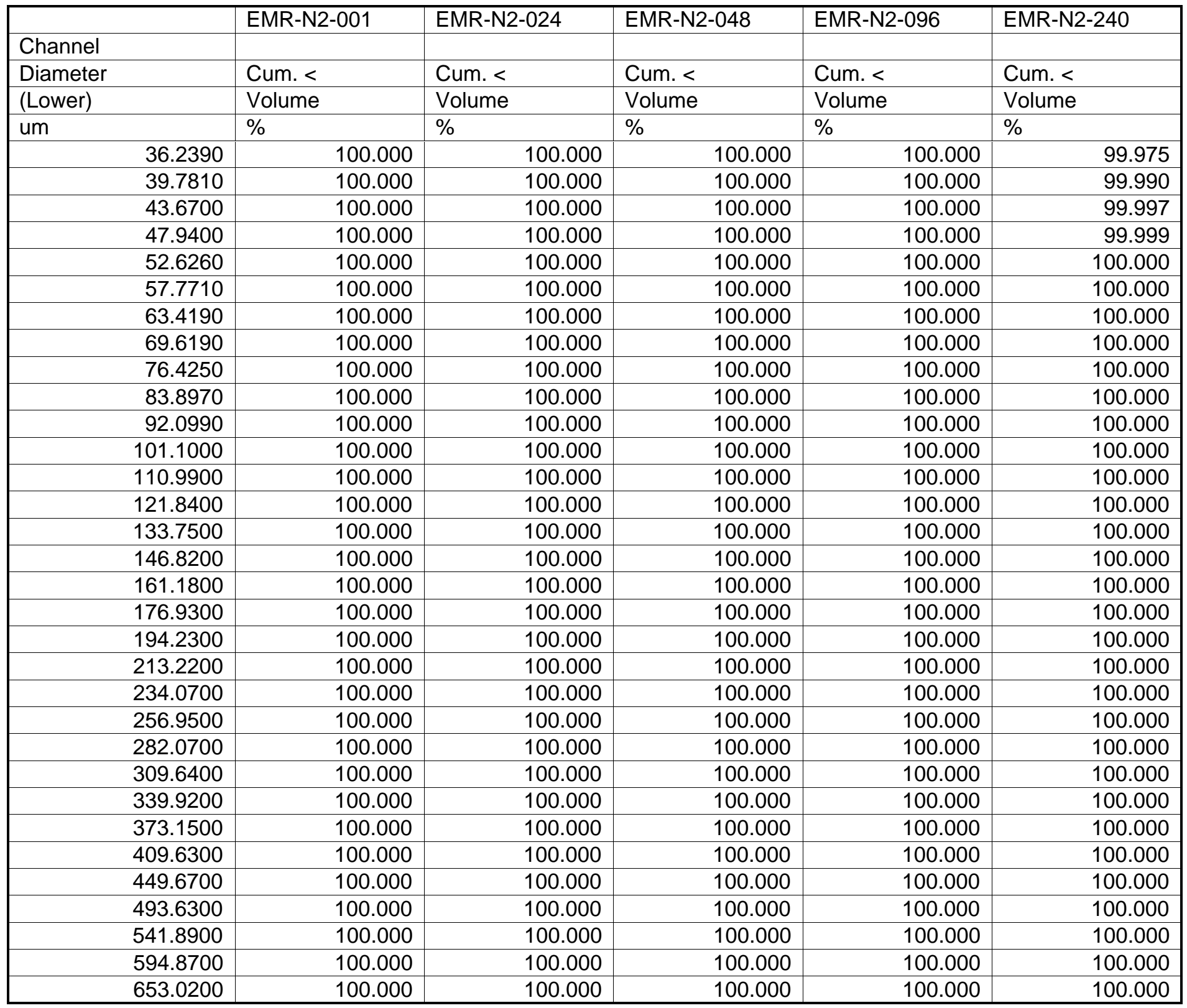




\begin{tabular}{|c|c|c|c|c|c|}
\hline \multirow{2}{*}{\multicolumn{6}{|c|}{ EMR-N2-096 }} \\
\hline & & & & & \\
\hline Diameter & Cum. $<$ & Cum. $<$ & Cum. $<$ & Cum. $<$ & Cum. $<$ \\
\hline (Lower) & Volume & Volume & Volume & Volume & Volume \\
\hline um & $\%$ & $\%$ & $\%$ & $\%$ & $\%$ \\
\hline 716.8700 & 100.000 & 100.000 & 100.000 & 100.000 & 100.000 \\
\hline 786.9500 & 100.000 & 100.000 & 100.000 & 100.000 & 100.000 \\
\hline 863.8800 & 100.000 & 100.000 & 100.000 & 100.000 & 100.000 \\
\hline 948.3400 & 100.000 & 100.000 & 100.000 & 100.000 & 100.000 \\
\hline
\end{tabular}




\section{Appendix D: Chemistry Data Reports}


Appendix D. Chemistry data reports

\section{USACE ERDC-EP-C \\ 3909 Halls Ferry Road \\ Vicksburg, MS 39180-6199}

03 September 2009

Tony Bednar

ERDC - ECB

$-,-$

RE: TVA Fly Ash Project

Enclosed are the results of analyses for samples received by the laboratory on 15-Jun-2009-27-Aug-2009. The samples associated with this report will be held for 90 days from the date of this report. The raw data associated with this report will be held for 5 years from the date of this report. If you need us to hold onto the samples or the data longer then these specified times, you will need to notify us in writing at least 30 days before the expiration dates. If you have any questions concerning this report, please feel free to contact me.

Sincerely,

DRAFT REPORT

DATA SUBJECT TO CHANGE 


\section{USACE ERDC-EP-C \\ 3909 Halls Ferry Road \\ Vicksburg, MS 39180-6199}

ERDC - ECB

Project: TVA Fly Ash Project

Reported:

$--$,

Project Manager: Tony Bednar

03-Sep-2009

WORK ORDER SUMMARY

\begin{tabular}{|c|c|c|c|c|}
\hline Sample ID & Laboratory ID & Matrix & Date Sampled & Date of Work Order \\
\hline DRAFT: EMR Sediment & $9061502-01$ & Soil/Sediment & 12-Jun-2009 & 15-Jun-2009 \\
\hline DRAFT: SLC Sediment & $9061502-02$ & Soil/Sediment & 12-Jun-2009 & 15-Jun-2009 \\
\hline DRAFT: RF1-SD-MT-A Sediment & $9061502-03$ & Soil/Sediment & 12-Jun-2009 & 15-Jun-2009 \\
\hline DRAFT: RF2-SD-MT-A Sediment & $9061502-04$ & Soil/Sediment & 12-Jun-2009 & 15-Jun-2009 \\
\hline DRAFT: RF3-SD-MT-A Sediment & $9061502-05$ & Soil/Sediment & 12-Jun-2009 & 15-Jun-2009 \\
\hline DRAFT: RF4-SD-MT-A Sediment & $9061502-06$ & Soil/Sediment & 12-Jun-2009 & 15-Jun-2009 \\
\hline DRAFT: RF5-SD-MT-A Sediment & $9061502-07$ & Soil/Sediment & 12-Jun-2009 & 15-Jun-2009 \\
\hline DRAFT: RF6-SD-MT-A Sediment & $9061502-08$ & Soil/Sediment & 12-Jun-2009 & 15-Jun-2009 \\
\hline DRAFT: PIL-FA-MT-A & $9061502-09$ & Soil/Sediment & 12-Jun-2009 & 15-Jun-2009 \\
\hline DRAFT: EFL - WA - MT - A (Effluent from Settling & $9061502-10$ & Water & 12-Jun-2009 & 15-Jun-2009 \\
\hline DRAFT: Emory River Water & $9061502-11$ & Water & 12-Jun-2009 & 15-Jun-2009 \\
\hline DRAFT: SLC Water & $9061502-12$ & Water & 12-Jun-2009 & 15-Jun-2009 \\
\hline DRAFT: EMR MT N2 Hour 1 Rep D & $9061502-16$ & Water & 12-Jun-2009 & 15-Jun-2009 \\
\hline DRAFT: EMR MT N2 Hour 1 Rep E & $9061502-17$ & Water & 12-Jun-2009 & 15-Jun-2009 \\
\hline DRAFT: EMR MT N2 Hour 1 Rep F & $9061502-18$ & Water & 12-Jun-2009 & 15-Jun-2009 \\
\hline DRAFT: EMR MT O2 Hour 1 Rep D & $9061502-22$ & Water & 12-Jun-2009 & 15-Jun-2009 \\
\hline DRAFT: EMR MT O2 Hour 1 Rep E & $9061502-23$ & Water & 12-Jun-2009 & 15-Jun-2009 \\
\hline DRAFT: EMR MT O2 Hour 1 Rep F & $9061502-24$ & Water & 12-Jun-2009 & 15-Jun-2009 \\
\hline DRAFT: SLC MT N2 Hour 1 Rep D & $9061502-28$ & Water & 12-Jun-2009 & 15-Jun-2009 \\
\hline DRAFT: SLC MT N2 Hour 1 Rep E & $9061502-29$ & Water & 12-Jun-2009 & 15-Jun-2009 \\
\hline DRAFT: SLC MT N2 Hour 1 Rep F & $9061502-30$ & Water & 12-Jun-2009 & 15-Jun-2009 \\
\hline DRAFT: SLC MT O2 Hour 1 Rep D & $9061502-34$ & Water & 12-Jun-2009 & 15-Jun-2009 \\
\hline DRAFT: SLC MT O2 Hour 1 Rep E & $9061502-35$ & Water & 12-Jun-2009 & 15-Jun-2009 \\
\hline DRAFT: SLC MT O2 Hour 1 Rep F & $9061502-36$ & Water & 12-Jun-2009 & 15-Jun-2009 \\
\hline DRAFT: EMR MT N2 Hour 24 Rep A & $9061502-40$ & Water & 12-Jun-2009 & 15-Jun-2009 \\
\hline DRAFT: EMR MT N2 Hour 24 Rep B & $9061502-41$ & Water & 12-Jun-2009 & 15-Jun-2009 \\
\hline DRAFT: EMR MT N2 Hour 24 Rep C & $9061502-42$ & Water & 12-Jun-2009 & 15-Jun-2009 \\
\hline DRAFT: EMR MT O2 Hour 24 Rep A & $9061502-46$ & Water & 12-Jun-2009 & 15-Jun-2009 \\
\hline DRAFT: EMR MT O2 Hour 24 Rep B & $9061502-47$ & Water & 12-Jun-2009 & 15-Jun-2009 \\
\hline DRAFT: EMR MT O2 Hour 24 Rep C & $9061502-48$ & Water & 12-Jun-2009 & 15-Jun-2009 \\
\hline DRAFT: SLC MT N2 Hour 24 Rep A & $9061502-52$ & Water & 12-Jun-2009 & 15-Jun-2009 \\
\hline
\end{tabular}




\section{USACE ERDC-EP-C \\ 3909 Halls Ferry Road \\ Vicksburg, MS 39180-6199}

ERDC - ECB

Project: TVA Fly Ash Project

Reported:

$--$,

Project Manager: Tony Bednar

03-Sep-2009

WORK ORDER SUMMARY

\begin{tabular}{|c|c|c|c|c|}
\hline Sample ID & Laboratory ID & Matrix & Date Sampled & Date of Work Order \\
\hline DRAFT: SLC MT N2 Hour 24 Rep B & $9061502-53$ & Water & 12-Jun-2009 & 15-Jun-2009 \\
\hline DRAFT: SLC MT N2 Hour 24 Rep C & $9061502-54$ & Water & 12-Jun-2009 & 15-Jun-2009 \\
\hline DRAFT: SLC MT O2 Hour 24 Rep A & $9061502-58$ & Water & 12-Jun-2009 & 15-Jun-2009 \\
\hline DRAFT: SLC MT O2 Hour 24 Rep B & $9061502-59$ & Water & 12-Jun-2009 & 15-Jun-2009 \\
\hline DRAFT: SLC MT O2 Hour 24 Rep C & $9061502-60$ & Water & 12-Jun-2009 & 15-Jun-2009 \\
\hline DRAFT: EFL - WA - MT - A (Effluent from Settling & $9061502-61$ & Water & 12-Jun-2009 & 15-Jun-2009 \\
\hline DRAFT: Emory River Water - Total Metals & $9061502-62$ & Water & 12-Jun-2009 & 15-Jun-2009 \\
\hline DRAFT: SLC Water - Total Metals & $9061502-63$ & Water & 12-Jun-2009 & 15-Jun-2009 \\
\hline DRAFT: EMR MT N2 Hour 1 Rep D - Total Metals & $9061502-64$ & Water & 12-Jun-2009 & 15-Jun-2009 \\
\hline DRAFT: EMR MT N2 Hour 1 Rep E - Total Metals & $9061502-65$ & Water & 12-Jun-2009 & 15-Jun-2009 \\
\hline DRAFT: EMR MT N2 Hour 1 Rep F - Total Metals & $9061502-66$ & Water & 12-Jun-2009 & 15-Jun-2009 \\
\hline DRAFT: EMR MT O2 Hour 1 Rep D - Total Metals & $9061502-67$ & Water & 12-Jun-2009 & 15-Jun-2009 \\
\hline DRAFT: EMR MT O2 Hour 1 Rep E - Total Metals & $9061502-68$ & Water & 12-Jun-2009 & 15-Jun-2009 \\
\hline DRAFT: EMR MT O2 Hour 1 Rep F - Total Metals & $9061502-69$ & Water & 12-Jun-2009 & 15-Jun-2009 \\
\hline DRAFT: SLC MT N2 Hour 1 Rep D - Total Metals & $9061502-70$ & Water & 12-Jun-2009 & 15-Jun-2009 \\
\hline DRAFT: SLC MT N2 Hour 1 Rep E - Total Metals & $9061502-71$ & Water & 12-Jun-2009 & 15-Jun-2009 \\
\hline DRAFT: SLC MT N2 Hour 1 Rep F - Total Metals & $9061502-72$ & Water & 12-Jun-2009 & 15-Jun-2009 \\
\hline DRAFT: SLC MT O2 Hour 1 Rep D - Total Metals & $9061502-73$ & Water & 12-Jun-2009 & 15-Jun-2009 \\
\hline DRAFT: SLC MT O2 Hour 1 Rep E - Total Metals & $9061502-74$ & Water & 12-Jun-2009 & 15-Jun-2009 \\
\hline DRAFT: SLC MT O2 Hour 1 Rep F - Total Metals & $9061502-75$ & Water & 12-Jun-2009 & 15-Jun-2009 \\
\hline DRAFT: EMR MT N2 Hour 24 Rep A - Total Metals & $9061502-76$ & Water & 12-Jun-2009 & 15-Jun-2009 \\
\hline DRAFT: EMR MT N2 Hour 24 Rep B - Total Metals & $9061502-77$ & Water & 12-Jun-2009 & 15-Jun-2009 \\
\hline DRAFT: EMR MT N2 Hour 24 Rep C - Total Metals & $9061502-78$ & Water & 12-Jun-2009 & 15-Jun-2009 \\
\hline DRAFT: EMR MT O2 Hour 24 Rep A - Total Metals & $9061502-79$ & Water & 12-Jun-2009 & 15-Jun-2009 \\
\hline DRAFT: EMR MT O2 Hour 24 Rep B - Total Metals & $9061502-80$ & Water & 12-Jun-2009 & 15-Jun-2009 \\
\hline DRAFT: EMR MT O2 Hour 24 Rep C - Total Metals & $9061502-81$ & Water & 12-Jun-2009 & 15-Jun-2009 \\
\hline DRAFT: SLC MT N2 Hour 24 Rep A - Total Metals & $9061502-82$ & Water & 12-Jun-2009 & 15-Jun-2009 \\
\hline DRAFT: SLC MT N2 Hour 24 Rep B - Total Metals & $9061502-83$ & Water & 12-Jun-2009 & 15-Jun-2009 \\
\hline DRAFT: SLC MT N2 Hour 24 Rep C - Total Metals & $9061502-84$ & Water & 12-Jun-2009 & 15-Jun-2009 \\
\hline DRAFT: SLC MT O2 Hour 24 Rep A - Total Metals & $9061502-85$ & Water & 12-Jun-2009 & 15-Jun-2009 \\
\hline DRAFT: SLC MT O2 Hour 24 Rep B - Total Metals & $9061502-86$ & Water & 12-Jun-2009 & 15-Jun-2009 \\
\hline DRAFT: SLC MT O2 Hour 24 Rep C - Total Metals & $9061502-87$ & Water & 12-Jun-2009 & 15-Jun-2009 \\
\hline
\end{tabular}




\section{USACE ERDC-EP-C \\ 3909 Halls Ferry Road \\ Vicksburg, MS 39180-6199}

ERDC - ECB

Project: TVA Fly Ash Project

Reported:

$--$,

Project Manager: Tony Bednar

03-Sep-2009

WORK ORDER SUMMARY

\begin{tabular}{|c|c|c|c|c|}
\hline Sample ID & Laboratory ID & Matrix & Date Sampled & Date of Work Order \\
\hline DRAFT: EMR MT N2 Hour 48 Rep A & $9061503-04$ & Water & 12-Jun-2009 & 15-Jun-2009 \\
\hline DRAFT: EMR MT N2 Hour 48 Rep B & $9061503-05$ & Water & 12-Jun-2009 & 15-Jun-2009 \\
\hline DRAFT: EMR MT N2 Hour 48 Rep C & 9061503-06 & Water & 12-Jun-2009 & 15-Jun-2009 \\
\hline DRAFT: EMR MT O2 Hour 48 Rep A & $9061503-10$ & Water & 12-Jun-2009 & 15-Jun-2009 \\
\hline DRAFT: EMR MT O2 Hour 48 Rep B & $9061503-11$ & Water & 12-Jun-2009 & 15-Jun-2009 \\
\hline DRAFT: EMR MT O2 Hour 48 Rep C & $9061503-12$ & Water & 12-Jun-2009 & 15-Jun-2009 \\
\hline DRAFT: SLC MT N2 Hour 48 Rep A & $9061503-16$ & Water & 12-Jun-2009 & 15-Jun-2009 \\
\hline DRAFT: SLC MT N2 Hour 48 Rep B & $9061503-17$ & Water & 12-Jun-2009 & 15-Jun-2009 \\
\hline DRAFT: SLC MT N2 Hour 48 Rep C & $9061503-18$ & Water & 12-Jun-2009 & 15-Jun-2009 \\
\hline DRAFT: SLC MT O2 Hour 48 Rep A & $9061503-22$ & Water & 12-Jun-2009 & 15-Jun-2009 \\
\hline DRAFT: SLC MT O2 Hour 48 Rep B & $9061503-23$ & Water & 12-Jun-2009 & 15-Jun-2009 \\
\hline DRAFT: SLC MT O2 Hour 48 Rep C & $9061503-24$ & Water & 12-Jun-2009 & 15-Jun-2009 \\
\hline DRAFT: EMR MT N2 Hour 48 Rep A - Total Metals & $9061503-25$ & Water & 15-Jun-2009 & 15-Jun-2009 \\
\hline DRAFT: EMR MT N2 Hour 48 Rep B - Total Metals & $9061503-26$ & Water & 15-Jun-2009 & 15-Jun-2009 \\
\hline DRAFT: EMR MT N2 Hour 48 Rep C - Total Metals & $9061503-27$ & Water & 15-Jun-2009 & 15-Jun-2009 \\
\hline DRAFT: EMR MT O2 Hour 48 Rep A - Total Metals & $9061503-28$ & Water & 15-Jun-2009 & 15-Jun-2009 \\
\hline DRAFT: EMR MT O2 Hour 48 Rep B - Total Metals & $9061503-29$ & Water & 15-Jun-2009 & 15-Jun-2009 \\
\hline DRAFT: EMR MT O2 Hour 48 Rep C - Total Metals & $9061503-30$ & Water & 15-Jun-2009 & 15-Jun-2009 \\
\hline DRAFT: SLC MT N2 Hour 48 Rep A - Total Metals & $9061503-31$ & Water & 12-Jun-2009 & 15-Jun-2009 \\
\hline DRAFT: SLC MT N2 Hour 48 Rep B - Total Metals & $9061503-32$ & Water & 12-Jun-2009 & 15-Jun-2009 \\
\hline DRAFT: SLC MT N2 Hour 48 Rep C - Total Metals & $9061503-33$ & Water & 12-Jun-2009 & 15-Jun-2009 \\
\hline DRAFT: SLC MT O2 Hour 48 Rep A - Total Metals & $9061503-34$ & Water & 12-Jun-2009 & 15-Jun-2009 \\
\hline DRAFT: SLC MT O2 Hour 48 Rep B - Total Metals & $9061503-35$ & Water & 12-Jun-2009 & 15-Jun-2009 \\
\hline DRAFT: SLC MT O2 Hour 48 Rep C - Total Metals & $9061503-36$ & Water & 12-Jun-2009 & 15-Jun-2009 \\
\hline DRAFT: EMR MT N2 Hour 96 Rep A & $9061701-01$ & Water & 17-Jun-2009 & 17-Jun-2009 \\
\hline DRAFT: EMR MT N2 Hour 96 Rep B & $9061701-02$ & Water & 17-Jun-2009 & 17-Jun-2009 \\
\hline DRAFT: EMR MT N2 Hour 96 Rep C & $9061701-03$ & Water & 17-Jun-2009 & 17-Jun-2009 \\
\hline DRAFT: EMR MT O2 Hour 96 Rep A & $9061701-04$ & Water & 17-Jun-2009 & 17-Jun-2009 \\
\hline DRAFT: EMR MT O2 Hour 96 Rep B & $9061701-05$ & Water & 17-Jun-2009 & 17-Jun-2009 \\
\hline DRAFT: EMR MT O2 Hour 96 Rep C & $9061701-06$ & Water & 17-Jun-2009 & 17-Jun-2009 \\
\hline DRAFT: SLC MT N2 Hour 96 Rep A & $9061701-07$ & Water & 17-Jun-2009 & 17-Jun-2009 \\
\hline
\end{tabular}




\section{USACE ERDC-EP-C \\ 3909 Halls Ferry Road \\ Vicksburg, MS 39180-6199}

ERDC - ECB

Project: TVA Fly Ash Project

Reported:

$--$,

Project Manager: Tony Bednar

03-Sep-2009

WORK ORDER SUMMARY

\begin{tabular}{|c|c|c|c|c|}
\hline Sample ID & Laboratory ID & Matrix & Date Sampled & Date of Work Order \\
\hline DRAFT: SLC MT N2 Hour 96 Rep B & 9061701-08 & Water & 17-Jun-2009 & 17-Jun-2009 \\
\hline DRAFT: SLC MT N2 Hour 96 Rep C & 9061701-09 & Water & 17-Jun-2009 & 17-Jun-2009 \\
\hline DRAFT: SLC MT O2 Hour 96 Rep A & $9061701-10$ & Water & 17-Jun-2009 & 17-Jun-2009 \\
\hline DRAFT: SLC MT O2 Hour 96 Rep B & $9061701-11$ & Water & 17-Jun-2009 & 17-Jun-2009 \\
\hline DRAFT: SLC MT O2 Hour 96 Rep C & $9061701-12$ & Water & 17-Jun-2009 & 17-Jun-2009 \\
\hline DRAFT: EMR MT N2 Hour 96 Rep A - Total Metals & $9061701-13$ & Water & 17-Jun-2009 & 17-Jun-2009 \\
\hline DRAFT: EMR MT N2 Hour 96 Rep B - Total Metals & $9061701-14$ & Water & 17-Jun-2009 & 17-Jun-2009 \\
\hline DRAFT: EMR MT N2 Hour 96 Rep C - Total Metals & $9061701-15$ & Water & 17-Jun-2009 & 17-Jun-2009 \\
\hline DRAFT: EMR MT O2 Hour 96 Rep A - Total Metals & $9061701-16$ & Water & 17-Jun-2009 & 17-Jun-2009 \\
\hline DRAFT: EMR MT O2 Hour 96 Rep B - Total Metals & $9061701-17$ & Water & 17-Jun-2009 & 17-Jun-2009 \\
\hline DRAFT: EMR MT O2 Hour 96 Rep C - Total Metals & $9061701-18$ & Water & 17-Jun-2009 & 17-Jun-2009 \\
\hline DRAFT: SLC MT N2 Hour 96 Rep A - Total Metals & $9061701-19$ & Water & 17-Jun-2009 & 17-Jun-2009 \\
\hline DRAFT: SLC MT N2 Hour 96 Rep B - Total Metals & $9061701-20$ & Water & 17-Jun-2009 & 17-Jun-2009 \\
\hline DRAFT: SLC MT N2 Hour 96 Rep C - Total Metals & $9061701-21$ & Water & 17-Jun-2009 & 17-Jun-2009 \\
\hline DRAFT: SLC MT O2 Hour 96 Rep A - Total Metals & $9061701-22$ & Water & 17-Jun-2009 & 17-Jun-2009 \\
\hline DRAFT: SLC MT O2 Hour 96 Rep B - Total Metals & $9061701-23$ & Water & 17-Jun-2009 & 17-Jun-2009 \\
\hline DRAFT: SLC MT O2 Hour 96 Rep C - Total Metals & $9061701-24$ & Water & 17-Jun-2009 & 17-Jun-2009 \\
\hline DRAFT: EMR MT N2 Hour 240 Rep A & $9062302-01$ & Water & 23-Jun-2009 & 23-Jun-2009 \\
\hline DRAFT: EMR MT N2 Hour 240 Rep B & $9062302-02$ & Water & 23-Jun-2009 & 23-Jun-2009 \\
\hline DRAFT: EMR MT N2 Hour 240 Rep C & $9062302-03$ & Water & 23-Jun-2009 & 23-Jun-2009 \\
\hline DRAFT: EMR MT O2 Hour 240 Rep A & $9062302-04$ & Water & 23-Jun-2009 & 23-Jun-2009 \\
\hline DRAFT: EMR MT O2 Hour 240 Rep B & $9062302-05$ & Water & 23-Jun-2009 & 23-Jun-2009 \\
\hline DRAFT: EMR MT O2 Hour 240 Rep C & $9062302-06$ & Water & 23-Jun-2009 & 23-Jun-2009 \\
\hline DRAFT: SLC MT N2 Hour 240 Rep A & $9062302-07$ & Water & 23-Jun-2009 & 23-Jun-2009 \\
\hline DRAFT: SLC MT N2 Hour 240 Rep B & $9062302-08$ & Water & 23-Jun-2009 & 23-Jun-2009 \\
\hline DRAFT: SLC MT N2 Hour 240 Rep C & $9062302-09$ & Water & 23-Jun-2009 & 23-Jun-2009 \\
\hline DRAFT: SLC MT O2 Hour 240 Rep A & $9062302-10$ & Water & 23-Jun-2009 & 23-Jun-2009 \\
\hline DRAFT: SLC MT O2 Hour 240 Rep B & $9062302-11$ & Water & 23-Jun-2009 & 23-Jun-2009 \\
\hline DRAFT: SLC MT O2 Hour 240 Rep C & $9062302-12$ & Water & 23-Jun-2009 & 23-Jun-2009 \\
\hline DRAFT: EMR MT N2 Hour 240 Rep A - Total Meta] & $9062302-13$ & Water & 23-Jun-2009 & 23-Jun-2009 \\
\hline DRAFT: EMR MT N2 Hour 240 Rep B - Total Metal & $9062302-14$ & Water & 23-Jun-2009 & 23-Jun-2009 \\
\hline DRAFT: EMR MT N2 Hour 240 Rep C - Total Metal & $9062302-15$ & Water & 23-Jun-2009 & 23-Jun-2009 \\
\hline
\end{tabular}




\section{USACE ERDC-EP-C \\ 3909 Halls Ferry Road \\ Vicksburg, MS 39180-6199}

ERDC - ECB

Project: TVA Fly Ash Project

Reported:

Project Manager: Tony Bednar

03-Sep-2009

WORK ORDER SUMMARY

\begin{tabular}{|c|c|c|c|c|}
\hline Sample ID & Laboratory ID & Matrix & Date Sampled & Date of Work Order \\
\hline DRAFT: EMR MT O2 Hour 240 Rep A - Total Metal & $9062302-16$ & Water & 23-Jun-2009 & 23-Jun-2009 \\
\hline DRAFT: EMR MT O2 Hour 240 Rep B - Total Metal & $9062302-17$ & Water & 23-Jun-2009 & 23-Jun-2009 \\
\hline DRAFT: EMR MT O2 Hour 240 Rep C - Total Metal & $9062302-18$ & Water & 23-Jun-2009 & 23-Jun-2009 \\
\hline DRAFT: SLC MT N2 Hour 240 Rep A - Total Metals & $9062302-19$ & Water & 23-Jun-2009 & 23-Jun-2009 \\
\hline DRAFT: SLC MT N2 Hour 240 Rep B - Total Metals & $9062302-20$ & Water & 23-Jun-2009 & 23-Jun-2009 \\
\hline DRAFT: SLC MT N2 Hour 240 Rep C - Total Metals & $9062302-21$ & Water & 23-Jun-2009 & 23-Jun-2009 \\
\hline DRAFT: SLC MT O2 Hour 240 Rep A - Total Metals & $9062302-22$ & Water & 23-Jun-2009 & 23-Jun-2009 \\
\hline DRAFT: SLC MT O2 Hour 240 Rep B - Total Metals & $9062302-23$ & Water & 23-Jun-2009 & 23-Jun-2009 \\
\hline DRAFT: SLC MT O2 Hour 240 Rep C - Total Metals & $9062302-24$ & Water & 23-Jun-2009 & 23-Jun-2009 \\
\hline DRAFT: EMR - EL - AF - 100 - A & $9071602-01$ & Tissue & 15-Jul-2009 & 16-Jul-2009 \\
\hline DRAFT: EMR - EL - AF - 100 - B & $9071602-02$ & Tissue & 15-Jul-2009 & 16-Jul-2009 \\
\hline DRAFT: EMR - EL - AF - 100 - C & $9071602-03$ & Tissue & 15-Jul-2009 & 16-Jul-2009 \\
\hline DRAFT: EMR - EL - AF - 100 - D & $9071602-04$ & Tissue & 15-Jul-2009 & 16-Jul-2009 \\
\hline DRAFT: EMR - EL - AF - 100 - E & $9071602-05$ & Tissue & 15-Jul-2009 & 16-Jul-2009 \\
\hline DRAFT: EMR - EL - AF - 50 - A & $9071602-06$ & Tissue & 15-Jul-2009 & 16-Jul-2009 \\
\hline DRAFT: EMR - EL - AF - 50 - B & $9071602-07$ & Tissue & 15-Jul-2009 & 16-Jul-2009 \\
\hline DRAFT: EMR - EL - AF - 50 - C & $9071602-08$ & Tissue & 15-Jul-2009 & 16-Jul-2009 \\
\hline DRAFT: EMR - EL - AF - 50 - D & $9071602-09$ & Tissue & 15-Jul-2009 & 16-Jul-2009 \\
\hline DRAFT: EMR - EL - AF - 50 - E & $9071602-10$ & Tissue & 15-Jul-2009 & 16-Jul-2009 \\
\hline DRAFT: EMR - EL - AF - 10 - A & $9071602-11$ & Tissue & 15-Jul-2009 & 16-Jul-2009 \\
\hline DRAFT: EMR - EL - AF - 10 - B & $9071602-12$ & Tissue & 15-Jul-2009 & 16-Jul-2009 \\
\hline DRAFT: EMR - EL - AF - 10 - C & $9071602-13$ & Tissue & 15-Jul-2009 & 16-Jul-2009 \\
\hline DRAFT: EMR - EL - AF - 10 - D & $9071602-14$ & Tissue & 15-Jul-2009 & 16-Jul-2009 \\
\hline DRAFT: EMR - EL - AF - 10 - E & $9071602-15$ & Tissue & 15-Jul-2009 & 16-Jul-2009 \\
\hline DRAFT: EMR - EL - AF - 0 - A & $9071602-16$ & Tissue & 15-Jul-2009 & 16-Jul-2009 \\
\hline DRAFT: EMR - EL - AF - 0 - B & $9071602-17$ & Tissue & 15-Jul-2009 & 16-Jul-2009 \\
\hline DRAFT: EMR - EL - AF - 0 - C & $9071602-18$ & Tissue & 15-Jul-2009 & 16-Jul-2009 \\
\hline DRAFT: EMR - EL - AF - 0 - D & $9071602-19$ & Tissue & 15-Jul-2009 & 16-Jul-2009 \\
\hline DRAFT: EMR - EL - AF - 0 - E & $9071602-20$ & Tissue & 15-Jul-2009 & 16-Jul-2009 \\
\hline DRAFT: Control - A & $9071602-21$ & Tissue & 15-Jul-2009 & 16-Jul-2009 \\
\hline DRAFT: Control - B & $9071602-22$ & Tissue & 15-Jul-2009 & 16-Jul-2009 \\
\hline
\end{tabular}

The results in this report apply to the samples analyzed in accordance with the chain of

custody document. This analytical report must be reproduced in its entirety. 


\section{USACE ERDC-EP-C \\ 3909 Halls Ferry Road \\ Vicksburg, MS 39180-6199}

ERDC - ECB

Project: TVA Fly Ash Project

Reported:

$--$,

Project Manager: Tony Bednar

03-Sep-2009

WORK ORDER SUMMARY

\begin{tabular}{|c|c|c|c|c|}
\hline Sample ID & Laboratory ID & Matrix & Date Sampled & Date of Work Order \\
\hline DRAFT: Control - C & $9071602-23$ & Tissue & 15-Jul-2009 & 16-Jul-2009 \\
\hline DRAFT: Control - D & $9071602-24$ & Tissue & 15-Jul-2009 & 16-Jul-2009 \\
\hline DRAFT: Control - E & $9071602-25$ & Tissue & 15-Jul-2009 & 16-Jul-2009 \\
\hline DRAFT: Larvae Food & $9071602-26$ & Solid & 15-Jul-2009 & 16-Jul-2009 \\
\hline DRAFT: Adult Food & $9071602-27$ & Solid & 15-Jul-2009 & 16-Jul-2009 \\
\hline DRAFT: Fish Tissue Dechlor 1 & 9082706-01 & Tissue & 26-Aug-2009 & 27-Aug-2009 \\
\hline DRAFT: Fish Tissue Dechlor 2 & $9082706-02$ & Tissue & 26-Aug-2009 & 27-Aug-2009 \\
\hline DRAFT: Fish Tissue Dechlor 3 & $9082706-03$ & Tissue & 26-Aug-2009 & 27-Aug-2009 \\
\hline DRAFT: Fish Tissue Dechlor 4 & 9082706-04 & Tissue & 26-Aug-2009 & 27-Aug-2009 \\
\hline DRAFT: Fish Tissue Dechlor 5 & $9082706-05$ & Tissue & 26-Aug-2009 & 27-Aug-2009 \\
\hline DRAFT: Fish Tissue EMR - WA 1 & $9082706-06$ & Tissue & 26-Aug-2009 & 27-Aug-2009 \\
\hline DRAFT: Fish Tissue EMR - WA 2 & $9082706-07$ & Tissue & 26-Aug-2009 & 27-Aug-2009 \\
\hline DRAFT: Fish Tissue EMR - WA 3 & $9082706-08$ & Tissue & 26-Aug-2009 & 27-Aug-2009 \\
\hline DRAFT: Fish Tissue EMR - WA 4 & 9082706-09 & Tissue & 26-Aug-2009 & 27-Aug-2009 \\
\hline DRAFT: Fish Tissue EMR - WA 5 & $9082706-10$ & Tissue & 26-Aug-2009 & 27-Aug-2009 \\
\hline DRAFT: Fish Tissue EMR - EL 1 & $9082706-11$ & Tissue & 26-Aug-2009 & 27-Aug-2009 \\
\hline DRAFT: Fish Tissue EMR - EL 2 & $9082706-12$ & Tissue & 26-Aug-2009 & 27-Aug-2009 \\
\hline DRAFT: Fish Tissue EMR - EL 3 & $9082706-13$ & Tissue & 26-Aug-2009 & 27-Aug-2009 \\
\hline DRAFT: Fish Tissue EMR - EL 4 & $9082706-14$ & Tissue & 26-Aug-2009 & 27-Aug-2009 \\
\hline DRAFT: Fish Tissue EMR - EL 5 & $9082706-15$ & Tissue & 26-Aug-2009 & 27-Aug-2009 \\
\hline DRAFT: Fish Gut Dechlor 1 & $9082706-16$ & Tissue & 26-Aug-2009 & 27-Aug-2009 \\
\hline DRAFT: Fish Gut Dechlor 2 & $9082706-17$ & Tissue & 26-Aug-2009 & 27-Aug-2009 \\
\hline DRAFT: Fish Gut Dechlor 3 & $9082706-18$ & Tissue & 26-Aug-2009 & 27-Aug-2009 \\
\hline DRAFT: Fish Gut Dechlor 4 & $9082706-19$ & Tissue & 26-Aug-2009 & 27-Aug-2009 \\
\hline DRAFT: Fish Gut Dechlor 5 & $9082706-20$ & Tissue & 26-Aug-2009 & 27-Aug-2009 \\
\hline DRAFT: Fish Gut EMR - WA 1 & $9082706-21$ & Tissue & 26-Aug-2009 & 27-Aug-2009 \\
\hline DRAFT: Fish Gut EMR - WA 2 & $9082706-22$ & Tissue & 26-Aug-2009 & 27-Aug-2009 \\
\hline DRAFT: Fish Gut EMR - WA 3 & $9082706-23$ & Tissue & 26-Aug-2009 & 27-Aug-2009 \\
\hline DRAFT: Fish Gut EMR - WA 4 & $9082706-24$ & Tissue & 26-Aug-2009 & 27-Aug-2009 \\
\hline DRAFT: Fish Gut EMR - WA 5 & $9082706-25$ & Tissue & 26-Aug-2009 & 27-Aug-2009 \\
\hline DRAFT: Fish Gut EMR - EL 1 & $9082706-26$ & Tissue & 26-Aug-2009 & 27-Aug-2009 \\
\hline DRAFT: Fish Gut EMR - EL 2 & $9082706-27$ & Tissue & 26-Aug-2009 & 27-Aug-2009 \\
\hline
\end{tabular}




\section{USACE ERDC-EP-C \\ 3909 Halls Ferry Road \\ Vicksburg, MS 39180-6199}

ERDC - ECB

Project: TVA Fly Ash Project

Reported:

$--$,

Project Manager: Tony Bednar

03-Sep-2009

WORK ORDER SUMMARY

\begin{tabular}{|c|c|c|c|c|}
\hline Sample ID & Laboratory ID & Matrix & Date Sampled & Date of Work Order \\
\hline DRAFT: Fish Gut EMR - EL 3 & $9082706-28$ & Tissue & 26-Aug-2009 & 27-Aug-2009 \\
\hline DRAFT: Fish Gut EMR - EL 5 & $9082706-30$ & Tissue & 26-Aug-2009 & 27-Aug-2009 \\
\hline
\end{tabular}




\section{USACE ERDC-EP-C \\ 3909 Halls Ferry Road \\ Vicksburg, MS 39180-6199}

ERDC - ECB

Project: TVA Fly Ash Project

Reported:

$--$,

Project Manager: Tony Bednar

03-Sep-2009

\section{DRAFT: EMR Sediment}

9061502-01 (Soil/Sediment)

\begin{tabular}{|c|c|c|c|c|c|c|c|c|}
\hline & & porting & & & & & & \\
\hline Analyte & Result & Limit & Units & Dilution & Prepared & Analyzed & Method & Notes \\
\hline
\end{tabular}

ERDC- EL-EP-C (Environmental Chemistry Branch)

DRAFT: Metals by EPA 6000/7000 Series Methods

\begin{tabular}{|c|c|c|c|c|c|c|c|c|}
\hline Mercury & 0.0732 & 0.00396 & $\mathrm{mg} / \mathrm{kg}$ & 1 & 06-Jul-2009 & 22-Jul-2009 & EPA 7471A & \\
\hline Strontium & 87.4 & 4.00 & $\mathrm{mg} / \mathrm{kg}$ & 2 & 25-Jun-2009 & 25-Jun-2009 & EPA $6010 B$ & \\
\hline Aluminum & 4890 & 2.00 & $\mathrm{mg} / \mathrm{kg}$ & 2 & 25-Jun-2009 & 25-Jun-2009 & SW 846/6010 & B \\
\hline Iron & 10400 & 2.00 & $\mathrm{mg} / \mathrm{kg}$ & 2 & 25-Jun-2009 & 25-Jun-2009 & SW 846/6010 & \\
\hline Magnesium & 559 & 2.00 & $\mathrm{mg} / \mathrm{kg}$ & 2 & 25-Jun-2009 & 25-Jun-2009 & SW 846/6010 & B \\
\hline Potassium & 625 & 2.00 & $\mathrm{mg} / \mathrm{kg}$ & 2 & 25-Jun-2009 & 25-Jun-2009 & SW 846/6010 & B \\
\hline Sodium & 65.2 & 2.00 & $\mathrm{mg} / \mathrm{kg}$ & 2 & 25-Jun-2009 & 25-Jun-2009 & SW 846/6010 & B \\
\hline Antimony & 0.233 & 0.0500 & $\mathrm{mg} / \mathrm{kg}$ & 10 & 25-Jun-2009 & 26-Jun-2009 & SW 846/6020 & B \\
\hline Arsenic & 65.8 & 0.0500 & $\mathrm{mg} / \mathrm{kg}$ & 10 & 25-Jun-2009 & 26-Jun-2009 & SW 846/6020 & \\
\hline Barium & 655 & 0.0500 & $\mathrm{mg} / \mathrm{kg}$ & 10 & 25-Jun-2009 & 26-Jun-2009 & SW 846/6020 & $\mathrm{B}$ \\
\hline Beryllium & 7.15 & 0.0500 & $\mathrm{mg} / \mathrm{kg}$ & 10 & 25-Jun-2009 & 26-Jun-2009 & SW 846/6020 & \\
\hline Cadmium & 0.576 & 0.0500 & $\mathrm{mg} / \mathrm{kg}$ & 10 & 25-Jun-2009 & 26-Jun-2009 & SW 846/6020 & \\
\hline Cobalt & 25.6 & 0.0500 & $\mathrm{mg} / \mathrm{kg}$ & 10 & 25-Jun-2009 & 26-Jun-2009 & SW 846/6020 & \\
\hline Copper & 84.1 & 0.0500 & $\mathrm{mg} / \mathrm{kg}$ & 10 & 25-Jun-2009 & 26-Jun-2009 & SW 846/6020 & \\
\hline Lead & 36.4 & 0.0500 & $\mathrm{mg} / \mathrm{kg}$ & 10 & 25-Jun-2009 & 26-Jun-2009 & SW 846/6020 & \\
\hline Manganese & 153 & 0.0500 & $\mathrm{mg} / \mathrm{kg}$ & 10 & 25-Jun-2009 & 26-Jun-2009 & SW 846/6020 & B \\
\hline Molybdenum & 4.04 & 0.0500 & $\mathrm{mg} / \mathrm{kg}$ & 10 & 25-Jun-2009 & 26-Jun-2009 & SW 846/6020 & \\
\hline Nickel & 52.6 & 0.0500 & $\mathrm{mg} / \mathrm{kg}$ & 10 & 25-Jun-2009 & 26-Jun-2009 & SW 846/6020 & \\
\hline Selenium & 6.97 & 0.0500 & $\mathrm{mg} / \mathrm{kg}$ & 10 & 25-Jun-2009 & 26-Jun-2009 & SW 846/6020 & \\
\hline Silver & 0.426 & 0.0500 & $\mathrm{mg} / \mathrm{kg}$ & 10 & 25-Jun-2009 & 26-Jun-2009 & SW 846/6020 & \\
\hline Thallium & 2.19 & 0.0500 & $\mathrm{mg} / \mathrm{kg}$ & 10 & 25-Jun-2009 & 26-Jun-2009 & SW 846/6020 & \\
\hline Vanadium & 133 & 0.0500 & $\mathrm{mg} / \mathrm{kg}$ & 10 & 25-Jun-2009 & 26-Jun-2009 & SW 846/6020 & \\
\hline Zinc & 77.4 & 0.0500 & $\mathrm{mg} / \mathrm{kg}$ & 10 & 25-Jun-2009 & 26-Jun-2009 & SW 846/6020 & B \\
\hline
\end{tabular}




\section{USACE ERDC-EP-C \\ 3909 Halls Ferry Road \\ Vicksburg, MS 39180-6199}

ERDC - ECB $--,-$

\section{DRAFT: SLC Sediment}

9061502-02 (Soil/Sediment)

\begin{tabular}{|c|c|c|c|c|c|c|c|c|}
\hline Analyte & Result & $\begin{array}{r}\text { porting } \\
\text { Limit }\end{array}$ & Units & Dilution & Prepared & Analyzed & Method & Notes \\
\hline
\end{tabular}

ERDC- EL-EP-C (Environmental Chemistry Branch)

DRAFT: Metals by EPA 6000/7000 Series Methods

\begin{tabular}{|c|c|c|c|c|c|c|c|c|}
\hline Mercury & 0.0294 & 0.00399 & $\mathrm{mg} / \mathrm{kg}$ & 1 & 06-Jul-2009 & 22-Jul-2009 & EPA 7471A & \\
\hline Strontium & 69.9 & 4.00 & $\mathrm{mg} / \mathrm{kg}$ & 2 & 25-Jun-2009 & 25-Jun-2009 & EPA 6010B & \\
\hline Aluminum & 29900 & 2.00 & $\mathrm{mg} / \mathrm{kg}$ & 2 & 25-Jun-2009 & 25-Jun-2009 & SW 846/6010 & B \\
\hline Calcium & 6510 & 2.00 & $\mathrm{mg} / \mathrm{kg}$ & 2 & 25-Jun-2009 & 25-Jun-2009 & SW 846/6010 & B \\
\hline Iron & 22200 & 2.00 & $\mathrm{mg} / \mathrm{kg}$ & 2 & 25-Jun-2009 & 25-Jun-2009 & SW 846/6010 & \\
\hline Magnesium & 2380 & 2.00 & $\mathrm{mg} / \mathrm{kg}$ & 2 & 25-Jun-2009 & 25-Jun-2009 & SW 846/6010 & B \\
\hline Potassium & 4890 & 2.00 & $\mathrm{mg} / \mathrm{kg}$ & 2 & 25-Jun-2009 & 25-Jun-2009 & SW 846/6010 & B \\
\hline Sodium & 608 & 2.00 & $\mathrm{mg} / \mathrm{kg}$ & 2 & 25-Jun-2009 & 25-Jun-2009 & SW 846/6010 & B \\
\hline Antimony & 0.212 & 0.0500 & $\mathrm{mg} / \mathrm{kg}$ & 10 & 25-Jun-2009 & 26-Jun-2009 & SW 846/6020 & B \\
\hline Arsenic & 76.2 & 0.0500 & $\mathrm{mg} / \mathrm{kg}$ & 10 & 25-Jun-2009 & 26-Jun-2009 & SW 846/6020 & \\
\hline Barium & 515 & 0.0500 & $\mathrm{mg} / \mathrm{kg}$ & 10 & 25-Jun-2009 & 26-Jun-2009 & SW 846/6020 & B \\
\hline Beryllium & 6.03 & 0.0500 & $\mathrm{mg} / \mathrm{kg}$ & 10 & 25-Jun-2009 & 26-Jun-2009 & SW 846/6020 & \\
\hline Cadmium & 0.631 & 0.0500 & $\mathrm{mg} / \mathrm{kg}$ & 10 & 25-Jun-2009 & 26-Jun-2009 & SW 846/6020 & \\
\hline Chromium & 49.2 & 0.0500 & $\mathrm{mg} / \mathrm{kg}$ & 10 & 25-Jun-2009 & 26-Jun-2009 & SW 846/6020 & B \\
\hline Cobalt & 23.1 & 0.0500 & $\mathrm{mg} / \mathrm{kg}$ & 10 & 25-Jun-2009 & 26-Jun-2009 & SW 846/6020 & \\
\hline Copper & 76.4 & 0.0500 & $\mathrm{mg} / \mathrm{kg}$ & 10 & 25-Jun-2009 & 26-Jun-2009 & SW 846/6020 & \\
\hline Lead & 33.1 & 0.0500 & $\mathrm{mg} / \mathrm{kg}$ & 10 & 25-Jun-2009 & 26-Jun-2009 & SW 846/6020 & \\
\hline Manganese & 187 & 0.0500 & $\mathrm{mg} / \mathrm{kg}$ & 10 & 25-Jun-2009 & 26-Jun-2009 & SW 846/6020 & B \\
\hline Molybdenum & 4.23 & 0.0500 & $\mathrm{mg} / \mathrm{kg}$ & 10 & 25-Jun-2009 & 26-Jun-2009 & SW 846/6020 & \\
\hline Nickel & 49.9 & 0.0500 & $\mathrm{mg} / \mathrm{kg}$ & 10 & 25-Jun-2009 & 26-Jun-2009 & SW 846/6020 & \\
\hline Selenium & 6.08 & 0.0500 & $\mathrm{mg} / \mathrm{kg}$ & 10 & 25-Jun-2009 & 26-Jun-2009 & SW 846/6020 & \\
\hline Silver & 0.335 & 0.0500 & $\mathrm{mg} / \mathrm{kg}$ & 10 & 25-Jun-2009 & 26-Jun-2009 & SW 846/6020 & \\
\hline Thallium & 2.04 & 0.0500 & $\mathrm{mg} / \mathrm{kg}$ & 10 & 25-Jun-2009 & 26-Jun-2009 & SW 846/6020 & \\
\hline Vanadium & 124 & 0.0500 & $\mathrm{mg} / \mathrm{kg}$ & 10 & 25-Jun-2009 & 26-Jun-2009 & SW 846/6020 & \\
\hline Zinc & 72.1 & 0.0500 & $\mathrm{mg} / \mathrm{kg}$ & 10 & 25-Jun-2009 & 26-Jun-2009 & SW 846/6020 & B \\
\hline
\end{tabular}




\section{USACE ERDC-EP-C \\ 3909 Halls Ferry Road \\ Vicksburg, MS 39180-6199}

ERDC - ECB $-,-$
Project: TVA Fly Ash Project

Project Manager: Tony Bednar
Reported:

03-Sep-2009

DRAFT: RF1-SD-MT-A Sediment

9061502-03 (Soil/Sediment)

\begin{tabular}{|c|c|c|c|c|c|c|c|c|}
\hline Analyte & Result & $\begin{array}{r}\text { porting } \\
\text { Limit }\end{array}$ & Units & Dilution & Prepared & Analyzed & Method & Notes \\
\hline
\end{tabular}

ERDC- EL-EP-C (Environmental Chemistry Branch)

DRAFT: Metals by EPA 6000/7000 Series Methods

\begin{tabular}{|c|c|c|c|c|c|c|c|c|}
\hline Mercury & 0.0211 & 0.00397 & $\mathrm{mg} / \mathrm{kg}$ & 1 & 06-Jul-2009 & 22-Jul-2009 & EPA 7471A & \\
\hline Strontium & 1.82 & 4.00 & $\mathrm{mg} / \mathrm{kg}$ & 2 & 25-Jun-2009 & 25-Jun-2009 & EPA 6010B & $\mathrm{J}$ \\
\hline Aluminum & 4650 & 2.00 & $\mathrm{mg} / \mathrm{kg}$ & 2 & 25-Jun-2009 & 25-Jun-2009 & SW 846/6010 & B \\
\hline Calcium & 1800 & 2.00 & $\mathrm{mg} / \mathrm{kg}$ & 2 & 25-Jun-2009 & 25-Jun-2009 & SW 846/6010 & B \\
\hline Iron & 7990 & 2.00 & $\mathrm{mg} / \mathrm{kg}$ & 2 & 25-Jun-2009 & 25-Jun-2009 & SW 846/6010 & \\
\hline Magnesium & 674 & 2.00 & $\mathrm{mg} / \mathrm{kg}$ & 2 & 25-Jun-2009 & 25-Jun-2009 & SW 846/6010 & B \\
\hline Potassium & 760 & 2.00 & $\mathrm{mg} / \mathrm{kg}$ & 2 & 25-Jun-2009 & 25-Jun-2009 & SW 846/6010 & B \\
\hline Sodium & 52.0 & 2.00 & $\mathrm{mg} / \mathrm{kg}$ & 2 & 25-Jun-2009 & 25-Jun-2009 & SW 846/6010 & B \\
\hline Antimony & 0.0262 & 0.0500 & $\mathrm{mg} / \mathrm{kg}$ & 10 & 25-Jun-2009 & 26-Jun-2009 & SW 846/6020 & $\mathrm{J}, \mathrm{B}$ \\
\hline Arsenic & 1.71 & 0.0500 & $\mathrm{mg} / \mathrm{kg}$ & 10 & 25-Jun-2009 & 26-Jun-2009 & SW 846/6020 & \\
\hline Barium & 48.0 & 0.0500 & $\mathrm{mg} / \mathrm{kg}$ & 10 & 25-Jun-2009 & 26-Jun-2009 & SW 846/6020 & B \\
\hline Beryllium & 0.584 & 0.0500 & $\mathrm{mg} / \mathrm{kg}$ & 10 & 25-Jun-2009 & 26-Jun-2009 & SW 846/6020 & \\
\hline Cadmium & 0.0991 & 0.0500 & $\mathrm{mg} / \mathrm{kg}$ & 10 & 25-Jun-2009 & 26-Jun-2009 & SW 846/6020 & \\
\hline Chromium & 8.25 & 0.0500 & $\mathrm{mg} / \mathrm{kg}$ & 10 & 25-Jun-2009 & 26-Jun-2009 & SW 846/6020 & B \\
\hline Cobalt & 7.01 & 0.0500 & $\mathrm{mg} / \mathrm{kg}$ & 10 & 25-Jun-2009 & 26-Jun-2009 & SW 846/6020 & \\
\hline Copper & 5.58 & 0.0500 & $\mathrm{mg} / \mathrm{kg}$ & 10 & 25-Jun-2009 & 26-Jun-2009 & SW 846/6020 & \\
\hline Lead & 6.68 & 0.0500 & $\mathrm{mg} / \mathrm{kg}$ & 10 & 25-Jun-2009 & 26-Jun-2009 & SW 846/6020 & \\
\hline Manganese & 313 & 0.0500 & $\mathrm{mg} / \mathrm{kg}$ & 10 & 25-Jun-2009 & 26-Jun-2009 & SW 846/6020 & B \\
\hline Molybdenum & 0.255 & 0.0500 & $\mathrm{mg} / \mathrm{kg}$ & 10 & 25-Jun-2009 & 26-Jun-2009 & SW 846/6020 & \\
\hline Nickel & 11.5 & 0.0500 & $\mathrm{mg} / \mathrm{kg}$ & 10 & 25-Jun-2009 & 26-Jun-2009 & SW 846/6020 & \\
\hline Selenium & 0.247 & 0.0500 & $\mathrm{mg} / \mathrm{kg}$ & 10 & 25-Jun-2009 & 26-Jun-2009 & SW 846/6020 & \\
\hline Silver & 0.0593 & 0.0500 & $\mathrm{mg} / \mathrm{kg}$ & 10 & 25-Jun-2009 & 26-Jun-2009 & SW 846/6020 & \\
\hline Thallium & 0.0776 & 0.0500 & $\mathrm{mg} / \mathrm{kg}$ & 10 & 25-Jun-2009 & 26-Jun-2009 & SW 846/6020 & \\
\hline Vanadium & 9.16 & 0.0500 & $\mathrm{mg} / \mathrm{kg}$ & 10 & 25-Jun-2009 & 26-Jun-2009 & SW 846/6020 & \\
\hline Zinc & 35.0 & 0.0500 & $\mathrm{mg} / \mathrm{kg}$ & 10 & 25-Jun-2009 & 26-Jun-2009 & SW 846/6020 & B \\
\hline
\end{tabular}




\section{USACE ERDC-EP-C \\ 3909 Halls Ferry Road \\ Vicksburg, MS 39180-6199}

ERDC - ECB

$-,-$
Project: TVA Fly Ash Project

Project Manager: Tony Bednar
Reported:

03-Sep-2009

DRAFT: RF2-SD-MT-A Sediment

9061502-04 (Soil/Sediment)

\begin{tabular}{|c|c|c|c|c|c|c|c|c|}
\hline Analyte & Result & $\begin{array}{r}\text { porting } \\
\text { Limit }\end{array}$ & Units & Dilution & Prepared & Analyzed & Method & Notes \\
\hline
\end{tabular}

ERDC- EL-EP-C (Environmental Chemistry Branch)

DRAFT: Metals by EPA 6000/7000 Series Methods

\begin{tabular}{|c|c|c|c|c|c|c|c|c|}
\hline Mercury & 0.0239 & 0.00399 & $\mathrm{mg} / \mathrm{kg}$ & 1 & 06-Jul-2009 & 22-Jul-2009 & EPA 7471A & \\
\hline Strontium & 87.9 & 4.00 & $\mathrm{mg} / \mathrm{kg}$ & 2 & 25-Jun-2009 & 25-Jun-2009 & EPA 6010B & \\
\hline Aluminum & 4950 & 2.00 & $\mathrm{mg} / \mathrm{kg}$ & 2 & 25-Jun-2009 & 25-Jun-2009 & SW 846/6010 & B \\
\hline Calcium & 2440 & 2.00 & $\mathrm{mg} / \mathrm{kg}$ & 2 & 25-Jun-2009 & 25-Jun-2009 & SW 846/6010 & B \\
\hline Iron & 10600 & 2.00 & $\mathrm{mg} / \mathrm{kg}$ & 2 & 25-Jun-2009 & 25-Jun-2009 & SW 846/6010 & \\
\hline Magnesium & 566 & 2.00 & $\mathrm{mg} / \mathrm{kg}$ & 2 & 25-Jun-2009 & 25-Jun-2009 & SW 846/6010 & B \\
\hline Potassium & 633 & 2.00 & $\mathrm{mg} / \mathrm{kg}$ & 2 & 25-Jun-2009 & 25-Jun-2009 & SW 846/6010 & B \\
\hline Sodium & 66.1 & 2.00 & $\mathrm{mg} / \mathrm{kg}$ & 2 & 25-Jun-2009 & 25-Jun-2009 & SW 846/6010 & B \\
\hline Antimony & 0.292 & 0.0500 & $\mathrm{mg} / \mathrm{kg}$ & 10 & 25-Jun-2009 & 26-Jun-2009 & SW 846/6020 & B \\
\hline Arsenic & 2.69 & 0.0500 & $\mathrm{mg} / \mathrm{kg}$ & 10 & 25-Jun-2009 & 26-Jun-2009 & SW 846/6020 & \\
\hline Barium & 73.5 & 0.0500 & $\mathrm{mg} / \mathrm{kg}$ & 10 & 25-Jun-2009 & 26-Jun-2009 & SW 846/6020 & B \\
\hline Beryllium & 0.528 & 0.0500 & $\mathrm{mg} / \mathrm{kg}$ & 10 & 25-Jun-2009 & 26-Jun-2009 & SW 846/6020 & \\
\hline Cadmium & 0.111 & 0.0500 & $\mathrm{mg} / \mathrm{kg}$ & 10 & 25-Jun-2009 & 26-Jun-2009 & SW 846/6020 & \\
\hline Chromium & 8.33 & 0.0500 & $\mathrm{mg} / \mathrm{kg}$ & 10 & 25-Jun-2009 & 26-Jun-2009 & SW 846/6020 & B \\
\hline Cobalt & 6.16 & 0.0500 & $\mathrm{mg} / \mathrm{kg}$ & 10 & 25-Jun-2009 & 26-Jun-2009 & SW 846/6020 & \\
\hline Copper & 5.33 & 0.0500 & $\mathrm{mg} / \mathrm{kg}$ & 10 & 25-Jun-2009 & 26-Jun-2009 & SW 846/6020 & \\
\hline Lead & 9.06 & 0.0500 & $\mathrm{mg} / \mathrm{kg}$ & 10 & 25-Jun-2009 & 26-Jun-2009 & SW 846/6020 & \\
\hline Manganese & 233 & 0.0500 & $\mathrm{mg} / \mathrm{kg}$ & 10 & 25-Jun-2009 & 26-Jun-2009 & SW 846/6020 & B \\
\hline Molybdenum & 0.273 & 0.0500 & $\mathrm{mg} / \mathrm{kg}$ & 10 & 25-Jun-2009 & 26-Jun-2009 & SW 846/6020 & \\
\hline Nickel & 9.96 & 0.0500 & $\mathrm{mg} / \mathrm{kg}$ & 10 & 25-Jun-2009 & 26-Jun-2009 & SW 846/6020 & \\
\hline Selenium & 0.271 & 0.0500 & $\mathrm{mg} / \mathrm{kg}$ & 10 & 25-Jun-2009 & 26-Jun-2009 & SW 846/6020 & \\
\hline Silver & 0.0779 & 0.0500 & $\mathrm{mg} / \mathrm{kg}$ & 10 & 25-Jun-2009 & 26-Jun-2009 & SW 846/6020 & \\
\hline Thallium & 0.103 & 0.0500 & $\mathrm{mg} / \mathrm{kg}$ & 10 & 25-Jun-2009 & 26-Jun-2009 & SW 846/6020 & \\
\hline Vanadium & 9.95 & 0.0500 & $\mathrm{mg} / \mathrm{kg}$ & 10 & 25-Jun-2009 & 26-Jun-2009 & SW 846/6020 & \\
\hline Zinc & 31.9 & 0.0500 & $\mathrm{mg} / \mathrm{kg}$ & 10 & 25-Jun-2009 & 26-Jun-2009 & SW 846/6020 & B \\
\hline
\end{tabular}




\section{USACE ERDC-EP-C \\ 3909 Halls Ferry Road \\ Vicksburg, MS 39180-6199}

ERDC - ECB $-,-$
Project: TVA Fly Ash Project

Project Manager: Tony Bednar
Reported:

03-Sep-2009

DRAFT: RF3-SD-MT-A Sediment

9061502-05 (Soil/Sediment)

\begin{tabular}{|c|c|c|c|c|c|c|c|c|}
\hline Analyte & Result & $\begin{array}{r}\text { porting } \\
\text { Limit }\end{array}$ & Units & Dilution & Prepared & Analyzed & Method & Notes \\
\hline
\end{tabular}

ERDC- EL-EP-C (Environmental Chemistry Branch)

DRAFT: Metals by EPA 6000/7000 Series Methods

\begin{tabular}{|c|c|c|c|c|c|c|c|c|}
\hline Mercury & 0.0110 & 0.00395 & $\mathrm{mg} / \mathrm{kg}$ & 1 & 06-Jul-2009 & 22-Jul-2009 & EPA 7471A & \\
\hline Strontium & 2.43 & 4.00 & $\mathrm{mg} / \mathrm{kg}$ & 2 & 25-Jun-2009 & 25-Jun-2009 & EPA $6010 \mathrm{~B}$ & J \\
\hline Aluminum & 4040 & 2.00 & $\mathrm{mg} / \mathrm{kg}$ & 2 & 25-Jun-2009 & 25-Jun-2009 & SW 846/6010 & B \\
\hline Calcium & 6150 & 2.00 & $\mathrm{mg} / \mathrm{kg}$ & 2 & 25-Jun-2009 & 25-Jun-2009 & SW 846/6010 & B \\
\hline Iron & 8670 & 2.00 & $\mathrm{mg} / \mathrm{kg}$ & 2 & 25-Jun-2009 & 25-Jun-2009 & SW 846/6010 & \\
\hline Magnesium & 1130 & 2.00 & $\mathrm{mg} / \mathrm{kg}$ & 2 & 25-Jun-2009 & 25-Jun-2009 & SW 846/6010 & B \\
\hline Potassium & 782 & 2.00 & $\mathrm{mg} / \mathrm{kg}$ & 2 & 25-Jun-2009 & 25-Jun-2009 & SW 846/6010 & B \\
\hline Sodium & 48.3 & 2.00 & $\mathrm{mg} / \mathrm{kg}$ & 2 & 25-Jun-2009 & 25-Jun-2009 & SW 846/6010 & B \\
\hline Antimony & 0.0162 & 0.0500 & $\mathrm{mg} / \mathrm{kg}$ & 10 & 25-Jun-2009 & 26-Jun-2009 & SW 846/6020 & $\mathrm{J}, \mathrm{B}$ \\
\hline Arsenic & 1.91 & 0.0500 & $\mathrm{mg} / \mathrm{kg}$ & 10 & 25-Jun-2009 & 26-Jun-2009 & SW 846/6020 & \\
\hline Barium & 33.6 & 0.0500 & $\mathrm{mg} / \mathrm{kg}$ & 10 & 25-Jun-2009 & 26-Jun-2009 & SW 846/6020 & B \\
\hline Beryllium & 0.496 & 0.0500 & $\mathrm{mg} / \mathrm{kg}$ & 10 & 25-Jun-2009 & 26-Jun-2009 & SW 846/6020 & \\
\hline Cadmium & 0.0971 & 0.0500 & $\mathrm{mg} / \mathrm{kg}$ & 10 & 25-Jun-2009 & 26-Jun-2009 & SW 846/6020 & \\
\hline Chromium & 9.11 & 0.0500 & $\mathrm{mg} / \mathrm{kg}$ & 10 & 25-Jun-2009 & 26-Jun-2009 & SW 846/6020 & B \\
\hline Cobalt & 6.27 & 0.0500 & $\mathrm{mg} / \mathrm{kg}$ & 10 & 25-Jun-2009 & 26-Jun-2009 & SW 846/6020 & \\
\hline Copper & 5.49 & 0.0500 & $\mathrm{mg} / \mathrm{kg}$ & 10 & 25-Jun-2009 & 26-Jun-2009 & SW 846/6020 & \\
\hline Lead & 9.33 & 0.0500 & $\mathrm{mg} / \mathrm{kg}$ & 10 & 25-Jun-2009 & 26-Jun-2009 & SW 846/6020 & \\
\hline Manganese & 209 & 0.0500 & $\mathrm{mg} / \mathrm{kg}$ & 10 & 25-Jun-2009 & 26-Jun-2009 & SW 846/6020 & B \\
\hline Molybdenum & 0.224 & 0.0500 & $\mathrm{mg} / \mathrm{kg}$ & 10 & 25-Jun-2009 & 26-Jun-2009 & SW 846/6020 & \\
\hline Nickel & 12.4 & 0.0500 & $\mathrm{mg} / \mathrm{kg}$ & 10 & 25-Jun-2009 & 26-Jun-2009 & SW 846/6020 & \\
\hline Selenium & 0.114 & 0.0500 & $\mathrm{mg} / \mathrm{kg}$ & 10 & 25-Jun-2009 & 26-Jun-2009 & SW 846/6020 & \\
\hline Silver & 0.0805 & 0.0500 & $\mathrm{mg} / \mathrm{kg}$ & 10 & 25-Jun-2009 & 26-Jun-2009 & SW 846/6020 & \\
\hline Thallium & 0.0595 & 0.0500 & $\mathrm{mg} / \mathrm{kg}$ & 10 & 25-Jun-2009 & 26-Jun-2009 & SW 846/6020 & \\
\hline Vanadium & 8.33 & 0.0500 & $\mathrm{mg} / \mathrm{kg}$ & 10 & 25-Jun-2009 & 26-Jun-2009 & SW 846/6020 & \\
\hline Zinc & 61.2 & 0.0500 & $\mathrm{mg} / \mathrm{kg}$ & 10 & 25-Jun-2009 & 26-Jun-2009 & SW 846/6020 & B \\
\hline
\end{tabular}




\section{USACE ERDC-EP-C \\ 3909 Halls Ferry Road \\ Vicksburg, MS 39180-6199}

ERDC - ECB

$-,-$
Project: TVA Fly Ash Project

Project Manager: Tony Bednar
Reported:

03-Sep-2009

DRAFT: RF4-SD-MT-A Sediment

9061502-06 (Soil/Sediment)

\begin{tabular}{|c|c|c|c|c|c|c|c|c|}
\hline Analyte & Result & $\begin{array}{r}\text { porting } \\
\text { Limit }\end{array}$ & Units & Dilution & Prepared & Analyzed & Method & Notes \\
\hline
\end{tabular}

ERDC- EL-EP-C (Environmental Chemistry Branch)

DRAFT: Metals by EPA 6000/7000 Series Methods

\begin{tabular}{|c|c|c|c|c|c|c|c|c|}
\hline Mercury & 0.0395 & 0.00399 & $\mathrm{mg} / \mathrm{kg}$ & 1 & 06-Jul-2009 & 22-Jul-2009 & EPA 7471A & \\
\hline Strontium & 3.25 & 4.00 & $\mathrm{mg} / \mathrm{kg}$ & 2 & 25-Jun-2009 & 25-Jun-2009 & EPA 6010B & $\mathrm{J}$ \\
\hline Aluminum & 8560 & 2.00 & $\mathrm{mg} / \mathrm{kg}$ & 2 & 25-Jun-2009 & 25-Jun-2009 & SW 846/6010 & B \\
\hline Calcium & 6860 & 2.00 & $\mathrm{mg} / \mathrm{kg}$ & 2 & 25-Jun-2009 & 25-Jun-2009 & SW 846/6010 & B \\
\hline Iron & 10900 & 2.00 & $\mathrm{mg} / \mathrm{kg}$ & 2 & 25-Jun-2009 & 25-Jun-2009 & SW 846/6010 & \\
\hline Magnesium & 1700 & 2.00 & $\mathrm{mg} / \mathrm{kg}$ & 2 & 25-Jun-2009 & 25-Jun-2009 & SW 846/6010 & B \\
\hline Potassium & 1080 & 2.00 & $\mathrm{mg} / \mathrm{kg}$ & 2 & 25-Jun-2009 & 25-Jun-2009 & SW 846/6010 & B \\
\hline Sodium & 49.9 & 2.00 & $\mathrm{mg} / \mathrm{kg}$ & 2 & 25-Jun-2009 & 25-Jun-2009 & SW 846/6010 & B \\
\hline Antimony & 0.0201 & 0.0500 & $\mathrm{mg} / \mathrm{kg}$ & 10 & 25-Jun-2009 & 26-Jun-2009 & SW 846/6020 & $\mathrm{J}, \mathrm{B}$ \\
\hline Arsenic & 2.42 & 0.0500 & $\mathrm{mg} / \mathrm{kg}$ & 10 & 25-Jun-2009 & 26-Jun-2009 & SW 846/6020 & \\
\hline Barium & 100 & 0.0500 & $\mathrm{mg} / \mathrm{kg}$ & 10 & 25-Jun-2009 & 26-Jun-2009 & SW 846/6020 & B \\
\hline Beryllium & 0.870 & 0.0500 & $\mathrm{mg} / \mathrm{kg}$ & 10 & 25-Jun-2009 & 26-Jun-2009 & SW 846/6020 & \\
\hline Cadmium & 0.248 & 0.0500 & $\mathrm{mg} / \mathrm{kg}$ & 10 & 25-Jun-2009 & 26-Jun-2009 & SW 846/6020 & \\
\hline Chromium & 13.0 & 0.0500 & $\mathrm{mg} / \mathrm{kg}$ & 10 & 25-Jun-2009 & 26-Jun-2009 & SW 846/6020 & B \\
\hline Cobalt & 8.76 & 0.0500 & $\mathrm{mg} / \mathrm{kg}$ & 10 & 25-Jun-2009 & 26-Jun-2009 & SW 846/6020 & \\
\hline Copper & 10.0 & 0.0500 & $\mathrm{mg} / \mathrm{kg}$ & 10 & 25-Jun-2009 & 26-Jun-2009 & SW 846/6020 & \\
\hline Lead & 16.8 & 0.0500 & $\mathrm{mg} / \mathrm{kg}$ & 10 & 25-Jun-2009 & 26-Jun-2009 & SW 846/6020 & \\
\hline Manganese & 724 & 0.0500 & $\mathrm{mg} / \mathrm{kg}$ & 10 & 25-Jun-2009 & 26-Jun-2009 & SW 846/6020 & B \\
\hline Molybdenum & 0.280 & 0.0500 & $\mathrm{mg} / \mathrm{kg}$ & 10 & 25-Jun-2009 & 26-Jun-2009 & SW 846/6020 & \\
\hline Nickel & 15.0 & 0.0500 & $\mathrm{mg} / \mathrm{kg}$ & 10 & 25-Jun-2009 & 26-Jun-2009 & SW 846/6020 & \\
\hline Selenium & 0.757 & 0.0500 & $\mathrm{mg} / \mathrm{kg}$ & 10 & 25-Jun-2009 & 26-Jun-2009 & SW 846/6020 & \\
\hline Silver & 0.104 & 0.0500 & $\mathrm{mg} / \mathrm{kg}$ & 10 & 25-Jun-2009 & 26-Jun-2009 & SW 846/6020 & \\
\hline Thallium & 0.131 & 0.0500 & $\mathrm{mg} / \mathrm{kg}$ & 10 & 25-Jun-2009 & 26-Jun-2009 & SW 846/6020 & \\
\hline Vanadium & 15.6 & 0.0500 & $\mathrm{mg} / \mathrm{kg}$ & 10 & 25-Jun-2009 & 26-Jun-2009 & SW 846/6020 & \\
\hline Zinc & 63.1 & 0.0500 & $\mathrm{mg} / \mathrm{kg}$ & 10 & 25-Jun-2009 & 26-Jun-2009 & SW 846/6020 & B \\
\hline
\end{tabular}




\section{USACE ERDC-EP-C \\ 3909 Halls Ferry Road \\ Vicksburg, MS 39180-6199}

ERDC - ECB

$-,-$
Project: TVA Fly Ash Project

Project Manager: Tony Bednar
Reported:

03-Sep-2009

DRAFT: RF5-SD-MT-A Sediment

9061502-07 (Soil/Sediment)

\begin{tabular}{|c|c|c|c|c|c|c|c|c|}
\hline Analyte & Result & $\begin{array}{r}\text { porting } \\
\text { Limit }\end{array}$ & Units & Dilution & Prepared & Analyzed & Method & Notes \\
\hline
\end{tabular}

ERDC- EL-EP-C (Environmental Chemistry Branch)

DRAFT: Metals by EPA 6000/7000 Series Methods

\begin{tabular}{|c|c|c|c|c|c|c|c|c|}
\hline Mercury & 0.0875 & 0.00399 & $\mathrm{mg} / \mathrm{kg}$ & 1 & 06-Jul-2009 & 22-Jul-2009 & EPA 7471A & \\
\hline Strontium & 27.0 & 4.00 & $\mathrm{mg} / \mathrm{kg}$ & 2 & 25-Jun-2009 & 25-Jun-2009 & EPA 6010B & \\
\hline Aluminum & 15100 & 2.00 & $\mathrm{mg} / \mathrm{kg}$ & 2 & 25-Jun-2009 & 25-Jun-2009 & SW 846/6010 & B \\
\hline Calcium & 14200 & 2.00 & $\mathrm{mg} / \mathrm{kg}$ & 2 & 25-Jun-2009 & 25-Jun-2009 & SW 846/6010 & B \\
\hline Iron & 39600 & 2.00 & $\mathrm{mg} / \mathrm{kg}$ & 2 & 25-Jun-2009 & 25-Jun-2009 & SW 846/6010 & \\
\hline Magnesium & 2070 & 2.00 & $\mathrm{mg} / \mathrm{kg}$ & 2 & 25-Jun-2009 & 25-Jun-2009 & SW 846/6010 & B \\
\hline Potassium & 1910 & 2.00 & $\mathrm{mg} / \mathrm{kg}$ & 2 & 25-Jun-2009 & 25-Jun-2009 & SW 846/6010 & B \\
\hline Sodium & 182 & 2.00 & $\mathrm{mg} / \mathrm{kg}$ & 2 & 25-Jun-2009 & 25-Jun-2009 & SW 846/6010 & B \\
\hline Antimony & 0.0500 & 0.0500 & $\mathrm{mg} / \mathrm{kg}$ & 10 & 25-Jun-2009 & 26-Jun-2009 & SW 846/6020 & B \\
\hline Arsenic & 10.9 & 0.0500 & $\mathrm{mg} / \mathrm{kg}$ & 10 & 25-Jun-2009 & 26-Jun-2009 & SW 846/6020 & \\
\hline Barium & 118 & 0.0500 & $\mathrm{mg} / \mathrm{kg}$ & 10 & 25-Jun-2009 & 26-Jun-2009 & SW 846/6020 & B \\
\hline Beryllium & 1.51 & 0.0500 & $\mathrm{mg} / \mathrm{kg}$ & 10 & 25-Jun-2009 & 26-Jun-2009 & SW 846/6020 & \\
\hline Cadmium & 0.208 & 0.0500 & $\mathrm{mg} / \mathrm{kg}$ & 10 & 25-Jun-2009 & 26-Jun-2009 & SW 846/6020 & \\
\hline Chromium & 27.9 & 0.0500 & $\mathrm{mg} / \mathrm{kg}$ & 10 & 25-Jun-2009 & 26-Jun-2009 & SW 846/6020 & B \\
\hline Cobalt & 9.92 & 0.0500 & $\mathrm{mg} / \mathrm{kg}$ & 10 & 25-Jun-2009 & 26-Jun-2009 & SW 846/6020 & \\
\hline Copper & 24.0 & 0.0500 & $\mathrm{mg} / \mathrm{kg}$ & 10 & 25-Jun-2009 & 26-Jun-2009 & SW 846/6020 & \\
\hline Lead & 45.3 & 0.0500 & $\mathrm{mg} / \mathrm{kg}$ & 10 & 25-Jun-2009 & 26-Jun-2009 & SW 846/6020 & \\
\hline Manganese & 518 & 0.0500 & $\mathrm{mg} / \mathrm{kg}$ & 10 & 25-Jun-2009 & 26-Jun-2009 & SW 846/6020 & B \\
\hline Molybdenum & 1.03 & 0.0500 & $\mathrm{mg} / \mathrm{kg}$ & 10 & 25-Jun-2009 & 26-Jun-2009 & SW 846/6020 & \\
\hline Nickel & 16.9 & 0.0500 & $\mathrm{mg} / \mathrm{kg}$ & 10 & 25-Jun-2009 & 26-Jun-2009 & SW 846/6020 & \\
\hline Selenium & 1.48 & 0.0500 & $\mathrm{mg} / \mathrm{kg}$ & 10 & 25-Jun-2009 & 26-Jun-2009 & SW 846/6020 & \\
\hline Silver & 0.172 & 0.0500 & $\mathrm{mg} / \mathrm{kg}$ & 10 & 25-Jun-2009 & 26-Jun-2009 & SW 846/6020 & \\
\hline Thallium & 0.297 & 0.0500 & $\mathrm{mg} / \mathrm{kg}$ & 10 & 25-Jun-2009 & 26-Jun-2009 & SW 846/6020 & \\
\hline Vanadium & 33.8 & 0.0500 & $\mathrm{mg} / \mathrm{kg}$ & 10 & 25-Jun-2009 & 26-Jun-2009 & SW 846/6020 & \\
\hline Zinc & 64.8 & 0.0500 & $\mathrm{mg} / \mathrm{kg}$ & 10 & 25-Jun-2009 & 26-Jun-2009 & SW 846/6020 & B \\
\hline
\end{tabular}




\section{USACE ERDC-EP-C \\ 3909 Halls Ferry Road \\ Vicksburg, MS 39180-6199}

ERDC - ECB

$-,-$
Project: TVA Fly Ash Project

Project Manager: Tony Bednar
Reported:

03-Sep-2009

DRAFT: RF6-SD-MT-A Sediment

9061502-08 (Soil/Sediment)

\begin{tabular}{|c|c|c|c|c|c|c|c|c|}
\hline Analyte & Result & $\begin{array}{r}\text { porting } \\
\text { Limit }\end{array}$ & Units & Dilution & Prepared & Analyzed & Method & Notes \\
\hline
\end{tabular}

ERDC- EL-EP-C (Environmental Chemistry Branch)

DRAFT: Metals by EPA 6000/7000 Series Methods

\begin{tabular}{|c|c|c|c|c|c|c|c|c|}
\hline Mercury & 0.0221 & 0.00397 & $\mathrm{mg} / \mathrm{kg}$ & 1 & 06-Jul-2009 & 22-Jul-2009 & EPA 7471A & \\
\hline Strontium & 3.08 & 4.00 & $\mathrm{mg} / \mathrm{kg}$ & 2 & 25-Jun-2009 & 25-Jun-2009 & EPA 6010B & $\mathrm{J}$ \\
\hline Aluminum & 6070 & 2.00 & $\mathrm{mg} / \mathrm{kg}$ & 2 & 25-Jun-2009 & 25-Jun-2009 & SW 846/6010 & B \\
\hline Calcium & 7320 & 2.00 & $\mathrm{mg} / \mathrm{kg}$ & 2 & 25-Jun-2009 & 25-Jun-2009 & SW 846/6010 & B \\
\hline Iron & 10900 & 2.00 & $\mathrm{mg} / \mathrm{kg}$ & 2 & 25-Jun-2009 & 25-Jun-2009 & SW 846/6010 & \\
\hline Magnesium & 1610 & 2.00 & $\mathrm{mg} / \mathrm{kg}$ & 2 & 25-Jun-2009 & 25-Jun-2009 & SW 846/6010 & B \\
\hline Potassium & 1060 & 2.00 & $\mathrm{mg} / \mathrm{kg}$ & 2 & 25-Jun-2009 & 25-Jun-2009 & SW 846/6010 & B \\
\hline Sodium & 54.1 & 2.00 & $\mathrm{mg} / \mathrm{kg}$ & 2 & 25-Jun-2009 & 25-Jun-2009 & SW 846/6010 & B \\
\hline Antimony & 0.0236 & 0.0500 & $\mathrm{mg} / \mathrm{kg}$ & 10 & 25-Jun-2009 & 26-Jun-2009 & SW 846/6020 & $\mathrm{J}, \mathrm{B}$ \\
\hline Arsenic & 3.15 & 0.0500 & $\mathrm{mg} / \mathrm{kg}$ & 10 & 25-Jun-2009 & 26-Jun-2009 & SW 846/6020 & \\
\hline Barium & 56.0 & 0.0500 & $\mathrm{mg} / \mathrm{kg}$ & 10 & 25-Jun-2009 & 26-Jun-2009 & SW 846/6020 & B \\
\hline Beryllium & 0.707 & 0.0500 & $\mathrm{mg} / \mathrm{kg}$ & 10 & 25-Jun-2009 & 26-Jun-2009 & SW 846/6020 & \\
\hline Cadmium & 0.117 & 0.0500 & $\mathrm{mg} / \mathrm{kg}$ & 10 & 25-Jun-2009 & 26-Jun-2009 & SW 846/6020 & \\
\hline Chromium & 11.1 & 0.0500 & $\mathrm{mg} / \mathrm{kg}$ & 10 & 25-Jun-2009 & 26-Jun-2009 & SW 846/6020 & B \\
\hline Cobalt & 8.41 & 0.0500 & $\mathrm{mg} / \mathrm{kg}$ & 10 & 25-Jun-2009 & 26-Jun-2009 & SW 846/6020 & \\
\hline Copper & 7.59 & 0.0500 & $\mathrm{mg} / \mathrm{kg}$ & 10 & 25-Jun-2009 & 26-Jun-2009 & SW 846/6020 & \\
\hline Lead & 12.9 & 0.0500 & $\mathrm{mg} / \mathrm{kg}$ & 10 & 25-Jun-2009 & 26-Jun-2009 & SW 846/6020 & \\
\hline Manganese & 453 & 0.0500 & $\mathrm{mg} / \mathrm{kg}$ & 10 & 25-Jun-2009 & 26-Jun-2009 & SW 846/6020 & B \\
\hline Molybdenum & 0.284 & 0.0500 & $\mathrm{mg} / \mathrm{kg}$ & 10 & 25-Jun-2009 & 26-Jun-2009 & SW 846/6020 & \\
\hline Nickel & 14.8 & 0.0500 & $\mathrm{mg} / \mathrm{kg}$ & 10 & 25-Jun-2009 & 26-Jun-2009 & SW 846/6020 & \\
\hline Selenium & 0.473 & 0.0500 & $\mathrm{mg} / \mathrm{kg}$ & 10 & 25-Jun-2009 & 26-Jun-2009 & SW 846/6020 & \\
\hline Silver & 0.0667 & 0.0500 & $\mathrm{mg} / \mathrm{kg}$ & 10 & 25-Jun-2009 & 26-Jun-2009 & SW 846/6020 & \\
\hline Thallium & 0.0947 & 0.0500 & $\mathrm{mg} / \mathrm{kg}$ & 10 & 25-Jun-2009 & 26-Jun-2009 & SW 846/6020 & \\
\hline Vanadium & 12.8 & 0.0500 & $\mathrm{mg} / \mathrm{kg}$ & 10 & 25-Jun-2009 & 26-Jun-2009 & SW 846/6020 & \\
\hline Zinc & 50.5 & 0.0500 & $\mathrm{mg} / \mathrm{kg}$ & 10 & 25-Jun-2009 & 26-Jun-2009 & SW 846/6020 & B \\
\hline
\end{tabular}




\section{USACE ERDC-EP-C \\ 3909 Halls Ferry Road \\ Vicksburg, MS 39180-6199}

ERDC - ECB

Project: TVA Fly Ash Project

$-$

Reported:

Project Manager: Tony Bednar

03-Sep-2009

DRAFT: PIL-FA-MT-A

9061502-09 (Soil/Sediment)

\begin{tabular}{|c|c|c|c|c|c|c|c|c|}
\hline Analyte & Result & porting & Units & Dilution & Prenared & Analyzed & Method & Notes \\
\hline Analyte & Kesult & Limit & & & Prepared & & Metnod & \\
\hline
\end{tabular}

ERDC- EL-EP-C (Environmental Chemistry Branch)

DRAFT: Metals by EPA 6000/7000 Series Methods

\begin{tabular}{|c|c|c|c|c|c|c|c|c|}
\hline Mercury & 0.0279 & 0.00396 & $\mathrm{mg} / \mathrm{kg}$ & 1 & 06-Jul-2009 & 22-Jul-2009 & EPA 7471A & \\
\hline Strontium & 64.3 & 4.00 & $\mathrm{mg} / \mathrm{kg}$ & 2 & 25-Jun-2009 & 25-Jun-2009 & EPA $6010 \mathrm{~B}$ & \\
\hline Aluminum & 18600 & 2.00 & $\mathrm{mg} / \mathrm{kg}$ & 2 & 25-Jun-2009 & 25-Jun-2009 & SW 846/6010 & B \\
\hline Calcium & 6780 & 2.00 & $\mathrm{mg} / \mathrm{kg}$ & 2 & 25-Jun-2009 & 25-Jun-2009 & SW 846/6010 & B \\
\hline Iron & 17500 & 2.00 & $\mathrm{mg} / \mathrm{kg}$ & 2 & 25-Jun-2009 & 25-Jun-2009 & SW 846/6010 & \\
\hline Magnesium & 1940 & 2.00 & $\mathrm{mg} / \mathrm{kg}$ & 2 & 25-Jun-2009 & 25-Jun-2009 & SW 846/6010 & B \\
\hline Potassium & 2760 & 2.00 & $\mathrm{mg} / \mathrm{kg}$ & 2 & 25-Jun-2009 & 25-Jun-2009 & SW 846/6010 & B \\
\hline Sodium & 482 & 2.00 & $\mathrm{mg} / \mathrm{kg}$ & 2 & 25-Jun-2009 & 25-Jun-2009 & SW 846/6010 & B \\
\hline Antimony & 0.145 & 0.0500 & $\mathrm{mg} / \mathrm{kg}$ & 10 & 25-Jun-2009 & 26-Jun-2009 & SW 846/6020 & B \\
\hline Arsenic & 46.9 & 0.0500 & $\mathrm{mg} / \mathrm{kg}$ & 10 & 25-Jun-2009 & 26-Jun-2009 & SW 846/6020 & \\
\hline Barium & 510 & 0.0500 & $\mathrm{mg} / \mathrm{kg}$ & 10 & 25-Jun-2009 & 26-Jun-2009 & SW 846/6020 & B \\
\hline Beryllium & 4.88 & 0.0500 & $\mathrm{mg} / \mathrm{kg}$ & 10 & 25-Jun-2009 & 26-Jun-2009 & SW 846/6020 & \\
\hline Cadmium & 0.272 & 0.0500 & $\mathrm{mg} / \mathrm{kg}$ & 10 & 25-Jun-2009 & 26-Jun-2009 & SW 846/6020 & \\
\hline Chromium & 33.5 & 0.0500 & $\mathrm{mg} / \mathrm{kg}$ & 10 & 25-Jun-2009 & 26-Jun-2009 & SW 846/6020 & B \\
\hline Cobalt & 16.9 & 0.0500 & $\mathrm{mg} / \mathrm{kg}$ & 10 & 25-Jun-2009 & 26-Jun-2009 & SW 846/6020 & \\
\hline Copper & 50.0 & 0.0500 & $\mathrm{mg} / \mathrm{kg}$ & 10 & 25-Jun-2009 & 26-Jun-2009 & SW 846/6020 & \\
\hline Lead & 20.2 & 0.0500 & $\mathrm{mg} / \mathrm{kg}$ & 10 & 25-Jun-2009 & 26-Jun-2009 & SW 846/6020 & \\
\hline Manganese & 88.7 & 0.0500 & $\mathrm{mg} / \mathrm{kg}$ & 10 & 25-Jun-2009 & 26-Jun-2009 & SW 846/6020 & B \\
\hline Molybdenum & 3.20 & 0.0500 & $\mathrm{mg} / \mathrm{kg}$ & 10 & 25-Jun-2009 & 26-Jun-2009 & SW 846/6020 & \\
\hline Nickel & 34.0 & 0.0500 & $\mathrm{mg} / \mathrm{kg}$ & 10 & 25-Jun-2009 & 26-Jun-2009 & SW 846/6020 & \\
\hline Selenium & 5.44 & 0.0500 & $\mathrm{mg} / \mathrm{kg}$ & 10 & 25-Jun-2009 & 26-Jun-2009 & SW 846/6020 & \\
\hline Silver & 0.220 & 0.0500 & $\mathrm{mg} / \mathrm{kg}$ & 10 & 25-Jun-2009 & 26-Jun-2009 & SW 846/6020 & \\
\hline Thallium & 1.35 & 0.0500 & $\mathrm{mg} / \mathrm{kg}$ & 10 & 25-Jun-2009 & 26-Jun-2009 & SW 846/6020 & \\
\hline Vanadium & 86.2 & 0.0500 & $\mathrm{mg} / \mathrm{kg}$ & 10 & 25-Jun-2009 & 26-Jun-2009 & SW 846/6020 & \\
\hline Zinc & 41.5 & 0.0500 & $\mathrm{mg} / \mathrm{kg}$ & 10 & 25-Jun-2009 & 26-Jun-2009 & SW 846/6020 & B \\
\hline
\end{tabular}




\section{USACE ERDC-EP-C \\ 3909 Halls Ferry Road \\ Vicksburg, MS 39180-6199}

ERDC - ECB

$-\cdot$,
Project: TVA Fly Ash Project

Project Manager: Tony Bednar
Reported:

03-Sep-2009

DRAFT: EFL - WA - MT - A (Effluent from Settling Pond)

9061502-10 (Water)

\begin{tabular}{|c|c|c|c|c|c|c|c|c|}
\hline Analyte & Result & $\begin{array}{r}\text { porting } \\
\text { Limit }\end{array}$ & Units & Dilution & Prepared & Analyzed & Method & Notes \\
\hline
\end{tabular}

ERDC- EL-EP-C (Environmental Chemistry Branch)

DRAFT: Wet Chemistry

\begin{tabular}{llllllll}
\hline Hardness & $\mathbf{1 1 7}$ & 1.32 & $\begin{array}{c}\text { mg equil } \\
\text { CaCO3/ }\end{array}$ & 1 & 25-Jun-2009 & 25-Jun-2009
\end{tabular}

DRAFT: Metals by EPA 6000/7000 Series Methods

\begin{tabular}{|c|c|c|c|c|c|c|c|c|}
\hline Mercury & 0.000014 & 0.000005 & $\mathrm{mg} / \mathrm{L}$ & 1 & 17-Jun-2009 & 01-Jul-2009 & EPA 7471A & \\
\hline Strontium & 0.353 & 0.0200 & $\mathrm{mg} / \mathrm{L}$ & 1 & 25-Jun-2009 & 25-Jun-2009 & EPA $6010 \mathrm{~B}$ & \\
\hline Aluminum & 0.281 & 0.200 & $\mathrm{mg} / \mathrm{L}$ & 1 & 25-Jun-2009 & 25-Jun-2009 & SW 846/6010 & \\
\hline Calcium & 34.7 & 0.200 & $\mathrm{mg} / \mathrm{L}$ & 1 & 25-Jun-2009 & 25-Jun-2009 & SW 846/6010 & \\
\hline Iron & ND & 0.200 & $\mathrm{mg} / \mathrm{L}$ & 1 & 25-Jun-2009 & 25-Jun-2009 & SW 846/6010 & $\mathrm{U}$ \\
\hline Magnesium & 7.34 & 0.200 & $\mathrm{mg} / \mathrm{L}$ & 1 & 25-Jun-2009 & 25-Jun-2009 & SW 846/6010 & \\
\hline Potassium & 2.30 & 0.200 & $\mathrm{mg} / \mathrm{L}$ & 1 & 25-Jun-2009 & 25-Jun-2009 & SW 846/6010 & \\
\hline Sodium & 7.79 & 0.200 & $\mathrm{mg} / \mathrm{L}$ & 1 & 25-Jun-2009 & 25-Jun-2009 & SW 846/6010 & \\
\hline Antimony & 0.0023 & 0.0010 & $\mathrm{mg} / \mathrm{L}$ & 2 & 16-Jun-2009 & 16-Jun-2009 & SW 846/6020 & \\
\hline Arsenic & 0.0208 & 0.0010 & $\mathrm{mg} / \mathrm{L}$ & 2 & 16-Jun-2009 & 16-Jun-2009 & SW 846/6020 & \\
\hline Barium & 0.175 & 0.0010 & $\mathrm{mg} / \mathrm{L}$ & 2 & 16-Jun-2009 & 16-Jun-2009 & SW 846/6020 & \\
\hline Beryllium & ND & 0.0010 & $\mathrm{mg} / \mathrm{L}$ & 2 & 16-Jun-2009 & 16-Jun-2009 & SW 846/6020 & $\mathrm{U}$ \\
\hline Cadmium & ND & 0.0010 & $\mathrm{mg} / \mathrm{L}$ & 2 & 16-Jun-2009 & 16-Jun-2009 & SW 846/6020 & $\mathrm{U}$ \\
\hline Chromium & 0.0041 & 0.0010 & $\mathrm{mg} / \mathrm{L}$ & 2 & 16-Jun-2009 & 16-Jun-2009 & SW 846/6020 & \\
\hline Cobalt & ND & 0.0010 & $\mathrm{mg} / \mathrm{L}$ & 2 & 16-Jun-2009 & 16-Jun-2009 & SW 846/6020 & $\mathrm{U}$ \\
\hline Copper & 0.0012 & 0.0010 & $\mathrm{mg} / \mathrm{L}$ & 2 & 16-Jun-2009 & 16-Jun-2009 & SW 846/6020 & \\
\hline Lead & ND & 0.0010 & $\mathrm{mg} / \mathrm{L}$ & 2 & 16-Jun-2009 & 16-Jun-2009 & SW 846/6020 & $\mathrm{U}$ \\
\hline Manganese & ND & 0.0010 & $\mathrm{mg} / \mathrm{L}$ & 2 & 16-Jun-2009 & 16-Jun-2009 & SW 846/6020 & $\mathrm{U}$ \\
\hline Molybdenum & 0.0236 & 0.0010 & $\mathrm{mg} / \mathrm{L}$ & 2 & 16-Jun-2009 & 16-Jun-2009 & SW 846/6020 & \\
\hline Nickel & 0.0013 & 0.0010 & $\mathrm{mg} / \mathrm{L}$ & 2 & 16-Jun-2009 & 16-Jun-2009 & SW 846/6020 & \\
\hline Selenium & 0.0054 & 0.0010 & $\mathrm{mg} / \mathrm{L}$ & 2 & 16-Jun-2009 & 16-Jun-2009 & SW 846/6020 & \\
\hline Silver & 0.0010 & 0.0010 & $\mathrm{mg} / \mathrm{L}$ & 2 & 16-Jun-2009 & 16-Jun-2009 & SW 846/6020 & B \\
\hline Thallium & 0.00029 & 0.0010 & $\mathrm{mg} / \mathrm{L}$ & 2 & 16-Jun-2009 & 16-Jun-2009 & SW 846/6020 & $\mathrm{J}$ \\
\hline Vanadium & 0.0317 & 0.0010 & $\mathrm{mg} / \mathrm{L}$ & 2 & 16-Jun-2009 & 16-Jun-2009 & SW 846/6020 & \\
\hline Zinc & 0.0064 & 0.0010 & $\mathrm{mg} / \mathrm{L}$ & 2 & 16-Jun-2009 & 16-Jun-2009 & SW 846/6020 & $\mathrm{B}$ \\
\hline
\end{tabular}




\section{USACE ERDC-EP-C \\ 3909 Halls Ferry Road \\ Vicksburg, MS 39180-6199}

ERDC - ECB

Project: TVA Fly Ash Project

Reported:

Project Manager: Tony Bednar

03-Sep-2009

DRAFT: EFL - WA - MT - A (Effluent from Settling Pond)

9061502-10 (Water)

\begin{tabular}{|lrrrrrrrr}
\hline & & & & & & & & \\
Analyte & Result & Leporting & & & & \\
Limit & Units & Dilution & Prepared & Analyzed & Method & Notes \\
\hline
\end{tabular}

ERDC- EL-EP-C (Environmental Chemistry Branch)

DRAFT: Miscellaneous Physical/Conventional Chemistry Parameters

\begin{tabular}{lrrrrrrr}
\hline Bromide & $\mathrm{ND}$ & 0.100 & $\mathrm{mg} / \mathrm{L}$ & 1 & 14-Jul-2009 & $14-J u l-2009$ & EPA 300.0 \\
Chloride & $\mathbf{7 . 6 3}$ & 1.00 & $\mathrm{mg} / \mathrm{L}$ & 1 & $14-J u l-2009$ & $14-J u l-2009$ & EPA 300.0 \\
Nitrate as N & $\mathrm{ND}$ & 0.100 & $\mathrm{mg} / \mathrm{L}$ & 1 & $14-J u l-2009$ & $14-J u l-2009$ & EPA 300.0 \\
Sulfate & $\mathbf{4 7 . 9}$ & 0.100 & $\mathrm{mg} / \mathrm{L}$ & 1 & $14-J u l-2009$ & $14-J u l-2009$ & EPA 300.0
\end{tabular}




\section{USACE ERDC-EP-C \\ 3909 Halls Ferry Road \\ Vicksburg, MS 39180-6199}

ERDC - ECB

Project: TVA Fly Ash Project

$-$

Project Manager: Tony Bednar

Reported:

$--,-$

03-Sep-2009

DRAFT: Emory River Water

9061502-11 (Water)

\begin{tabular}{|c|c|c|c|c|c|c|c|c|}
\hline & & porting & & & & & & \\
\hline Analyte & Result & Limit & Units & Dilution & Prepared & Analyzed & Method & Notes \\
\hline
\end{tabular}

ERDC- EL-EP-C (Environmental Chemistry Branch)

DRAFT: Wet Chemistry

\begin{tabular}{llllllll}
\hline Hardness & $\mathbf{3 0 . 0}$ & 1.32 & $\begin{array}{c}\mathrm{mg} \text { equil } \\
\text { CaCO3/ }\end{array}$ & 1 & 25-Jun-2009 & 25-Jun-2009 & Hardness
\end{tabular}

$\mathrm{L}$

DRAFT: Metals by EPA 6000/7000 Series Methods

\begin{tabular}{|c|c|c|c|c|c|c|c|c|}
\hline Mercury & ND & 0.000005 & $\mathrm{mg} / \mathrm{L}$ & 1 & 17-Jun-2009 & 01-Jul-2009 & EPA 7471A & $\mathrm{U}$ \\
\hline Strontium & 0.0424 & 0.0200 & $\mathrm{mg} / \mathrm{L}$ & 1 & 25-Jun-2009 & 25-Jun-2009 & EPA 6010B & \\
\hline Aluminum & ND & 0.200 & $\mathrm{mg} / \mathrm{L}$ & 1 & 25-Jun-2009 & 25-Jun-2009 & SW 846/6010 & $\mathrm{U}$ \\
\hline Calcium & 8.55 & 0.200 & $\mathrm{mg} / \mathrm{L}$ & 1 & 25-Jun-2009 & 25-Jun-2009 & SW 846/6010 & \\
\hline Iron & 0.0525 & 0.200 & $\mathrm{mg} / \mathrm{L}$ & 1 & 25-Jun-2009 & 25-Jun-2009 & SW 846/6010 & $\mathrm{J}$ \\
\hline Magnesium & 2.10 & 0.200 & $\mathrm{mg} / \mathrm{L}$ & 1 & 25-Jun-2009 & 25-Jun-2009 & SW 846/6010 & \\
\hline Potassium & 1.29 & 0.200 & $\mathrm{mg} / \mathrm{L}$ & 1 & 25-Jun-2009 & 25-Jun-2009 & SW 846/6010 & \\
\hline Sodium & 2.44 & 0.200 & $\mathrm{mg} / \mathrm{L}$ & 1 & 25-Jun-2009 & 25-Jun-2009 & SW 846/6010 & \\
\hline Antimony & ND & 0.0010 & $\mathrm{mg} / \mathrm{L}$ & 2 & 16-Jun-2009 & 16-Jun-2009 & SW 846/6020 & $\mathrm{U}$ \\
\hline Arsenic & 0.0008 & 0.0010 & $\mathrm{mg} / \mathrm{L}$ & 2 & 16-Jun-2009 & 16-Jun-2009 & SW 846/6020 & J \\
\hline Barium & 0.0307 & 0.0010 & $\mathrm{mg} / \mathrm{L}$ & 2 & 16-Jun-2009 & 16-Jun-2009 & SW 846/6020 & \\
\hline Beryllium & ND & 0.0010 & $\mathrm{mg} / \mathrm{L}$ & 2 & 16-Jun-2009 & 16-Jun-2009 & SW 846/6020 & $\mathrm{U}$ \\
\hline Cadmium & ND & 0.0010 & $\mathrm{mg} / \mathrm{L}$ & 2 & 16-Jun-2009 & 16-Jun-2009 & SW 846/6020 & $\mathrm{U}$ \\
\hline Chromium & ND & 0.0010 & $\mathrm{mg} / \mathrm{L}$ & 2 & 16-Jun-2009 & 16-Jun-2009 & SW 846/6020 & $\mathrm{U}$ \\
\hline Cobalt & ND & 0.0010 & $\mathrm{mg} / \mathrm{L}$ & 2 & 16-Jun-2009 & 16-Jun-2009 & SW 846/6020 & $\mathrm{U}$ \\
\hline Copper & 0.0005 & 0.0010 & $\mathrm{mg} / \mathrm{L}$ & 2 & 16-Jun-2009 & 16-Jun-2009 & SW 846/6020 & $\mathrm{J}$ \\
\hline Lead & ND & 0.0010 & $\mathrm{mg} / \mathrm{L}$ & 2 & 16-Jun-2009 & 16-Jun-2009 & SW 846/6020 & $\mathrm{U}$ \\
\hline Manganese & 0.0005 & 0.0010 & $\mathrm{mg} / \mathrm{L}$ & 2 & 16-Jun-2009 & 16-Jun-2009 & SW 846/6020 & $\mathrm{J}$ \\
\hline Molybdenum & ND & 0.0010 & $\mathrm{mg} / \mathrm{L}$ & 2 & 16-Jun-2009 & 16-Jun-2009 & SW 846/6020 & $\mathrm{U}$ \\
\hline Nickel & 0.0009 & 0.0010 & $\mathrm{mg} / \mathrm{L}$ & 2 & 16-Jun-2009 & 16-Jun-2009 & SW 846/6020 & $\mathrm{J}$ \\
\hline Selenium & ND & 0.0010 & $\mathrm{mg} / \mathrm{L}$ & 2 & 16-Jun-2009 & 16-Jun-2009 & SW 846/6020 & $\mathrm{U}$ \\
\hline Silver & 0.0005 & 0.0010 & $\mathrm{mg} / \mathrm{L}$ & 2 & 16-Jun-2009 & 16-Jun-2009 & SW 846/6020 & $\mathrm{J}, \mathrm{B}$ \\
\hline Thallium & ND & 0.0010 & $\mathrm{mg} / \mathrm{L}$ & 2 & 16-Jun-2009 & 16-Jun-2009 & SW 846/6020 & $\mathrm{U}$ \\
\hline Vanadium & ND & 0.0010 & $\mathrm{mg} / \mathrm{L}$ & 2 & 16-Jun-2009 & 16-Jun-2009 & SW 846/6020 & $\mathrm{U}$ \\
\hline Zinc & 0.0057 & 0.0010 & $\mathrm{mg} / \mathrm{L}$ & 2 & 16-Jun-2009 & 16-Jun-2009 & SW 846/6020 & B \\
\hline
\end{tabular}




\section{USACE ERDC-EP-C \\ 3909 Halls Ferry Road \\ Vicksburg, MS 39180-6199}

\begin{tabular}{|c|c|c|}
\hline ERDC - ECB & Project: TVA Fly Ash Project & \\
\hline- & & Reported: \\
\hline,--- & Project Manager: Tony Bednar & 03-Sep-2009 \\
\hline
\end{tabular}

DRAFT: Emory River Water

9061502-11 (Water)

\begin{tabular}{|c|c|c|c|c|c|c|c|c|}
\hline & & porting & & & & & & \\
\hline Analyte & Result & Limit & Units & Dilution & Prepared & Analyzed & Method & Notes \\
\hline
\end{tabular}

ERDC- EL-EP-C (Environmental Chemistry Branch)

DRAFT: Miscellaneous Physical/Conventional Chemistry Parameters

\begin{tabular}{lrrrrrrr}
\hline Bromide & $\mathrm{ND}$ & 0.100 & $\mathrm{mg} / \mathrm{L}$ & 1 & 14-Jul-2009 & 14-Jul-2009 & EPA 300.0 \\
Chloride & $\mathbf{2 . 2 6}$ & 1.00 & $\mathrm{mg} / \mathrm{L}$ & 1 & $14-J u l-2009$ & $14-\mathrm{Jul}-2009$ & EPA 300.0 \\
Nitrate as N & $\mathbf{0 . 3 6 5}$ & 0.100 & $\mathrm{mg} / \mathrm{L}$ & 1 & $14-J u l-2009$ & $14-J u l-2009$ & EPA 300.0 \\
Sulfate & $\mathbf{1 1 . 5}$ & 0.100 & $\mathrm{mg} / \mathrm{L}$ & 1 & $14-J u l-2009$ & $14-J u l-2009$ & EPA 300.0
\end{tabular}




\section{USACE ERDC-EP-C \\ 3909 Halls Ferry Road \\ Vicksburg, MS 39180-6199}

ERDC - ECB

Project: TVA Fly Ash Project

Project Manager: Tony Bednar

Reported:

03-Sep-2009

DRAFT: SLC Water

9061502-12 (Water)

\begin{tabular}{|c|c|c|c|c|c|c|c|c|}
\hline Analyte & Result & $\begin{array}{r}\text { porting } \\
\text { Limit }\end{array}$ & Units & Dilution & Prepared & Analyzed & Method & Notes \\
\hline
\end{tabular}

ERDC- EL-EP-C (Environmental Chemistry Branch)

DRAFT: Wet Chemistry

\begin{tabular}{llllllll}
\hline Hardness & $\mathbf{9 7 . 9}$ & 1.32 & $\begin{array}{c}\text { mg equil } \\
\text { CaCO3/ }\end{array}$ & 1 & 25-Jun-2009 & 25-Jun-2009 & Hardness
\end{tabular}

$\mathrm{L}$

DRAFT: Metals by EPA 6000/7000 Series Methods

\begin{tabular}{|c|c|c|c|c|c|c|c|c|}
\hline Mercury & ND & 0.000005 & $\mathrm{mg} / \mathrm{L}$ & 1 & 17-Jun-2009 & 01-Jul-2009 & EPA 7471A & $\mathrm{U}$ \\
\hline Strontium & 0.544 & 0.0200 & $\mathrm{mg} / \mathrm{L}$ & 1 & 25-Jun-2009 & 25-Jun-2009 & EPA 6010B & \\
\hline Aluminum & 0.252 & 0.200 & $\mathrm{mg} / \mathrm{L}$ & 1 & 25-Jun-2009 & 25-Jun-2009 & SW 846/6010 & \\
\hline Calcium & 30.7 & 0.200 & $\mathrm{mg} / \mathrm{L}$ & 1 & 25-Jun-2009 & 25-Jun-2009 & SW 846/6010 & \\
\hline Iron & ND & 0.200 & $\mathrm{mg} / \mathrm{L}$ & 1 & 25-Jun-2009 & 25-Jun-2009 & SW 846/6010 & $\mathrm{U}$ \\
\hline Magnesium & 5.15 & 0.200 & $\mathrm{mg} / \mathrm{L}$ & 1 & 25-Jun-2009 & 25-Jun-2009 & SW 846/6010 & \\
\hline Potassium & 1.88 & 0.200 & $\mathrm{mg} / \mathrm{L}$ & 1 & 25-Jun-2009 & 25-Jun-2009 & SW 846/6010 & \\
\hline Sodium & 4.05 & 0.200 & $\mathrm{mg} / \mathrm{L}$ & 1 & 25-Jun-2009 & 25-Jun-2009 & SW 846/6010 & \\
\hline Antimony & 0.0081 & 0.0010 & $\mathrm{mg} / \mathrm{L}$ & 2 & 16-Jun-2009 & 16-Jun-2009 & SW 846/6020 & \\
\hline Arsenic & 0.0779 & 0.0010 & $\mathrm{mg} / \mathrm{L}$ & 2 & 16-Jun-2009 & 16-Jun-2009 & SW 846/6020 & \\
\hline Barium & 0.107 & 0.0010 & $\mathrm{mg} / \mathrm{L}$ & 2 & 16-Jun-2009 & 16-Jun-2009 & SW 846/6020 & \\
\hline Beryllium & ND & 0.0010 & $\mathrm{mg} / \mathrm{L}$ & 2 & 16-Jun-2009 & 16-Jun-2009 & SW 846/6020 & U \\
\hline Cadmium & 0.0003 & 0.0010 & $\mathrm{mg} / \mathrm{L}$ & 2 & 16-Jun-2009 & 16-Jun-2009 & SW 846/6020 & $\mathrm{J}$ \\
\hline Chromium & ND & 0.0010 & $\mathrm{mg} / \mathrm{L}$ & 2 & 16-Jun-2009 & 16-Jun-2009 & SW 846/6020 & $\mathrm{U}$ \\
\hline Cobalt & ND & 0.0010 & $\mathrm{mg} / \mathrm{L}$ & 2 & 16-Jun-2009 & 16-Jun-2009 & SW 846/6020 & U \\
\hline Copper & 0.0005 & 0.0010 & $\mathrm{mg} / \mathrm{L}$ & 2 & 16-Jun-2009 & 16-Jun-2009 & SW 846/6020 & $\mathrm{J}$ \\
\hline Lead & ND & 0.0010 & $\mathrm{mg} / \mathrm{L}$ & 2 & 16-Jun-2009 & 16-Jun-2009 & SW 846/6020 & U \\
\hline Manganese & 0.0296 & 0.0010 & $\mathrm{mg} / \mathrm{L}$ & 2 & 16-Jun-2009 & 16-Jun-2009 & SW 846/6020 & \\
\hline Molybdenum & 0.0733 & 0.0010 & $\mathrm{mg} / \mathrm{L}$ & 2 & 16-Jun-2009 & 16-Jun-2009 & SW 846/6020 & \\
\hline Nickel & 0.0005 & 0.0010 & $\mathrm{mg} / \mathrm{L}$ & 2 & 16-Jun-2009 & 16-Jun-2009 & SW 846/6020 & $\mathrm{J}$ \\
\hline Selenium & 0.0059 & 0.0010 & $\mathrm{mg} / \mathrm{L}$ & 2 & 16-Jun-2009 & 16-Jun-2009 & SW 846/6020 & \\
\hline Silver & 0.0004 & 0.0010 & $\mathrm{mg} / \mathrm{L}$ & 2 & 16-Jun-2009 & 16-Jun-2009 & SW 846/6020 & $\mathrm{J}, \mathrm{B}$ \\
\hline Thallium & 0.0005 & 0.0010 & $\mathrm{mg} / \mathrm{L}$ & 2 & 16-Jun-2009 & 16-Jun-2009 & SW 846/6020 & $\mathrm{J}$ \\
\hline Vanadium & 0.0509 & 0.0010 & $\mathrm{mg} / \mathrm{L}$ & 2 & 16-Jun-2009 & 16-Jun-2009 & SW 846/6020 & \\
\hline Zinc & 0.0058 & 0.0010 & $\mathrm{mg} / \mathrm{L}$ & 2 & 16-Jun-2009 & 16-Jun-2009 & SW 846/6020 & B \\
\hline
\end{tabular}




\section{USACE ERDC-EP-C \\ 3909 Halls Ferry Road \\ Vicksburg, MS 39180-6199}

\begin{tabular}{|c|c|c|}
\hline ERDC - ECB & Project: TVA Fly Ash Project & \\
\hline - & & Reported: \\
\hline,--- & Project Manager: Tony Bednar & 3-Sep-20 \\
\hline
\end{tabular}

\section{DRAFT: SLC Water}

9061502-12 (Water)

\begin{tabular}{|c|c|c|c|c|c|c|c|c|}
\hline & & porting & & & & & & \\
\hline Analyte & Result & Limit & Units & Dilution & Prepared & Analyzed & Method & Notes \\
\hline
\end{tabular}

ERDC- EL-EP-C (Environmental Chemistry Branch)

DRAFT: Miscellaneous Physical/Conventional Chemistry Parameters

\begin{tabular}{lrrrrrrr}
\hline Bromide & $\mathrm{ND}$ & 0.100 & $\mathrm{mg} / \mathrm{L}$ & 1 & 14-Jul-2009 & 14-Jul-2009 & EPA 300.0 \\
Chloride & $\mathbf{3 . 7 1}$ & 1.00 & $\mathrm{mg} / \mathrm{L}$ & 1 & $14-J u l-2009$ & $14-J u l-2009$ & EPA 300.0 \\
Nitrate as N & $\mathbf{0 . 3 6 0}$ & 0.100 & $\mathrm{mg} / \mathrm{L}$ & 1 & $14-J u l-2009$ & $14-J u l-2009$ & EPA 300.0 \\
Sulfate & $\mathbf{3 8 . 1}$ & 0.100 & $\mathrm{mg} / \mathrm{L}$ & 1 & 14-Jul-2009 & $14-J u l-2009$ & EPA 300.0
\end{tabular}




\section{USACE ERDC-EP-C \\ 3909 Halls Ferry Road \\ Vicksburg, MS 39180-6199}

ERDC - ECB

$-,-$
Project: TVA Fly Ash Project

Project Manager: Tony Bednar
Reported:

03-Sep-2009

DRAFT: EMR MT N2 Hour 1 Rep D

9061502-16 (Water)

\begin{tabular}{|c|c|c|c|c|c|c|c|c|}
\hline Analyte & Result & $\begin{array}{r}\text { porting } \\
\text { Limit }\end{array}$ & Units & Dilution & Prepared & Analyzed & Method & Notes \\
\hline
\end{tabular}

ERDC- EL-EP-C (Environmental Chemistry Branch)

DRAFT: Wet Chemistry

\begin{tabular}{llllllll}
\hline Hardness & $\mathbf{3 6 . 1}$ & 1.32 & $\begin{array}{c}\text { mg equil } \\
\text { CaCO3/ }\end{array}$ & 1 & 25-Jun-2009 & 25-Jun-2009
\end{tabular}

$\mathrm{L}$

DRAFT: Metals by EPA 6000/7000 Series Methods

\begin{tabular}{|c|c|c|c|c|c|c|c|c|}
\hline Mercury & ND & 0.000005 & $\mathrm{mg} / \mathrm{L}$ & 1 & 17-Jun-2009 & 01-Jul-2009 & EPA 7471A & $\mathrm{U}$ \\
\hline Strontium & 0.121 & 0.0200 & $\mathrm{mg} / \mathrm{L}$ & 1 & 25-Jun-2009 & 25-Jun-2009 & EPA 6010B & \\
\hline Aluminum & 0.0604 & 0.200 & $\mathrm{mg} / \mathrm{L}$ & 1 & 25-Jun-2009 & 25-Jun-2009 & SW 846/6010 & $\mathrm{J}$ \\
\hline Calcium & 10.8 & 0.200 & $\mathrm{mg} / \mathrm{L}$ & 1 & 25-Jun-2009 & 25-Jun-2009 & SW 846/6010 & \\
\hline Iron & ND & 0.200 & $\mathrm{mg} / \mathrm{L}$ & 1 & 25-Jun-2009 & 25-Jun-2009 & SW 846/6010 & $\mathrm{U}$ \\
\hline Magnesium & 2.24 & 0.200 & $\mathrm{mg} / \mathrm{L}$ & 1 & 25-Jun-2009 & 25-Jun-2009 & SW 846/6010 & \\
\hline Potassium & 1.33 & 0.200 & $\mathrm{mg} / \mathrm{L}$ & 1 & 25-Jun-2009 & 25-Jun-2009 & SW 846/6010 & \\
\hline Sodium & 2.47 & 0.200 & $\mathrm{mg} / \mathrm{L}$ & 1 & 25-Jun-2009 & 25-Jun-2009 & SW 846/6010 & \\
\hline Antimony & 0.0004 & 0.0010 & $\mathrm{mg} / \mathrm{L}$ & 2 & 16-Jun-2009 & 16-Jun-2009 & SW 846/6020 & J \\
\hline Arsenic & 0.0278 & 0.0010 & $\mathrm{mg} / \mathrm{L}$ & 2 & 16-Jun-2009 & 16-Jun-2009 & SW 846/6020 & \\
\hline Barium & 0.0823 & 0.0010 & $\mathrm{mg} / \mathrm{L}$ & 2 & 16-Jun-2009 & 16-Jun-2009 & SW 846/6020 & \\
\hline Beryllium & ND & 0.0010 & $\mathrm{mg} / \mathrm{L}$ & 2 & 16-Jun-2009 & 16-Jun-2009 & SW 846/6020 & $\mathrm{U}$ \\
\hline Cadmium & ND & 0.0010 & $\mathrm{mg} / \mathrm{L}$ & 2 & 16-Jun-2009 & 16-Jun-2009 & SW 846/6020 & $\mathrm{U}$ \\
\hline Chromium & ND & 0.0010 & $\mathrm{mg} / \mathrm{L}$ & 2 & 16-Jun-2009 & 16-Jun-2009 & SW 846/6020 & $\mathrm{U}$ \\
\hline Cobalt & ND & 0.0010 & $\mathrm{mg} / \mathrm{L}$ & 2 & 16-Jun-2009 & 16-Jun-2009 & SW 846/6020 & $\mathrm{U}$ \\
\hline Copper & 0.0012 & 0.0010 & $\mathrm{mg} / \mathrm{L}$ & 2 & 16-Jun-2009 & 16-Jun-2009 & SW 846/6020 & \\
\hline Lead & ND & 0.0010 & $\mathrm{mg} / \mathrm{L}$ & 2 & 16-Jun-2009 & 16-Jun-2009 & SW 846/6020 & U \\
\hline Manganese & 0.0057 & 0.0010 & $\mathrm{mg} / \mathrm{L}$ & 2 & 16-Jun-2009 & 16-Jun-2009 & SW 846/6020 & \\
\hline Molybdenum & 0.0015 & 0.0010 & $\mathrm{mg} / \mathrm{L}$ & 2 & 16-Jun-2009 & 16-Jun-2009 & SW 846/6020 & \\
\hline Nickel & 0.0006 & 0.0010 & $\mathrm{mg} / \mathrm{L}$ & 2 & 16-Jun-2009 & 16-Jun-2009 & SW 846/6020 & $\mathrm{J}$ \\
\hline Selenium & 0.0010 & 0.0010 & $\mathrm{mg} / \mathrm{L}$ & 2 & 16-Jun-2009 & 16-Jun-2009 & SW 846/6020 & \\
\hline Silver & ND & 0.0010 & $\mathrm{mg} / \mathrm{L}$ & 2 & 16-Jun-2009 & 16-Jun-2009 & SW 846/6020 & $\mathrm{U}$ \\
\hline Thallium & 0.0004 & 0.0010 & $\mathrm{mg} / \mathrm{L}$ & 2 & 16-Jun-2009 & 16-Jun-2009 & SW 846/6020 & $\mathrm{J}$ \\
\hline Vanadium & 0.0173 & 0.0010 & $\mathrm{mg} / \mathrm{L}$ & 2 & 16-Jun-2009 & 16-Jun-2009 & SW 846/6020 & \\
\hline Zinc & 0.0057 & 0.0010 & $\mathrm{mg} / \mathrm{L}$ & 2 & 16-Jun-2009 & 16-Jun-2009 & SW 846/6020 & B \\
\hline
\end{tabular}




\section{USACE ERDC-EP-C \\ 3909 Halls Ferry Road \\ Vicksburg, MS 39180-6199}

ERDC - ECB

Project: TVA Fly Ash Project

Reported:

$-$

Project Manager: Tony Bednar

03-Sep-2009

DRAFT: EMR MT N2 Hour 1 Rep D

9061502-16 (Water)

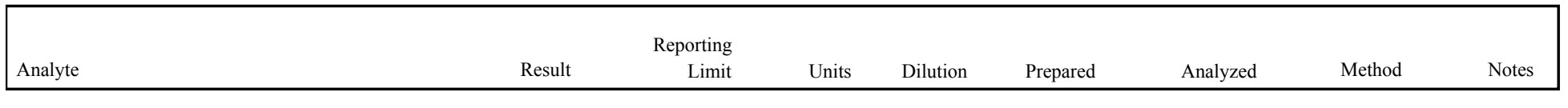

ERDC- EL-EP-C (Environmental Chemistry Branch)

DRAFT: Miscellaneous Physical/Conventional Chemistry Parameters

\begin{tabular}{lrrrrrrr}
\hline Bromide & $\mathrm{ND}$ & 0.100 & $\mathrm{mg} / \mathrm{L}$ & 1 & 14-Jul-2009 & $14-\mathrm{Jul}-2009$ & EPA 300.0 \\
Chloride & $\mathbf{2 . 3 2}$ & 1.00 & $\mathrm{mg} / \mathrm{L}$ & 1 & $14-J u l-2009$ & $14-\mathrm{Jul}-2009$ & EPA 300.0 \\
Nitrate as N & $\mathbf{0 . 1 5 0}$ & 0.100 & $\mathrm{mg} / \mathrm{L}$ & 1 & $14-J u l-2009$ & $14-J u l-2009$ & EPA 300.0 \\
Sulfate & $\mathbf{1 3 . 6}$ & 0.100 & $\mathrm{mg} / \mathrm{L}$ & 1 & $14-J u l-2009$ & $14-J u l-2009$ & EPA 300.0
\end{tabular}




\section{USACE ERDC-EP-C \\ 3909 Halls Ferry Road \\ Vicksburg, MS 39180-6199}

ERDC - ECB

$-,-$
Project: TVA Fly Ash Project

Project Manager: Tony Bednar
Reported:

03-Sep-2009

DRAFT: EMR MT N2 Hour 1 Rep E

9061502-17 (Water)

\begin{tabular}{|c|c|c|c|c|c|c|c|c|}
\hline Analyte & Result & $\begin{array}{r}\text { porting } \\
\text { Limit }\end{array}$ & Units & Dilution & Prepared & Analyzed & Method & Notes \\
\hline
\end{tabular}

ERDC- EL-EP-C (Environmental Chemistry Branch)

DRAFT: Wet Chemistry

\begin{tabular}{llllllll}
\hline Hardness & $\mathbf{3 4 . 9}$ & 1.32 & $\begin{array}{c}\text { mg equil } \\
\text { CaCO3/ }\end{array}$ & 1 & 25-Jun-2009 & 25-Jun-2009
\end{tabular}

$\mathrm{L}$

DRAFT: Metals by EPA 6000/7000 Series Methods

\begin{tabular}{|c|c|c|c|c|c|c|c|c|}
\hline Mercury & 0.000012 & 0.000005 & $\mathrm{mg} / \mathrm{L}$ & 1 & 17-Jun-2009 & 01-Jul-2009 & EPA 7471A & \\
\hline Strontium & 0.111 & 0.0200 & $\mathrm{mg} / \mathrm{L}$ & 1 & 25-Jun-2009 & 25-Jun-2009 & EPA $6010 B$ & \\
\hline Aluminum & 0.0515 & 0.200 & $\mathrm{mg} / \mathrm{L}$ & 1 & 25-Jun-2009 & 25-Jun-2009 & SW 846/6010 & $\mathrm{J}$ \\
\hline Calcium & 10.4 & 0.200 & $\mathrm{mg} / \mathrm{L}$ & 1 & 25-Jun-2009 & 25-Jun-2009 & SW 846/6010 & \\
\hline Iron & ND & 0.200 & $\mathrm{mg} / \mathrm{L}$ & 1 & 25-Jun-2009 & 25-Jun-2009 & SW 846/6010 & $\mathrm{U}$ \\
\hline Magnesium & 2.18 & 0.200 & $\mathrm{mg} / \mathrm{L}$ & 1 & 25-Jun-2009 & 25-Jun-2009 & SW 846/6010 & \\
\hline Potassium & 1.33 & 0.200 & $\mathrm{mg} / \mathrm{L}$ & 1 & 25-Jun-2009 & 25-Jun-2009 & SW 846/6010 & \\
\hline Sodium & 2.49 & 0.200 & $\mathrm{mg} / \mathrm{L}$ & 1 & 25-Jun-2009 & 25-Jun-2009 & SW 846/6010 & \\
\hline Antimony & 0.0004 & 0.0010 & $\mathrm{mg} / \mathrm{L}$ & 2 & 16-Jun-2009 & 16-Jun-2009 & SW 846/6020 & $\mathrm{J}$ \\
\hline Arsenic & 0.0239 & 0.0010 & $\mathrm{mg} / \mathrm{L}$ & 2 & 16-Jun-2009 & 16-Jun-2009 & SW 846/6020 & \\
\hline Barium & 0.0791 & 0.0010 & $\mathrm{mg} / \mathrm{L}$ & 2 & 16-Jun-2009 & 16-Jun-2009 & SW 846/6020 & \\
\hline Beryllium & ND & 0.0010 & $\mathrm{mg} / \mathrm{L}$ & 2 & 16-Jun-2009 & 16-Jun-2009 & SW 846/6020 & $\mathrm{U}$ \\
\hline Cadmium & ND & 0.0010 & $\mathrm{mg} / \mathrm{L}$ & 2 & 16-Jun-2009 & 16-Jun-2009 & SW 846/6020 & $\mathrm{U}$ \\
\hline Chromium & ND & 0.0010 & $\mathrm{mg} / \mathrm{L}$ & 2 & 16-Jun-2009 & 16-Jun-2009 & SW 846/6020 & $\mathrm{U}$ \\
\hline Cobalt & ND & 0.0010 & $\mathrm{mg} / \mathrm{L}$ & 2 & 16-Jun-2009 & 16-Jun-2009 & SW 846/6020 & $\mathrm{U}$ \\
\hline Copper & 0.0013 & 0.0010 & $\mathrm{mg} / \mathrm{L}$ & 2 & 16-Jun-2009 & 16-Jun-2009 & SW 846/6020 & \\
\hline Lead & ND & 0.0010 & $\mathrm{mg} / \mathrm{L}$ & 2 & 16-Jun-2009 & 16-Jun-2009 & SW 846/6020 & $\mathrm{U}$ \\
\hline Manganese & 0.0054 & 0.0010 & $\mathrm{mg} / \mathrm{L}$ & 2 & 16-Jun-2009 & 16-Jun-2009 & SW 846/6020 & \\
\hline Molybdenum & 0.0013 & 0.0010 & $\mathrm{mg} / \mathrm{L}$ & 2 & 16-Jun-2009 & 16-Jun-2009 & SW 846/6020 & \\
\hline Nickel & 0.0007 & 0.0010 & $\mathrm{mg} / \mathrm{L}$ & 2 & 16-Jun-2009 & 16-Jun-2009 & SW 846/6020 & J \\
\hline Selenium & 0.0009 & 0.0010 & $\mathrm{mg} / \mathrm{L}$ & 2 & 16-Jun-2009 & 16-Jun-2009 & SW 846/6020 & $\mathrm{J}$ \\
\hline Silver & ND & 0.0010 & $\mathrm{mg} / \mathrm{L}$ & 2 & 16-Jun-2009 & 16-Jun-2009 & SW 846/6020 & $\mathrm{U}$ \\
\hline Thallium & 0.0003 & 0.0010 & $\mathrm{mg} / \mathrm{L}$ & 2 & 16-Jun-2009 & 16-Jun-2009 & SW 846/6020 & J \\
\hline Vanadium & 0.0152 & 0.0010 & $\mathrm{mg} / \mathrm{L}$ & 2 & 16-Jun-2009 & 16-Jun-2009 & SW 846/6020 & \\
\hline Zinc & 0.0062 & 0.0010 & $\mathrm{mg} / \mathrm{L}$ & 2 & 16-Jun-2009 & 16-Jun-2009 & SW 846/6020 & B \\
\hline
\end{tabular}




\section{USACE ERDC-EP-C \\ 3909 Halls Ferry Road \\ Vicksburg, MS 39180-6199}

ERDC - ECB

Project: TVA Fly Ash Project

Reported:

Project Manager: Tony Bednar

03-Sep-2009

DRAFT: EMR MT N2 Hour 1 Rep E

9061502-17 (Water)

\begin{tabular}{|c|c|c|c|c|c|c|c|c|}
\hline & & orting & & & & & & \\
\hline Analyte & Result & Limit & Units & Dilution & Prepared & Analyzed & Method & Notes \\
\hline
\end{tabular}

ERDC- EL-EP-C (Environmental Chemistry Branch)

DRAFT: Miscellaneous Physical/Conventional Chemistry Parameters

\begin{tabular}{lrrrrrrr}
\hline Bromide & $\mathrm{ND}$ & 0.100 & $\mathrm{mg} / \mathrm{L}$ & 1 & 14-Jul-2009 & $14-\mathrm{Jul}-2009$ & EPA 300.0 \\
Chloride & $\mathbf{2 . 4 0}$ & 1.00 & $\mathrm{mg} / \mathrm{L}$ & 1 & $14-J u l-2009$ & $14-\mathrm{Jul}-2009$ & EPA 300.0 \\
Nitrate as N & $\mathbf{0 . 3 6 0}$ & 0.100 & $\mathrm{mg} / \mathrm{L}$ & 1 & $14-J u l-2009$ & $14-J u l-2009$ & EPA 300.0 \\
Sulfate & $\mathbf{1 4 . 0}$ & 0.100 & $\mathrm{mg} / \mathrm{L}$ & 1 & $14-J u l-2009$ & $14-J u l-2009$ & EPA 300.0
\end{tabular}




\section{USACE ERDC-EP-C \\ 3909 Halls Ferry Road \\ Vicksburg, MS 39180-6199}

ERDC - ECB

$-,-$
Project: TVA Fly Ash Project

Project Manager: Tony Bednar
Reported:

03-Sep-2009

DRAFT: EMR MT N2 Hour 1 Rep F

9061502-18 (Water)

\begin{tabular}{|c|c|c|c|c|c|c|c|c|}
\hline Analyte & Result & $\begin{array}{r}\text { porting } \\
\text { Limit }\end{array}$ & Units & Dilution & Prepared & Analyzed & Method & Notes \\
\hline
\end{tabular}

ERDC- EL-EP-C (Environmental Chemistry Branch)

DRAFT: Wet Chemistry

\begin{tabular}{llllllll}
\hline Hardness & $\mathbf{3 5 . 1}$ & 1.32 & $\begin{array}{c}\text { mg equil } \\
\text { CaCO3/ }\end{array}$ & 1 & 25-Jun-2009 & 25-Jun-2009
\end{tabular}

$\mathrm{L}$

DRAFT: Metals by EPA 6000/7000 Series Methods

\begin{tabular}{|c|c|c|c|c|c|c|c|c|}
\hline Mercury & ND & 0.000005 & $\mathrm{mg} / \mathrm{L}$ & 1 & 17-Jun-2009 & 01-Jul-2009 & EPA 7471A & $\mathrm{U}$ \\
\hline Strontium & 0.110 & 0.0200 & $\mathrm{mg} / \mathrm{L}$ & 1 & 25-Jun-2009 & 25-Jun-2009 & EPA 6010B & \\
\hline Aluminum & ND & 0.200 & $\mathrm{mg} / \mathrm{L}$ & 1 & 25-Jun-2009 & 25-Jun-2009 & SW 846/6010 & $\mathrm{U}$ \\
\hline Calcium & 10.4 & 0.200 & $\mathrm{mg} / \mathrm{L}$ & 1 & 25-Jun-2009 & 25-Jun-2009 & SW 846/6010 & \\
\hline Iron & ND & 0.200 & $\mathrm{mg} / \mathrm{L}$ & 1 & 25-Jun-2009 & 25-Jun-2009 & SW 846/6010 & $\mathrm{U}$ \\
\hline Magnesium & 2.20 & 0.200 & $\mathrm{mg} / \mathrm{L}$ & 1 & 25-Jun-2009 & 25-Jun-2009 & SW 846/6010 & \\
\hline Potassium & 1.35 & 0.200 & $\mathrm{mg} / \mathrm{L}$ & 1 & 25-Jun-2009 & 25-Jun-2009 & SW 846/6010 & \\
\hline Sodium & 2.51 & 0.200 & $\mathrm{mg} / \mathrm{L}$ & 1 & 25-Jun-2009 & 25-Jun-2009 & SW 846/6010 & \\
\hline Antimony & 0.0004 & 0.0010 & $\mathrm{mg} / \mathrm{L}$ & 2 & 16-Jun-2009 & 16-Jun-2009 & SW 846/6020 & J \\
\hline Arsenic & 0.0232 & 0.0010 & $\mathrm{mg} / \mathrm{L}$ & 2 & 16-Jun-2009 & 16-Jun-2009 & SW 846/6020 & \\
\hline Barium & 0.0790 & 0.0010 & $\mathrm{mg} / \mathrm{L}$ & 2 & 16-Jun-2009 & 16-Jun-2009 & SW 846/6020 & \\
\hline Beryllium & ND & 0.0010 & $\mathrm{mg} / \mathrm{L}$ & 2 & 16-Jun-2009 & 16-Jun-2009 & SW 846/6020 & $\mathrm{U}$ \\
\hline Cadmium & ND & 0.0010 & $\mathrm{mg} / \mathrm{L}$ & 2 & 16-Jun-2009 & 16-Jun-2009 & SW 846/6020 & $\mathrm{U}$ \\
\hline Chromium & ND & 0.0010 & $\mathrm{mg} / \mathrm{L}$ & 2 & 16-Jun-2009 & 16-Jun-2009 & SW 846/6020 & $\mathrm{U}$ \\
\hline Cobalt & ND & 0.0010 & $\mathrm{mg} / \mathrm{L}$ & 2 & 16-Jun-2009 & 16-Jun-2009 & SW 846/6020 & $\mathrm{U}$ \\
\hline Copper & 0.0013 & 0.0010 & $\mathrm{mg} / \mathrm{L}$ & 2 & 16-Jun-2009 & 16-Jun-2009 & SW 846/6020 & \\
\hline Lead & ND & 0.0010 & $\mathrm{mg} / \mathrm{L}$ & 2 & 16-Jun-2009 & 16-Jun-2009 & SW 846/6020 & $\mathrm{U}$ \\
\hline Manganese & 0.0045 & 0.0010 & $\mathrm{mg} / \mathrm{L}$ & 2 & 16-Jun-2009 & 16-Jun-2009 & SW 846/6020 & \\
\hline Molybdenum & 0.0014 & 0.0010 & $\mathrm{mg} / \mathrm{L}$ & 2 & 16-Jun-2009 & 16-Jun-2009 & SW 846/6020 & \\
\hline Nickel & 0.00067 & 0.0010 & $\mathrm{mg} / \mathrm{L}$ & 2 & 16-Jun-2009 & 16-Jun-2009 & SW 846/6020 & $\mathrm{J}$ \\
\hline Selenium & 0.0008 & 0.0010 & $\mathrm{mg} / \mathrm{L}$ & 2 & 16-Jun-2009 & 16-Jun-2009 & SW 846/6020 & $\mathrm{J}$ \\
\hline Silver & ND & 0.0010 & $\mathrm{mg} / \mathrm{L}$ & 2 & 16-Jun-2009 & 16-Jun-2009 & SW 846/6020 & $\mathrm{U}$ \\
\hline Thallium & 0.0003 & 0.0010 & $\mathrm{mg} / \mathrm{L}$ & 2 & 16-Jun-2009 & 16-Jun-2009 & SW 846/6020 & J \\
\hline Vanadium & 0.0147 & 0.0010 & $\mathrm{mg} / \mathrm{L}$ & 2 & 16-Jun-2009 & 16-Jun-2009 & SW 846/6020 & \\
\hline Zinc & 0.0064 & 0.0010 & $\mathrm{mg} / \mathrm{L}$ & 2 & 16-Jun-2009 & 16-Jun-2009 & SW 846/6020 & B \\
\hline
\end{tabular}




\section{USACE ERDC-EP-C \\ 3909 Halls Ferry Road \\ Vicksburg, MS 39180-6199}

ERDC - ECB

Project: TVA Fly Ash Project

Reported:

Project Manager: Tony Bednar

03-Sep-2009

DRAFT: EMR MT N2 Hour 1 Rep F

9061502-18 (Water)

\begin{tabular}{|c|c|c|c|c|c|c|c|c|}
\hline & & orting & & & & & & \\
\hline Analyte & Result & Limit & Units & Dilution & Prepared & Analyzed & Method & Notes \\
\hline
\end{tabular}

ERDC- EL-EP-C (Environmental Chemistry Branch)

DRAFT: Miscellaneous Physical/Conventional Chemistry Parameters

\begin{tabular}{lrrrrrrr}
\hline Bromide & $\mathrm{ND}$ & 0.100 & $\mathrm{mg} / \mathrm{L}$ & 1 & 14-Jul-2009 & 14-Jul-2009 & EPA 300.0 \\
Chloride & $\mathbf{2 . 3 8}$ & 1.00 & $\mathrm{mg} / \mathrm{L}$ & 1 & $14-J u l-2009$ & $14-J u l-2009$ & EPA 300.0 \\
Nitrate as N & $\mathbf{0 . 1 0 4}$ & 0.100 & $\mathrm{mg} / \mathrm{L}$ & 1 & $14-J u l-2009$ & $14-J u l-2009$ & EPA 300.0 \\
Sulfate & $\mathbf{1 2 . 5}$ & 0.100 & $\mathrm{mg} / \mathrm{L}$ & 1 & $14-J u l-2009$ & $14-J u l-2009$ & EPA 300.0
\end{tabular}




\section{USACE ERDC-EP-C \\ 3909 Halls Ferry Road \\ Vicksburg, MS 39180-6199}

ERDC - ECB

$-\cdot$,
Project: TVA Fly Ash Project

Project Manager: Tony Bednar
Reported:

03-Sep-2009

DRAFT: EMR MT O2 Hour 1 Rep D

9061502-22 (Water)

\begin{tabular}{|c|c|c|c|c|c|c|c|c|}
\hline Analyte & Result & $\begin{array}{r}\text { porting } \\
\text { Limit }\end{array}$ & Units & Dilution & Prepared & Analyzed & Method & Notes \\
\hline
\end{tabular}

ERDC- EL-EP-C (Environmental Chemistry Branch)

DRAFT: Wet Chemistry

\begin{tabular}{llllllll}
\hline Hardness & $\mathbf{3 4 . 8}$ & 1.32 & $\begin{array}{c}\text { mg equil } \\
\text { CaCO3/ }\end{array}$ & 1 & 25-Jun-2009 & 25-Jun-2009
\end{tabular}

$\mathrm{L}$

DRAFT: Metals by EPA 6000/7000 Series Methods

\begin{tabular}{|c|c|c|c|c|c|c|c|c|}
\hline Mercury & 0.000014 & 0.000005 & $\mathrm{mg} / \mathrm{L}$ & 1 & 17-Jun-2009 & 01-Jul-2009 & EPA 7471A & \\
\hline Strontium & 0.0998 & 0.0200 & $\mathrm{mg} / \mathrm{L}$ & 1 & 25-Jun-2009 & 25-Jun-2009 & EPA 6010B & \\
\hline Aluminum & ND & 0.200 & $\mathrm{mg} / \mathrm{L}$ & 1 & 25-Jun-2009 & 25-Jun-2009 & SW 846/6010 & $\mathrm{U}$ \\
\hline Calcium & 10.2 & 0.200 & $\mathrm{mg} / \mathrm{L}$ & 1 & 25-Jun-2009 & 25-Jun-2009 & SW 846/6010 & \\
\hline Iron & ND & 0.200 & $\mathrm{mg} / \mathrm{L}$ & 1 & 25-Jun-2009 & 25-Jun-2009 & SW 846/6010 & $\mathrm{U}$ \\
\hline Magnesium & 2.23 & 0.200 & $\mathrm{mg} / \mathrm{L}$ & 1 & 25-Jun-2009 & 25-Jun-2009 & SW 846/6010 & \\
\hline Potassium & 1.34 & 0.200 & $\mathrm{mg} / \mathrm{L}$ & 1 & 25-Jun-2009 & 25-Jun-2009 & SW 846/6010 & \\
\hline Sodium & 2.48 & 0.200 & $\mathrm{mg} / \mathrm{L}$ & 1 & 25-Jun-2009 & 25-Jun-2009 & SW 846/6010 & \\
\hline Antimony & 0.0003 & 0.0010 & $\mathrm{mg} / \mathrm{L}$ & 2 & 16-Jun-2009 & 16-Jun-2009 & SW 846/6020 & J \\
\hline Arsenic & 0.0198 & 0.0010 & $\mathrm{mg} / \mathrm{L}$ & 2 & 16-Jun-2009 & 16-Jun-2009 & SW 846/6020 & \\
\hline Barium & 0.0768 & 0.0010 & $\mathrm{mg} / \mathrm{L}$ & 2 & 16-Jun-2009 & 16-Jun-2009 & SW 846/6020 & \\
\hline Beryllium & ND & 0.0010 & $\mathrm{mg} / \mathrm{L}$ & 2 & 16-Jun-2009 & 16-Jun-2009 & SW 846/6020 & U \\
\hline Cadmium & ND & 0.0010 & $\mathrm{mg} / \mathrm{L}$ & 2 & 16-Jun-2009 & 16-Jun-2009 & SW 846/6020 & U \\
\hline Chromium & ND & 0.0010 & $\mathrm{mg} / \mathrm{L}$ & 2 & 16-Jun-2009 & 16-Jun-2009 & SW 846/6020 & U \\
\hline Cobalt & ND & 0.0010 & $\mathrm{mg} / \mathrm{L}$ & 2 & 16-Jun-2009 & 16-Jun-2009 & SW 846/6020 & U \\
\hline Copper & 0.0016 & 0.0010 & $\mathrm{mg} / \mathrm{L}$ & 2 & 16-Jun-2009 & 16-Jun-2009 & SW 846/6020 & \\
\hline Lead & ND & 0.0010 & $\mathrm{mg} / \mathrm{L}$ & 2 & 16-Jun-2009 & 16-Jun-2009 & SW 846/6020 & U \\
\hline Manganese & 0.0048 & 0.0010 & $\mathrm{mg} / \mathrm{L}$ & 2 & 16-Jun-2009 & 16-Jun-2009 & SW 846/6020 & \\
\hline Molybdenum & 0.0011 & 0.0010 & $\mathrm{mg} / \mathrm{L}$ & 2 & 16-Jun-2009 & 16-Jun-2009 & SW 846/6020 & \\
\hline Nickel & 0.0007 & 0.0010 & $\mathrm{mg} / \mathrm{L}$ & 2 & 16-Jun-2009 & 16-Jun-2009 & SW 846/6020 & $\mathrm{J}$ \\
\hline Selenium & 0.0008 & 0.0010 & $\mathrm{mg} / \mathrm{L}$ & 2 & 16-Jun-2009 & 16-Jun-2009 & SW 846/6020 & $\mathrm{J}$ \\
\hline Silver & ND & 0.0010 & $\mathrm{mg} / \mathrm{L}$ & 2 & 16-Jun-2009 & 16-Jun-2009 & SW 846/6020 & U \\
\hline Thallium & 0.0003 & 0.0010 & $\mathrm{mg} / \mathrm{L}$ & 2 & 16-Jun-2009 & 16-Jun-2009 & SW 846/6020 & J \\
\hline Vanadium & 0.0128 & 0.0010 & $\mathrm{mg} / \mathrm{L}$ & 2 & 16-Jun-2009 & 16-Jun-2009 & SW 846/6020 & \\
\hline Zinc & 0.0062 & 0.0010 & $\mathrm{mg} / \mathrm{L}$ & 2 & 16-Jun-2009 & 16-Jun-2009 & SW 846/6020 & B \\
\hline
\end{tabular}




\section{USACE ERDC-EP-C \\ 3909 Halls Ferry Road \\ Vicksburg, MS 39180-6199}

ERDC - ECB

Project: TVA Fly Ash Project

Reported:

$--,-$

Project Manager: Tony Bednar

03-Sep-2009

DRAFT: EMR MT O2 Hour 1 Rep D

9061502-22 (Water)

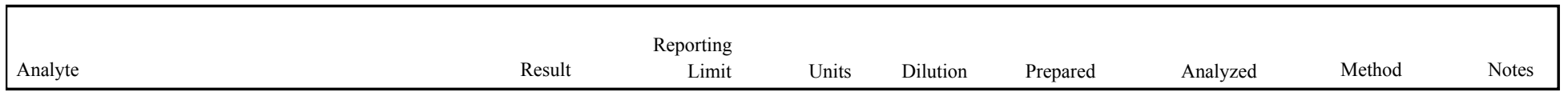

ERDC- EL-EP-C (Environmental Chemistry Branch)

DRAFT: Miscellaneous Physical/Conventional Chemistry Parameters

\begin{tabular}{lrrrrrrr}
\hline Bromide & $\mathrm{ND}$ & 0.100 & $\mathrm{mg} / \mathrm{L}$ & 1 & 14-Jul-2009 & $14-\mathrm{Jul}-2009$ & EPA 300.0 \\
Chloride & $\mathbf{2 . 3 6}$ & 1.00 & $\mathrm{mg} / \mathrm{L}$ & 1 & $14-J u l-2009$ & $14-\mathrm{Jul}-2009$ & EPA 300.0 \\
Nitrate as N & $\mathbf{0 . 4 1 3}$ & 0.100 & $\mathrm{mg} / \mathrm{L}$ & 1 & $14-J u l-2009$ & $14-J u l-2009$ & EPA 300.0 \\
Sulfate & $\mathbf{1 2 . 3}$ & 0.100 & $\mathrm{mg} / \mathrm{L}$ & 1 & $14-J u l-2009$ & $14-J u l-2009$ & EPA 300.0
\end{tabular}




\section{USACE ERDC-EP-C \\ 3909 Halls Ferry Road \\ Vicksburg, MS 39180-6199}

ERDC - ECB

$-,-$
Project: TVA Fly Ash Project

Project Manager: Tony Bednar
Reported:

03-Sep-2009

DRAFT: EMR MT O2 Hour 1 Rep E

9061502-23 (Water)

\begin{tabular}{|c|c|c|c|c|c|c|c|c|}
\hline Analyte & Result & $\begin{array}{r}\text { porting } \\
\text { Limit }\end{array}$ & Units & Dilution & Prepared & Analyzed & Method & Notes \\
\hline
\end{tabular}

ERDC- EL-EP-C (Environmental Chemistry Branch)

DRAFT: Wet Chemistry

\begin{tabular}{llllllll}
\hline Hardness & $\mathbf{3 3 . 8}$ & 1.32 & $\begin{array}{c}\text { mg equil } \\
\text { CaCO3/ }\end{array}$ & 1 & 25-Jun-2009 & 25-Jun-2009
\end{tabular}

$\mathrm{L}$

DRAFT: Metals by EPA 6000/7000 Series Methods

\begin{tabular}{|c|c|c|c|c|c|c|c|c|}
\hline Mercury & 0.000014 & 0.000005 & $\mathrm{mg} / \mathrm{L}$ & 1 & 17-Jun-2009 & 01-Jul-2009 & EPA 7471A & \\
\hline Strontium & 0.0915 & 0.0200 & $\mathrm{mg} / \mathrm{L}$ & 1 & 25-Jun-2009 & 25-Jun-2009 & EPA 6010B & \\
\hline Aluminum & ND & 0.200 & $\mathrm{mg} / \mathrm{L}$ & 1 & 25-Jun-2009 & 25-Jun-2009 & SW 846/6010 & $\mathrm{U}$ \\
\hline Calcium & 9.95 & 0.200 & $\mathrm{mg} / \mathrm{L}$ & 1 & 25-Jun-2009 & 25-Jun-2009 & SW 846/6010 & \\
\hline Iron & ND & 0.200 & $\mathrm{mg} / \mathrm{L}$ & 1 & 25-Jun-2009 & 25-Jun-2009 & SW 846/6010 & $\mathrm{U}$ \\
\hline Magnesium & 2.19 & 0.200 & $\mathrm{mg} / \mathrm{L}$ & 1 & 25-Jun-2009 & 25-Jun-2009 & SW 846/6010 & \\
\hline Potassium & 1.31 & 0.200 & $\mathrm{mg} / \mathrm{L}$ & 1 & 25-Jun-2009 & 25-Jun-2009 & SW 846/6010 & \\
\hline Sodium & 2.44 & 0.200 & $\mathrm{mg} / \mathrm{L}$ & 1 & 25-Jun-2009 & 25-Jun-2009 & SW 846/6010 & \\
\hline Antimony & 0.0002 & 0.0010 & $\mathrm{mg} / \mathrm{L}$ & 2 & 16-Jun-2009 & 16-Jun-2009 & SW 846/6020 & J \\
\hline Arsenic & 0.0177 & 0.0010 & $\mathrm{mg} / \mathrm{L}$ & 2 & 16-Jun-2009 & 16-Jun-2009 & SW 846/6020 & \\
\hline Barium & 0.0731 & 0.0010 & $\mathrm{mg} / \mathrm{L}$ & 2 & 16-Jun-2009 & 16-Jun-2009 & SW 846/6020 & \\
\hline Beryllium & ND & 0.0010 & $\mathrm{mg} / \mathrm{L}$ & 2 & 16-Jun-2009 & 16-Jun-2009 & SW 846/6020 & U \\
\hline Cadmium & ND & 0.0010 & $\mathrm{mg} / \mathrm{L}$ & 2 & 16-Jun-2009 & 16-Jun-2009 & SW 846/6020 & U \\
\hline Chromium & ND & 0.0010 & $\mathrm{mg} / \mathrm{L}$ & 2 & 16-Jun-2009 & 16-Jun-2009 & SW 846/6020 & U \\
\hline Cobalt & ND & 0.0010 & $\mathrm{mg} / \mathrm{L}$ & 2 & 16-Jun-2009 & 16-Jun-2009 & SW 846/6020 & U \\
\hline Copper & 0.0013 & 0.0010 & $\mathrm{mg} / \mathrm{L}$ & 2 & 16-Jun-2009 & 16-Jun-2009 & SW 846/6020 & \\
\hline Lead & ND & 0.0010 & $\mathrm{mg} / \mathrm{L}$ & 2 & 16-Jun-2009 & 16-Jun-2009 & SW 846/6020 & U \\
\hline Manganese & 0.0032 & 0.0010 & $\mathrm{mg} / \mathrm{L}$ & 2 & 16-Jun-2009 & 16-Jun-2009 & SW 846/6020 & \\
\hline Molybdenum & 0.0010 & 0.0010 & $\mathrm{mg} / \mathrm{L}$ & 2 & 16-Jun-2009 & 16-Jun-2009 & SW 846/6020 & \\
\hline Nickel & 0.0007 & 0.0010 & $\mathrm{mg} / \mathrm{L}$ & 2 & 16-Jun-2009 & 16-Jun-2009 & SW 846/6020 & $\mathrm{J}$ \\
\hline Selenium & 0.0006 & 0.0010 & $\mathrm{mg} / \mathrm{L}$ & 2 & 16-Jun-2009 & 16-Jun-2009 & SW 846/6020 & $\mathrm{J}$ \\
\hline Silver & ND & 0.0010 & $\mathrm{mg} / \mathrm{L}$ & 2 & 16-Jun-2009 & 16-Jun-2009 & SW 846/6020 & U \\
\hline Thallium & 0.0003 & 0.0010 & $\mathrm{mg} / \mathrm{L}$ & 2 & 16-Jun-2009 & 16-Jun-2009 & SW 846/6020 & J \\
\hline Vanadium & 0.0109 & 0.0010 & $\mathrm{mg} / \mathrm{L}$ & 2 & 16-Jun-2009 & 16-Jun-2009 & SW 846/6020 & \\
\hline Zinc & 0.0055 & 0.0010 & $\mathrm{mg} / \mathrm{L}$ & 2 & 16-Jun-2009 & 16-Jun-2009 & SW 846/6020 & B \\
\hline
\end{tabular}




\section{USACE ERDC-EP-C \\ 3909 Halls Ferry Road \\ Vicksburg, MS 39180-6199}

ERDC - ECB

Project: TVA Fly Ash Project

Reported:

$-$

Project Manager: Tony Bednar

03-Sep-2009

DRAFT: EMR MT O2 Hour 1 Rep E

9061502-23 (Water)

\begin{tabular}{|c|c|c|c|c|c|c|c|c|}
\hline & & orting & & & & & & \\
\hline Analyte & Result & Limit & Units & Dilution & Prepared & Analyzed & Method & Notes \\
\hline
\end{tabular}

ERDC- EL-EP-C (Environmental Chemistry Branch)

DRAFT: Miscellaneous Physical/Conventional Chemistry Parameters

\begin{tabular}{lrrrrrrr}
\hline Bromide & $\mathrm{ND}$ & 0.100 & $\mathrm{mg} / \mathrm{L}$ & 1 & 14-Jul-2009 & $14-\mathrm{Jul}-2009$ & EPA 300.0 \\
Chloride & $\mathbf{2 . 3 2}$ & 1.00 & $\mathrm{mg} / \mathrm{L}$ & 1 & $14-J u l-2009$ & $14-\mathrm{Jul}-2009$ & EPA 300.0 \\
Nitrate as N & $\mathbf{0 . 4 1 0}$ & 0.100 & $\mathrm{mg} / \mathrm{L}$ & 1 & $14-J u l-2009$ & $14-J u l-2009$ & EPA 300.0 \\
Sulfate & $\mathbf{1 2 . 2}$ & 0.100 & $\mathrm{mg} / \mathrm{L}$ & 1 & $14-J u l-2009$ & $14-J u l-2009$ & EPA 300.0
\end{tabular}




\section{USACE ERDC-EP-C \\ 3909 Halls Ferry Road \\ Vicksburg, MS 39180-6199}

ERDC - ECB

$-,-$
Project: TVA Fly Ash Project

Project Manager: Tony Bednar
Reported:

03-Sep-2009

DRAFT: EMR MT O2 Hour 1 Rep F

9061502-24 (Water)

\begin{tabular}{|c|c|c|c|c|c|c|c|c|}
\hline Analyte & Result & $\begin{array}{r}\text { porting } \\
\text { Limit }\end{array}$ & Units & Dilution & Prepared & Analyzed & Method & Notes \\
\hline
\end{tabular}

ERDC- EL-EP-C (Environmental Chemistry Branch)

DRAFT: Wet Chemistry

\begin{tabular}{llllllll}
\hline Hardness & $\mathbf{3 5 . 5}$ & 1.32 & $\begin{array}{c}\text { mg equil } \\
\text { CaCO3/ }\end{array}$ & 1 & 25-Jun-2009 & 25-Jun-2009
\end{tabular}

$\mathrm{L}$

DRAFT: Metals by EPA 6000/7000 Series Methods

\begin{tabular}{|c|c|c|c|c|c|c|c|c|}
\hline Mercury & 0.000013 & 0.000005 & $\mathrm{mg} / \mathrm{L}$ & 1 & 17-Jun-2009 & 01-Jul-2009 & EPA 7471A & \\
\hline Strontium & 0.116 & 0.0200 & $\mathrm{mg} / \mathrm{L}$ & 1 & 25-Jun-2009 & 25-Jun-2009 & EPA 6010B & \\
\hline Aluminum & 0.0516 & 0.200 & $\mathrm{mg} / \mathrm{L}$ & 1 & 25-Jun-2009 & 25-Jun-2009 & SW 846/6010 & J \\
\hline Calcium & 10.6 & 0.200 & $\mathrm{mg} / \mathrm{L}$ & 1 & 25-Jun-2009 & 25-Jun-2009 & SW 846/6010 & \\
\hline Iron & ND & 0.200 & $\mathrm{mg} / \mathrm{L}$ & 1 & 25-Jun-2009 & 25-Jun-2009 & SW 846/6010 & $\mathrm{U}$ \\
\hline Magnesium & 2.20 & 0.200 & $\mathrm{mg} / \mathrm{L}$ & 1 & 25-Jun-2009 & 25-Jun-2009 & SW 846/6010 & \\
\hline Potassium & 1.34 & 0.200 & $\mathrm{mg} / \mathrm{L}$ & 1 & 25-Jun-2009 & 25-Jun-2009 & SW 846/6010 & \\
\hline Sodium & 2.44 & 0.200 & $\mathrm{mg} / \mathrm{L}$ & 1 & 25-Jun-2009 & 25-Jun-2009 & SW 846/6010 & \\
\hline Antimony & 0.0004 & 0.0010 & $\mathrm{mg} / \mathrm{L}$ & 2 & 16-Jun-2009 & 16-Jun-2009 & SW 846/6020 & $\mathrm{J}$ \\
\hline Arsenic & 0.0252 & 0.0010 & $\mathrm{mg} / \mathrm{L}$ & 2 & 16-Jun-2009 & 16-Jun-2009 & SW 846/6020 & \\
\hline Barium & 0.0832 & 0.0010 & $\mathrm{mg} / \mathrm{L}$ & 2 & 16-Jun-2009 & 16-Jun-2009 & SW 846/6020 & \\
\hline Beryllium & ND & 0.0010 & $\mathrm{mg} / \mathrm{L}$ & 2 & 16-Jun-2009 & 16-Jun-2009 & SW 846/6020 & U \\
\hline Cadmium & ND & 0.0010 & $\mathrm{mg} / \mathrm{L}$ & 2 & 16-Jun-2009 & 16-Jun-2009 & SW 846/6020 & $\mathrm{U}$ \\
\hline Chromium & ND & 0.0010 & $\mathrm{mg} / \mathrm{L}$ & 2 & 16-Jun-2009 & 16-Jun-2009 & SW 846/6020 & U \\
\hline Cobalt & ND & 0.0010 & $\mathrm{mg} / \mathrm{L}$ & 2 & 16-Jun-2009 & 16-Jun-2009 & SW 846/6020 & U \\
\hline Copper & 0.0013 & 0.0010 & $\mathrm{mg} / \mathrm{L}$ & 2 & 16-Jun-2009 & 16-Jun-2009 & SW 846/6020 & \\
\hline Lead & ND & 0.0010 & $\mathrm{mg} / \mathrm{L}$ & 2 & 16-Jun-2009 & 16-Jun-2009 & SW 846/6020 & U \\
\hline Manganese & 0.0063 & 0.0010 & $\mathrm{mg} / \mathrm{L}$ & 2 & 16-Jun-2009 & 16-Jun-2009 & SW 846/6020 & \\
\hline Molybdenum & 0.0013 & 0.0010 & $\mathrm{mg} / \mathrm{L}$ & 2 & 16-Jun-2009 & 16-Jun-2009 & SW 846/6020 & \\
\hline Nickel & 0.00054 & 0.0010 & $\mathrm{mg} / \mathrm{L}$ & 2 & 16-Jun-2009 & 16-Jun-2009 & SW 846/6020 & J \\
\hline Selenium & 0.0008 & 0.0010 & $\mathrm{mg} / \mathrm{L}$ & 2 & 16-Jun-2009 & 16-Jun-2009 & SW 846/6020 & $\mathrm{J}$ \\
\hline Silver & ND & 0.0010 & $\mathrm{mg} / \mathrm{L}$ & 2 & 16-Jun-2009 & 16-Jun-2009 & SW 846/6020 & U \\
\hline Thallium & 0.0004 & 0.0010 & $\mathrm{mg} / \mathrm{L}$ & 2 & 16-Jun-2009 & 16-Jun-2009 & SW 846/6020 & J \\
\hline Vanadium & 0.0163 & 0.0010 & $\mathrm{mg} / \mathrm{L}$ & 2 & 16-Jun-2009 & 16-Jun-2009 & SW 846/6020 & \\
\hline Zinc & 0.0056 & 0.0010 & $\mathrm{mg} / \mathrm{L}$ & 2 & 16-Jun-2009 & 16-Jun-2009 & SW 846/6020 & B \\
\hline
\end{tabular}




\section{USACE ERDC-EP-C \\ 3909 Halls Ferry Road \\ Vicksburg, MS 39180-6199}

ERDC - ECB

Project: TVA Fly Ash Project

Reported:

$--,-$

Project Manager: Tony Bednar

03-Sep-2009

DRAFT: EMR MT O2 Hour 1 Rep F

9061502-24 (Water)

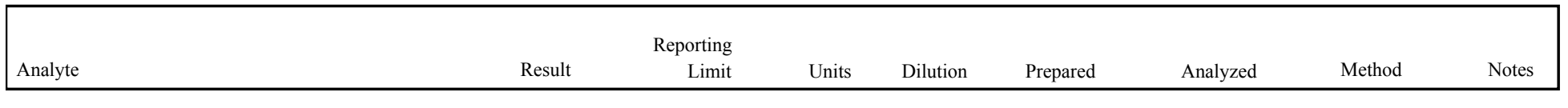

ERDC- EL-EP-C (Environmental Chemistry Branch)

DRAFT: Miscellaneous Physical/Conventional Chemistry Parameters

\begin{tabular}{lrrrrrrr}
\hline Bromide & $\mathrm{ND}$ & 0.100 & $\mathrm{mg} / \mathrm{L}$ & 1 & 14-Jul-2009 & $14-\mathrm{Jul}-2009$ & EPA 300.0 \\
Chloride & $\mathbf{2 . 3 6}$ & 1.00 & $\mathrm{mg} / \mathrm{L}$ & 1 & $14-J u l-2009$ & $14-\mathrm{Jul}-2009$ & EPA 300.0 \\
Nitrate as N & $\mathbf{0 . 4 0 0}$ & 0.100 & $\mathrm{mg} / \mathrm{L}$ & 1 & $14-J u l-2009$ & $14-J u l-2009$ & EPA 300.0 \\
Sulfate & $\mathbf{1 2 . 5}$ & 0.100 & $\mathrm{mg} / \mathrm{L}$ & 1 & $14-J u l-2009$ & $14-J u l-2009$ & EPA 300.0
\end{tabular}




\section{USACE ERDC-EP-C \\ 3909 Halls Ferry Road \\ Vicksburg, MS 39180-6199}

ERDC - ECB

$-,-$
Project: TVA Fly Ash Project

Project Manager: Tony Bednar
Reported:

03-Sep-2009

DRAFT: SLC MT N2 Hour 1 Rep D

9061502-28 (Water)

\begin{tabular}{|c|c|c|c|c|c|c|c|c|}
\hline Analyte & Result & $\begin{array}{r}\text { porting } \\
\text { Limit }\end{array}$ & Units & Dilution & Prepared & Analyzed & Method & Notes \\
\hline
\end{tabular}

ERDC- EL-EP-C (Environmental Chemistry Branch)

DRAFT: Wet Chemistry

\begin{tabular}{llllllll}
\hline Hardness & $\mathbf{9 6 . 8}$ & 1.32 & $\begin{array}{c}\text { mg equil } \\
\text { CaCO3/ }\end{array}$ & 1 & 25-Jun-2009 & 25-Jun-2009
\end{tabular}

$\mathrm{L}$

DRAFT: Metals by EPA 6000/7000 Series Methods

\begin{tabular}{|c|c|c|c|c|c|c|c|c|}
\hline Mercury & 0.000012 & 0.000005 & $\mathrm{mg} / \mathrm{L}$ & 1 & 17-Jun-2009 & 01-Jul-2009 & EPA 7471A & \\
\hline Strontium & 0.538 & 0.0200 & $\mathrm{mg} / \mathrm{L}$ & 1 & 25-Jun-2009 & 25-Jun-2009 & EPA 6010B & \\
\hline Aluminum & 0.118 & 0.200 & $\mathrm{mg} / \mathrm{L}$ & 1 & 25-Jun-2009 & 25-Jun-2009 & SW 846/6010 & J \\
\hline Calcium & 30.4 & 0.200 & $\mathrm{mg} / \mathrm{L}$ & 1 & 25-Jun-2009 & 25-Jun-2009 & SW 846/6010 & \\
\hline Iron & ND & 0.200 & $\mathrm{mg} / \mathrm{L}$ & 1 & 25-Jun-2009 & 25-Jun-2009 & SW 846/6010 & $\mathrm{U}$ \\
\hline Magnesium & 5.04 & 0.200 & $\mathrm{mg} / \mathrm{L}$ & 1 & 25-Jun-2009 & 25-Jun-2009 & SW 846/6010 & \\
\hline Potassium & 1.85 & 0.200 & $\mathrm{mg} / \mathrm{L}$ & 1 & 25-Jun-2009 & 25-Jun-2009 & SW 846/6010 & \\
\hline Sodium & 4.06 & 0.200 & $\mathrm{mg} / \mathrm{L}$ & 1 & 25-Jun-2009 & 25-Jun-2009 & SW 846/6010 & \\
\hline Antimony & 0.0077 & 0.0010 & $\mathrm{mg} / \mathrm{L}$ & 2 & 16-Jun-2009 & 16-Jun-2009 & SW 846/6020 & \\
\hline Arsenic & 0.0771 & 0.0010 & $\mathrm{mg} / \mathrm{L}$ & 2 & 16-Jun-2009 & 16-Jun-2009 & SW 846/6020 & \\
\hline Barium & 0.0992 & 0.0010 & $\mathrm{mg} / \mathrm{L}$ & 2 & 16-Jun-2009 & 16-Jun-2009 & SW 846/6020 & \\
\hline Beryllium & ND & 0.0010 & $\mathrm{mg} / \mathrm{L}$ & 2 & 16-Jun-2009 & 16-Jun-2009 & SW 846/6020 & U \\
\hline Cadmium & 0.0003 & 0.0010 & $\mathrm{mg} / \mathrm{L}$ & 2 & 16-Jun-2009 & 16-Jun-2009 & SW 846/6020 & J \\
\hline Chromium & ND & 0.0010 & $\mathrm{mg} / \mathrm{L}$ & 2 & 16-Jun-2009 & 16-Jun-2009 & SW 846/6020 & U \\
\hline Cobalt & ND & 0.0010 & $\mathrm{mg} / \mathrm{L}$ & 2 & 16-Jun-2009 & 16-Jun-2009 & SW 846/6020 & U \\
\hline Copper & 0.0004 & 0.0010 & $\mathrm{mg} / \mathrm{L}$ & 2 & 16-Jun-2009 & 16-Jun-2009 & SW 846/6020 & J \\
\hline Lead & ND & 0.0010 & $\mathrm{mg} / \mathrm{L}$ & 2 & 16-Jun-2009 & 16-Jun-2009 & SW 846/6020 & U \\
\hline Manganese & 0.0345 & 0.0010 & $\mathrm{mg} / \mathrm{L}$ & 2 & 16-Jun-2009 & 16-Jun-2009 & SW 846/6020 & \\
\hline Molybdenum & 0.0720 & 0.0010 & $\mathrm{mg} / \mathrm{L}$ & 2 & 16-Jun-2009 & 16-Jun-2009 & SW 846/6020 & \\
\hline Nickel & 0.0006 & 0.0010 & $\mathrm{mg} / \mathrm{L}$ & 2 & 16-Jun-2009 & 16-Jun-2009 & SW 846/6020 & J \\
\hline Selenium & 0.0055 & 0.0010 & $\mathrm{mg} / \mathrm{L}$ & 2 & 16-Jun-2009 & 16-Jun-2009 & SW 846/6020 & \\
\hline Silver & ND & 0.0010 & $\mathrm{mg} / \mathrm{L}$ & 2 & 16-Jun-2009 & 16-Jun-2009 & SW 846/6020 & $\mathrm{U}$ \\
\hline Thallium & 0.0006 & 0.0010 & $\mathrm{mg} / \mathrm{L}$ & 2 & 16-Jun-2009 & 16-Jun-2009 & SW 846/6020 & J \\
\hline Vanadium & 0.0540 & 0.0010 & $\mathrm{mg} / \mathrm{L}$ & 2 & 16-Jun-2009 & 16-Jun-2009 & SW 846/6020 & \\
\hline Zinc & 0.0054 & 0.0010 & $\mathrm{mg} / \mathrm{L}$ & 2 & 16-Jun-2009 & 16-Jun-2009 & SW 846/6020 & $\mathrm{B}$ \\
\hline
\end{tabular}




\section{USACE ERDC-EP-C \\ 3909 Halls Ferry Road \\ Vicksburg, MS 39180-6199}

ERDC - ECB

Project: TVA Fly Ash Project

Reported:

$--,-$

Project Manager: Tony Bednar

03-Sep-2009

DRAFT: SLC MT N2 Hour 1 Rep D

9061502-28 (Water)

\begin{tabular}{|c|c|c|c|c|c|c|c|c|}
\hline & & orting & & & & & & \\
\hline Analyte & Result & Limit & Units & Dilution & Prepared & Analyzed & Method & Notes \\
\hline
\end{tabular}

ERDC- EL-EP-C (Environmental Chemistry Branch)

DRAFT: Miscellaneous Physical/Conventional Chemistry Parameters

\begin{tabular}{lrrrrrrr}
\hline Bromide & $\mathrm{ND}$ & 0.100 & $\mathrm{mg} / \mathrm{L}$ & 1 & 14-Jul-2009 & $14-\mathrm{Jul}-2009$ & EPA 300.0 \\
Chloride & $\mathbf{3 . 7 8}$ & 1.00 & $\mathrm{mg} / \mathrm{L}$ & 1 & $14-J u l-2009$ & $14-\mathrm{Jul}-2009$ & EPA 300.0 \\
Nitrate as N & $\mathbf{0 . 3 7 0}$ & 0.100 & $\mathrm{mg} / \mathrm{L}$ & 1 & $14-J u l-2009$ & $14-J u l-2009$ & EPA 300.0 \\
Sulfate & $\mathbf{3 6 . 6}$ & 0.100 & $\mathrm{mg} / \mathrm{L}$ & 1 & $14-J u l-2009$ & $14-J u l-2009$ & EPA 300.0
\end{tabular}




\section{USACE ERDC-EP-C \\ 3909 Halls Ferry Road \\ Vicksburg, MS 39180-6199}

ERDC - ECB

$-,-$
Project: TVA Fly Ash Project

Project Manager: Tony Bednar
Reported:

03-Sep-2009

DRAFT: SLC MT N2 Hour 1 Rep E

9061502-29 (Water)

\begin{tabular}{|c|c|c|c|c|c|c|c|c|}
\hline Analyte & Result & $\begin{array}{r}\text { porting } \\
\text { Limit }\end{array}$ & Units & Dilution & Prepared & Analyzed & Method & Notes \\
\hline
\end{tabular}

ERDC- EL-EP-C (Environmental Chemistry Branch)

DRAFT: Wet Chemistry

\begin{tabular}{llllllll}
\hline Hardness & $\mathbf{9 7 . 7}$ & 1.32 & $\begin{array}{c}\text { mg equil } \\
\text { CaCO3/ }\end{array}$ & 1 & 25-Jun-2009 & 25-Jun-2009 & Hardness
\end{tabular}

$\mathrm{L}$

DRAFT: Metals by EPA 6000/7000 Series Methods

\begin{tabular}{|c|c|c|c|c|c|c|c|c|}
\hline Mercury & 0.00001 & 0.000005 & $\mathrm{mg} / \mathrm{L}$ & 1 & 17-Jun-2009 & 01-Jul-2009 & EPA 7471A & \\
\hline Strontium & 0.535 & 0.0200 & $\mathrm{mg} / \mathrm{L}$ & 1 & 25-Jun-2009 & 25-Jun-2009 & EPA $6010 B$ & \\
\hline Aluminum & 0.122 & 0.200 & $\mathrm{mg} / \mathrm{L}$ & 1 & 25-Jun-2009 & 25-Jun-2009 & SW 846/6010 & $\mathrm{J}$ \\
\hline Calcium & 30.6 & 0.200 & $\mathrm{mg} / \mathrm{L}$ & 1 & 25-Jun-2009 & 25-Jun-2009 & SW 846/6010 & \\
\hline Iron & ND & 0.200 & $\mathrm{mg} / \mathrm{L}$ & 1 & 25-Jun-2009 & 25-Jun-2009 & SW 846/6010 & $\mathrm{U}$ \\
\hline Magnesium & 5.19 & 0.200 & $\mathrm{mg} / \mathrm{L}$ & 1 & 25-Jun-2009 & 25-Jun-2009 & SW 846/6010 & \\
\hline Potassium & 1.90 & 0.200 & $\mathrm{mg} / \mathrm{L}$ & 1 & 25-Jun-2009 & 25-Jun-2009 & SW 846/6010 & \\
\hline Sodium & 4.02 & 0.200 & $\mathrm{mg} / \mathrm{L}$ & 1 & 25-Jun-2009 & 25-Jun-2009 & SW 846/6010 & \\
\hline Antimony & 0.0080 & 0.0010 & $\mathrm{mg} / \mathrm{L}$ & 2 & 16-Jun-2009 & 16-Jun-2009 & SW 846/6020 & \\
\hline Arsenic & 0.0776 & 0.0010 & $\mathrm{mg} / \mathrm{L}$ & 2 & 16-Jun-2009 & 16-Jun-2009 & SW 846/6020 & \\
\hline Barium & 0.103 & 0.0010 & $\mathrm{mg} / \mathrm{L}$ & 2 & 16-Jun-2009 & 16-Jun-2009 & SW 846/6020 & \\
\hline Beryllium & ND & 0.0010 & $\mathrm{mg} / \mathrm{L}$ & 2 & 16-Jun-2009 & 16-Jun-2009 & SW 846/6020 & $\mathrm{U}$ \\
\hline Cadmium & 0.0003 & 0.0010 & $\mathrm{mg} / \mathrm{L}$ & 2 & 16-Jun-2009 & 16-Jun-2009 & SW 846/6020 & J \\
\hline Chromium & ND & 0.0010 & $\mathrm{mg} / \mathrm{L}$ & 2 & 16-Jun-2009 & 16-Jun-2009 & SW 846/6020 & $\mathrm{U}$ \\
\hline Cobalt & ND & 0.0010 & $\mathrm{mg} / \mathrm{L}$ & 2 & 16-Jun-2009 & 16-Jun-2009 & SW 846/6020 & $\mathrm{U}$ \\
\hline Copper & 1.81 & 0.0010 & $\mathrm{mg} / \mathrm{L}$ & 2 & 16-Jun-2009 & 16-Jun-2009 & SW 846/6020 & \\
\hline Lead & 0.0366 & 0.0010 & $\mathrm{mg} / \mathrm{L}$ & 2 & 16-Jun-2009 & 16-Jun-2009 & SW 846/6020 & \\
\hline Manganese & 0.0353 & 0.0010 & $\mathrm{mg} / \mathrm{L}$ & 2 & 16-Jun-2009 & 16-Jun-2009 & SW 846/6020 & \\
\hline Molybdenum & 0.0738 & 0.0010 & $\mathrm{mg} / \mathrm{L}$ & 2 & 16-Jun-2009 & 16-Jun-2009 & SW 846/6020 & \\
\hline Nickel & 0.0029 & 0.0010 & $\mathrm{mg} / \mathrm{L}$ & 2 & 16-Jun-2009 & 16-Jun-2009 & SW 846/6020 & \\
\hline Selenium & 0.0058 & 0.0010 & $\mathrm{mg} / \mathrm{L}$ & 2 & 16-Jun-2009 & 16-Jun-2009 & SW 846/6020 & \\
\hline Silver & ND & 0.0010 & $\mathrm{mg} / \mathrm{L}$ & 2 & 16-Jun-2009 & 16-Jun-2009 & SW 846/6020 & $\mathrm{U}$ \\
\hline Thallium & 0.0006 & 0.0010 & $\mathrm{mg} / \mathrm{L}$ & 2 & 16-Jun-2009 & 16-Jun-2009 & SW 846/6020 & $\mathrm{J}$ \\
\hline Vanadium & 0.0536 & 0.0010 & $\mathrm{mg} / \mathrm{L}$ & 2 & 16-Jun-2009 & 16-Jun-2009 & SW 846/6020 & \\
\hline Zinc & 1.01 & 0.0010 & $\mathrm{mg} / \mathrm{L}$ & 2 & 16-Jun-2009 & 16-Jun-2009 & SW 846/6020 & $\mathrm{B}$ \\
\hline
\end{tabular}




\section{USACE ERDC-EP-C \\ 3909 Halls Ferry Road \\ Vicksburg, MS 39180-6199}

ERDC - ECB

Project: TVA Fly Ash Project

Reported:

Project Manager: Tony Bednar

03-Sep-2009

DRAFT: SLC MT N2 Hour 1 Rep E

9061502-29 (Water)

\begin{tabular}{|c|c|c|c|c|c|c|c|c|}
\hline & & orting & & & & & & \\
\hline Analyte & Result & Limit & Units & Dilution & Prepared & Analyzed & Method & Notes \\
\hline
\end{tabular}

ERDC- EL-EP-C (Environmental Chemistry Branch)

DRAFT: Miscellaneous Physical/Conventional Chemistry Parameters

\begin{tabular}{lrrrrrrr}
\hline Bromide & $\mathrm{ND}$ & 0.100 & $\mathrm{mg} / \mathrm{L}$ & 1 & 14-Jul-2009 & $14-\mathrm{Jul}-2009$ & EPA 300.0 \\
Chloride & $\mathbf{3 . 7 9}$ & 1.00 & $\mathrm{mg} / \mathrm{L}$ & 1 & $14-J u l-2009$ & $14-\mathrm{Jul}-2009$ & EPA 300.0 \\
Nitrate as N & $\mathbf{0 . 3 9 0}$ & 0.100 & $\mathrm{mg} / \mathrm{L}$ & 1 & $14-J u l-2009$ & $14-J u l-2009$ & EPA 300.0 \\
Sulfate & $\mathbf{3 6 . 6}$ & 0.100 & $\mathrm{mg} / \mathrm{L}$ & 1 & $14-J u l-2009$ & $14-J u l-2009$ & EPA 300.0
\end{tabular}




\section{USACE ERDC-EP-C \\ 3909 Halls Ferry Road \\ Vicksburg, MS 39180-6199}

ERDC - ECB

$-,-$
Project: TVA Fly Ash Project

Project Manager: Tony Bednar
Reported:

03-Sep-2009

DRAFT: SLC MT N2 Hour 1 Rep F

9061502-30 (Water)

\begin{tabular}{|c|c|c|c|c|c|c|c|c|}
\hline Analyte & Result & $\begin{array}{r}\text { porting } \\
\text { Limit }\end{array}$ & Units & Dilution & Prepared & Analyzed & Method & Notes \\
\hline
\end{tabular}

ERDC- EL-EP-C (Environmental Chemistry Branch)

DRAFT: Wet Chemistry

\begin{tabular}{lccccccc}
\hline Hardness & $\mathbf{9 7 . 5}$ & 1.32 & $\begin{array}{c}\text { mg equil } \\
\text { CaCO3/ }\end{array}$ & 1 & 25-Jun-2009 & 25-Jun-2009
\end{tabular}

$\mathrm{L}$

DRAFT: Metals by EPA 6000/7000 Series Methods

\begin{tabular}{|c|c|c|c|c|c|c|c|c|}
\hline Mercury & ND & 0.000005 & $\mathrm{mg} / \mathrm{L}$ & 1 & 17-Jun-2009 & 01-Jul-2009 & EPA 7471A & $\mathrm{U}$ \\
\hline Strontium & 0.536 & 0.0200 & $\mathrm{mg} / \mathrm{L}$ & 1 & 25-Jun-2009 & 25-Jun-2009 & EPA 6010B & \\
\hline Aluminum & 0.124 & 0.200 & $\mathrm{mg} / \mathrm{L}$ & 1 & 25-Jun-2009 & 25-Jun-2009 & SW 846/6010 & $\mathrm{J}$ \\
\hline Calcium & 30.6 & 0.200 & $\mathrm{mg} / \mathrm{L}$ & 1 & 25-Jun-2009 & 25-Jun-2009 & SW 846/6010 & \\
\hline Iron & ND & 0.200 & $\mathrm{mg} / \mathrm{L}$ & 1 & 25-Jun-2009 & 25-Jun-2009 & SW 846/6010 & $\mathrm{U}$ \\
\hline Magnesium & 5.10 & 0.200 & $\mathrm{mg} / \mathrm{L}$ & 1 & 25-Jun-2009 & 25-Jun-2009 & SW 846/6010 & \\
\hline Potassium & 1.90 & 0.200 & $\mathrm{mg} / \mathrm{L}$ & 1 & 25-Jun-2009 & 25-Jun-2009 & SW 846/6010 & \\
\hline Sodium & 4.04 & 0.200 & $\mathrm{mg} / \mathrm{L}$ & 1 & 25-Jun-2009 & 25-Jun-2009 & SW 846/6010 & \\
\hline Antimony & 0.0078 & 0.0010 & $\mathrm{mg} / \mathrm{L}$ & 2 & 16-Jun-2009 & 16-Jun-2009 & SW 846/6020 & \\
\hline Arsenic & 0.0763 & 0.0010 & $\mathrm{mg} / \mathrm{L}$ & 2 & 16-Jun-2009 & 16-Jun-2009 & SW 846/6020 & \\
\hline Barium & 0.103 & 0.0010 & $\mathrm{mg} / \mathrm{L}$ & 2 & 16-Jun-2009 & 16-Jun-2009 & SW 846/6020 & \\
\hline Beryllium & ND & 0.0010 & $\mathrm{mg} / \mathrm{L}$ & 2 & 16-Jun-2009 & 16-Jun-2009 & SW 846/6020 & $\mathrm{U}$ \\
\hline Cadmium & 0.0003 & 0.0010 & $\mathrm{mg} / \mathrm{L}$ & 2 & 16-Jun-2009 & 16-Jun-2009 & SW 846/6020 & J \\
\hline Chromium & ND & 0.0010 & $\mathrm{mg} / \mathrm{L}$ & 2 & 16-Jun-2009 & 16-Jun-2009 & SW 846/6020 & $\mathrm{U}$ \\
\hline Cobalt & ND & 0.0010 & $\mathrm{mg} / \mathrm{L}$ & 2 & 16-Jun-2009 & 16-Jun-2009 & SW 846/6020 & $\mathrm{U}$ \\
\hline Copper & 0.0006 & 0.0010 & $\mathrm{mg} / \mathrm{L}$ & 2 & 16-Jun-2009 & 16-Jun-2009 & SW 846/6020 & J \\
\hline Lead & ND & 0.0010 & $\mathrm{mg} / \mathrm{L}$ & 2 & 16-Jun-2009 & 16-Jun-2009 & SW 846/6020 & $\mathrm{U}$ \\
\hline Manganese & 0.0351 & 0.0010 & $\mathrm{mg} / \mathrm{L}$ & 2 & 16-Jun-2009 & 16-Jun-2009 & SW 846/6020 & \\
\hline Molybdenum & 0.0732 & 0.0010 & $\mathrm{mg} / \mathrm{L}$ & 2 & 16-Jun-2009 & 16-Jun-2009 & SW 846/6020 & \\
\hline Nickel & 0.0005 & 0.0010 & $\mathrm{mg} / \mathrm{L}$ & 2 & 16-Jun-2009 & 16-Jun-2009 & SW 846/6020 & $\mathrm{J}$ \\
\hline Selenium & 0.0057 & 0.0010 & $\mathrm{mg} / \mathrm{L}$ & 2 & 16-Jun-2009 & 16-Jun-2009 & SW 846/6020 & \\
\hline Silver & ND & 0.0010 & $\mathrm{mg} / \mathrm{L}$ & 2 & 16-Jun-2009 & 16-Jun-2009 & SW 846/6020 & $\mathrm{U}$ \\
\hline Thallium & 0.0006 & 0.0010 & $\mathrm{mg} / \mathrm{L}$ & 2 & 16-Jun-2009 & 16-Jun-2009 & SW 846/6020 & $\mathrm{J}$ \\
\hline Vanadium & 0.0542 & 0.0010 & $\mathrm{mg} / \mathrm{L}$ & 2 & 16-Jun-2009 & 16-Jun-2009 & SW 846/6020 & \\
\hline Zinc & 0.0052 & 0.0010 & $\mathrm{mg} / \mathrm{L}$ & 2 & 16-Jun-2009 & 16-Jun-2009 & SW 846/6020 & B \\
\hline
\end{tabular}




\section{USACE ERDC-EP-C \\ 3909 Halls Ferry Road \\ Vicksburg, MS 39180-6199}

ERDC - ECB

Project: TVA Fly Ash Project

Reported:

$-$

Project Manager: Tony Bednar

03-Sep-2009

DRAFT: SLC MT N2 Hour 1 Rep F

9061502-30 (Water)

\begin{tabular}{|lrrrrrrrr}
\hline & & & & & & & & \\
Analyte & Result & Limit & Units & Dilution & Prepared & Analyzed & Method & Notes \\
\hline
\end{tabular}

ERDC- EL-EP-C (Environmental Chemistry Branch)

DRAFT: Miscellaneous Physical/Conventional Chemistry Parameters

\begin{tabular}{lrrrrrrr}
\hline Bromide & $\mathrm{ND}$ & 0.100 & $\mathrm{mg} / \mathrm{L}$ & 1 & 14-Jul-2009 & $14-\mathrm{Jul}-2009$ & EPA 300.0 \\
Chloride & $\mathbf{3 . 7 6}$ & 1.00 & $\mathrm{mg} / \mathrm{L}$ & 1 & $14-J u l-2009$ & $14-\mathrm{Jul}-2009$ & EPA 300.0 \\
Nitrate as N & $\mathbf{0 . 3 7 0}$ & 0.100 & $\mathrm{mg} / \mathrm{L}$ & 1 & $14-J u l-2009$ & $14-J u l-2009$ & EPA 300.0 \\
Sulfate & $\mathbf{3 5 . 9}$ & 0.100 & $\mathrm{mg} / \mathrm{L}$ & 1 & $14-J u l-2009$ & $14-J u l-2009$ & EPA 300.0
\end{tabular}




\section{USACE ERDC-EP-C \\ 3909 Halls Ferry Road \\ Vicksburg, MS 39180-6199}

ERDC - ECB

$-,-$
Project: TVA Fly Ash Project

Project Manager: Tony Bednar
Reported:

03-Sep-2009

DRAFT: SLC MT O2 Hour 1 Rep D

9061502-34 (Water)

\begin{tabular}{|c|c|c|c|c|c|c|c|c|}
\hline Analyte & Result & $\begin{array}{r}\text { porting } \\
\text { Limit }\end{array}$ & Units & Dilution & Prepared & Analyzed & Method & Notes \\
\hline
\end{tabular}

ERDC- EL-EP-C (Environmental Chemistry Branch)

DRAFT: Wet Chemistry

\begin{tabular}{llllllll}
\hline Hardness & $\mathbf{9 7 . 1}$ & 1.32 & $\begin{array}{c}\text { mg equil } \\
\text { CaCO3/ }\end{array}$ & 1 & 25-Jun-2009 & 25-Jun-2009
\end{tabular}

$\mathrm{L}$

DRAFT: Metals by EPA 6000/7000 Series Methods

\begin{tabular}{|c|c|c|c|c|c|c|c|c|}
\hline Mercury & 0.000013 & 0.000005 & $\mathrm{mg} / \mathrm{L}$ & 1 & 17-Jun-2009 & 01-Jul-2009 & EPA 7471A & \\
\hline Strontium & 0.533 & 0.0200 & $\mathrm{mg} / \mathrm{L}$ & 1 & 25-Jun-2009 & 25-Jun-2009 & EPA 6010B & \\
\hline Aluminum & 0.105 & 0.200 & $\mathrm{mg} / \mathrm{L}$ & 1 & 25-Jun-2009 & 25-Jun-2009 & SW 846/6010 & J \\
\hline Calcium & 30.5 & 0.200 & $\mathrm{mg} / \mathrm{L}$ & 1 & 25-Jun-2009 & 25-Jun-2009 & SW 846/6010 & \\
\hline Iron & ND & 0.200 & $\mathrm{mg} / \mathrm{L}$ & 1 & 25-Jun-2009 & 25-Jun-2009 & SW 846/6010 & $\mathrm{U}$ \\
\hline Magnesium & 5.10 & 0.200 & $\mathrm{mg} / \mathrm{L}$ & 1 & 25-Jun-2009 & 25-Jun-2009 & SW 846/6010 & \\
\hline Potassium & 1.92 & 0.200 & $\mathrm{mg} / \mathrm{L}$ & 1 & 25-Jun-2009 & 25-Jun-2009 & SW 846/6010 & \\
\hline Sodium & 4.09 & 0.200 & $\mathrm{mg} / \mathrm{L}$ & 1 & 25-Jun-2009 & 25-Jun-2009 & SW 846/6010 & \\
\hline Antimony & 0.0075 & 0.0010 & $\mathrm{mg} / \mathrm{L}$ & 2 & 16-Jun-2009 & 16-Jun-2009 & SW 846/6020 & \\
\hline Arsenic & 0.0723 & 0.0010 & $\mathrm{mg} / \mathrm{L}$ & 2 & 16-Jun-2009 & 16-Jun-2009 & SW 846/6020 & \\
\hline Barium & 0.0994 & 0.0010 & $\mathrm{mg} / \mathrm{L}$ & 2 & 16-Jun-2009 & 16-Jun-2009 & SW 846/6020 & \\
\hline Beryllium & ND & 0.0010 & $\mathrm{mg} / \mathrm{L}$ & 2 & 16-Jun-2009 & 16-Jun-2009 & SW 846/6020 & U \\
\hline Cadmium & 0.0003 & 0.0010 & $\mathrm{mg} / \mathrm{L}$ & 2 & 16-Jun-2009 & 16-Jun-2009 & SW 846/6020 & J \\
\hline Chromium & ND & 0.0010 & $\mathrm{mg} / \mathrm{L}$ & 2 & 16-Jun-2009 & 16-Jun-2009 & SW 846/6020 & U \\
\hline Cobalt & ND & 0.0010 & $\mathrm{mg} / \mathrm{L}$ & 2 & 16-Jun-2009 & 16-Jun-2009 & SW 846/6020 & U \\
\hline Copper & 0.0008 & 0.0010 & $\mathrm{mg} / \mathrm{L}$ & 2 & 16-Jun-2009 & 16-Jun-2009 & SW 846/6020 & J \\
\hline Lead & ND & 0.0010 & $\mathrm{mg} / \mathrm{L}$ & 2 & 16-Jun-2009 & 16-Jun-2009 & SW 846/6020 & U \\
\hline Manganese & 0.0358 & 0.0010 & $\mathrm{mg} / \mathrm{L}$ & 2 & 16-Jun-2009 & 16-Jun-2009 & SW 846/6020 & \\
\hline Molybdenum & 0.0692 & 0.0010 & $\mathrm{mg} / \mathrm{L}$ & 2 & 16-Jun-2009 & 16-Jun-2009 & SW 846/6020 & \\
\hline Nickel & 0.00067 & 0.0010 & $\mathrm{mg} / \mathrm{L}$ & 2 & 16-Jun-2009 & 16-Jun-2009 & SW 846/6020 & J \\
\hline Selenium & 0.0058 & 0.0010 & $\mathrm{mg} / \mathrm{L}$ & 2 & 16-Jun-2009 & 16-Jun-2009 & SW 846/6020 & \\
\hline Silver & ND & 0.0010 & $\mathrm{mg} / \mathrm{L}$ & 2 & 16-Jun-2009 & 16-Jun-2009 & SW 846/6020 & $\mathrm{U}$ \\
\hline Thallium & 0.0006 & 0.0010 & $\mathrm{mg} / \mathrm{L}$ & 2 & 16-Jun-2009 & 16-Jun-2009 & SW 846/6020 & J \\
\hline Vanadium & 0.0537 & 0.0010 & $\mathrm{mg} / \mathrm{L}$ & 2 & 16-Jun-2009 & 16-Jun-2009 & SW 846/6020 & \\
\hline Zinc & 0.0059 & 0.0010 & $\mathrm{mg} / \mathrm{L}$ & 2 & 16-Jun-2009 & 16-Jun-2009 & SW 846/6020 & $\mathrm{B}$ \\
\hline
\end{tabular}




\section{USACE ERDC-EP-C \\ 3909 Halls Ferry Road \\ Vicksburg, MS 39180-6199}

ERDC - ECB

Project: TVA Fly Ash Project

Reported:

$--,-$

Project Manager: Tony Bednar

03-Sep-2009

DRAFT: SLC MT O2 Hour 1 Rep D

9061502-34 (Water)

\begin{tabular}{|lrrrrrrrr}
\hline & & & & & & & & \\
Analyte & Result & Leporting & & & & \\
Limit & Units & Dilution & Prepared & Analyzed & Method & Notes \\
\hline
\end{tabular}

ERDC- EL-EP-C (Environmental Chemistry Branch)

DRAFT: Miscellaneous Physical/Conventional Chemistry Parameters

\begin{tabular}{lrrrrrrr}
\hline Bromide & $\mathrm{ND}$ & 0.100 & $\mathrm{mg} / \mathrm{L}$ & 1 & 14-Jul-2009 & 14-Jul-2009 & EPA 300.0 \\
Chloride & $\mathbf{3 . 8 7}$ & 1.00 & $\mathrm{mg} / \mathrm{L}$ & 1 & $14-J u l-2009$ & $14-\mathrm{Jul}-2009$ & EPA 300.0 \\
Nitrate as N & $\mathbf{0 . 3 8 0}$ & 0.100 & $\mathrm{mg} / \mathrm{L}$ & 1 & $14-J u l-2009$ & $14-J u l-2009$ & EPA 300.0 \\
Sulfate & $\mathbf{3 6 . 2}$ & 0.100 & $\mathrm{mg} / \mathrm{L}$ & 1 & $14-J u l-2009$ & $14-J u l-2009$ & EPA 300.0
\end{tabular}




\section{USACE ERDC-EP-C \\ 3909 Halls Ferry Road \\ Vicksburg, MS 39180-6199}

ERDC - ECB

$-,-$
Project: TVA Fly Ash Project

Project Manager: Tony Bednar
Reported:

03-Sep-2009

DRAFT: SLC MT O2 Hour 1 Rep E

9061502-35 (Water)

\begin{tabular}{|c|c|c|c|c|c|c|c|c|}
\hline Analyte & Result & $\begin{array}{r}\text { porting } \\
\text { Limit }\end{array}$ & Units & Dilution & Prepared & Analyzed & Method & Notes \\
\hline
\end{tabular}

ERDC- EL-EP-C (Environmental Chemistry Branch)

DRAFT: Wet Chemistry

\begin{tabular}{lccccccc}
\hline Hardness & $\mathbf{9 7 . 8}$ & 1.32 & $\begin{array}{c}\text { mg equil } \\
\text { CaCO3/ }\end{array}$ & 1 & 25-Jun-2009 & 25-Jun-2009 & Hardness
\end{tabular}

$\mathrm{L}$

DRAFT: Metals by EPA 6000/7000 Series Methods

\begin{tabular}{|c|c|c|c|c|c|c|c|c|}
\hline Mercury & ND & 0.000005 & $\mathrm{mg} / \mathrm{L}$ & 1 & 17-Jun-2009 & 01-Jul-2009 & EPA 7471A & $\mathrm{U}$ \\
\hline Strontium & 0.534 & 0.0200 & $\mathrm{mg} / \mathrm{L}$ & 1 & 25-Jun-2009 & 25-Jun-2009 & EPA 6010B & \\
\hline Aluminum & 0.0883 & 0.200 & $\mathrm{mg} / \mathrm{L}$ & 1 & 25-Jun-2009 & 25-Jun-2009 & SW 846/6010 & J \\
\hline Calcium & 30.7 & 0.200 & $\mathrm{mg} / \mathrm{L}$ & 1 & 25-Jun-2009 & 25-Jun-2009 & SW 846/6010 & \\
\hline Iron & ND & 0.200 & $\mathrm{mg} / \mathrm{L}$ & 1 & 25-Jun-2009 & 25-Jun-2009 & SW 846/6010 & $\mathrm{U}$ \\
\hline Magnesium & 5.16 & 0.200 & $\mathrm{mg} / \mathrm{L}$ & 1 & 25-Jun-2009 & 25-Jun-2009 & SW 846/6010 & \\
\hline Potassium & 1.94 & 0.200 & $\mathrm{mg} / \mathrm{L}$ & 1 & 25-Jun-2009 & 25-Jun-2009 & SW 846/6010 & \\
\hline Sodium & 4.07 & 0.200 & $\mathrm{mg} / \mathrm{L}$ & 1 & 25-Jun-2009 & 25-Jun-2009 & SW 846/6010 & \\
\hline Antimony & 0.0075 & 0.0010 & $\mathrm{mg} / \mathrm{L}$ & 2 & 16-Jun-2009 & 16-Jun-2009 & SW 846/6020 & \\
\hline Arsenic & 0.0746 & 0.0010 & $\mathrm{mg} / \mathrm{L}$ & 2 & 16-Jun-2009 & 16-Jun-2009 & SW 846/6020 & \\
\hline Barium & 0.0998 & 0.0010 & $\mathrm{mg} / \mathrm{L}$ & 2 & 16-Jun-2009 & 16-Jun-2009 & SW 846/6020 & \\
\hline Beryllium & ND & 0.0010 & $\mathrm{mg} / \mathrm{L}$ & 2 & 16-Jun-2009 & 16-Jun-2009 & SW 846/6020 & $\mathrm{U}$ \\
\hline Cadmium & 0.0003 & 0.0010 & $\mathrm{mg} / \mathrm{L}$ & 2 & 16-Jun-2009 & 16-Jun-2009 & SW 846/6020 & J \\
\hline Chromium & ND & 0.0010 & $\mathrm{mg} / \mathrm{L}$ & 2 & 16-Jun-2009 & 16-Jun-2009 & SW 846/6020 & $\mathrm{U}$ \\
\hline Cobalt & ND & 0.0010 & $\mathrm{mg} / \mathrm{L}$ & 2 & 16-Jun-2009 & 16-Jun-2009 & SW 846/6020 & $\mathrm{U}$ \\
\hline Copper & 0.0005 & 0.0010 & $\mathrm{mg} / \mathrm{L}$ & 2 & 16-Jun-2009 & 16-Jun-2009 & SW 846/6020 & $\mathrm{J}$ \\
\hline Lead & ND & 0.0010 & $\mathrm{mg} / \mathrm{L}$ & 2 & 16-Jun-2009 & 16-Jun-2009 & SW 846/6020 & $\mathrm{U}$ \\
\hline Manganese & 0.0355 & 0.0010 & $\mathrm{mg} / \mathrm{L}$ & 2 & 16-Jun-2009 & 16-Jun-2009 & SW 846/6020 & \\
\hline Molybdenum & 0.0686 & 0.0010 & $\mathrm{mg} / \mathrm{L}$ & 2 & 16-Jun-2009 & 16-Jun-2009 & SW 846/6020 & \\
\hline Nickel & 0.0006 & 0.0010 & $\mathrm{mg} / \mathrm{L}$ & 2 & 16-Jun-2009 & 16-Jun-2009 & SW 846/6020 & $\mathrm{J}$ \\
\hline Selenium & 0.0058 & 0.0010 & $\mathrm{mg} / \mathrm{L}$ & 2 & 16-Jun-2009 & 16-Jun-2009 & SW 846/6020 & \\
\hline Silver & ND & 0.0010 & $\mathrm{mg} / \mathrm{L}$ & 2 & 16-Jun-2009 & 16-Jun-2009 & SW 846/6020 & $\mathrm{U}$ \\
\hline Thallium & 0.0006 & 0.0010 & $\mathrm{mg} / \mathrm{L}$ & 2 & 16-Jun-2009 & 16-Jun-2009 & SW 846/6020 & $\mathrm{J}$ \\
\hline Vanadium & 0.0537 & 0.0010 & $\mathrm{mg} / \mathrm{L}$ & 2 & 16-Jun-2009 & 16-Jun-2009 & SW 846/6020 & \\
\hline Zinc & 0.0085 & 0.0010 & $\mathrm{mg} / \mathrm{L}$ & 2 & 16-Jun-2009 & 16-Jun-2009 & SW 846/6020 & B \\
\hline
\end{tabular}




\section{USACE ERDC-EP-C \\ 3909 Halls Ferry Road \\ Vicksburg, MS 39180-6199}

ERDC - ECB

Project: TVA Fly Ash Project

Reported:

Project Manager: Tony Bednar

03-Sep-2009

DRAFT: SLC MT O2 Hour 1 Rep E

9061502-35 (Water)

\begin{tabular}{|c|c|c|c|c|c|c|c|c|}
\hline & & orting & & & & & & \\
\hline Analyte & Result & Limit & Units & Dilution & Prepared & Analyzed & Method & Notes \\
\hline
\end{tabular}

ERDC- EL-EP-C (Environmental Chemistry Branch)

DRAFT: Miscellaneous Physical/Conventional Chemistry Parameters

\begin{tabular}{lrrrrrrr}
\hline Bromide & $\mathrm{ND}$ & 0.100 & $\mathrm{mg} / \mathrm{L}$ & 1 & 14-Jul-2009 & 14-Jul-2009 & EPA 300.0 \\
Chloride & $\mathbf{3 . 7 7}$ & 1.00 & $\mathrm{mg} / \mathrm{L}$ & 1 & $14-J u l-2009$ & $14-\mathrm{Jul}-2009$ & EPA 300.0 \\
Nitrate as N & $\mathbf{0 . 3 9 0}$ & 0.100 & $\mathrm{mg} / \mathrm{L}$ & 1 & $14-J u l-2009$ & $14-J u l-2009$ & EPA 300.0 \\
Sulfate & $\mathbf{3 6 . 0}$ & 0.100 & $\mathrm{mg} / \mathrm{L}$ & 1 & $14-J u l-2009$ & $14-J u l-2009$ & EPA 300.0
\end{tabular}




\section{USACE ERDC-EP-C \\ 3909 Halls Ferry Road \\ Vicksburg, MS 39180-6199}

ERDC - ECB

$-,-$
Project: TVA Fly Ash Project

Project Manager: Tony Bednar
Reported:

03-Sep-2009

DRAFT: SLC MT O2 Hour 1 Rep F

9061502-36 (Water)

\begin{tabular}{|c|c|c|c|c|c|c|c|c|}
\hline Analyte & Result & $\begin{array}{r}\text { porting } \\
\text { Limit }\end{array}$ & Units & Dilution & Prepared & Analyzed & Method & Notes \\
\hline
\end{tabular}

ERDC- EL-EP-C (Environmental Chemistry Branch)

DRAFT: Wet Chemistry

\begin{tabular}{lccccccc}
\hline Hardness & $\mathbf{9 7 . 6}$ & 1.32 & $\begin{array}{c}\text { mg equil } \\
\text { CaCO3/ }\end{array}$ & 1 & 25-Jun-2009 & 25-Jun-2009 & Hardness
\end{tabular}

$\mathrm{L}$

DRAFT: Metals by EPA 6000/7000 Series Methods

\begin{tabular}{|c|c|c|c|c|c|c|c|c|}
\hline Mercury & $8.30 \mathrm{E}-6$ & 0.000005 & $\mathrm{mg} / \mathrm{L}$ & 1 & 17-Jun-2009 & 01-Jul-2009 & EPA 7471A & \\
\hline Strontium & 0.536 & 0.0200 & $\mathrm{mg} / \mathrm{L}$ & 1 & 25-Jun-2009 & 25-Jun-2009 & EPA 6010B & \\
\hline Aluminum & 0.0901 & 0.200 & $\mathrm{mg} / \mathrm{L}$ & 1 & 25-Jun-2009 & 25-Jun-2009 & SW 846/6010 & $\mathrm{J}$ \\
\hline Calcium & 30.6 & 0.200 & $\mathrm{mg} / \mathrm{L}$ & 1 & 25-Jun-2009 & 25-Jun-2009 & SW 846/6010 & \\
\hline Iron & ND & 0.200 & $\mathrm{mg} / \mathrm{L}$ & 1 & 25-Jun-2009 & 25-Jun-2009 & SW 846/6010 & U \\
\hline Magnesium & 5.12 & 0.200 & $\mathrm{mg} / \mathrm{L}$ & 1 & 25-Jun-2009 & 25-Jun-2009 & SW 846/6010 & \\
\hline Potassium & 1.89 & 0.200 & $\mathrm{mg} / \mathrm{L}$ & 1 & 25-Jun-2009 & 25-Jun-2009 & SW 846/6010 & \\
\hline Sodium & 4.04 & 0.200 & $\mathrm{mg} / \mathrm{L}$ & 1 & 25-Jun-2009 & 25-Jun-2009 & SW 846/6010 & \\
\hline Antimony & 0.0078 & 0.0010 & $\mathrm{mg} / \mathrm{L}$ & 2 & 16-Jun-2009 & 16-Jun-2009 & SW 846/6020 & \\
\hline Arsenic & 0.0782 & 0.0010 & $\mathrm{mg} / \mathrm{L}$ & 2 & 16-Jun-2009 & 16-Jun-2009 & SW 846/6020 & \\
\hline Barium & 0.0989 & 0.0010 & $\mathrm{mg} / \mathrm{L}$ & 2 & 16-Jun-2009 & 16-Jun-2009 & SW 846/6020 & \\
\hline Beryllium & ND & 0.0010 & $\mathrm{mg} / \mathrm{L}$ & 2 & 16-Jun-2009 & 16-Jun-2009 & SW 846/6020 & U \\
\hline Cadmium & 0.0003 & 0.0010 & $\mathrm{mg} / \mathrm{L}$ & 2 & 16-Jun-2009 & 16-Jun-2009 & SW 846/6020 & J \\
\hline Chromium & ND & 0.0010 & $\mathrm{mg} / \mathrm{L}$ & 2 & 16-Jun-2009 & 16-Jun-2009 & SW 846/6020 & U \\
\hline Cobalt & ND & 0.0010 & $\mathrm{mg} / \mathrm{L}$ & 2 & 16-Jun-2009 & 16-Jun-2009 & SW 846/6020 & U \\
\hline Copper & 0.0022 & 0.0010 & $\mathrm{mg} / \mathrm{L}$ & 2 & 16-Jun-2009 & 16-Jun-2009 & SW 846/6020 & \\
\hline Lead & ND & 0.0010 & $\mathrm{mg} / \mathrm{L}$ & 2 & 16-Jun-2009 & 16-Jun-2009 & SW 846/6020 & $\mathrm{U}$ \\
\hline Manganese & 0.0369 & 0.0010 & $\mathrm{mg} / \mathrm{L}$ & 2 & 16-Jun-2009 & 16-Jun-2009 & SW 846/6020 & \\
\hline Molybdenum & 0.0729 & 0.0010 & $\mathrm{mg} / \mathrm{L}$ & 2 & 16-Jun-2009 & 16-Jun-2009 & SW 846/6020 & \\
\hline Nickel & 0.0008 & 0.0010 & $\mathrm{mg} / \mathrm{L}$ & 2 & 16-Jun-2009 & 16-Jun-2009 & SW 846/6020 & J \\
\hline Selenium & 0.0053 & 0.0010 & $\mathrm{mg} / \mathrm{L}$ & 2 & 16-Jun-2009 & 16-Jun-2009 & SW 846/6020 & \\
\hline Silver & ND & 0.0010 & $\mathrm{mg} / \mathrm{L}$ & 2 & 16-Jun-2009 & 16-Jun-2009 & SW 846/6020 & $\mathrm{U}$ \\
\hline Thallium & 0.00058 & 0.0010 & $\mathrm{mg} / \mathrm{L}$ & 2 & 16-Jun-2009 & 16-Jun-2009 & SW 846/6020 & $\mathrm{J}$ \\
\hline Vanadium & 0.0531 & 0.0010 & $\mathrm{mg} / \mathrm{L}$ & 2 & 16-Jun-2009 & 16-Jun-2009 & SW 846/6020 & \\
\hline Zinc & 0.0080 & 0.0010 & $\mathrm{mg} / \mathrm{L}$ & 2 & 16-Jun-2009 & 16-Jun-2009 & SW 846/6020 & B \\
\hline
\end{tabular}




\section{USACE ERDC-EP-C \\ 3909 Halls Ferry Road \\ Vicksburg, MS 39180-6199}

ERDC - ECB

Project: TVA Fly Ash Project

Reported:

Project Manager: Tony Bednar

03-Sep-2009

DRAFT: SLC MT O2 Hour 1 Rep F

9061502-36 (Water)

\begin{tabular}{|lrrrrrrrr}
\hline & & & & & & & & \\
Analyte & Result & Limit & Units & Dilution & Prepared & Analyzed & Method & Notes \\
\hline
\end{tabular}

ERDC- EL-EP-C (Environmental Chemistry Branch)

DRAFT: Miscellaneous Physical/Conventional Chemistry Parameters

\begin{tabular}{lrrrrrrr}
\hline Bromide & $\mathrm{ND}$ & 0.100 & $\mathrm{mg} / \mathrm{L}$ & 1 & 14-Jul-2009 & 14-Jul-2009 & EPA 300.0 \\
Chloride & $\mathbf{3 . 7 4}$ & 1.00 & $\mathrm{mg} / \mathrm{L}$ & 1 & $14-J u l-2009$ & $14-\mathrm{Jul}-2009$ & EPA 300.0 \\
Nitrate as N & $\mathbf{0 . 3 8 0}$ & 0.100 & $\mathrm{mg} / \mathrm{L}$ & 1 & $14-J u l-2009$ & $14-J u l-2009$ & EPA 300.0 \\
Sulfate & $\mathbf{3 5 . 7}$ & 0.100 & $\mathrm{mg} / \mathrm{L}$ & 1 & $14-J u l-2009$ & $14-J u l-2009$ & EPA 300.0
\end{tabular}




\section{USACE ERDC-EP-C \\ 3909 Halls Ferry Road \\ Vicksburg, MS 39180-6199}

ERDC - ECB

$-\cdot$,
Project: TVA Fly Ash Project

Project Manager: Tony Bednar
Reported:

03-Sep-2009

DRAFT: EMR MT N2 Hour 24 Rep A

9061502-40 (Water)

\begin{tabular}{|c|c|c|c|c|c|c|c|c|}
\hline Analyte & Result & $\begin{array}{r}\text { porting } \\
\text { Limit }\end{array}$ & Units & Dilution & Prepared & Analyzed & Method & Notes \\
\hline
\end{tabular}

ERDC- EL-EP-C (Environmental Chemistry Branch)

DRAFT: Wet Chemistry

\begin{tabular}{llllllll}
\hline Hardness & $\mathbf{3 7 . 7}$ & 1.32 & $\begin{array}{c}\text { mg equil } \\
\text { CaCO3/ }\end{array}$ & 1 & 25-Jun-2009 & 25-Jun-2009
\end{tabular}

DRAFT: Metals by EPA 6000/7000 Series Methods

\begin{tabular}{|c|c|c|c|c|c|c|c|c|}
\hline Mercury & 0.000011 & 0.000005 & $\mathrm{mg} / \mathrm{L}$ & 1 & 17-Jun-2009 & 01-Jul-2009 & EPA 7471A & \\
\hline Strontium & 0.135 & 0.0200 & $\mathrm{mg} / \mathrm{L}$ & 1 & 25-Jun-2009 & 25-Jun-2009 & EPA 6010B & \\
\hline Aluminum & 0.0583 & 0.200 & $\mathrm{mg} / \mathrm{L}$ & 1 & 25-Jun-2009 & 25-Jun-2009 & SW 846/6010 & $\mathrm{J}$ \\
\hline Calcium & 11.3 & 0.200 & $\mathrm{mg} / \mathrm{L}$ & 1 & 25-Jun-2009 & 25-Jun-2009 & SW 846/6010 & \\
\hline Iron & ND & 0.200 & $\mathrm{mg} / \mathrm{L}$ & 1 & 25-Jun-2009 & 25-Jun-2009 & SW 846/6010 & $\mathrm{U}$ \\
\hline Magnesium & 2.28 & 0.200 & $\mathrm{mg} / \mathrm{L}$ & 1 & 25-Jun-2009 & 25-Jun-2009 & SW 846/6010 & \\
\hline Potassium & 1.36 & 0.200 & $\mathrm{mg} / \mathrm{L}$ & 1 & 25-Jun-2009 & 25-Jun-2009 & SW 846/6010 & \\
\hline Sodium & 2.51 & 0.200 & $\mathrm{mg} / \mathrm{L}$ & 1 & 25-Jun-2009 & 25-Jun-2009 & SW 846/6010 & \\
\hline Antimony & 0.0006 & 0.0010 & $\mathrm{mg} / \mathrm{L}$ & 2 & 16-Jun-2009 & 16-Jun-2009 & SW 846/6020 & J \\
\hline Arsenic & 0.0359 & 0.0010 & $\mathrm{mg} / \mathrm{L}$ & 2 & 16-Jun-2009 & 16-Jun-2009 & SW 846/6020 & \\
\hline Barium & 0.0879 & 0.0010 & $\mathrm{mg} / \mathrm{L}$ & 2 & 16-Jun-2009 & 16-Jun-2009 & SW 846/6020 & \\
\hline Beryllium & ND & 0.0010 & $\mathrm{mg} / \mathrm{L}$ & 2 & 16-Jun-2009 & 16-Jun-2009 & SW 846/6020 & $\mathrm{U}$ \\
\hline Cadmium & ND & 0.0010 & $\mathrm{mg} / \mathrm{L}$ & 2 & 16-Jun-2009 & 16-Jun-2009 & SW 846/6020 & $\mathrm{U}$ \\
\hline Chromium & ND & 0.0010 & $\mathrm{mg} / \mathrm{L}$ & 2 & 16-Jun-2009 & 16-Jun-2009 & SW 846/6020 & $\mathrm{U}$ \\
\hline Cobalt & ND & 0.0010 & $\mathrm{mg} / \mathrm{L}$ & 2 & 16-Jun-2009 & 16-Jun-2009 & SW 846/6020 & $\mathrm{U}$ \\
\hline Copper & 0.0014 & 0.0010 & $\mathrm{mg} / \mathrm{L}$ & 2 & 16-Jun-2009 & 16-Jun-2009 & SW 846/6020 & \\
\hline Lead & ND & 0.0010 & $\mathrm{mg} / \mathrm{L}$ & 2 & 16-Jun-2009 & 16-Jun-2009 & SW 846/6020 & $\mathrm{U}$ \\
\hline Manganese & 0.0033 & 0.0010 & $\mathrm{mg} / \mathrm{L}$ & 2 & 16-Jun-2009 & 16-Jun-2009 & SW 846/6020 & \\
\hline Molybdenum & 0.0018 & 0.0010 & $\mathrm{mg} / \mathrm{L}$ & 2 & 16-Jun-2009 & 16-Jun-2009 & SW 846/6020 & \\
\hline Nickel & 0.0006 & 0.0010 & $\mathrm{mg} / \mathrm{L}$ & 2 & 16-Jun-2009 & 16-Jun-2009 & SW 846/6020 & $\mathrm{J}$ \\
\hline Selenium & 0.0013 & 0.0010 & $\mathrm{mg} / \mathrm{L}$ & 2 & 16-Jun-2009 & 16-Jun-2009 & SW 846/6020 & \\
\hline Silver & ND & 0.0010 & $\mathrm{mg} / \mathrm{L}$ & 2 & 16-Jun-2009 & 16-Jun-2009 & SW 846/6020 & $\mathrm{U}$ \\
\hline Thallium & 0.0004 & 0.0010 & $\mathrm{mg} / \mathrm{L}$ & 2 & 16-Jun-2009 & 16-Jun-2009 & SW 846/6020 & $\mathrm{J}$ \\
\hline Vanadium & 0.0235 & 0.0010 & $\mathrm{mg} / \mathrm{L}$ & 2 & 16-Jun-2009 & 16-Jun-2009 & SW 846/6020 & \\
\hline Zinc & 0.0060 & 0.0010 & $\mathrm{mg} / \mathrm{L}$ & 2 & 16-Jun-2009 & 16-Jun-2009 & SW 846/6020 & B \\
\hline
\end{tabular}




\section{USACE ERDC-EP-C \\ 3909 Halls Ferry Road \\ Vicksburg, MS 39180-6199}

ERDC - ECB

Project: TVA Fly Ash Project

Reported:

$--,-$

Project Manager: Tony Bednar

03-Sep-2009

DRAFT: EMR MT N2 Hour 24 Rep A

9061502-40 (Water)

\begin{tabular}{|c|c|c|c|c|c|c|c|c|}
\hline & & orting & & & & & & \\
\hline Analyte & Result & Limit & Units & Dilution & Prepared & Analyzed & Method & Notes \\
\hline
\end{tabular}

ERDC- EL-EP-C (Environmental Chemistry Branch)

DRAFT: Miscellaneous Physical/Conventional Chemistry Parameters

\begin{tabular}{lrrrrrrr}
\hline Bromide & $\mathrm{ND}$ & 0.100 & $\mathrm{mg} / \mathrm{L}$ & 1 & $14-J u l-2009$ & $14-\mathrm{Jul}-2009$ & EPA 300.0 \\
Chloride & $\mathbf{2 . 6 1}$ & 1.00 & $\mathrm{mg} / \mathrm{L}$ & 1 & $14-J u l-2009$ & $14-\mathrm{Jul}-2009$ & EPA 300.0 \\
Nitrate as N & $\mathbf{0 . 3 8 0}$ & 0.100 & $\mathrm{mg} / \mathrm{L}$ & 1 & $14-J u l-2009$ & $14-J u l-2009$ & EPA 300.0 \\
Sulfate & $\mathbf{1 4 . 6}$ & 0.100 & $\mathrm{mg} / \mathrm{L}$ & 1 & $14-J u l-2009$ & $14-J u l-2009$ & EPA 300.0
\end{tabular}




\section{USACE ERDC-EP-C \\ 3909 Halls Ferry Road \\ Vicksburg, MS 39180-6199}

ERDC - ECB

$-\cdot$,
Project: TVA Fly Ash Project

Project Manager: Tony Bednar
Reported:

03-Sep-2009

DRAFT: EMR MT N2 Hour 24 Rep B

9061502-41 (Water)

\begin{tabular}{|c|c|c|c|c|c|c|c|c|}
\hline Analyte & Result & $\begin{array}{r}\text { porting } \\
\text { Limit }\end{array}$ & Units & Dilution & Prepared & Analyzed & Method & Notes \\
\hline
\end{tabular}

ERDC- EL-EP-C (Environmental Chemistry Branch)

DRAFT: Wet Chemistry

\begin{tabular}{llcccccc}
\hline Hardness & $\mathbf{3 7 . 1}$ & 1.32 & $\begin{array}{c}\text { mg equil } \\
\text { CaCO3/ }\end{array}$ & 1 & 25-Jun-2009 & 25-Jun-2009
\end{tabular}

$\mathrm{L}$

DRAFT: Metals by EPA 6000/7000 Series Methods

\begin{tabular}{|c|c|c|c|c|c|c|c|c|}
\hline Mercury & 0.000006 & 0.000005 & $\mathrm{mg} / \mathrm{L}$ & 1 & 17-Jun-2009 & 01-Jul-2009 & EPA 7471A & \\
\hline Strontium & 0.127 & 0.0200 & $\mathrm{mg} / \mathrm{L}$ & 1 & 25-Jun-2009 & 25-Jun-2009 & EPA 6010B & \\
\hline Aluminum & 0.0591 & 0.200 & $\mathrm{mg} / \mathrm{L}$ & 1 & 25-Jun-2009 & 25-Jun-2009 & SW 846/6010 & J \\
\hline Calcium & 11.1 & 0.200 & $\mathrm{mg} / \mathrm{L}$ & 1 & 25-Jun-2009 & 25-Jun-2009 & SW 846/6010 & \\
\hline Iron & ND & 0.200 & $\mathrm{mg} / \mathrm{L}$ & 1 & 25-Jun-2009 & 25-Jun-2009 & SW 846/6010 & $\mathrm{U}$ \\
\hline Magnesium & 2.29 & 0.200 & $\mathrm{mg} / \mathrm{L}$ & 1 & 25-Jun-2009 & 25-Jun-2009 & SW 846/6010 & \\
\hline Potassium & 1.36 & 0.200 & $\mathrm{mg} / \mathrm{L}$ & 1 & 25-Jun-2009 & 25-Jun-2009 & SW 846/6010 & \\
\hline Sodium & 2.56 & 0.200 & $\mathrm{mg} / \mathrm{L}$ & 1 & 25-Jun-2009 & 25-Jun-2009 & SW 846/6010 & \\
\hline Antimony & 0.0009 & 0.0010 & $\mathrm{mg} / \mathrm{L}$ & 2 & 16-Jun-2009 & 16-Jun-2009 & SW 846/6020 & J \\
\hline Arsenic & 0.0330 & 0.0010 & $\mathrm{mg} / \mathrm{L}$ & 2 & 16-Jun-2009 & 16-Jun-2009 & SW 846/6020 & \\
\hline Barium & 0.0839 & 0.0010 & $\mathrm{mg} / \mathrm{L}$ & 2 & 16-Jun-2009 & 16-Jun-2009 & SW 846/6020 & \\
\hline Beryllium & ND & 0.0010 & $\mathrm{mg} / \mathrm{L}$ & 2 & 16-Jun-2009 & 16-Jun-2009 & SW 846/6020 & $\mathrm{U}$ \\
\hline Cadmium & ND & 0.0010 & $\mathrm{mg} / \mathrm{L}$ & 2 & 16-Jun-2009 & 16-Jun-2009 & SW 846/6020 & $\mathrm{U}$ \\
\hline Chromium & ND & 0.0010 & $\mathrm{mg} / \mathrm{L}$ & 2 & 16-Jun-2009 & 16-Jun-2009 & SW 846/6020 & $\mathrm{U}$ \\
\hline Cobalt & ND & 0.0010 & $\mathrm{mg} / \mathrm{L}$ & 2 & 16-Jun-2009 & 16-Jun-2009 & SW 846/6020 & U \\
\hline Copper & 0.0016 & 0.0010 & $\mathrm{mg} / \mathrm{L}$ & 2 & 16-Jun-2009 & 16-Jun-2009 & SW 846/6020 & \\
\hline Lead & ND & 0.0010 & $\mathrm{mg} / \mathrm{L}$ & 2 & 16-Jun-2009 & 16-Jun-2009 & SW 846/6020 & $\mathrm{U}$ \\
\hline Manganese & 0.0017 & 0.0010 & $\mathrm{mg} / \mathrm{L}$ & 2 & 16-Jun-2009 & 16-Jun-2009 & SW 846/6020 & \\
\hline Molybdenum & 0.0017 & 0.0010 & $\mathrm{mg} / \mathrm{L}$ & 2 & 16-Jun-2009 & 16-Jun-2009 & SW 846/6020 & \\
\hline Nickel & 0.0006 & 0.0010 & $\mathrm{mg} / \mathrm{L}$ & 2 & 16-Jun-2009 & 16-Jun-2009 & SW 846/6020 & J \\
\hline Selenium & 0.0012 & 0.0010 & $\mathrm{mg} / \mathrm{L}$ & 2 & 16-Jun-2009 & 16-Jun-2009 & SW 846/6020 & \\
\hline Silver & ND & 0.0010 & $\mathrm{mg} / \mathrm{L}$ & 2 & 16-Jun-2009 & 16-Jun-2009 & SW 846/6020 & $\mathrm{U}$ \\
\hline Thallium & 0.0004 & 0.0010 & $\mathrm{mg} / \mathrm{L}$ & 2 & 16-Jun-2009 & 16-Jun-2009 & SW 846/6020 & J \\
\hline Vanadium & 0.0217 & 0.0010 & $\mathrm{mg} / \mathrm{L}$ & 2 & 16-Jun-2009 & 16-Jun-2009 & SW 846/6020 & \\
\hline Zinc & 0.0104 & 0.0010 & $\mathrm{mg} / \mathrm{L}$ & 2 & 16-Jun-2009 & 16-Jun-2009 & SW 846/6020 & B \\
\hline
\end{tabular}




\section{USACE ERDC-EP-C \\ 3909 Halls Ferry Road \\ Vicksburg, MS 39180-6199}

ERDC - ECB

Project: TVA Fly Ash Project

Reported:

$--,-$

Project Manager: Tony Bednar

03-Sep-2009

DRAFT: EMR MT N2 Hour 24 Rep B

9061502-41 (Water)

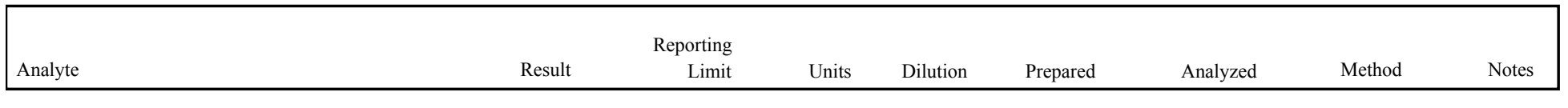

ERDC- EL-EP-C (Environmental Chemistry Branch)

DRAFT: Miscellaneous Physical/Conventional Chemistry Parameters

\begin{tabular}{lrrrrrrr}
\hline Bromide & $\mathrm{ND}$ & 0.100 & $\mathrm{mg} / \mathrm{L}$ & 1 & 14-Jul-2009 & $14-\mathrm{Jul}-2009$ & EPA 300.0 \\
Chloride & $\mathbf{2 . 6 0}$ & 1.00 & $\mathrm{mg} / \mathrm{L}$ & 1 & $14-J u l-2009$ & $14-\mathrm{Jul}-2009$ & EPA 300.0 \\
Nitrate as N & $\mathbf{0 . 3 9 6}$ & 0.100 & $\mathrm{mg} / \mathrm{L}$ & 1 & $14-J u l-2009$ & $14-J u l-2009$ & EPA 300.0 \\
Sulfate & $\mathbf{1 3 . 2}$ & 0.100 & $\mathrm{mg} / \mathrm{L}$ & 1 & $14-J u l-2009$ & $14-J u l-2009$ & EPA 300.0
\end{tabular}




\section{USACE ERDC-EP-C \\ 3909 Halls Ferry Road \\ Vicksburg, MS 39180-6199}

ERDC - ECB

$-\cdot$,
Project: TVA Fly Ash Project

Project Manager: Tony Bednar
Reported:

03-Sep-2009

DRAFT: EMR MT N2 Hour 24 Rep C

9061502-42 (Water)

\begin{tabular}{|c|c|c|c|c|c|c|c|c|}
\hline Analyte & Result & $\begin{array}{r}\text { porting } \\
\text { Limit }\end{array}$ & Units & Dilution & Prepared & Analyzed & Method & Notes \\
\hline
\end{tabular}

ERDC- EL-EP-C (Environmental Chemistry Branch)

DRAFT: Wet Chemistry

\begin{tabular}{llllllll}
\hline Hardness & $\mathbf{3 7 . 5}$ & 1.32 & $\begin{array}{c}\text { mg equil } \\
\text { CaCO3/ }\end{array}$ & 1 & 25-Jun-2009 & 25-Jun-2009
\end{tabular}

$\mathrm{L}$

DRAFT: Metals by EPA 6000/7000 Series Methods

\begin{tabular}{|c|c|c|c|c|c|c|c|c|}
\hline Mercury & ND & 0.000005 & $\mathrm{mg} / \mathrm{L}$ & 1 & 17-Jun-2009 & 01-Jul-2009 & EPA 7471A & $\mathrm{U}$ \\
\hline Strontium & 0.134 & 0.0200 & $\mathrm{mg} / \mathrm{L}$ & 1 & 25-Jun-2009 & 25-Jun-2009 & EPA 6010B & \\
\hline Aluminum & 0.0637 & 0.200 & $\mathrm{mg} / \mathrm{L}$ & 1 & 25-Jun-2009 & 25-Jun-2009 & SW 846/6010 & $\mathrm{J}$ \\
\hline Calcium & 11.3 & 0.200 & $\mathrm{mg} / \mathrm{L}$ & 1 & 25-Jun-2009 & 25-Jun-2009 & SW 846/6010 & \\
\hline Iron & ND & 0.200 & $\mathrm{mg} / \mathrm{L}$ & 1 & 25-Jun-2009 & 25-Jun-2009 & SW 846/6010 & $\mathrm{U}$ \\
\hline Magnesium & 2.29 & 0.200 & $\mathrm{mg} / \mathrm{L}$ & 1 & 25-Jun-2009 & 25-Jun-2009 & SW 846/6010 & \\
\hline Potassium & 1.37 & 0.200 & $\mathrm{mg} / \mathrm{L}$ & 1 & 25-Jun-2009 & 25-Jun-2009 & SW 846/6010 & \\
\hline Sodium & 2.66 & 0.200 & $\mathrm{mg} / \mathrm{L}$ & 1 & 25-Jun-2009 & 25-Jun-2009 & SW 846/6010 & \\
\hline Antimony & 0.0008 & 0.0010 & $\mathrm{mg} / \mathrm{L}$ & 2 & 16-Jun-2009 & 16-Jun-2009 & SW 846/6020 & J \\
\hline Arsenic & 0.0355 & 0.0010 & $\mathrm{mg} / \mathrm{L}$ & 2 & 16-Jun-2009 & 16-Jun-2009 & SW 846/6020 & \\
\hline Barium & 0.0838 & 0.0010 & $\mathrm{mg} / \mathrm{L}$ & 2 & 16-Jun-2009 & 16-Jun-2009 & SW 846/6020 & \\
\hline Beryllium & ND & 0.0010 & $\mathrm{mg} / \mathrm{L}$ & 2 & 16-Jun-2009 & 16-Jun-2009 & SW 846/6020 & $\mathrm{U}$ \\
\hline Cadmium & ND & 0.0010 & $\mathrm{mg} / \mathrm{L}$ & 2 & 16-Jun-2009 & 16-Jun-2009 & SW 846/6020 & $\mathrm{U}$ \\
\hline Chromium & ND & 0.0010 & $\mathrm{mg} / \mathrm{L}$ & 2 & 16-Jun-2009 & 16-Jun-2009 & SW 846/6020 & $\mathrm{U}$ \\
\hline Cobalt & ND & 0.0010 & $\mathrm{mg} / \mathrm{L}$ & 2 & 16-Jun-2009 & 16-Jun-2009 & SW 846/6020 & $\mathrm{U}$ \\
\hline Copper & 0.0014 & 0.0010 & $\mathrm{mg} / \mathrm{L}$ & 2 & 16-Jun-2009 & 16-Jun-2009 & SW 846/6020 & \\
\hline Lead & ND & 0.0010 & $\mathrm{mg} / \mathrm{L}$ & 2 & 16-Jun-2009 & 16-Jun-2009 & SW 846/6020 & $\mathrm{U}$ \\
\hline Manganese & 0.0026 & 0.0010 & $\mathrm{mg} / \mathrm{L}$ & 2 & 16-Jun-2009 & 16-Jun-2009 & SW 846/6020 & \\
\hline Molybdenum & 0.0017 & 0.0010 & $\mathrm{mg} / \mathrm{L}$ & 2 & 16-Jun-2009 & 16-Jun-2009 & SW 846/6020 & \\
\hline Nickel & 0.0006 & 0.0010 & $\mathrm{mg} / \mathrm{L}$ & 2 & 16-Jun-2009 & 16-Jun-2009 & SW 846/6020 & $\mathrm{J}$ \\
\hline Selenium & 0.0014 & 0.0010 & $\mathrm{mg} / \mathrm{L}$ & 2 & 16-Jun-2009 & 16-Jun-2009 & SW 846/6020 & \\
\hline Silver & ND & 0.0010 & $\mathrm{mg} / \mathrm{L}$ & 2 & 16-Jun-2009 & 16-Jun-2009 & SW 846/6020 & $\mathrm{U}$ \\
\hline Thallium & 0.0004 & 0.0010 & $\mathrm{mg} / \mathrm{L}$ & 2 & 16-Jun-2009 & 16-Jun-2009 & SW 846/6020 & $\mathrm{J}$ \\
\hline Vanadium & 0.0233 & 0.0010 & $\mathrm{mg} / \mathrm{L}$ & 2 & 16-Jun-2009 & 16-Jun-2009 & SW 846/6020 & \\
\hline Zinc & 0.0053 & 0.0010 & $\mathrm{mg} / \mathrm{L}$ & 2 & 16-Jun-2009 & 16-Jun-2009 & SW 846/6020 & B \\
\hline
\end{tabular}




\section{USACE ERDC-EP-C \\ 3909 Halls Ferry Road \\ Vicksburg, MS 39180-6199}

ERDC - ECB

Project: TVA Fly Ash Project

Reported:

$--,-$

Project Manager: Tony Bednar

03-Sep-2009

DRAFT: EMR MT N2 Hour 24 Rep C

9061502-42 (Water)

\begin{tabular}{|c|c|c|c|c|c|c|c|c|}
\hline & & orting & & & & & & \\
\hline Analyte & Result & Limit & Units & Dilution & Prepared & Analyzed & Method & Notes \\
\hline
\end{tabular}

ERDC- EL-EP-C (Environmental Chemistry Branch)

DRAFT: Miscellaneous Physical/Conventional Chemistry Parameters

\begin{tabular}{lrrrrrrr}
\hline Bromide & $\mathrm{ND}$ & 0.100 & $\mathrm{mg} / \mathrm{L}$ & 1 & 14-Jul-2009 & $14-\mathrm{Jul}-2009$ & EPA 300.0 \\
Chloride & $\mathbf{2 . 8 0}$ & 1.00 & $\mathrm{mg} / \mathrm{L}$ & 1 & $14-J u l-2009$ & $14-\mathrm{Jul}-2009$ & EPA 300.0 \\
Nitrate as N & $\mathbf{0 . 3 9 0}$ & 0.100 & $\mathrm{mg} / \mathrm{L}$ & 1 & $14-J u l-2009$ & $14-J u l-2009$ & EPA 300.0 \\
Sulfate & $\mathbf{1 3 . 4}$ & 0.100 & $\mathrm{mg} / \mathrm{L}$ & 1 & $14-J u l-2009$ & $14-J u l-2009$ & EPA 300.0
\end{tabular}




\section{USACE ERDC-EP-C \\ 3909 Halls Ferry Road \\ Vicksburg, MS 39180-6199}

ERDC - ECB

$-\cdot$,
Project: TVA Fly Ash Project

Project Manager: Tony Bednar
Reported:

03-Sep-2009

DRAFT: EMR MT O2 Hour 24 Rep A

9061502-46 (Water)

\begin{tabular}{|c|c|c|c|c|c|c|c|c|}
\hline Analyte & Result & $\begin{array}{r}\text { porting } \\
\text { Limit }\end{array}$ & Units & Dilution & Prepared & Analyzed & Method & Notes \\
\hline
\end{tabular}

ERDC- EL-EP-C (Environmental Chemistry Branch)

DRAFT: Wet Chemistry

\begin{tabular}{llcccccc}
\hline Hardness & $\mathbf{3 6 . 3}$ & 1.32 & $\begin{array}{c}\text { mg equil } \\
\text { CaCO3/ }\end{array}$ & 1 & 25-Jun-2009 & 25-Jun-2009
\end{tabular}

DRAFT: Metals by EPA 6000/7000 Series Methods

\begin{tabular}{|c|c|c|c|c|c|c|c|c|}
\hline Mercury & ND & 0.000005 & $\mathrm{mg} / \mathrm{L}$ & 1 & 17-Jun-2009 & 01-Jul-2009 & EPA 7471A & $\mathrm{U}$ \\
\hline Strontium & 0.116 & 0.0200 & $\mathrm{mg} / \mathrm{L}$ & 1 & 25-Jun-2009 & 25-Jun-2009 & EPA $6010 \mathrm{~B}$ & \\
\hline Aluminum & ND & 0.200 & $\mathrm{mg} / \mathrm{L}$ & 1 & 25-Jun-2009 & 25-Jun-2009 & SW 846/6010 & U \\
\hline Calcium & 10.8 & 0.200 & $\mathrm{mg} / \mathrm{L}$ & 1 & 25-Jun-2009 & 25-Jun-2009 & SW 846/6010 & \\
\hline Iron & ND & 0.200 & $\mathrm{mg} / \mathrm{L}$ & 1 & 25-Jun-2009 & 25-Jun-2009 & SW 846/6010 & $\mathrm{U}$ \\
\hline Magnesium & 2.26 & 0.200 & $\mathrm{mg} / \mathrm{L}$ & 1 & 25-Jun-2009 & 25-Jun-2009 & SW 846/6010 & \\
\hline Potassium & 1.47 & 0.200 & $\mathrm{mg} / \mathrm{L}$ & 1 & 25-Jun-2009 & 25-Jun-2009 & SW 846/6010 & \\
\hline Sodium & 2.63 & 0.200 & $\mathrm{mg} / \mathrm{L}$ & 1 & 25-Jun-2009 & 25-Jun-2009 & SW 846/6010 & \\
\hline Antimony & 0.0006 & 0.0010 & $\mathrm{mg} / \mathrm{L}$ & 2 & 16-Jun-2009 & 16-Jun-2009 & SW 846/6020 & J \\
\hline Arsenic & 0.0270 & 0.0010 & $\mathrm{mg} / \mathrm{L}$ & 2 & 16-Jun-2009 & 16-Jun-2009 & SW 846/6020 & \\
\hline Barium & 0.0853 & 0.0010 & $\mathrm{mg} / \mathrm{L}$ & 2 & 16-Jun-2009 & 16-Jun-2009 & SW 846/6020 & \\
\hline Beryllium & ND & 0.0010 & $\mathrm{mg} / \mathrm{L}$ & 2 & 16-Jun-2009 & 16-Jun-2009 & SW 846/6020 & $\mathrm{U}$ \\
\hline Cadmium & ND & 0.0010 & $\mathrm{mg} / \mathrm{L}$ & 2 & 16-Jun-2009 & 16-Jun-2009 & SW 846/6020 & $\mathrm{U}$ \\
\hline Chromium & ND & 0.0010 & $\mathrm{mg} / \mathrm{L}$ & 2 & 16-Jun-2009 & 16-Jun-2009 & SW 846/6020 & $\mathrm{U}$ \\
\hline Cobalt & ND & 0.0010 & $\mathrm{mg} / \mathrm{L}$ & 2 & 16-Jun-2009 & 16-Jun-2009 & SW 846/6020 & $\mathrm{U}$ \\
\hline Copper & 0.0017 & 0.0010 & $\mathrm{mg} / \mathrm{L}$ & 2 & 16-Jun-2009 & 16-Jun-2009 & SW 846/6020 & \\
\hline Lead & ND & 0.0010 & $\mathrm{mg} / \mathrm{L}$ & 2 & 16-Jun-2009 & 16-Jun-2009 & SW 846/6020 & $\mathrm{U}$ \\
\hline Manganese & 0.0026 & 0.0010 & $\mathrm{mg} / \mathrm{L}$ & 2 & 16-Jun-2009 & 16-Jun-2009 & SW 846/6020 & \\
\hline Molybdenum & 0.0015 & 0.0010 & $\mathrm{mg} / \mathrm{L}$ & 2 & 16-Jun-2009 & 16-Jun-2009 & SW 846/6020 & \\
\hline Nickel & 0.0008 & 0.0010 & $\mathrm{mg} / \mathrm{L}$ & 2 & 16-Jun-2009 & 16-Jun-2009 & SW 846/6020 & $\mathrm{J}$ \\
\hline Selenium & 0.0012 & 0.0010 & $\mathrm{mg} / \mathrm{L}$ & 2 & 16-Jun-2009 & 16-Jun-2009 & SW 846/6020 & \\
\hline Silver & ND & 0.0010 & $\mathrm{mg} / \mathrm{L}$ & 2 & 16-Jun-2009 & 16-Jun-2009 & SW 846/6020 & $\mathrm{U}$ \\
\hline Thallium & 0.0004 & 0.0010 & $\mathrm{mg} / \mathrm{L}$ & 2 & 16-Jun-2009 & 16-Jun-2009 & SW 846/6020 & $\mathrm{J}$ \\
\hline Vanadium & 0.0180 & 0.0010 & $\mathrm{mg} / \mathrm{L}$ & 2 & 16-Jun-2009 & 16-Jun-2009 & SW 846/6020 & \\
\hline Zinc & 0.0072 & 0.0010 & $\mathrm{mg} / \mathrm{L}$ & 2 & 16-Jun-2009 & 16-Jun-2009 & SW 846/6020 & B \\
\hline
\end{tabular}




\section{USACE ERDC-EP-C \\ 3909 Halls Ferry Road \\ Vicksburg, MS 39180-6199}

ERDC - ECB

Project: TVA Fly Ash Project

Reported:

$--$,

Project Manager: Tony Bednar

03-Sep-2009

DRAFT: EMR MT O2 Hour 24 Rep A

9061502-46 (Water)

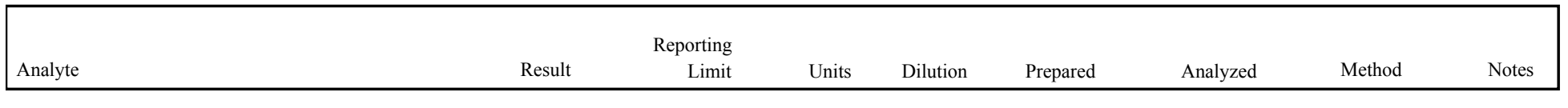

ERDC- EL-EP-C (Environmental Chemistry Branch)

DRAFT: Miscellaneous Physical/Conventional Chemistry Parameters

\begin{tabular}{lrrrrrrr}
\hline Bromide & $\mathrm{ND}$ & 0.100 & $\mathrm{mg} / \mathrm{L}$ & 1 & 14-Jul-2009 & $14-\mathrm{Jul}-2009$ & EPA 300.0 \\
Chloride & $\mathbf{2 . 6 5}$ & 1.00 & $\mathrm{mg} / \mathrm{L}$ & 1 & $14-J u l-2009$ & $14-\mathrm{Jul}-2009$ & EPA 300.0 \\
Nitrate as N & $\mathbf{0 . 3 9 0}$ & 0.100 & $\mathrm{mg} / \mathrm{L}$ & 1 & $14-J u l-2009$ & $14-J u l-2009$ & EPA 300.0 \\
Sulfate & $\mathbf{1 3 . 2}$ & 0.100 & $\mathrm{mg} / \mathrm{L}$ & 1 & $14-J u l-2009$ & $14-J u l-2009$ & EPA 300.0
\end{tabular}




\section{USACE ERDC-EP-C \\ 3909 Halls Ferry Road \\ Vicksburg, MS 39180-6199}

ERDC - ECB

$-\cdot$,
Project: TVA Fly Ash Project

Project Manager: Tony Bednar
Reported:

03-Sep-2009

DRAFT: EMR MT O2 Hour 24 Rep B

9061502-47 (Water)

\begin{tabular}{|c|c|c|c|c|c|c|c|c|}
\hline Analyte & Result & $\begin{array}{r}\text { porting } \\
\text { Limit }\end{array}$ & Units & Dilution & Prepared & Analyzed & Method & Notes \\
\hline
\end{tabular}

ERDC- EL-EP-C (Environmental Chemistry Branch)

DRAFT: Wet Chemistry

\begin{tabular}{llllllll}
\hline Hardness & $\mathbf{3 5 . 1}$ & 1.32 & $\begin{array}{c}\text { mg equil } \\
\text { CaCO3/ }\end{array}$ & 1 & 25-Jun-2009 & 25-Jun-2009
\end{tabular}

$\mathrm{L}$

DRAFT: Metals by EPA 6000/7000 Series Methods

\begin{tabular}{|c|c|c|c|c|c|c|c|c|}
\hline Mercury & 0.000016 & 0.000005 & $\mathrm{mg} / \mathrm{L}$ & 1 & 17-Jun-2009 & 01-Jul-2009 & EPA 7471A & \\
\hline Strontium & 0.104 & 0.0200 & $\mathrm{mg} / \mathrm{L}$ & 1 & 25-Jun-2009 & 25-Jun-2009 & EPA $6010 B$ & \\
\hline Aluminum & ND & 0.200 & $\mathrm{mg} / \mathrm{L}$ & 1 & 25-Jun-2009 & 25-Jun-2009 & SW 846/6010 & $\mathrm{U}$ \\
\hline Calcium & 10.4 & 0.200 & $\mathrm{mg} / \mathrm{L}$ & 1 & 25-Jun-2009 & 25-Jun-2009 & SW 846/6010 & \\
\hline Iron & ND & 0.200 & $\mathrm{mg} / \mathrm{L}$ & 1 & 25-Jun-2009 & 25-Jun-2009 & SW 846/6010 & $\mathrm{U}$ \\
\hline Magnesium & 2.21 & 0.200 & $\mathrm{mg} / \mathrm{L}$ & 1 & 25-Jun-2009 & 25-Jun-2009 & SW 846/6010 & \\
\hline Potassium & 1.36 & 0.200 & $\mathrm{mg} / \mathrm{L}$ & 1 & 25-Jun-2009 & 25-Jun-2009 & SW 846/6010 & \\
\hline Sodium & 2.47 & 0.200 & $\mathrm{mg} / \mathrm{L}$ & 1 & 25-Jun-2009 & 25-Jun-2009 & SW 846/6010 & \\
\hline Antimony & 0.0004 & 0.0010 & $\mathrm{mg} / \mathrm{L}$ & 2 & 16-Jun-2009 & 16-Jun-2009 & SW 846/6020 & J \\
\hline Arsenic & 0.0224 & 0.0010 & $\mathrm{mg} / \mathrm{L}$ & 2 & 16-Jun-2009 & 16-Jun-2009 & SW 846/6020 & \\
\hline Barium & 0.0784 & 0.0010 & $\mathrm{mg} / \mathrm{L}$ & 2 & 16-Jun-2009 & 16-Jun-2009 & SW 846/6020 & \\
\hline Beryllium & ND & 0.0010 & $\mathrm{mg} / \mathrm{L}$ & 2 & 16-Jun-2009 & 16-Jun-2009 & SW 846/6020 & $\mathrm{U}$ \\
\hline Cadmium & ND & 0.0010 & $\mathrm{mg} / \mathrm{L}$ & 2 & 16-Jun-2009 & 16-Jun-2009 & SW 846/6020 & $\mathrm{U}$ \\
\hline Chromium & ND & 0.0010 & $\mathrm{mg} / \mathrm{L}$ & 2 & 16-Jun-2009 & 16-Jun-2009 & SW 846/6020 & $\mathrm{U}$ \\
\hline Cobalt & ND & 0.0010 & $\mathrm{mg} / \mathrm{L}$ & 2 & 16-Jun-2009 & 16-Jun-2009 & SW 846/6020 & $\mathrm{U}$ \\
\hline Copper & 0.0014 & 0.0010 & $\mathrm{mg} / \mathrm{L}$ & 2 & 16-Jun-2009 & 16-Jun-2009 & SW 846/6020 & \\
\hline Lead & ND & 0.0010 & $\mathrm{mg} / \mathrm{L}$ & 2 & 16-Jun-2009 & 16-Jun-2009 & SW 846/6020 & $\mathrm{U}$ \\
\hline Manganese & 0.0020 & 0.0010 & $\mathrm{mg} / \mathrm{L}$ & 2 & 16-Jun-2009 & 16-Jun-2009 & SW 846/6020 & \\
\hline Molybdenum & 0.0015 & 0.0010 & $\mathrm{mg} / \mathrm{L}$ & 2 & 16-Jun-2009 & 16-Jun-2009 & SW 846/6020 & \\
\hline Nickel & 0.0007 & 0.0010 & $\mathrm{mg} / \mathrm{L}$ & 2 & 16-Jun-2009 & 16-Jun-2009 & SW 846/6020 & $\mathrm{J}$ \\
\hline Selenium & 0.0010 & 0.0010 & $\mathrm{mg} / \mathrm{L}$ & 2 & 16-Jun-2009 & 16-Jun-2009 & SW 846/6020 & \\
\hline Silver & ND & 0.0010 & $\mathrm{mg} / \mathrm{L}$ & 2 & 16-Jun-2009 & 16-Jun-2009 & SW 846/6020 & $\mathrm{U}$ \\
\hline Thallium & 0.0003 & 0.0010 & $\mathrm{mg} / \mathrm{L}$ & 2 & 16-Jun-2009 & 16-Jun-2009 & SW 846/6020 & $\mathrm{J}$ \\
\hline Vanadium & 0.0167 & 0.0010 & $\mathrm{mg} / \mathrm{L}$ & 2 & 16-Jun-2009 & 16-Jun-2009 & SW 846/6020 & \\
\hline Zinc & 0.0062 & 0.0010 & $\mathrm{mg} / \mathrm{L}$ & 2 & 16-Jun-2009 & 16-Jun-2009 & SW 846/6020 & B \\
\hline
\end{tabular}




\section{USACE ERDC-EP-C \\ 3909 Halls Ferry Road \\ Vicksburg, MS 39180-6199}

ERDC - ECB

Project: TVA Fly Ash Project

Reported:

$--,-$

Project Manager: Tony Bednar

03-Sep-2009

DRAFT: EMR MT O2 Hour 24 Rep B

9061502-47 (Water)

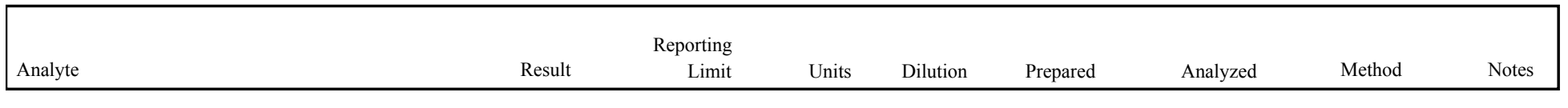

ERDC- EL-EP-C (Environmental Chemistry Branch)

DRAFT: Miscellaneous Physical/Conventional Chemistry Parameters

\begin{tabular}{lrrrrrrr}
\hline Bromide & $\mathrm{ND}$ & 0.100 & $\mathrm{mg} / \mathrm{L}$ & 1 & 14-Jul-2009 & $14-\mathrm{Jul}-2009$ & EPA 300.0 \\
Chloride & $\mathbf{2 . 4 9}$ & 1.00 & $\mathrm{mg} / \mathrm{L}$ & 1 & $14-J u l-2009$ & $14-\mathrm{Jul}-2009$ & EPA 300.0 \\
Nitrate as N & $\mathbf{0 . 3 8 0}$ & 0.100 & $\mathrm{mg} / \mathrm{L}$ & 1 & $14-J u l-2009$ & $14-J u l-2009$ & EPA 300.0 \\
Sulfate & $\mathbf{1 3 . 1}$ & 0.100 & $\mathrm{mg} / \mathrm{L}$ & 1 & $14-J u l-2009$ & $14-J u l-2009$ & EPA 300.0
\end{tabular}




\section{USACE ERDC-EP-C \\ 3909 Halls Ferry Road \\ Vicksburg, MS 39180-6199}

ERDC - ECB

$-\cdot$,
Project: TVA Fly Ash Project

Project Manager: Tony Bednar
Reported:

03-Sep-2009

DRAFT: EMR MT O2 Hour 24 Rep C

9061502-48 (Water)

\begin{tabular}{|c|c|c|c|c|c|c|c|c|}
\hline Analyte & Result & $\begin{array}{r}\text { porting } \\
\text { Limit }\end{array}$ & Units & Dilution & Prepared & Analyzed & Method & Notes \\
\hline
\end{tabular}

ERDC- EL-EP-C (Environmental Chemistry Branch)

DRAFT: Wet Chemistry

\begin{tabular}{llllllll}
\hline Hardness & $\mathbf{3 7 . 2}$ & 1.32 & $\begin{array}{c}\text { mg equil } \\
\text { CaCO3/ }\end{array}$ & 1 & 25-Jun-2009 & 25-Jun-2009
\end{tabular}

$\mathrm{L}$

DRAFT: Metals by EPA 6000/7000 Series Methods

\begin{tabular}{|c|c|c|c|c|c|c|c|c|}
\hline Mercury & 0.000014 & 0.000005 & $\mathrm{mg} / \mathrm{L}$ & 1 & 17-Jun-2009 & 01-Jul-2009 & EPA 7471A & \\
\hline Strontium & 0.129 & 0.0200 & $\mathrm{mg} / \mathrm{L}$ & 1 & 25-Jun-2009 & 25-Jun-2009 & EPA $6010 \mathrm{~B}$ & \\
\hline Aluminum & ND & 0.200 & $\mathrm{mg} / \mathrm{L}$ & 1 & 25-Jun-2009 & 25-Jun-2009 & SW 846/6010 & $\mathrm{U}$ \\
\hline Calcium & 11.2 & 0.200 & $\mathrm{mg} / \mathrm{L}$ & 1 & 25-Jun-2009 & 25-Jun-2009 & SW 846/6010 & \\
\hline Iron & ND & 0.200 & $\mathrm{mg} / \mathrm{L}$ & 1 & 25-Jun-2009 & 25-Jun-2009 & SW 846/6010 & $\mathrm{U}$ \\
\hline Magnesium & 2.27 & 0.200 & $\mathrm{mg} / \mathrm{L}$ & 1 & 25-Jun-2009 & 25-Jun-2009 & SW 846/6010 & \\
\hline Potassium & 1.35 & 0.200 & $\mathrm{mg} / \mathrm{L}$ & 1 & 25-Jun-2009 & 25-Jun-2009 & SW 846/6010 & \\
\hline Sodium & 2.48 & 0.200 & $\mathrm{mg} / \mathrm{L}$ & 1 & 25-Jun-2009 & 25-Jun-2009 & SW 846/6010 & \\
\hline Antimony & 0.0006 & 0.0010 & $\mathrm{mg} / \mathrm{L}$ & 2 & 16-Jun-2009 & 16-Jun-2009 & SW 846/6020 & J \\
\hline Arsenic & 0.0308 & 0.0010 & $\mathrm{mg} / \mathrm{L}$ & 2 & 16-Jun-2009 & 16-Jun-2009 & SW 846/6020 & \\
\hline Barium & 0.0928 & 0.0010 & $\mathrm{mg} / \mathrm{L}$ & 2 & 16-Jun-2009 & 16-Jun-2009 & SW 846/6020 & \\
\hline Beryllium & ND & 0.0010 & $\mathrm{mg} / \mathrm{L}$ & 2 & 16-Jun-2009 & 16-Jun-2009 & SW 846/6020 & $\mathrm{U}$ \\
\hline Cadmium & ND & 0.0010 & $\mathrm{mg} / \mathrm{L}$ & 2 & 16-Jun-2009 & 16-Jun-2009 & SW 846/6020 & $\mathrm{U}$ \\
\hline Chromium & ND & 0.0010 & $\mathrm{mg} / \mathrm{L}$ & 2 & 16-Jun-2009 & 16-Jun-2009 & SW 846/6020 & $\mathrm{U}$ \\
\hline Cobalt & ND & 0.0010 & $\mathrm{mg} / \mathrm{L}$ & 2 & 16-Jun-2009 & 16-Jun-2009 & SW 846/6020 & $\mathrm{U}$ \\
\hline Copper & 0.0014 & 0.0010 & $\mathrm{mg} / \mathrm{L}$ & 2 & 16-Jun-2009 & 16-Jun-2009 & SW 846/6020 & \\
\hline Lead & ND & 0.0010 & $\mathrm{mg} / \mathrm{L}$ & 2 & 16-Jun-2009 & 16-Jun-2009 & SW 846/6020 & $\mathrm{U}$ \\
\hline Manganese & 0.0043 & 0.0010 & $\mathrm{mg} / \mathrm{L}$ & 2 & 16-Jun-2009 & 16-Jun-2009 & SW 846/6020 & \\
\hline Molybdenum & 0.0016 & 0.0010 & $\mathrm{mg} / \mathrm{L}$ & 2 & 16-Jun-2009 & 16-Jun-2009 & SW 846/6020 & \\
\hline Nickel & 0.0006 & 0.0010 & $\mathrm{mg} / \mathrm{L}$ & 2 & 16-Jun-2009 & 16-Jun-2009 & SW 846/6020 & $\mathrm{J}$ \\
\hline Selenium & 0.0011 & 0.0010 & $\mathrm{mg} / \mathrm{L}$ & 2 & 16-Jun-2009 & 16-Jun-2009 & SW 846/6020 & \\
\hline Silver & ND & 0.0010 & $\mathrm{mg} / \mathrm{L}$ & 2 & 16-Jun-2009 & 16-Jun-2009 & SW 846/6020 & $\mathrm{U}$ \\
\hline Thallium & 0.0004 & 0.0010 & $\mathrm{mg} / \mathrm{L}$ & 2 & 16-Jun-2009 & 16-Jun-2009 & SW 846/6020 & $\mathrm{J}$ \\
\hline Vanadium & 0.0213 & 0.0010 & $\mathrm{mg} / \mathrm{L}$ & 2 & 16-Jun-2009 & 16-Jun-2009 & SW 846/6020 & \\
\hline Zinc & 0.0060 & 0.0010 & $\mathrm{mg} / \mathrm{L}$ & 2 & 16-Jun-2009 & 16-Jun-2009 & SW 846/6020 & B \\
\hline
\end{tabular}




\section{USACE ERDC-EP-C \\ 3909 Halls Ferry Road \\ Vicksburg, MS 39180-6199}

ERDC - ECB

Project: TVA Fly Ash Project

Reported:

$--,-$

Project Manager: Tony Bednar

03-Sep-2009

DRAFT: EMR MT O2 Hour 24 Rep C

9061502-48 (Water)

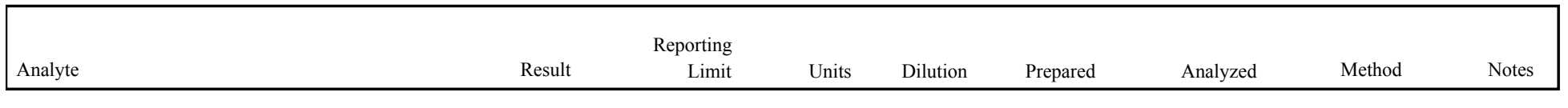

ERDC- EL-EP-C (Environmental Chemistry Branch)

DRAFT: Miscellaneous Physical/Conventional Chemistry Parameters

\begin{tabular}{lrrrrrrr}
\hline Bromide & $\mathrm{ND}$ & 0.100 & $\mathrm{mg} / \mathrm{L}$ & 1 & 14-Jul-2009 & $14-\mathrm{Jul}-2009$ & EPA 300.0 \\
Chloride & $\mathbf{2 . 5 1}$ & 1.00 & $\mathrm{mg} / \mathrm{L}$ & 1 & $14-J u l-2009$ & $14-\mathrm{Jul}-2009$ & EPA 300.0 \\
Nitrate as N & $\mathbf{0 . 2 8 0}$ & 0.100 & $\mathrm{mg} / \mathrm{L}$ & 1 & $14-J u l-2009$ & $14-J u l-2009$ & EPA 300.0 \\
Sulfate & $\mathbf{1 3 . 2}$ & 0.100 & $\mathrm{mg} / \mathrm{L}$ & 1 & $14-J u l-2009$ & $14-J u l-2009$ & EPA 300.0
\end{tabular}




\section{USACE ERDC-EP-C \\ 3909 Halls Ferry Road \\ Vicksburg, MS 39180-6199}

ERDC - ECB

$-,-$
Project: TVA Fly Ash Project

Project Manager: Tony Bednar
Reported:

03-Sep-2009

DRAFT: SLC MT N2 Hour $24 \operatorname{Rep} A$

9061502-52 (Water)

\begin{tabular}{|c|c|c|c|c|c|c|c|c|}
\hline Analyte & Result & $\begin{array}{r}\text { porting } \\
\text { Limit }\end{array}$ & Units & Dilution & Prepared & Analyzed & Method & Notes \\
\hline
\end{tabular}

ERDC- EL-EP-C (Environmental Chemistry Branch)

DRAFT: Wet Chemistry

\begin{tabular}{llllllll}
\hline Hardness & $\mathbf{9 8 . 5}$ & 1.32 & $\begin{array}{c}\text { mg equil } \\
\text { CaCO3/ }\end{array}$ & 1 & 25-Jun-2009 & 25-Jun-2009
\end{tabular}

$\mathrm{L}$

DRAFT: Metals by EPA 6000/7000 Series Methods

\begin{tabular}{|c|c|c|c|c|c|c|c|c|}
\hline Mercury & 0.00003 & 0.000005 & $\mathrm{mg} / \mathrm{L}$ & 1 & 17-Jun-2009 & 01-Jul-2009 & EPA 7471A & \\
\hline Strontium & 0.539 & 0.0200 & $\mathrm{mg} / \mathrm{L}$ & 1 & 25-Jun-2009 & 25-Jun-2009 & EPA 6010B & \\
\hline Aluminum & 0.192 & 0.200 & $\mathrm{mg} / \mathrm{L}$ & 1 & 25-Jun-2009 & 25-Jun-2009 & SW 846/6010 & J \\
\hline Calcium & 30.8 & 0.200 & $\mathrm{mg} / \mathrm{L}$ & 1 & 25-Jun-2009 & 25-Jun-2009 & SW 846/6010 & \\
\hline Iron & ND & 0.200 & $\mathrm{mg} / \mathrm{L}$ & 1 & 25-Jun-2009 & 25-Jun-2009 & SW 846/6010 & $\mathrm{U}$ \\
\hline Magnesium & 5.27 & 0.200 & $\mathrm{mg} / \mathrm{L}$ & 1 & 25-Jun-2009 & 25-Jun-2009 & SW 846/6010 & \\
\hline Potassium & 1.94 & 0.200 & $\mathrm{mg} / \mathrm{L}$ & 1 & 25-Jun-2009 & 25-Jun-2009 & SW 846/6010 & \\
\hline Sodium & 4.13 & 0.200 & $\mathrm{mg} / \mathrm{L}$ & 1 & 25-Jun-2009 & 25-Jun-2009 & SW 846/6010 & \\
\hline Antimony & 0.0080 & 0.0010 & $\mathrm{mg} / \mathrm{L}$ & 2 & 16-Jun-2009 & 16-Jun-2009 & SW 846/6020 & \\
\hline Arsenic & 0.0808 & 0.0010 & $\mathrm{mg} / \mathrm{L}$ & 2 & 16-Jun-2009 & 16-Jun-2009 & SW 846/6020 & \\
\hline Barium & 0.0970 & 0.0010 & $\mathrm{mg} / \mathrm{L}$ & 2 & 16-Jun-2009 & 16-Jun-2009 & SW 846/6020 & \\
\hline Beryllium & ND & 0.0010 & $\mathrm{mg} / \mathrm{L}$ & 2 & 16-Jun-2009 & 16-Jun-2009 & SW 846/6020 & U \\
\hline Cadmium & 0.0003 & 0.0010 & $\mathrm{mg} / \mathrm{L}$ & 2 & 16-Jun-2009 & 16-Jun-2009 & SW 846/6020 & J \\
\hline Chromium & ND & 0.0010 & $\mathrm{mg} / \mathrm{L}$ & 2 & 16-Jun-2009 & 16-Jun-2009 & SW 846/6020 & U \\
\hline Cobalt & ND & 0.0010 & $\mathrm{mg} / \mathrm{L}$ & 2 & 16-Jun-2009 & 16-Jun-2009 & SW 846/6020 & U \\
\hline Copper & 0.0012 & 0.0010 & $\mathrm{mg} / \mathrm{L}$ & 2 & 16-Jun-2009 & 16-Jun-2009 & SW 846/6020 & \\
\hline Lead & ND & 0.0010 & $\mathrm{mg} / \mathrm{L}$ & 2 & 16-Jun-2009 & 16-Jun-2009 & SW 846/6020 & U \\
\hline Manganese & 0.0284 & 0.0010 & $\mathrm{mg} / \mathrm{L}$ & 2 & 16-Jun-2009 & 16-Jun-2009 & SW 846/6020 & \\
\hline Molybdenum & 0.0733 & 0.0010 & $\mathrm{mg} / \mathrm{L}$ & 2 & 16-Jun-2009 & 16-Jun-2009 & SW 846/6020 & \\
\hline Nickel & 0.0007 & 0.0010 & $\mathrm{mg} / \mathrm{L}$ & 2 & 16-Jun-2009 & 16-Jun-2009 & SW 846/6020 & J \\
\hline Selenium & 0.0064 & 0.0010 & $\mathrm{mg} / \mathrm{L}$ & 2 & 16-Jun-2009 & 16-Jun-2009 & SW 846/6020 & \\
\hline Silver & ND & 0.0010 & $\mathrm{mg} / \mathrm{L}$ & 2 & 16-Jun-2009 & 16-Jun-2009 & SW 846/6020 & $\mathrm{U}$ \\
\hline Thallium & 0.0006 & 0.0010 & $\mathrm{mg} / \mathrm{L}$ & 2 & 16-Jun-2009 & 16-Jun-2009 & SW 846/6020 & J \\
\hline Vanadium & 0.0558 & 0.0010 & $\mathrm{mg} / \mathrm{L}$ & 2 & 16-Jun-2009 & 16-Jun-2009 & SW 846/6020 & \\
\hline Zinc & 0.0066 & 0.0010 & $\mathrm{mg} / \mathrm{L}$ & 2 & 16-Jun-2009 & 16-Jun-2009 & SW 846/6020 & $\mathrm{B}$ \\
\hline
\end{tabular}




\section{USACE ERDC-EP-C \\ 3909 Halls Ferry Road \\ Vicksburg, MS 39180-6199}

ERDC - ECB

Project: TVA Fly Ash Project

Reported:

Project Manager: Tony Bednar

03-Sep-2009

DRAFT: SLC MT N2 Hour $24 \operatorname{Rep} A$

9061502-52 (Water)

\begin{tabular}{|c|c|c|c|c|c|c|c|c|}
\hline & & orting & & & & & & \\
\hline Analyte & Result & Limit & Units & Dilution & Prepared & Analyzed & Method & Notes \\
\hline
\end{tabular}

ERDC- EL-EP-C (Environmental Chemistry Branch)

DRAFT: Miscellaneous Physical/Conventional Chemistry Parameters

\begin{tabular}{lrrrrrrr}
\hline Bromide & $\mathrm{ND}$ & 0.100 & $\mathrm{mg} / \mathrm{L}$ & 1 & $14-J u l-2009$ & $14-\mathrm{Jul}-2009$ & EPA 300.0 \\
Chloride & $\mathbf{4 . 0 5}$ & 1.00 & $\mathrm{mg} / \mathrm{L}$ & 1 & $14-J u l-2009$ & $14-\mathrm{Jul}-2009$ & EPA 300.0 \\
Nitrate as N & $\mathbf{0 . 3 8 0}$ & 0.100 & $\mathrm{mg} / \mathrm{L}$ & 1 & $14-J u l-2009$ & $14-J u l-2009$ & EPA 300.0 \\
Sulfate & $\mathbf{4 3 . 5}$ & 0.100 & $\mathrm{mg} / \mathrm{L}$ & 1 & $14-J u l-2009$ & $14-J u l-2009$ & EPA 300.0
\end{tabular}




\section{USACE ERDC-EP-C \\ 3909 Halls Ferry Road \\ Vicksburg, MS 39180-6199}

ERDC - ECB

$-,-$
Project: TVA Fly Ash Project

Project Manager: Tony Bednar
Reported:

03-Sep-2009

DRAFT: SLC MT N2 Hour 24 Rep B

9061502-53 (Water)

\begin{tabular}{|c|c|c|c|c|c|c|c|c|}
\hline Analyte & Result & $\begin{array}{r}\text { porting } \\
\text { Limit }\end{array}$ & Units & Dilution & Prepared & Analyzed & Method & Notes \\
\hline
\end{tabular}

ERDC- EL-EP-C (Environmental Chemistry Branch)

DRAFT: Wet Chemistry

\begin{tabular}{llllllll}
\hline Hardness & $\mathbf{9 8 . 5}$ & 1.32 & $\begin{array}{c}\text { mg equil } \\
\text { CaCO3/ }\end{array}$ & 1 & 25-Jun-2009 & 25-Jun-2009
\end{tabular}

$\mathrm{L}$

DRAFT: Metals by EPA 6000/7000 Series Methods

\begin{tabular}{|c|c|c|c|c|c|c|c|c|}
\hline Mercury & ND & 0.000005 & $\mathrm{mg} / \mathrm{L}$ & 1 & 17-Jun-2009 & 01-Jul-2009 & EPA 7471A & $\mathrm{U}$ \\
\hline Strontium & 0.538 & 0.0200 & $\mathrm{mg} / \mathrm{L}$ & 1 & 25-Jun-2009 & 25-Jun-2009 & EPA 6010B & \\
\hline Aluminum & 0.200 & 0.200 & $\mathrm{mg} / \mathrm{L}$ & 1 & 25-Jun-2009 & 25-Jun-2009 & SW 846/6010 & \\
\hline Calcium & 30.9 & 0.200 & $\mathrm{mg} / \mathrm{L}$ & 1 & 25-Jun-2009 & 25-Jun-2009 & SW 846/6010 & \\
\hline Iron & ND & 0.200 & $\mathrm{mg} / \mathrm{L}$ & 1 & 25-Jun-2009 & 25-Jun-2009 & SW 846/6010 & $\mathrm{U}$ \\
\hline Magnesium & 5.19 & 0.200 & $\mathrm{mg} / \mathrm{L}$ & 1 & 25-Jun-2009 & 25-Jun-2009 & SW 846/6010 & \\
\hline Potassium & 1.91 & 0.200 & $\mathrm{mg} / \mathrm{L}$ & 1 & 25-Jun-2009 & 25-Jun-2009 & SW 846/6010 & \\
\hline Sodium & 4.09 & 0.200 & $\mathrm{mg} / \mathrm{L}$ & 1 & 25-Jun-2009 & 25-Jun-2009 & SW 846/6010 & \\
\hline Antimony & 0.0078 & 0.0010 & $\mathrm{mg} / \mathrm{L}$ & 2 & 16-Jun-2009 & 16-Jun-2009 & SW 846/6020 & \\
\hline Arsenic & 0.0837 & 0.0010 & $\mathrm{mg} / \mathrm{L}$ & 2 & 16-Jun-2009 & 16-Jun-2009 & SW 846/6020 & \\
\hline Barium & 0.0984 & 0.0010 & $\mathrm{mg} / \mathrm{L}$ & 2 & 16-Jun-2009 & 16-Jun-2009 & SW 846/6020 & \\
\hline Beryllium & ND & 0.0010 & $\mathrm{mg} / \mathrm{L}$ & 2 & 16-Jun-2009 & 16-Jun-2009 & SW 846/6020 & $\mathrm{U}$ \\
\hline Cadmium & 0.0003 & 0.0010 & $\mathrm{mg} / \mathrm{L}$ & 2 & 16-Jun-2009 & 16-Jun-2009 & SW 846/6020 & $\mathrm{J}$ \\
\hline Chromium & ND & 0.0010 & $\mathrm{mg} / \mathrm{L}$ & 2 & 16-Jun-2009 & 16-Jun-2009 & SW 846/6020 & $\mathrm{U}$ \\
\hline Cobalt & ND & 0.0010 & $\mathrm{mg} / \mathrm{L}$ & 2 & 16-Jun-2009 & 16-Jun-2009 & SW 846/6020 & $\mathrm{U}$ \\
\hline Copper & 0.0027 & 0.0010 & $\mathrm{mg} / \mathrm{L}$ & 2 & 16-Jun-2009 & 16-Jun-2009 & SW 846/6020 & \\
\hline Lead & ND & 0.0010 & $\mathrm{mg} / \mathrm{L}$ & 2 & 16-Jun-2009 & 16-Jun-2009 & SW 846/6020 & $\mathrm{U}$ \\
\hline Manganese & 0.0271 & 0.0010 & $\mathrm{mg} / \mathrm{L}$ & 2 & 16-Jun-2009 & 16-Jun-2009 & SW 846/6020 & \\
\hline Molybdenum & 0.0726 & 0.0010 & $\mathrm{mg} / \mathrm{L}$ & 2 & 16-Jun-2009 & 16-Jun-2009 & SW 846/6020 & \\
\hline Nickel & 0.0005 & 0.0010 & $\mathrm{mg} / \mathrm{L}$ & 2 & 16-Jun-2009 & 16-Jun-2009 & SW 846/6020 & $\mathrm{J}$ \\
\hline Selenium & 0.0061 & 0.0010 & $\mathrm{mg} / \mathrm{L}$ & 2 & 16-Jun-2009 & 16-Jun-2009 & SW 846/6020 & \\
\hline Silver & ND & 0.0010 & $\mathrm{mg} / \mathrm{L}$ & 2 & 16-Jun-2009 & 16-Jun-2009 & SW 846/6020 & $\mathrm{U}$ \\
\hline Thallium & 0.0006 & 0.0010 & $\mathrm{mg} / \mathrm{L}$ & 2 & 16-Jun-2009 & 16-Jun-2009 & SW 846/6020 & $\mathrm{J}$ \\
\hline Vanadium & 0.0561 & 0.0010 & $\mathrm{mg} / \mathrm{L}$ & 2 & 16-Jun-2009 & 16-Jun-2009 & SW 846/6020 & \\
\hline Zinc & 0.0057 & 0.0010 & $\mathrm{mg} / \mathrm{L}$ & 2 & 16-Jun-2009 & 16-Jun-2009 & SW 846/6020 & B \\
\hline
\end{tabular}




\section{USACE ERDC-EP-C \\ 3909 Halls Ferry Road \\ Vicksburg, MS 39180-6199}

ERDC - ECB

Project: TVA Fly Ash Project

Reported:

$--,-$

Project Manager: Tony Bednar

03-Sep-2009

DRAFT: SLC MT N2 Hour 24 Rep B

9061502-53 (Water)

\begin{tabular}{|c|c|c|c|c|c|c|c|c|}
\hline & & orting & & & & & & \\
\hline Analyte & Result & Limit & Units & Dilution & Prepared & Analyzed & Method & Notes \\
\hline
\end{tabular}

ERDC- EL-EP-C (Environmental Chemistry Branch)

DRAFT: Miscellaneous Physical/Conventional Chemistry Parameters

\begin{tabular}{lrrrrrrr}
\hline Bromide & $\mathrm{ND}$ & 0.100 & $\mathrm{mg} / \mathrm{L}$ & 1 & 14-Jul-2009 & $14-\mathrm{Jul}-2009$ & EPA 300.0 \\
Chloride & $\mathbf{4 . 0 0}$ & 1.00 & $\mathrm{mg} / \mathrm{L}$ & 1 & $14-J u l-2009$ & $14-\mathrm{Jul}-2009$ & EPA 300.0 \\
Nitrate as N & $\mathbf{0 . 3 7 0}$ & 0.100 & $\mathrm{mg} / \mathrm{L}$ & 1 & $14-J u l-2009$ & $14-J u l-2009$ & EPA 300.0 \\
Sulfate & $\mathbf{4 2 . 7}$ & 0.100 & $\mathrm{mg} / \mathrm{L}$ & 1 & $14-J u l-2009$ & $14-J u l-2009$ & EPA 300.0
\end{tabular}




\section{USACE ERDC-EP-C \\ 3909 Halls Ferry Road \\ Vicksburg, MS 39180-6199}

ERDC - ECB

$-,-$
Project: TVA Fly Ash Project

Project Manager: Tony Bednar
Reported:

03-Sep-2009

DRAFT: SLC MT N2 Hour 24 Rep C

9061502-54 (Water)

\begin{tabular}{|c|c|c|c|c|c|c|c|c|}
\hline Analyte & Result & $\begin{array}{r}\text { porting } \\
\text { Limit }\end{array}$ & Units & Dilution & Prepared & Analyzed & Method & Notes \\
\hline
\end{tabular}

ERDC- EL-EP-C (Environmental Chemistry Branch)

DRAFT: Wet Chemistry

\begin{tabular}{llllllll}
\hline Hardness & $\mathbf{9 8 . 7}$ & 1.32 & $\begin{array}{c}\text { mg equil } \\
\text { CaCO3/ }\end{array}$ & 1 & 25-Jun-2009 & 25-Jun-2009
\end{tabular}

$\mathrm{L}$

DRAFT: Metals by EPA 6000/7000 Series Methods

\begin{tabular}{|c|c|c|c|c|c|c|c|c|}
\hline Mercury & ND & 0.000005 & $\mathrm{mg} / \mathrm{L}$ & 1 & 17-Jun-2009 & 01-Jul-2009 & EPA 7471A & $\mathrm{U}$ \\
\hline Strontium & 0.539 & 0.0200 & $\mathrm{mg} / \mathrm{L}$ & 1 & 25-Jun-2009 & 25-Jun-2009 & EPA 6010B & \\
\hline Aluminum & 0.201 & 0.200 & $\mathrm{mg} / \mathrm{L}$ & 1 & 25-Jun-2009 & 25-Jun-2009 & SW 846/6010 & \\
\hline Calcium & 30.9 & 0.200 & $\mathrm{mg} / \mathrm{L}$ & 1 & 25-Jun-2009 & 25-Jun-2009 & SW 846/6010 & \\
\hline Iron & ND & 0.200 & $\mathrm{mg} / \mathrm{L}$ & 1 & 25-Jun-2009 & 25-Jun-2009 & SW 846/6010 & $\mathrm{U}$ \\
\hline Magnesium & 5.24 & 0.200 & $\mathrm{mg} / \mathrm{L}$ & 1 & 25-Jun-2009 & 25-Jun-2009 & SW 846/6010 & \\
\hline Potassium & 1.94 & 0.200 & $\mathrm{mg} / \mathrm{L}$ & 1 & 25-Jun-2009 & 25-Jun-2009 & SW 846/6010 & \\
\hline Sodium & 4.11 & 0.200 & $\mathrm{mg} / \mathrm{L}$ & 1 & 25-Jun-2009 & 25-Jun-2009 & SW 846/6010 & \\
\hline Antimony & 0.0078 & 0.0010 & $\mathrm{mg} / \mathrm{L}$ & 2 & 16-Jun-2009 & 16-Jun-2009 & SW 846/6020 & \\
\hline Arsenic & 0.0806 & 0.0010 & $\mathrm{mg} / \mathrm{L}$ & 2 & 16-Jun-2009 & 16-Jun-2009 & SW 846/6020 & \\
\hline Barium & 0.0976 & 0.0010 & $\mathrm{mg} / \mathrm{L}$ & 2 & 16-Jun-2009 & 16-Jun-2009 & SW 846/6020 & \\
\hline Beryllium & ND & 0.0010 & $\mathrm{mg} / \mathrm{L}$ & 2 & 16-Jun-2009 & 16-Jun-2009 & SW 846/6020 & $\mathrm{U}$ \\
\hline Cadmium & 0.0003 & 0.0010 & $\mathrm{mg} / \mathrm{L}$ & 2 & 16-Jun-2009 & 16-Jun-2009 & SW 846/6020 & $\mathrm{J}$ \\
\hline Chromium & ND & 0.0010 & $\mathrm{mg} / \mathrm{L}$ & 2 & 16-Jun-2009 & 16-Jun-2009 & SW 846/6020 & $\mathrm{U}$ \\
\hline Cobalt & ND & 0.0010 & $\mathrm{mg} / \mathrm{L}$ & 2 & 16-Jun-2009 & 16-Jun-2009 & SW 846/6020 & $\mathrm{U}$ \\
\hline Copper & 0.0005 & 0.0010 & $\mathrm{mg} / \mathrm{L}$ & 2 & 16-Jun-2009 & 16-Jun-2009 & SW 846/6020 & $\mathrm{J}$ \\
\hline Lead & ND & 0.0010 & $\mathrm{mg} / \mathrm{L}$ & 2 & 16-Jun-2009 & 16-Jun-2009 & SW 846/6020 & $\mathrm{U}$ \\
\hline Manganese & 0.0293 & 0.0010 & $\mathrm{mg} / \mathrm{L}$ & 2 & 16-Jun-2009 & 16-Jun-2009 & SW 846/6020 & \\
\hline Molybdenum & 0.0708 & 0.0010 & $\mathrm{mg} / \mathrm{L}$ & 2 & 16-Jun-2009 & 16-Jun-2009 & SW 846/6020 & \\
\hline Nickel & 0.00057 & 0.0010 & $\mathrm{mg} / \mathrm{L}$ & 2 & 16-Jun-2009 & 16-Jun-2009 & SW 846/6020 & $\mathrm{J}$ \\
\hline Selenium & 0.0065 & 0.0010 & $\mathrm{mg} / \mathrm{L}$ & 2 & 16-Jun-2009 & 16-Jun-2009 & SW 846/6020 & \\
\hline Silver & ND & 0.0010 & $\mathrm{mg} / \mathrm{L}$ & 2 & 16-Jun-2009 & 16-Jun-2009 & SW 846/6020 & $\mathrm{U}$ \\
\hline Thallium & 0.0006 & 0.0010 & $\mathrm{mg} / \mathrm{L}$ & 2 & 16-Jun-2009 & 16-Jun-2009 & SW 846/6020 & $\mathrm{J}$ \\
\hline Vanadium & 0.0569 & 0.0010 & $\mathrm{mg} / \mathrm{L}$ & 2 & 16-Jun-2009 & 16-Jun-2009 & SW 846/6020 & \\
\hline Zinc & 0.0066 & 0.0010 & $\mathrm{mg} / \mathrm{L}$ & 2 & 16-Jun-2009 & 16-Jun-2009 & SW 846/6020 & B \\
\hline
\end{tabular}




\section{USACE ERDC-EP-C \\ 3909 Halls Ferry Road \\ Vicksburg, MS 39180-6199}

ERDC - ECB

Project: TVA Fly Ash Project

Reported:

Project Manager: Tony Bednar

03-Sep-2009

DRAFT: SLC MT N2 Hour 24 Rep C

9061502-54 (Water)

\begin{tabular}{|c|c|c|c|c|c|c|c|c|}
\hline & & orting & & & & & & \\
\hline Analyte & Result & Limit & Units & Dilution & Prepared & Analyzed & Method & Notes \\
\hline
\end{tabular}

ERDC- EL-EP-C (Environmental Chemistry Branch)

DRAFT: Miscellaneous Physical/Conventional Chemistry Parameters

\begin{tabular}{lrrrrrrr}
\hline Bromide & $\mathrm{ND}$ & 0.100 & $\mathrm{mg} / \mathrm{L}$ & 1 & 14-Jul-2009 & $14-\mathrm{Jul}-2009$ & EPA 300.0 \\
Chloride & $\mathbf{4 . 0 3}$ & 1.00 & $\mathrm{mg} / \mathrm{L}$ & 1 & $14-J u l-2009$ & $14-\mathrm{Jul}-2009$ & EPA 300.0 \\
Nitrate as N & $\mathbf{0 . 3 7 0}$ & 0.100 & $\mathrm{mg} / \mathrm{L}$ & 1 & $14-J u l-2009$ & $14-J u l-2009$ & EPA 300.0 \\
Sulfate & $\mathbf{4 3 . 6}$ & 0.100 & $\mathrm{mg} / \mathrm{L}$ & 1 & $14-J u l-2009$ & $14-J u l-2009$ & EPA 300.0
\end{tabular}




\section{USACE ERDC-EP-C \\ 3909 Halls Ferry Road \\ Vicksburg, MS 39180-6199}

ERDC - ECB

$-\cdot$,
Project: TVA Fly Ash Project

Project Manager: Tony Bednar
Reported:

03-Sep-2009

DRAFT: SLC MT O2 Hour 24 Rep A

9061502-58 (Water)

\begin{tabular}{|c|c|c|c|c|c|c|c|c|}
\hline Analyte & Result & $\begin{array}{r}\text { porting } \\
\text { Limit }\end{array}$ & Units & Dilution & Prepared & Analyzed & Method & Notes \\
\hline
\end{tabular}

ERDC- EL-EP-C (Environmental Chemistry Branch)

DRAFT: Wet Chemistry

\begin{tabular}{llllllll}
\hline Hardness & $\mathbf{9 8 . 1}$ & 1.32 & $\begin{array}{c}\text { mg equil } \\
\text { CaCO3/ }\end{array}$ & 1 & 25-Jun-2009 & 25-Jun-2009
\end{tabular}

$\mathrm{L}$

DRAFT: Metals by EPA 6000/7000 Series Methods

\begin{tabular}{|c|c|c|c|c|c|c|c|c|}
\hline Mercury & 0.000016 & 0.000005 & $\mathrm{mg} / \mathrm{L}$ & 1 & 17-Jun-2009 & 01-Jul-2009 & EPA 7471A & \\
\hline Strontium & 0.536 & 0.0200 & $\mathrm{mg} / \mathrm{L}$ & 1 & 25-Jun-2009 & 25-Jun-2009 & EPA 6010B & \\
\hline Aluminum & 0.142 & 0.200 & $\mathrm{mg} / \mathrm{L}$ & 1 & 25-Jun-2009 & 25-Jun-2009 & SW 846/6010 & J \\
\hline Calcium & 30.7 & 0.200 & $\mathrm{mg} / \mathrm{L}$ & 1 & 25-Jun-2009 & 25-Jun-2009 & SW 846/6010 & \\
\hline Iron & ND & 0.200 & $\mathrm{mg} / \mathrm{L}$ & 1 & 25-Jun-2009 & 25-Jun-2009 & SW 846/6010 & $\mathrm{U}$ \\
\hline Magnesium & 5.18 & 0.200 & $\mathrm{mg} / \mathrm{L}$ & 1 & 25-Jun-2009 & 25-Jun-2009 & SW 846/6010 & \\
\hline Potassium & 1.95 & 0.200 & $\mathrm{mg} / \mathrm{L}$ & 1 & 25-Jun-2009 & 25-Jun-2009 & SW 846/6010 & \\
\hline Sodium & 4.17 & 0.200 & $\mathrm{mg} / \mathrm{L}$ & 1 & 25-Jun-2009 & 25-Jun-2009 & SW 846/6010 & \\
\hline Antimony & 0.0075 & 0.0010 & $\mathrm{mg} / \mathrm{L}$ & 2 & 16-Jun-2009 & 16-Jun-2009 & SW 846/6020 & \\
\hline Arsenic & 0.0780 & 0.0010 & $\mathrm{mg} / \mathrm{L}$ & 2 & 16-Jun-2009 & 16-Jun-2009 & SW 846/6020 & \\
\hline Barium & 0.0975 & 0.0010 & $\mathrm{mg} / \mathrm{L}$ & 2 & 16-Jun-2009 & 16-Jun-2009 & SW 846/6020 & \\
\hline Beryllium & ND & 0.0010 & $\mathrm{mg} / \mathrm{L}$ & 2 & 16-Jun-2009 & 16-Jun-2009 & SW 846/6020 & U \\
\hline Cadmium & 0.0003 & 0.0010 & $\mathrm{mg} / \mathrm{L}$ & 2 & 16-Jun-2009 & 16-Jun-2009 & SW 846/6020 & J \\
\hline Chromium & ND & 0.0010 & $\mathrm{mg} / \mathrm{L}$ & 2 & 16-Jun-2009 & 16-Jun-2009 & SW 846/6020 & U \\
\hline Cobalt & ND & 0.0010 & $\mathrm{mg} / \mathrm{L}$ & 2 & 16-Jun-2009 & 16-Jun-2009 & SW 846/6020 & U \\
\hline Copper & 0.0007 & 0.0010 & $\mathrm{mg} / \mathrm{L}$ & 2 & 16-Jun-2009 & 16-Jun-2009 & SW 846/6020 & J \\
\hline Lead & ND & 0.0010 & $\mathrm{mg} / \mathrm{L}$ & 2 & 16-Jun-2009 & 16-Jun-2009 & SW 846/6020 & U \\
\hline Manganese & 0.0299 & 0.0010 & $\mathrm{mg} / \mathrm{L}$ & 2 & 16-Jun-2009 & 16-Jun-2009 & SW 846/6020 & \\
\hline Molybdenum & 0.0727 & 0.0010 & $\mathrm{mg} / \mathrm{L}$ & 2 & 16-Jun-2009 & 16-Jun-2009 & SW 846/6020 & \\
\hline Nickel & 0.0006 & 0.0010 & $\mathrm{mg} / \mathrm{L}$ & 2 & 16-Jun-2009 & 16-Jun-2009 & SW 846/6020 & J \\
\hline Selenium & 0.0064 & 0.0010 & $\mathrm{mg} / \mathrm{L}$ & 2 & 16-Jun-2009 & 16-Jun-2009 & SW 846/6020 & \\
\hline Silver & ND & 0.0010 & $\mathrm{mg} / \mathrm{L}$ & 2 & 16-Jun-2009 & 16-Jun-2009 & SW 846/6020 & $\mathrm{U}$ \\
\hline Thallium & 0.0006 & 0.0010 & $\mathrm{mg} / \mathrm{L}$ & 2 & 16-Jun-2009 & 16-Jun-2009 & SW 846/6020 & J \\
\hline Vanadium & 0.0567 & 0.0010 & $\mathrm{mg} / \mathrm{L}$ & 2 & 16-Jun-2009 & 16-Jun-2009 & SW 846/6020 & \\
\hline Zinc & 0.0068 & 0.0010 & $\mathrm{mg} / \mathrm{L}$ & 2 & 16-Jun-2009 & 16-Jun-2009 & SW 846/6020 & $\mathrm{B}$ \\
\hline
\end{tabular}




\section{USACE ERDC-EP-C \\ 3909 Halls Ferry Road \\ Vicksburg, MS 39180-6199}

ERDC - ECB

Project: TVA Fly Ash Project

Reported:

Project Manager: Tony Bednar

03-Sep-2009

DRAFT: SLC MT O2 Hour 24 Rep A

9061502-58 (Water)

\begin{tabular}{|c|c|c|c|c|c|c|c|c|}
\hline & & orting & & & & & & \\
\hline Analyte & Result & Limit & Units & Dilution & Prepared & Analyzed & Method & Notes \\
\hline
\end{tabular}

ERDC- EL-EP-C (Environmental Chemistry Branch)

DRAFT: Miscellaneous Physical/Conventional Chemistry Parameters

\begin{tabular}{lrrrrrrr}
\hline Bromide & $\mathrm{ND}$ & 0.100 & $\mathrm{mg} / \mathrm{L}$ & 1 & 14-Jul-2009 & $14-\mathrm{Jul}-2009$ & EPA 300.0 \\
Chloride & $\mathbf{4 . 1 3}$ & 1.00 & $\mathrm{mg} / \mathrm{L}$ & 1 & $14-J u l-2009$ & $14-\mathrm{Jul}-2009$ & EPA 300.0 \\
Nitrate as N & $\mathbf{0 . 3 7 0}$ & 0.100 & $\mathrm{mg} / \mathrm{L}$ & 1 & $14-J u l-2009$ & $14-J u l-2009$ & EPA 300.0 \\
Sulfate & $\mathbf{4 3 . 3}$ & 0.100 & $\mathrm{mg} / \mathrm{L}$ & 1 & $14-J u l-2009$ & $14-J u l-2009$ & EPA 300.0
\end{tabular}




\section{USACE ERDC-EP-C \\ 3909 Halls Ferry Road \\ Vicksburg, MS 39180-6199}

ERDC - ECB

$-,-$
Project: TVA Fly Ash Project

Project Manager: Tony Bednar
Reported:

03-Sep-2009

DRAFT: SLC MT O2 Hour 24 Rep B

9061502-59 (Water)

\begin{tabular}{|c|c|c|c|c|c|c|c|c|}
\hline Analyte & Result & $\begin{array}{r}\text { porting } \\
\text { Limit }\end{array}$ & Units & Dilution & Prepared & Analyzed & Method & Notes \\
\hline
\end{tabular}

ERDC- EL-EP-C (Environmental Chemistry Branch)

DRAFT: Wet Chemistry

\begin{tabular}{llllllll}
\hline Hardness & $\mathbf{9 8 . 2}$ & 1.32 & $\begin{array}{c}\text { mg equil } \\
\text { CaCO3/ }\end{array}$ & 1 & 25-Jun-2009 & 25-Jun-2009
\end{tabular}

$\mathrm{L}$

DRAFT: Metals by EPA 6000/7000 Series Methods

\begin{tabular}{|c|c|c|c|c|c|c|c|c|}
\hline Mercury & ND & 0.000005 & $\mathrm{mg} / \mathrm{L}$ & 1 & 17-Jun-2009 & 01-Jul-2009 & EPA 7471A & $\mathrm{U}$ \\
\hline Strontium & 0.536 & 0.0200 & $\mathrm{mg} / \mathrm{L}$ & 1 & 25-Jun-2009 & 25-Jun-2009 & EPA 6010B & \\
\hline Aluminum & 0.126 & 0.200 & $\mathrm{mg} / \mathrm{L}$ & 1 & 25-Jun-2009 & 25-Jun-2009 & SW 846/6010 & J \\
\hline Calcium & 30.8 & 0.200 & $\mathrm{mg} / \mathrm{L}$ & 1 & 25-Jun-2009 & 25-Jun-2009 & SW 846/6010 & \\
\hline Iron & ND & 0.200 & $\mathrm{mg} / \mathrm{L}$ & 1 & 25-Jun-2009 & 25-Jun-2009 & SW 846/6010 & $\mathrm{U}$ \\
\hline Magnesium & 5.19 & 0.200 & $\mathrm{mg} / \mathrm{L}$ & 1 & 25-Jun-2009 & 25-Jun-2009 & SW 846/6010 & \\
\hline Potassium & 1.95 & 0.200 & $\mathrm{mg} / \mathrm{L}$ & 1 & 25-Jun-2009 & 25-Jun-2009 & SW 846/6010 & \\
\hline Sodium & 4.13 & 0.200 & $\mathrm{mg} / \mathrm{L}$ & 1 & 25-Jun-2009 & 25-Jun-2009 & SW 846/6010 & \\
\hline Antimony & 0.0079 & 0.0010 & $\mathrm{mg} / \mathrm{L}$ & 2 & 16-Jun-2009 & 16-Jun-2009 & SW 846/6020 & \\
\hline Arsenic & 0.0772 & 0.0010 & $\mathrm{mg} / \mathrm{L}$ & 2 & 16-Jun-2009 & 16-Jun-2009 & SW 846/6020 & \\
\hline Barium & 0.101 & 0.0010 & $\mathrm{mg} / \mathrm{L}$ & 2 & 16-Jun-2009 & 16-Jun-2009 & SW 846/6020 & \\
\hline Beryllium & ND & 0.0010 & $\mathrm{mg} / \mathrm{L}$ & 2 & 16-Jun-2009 & 16-Jun-2009 & SW 846/6020 & $\mathrm{U}$ \\
\hline Cadmium & 0.0004 & 0.0010 & $\mathrm{mg} / \mathrm{L}$ & 2 & 16-Jun-2009 & 16-Jun-2009 & SW 846/6020 & J \\
\hline Chromium & ND & 0.0010 & $\mathrm{mg} / \mathrm{L}$ & 2 & 16-Jun-2009 & 16-Jun-2009 & SW 846/6020 & $\mathrm{U}$ \\
\hline Cobalt & ND & 0.0010 & $\mathrm{mg} / \mathrm{L}$ & 2 & 16-Jun-2009 & 16-Jun-2009 & SW 846/6020 & $\mathrm{U}$ \\
\hline Copper & 0.0009 & 0.0010 & $\mathrm{mg} / \mathrm{L}$ & 2 & 16-Jun-2009 & 16-Jun-2009 & SW 846/6020 & $\mathrm{J}$ \\
\hline Lead & ND & 0.0010 & $\mathrm{mg} / \mathrm{L}$ & 2 & 16-Jun-2009 & 16-Jun-2009 & SW 846/6020 & $\mathrm{U}$ \\
\hline Manganese & 0.0303 & 0.0010 & $\mathrm{mg} / \mathrm{L}$ & 2 & 16-Jun-2009 & 16-Jun-2009 & SW 846/6020 & \\
\hline Molybdenum & 0.0703 & 0.0010 & $\mathrm{mg} / \mathrm{L}$ & 2 & 16-Jun-2009 & 16-Jun-2009 & SW 846/6020 & \\
\hline Nickel & 0.0007 & 0.0010 & $\mathrm{mg} / \mathrm{L}$ & 2 & 16-Jun-2009 & 16-Jun-2009 & SW 846/6020 & $\mathrm{J}$ \\
\hline Selenium & 0.0065 & 0.0010 & $\mathrm{mg} / \mathrm{L}$ & 2 & 16-Jun-2009 & 16-Jun-2009 & SW 846/6020 & \\
\hline Silver & ND & 0.0010 & $\mathrm{mg} / \mathrm{L}$ & 2 & 16-Jun-2009 & 16-Jun-2009 & SW 846/6020 & $\mathrm{U}$ \\
\hline Thallium & 0.0007 & 0.0010 & $\mathrm{mg} / \mathrm{L}$ & 2 & 16-Jun-2009 & 16-Jun-2009 & SW 846/6020 & $\mathrm{J}$ \\
\hline Vanadium & 0.0570 & 0.0010 & $\mathrm{mg} / \mathrm{L}$ & 2 & 16-Jun-2009 & 16-Jun-2009 & SW 846/6020 & \\
\hline Zinc & 0.0055 & 0.0010 & $\mathrm{mg} / \mathrm{L}$ & 2 & 16-Jun-2009 & 16-Jun-2009 & SW 846/6020 & B \\
\hline
\end{tabular}




\section{USACE ERDC-EP-C \\ 3909 Halls Ferry Road \\ Vicksburg, MS 39180-6199}

ERDC - ECB

Project: TVA Fly Ash Project

Reported:

Project Manager: Tony Bednar

03-Sep-2009

DRAFT: SLC MT O2 Hour 24 Rep B

9061502-59 (Water)

\begin{tabular}{|c|c|c|c|c|c|c|c|c|}
\hline & & orting & & & & & & \\
\hline Analyte & Result & Limit & Units & Dilution & Prepared & Analyzed & Method & Notes \\
\hline
\end{tabular}

ERDC- EL-EP-C (Environmental Chemistry Branch)

DRAFT: Miscellaneous Physical/Conventional Chemistry Parameters

\begin{tabular}{lrrrrrrr}
\hline Bromide & $\mathrm{ND}$ & 0.100 & $\mathrm{mg} / \mathrm{L}$ & 1 & 14-Jul-2009 & 14-Jul-2009 & EPA 300.0 \\
Chloride & $\mathbf{3 . 8 6}$ & 1.00 & $\mathrm{mg} / \mathrm{L}$ & 1 & $14-J u l-2009$ & $14-\mathrm{Jul}-2009$ & EPA 300.0 \\
Nitrate as N & $\mathbf{0 . 3 6 0}$ & 0.100 & $\mathrm{mg} / \mathrm{L}$ & 1 & $14-J u l-2009$ & $14-J u l-2009$ & EPA 300.0 \\
Sulfate & $\mathbf{3 8 . 1}$ & 0.100 & $\mathrm{mg} / \mathrm{L}$ & 1 & $14-J u l-2009$ & $14-J u l-2009$ & EPA 300.0
\end{tabular}




\section{USACE ERDC-EP-C \\ 3909 Halls Ferry Road \\ Vicksburg, MS 39180-6199}

ERDC - ECB

$-\cdot$,
Project: TVA Fly Ash Project

Project Manager: Tony Bednar
Reported:

03-Sep-2009

DRAFT: SLC MT O2 Hour 24 Rep C

9061502-60 (Water)

\begin{tabular}{|c|c|c|c|c|c|c|c|c|}
\hline Analyte & Result & $\begin{array}{r}\text { porting } \\
\text { Limit }\end{array}$ & Units & Dilution & Prepared & Analyzed & Method & Notes \\
\hline
\end{tabular}

ERDC- EL-EP-C (Environmental Chemistry Branch)

DRAFT: Wet Chemistry

\begin{tabular}{llllllll}
\hline Hardness & $\mathbf{9 7 . 9}$ & 1.32 & $\begin{array}{c}\text { mg equil } \\
\text { CaCO3/ }\end{array}$ & 1 & 25-Jun-2009 & 25-Jun-2009
\end{tabular}

$\mathrm{L}$

DRAFT: Metals by EPA 6000/7000 Series Methods

\begin{tabular}{|c|c|c|c|c|c|c|c|c|}
\hline Mercury & 0.000011 & 0.000005 & $\mathrm{mg} / \mathrm{L}$ & 1 & 17-Jun-2009 & 01-Jul-2009 & EPA 7471A & \\
\hline Strontium & 0.542 & 0.0200 & $\mathrm{mg} / \mathrm{L}$ & 1 & 25-Jun-2009 & 25-Jun-2009 & EPA 6010B & \\
\hline Aluminum & 0.143 & 0.200 & $\mathrm{mg} / \mathrm{L}$ & 1 & 25-Jun-2009 & 25-Jun-2009 & SW 846/6010 & J \\
\hline Calcium & 30.8 & 0.200 & $\mathrm{mg} / \mathrm{L}$ & 1 & 25-Jun-2009 & 25-Jun-2009 & SW 846/6010 & \\
\hline Iron & ND & 0.200 & $\mathrm{mg} / \mathrm{L}$ & 1 & 25-Jun-2009 & 25-Jun-2009 & SW 846/6010 & $\mathrm{U}$ \\
\hline Magnesium & 5.08 & 0.200 & $\mathrm{mg} / \mathrm{L}$ & 1 & 25-Jun-2009 & 25-Jun-2009 & SW 846/6010 & \\
\hline Potassium & 1.87 & 0.200 & $\mathrm{mg} / \mathrm{L}$ & 1 & 25-Jun-2009 & 25-Jun-2009 & SW 846/6010 & \\
\hline Sodium & 4.10 & 0.200 & $\mathrm{mg} / \mathrm{L}$ & 1 & 25-Jun-2009 & 25-Jun-2009 & SW 846/6010 & \\
\hline Antimony & 0.0077 & 0.0010 & $\mathrm{mg} / \mathrm{L}$ & 2 & 16-Jun-2009 & 16-Jun-2009 & SW 846/6020 & \\
\hline Arsenic & 0.0805 & 0.0010 & $\mathrm{mg} / \mathrm{L}$ & 2 & 16-Jun-2009 & 16-Jun-2009 & SW 846/6020 & \\
\hline Barium & 0.0973 & 0.0010 & $\mathrm{mg} / \mathrm{L}$ & 2 & 16-Jun-2009 & 16-Jun-2009 & SW 846/6020 & \\
\hline Beryllium & ND & 0.0010 & $\mathrm{mg} / \mathrm{L}$ & 2 & 16-Jun-2009 & 16-Jun-2009 & SW 846/6020 & U \\
\hline Cadmium & 0.0003 & 0.0010 & $\mathrm{mg} / \mathrm{L}$ & 2 & 16-Jun-2009 & 16-Jun-2009 & SW 846/6020 & J \\
\hline Chromium & ND & 0.0010 & $\mathrm{mg} / \mathrm{L}$ & 2 & 16-Jun-2009 & 16-Jun-2009 & SW 846/6020 & U \\
\hline Cobalt & ND & 0.0010 & $\mathrm{mg} / \mathrm{L}$ & 2 & 16-Jun-2009 & 16-Jun-2009 & SW 846/6020 & U \\
\hline Copper & 0.0006 & 0.0010 & $\mathrm{mg} / \mathrm{L}$ & 2 & 16-Jun-2009 & 16-Jun-2009 & SW 846/6020 & J \\
\hline Lead & ND & 0.0010 & $\mathrm{mg} / \mathrm{L}$ & 2 & 16-Jun-2009 & 16-Jun-2009 & SW 846/6020 & U \\
\hline Manganese & 0.0301 & 0.0010 & $\mathrm{mg} / \mathrm{L}$ & 2 & 16-Jun-2009 & 16-Jun-2009 & SW 846/6020 & \\
\hline Molybdenum & 0.0714 & 0.0010 & $\mathrm{mg} / \mathrm{L}$ & 2 & 16-Jun-2009 & 16-Jun-2009 & SW 846/6020 & \\
\hline Nickel & 0.0005 & 0.0010 & $\mathrm{mg} / \mathrm{L}$ & 2 & 16-Jun-2009 & 16-Jun-2009 & SW 846/6020 & J \\
\hline Selenium & 0.0060 & 0.0010 & $\mathrm{mg} / \mathrm{L}$ & 2 & 16-Jun-2009 & 16-Jun-2009 & SW 846/6020 & \\
\hline Silver & ND & 0.0010 & $\mathrm{mg} / \mathrm{L}$ & 2 & 16-Jun-2009 & 16-Jun-2009 & SW 846/6020 & $\mathrm{U}$ \\
\hline Thallium & 0.0007 & 0.0010 & $\mathrm{mg} / \mathrm{L}$ & 2 & 16-Jun-2009 & 16-Jun-2009 & SW 846/6020 & J \\
\hline Vanadium & 0.0560 & 0.0010 & $\mathrm{mg} / \mathrm{L}$ & 2 & 16-Jun-2009 & 16-Jun-2009 & SW 846/6020 & \\
\hline Zinc & 0.0057 & 0.0010 & $\mathrm{mg} / \mathrm{L}$ & 2 & 16-Jun-2009 & 16-Jun-2009 & SW 846/6020 & $\mathrm{B}$ \\
\hline
\end{tabular}




\section{USACE ERDC-EP-C \\ 3909 Halls Ferry Road \\ Vicksburg, MS 39180-6199}

ERDC - ECB

Project: TVA Fly Ash Project

Reported:

$--,-$

Project Manager: Tony Bednar

03-Sep-2009

DRAFT: SLC MT O2 Hour 24 Rep C

9061502-60 (Water)

\begin{tabular}{|c|c|c|c|c|c|c|c|c|}
\hline & & orting & & & & & & \\
\hline Analyte & Result & Limit & Units & Dilution & Prepared & Analyzed & Method & Notes \\
\hline
\end{tabular}

ERDC- EL-EP-C (Environmental Chemistry Branch)

DRAFT: Miscellaneous Physical/Conventional Chemistry Parameters

\begin{tabular}{lrrrrrrr}
\hline Bromide & $\mathrm{ND}$ & 0.100 & $\mathrm{mg} / \mathrm{L}$ & 1 & 14-Jul-2009 & 14-Jul-2009 & EPA 300.0 \\
Chloride & $\mathbf{3 . 8 0}$ & 1.00 & $\mathrm{mg} / \mathrm{L}$ & 1 & $14-J u l-2009$ & $14-\mathrm{Jul}-2009$ & EPA 300.0 \\
Nitrate as N & $\mathbf{0 . 4 0 0}$ & 0.100 & $\mathrm{mg} / \mathrm{L}$ & 1 & $14-J u l-2009$ & $14-J u l-2009$ & EPA 300.0 \\
Sulfate & $\mathbf{3 8 . 4}$ & 0.100 & $\mathrm{mg} / \mathrm{L}$ & 1 & $14-J u l-2009$ & $14-J u l-2009$ & EPA 300.0
\end{tabular}




\section{USACE ERDC-EP-C \\ 3909 Halls Ferry Road \\ Vicksburg, MS 39180-6199}

ERDC - ECB

Project: TVA Fly Ash Project

Project Manager: Tony Bednar

Reported:

03-Sep-2009

DRAFT: EFL - WA - MT - A (Effluent from Settling Pond) - Total Metals

9061502-61 (Water)

\begin{tabular}{|c|c|c|c|c|c|c|c|c|}
\hline Analyte & Result & $\begin{array}{r}\text { porting } \\
\text { Limit }\end{array}$ & Units & Dilution & Prepared & Analyzed & Method & Notes \\
\hline
\end{tabular}

ERDC- EL-EP-C (Environmental Chemistry Branch)

DRAFT: Wet Chemistry

\begin{tabular}{llllllll}
\hline Hardness & 116 & 1.32 & $\begin{array}{c}\text { mg equil } \\
\text { CaCO3/ }\end{array}$ & 4 & 25-Jun-2009 & 25-Jun-2009
\end{tabular}

$\mathrm{L}$

DRAFT: Metals by EPA 6000/7000 Series Methods

\begin{tabular}{|c|c|c|c|c|c|c|c|c|}
\hline Mercury & 0.000431 & 0.000005 & $\mathrm{mg} / \mathrm{L}$ & 2 & 24-Jul-2009 & 24-Jul-2009 & EPA 7471A & \\
\hline Strontium & 0.352 & 0.0800 & $\mathrm{mg} / \mathrm{L}$ & 4 & 25-Jun-2009 & 25-Jun-2009 & EPA 6010B & \\
\hline Aluminum & 1.14 & 0.200 & $\mathrm{mg} / \mathrm{L}$ & 4 & 25-Jun-2009 & 25-Jun-2009 & SW 846/6010 & \\
\hline Calcium & 34.4 & 0.200 & $\mathrm{mg} / \mathrm{L}$ & 4 & 25-Jun-2009 & 25-Jun-2009 & SW 846/6010 & B \\
\hline Iron & 0.333 & 0.200 & $\mathrm{mg} / \mathrm{L}$ & 4 & 25-Jun-2009 & 25-Jun-2009 & SW 846/6010 & \\
\hline Magnesium & 7.38 & 0.200 & $\mathrm{mg} / \mathrm{L}$ & 4 & 25-Jun-2009 & 25-Jun-2009 & SW 846/6010 & \\
\hline Potassium & 2.49 & 0.200 & $\mathrm{mg} / \mathrm{L}$ & 4 & 25-Jun-2009 & 25-Jun-2009 & SW 846/6010 & B \\
\hline Sodium & 8.23 & 0.200 & $\mathrm{mg} / \mathrm{L}$ & 4 & 25-Jun-2009 & 25-Jun-2009 & SW 846/6010 & B \\
\hline Antimony & 0.0088 & 0.0020 & $\mathrm{mg} / \mathrm{L}$ & 4 & 17-Jun-2009 & 17-Jun-2009 & SW 846/6020 & \\
\hline Arsenic & 0.0192 & 0.0020 & $\mathrm{mg} / \mathrm{L}$ & 4 & 17-Jun-2009 & 17-Jun-2009 & SW 846/6020 & \\
\hline Barium & 0.182 & 0.0020 & $\mathrm{mg} / \mathrm{L}$ & 4 & 17-Jun-2009 & 17-Jun-2009 & SW 846/6020 & B \\
\hline Beryllium & ND & 0.0020 & $\mathrm{mg} / \mathrm{L}$ & 4 & 17-Jun-2009 & 17-Jun-2009 & SW 846/6020 & U \\
\hline Cadmium & ND & 0.0020 & $\mathrm{mg} / \mathrm{L}$ & 4 & 17-Jun-2009 & 17-Jun-2009 & SW 846/6020 & U \\
\hline Chromium & 0.0081 & 0.0020 & $\mathrm{mg} / \mathrm{L}$ & 4 & 17-Jun-2009 & 17-Jun-2009 & SW 846/6020 & \\
\hline Cobalt & 0.0012 & 0.0020 & $\mathrm{mg} / \mathrm{L}$ & 4 & 17-Jun-2009 & 17-Jun-2009 & SW 846/6020 & J \\
\hline Copper & 0.0090 & 0.0020 & $\mathrm{mg} / \mathrm{L}$ & 4 & 17-Jun-2009 & 17-Jun-2009 & SW 846/6020 & B \\
\hline Lead & 0.0017 & 0.0020 & $\mathrm{mg} / \mathrm{L}$ & 4 & 17-Jun-2009 & 17-Jun-2009 & SW 846/6020 & $\mathrm{J}$ \\
\hline Manganese & 0.0235 & 0.0020 & $\mathrm{mg} / \mathrm{L}$ & 4 & 17-Jun-2009 & 17-Jun-2009 & SW 846/6020 & \\
\hline Molybdenum & 0.0236 & 0.0020 & $\mathrm{mg} / \mathrm{L}$ & 4 & 17-Jun-2009 & 17-Jun-2009 & SW 846/6020 & \\
\hline Nickel & 0.0055 & 0.0020 & $\mathrm{mg} / \mathrm{L}$ & 4 & 17-Jun-2009 & 17-Jun-2009 & SW 846/6020 & \\
\hline Selenium & 0.0043 & 0.0020 & $\mathrm{mg} / \mathrm{L}$ & 4 & 17-Jun-2009 & 17-Jun-2009 & SW 846/6020 & \\
\hline Silver & 0.0023 & 0.0020 & $\mathrm{mg} / \mathrm{L}$ & 4 & 17-Jun-2009 & 17-Jun-2009 & SW 846/6020 & B \\
\hline Thallium & ND & 0.0020 & $\mathrm{mg} / \mathrm{L}$ & 4 & 17-Jun-2009 & 17-Jun-2009 & SW 846/6020 & $\mathrm{U}$ \\
\hline Vanadium & 0.0390 & 0.0020 & $\mathrm{mg} / \mathrm{L}$ & 4 & 17-Jun-2009 & 17-Jun-2009 & SW 846/6020 & \\
\hline Zinc & 0.0285 & 0.0020 & $\mathrm{mg} / \mathrm{L}$ & 4 & 17-Jun-2009 & 17-Jun-2009 & SW 846/6020 & $\mathrm{B}$ \\
\hline
\end{tabular}




\section{USACE ERDC-EP-C \\ 3909 Halls Ferry Road \\ Vicksburg, MS 39180-6199}

ERDC - ECB

$-\cdot$,
Project: TVA Fly Ash Project

Project Manager: Tony Bednar
Reported:

03-Sep-2009

DRAFT: Emory River Water - Total Metals

9061502-62 (Water)

\begin{tabular}{|c|c|c|c|c|c|c|c|c|}
\hline Analyte & Result & $\begin{array}{r}\text { porting } \\
\text { Limit }\end{array}$ & Units & Dilution & Prepared & Analyzed & Method & Notes \\
\hline
\end{tabular}

ERDC- EL-EP-C (Environmental Chemistry Branch)

DRAFT: Wet Chemistry

\begin{tabular}{llllllll}
\hline Hardness & $\mathbf{3 1 . 2}$ & 1.32 & $\begin{array}{c}\text { mg equil } \\
\text { CaCO3/ }\end{array}$ & 4 & 25-Jun-2009 & 25-Jun-2009
\end{tabular}

$\mathrm{L}$

DRAFT: Metals by EPA 6000/7000 Series Methods

\begin{tabular}{|c|c|c|c|c|c|c|c|c|}
\hline Mercury & 0.000148 & 0.000005 & $\mathrm{mg} / \mathrm{L}$ & 2 & 24-Jul-2009 & 24-Jul-2009 & EPA 7471A & \\
\hline Strontium & 0.0429 & 0.0800 & $\mathrm{mg} / \mathrm{L}$ & 4 & 25-Jun-2009 & 25-Jun-2009 & EPA $6010 B$ & $\mathrm{~J}$ \\
\hline Aluminum & 0.228 & 0.200 & $\mathrm{mg} / \mathrm{L}$ & 4 & 25-Jun-2009 & 25-Jun-2009 & SW 846/6010 & \\
\hline Calcium & 8.93 & 0.200 & $\mathrm{mg} / \mathrm{L}$ & 4 & 25-Jun-2009 & 25-Jun-2009 & SW 846/6010 & B \\
\hline Iron & 0.253 & 0.200 & $\mathrm{mg} / \mathrm{L}$ & 4 & 25-Jun-2009 & 25-Jun-2009 & SW 846/6010 & \\
\hline Magnesium & 2.17 & 0.200 & $\mathrm{mg} / \mathrm{L}$ & 4 & 25-Jun-2009 & 25-Jun-2009 & SW 846/6010 & \\
\hline Potassium & 1.71 & 0.200 & $\mathrm{mg} / \mathrm{L}$ & 4 & 25-Jun-2009 & 25-Jun-2009 & SW 846/6010 & B \\
\hline Sodium & 3.01 & 0.200 & $\mathrm{mg} / \mathrm{L}$ & 4 & 25-Jun-2009 & 25-Jun-2009 & SW 846/6010 & B \\
\hline Antimony & 0.0088 & 0.0020 & $\mathrm{mg} / \mathrm{L}$ & 4 & 17-Jun-2009 & 17-Jun-2009 & SW 846/6020 & \\
\hline Arsenic & 0.0011 & 0.0020 & $\mathrm{mg} / \mathrm{L}$ & 4 & 17-Jun-2009 & 17-Jun-2009 & SW 846/6020 & J \\
\hline Barium & 0.0340 & 0.0020 & $\mathrm{mg} / \mathrm{L}$ & 4 & 17-Jun-2009 & 17-Jun-2009 & SW 846/6020 & B \\
\hline Beryllium & ND & 0.0020 & $\mathrm{mg} / \mathrm{L}$ & 4 & 17-Jun-2009 & 17-Jun-2009 & SW 846/6020 & $\mathrm{U}$ \\
\hline Cadmium & ND & 0.0020 & $\mathrm{mg} / \mathrm{L}$ & 4 & 17-Jun-2009 & 17-Jun-2009 & SW 846/6020 & $\mathrm{U}$ \\
\hline Chromium & 0.00095 & 0.0020 & $\mathrm{mg} / \mathrm{L}$ & 4 & 17-Jun-2009 & 17-Jun-2009 & SW 846/6020 & $\mathrm{J}$ \\
\hline Cobalt & ND & 0.0020 & $\mathrm{mg} / \mathrm{L}$ & 4 & 17-Jun-2009 & 17-Jun-2009 & SW 846/6020 & $\mathrm{U}$ \\
\hline Copper & 0.0040 & 0.0020 & $\mathrm{mg} / \mathrm{L}$ & 4 & 17-Jun-2009 & 17-Jun-2009 & SW 846/6020 & B \\
\hline Lead & 0.0007 & 0.0020 & $\mathrm{mg} / \mathrm{L}$ & 4 & 17-Jun-2009 & 17-Jun-2009 & SW 846/6020 & $\mathrm{J}$ \\
\hline Manganese & 0.0351 & 0.0020 & $\mathrm{mg} / \mathrm{L}$ & 4 & 17-Jun-2009 & 17-Jun-2009 & SW 846/6020 & \\
\hline Molybdenum & ND & 0.0020 & $\mathrm{mg} / \mathrm{L}$ & 4 & 17-Jun-2009 & 17-Jun-2009 & SW 846/6020 & $\mathrm{U}$ \\
\hline Nickel & 0.0027 & 0.0020 & $\mathrm{mg} / \mathrm{L}$ & 4 & 17-Jun-2009 & 17-Jun-2009 & SW 846/6020 & \\
\hline Selenium & ND & 0.0020 & $\mathrm{mg} / \mathrm{L}$ & 4 & 17-Jun-2009 & 17-Jun-2009 & SW 846/6020 & $\mathrm{U}$ \\
\hline Silver & 0.0016 & 0.0020 & $\mathrm{mg} / \mathrm{L}$ & 4 & 17-Jun-2009 & 17-Jun-2009 & SW 846/6020 & $\mathrm{J}, \mathrm{B}$ \\
\hline Thallium & ND & 0.0020 & $\mathrm{mg} / \mathrm{L}$ & 4 & 17-Jun-2009 & 17-Jun-2009 & SW 846/6020 & $\mathrm{U}$ \\
\hline Vanadium & 0.0005 & 0.0020 & $\mathrm{mg} / \mathrm{L}$ & 4 & 17-Jun-2009 & 17-Jun-2009 & SW 846/6020 & $\mathrm{J}$ \\
\hline Zinc & 0.0210 & 0.0020 & $\mathrm{mg} / \mathrm{L}$ & 4 & 17-Jun-2009 & 17-Jun-2009 & SW 846/6020 & B \\
\hline
\end{tabular}




\section{USACE ERDC-EP-C \\ 3909 Halls Ferry Road \\ Vicksburg, MS 39180-6199}

ERDC - ECB

$-,-$
Project: TVA Fly Ash Project

Project Manager: Tony Bednar
Reported:

03-Sep-2009

DRAFT: SLC Water - Total Metals

9061502-63 (Water)

\begin{tabular}{|c|c|c|c|c|c|c|c|c|}
\hline Analyte & Result & $\begin{array}{r}\text { porting } \\
\text { Limit }\end{array}$ & Units & Dilution & Prepared & Analyzed & Method & Notes \\
\hline
\end{tabular}

ERDC- EL-EP-C (Environmental Chemistry Branch)

DRAFT: Wet Chemistry

\begin{tabular}{llllllll}
\hline Hardness & $\mathbf{9 9 . 9}$ & 1.32 & $\begin{array}{c}\text { mg equil } \\
\text { CaCO3/ }\end{array}$ & 4 & 25-Jun-2009 & 25-Jun-2009
\end{tabular}

$\mathrm{L}$

DRAFT: Metals by EPA 6000/7000 Series Methods

\begin{tabular}{|c|c|c|c|c|c|c|c|c|}
\hline Mercury & 0.000306 & 0.000005 & $\mathrm{mg} / \mathrm{L}$ & 2 & 24-Jul-2009 & 24-Jul-2009 & EPA 7471A & \\
\hline Strontium & 0.571 & 0.0800 & $\mathrm{mg} / \mathrm{L}$ & 4 & 25-Jun-2009 & 25-Jun-2009 & EPA 6010B & \\
\hline Aluminum & 5.58 & 0.200 & $\mathrm{mg} / \mathrm{L}$ & 4 & 25-Jun-2009 & 25-Jun-2009 & SW 846/6010 & \\
\hline Calcium & 31.0 & 0.200 & $\mathrm{mg} / \mathrm{L}$ & 4 & 25-Jun-2009 & 25-Jun-2009 & SW 846/6010 & B \\
\hline Iron & 2.00 & 0.200 & $\mathrm{mg} / \mathrm{L}$ & 4 & 25-Jun-2009 & 25-Jun-2009 & SW 846/6010 & \\
\hline Magnesium & 5.47 & 0.200 & $\mathrm{mg} / \mathrm{L}$ & 4 & 25-Jun-2009 & 25-Jun-2009 & SW 846/6010 & \\
\hline Potassium & 4.09 & 0.200 & $\mathrm{mg} / \mathrm{L}$ & 4 & 25-Jun-2009 & 25-Jun-2009 & SW 846/6010 & B \\
\hline Sodium & 4.79 & 0.200 & $\mathrm{mg} / \mathrm{L}$ & 4 & 25-Jun-2009 & 25-Jun-2009 & SW 846/6010 & B \\
\hline Antimony & 0.0411 & 0.0020 & $\mathrm{mg} / \mathrm{L}$ & 4 & 17-Jun-2009 & 17-Jun-2009 & SW 846/6020 & \\
\hline Arsenic & 0.0767 & 0.0020 & $\mathrm{mg} / \mathrm{L}$ & 4 & 17-Jun-2009 & 17-Jun-2009 & SW 846/6020 & \\
\hline Barium & 0.167 & 0.0020 & $\mathrm{mg} / \mathrm{L}$ & 4 & 17-Jun-2009 & 17-Jun-2009 & SW 846/6020 & B \\
\hline Beryllium & 0.0009 & 0.0020 & $\mathrm{mg} / \mathrm{L}$ & 4 & 17-Jun-2009 & 17-Jun-2009 & SW 846/6020 & $\mathrm{J}$ \\
\hline Cadmium & ND & 0.0020 & $\mathrm{mg} / \mathrm{L}$ & 4 & 17-Jun-2009 & 17-Jun-2009 & SW 846/6020 & $\mathrm{U}$ \\
\hline Chromium & 0.0063 & 0.0020 & $\mathrm{mg} / \mathrm{L}$ & 4 & 17-Jun-2009 & 17-Jun-2009 & SW 846/6020 & \\
\hline Cobalt & 0.0034 & 0.0020 & $\mathrm{mg} / \mathrm{L}$ & 4 & 17-Jun-2009 & 17-Jun-2009 & SW 846/6020 & \\
\hline Copper & 0.0190 & 0.0020 & $\mathrm{mg} / \mathrm{L}$ & 4 & 17-Jun-2009 & 17-Jun-2009 & SW 846/6020 & B \\
\hline Lead & 0.0065 & 0.0020 & $\mathrm{mg} / \mathrm{L}$ & 4 & 17-Jun-2009 & 17-Jun-2009 & SW 846/6020 & \\
\hline Manganese & 0.0482 & 0.0020 & $\mathrm{mg} / \mathrm{L}$ & 4 & 17-Jun-2009 & 17-Jun-2009 & SW 846/6020 & \\
\hline Molybdenum & 0.0738 & 0.0020 & $\mathrm{mg} / \mathrm{L}$ & 4 & 17-Jun-2009 & 17-Jun-2009 & SW 846/6020 & \\
\hline Nickel & 0.0115 & 0.0020 & $\mathrm{mg} / \mathrm{L}$ & 4 & 17-Jun-2009 & 17-Jun-2009 & SW 846/6020 & \\
\hline Selenium & 0.0046 & 0.0020 & $\mathrm{mg} / \mathrm{L}$ & 4 & 17-Jun-2009 & 17-Jun-2009 & SW 846/6020 & \\
\hline Silver & 0.0010 & 0.0020 & $\mathrm{mg} / \mathrm{L}$ & 4 & 17-Jun-2009 & 17-Jun-2009 & SW 846/6020 & $\mathrm{J}, \mathrm{B}$ \\
\hline Thallium & 0.0008 & 0.0020 & $\mathrm{mg} / \mathrm{L}$ & 4 & 17-Jun-2009 & 17-Jun-2009 & SW 846/6020 & $\mathrm{J}$ \\
\hline Vanadium & 0.0769 & 0.0020 & $\mathrm{mg} / \mathrm{L}$ & 4 & 17-Jun-2009 & 17-Jun-2009 & SW 846/6020 & \\
\hline Zinc & 0.0334 & 0.0020 & $\mathrm{mg} / \mathrm{L}$ & 4 & 17-Jun-2009 & 17-Jun-2009 & SW 846/6020 & B \\
\hline
\end{tabular}




\section{USACE ERDC-EP-C \\ 3909 Halls Ferry Road \\ Vicksburg, MS 39180-6199}

ERDC - ECB

$-\cdot$,
Project: TVA Fly Ash Project

Project Manager: Tony Bednar
Reported:

03-Sep-2009

DRAFT: EMR MT N2 Hour 1 Rep D - Total Metals

9061502-64 (Water)

\begin{tabular}{|c|c|c|c|c|c|c|c|c|}
\hline Analyte & Result & $\begin{array}{r}\text { porting } \\
\text { Limit }\end{array}$ & Units & Dilution & Prepared & Analyzed & Method & Notes \\
\hline
\end{tabular}

ERDC- EL-EP-C (Environmental Chemistry Branch)

DRAFT: Wet Chemistry

\begin{tabular}{llllllll}
\hline Hardness & $\mathbf{6 8 . 5}$ & 1.32 & $\begin{array}{c}\text { mg equil } \\
\text { CaCO3/ }\end{array}$ & 4 & 25-Jun-2009 & 25-Jun-2009
\end{tabular}

$\mathrm{L}$

DRAFT: Metals by EPA 6000/7000 Series Methods

\begin{tabular}{|c|c|c|c|c|c|c|c|c|}
\hline Mercury & 0.000553 & 0.000005 & $\mathrm{mg} / \mathrm{L}$ & 2 & 24-Jul-2009 & 24-Jul-2009 & EPA 7471A & \\
\hline Strontium & 0.490 & 0.0800 & $\mathrm{mg} / \mathrm{L}$ & 4 & 25-Jun-2009 & 25-Jun-2009 & EPA 6010B & \\
\hline Aluminum & 38.1 & 0.200 & $\mathrm{mg} / \mathrm{L}$ & 4 & 25-Jun-2009 & 25-Jun-2009 & SW 846/6010 & \\
\hline Calcium & 18.4 & 0.200 & $\mathrm{mg} / \mathrm{L}$ & 4 & 25-Jun-2009 & 25-Jun-2009 & SW 846/6010 & B \\
\hline Iron & 15.4 & 0.200 & $\mathrm{mg} / \mathrm{L}$ & 4 & 25-Jun-2009 & 25-Jun-2009 & SW 846/6010 & \\
\hline Magnesium & 5.44 & 0.200 & $\mathrm{mg} / \mathrm{L}$ & 4 & 25-Jun-2009 & 25-Jun-2009 & SW 846/6010 & \\
\hline Potassium & 8.73 & 0.200 & $\mathrm{mg} / \mathrm{L}$ & 4 & 25-Jun-2009 & 25-Jun-2009 & SW 846/6010 & B \\
\hline Sodium & 4.82 & 0.200 & $\mathrm{mg} / \mathrm{L}$ & 4 & 25-Jun-2009 & 25-Jun-2009 & SW 846/6010 & B \\
\hline Antimony & 0.0066 & 0.0020 & $\mathrm{mg} / \mathrm{L}$ & 4 & 17-Jun-2009 & 17-Jun-2009 & SW 846/6020 & \\
\hline Arsenic & 0.139 & 0.0020 & $\mathrm{mg} / \mathrm{L}$ & 4 & 17-Jun-2009 & 17-Jun-2009 & SW 846/6020 & \\
\hline Barium & 0.765 & 0.0020 & $\mathrm{mg} / \mathrm{L}$ & 4 & 17-Jun-2009 & 17-Jun-2009 & SW 846/6020 & B \\
\hline Beryllium & 0.0062 & 0.0020 & $\mathrm{mg} / \mathrm{L}$ & 4 & 17-Jun-2009 & 17-Jun-2009 & SW 846/6020 & \\
\hline Cadmium & 0.0013 & 0.0020 & $\mathrm{mg} / \mathrm{L}$ & 4 & 17-Jun-2009 & 17-Jun-2009 & SW 846/6020 & J \\
\hline Chromium & 0.0462 & 0.0020 & $\mathrm{mg} / \mathrm{L}$ & 4 & 17-Jun-2009 & 17-Jun-2009 & SW 846/6020 & \\
\hline Cobalt & 0.0284 & 0.0020 & $\mathrm{mg} / \mathrm{L}$ & 4 & 17-Jun-2009 & 17-Jun-2009 & SW 846/6020 & \\
\hline Copper & 0.0949 & 0.0020 & $\mathrm{mg} / \mathrm{L}$ & 4 & 17-Jun-2009 & 17-Jun-2009 & SW 846/6020 & B \\
\hline Lead & 0.0661 & 0.0020 & $\mathrm{mg} / \mathrm{L}$ & 4 & 17-Jun-2009 & 17-Jun-2009 & SW 846/6020 & \\
\hline Manganese & 0.132 & 0.0020 & $\mathrm{mg} / \mathrm{L}$ & 4 & 17-Jun-2009 & 17-Jun-2009 & SW 846/6020 & \\
\hline Molybdenum & 0.0076 & 0.0020 & $\mathrm{mg} / \mathrm{L}$ & 4 & 17-Jun-2009 & 17-Jun-2009 & SW 846/6020 & \\
\hline Nickel & 0.0570 & 0.0020 & $\mathrm{mg} / \mathrm{L}$ & 4 & 17-Jun-2009 & 17-Jun-2009 & SW 846/6020 & \\
\hline Selenium & 0.0061 & 0.0020 & $\mathrm{mg} / \mathrm{L}$ & 4 & 17-Jun-2009 & 17-Jun-2009 & SW 846/6020 & \\
\hline Silver & 0.0031 & 0.0020 & $\mathrm{mg} / \mathrm{L}$ & 4 & 17-Jun-2009 & 17-Jun-2009 & SW 846/6020 & B \\
\hline Thallium & 0.0039 & 0.0020 & $\mathrm{mg} / \mathrm{L}$ & 4 & 17-Jun-2009 & 17-Jun-2009 & SW 846/6020 & \\
\hline Vanadium & 0.142 & 0.0020 & $\mathrm{mg} / \mathrm{L}$ & 4 & 17-Jun-2009 & 17-Jun-2009 & SW 846/6020 & \\
\hline Zinc & 0.103 & 0.0020 & $\mathrm{mg} / \mathrm{L}$ & 4 & 17-Jun-2009 & 17-Jun-2009 & SW 846/6020 & B \\
\hline
\end{tabular}




\section{USACE ERDC-EP-C \\ 3909 Halls Ferry Road \\ Vicksburg, MS 39180-6199}

ERDC - ECB

$-\cdot$,
Project: TVA Fly Ash Project

Project Manager: Tony Bednar
Reported:

03-Sep-2009

DRAFT: EMR MT N2 Hour 1 Rep E - Total Metals

9061502-65 (Water)

\begin{tabular}{|c|c|c|c|c|c|c|c|c|}
\hline Analyte & Result & $\begin{array}{r}\text { porting } \\
\text { Limit }\end{array}$ & Units & Dilution & Prepared & Analyzed & Method & Notes \\
\hline
\end{tabular}

ERDC- EL-EP-C (Environmental Chemistry Branch)

DRAFT: Wet Chemistry

\begin{tabular}{llllllll}
\hline Hardness & $\mathbf{6 2 . 2}$ & 1.32 & $\begin{array}{c}\text { mg equil } \\
\text { CaCO3/ }\end{array}$ & 4 & 25-Jun-2009 & 25-Jun-2009
\end{tabular}

$\mathrm{L}$

DRAFT: Metals by EPA 6000/7000 Series Methods

\begin{tabular}{|c|c|c|c|c|c|c|c|c|}
\hline Mercury & 0.000477 & 0.000005 & $\mathrm{mg} / \mathrm{L}$ & 2 & 24-Jul-2009 & 24-Jul-2009 & EPA 7471A & \\
\hline Strontium & 0.417 & 0.0800 & $\mathrm{mg} / \mathrm{L}$ & 4 & 25-Jun-2009 & 25-Jun-2009 & EPA 6010B & \\
\hline Aluminum & 32.9 & 0.200 & $\mathrm{mg} / \mathrm{L}$ & 4 & 25-Jun-2009 & 25-Jun-2009 & SW 846/6010 & \\
\hline Calcium & 16.8 & 0.200 & $\mathrm{mg} / \mathrm{L}$ & 4 & 25-Jun-2009 & 25-Jun-2009 & SW 846/6010 & B \\
\hline Iron & 13.0 & 0.200 & $\mathrm{mg} / \mathrm{L}$ & 4 & 25-Jun-2009 & 25-Jun-2009 & SW 846/6010 & \\
\hline Magnesium & 4.94 & 0.200 & $\mathrm{mg} / \mathrm{L}$ & 4 & 25-Jun-2009 & 25-Jun-2009 & SW 846/6010 & \\
\hline Potassium & 8.29 & 0.200 & $\mathrm{mg} / \mathrm{L}$ & 4 & 25-Jun-2009 & 25-Jun-2009 & SW 846/6010 & B \\
\hline Sodium & 3.86 & 0.200 & $\mathrm{mg} / \mathrm{L}$ & 4 & 25-Jun-2009 & 25-Jun-2009 & SW 846/6010 & B \\
\hline Antimony & 0.0053 & 0.0020 & $\mathrm{mg} / \mathrm{L}$ & 4 & 17-Jun-2009 & 17-Jun-2009 & SW 846/6020 & \\
\hline Arsenic & 0.114 & 0.0020 & $\mathrm{mg} / \mathrm{L}$ & 4 & 17-Jun-2009 & 17-Jun-2009 & SW 846/6020 & \\
\hline Barium & 0.619 & 0.0020 & $\mathrm{mg} / \mathrm{L}$ & 4 & 17-Jun-2009 & 17-Jun-2009 & SW 846/6020 & B \\
\hline Beryllium & 0.0055 & 0.0020 & $\mathrm{mg} / \mathrm{L}$ & 4 & 17-Jun-2009 & 17-Jun-2009 & SW 846/6020 & \\
\hline Cadmium & 0.0009 & 0.0020 & $\mathrm{mg} / \mathrm{L}$ & 4 & 17-Jun-2009 & 17-Jun-2009 & SW 846/6020 & $\mathrm{J}$ \\
\hline Chromium & 0.0427 & 0.0020 & $\mathrm{mg} / \mathrm{L}$ & 4 & 17-Jun-2009 & 17-Jun-2009 & SW 846/6020 & \\
\hline Cobalt & 0.0239 & 0.0020 & $\mathrm{mg} / \mathrm{L}$ & 4 & 17-Jun-2009 & 17-Jun-2009 & SW 846/6020 & \\
\hline Copper & 0.0843 & 0.0020 & $\mathrm{mg} / \mathrm{L}$ & 4 & 17-Jun-2009 & 17-Jun-2009 & SW 846/6020 & B \\
\hline Lead & 0.0537 & 0.0020 & $\mathrm{mg} / \mathrm{L}$ & 4 & 17-Jun-2009 & 17-Jun-2009 & SW 846/6020 & \\
\hline Manganese & 0.129 & 0.0020 & $\mathrm{mg} / \mathrm{L}$ & 4 & 17-Jun-2009 & 17-Jun-2009 & SW 846/6020 & \\
\hline Molybdenum & 0.0061 & 0.0020 & $\mathrm{mg} / \mathrm{L}$ & 4 & 17-Jun-2009 & 17-Jun-2009 & SW 846/6020 & \\
\hline Nickel & 0.0484 & 0.0020 & $\mathrm{mg} / \mathrm{L}$ & 4 & 17-Jun-2009 & 17-Jun-2009 & SW 846/6020 & \\
\hline Selenium & 0.0050 & 0.0020 & $\mathrm{mg} / \mathrm{L}$ & 4 & 17-Jun-2009 & 17-Jun-2009 & SW 846/6020 & \\
\hline Silver & 0.0025 & 0.0020 & $\mathrm{mg} / \mathrm{L}$ & 4 & 17-Jun-2009 & 17-Jun-2009 & SW 846/6020 & B \\
\hline Thallium & 0.0031 & 0.0020 & $\mathrm{mg} / \mathrm{L}$ & 4 & 17-Jun-2009 & 17-Jun-2009 & SW 846/6020 & \\
\hline Vanadium & 0.125 & 0.0020 & $\mathrm{mg} / \mathrm{L}$ & 4 & 17-Jun-2009 & 17-Jun-2009 & SW 846/6020 & \\
\hline Zinc & 0.0937 & 0.0020 & $\mathrm{mg} / \mathrm{L}$ & 4 & 17-Jun-2009 & 17-Jun-2009 & SW 846/6020 & B \\
\hline
\end{tabular}




\section{USACE ERDC-EP-C \\ 3909 Halls Ferry Road \\ Vicksburg, MS 39180-6199}

ERDC - ECB

$-,-$
Project: TVA Fly Ash Project

Project Manager: Tony Bednar
Reported:

03-Sep-2009

DRAFT: EMR MT N2 Hour 1 Rep F - Total Metals

9061502-66 (Water)

\begin{tabular}{|c|c|c|c|c|c|c|c|c|}
\hline & & porting & & & & & & \\
\hline Analyte & Result & Limit & Units & Dilution & Prepared & Analyzed & Method & Notes \\
\hline
\end{tabular}

ERDC- EL-EP-C (Environmental Chemistry Branch)

DRAFT: Wet Chemistry

\begin{tabular}{llllllll}
\hline Hardness & $\mathbf{6 3 . 8}$ & 1.32 & $\begin{array}{c}\text { mg equil } \\
\text { CaCO3/ }\end{array}$ & 4 & 25-Jun-2009 & 25-Jun-2009
\end{tabular}

$\mathrm{L}$

DRAFT: Metals by EPA 6000/7000 Series Methods

\begin{tabular}{|c|c|c|c|c|c|c|c|c|}
\hline Mercury & 0.000388 & 0.000005 & $\mathrm{mg} / \mathrm{L}$ & 2 & 24-Jul-2009 & 24-Jul-2009 & EPA 7471A & \\
\hline Strontium & 0.437 & 0.0800 & $\mathrm{mg} / \mathrm{L}$ & 4 & 25-Jun-2009 & 25-Jun-2009 & EPA 6010B & \\
\hline Aluminum & 34.8 & 0.200 & $\mathrm{mg} / \mathrm{L}$ & 4 & 25-Jun-2009 & 25-Jun-2009 & SW 846/6010 & \\
\hline Calcium & 17.1 & 0.200 & $\mathrm{mg} / \mathrm{L}$ & 4 & 25-Jun-2009 & 25-Jun-2009 & SW 846/6010 & B \\
\hline Iron & 13.8 & 0.200 & $\mathrm{mg} / \mathrm{L}$ & 4 & 25-Jun-2009 & 25-Jun-2009 & SW 846/6010 & \\
\hline Magnesium & 5.09 & 0.200 & $\mathrm{mg} / \mathrm{L}$ & 4 & 25-Jun-2009 & 25-Jun-2009 & SW 846/6010 & \\
\hline Potassium & 7.68 & 0.200 & $\mathrm{mg} / \mathrm{L}$ & 4 & 25-Jun-2009 & 25-Jun-2009 & SW 846/6010 & B \\
\hline Sodium & 3.88 & 0.200 & $\mathrm{mg} / \mathrm{L}$ & 4 & 25-Jun-2009 & 25-Jun-2009 & SW 846/6010 & B \\
\hline Antimony & 0.0054 & 0.0020 & $\mathrm{mg} / \mathrm{L}$ & 4 & 17-Jun-2009 & 17-Jun-2009 & SW 846/6020 & \\
\hline Arsenic & 0.118 & 0.0020 & $\mathrm{mg} / \mathrm{L}$ & 4 & 17-Jun-2009 & 17-Jun-2009 & SW 846/6020 & \\
\hline Barium & 0.686 & 0.0020 & $\mathrm{mg} / \mathrm{L}$ & 4 & 17-Jun-2009 & 17-Jun-2009 & SW 846/6020 & B \\
\hline Beryllium & 0.0055 & 0.0020 & $\mathrm{mg} / \mathrm{L}$ & 4 & 17-Jun-2009 & 17-Jun-2009 & SW 846/6020 & \\
\hline Cadmium & 0.0010 & 0.0020 & $\mathrm{mg} / \mathrm{L}$ & 4 & 17-Jun-2009 & 17-Jun-2009 & SW 846/6020 & $\mathrm{J}$ \\
\hline Chromium & 0.0413 & 0.0020 & $\mathrm{mg} / \mathrm{L}$ & 4 & 17-Jun-2009 & 17-Jun-2009 & SW 846/6020 & \\
\hline Cobalt & 0.0256 & 0.0020 & $\mathrm{mg} / \mathrm{L}$ & 4 & 17-Jun-2009 & 17-Jun-2009 & SW 846/6020 & \\
\hline Copper & 0.0881 & 0.0020 & $\mathrm{mg} / \mathrm{L}$ & 4 & 17-Jun-2009 & 17-Jun-2009 & SW 846/6020 & B \\
\hline Lead & 0.0575 & 0.0020 & $\mathrm{mg} / \mathrm{L}$ & 4 & 17-Jun-2009 & 17-Jun-2009 & SW 846/6020 & \\
\hline Manganese & 0.125 & 0.0020 & $\mathrm{mg} / \mathrm{L}$ & 4 & 17-Jun-2009 & 17-Jun-2009 & SW 846/6020 & \\
\hline Molybdenum & 0.0065 & 0.0020 & $\mathrm{mg} / \mathrm{L}$ & 4 & 17-Jun-2009 & 17-Jun-2009 & SW 846/6020 & \\
\hline Nickel & 0.0498 & 0.0020 & $\mathrm{mg} / \mathrm{L}$ & 4 & 17-Jun-2009 & 17-Jun-2009 & SW 846/6020 & \\
\hline Selenium & 0.0063 & 0.0020 & $\mathrm{mg} / \mathrm{L}$ & 4 & 17-Jun-2009 & 17-Jun-2009 & SW 846/6020 & \\
\hline Silver & 0.0014 & 0.0020 & $\mathrm{mg} / \mathrm{L}$ & 4 & 17-Jun-2009 & 17-Jun-2009 & SW 846/6020 & $\mathrm{J}, \mathrm{B}$ \\
\hline Thallium & 0.0034 & 0.0020 & $\mathrm{mg} / \mathrm{L}$ & 4 & 17-Jun-2009 & 17-Jun-2009 & SW 846/6020 & \\
\hline Vanadium & 0.142 & 0.0020 & $\mathrm{mg} / \mathrm{L}$ & 4 & 17-Jun-2009 & 17-Jun-2009 & SW 846/6020 & \\
\hline Zinc & 0.0933 & 0.0020 & $\mathrm{mg} / \mathrm{L}$ & 4 & 17-Jun-2009 & 17-Jun-2009 & SW 846/6020 & B \\
\hline
\end{tabular}




\section{USACE ERDC-EP-C \\ 3909 Halls Ferry Road \\ Vicksburg, MS 39180-6199}

ERDC - ECB

$-\cdot$,
Project: TVA Fly Ash Project

Project Manager: Tony Bednar
Reported:

03-Sep-2009

DRAFT: EMR MT O2 Hour 1 Rep D - Total Metals

9061502-67 (Water)

\begin{tabular}{|c|c|c|c|c|c|c|c|c|}
\hline Analyte & Result & $\begin{array}{r}\text { porting } \\
\text { Limit }\end{array}$ & Units & Dilution & Prepared & Analyzed & Method & Notes \\
\hline
\end{tabular}

ERDC- EL-EP-C (Environmental Chemistry Branch)

DRAFT: Wet Chemistry

\begin{tabular}{llllllll}
\hline Hardness & $\mathbf{5 6 . 5}$ & 1.32 & $\begin{array}{c}\text { mg equil } \\
\text { CaCO3/ }\end{array}$ & 4 & 25-Jun-2009 & 25-Jun-2009
\end{tabular}

$\mathrm{L}$

DRAFT: Metals by EPA 6000/7000 Series Methods

\begin{tabular}{|c|c|c|c|c|c|c|c|c|}
\hline Mercury & 0.000354 & 0.000005 & $\mathrm{mg} / \mathrm{L}$ & 2 & 24-Jul-2009 & 24-Jul-2009 & EPA 7471A & \\
\hline Strontium & 0.335 & 0.0800 & $\mathrm{mg} / \mathrm{L}$ & 4 & 25-Jun-2009 & 25-Jun-2009 & EPA 6010B & \\
\hline Aluminum & 25.3 & 0.200 & $\mathrm{mg} / \mathrm{L}$ & 4 & 25-Jun-2009 & 25-Jun-2009 & SW 846/6010 & \\
\hline Calcium & 15.4 & 0.200 & $\mathrm{mg} / \mathrm{L}$ & 4 & 25-Jun-2009 & 25-Jun-2009 & SW 846/6010 & B \\
\hline Iron & 10.1 & 0.200 & $\mathrm{mg} / \mathrm{L}$ & 4 & 25-Jun-2009 & 25-Jun-2009 & SW 846/6010 & \\
\hline Magnesium & 4.37 & 0.200 & $\mathrm{mg} / \mathrm{L}$ & 4 & 25-Jun-2009 & 25-Jun-2009 & SW 846/6010 & \\
\hline Potassium & 6.00 & 0.200 & $\mathrm{mg} / \mathrm{L}$ & 4 & 25-Jun-2009 & 25-Jun-2009 & SW 846/6010 & B \\
\hline Sodium & 3.89 & 0.200 & $\mathrm{mg} / \mathrm{L}$ & 4 & 25-Jun-2009 & 25-Jun-2009 & SW 846/6010 & B \\
\hline Antimony & 0.0040 & 0.0020 & $\mathrm{mg} / \mathrm{L}$ & 4 & 17-Jun-2009 & 17-Jun-2009 & SW 846/6020 & \\
\hline Arsenic & 0.0899 & 0.0020 & $\mathrm{mg} / \mathrm{L}$ & 4 & 17-Jun-2009 & 17-Jun-2009 & SW 846/6020 & \\
\hline Barium & 0.503 & 0.0020 & $\mathrm{mg} / \mathrm{L}$ & 4 & 17-Jun-2009 & 17-Jun-2009 & SW 846/6020 & B \\
\hline Beryllium & 0.0042 & 0.0020 & $\mathrm{mg} / \mathrm{L}$ & 4 & 17-Jun-2009 & 17-Jun-2009 & SW 846/6020 & \\
\hline Cadmium & 0.0008 & 0.0020 & $\mathrm{mg} / \mathrm{L}$ & 4 & 17-Jun-2009 & 17-Jun-2009 & SW 846/6020 & $\mathrm{J}$ \\
\hline Chromium & 0.0369 & 0.0020 & $\mathrm{mg} / \mathrm{L}$ & 4 & 17-Jun-2009 & 17-Jun-2009 & SW 846/6020 & \\
\hline Cobalt & 0.0191 & 0.0020 & $\mathrm{mg} / \mathrm{L}$ & 4 & 17-Jun-2009 & 17-Jun-2009 & SW 846/6020 & \\
\hline Copper & 0.0708 & 0.0020 & $\mathrm{mg} / \mathrm{L}$ & 4 & 17-Jun-2009 & 17-Jun-2009 & SW 846/6020 & B \\
\hline Lead & 0.0413 & 0.0020 & $\mathrm{mg} / \mathrm{L}$ & 4 & 17-Jun-2009 & 17-Jun-2009 & SW 846/6020 & \\
\hline Manganese & 0.102 & 0.0020 & $\mathrm{mg} / \mathrm{L}$ & 4 & 17-Jun-2009 & 17-Jun-2009 & SW 846/6020 & \\
\hline Molybdenum & 0.0049 & 0.0020 & $\mathrm{mg} / \mathrm{L}$ & 4 & 17-Jun-2009 & 17-Jun-2009 & SW 846/6020 & \\
\hline Nickel & 0.0407 & 0.0020 & $\mathrm{mg} / \mathrm{L}$ & 4 & 17-Jun-2009 & 17-Jun-2009 & SW 846/6020 & \\
\hline Selenium & 0.0037 & 0.0020 & $\mathrm{mg} / \mathrm{L}$ & 4 & 17-Jun-2009 & 17-Jun-2009 & SW 846/6020 & \\
\hline Silver & 0.0011 & 0.0020 & $\mathrm{mg} / \mathrm{L}$ & 4 & 17-Jun-2009 & 17-Jun-2009 & SW 846/6020 & $\mathrm{J}, \mathrm{B}$ \\
\hline Thallium & 0.0024 & 0.0020 & $\mathrm{mg} / \mathrm{L}$ & 4 & 17-Jun-2009 & 17-Jun-2009 & SW 846/6020 & \\
\hline Vanadium & 0.109 & 0.0020 & $\mathrm{mg} / \mathrm{L}$ & 4 & 17-Jun-2009 & 17-Jun-2009 & SW 846/6020 & \\
\hline Zinc & 0.0941 & 0.0020 & $\mathrm{mg} / \mathrm{L}$ & 4 & 17-Jun-2009 & 17-Jun-2009 & SW 846/6020 & B \\
\hline
\end{tabular}




\section{USACE ERDC-EP-C \\ 3909 Halls Ferry Road \\ Vicksburg, MS 39180-6199}

ERDC - ECB

$-\cdot$,
Project: TVA Fly Ash Project

Project Manager: Tony Bednar
Reported:

03-Sep-2009

DRAFT: EMR MT O2 Hour 1 Rep E - Total Metals

9061502-68 (Water)

\begin{tabular}{|c|c|c|c|c|c|c|c|c|}
\hline Analyte & Result & $\begin{array}{r}\text { porting } \\
\text { Limit }\end{array}$ & Units & Dilution & Prepared & Analyzed & Method & Notes \\
\hline
\end{tabular}

ERDC- EL-EP-C (Environmental Chemistry Branch)

DRAFT: Wet Chemistry

\begin{tabular}{llllllll}
\hline Hardness & $\mathbf{5 3 . 3}$ & 1.32 & $\begin{array}{c}\text { mg equil } \\
\text { CaCO3/ }\end{array}$ & 4 & 25-Jun-2009 & 25-Jun-2009
\end{tabular}

$\mathrm{L}$

DRAFT: Metals by EPA 6000/7000 Series Methods

\begin{tabular}{|c|c|c|c|c|c|c|c|c|}
\hline Mercury & 0.000461 & 0.000005 & $\mathrm{mg} / \mathrm{L}$ & 2 & 24-Jul-2009 & 24-Jul-2009 & EPA 7471A & \\
\hline Strontium & 0.310 & 0.0800 & $\mathrm{mg} / \mathrm{L}$ & 4 & 25-Jun-2009 & 25-Jun-2009 & EPA $6010 \mathrm{~B}$ & \\
\hline Aluminum & 23.2 & 0.200 & $\mathrm{mg} / \mathrm{L}$ & 4 & 25-Jun-2009 & 25-Jun-2009 & SW 846/6010 & \\
\hline Calcium & 14.5 & 0.200 & $\mathrm{mg} / \mathrm{L}$ & 4 & 25-Jun-2009 & 25-Jun-2009 & SW 846/6010 & B \\
\hline Iron & 9.25 & 0.200 & $\mathrm{mg} / \mathrm{L}$ & 4 & 25-Jun-2009 & 25-Jun-2009 & SW 846/6010 & \\
\hline Magnesium & 4.14 & 0.200 & $\mathrm{mg} / \mathrm{L}$ & 4 & 25-Jun-2009 & 25-Jun-2009 & SW 846/6010 & \\
\hline Potassium & 6.41 & 0.200 & $\mathrm{mg} / \mathrm{L}$ & 4 & 25-Jun-2009 & 25-Jun-2009 & SW 846/6010 & B \\
\hline Sodium & 3.81 & 0.200 & $\mathrm{mg} / \mathrm{L}$ & 4 & 25-Jun-2009 & 25-Jun-2009 & SW 846/6010 & B \\
\hline Antimony & 0.0036 & 0.0020 & $\mathrm{mg} / \mathrm{L}$ & 4 & 17-Jun-2009 & 17-Jun-2009 & SW 846/6020 & \\
\hline Arsenic & 0.0767 & 0.0020 & $\mathrm{mg} / \mathrm{L}$ & 4 & 17-Jun-2009 & 17-Jun-2009 & SW 846/6020 & \\
\hline Barium & 0.449 & 0.0020 & $\mathrm{mg} / \mathrm{L}$ & 4 & 17-Jun-2009 & 17-Jun-2009 & SW 846/6020 & B \\
\hline Beryllium & 0.0041 & 0.0020 & $\mathrm{mg} / \mathrm{L}$ & 4 & 17-Jun-2009 & 17-Jun-2009 & SW 846/6020 & \\
\hline Cadmium & 0.0006 & 0.0020 & $\mathrm{mg} / \mathrm{L}$ & 4 & 17-Jun-2009 & 17-Jun-2009 & SW 846/6020 & $\mathrm{J}$ \\
\hline Chromium & 0.0313 & 0.0020 & $\mathrm{mg} / \mathrm{L}$ & 4 & 17-Jun-2009 & 17-Jun-2009 & SW 846/6020 & \\
\hline Cobalt & 0.0178 & 0.0020 & $\mathrm{mg} / \mathrm{L}$ & 4 & 17-Jun-2009 & 17-Jun-2009 & SW 846/6020 & \\
\hline Copper & 0.0617 & 0.0020 & $\mathrm{mg} / \mathrm{L}$ & 4 & 17-Jun-2009 & 17-Jun-2009 & SW 846/6020 & B \\
\hline Lead & 0.0361 & 0.0020 & $\mathrm{mg} / \mathrm{L}$ & 4 & 17-Jun-2009 & 17-Jun-2009 & SW 846/6020 & \\
\hline Manganese & 0.104 & 0.0020 & $\mathrm{mg} / \mathrm{L}$ & 4 & 17-Jun-2009 & 17-Jun-2009 & SW 846/6020 & \\
\hline Molybdenum & 0.0043 & 0.0020 & $\mathrm{mg} / \mathrm{L}$ & 4 & 17-Jun-2009 & 17-Jun-2009 & SW 846/6020 & \\
\hline Nickel & 0.0364 & 0.0020 & $\mathrm{mg} / \mathrm{L}$ & 4 & 17-Jun-2009 & 17-Jun-2009 & SW 846/6020 & \\
\hline Selenium & 0.0027 & 0.0020 & $\mathrm{mg} / \mathrm{L}$ & 4 & 17-Jun-2009 & 17-Jun-2009 & SW 846/6020 & \\
\hline Silver & 0.0006 & 0.0020 & $\mathrm{mg} / \mathrm{L}$ & 4 & 17-Jun-2009 & 17-Jun-2009 & SW 846/6020 & $\mathrm{J}, \mathrm{B}$ \\
\hline Thallium & 0.0021 & 0.0020 & $\mathrm{mg} / \mathrm{L}$ & 4 & 17-Jun-2009 & 17-Jun-2009 & SW 846/6020 & \\
\hline Vanadium & 0.0915 & 0.0020 & $\mathrm{mg} / \mathrm{L}$ & 4 & 17-Jun-2009 & 17-Jun-2009 & SW 846/6020 & \\
\hline Zinc & 0.0907 & 0.0020 & $\mathrm{mg} / \mathrm{L}$ & 4 & 17-Jun-2009 & 17-Jun-2009 & SW 846/6020 & B \\
\hline
\end{tabular}




\section{USACE ERDC-EP-C \\ 3909 Halls Ferry Road \\ Vicksburg, MS 39180-6199}

ERDC - ECB

$-\cdot$,
Project: TVA Fly Ash Project

Project Manager: Tony Bednar
Reported:

03-Sep-2009

DRAFT: EMR MT O2 Hour 1 Rep F - Total Metals

9061502-69 (Water)

\begin{tabular}{|c|c|c|c|c|c|c|c|c|}
\hline Analyte & Result & $\begin{array}{r}\text { porting } \\
\text { Limit }\end{array}$ & Units & Dilution & Prepared & Analyzed & Method & Notes \\
\hline
\end{tabular}

ERDC- EL-EP-C (Environmental Chemistry Branch)

DRAFT: Wet Chemistry

\begin{tabular}{llllllll}
\hline Hardness & $\mathbf{6 7 . 9}$ & 1.32 & $\begin{array}{c}\text { mg equil } \\
\text { CaCO3/ }\end{array}$ & 4 & 25-Jun-2009 & 25-Jun-2009
\end{tabular}

$\mathrm{L}$

DRAFT: Metals by EPA 6000/7000 Series Methods

\begin{tabular}{|c|c|c|c|c|c|c|c|c|}
\hline Mercury & 0.000359 & 0.000005 & $\mathrm{mg} / \mathrm{L}$ & 2 & 24-Jul-2009 & 24-Jul-2009 & EPA 7471A & \\
\hline Strontium & 0.475 & 0.0800 & $\mathrm{mg} / \mathrm{L}$ & 4 & 25-Jun-2009 & 25-Jun-2009 & EPA $6010 \mathrm{~B}$ & \\
\hline Aluminum & 37.0 & 0.200 & $\mathrm{mg} / \mathrm{L}$ & 4 & 25-Jun-2009 & 25-Jun-2009 & SW 846/6010 & \\
\hline Calcium & 18.3 & 0.200 & $\mathrm{mg} / \mathrm{L}$ & 4 & 25-Jun-2009 & 25-Jun-2009 & SW 846/6010 & B \\
\hline Iron & 14.8 & 0.200 & $\mathrm{mg} / \mathrm{L}$ & 4 & 25-Jun-2009 & 25-Jun-2009 & SW 846/6010 & \\
\hline Magnesium & 5.40 & 0.200 & $\mathrm{mg} / \mathrm{L}$ & 4 & 25-Jun-2009 & 25-Jun-2009 & SW 846/6010 & \\
\hline Potassium & 8.12 & 0.200 & $\mathrm{mg} / \mathrm{L}$ & 4 & 25-Jun-2009 & 25-Jun-2009 & SW 846/6010 & B \\
\hline Sodium & 4.25 & 0.200 & $\mathrm{mg} / \mathrm{L}$ & 4 & 25-Jun-2009 & 25-Jun-2009 & SW 846/6010 & B \\
\hline Antimony & 0.0055 & 0.0020 & $\mathrm{mg} / \mathrm{L}$ & 4 & 17-Jun-2009 & 17-Jun-2009 & SW 846/6020 & \\
\hline Arsenic & 0.129 & 0.0020 & $\mathrm{mg} / \mathrm{L}$ & 4 & 17-Jun-2009 & 17-Jun-2009 & SW 846/6020 & \\
\hline Barium & 0.709 & 0.0020 & $\mathrm{mg} / \mathrm{L}$ & 4 & 17-Jun-2009 & 17-Jun-2009 & SW 846/6020 & B \\
\hline Beryllium & 0.0060 & 0.0020 & $\mathrm{mg} / \mathrm{L}$ & 4 & 17-Jun-2009 & 17-Jun-2009 & SW 846/6020 & \\
\hline Cadmium & 0.0012 & 0.0020 & $\mathrm{mg} / \mathrm{L}$ & 4 & 17-Jun-2009 & 17-Jun-2009 & SW 846/6020 & $\mathrm{J}$ \\
\hline Chromium & 0.0469 & 0.0020 & $\mathrm{mg} / \mathrm{L}$ & 4 & 17-Jun-2009 & 17-Jun-2009 & SW 846/6020 & \\
\hline Cobalt & 0.0279 & 0.0020 & $\mathrm{mg} / \mathrm{L}$ & 4 & 17-Jun-2009 & 17-Jun-2009 & SW 846/6020 & \\
\hline Copper & 0.0954 & 0.0020 & $\mathrm{mg} / \mathrm{L}$ & 4 & 17-Jun-2009 & 17-Jun-2009 & SW 846/6020 & B \\
\hline Lead & 0.0602 & 0.0020 & $\mathrm{mg} / \mathrm{L}$ & 4 & 17-Jun-2009 & 17-Jun-2009 & SW 846/6020 & \\
\hline Manganese & 0.139 & 0.0020 & $\mathrm{mg} / \mathrm{L}$ & 4 & 17-Jun-2009 & 17-Jun-2009 & SW 846/6020 & \\
\hline Molybdenum & 0.0069 & 0.0020 & $\mathrm{mg} / \mathrm{L}$ & 4 & 17-Jun-2009 & 17-Jun-2009 & SW 846/6020 & \\
\hline Nickel & 0.0544 & 0.0020 & $\mathrm{mg} / \mathrm{L}$ & 4 & 17-Jun-2009 & 17-Jun-2009 & SW 846/6020 & \\
\hline Selenium & 0.0067 & 0.0020 & $\mathrm{mg} / \mathrm{L}$ & 4 & 17-Jun-2009 & 17-Jun-2009 & SW 846/6020 & \\
\hline Silver & 0.0006 & 0.0020 & $\mathrm{mg} / \mathrm{L}$ & 4 & 17-Jun-2009 & 17-Jun-2009 & SW 846/6020 & $\mathrm{J}, \mathrm{B}$ \\
\hline Thallium & 0.0033 & 0.0020 & $\mathrm{mg} / \mathrm{L}$ & 4 & 17-Jun-2009 & 17-Jun-2009 & SW 846/6020 & \\
\hline Vanadium & 0.139 & 0.0020 & $\mathrm{mg} / \mathrm{L}$ & 4 & 17-Jun-2009 & 17-Jun-2009 & SW 846/6020 & \\
\hline Zinc & 0.164 & 0.0020 & $\mathrm{mg} / \mathrm{L}$ & 4 & 17-Jun-2009 & 17-Jun-2009 & SW 846/6020 & B \\
\hline
\end{tabular}




\section{USACE ERDC-EP-C \\ 3909 Halls Ferry Road \\ Vicksburg, MS 39180-6199}

ERDC - ECB

$-\cdot$,
Project: TVA Fly Ash Project

Project Manager: Tony Bednar
Reported:

03-Sep-2009

DRAFT: SLC MT N2 Hour 1 Rep D - Total Metals

9061502-70 (Water)

\begin{tabular}{|c|c|c|c|c|c|c|c|c|}
\hline Analyte & Result & $\begin{array}{r}\text { porting } \\
\text { Limit }\end{array}$ & Units & Dilution & Prepared & Analyzed & Method & Notes \\
\hline
\end{tabular}

ERDC- EL-EP-C (Environmental Chemistry Branch)

DRAFT: Wet Chemistry

\begin{tabular}{llllllll}
\hline Hardness & $\mathbf{1 0 8}$ & 1.32 & $\begin{array}{c}\text { mg equil } \\
\text { CaCO3/ }\end{array}$ & 4 & 25-Jun-2009 & 25-Jun-2009
\end{tabular}

$\mathrm{L}$

DRAFT: Metals by EPA 6000/7000 Series Methods

\begin{tabular}{|c|c|c|c|c|c|c|c|c|}
\hline Mercury & 0.000395 & 0.000005 & $\mathrm{mg} / \mathrm{L}$ & 2 & 24-Jul-2009 & 24-Jul-2009 & EPA 7471A & \\
\hline Strontium & 0.637 & 0.0800 & $\mathrm{mg} / \mathrm{L}$ & 4 & 25-Jun-2009 & 25-Jun-2009 & EPA 6010B & \\
\hline Aluminum & 11.9 & 0.200 & $\mathrm{mg} / \mathrm{L}$ & 4 & 25-Jun-2009 & 25-Jun-2009 & SW 846/6010 & \\
\hline Calcium & 33.2 & 0.200 & $\mathrm{mg} / \mathrm{L}$ & 4 & 25-Jun-2009 & 25-Jun-2009 & SW 846/6010 & B \\
\hline Iron & 4.98 & 0.200 & $\mathrm{mg} / \mathrm{L}$ & 4 & 25-Jun-2009 & 25-Jun-2009 & SW 846/6010 & \\
\hline Magnesium & 6.03 & 0.200 & $\mathrm{mg} / \mathrm{L}$ & 4 & 25-Jun-2009 & 25-Jun-2009 & SW 846/6010 & \\
\hline Potassium & 4.09 & 0.200 & $\mathrm{mg} / \mathrm{L}$ & 4 & 25-Jun-2009 & 25-Jun-2009 & SW 846/6010 & B \\
\hline Sodium & 4.99 & 0.200 & $\mathrm{mg} / \mathrm{L}$ & 4 & 25-Jun-2009 & 25-Jun-2009 & SW 846/6010 & B \\
\hline Antimony & 0.0081 & 0.0020 & $\mathrm{mg} / \mathrm{L}$ & 4 & 17-Jun-2009 & 17-Jun-2009 & SW 846/6020 & \\
\hline Arsenic & 0.108 & 0.0020 & $\mathrm{mg} / \mathrm{L}$ & 4 & 17-Jun-2009 & 17-Jun-2009 & SW 846/6020 & \\
\hline Barium & 0.276 & 0.0020 & $\mathrm{mg} / \mathrm{L}$ & 4 & 17-Jun-2009 & 17-Jun-2009 & SW 846/6020 & B \\
\hline Beryllium & 0.0019 & 0.0020 & $\mathrm{mg} / \mathrm{L}$ & 4 & 17-Jun-2009 & 17-Jun-2009 & SW 846/6020 & J \\
\hline Cadmium & 0.00071 & 0.0020 & $\mathrm{mg} / \mathrm{L}$ & 4 & 17-Jun-2009 & 17-Jun-2009 & SW 846/6020 & J \\
\hline Chromium & 0.0179 & 0.0020 & $\mathrm{mg} / \mathrm{L}$ & 4 & 17-Jun-2009 & 17-Jun-2009 & SW 846/6020 & \\
\hline Cobalt & 0.0096 & 0.0020 & $\mathrm{mg} / \mathrm{L}$ & 4 & 17-Jun-2009 & 17-Jun-2009 & SW 846/6020 & \\
\hline Copper & 0.0358 & 0.0020 & $\mathrm{mg} / \mathrm{L}$ & 4 & 17-Jun-2009 & 17-Jun-2009 & SW 846/6020 & B \\
\hline Lead & 0.0173 & 0.0020 & $\mathrm{mg} / \mathrm{L}$ & 4 & 17-Jun-2009 & 17-Jun-2009 & SW 846/6020 & \\
\hline Manganese & 0.0917 & 0.0020 & $\mathrm{mg} / \mathrm{L}$ & 4 & 17-Jun-2009 & 17-Jun-2009 & SW 846/6020 & \\
\hline Molybdenum & 0.0770 & 0.0020 & $\mathrm{mg} / \mathrm{L}$ & 4 & 17-Jun-2009 & 17-Jun-2009 & SW 846/6020 & \\
\hline Nickel & 0.0222 & 0.0020 & $\mathrm{mg} / \mathrm{L}$ & 4 & 17-Jun-2009 & 17-Jun-2009 & SW 846/6020 & \\
\hline Selenium & 0.0037 & 0.0020 & $\mathrm{mg} / \mathrm{L}$ & 4 & 17-Jun-2009 & 17-Jun-2009 & SW 846/6020 & \\
\hline Silver & ND & 0.0020 & $\mathrm{mg} / \mathrm{L}$ & 4 & 17-Jun-2009 & 17-Jun-2009 & SW 846/6020 & $\mathrm{U}$ \\
\hline Thallium & 0.0015 & 0.0020 & $\mathrm{mg} / \mathrm{L}$ & 4 & 17-Jun-2009 & 17-Jun-2009 & SW 846/6020 & J \\
\hline Vanadium & 0.0999 & 0.0020 & $\mathrm{mg} / \mathrm{L}$ & 4 & 17-Jun-2009 & 17-Jun-2009 & SW 846/6020 & \\
\hline Zinc & 0.0648 & 0.0020 & $\mathrm{mg} / \mathrm{L}$ & 4 & 17-Jun-2009 & 17-Jun-2009 & SW 846/6020 & B \\
\hline
\end{tabular}




\section{USACE ERDC-EP-C \\ 3909 Halls Ferry Road \\ Vicksburg, MS 39180-6199}

ERDC - ECB

$-\cdot$,
Project: TVA Fly Ash Project

Project Manager: Tony Bednar
Reported:

03-Sep-2009

DRAFT: SLC MT N2 Hour 1 Rep E - Total Metals

9061502-71 (Water)

\begin{tabular}{|c|c|c|c|c|c|c|c|c|}
\hline Analyte & Result & $\begin{array}{r}\text { porting } \\
\text { Limit }\end{array}$ & Units & Dilution & Prepared & Analyzed & Method & Notes \\
\hline
\end{tabular}

ERDC- EL-EP-C (Environmental Chemistry Branch)

DRAFT: Wet Chemistry

\begin{tabular}{llllllll}
\hline Hardness & $\mathbf{1 0 8}$ & 1.32 & $\begin{array}{c}\text { mg equil } \\
\text { CaCO3/ }\end{array}$ & 4 & 25-Jun-2009 & 25-Jun-2009
\end{tabular}

DRAFT: Metals by EPA 6000/7000 Series Methods

\begin{tabular}{|c|c|c|c|c|c|c|c|c|}
\hline Mercury & 0.000266 & 0.000005 & $\mathrm{mg} / \mathrm{L}$ & 2 & 24-Jul-2009 & 24-Jul-2009 & EPA 7471A & \\
\hline Strontium & 0.629 & 0.0800 & $\mathrm{mg} / \mathrm{L}$ & 4 & 25-Jun-2009 & 25-Jun-2009 & EPA 6010B & \\
\hline Aluminum & 11.8 & 0.200 & $\mathrm{mg} / \mathrm{L}$ & 4 & 25-Jun-2009 & 25-Jun-2009 & SW 846/6010 & \\
\hline Calcium & 33.3 & 0.200 & $\mathrm{mg} / \mathrm{L}$ & 4 & 25-Jun-2009 & 25-Jun-2009 & SW 846/6010 & B \\
\hline Iron & 5.04 & 0.200 & $\mathrm{mg} / \mathrm{L}$ & 4 & 25-Jun-2009 & 25-Jun-2009 & SW 846/6010 & \\
\hline Magnesium & 5.96 & 0.200 & $\mathrm{mg} / \mathrm{L}$ & 4 & 25-Jun-2009 & 25-Jun-2009 & SW 846/6010 & \\
\hline Potassium & 4.10 & 0.200 & $\mathrm{mg} / \mathrm{L}$ & 4 & 25-Jun-2009 & 25-Jun-2009 & SW 846/6010 & B \\
\hline Sodium & 4.92 & 0.200 & $\mathrm{mg} / \mathrm{L}$ & 4 & 25-Jun-2009 & 25-Jun-2009 & SW 846/6010 & B \\
\hline Antimony & 0.0155 & 0.0020 & $\mathrm{mg} / \mathrm{L}$ & 4 & 17-Jun-2009 & 17-Jun-2009 & SW 846/6020 & \\
\hline Arsenic & 0.101 & 0.0020 & $\mathrm{mg} / \mathrm{L}$ & 4 & 17-Jun-2009 & 17-Jun-2009 & SW 846/6020 & \\
\hline Barium & 0.274 & 0.0020 & $\mathrm{mg} / \mathrm{L}$ & 4 & 17-Jun-2009 & 17-Jun-2009 & SW 846/6020 & B \\
\hline Beryllium & 0.0017 & 0.0020 & $\mathrm{mg} / \mathrm{L}$ & 4 & 17-Jun-2009 & 17-Jun-2009 & SW 846/6020 & J \\
\hline Cadmium & 0.0007 & 0.0020 & $\mathrm{mg} / \mathrm{L}$ & 4 & 17-Jun-2009 & 17-Jun-2009 & SW 846/6020 & J \\
\hline Chromium & 0.0171 & 0.0020 & $\mathrm{mg} / \mathrm{L}$ & 4 & 17-Jun-2009 & 17-Jun-2009 & SW 846/6020 & \\
\hline Cobalt & 0.0090 & 0.0020 & $\mathrm{mg} / \mathrm{L}$ & 4 & 17-Jun-2009 & 17-Jun-2009 & SW 846/6020 & \\
\hline Copper & 0.0337 & 0.0020 & $\mathrm{mg} / \mathrm{L}$ & 4 & 17-Jun-2009 & 17-Jun-2009 & SW 846/6020 & B \\
\hline Lead & 0.0181 & 0.0020 & $\mathrm{mg} / \mathrm{L}$ & 4 & 17-Jun-2009 & 17-Jun-2009 & SW 846/6020 & \\
\hline Manganese & 0.0850 & 0.0020 & $\mathrm{mg} / \mathrm{L}$ & 4 & 17-Jun-2009 & 17-Jun-2009 & SW 846/6020 & \\
\hline Molybdenum & 0.0758 & 0.0020 & $\mathrm{mg} / \mathrm{L}$ & 4 & 17-Jun-2009 & 17-Jun-2009 & SW 846/6020 & \\
\hline Nickel & 0.0203 & 0.0020 & $\mathrm{mg} / \mathrm{L}$ & 4 & 17-Jun-2009 & 17-Jun-2009 & SW 846/6020 & \\
\hline Selenium & 0.0047 & 0.0020 & $\mathrm{mg} / \mathrm{L}$ & 4 & 17-Jun-2009 & 17-Jun-2009 & SW 846/6020 & \\
\hline Silver & ND & 0.0020 & $\mathrm{mg} / \mathrm{L}$ & 4 & 17-Jun-2009 & 17-Jun-2009 & SW 846/6020 & $\mathrm{U}$ \\
\hline Thallium & 0.0016 & 0.0020 & $\mathrm{mg} / \mathrm{L}$ & 4 & 17-Jun-2009 & 17-Jun-2009 & SW 846/6020 & $\mathrm{J}$ \\
\hline Vanadium & 0.0950 & 0.0020 & $\mathrm{mg} / \mathrm{L}$ & 4 & 17-Jun-2009 & 17-Jun-2009 & SW 846/6020 & \\
\hline Zinc & 0.0725 & 0.0020 & $\mathrm{mg} / \mathrm{L}$ & 4 & 17-Jun-2009 & 17-Jun-2009 & SW 846/6020 & B \\
\hline
\end{tabular}




\section{USACE ERDC-EP-C \\ 3909 Halls Ferry Road \\ Vicksburg, MS 39180-6199}

ERDC - ECB

$--,-$
Project: TVA Fly Ash Project

Project Manager: Tony Bednar
Reported:

03-Sep-2009

DRAFT: SLC MT N2 Hour 1 Rep F - Total Metals

9061502-72 (Water)

\begin{tabular}{|c|c|c|c|c|c|c|c|c|}
\hline Analyte & Result & $\begin{array}{r}\text { porting } \\
\text { Limit }\end{array}$ & Units & Dilution & Prepared & Analyzed & Method & Notes \\
\hline
\end{tabular}

ERDC- EL-EP-C (Environmental Chemistry Branch)

DRAFT: Wet Chemistry

\begin{tabular}{llllllll}
\hline Hardness & $\mathbf{1 0 5}$ & 1.32 & $\begin{array}{c}\text { mg equil } \\
\text { CaCO3/ }\end{array}$ & 4 & 25-Jun-2009 & 25-Jun-2009
\end{tabular}

$\mathrm{L}$

DRAFT: Metals by EPA 6000/7000 Series Methods

\begin{tabular}{|c|c|c|c|c|c|c|c|c|}
\hline Mercury & 0.000259 & 0.000005 & $\mathrm{mg} / \mathrm{L}$ & 2 & 24-Jul-2009 & 24-Jul-2009 & EPA 7471A & \\
\hline Strontium & 0.617 & 0.0800 & $\mathrm{mg} / \mathrm{L}$ & 4 & 25-Jun-2009 & 25-Jun-2009 & EPA 6010B & \\
\hline Aluminum & 11.0 & 0.200 & $\mathrm{mg} / \mathrm{L}$ & 4 & 25-Jun-2009 & 25-Jun-2009 & SW 846/6010 & \\
\hline Calcium & 32.4 & 0.200 & $\mathrm{mg} / \mathrm{L}$ & 4 & 25-Jun-2009 & 25-Jun-2009 & SW 846/6010 & B \\
\hline Iron & 4.66 & 0.200 & $\mathrm{mg} / \mathrm{L}$ & 4 & 25-Jun-2009 & 25-Jun-2009 & SW 846/6010 & \\
\hline Magnesium & 5.84 & 0.200 & $\mathrm{mg} / \mathrm{L}$ & 4 & 25-Jun-2009 & 25-Jun-2009 & SW 846/6010 & \\
\hline Potassium & 3.97 & 0.200 & $\mathrm{mg} / \mathrm{L}$ & 4 & 25-Jun-2009 & 25-Jun-2009 & SW 846/6010 & B \\
\hline Sodium & 4.57 & 0.200 & $\mathrm{mg} / \mathrm{L}$ & 4 & 25-Jun-2009 & 25-Jun-2009 & SW 846/6010 & B \\
\hline Antimony & 0.0079 & 0.0020 & $\mathrm{mg} / \mathrm{L}$ & 4 & 17-Jun-2009 & 17-Jun-2009 & SW 846/6020 & \\
\hline Arsenic & 0.105 & 0.0020 & $\mathrm{mg} / \mathrm{L}$ & 4 & 17-Jun-2009 & 17-Jun-2009 & SW 846/6020 & \\
\hline Barium & 0.263 & 0.0020 & $\mathrm{mg} / \mathrm{L}$ & 4 & 17-Jun-2009 & 17-Jun-2009 & SW 846/6020 & B \\
\hline Beryllium & 0.0019 & 0.0020 & $\mathrm{mg} / \mathrm{L}$ & 4 & 17-Jun-2009 & 17-Jun-2009 & SW 846/6020 & J \\
\hline Cadmium & 0.0006 & 0.0020 & $\mathrm{mg} / \mathrm{L}$ & 4 & 17-Jun-2009 & 17-Jun-2009 & SW 846/6020 & J \\
\hline Chromium & 0.0161 & 0.0020 & $\mathrm{mg} / \mathrm{L}$ & 4 & 17-Jun-2009 & 17-Jun-2009 & SW 846/6020 & \\
\hline Cobalt & 0.0085 & 0.0020 & $\mathrm{mg} / \mathrm{L}$ & 4 & 17-Jun-2009 & 17-Jun-2009 & SW 846/6020 & \\
\hline Copper & 0.0315 & 0.0020 & $\mathrm{mg} / \mathrm{L}$ & 4 & 17-Jun-2009 & 17-Jun-2009 & SW 846/6020 & B \\
\hline Lead & 0.0162 & 0.0020 & $\mathrm{mg} / \mathrm{L}$ & 4 & 17-Jun-2009 & 17-Jun-2009 & SW 846/6020 & \\
\hline Manganese & 0.0842 & 0.0020 & $\mathrm{mg} / \mathrm{L}$ & 4 & 17-Jun-2009 & 17-Jun-2009 & SW 846/6020 & \\
\hline Molybdenum & 0.0752 & 0.0020 & $\mathrm{mg} / \mathrm{L}$ & 4 & 17-Jun-2009 & 17-Jun-2009 & SW 846/6020 & \\
\hline Nickel & 0.0188 & 0.0020 & $\mathrm{mg} / \mathrm{L}$ & 4 & 17-Jun-2009 & 17-Jun-2009 & SW 846/6020 & \\
\hline Selenium & 0.0039 & 0.0020 & $\mathrm{mg} / \mathrm{L}$ & 4 & 17-Jun-2009 & 17-Jun-2009 & SW 846/6020 & \\
\hline Silver & ND & 0.0020 & $\mathrm{mg} / \mathrm{L}$ & 4 & 17-Jun-2009 & 17-Jun-2009 & SW 846/6020 & $\mathrm{U}$ \\
\hline Thallium & 0.0014 & 0.0020 & $\mathrm{mg} / \mathrm{L}$ & 4 & 17-Jun-2009 & 17-Jun-2009 & SW 846/6020 & $\mathrm{J}$ \\
\hline Vanadium & 0.0982 & 0.0020 & $\mathrm{mg} / \mathrm{L}$ & 4 & 17-Jun-2009 & 17-Jun-2009 & SW 846/6020 & \\
\hline Zinc & 0.0461 & 0.0020 & $\mathrm{mg} / \mathrm{L}$ & 4 & 17-Jun-2009 & 17-Jun-2009 & SW 846/6020 & B \\
\hline
\end{tabular}




\section{USACE ERDC-EP-C \\ 3909 Halls Ferry Road \\ Vicksburg, MS 39180-6199}

ERDC - ECB

$-\cdot$,
Project: TVA Fly Ash Project

Project Manager: Tony Bednar
Reported:

03-Sep-2009

DRAFT: SLC MT O2 Hour 1 Rep D - Total Metals

9061502-73 (Water)

\begin{tabular}{|c|c|c|c|c|c|c|c|c|}
\hline Analyte & Result & $\begin{array}{r}\text { porting } \\
\text { Limit }\end{array}$ & Units & Dilution & Prepared & Analyzed & Method & Notes \\
\hline
\end{tabular}

ERDC- EL-EP-C (Environmental Chemistry Branch)

DRAFT: Wet Chemistry

\begin{tabular}{llllllll}
\hline Hardness & $\mathbf{1 0 5}$ & 1.32 & $\begin{array}{c}\text { mg equil } \\
\text { CaCO3/ }\end{array}$ & 4 & 25-Jun-2009 & 25-Jun-2009
\end{tabular}

$\mathrm{L}$

DRAFT: Metals by EPA 6000/7000 Series Methods

\begin{tabular}{|c|c|c|c|c|c|c|c|c|}
\hline Mercury & 0.0001 & 0.000005 & $\mathrm{mg} / \mathrm{L}$ & 2 & 24-Jul-2009 & 24-Jul-2009 & EPA 7471A & \\
\hline Strontium & 0.616 & 0.0800 & $\mathrm{mg} / \mathrm{L}$ & 4 & 25-Jun-2009 & 25-Jun-2009 & EPA 6010B & \\
\hline Aluminum & 10.5 & 0.200 & $\mathrm{mg} / \mathrm{L}$ & 4 & 25-Jun-2009 & 25-Jun-2009 & SW 846/6010 & \\
\hline Calcium & 32.4 & 0.200 & $\mathrm{mg} / \mathrm{L}$ & 4 & 25-Jun-2009 & 25-Jun-2009 & SW 846/6010 & B \\
\hline Iron & 4.32 & 0.200 & $\mathrm{mg} / \mathrm{L}$ & 4 & 25-Jun-2009 & 25-Jun-2009 & SW 846/6010 & \\
\hline Magnesium & 5.85 & 0.200 & $\mathrm{mg} / \mathrm{L}$ & 4 & 25-Jun-2009 & 25-Jun-2009 & SW 846/6010 & \\
\hline Potassium & 4.00 & 0.200 & $\mathrm{mg} / \mathrm{L}$ & 4 & 25-Jun-2009 & 25-Jun-2009 & SW 846/6010 & B \\
\hline Sodium & 4.86 & 0.200 & $\mathrm{mg} / \mathrm{L}$ & 4 & 25-Jun-2009 & 25-Jun-2009 & SW 846/6010 & B \\
\hline Antimony & 0.0084 & 0.0020 & $\mathrm{mg} / \mathrm{L}$ & 4 & 17-Jun-2009 & 17-Jun-2009 & SW 846/6020 & \\
\hline Arsenic & 0.0995 & 0.0020 & $\mathrm{mg} / \mathrm{L}$ & 4 & 17-Jun-2009 & 17-Jun-2009 & SW 846/6020 & \\
\hline Barium & 0.252 & 0.0020 & $\mathrm{mg} / \mathrm{L}$ & 4 & 17-Jun-2009 & 17-Jun-2009 & SW 846/6020 & B \\
\hline Beryllium & 0.0017 & 0.0020 & $\mathrm{mg} / \mathrm{L}$ & 4 & 17-Jun-2009 & 17-Jun-2009 & SW 846/6020 & $\mathrm{J}$ \\
\hline Cadmium & 0.0007 & 0.0020 & $\mathrm{mg} / \mathrm{L}$ & 4 & 17-Jun-2009 & 17-Jun-2009 & SW 846/6020 & J \\
\hline Chromium & 0.0166 & 0.0020 & $\mathrm{mg} / \mathrm{L}$ & 4 & 17-Jun-2009 & 17-Jun-2009 & SW 846/6020 & \\
\hline Cobalt & 0.0080 & 0.0020 & $\mathrm{mg} / \mathrm{L}$ & 4 & 17-Jun-2009 & 17-Jun-2009 & SW 846/6020 & \\
\hline Copper & 0.0305 & 0.0020 & $\mathrm{mg} / \mathrm{L}$ & 4 & 17-Jun-2009 & 17-Jun-2009 & SW 846/6020 & B \\
\hline Lead & 0.0154 & 0.0020 & $\mathrm{mg} / \mathrm{L}$ & 4 & 17-Jun-2009 & 17-Jun-2009 & SW 846/6020 & \\
\hline Manganese & 0.0804 & 0.0020 & $\mathrm{mg} / \mathrm{L}$ & 4 & 17-Jun-2009 & 17-Jun-2009 & SW 846/6020 & \\
\hline Molybdenum & 0.0765 & 0.0020 & $\mathrm{mg} / \mathrm{L}$ & 4 & 17-Jun-2009 & 17-Jun-2009 & SW 846/6020 & \\
\hline Nickel & 0.0192 & 0.0020 & $\mathrm{mg} / \mathrm{L}$ & 4 & 17-Jun-2009 & 17-Jun-2009 & SW 846/6020 & \\
\hline Selenium & 0.0044 & 0.0020 & $\mathrm{mg} / \mathrm{L}$ & 4 & 17-Jun-2009 & 17-Jun-2009 & SW 846/6020 & \\
\hline Silver & ND & 0.0020 & $\mathrm{mg} / \mathrm{L}$ & 4 & 17-Jun-2009 & 17-Jun-2009 & SW 846/6020 & $\mathrm{U}$ \\
\hline Thallium & 0.0014 & 0.0020 & $\mathrm{mg} / \mathrm{L}$ & 4 & 17-Jun-2009 & 17-Jun-2009 & SW 846/6020 & J \\
\hline Vanadium & 0.0937 & 0.0020 & $\mathrm{mg} / \mathrm{L}$ & 4 & 17-Jun-2009 & 17-Jun-2009 & SW 846/6020 & \\
\hline Zinc & 0.0487 & 0.0020 & $\mathrm{mg} / \mathrm{L}$ & 4 & 17-Jun-2009 & 17-Jun-2009 & SW 846/6020 & B \\
\hline
\end{tabular}




\section{USACE ERDC-EP-C \\ 3909 Halls Ferry Road \\ Vicksburg, MS 39180-6199}

ERDC - ECB

$-\cdot$,
Project: TVA Fly Ash Project

Project Manager: Tony Bednar
Reported:

03-Sep-2009

DRAFT: SLC MT O2 Hour 1 Rep E - Total Metals

9061502-74 (Water)

\begin{tabular}{|c|c|c|c|c|c|c|c|c|}
\hline Analyte & Result & $\begin{array}{r}\text { porting } \\
\text { Limit }\end{array}$ & Units & Dilution & Prepared & Analyzed & Method & Notes \\
\hline
\end{tabular}

ERDC- EL-EP-C (Environmental Chemistry Branch)

DRAFT: Wet Chemistry

\begin{tabular}{llllllll}
\hline Hardness & $\mathbf{1 0 5}$ & 1.32 & $\begin{array}{c}\text { mg equil } \\
\text { CaCO3/ }\end{array}$ & 4 & 25-Jun-2009 & 25-Jun-2009
\end{tabular}

$\mathrm{L}$

DRAFT: Metals by EPA 6000/7000 Series Methods

\begin{tabular}{|c|c|c|c|c|c|c|c|c|}
\hline Mercury & 0.000308 & 0.000005 & $\mathrm{mg} / \mathrm{L}$ & 2 & 24-Jul-2009 & 24-Jul-2009 & EPA 7471A & \\
\hline Strontium & 0.628 & 0.0800 & $\mathrm{mg} / \mathrm{L}$ & 4 & 25-Jun-2009 & 25-Jun-2009 & EPA $6010 B$ & \\
\hline Aluminum & 13.3 & 0.200 & $\mathrm{mg} / \mathrm{L}$ & 4 & 25-Jun-2009 & 25-Jun-2009 & SW 846/6010 & \\
\hline Calcium & 32.2 & 0.200 & $\mathrm{mg} / \mathrm{L}$ & 4 & 25-Jun-2009 & 25-Jun-2009 & SW 846/6010 & B \\
\hline Iron & 5.60 & 0.200 & $\mathrm{mg} / \mathrm{L}$ & 4 & 25-Jun-2009 & 25-Jun-2009 & SW 846/6010 & \\
\hline Magnesium & 6.02 & 0.200 & $\mathrm{mg} / \mathrm{L}$ & 4 & 25-Jun-2009 & 25-Jun-2009 & SW 846/6010 & \\
\hline Potassium & 4.61 & 0.200 & $\mathrm{mg} / \mathrm{L}$ & 4 & 25-Jun-2009 & 25-Jun-2009 & SW 846/6010 & B \\
\hline Sodium & 4.65 & 0.200 & $\mathrm{mg} / \mathrm{L}$ & 4 & 25-Jun-2009 & 25-Jun-2009 & SW 846/6010 & B \\
\hline Antimony & 0.0084 & 0.0020 & $\mathrm{mg} / \mathrm{L}$ & 4 & 17-Jun-2009 & 17-Jun-2009 & SW 846/6020 & \\
\hline Arsenic & 0.109 & 0.0020 & $\mathrm{mg} / \mathrm{L}$ & 4 & 17-Jun-2009 & 17-Jun-2009 & SW 846/6020 & \\
\hline Barium & 0.295 & 0.0020 & $\mathrm{mg} / \mathrm{L}$ & 4 & 17-Jun-2009 & 17-Jun-2009 & SW 846/6020 & B \\
\hline Beryllium & 0.0022 & 0.0020 & $\mathrm{mg} / \mathrm{L}$ & 4 & 17-Jun-2009 & 17-Jun-2009 & SW 846/6020 & \\
\hline Cadmium & 0.0008 & 0.0020 & $\mathrm{mg} / \mathrm{L}$ & 4 & 17-Jun-2009 & 17-Jun-2009 & SW 846/6020 & $\mathrm{J}$ \\
\hline Chromium & 0.0194 & 0.0020 & $\mathrm{mg} / \mathrm{L}$ & 4 & 17-Jun-2009 & 17-Jun-2009 & SW 846/6020 & \\
\hline Cobalt & 0.0101 & 0.0020 & $\mathrm{mg} / \mathrm{L}$ & 4 & 17-Jun-2009 & 17-Jun-2009 & SW 846/6020 & \\
\hline Copper & 0.0379 & 0.0020 & $\mathrm{mg} / \mathrm{L}$ & 4 & 17-Jun-2009 & 17-Jun-2009 & SW 846/6020 & B \\
\hline Lead & 0.0200 & 0.0020 & $\mathrm{mg} / \mathrm{L}$ & 4 & 17-Jun-2009 & 17-Jun-2009 & SW 846/6020 & \\
\hline Manganese & 0.0895 & 0.0020 & $\mathrm{mg} / \mathrm{L}$ & 4 & 17-Jun-2009 & 17-Jun-2009 & SW 846/6020 & \\
\hline Molybdenum & 0.0745 & 0.0020 & $\mathrm{mg} / \mathrm{L}$ & 4 & 17-Jun-2009 & 17-Jun-2009 & SW 846/6020 & \\
\hline Nickel & 0.0231 & 0.0020 & $\mathrm{mg} / \mathrm{L}$ & 4 & 17-Jun-2009 & 17-Jun-2009 & SW 846/6020 & \\
\hline Selenium & 0.0054 & 0.0020 & $\mathrm{mg} / \mathrm{L}$ & 4 & 17-Jun-2009 & 17-Jun-2009 & SW 846/6020 & \\
\hline Silver & ND & 0.0020 & $\mathrm{mg} / \mathrm{L}$ & 4 & 17-Jun-2009 & 17-Jun-2009 & SW 846/6020 & $\mathrm{U}$ \\
\hline Thallium & 0.0016 & 0.0020 & $\mathrm{mg} / \mathrm{L}$ & 4 & 17-Jun-2009 & 17-Jun-2009 & SW 846/6020 & $\mathrm{J}$ \\
\hline Vanadium & 0.104 & 0.0020 & $\mathrm{mg} / \mathrm{L}$ & 4 & 17-Jun-2009 & 17-Jun-2009 & SW 846/6020 & \\
\hline Zinc & 0.0520 & 0.0020 & $\mathrm{mg} / \mathrm{L}$ & 4 & 17-Jun-2009 & 17-Jun-2009 & SW 846/6020 & B \\
\hline
\end{tabular}




\section{USACE ERDC-EP-C \\ 3909 Halls Ferry Road \\ Vicksburg, MS 39180-6199}

ERDC - ECB

$-\cdot$,
Project: TVA Fly Ash Project

Project Manager: Tony Bednar
Reported:

03-Sep-2009

DRAFT: SLC MT O2 Hour 1 Rep F - Total Metals

9061502-75 (Water)

\begin{tabular}{|c|c|c|c|c|c|c|c|c|}
\hline Analyte & Result & $\begin{array}{r}\text { porting } \\
\text { Limit }\end{array}$ & Units & Dilution & Prepared & Analyzed & Method & Notes \\
\hline
\end{tabular}

ERDC- EL-EP-C (Environmental Chemistry Branch)

DRAFT: Wet Chemistry

\begin{tabular}{llllllll}
\hline Hardness & $\mathbf{1 0 8}$ & 1.32 & $\begin{array}{c}\text { mg equil } \\
\text { CaCO3/ }\end{array}$ & 4 & 25-Jun-2009 & 25-Jun-2009
\end{tabular}

$\mathrm{L}$

DRAFT: Metals by EPA 6000/7000 Series Methods

\begin{tabular}{|c|c|c|c|c|c|c|c|c|}
\hline Mercury & 0.000218 & 0.000005 & $\mathrm{mg} / \mathrm{L}$ & 2 & 24-Jul-2009 & 24-Jul-2009 & EPA 7471A & \\
\hline Strontium & 0.651 & 0.0800 & $\mathrm{mg} / \mathrm{L}$ & 4 & 25-Jun-2009 & 25-Jun-2009 & EPA 6010B & \\
\hline Aluminum & 13.9 & 0.200 & $\mathrm{mg} / \mathrm{L}$ & 4 & 25-Jun-2009 & 25-Jun-2009 & SW 846/6010 & \\
\hline Calcium & 33.2 & 0.200 & $\mathrm{mg} / \mathrm{L}$ & 4 & 25-Jun-2009 & 25-Jun-2009 & SW 846/6010 & B \\
\hline Iron & 5.92 & 0.200 & $\mathrm{mg} / \mathrm{L}$ & 4 & 25-Jun-2009 & 25-Jun-2009 & SW 846/6010 & \\
\hline Magnesium & 6.16 & 0.200 & $\mathrm{mg} / \mathrm{L}$ & 4 & 25-Jun-2009 & 25-Jun-2009 & SW 846/6010 & \\
\hline Potassium & 4.87 & 0.200 & $\mathrm{mg} / \mathrm{L}$ & 4 & 25-Jun-2009 & 25-Jun-2009 & SW 846/6010 & B \\
\hline Sodium & 4.85 & 0.200 & $\mathrm{mg} / \mathrm{L}$ & 4 & 25-Jun-2009 & 25-Jun-2009 & SW 846/6010 & B \\
\hline Antimony & 0.0090 & 0.0020 & $\mathrm{mg} / \mathrm{L}$ & 4 & 17-Jun-2009 & 17-Jun-2009 & SW 846/6020 & \\
\hline Arsenic & 0.112 & 0.0020 & $\mathrm{mg} / \mathrm{L}$ & 4 & 17-Jun-2009 & 17-Jun-2009 & SW 846/6020 & \\
\hline Barium & 0.309 & 0.0020 & $\mathrm{mg} / \mathrm{L}$ & 4 & 17-Jun-2009 & 17-Jun-2009 & SW 846/6020 & B \\
\hline Beryllium & 0.0022 & 0.0020 & $\mathrm{mg} / \mathrm{L}$ & 4 & 17-Jun-2009 & 17-Jun-2009 & SW 846/6020 & \\
\hline Cadmium & 0.0009 & 0.0020 & $\mathrm{mg} / \mathrm{L}$ & 4 & 17-Jun-2009 & 17-Jun-2009 & SW 846/6020 & J \\
\hline Chromium & 0.0198 & 0.0020 & $\mathrm{mg} / \mathrm{L}$ & 4 & 17-Jun-2009 & 17-Jun-2009 & SW 846/6020 & \\
\hline Cobalt & 0.0107 & 0.0020 & $\mathrm{mg} / \mathrm{L}$ & 4 & 17-Jun-2009 & 17-Jun-2009 & SW 846/6020 & \\
\hline Copper & 0.0382 & 0.0020 & $\mathrm{mg} / \mathrm{L}$ & 4 & 17-Jun-2009 & 17-Jun-2009 & SW 846/6020 & B \\
\hline Lead & 0.0211 & 0.0020 & $\mathrm{mg} / \mathrm{L}$ & 4 & 17-Jun-2009 & 17-Jun-2009 & SW 846/6020 & \\
\hline Manganese & 0.0978 & 0.0020 & $\mathrm{mg} / \mathrm{L}$ & 4 & 17-Jun-2009 & 17-Jun-2009 & SW 846/6020 & \\
\hline Molybdenum & 0.0749 & 0.0020 & $\mathrm{mg} / \mathrm{L}$ & 4 & 17-Jun-2009 & 17-Jun-2009 & SW 846/6020 & \\
\hline Nickel & 0.0235 & 0.0020 & $\mathrm{mg} / \mathrm{L}$ & 4 & 17-Jun-2009 & 17-Jun-2009 & SW 846/6020 & \\
\hline Selenium & 0.0055 & 0.0020 & $\mathrm{mg} / \mathrm{L}$ & 4 & 17-Jun-2009 & 17-Jun-2009 & SW 846/6020 & \\
\hline Silver & ND & 0.0020 & $\mathrm{mg} / \mathrm{L}$ & 4 & 17-Jun-2009 & 17-Jun-2009 & SW 846/6020 & $\mathrm{U}$ \\
\hline Thallium & 0.0017 & 0.0020 & $\mathrm{mg} / \mathrm{L}$ & 4 & 17-Jun-2009 & 17-Jun-2009 & SW 846/6020 & J \\
\hline Vanadium & 0.107 & 0.0020 & $\mathrm{mg} / \mathrm{L}$ & 4 & 17-Jun-2009 & 17-Jun-2009 & SW 846/6020 & \\
\hline Zinc & 0.0533 & 0.0020 & $\mathrm{mg} / \mathrm{L}$ & 4 & 17-Jun-2009 & 17-Jun-2009 & SW 846/6020 & B \\
\hline
\end{tabular}




\section{USACE ERDC-EP-C \\ 3909 Halls Ferry Road \\ Vicksburg, MS 39180-6199}

ERDC - ECB

$-\cdot$,
Project: TVA Fly Ash Project

Project Manager: Tony Bednar
Reported:

03-Sep-2009

DRAFT: EMR MT N2 Hour 24 Rep A - Total Metals

9061502-76 (Water)

\begin{tabular}{|c|c|c|c|c|c|c|c|c|}
\hline Analyte & Result & $\begin{array}{r}\text { porting } \\
\text { Limit }\end{array}$ & Units & Dilution & Prepared & Analyzed & Method & Notes \\
\hline
\end{tabular}

ERDC- EL-EP-C (Environmental Chemistry Branch)

DRAFT: Wet Chemistry

\begin{tabular}{llllllll}
\hline Hardness & $\mathbf{5 7 . 4}$ & 1.32 & $\begin{array}{c}\text { mg equil } \\
\text { CaCO3/ }\end{array}$ & 4 & 25-Jun-2009 & 25-Jun-2009
\end{tabular}

$\mathrm{L}$

DRAFT: Metals by EPA 6000/7000 Series Methods

\begin{tabular}{|c|c|c|c|c|c|c|c|c|}
\hline Mercury & 0.000445 & 0.000005 & $\mathrm{mg} / \mathrm{L}$ & 2 & 24-Jul-2009 & 24-Jul-2009 & EPA 7471A & \\
\hline Strontium & 0.359 & 0.0800 & $\mathrm{mg} / \mathrm{L}$ & 4 & 25-Jun-2009 & 25-Jun-2009 & EPA 6010B & \\
\hline Aluminum & 23.3 & 0.200 & $\mathrm{mg} / \mathrm{L}$ & 4 & 25-Jun-2009 & 25-Jun-2009 & SW 846/6010 & \\
\hline Calcium & 16.0 & 0.200 & $\mathrm{mg} / \mathrm{L}$ & 4 & 25-Jun-2009 & 25-Jun-2009 & SW 846/6010 & B \\
\hline Iron & 9.51 & 0.200 & $\mathrm{mg} / \mathrm{L}$ & 4 & 25-Jun-2009 & 25-Jun-2009 & SW 846/6010 & \\
\hline Magnesium & 4.21 & 0.200 & $\mathrm{mg} / \mathrm{L}$ & 4 & 25-Jun-2009 & 25-Jun-2009 & SW 846/6010 & \\
\hline Potassium & 5.50 & 0.200 & $\mathrm{mg} / \mathrm{L}$ & 4 & 25-Jun-2009 & 25-Jun-2009 & SW 846/6010 & B \\
\hline Sodium & 3.49 & 0.200 & $\mathrm{mg} / \mathrm{L}$ & 4 & 25-Jun-2009 & 25-Jun-2009 & SW 846/6010 & B \\
\hline Antimony & 0.0041 & 0.0020 & $\mathrm{mg} / \mathrm{L}$ & 4 & 17-Jun-2009 & 17-Jun-2009 & SW 846/6020 & \\
\hline Arsenic & 0.106 & 0.0020 & $\mathrm{mg} / \mathrm{L}$ & 4 & 17-Jun-2009 & 17-Jun-2009 & SW 846/6020 & \\
\hline Barium & 0.513 & 0.0020 & $\mathrm{mg} / \mathrm{L}$ & 4 & 17-Jun-2009 & 17-Jun-2009 & SW 846/6020 & B \\
\hline Beryllium & 0.0042 & 0.0020 & $\mathrm{mg} / \mathrm{L}$ & 4 & 17-Jun-2009 & 17-Jun-2009 & SW 846/6020 & \\
\hline Cadmium & 0.0010 & 0.0020 & $\mathrm{mg} / \mathrm{L}$ & 4 & 17-Jun-2009 & 17-Jun-2009 & SW 846/6020 & J \\
\hline Chromium & 0.0338 & 0.0020 & $\mathrm{mg} / \mathrm{L}$ & 4 & 17-Jun-2009 & 17-Jun-2009 & SW 846/6020 & \\
\hline Cobalt & 0.0188 & 0.0020 & $\mathrm{mg} / \mathrm{L}$ & 4 & 17-Jun-2009 & 17-Jun-2009 & SW 846/6020 & \\
\hline Copper & 0.0675 & 0.0020 & $\mathrm{mg} / \mathrm{L}$ & 4 & 17-Jun-2009 & 17-Jun-2009 & SW 846/6020 & B \\
\hline Lead & 0.0380 & 0.0020 & $\mathrm{mg} / \mathrm{L}$ & 4 & 17-Jun-2009 & 17-Jun-2009 & SW 846/6020 & \\
\hline Manganese & 0.104 & 0.0020 & $\mathrm{mg} / \mathrm{L}$ & 4 & 17-Jun-2009 & 17-Jun-2009 & SW 846/6020 & \\
\hline Molybdenum & 0.0055 & 0.0020 & $\mathrm{mg} / \mathrm{L}$ & 4 & 17-Jun-2009 & 17-Jun-2009 & SW 846/6020 & \\
\hline Nickel & 0.0377 & 0.0020 & $\mathrm{mg} / \mathrm{L}$ & 4 & 17-Jun-2009 & 17-Jun-2009 & SW 846/6020 & \\
\hline Selenium & 0.0044 & 0.0020 & $\mathrm{mg} / \mathrm{L}$ & 4 & 17-Jun-2009 & 17-Jun-2009 & SW 846/6020 & \\
\hline Silver & ND & 0.0020 & $\mathrm{mg} / \mathrm{L}$ & 4 & 17-Jun-2009 & 17-Jun-2009 & SW 846/6020 & $\mathrm{U}$ \\
\hline Thallium & 0.0025 & 0.0020 & $\mathrm{mg} / \mathrm{L}$ & 4 & 17-Jun-2009 & 17-Jun-2009 & SW 846/6020 & \\
\hline Vanadium & 0.110 & 0.0020 & $\mathrm{mg} / \mathrm{L}$ & 4 & 17-Jun-2009 & 17-Jun-2009 & SW 846/6020 & \\
\hline Zinc & 0.0917 & 0.0020 & $\mathrm{mg} / \mathrm{L}$ & 4 & 17-Jun-2009 & 17-Jun-2009 & SW 846/6020 & B \\
\hline
\end{tabular}




\section{USACE ERDC-EP-C \\ 3909 Halls Ferry Road \\ Vicksburg, MS 39180-6199}

ERDC - ECB

$-\cdot$,
Project: TVA Fly Ash Project

Project Manager: Tony Bednar
Reported:

03-Sep-2009

DRAFT: EMR MT N2 Hour 24 Rep B - Total Metals

9061502-77 (Water)

\begin{tabular}{|c|c|c|c|c|c|c|c|c|}
\hline Analyte & Result & $\begin{array}{r}\text { porting } \\
\text { Limit }\end{array}$ & Units & Dilution & Prepared & Analyzed & Method & Notes \\
\hline
\end{tabular}

ERDC- EL-EP-C (Environmental Chemistry Branch)

DRAFT: Wet Chemistry

\begin{tabular}{llllllll}
\hline Hardness & $\mathbf{5 5 . 6}$ & 1.32 & $\begin{array}{c}\text { mg equil } \\
\text { CaCO3/ }\end{array}$ & 4 & 25-Jun-2009 & 25-Jun-2009
\end{tabular}

$\mathrm{L}$

DRAFT: Metals by EPA 6000/7000 Series Methods

\begin{tabular}{|c|c|c|c|c|c|c|c|c|}
\hline Mercury & 0.000412 & 0.000005 & $\mathrm{mg} / \mathrm{L}$ & 2 & 24-Jul-2009 & 24-Jul-2009 & EPA 7471A & \\
\hline Strontium & 0.337 & 0.0800 & $\mathrm{mg} / \mathrm{L}$ & 4 & 25-Jun-2009 & 25-Jun-2009 & EPA 6010B & \\
\hline Aluminum & 22.4 & 0.200 & $\mathrm{mg} / \mathrm{L}$ & 4 & 25-Jun-2009 & 25-Jun-2009 & SW 846/6010 & \\
\hline Calcium & 15.4 & 0.200 & $\mathrm{mg} / \mathrm{L}$ & 4 & 25-Jun-2009 & 25-Jun-2009 & SW 846/6010 & B \\
\hline Iron & 9.20 & 0.200 & $\mathrm{mg} / \mathrm{L}$ & 4 & 25-Jun-2009 & 25-Jun-2009 & SW 846/6010 & \\
\hline Magnesium & 4.16 & 0.200 & $\mathrm{mg} / \mathrm{L}$ & 4 & 25-Jun-2009 & 25-Jun-2009 & SW 846/6010 & \\
\hline Potassium & 5.69 & 0.200 & $\mathrm{mg} / \mathrm{L}$ & 4 & 25-Jun-2009 & 25-Jun-2009 & SW 846/6010 & B \\
\hline Sodium & 3.56 & 0.200 & $\mathrm{mg} / \mathrm{L}$ & 4 & 25-Jun-2009 & 25-Jun-2009 & SW 846/6010 & B \\
\hline Antimony & 0.0076 & 0.0020 & $\mathrm{mg} / \mathrm{L}$ & 4 & 17-Jun-2009 & 17-Jun-2009 & SW 846/6020 & \\
\hline Arsenic & 0.0986 & 0.0020 & $\mathrm{mg} / \mathrm{L}$ & 4 & 17-Jun-2009 & 17-Jun-2009 & SW 846/6020 & \\
\hline Barium & 0.476 & 0.0020 & $\mathrm{mg} / \mathrm{L}$ & 4 & 17-Jun-2009 & 17-Jun-2009 & SW 846/6020 & B \\
\hline Beryllium & 0.0039 & 0.0020 & $\mathrm{mg} / \mathrm{L}$ & 4 & 17-Jun-2009 & 17-Jun-2009 & SW 846/6020 & \\
\hline Cadmium & 0.0007 & 0.0020 & $\mathrm{mg} / \mathrm{L}$ & 4 & 17-Jun-2009 & 17-Jun-2009 & SW 846/6020 & $\mathrm{J}$ \\
\hline Chromium & 0.0299 & 0.0020 & $\mathrm{mg} / \mathrm{L}$ & 4 & 17-Jun-2009 & 17-Jun-2009 & SW 846/6020 & \\
\hline Cobalt & 0.0169 & 0.0020 & $\mathrm{mg} / \mathrm{L}$ & 4 & 17-Jun-2009 & 17-Jun-2009 & SW 846/6020 & \\
\hline Copper & 0.0635 & 0.0020 & $\mathrm{mg} / \mathrm{L}$ & 4 & 17-Jun-2009 & 17-Jun-2009 & SW 846/6020 & B \\
\hline Lead & 0.0342 & 0.0020 & $\mathrm{mg} / \mathrm{L}$ & 4 & 17-Jun-2009 & 17-Jun-2009 & SW 846/6020 & \\
\hline Manganese & 0.0953 & 0.0020 & $\mathrm{mg} / \mathrm{L}$ & 4 & 17-Jun-2009 & 17-Jun-2009 & SW 846/6020 & \\
\hline Molybdenum & 0.0054 & 0.0020 & $\mathrm{mg} / \mathrm{L}$ & 4 & 17-Jun-2009 & 17-Jun-2009 & SW 846/6020 & \\
\hline Nickel & 0.0331 & 0.0020 & $\mathrm{mg} / \mathrm{L}$ & 4 & 17-Jun-2009 & 17-Jun-2009 & SW 846/6020 & \\
\hline Selenium & 0.0056 & 0.0020 & $\mathrm{mg} / \mathrm{L}$ & 4 & 17-Jun-2009 & 17-Jun-2009 & SW 846/6020 & \\
\hline Silver & ND & 0.0020 & $\mathrm{mg} / \mathrm{L}$ & 4 & 17-Jun-2009 & 17-Jun-2009 & SW 846/6020 & $\mathrm{U}$ \\
\hline Thallium & 0.0023 & 0.0020 & $\mathrm{mg} / \mathrm{L}$ & 4 & 17-Jun-2009 & 17-Jun-2009 & SW 846/6020 & \\
\hline Vanadium & 0.0989 & 0.0020 & $\mathrm{mg} / \mathrm{L}$ & 4 & 17-Jun-2009 & 17-Jun-2009 & SW 846/6020 & \\
\hline Zinc & 0.0701 & 0.0020 & $\mathrm{mg} / \mathrm{L}$ & 4 & 17-Jun-2009 & 17-Jun-2009 & SW 846/6020 & B \\
\hline
\end{tabular}




\section{USACE ERDC-EP-C \\ 3909 Halls Ferry Road \\ Vicksburg, MS 39180-6199}

ERDC - ECB

$-\cdot$,
Project: TVA Fly Ash Project

Project Manager: Tony Bednar
Reported:

03-Sep-2009

DRAFT: EMR MT N2 Hour 24 Rep C - Total Metals

9061502-78 (Water)

\begin{tabular}{|c|c|c|c|c|c|c|c|c|}
\hline Analyte & Result & $\begin{array}{r}\text { porting } \\
\text { Limit }\end{array}$ & Units & Dilution & Prepared & Analyzed & Method & Notes \\
\hline
\end{tabular}

ERDC- EL-EP-C (Environmental Chemistry Branch)

DRAFT: Wet Chemistry

\begin{tabular}{llllllll}
\hline Hardness & $\mathbf{6 5 . 1}$ & 1.32 & $\begin{array}{c}\text { mg equil } \\
\text { CaCO3/ }\end{array}$ & 4 & 25-Jun-2009 & 25-Jun-2009
\end{tabular}

$\mathrm{L}$

DRAFT: Metals by EPA 6000/7000 Series Methods

\begin{tabular}{|c|c|c|c|c|c|c|c|c|}
\hline Mercury & 0.000407 & 0.000005 & $\mathrm{mg} / \mathrm{L}$ & 2 & 24-Jul-2009 & 24-Jul-2009 & EPA 7471A & \\
\hline Strontium & 0.446 & 0.0800 & $\mathrm{mg} / \mathrm{L}$ & 4 & 25-Jun-2009 & 25-Jun-2009 & EPA 6010B & \\
\hline Aluminum & 33.1 & 0.200 & $\mathrm{mg} / \mathrm{L}$ & 4 & 25-Jun-2009 & 25-Jun-2009 & SW 846/6010 & \\
\hline Calcium & 17.8 & 0.200 & $\mathrm{mg} / \mathrm{L}$ & 4 & 25-Jun-2009 & 25-Jun-2009 & SW 846/6010 & B \\
\hline Iron & 13.2 & 0.200 & $\mathrm{mg} / \mathrm{L}$ & 4 & 25-Jun-2009 & 25-Jun-2009 & SW 846/6010 & \\
\hline Magnesium & 5.05 & 0.200 & $\mathrm{mg} / \mathrm{L}$ & 4 & 25-Jun-2009 & 25-Jun-2009 & SW 846/6010 & \\
\hline Potassium & 7.43 & 0.200 & $\mathrm{mg} / \mathrm{L}$ & 4 & 25-Jun-2009 & 25-Jun-2009 & SW 846/6010 & B \\
\hline Sodium & 4.03 & 0.200 & $\mathrm{mg} / \mathrm{L}$ & 4 & 25-Jun-2009 & 25-Jun-2009 & SW 846/6010 & B \\
\hline Antimony & 0.0060 & 0.0020 & $\mathrm{mg} / \mathrm{L}$ & 4 & 17-Jun-2009 & 17-Jun-2009 & SW 846/6020 & \\
\hline Arsenic & 0.136 & 0.0020 & $\mathrm{mg} / \mathrm{L}$ & 4 & 17-Jun-2009 & 17-Jun-2009 & SW 846/6020 & \\
\hline Barium & 0.639 & 0.0020 & $\mathrm{mg} / \mathrm{L}$ & 4 & 17-Jun-2009 & 17-Jun-2009 & SW 846/6020 & B \\
\hline Beryllium & 0.0055 & 0.0020 & $\mathrm{mg} / \mathrm{L}$ & 4 & 17-Jun-2009 & 17-Jun-2009 & SW 846/6020 & \\
\hline Cadmium & 0.0011 & 0.0020 & $\mathrm{mg} / \mathrm{L}$ & 4 & 17-Jun-2009 & 17-Jun-2009 & SW 846/6020 & $\mathrm{J}$ \\
\hline Chromium & 0.0453 & 0.0020 & $\mathrm{mg} / \mathrm{L}$ & 4 & 17-Jun-2009 & 17-Jun-2009 & SW 846/6020 & \\
\hline Cobalt & 0.0248 & 0.0020 & $\mathrm{mg} / \mathrm{L}$ & 4 & 17-Jun-2009 & 17-Jun-2009 & SW 846/6020 & \\
\hline Copper & 0.0862 & 0.0020 & $\mathrm{mg} / \mathrm{L}$ & 4 & 17-Jun-2009 & 17-Jun-2009 & SW 846/6020 & B \\
\hline Lead & 0.0504 & 0.0020 & $\mathrm{mg} / \mathrm{L}$ & 4 & 17-Jun-2009 & 17-Jun-2009 & SW 846/6020 & \\
\hline Manganese & 0.114 & 0.0020 & $\mathrm{mg} / \mathrm{L}$ & 4 & 17-Jun-2009 & 17-Jun-2009 & SW 846/6020 & \\
\hline Molybdenum & 0.0068 & 0.0020 & $\mathrm{mg} / \mathrm{L}$ & 4 & 17-Jun-2009 & 17-Jun-2009 & SW 846/6020 & \\
\hline Nickel & 0.0493 & 0.0020 & $\mathrm{mg} / \mathrm{L}$ & 4 & 17-Jun-2009 & 17-Jun-2009 & SW 846/6020 & \\
\hline Selenium & 0.0054 & 0.0020 & $\mathrm{mg} / \mathrm{L}$ & 4 & 17-Jun-2009 & 17-Jun-2009 & SW 846/6020 & \\
\hline Silver & ND & 0.0020 & $\mathrm{mg} / \mathrm{L}$ & 4 & 17-Jun-2009 & 17-Jun-2009 & SW 846/6020 & $\mathrm{U}$ \\
\hline Thallium & 0.0029 & 0.0020 & $\mathrm{mg} / \mathrm{L}$ & 4 & 17-Jun-2009 & 17-Jun-2009 & SW 846/6020 & \\
\hline Vanadium & 0.149 & 0.0020 & $\mathrm{mg} / \mathrm{L}$ & 4 & 17-Jun-2009 & 17-Jun-2009 & SW 846/6020 & \\
\hline Zinc & 0.0975 & 0.0020 & $\mathrm{mg} / \mathrm{L}$ & 4 & 17-Jun-2009 & 17-Jun-2009 & SW 846/6020 & B \\
\hline
\end{tabular}




\section{USACE ERDC-EP-C \\ 3909 Halls Ferry Road \\ Vicksburg, MS 39180-6199}

ERDC - ECB

$-\cdot$,
Project: TVA Fly Ash Project

Project Manager: Tony Bednar
Reported:

03-Sep-2009

DRAFT: EMR MT O2 Hour 24 Rep A - Total Metals

9061502-79 (Water)

\begin{tabular}{|c|c|c|c|c|c|c|c|c|}
\hline Analyte & Result & $\begin{array}{r}\text { porting } \\
\text { Limit }\end{array}$ & Units & Dilution & Prepared & Analyzed & Method & Notes \\
\hline
\end{tabular}

ERDC- EL-EP-C (Environmental Chemistry Branch)

DRAFT: Wet Chemistry

\begin{tabular}{llllllll}
\hline Hardness & $\mathbf{5 2 . 3}$ & 1.32 & $\begin{array}{c}\text { mg equil } \\
\text { CaCO3/ }\end{array}$ & 4 & 25-Jun-2009 & 25-Jun-2009
\end{tabular}

$\mathrm{L}$

DRAFT: Metals by EPA 6000/7000 Series Methods

\begin{tabular}{|c|c|c|c|c|c|c|c|c|}
\hline Mercury & 0.000181 & 0.000005 & $\mathrm{mg} / \mathrm{L}$ & 2 & 24-Jul-2009 & 24-Jul-2009 & EPA 7471A & \\
\hline Strontium & 0.294 & 0.0800 & $\mathrm{mg} / \mathrm{L}$ & 4 & 25-Jun-2009 & 25-Jun-2009 & EPA 6010B & \\
\hline Aluminum & 18.4 & 0.200 & $\mathrm{mg} / \mathrm{L}$ & 4 & 25-Jun-2009 & 25-Jun-2009 & SW 846/6010 & \\
\hline Calcium & 14.7 & 0.200 & $\mathrm{mg} / \mathrm{L}$ & 4 & 25-Jun-2009 & 25-Jun-2009 & SW 846/6010 & B \\
\hline Iron & 7.62 & 0.200 & $\mathrm{mg} / \mathrm{L}$ & 4 & 25-Jun-2009 & 25-Jun-2009 & SW 846/6010 & \\
\hline Magnesium & 3.81 & 0.200 & $\mathrm{mg} / \mathrm{L}$ & 4 & 25-Jun-2009 & 25-Jun-2009 & SW 846/6010 & \\
\hline Potassium & 5.05 & 0.200 & $\mathrm{mg} / \mathrm{L}$ & 4 & 25-Jun-2009 & 25-Jun-2009 & SW 846/6010 & B \\
\hline Sodium & 3.64 & 0.200 & $\mathrm{mg} / \mathrm{L}$ & 4 & 25-Jun-2009 & 25-Jun-2009 & SW 846/6010 & B \\
\hline Antimony & 0.0083 & 0.0020 & $\mathrm{mg} / \mathrm{L}$ & 4 & 17-Jun-2009 & 17-Jun-2009 & SW 846/6020 & \\
\hline Arsenic & 0.0850 & 0.0020 & $\mathrm{mg} / \mathrm{L}$ & 4 & 17-Jun-2009 & 17-Jun-2009 & SW 846/6020 & \\
\hline Barium & 0.409 & 0.0020 & $\mathrm{mg} / \mathrm{L}$ & 4 & 17-Jun-2009 & 17-Jun-2009 & SW 846/6020 & B \\
\hline Beryllium & 0.0041 & 0.0020 & $\mathrm{mg} / \mathrm{L}$ & 4 & 17-Jun-2009 & 17-Jun-2009 & SW 846/6020 & \\
\hline Cadmium & 0.0006 & 0.0020 & $\mathrm{mg} / \mathrm{L}$ & 4 & 17-Jun-2009 & 17-Jun-2009 & SW 846/6020 & J \\
\hline Chromium & 0.0270 & 0.0020 & $\mathrm{mg} / \mathrm{L}$ & 4 & 17-Jun-2009 & 17-Jun-2009 & SW 846/6020 & \\
\hline Cobalt & 0.0160 & 0.0020 & $\mathrm{mg} / \mathrm{L}$ & 4 & 17-Jun-2009 & 17-Jun-2009 & SW 846/6020 & \\
\hline Copper & 0.0588 & 0.0020 & $\mathrm{mg} / \mathrm{L}$ & 4 & 17-Jun-2009 & 17-Jun-2009 & SW 846/6020 & B \\
\hline Lead & 0.0304 & 0.0020 & $\mathrm{mg} / \mathrm{L}$ & 4 & 17-Jun-2009 & 17-Jun-2009 & SW 846/6020 & \\
\hline Manganese & 0.0887 & 0.0020 & $\mathrm{mg} / \mathrm{L}$ & 4 & 17-Jun-2009 & 17-Jun-2009 & SW 846/6020 & \\
\hline Molybdenum & 0.0047 & 0.0020 & $\mathrm{mg} / \mathrm{L}$ & 4 & 17-Jun-2009 & 17-Jun-2009 & SW 846/6020 & \\
\hline Nickel & 0.0315 & 0.0020 & $\mathrm{mg} / \mathrm{L}$ & 4 & 17-Jun-2009 & 17-Jun-2009 & SW 846/6020 & \\
\hline Selenium & 0.0038 & 0.0020 & $\mathrm{mg} / \mathrm{L}$ & 4 & 17-Jun-2009 & 17-Jun-2009 & SW 846/6020 & \\
\hline Silver & ND & 0.0020 & $\mathrm{mg} / \mathrm{L}$ & 4 & 17-Jun-2009 & 17-Jun-2009 & SW 846/6020 & $\mathrm{U}$ \\
\hline Thallium & 0.0020 & 0.0020 & $\mathrm{mg} / \mathrm{L}$ & 4 & 17-Jun-2009 & 17-Jun-2009 & SW 846/6020 & \\
\hline Vanadium & 0.0918 & 0.0020 & $\mathrm{mg} / \mathrm{L}$ & 4 & 17-Jun-2009 & 17-Jun-2009 & SW 846/6020 & \\
\hline Zinc & 0.0752 & 0.0020 & $\mathrm{mg} / \mathrm{L}$ & 4 & 17-Jun-2009 & 17-Jun-2009 & SW 846/6020 & B \\
\hline
\end{tabular}




\section{USACE ERDC-EP-C \\ 3909 Halls Ferry Road \\ Vicksburg, MS 39180-6199}

ERDC - ECB

$-\cdot$,
Project: TVA Fly Ash Project

Project Manager: Tony Bednar
Reported:

03-Sep-2009

DRAFT: EMR MT O2 Hour 24 Rep B - Total Metals

9061502-80 (Water)

\begin{tabular}{|c|c|c|c|c|c|c|c|c|}
\hline Analyte & Result & $\begin{array}{r}\text { porting } \\
\text { Limit }\end{array}$ & Units & Dilution & Prepared & Analyzed & Method & Notes \\
\hline
\end{tabular}

ERDC- EL-EP-C (Environmental Chemistry Branch)

DRAFT: Wet Chemistry

\begin{tabular}{llllllll}
\hline Hardness & $\mathbf{4 4 . 0}$ & 1.32 & $\begin{array}{c}\text { mg equil } \\
\text { CaCO3/ }\end{array}$ & 4 & 25-Jun-2009 & 25-Jun-2009
\end{tabular}

$\mathrm{L}$

DRAFT: Metals by EPA 6000/7000 Series Methods

\begin{tabular}{|c|c|c|c|c|c|c|c|c|}
\hline Mercury & 0.000509 & 0.000005 & $\mathrm{mg} / \mathrm{L}$ & 2 & 24-Jul-2009 & 24-Jul-2009 & EPA 7471A & \\
\hline Strontium & 0.183 & 0.0800 & $\mathrm{mg} / \mathrm{L}$ & 4 & 25-Jun-2009 & 25-Jun-2009 & EPA 6010B & \\
\hline Aluminum & 8.12 & 0.200 & $\mathrm{mg} / \mathrm{L}$ & 4 & 25-Jun-2009 & 25-Jun-2009 & SW 846/6010 & \\
\hline Calcium & 12.6 & 0.200 & $\mathrm{mg} / \mathrm{L}$ & 4 & 25-Jun-2009 & 25-Jun-2009 & SW 846/6010 & B \\
\hline Iron & 3.55 & 0.200 & $\mathrm{mg} / \mathrm{L}$ & 4 & 25-Jun-2009 & 25-Jun-2009 & SW 846/6010 & \\
\hline Magnesium & 3.02 & 0.200 & $\mathrm{mg} / \mathrm{L}$ & 4 & 25-Jun-2009 & 25-Jun-2009 & SW 846/6010 & \\
\hline Potassium & 2.80 & 0.200 & $\mathrm{mg} / \mathrm{L}$ & 4 & 25-Jun-2009 & 25-Jun-2009 & SW 846/6010 & B \\
\hline Sodium & 3.39 & 0.200 & $\mathrm{mg} / \mathrm{L}$ & 4 & 25-Jun-2009 & 25-Jun-2009 & SW 846/6010 & B \\
\hline Antimony & 0.0140 & 0.0020 & $\mathrm{mg} / \mathrm{L}$ & 4 & 17-Jun-2009 & 17-Jun-2009 & SW 846/6020 & \\
\hline Arsenic & 0.0515 & 0.0020 & $\mathrm{mg} / \mathrm{L}$ & 4 & 17-Jun-2009 & 17-Jun-2009 & SW 846/6020 & \\
\hline Barium & 0.230 & 0.0020 & $\mathrm{mg} / \mathrm{L}$ & 4 & 17-Jun-2009 & 17-Jun-2009 & SW 846/6020 & B \\
\hline Beryllium & 0.0019 & 0.0020 & $\mathrm{mg} / \mathrm{L}$ & 4 & 17-Jun-2009 & 17-Jun-2009 & SW 846/6020 & J \\
\hline Cadmium & 0.0005 & 0.0020 & $\mathrm{mg} / \mathrm{L}$ & 4 & 17-Jun-2009 & 17-Jun-2009 & SW 846/6020 & J \\
\hline Chromium & 0.0167 & 0.0020 & $\mathrm{mg} / \mathrm{L}$ & 4 & 17-Jun-2009 & 17-Jun-2009 & SW 846/6020 & \\
\hline Cobalt & 0.0078 & 0.0020 & $\mathrm{mg} / \mathrm{L}$ & 4 & 17-Jun-2009 & 17-Jun-2009 & SW 846/6020 & \\
\hline Copper & 0.0314 & 0.0020 & $\mathrm{mg} / \mathrm{L}$ & 4 & 17-Jun-2009 & 17-Jun-2009 & SW 846/6020 & B \\
\hline Lead & 0.0162 & 0.0020 & $\mathrm{mg} / \mathrm{L}$ & 4 & 17-Jun-2009 & 17-Jun-2009 & SW 846/6020 & \\
\hline Manganese & 0.0569 & 0.0020 & $\mathrm{mg} / \mathrm{L}$ & 4 & 17-Jun-2009 & 17-Jun-2009 & SW 846/6020 & \\
\hline Molybdenum & 0.0031 & 0.0020 & $\mathrm{mg} / \mathrm{L}$ & 4 & 17-Jun-2009 & 17-Jun-2009 & SW 846/6020 & \\
\hline Nickel & 0.0183 & 0.0020 & $\mathrm{mg} / \mathrm{L}$ & 4 & 17-Jun-2009 & 17-Jun-2009 & SW 846/6020 & \\
\hline Selenium & 0.0014 & 0.0020 & $\mathrm{mg} / \mathrm{L}$ & 4 & 17-Jun-2009 & 17-Jun-2009 & SW 846/6020 & J \\
\hline Silver & ND & 0.0020 & $\mathrm{mg} / \mathrm{L}$ & 4 & 17-Jun-2009 & 17-Jun-2009 & SW 846/6020 & $\mathrm{U}$ \\
\hline Thallium & 0.0012 & 0.0020 & $\mathrm{mg} / \mathrm{L}$ & 4 & 17-Jun-2009 & 17-Jun-2009 & SW 846/6020 & J \\
\hline Vanadium & 0.0540 & 0.0020 & $\mathrm{mg} / \mathrm{L}$ & 4 & 17-Jun-2009 & 17-Jun-2009 & SW 846/6020 & \\
\hline Zinc & 0.0547 & 0.0020 & $\mathrm{mg} / \mathrm{L}$ & 4 & 17-Jun-2009 & 17-Jun-2009 & SW 846/6020 & B \\
\hline
\end{tabular}




\section{USACE ERDC-EP-C \\ 3909 Halls Ferry Road \\ Vicksburg, MS 39180-6199}

ERDC - ECB

$-\cdot$,
Project: TVA Fly Ash Project

Project Manager: Tony Bednar
Reported:

03-Sep-2009

DRAFT: EMR MT O2 Hour 24 Rep C - Total Metals

9061502-81 (Water)

\begin{tabular}{|c|c|c|c|c|c|c|c|c|}
\hline Analyte & Result & $\begin{array}{r}\text { porting } \\
\text { Limit }\end{array}$ & Units & Dilution & Prepared & Analyzed & Method & Notes \\
\hline
\end{tabular}

ERDC- EL-EP-C (Environmental Chemistry Branch)

DRAFT: Wet Chemistry

\begin{tabular}{llllllll}
\hline Hardness & $\mathbf{5 1 . 4}$ & 1.32 & $\begin{array}{c}\text { mg equil } \\
\text { CaCO3/ }\end{array}$ & 4 & 25-Jun-2009 & 25-Jun-2009
\end{tabular}

$\mathrm{L}$

DRAFT: Metals by EPA 6000/7000 Series Methods

\begin{tabular}{|c|c|c|c|c|c|c|c|c|}
\hline Mercury & 0.000358 & 0.000005 & $\mathrm{mg} / \mathrm{L}$ & 2 & 24-Jul-2009 & 24-Jul-2009 & EPA 7471A & \\
\hline Strontium & 0.293 & 0.0800 & $\mathrm{mg} / \mathrm{L}$ & 4 & 25-Jun-2009 & 25-Jun-2009 & EPA 6010B & \\
\hline Aluminum & 17.4 & 0.200 & $\mathrm{mg} / \mathrm{L}$ & 4 & 25-Jun-2009 & 25-Jun-2009 & SW 846/6010 & \\
\hline Calcium & 14.4 & 0.200 & $\mathrm{mg} / \mathrm{L}$ & 4 & 25-Jun-2009 & 25-Jun-2009 & SW 846/6010 & B \\
\hline Iron & 7.30 & 0.200 & $\mathrm{mg} / \mathrm{L}$ & 4 & 25-Jun-2009 & 25-Jun-2009 & SW 846/6010 & \\
\hline Magnesium & 3.72 & 0.200 & $\mathrm{mg} / \mathrm{L}$ & 4 & 25-Jun-2009 & 25-Jun-2009 & SW 846/6010 & \\
\hline Potassium & 4.70 & 0.200 & $\mathrm{mg} / \mathrm{L}$ & 4 & 25-Jun-2009 & 25-Jun-2009 & SW 846/6010 & B \\
\hline Sodium & 3.46 & 0.200 & $\mathrm{mg} / \mathrm{L}$ & 4 & 25-Jun-2009 & 25-Jun-2009 & SW 846/6010 & B \\
\hline Antimony & 0.0039 & 0.0020 & $\mathrm{mg} / \mathrm{L}$ & 4 & 17-Jun-2009 & 17-Jun-2009 & SW 846/6020 & \\
\hline Arsenic & 0.0915 & 0.0020 & $\mathrm{mg} / \mathrm{L}$ & 4 & 17-Jun-2009 & 17-Jun-2009 & SW 846/6020 & \\
\hline Barium & 0.413 & 0.0020 & $\mathrm{mg} / \mathrm{L}$ & 4 & 17-Jun-2009 & 17-Jun-2009 & SW 846/6020 & B \\
\hline Beryllium & 0.0036 & 0.0020 & $\mathrm{mg} / \mathrm{L}$ & 4 & 17-Jun-2009 & 17-Jun-2009 & SW 846/6020 & \\
\hline Cadmium & 0.0006 & 0.0020 & $\mathrm{mg} / \mathrm{L}$ & 4 & 17-Jun-2009 & 17-Jun-2009 & SW 846/6020 & $\mathrm{J}$ \\
\hline Chromium & 0.0307 & 0.0020 & $\mathrm{mg} / \mathrm{L}$ & 4 & 17-Jun-2009 & 17-Jun-2009 & SW 846/6020 & \\
\hline Cobalt & 0.0148 & 0.0020 & $\mathrm{mg} / \mathrm{L}$ & 4 & 17-Jun-2009 & 17-Jun-2009 & SW 846/6020 & \\
\hline Copper & 0.0546 & 0.0020 & $\mathrm{mg} / \mathrm{L}$ & 4 & 17-Jun-2009 & 17-Jun-2009 & SW 846/6020 & B \\
\hline Lead & 0.0302 & 0.0020 & $\mathrm{mg} / \mathrm{L}$ & 4 & 17-Jun-2009 & 17-Jun-2009 & SW 846/6020 & \\
\hline Manganese & 0.0854 & 0.0020 & $\mathrm{mg} / \mathrm{L}$ & 4 & 17-Jun-2009 & 17-Jun-2009 & SW 846/6020 & \\
\hline Molybdenum & 0.0046 & 0.0020 & $\mathrm{mg} / \mathrm{L}$ & 4 & 17-Jun-2009 & 17-Jun-2009 & SW 846/6020 & \\
\hline Nickel & 0.0304 & 0.0020 & $\mathrm{mg} / \mathrm{L}$ & 4 & 17-Jun-2009 & 17-Jun-2009 & SW 846/6020 & \\
\hline Selenium & 0.0047 & 0.0020 & $\mathrm{mg} / \mathrm{L}$ & 4 & 17-Jun-2009 & 17-Jun-2009 & SW 846/6020 & \\
\hline Silver & ND & 0.0020 & $\mathrm{mg} / \mathrm{L}$ & 4 & 17-Jun-2009 & 17-Jun-2009 & SW 846/6020 & $\mathrm{U}$ \\
\hline Thallium & 0.0022 & 0.0020 & $\mathrm{mg} / \mathrm{L}$ & 4 & 17-Jun-2009 & 17-Jun-2009 & SW 846/6020 & \\
\hline Vanadium & 0.101 & 0.0020 & $\mathrm{mg} / \mathrm{L}$ & 4 & 17-Jun-2009 & 17-Jun-2009 & SW 846/6020 & \\
\hline Zinc & 0.0689 & 0.0020 & $\mathrm{mg} / \mathrm{L}$ & 4 & 17-Jun-2009 & 17-Jun-2009 & SW 846/6020 & B \\
\hline
\end{tabular}




\section{USACE ERDC-EP-C \\ 3909 Halls Ferry Road \\ Vicksburg, MS 39180-6199}

ERDC - ECB

$-\cdot$,
Project: TVA Fly Ash Project

Project Manager: Tony Bednar
Reported:

03-Sep-2009

DRAFT: SLC MT N2 Hour 24 Rep A - Total Metals

9061502-82 (Water)

\begin{tabular}{|c|c|c|c|c|c|c|c|c|}
\hline Analyte & Result & $\begin{array}{r}\text { porting } \\
\text { Limit }\end{array}$ & Units & Dilution & Prepared & Analyzed & Method & Notes \\
\hline
\end{tabular}

ERDC- EL-EP-C (Environmental Chemistry Branch)

DRAFT: Wet Chemistry

\begin{tabular}{llllllll}
\hline Hardness & $\mathbf{1 0 4}$ & 1.32 & $\begin{array}{c}\text { mg equil } \\
\text { CaCO3/ }\end{array}$ & 4 & 25-Jun-2009 & 25-Jun-2009
\end{tabular}

$\mathrm{L}$

DRAFT: Metals by EPA 6000/7000 Series Methods

\begin{tabular}{|c|c|c|c|c|c|c|c|c|}
\hline Mercury & 0.000508 & 0.000005 & $\mathrm{mg} / \mathrm{L}$ & 2 & 24-Jul-2009 & 24-Jul-2009 & EPA 7471A & \\
\hline Strontium & 0.615 & 0.0800 & $\mathrm{mg} / \mathrm{L}$ & 4 & 25-Jun-2009 & 25-Jun-2009 & EPA 6010B & \\
\hline Aluminum & 13.0 & 0.200 & $\mathrm{mg} / \mathrm{L}$ & 4 & 25-Jun-2009 & 25-Jun-2009 & SW 846/6010 & \\
\hline Calcium & 31.6 & 0.200 & $\mathrm{mg} / \mathrm{L}$ & 4 & 25-Jun-2009 & 25-Jun-2009 & SW 846/6010 & B \\
\hline Iron & 5.49 & 0.200 & $\mathrm{mg} / \mathrm{L}$ & 4 & 25-Jun-2009 & 25-Jun-2009 & SW 846/6010 & \\
\hline Magnesium & 5.97 & 0.200 & $\mathrm{mg} / \mathrm{L}$ & 4 & 25-Jun-2009 & 25-Jun-2009 & SW 846/6010 & \\
\hline Potassium & 5.36 & 0.200 & $\mathrm{mg} / \mathrm{L}$ & 4 & 25-Jun-2009 & 25-Jun-2009 & SW 846/6010 & B \\
\hline Sodium & 4.82 & 0.200 & $\mathrm{mg} / \mathrm{L}$ & 4 & 25-Jun-2009 & 25-Jun-2009 & SW 846/6010 & B \\
\hline Antimony & 0.0087 & 0.0020 & $\mathrm{mg} / \mathrm{L}$ & 4 & 17-Jun-2009 & 17-Jun-2009 & SW 846/6020 & \\
\hline Arsenic & 0.113 & 0.0020 & $\mathrm{mg} / \mathrm{L}$ & 4 & 17-Jun-2009 & 17-Jun-2009 & SW 846/6020 & \\
\hline Barium & 0.281 & 0.0020 & $\mathrm{mg} / \mathrm{L}$ & 4 & 17-Jun-2009 & 17-Jun-2009 & SW 846/6020 & B \\
\hline Beryllium & 0.0022 & 0.0020 & $\mathrm{mg} / \mathrm{L}$ & 4 & 17-Jun-2009 & 17-Jun-2009 & SW 846/6020 & \\
\hline Cadmium & 0.0007 & 0.0020 & $\mathrm{mg} / \mathrm{L}$ & 4 & 17-Jun-2009 & 17-Jun-2009 & SW 846/6020 & J \\
\hline Chromium & 0.0188 & 0.0020 & $\mathrm{mg} / \mathrm{L}$ & 4 & 17-Jun-2009 & 17-Jun-2009 & SW 846/6020 & \\
\hline Cobalt & 0.0098 & 0.0020 & $\mathrm{mg} / \mathrm{L}$ & 4 & 17-Jun-2009 & 17-Jun-2009 & SW 846/6020 & \\
\hline Copper & 0.0347 & 0.0020 & $\mathrm{mg} / \mathrm{L}$ & 4 & 17-Jun-2009 & 17-Jun-2009 & SW 846/6020 & B \\
\hline Lead & 0.0188 & 0.0020 & $\mathrm{mg} / \mathrm{L}$ & 4 & 17-Jun-2009 & 17-Jun-2009 & SW 846/6020 & \\
\hline Manganese & 0.0824 & 0.0020 & $\mathrm{mg} / \mathrm{L}$ & 4 & 17-Jun-2009 & 17-Jun-2009 & SW 846/6020 & \\
\hline Molybdenum & 0.0761 & 0.0020 & $\mathrm{mg} / \mathrm{L}$ & 4 & 17-Jun-2009 & 17-Jun-2009 & SW 846/6020 & \\
\hline Nickel & 0.0214 & 0.0020 & $\mathrm{mg} / \mathrm{L}$ & 4 & 17-Jun-2009 & 17-Jun-2009 & SW 846/6020 & \\
\hline Selenium & 0.0060 & 0.0020 & $\mathrm{mg} / \mathrm{L}$ & 4 & 17-Jun-2009 & 17-Jun-2009 & SW 846/6020 & \\
\hline Silver & ND & 0.0020 & $\mathrm{mg} / \mathrm{L}$ & 4 & 17-Jun-2009 & 17-Jun-2009 & SW 846/6020 & $\mathrm{U}$ \\
\hline Thallium & 0.0016 & 0.0020 & $\mathrm{mg} / \mathrm{L}$ & 4 & 17-Jun-2009 & 17-Jun-2009 & SW 846/6020 & J \\
\hline Vanadium & 0.102 & 0.0020 & $\mathrm{mg} / \mathrm{L}$ & 4 & 17-Jun-2009 & 17-Jun-2009 & SW 846/6020 & \\
\hline Zinc & 0.0503 & 0.0020 & $\mathrm{mg} / \mathrm{L}$ & 4 & 17-Jun-2009 & 17-Jun-2009 & SW 846/6020 & B \\
\hline
\end{tabular}




\section{USACE ERDC-EP-C \\ 3909 Halls Ferry Road \\ Vicksburg, MS 39180-6199}

ERDC - ECB

$-\cdot$,
Project: TVA Fly Ash Project

Project Manager: Tony Bednar
Reported:

03-Sep-2009

DRAFT: SLC MT N2 Hour 24 Rep B - Total Metals

9061502-83 (Water)

\begin{tabular}{|c|c|c|c|c|c|c|c|c|}
\hline Analyte & Result & $\begin{array}{r}\text { porting } \\
\text { Limit }\end{array}$ & Units & Dilution & Prepared & Analyzed & Method & Notes \\
\hline
\end{tabular}

ERDC- EL-EP-C (Environmental Chemistry Branch)

DRAFT: Wet Chemistry

\begin{tabular}{llllllll}
\hline Hardness & $\mathbf{1 0 3}$ & 1.32 & $\begin{array}{c}\text { mg equil } \\
\text { CaCO3/ }\end{array}$ & 4 & 25-Jun-2009 & 25-Jun-2009
\end{tabular}

$\mathrm{L}$

DRAFT: Metals by EPA 6000/7000 Series Methods

\begin{tabular}{|c|c|c|c|c|c|c|c|c|}
\hline Mercury & 0.000374 & 0.000005 & $\mathrm{mg} / \mathrm{L}$ & 2 & 24-Jul-2009 & 24-Jul-2009 & EPA 7471A & \\
\hline Strontium & 0.613 & 0.0800 & $\mathrm{mg} / \mathrm{L}$ & 4 & 25-Jun-2009 & 25-Jun-2009 & EPA 6010B & \\
\hline Aluminum & 12.5 & 0.200 & $\mathrm{mg} / \mathrm{L}$ & 4 & 25-Jun-2009 & 25-Jun-2009 & SW 846/6010 & \\
\hline Calcium & 31.6 & 0.200 & $\mathrm{mg} / \mathrm{L}$ & 4 & 25-Jun-2009 & 25-Jun-2009 & SW 846/6010 & B \\
\hline Iron & 5.23 & 0.200 & $\mathrm{mg} / \mathrm{L}$ & 4 & 25-Jun-2009 & 25-Jun-2009 & SW 846/6010 & \\
\hline Magnesium & 5.77 & 0.200 & $\mathrm{mg} / \mathrm{L}$ & 4 & 25-Jun-2009 & 25-Jun-2009 & SW 846/6010 & \\
\hline Potassium & 4.52 & 0.200 & $\mathrm{mg} / \mathrm{L}$ & 4 & 25-Jun-2009 & 25-Jun-2009 & SW 846/6010 & B \\
\hline Sodium & 4.68 & 0.200 & $\mathrm{mg} / \mathrm{L}$ & 4 & 25-Jun-2009 & 25-Jun-2009 & SW 846/6010 & B \\
\hline Antimony & 0.0086 & 0.0020 & $\mathrm{mg} / \mathrm{L}$ & 4 & 17-Jun-2009 & 17-Jun-2009 & SW 846/6020 & \\
\hline Arsenic & 0.111 & 0.0020 & $\mathrm{mg} / \mathrm{L}$ & 4 & 17-Jun-2009 & 17-Jun-2009 & SW 846/6020 & \\
\hline Barium & 0.285 & 0.0020 & $\mathrm{mg} / \mathrm{L}$ & 4 & 17-Jun-2009 & 17-Jun-2009 & SW 846/6020 & B \\
\hline Beryllium & 0.0022 & 0.0020 & $\mathrm{mg} / \mathrm{L}$ & 4 & 17-Jun-2009 & 17-Jun-2009 & SW 846/6020 & \\
\hline Cadmium & 0.0007 & 0.0020 & $\mathrm{mg} / \mathrm{L}$ & 4 & 17-Jun-2009 & 17-Jun-2009 & SW 846/6020 & $\mathrm{J}$ \\
\hline Chromium & 0.0250 & 0.0020 & $\mathrm{mg} / \mathrm{L}$ & 4 & 17-Jun-2009 & 17-Jun-2009 & SW 846/6020 & \\
\hline Cobalt & 0.0102 & 0.0020 & $\mathrm{mg} / \mathrm{L}$ & 4 & 17-Jun-2009 & 17-Jun-2009 & SW 846/6020 & \\
\hline Copper & 0.0366 & 0.0020 & $\mathrm{mg} / \mathrm{L}$ & 4 & 17-Jun-2009 & 17-Jun-2009 & SW 846/6020 & B \\
\hline Lead & 0.0194 & 0.0020 & $\mathrm{mg} / \mathrm{L}$ & 4 & 17-Jun-2009 & 17-Jun-2009 & SW 846/6020 & \\
\hline Manganese & 0.0831 & 0.0020 & $\mathrm{mg} / \mathrm{L}$ & 4 & 17-Jun-2009 & 17-Jun-2009 & SW 846/6020 & \\
\hline Molybdenum & 0.0735 & 0.0020 & $\mathrm{mg} / \mathrm{L}$ & 4 & 17-Jun-2009 & 17-Jun-2009 & SW 846/6020 & \\
\hline Nickel & 0.0221 & 0.0020 & $\mathrm{mg} / \mathrm{L}$ & 4 & 17-Jun-2009 & 17-Jun-2009 & SW 846/6020 & \\
\hline Selenium & 0.0059 & 0.0020 & $\mathrm{mg} / \mathrm{L}$ & 4 & 17-Jun-2009 & 17-Jun-2009 & SW 846/6020 & \\
\hline Silver & ND & 0.0020 & $\mathrm{mg} / \mathrm{L}$ & 4 & 17-Jun-2009 & 17-Jun-2009 & SW 846/6020 & $\mathrm{U}$ \\
\hline Thallium & 0.0017 & 0.0020 & $\mathrm{mg} / \mathrm{L}$ & 4 & 17-Jun-2009 & 17-Jun-2009 & SW 846/6020 & $\mathrm{J}$ \\
\hline Vanadium & 0.108 & 0.0020 & $\mathrm{mg} / \mathrm{L}$ & 4 & 17-Jun-2009 & 17-Jun-2009 & SW 846/6020 & \\
\hline Zinc & 0.0551 & 0.0020 & $\mathrm{mg} / \mathrm{L}$ & 4 & 17-Jun-2009 & 17-Jun-2009 & SW 846/6020 & B \\
\hline
\end{tabular}




\section{USACE ERDC-EP-C \\ 3909 Halls Ferry Road \\ Vicksburg, MS 39180-6199}

ERDC - ECB

$-\cdot$,
Project: TVA Fly Ash Project

Project Manager: Tony Bednar
Reported:

03-Sep-2009

DRAFT: SLC MT N2 Hour 24 Rep C - Total Metals

9061502-84 (Water)

\begin{tabular}{|c|c|c|c|c|c|c|c|c|}
\hline Analyte & Result & $\begin{array}{r}\text { porting } \\
\text { Limit }\end{array}$ & Units & Dilution & Prepared & Analyzed & Method & Notes \\
\hline
\end{tabular}

ERDC- EL-EP-C (Environmental Chemistry Branch)

DRAFT: Wet Chemistry

\begin{tabular}{llllllll}
\hline Hardness & $\mathbf{1 0 5}$ & 1.32 & $\begin{array}{c}\text { mg equil } \\
\text { CaCO3/ }\end{array}$ & 4 & 25-Jun-2009 & 25-Jun-2009
\end{tabular}

$\mathrm{L}$

DRAFT: Metals by EPA 6000/7000 Series Methods

\begin{tabular}{|c|c|c|c|c|c|c|c|c|}
\hline Mercury & 0.000358 & 0.000005 & $\mathrm{mg} / \mathrm{L}$ & 2 & 24-Jul-2009 & 24-Jul-2009 & EPA 7471A & \\
\hline Strontium & 0.618 & 0.0800 & $\mathrm{mg} / \mathrm{L}$ & 4 & 25-Jun-2009 & 25-Jun-2009 & EPA 6010B & \\
\hline Aluminum & 13.2 & 0.200 & $\mathrm{mg} / \mathrm{L}$ & 4 & 25-Jun-2009 & 25-Jun-2009 & SW 846/6010 & \\
\hline Calcium & 32.1 & 0.200 & $\mathrm{mg} / \mathrm{L}$ & 4 & 25-Jun-2009 & 25-Jun-2009 & SW 846/6010 & B \\
\hline Iron & 5.48 & 0.200 & $\mathrm{mg} / \mathrm{L}$ & 4 & 25-Jun-2009 & 25-Jun-2009 & SW 846/6010 & \\
\hline Magnesium & 6.02 & 0.200 & $\mathrm{mg} / \mathrm{L}$ & 4 & 25-Jun-2009 & 25-Jun-2009 & SW 846/6010 & \\
\hline Potassium & 5.43 & 0.200 & $\mathrm{mg} / \mathrm{L}$ & 4 & 25-Jun-2009 & 25-Jun-2009 & SW 846/6010 & B \\
\hline Sodium & 4.95 & 0.200 & $\mathrm{mg} / \mathrm{L}$ & 4 & 25-Jun-2009 & 25-Jun-2009 & SW 846/6010 & B \\
\hline Antimony & 0.0092 & 0.0020 & $\mathrm{mg} / \mathrm{L}$ & 4 & 17-Jun-2009 & 17-Jun-2009 & SW 846/6020 & \\
\hline Arsenic & 0.110 & 0.0020 & $\mathrm{mg} / \mathrm{L}$ & 4 & 17-Jun-2009 & 17-Jun-2009 & SW 846/6020 & \\
\hline Barium & 0.284 & 0.0020 & $\mathrm{mg} / \mathrm{L}$ & 4 & 17-Jun-2009 & 17-Jun-2009 & SW 846/6020 & B \\
\hline Beryllium & 0.0022 & 0.0020 & $\mathrm{mg} / \mathrm{L}$ & 4 & 17-Jun-2009 & 17-Jun-2009 & SW 846/6020 & \\
\hline Cadmium & 0.0008 & 0.0020 & $\mathrm{mg} / \mathrm{L}$ & 4 & 17-Jun-2009 & 17-Jun-2009 & SW 846/6020 & J \\
\hline Chromium & 0.0177 & 0.0020 & $\mathrm{mg} / \mathrm{L}$ & 4 & 17-Jun-2009 & 17-Jun-2009 & SW 846/6020 & \\
\hline Cobalt & 0.0094 & 0.0020 & $\mathrm{mg} / \mathrm{L}$ & 4 & 17-Jun-2009 & 17-Jun-2009 & SW 846/6020 & \\
\hline Copper & 0.0360 & 0.0020 & $\mathrm{mg} / \mathrm{L}$ & 4 & 17-Jun-2009 & 17-Jun-2009 & SW 846/6020 & B \\
\hline Lead & 0.0188 & 0.0020 & $\mathrm{mg} / \mathrm{L}$ & 4 & 17-Jun-2009 & 17-Jun-2009 & SW 846/6020 & \\
\hline Manganese & 0.0873 & 0.0020 & $\mathrm{mg} / \mathrm{L}$ & 4 & 17-Jun-2009 & 17-Jun-2009 & SW 846/6020 & \\
\hline Molybdenum & 0.0762 & 0.0020 & $\mathrm{mg} / \mathrm{L}$ & 4 & 17-Jun-2009 & 17-Jun-2009 & SW 846/6020 & \\
\hline Nickel & 0.0210 & 0.0020 & $\mathrm{mg} / \mathrm{L}$ & 4 & 17-Jun-2009 & 17-Jun-2009 & SW 846/6020 & \\
\hline Selenium & 0.0063 & 0.0020 & $\mathrm{mg} / \mathrm{L}$ & 4 & 17-Jun-2009 & 17-Jun-2009 & SW 846/6020 & \\
\hline Silver & ND & 0.0020 & $\mathrm{mg} / \mathrm{L}$ & 4 & 17-Jun-2009 & 17-Jun-2009 & SW 846/6020 & $\mathrm{U}$ \\
\hline Thallium & 0.0016 & 0.0020 & $\mathrm{mg} / \mathrm{L}$ & 4 & 17-Jun-2009 & 17-Jun-2009 & SW 846/6020 & J \\
\hline Vanadium & 0.0999 & 0.0020 & $\mathrm{mg} / \mathrm{L}$ & 4 & 17-Jun-2009 & 17-Jun-2009 & SW 846/6020 & \\
\hline Zinc & 0.0535 & 0.0020 & $\mathrm{mg} / \mathrm{L}$ & 4 & 17-Jun-2009 & 17-Jun-2009 & SW 846/6020 & B \\
\hline
\end{tabular}




\section{USACE ERDC-EP-C \\ 3909 Halls Ferry Road \\ Vicksburg, MS 39180-6199}

ERDC - ECB

$-\cdot$,
Project: TVA Fly Ash Project

Project Manager: Tony Bednar
Reported:

03-Sep-2009

DRAFT: SLC MT O2 Hour 24 Rep A - Total Metals

9061502-85 (Water)

\begin{tabular}{|c|c|c|c|c|c|c|c|c|}
\hline Analyte & Result & $\begin{array}{r}\text { porting } \\
\text { Limit }\end{array}$ & Units & Dilution & Prepared & Analyzed & Method & Notes \\
\hline
\end{tabular}

ERDC- EL-EP-C (Environmental Chemistry Branch)

DRAFT: Wet Chemistry

\begin{tabular}{llllllll}
\hline Hardness & $\mathbf{1 0 6}$ & 1.32 & $\begin{array}{c}\text { mg equil } \\
\text { CaCO3/ }\end{array}$ & 4 & 25-Jun-2009 & 25-Jun-2009
\end{tabular}

$\mathrm{L}$

DRAFT: Metals by EPA 6000/7000 Series Methods

\begin{tabular}{|c|c|c|c|c|c|c|c|c|}
\hline Mercury & 0.000409 & 0.000005 & $\mathrm{mg} / \mathrm{L}$ & 2 & 24-Jul-2009 & 24-Jul-2009 & EPA 7471A & \\
\hline Strontium & 0.626 & 0.0800 & $\mathrm{mg} / \mathrm{L}$ & 4 & 25-Jun-2009 & 25-Jun-2009 & EPA 6010B & \\
\hline Aluminum & 11.1 & 0.200 & $\mathrm{mg} / \mathrm{L}$ & 4 & 25-Jun-2009 & 25-Jun-2009 & SW 846/6010 & \\
\hline Calcium & 32.6 & 0.200 & $\mathrm{mg} / \mathrm{L}$ & 4 & 25-Jun-2009 & 25-Jun-2009 & SW 846/6010 & B \\
\hline Iron & 4.66 & 0.200 & $\mathrm{mg} / \mathrm{L}$ & 4 & 25-Jun-2009 & 25-Jun-2009 & SW 846/6010 & \\
\hline Magnesium & 5.94 & 0.200 & $\mathrm{mg} / \mathrm{L}$ & 4 & 25-Jun-2009 & 25-Jun-2009 & SW 846/6010 & \\
\hline Potassium & 4.35 & 0.200 & $\mathrm{mg} / \mathrm{L}$ & 4 & 25-Jun-2009 & 25-Jun-2009 & SW 846/6010 & B \\
\hline Sodium & 4.72 & 0.200 & $\mathrm{mg} / \mathrm{L}$ & 4 & 25-Jun-2009 & 25-Jun-2009 & SW 846/6010 & B \\
\hline Antimony & 0.0087 & 0.0020 & $\mathrm{mg} / \mathrm{L}$ & 4 & 17-Jun-2009 & 17-Jun-2009 & SW 846/6020 & \\
\hline Arsenic & 0.109 & 0.0020 & $\mathrm{mg} / \mathrm{L}$ & 4 & 17-Jun-2009 & 17-Jun-2009 & SW 846/6020 & \\
\hline Barium & 0.275 & 0.0020 & $\mathrm{mg} / \mathrm{L}$ & 4 & 17-Jun-2009 & 17-Jun-2009 & SW 846/6020 & B \\
\hline Beryllium & 0.0019 & 0.0020 & $\mathrm{mg} / \mathrm{L}$ & 4 & 17-Jun-2009 & 17-Jun-2009 & SW 846/6020 & $\mathrm{J}$ \\
\hline Cadmium & 0.0007 & 0.0020 & $\mathrm{mg} / \mathrm{L}$ & 4 & 17-Jun-2009 & 17-Jun-2009 & SW 846/6020 & $\mathrm{J}$ \\
\hline Chromium & 0.0168 & 0.0020 & $\mathrm{mg} / \mathrm{L}$ & 4 & 17-Jun-2009 & 17-Jun-2009 & SW 846/6020 & \\
\hline Cobalt & 0.0087 & 0.0020 & $\mathrm{mg} / \mathrm{L}$ & 4 & 17-Jun-2009 & 17-Jun-2009 & SW 846/6020 & \\
\hline Copper & 0.0325 & 0.0020 & $\mathrm{mg} / \mathrm{L}$ & 4 & 17-Jun-2009 & 17-Jun-2009 & SW 846/6020 & B \\
\hline Lead & 0.0168 & 0.0020 & $\mathrm{mg} / \mathrm{L}$ & 4 & 17-Jun-2009 & 17-Jun-2009 & SW 846/6020 & \\
\hline Manganese & 0.0858 & 0.0020 & $\mathrm{mg} / \mathrm{L}$ & 4 & 17-Jun-2009 & 17-Jun-2009 & SW 846/6020 & \\
\hline Molybdenum & 0.0780 & 0.0020 & $\mathrm{mg} / \mathrm{L}$ & 4 & 17-Jun-2009 & 17-Jun-2009 & SW 846/6020 & \\
\hline Nickel & 0.0197 & 0.0020 & $\mathrm{mg} / \mathrm{L}$ & 4 & 17-Jun-2009 & 17-Jun-2009 & SW 846/6020 & \\
\hline Selenium & 0.0063 & 0.0020 & $\mathrm{mg} / \mathrm{L}$ & 4 & 17-Jun-2009 & 17-Jun-2009 & SW 846/6020 & \\
\hline Silver & ND & 0.0020 & $\mathrm{mg} / \mathrm{L}$ & 4 & 17-Jun-2009 & 17-Jun-2009 & SW 846/6020 & $\mathrm{U}$ \\
\hline Thallium & 0.0016 & 0.0020 & $\mathrm{mg} / \mathrm{L}$ & 4 & 17-Jun-2009 & 17-Jun-2009 & SW 846/6020 & $\mathrm{J}$ \\
\hline Vanadium & 0.103 & 0.0020 & $\mathrm{mg} / \mathrm{L}$ & 4 & 17-Jun-2009 & 17-Jun-2009 & SW 846/6020 & \\
\hline Zinc & 0.0442 & 0.0020 & $\mathrm{mg} / \mathrm{L}$ & 4 & 17-Jun-2009 & 17-Jun-2009 & SW 846/6020 & B \\
\hline
\end{tabular}




\section{USACE ERDC-EP-C \\ 3909 Halls Ferry Road \\ Vicksburg, MS 39180-6199}

ERDC - ECB

$-\cdot$,
Project: TVA Fly Ash Project

Project Manager: Tony Bednar
Reported:

03-Sep-2009

DRAFT: SLC MT O2 Hour 24 Rep B - Total Metals

9061502-86 (Water)

\begin{tabular}{|c|c|c|c|c|c|c|c|c|}
\hline Analyte & Result & $\begin{array}{r}\text { porting } \\
\text { Limit }\end{array}$ & Units & Dilution & Prepared & Analyzed & Method & Notes \\
\hline
\end{tabular}

ERDC- EL-EP-C (Environmental Chemistry Branch)

DRAFT: Wet Chemistry

\begin{tabular}{llllllll}
\hline Hardness & $\mathbf{1 0 4}$ & 1.32 & $\begin{array}{c}\text { mg equil } \\
\text { CaCO3/ }\end{array}$ & 4 & 25-Jun-2009 & 25-Jun-2009
\end{tabular}

$\mathrm{L}$

DRAFT: Metals by EPA 6000/7000 Series Methods

\begin{tabular}{|c|c|c|c|c|c|c|c|c|}
\hline Mercury & 0.000356 & 0.000005 & $\mathrm{mg} / \mathrm{L}$ & 2 & 24-Jul-2009 & 24-Jul-2009 & EPA 7471A & \\
\hline Strontium & 0.629 & 0.0800 & $\mathrm{mg} / \mathrm{L}$ & 4 & 25-Jun-2009 & 25-Jun-2009 & EPA 6010B & \\
\hline Aluminum & 14.1 & 0.200 & $\mathrm{mg} / \mathrm{L}$ & 4 & 25-Jun-2009 & 25-Jun-2009 & SW 846/6010 & \\
\hline Calcium & 31.8 & 0.200 & $\mathrm{mg} / \mathrm{L}$ & 4 & 25-Jun-2009 & 25-Jun-2009 & SW 846/6010 & B \\
\hline Iron & 5.83 & 0.200 & $\mathrm{mg} / \mathrm{L}$ & 4 & 25-Jun-2009 & 25-Jun-2009 & SW 846/6010 & \\
\hline Magnesium & 5.95 & 0.200 & $\mathrm{mg} / \mathrm{L}$ & 4 & 25-Jun-2009 & 25-Jun-2009 & SW 846/6010 & \\
\hline Potassium & 5.34 & 0.200 & $\mathrm{mg} / \mathrm{L}$ & 4 & 25-Jun-2009 & 25-Jun-2009 & SW 846/6010 & B \\
\hline Sodium & 5.14 & 0.200 & $\mathrm{mg} / \mathrm{L}$ & 4 & 25-Jun-2009 & 25-Jun-2009 & SW 846/6010 & B \\
\hline Antimony & 0.0228 & 0.0020 & $\mathrm{mg} / \mathrm{L}$ & 4 & 17-Jun-2009 & 17-Jun-2009 & SW 846/6020 & \\
\hline Arsenic & 0.111 & 0.0020 & $\mathrm{mg} / \mathrm{L}$ & 4 & 17-Jun-2009 & 17-Jun-2009 & SW 846/6020 & \\
\hline Barium & 0.299 & 0.0020 & $\mathrm{mg} / \mathrm{L}$ & 4 & 17-Jun-2009 & 17-Jun-2009 & SW 846/6020 & B \\
\hline Beryllium & 0.0023 & 0.0020 & $\mathrm{mg} / \mathrm{L}$ & 4 & 17-Jun-2009 & 17-Jun-2009 & SW 846/6020 & \\
\hline Cadmium & 0.0007 & 0.0020 & $\mathrm{mg} / \mathrm{L}$ & 4 & 17-Jun-2009 & 17-Jun-2009 & SW 846/6020 & J \\
\hline Chromium & 0.0224 & 0.0020 & $\mathrm{mg} / \mathrm{L}$ & 4 & 17-Jun-2009 & 17-Jun-2009 & SW 846/6020 & \\
\hline Cobalt & 0.0110 & 0.0020 & $\mathrm{mg} / \mathrm{L}$ & 4 & 17-Jun-2009 & 17-Jun-2009 & SW 846/6020 & \\
\hline Copper & 0.0401 & 0.0020 & $\mathrm{mg} / \mathrm{L}$ & 4 & 17-Jun-2009 & 17-Jun-2009 & SW 846/6020 & B \\
\hline Lead & 0.0198 & 0.0020 & $\mathrm{mg} / \mathrm{L}$ & 4 & 17-Jun-2009 & 17-Jun-2009 & SW 846/6020 & \\
\hline Manganese & 0.0906 & 0.0020 & $\mathrm{mg} / \mathrm{L}$ & 4 & 17-Jun-2009 & 17-Jun-2009 & SW 846/6020 & \\
\hline Molybdenum & 0.0717 & 0.0020 & $\mathrm{mg} / \mathrm{L}$ & 4 & 17-Jun-2009 & 17-Jun-2009 & SW 846/6020 & \\
\hline Nickel & 0.0240 & 0.0020 & $\mathrm{mg} / \mathrm{L}$ & 4 & 17-Jun-2009 & 17-Jun-2009 & SW 846/6020 & \\
\hline Selenium & 0.0066 & 0.0020 & $\mathrm{mg} / \mathrm{L}$ & 4 & 17-Jun-2009 & 17-Jun-2009 & SW 846/6020 & \\
\hline Silver & ND & 0.0020 & $\mathrm{mg} / \mathrm{L}$ & 4 & 17-Jun-2009 & 17-Jun-2009 & SW 846/6020 & $\mathrm{U}$ \\
\hline Thallium & 0.0017 & 0.0020 & $\mathrm{mg} / \mathrm{L}$ & 4 & 17-Jun-2009 & 17-Jun-2009 & SW 846/6020 & J \\
\hline Vanadium & 0.110 & 0.0020 & $\mathrm{mg} / \mathrm{L}$ & 4 & 17-Jun-2009 & 17-Jun-2009 & SW 846/6020 & \\
\hline Zinc & 0.0550 & 0.0020 & $\mathrm{mg} / \mathrm{L}$ & 4 & 17-Jun-2009 & 17-Jun-2009 & SW 846/6020 & B \\
\hline
\end{tabular}




\section{USACE ERDC-EP-C \\ 3909 Halls Ferry Road \\ Vicksburg, MS 39180-6199}

ERDC - ECB

$-\cdot$,
Project: TVA Fly Ash Project

Project Manager: Tony Bednar
Reported:

03-Sep-2009

DRAFT: SLC MT O2 Hour 24 Rep C - Total Metals

9061502-87 (Water)

\begin{tabular}{|c|c|c|c|c|c|c|c|c|}
\hline Analyte & Result & $\begin{array}{r}\text { porting } \\
\text { Limit }\end{array}$ & Units & Dilution & Prepared & Analyzed & Method & Notes \\
\hline
\end{tabular}

ERDC- EL-EP-C (Environmental Chemistry Branch)

DRAFT: Wet Chemistry

\begin{tabular}{llllllll}
\hline Hardness & $\mathbf{1 0 9}$ & 1.32 & $\begin{array}{c}\text { mg equil } \\
\text { CaCO3/ }\end{array}$ & 4 & 25-Jun-2009 & 25-Jun-2009
\end{tabular}

$\mathrm{L}$

DRAFT: Metals by EPA 6000/7000 Series Methods

\begin{tabular}{|c|c|c|c|c|c|c|c|c|}
\hline Mercury & 0.000292 & 0.000005 & $\mathrm{mg} / \mathrm{L}$ & 2 & 24-Jul-2009 & 24-Jul-2009 & EPA 7471A & \\
\hline Strontium & 0.656 & 0.0800 & $\mathrm{mg} / \mathrm{L}$ & 4 & 25-Jun-2009 & 25-Jun-2009 & EPA 6010B & \\
\hline Aluminum & 15.5 & 0.200 & $\mathrm{mg} / \mathrm{L}$ & 4 & 25-Jun-2009 & 25-Jun-2009 & SW 846/6010 & \\
\hline Calcium & 33.2 & 0.200 & $\mathrm{mg} / \mathrm{L}$ & 4 & 25-Jun-2009 & 25-Jun-2009 & SW 846/6010 & B \\
\hline Iron & 6.44 & 0.200 & $\mathrm{mg} / \mathrm{L}$ & 4 & 25-Jun-2009 & 25-Jun-2009 & SW 846/6010 & \\
\hline Magnesium & 6.24 & 0.200 & $\mathrm{mg} / \mathrm{L}$ & 4 & 25-Jun-2009 & 25-Jun-2009 & SW 846/6010 & \\
\hline Potassium & 4.84 & 0.200 & $\mathrm{mg} / \mathrm{L}$ & 4 & 25-Jun-2009 & 25-Jun-2009 & SW 846/6010 & B \\
\hline Sodium & 5.02 & 0.200 & $\mathrm{mg} / \mathrm{L}$ & 4 & 25-Jun-2009 & 25-Jun-2009 & SW 846/6010 & B \\
\hline Antimony & 0.0085 & 0.0020 & $\mathrm{mg} / \mathrm{L}$ & 4 & 17-Jun-2009 & 17-Jun-2009 & SW 846/6020 & \\
\hline Arsenic & 0.117 & 0.0020 & $\mathrm{mg} / \mathrm{L}$ & 4 & 17-Jun-2009 & 17-Jun-2009 & SW 846/6020 & \\
\hline Barium & 0.324 & 0.0020 & $\mathrm{mg} / \mathrm{L}$ & 4 & 17-Jun-2009 & 17-Jun-2009 & SW 846/6020 & B \\
\hline Beryllium & 0.0024 & 0.0020 & $\mathrm{mg} / \mathrm{L}$ & 4 & 17-Jun-2009 & 17-Jun-2009 & SW 846/6020 & \\
\hline Cadmium & 0.0012 & 0.0020 & $\mathrm{mg} / \mathrm{L}$ & 4 & 17-Jun-2009 & 17-Jun-2009 & SW 846/6020 & $\mathrm{J}$ \\
\hline Chromium & 0.0209 & 0.0020 & $\mathrm{mg} / \mathrm{L}$ & 4 & 17-Jun-2009 & 17-Jun-2009 & SW 846/6020 & \\
\hline Cobalt & 0.0110 & 0.0020 & $\mathrm{mg} / \mathrm{L}$ & 4 & 17-Jun-2009 & 17-Jun-2009 & SW 846/6020 & \\
\hline Copper & 0.0413 & 0.0020 & $\mathrm{mg} / \mathrm{L}$ & 4 & 17-Jun-2009 & 17-Jun-2009 & SW 846/6020 & B \\
\hline Lead & 0.0210 & 0.0020 & $\mathrm{mg} / \mathrm{L}$ & 4 & 17-Jun-2009 & 17-Jun-2009 & SW 846/6020 & \\
\hline Manganese & 0.0928 & 0.0020 & $\mathrm{mg} / \mathrm{L}$ & 4 & 17-Jun-2009 & 17-Jun-2009 & SW 846/6020 & \\
\hline Molybdenum & 0.0759 & 0.0020 & $\mathrm{mg} / \mathrm{L}$ & 4 & 17-Jun-2009 & 17-Jun-2009 & SW 846/6020 & \\
\hline Nickel & 0.0256 & 0.0020 & $\mathrm{mg} / \mathrm{L}$ & 4 & 17-Jun-2009 & 17-Jun-2009 & SW 846/6020 & \\
\hline Selenium & 0.0086 & 0.0020 & $\mathrm{mg} / \mathrm{L}$ & 4 & 17-Jun-2009 & 17-Jun-2009 & SW 846/6020 & \\
\hline Silver & ND & 0.0020 & $\mathrm{mg} / \mathrm{L}$ & 4 & 17-Jun-2009 & 17-Jun-2009 & SW 846/6020 & $\mathrm{U}$ \\
\hline Thallium & 0.0018 & 0.0020 & $\mathrm{mg} / \mathrm{L}$ & 4 & 17-Jun-2009 & 17-Jun-2009 & SW 846/6020 & J \\
\hline Vanadium & 0.113 & 0.0020 & $\mathrm{mg} / \mathrm{L}$ & 4 & 17-Jun-2009 & 17-Jun-2009 & SW 846/6020 & \\
\hline Zinc & 0.0564 & 0.0020 & $\mathrm{mg} / \mathrm{L}$ & 4 & 17-Jun-2009 & 17-Jun-2009 & SW 846/6020 & B \\
\hline
\end{tabular}




\section{USACE ERDC-EP-C \\ 3909 Halls Ferry Road \\ Vicksburg, MS 39180-6199}

ERDC - ECB

Project: TVA Fly Ash Project

Reported:

Project Manager: Tony Bednar

03-Sep-2009

DRAFT: EMR MT N2 Hour 48 Rep A

9061503-04 (Water)

\begin{tabular}{|c|c|c|c|c|c|c|c|c|}
\hline & & porting & & & & & & \\
\hline Analyte & Result & Limit & Units & Dilution & Prepared & Analyzed & Method & Notes \\
\hline
\end{tabular}

ERDC- EL-EP-C (Environmental Chemistry Branch)

DRAFT: Wet Chemistry

\begin{tabular}{llllllll}
\hline Hardness & $\mathbf{3 9 . 5}$ & 1.32 & $\begin{array}{c}\text { mg equil } \\
\text { CaCO3/ }\end{array}$ & 1 & 25-Jun-2009 & 25-Jun-2009
\end{tabular}

DRAFT: Metals by EPA 6000/7000 Series Methods

\begin{tabular}{|c|c|c|c|c|c|c|c|c|}
\hline Mercury & ND & 0.000005 & $\mathrm{mg} / \mathrm{L}$ & 1 & 17-Jun-2009 & 01-Jul-2009 & EPA 7471A & $\mathrm{U}$ \\
\hline Strontium & 0.150 & 0.0200 & $\mathrm{mg} / \mathrm{L}$ & 1 & 25-Jun-2009 & 25-Jun-2009 & EPA $6010 \mathrm{~B}$ & \\
\hline Aluminum & 0.0733 & 0.200 & $\mathrm{mg} / \mathrm{L}$ & 1 & 25-Jun-2009 & 25-Jun-2009 & SW 846/6010 & J \\
\hline Calcium & 12.0 & 0.200 & $\mathrm{mg} / \mathrm{L}$ & 1 & 25-Jun-2009 & 25-Jun-2009 & SW 846/6010 & \\
\hline Iron & ND & 0.200 & $\mathrm{mg} / \mathrm{L}$ & 1 & 25-Jun-2009 & 25-Jun-2009 & SW 846/6010 & $\mathrm{U}$ \\
\hline Magnesium & 2.32 & 0.200 & $\mathrm{mg} / \mathrm{L}$ & 1 & 25-Jun-2009 & 25-Jun-2009 & SW 846/6010 & \\
\hline Potassium & 1.38 & 0.200 & $\mathrm{mg} / \mathrm{L}$ & 1 & 25-Jun-2009 & 25-Jun-2009 & SW 846/6010 & \\
\hline Sodium & 2.51 & 0.200 & $\mathrm{mg} / \mathrm{L}$ & 1 & 25-Jun-2009 & 25-Jun-2009 & SW 846/6010 & \\
\hline Antimony & 0.0010 & 0.0010 & $\mathrm{mg} / \mathrm{L}$ & 2 & 16-Jun-2009 & 16-Jun-2009 & SW 846/6020 & \\
\hline Arsenic & 0.0433 & 0.0010 & $\mathrm{mg} / \mathrm{L}$ & 2 & 16-Jun-2009 & 16-Jun-2009 & SW 846/6020 & \\
\hline Barium & 0.0975 & 0.0010 & $\mathrm{mg} / \mathrm{L}$ & 2 & 16-Jun-2009 & 16-Jun-2009 & SW 846/6020 & \\
\hline Beryllium & ND & 0.0010 & $\mathrm{mg} / \mathrm{L}$ & 2 & 16-Jun-2009 & 16-Jun-2009 & SW 846/6020 & $\mathrm{U}$ \\
\hline Cadmium & ND & 0.0010 & $\mathrm{mg} / \mathrm{L}$ & 2 & 16-Jun-2009 & 16-Jun-2009 & SW 846/6020 & $\mathrm{U}$ \\
\hline Chromium & ND & 0.0010 & $\mathrm{mg} / \mathrm{L}$ & 2 & 16-Jun-2009 & 16-Jun-2009 & SW 846/6020 & $\mathrm{U}$ \\
\hline Cobalt & ND & 0.0010 & $\mathrm{mg} / \mathrm{L}$ & 2 & 16-Jun-2009 & 16-Jun-2009 & SW 846/6020 & $\mathrm{U}$ \\
\hline Copper & 0.0017 & 0.0010 & $\mathrm{mg} / \mathrm{L}$ & 2 & 16-Jun-2009 & 16-Jun-2009 & SW 846/6020 & \\
\hline Lead & ND & 0.0010 & $\mathrm{mg} / \mathrm{L}$ & 2 & 16-Jun-2009 & 16-Jun-2009 & SW 846/6020 & $\mathrm{U}$ \\
\hline Manganese & 0.0023 & 0.0010 & $\mathrm{mg} / \mathrm{L}$ & 2 & 16-Jun-2009 & 16-Jun-2009 & SW 846/6020 & \\
\hline Molybdenum & 0.0019 & 0.0010 & $\mathrm{mg} / \mathrm{L}$ & 2 & 16-Jun-2009 & 16-Jun-2009 & SW 846/6020 & \\
\hline Nickel & 0.0006 & 0.0010 & $\mathrm{mg} / \mathrm{L}$ & 2 & 16-Jun-2009 & 16-Jun-2009 & SW 846/6020 & $\mathrm{J}$ \\
\hline Selenium & 0.0018 & 0.0010 & $\mathrm{mg} / \mathrm{L}$ & 2 & 16-Jun-2009 & 16-Jun-2009 & SW 846/6020 & \\
\hline Silver & ND & 0.0010 & $\mathrm{mg} / \mathrm{L}$ & 2 & 16-Jun-2009 & 16-Jun-2009 & SW 846/6020 & $\mathrm{U}$ \\
\hline Thallium & 0.0005 & 0.0010 & $\mathrm{mg} / \mathrm{L}$ & 2 & 16-Jun-2009 & 16-Jun-2009 & SW 846/6020 & $\mathrm{J}$ \\
\hline Vanadium & 0.0286 & 0.0010 & $\mathrm{mg} / \mathrm{L}$ & 2 & 16-Jun-2009 & 16-Jun-2009 & SW 846/6020 & \\
\hline Zinc & 0.0074 & 0.0010 & $\mathrm{mg} / \mathrm{L}$ & 2 & 16-Jun-2009 & 16-Jun-2009 & SW 846/6020 & B \\
\hline
\end{tabular}




\section{USACE ERDC-EP-C \\ 3909 Halls Ferry Road \\ Vicksburg, MS 39180-6199}

ERDC - ECB

Project: TVA Fly Ash Project

Reported:

$--,-$

Project Manager: Tony Bednar

03-Sep-2009

DRAFT: EMR MT N2 Hour 48 Rep A

9061503-04 (Water)

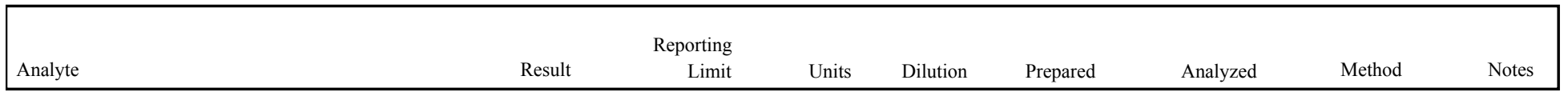

ERDC- EL-EP-C (Environmental Chemistry Branch)

DRAFT: Miscellaneous Physical/Conventional Chemistry Parameters

\begin{tabular}{lrrrrrrr}
\hline Bromide & $\mathrm{ND}$ & 0.100 & $\mathrm{mg} / \mathrm{L}$ & 1 & $15-\mathrm{Jul}-2009$ & $15-\mathrm{Jul}-2009$ & EPA 300.0 \\
Chloride & $\mathbf{2 . 3 9}$ & 1.00 & $\mathrm{mg} / \mathrm{L}$ & 1 & $15-\mathrm{Jul}-2009$ & $15-\mathrm{Jul}-2009$ & EPA 300.0 \\
Nitrate as N & $\mathbf{0 . 4 2 4}$ & 0.100 & $\mathrm{mg} / \mathrm{L}$ & 1 & $15-J u l-2009$ & $15-\mathrm{Jul}-2009$ & EPA 300.0 \\
Sulfate & $\mathbf{1 2 . 7}$ & 0.100 & $\mathrm{mg} / \mathrm{L}$ & 1 & $15-J u l-2009$ & $15-J u l-2009$ & EPA 300.0
\end{tabular}




\section{USACE ERDC-EP-C \\ 3909 Halls Ferry Road \\ Vicksburg, MS 39180-6199}

ERDC - ECB

$-\cdot$,
Project: TVA Fly Ash Project

Project Manager: Tony Bednar
Reported:

03-Sep-2009

DRAFT: EMR MT N2 Hour 48 Rep B

9061503-05 (Water)

\begin{tabular}{|c|c|c|c|c|c|c|c|c|}
\hline Analyte & Result & $\begin{array}{r}\text { porting } \\
\text { Limit }\end{array}$ & Units & Dilution & Prepared & Analyzed & Method & Notes \\
\hline
\end{tabular}

ERDC- EL-EP-C (Environmental Chemistry Branch)

DRAFT: Wet Chemistry

\begin{tabular}{llllllll}
\hline Hardness & $\mathbf{3 8 . 8}$ & 1.32 & $\begin{array}{c}\text { mg equil } \\
\text { CaCO3/ }\end{array}$ & 1 & 25-Jun-2009 & 25-Jun-2009
\end{tabular}

$\mathrm{L}$

DRAFT: Metals by EPA 6000/7000 Series Methods

\begin{tabular}{|c|c|c|c|c|c|c|c|c|}
\hline Mercury & ND & 0.000005 & $\mathrm{mg} / \mathrm{L}$ & 1 & 17-Jun-2009 & 01-Jul-2009 & EPA 7471A & $\mathrm{U}$ \\
\hline Strontium & 0.145 & 0.0200 & $\mathrm{mg} / \mathrm{L}$ & 1 & 25-Jun-2009 & 25-Jun-2009 & EPA 6010B & \\
\hline Aluminum & 0.0601 & 0.200 & $\mathrm{mg} / \mathrm{L}$ & 1 & 25-Jun-2009 & 25-Jun-2009 & SW 846/6010 & $\mathrm{J}$ \\
\hline Calcium & 11.7 & 0.200 & $\mathrm{mg} / \mathrm{L}$ & 1 & 25-Jun-2009 & 25-Jun-2009 & SW 846/6010 & \\
\hline Iron & ND & 0.200 & $\mathrm{mg} / \mathrm{L}$ & 1 & 25-Jun-2009 & 25-Jun-2009 & SW 846/6010 & $\mathrm{U}$ \\
\hline Magnesium & 2.32 & 0.200 & $\mathrm{mg} / \mathrm{L}$ & 1 & 25-Jun-2009 & 25-Jun-2009 & SW 846/6010 & \\
\hline Potassium & 1.38 & 0.200 & $\mathrm{mg} / \mathrm{L}$ & 1 & 25-Jun-2009 & 25-Jun-2009 & SW 846/6010 & \\
\hline Sodium & 2.67 & 0.200 & $\mathrm{mg} / \mathrm{L}$ & 1 & 25-Jun-2009 & 25-Jun-2009 & SW 846/6010 & \\
\hline Antimony & 0.0009 & 0.0010 & $\mathrm{mg} / \mathrm{L}$ & 2 & 16-Jun-2009 & 16-Jun-2009 & SW 846/6020 & J \\
\hline Arsenic & 0.0400 & 0.0010 & $\mathrm{mg} / \mathrm{L}$ & 2 & 16-Jun-2009 & 16-Jun-2009 & SW 846/6020 & \\
\hline Barium & 0.0952 & 0.0010 & $\mathrm{mg} / \mathrm{L}$ & 2 & 16-Jun-2009 & 16-Jun-2009 & SW 846/6020 & \\
\hline Beryllium & ND & 0.0010 & $\mathrm{mg} / \mathrm{L}$ & 2 & 16-Jun-2009 & 16-Jun-2009 & SW 846/6020 & $\mathrm{U}$ \\
\hline Cadmium & ND & 0.0010 & $\mathrm{mg} / \mathrm{L}$ & 2 & 16-Jun-2009 & 16-Jun-2009 & SW 846/6020 & $\mathrm{U}$ \\
\hline Chromium & ND & 0.0010 & $\mathrm{mg} / \mathrm{L}$ & 2 & 16-Jun-2009 & 16-Jun-2009 & SW 846/6020 & $\mathrm{U}$ \\
\hline Cobalt & ND & 0.0010 & $\mathrm{mg} / \mathrm{L}$ & 2 & 16-Jun-2009 & 16-Jun-2009 & SW 846/6020 & $\mathrm{U}$ \\
\hline Copper & 0.0014 & 0.0010 & $\mathrm{mg} / \mathrm{L}$ & 2 & 16-Jun-2009 & 16-Jun-2009 & SW 846/6020 & \\
\hline Lead & ND & 0.0010 & $\mathrm{mg} / \mathrm{L}$ & 2 & 16-Jun-2009 & 16-Jun-2009 & SW 846/6020 & $\mathrm{U}$ \\
\hline Manganese & 0.0019 & 0.0010 & $\mathrm{mg} / \mathrm{L}$ & 2 & 16-Jun-2009 & 16-Jun-2009 & SW 846/6020 & \\
\hline Molybdenum & 0.0019 & 0.0010 & $\mathrm{mg} / \mathrm{L}$ & 2 & 16-Jun-2009 & 16-Jun-2009 & SW 846/6020 & \\
\hline Nickel & 0.0005 & 0.0010 & $\mathrm{mg} / \mathrm{L}$ & 2 & 16-Jun-2009 & 16-Jun-2009 & SW 846/6020 & $\mathrm{J}$ \\
\hline Selenium & 0.0017 & 0.0010 & $\mathrm{mg} / \mathrm{L}$ & 2 & 16-Jun-2009 & 16-Jun-2009 & SW 846/6020 & \\
\hline Silver & ND & 0.0010 & $\mathrm{mg} / \mathrm{L}$ & 2 & 16-Jun-2009 & 16-Jun-2009 & SW 846/6020 & $\mathrm{U}$ \\
\hline Thallium & 0.0005 & 0.0010 & $\mathrm{mg} / \mathrm{L}$ & 2 & 16-Jun-2009 & 16-Jun-2009 & SW 846/6020 & $\mathrm{J}$ \\
\hline Vanadium & 0.0274 & 0.0010 & $\mathrm{mg} / \mathrm{L}$ & 2 & 16-Jun-2009 & 16-Jun-2009 & SW 846/6020 & \\
\hline Zinc & 0.0049 & 0.0010 & $\mathrm{mg} / \mathrm{L}$ & 2 & 16-Jun-2009 & 16-Jun-2009 & SW 846/6020 & B \\
\hline
\end{tabular}




\section{USACE ERDC-EP-C \\ 3909 Halls Ferry Road \\ Vicksburg, MS 39180-6199}

ERDC - ECB

Project: TVA Fly Ash Project

Reported:

$--,-$

Project Manager: Tony Bednar

03-Sep-2009

DRAFT: EMR MT N2 Hour 48 Rep B

9061503-05 (Water)

\begin{tabular}{|c|c|c|c|c|c|c|c|c|}
\hline & & orting & & & & & & \\
\hline Analyte & Result & Limit & Units & Dilution & Prepared & Analyzed & Method & Notes \\
\hline
\end{tabular}

ERDC- EL-EP-C (Environmental Chemistry Branch)

DRAFT: Miscellaneous Physical/Conventional Chemistry Parameters

\begin{tabular}{lrrrrrrr}
\hline Bromide & $\mathrm{ND}$ & 0.100 & $\mathrm{mg} / \mathrm{L}$ & 1 & $15-J u l-2009$ & $15-\mathrm{Jul}-2009$ & EPA 300.0 \\
Chloride & $\mathbf{2 . 7 1}$ & 1.00 & $\mathrm{mg} / \mathrm{L}$ & 1 & $15-J u l-2009$ & $15-\mathrm{Jul}-2009$ & EPA 300.0 \\
Nitrate as N & $\mathbf{0 . 3 9 6}$ & 0.100 & $\mathrm{mg} / \mathrm{L}$ & 1 & $15-J u l-2009$ & $15-J u l-2009$ & EPA 300.0 \\
Sulfate & $\mathbf{1 2 . 7}$ & 0.100 & $\mathrm{mg} / \mathrm{L}$ & 1 & $15-J u l-2009$ & $15-J u l-2009$ & EPA 300.0
\end{tabular}




\section{USACE ERDC-EP-C \\ 3909 Halls Ferry Road \\ Vicksburg, MS 39180-6199}

ERDC - ECB

$-\cdot$,
Project: TVA Fly Ash Project

Project Manager: Tony Bednar
Reported:

03-Sep-2009

DRAFT: EMR MT N2 Hour 48 Rep C

9061503-06 (Water)

\begin{tabular}{|c|c|c|c|c|c|c|c|c|}
\hline Analyte & Result & $\begin{array}{r}\text { porting } \\
\text { Limit }\end{array}$ & Units & Dilution & Prepared & Analyzed & Method & Notes \\
\hline
\end{tabular}

ERDC- EL-EP-C (Environmental Chemistry Branch)

DRAFT: Wet Chemistry

\begin{tabular}{llllllll}
\hline Hardness & $\mathbf{3 8 . 7}$ & 1.32 & $\begin{array}{c}\text { mg equil } \\
\text { CaCO3/ }\end{array}$ & 1 & 25-Jun-2009 & 25-Jun-2009
\end{tabular}

DRAFT: Metals by EPA 6000/7000 Series Methods

\begin{tabular}{|c|c|c|c|c|c|c|c|c|}
\hline Mercury & 0.000011 & 0.000005 & $\mathrm{mg} / \mathrm{L}$ & 1 & 17-Jun-2009 & 01-Jul-2009 & EPA 7471A & \\
\hline Strontium & 0.141 & 0.0200 & $\mathrm{mg} / \mathrm{L}$ & 1 & 25-Jun-2009 & 25-Jun-2009 & EPA 6010B & \\
\hline Aluminum & 0.0672 & 0.200 & $\mathrm{mg} / \mathrm{L}$ & 1 & 25-Jun-2009 & 25-Jun-2009 & SW 846/6010 & J \\
\hline Calcium & 11.7 & 0.200 & $\mathrm{mg} / \mathrm{L}$ & 1 & 25-Jun-2009 & 25-Jun-2009 & SW 846/6010 & \\
\hline Iron & ND & 0.200 & $\mathrm{mg} / \mathrm{L}$ & 1 & 25-Jun-2009 & 25-Jun-2009 & SW 846/6010 & $\mathrm{U}$ \\
\hline Magnesium & 2.32 & 0.200 & $\mathrm{mg} / \mathrm{L}$ & 1 & 25-Jun-2009 & 25-Jun-2009 & SW 846/6010 & \\
\hline Potassium & 1.39 & 0.200 & $\mathrm{mg} / \mathrm{L}$ & 1 & 25-Jun-2009 & 25-Jun-2009 & SW 846/6010 & \\
\hline Sodium & 2.58 & 0.200 & $\mathrm{mg} / \mathrm{L}$ & 1 & 25-Jun-2009 & 25-Jun-2009 & SW 846/6010 & \\
\hline Antimony & 0.0008 & 0.0010 & $\mathrm{mg} / \mathrm{L}$ & 2 & 16-Jun-2009 & 16-Jun-2009 & SW 846/6020 & J \\
\hline Arsenic & 0.0414 & 0.0010 & $\mathrm{mg} / \mathrm{L}$ & 2 & 16-Jun-2009 & 16-Jun-2009 & SW 846/6020 & \\
\hline Barium & 0.0918 & 0.0010 & $\mathrm{mg} / \mathrm{L}$ & 2 & 16-Jun-2009 & 16-Jun-2009 & SW 846/6020 & \\
\hline Beryllium & ND & 0.0010 & $\mathrm{mg} / \mathrm{L}$ & 2 & 16-Jun-2009 & 16-Jun-2009 & SW 846/6020 & $\mathrm{U}$ \\
\hline Cadmium & ND & 0.0010 & $\mathrm{mg} / \mathrm{L}$ & 2 & 16-Jun-2009 & 16-Jun-2009 & SW 846/6020 & $\mathrm{U}$ \\
\hline Chromium & ND & 0.0010 & $\mathrm{mg} / \mathrm{L}$ & 2 & 16-Jun-2009 & 16-Jun-2009 & SW 846/6020 & $\mathrm{U}$ \\
\hline Cobalt & ND & 0.0010 & $\mathrm{mg} / \mathrm{L}$ & 2 & 16-Jun-2009 & 16-Jun-2009 & SW 846/6020 & U \\
\hline Copper & 0.0016 & 0.0010 & $\mathrm{mg} / \mathrm{L}$ & 2 & 16-Jun-2009 & 16-Jun-2009 & SW 846/6020 & \\
\hline Lead & ND & 0.0010 & $\mathrm{mg} / \mathrm{L}$ & 2 & 16-Jun-2009 & 16-Jun-2009 & SW 846/6020 & $\mathrm{U}$ \\
\hline Manganese & 0.0016 & 0.0010 & $\mathrm{mg} / \mathrm{L}$ & 2 & 16-Jun-2009 & 16-Jun-2009 & SW 846/6020 & \\
\hline Molybdenum & 0.0019 & 0.0010 & $\mathrm{mg} / \mathrm{L}$ & 2 & 16-Jun-2009 & 16-Jun-2009 & SW 846/6020 & \\
\hline Nickel & 0.0006 & 0.0010 & $\mathrm{mg} / \mathrm{L}$ & 2 & 16-Jun-2009 & 16-Jun-2009 & SW 846/6020 & J \\
\hline Selenium & 0.0017 & 0.0010 & $\mathrm{mg} / \mathrm{L}$ & 2 & 16-Jun-2009 & 16-Jun-2009 & SW 846/6020 & \\
\hline Silver & ND & 0.0010 & $\mathrm{mg} / \mathrm{L}$ & 2 & 16-Jun-2009 & 16-Jun-2009 & SW 846/6020 & $\mathrm{U}$ \\
\hline Thallium & 0.00044 & 0.0010 & $\mathrm{mg} / \mathrm{L}$ & 2 & 16-Jun-2009 & 16-Jun-2009 & SW 846/6020 & J \\
\hline Vanadium & 0.0287 & 0.0010 & $\mathrm{mg} / \mathrm{L}$ & 2 & 16-Jun-2009 & 16-Jun-2009 & SW 846/6020 & \\
\hline Zinc & 0.0054 & 0.0010 & $\mathrm{mg} / \mathrm{L}$ & 2 & 16-Jun-2009 & 16-Jun-2009 & SW 846/6020 & B \\
\hline
\end{tabular}




\section{USACE ERDC-EP-C \\ 3909 Halls Ferry Road \\ Vicksburg, MS 39180-6199}

ERDC - ECB

Project: TVA Fly Ash Project

Reported:

$--,-$

Project Manager: Tony Bednar

03-Sep-2009

DRAFT: EMR MT N2 Hour 48 Rep C

9061503-06 (Water)

\begin{tabular}{|c|c|c|c|c|c|c|c|c|}
\hline & & orting & & & & & & \\
\hline Analyte & Result & Limit & Units & Dilution & Prepared & Analyzed & Method & Notes \\
\hline
\end{tabular}

ERDC- EL-EP-C (Environmental Chemistry Branch)

DRAFT: Miscellaneous Physical/Conventional Chemistry Parameters

\begin{tabular}{lrrrrrrr}
\hline Bromide & $\mathrm{ND}$ & 0.100 & $\mathrm{mg} / \mathrm{L}$ & 1 & $15-J u l-2009$ & $15-\mathrm{Jul}-2009$ & EPA 300.0 \\
Chloride & $\mathbf{2 . 4 9}$ & 1.00 & $\mathrm{mg} / \mathrm{L}$ & 1 & $15-J u l-2009$ & $15-\mathrm{Jul}-2009$ & EPA 300.0 \\
Nitrate as N & $\mathbf{0 . 4 1 5}$ & 0.100 & $\mathrm{mg} / \mathrm{L}$ & 1 & $15-J u l-2009$ & $15-J u l-2009$ & EPA 300.0 \\
Sulfate & $\mathbf{1 2 . 6}$ & 0.100 & $\mathrm{mg} / \mathrm{L}$ & 1 & $15-J u l-2009$ & $15-J u l-2009$ & EPA 300.0
\end{tabular}




\section{USACE ERDC-EP-C \\ 3909 Halls Ferry Road \\ Vicksburg, MS 39180-6199}

ERDC - ECB

$-\cdot$,
Project: TVA Fly Ash Project

Project Manager: Tony Bednar
Reported:

03-Sep-2009

DRAFT: EMR MT O2 Hour 48 Rep A

9061503-10 (Water)

\begin{tabular}{|c|c|c|c|c|c|c|c|c|}
\hline Analyte & Result & $\begin{array}{r}\text { porting } \\
\text { Limit }\end{array}$ & Units & Dilution & Prepared & Analyzed & Method & Notes \\
\hline
\end{tabular}

ERDC- EL-EP-C (Environmental Chemistry Branch)

DRAFT: Wet Chemistry

\begin{tabular}{llllllll}
\hline Hardness & $\mathbf{3 7 . 1}$ & 1.32 & $\begin{array}{c}\text { mg equil } \\
\text { CaCO3/ }\end{array}$ & 1 & 25-Jun-2009 & 25-Jun-2009
\end{tabular}

DRAFT: Metals by EPA 6000/7000 Series Methods

\begin{tabular}{|c|c|c|c|c|c|c|c|c|}
\hline Mercury & 0.000016 & 0.000005 & $\mathrm{mg} / \mathrm{L}$ & 1 & 17-Jun-2009 & 01-Jul-2009 & EPA 7471A & \\
\hline Strontium & 0.124 & 0.0200 & $\mathrm{mg} / \mathrm{L}$ & 1 & 25-Jun-2009 & 25-Jun-2009 & EPA $6010 B$ & \\
\hline Aluminum & ND & 0.200 & $\mathrm{mg} / \mathrm{L}$ & 1 & 25-Jun-2009 & 25-Jun-2009 & SW 846/6010 & $\mathrm{U}$ \\
\hline Calcium & 11.2 & 0.200 & $\mathrm{mg} / \mathrm{L}$ & 1 & 25-Jun-2009 & 25-Jun-2009 & SW 846/6010 & \\
\hline Iron & ND & 0.200 & $\mathrm{mg} / \mathrm{L}$ & 1 & 25-Jun-2009 & 25-Jun-2009 & SW 846/6010 & $\mathrm{U}$ \\
\hline Magnesium & 2.25 & 0.200 & $\mathrm{mg} / \mathrm{L}$ & 1 & 25-Jun-2009 & 25-Jun-2009 & SW 846/6010 & \\
\hline Potassium & 1.43 & 0.200 & $\mathrm{mg} / \mathrm{L}$ & 1 & 25-Jun-2009 & 25-Jun-2009 & SW 846/6010 & \\
\hline Sodium & 2.57 & 0.200 & $\mathrm{mg} / \mathrm{L}$ & 1 & 25-Jun-2009 & 25-Jun-2009 & SW 846/6010 & \\
\hline Antimony & 0.0006 & 0.0010 & $\mathrm{mg} / \mathrm{L}$ & 2 & 16-Jun-2009 & 16-Jun-2009 & SW 846/6020 & J \\
\hline Arsenic & 0.0310 & 0.0010 & $\mathrm{mg} / \mathrm{L}$ & 2 & 16-Jun-2009 & 16-Jun-2009 & SW 846/6020 & \\
\hline Barium & 0.0920 & 0.0010 & $\mathrm{mg} / \mathrm{L}$ & 2 & 16-Jun-2009 & 16-Jun-2009 & SW 846/6020 & \\
\hline Beryllium & ND & 0.0010 & $\mathrm{mg} / \mathrm{L}$ & 2 & 16-Jun-2009 & 16-Jun-2009 & SW 846/6020 & $\mathrm{U}$ \\
\hline Cadmium & ND & 0.0010 & $\mathrm{mg} / \mathrm{L}$ & 2 & 16-Jun-2009 & 16-Jun-2009 & SW 846/6020 & $\mathrm{U}$ \\
\hline Chromium & ND & 0.0010 & $\mathrm{mg} / \mathrm{L}$ & 2 & 16-Jun-2009 & 16-Jun-2009 & SW 846/6020 & $\mathrm{U}$ \\
\hline Cobalt & ND & 0.0010 & $\mathrm{mg} / \mathrm{L}$ & 2 & 16-Jun-2009 & 16-Jun-2009 & SW 846/6020 & $\mathrm{U}$ \\
\hline Copper & 0.0016 & 0.0010 & $\mathrm{mg} / \mathrm{L}$ & 2 & 16-Jun-2009 & 16-Jun-2009 & SW 846/6020 & \\
\hline Lead & ND & 0.0010 & $\mathrm{mg} / \mathrm{L}$ & 2 & 16-Jun-2009 & 16-Jun-2009 & SW 846/6020 & $\mathrm{U}$ \\
\hline Manganese & 0.0020 & 0.0010 & $\mathrm{mg} / \mathrm{L}$ & 2 & 16-Jun-2009 & 16-Jun-2009 & SW 846/6020 & \\
\hline Molybdenum & 0.0017 & 0.0010 & $\mathrm{mg} / \mathrm{L}$ & 2 & 16-Jun-2009 & 16-Jun-2009 & SW 846/6020 & \\
\hline Nickel & 0.0007 & 0.0010 & $\mathrm{mg} / \mathrm{L}$ & 2 & 16-Jun-2009 & 16-Jun-2009 & SW 846/6020 & $\mathrm{J}$ \\
\hline Selenium & 0.0013 & 0.0010 & $\mathrm{mg} / \mathrm{L}$ & 2 & 16-Jun-2009 & 16-Jun-2009 & SW 846/6020 & \\
\hline Silver & ND & 0.0010 & $\mathrm{mg} / \mathrm{L}$ & 2 & 16-Jun-2009 & 16-Jun-2009 & SW 846/6020 & $\mathrm{U}$ \\
\hline Thallium & 0.0004 & 0.0010 & $\mathrm{mg} / \mathrm{L}$ & 2 & 16-Jun-2009 & 16-Jun-2009 & SW 846/6020 & $\mathrm{J}$ \\
\hline Vanadium & 0.0217 & 0.0010 & $\mathrm{mg} / \mathrm{L}$ & 2 & 16-Jun-2009 & 16-Jun-2009 & SW 846/6020 & \\
\hline Zinc & 0.0055 & 0.0010 & $\mathrm{mg} / \mathrm{L}$ & 2 & 16-Jun-2009 & 16-Jun-2009 & SW 846/6020 & B \\
\hline
\end{tabular}




\section{USACE ERDC-EP-C \\ 3909 Halls Ferry Road \\ Vicksburg, MS 39180-6199}

ERDC - ECB

Project: TVA Fly Ash Project

Reported:

$--,-$

Project Manager: Tony Bednar

03-Sep-2009

DRAFT: EMR MT O2 Hour 48 Rep A

9061503-10 (Water)

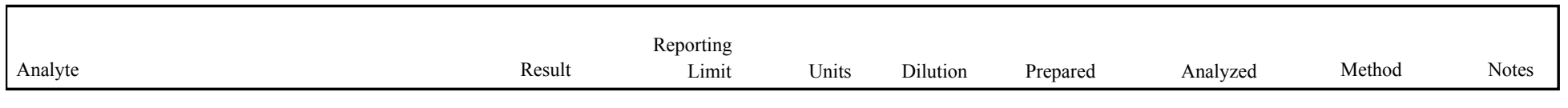

ERDC- EL-EP-C (Environmental Chemistry Branch)

DRAFT: Miscellaneous Physical/Conventional Chemistry Parameters

\begin{tabular}{lrrrrrrr}
\hline Bromide & $\mathrm{ND}$ & 0.100 & $\mathrm{mg} / \mathrm{L}$ & 1 & $15-J u l-2009$ & $15-\mathrm{Jul}-2009$ & EPA 300.0 \\
Chloride & $\mathbf{2 . 4 9}$ & 1.00 & $\mathrm{mg} / \mathrm{L}$ & 1 & $15-J u l-2009$ & $15-\mathrm{Jul}-2009$ & EPA 300.0 \\
Nitrate as N & $\mathbf{0 . 3 4 7}$ & 0.100 & $\mathrm{mg} / \mathrm{L}$ & 1 & $15-J u l-2009$ & $15-\mathrm{Jul}-2009$ & EPA 300.0 \\
Sulfate & $\mathbf{1 2 . 4}$ & 0.100 & $\mathrm{mg} / \mathrm{L}$ & 1 & $15-J u l-2009$ & $15-J u l-2009$ & EPA 300.0
\end{tabular}




\section{USACE ERDC-EP-C \\ 3909 Halls Ferry Road \\ Vicksburg, MS 39180-6199}

ERDC - ECB

$-\cdot$,
Project: TVA Fly Ash Project

Project Manager: Tony Bednar
Reported:

03-Sep-2009

DRAFT: EMR MT O2 Hour 48 Rep B

9061503-11 (Water)

\begin{tabular}{|c|c|c|c|c|c|c|c|c|}
\hline Analyte & Result & $\begin{array}{r}\text { porting } \\
\text { Limit }\end{array}$ & Units & Dilution & Prepared & Analyzed & Method & Notes \\
\hline
\end{tabular}

ERDC- EL-EP-C (Environmental Chemistry Branch)

DRAFT: Wet Chemistry

\begin{tabular}{llcccccc}
\hline Hardness & $\mathbf{3 6 . 5}$ & 1.32 & $\begin{array}{c}\text { mg equil } \\
\text { CaCO3/ }\end{array}$ & 1 & 25-Jun-2009 & 25-Jun-2009
\end{tabular}

$\mathrm{L}$

DRAFT: Metals by EPA 6000/7000 Series Methods

\begin{tabular}{|c|c|c|c|c|c|c|c|c|}
\hline Mercury & ND & 0.000005 & $\mathrm{mg} / \mathrm{L}$ & 1 & 17-Jun-2009 & 01-Jul-2009 & EPA 7471A & $\mathrm{U}$ \\
\hline Strontium & 0.115 & 0.0200 & $\mathrm{mg} / \mathrm{L}$ & 1 & 25-Jun-2009 & 25-Jun-2009 & EPA 6010B & \\
\hline Aluminum & ND & 0.200 & $\mathrm{mg} / \mathrm{L}$ & 1 & 25-Jun-2009 & 25-Jun-2009 & SW 846/6010 & $\mathrm{U}$ \\
\hline Calcium & 10.9 & 0.200 & $\mathrm{mg} / \mathrm{L}$ & 1 & 25-Jun-2009 & 25-Jun-2009 & SW 846/6010 & \\
\hline Iron & ND & 0.200 & $\mathrm{mg} / \mathrm{L}$ & 1 & 25-Jun-2009 & 25-Jun-2009 & SW 846/6010 & $\mathrm{U}$ \\
\hline Magnesium & 2.28 & 0.200 & $\mathrm{mg} / \mathrm{L}$ & 1 & 25-Jun-2009 & 25-Jun-2009 & SW 846/6010 & \\
\hline Potassium & 1.39 & 0.200 & $\mathrm{mg} / \mathrm{L}$ & 1 & 25-Jun-2009 & 25-Jun-2009 & SW 846/6010 & \\
\hline Sodium & 2.54 & 0.200 & $\mathrm{mg} / \mathrm{L}$ & 1 & 25-Jun-2009 & 25-Jun-2009 & SW 846/6010 & \\
\hline Antimony & 0.0006 & 0.0010 & $\mathrm{mg} / \mathrm{L}$ & 2 & 16-Jun-2009 & 16-Jun-2009 & SW 846/6020 & J \\
\hline Arsenic & 0.0279 & 0.0010 & $\mathrm{mg} / \mathrm{L}$ & 2 & 16-Jun-2009 & 16-Jun-2009 & SW 846/6020 & \\
\hline Barium & 0.0861 & 0.0010 & $\mathrm{mg} / \mathrm{L}$ & 2 & 16-Jun-2009 & 16-Jun-2009 & SW 846/6020 & \\
\hline Beryllium & ND & 0.0010 & $\mathrm{mg} / \mathrm{L}$ & 2 & 16-Jun-2009 & 16-Jun-2009 & SW 846/6020 & $\mathrm{U}$ \\
\hline Cadmium & ND & 0.0010 & $\mathrm{mg} / \mathrm{L}$ & 2 & 16-Jun-2009 & 16-Jun-2009 & SW 846/6020 & $\mathrm{U}$ \\
\hline Chromium & ND & 0.0010 & $\mathrm{mg} / \mathrm{L}$ & 2 & 16-Jun-2009 & 16-Jun-2009 & SW 846/6020 & $\mathrm{U}$ \\
\hline Cobalt & ND & 0.0010 & $\mathrm{mg} / \mathrm{L}$ & 2 & 16-Jun-2009 & 16-Jun-2009 & SW 846/6020 & $\mathrm{U}$ \\
\hline Copper & 0.0017 & 0.0010 & $\mathrm{mg} / \mathrm{L}$ & 2 & 16-Jun-2009 & 16-Jun-2009 & SW 846/6020 & \\
\hline Lead & ND & 0.0010 & $\mathrm{mg} / \mathrm{L}$ & 2 & 16-Jun-2009 & 16-Jun-2009 & SW 846/6020 & $\mathrm{U}$ \\
\hline Manganese & 0.0015 & 0.0010 & $\mathrm{mg} / \mathrm{L}$ & 2 & 16-Jun-2009 & 16-Jun-2009 & SW 846/6020 & \\
\hline Molybdenum & 0.0016 & 0.0010 & $\mathrm{mg} / \mathrm{L}$ & 2 & 16-Jun-2009 & 16-Jun-2009 & SW 846/6020 & \\
\hline Nickel & 0.0007 & 0.0010 & $\mathrm{mg} / \mathrm{L}$ & 2 & 16-Jun-2009 & 16-Jun-2009 & SW 846/6020 & $\mathrm{J}$ \\
\hline Selenium & 0.0013 & 0.0010 & $\mathrm{mg} / \mathrm{L}$ & 2 & 16-Jun-2009 & 16-Jun-2009 & SW 846/6020 & \\
\hline Silver & ND & 0.0010 & $\mathrm{mg} / \mathrm{L}$ & 2 & 16-Jun-2009 & 16-Jun-2009 & SW 846/6020 & $\mathrm{U}$ \\
\hline Thallium & 0.0004 & 0.0010 & $\mathrm{mg} / \mathrm{L}$ & 2 & 16-Jun-2009 & 16-Jun-2009 & SW 846/6020 & $\mathrm{J}$ \\
\hline Vanadium & 0.0194 & 0.0010 & $\mathrm{mg} / \mathrm{L}$ & 2 & 16-Jun-2009 & 16-Jun-2009 & SW 846/6020 & \\
\hline Zinc & 0.0063 & 0.0010 & $\mathrm{mg} / \mathrm{L}$ & 2 & 16-Jun-2009 & 16-Jun-2009 & SW 846/6020 & B \\
\hline
\end{tabular}




\section{USACE ERDC-EP-C \\ 3909 Halls Ferry Road \\ Vicksburg, MS 39180-6199}

ERDC - ECB

Project: TVA Fly Ash Project

Reported:

$--,-$

Project Manager: Tony Bednar

03-Sep-2009

DRAFT: EMR MT O2 Hour 48 Rep B

9061503-11 (Water)

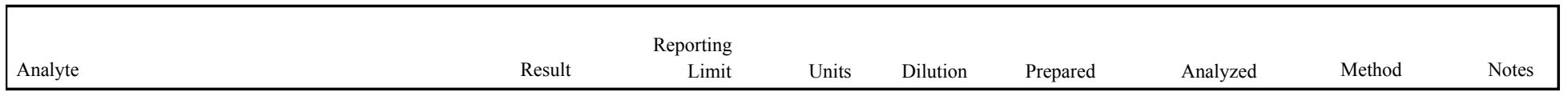

ERDC- EL-EP-C (Environmental Chemistry Branch)

DRAFT: Miscellaneous Physical/Conventional Chemistry Parameters

\begin{tabular}{lrrrrrrr}
\hline Bromide & $\mathrm{ND}$ & 0.100 & $\mathrm{mg} / \mathrm{L}$ & 1 & $15-\mathrm{Jul}-2009$ & $15-\mathrm{Jul}-2009$ & EPA 300.0 \\
Chloride & $\mathbf{2 . 5 0}$ & 1.00 & $\mathrm{mg} / \mathrm{L}$ & 1 & $15-\mathrm{Jul}-2009$ & $15-\mathrm{Jul}-2009$ & EPA 300.0 \\
Nitrate as N & $\mathbf{0 . 3 7 6}$ & 0.100 & $\mathrm{mg} / \mathrm{L}$ & 1 & $15-J u l-2009$ & $15-\mathrm{Jul}-2009$ & EPA 300.0 \\
Sulfate & $\mathbf{1 2 . 4}$ & 0.100 & $\mathrm{mg} / \mathrm{L}$ & 1 & $15-J u l-2009$ & $15-J u l-2009$ & EPA 300.0
\end{tabular}




\section{USACE ERDC-EP-C \\ 3909 Halls Ferry Road \\ Vicksburg, MS 39180-6199}

ERDC - ECB

$-,-$
Project: TVA Fly Ash Project

Project Manager: Tony Bednar
Reported:

03-Sep-2009

DRAFT: EMR MT O2 Hour 48 Rep C

9061503-12 (Water)

\begin{tabular}{|c|c|c|c|c|c|c|c|c|}
\hline Analyte & Result & $\begin{array}{r}\text { porting } \\
\text { Limit }\end{array}$ & Units & Dilution & Prepared & Analyzed & Method & Notes \\
\hline
\end{tabular}

ERDC- EL-EP-C (Environmental Chemistry Branch)

DRAFT: Wet Chemistry

\begin{tabular}{llllllll}
\hline Hardness & $\mathbf{3 8 . 4}$ & 1.32 & $\begin{array}{c}\text { mg equil } \\
\text { CaCO3/ }\end{array}$ & 1 & 25-Jun-2009 & 25-Jun-2009
\end{tabular}

DRAFT: Metals by EPA 6000/7000 Series Methods

\begin{tabular}{|c|c|c|c|c|c|c|c|c|}
\hline Mercury & 0.000008 & 0.000005 & $\mathrm{mg} / \mathrm{L}$ & 1 & 17-Jun-2009 & 01-Jul-2009 & EPA 7471A & \\
\hline Strontium & 0.141 & 0.0200 & $\mathrm{mg} / \mathrm{L}$ & 1 & 25-Jun-2009 & 25-Jun-2009 & EPA 6010B & \\
\hline Aluminum & 0.0507 & 0.200 & $\mathrm{mg} / \mathrm{L}$ & 1 & 25-Jun-2009 & 25-Jun-2009 & SW 846/6010 & J \\
\hline Calcium & 11.6 & 0.200 & $\mathrm{mg} / \mathrm{L}$ & 1 & 25-Jun-2009 & 25-Jun-2009 & SW 846/6010 & \\
\hline Iron & ND & 0.200 & $\mathrm{mg} / \mathrm{L}$ & 1 & 25-Jun-2009 & 25-Jun-2009 & SW 846/6010 & $\mathrm{U}$ \\
\hline Magnesium & 2.30 & 0.200 & $\mathrm{mg} / \mathrm{L}$ & 1 & 25-Jun-2009 & 25-Jun-2009 & SW 846/6010 & \\
\hline Potassium & 1.42 & 0.200 & $\mathrm{mg} / \mathrm{L}$ & 1 & 25-Jun-2009 & 25-Jun-2009 & SW 846/6010 & \\
\hline Sodium & 2.58 & 0.200 & $\mathrm{mg} / \mathrm{L}$ & 1 & 25-Jun-2009 & 25-Jun-2009 & SW 846/6010 & \\
\hline Antimony & 0.0007 & 0.0010 & $\mathrm{mg} / \mathrm{L}$ & 2 & 16-Jun-2009 & 16-Jun-2009 & SW 846/6020 & J \\
\hline Arsenic & 0.0358 & 0.0010 & $\mathrm{mg} / \mathrm{L}$ & 2 & 16-Jun-2009 & 16-Jun-2009 & SW 846/6020 & \\
\hline Barium & 0.0973 & 0.0010 & $\mathrm{mg} / \mathrm{L}$ & 2 & 16-Jun-2009 & 16-Jun-2009 & SW 846/6020 & \\
\hline Beryllium & ND & 0.0010 & $\mathrm{mg} / \mathrm{L}$ & 2 & 16-Jun-2009 & 16-Jun-2009 & SW 846/6020 & $\mathrm{U}$ \\
\hline Cadmium & ND & 0.0010 & $\mathrm{mg} / \mathrm{L}$ & 2 & 16-Jun-2009 & 16-Jun-2009 & SW 846/6020 & $\mathrm{U}$ \\
\hline Chromium & ND & 0.0010 & $\mathrm{mg} / \mathrm{L}$ & 2 & 16-Jun-2009 & 16-Jun-2009 & SW 846/6020 & $\mathrm{U}$ \\
\hline Cobalt & ND & 0.0010 & $\mathrm{mg} / \mathrm{L}$ & 2 & 16-Jun-2009 & 16-Jun-2009 & SW 846/6020 & U \\
\hline Copper & 0.0024 & 0.0010 & $\mathrm{mg} / \mathrm{L}$ & 2 & 16-Jun-2009 & 16-Jun-2009 & SW 846/6020 & \\
\hline Lead & ND & 0.0010 & $\mathrm{mg} / \mathrm{L}$ & 2 & 16-Jun-2009 & 16-Jun-2009 & SW 846/6020 & $\mathrm{U}$ \\
\hline Manganese & 0.0028 & 0.0010 & $\mathrm{mg} / \mathrm{L}$ & 2 & 16-Jun-2009 & 16-Jun-2009 & SW 846/6020 & \\
\hline Molybdenum & 0.0018 & 0.0010 & $\mathrm{mg} / \mathrm{L}$ & 2 & 16-Jun-2009 & 16-Jun-2009 & SW 846/6020 & \\
\hline Nickel & 0.0008 & 0.0010 & $\mathrm{mg} / \mathrm{L}$ & 2 & 16-Jun-2009 & 16-Jun-2009 & SW 846/6020 & J \\
\hline Selenium & 0.0017 & 0.0010 & $\mathrm{mg} / \mathrm{L}$ & 2 & 16-Jun-2009 & 16-Jun-2009 & SW 846/6020 & \\
\hline Silver & ND & 0.0010 & $\mathrm{mg} / \mathrm{L}$ & 2 & 16-Jun-2009 & 16-Jun-2009 & SW 846/6020 & $\mathrm{U}$ \\
\hline Thallium & 0.0005 & 0.0010 & $\mathrm{mg} / \mathrm{L}$ & 2 & 16-Jun-2009 & 16-Jun-2009 & SW 846/6020 & J \\
\hline Vanadium & 0.0269 & 0.0010 & $\mathrm{mg} / \mathrm{L}$ & 2 & 16-Jun-2009 & 16-Jun-2009 & SW 846/6020 & \\
\hline Zinc & 0.0061 & 0.0010 & $\mathrm{mg} / \mathrm{L}$ & 2 & 16-Jun-2009 & 16-Jun-2009 & SW 846/6020 & B \\
\hline
\end{tabular}




\section{USACE ERDC-EP-C \\ 3909 Halls Ferry Road \\ Vicksburg, MS 39180-6199}

ERDC - ECB

Project: TVA Fly Ash Project

Reported:

$--,-$

Project Manager: Tony Bednar

03-Sep-2009

DRAFT: EMR MT O2 Hour 48 Rep C

9061503-12 (Water)

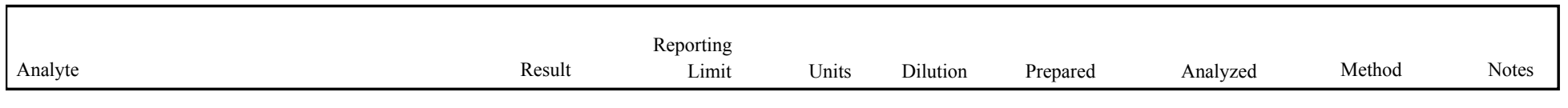

ERDC- EL-EP-C (Environmental Chemistry Branch)

DRAFT: Miscellaneous Physical/Conventional Chemistry Parameters

\begin{tabular}{lrrrrrrr}
\hline Bromide & $\mathrm{ND}$ & 0.100 & $\mathrm{mg} / \mathrm{L}$ & 1 & $15-\mathrm{Jul}-2009$ & $15-\mathrm{Jul}-2009$ & EPA 300.0 \\
Chloride & $\mathbf{2 . 5 1}$ & 1.00 & $\mathrm{mg} / \mathrm{L}$ & 1 & $15-\mathrm{Jul}-2009$ & $15-\mathrm{Jul}-2009$ & EPA 300.0 \\
Nitrate as N & $\mathbf{0 . 3 9 0}$ & 0.100 & $\mathrm{mg} / \mathrm{L}$ & 1 & $15-J u l-2009$ & $15-\mathrm{Jul}-2009$ & EPA 300.0 \\
Sulfate & $\mathbf{1 2 . 5}$ & 0.100 & $\mathrm{mg} / \mathrm{L}$ & 1 & $15-J u l-2009$ & $15-J u l-2009$ & EPA 300.0
\end{tabular}




\section{USACE ERDC-EP-C \\ 3909 Halls Ferry Road \\ Vicksburg, MS 39180-6199}

ERDC - ECB

$-\cdot$,
Project: TVA Fly Ash Project

Project Manager: Tony Bednar
Reported:

03-Sep-2009

DRAFT: SLC MT N2 Hour $48 \operatorname{Rep} A$

9061503-16 (Water)

\begin{tabular}{|c|c|c|c|c|c|c|c|c|}
\hline Analyte & Result & $\begin{array}{r}\text { porting } \\
\text { Limit }\end{array}$ & Units & Dilution & Prepared & Analyzed & Method & Notes \\
\hline
\end{tabular}

ERDC- EL-EP-C (Environmental Chemistry Branch)

DRAFT: Wet Chemistry

\begin{tabular}{llllllll}
\hline Hardness & $\mathbf{9 9 . 3}$ & 1.32 & $\begin{array}{c}\text { mg equil } \\
\text { CaCO3/ }\end{array}$ & 1 & 25-Jun-2009 & 25-Jun-2009
\end{tabular}

$\mathrm{L}$

DRAFT: Metals by EPA 6000/7000 Series Methods

\begin{tabular}{|c|c|c|c|c|c|c|c|c|}
\hline Mercury & ND & 0.000005 & $\mathrm{mg} / \mathrm{L}$ & 1 & 17-Jun-2009 & 01-Jul-2009 & EPA 7471A & $\mathrm{U}$ \\
\hline Strontium & 0.544 & 0.0200 & $\mathrm{mg} / \mathrm{L}$ & 1 & 25-Jun-2009 & 25-Jun-2009 & EPA 6010B & \\
\hline Aluminum & 0.139 & 0.200 & $\mathrm{mg} / \mathrm{L}$ & 1 & 25-Jun-2009 & 25-Jun-2009 & SW 846/6010 & J \\
\hline Calcium & 31.2 & 0.200 & $\mathrm{mg} / \mathrm{L}$ & 1 & 25-Jun-2009 & 25-Jun-2009 & SW 846/6010 & \\
\hline Iron & ND & 0.200 & $\mathrm{mg} / \mathrm{L}$ & 1 & 25-Jun-2009 & 25-Jun-2009 & SW 846/6010 & $\mathrm{U}$ \\
\hline Magnesium & 5.19 & 0.200 & $\mathrm{mg} / \mathrm{L}$ & 1 & 25-Jun-2009 & 25-Jun-2009 & SW 846/6010 & \\
\hline Potassium & 1.91 & 0.200 & $\mathrm{mg} / \mathrm{L}$ & 1 & 25-Jun-2009 & 25-Jun-2009 & SW 846/6010 & \\
\hline Sodium & 4.13 & 0.200 & $\mathrm{mg} / \mathrm{L}$ & 1 & 25-Jun-2009 & 25-Jun-2009 & SW 846/6010 & \\
\hline Antimony & 0.0078 & 0.0010 & $\mathrm{mg} / \mathrm{L}$ & 2 & 16-Jun-2009 & 16-Jun-2009 & SW 846/6020 & \\
\hline Arsenic & 0.0828 & 0.0010 & $\mathrm{mg} / \mathrm{L}$ & 2 & 16-Jun-2009 & 16-Jun-2009 & SW 846/6020 & \\
\hline Barium & 0.0978 & 0.0010 & $\mathrm{mg} / \mathrm{L}$ & 2 & 16-Jun-2009 & 16-Jun-2009 & SW 846/6020 & \\
\hline Beryllium & ND & 0.0010 & $\mathrm{mg} / \mathrm{L}$ & 2 & 16-Jun-2009 & 16-Jun-2009 & SW 846/6020 & $\mathrm{U}$ \\
\hline Cadmium & 0.0003 & 0.0010 & $\mathrm{mg} / \mathrm{L}$ & 2 & 16-Jun-2009 & 16-Jun-2009 & SW 846/6020 & J \\
\hline Chromium & ND & 0.0010 & $\mathrm{mg} / \mathrm{L}$ & 2 & 16-Jun-2009 & 16-Jun-2009 & SW 846/6020 & $\mathrm{U}$ \\
\hline Cobalt & ND & 0.0010 & $\mathrm{mg} / \mathrm{L}$ & 2 & 16-Jun-2009 & 16-Jun-2009 & SW 846/6020 & $\mathrm{U}$ \\
\hline Copper & 0.0007 & 0.0010 & $\mathrm{mg} / \mathrm{L}$ & 2 & 16-Jun-2009 & 16-Jun-2009 & SW 846/6020 & $\mathrm{J}$ \\
\hline Lead & ND & 0.0010 & $\mathrm{mg} / \mathrm{L}$ & 2 & 16-Jun-2009 & 16-Jun-2009 & SW 846/6020 & $\mathrm{U}$ \\
\hline Manganese & 0.0220 & 0.0010 & $\mathrm{mg} / \mathrm{L}$ & 2 & 16-Jun-2009 & 16-Jun-2009 & SW 846/6020 & \\
\hline Molybdenum & 0.0718 & 0.0010 & $\mathrm{mg} / \mathrm{L}$ & 2 & 16-Jun-2009 & 16-Jun-2009 & SW 846/6020 & \\
\hline Nickel & 0.0007 & 0.0010 & $\mathrm{mg} / \mathrm{L}$ & 2 & 16-Jun-2009 & 16-Jun-2009 & SW 846/6020 & $\mathrm{J}$ \\
\hline Selenium & 0.0068 & 0.0010 & $\mathrm{mg} / \mathrm{L}$ & 2 & 16-Jun-2009 & 16-Jun-2009 & SW 846/6020 & \\
\hline Silver & ND & 0.0010 & $\mathrm{mg} / \mathrm{L}$ & 2 & 16-Jun-2009 & 16-Jun-2009 & SW 846/6020 & $\mathrm{U}$ \\
\hline Thallium & 0.0006 & 0.0010 & $\mathrm{mg} / \mathrm{L}$ & 2 & 16-Jun-2009 & 16-Jun-2009 & SW 846/6020 & $\mathrm{J}$ \\
\hline Vanadium & 0.0534 & 0.0010 & $\mathrm{mg} / \mathrm{L}$ & 2 & 16-Jun-2009 & 16-Jun-2009 & SW 846/6020 & \\
\hline Zinc & 0.0058 & 0.0010 & $\mathrm{mg} / \mathrm{L}$ & 2 & 16-Jun-2009 & 16-Jun-2009 & SW 846/6020 & B \\
\hline
\end{tabular}




\section{USACE ERDC-EP-C \\ 3909 Halls Ferry Road \\ Vicksburg, MS 39180-6199}

ERDC - ECB

Project: TVA Fly Ash Project

Reported:

Project Manager: Tony Bednar

03-Sep-2009

DRAFT: SLC MT N2 Hour $48 \operatorname{Rep} A$

9061503-16 (Water)

\begin{tabular}{|c|c|c|c|c|c|c|c|c|}
\hline & & orting & & & & & & \\
\hline Analyte & Result & Limit & Units & Dilution & Prepared & Analyzed & Method & Notes \\
\hline
\end{tabular}

ERDC- EL-EP-C (Environmental Chemistry Branch)

DRAFT: Miscellaneous Physical/Conventional Chemistry Parameters

\begin{tabular}{lrrrrrrr}
\hline Bromide & $\mathrm{ND}$ & 0.100 & $\mathrm{mg} / \mathrm{L}$ & 1 & $15-J u l-2009$ & $15-\mathrm{Jul}-2009$ & EPA 300.0 \\
Chloride & $\mathbf{3 . 8 5}$ & 1.00 & $\mathrm{mg} / \mathrm{L}$ & 1 & $15-J u l-2009$ & $15-\mathrm{Jul}-2009$ & EPA 300.0 \\
Nitrate as N & $\mathbf{0 . 3 5 9}$ & 0.100 & $\mathrm{mg} / \mathrm{L}$ & 1 & $15-J u l-2009$ & $15-J u l-2009$ & EPA 300.0 \\
Sulfate & $\mathbf{3 8 . 7}$ & 0.100 & $\mathrm{mg} / \mathrm{L}$ & 1 & $15-J u l-2009$ & $15-J u l-2009$ & EPA 300.0
\end{tabular}




\section{USACE ERDC-EP-C \\ 3909 Halls Ferry Road \\ Vicksburg, MS 39180-6199}

ERDC - ECB

$-,-$
Project: TVA Fly Ash Project

Project Manager: Tony Bednar
Reported:

03-Sep-2009

DRAFT: SLC MT N2 Hour 48 Rep B

9061503-17 (Water)

\begin{tabular}{|c|c|c|c|c|c|c|c|c|}
\hline Analyte & Result & $\begin{array}{r}\text { porting } \\
\text { Limit }\end{array}$ & Units & Dilution & Prepared & Analyzed & Method & Notes \\
\hline
\end{tabular}

ERDC- EL-EP-C (Environmental Chemistry Branch)

DRAFT: Wet Chemistry

\begin{tabular}{llllllll}
\hline Hardness & $\mathbf{9 8 . 6}$ & 1.32 & $\begin{array}{c}\text { mg equil } \\
\text { CaCO3/ }\end{array}$ & 1 & 25-Jun-2009 & 25-Jun-2009
\end{tabular}

$\mathrm{L}$

DRAFT: Metals by EPA 6000/7000 Series Methods

\begin{tabular}{|c|c|c|c|c|c|c|c|c|}
\hline Mercury & 0.000028 & 0.000005 & $\mathrm{mg} / \mathrm{L}$ & 1 & 17-Jun-2009 & 01-Jul-2009 & EPA 7471A & \\
\hline Strontium & 0.537 & 0.0200 & $\mathrm{mg} / \mathrm{L}$ & 1 & 25-Jun-2009 & 25-Jun-2009 & EPA 6010B & \\
\hline Aluminum & 0.148 & 0.200 & $\mathrm{mg} / \mathrm{L}$ & 1 & 25-Jun-2009 & 25-Jun-2009 & SW 846/6010 & J \\
\hline Calcium & 30.9 & 0.200 & $\mathrm{mg} / \mathrm{L}$ & 1 & 25-Jun-2009 & 25-Jun-2009 & SW 846/6010 & \\
\hline Iron & ND & 0.200 & $\mathrm{mg} / \mathrm{L}$ & 1 & 25-Jun-2009 & 25-Jun-2009 & SW 846/6010 & $\mathrm{U}$ \\
\hline Magnesium & 5.22 & 0.200 & $\mathrm{mg} / \mathrm{L}$ & 1 & 25-Jun-2009 & 25-Jun-2009 & SW 846/6010 & \\
\hline Potassium & 1.91 & 0.200 & $\mathrm{mg} / \mathrm{L}$ & 1 & 25-Jun-2009 & 25-Jun-2009 & SW 846/6010 & \\
\hline Sodium & 4.05 & 0.200 & $\mathrm{mg} / \mathrm{L}$ & 1 & 25-Jun-2009 & 25-Jun-2009 & SW 846/6010 & \\
\hline Antimony & 0.0081 & 0.0010 & $\mathrm{mg} / \mathrm{L}$ & 2 & 16-Jun-2009 & 16-Jun-2009 & SW 846/6020 & \\
\hline Arsenic & 0.0855 & 0.0010 & $\mathrm{mg} / \mathrm{L}$ & 2 & 16-Jun-2009 & 16-Jun-2009 & SW 846/6020 & \\
\hline Barium & 0.0991 & 0.0010 & $\mathrm{mg} / \mathrm{L}$ & 2 & 16-Jun-2009 & 16-Jun-2009 & SW 846/6020 & \\
\hline Beryllium & ND & 0.0010 & $\mathrm{mg} / \mathrm{L}$ & 2 & 16-Jun-2009 & 16-Jun-2009 & SW 846/6020 & U \\
\hline Cadmium & 0.0003 & 0.0010 & $\mathrm{mg} / \mathrm{L}$ & 2 & 16-Jun-2009 & 16-Jun-2009 & SW 846/6020 & J \\
\hline Chromium & ND & 0.0010 & $\mathrm{mg} / \mathrm{L}$ & 2 & 16-Jun-2009 & 16-Jun-2009 & SW 846/6020 & U \\
\hline Cobalt & ND & 0.0010 & $\mathrm{mg} / \mathrm{L}$ & 2 & 16-Jun-2009 & 16-Jun-2009 & SW 846/6020 & U \\
\hline Copper & 0.0005 & 0.0010 & $\mathrm{mg} / \mathrm{L}$ & 2 & 16-Jun-2009 & 16-Jun-2009 & SW 846/6020 & J \\
\hline Lead & ND & 0.0010 & $\mathrm{mg} / \mathrm{L}$ & 2 & 16-Jun-2009 & 16-Jun-2009 & SW 846/6020 & U \\
\hline Manganese & 0.0228 & 0.0010 & $\mathrm{mg} / \mathrm{L}$ & 2 & 16-Jun-2009 & 16-Jun-2009 & SW 846/6020 & \\
\hline Molybdenum & 0.0741 & 0.0010 & $\mathrm{mg} / \mathrm{L}$ & 2 & 16-Jun-2009 & 16-Jun-2009 & SW 846/6020 & \\
\hline Nickel & 0.0005 & 0.0010 & $\mathrm{mg} / \mathrm{L}$ & 2 & 16-Jun-2009 & 16-Jun-2009 & SW 846/6020 & J \\
\hline Selenium & 0.0071 & 0.0010 & $\mathrm{mg} / \mathrm{L}$ & 2 & 16-Jun-2009 & 16-Jun-2009 & SW 846/6020 & \\
\hline Silver & ND & 0.0010 & $\mathrm{mg} / \mathrm{L}$ & 2 & 16-Jun-2009 & 16-Jun-2009 & SW 846/6020 & $\mathrm{U}$ \\
\hline Thallium & 0.0006 & 0.0010 & $\mathrm{mg} / \mathrm{L}$ & 2 & 16-Jun-2009 & 16-Jun-2009 & SW 846/6020 & J \\
\hline Vanadium & 0.0578 & 0.0010 & $\mathrm{mg} / \mathrm{L}$ & 2 & 16-Jun-2009 & 16-Jun-2009 & SW 846/6020 & \\
\hline Zinc & 0.0055 & 0.0010 & $\mathrm{mg} / \mathrm{L}$ & 2 & 16-Jun-2009 & 16-Jun-2009 & SW 846/6020 & $\mathrm{B}$ \\
\hline
\end{tabular}




\section{USACE ERDC-EP-C \\ 3909 Halls Ferry Road \\ Vicksburg, MS 39180-6199}

ERDC - ECB

Project: TVA Fly Ash Project

Reported:

Project Manager: Tony Bednar

03-Sep-2009

DRAFT: SLC MT N2 Hour 48 Rep B

9061503-17 (Water)

\begin{tabular}{|c|c|c|c|c|c|c|c|c|}
\hline & & orting & & & & & & \\
\hline Analyte & Result & Limit & Units & Dilution & Prepared & Analyzed & Method & Notes \\
\hline
\end{tabular}

ERDC- EL-EP-C (Environmental Chemistry Branch)

DRAFT: Miscellaneous Physical/Conventional Chemistry Parameters

\begin{tabular}{lrrrrrrr}
\hline Bromide & $\mathrm{ND}$ & 0.100 & $\mathrm{mg} / \mathrm{L}$ & 1 & $15-\mathrm{Jul}-2009$ & $15-\mathrm{Jul}-2009$ & EPA 300.0 \\
Chloride & $\mathbf{3 . 7 4}$ & 1.00 & $\mathrm{mg} / \mathrm{L}$ & 1 & $15-\mathrm{Jul}-2009$ & $15-\mathrm{Jul}-2009$ & EPA 300.0 \\
Nitrate as N & $\mathbf{0 . 3 5 9}$ & 0.100 & $\mathrm{mg} / \mathrm{L}$ & 1 & $15-\mathrm{Jul}-2009$ & $15-\mathrm{Jul}-2009$ & EPA 300.0 \\
Sulfate & $\mathbf{3 8 . 2}$ & 0.100 & $\mathrm{mg} / \mathrm{L}$ & 1 & $15-J u l-2009$ & $15-\mathrm{Jul}-2009$ & EPA 300.0
\end{tabular}




\section{USACE ERDC-EP-C \\ 3909 Halls Ferry Road \\ Vicksburg, MS 39180-6199}

ERDC - ECB

$-,-$
Project: TVA Fly Ash Project

Project Manager: Tony Bednar
Reported:

03-Sep-2009

DRAFT: SLC MT N2 Hour 48 Rep C

9061503-18 (Water)

\begin{tabular}{|c|c|c|c|c|c|c|c|c|}
\hline Analyte & Result & $\begin{array}{r}\text { porting } \\
\text { Limit }\end{array}$ & Units & Dilution & Prepared & Analyzed & Method & Notes \\
\hline
\end{tabular}

ERDC- EL-EP-C (Environmental Chemistry Branch)

DRAFT: Wet Chemistry

\begin{tabular}{llllllll}
\hline Hardness & $\mathbf{9 8 . 5}$ & 1.32 & $\begin{array}{c}\text { mg equil } \\
\text { CaCO3/ }\end{array}$ & 1 & 25-Jun-2009 & 25-Jun-2009
\end{tabular}

$\mathrm{L}$

DRAFT: Metals by EPA 6000/7000 Series Methods

\begin{tabular}{|c|c|c|c|c|c|c|c|c|}
\hline Mercury & ND & 0.000005 & $\mathrm{mg} / \mathrm{L}$ & 1 & 17-Jun-2009 & 01-Jul-2009 & EPA 7471A & $\mathrm{U}$ \\
\hline Strontium & 0.542 & 0.0200 & $\mathrm{mg} / \mathrm{L}$ & 1 & 25-Jun-2009 & 25-Jun-2009 & EPA 6010B & \\
\hline Aluminum & 0.160 & 0.200 & $\mathrm{mg} / \mathrm{L}$ & 1 & 25-Jun-2009 & 25-Jun-2009 & SW 846/6010 & J \\
\hline Calcium & 31.0 & 0.200 & $\mathrm{mg} / \mathrm{L}$ & 1 & 25-Jun-2009 & 25-Jun-2009 & SW 846/6010 & \\
\hline Iron & ND & 0.200 & $\mathrm{mg} / \mathrm{L}$ & 1 & 25-Jun-2009 & 25-Jun-2009 & SW 846/6010 & $\mathrm{U}$ \\
\hline Magnesium & 5.13 & 0.200 & $\mathrm{mg} / \mathrm{L}$ & 1 & 25-Jun-2009 & 25-Jun-2009 & SW 846/6010 & \\
\hline Potassium & 1.87 & 0.200 & $\mathrm{mg} / \mathrm{L}$ & 1 & 25-Jun-2009 & 25-Jun-2009 & SW 846/6010 & \\
\hline Sodium & 4.08 & 0.200 & $\mathrm{mg} / \mathrm{L}$ & 1 & 25-Jun-2009 & 25-Jun-2009 & SW 846/6010 & \\
\hline Antimony & 0.0082 & 0.0010 & $\mathrm{mg} / \mathrm{L}$ & 2 & 16-Jun-2009 & 16-Jun-2009 & SW 846/6020 & \\
\hline Arsenic & 0.0851 & 0.0010 & $\mathrm{mg} / \mathrm{L}$ & 2 & 16-Jun-2009 & 16-Jun-2009 & SW 846/6020 & \\
\hline Barium & 0.0981 & 0.0010 & $\mathrm{mg} / \mathrm{L}$ & 2 & 16-Jun-2009 & 16-Jun-2009 & SW 846/6020 & \\
\hline Beryllium & ND & 0.0010 & $\mathrm{mg} / \mathrm{L}$ & 2 & 16-Jun-2009 & 16-Jun-2009 & SW 846/6020 & $\mathrm{U}$ \\
\hline Cadmium & 0.0003 & 0.0010 & $\mathrm{mg} / \mathrm{L}$ & 2 & 16-Jun-2009 & 16-Jun-2009 & SW 846/6020 & J \\
\hline Chromium & ND & 0.0010 & $\mathrm{mg} / \mathrm{L}$ & 2 & 16-Jun-2009 & 16-Jun-2009 & SW 846/6020 & $\mathrm{U}$ \\
\hline Cobalt & ND & 0.0010 & $\mathrm{mg} / \mathrm{L}$ & 2 & 16-Jun-2009 & 16-Jun-2009 & SW 846/6020 & $\mathrm{U}$ \\
\hline Copper & 0.0007 & 0.0010 & $\mathrm{mg} / \mathrm{L}$ & 2 & 16-Jun-2009 & 16-Jun-2009 & SW 846/6020 & $\mathrm{J}$ \\
\hline Lead & ND & 0.0010 & $\mathrm{mg} / \mathrm{L}$ & 2 & 16-Jun-2009 & 16-Jun-2009 & SW 846/6020 & $\mathrm{U}$ \\
\hline Manganese & 0.0236 & 0.0010 & $\mathrm{mg} / \mathrm{L}$ & 2 & 16-Jun-2009 & 16-Jun-2009 & SW 846/6020 & \\
\hline Molybdenum & 0.0730 & 0.0010 & $\mathrm{mg} / \mathrm{L}$ & 2 & 16-Jun-2009 & 16-Jun-2009 & SW 846/6020 & \\
\hline Nickel & 0.0006 & 0.0010 & $\mathrm{mg} / \mathrm{L}$ & 2 & 16-Jun-2009 & 16-Jun-2009 & SW 846/6020 & $\mathrm{J}$ \\
\hline Selenium & 0.0068 & 0.0010 & $\mathrm{mg} / \mathrm{L}$ & 2 & 16-Jun-2009 & 16-Jun-2009 & SW 846/6020 & \\
\hline Silver & ND & 0.0010 & $\mathrm{mg} / \mathrm{L}$ & 2 & 16-Jun-2009 & 16-Jun-2009 & SW 846/6020 & $\mathrm{U}$ \\
\hline Thallium & 0.0007 & 0.0010 & $\mathrm{mg} / \mathrm{L}$ & 2 & 16-Jun-2009 & 16-Jun-2009 & SW 846/6020 & $\mathrm{J}$ \\
\hline Vanadium & 0.0596 & 0.0010 & $\mathrm{mg} / \mathrm{L}$ & 2 & 16-Jun-2009 & 16-Jun-2009 & SW 846/6020 & \\
\hline Zinc & 0.0083 & 0.0010 & $\mathrm{mg} / \mathrm{L}$ & 2 & 16-Jun-2009 & 16-Jun-2009 & SW 846/6020 & B \\
\hline
\end{tabular}




\section{USACE ERDC-EP-C \\ 3909 Halls Ferry Road \\ Vicksburg, MS 39180-6199}

ERDC - ECB

Project: TVA Fly Ash Project

Reported:

Project Manager: Tony Bednar

03-Sep-2009

DRAFT: SLC MT N2 Hour 48 Rep C

9061503-18 (Water)

\begin{tabular}{|c|c|c|c|c|c|c|c|c|}
\hline & & orting & & & & & & \\
\hline Analyte & Result & Limit & Units & Dilution & Prepared & Analyzed & Method & Notes \\
\hline
\end{tabular}

ERDC- EL-EP-C (Environmental Chemistry Branch)

DRAFT: Miscellaneous Physical/Conventional Chemistry Parameters

\begin{tabular}{lrrrrrrr}
\hline Bromide & $\mathrm{ND}$ & 0.100 & $\mathrm{mg} / \mathrm{L}$ & 1 & $15-\mathrm{Jul}-2009$ & $15-\mathrm{Jul}-2009$ & EPA 300.0 \\
Chloride & $\mathbf{3 . 7 7}$ & 1.00 & $\mathrm{mg} / \mathrm{L}$ & 1 & $15-\mathrm{Jul}-2009$ & $15-\mathrm{Jul}-2009$ & EPA 300.0 \\
Nitrate as N & $\mathbf{0 . 3 6 6}$ & 0.100 & $\mathrm{mg} / \mathrm{L}$ & 1 & $15-\mathrm{Jul}-2009$ & $15-\mathrm{Jul}-2009$ & EPA 300.0 \\
Sulfate & $\mathbf{3 8 . 4}$ & 0.100 & $\mathrm{mg} / \mathrm{L}$ & 1 & $15-J u l-2009$ & $15-\mathrm{Jul}-2009$ & EPA 300.0
\end{tabular}




\section{USACE ERDC-EP-C \\ 3909 Halls Ferry Road \\ Vicksburg, MS 39180-6199}

ERDC - ECB

$-\cdot$,
Project: TVA Fly Ash Project

Project Manager: Tony Bednar
Reported:

03-Sep-2009

DRAFT: SLC MT O2 Hour 48 Rep A

9061503-22 (Water)

\begin{tabular}{|c|c|c|c|c|c|c|c|c|}
\hline Analyte & Result & $\begin{array}{r}\text { porting } \\
\text { Limit }\end{array}$ & Units & Dilution & Prepared & Analyzed & Method & Notes \\
\hline
\end{tabular}

ERDC- EL-EP-C (Environmental Chemistry Branch)

DRAFT: Wet Chemistry

\begin{tabular}{lccccccc}
\hline Hardness & $\mathbf{9 8 . 2}$ & 1.32 & $\begin{array}{c}\text { mg equil } \\
\text { CaCO3/ }\end{array}$ & 1 & 25-Jun-2009 & 25-Jun-2009
\end{tabular}

$\mathrm{L}$

DRAFT: Metals by EPA 6000/7000 Series Methods

\begin{tabular}{|c|c|c|c|c|c|c|c|c|}
\hline Mercury & 0.000011 & 0.000005 & $\mathrm{mg} / \mathrm{L}$ & 1 & 17-Jun-2009 & 01-Jul-2009 & EPA 7471A & \\
\hline Strontium & 0.539 & 0.0200 & $\mathrm{mg} / \mathrm{L}$ & 1 & 25-Jun-2009 & 25-Jun-2009 & EPA 6010B & \\
\hline Aluminum & 0.0938 & 0.200 & $\mathrm{mg} / \mathrm{L}$ & 1 & 25-Jun-2009 & 25-Jun-2009 & SW 846/6010 & J \\
\hline Calcium & 30.9 & 0.200 & $\mathrm{mg} / \mathrm{L}$ & 1 & 25-Jun-2009 & 25-Jun-2009 & SW 846/6010 & \\
\hline Iron & ND & 0.200 & $\mathrm{mg} / \mathrm{L}$ & 1 & 25-Jun-2009 & 25-Jun-2009 & SW 846/6010 & $\mathrm{U}$ \\
\hline Magnesium & 5.12 & 0.200 & $\mathrm{mg} / \mathrm{L}$ & 1 & 25-Jun-2009 & 25-Jun-2009 & SW 846/6010 & \\
\hline Potassium & 2.07 & 0.200 & $\mathrm{mg} / \mathrm{L}$ & 1 & 25-Jun-2009 & 25-Jun-2009 & SW 846/6010 & \\
\hline Sodium & 4.27 & 0.200 & $\mathrm{mg} / \mathrm{L}$ & 1 & 25-Jun-2009 & 25-Jun-2009 & SW 846/6010 & \\
\hline Antimony & 0.0080 & 0.0010 & $\mathrm{mg} / \mathrm{L}$ & 2 & 16-Jun-2009 & 16-Jun-2009 & SW 846/6020 & \\
\hline Arsenic & 0.0796 & 0.0010 & $\mathrm{mg} / \mathrm{L}$ & 2 & 16-Jun-2009 & 16-Jun-2009 & SW 846/6020 & \\
\hline Barium & 0.101 & 0.0010 & $\mathrm{mg} / \mathrm{L}$ & 2 & 16-Jun-2009 & 16-Jun-2009 & SW 846/6020 & \\
\hline Beryllium & ND & 0.0010 & $\mathrm{mg} / \mathrm{L}$ & 2 & 16-Jun-2009 & 16-Jun-2009 & SW 846/6020 & U \\
\hline Cadmium & 0.0003 & 0.0010 & $\mathrm{mg} / \mathrm{L}$ & 2 & 16-Jun-2009 & 16-Jun-2009 & SW 846/6020 & J \\
\hline Chromium & ND & 0.0010 & $\mathrm{mg} / \mathrm{L}$ & 2 & 16-Jun-2009 & 16-Jun-2009 & SW 846/6020 & U \\
\hline Cobalt & ND & 0.0010 & $\mathrm{mg} / \mathrm{L}$ & 2 & 16-Jun-2009 & 16-Jun-2009 & SW 846/6020 & U \\
\hline Copper & 0.0015 & 0.0010 & $\mathrm{mg} / \mathrm{L}$ & 2 & 16-Jun-2009 & 16-Jun-2009 & SW 846/6020 & \\
\hline Lead & ND & 0.0010 & $\mathrm{mg} / \mathrm{L}$ & 2 & 16-Jun-2009 & 16-Jun-2009 & SW 846/6020 & U \\
\hline Manganese & 0.0269 & 0.0010 & $\mathrm{mg} / \mathrm{L}$ & 2 & 16-Jun-2009 & 16-Jun-2009 & SW 846/6020 & \\
\hline Molybdenum & 0.0713 & 0.0010 & $\mathrm{mg} / \mathrm{L}$ & 2 & 16-Jun-2009 & 16-Jun-2009 & SW 846/6020 & \\
\hline Nickel & 0.0008 & 0.0010 & $\mathrm{mg} / \mathrm{L}$ & 2 & 16-Jun-2009 & 16-Jun-2009 & SW 846/6020 & J \\
\hline Selenium & 0.0064 & 0.0010 & $\mathrm{mg} / \mathrm{L}$ & 2 & 16-Jun-2009 & 16-Jun-2009 & SW 846/6020 & \\
\hline Silver & ND & 0.0010 & $\mathrm{mg} / \mathrm{L}$ & 2 & 16-Jun-2009 & 16-Jun-2009 & SW 846/6020 & $\mathrm{U}$ \\
\hline Thallium & 0.0007 & 0.0010 & $\mathrm{mg} / \mathrm{L}$ & 2 & 16-Jun-2009 & 16-Jun-2009 & SW 846/6020 & J \\
\hline Vanadium & 0.0571 & 0.0010 & $\mathrm{mg} / \mathrm{L}$ & 2 & 16-Jun-2009 & 16-Jun-2009 & SW 846/6020 & \\
\hline Zinc & 0.0056 & 0.0010 & $\mathrm{mg} / \mathrm{L}$ & 2 & 16-Jun-2009 & 16-Jun-2009 & SW 846/6020 & $\mathrm{B}$ \\
\hline
\end{tabular}




\section{USACE ERDC-EP-C \\ 3909 Halls Ferry Road \\ Vicksburg, MS 39180-6199}

ERDC - ECB

Project: TVA Fly Ash Project

Reported:

Project Manager: Tony Bednar

03-Sep-2009

DRAFT: SLC MT O2 Hour 48 Rep A

9061503-22 (Water)

\begin{tabular}{|c|c|c|c|c|c|c|c|c|}
\hline & & orting & & & & & & \\
\hline Analyte & Result & Limit & Units & Dilution & Prepared & Analyzed & Method & Notes \\
\hline
\end{tabular}

ERDC- EL-EP-C (Environmental Chemistry Branch)

DRAFT: Miscellaneous Physical/Conventional Chemistry Parameters

\begin{tabular}{lrrrrrrr}
\hline Bromide & $\mathrm{ND}$ & 0.100 & $\mathrm{mg} / \mathrm{L}$ & 1 & $15-\mathrm{Jul}-2009$ & $15-\mathrm{Jul}-2009$ & EPA 300.0 \\
Chloride & $\mathbf{4 . 0 9}$ & 1.00 & $\mathrm{mg} / \mathrm{L}$ & 1 & $15-\mathrm{Jul}-2009$ & $15-\mathrm{Jul}-2009$ & EPA 300.0 \\
Nitrate as N & $\mathbf{0 . 3 9 2}$ & 0.100 & $\mathrm{mg} / \mathrm{L}$ & 1 & $15-\mathrm{Jul}-2009$ & $15-\mathrm{Jul}-2009$ & EPA 300.0 \\
Sulfate & $\mathbf{3 8 . 0}$ & 0.100 & $\mathrm{mg} / \mathrm{L}$ & 1 & $15-J u l-2009$ & $15-\mathrm{Jul}-2009$ & EPA 300.0
\end{tabular}




\section{USACE ERDC-EP-C \\ 3909 Halls Ferry Road \\ Vicksburg, MS 39180-6199}

ERDC - ECB

$-,-$
Project: TVA Fly Ash Project

Project Manager: Tony Bednar
Reported:

03-Sep-2009

DRAFT: SLC MT O2 Hour 48 Rep B

9061503-23 (Water)

\begin{tabular}{|c|c|c|c|c|c|c|c|c|}
\hline Analyte & Result & $\begin{array}{r}\text { porting } \\
\text { Limit }\end{array}$ & Units & Dilution & Prepared & Analyzed & Method & Notes \\
\hline
\end{tabular}

ERDC- EL-EP-C (Environmental Chemistry Branch)

DRAFT: Wet Chemistry

\begin{tabular}{llllllll}
\hline Hardness & $\mathbf{9 9 . 3}$ & 1.32 & $\begin{array}{c}\text { mg equil } \\
\text { CaCO3/ }\end{array}$ & 1 & 25-Jun-2009 & 25-Jun-2009
\end{tabular}

$\mathrm{L}$

DRAFT: Metals by EPA 6000/7000 Series Methods

\begin{tabular}{|c|c|c|c|c|c|c|c|c|}
\hline Mercury & ND & 0.000005 & $\mathrm{mg} / \mathrm{L}$ & 1 & 17-Jun-2009 & 01-Jul-2009 & EPA 7471A & $\mathrm{U}$ \\
\hline Strontium & 0.541 & 0.0200 & $\mathrm{mg} / \mathrm{L}$ & 1 & 25-Jun-2009 & 25-Jun-2009 & EPA 6010B & \\
\hline Aluminum & 0.117 & 0.200 & $\mathrm{mg} / \mathrm{L}$ & 1 & 25-Jun-2009 & 25-Jun-2009 & SW 846/6010 & J \\
\hline Calcium & 31.2 & 0.200 & $\mathrm{mg} / \mathrm{L}$ & 1 & 25-Jun-2009 & 25-Jun-2009 & SW 846/6010 & \\
\hline Iron & ND & 0.200 & $\mathrm{mg} / \mathrm{L}$ & 1 & 25-Jun-2009 & 25-Jun-2009 & SW 846/6010 & $\mathrm{U}$ \\
\hline Magnesium & 5.22 & 0.200 & $\mathrm{mg} / \mathrm{L}$ & 1 & 25-Jun-2009 & 25-Jun-2009 & SW 846/6010 & \\
\hline Potassium & 1.92 & 0.200 & $\mathrm{mg} / \mathrm{L}$ & 1 & 25-Jun-2009 & 25-Jun-2009 & SW 846/6010 & \\
\hline Sodium & 4.10 & 0.200 & $\mathrm{mg} / \mathrm{L}$ & 1 & 25-Jun-2009 & 25-Jun-2009 & SW 846/6010 & \\
\hline Antimony & 0.0079 & 0.0010 & $\mathrm{mg} / \mathrm{L}$ & 2 & 16-Jun-2009 & 16-Jun-2009 & SW 846/6020 & \\
\hline Arsenic & 0.0820 & 0.0010 & $\mathrm{mg} / \mathrm{L}$ & 2 & 16-Jun-2009 & 16-Jun-2009 & SW 846/6020 & \\
\hline Barium & 0.101 & 0.0010 & $\mathrm{mg} / \mathrm{L}$ & 2 & 16-Jun-2009 & 16-Jun-2009 & SW 846/6020 & \\
\hline Beryllium & ND & 0.0010 & $\mathrm{mg} / \mathrm{L}$ & 2 & 16-Jun-2009 & 16-Jun-2009 & SW 846/6020 & $\mathrm{U}$ \\
\hline Cadmium & 0.0003 & 0.0010 & $\mathrm{mg} / \mathrm{L}$ & 2 & 16-Jun-2009 & 16-Jun-2009 & SW 846/6020 & $\mathrm{J}$ \\
\hline Chromium & ND & 0.0010 & $\mathrm{mg} / \mathrm{L}$ & 2 & 16-Jun-2009 & 16-Jun-2009 & SW 846/6020 & $\mathrm{U}$ \\
\hline Cobalt & ND & 0.0010 & $\mathrm{mg} / \mathrm{L}$ & 2 & 16-Jun-2009 & 16-Jun-2009 & SW 846/6020 & $\mathrm{U}$ \\
\hline Copper & 0.00051 & 0.0010 & $\mathrm{mg} / \mathrm{L}$ & 2 & 16-Jun-2009 & 16-Jun-2009 & SW 846/6020 & $\mathrm{J}$ \\
\hline Lead & ND & 0.0010 & $\mathrm{mg} / \mathrm{L}$ & 2 & 16-Jun-2009 & 16-Jun-2009 & SW 846/6020 & $\mathrm{U}$ \\
\hline Manganese & 0.0249 & 0.0010 & $\mathrm{mg} / \mathrm{L}$ & 2 & 16-Jun-2009 & 16-Jun-2009 & SW 846/6020 & \\
\hline Molybdenum & 0.0738 & 0.0010 & $\mathrm{mg} / \mathrm{L}$ & 2 & 16-Jun-2009 & 16-Jun-2009 & SW 846/6020 & \\
\hline Nickel & 0.00067 & 0.0010 & $\mathrm{mg} / \mathrm{L}$ & 2 & 16-Jun-2009 & 16-Jun-2009 & SW 846/6020 & $\mathrm{J}$ \\
\hline Selenium & 0.0073 & 0.0010 & $\mathrm{mg} / \mathrm{L}$ & 2 & 16-Jun-2009 & 16-Jun-2009 & SW 846/6020 & \\
\hline Silver & ND & 0.0010 & $\mathrm{mg} / \mathrm{L}$ & 2 & 16-Jun-2009 & 16-Jun-2009 & SW 846/6020 & $\mathrm{U}$ \\
\hline Thallium & 0.0007 & 0.0010 & $\mathrm{mg} / \mathrm{L}$ & 2 & 16-Jun-2009 & 16-Jun-2009 & SW 846/6020 & $\mathrm{J}$ \\
\hline Vanadium & 0.0570 & 0.0010 & $\mathrm{mg} / \mathrm{L}$ & 2 & 16-Jun-2009 & 16-Jun-2009 & SW 846/6020 & \\
\hline Zinc & 0.0060 & 0.0010 & $\mathrm{mg} / \mathrm{L}$ & 2 & 16-Jun-2009 & 16-Jun-2009 & SW 846/6020 & B \\
\hline
\end{tabular}




\section{USACE ERDC-EP-C \\ 3909 Halls Ferry Road \\ Vicksburg, MS 39180-6199}

ERDC - ECB

Project: TVA Fly Ash Project

Reported:

Project Manager: Tony Bednar

03-Sep-2009

DRAFT: SLC MT O2 Hour 48 Rep B

9061503-23 (Water)

\begin{tabular}{|c|c|c|c|c|c|c|c|c|}
\hline & & orting & & & & & & \\
\hline Analyte & Result & Limit & Units & Dilution & Prepared & Analyzed & Method & Notes \\
\hline
\end{tabular}

ERDC- EL-EP-C (Environmental Chemistry Branch)

DRAFT: Miscellaneous Physical/Conventional Chemistry Parameters

\begin{tabular}{lrrrrrrr}
\hline Bromide & $\mathrm{ND}$ & 0.100 & $\mathrm{mg} / \mathrm{L}$ & 1 & $15-\mathrm{Jul}-2009$ & $15-\mathrm{Jul}-2009$ & EPA 300.0 \\
Chloride & $\mathbf{3 . 7 6}$ & 1.00 & $\mathrm{mg} / \mathrm{L}$ & 1 & $15-\mathrm{Jul}-2009$ & $15-\mathrm{Jul}-2009$ & EPA 300.0 \\
Nitrate as N & $\mathbf{0 . 3 6 9}$ & 0.100 & $\mathrm{mg} / \mathrm{L}$ & 1 & $15-\mathrm{Jul}-2009$ & $15-\mathrm{Jul}-2009$ & EPA 300.0 \\
Sulfate & $\mathbf{3 7 . 8}$ & 0.100 & $\mathrm{mg} / \mathrm{L}$ & 1 & $15-J u l-2009$ & $15-\mathrm{Jul}-2009$ & EPA 300.0
\end{tabular}




\section{USACE ERDC-EP-C \\ 3909 Halls Ferry Road \\ Vicksburg, MS 39180-6199}

ERDC - ECB

$-\cdot$,
Project: TVA Fly Ash Project

Project Manager: Tony Bednar
Reported:

03-Sep-2009

DRAFT: SLC MT O2 Hour 48 Rep C

9061503-24 (Water)

\begin{tabular}{|c|c|c|c|c|c|c|c|c|}
\hline Analyte & Result & $\begin{array}{r}\text { porting } \\
\text { Limit }\end{array}$ & Units & Dilution & Prepared & Analyzed & Method & Notes \\
\hline
\end{tabular}

ERDC- EL-EP-C (Environmental Chemistry Branch)

DRAFT: Wet Chemistry

\begin{tabular}{llllllll}
\hline Hardness & $\mathbf{9 9 . 4}$ & 1.32 & $\begin{array}{c}\text { mg equil } \\
\text { CaCO3/ }\end{array}$ & 1 & 25-Jun-2009 & 25-Jun-2009
\end{tabular}

$\mathrm{L}$

DRAFT: Metals by EPA 6000/7000 Series Methods

\begin{tabular}{|c|c|c|c|c|c|c|c|c|}
\hline Mercury & 0.00001 & 0.000005 & $\mathrm{mg} / \mathrm{L}$ & 1 & 17-Jun-2009 & 01-Jul-2009 & EPA 7471A & \\
\hline Strontium & 0.544 & 0.0200 & $\mathrm{mg} / \mathrm{L}$ & 1 & 25-Jun-2009 & 25-Jun-2009 & EPA $6010 B$ & \\
\hline Aluminum & 0.116 & 0.200 & $\mathrm{mg} / \mathrm{L}$ & 1 & 25-Jun-2009 & 25-Jun-2009 & SW 846/6010 & $\mathrm{J}$ \\
\hline Calcium & 31.2 & 0.200 & $\mathrm{mg} / \mathrm{L}$ & 1 & 25-Jun-2009 & 25-Jun-2009 & SW 846/6010 & \\
\hline Iron & ND & 0.200 & $\mathrm{mg} / \mathrm{L}$ & 1 & 25-Jun-2009 & 25-Jun-2009 & SW 846/6010 & $\mathrm{U}$ \\
\hline Magnesium & 5.20 & 0.200 & $\mathrm{mg} / \mathrm{L}$ & 1 & 25-Jun-2009 & 25-Jun-2009 & SW 846/6010 & \\
\hline Potassium & 1.95 & 0.200 & $\mathrm{mg} / \mathrm{L}$ & 1 & 25-Jun-2009 & 25-Jun-2009 & SW 846/6010 & \\
\hline Sodium & 4.13 & 0.200 & $\mathrm{mg} / \mathrm{L}$ & 1 & 25-Jun-2009 & 25-Jun-2009 & SW 846/6010 & \\
\hline Antimony & 0.0080 & 0.0010 & $\mathrm{mg} / \mathrm{L}$ & 2 & 16-Jun-2009 & 16-Jun-2009 & SW 846/6020 & \\
\hline Arsenic & 0.0794 & 0.0010 & $\mathrm{mg} / \mathrm{L}$ & 2 & 16-Jun-2009 & 16-Jun-2009 & SW 846/6020 & \\
\hline Barium & 0.103 & 0.0010 & $\mathrm{mg} / \mathrm{L}$ & 2 & 16-Jun-2009 & 16-Jun-2009 & SW 846/6020 & \\
\hline Beryllium & ND & 0.0010 & $\mathrm{mg} / \mathrm{L}$ & 2 & 16-Jun-2009 & 16-Jun-2009 & SW 846/6020 & $\mathrm{U}$ \\
\hline Cadmium & 0.0003 & 0.0010 & $\mathrm{mg} / \mathrm{L}$ & 2 & 16-Jun-2009 & 16-Jun-2009 & SW 846/6020 & J \\
\hline Chromium & ND & 0.0010 & $\mathrm{mg} / \mathrm{L}$ & 2 & 16-Jun-2009 & 16-Jun-2009 & SW 846/6020 & $\mathrm{U}$ \\
\hline Cobalt & ND & 0.0010 & $\mathrm{mg} / \mathrm{L}$ & 2 & 16-Jun-2009 & 16-Jun-2009 & SW 846/6020 & $\mathrm{U}$ \\
\hline Copper & 0.0007 & 0.0010 & $\mathrm{mg} / \mathrm{L}$ & 2 & 16-Jun-2009 & 16-Jun-2009 & SW 846/6020 & J \\
\hline Lead & ND & 0.0010 & $\mathrm{mg} / \mathrm{L}$ & 2 & 16-Jun-2009 & 16-Jun-2009 & SW 846/6020 & $\mathrm{U}$ \\
\hline Manganese & 0.0248 & 0.0010 & $\mathrm{mg} / \mathrm{L}$ & 2 & 16-Jun-2009 & 16-Jun-2009 & SW 846/6020 & \\
\hline Molybdenum & 0.0738 & 0.0010 & $\mathrm{mg} / \mathrm{L}$ & 2 & 16-Jun-2009 & 16-Jun-2009 & SW 846/6020 & \\
\hline Nickel & 0.0006 & 0.0010 & $\mathrm{mg} / \mathrm{L}$ & 2 & 16-Jun-2009 & 16-Jun-2009 & SW 846/6020 & $\mathrm{J}$ \\
\hline Selenium & 0.0074 & 0.0010 & $\mathrm{mg} / \mathrm{L}$ & 2 & 16-Jun-2009 & 16-Jun-2009 & SW 846/6020 & \\
\hline Silver & ND & 0.0010 & $\mathrm{mg} / \mathrm{L}$ & 2 & 16-Jun-2009 & 16-Jun-2009 & SW 846/6020 & $\mathrm{U}$ \\
\hline Thallium & 0.0007 & 0.0010 & $\mathrm{mg} / \mathrm{L}$ & 2 & 16-Jun-2009 & 16-Jun-2009 & SW 846/6020 & $\mathrm{J}$ \\
\hline Vanadium & 0.0573 & 0.0010 & $\mathrm{mg} / \mathrm{L}$ & 2 & 16-Jun-2009 & 16-Jun-2009 & SW 846/6020 & \\
\hline Zinc & 0.0060 & 0.0010 & $\mathrm{mg} / \mathrm{L}$ & 2 & 16-Jun-2009 & 16-Jun-2009 & SW 846/6020 & $\mathrm{B}$ \\
\hline
\end{tabular}




\section{USACE ERDC-EP-C \\ 3909 Halls Ferry Road \\ Vicksburg, MS 39180-6199}

ERDC - ECB

Project: TVA Fly Ash Project

Reported:

Project Manager: Tony Bednar

03-Sep-2009

DRAFT: SLC MT O2 Hour 48 Rep C

9061503-24 (Water)

\begin{tabular}{|c|c|c|c|c|c|c|c|c|}
\hline & & orting & & & & & & \\
\hline Analyte & Result & Limit & Units & Dilution & Prepared & Analyzed & Method & Notes \\
\hline
\end{tabular}

ERDC- EL-EP-C (Environmental Chemistry Branch)

DRAFT: Miscellaneous Physical/Conventional Chemistry Parameters

\begin{tabular}{lrrrrrrr}
\hline Bromide & $\mathrm{ND}$ & 0.100 & $\mathrm{mg} / \mathrm{L}$ & 1 & $15-\mathrm{Jul}-2009$ & $15-\mathrm{Jul}-2009$ & EPA 300.0 \\
Chloride & $\mathbf{3 . 8 0}$ & 1.00 & $\mathrm{mg} / \mathrm{L}$ & 1 & $15-\mathrm{Jul}-2009$ & $15-\mathrm{Jul}-2009$ & EPA 300.0 \\
Nitrate as N & $\mathbf{0 . 3 8 4}$ & 0.100 & $\mathrm{mg} / \mathrm{L}$ & 1 & $15-J u l-2009$ & $15-\mathrm{Jul}-2009$ & EPA 300.0 \\
Sulfate & $\mathbf{3 7 . 9}$ & 0.100 & $\mathrm{mg} / \mathrm{L}$ & 1 & $15-J u l-2009$ & $15-J u l-2009$ & EPA 300.0
\end{tabular}




\section{USACE ERDC-EP-C \\ 3909 Halls Ferry Road \\ Vicksburg, MS 39180-6199}

ERDC - ECB

$-,-$
Project: TVA Fly Ash Project

Project Manager: Tony Bednar
Reported:

03-Sep-2009

DRAFT: EMR MT N2 Hour 48 Rep A - Total Metals

9061503-25 (Water)

\begin{tabular}{|c|c|c|c|c|c|c|c|c|}
\hline Analyte & Result & $\begin{array}{r}\text { porting } \\
\text { Limit }\end{array}$ & Units & Dilution & Prepared & Analyzed & Method & Notes \\
\hline
\end{tabular}

ERDC- EL-EP-C (Environmental Chemistry Branch)

DRAFT: Wet Chemistry

\begin{tabular}{llllllll}
\hline Hardness & $\mathbf{6 7 . 6}$ & 1.32 & $\begin{array}{c}\text { mg equil } \\
\text { CaCO3/ }\end{array}$ & 4 & 25-Jun-2009 & 25-Jun-2009 & Hardness
\end{tabular}

$\mathrm{L}$

DRAFT: Metals by EPA 6000/7000 Series Methods

\begin{tabular}{|c|c|c|c|c|c|c|c|c|}
\hline Mercury & 0.000435 & 0.000005 & $\mathrm{mg} / \mathrm{L}$ & 2 & 24-Jul-2009 & 24-Jul-2009 & EPA 7471A & \\
\hline Strontium & 0.450 & 0.0800 & $\mathrm{mg} / \mathrm{L}$ & 4 & 25-Jun-2009 & 25-Jun-2009 & EPA 6010B & \\
\hline Aluminum & 31.1 & 0.200 & $\mathrm{mg} / \mathrm{L}$ & 4 & 25-Jun-2009 & 25-Jun-2009 & SW 846/6010 & \\
\hline Calcium & 18.6 & 0.200 & $\mathrm{mg} / \mathrm{L}$ & 4 & 25-Jun-2009 & 25-Jun-2009 & SW 846/6010 & B \\
\hline Iron & 12.7 & 0.200 & $\mathrm{mg} / \mathrm{L}$ & 4 & 25-Jun-2009 & 25-Jun-2009 & SW 846/6010 & \\
\hline Magnesium & 5.15 & 0.200 & $\mathrm{mg} / \mathrm{L}$ & 4 & 25-Jun-2009 & 25-Jun-2009 & SW 846/6010 & \\
\hline Potassium & 7.34 & 0.200 & $\mathrm{mg} / \mathrm{L}$ & 4 & 25-Jun-2009 & 25-Jun-2009 & SW 846/6010 & B \\
\hline Sodium & 4.05 & 0.200 & $\mathrm{mg} / \mathrm{L}$ & 4 & 25-Jun-2009 & 25-Jun-2009 & SW 846/6010 & B \\
\hline Antimony & 0.0056 & 0.0020 & $\mathrm{mg} / \mathrm{L}$ & 4 & 17-Jun-2009 & 17-Jun-2009 & SW 846/6020 & \\
\hline Arsenic & 0.146 & 0.0020 & $\mathrm{mg} / \mathrm{L}$ & 4 & 17-Jun-2009 & 17-Jun-2009 & SW 846/6020 & \\
\hline Barium & 0.665 & 0.0020 & $\mathrm{mg} / \mathrm{L}$ & 4 & 17-Jun-2009 & 17-Jun-2009 & SW 846/6020 & B \\
\hline Beryllium & 0.0059 & 0.0020 & $\mathrm{mg} / \mathrm{L}$ & 4 & 17-Jun-2009 & 17-Jun-2009 & SW 846/6020 & \\
\hline Cadmium & 0.0009 & 0.0020 & $\mathrm{mg} / \mathrm{L}$ & 4 & 17-Jun-2009 & 17-Jun-2009 & SW 846/6020 & J \\
\hline Chromium & 0.0402 & 0.0020 & $\mathrm{mg} / \mathrm{L}$ & 4 & 17-Jun-2009 & 17-Jun-2009 & SW 846/6020 & \\
\hline Cobalt & 0.0225 & 0.0020 & $\mathrm{mg} / \mathrm{L}$ & 4 & 17-Jun-2009 & 17-Jun-2009 & SW 846/6020 & \\
\hline Copper & 0.0819 & 0.0020 & $\mathrm{mg} / \mathrm{L}$ & 4 & 17-Jun-2009 & 17-Jun-2009 & SW 846/6020 & B \\
\hline Lead & 0.0490 & 0.0020 & $\mathrm{mg} / \mathrm{L}$ & 4 & 17-Jun-2009 & 17-Jun-2009 & SW 846/6020 & \\
\hline Manganese & 0.122 & 0.0020 & $\mathrm{mg} / \mathrm{L}$ & 4 & 17-Jun-2009 & 17-Jun-2009 & SW 846/6020 & \\
\hline Molybdenum & 0.0074 & 0.0020 & $\mathrm{mg} / \mathrm{L}$ & 4 & 17-Jun-2009 & 17-Jun-2009 & SW 846/6020 & \\
\hline Nickel & 0.0445 & 0.0020 & $\mathrm{mg} / \mathrm{L}$ & 4 & 17-Jun-2009 & 17-Jun-2009 & SW 846/6020 & \\
\hline Selenium & 0.0077 & 0.0020 & $\mathrm{mg} / \mathrm{L}$ & 4 & 17-Jun-2009 & 17-Jun-2009 & SW 846/6020 & \\
\hline Silver & ND & 0.0020 & $\mathrm{mg} / \mathrm{L}$ & 4 & 17-Jun-2009 & 17-Jun-2009 & SW 846/6020 & $\mathrm{U}$ \\
\hline Thallium & 0.0032 & 0.0020 & $\mathrm{mg} / \mathrm{L}$ & 4 & 17-Jun-2009 & 17-Jun-2009 & SW 846/6020 & \\
\hline Vanadium & 0.136 & 0.0020 & $\mathrm{mg} / \mathrm{L}$ & 4 & 17-Jun-2009 & 17-Jun-2009 & SW 846/6020 & \\
\hline Zinc & 0.0964 & 0.0020 & $\mathrm{mg} / \mathrm{L}$ & 4 & 17-Jun-2009 & 17-Jun-2009 & SW 846/6020 & B \\
\hline
\end{tabular}




\section{USACE ERDC-EP-C \\ 3909 Halls Ferry Road \\ Vicksburg, MS 39180-6199}

ERDC - ECB

$-\cdot$,
Project: TVA Fly Ash Project

Project Manager: Tony Bednar
Reported:

03-Sep-2009

DRAFT: EMR MT N2 Hour 48 Rep B - Total Metals

9061503-26 (Water)

\begin{tabular}{|c|c|c|c|c|c|c|c|c|}
\hline Analyte & Result & $\begin{array}{r}\text { porting } \\
\text { Limit }\end{array}$ & Units & Dilution & Prepared & Analyzed & Method & Notes \\
\hline
\end{tabular}

ERDC- EL-EP-C (Environmental Chemistry Branch)

DRAFT: Wet Chemistry

\begin{tabular}{llllllll}
\hline Hardness & $\mathbf{5 6 . 2}$ & 1.32 & $\begin{array}{c}\text { mg equil } \\
\text { CaCO3/ }\end{array}$ & 4 & 25-Jun-2009 & 25-Jun-2009
\end{tabular}

$\mathrm{L}$

DRAFT: Metals by EPA 6000/7000 Series Methods

\begin{tabular}{|c|c|c|c|c|c|c|c|c|}
\hline Mercury & 0.000435 & 0.000005 & $\mathrm{mg} / \mathrm{L}$ & 2 & 24-Jul-2009 & 24-Jul-2009 & EPA 7471A & \\
\hline Strontium & 0.320 & 0.0800 & $\mathrm{mg} / \mathrm{L}$ & 4 & 25-Jun-2009 & 25-Jun-2009 & EPA 6010B & \\
\hline Aluminum & 18.0 & 0.200 & $\mathrm{mg} / \mathrm{L}$ & 4 & 25-Jun-2009 & 25-Jun-2009 & SW 846/6010 & \\
\hline Calcium & 15.9 & 0.200 & $\mathrm{mg} / \mathrm{L}$ & 4 & 25-Jun-2009 & 25-Jun-2009 & SW 846/6010 & B \\
\hline Iron & 7.60 & 0.200 & $\mathrm{mg} / \mathrm{L}$ & 4 & 25-Jun-2009 & 25-Jun-2009 & SW 846/6010 & \\
\hline Magnesium & 4.01 & 0.200 & $\mathrm{mg} / \mathrm{L}$ & 4 & 25-Jun-2009 & 25-Jun-2009 & SW 846/6010 & \\
\hline Potassium & 4.99 & 0.200 & $\mathrm{mg} / \mathrm{L}$ & 4 & 25-Jun-2009 & 25-Jun-2009 & SW 846/6010 & B \\
\hline Sodium & 4.04 & 0.200 & $\mathrm{mg} / \mathrm{L}$ & 4 & 25-Jun-2009 & 25-Jun-2009 & SW 846/6010 & B \\
\hline Antimony & 0.0042 & 0.0020 & $\mathrm{mg} / \mathrm{L}$ & 4 & 17-Jun-2009 & 17-Jun-2009 & SW 846/6020 & \\
\hline Arsenic & 0.103 & 0.0020 & $\mathrm{mg} / \mathrm{L}$ & 4 & 17-Jun-2009 & 17-Jun-2009 & SW 846/6020 & \\
\hline Barium & 0.424 & 0.0020 & $\mathrm{mg} / \mathrm{L}$ & 4 & 17-Jun-2009 & 17-Jun-2009 & SW 846/6020 & B \\
\hline Beryllium & 0.0035 & 0.0020 & $\mathrm{mg} / \mathrm{L}$ & 4 & 17-Jun-2009 & 17-Jun-2009 & SW 846/6020 & \\
\hline Cadmium & 0.0007 & 0.0020 & $\mathrm{mg} / \mathrm{L}$ & 4 & 17-Jun-2009 & 17-Jun-2009 & SW 846/6020 & $\mathrm{J}$ \\
\hline Chromium & 0.0273 & 0.0020 & $\mathrm{mg} / \mathrm{L}$ & 4 & 17-Jun-2009 & 17-Jun-2009 & SW 846/6020 & \\
\hline Cobalt & 0.0145 & 0.0020 & $\mathrm{mg} / \mathrm{L}$ & 4 & 17-Jun-2009 & 17-Jun-2009 & SW 846/6020 & \\
\hline Copper & 0.0552 & 0.0020 & $\mathrm{mg} / \mathrm{L}$ & 4 & 17-Jun-2009 & 17-Jun-2009 & SW 846/6020 & B \\
\hline Lead & 0.0287 & 0.0020 & $\mathrm{mg} / \mathrm{L}$ & 4 & 17-Jun-2009 & 17-Jun-2009 & SW 846/6020 & \\
\hline Manganese & 0.0780 & 0.0020 & $\mathrm{mg} / \mathrm{L}$ & 4 & 17-Jun-2009 & 17-Jun-2009 & SW 846/6020 & \\
\hline Molybdenum & 0.0051 & 0.0020 & $\mathrm{mg} / \mathrm{L}$ & 4 & 17-Jun-2009 & 17-Jun-2009 & SW 846/6020 & \\
\hline Nickel & 0.0296 & 0.0020 & $\mathrm{mg} / \mathrm{L}$ & 4 & 17-Jun-2009 & 17-Jun-2009 & SW 846/6020 & \\
\hline Selenium & 0.0059 & 0.0020 & $\mathrm{mg} / \mathrm{L}$ & 4 & 17-Jun-2009 & 17-Jun-2009 & SW 846/6020 & \\
\hline Silver & ND & 0.0020 & $\mathrm{mg} / \mathrm{L}$ & 4 & 17-Jun-2009 & 17-Jun-2009 & SW 846/6020 & $\mathrm{U}$ \\
\hline Thallium & 0.0021 & 0.0020 & $\mathrm{mg} / \mathrm{L}$ & 4 & 17-Jun-2009 & 17-Jun-2009 & SW 846/6020 & \\
\hline Vanadium & 0.0964 & 0.0020 & $\mathrm{mg} / \mathrm{L}$ & 4 & 17-Jun-2009 & 17-Jun-2009 & SW 846/6020 & \\
\hline Zinc & 0.0756 & 0.0020 & $\mathrm{mg} / \mathrm{L}$ & 4 & 17-Jun-2009 & 17-Jun-2009 & SW 846/6020 & B \\
\hline
\end{tabular}




\section{USACE ERDC-EP-C \\ 3909 Halls Ferry Road \\ Vicksburg, MS 39180-6199}

ERDC - ECB

$-\cdot$,
Project: TVA Fly Ash Project

Project Manager: Tony Bednar
Reported:

03-Sep-2009

DRAFT: EMR MT N2 Hour 48 Rep C - Total Metals

9061503-27 (Water)

\begin{tabular}{|c|c|c|c|c|c|c|c|c|}
\hline Analyte & Result & $\begin{array}{r}\text { porting } \\
\text { Limit }\end{array}$ & Units & Dilution & Prepared & Analyzed & Method & Notes \\
\hline
\end{tabular}

ERDC- EL-EP-C (Environmental Chemistry Branch)

DRAFT: Wet Chemistry

\begin{tabular}{llllllll}
\hline Hardness & $\mathbf{5 3 . 3}$ & 1.32 & $\begin{array}{c}\text { mg equil } \\
\text { CaCO3/ }\end{array}$ & 4 & 25-Jun-2009 & 25-Jun-2009
\end{tabular}

$\mathrm{L}$

DRAFT: Metals by EPA 6000/7000 Series Methods

\begin{tabular}{|c|c|c|c|c|c|c|c|c|}
\hline Mercury & 0.000375 & 0.000005 & $\mathrm{mg} / \mathrm{L}$ & 2 & 24-Jul-2009 & 24-Jul-2009 & EPA 7471A & \\
\hline Strontium & 0.310 & 0.0800 & $\mathrm{mg} / \mathrm{L}$ & 4 & 25-Jun-2009 & 25-Jun-2009 & EPA 6010B & \\
\hline Aluminum & 17.2 & 0.200 & $\mathrm{mg} / \mathrm{L}$ & 4 & 25-Jun-2009 & 25-Jun-2009 & SW 846/6010 & \\
\hline Calcium & 15.2 & 0.200 & $\mathrm{mg} / \mathrm{L}$ & 4 & 25-Jun-2009 & 25-Jun-2009 & SW 846/6010 & B \\
\hline Iron & 7.28 & 0.200 & $\mathrm{mg} / \mathrm{L}$ & 4 & 25-Jun-2009 & 25-Jun-2009 & SW 846/6010 & \\
\hline Magnesium & 3.76 & 0.200 & $\mathrm{mg} / \mathrm{L}$ & 4 & 25-Jun-2009 & 25-Jun-2009 & SW 846/6010 & \\
\hline Potassium & 4.56 & 0.200 & $\mathrm{mg} / \mathrm{L}$ & 4 & 25-Jun-2009 & 25-Jun-2009 & SW 846/6010 & B \\
\hline Sodium & 3.57 & 0.200 & $\mathrm{mg} / \mathrm{L}$ & 4 & 25-Jun-2009 & 25-Jun-2009 & SW 846/6010 & B \\
\hline Antimony & 0.0038 & 0.0020 & $\mathrm{mg} / \mathrm{L}$ & 4 & 17-Jun-2009 & 17-Jun-2009 & SW 846/6020 & \\
\hline Arsenic & 0.101 & 0.0020 & $\mathrm{mg} / \mathrm{L}$ & 4 & 17-Jun-2009 & 17-Jun-2009 & SW 846/6020 & \\
\hline Barium & 0.421 & 0.0020 & $\mathrm{mg} / \mathrm{L}$ & 4 & 17-Jun-2009 & 17-Jun-2009 & SW 846/6020 & B \\
\hline Beryllium & 0.0032 & 0.0020 & $\mathrm{mg} / \mathrm{L}$ & 4 & 17-Jun-2009 & 17-Jun-2009 & SW 846/6020 & \\
\hline Cadmium & 0.0007 & 0.0020 & $\mathrm{mg} / \mathrm{L}$ & 4 & 17-Jun-2009 & 17-Jun-2009 & SW 846/6020 & $\mathrm{J}$ \\
\hline Chromium & 0.0260 & 0.0020 & $\mathrm{mg} / \mathrm{L}$ & 4 & 17-Jun-2009 & 17-Jun-2009 & SW 846/6020 & \\
\hline Cobalt & 0.0131 & 0.0020 & $\mathrm{mg} / \mathrm{L}$ & 4 & 17-Jun-2009 & 17-Jun-2009 & SW 846/6020 & \\
\hline Copper & 0.0509 & 0.0020 & $\mathrm{mg} / \mathrm{L}$ & 4 & 17-Jun-2009 & 17-Jun-2009 & SW 846/6020 & B \\
\hline Lead & 0.0283 & 0.0020 & $\mathrm{mg} / \mathrm{L}$ & 4 & 17-Jun-2009 & 17-Jun-2009 & SW 846/6020 & \\
\hline Manganese & 0.0746 & 0.0020 & $\mathrm{mg} / \mathrm{L}$ & 4 & 17-Jun-2009 & 17-Jun-2009 & SW 846/6020 & \\
\hline Molybdenum & 0.0052 & 0.0020 & $\mathrm{mg} / \mathrm{L}$ & 4 & 17-Jun-2009 & 17-Jun-2009 & SW 846/6020 & \\
\hline Nickel & 0.0265 & 0.0020 & $\mathrm{mg} / \mathrm{L}$ & 4 & 17-Jun-2009 & 17-Jun-2009 & SW 846/6020 & \\
\hline Selenium & 0.0058 & 0.0020 & $\mathrm{mg} / \mathrm{L}$ & 4 & 17-Jun-2009 & 17-Jun-2009 & SW 846/6020 & \\
\hline Silver & ND & 0.0020 & $\mathrm{mg} / \mathrm{L}$ & 4 & 17-Jun-2009 & 17-Jun-2009 & SW 846/6020 & $\mathrm{U}$ \\
\hline Thallium & 0.0021 & 0.0020 & $\mathrm{mg} / \mathrm{L}$ & 4 & 17-Jun-2009 & 17-Jun-2009 & SW 846/6020 & \\
\hline Vanadium & 0.0880 & 0.0020 & $\mathrm{mg} / \mathrm{L}$ & 4 & 17-Jun-2009 & 17-Jun-2009 & SW 846/6020 & \\
\hline Zinc & 0.0667 & 0.0020 & $\mathrm{mg} / \mathrm{L}$ & 4 & 17-Jun-2009 & 17-Jun-2009 & SW 846/6020 & B \\
\hline
\end{tabular}




\section{USACE ERDC-EP-C \\ 3909 Halls Ferry Road \\ Vicksburg, MS 39180-6199}

ERDC - ECB

$-\cdot$,
Project: TVA Fly Ash Project

Project Manager: Tony Bednar
Reported:

03-Sep-2009

DRAFT: EMR MT O2 Hour 48 Rep A - Total Metals

9061503-28 (Water)

\begin{tabular}{|c|c|c|c|c|c|c|c|c|}
\hline Analyte & Result & $\begin{array}{r}\text { porting } \\
\text { Limit }\end{array}$ & Units & Dilution & Prepared & Analyzed & Method & Notes \\
\hline
\end{tabular}

ERDC- EL-EP-C (Environmental Chemistry Branch)

DRAFT: Wet Chemistry

\begin{tabular}{llcccccc}
\hline Hardness & $\mathbf{4 6 . 6}$ & 1.32 & $\begin{array}{c}\text { mg equil } \\
\text { CaCO3/ }\end{array}$ & 4 & 25-Jun-2009 & 25-Jun-2009
\end{tabular}

$\mathrm{L}$

DRAFT: Metals by EPA 6000/7000 Series Methods

\begin{tabular}{|c|c|c|c|c|c|c|c|c|}
\hline Mercury & 0.000456 & 0.000005 & $\mathrm{mg} / \mathrm{L}$ & 2 & 24-Jul-2009 & 24-Jul-2009 & EPA 7471A & \\
\hline Strontium & 0.218 & 0.0800 & $\mathrm{mg} / \mathrm{L}$ & 4 & 25-Jun-2009 & 25-Jun-2009 & EPA 6010B & \\
\hline Aluminum & 9.51 & 0.200 & $\mathrm{mg} / \mathrm{L}$ & 4 & 25-Jun-2009 & 25-Jun-2009 & SW 846/6010 & \\
\hline Calcium & 13.4 & 0.200 & $\mathrm{mg} / \mathrm{L}$ & 4 & 25-Jun-2009 & 25-Jun-2009 & SW 846/6010 & B \\
\hline Iron & 4.17 & 0.200 & $\mathrm{mg} / \mathrm{L}$ & 4 & 25-Jun-2009 & 25-Jun-2009 & SW 846/6010 & \\
\hline Magnesium & 3.21 & 0.200 & $\mathrm{mg} / \mathrm{L}$ & 4 & 25-Jun-2009 & 25-Jun-2009 & SW 846/6010 & \\
\hline Potassium & 3.47 & 0.200 & $\mathrm{mg} / \mathrm{L}$ & 4 & 25-Jun-2009 & 25-Jun-2009 & SW 846/6010 & B \\
\hline Sodium & 3.35 & 0.200 & $\mathrm{mg} / \mathrm{L}$ & 4 & 25-Jun-2009 & 25-Jun-2009 & SW 846/6010 & B \\
\hline Antimony & 0.0025 & 0.0020 & $\mathrm{mg} / \mathrm{L}$ & 4 & 17-Jun-2009 & 17-Jun-2009 & SW 846/6020 & \\
\hline Arsenic & 0.0673 & 0.0020 & $\mathrm{mg} / \mathrm{L}$ & 4 & 17-Jun-2009 & 17-Jun-2009 & SW 846/6020 & \\
\hline Barium & 0.277 & 0.0020 & $\mathrm{mg} / \mathrm{L}$ & 4 & 17-Jun-2009 & 17-Jun-2009 & SW 846/6020 & B \\
\hline Beryllium & 0.0020 & 0.0020 & $\mathrm{mg} / \mathrm{L}$ & 4 & 17-Jun-2009 & 17-Jun-2009 & SW 846/6020 & $\mathrm{J}$ \\
\hline Cadmium & 0.0008 & 0.0020 & $\mathrm{mg} / \mathrm{L}$ & 4 & 17-Jun-2009 & 17-Jun-2009 & SW 846/6020 & J \\
\hline Chromium & 0.0179 & 0.0020 & $\mathrm{mg} / \mathrm{L}$ & 4 & 17-Jun-2009 & 17-Jun-2009 & SW 846/6020 & \\
\hline Cobalt & 0.0085 & 0.0020 & $\mathrm{mg} / \mathrm{L}$ & 4 & 17-Jun-2009 & 17-Jun-2009 & SW 846/6020 & \\
\hline Copper & 0.0341 & 0.0020 & $\mathrm{mg} / \mathrm{L}$ & 4 & 17-Jun-2009 & 17-Jun-2009 & SW 846/6020 & B \\
\hline Lead & 0.0163 & 0.0020 & $\mathrm{mg} / \mathrm{L}$ & 4 & 17-Jun-2009 & 17-Jun-2009 & SW 846/6020 & \\
\hline Manganese & 0.0589 & 0.0020 & $\mathrm{mg} / \mathrm{L}$ & 4 & 17-Jun-2009 & 17-Jun-2009 & SW 846/6020 & \\
\hline Molybdenum & 0.0037 & 0.0020 & $\mathrm{mg} / \mathrm{L}$ & 4 & 17-Jun-2009 & 17-Jun-2009 & SW 846/6020 & \\
\hline Nickel & 0.0187 & 0.0020 & $\mathrm{mg} / \mathrm{L}$ & 4 & 17-Jun-2009 & 17-Jun-2009 & SW 846/6020 & \\
\hline Selenium & 0.0047 & 0.0020 & $\mathrm{mg} / \mathrm{L}$ & 4 & 17-Jun-2009 & 17-Jun-2009 & SW 846/6020 & \\
\hline Silver & ND & 0.0020 & $\mathrm{mg} / \mathrm{L}$ & 4 & 17-Jun-2009 & 17-Jun-2009 & SW 846/6020 & $\mathrm{U}$ \\
\hline Thallium & 0.0014 & 0.0020 & $\mathrm{mg} / \mathrm{L}$ & 4 & 17-Jun-2009 & 17-Jun-2009 & SW 846/6020 & J \\
\hline Vanadium & 0.0658 & 0.0020 & $\mathrm{mg} / \mathrm{L}$ & 4 & 17-Jun-2009 & 17-Jun-2009 & SW 846/6020 & \\
\hline Zinc & 0.0457 & 0.0020 & $\mathrm{mg} / \mathrm{L}$ & 4 & 17-Jun-2009 & 17-Jun-2009 & SW 846/6020 & B \\
\hline
\end{tabular}




\section{USACE ERDC-EP-C \\ 3909 Halls Ferry Road \\ Vicksburg, MS 39180-6199}

ERDC - ECB

$-\cdot$,
Project: TVA Fly Ash Project

Project Manager: Tony Bednar
Reported:

03-Sep-2009

DRAFT: EMR MT O2 Hour 48 Rep B - Total Metals

9061503-29 (Water)

\begin{tabular}{|c|c|c|c|c|c|c|c|c|}
\hline Analyte & Result & $\begin{array}{r}\text { porting } \\
\text { Limit }\end{array}$ & Units & Dilution & Prepared & Analyzed & Method & Notes \\
\hline
\end{tabular}

ERDC- EL-EP-C (Environmental Chemistry Branch)

DRAFT: Wet Chemistry

\begin{tabular}{llllllll}
\hline Hardness & $\mathbf{4 2 . 9}$ & 1.32 & $\begin{array}{c}\text { mg equil } \\
\text { CaCO3/ }\end{array}$ & 4 & 25-Jun-2009 & 25-Jun-2009
\end{tabular}

$\mathrm{L}$

DRAFT: Metals by EPA 6000/7000 Series Methods

\begin{tabular}{|c|c|c|c|c|c|c|c|c|}
\hline Mercury & 0.000429 & 0.000005 & $\mathrm{mg} / \mathrm{L}$ & 2 & 24-Jul-2009 & 24-Jul-2009 & EPA 7471A & \\
\hline Strontium & 0.179 & 0.0800 & $\mathrm{mg} / \mathrm{L}$ & 4 & 25-Jun-2009 & 25-Jun-2009 & EPA 6010B & \\
\hline Aluminum & 6.60 & 0.200 & $\mathrm{mg} / \mathrm{L}$ & 4 & 25-Jun-2009 & 25-Jun-2009 & SW 846/6010 & \\
\hline Calcium & 12.5 & 0.200 & $\mathrm{mg} / \mathrm{L}$ & 4 & 25-Jun-2009 & 25-Jun-2009 & SW 846/6010 & B \\
\hline Iron & 2.91 & 0.200 & $\mathrm{mg} / \mathrm{L}$ & 4 & 25-Jun-2009 & 25-Jun-2009 & SW 846/6010 & \\
\hline Magnesium & 2.86 & 0.200 & $\mathrm{mg} / \mathrm{L}$ & 4 & 25-Jun-2009 & 25-Jun-2009 & SW 846/6010 & \\
\hline Potassium & 2.73 & 0.200 & $\mathrm{mg} / \mathrm{L}$ & 4 & 25-Jun-2009 & 25-Jun-2009 & SW 846/6010 & B \\
\hline Sodium & 3.29 & 0.200 & $\mathrm{mg} / \mathrm{L}$ & 4 & 25-Jun-2009 & 25-Jun-2009 & SW 846/6010 & B \\
\hline Antimony & 0.0018 & 0.0020 & $\mathrm{mg} / \mathrm{L}$ & 4 & 17-Jun-2009 & 17-Jun-2009 & SW 846/6020 & $\mathrm{J}$ \\
\hline Arsenic & 0.0516 & 0.0020 & $\mathrm{mg} / \mathrm{L}$ & 4 & 17-Jun-2009 & 17-Jun-2009 & SW 846/6020 & \\
\hline Barium & 0.211 & 0.0020 & $\mathrm{mg} / \mathrm{L}$ & 4 & 17-Jun-2009 & 17-Jun-2009 & SW 846/6020 & B \\
\hline Beryllium & 0.0015 & 0.0020 & $\mathrm{mg} / \mathrm{L}$ & 4 & 17-Jun-2009 & 17-Jun-2009 & SW 846/6020 & J \\
\hline Cadmium & ND & 0.0020 & $\mathrm{mg} / \mathrm{L}$ & 4 & 17-Jun-2009 & 17-Jun-2009 & SW 846/6020 & $\mathrm{U}$ \\
\hline Chromium & 0.0133 & 0.0020 & $\mathrm{mg} / \mathrm{L}$ & 4 & 17-Jun-2009 & 17-Jun-2009 & SW 846/6020 & \\
\hline Cobalt & 0.0062 & 0.0020 & $\mathrm{mg} / \mathrm{L}$ & 4 & 17-Jun-2009 & 17-Jun-2009 & SW 846/6020 & \\
\hline Copper & 0.0256 & 0.0020 & $\mathrm{mg} / \mathrm{L}$ & 4 & 17-Jun-2009 & 17-Jun-2009 & SW 846/6020 & B \\
\hline Lead & 0.0109 & 0.0020 & $\mathrm{mg} / \mathrm{L}$ & 4 & 17-Jun-2009 & 17-Jun-2009 & SW 846/6020 & \\
\hline Manganese & 0.0436 & 0.0020 & $\mathrm{mg} / \mathrm{L}$ & 4 & 17-Jun-2009 & 17-Jun-2009 & SW 846/6020 & \\
\hline Molybdenum & 0.0030 & 0.0020 & $\mathrm{mg} / \mathrm{L}$ & 4 & 17-Jun-2009 & 17-Jun-2009 & SW 846/6020 & \\
\hline Nickel & 0.0137 & 0.0020 & $\mathrm{mg} / \mathrm{L}$ & 4 & 17-Jun-2009 & 17-Jun-2009 & SW 846/6020 & \\
\hline Selenium & 0.0026 & 0.0020 & $\mathrm{mg} / \mathrm{L}$ & 4 & 17-Jun-2009 & 17-Jun-2009 & SW 846/6020 & \\
\hline Silver & ND & 0.0020 & $\mathrm{mg} / \mathrm{L}$ & 4 & 17-Jun-2009 & 17-Jun-2009 & SW 846/6020 & $\mathrm{U}$ \\
\hline Thallium & 0.0011 & 0.0020 & $\mathrm{mg} / \mathrm{L}$ & 4 & 17-Jun-2009 & 17-Jun-2009 & SW 846/6020 & J \\
\hline Vanadium & 0.0502 & 0.0020 & $\mathrm{mg} / \mathrm{L}$ & 4 & 17-Jun-2009 & 17-Jun-2009 & SW 846/6020 & \\
\hline Zinc & 0.0410 & 0.0020 & $\mathrm{mg} / \mathrm{L}$ & 4 & 17-Jun-2009 & 17-Jun-2009 & SW 846/6020 & B \\
\hline
\end{tabular}




\section{USACE ERDC-EP-C \\ 3909 Halls Ferry Road \\ Vicksburg, MS 39180-6199}

ERDC - ECB

$-\cdot$,
Project: TVA Fly Ash Project

Project Manager: Tony Bednar
Reported:

03-Sep-2009

DRAFT: EMR MT O2 Hour 48 Rep C - Total Metals

9061503-30 (Water)

\begin{tabular}{|c|c|c|c|c|c|c|c|c|}
\hline Analyte & Result & $\begin{array}{r}\text { porting } \\
\text { Limit }\end{array}$ & Units & Dilution & Prepared & Analyzed & Method & Notes \\
\hline
\end{tabular}

ERDC- EL-EP-C (Environmental Chemistry Branch)

DRAFT: Wet Chemistry

\begin{tabular}{llllllll}
\hline Hardness & $\mathbf{6 0 . 2}$ & 1.32 & $\begin{array}{c}\text { mg equil } \\
\text { CaCO3/ }\end{array}$ & 4 & 25-Jun-2009 & 25-Jun-2009
\end{tabular}

$\mathrm{L}$

DRAFT: Metals by EPA 6000/7000 Series Methods

\begin{tabular}{|c|c|c|c|c|c|c|c|c|}
\hline Mercury & 0.000557 & 0.000005 & $\mathrm{mg} / \mathrm{L}$ & 2 & 24-Jul-2009 & 24-Jul-2009 & EPA 7471A & \\
\hline Strontium & 0.390 & 0.0800 & $\mathrm{mg} / \mathrm{L}$ & 4 & 25-Jun-2009 & 25-Jun-2009 & EPA 6010B & \\
\hline Aluminum & 24.3 & 0.200 & $\mathrm{mg} / \mathrm{L}$ & 4 & 25-Jun-2009 & 25-Jun-2009 & SW 846/6010 & \\
\hline Calcium & 16.8 & 0.200 & $\mathrm{mg} / \mathrm{L}$ & 4 & 25-Jun-2009 & 25-Jun-2009 & SW 846/6010 & B \\
\hline Iron & 9.95 & 0.200 & $\mathrm{mg} / \mathrm{L}$ & 4 & 25-Jun-2009 & 25-Jun-2009 & SW 846/6010 & \\
\hline Magnesium & 4.40 & 0.200 & $\mathrm{mg} / \mathrm{L}$ & 4 & 25-Jun-2009 & 25-Jun-2009 & SW 846/6010 & \\
\hline Potassium & 6.04 & 0.200 & $\mathrm{mg} / \mathrm{L}$ & 4 & 25-Jun-2009 & 25-Jun-2009 & SW 846/6010 & B \\
\hline Sodium & 3.92 & 0.200 & $\mathrm{mg} / \mathrm{L}$ & 4 & 25-Jun-2009 & 25-Jun-2009 & SW 846/6010 & B \\
\hline Antimony & 0.0043 & 0.0020 & $\mathrm{mg} / \mathrm{L}$ & 4 & 17-Jun-2009 & 17-Jun-2009 & SW 846/6020 & \\
\hline Arsenic & 0.114 & 0.0020 & $\mathrm{mg} / \mathrm{L}$ & 4 & 17-Jun-2009 & 17-Jun-2009 & SW 846/6020 & \\
\hline Barium & 0.546 & 0.0020 & $\mathrm{mg} / \mathrm{L}$ & 4 & 17-Jun-2009 & 17-Jun-2009 & SW 846/6020 & B \\
\hline Beryllium & 0.0046 & 0.0020 & $\mathrm{mg} / \mathrm{L}$ & 4 & 17-Jun-2009 & 17-Jun-2009 & SW 846/6020 & \\
\hline Cadmium & 0.0007 & 0.0020 & $\mathrm{mg} / \mathrm{L}$ & 4 & 17-Jun-2009 & 17-Jun-2009 & SW 846/6020 & J \\
\hline Chromium & 0.0348 & 0.0020 & $\mathrm{mg} / \mathrm{L}$ & 4 & 17-Jun-2009 & 17-Jun-2009 & SW 846/6020 & \\
\hline Cobalt & 0.0193 & 0.0020 & $\mathrm{mg} / \mathrm{L}$ & 4 & 17-Jun-2009 & 17-Jun-2009 & SW 846/6020 & \\
\hline Copper & 0.0696 & 0.0020 & $\mathrm{mg} / \mathrm{L}$ & 4 & 17-Jun-2009 & 17-Jun-2009 & SW 846/6020 & B \\
\hline Lead & 0.0384 & 0.0020 & $\mathrm{mg} / \mathrm{L}$ & 4 & 17-Jun-2009 & 17-Jun-2009 & SW 846/6020 & \\
\hline Manganese & 0.112 & 0.0020 & $\mathrm{mg} / \mathrm{L}$ & 4 & 17-Jun-2009 & 17-Jun-2009 & SW 846/6020 & \\
\hline Molybdenum & 0.0059 & 0.0020 & $\mathrm{mg} / \mathrm{L}$ & 4 & 17-Jun-2009 & 17-Jun-2009 & SW 846/6020 & \\
\hline Nickel & 0.0390 & 0.0020 & $\mathrm{mg} / \mathrm{L}$ & 4 & 17-Jun-2009 & 17-Jun-2009 & SW 846/6020 & \\
\hline Selenium & 0.0071 & 0.0020 & $\mathrm{mg} / \mathrm{L}$ & 4 & 17-Jun-2009 & 17-Jun-2009 & SW 846/6020 & \\
\hline Silver & ND & 0.0020 & $\mathrm{mg} / \mathrm{L}$ & 4 & 17-Jun-2009 & 17-Jun-2009 & SW 846/6020 & $\mathrm{U}$ \\
\hline Thallium & 0.0026 & 0.0020 & $\mathrm{mg} / \mathrm{L}$ & 4 & 17-Jun-2009 & 17-Jun-2009 & SW 846/6020 & \\
\hline Vanadium & 0.124 & 0.0020 & $\mathrm{mg} / \mathrm{L}$ & 4 & 17-Jun-2009 & 17-Jun-2009 & SW 846/6020 & \\
\hline Zinc & 0.0810 & 0.0020 & $\mathrm{mg} / \mathrm{L}$ & 4 & 17-Jun-2009 & 17-Jun-2009 & SW 846/6020 & B \\
\hline
\end{tabular}




\section{USACE ERDC-EP-C \\ 3909 Halls Ferry Road \\ Vicksburg, MS 39180-6199}

ERDC - ECB

$-\cdot$,
Project: TVA Fly Ash Project

Project Manager: Tony Bednar
Reported:

03-Sep-2009

DRAFT: SLC MT N2 Hour 48 Rep A - Total Metals

9061503-31 (Water)

\begin{tabular}{|c|c|c|c|c|c|c|c|c|}
\hline Analyte & Result & $\begin{array}{r}\text { porting } \\
\text { Limit }\end{array}$ & Units & Dilution & Prepared & Analyzed & Method & Notes \\
\hline
\end{tabular}

ERDC- EL-EP-C (Environmental Chemistry Branch)

DRAFT: Wet Chemistry

\begin{tabular}{llllllll}
\hline Hardness & $\mathbf{1 0 4}$ & 1.32 & $\begin{array}{c}\text { mg equil } \\
\text { CaCO3/ }\end{array}$ & 4 & 25-Jun-2009 & 25-Jun-2009
\end{tabular}

$\mathrm{L}$

DRAFT: Metals by EPA 6000/7000 Series Methods

\begin{tabular}{|c|c|c|c|c|c|c|c|c|}
\hline Mercury & 0.000134 & 0.000005 & $\mathrm{mg} / \mathrm{L}$ & 2 & 24-Jul-2009 & 24-Jul-2009 & EPA 7471A & \\
\hline Strontium & 0.611 & 0.0800 & $\mathrm{mg} / \mathrm{L}$ & 4 & 25-Jun-2009 & 25-Jun-2009 & EPA 6010B & \\
\hline Aluminum & 10.9 & 0.200 & $\mathrm{mg} / \mathrm{L}$ & 4 & 25-Jun-2009 & 25-Jun-2009 & SW 846/6010 & \\
\hline Calcium & 32.1 & 0.200 & $\mathrm{mg} / \mathrm{L}$ & 4 & 25-Jun-2009 & 25-Jun-2009 & SW 846/6010 & B \\
\hline Iron & 4.80 & 0.200 & $\mathrm{mg} / \mathrm{L}$ & 4 & 25-Jun-2009 & 25-Jun-2009 & SW 846/6010 & \\
\hline Magnesium & 5.85 & 0.200 & $\mathrm{mg} / \mathrm{L}$ & 4 & 25-Jun-2009 & 25-Jun-2009 & SW 846/6010 & \\
\hline Potassium & 4.02 & 0.200 & $\mathrm{mg} / \mathrm{L}$ & 4 & 25-Jun-2009 & 25-Jun-2009 & SW 846/6010 & B \\
\hline Sodium & 4.86 & 0.200 & $\mathrm{mg} / \mathrm{L}$ & 4 & 25-Jun-2009 & 25-Jun-2009 & SW 846/6010 & B \\
\hline Antimony & 0.0085 & 0.0020 & $\mathrm{mg} / \mathrm{L}$ & 4 & 17-Jun-2009 & 17-Jun-2009 & SW 846/6020 & \\
\hline Arsenic & 0.112 & 0.0020 & $\mathrm{mg} / \mathrm{L}$ & 4 & 17-Jun-2009 & 17-Jun-2009 & SW 846/6020 & \\
\hline Barium & 0.266 & 0.0020 & $\mathrm{mg} / \mathrm{L}$ & 4 & 17-Jun-2009 & 17-Jun-2009 & SW 846/6020 & B \\
\hline Beryllium & 0.0020 & 0.0020 & $\mathrm{mg} / \mathrm{L}$ & 4 & 17-Jun-2009 & 17-Jun-2009 & SW 846/6020 & J \\
\hline Cadmium & 0.0007 & 0.0020 & $\mathrm{mg} / \mathrm{L}$ & 4 & 17-Jun-2009 & 17-Jun-2009 & SW 846/6020 & J \\
\hline Chromium & 0.0184 & 0.0020 & $\mathrm{mg} / \mathrm{L}$ & 4 & 17-Jun-2009 & 17-Jun-2009 & SW 846/6020 & \\
\hline Cobalt & 0.0084 & 0.0020 & $\mathrm{mg} / \mathrm{L}$ & 4 & 17-Jun-2009 & 17-Jun-2009 & SW 846/6020 & \\
\hline Copper & 0.0344 & 0.0020 & $\mathrm{mg} / \mathrm{L}$ & 4 & 17-Jun-2009 & 17-Jun-2009 & SW 846/6020 & B \\
\hline Lead & 0.0169 & 0.0020 & $\mathrm{mg} / \mathrm{L}$ & 4 & 17-Jun-2009 & 17-Jun-2009 & SW 846/6020 & \\
\hline Manganese & 0.0801 & 0.0020 & $\mathrm{mg} / \mathrm{L}$ & 4 & 17-Jun-2009 & 17-Jun-2009 & SW 846/6020 & \\
\hline Molybdenum & 0.0750 & 0.0020 & $\mathrm{mg} / \mathrm{L}$ & 4 & 17-Jun-2009 & 17-Jun-2009 & SW 846/6020 & \\
\hline Nickel & 0.0193 & 0.0020 & $\mathrm{mg} / \mathrm{L}$ & 4 & 17-Jun-2009 & 17-Jun-2009 & SW 846/6020 & \\
\hline Selenium & 0.0074 & 0.0020 & $\mathrm{mg} / \mathrm{L}$ & 4 & 17-Jun-2009 & 17-Jun-2009 & SW 846/6020 & \\
\hline Silver & ND & 0.0020 & $\mathrm{mg} / \mathrm{L}$ & 4 & 17-Jun-2009 & 17-Jun-2009 & SW 846/6020 & $\mathrm{U}$ \\
\hline Thallium & 0.0016 & 0.0020 & $\mathrm{mg} / \mathrm{L}$ & 4 & 17-Jun-2009 & 17-Jun-2009 & SW 846/6020 & $\mathrm{J}$ \\
\hline Vanadium & 0.100 & 0.0020 & $\mathrm{mg} / \mathrm{L}$ & 4 & 17-Jun-2009 & 17-Jun-2009 & SW 846/6020 & \\
\hline Zinc & 0.0589 & 0.0020 & $\mathrm{mg} / \mathrm{L}$ & 4 & 17-Jun-2009 & 17-Jun-2009 & SW 846/6020 & B \\
\hline
\end{tabular}




\section{USACE ERDC-EP-C \\ 3909 Halls Ferry Road \\ Vicksburg, MS 39180-6199}

ERDC - ECB

$-\cdot$,
Project: TVA Fly Ash Project

Project Manager: Tony Bednar
Reported:

03-Sep-2009

DRAFT: SLC MT N2 Hour 48 Rep B - Total Metals

9061503-32 (Water)

\begin{tabular}{|c|c|c|c|c|c|c|c|c|}
\hline Analyte & Result & $\begin{array}{r}\text { porting } \\
\text { Limit }\end{array}$ & Units & Dilution & Prepared & Analyzed & Method & Notes \\
\hline
\end{tabular}

ERDC- EL-EP-C (Environmental Chemistry Branch)

DRAFT: Wet Chemistry

\begin{tabular}{llllllll}
\hline Hardness & $\mathbf{1 0 5}$ & 1.32 & $\begin{array}{c}\text { mg equil } \\
\text { CaCO3/ }\end{array}$ & 4 & 25-Jun-2009 & 25-Jun-2009
\end{tabular}

$\mathrm{L}$

DRAFT: Metals by EPA 6000/7000 Series Methods

\begin{tabular}{|c|c|c|c|c|c|c|c|c|}
\hline Mercury & 0.000093 & 0.000005 & $\mathrm{mg} / \mathrm{L}$ & 2 & 24-Jul-2009 & 24-Jul-2009 & EPA 7471A & \\
\hline Strontium & 0.623 & 0.0800 & $\mathrm{mg} / \mathrm{L}$ & 4 & 25-Jun-2009 & 25-Jun-2009 & EPA 6010B & \\
\hline Aluminum & 11.1 & 0.200 & $\mathrm{mg} / \mathrm{L}$ & 4 & 25-Jun-2009 & 25-Jun-2009 & SW 846/6010 & \\
\hline Calcium & 32.2 & 0.200 & $\mathrm{mg} / \mathrm{L}$ & 4 & 25-Jun-2009 & 25-Jun-2009 & SW 846/6010 & B \\
\hline Iron & 4.84 & 0.200 & $\mathrm{mg} / \mathrm{L}$ & 4 & 25-Jun-2009 & 25-Jun-2009 & SW 846/6010 & \\
\hline Magnesium & 5.94 & 0.200 & $\mathrm{mg} / \mathrm{L}$ & 4 & 25-Jun-2009 & 25-Jun-2009 & SW 846/6010 & \\
\hline Potassium & 5.17 & 0.200 & $\mathrm{mg} / \mathrm{L}$ & 4 & 25-Jun-2009 & 25-Jun-2009 & SW 846/6010 & B \\
\hline Sodium & 5.07 & 0.200 & $\mathrm{mg} / \mathrm{L}$ & 4 & 25-Jun-2009 & 25-Jun-2009 & SW 846/6010 & B \\
\hline Antimony & 0.0083 & 0.0020 & $\mathrm{mg} / \mathrm{L}$ & 4 & 17-Jun-2009 & 17-Jun-2009 & SW 846/6020 & \\
\hline Arsenic & 0.112 & 0.0020 & $\mathrm{mg} / \mathrm{L}$ & 4 & 17-Jun-2009 & 17-Jun-2009 & SW 846/6020 & \\
\hline Barium & 0.263 & 0.0020 & $\mathrm{mg} / \mathrm{L}$ & 4 & 17-Jun-2009 & 17-Jun-2009 & SW 846/6020 & B \\
\hline Beryllium & 0.0019 & 0.0020 & $\mathrm{mg} / \mathrm{L}$ & 4 & 17-Jun-2009 & 17-Jun-2009 & SW 846/6020 & J \\
\hline Cadmium & 0.00067 & 0.0020 & $\mathrm{mg} / \mathrm{L}$ & 4 & 17-Jun-2009 & 17-Jun-2009 & SW 846/6020 & J \\
\hline Chromium & 0.0178 & 0.0020 & $\mathrm{mg} / \mathrm{L}$ & 4 & 17-Jun-2009 & 17-Jun-2009 & SW 846/6020 & \\
\hline Cobalt & 0.0087 & 0.0020 & $\mathrm{mg} / \mathrm{L}$ & 4 & 17-Jun-2009 & 17-Jun-2009 & SW 846/6020 & \\
\hline Copper & 0.0316 & 0.0020 & $\mathrm{mg} / \mathrm{L}$ & 4 & 17-Jun-2009 & 17-Jun-2009 & SW 846/6020 & B \\
\hline Lead & 0.0158 & 0.0020 & $\mathrm{mg} / \mathrm{L}$ & 4 & 17-Jun-2009 & 17-Jun-2009 & SW 846/6020 & \\
\hline Manganese & 0.0853 & 0.0020 & $\mathrm{mg} / \mathrm{L}$ & 4 & 17-Jun-2009 & 17-Jun-2009 & SW 846/6020 & \\
\hline Molybdenum & 0.0751 & 0.0020 & $\mathrm{mg} / \mathrm{L}$ & 4 & 17-Jun-2009 & 17-Jun-2009 & SW 846/6020 & \\
\hline Nickel & 0.0198 & 0.0020 & $\mathrm{mg} / \mathrm{L}$ & 4 & 17-Jun-2009 & 17-Jun-2009 & SW 846/6020 & \\
\hline Selenium & 0.0080 & 0.0020 & $\mathrm{mg} / \mathrm{L}$ & 4 & 17-Jun-2009 & 17-Jun-2009 & SW 846/6020 & \\
\hline Silver & ND & 0.0020 & $\mathrm{mg} / \mathrm{L}$ & 4 & 17-Jun-2009 & 17-Jun-2009 & SW 846/6020 & $\mathrm{U}$ \\
\hline Thallium & 0.0015 & 0.0020 & $\mathrm{mg} / \mathrm{L}$ & 4 & 17-Jun-2009 & 17-Jun-2009 & SW 846/6020 & J \\
\hline Vanadium & 0.100 & 0.0020 & $\mathrm{mg} / \mathrm{L}$ & 4 & 17-Jun-2009 & 17-Jun-2009 & SW 846/6020 & \\
\hline Zinc & 0.0461 & 0.0020 & $\mathrm{mg} / \mathrm{L}$ & 4 & 17-Jun-2009 & 17-Jun-2009 & SW 846/6020 & B \\
\hline
\end{tabular}




\section{USACE ERDC-EP-C \\ 3909 Halls Ferry Road \\ Vicksburg, MS 39180-6199}

ERDC - ECB

$-\cdot$,
Project: TVA Fly Ash Project

Project Manager: Tony Bednar
Reported:

03-Sep-2009

DRAFT: SLC MT N2 Hour 48 Rep C - Total Metals

9061503-33 (Water)

\begin{tabular}{|c|c|c|c|c|c|c|c|c|}
\hline Analyte & Result & $\begin{array}{r}\text { porting } \\
\text { Limit }\end{array}$ & Units & Dilution & Prepared & Analyzed & Method & Notes \\
\hline
\end{tabular}

ERDC- EL-EP-C (Environmental Chemistry Branch)

DRAFT: Wet Chemistry

\begin{tabular}{llllllll}
\hline Hardness & $\mathbf{1 0 8}$ & 1.32 & $\begin{array}{c}\text { mg equil } \\
\text { CaCO3/ }\end{array}$ & 4 & 25-Jun-2009 & 25-Jun-2009
\end{tabular}

$\mathrm{L}$

DRAFT: Metals by EPA 6000/7000 Series Methods

\begin{tabular}{|c|c|c|c|c|c|c|c|c|}
\hline Mercury & 0.000197 & 0.000005 & $\mathrm{mg} / \mathrm{L}$ & 2 & 24-Jul-2009 & 24-Jul-2009 & EPA 7471A & \\
\hline Strontium & 0.630 & 0.0800 & $\mathrm{mg} / \mathrm{L}$ & 4 & 25-Jun-2009 & 25-Jun-2009 & EPA 6010B & \\
\hline Aluminum & 11.3 & 0.200 & $\mathrm{mg} / \mathrm{L}$ & 4 & 25-Jun-2009 & 25-Jun-2009 & SW 846/6010 & \\
\hline Calcium & 33.3 & 0.200 & $\mathrm{mg} / \mathrm{L}$ & 4 & 25-Jun-2009 & 25-Jun-2009 & SW 846/6010 & B \\
\hline Iron & 4.90 & 0.200 & $\mathrm{mg} / \mathrm{L}$ & 4 & 25-Jun-2009 & 25-Jun-2009 & SW 846/6010 & \\
\hline Magnesium & 6.03 & 0.200 & $\mathrm{mg} / \mathrm{L}$ & 4 & 25-Jun-2009 & 25-Jun-2009 & SW 846/6010 & \\
\hline Potassium & 4.09 & 0.200 & $\mathrm{mg} / \mathrm{L}$ & 4 & 25-Jun-2009 & 25-Jun-2009 & SW 846/6010 & B \\
\hline Sodium & 4.94 & 0.200 & $\mathrm{mg} / \mathrm{L}$ & 4 & 25-Jun-2009 & 25-Jun-2009 & SW 846/6010 & B \\
\hline Antimony & 0.0086 & 0.0020 & $\mathrm{mg} / \mathrm{L}$ & 4 & 17-Jun-2009 & 17-Jun-2009 & SW 846/6020 & \\
\hline Arsenic & 0.111 & 0.0020 & $\mathrm{mg} / \mathrm{L}$ & 4 & 17-Jun-2009 & 17-Jun-2009 & SW 846/6020 & \\
\hline Barium & 0.272 & 0.0020 & $\mathrm{mg} / \mathrm{L}$ & 4 & 17-Jun-2009 & 17-Jun-2009 & SW 846/6020 & B \\
\hline Beryllium & 0.0020 & 0.0020 & $\mathrm{mg} / \mathrm{L}$ & 4 & 17-Jun-2009 & 17-Jun-2009 & SW 846/6020 & \\
\hline Cadmium & 0.0006 & 0.0020 & $\mathrm{mg} / \mathrm{L}$ & 4 & 17-Jun-2009 & 17-Jun-2009 & SW 846/6020 & $\mathrm{J}$ \\
\hline Chromium & 0.0178 & 0.0020 & $\mathrm{mg} / \mathrm{L}$ & 4 & 17-Jun-2009 & 17-Jun-2009 & SW 846/6020 & \\
\hline Cobalt & 0.0085 & 0.0020 & $\mathrm{mg} / \mathrm{L}$ & 4 & 17-Jun-2009 & 17-Jun-2009 & SW 846/6020 & \\
\hline Copper & 0.0326 & 0.0020 & $\mathrm{mg} / \mathrm{L}$ & 4 & 17-Jun-2009 & 17-Jun-2009 & SW 846/6020 & B \\
\hline Lead & 0.0176 & 0.0020 & $\mathrm{mg} / \mathrm{L}$ & 4 & 17-Jun-2009 & 17-Jun-2009 & SW 846/6020 & \\
\hline Manganese & 0.0784 & 0.0020 & $\mathrm{mg} / \mathrm{L}$ & 4 & 17-Jun-2009 & 17-Jun-2009 & SW 846/6020 & \\
\hline Molybdenum & 0.0785 & 0.0020 & $\mathrm{mg} / \mathrm{L}$ & 4 & 17-Jun-2009 & 17-Jun-2009 & SW 846/6020 & \\
\hline Nickel & 0.0192 & 0.0020 & $\mathrm{mg} / \mathrm{L}$ & 4 & 17-Jun-2009 & 17-Jun-2009 & SW 846/6020 & \\
\hline Selenium & 0.0075 & 0.0020 & $\mathrm{mg} / \mathrm{L}$ & 4 & 17-Jun-2009 & 17-Jun-2009 & SW 846/6020 & \\
\hline Silver & ND & 0.0020 & $\mathrm{mg} / \mathrm{L}$ & 4 & 17-Jun-2009 & 17-Jun-2009 & SW 846/6020 & $\mathrm{U}$ \\
\hline Thallium & 0.0016 & 0.0020 & $\mathrm{mg} / \mathrm{L}$ & 4 & 17-Jun-2009 & 17-Jun-2009 & SW 846/6020 & $\mathrm{J}$ \\
\hline Vanadium & 0.102 & 0.0020 & $\mathrm{mg} / \mathrm{L}$ & 4 & 17-Jun-2009 & 17-Jun-2009 & SW 846/6020 & \\
\hline Zinc & 0.0721 & 0.0020 & $\mathrm{mg} / \mathrm{L}$ & 4 & 17-Jun-2009 & 17-Jun-2009 & SW 846/6020 & B \\
\hline
\end{tabular}




\section{USACE ERDC-EP-C \\ 3909 Halls Ferry Road \\ Vicksburg, MS 39180-6199}

ERDC - ECB

$-\cdot$,
Project: TVA Fly Ash Project

Project Manager: Tony Bednar
Reported:

03-Sep-2009

DRAFT: SLC MT O2 Hour 48 Rep A - Total Metals

9061503-34 (Water)

\begin{tabular}{|c|c|c|c|c|c|c|c|c|}
\hline Analyte & Result & $\begin{array}{r}\text { porting } \\
\text { Limit }\end{array}$ & Units & Dilution & Prepared & Analyzed & Method & Notes \\
\hline
\end{tabular}

ERDC- EL-EP-C (Environmental Chemistry Branch)

DRAFT: Wet Chemistry

\begin{tabular}{llllllll}
\hline Hardness & $\mathbf{1 0 5}$ & 1.32 & $\begin{array}{c}\text { mg equil } \\
\text { CaCO3/ }\end{array}$ & 4 & 25-Jun-2009 & 25-Jun-2009
\end{tabular}

$\mathrm{L}$

DRAFT: Metals by EPA 6000/7000 Series Methods

\begin{tabular}{|c|c|c|c|c|c|c|c|c|}
\hline Mercury & 0.000356 & 0.000005 & $\mathrm{mg} / \mathrm{L}$ & 2 & 24-Jul-2009 & 24-Jul-2009 & EPA 7471A & \\
\hline Strontium & 0.617 & 0.0800 & $\mathrm{mg} / \mathrm{L}$ & 4 & 25-Jun-2009 & 25-Jun-2009 & EPA 6010B & \\
\hline Aluminum & 11.9 & 0.200 & $\mathrm{mg} / \mathrm{L}$ & 4 & 25-Jun-2009 & 25-Jun-2009 & SW 846/6010 & \\
\hline Calcium & 32.1 & 0.200 & $\mathrm{mg} / \mathrm{L}$ & 4 & 25-Jun-2009 & 25-Jun-2009 & SW 846/6010 & B \\
\hline Iron & 5.20 & 0.200 & $\mathrm{mg} / \mathrm{L}$ & 4 & 25-Jun-2009 & 25-Jun-2009 & SW 846/6010 & \\
\hline Magnesium & 5.96 & 0.200 & $\mathrm{mg} / \mathrm{L}$ & 4 & 25-Jun-2009 & 25-Jun-2009 & SW 846/6010 & \\
\hline Potassium & 4.82 & 0.200 & $\mathrm{mg} / \mathrm{L}$ & 4 & 25-Jun-2009 & 25-Jun-2009 & SW 846/6010 & B \\
\hline Sodium & 5.15 & 0.200 & $\mathrm{mg} / \mathrm{L}$ & 4 & 25-Jun-2009 & 25-Jun-2009 & SW 846/6010 & B \\
\hline Antimony & 0.0084 & 0.0020 & $\mathrm{mg} / \mathrm{L}$ & 4 & 17-Jun-2009 & 17-Jun-2009 & SW 846/6020 & \\
\hline Arsenic & 0.114 & 0.0020 & $\mathrm{mg} / \mathrm{L}$ & 4 & 17-Jun-2009 & 17-Jun-2009 & SW 846/6020 & \\
\hline Barium & 0.276 & 0.0020 & $\mathrm{mg} / \mathrm{L}$ & 4 & 17-Jun-2009 & 17-Jun-2009 & SW 846/6020 & B \\
\hline Beryllium & 0.0021 & 0.0020 & $\mathrm{mg} / \mathrm{L}$ & 4 & 17-Jun-2009 & 17-Jun-2009 & SW 846/6020 & \\
\hline Cadmium & 0.0007 & 0.0020 & $\mathrm{mg} / \mathrm{L}$ & 4 & 17-Jun-2009 & 17-Jun-2009 & SW 846/6020 & J \\
\hline Chromium & 0.0206 & 0.0020 & $\mathrm{mg} / \mathrm{L}$ & 4 & 17-Jun-2009 & 17-Jun-2009 & SW 846/6020 & \\
\hline Cobalt & 0.0087 & 0.0020 & $\mathrm{mg} / \mathrm{L}$ & 4 & 17-Jun-2009 & 17-Jun-2009 & SW 846/6020 & \\
\hline Copper & 0.0361 & 0.0020 & $\mathrm{mg} / \mathrm{L}$ & 4 & 17-Jun-2009 & 17-Jun-2009 & SW 846/6020 & B \\
\hline Lead & 0.0171 & 0.0020 & $\mathrm{mg} / \mathrm{L}$ & 4 & 17-Jun-2009 & 17-Jun-2009 & SW 846/6020 & \\
\hline Manganese & 0.0835 & 0.0020 & $\mathrm{mg} / \mathrm{L}$ & 4 & 17-Jun-2009 & 17-Jun-2009 & SW 846/6020 & \\
\hline Molybdenum & 0.0759 & 0.0020 & $\mathrm{mg} / \mathrm{L}$ & 4 & 17-Jun-2009 & 17-Jun-2009 & SW 846/6020 & \\
\hline Nickel & 0.0210 & 0.0020 & $\mathrm{mg} / \mathrm{L}$ & 4 & 17-Jun-2009 & 17-Jun-2009 & SW 846/6020 & \\
\hline Selenium & 0.0088 & 0.0020 & $\mathrm{mg} / \mathrm{L}$ & 4 & 17-Jun-2009 & 17-Jun-2009 & SW 846/6020 & \\
\hline Silver & ND & 0.0020 & $\mathrm{mg} / \mathrm{L}$ & 4 & 17-Jun-2009 & 17-Jun-2009 & SW 846/6020 & $\mathrm{U}$ \\
\hline Thallium & 0.0018 & 0.0020 & $\mathrm{mg} / \mathrm{L}$ & 4 & 17-Jun-2009 & 17-Jun-2009 & SW 846/6020 & J \\
\hline Vanadium & 0.104 & 0.0020 & $\mathrm{mg} / \mathrm{L}$ & 4 & 17-Jun-2009 & 17-Jun-2009 & SW 846/6020 & \\
\hline Zinc & 0.0496 & 0.0020 & $\mathrm{mg} / \mathrm{L}$ & 4 & 17-Jun-2009 & 17-Jun-2009 & SW 846/6020 & B \\
\hline
\end{tabular}




\section{USACE ERDC-EP-C \\ 3909 Halls Ferry Road \\ Vicksburg, MS 39180-6199}

ERDC - ECB

$-\cdot$,
Project: TVA Fly Ash Project

Project Manager: Tony Bednar
Reported:

03-Sep-2009

DRAFT: SLC MT O2 Hour 48 Rep B - Total Metals

9061503-35 (Water)

\begin{tabular}{|c|c|c|c|c|c|c|c|c|}
\hline Analyte & Result & $\begin{array}{r}\text { porting } \\
\text { Limit }\end{array}$ & Units & Dilution & Prepared & Analyzed & Method & Notes \\
\hline
\end{tabular}

ERDC- EL-EP-C (Environmental Chemistry Branch)

DRAFT: Wet Chemistry

\begin{tabular}{llllllll}
\hline Hardness & $\mathbf{1 0 8}$ & 1.32 & $\begin{array}{c}\text { mg equil } \\
\text { CaCO3/ }\end{array}$ & 4 & 25-Jun-2009 & 25-Jun-2009
\end{tabular}

$\mathrm{L}$

DRAFT: Metals by EPA 6000/7000 Series Methods

\begin{tabular}{|c|c|c|c|c|c|c|c|c|}
\hline Mercury & 0.00034 & 0.000005 & $\mathrm{mg} / \mathrm{L}$ & 2 & 24-Jul-2009 & 24-Jul-2009 & EPA 7471A & \\
\hline Strontium & 0.645 & 0.0800 & $\mathrm{mg} / \mathrm{L}$ & 4 & 25-Jun-2009 & 25-Jun-2009 & EPA 6010B & \\
\hline Aluminum & 15.3 & 0.200 & $\mathrm{mg} / \mathrm{L}$ & 4 & 25-Jun-2009 & 25-Jun-2009 & SW 846/6010 & \\
\hline Calcium & 32.8 & 0.200 & $\mathrm{mg} / \mathrm{L}$ & 4 & 25-Jun-2009 & 25-Jun-2009 & SW 846/6010 & B \\
\hline Iron & 6.70 & 0.200 & $\mathrm{mg} / \mathrm{L}$ & 4 & 25-Jun-2009 & 25-Jun-2009 & SW 846/6010 & \\
\hline Magnesium & 6.27 & 0.200 & $\mathrm{mg} / \mathrm{L}$ & 4 & 25-Jun-2009 & 25-Jun-2009 & SW 846/6010 & \\
\hline Potassium & 4.92 & 0.200 & $\mathrm{mg} / \mathrm{L}$ & 4 & 25-Jun-2009 & 25-Jun-2009 & SW 846/6010 & B \\
\hline Sodium & 4.89 & 0.200 & $\mathrm{mg} / \mathrm{L}$ & 4 & 25-Jun-2009 & 25-Jun-2009 & SW 846/6010 & B \\
\hline Antimony & 0.0087 & 0.0020 & $\mathrm{mg} / \mathrm{L}$ & 4 & 17-Jun-2009 & 17-Jun-2009 & SW 846/6020 & \\
\hline Arsenic & 0.125 & 0.0020 & $\mathrm{mg} / \mathrm{L}$ & 4 & 17-Jun-2009 & 17-Jun-2009 & SW 846/6020 & \\
\hline Barium & 0.321 & 0.0020 & $\mathrm{mg} / \mathrm{L}$ & 4 & 17-Jun-2009 & 17-Jun-2009 & SW 846/6020 & B \\
\hline Beryllium & 0.0026 & 0.0020 & $\mathrm{mg} / \mathrm{L}$ & 4 & 17-Jun-2009 & 17-Jun-2009 & SW 846/6020 & \\
\hline Cadmium & 0.0007 & 0.0020 & $\mathrm{mg} / \mathrm{L}$ & 4 & 17-Jun-2009 & 17-Jun-2009 & SW 846/6020 & J \\
\hline Chromium & 0.0230 & 0.0020 & $\mathrm{mg} / \mathrm{L}$ & 4 & 17-Jun-2009 & 17-Jun-2009 & SW 846/6020 & \\
\hline Cobalt & 0.0112 & 0.0020 & $\mathrm{mg} / \mathrm{L}$ & 4 & 17-Jun-2009 & 17-Jun-2009 & SW 846/6020 & \\
\hline Copper & 0.0428 & 0.0020 & $\mathrm{mg} / \mathrm{L}$ & 4 & 17-Jun-2009 & 17-Jun-2009 & SW 846/6020 & B \\
\hline Lead & 0.0212 & 0.0020 & $\mathrm{mg} / \mathrm{L}$ & 4 & 17-Jun-2009 & 17-Jun-2009 & SW 846/6020 & \\
\hline Manganese & 0.0969 & 0.0020 & $\mathrm{mg} / \mathrm{L}$ & 4 & 17-Jun-2009 & 17-Jun-2009 & SW 846/6020 & \\
\hline Molybdenum & 0.0770 & 0.0020 & $\mathrm{mg} / \mathrm{L}$ & 4 & 17-Jun-2009 & 17-Jun-2009 & SW 846/6020 & \\
\hline Nickel & 0.0241 & 0.0020 & $\mathrm{mg} / \mathrm{L}$ & 4 & 17-Jun-2009 & 17-Jun-2009 & SW 846/6020 & \\
\hline Selenium & 0.0093 & 0.0020 & $\mathrm{mg} / \mathrm{L}$ & 4 & 17-Jun-2009 & 17-Jun-2009 & SW 846/6020 & \\
\hline Silver & ND & 0.0020 & $\mathrm{mg} / \mathrm{L}$ & 4 & 17-Jun-2009 & 17-Jun-2009 & SW 846/6020 & $\mathrm{U}$ \\
\hline Thallium & 0.0020 & 0.0020 & $\mathrm{mg} / \mathrm{L}$ & 4 & 17-Jun-2009 & 17-Jun-2009 & SW 846/6020 & $\mathrm{J}$ \\
\hline Vanadium & 0.115 & 0.0020 & $\mathrm{mg} / \mathrm{L}$ & 4 & 17-Jun-2009 & 17-Jun-2009 & SW 846/6020 & \\
\hline Zinc & 0.0565 & 0.0020 & $\mathrm{mg} / \mathrm{L}$ & 4 & 17-Jun-2009 & 17-Jun-2009 & SW 846/6020 & B \\
\hline
\end{tabular}




\section{USACE ERDC-EP-C \\ 3909 Halls Ferry Road \\ Vicksburg, MS 39180-6199}

ERDC - ECB

$-\cdot$,
Project: TVA Fly Ash Project

Project Manager: Tony Bednar
Reported:

03-Sep-2009

DRAFT: SLC MT O2 Hour 48 Rep C - Total Metals

9061503-36 (Water)

\begin{tabular}{|c|c|c|c|c|c|c|c|c|}
\hline Analyte & Result & $\begin{array}{r}\text { porting } \\
\text { Limit }\end{array}$ & Units & Dilution & Prepared & Analyzed & Method & Notes \\
\hline
\end{tabular}

ERDC- EL-EP-C (Environmental Chemistry Branch)

DRAFT: Wet Chemistry

\begin{tabular}{llllllll}
\hline Hardness & $\mathbf{1 0 9}$ & 1.32 & $\begin{array}{c}\text { mg equil } \\
\text { CaCO3/ }\end{array}$ & 4 & 25-Jun-2009 & 25-Jun-2009
\end{tabular}

$\mathrm{L}$

DRAFT: Metals by EPA 6000/7000 Series Methods

\begin{tabular}{|c|c|c|c|c|c|c|c|c|}
\hline Mercury & 0.000277 & 0.000005 & $\mathrm{mg} / \mathrm{L}$ & 2 & 24-Jul-2009 & 24-Jul-2009 & EPA 7471A & \\
\hline Strontium & 0.651 & 0.0800 & $\mathrm{mg} / \mathrm{L}$ & 4 & 25-Jun-2009 & 25-Jun-2009 & EPA 6010B & \\
\hline Aluminum & 16.7 & 0.200 & $\mathrm{mg} / \mathrm{L}$ & 4 & 25-Jun-2009 & 25-Jun-2009 & SW 846/6010 & \\
\hline Calcium & 33.0 & 0.200 & $\mathrm{mg} / \mathrm{L}$ & 4 & 25-Jun-2009 & 25-Jun-2009 & SW 846/6010 & B \\
\hline Iron & 7.29 & 0.200 & $\mathrm{mg} / \mathrm{L}$ & 4 & 25-Jun-2009 & 25-Jun-2009 & SW 846/6010 & \\
\hline Magnesium & 6.39 & 0.200 & $\mathrm{mg} / \mathrm{L}$ & 4 & 25-Jun-2009 & 25-Jun-2009 & SW 846/6010 & \\
\hline Potassium & 5.14 & 0.200 & $\mathrm{mg} / \mathrm{L}$ & 4 & 25-Jun-2009 & 25-Jun-2009 & SW 846/6010 & B \\
\hline Sodium & 4.65 & 0.200 & $\mathrm{mg} / \mathrm{L}$ & 4 & 25-Jun-2009 & 25-Jun-2009 & SW 846/6010 & B \\
\hline Antimony & 0.0088 & 0.0020 & $\mathrm{mg} / \mathrm{L}$ & 4 & 17-Jun-2009 & 17-Jun-2009 & SW 846/6020 & \\
\hline Arsenic & 0.130 & 0.0020 & $\mathrm{mg} / \mathrm{L}$ & 4 & 17-Jun-2009 & 17-Jun-2009 & SW 846/6020 & \\
\hline Barium & 0.336 & 0.0020 & $\mathrm{mg} / \mathrm{L}$ & 4 & 17-Jun-2009 & 17-Jun-2009 & SW 846/6020 & B \\
\hline Beryllium & 0.0028 & 0.0020 & $\mathrm{mg} / \mathrm{L}$ & 4 & 17-Jun-2009 & 17-Jun-2009 & SW 846/6020 & \\
\hline Cadmium & 0.0007 & 0.0020 & $\mathrm{mg} / \mathrm{L}$ & 4 & 17-Jun-2009 & 17-Jun-2009 & SW 846/6020 & J \\
\hline Chromium & 0.0229 & 0.0020 & $\mathrm{mg} / \mathrm{L}$ & 4 & 17-Jun-2009 & 17-Jun-2009 & SW 846/6020 & \\
\hline Cobalt & 0.0119 & 0.0020 & $\mathrm{mg} / \mathrm{L}$ & 4 & 17-Jun-2009 & 17-Jun-2009 & SW 846/6020 & \\
\hline Copper & 0.0435 & 0.0020 & $\mathrm{mg} / \mathrm{L}$ & 4 & 17-Jun-2009 & 17-Jun-2009 & SW 846/6020 & B \\
\hline Lead & 0.0228 & 0.0020 & $\mathrm{mg} / \mathrm{L}$ & 4 & 17-Jun-2009 & 17-Jun-2009 & SW 846/6020 & \\
\hline Manganese & 0.0985 & 0.0020 & $\mathrm{mg} / \mathrm{L}$ & 4 & 17-Jun-2009 & 17-Jun-2009 & SW 846/6020 & \\
\hline Molybdenum & 0.0767 & 0.0020 & $\mathrm{mg} / \mathrm{L}$ & 4 & 17-Jun-2009 & 17-Jun-2009 & SW 846/6020 & \\
\hline Nickel & 0.0258 & 0.0020 & $\mathrm{mg} / \mathrm{L}$ & 4 & 17-Jun-2009 & 17-Jun-2009 & SW 846/6020 & \\
\hline Selenium & 0.0093 & 0.0020 & $\mathrm{mg} / \mathrm{L}$ & 4 & 17-Jun-2009 & 17-Jun-2009 & SW 846/6020 & \\
\hline Silver & ND & 0.0020 & $\mathrm{mg} / \mathrm{L}$ & 4 & 17-Jun-2009 & 17-Jun-2009 & SW 846/6020 & $\mathrm{U}$ \\
\hline Thallium & 0.0020 & 0.0020 & $\mathrm{mg} / \mathrm{L}$ & 4 & 17-Jun-2009 & 17-Jun-2009 & SW 846/6020 & \\
\hline Vanadium & 0.118 & 0.0020 & $\mathrm{mg} / \mathrm{L}$ & 4 & 17-Jun-2009 & 17-Jun-2009 & SW 846/6020 & \\
\hline Zinc & 0.0541 & 0.0020 & $\mathrm{mg} / \mathrm{L}$ & 4 & 17-Jun-2009 & 17-Jun-2009 & SW 846/6020 & B \\
\hline
\end{tabular}




\section{USACE ERDC-EP-C \\ 3909 Halls Ferry Road \\ Vicksburg, MS 39180-6199}

ERDC - ECB

$-\cdot$,
Project: TVA Fly Ash Project

Project Manager: Tony Bednar
Reported:

03-Sep-2009

DRAFT: EMR MT N2 Hour 96 Rep A

9061701-01 (Water)

\begin{tabular}{|c|c|c|c|c|c|c|c|c|}
\hline Analyte & Result & $\begin{array}{r}\text { porting } \\
\text { Limit }\end{array}$ & Units & Dilution & Prepared & Analyzed & Method & Notes \\
\hline
\end{tabular}

ERDC- EL-EP-C (Environmental Chemistry Branch)

DRAFT: Wet Chemistry

\begin{tabular}{llllllll}
\hline Hardness & $\mathbf{4 2 . 8}$ & 1.32 & $\begin{array}{c}\text { mg equil } \\
\text { CaCO3/ }\end{array}$ & 1 & 25-Jun-2009 & 25-Jun-2009 & Hardness
\end{tabular}

$\mathrm{L}$

DRAFT: Metals by EPA 6000/7000 Series Methods

\begin{tabular}{|c|c|c|c|c|c|c|c|c|}
\hline Mercury & ND & 0.000005 & $\mathrm{mg} / \mathrm{L}$ & 1 & 17-Jun-2009 & 01-Jul-2009 & EPA 7471A & $\mathrm{U}$ \\
\hline Strontium & 0.171 & 0.0200 & $\mathrm{mg} / \mathrm{L}$ & 1 & 25-Jun-2009 & 25-Jun-2009 & EPA 6010B & \\
\hline Aluminum & 0.0542 & 0.200 & $\mathrm{mg} / \mathrm{L}$ & 1 & 25-Jun-2009 & 25-Jun-2009 & SW 846/6010 & $\mathrm{J}$ \\
\hline Calcium & 13.1 & 0.200 & $\mathrm{mg} / \mathrm{L}$ & 1 & 25-Jun-2009 & 25-Jun-2009 & SW 846/6010 & \\
\hline Iron & ND & 0.200 & $\mathrm{mg} / \mathrm{L}$ & 1 & 25-Jun-2009 & 25-Jun-2009 & SW 846/6010 & $\mathrm{U}$ \\
\hline Magnesium & 2.45 & 0.200 & $\mathrm{mg} / \mathrm{L}$ & 1 & 25-Jun-2009 & 25-Jun-2009 & SW 846/6010 & \\
\hline Potassium & 1.41 & 0.200 & $\mathrm{mg} / \mathrm{L}$ & 1 & 25-Jun-2009 & 25-Jun-2009 & SW 846/6010 & \\
\hline Sodium & 2.58 & 0.200 & $\mathrm{mg} / \mathrm{L}$ & 1 & 25-Jun-2009 & 25-Jun-2009 & SW 846/6010 & \\
\hline Antimony & 0.0017 & 0.0010 & $\mathrm{mg} / \mathrm{L}$ & 2 & 19-Jun-2009 & 19-Jun-2009 & SW 846/6020 & \\
\hline Arsenic & 0.0508 & 0.0010 & $\mathrm{mg} / \mathrm{L}$ & 2 & 19-Jun-2009 & 19-Jun-2009 & SW 846/6020 & \\
\hline Barium & 0.109 & 0.0010 & $\mathrm{mg} / \mathrm{L}$ & 2 & 19-Jun-2009 & 19-Jun-2009 & SW 846/6020 & \\
\hline Beryllium & ND & 0.0010 & $\mathrm{mg} / \mathrm{L}$ & 2 & 19-Jun-2009 & 19-Jun-2009 & SW 846/6020 & $\mathrm{U}$ \\
\hline Cadmium & ND & 0.0010 & $\mathrm{mg} / \mathrm{L}$ & 2 & 19-Jun-2009 & 19-Jun-2009 & SW 846/6020 & $\mathrm{U}$ \\
\hline Chromium & 0.0012 & 0.0010 & $\mathrm{mg} / \mathrm{L}$ & 2 & 19-Jun-2009 & 19-Jun-2009 & SW 846/6020 & \\
\hline Cobalt & ND & 0.0010 & $\mathrm{mg} / \mathrm{L}$ & 2 & 19-Jun-2009 & 19-Jun-2009 & SW 846/6020 & $\mathrm{U}$ \\
\hline Copper & 0.0025 & 0.0010 & $\mathrm{mg} / \mathrm{L}$ & 2 & 19-Jun-2009 & 19-Jun-2009 & SW 846/6020 & \\
\hline Lead & ND & 0.0010 & $\mathrm{mg} / \mathrm{L}$ & 2 & 19-Jun-2009 & 19-Jun-2009 & SW 846/6020 & U \\
\hline Manganese & 0.0012 & 0.0010 & $\mathrm{mg} / \mathrm{L}$ & 2 & 19-Jun-2009 & 19-Jun-2009 & SW 846/6020 & \\
\hline Molybdenum & 0.0022 & 0.0010 & $\mathrm{mg} / \mathrm{L}$ & 2 & 19-Jun-2009 & 19-Jun-2009 & SW 846/6020 & \\
\hline Nickel & 0.0008 & 0.0010 & $\mathrm{mg} / \mathrm{L}$ & 2 & 19-Jun-2009 & 19-Jun-2009 & SW 846/6020 & $\mathrm{J}$ \\
\hline Selenium & 0.0034 & 0.0010 & $\mathrm{mg} / \mathrm{L}$ & 2 & 19-Jun-2009 & 19-Jun-2009 & SW 846/6020 & \\
\hline Silver & 0.0008 & 0.0010 & $\mathrm{mg} / \mathrm{L}$ & 2 & 19-Jun-2009 & 19-Jun-2009 & SW 846/6020 & $\mathrm{J}, \mathrm{B}$ \\
\hline Thallium & 0.0005 & 0.0010 & $\mathrm{mg} / \mathrm{L}$ & 2 & 19-Jun-2009 & 19-Jun-2009 & SW 846/6020 & $\mathrm{J}$ \\
\hline Vanadium & 0.0298 & 0.0010 & $\mathrm{mg} / \mathrm{L}$ & 2 & 19-Jun-2009 & 19-Jun-2009 & SW 846/6020 & \\
\hline Zinc & 0.0058 & 0.0010 & $\mathrm{mg} / \mathrm{L}$ & 2 & 19-Jun-2009 & 19-Jun-2009 & SW 846/6020 & B \\
\hline
\end{tabular}




\section{USACE ERDC-EP-C \\ 3909 Halls Ferry Road \\ Vicksburg, MS 39180-6199}

ERDC - ECB

Project: TVA Fly Ash Project

Reported:

$--,-$

Project Manager: Tony Bednar

03-Sep-2009

DRAFT: EMR MT N2 Hour 96 Rep A

9061701-01 (Water)

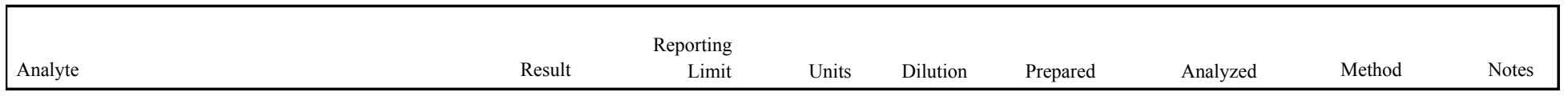

ERDC- EL-EP-C (Environmental Chemistry Branch)

DRAFT: Miscellaneous Physical/Conventional Chemistry Parameters

\begin{tabular}{lrrrrrrr}
\hline Bromide & $\mathrm{ND}$ & 0.100 & $\mathrm{mg} / \mathrm{L}$ & 1 & $15-J u l-2009$ & $16-\mathrm{Jul}-2009$ & EPA 300.0 \\
Chloride & $\mathbf{2 . 7 1}$ & 1.00 & $\mathrm{mg} / \mathrm{L}$ & 1 & $15-J u l-2009$ & $16-\mathrm{Jul}-2009$ & EPA 300.0 \\
Nitrate as N & $\mathbf{0 . 3 9 9}$ & 0.100 & $\mathrm{mg} / \mathrm{L}$ & 1 & $15-J u l-2009$ & $16-J u l-2009$ & EPA 300.0 \\
Sulfate & $\mathbf{1 3 . 0}$ & 0.100 & $\mathrm{mg} / \mathrm{L}$ & 1 & $15-J u l-2009$ & $16-J u l-2009$ & EPA 300.0
\end{tabular}




\section{USACE ERDC-EP-C \\ 3909 Halls Ferry Road \\ Vicksburg, MS 39180-6199}

ERDC - ECB

$-\cdot$,
Project: TVA Fly Ash Project

Project Manager: Tony Bednar
Reported:

03-Sep-2009

DRAFT: EMR MT N2 Hour 96 Rep B

9061701-02 (Water)

\begin{tabular}{|c|c|c|c|c|c|c|c|c|}
\hline Analyte & Result & $\begin{array}{r}\text { porting } \\
\text { Limit }\end{array}$ & Units & Dilution & Prepared & Analyzed & Method & Notes \\
\hline
\end{tabular}

ERDC- EL-EP-C (Environmental Chemistry Branch)

DRAFT: Wet Chemistry

\begin{tabular}{llllllll}
\hline Hardness & $\mathbf{4 2 . 2}$ & 1.32 & $\begin{array}{c}\text { mg equil } \\
\text { CaCO3/ }\end{array}$ & 1 & 25-Jun-2009 & 25-Jun-2009
\end{tabular}

DRAFT: Metals by EPA 6000/7000 Series Methods

\begin{tabular}{|c|c|c|c|c|c|c|c|c|}
\hline Mercury & 0.000024 & 0.000005 & $\mathrm{mg} / \mathrm{L}$ & 1 & 17-Jun-2009 & 01-Jul-2009 & EPA 7471A & \\
\hline Strontium & 0.171 & 0.0200 & $\mathrm{mg} / \mathrm{L}$ & 1 & 25-Jun-2009 & 25-Jun-2009 & EPA 6010B & \\
\hline Aluminum & 0.0584 & 0.200 & $\mathrm{mg} / \mathrm{L}$ & 1 & 25-Jun-2009 & 25-Jun-2009 & SW 846/6010 & $\mathrm{J}$ \\
\hline Calcium & 12.9 & 0.200 & $\mathrm{mg} / \mathrm{L}$ & 1 & 25-Jun-2009 & 25-Jun-2009 & SW 846/6010 & \\
\hline Iron & ND & 0.200 & $\mathrm{mg} / \mathrm{L}$ & 1 & 25-Jun-2009 & 25-Jun-2009 & SW 846/6010 & $\mathrm{U}$ \\
\hline Magnesium & 2.42 & 0.200 & $\mathrm{mg} / \mathrm{L}$ & 1 & 25-Jun-2009 & 25-Jun-2009 & SW 846/6010 & \\
\hline Potassium & 1.42 & 0.200 & $\mathrm{mg} / \mathrm{L}$ & 1 & 25-Jun-2009 & 25-Jun-2009 & SW 846/6010 & \\
\hline Sodium & 2.84 & 0.200 & $\mathrm{mg} / \mathrm{L}$ & 1 & 25-Jun-2009 & 25-Jun-2009 & SW 846/6010 & \\
\hline Antimony & 0.0025 & 0.0010 & $\mathrm{mg} / \mathrm{L}$ & 2 & 19-Jun-2009 & 19-Jun-2009 & SW 846/6020 & \\
\hline Arsenic & 0.0494 & 0.0010 & $\mathrm{mg} / \mathrm{L}$ & 2 & 19-Jun-2009 & 19-Jun-2009 & SW 846/6020 & \\
\hline Barium & 0.109 & 0.0010 & $\mathrm{mg} / \mathrm{L}$ & 2 & 19-Jun-2009 & 19-Jun-2009 & SW 846/6020 & \\
\hline Beryllium & ND & 0.0010 & $\mathrm{mg} / \mathrm{L}$ & 2 & 19-Jun-2009 & 19-Jun-2009 & SW 846/6020 & $\mathrm{U}$ \\
\hline Cadmium & ND & 0.0010 & $\mathrm{mg} / \mathrm{L}$ & 2 & 19-Jun-2009 & 19-Jun-2009 & SW 846/6020 & $\mathrm{U}$ \\
\hline Chromium & 0.0013 & 0.0010 & $\mathrm{mg} / \mathrm{L}$ & 2 & 19-Jun-2009 & 19-Jun-2009 & SW 846/6020 & \\
\hline Cobalt & ND & 0.0010 & $\mathrm{mg} / \mathrm{L}$ & 2 & 19-Jun-2009 & 19-Jun-2009 & SW 846/6020 & U \\
\hline Copper & 0.0023 & 0.0010 & $\mathrm{mg} / \mathrm{L}$ & 2 & 19-Jun-2009 & 19-Jun-2009 & SW 846/6020 & \\
\hline Lead & ND & 0.0010 & $\mathrm{mg} / \mathrm{L}$ & 2 & 19-Jun-2009 & 19-Jun-2009 & SW 846/6020 & $\mathrm{U}$ \\
\hline Manganese & 0.0012 & 0.0010 & $\mathrm{mg} / \mathrm{L}$ & 2 & 19-Jun-2009 & 19-Jun-2009 & SW 846/6020 & \\
\hline Molybdenum & 0.0022 & 0.0010 & $\mathrm{mg} / \mathrm{L}$ & 2 & 19-Jun-2009 & 19-Jun-2009 & SW 846/6020 & \\
\hline Nickel & 0.00062 & 0.0010 & $\mathrm{mg} / \mathrm{L}$ & 2 & 19-Jun-2009 & 19-Jun-2009 & SW 846/6020 & $\mathrm{J}$ \\
\hline Selenium & 0.0040 & 0.0010 & $\mathrm{mg} / \mathrm{L}$ & 2 & 19-Jun-2009 & 19-Jun-2009 & SW 846/6020 & \\
\hline Silver & 0.0003 & 0.0010 & $\mathrm{mg} / \mathrm{L}$ & 2 & 19-Jun-2009 & 19-Jun-2009 & SW 846/6020 & $\mathrm{J}, \mathrm{B}$ \\
\hline Thallium & 0.0006 & 0.0010 & $\mathrm{mg} / \mathrm{L}$ & 2 & 19-Jun-2009 & 19-Jun-2009 & SW 846/6020 & $\mathrm{J}$ \\
\hline Vanadium & 0.0297 & 0.0010 & $\mathrm{mg} / \mathrm{L}$ & 2 & 19-Jun-2009 & 19-Jun-2009 & SW 846/6020 & \\
\hline Zinc & 0.0049 & 0.0010 & $\mathrm{mg} / \mathrm{L}$ & 2 & 19-Jun-2009 & 19-Jun-2009 & SW 846/6020 & B \\
\hline
\end{tabular}




\section{USACE ERDC-EP-C \\ 3909 Halls Ferry Road \\ Vicksburg, MS 39180-6199}

ERDC - ECB

Project: TVA Fly Ash Project

Reported:

$--,-$

Project Manager: Tony Bednar

03-Sep-2009

DRAFT: EMR MT N2 Hour 96 Rep B

9061701-02 (Water)

\begin{tabular}{|c|c|c|c|c|c|c|c|c|}
\hline & & orting & & & & & & \\
\hline Analyte & Result & Limit & Units & Dilution & Prepared & Analyzed & Method & Notes \\
\hline
\end{tabular}

ERDC- EL-EP-C (Environmental Chemistry Branch)

DRAFT: Miscellaneous Physical/Conventional Chemistry Parameters

\begin{tabular}{lrrrrrrr}
\hline Bromide & $\mathrm{ND}$ & 0.100 & $\mathrm{mg} / \mathrm{L}$ & 1 & $15-J u l-2009$ & $16-\mathrm{Jul}-2009$ & EPA 300.0 \\
Chloride & $\mathbf{2 . 8 8}$ & 1.00 & $\mathrm{mg} / \mathrm{L}$ & 1 & $15-J u l-2009$ & $16-\mathrm{Jul}-2009$ & EPA 300.0 \\
Nitrate as N & $\mathbf{0 . 4 1 4}$ & 0.100 & $\mathrm{mg} / \mathrm{L}$ & 1 & $15-J u l-2009$ & $16-J u l-2009$ & EPA 300.0 \\
Sulfate & $\mathbf{1 2 . 9}$ & 0.100 & $\mathrm{mg} / \mathrm{L}$ & 1 & $15-J u l-2009$ & $16-J u l-2009$ & EPA 300.0
\end{tabular}




\section{USACE ERDC-EP-C \\ 3909 Halls Ferry Road \\ Vicksburg, MS 39180-6199}

ERDC - ECB

$-\cdot$,
Project: TVA Fly Ash Project

Project Manager: Tony Bednar
Reported:

03-Sep-2009

DRAFT: EMR MT N2 Hour 96 Rep C

9061701-03 (Water)

\begin{tabular}{|c|c|c|c|c|c|c|c|c|}
\hline Analyte & Result & $\begin{array}{r}\text { porting } \\
\text { Limit }\end{array}$ & Units & Dilution & Prepared & Analyzed & Method & Notes \\
\hline
\end{tabular}

ERDC- EL-EP-C (Environmental Chemistry Branch)

DRAFT: Wet Chemistry

\begin{tabular}{llllllll}
\hline Hardness & $\mathbf{4 2 . 4}$ & 1.32 & $\begin{array}{c}\text { mg equil } \\
\text { CaCO3/ }\end{array}$ & 1 & 25-Jun-2009 & 25-Jun-2009 & Hardness
\end{tabular}

DRAFT: Metals by EPA 6000/7000 Series Methods

\begin{tabular}{|c|c|c|c|c|c|c|c|c|}
\hline Mercury & ND & 0.000005 & $\mathrm{mg} / \mathrm{L}$ & 1 & 17-Jun-2009 & 01-Jul-2009 & EPA 7471A & $\mathrm{U}$ \\
\hline Strontium & 0.172 & 0.0200 & $\mathrm{mg} / \mathrm{L}$ & 1 & 25-Jun-2009 & 25-Jun-2009 & EPA 6010B & \\
\hline Aluminum & ND & 0.200 & $\mathrm{mg} / \mathrm{L}$ & 1 & 25-Jun-2009 & 25-Jun-2009 & SW 846/6010 & $\mathrm{U}$ \\
\hline Calcium & 13.0 & 0.200 & $\mathrm{mg} / \mathrm{L}$ & 1 & 25-Jun-2009 & 25-Jun-2009 & SW 846/6010 & \\
\hline Iron & ND & 0.200 & $\mathrm{mg} / \mathrm{L}$ & 1 & 25-Jun-2009 & 25-Jun-2009 & SW 846/6010 & $\mathrm{U}$ \\
\hline Magnesium & 2.44 & 0.200 & $\mathrm{mg} / \mathrm{L}$ & 1 & 25-Jun-2009 & 25-Jun-2009 & SW 846/6010 & \\
\hline Potassium & 1.50 & 0.200 & $\mathrm{mg} / \mathrm{L}$ & 1 & 25-Jun-2009 & 25-Jun-2009 & SW 846/6010 & \\
\hline Sodium & 2.73 & 0.200 & $\mathrm{mg} / \mathrm{L}$ & 1 & 25-Jun-2009 & 25-Jun-2009 & SW 846/6010 & \\
\hline Antimony & 0.0016 & 0.0010 & $\mathrm{mg} / \mathrm{L}$ & 2 & 19-Jun-2009 & 19-Jun-2009 & SW 846/6020 & \\
\hline Arsenic & 0.0502 & 0.0010 & $\mathrm{mg} / \mathrm{L}$ & 2 & 19-Jun-2009 & 19-Jun-2009 & SW 846/6020 & \\
\hline Barium & 0.107 & 0.0010 & $\mathrm{mg} / \mathrm{L}$ & 2 & 19-Jun-2009 & 19-Jun-2009 & SW 846/6020 & \\
\hline Beryllium & ND & 0.0010 & $\mathrm{mg} / \mathrm{L}$ & 2 & 19-Jun-2009 & 19-Jun-2009 & SW 846/6020 & $\mathrm{U}$ \\
\hline Cadmium & ND & 0.0010 & $\mathrm{mg} / \mathrm{L}$ & 2 & 19-Jun-2009 & 19-Jun-2009 & SW 846/6020 & $\mathrm{U}$ \\
\hline Chromium & 0.0013 & 0.0010 & $\mathrm{mg} / \mathrm{L}$ & 2 & 19-Jun-2009 & 19-Jun-2009 & SW 846/6020 & \\
\hline Cobalt & ND & 0.0010 & $\mathrm{mg} / \mathrm{L}$ & 2 & 19-Jun-2009 & 19-Jun-2009 & SW 846/6020 & $\mathrm{U}$ \\
\hline Copper & 0.0026 & 0.0010 & $\mathrm{mg} / \mathrm{L}$ & 2 & 19-Jun-2009 & 19-Jun-2009 & SW 846/6020 & \\
\hline Lead & ND & 0.0010 & $\mathrm{mg} / \mathrm{L}$ & 2 & 19-Jun-2009 & 19-Jun-2009 & SW 846/6020 & $\mathrm{U}$ \\
\hline Manganese & 0.0012 & 0.0010 & $\mathrm{mg} / \mathrm{L}$ & 2 & 19-Jun-2009 & 19-Jun-2009 & SW 846/6020 & \\
\hline Molybdenum & 0.0022 & 0.0010 & $\mathrm{mg} / \mathrm{L}$ & 2 & 19-Jun-2009 & 19-Jun-2009 & SW 846/6020 & \\
\hline Nickel & 0.0007 & 0.0010 & $\mathrm{mg} / \mathrm{L}$ & 2 & 19-Jun-2009 & 19-Jun-2009 & SW 846/6020 & $\mathrm{J}$ \\
\hline Selenium & 0.0037 & 0.0010 & $\mathrm{mg} / \mathrm{L}$ & 2 & 19-Jun-2009 & 19-Jun-2009 & SW 846/6020 & \\
\hline Silver & ND & 0.0010 & $\mathrm{mg} / \mathrm{L}$ & 2 & 19-Jun-2009 & 19-Jun-2009 & SW 846/6020 & $\mathrm{U}$ \\
\hline Thallium & 0.0006 & 0.0010 & $\mathrm{mg} / \mathrm{L}$ & 2 & 19-Jun-2009 & 19-Jun-2009 & SW 846/6020 & $\mathrm{J}$ \\
\hline Vanadium & 0.0310 & 0.0010 & $\mathrm{mg} / \mathrm{L}$ & 2 & 19-Jun-2009 & 19-Jun-2009 & SW 846/6020 & \\
\hline Zinc & 0.0052 & 0.0010 & $\mathrm{mg} / \mathrm{L}$ & 2 & 19-Jun-2009 & 19-Jun-2009 & SW 846/6020 & B \\
\hline
\end{tabular}




\section{USACE ERDC-EP-C \\ 3909 Halls Ferry Road \\ Vicksburg, MS 39180-6199}

ERDC - ECB

Project: TVA Fly Ash Project

Reported:

$-$

Project Manager: Tony Bednar

03-Sep-2009

DRAFT: EMR MT N2 Hour 96 Rep C

9061701-03 (Water)

\begin{tabular}{|c|c|c|c|c|c|c|c|c|}
\hline & & orting & & & & & & \\
\hline Analyte & Result & Limit & Units & Dilution & Prepared & Analyzed & Method & Notes \\
\hline
\end{tabular}

ERDC- EL-EP-C (Environmental Chemistry Branch)

DRAFT: Miscellaneous Physical/Conventional Chemistry Parameters

\begin{tabular}{lrrrrrrr}
\hline Bromide & $\mathrm{ND}$ & 0.100 & $\mathrm{mg} / \mathrm{L}$ & 1 & $15-J u l-2009$ & $16-\mathrm{Jul}-2009$ & EPA 300.0 \\
Chloride & $\mathbf{2 . 6 8}$ & 1.00 & $\mathrm{mg} / \mathrm{L}$ & 1 & $15-J u l-2009$ & $16-\mathrm{Jul}-2009$ & EPA 300.0 \\
Nitrate as N & $\mathbf{0 . 3 9 3}$ & 0.100 & $\mathrm{mg} / \mathrm{L}$ & 1 & $15-J u l-2009$ & $16-J u l-2009$ & EPA 300.0 \\
Sulfate & $\mathbf{1 2 . 9}$ & 0.100 & $\mathrm{mg} / \mathrm{L}$ & 1 & $15-J u l-2009$ & $16-J u l-2009$ & EPA 300.0
\end{tabular}




\section{USACE ERDC-EP-C \\ 3909 Halls Ferry Road \\ Vicksburg, MS 39180-6199}

ERDC - ECB

$-\cdot$,
Project: TVA Fly Ash Project

Project Manager: Tony Bednar
Reported:

03-Sep-2009

DRAFT: EMR MT O2 Hour 96 Rep A

9061701-04 (Water)

\begin{tabular}{|c|c|c|c|c|c|c|c|c|}
\hline Analyte & Result & $\begin{array}{r}\text { porting } \\
\text { Limit }\end{array}$ & Units & Dilution & Prepared & Analyzed & Method & Notes \\
\hline
\end{tabular}

ERDC- EL-EP-C (Environmental Chemistry Branch)

DRAFT: Wet Chemistry

\begin{tabular}{llllllll}
\hline Hardness & $\mathbf{4 0 . 5}$ & 1.32 & $\begin{array}{c}\text { mg equil } \\
\text { CaCO3/ }\end{array}$ & 1 & 25-Jun-2009 & 25-Jun-2009
\end{tabular}

DRAFT: Metals by EPA 6000/7000 Series Methods

\begin{tabular}{|c|c|c|c|c|c|c|c|c|}
\hline Mercury & ND & 0.000005 & $\mathrm{mg} / \mathrm{L}$ & 1 & 17-Jun-2009 & 01-Jul-2009 & EPA 7471A & $\mathrm{U}$ \\
\hline Strontium & 0.156 & 0.0200 & $\mathrm{mg} / \mathrm{L}$ & 1 & 25-Jun-2009 & 25-Jun-2009 & EPA 6010B & \\
\hline Aluminum & 0.0591 & 0.200 & $\mathrm{mg} / \mathrm{L}$ & 1 & 25-Jun-2009 & 25-Jun-2009 & SW 846/6010 & J \\
\hline Calcium & 12.3 & 0.200 & $\mathrm{mg} / \mathrm{L}$ & 1 & 25-Jun-2009 & 25-Jun-2009 & SW 846/6010 & \\
\hline Iron & ND & 0.200 & $\mathrm{mg} / \mathrm{L}$ & 1 & 25-Jun-2009 & 25-Jun-2009 & SW 846/6010 & $\mathrm{U}$ \\
\hline Magnesium & 2.39 & 0.200 & $\mathrm{mg} / \mathrm{L}$ & 1 & 25-Jun-2009 & 25-Jun-2009 & SW 846/6010 & \\
\hline Potassium & 1.37 & 0.200 & $\mathrm{mg} / \mathrm{L}$ & 1 & 25-Jun-2009 & 25-Jun-2009 & SW 846/6010 & \\
\hline Sodium & 2.57 & 0.200 & $\mathrm{mg} / \mathrm{L}$ & 1 & 25-Jun-2009 & 25-Jun-2009 & SW 846/6010 & \\
\hline Antimony & 0.0015 & 0.0010 & $\mathrm{mg} / \mathrm{L}$ & 2 & 19-Jun-2009 & 19-Jun-2009 & SW 846/6020 & \\
\hline Arsenic & 0.0428 & 0.0010 & $\mathrm{mg} / \mathrm{L}$ & 2 & 19-Jun-2009 & 19-Jun-2009 & SW 846/6020 & \\
\hline Barium & 0.105 & 0.0010 & $\mathrm{mg} / \mathrm{L}$ & 2 & 19-Jun-2009 & 19-Jun-2009 & SW 846/6020 & \\
\hline Beryllium & ND & 0.0010 & $\mathrm{mg} / \mathrm{L}$ & 2 & 19-Jun-2009 & 19-Jun-2009 & SW 846/6020 & $\mathrm{U}$ \\
\hline Cadmium & ND & 0.0010 & $\mathrm{mg} / \mathrm{L}$ & 2 & 19-Jun-2009 & 19-Jun-2009 & SW 846/6020 & $\mathrm{U}$ \\
\hline Chromium & 0.0011 & 0.0010 & $\mathrm{mg} / \mathrm{L}$ & 2 & 19-Jun-2009 & 19-Jun-2009 & SW 846/6020 & \\
\hline Cobalt & ND & 0.0010 & $\mathrm{mg} / \mathrm{L}$ & 2 & 19-Jun-2009 & 19-Jun-2009 & SW 846/6020 & $\mathrm{U}$ \\
\hline Copper & 0.0022 & 0.0010 & $\mathrm{mg} / \mathrm{L}$ & 2 & 19-Jun-2009 & 19-Jun-2009 & SW 846/6020 & \\
\hline Lead & ND & 0.0010 & $\mathrm{mg} / \mathrm{L}$ & 2 & 19-Jun-2009 & 19-Jun-2009 & SW 846/6020 & $\mathrm{U}$ \\
\hline Manganese & 0.0017 & 0.0010 & $\mathrm{mg} / \mathrm{L}$ & 2 & 19-Jun-2009 & 19-Jun-2009 & SW 846/6020 & \\
\hline Molybdenum & 0.0020 & 0.0010 & $\mathrm{mg} / \mathrm{L}$ & 2 & 19-Jun-2009 & 19-Jun-2009 & SW 846/6020 & \\
\hline Nickel & 0.0006 & 0.0010 & $\mathrm{mg} / \mathrm{L}$ & 2 & 19-Jun-2009 & 19-Jun-2009 & SW 846/6020 & $\mathrm{J}$ \\
\hline Selenium & 0.0031 & 0.0010 & $\mathrm{mg} / \mathrm{L}$ & 2 & 19-Jun-2009 & 19-Jun-2009 & SW 846/6020 & \\
\hline Silver & ND & 0.0010 & $\mathrm{mg} / \mathrm{L}$ & 2 & 19-Jun-2009 & 19-Jun-2009 & SW 846/6020 & $\mathrm{U}$ \\
\hline Thallium & 0.0005 & 0.0010 & $\mathrm{mg} / \mathrm{L}$ & 2 & 19-Jun-2009 & 19-Jun-2009 & SW 846/6020 & $\mathrm{J}$ \\
\hline Vanadium & 0.0266 & 0.0010 & $\mathrm{mg} / \mathrm{L}$ & 2 & 19-Jun-2009 & 19-Jun-2009 & SW 846/6020 & \\
\hline Zinc & 0.0053 & 0.0010 & $\mathrm{mg} / \mathrm{L}$ & 2 & 19-Jun-2009 & 19-Jun-2009 & SW 846/6020 & B \\
\hline
\end{tabular}




\section{USACE ERDC-EP-C \\ 3909 Halls Ferry Road \\ Vicksburg, MS 39180-6199}

ERDC - ECB

Project: TVA Fly Ash Project

Reported:

$--,-$

Project Manager: Tony Bednar

03-Sep-2009

DRAFT: EMR MT O2 Hour 96 Rep A

9061701-04 (Water)

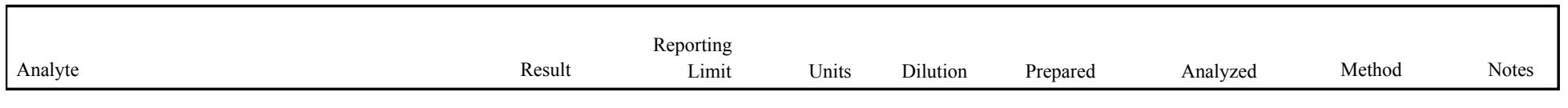

ERDC- EL-EP-C (Environmental Chemistry Branch)

DRAFT: Miscellaneous Physical/Conventional Chemistry Parameters

\begin{tabular}{lrrrrrrr}
\hline Bromide & $\mathrm{ND}$ & 0.100 & $\mathrm{mg} / \mathrm{L}$ & 1 & $15-J u l-2009$ & $16-\mathrm{Jul}-2009$ & EPA 300.0 \\
Chloride & $\mathbf{2 . 4 0}$ & 1.00 & $\mathrm{mg} / \mathrm{L}$ & 1 & $15-J u l-2009$ & $16-\mathrm{Jul}-2009$ & EPA 300.0 \\
Nitrate as N & $\mathbf{0 . 1 7 4}$ & 0.100 & $\mathrm{mg} / \mathrm{L}$ & 1 & $15-J u l-2009$ & $16-J u l-2009$ & EPA 300.0 \\
Sulfate & $\mathbf{1 2 . 5}$ & 0.100 & $\mathrm{mg} / \mathrm{L}$ & 1 & $15-J u l-2009$ & $16-J u l-2009$ & EPA 300.0
\end{tabular}




\section{USACE ERDC-EP-C \\ 3909 Halls Ferry Road \\ Vicksburg, MS 39180-6199}

ERDC - ECB

$-\cdot$,
Project: TVA Fly Ash Project

Project Manager: Tony Bednar
Reported:

03-Sep-2009

DRAFT: EMR MT O2 Hour 96 Rep B

9061701-05 (Water)

\begin{tabular}{|c|c|c|c|c|c|c|c|c|}
\hline Analyte & Result & $\begin{array}{r}\text { porting } \\
\text { Limit }\end{array}$ & Units & Dilution & Prepared & Analyzed & Method & Notes \\
\hline
\end{tabular}

ERDC- EL-EP-C (Environmental Chemistry Branch)

DRAFT: Wet Chemistry

\begin{tabular}{llcccccc}
\hline Hardness & $\mathbf{4 1 . 2}$ & 1.32 & $\begin{array}{c}\text { mg equil } \\
\text { CaCO3/ }\end{array}$ & 1 & 25-Jun-2009 & 25-Jun-2009
\end{tabular}

$\mathrm{L}$

DRAFT: Metals by EPA 6000/7000 Series Methods

\begin{tabular}{|c|c|c|c|c|c|c|c|c|}
\hline Mercury & ND & 0.000005 & $\mathrm{mg} / \mathrm{L}$ & 1 & 17-Jun-2009 & 01-Jul-2009 & EPA 7471A & $\mathrm{U}$ \\
\hline Strontium & 0.163 & 0.0200 & $\mathrm{mg} / \mathrm{L}$ & 1 & 25-Jun-2009 & 25-Jun-2009 & EPA 6010B & \\
\hline Aluminum & 0.0529 & 0.200 & $\mathrm{mg} / \mathrm{L}$ & 1 & 25-Jun-2009 & 25-Jun-2009 & SW 846/6010 & J \\
\hline Calcium & 12.6 & 0.200 & $\mathrm{mg} / \mathrm{L}$ & 1 & 25-Jun-2009 & 25-Jun-2009 & SW 846/6010 & \\
\hline Iron & ND & 0.200 & $\mathrm{mg} / \mathrm{L}$ & 1 & 25-Jun-2009 & 25-Jun-2009 & SW 846/6010 & $\mathrm{U}$ \\
\hline Magnesium & 2.36 & 0.200 & $\mathrm{mg} / \mathrm{L}$ & 1 & 25-Jun-2009 & 25-Jun-2009 & SW 846/6010 & \\
\hline Potassium & 1.39 & 0.200 & $\mathrm{mg} / \mathrm{L}$ & 1 & 25-Jun-2009 & 25-Jun-2009 & SW 846/6010 & \\
\hline Sodium & 2.53 & 0.200 & $\mathrm{mg} / \mathrm{L}$ & 1 & 25-Jun-2009 & 25-Jun-2009 & SW 846/6010 & \\
\hline Antimony & 0.0015 & 0.0010 & $\mathrm{mg} / \mathrm{L}$ & 2 & 19-Jun-2009 & 19-Jun-2009 & SW 846/6020 & \\
\hline Arsenic & 0.0457 & 0.0010 & $\mathrm{mg} / \mathrm{L}$ & 2 & 19-Jun-2009 & 19-Jun-2009 & SW 846/6020 & \\
\hline Barium & 0.114 & 0.0010 & $\mathrm{mg} / \mathrm{L}$ & 2 & 19-Jun-2009 & 19-Jun-2009 & SW 846/6020 & \\
\hline Beryllium & ND & 0.0010 & $\mathrm{mg} / \mathrm{L}$ & 2 & 19-Jun-2009 & 19-Jun-2009 & SW 846/6020 & $\mathrm{U}$ \\
\hline Cadmium & ND & 0.0010 & $\mathrm{mg} / \mathrm{L}$ & 2 & 19-Jun-2009 & 19-Jun-2009 & SW 846/6020 & $\mathrm{U}$ \\
\hline Chromium & 0.0012 & 0.0010 & $\mathrm{mg} / \mathrm{L}$ & 2 & 19-Jun-2009 & 19-Jun-2009 & SW 846/6020 & \\
\hline Cobalt & ND & 0.0010 & $\mathrm{mg} / \mathrm{L}$ & 2 & 19-Jun-2009 & 19-Jun-2009 & SW 846/6020 & $\mathrm{U}$ \\
\hline Copper & 0.0020 & 0.0010 & $\mathrm{mg} / \mathrm{L}$ & 2 & 19-Jun-2009 & 19-Jun-2009 & SW 846/6020 & \\
\hline Lead & ND & 0.0010 & $\mathrm{mg} / \mathrm{L}$ & 2 & 19-Jun-2009 & 19-Jun-2009 & SW 846/6020 & $\mathrm{U}$ \\
\hline Manganese & 0.0014 & 0.0010 & $\mathrm{mg} / \mathrm{L}$ & 2 & 19-Jun-2009 & 19-Jun-2009 & SW 846/6020 & \\
\hline Molybdenum & 0.0020 & 0.0010 & $\mathrm{mg} / \mathrm{L}$ & 2 & 19-Jun-2009 & 19-Jun-2009 & SW 846/6020 & \\
\hline Nickel & 0.0005 & 0.0010 & $\mathrm{mg} / \mathrm{L}$ & 2 & 19-Jun-2009 & 19-Jun-2009 & SW 846/6020 & $\mathrm{J}$ \\
\hline Selenium & 0.0036 & 0.0010 & $\mathrm{mg} / \mathrm{L}$ & 2 & 19-Jun-2009 & 19-Jun-2009 & SW 846/6020 & \\
\hline Silver & ND & 0.0010 & $\mathrm{mg} / \mathrm{L}$ & 2 & 19-Jun-2009 & 19-Jun-2009 & SW 846/6020 & $\mathrm{U}$ \\
\hline Thallium & 0.00057 & 0.0010 & $\mathrm{mg} / \mathrm{L}$ & 2 & 19-Jun-2009 & 19-Jun-2009 & SW 846/6020 & $\mathrm{J}$ \\
\hline Vanadium & 0.0286 & 0.0010 & $\mathrm{mg} / \mathrm{L}$ & 2 & 19-Jun-2009 & 19-Jun-2009 & SW 846/6020 & \\
\hline Zinc & 0.0056 & 0.0010 & $\mathrm{mg} / \mathrm{L}$ & 2 & 19-Jun-2009 & 19-Jun-2009 & SW 846/6020 & B \\
\hline
\end{tabular}




\section{USACE ERDC-EP-C \\ 3909 Halls Ferry Road \\ Vicksburg, MS 39180-6199}

ERDC - ECB

Project: TVA Fly Ash Project

Reported:

$--,-$

Project Manager: Tony Bednar

03-Sep-2009

DRAFT: EMR MT O2 Hour 96 Rep B

9061701-05 (Water)

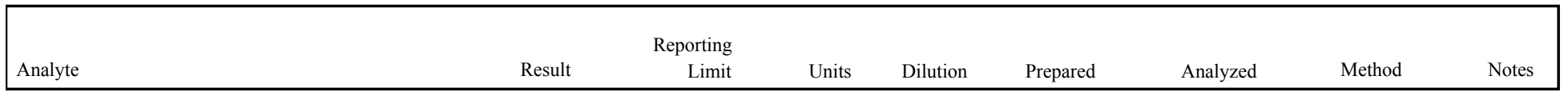

ERDC- EL-EP-C (Environmental Chemistry Branch)

DRAFT: Miscellaneous Physical/Conventional Chemistry Parameters

\begin{tabular}{lrrrrrrr}
\hline Bromide & $\mathrm{ND}$ & 0.100 & $\mathrm{mg} / \mathrm{L}$ & 1 & $15-J u l-2009$ & $16-\mathrm{Jul}-2009$ & EPA 300.0 \\
Chloride & $\mathbf{2 . 4 3}$ & 1.00 & $\mathrm{mg} / \mathrm{L}$ & 1 & $15-J u l-2009$ & $16-\mathrm{Jul}-2009$ & EPA 300.0 \\
Nitrate as N & $\mathbf{0 . 2 2 0}$ & 0.100 & $\mathrm{mg} / \mathrm{L}$ & 1 & $15-J u l-2009$ & $16-J u l-2009$ & EPA 300.0 \\
Sulfate & $\mathbf{1 2 . 6}$ & 0.100 & $\mathrm{mg} / \mathrm{L}$ & 1 & $15-J u l-2009$ & $16-J u l-2009$ & EPA 300.0
\end{tabular}




\section{USACE ERDC-EP-C \\ 3909 Halls Ferry Road \\ Vicksburg, MS 39180-6199}

ERDC - ECB

$-\cdot$,
Project: TVA Fly Ash Project

Project Manager: Tony Bednar
Reported:

03-Sep-2009

DRAFT: EMR MT O2 Hour 96 Rep C

9061701-06 (Water)

\begin{tabular}{|c|c|c|c|c|c|c|c|c|}
\hline Analyte & Result & $\begin{array}{r}\text { porting } \\
\text { Limit }\end{array}$ & Units & Dilution & Prepared & Analyzed & Method & Notes \\
\hline
\end{tabular}

ERDC- EL-EP-C (Environmental Chemistry Branch)

DRAFT: Wet Chemistry

\begin{tabular}{llllllll}
\hline Hardness & $\mathbf{4 1 . 2}$ & 1.32 & $\begin{array}{c}\text { mg equil } \\
\text { CaCO3/ }\end{array}$ & 1 & 25-Jun-2009 & 25-Jun-2009
\end{tabular}

$\mathrm{L}$

DRAFT: Metals by EPA 6000/7000 Series Methods

\begin{tabular}{|c|c|c|c|c|c|c|c|c|}
\hline Mercury & ND & 0.000005 & $\mathrm{mg} / \mathrm{L}$ & 1 & 17-Jun-2009 & 01-Jul-2009 & EPA 7471A & $\mathrm{U}$ \\
\hline Strontium & 0.159 & 0.0200 & $\mathrm{mg} / \mathrm{L}$ & 1 & 25-Jun-2009 & 25-Jun-2009 & EPA $6010 B$ & \\
\hline Aluminum & 0.0588 & 0.200 & $\mathrm{mg} / \mathrm{L}$ & 1 & 25-Jun-2009 & 25-Jun-2009 & SW 846/6010 & J \\
\hline Calcium & 12.5 & 0.200 & $\mathrm{mg} / \mathrm{L}$ & 1 & 25-Jun-2009 & 25-Jun-2009 & SW 846/6010 & \\
\hline Iron & ND & 0.200 & $\mathrm{mg} / \mathrm{L}$ & 1 & 25-Jun-2009 & 25-Jun-2009 & SW 846/6010 & $\mathrm{U}$ \\
\hline Magnesium & 2.43 & 0.200 & $\mathrm{mg} / \mathrm{L}$ & 1 & 25-Jun-2009 & 25-Jun-2009 & SW 846/6010 & \\
\hline Potassium & 1.45 & 0.200 & $\mathrm{mg} / \mathrm{L}$ & 1 & 25-Jun-2009 & 25-Jun-2009 & SW 846/6010 & \\
\hline Sodium & 2.61 & 0.200 & $\mathrm{mg} / \mathrm{L}$ & 1 & 25-Jun-2009 & 25-Jun-2009 & SW 846/6010 & \\
\hline Antimony & 0.0013 & 0.0010 & $\mathrm{mg} / \mathrm{L}$ & 2 & 19-Jun-2009 & 19-Jun-2009 & SW 846/6020 & \\
\hline Arsenic & 0.0432 & 0.0010 & $\mathrm{mg} / \mathrm{L}$ & 2 & 19-Jun-2009 & 19-Jun-2009 & SW 846/6020 & \\
\hline Barium & 0.111 & 0.0010 & $\mathrm{mg} / \mathrm{L}$ & 2 & 19-Jun-2009 & 19-Jun-2009 & SW 846/6020 & \\
\hline Beryllium & ND & 0.0010 & $\mathrm{mg} / \mathrm{L}$ & 2 & 19-Jun-2009 & 19-Jun-2009 & SW 846/6020 & $\mathrm{U}$ \\
\hline Cadmium & ND & 0.0010 & $\mathrm{mg} / \mathrm{L}$ & 2 & 19-Jun-2009 & 19-Jun-2009 & SW 846/6020 & $\mathrm{U}$ \\
\hline Chromium & 0.0012 & 0.0010 & $\mathrm{mg} / \mathrm{L}$ & 2 & 19-Jun-2009 & 19-Jun-2009 & SW 846/6020 & \\
\hline Cobalt & ND & 0.0010 & $\mathrm{mg} / \mathrm{L}$ & 2 & 19-Jun-2009 & 19-Jun-2009 & SW 846/6020 & $\mathrm{U}$ \\
\hline Copper & 0.0023 & 0.0010 & $\mathrm{mg} / \mathrm{L}$ & 2 & 19-Jun-2009 & 19-Jun-2009 & SW 846/6020 & \\
\hline Lead & ND & 0.0010 & $\mathrm{mg} / \mathrm{L}$ & 2 & 19-Jun-2009 & 19-Jun-2009 & SW 846/6020 & $\mathrm{U}$ \\
\hline Manganese & 0.0015 & 0.0010 & $\mathrm{mg} / \mathrm{L}$ & 2 & 19-Jun-2009 & 19-Jun-2009 & SW 846/6020 & \\
\hline Molybdenum & 0.0020 & 0.0010 & $\mathrm{mg} / \mathrm{L}$ & 2 & 19-Jun-2009 & 19-Jun-2009 & SW 846/6020 & \\
\hline Nickel & 0.0006 & 0.0010 & $\mathrm{mg} / \mathrm{L}$ & 2 & 19-Jun-2009 & 19-Jun-2009 & SW 846/6020 & $\mathrm{J}$ \\
\hline Selenium & 0.0033 & 0.0010 & $\mathrm{mg} / \mathrm{L}$ & 2 & 19-Jun-2009 & 19-Jun-2009 & SW 846/6020 & \\
\hline Silver & ND & 0.0010 & $\mathrm{mg} / \mathrm{L}$ & 2 & 19-Jun-2009 & 19-Jun-2009 & SW 846/6020 & $\mathrm{U}$ \\
\hline Thallium & 0.00055 & 0.0010 & $\mathrm{mg} / \mathrm{L}$ & 2 & 19-Jun-2009 & 19-Jun-2009 & SW 846/6020 & $\mathrm{J}$ \\
\hline Vanadium & 0.0285 & 0.0010 & $\mathrm{mg} / \mathrm{L}$ & 2 & 19-Jun-2009 & 19-Jun-2009 & SW 846/6020 & \\
\hline Zinc & 0.0054 & 0.0010 & $\mathrm{mg} / \mathrm{L}$ & 2 & 19-Jun-2009 & 19-Jun-2009 & SW 846/6020 & B \\
\hline
\end{tabular}




\section{USACE ERDC-EP-C \\ 3909 Halls Ferry Road \\ Vicksburg, MS 39180-6199}

ERDC - ECB

Project: TVA Fly Ash Project

Reported:

$--,-$

Project Manager: Tony Bednar

03-Sep-2009

DRAFT: EMR MT O2 Hour 96 Rep C

9061701-06 (Water)

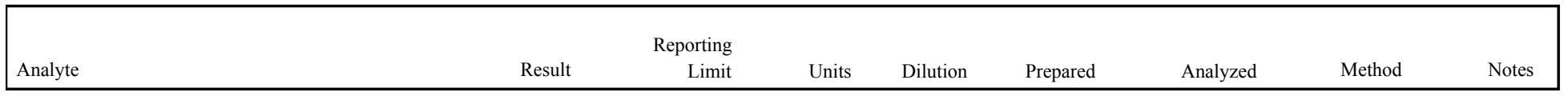

ERDC- EL-EP-C (Environmental Chemistry Branch)

DRAFT: Miscellaneous Physical/Conventional Chemistry Parameters

\begin{tabular}{lrrrrrrr}
\hline Bromide & $\mathrm{ND}$ & 0.100 & $\mathrm{mg} / \mathrm{L}$ & 1 & $15-J u l-2009$ & $16-\mathrm{Jul}-2009$ & EPA 300.0 \\
Chloride & $\mathbf{2 . 5 2}$ & 1.00 & $\mathrm{mg} / \mathrm{L}$ & 1 & $15-J u l-2009$ & $16-\mathrm{Jul}-2009$ & EPA 300.0 \\
Nitrate as N & $\mathbf{0 . 3 4 8}$ & 0.100 & $\mathrm{mg} / \mathrm{L}$ & 1 & $15-J u l-2009$ & $16-J u l-2009$ & EPA 300.0 \\
Sulfate & $\mathbf{1 2 . 6}$ & 0.100 & $\mathrm{mg} / \mathrm{L}$ & 1 & $15-J u l-2009$ & $16-J u l-2009$ & EPA 300.0
\end{tabular}




\section{USACE ERDC-EP-C \\ 3909 Halls Ferry Road \\ Vicksburg, MS 39180-6199}

ERDC - ECB

$-\cdot$,
Project: TVA Fly Ash Project

Project Manager: Tony Bednar
Reported:

03-Sep-2009

DRAFT: SLC MT N2 Hour 96 Rep A

9061701-07 (Water)

\begin{tabular}{|c|c|c|c|c|c|c|c|c|}
\hline Analyte & Result & $\begin{array}{r}\text { porting } \\
\text { Limit }\end{array}$ & Units & Dilution & Prepared & Analyzed & Method & Notes \\
\hline
\end{tabular}

ERDC- EL-EP-C (Environmental Chemistry Branch)

DRAFT: Wet Chemistry

\begin{tabular}{llllllll}
\hline Hardness & $\mathbf{1 0 1}$ & 1.32 & $\begin{array}{c}\text { mg equil } \\
\text { CaCO3/ }\end{array}$ & 1 & 25-Jun-2009 & 25-Jun-2009
\end{tabular}

$\mathrm{L}$

DRAFT: Metals by EPA 6000/7000 Series Methods

\begin{tabular}{|c|c|c|c|c|c|c|c|c|}
\hline Mercury & ND & 0.000005 & $\mathrm{mg} / \mathrm{L}$ & 1 & 17-Jun-2009 & 01-Jul-2009 & EPA 7471A & $\mathrm{U}$ \\
\hline Strontium & 0.550 & 0.0200 & $\mathrm{mg} / \mathrm{L}$ & 1 & 25-Jun-2009 & 25-Jun-2009 & EPA 6010B & \\
\hline Aluminum & 0.0956 & 0.200 & $\mathrm{mg} / \mathrm{L}$ & 1 & 25-Jun-2009 & 25-Jun-2009 & SW 846/6010 & $\mathrm{J}$ \\
\hline Calcium & 31.5 & 0.200 & $\mathrm{mg} / \mathrm{L}$ & 1 & 25-Jun-2009 & 25-Jun-2009 & SW 846/6010 & \\
\hline Iron & ND & 0.200 & $\mathrm{mg} / \mathrm{L}$ & 1 & 25-Jun-2009 & 25-Jun-2009 & SW 846/6010 & $\mathrm{U}$ \\
\hline Magnesium & 5.33 & 0.200 & $\mathrm{mg} / \mathrm{L}$ & 1 & 25-Jun-2009 & 25-Jun-2009 & SW 846/6010 & \\
\hline Potassium & 2.01 & 0.200 & $\mathrm{mg} / \mathrm{L}$ & 1 & 25-Jun-2009 & 25-Jun-2009 & SW 846/6010 & \\
\hline Sodium & 4.26 & 0.200 & $\mathrm{mg} / \mathrm{L}$ & 1 & 25-Jun-2009 & 25-Jun-2009 & SW 846/6010 & \\
\hline Antimony & 0.0089 & 0.0010 & $\mathrm{mg} / \mathrm{L}$ & 2 & 19-Jun-2009 & 19-Jun-2009 & SW 846/6020 & \\
\hline Arsenic & 0.0851 & 0.0010 & $\mathrm{mg} / \mathrm{L}$ & 2 & 19-Jun-2009 & 19-Jun-2009 & SW 846/6020 & \\
\hline Barium & 0.105 & 0.0010 & $\mathrm{mg} / \mathrm{L}$ & 2 & 19-Jun-2009 & 19-Jun-2009 & SW 846/6020 & \\
\hline Beryllium & ND & 0.0010 & $\mathrm{mg} / \mathrm{L}$ & 2 & 19-Jun-2009 & 19-Jun-2009 & SW 846/6020 & $\mathrm{U}$ \\
\hline Cadmium & 0.0003 & 0.0010 & $\mathrm{mg} / \mathrm{L}$ & 2 & 19-Jun-2009 & 19-Jun-2009 & SW 846/6020 & $\mathrm{J}$ \\
\hline Chromium & 0.0004 & 0.0010 & $\mathrm{mg} / \mathrm{L}$ & 2 & 19-Jun-2009 & 19-Jun-2009 & SW 846/6020 & $\mathrm{J}$ \\
\hline Cobalt & ND & 0.0010 & $\mathrm{mg} / \mathrm{L}$ & 2 & 19-Jun-2009 & 19-Jun-2009 & SW 846/6020 & $\mathrm{U}$ \\
\hline Copper & 0.0014 & 0.0010 & $\mathrm{mg} / \mathrm{L}$ & 2 & 19-Jun-2009 & 19-Jun-2009 & SW 846/6020 & \\
\hline Lead & ND & 0.0010 & $\mathrm{mg} / \mathrm{L}$ & 2 & 19-Jun-2009 & 19-Jun-2009 & SW 846/6020 & $\mathrm{U}$ \\
\hline Manganese & 0.0088 & 0.0010 & $\mathrm{mg} / \mathrm{L}$ & 2 & 19-Jun-2009 & 19-Jun-2009 & SW 846/6020 & \\
\hline Molybdenum & 0.0728 & 0.0010 & $\mathrm{mg} / \mathrm{L}$ & 2 & 19-Jun-2009 & 19-Jun-2009 & SW 846/6020 & \\
\hline Nickel & 0.0007 & 0.0010 & $\mathrm{mg} / \mathrm{L}$ & 2 & 19-Jun-2009 & 19-Jun-2009 & SW 846/6020 & $\mathrm{J}$ \\
\hline Selenium & 0.0098 & 0.0010 & $\mathrm{mg} / \mathrm{L}$ & 2 & 19-Jun-2009 & 19-Jun-2009 & SW 846/6020 & \\
\hline Silver & ND & 0.0010 & $\mathrm{mg} / \mathrm{L}$ & 2 & 19-Jun-2009 & 19-Jun-2009 & SW 846/6020 & $\mathrm{U}$ \\
\hline Thallium & 0.0008 & 0.0010 & $\mathrm{mg} / \mathrm{L}$ & 2 & 19-Jun-2009 & 19-Jun-2009 & SW 846/6020 & $\mathrm{J}$ \\
\hline Vanadium & 0.0516 & 0.0010 & $\mathrm{mg} / \mathrm{L}$ & 2 & 19-Jun-2009 & 19-Jun-2009 & SW 846/6020 & \\
\hline Zinc & 0.0056 & 0.0010 & $\mathrm{mg} / \mathrm{L}$ & 2 & 19-Jun-2009 & 19-Jun-2009 & SW 846/6020 & B \\
\hline
\end{tabular}




\section{USACE ERDC-EP-C \\ 3909 Halls Ferry Road \\ Vicksburg, MS 39180-6199}

ERDC - ECB

Project: TVA Fly Ash Project

Reported:

$--,-$

Project Manager: Tony Bednar

03-Sep-2009

DRAFT: SLC MT N2 Hour $96 \operatorname{Rep} A$

9061701-07 (Water)

\begin{tabular}{|c|c|c|c|c|c|c|c|c|}
\hline & & orting & & & & & & \\
\hline Analyte & Result & Limit & Units & Dilution & Prepared & Analyzed & Method & Notes \\
\hline
\end{tabular}

ERDC- EL-EP-C (Environmental Chemistry Branch)

DRAFT: Miscellaneous Physical/Conventional Chemistry Parameters

\begin{tabular}{lrrrrrrr}
\hline Bromide & $\mathrm{ND}$ & 0.100 & $\mathrm{mg} / \mathrm{L}$ & 1 & $15-J u l-2009$ & $16-\mathrm{Jul}-2009$ & EPA 300.0 \\
Chloride & $\mathbf{3 . 9 0}$ & 1.00 & $\mathrm{mg} / \mathrm{L}$ & 1 & $15-J u l-2009$ & $16-J u l-2009$ & EPA 300.0 \\
Nitrate as N & $\mathbf{0 . 3 9 0}$ & 0.100 & $\mathrm{mg} / \mathrm{L}$ & 1 & $15-J u l-2009$ & $16-J u l-2009$ & EPA 300.0
\end{tabular}




\section{USACE ERDC-EP-C \\ 3909 Halls Ferry Road \\ Vicksburg, MS 39180-6199}

ERDC - ECB

$-\cdot$,
Project: TVA Fly Ash Project

Project Manager: Tony Bednar
Reported:

03-Sep-2009

DRAFT: SLC MT N2 Hour 96 Rep B

9061701-08 (Water)

\begin{tabular}{|c|c|c|c|c|c|c|c|c|}
\hline Analyte & Result & $\begin{array}{r}\text { porting } \\
\text { Limit }\end{array}$ & Units & Dilution & Prepared & Analyzed & Method & Notes \\
\hline
\end{tabular}

ERDC- EL-EP-C (Environmental Chemistry Branch)

DRAFT: Wet Chemistry

\begin{tabular}{llllllll}
\hline Hardness & $\mathbf{1 0 0}$ & 1.32 & $\begin{array}{c}\text { mg equil } \\
\text { CaCO3/ }\end{array}$ & 1 & 25-Jun-2009 & 25-Jun-2009
\end{tabular}

$\mathrm{L}$

DRAFT: Metals by EPA 6000/7000 Series Methods

\begin{tabular}{|c|c|c|c|c|c|c|c|c|}
\hline Mercury & ND & 0.000005 & $\mathrm{mg} / \mathrm{L}$ & 1 & 17-Jun-2009 & 01-Jul-2009 & EPA 7471A & $\mathrm{U}$ \\
\hline Strontium & 0.551 & 0.0200 & $\mathrm{mg} / \mathrm{L}$ & 1 & 25-Jun-2009 & 25-Jun-2009 & EPA $6010 \mathrm{~B}$ & \\
\hline Aluminum & 0.112 & 0.200 & $\mathrm{mg} / \mathrm{L}$ & 1 & 25-Jun-2009 & 25-Jun-2009 & SW 846/6010 & $\mathrm{J}$ \\
\hline Calcium & 31.5 & 0.200 & $\mathrm{mg} / \mathrm{L}$ & 1 & 25-Jun-2009 & 25-Jun-2009 & SW 846/6010 & \\
\hline Iron & ND & 0.200 & $\mathrm{mg} / \mathrm{L}$ & 1 & 25-Jun-2009 & 25-Jun-2009 & SW 846/6010 & $\mathrm{U}$ \\
\hline Magnesium & 5.30 & 0.200 & $\mathrm{mg} / \mathrm{L}$ & 1 & 25-Jun-2009 & 25-Jun-2009 & SW 846/6010 & \\
\hline Potassium & 1.94 & 0.200 & $\mathrm{mg} / \mathrm{L}$ & 1 & 25-Jun-2009 & 25-Jun-2009 & SW 846/6010 & \\
\hline Sodium & 4.16 & 0.200 & $\mathrm{mg} / \mathrm{L}$ & 1 & 25-Jun-2009 & 25-Jun-2009 & SW 846/6010 & \\
\hline Antimony & 0.0090 & 0.0010 & $\mathrm{mg} / \mathrm{L}$ & 2 & 19-Jun-2009 & 19-Jun-2009 & SW 846/6020 & \\
\hline Arsenic & 0.0841 & 0.0010 & $\mathrm{mg} / \mathrm{L}$ & 2 & 19-Jun-2009 & 19-Jun-2009 & SW 846/6020 & \\
\hline Barium & 0.106 & 0.0010 & $\mathrm{mg} / \mathrm{L}$ & 2 & 19-Jun-2009 & 19-Jun-2009 & SW 846/6020 & \\
\hline Beryllium & ND & 0.0010 & $\mathrm{mg} / \mathrm{L}$ & 2 & 19-Jun-2009 & 19-Jun-2009 & SW 846/6020 & $\mathrm{U}$ \\
\hline Cadmium & 0.0004 & 0.0010 & $\mathrm{mg} / \mathrm{L}$ & 2 & 19-Jun-2009 & 19-Jun-2009 & SW 846/6020 & $\mathrm{J}$ \\
\hline Chromium & 0.0004 & 0.0010 & $\mathrm{mg} / \mathrm{L}$ & 2 & 19-Jun-2009 & 19-Jun-2009 & SW 846/6020 & J \\
\hline Cobalt & ND & 0.0010 & $\mathrm{mg} / \mathrm{L}$ & 2 & 19-Jun-2009 & 19-Jun-2009 & SW 846/6020 & $\mathrm{U}$ \\
\hline Copper & 0.0010 & 0.0010 & $\mathrm{mg} / \mathrm{L}$ & 2 & 19-Jun-2009 & 19-Jun-2009 & SW 846/6020 & \\
\hline Lead & ND & 0.0010 & $\mathrm{mg} / \mathrm{L}$ & 2 & 19-Jun-2009 & 19-Jun-2009 & SW 846/6020 & $\mathrm{U}$ \\
\hline Manganese & 0.0089 & 0.0010 & $\mathrm{mg} / \mathrm{L}$ & 2 & 19-Jun-2009 & 19-Jun-2009 & SW 846/6020 & \\
\hline Molybdenum & 0.0732 & 0.0010 & $\mathrm{mg} / \mathrm{L}$ & 2 & 19-Jun-2009 & 19-Jun-2009 & SW 846/6020 & \\
\hline Nickel & 0.0006 & 0.0010 & $\mathrm{mg} / \mathrm{L}$ & 2 & 19-Jun-2009 & 19-Jun-2009 & SW 846/6020 & $\mathrm{J}$ \\
\hline Selenium & 0.0091 & 0.0010 & $\mathrm{mg} / \mathrm{L}$ & 2 & 19-Jun-2009 & 19-Jun-2009 & SW 846/6020 & \\
\hline Silver & ND & 0.0010 & $\mathrm{mg} / \mathrm{L}$ & 2 & 19-Jun-2009 & 19-Jun-2009 & SW 846/6020 & $\mathrm{U}$ \\
\hline Thallium & 0.0008 & 0.0010 & $\mathrm{mg} / \mathrm{L}$ & 2 & 19-Jun-2009 & 19-Jun-2009 & SW 846/6020 & $\mathrm{J}$ \\
\hline Vanadium & 0.0512 & 0.0010 & $\mathrm{mg} / \mathrm{L}$ & 2 & 19-Jun-2009 & 19-Jun-2009 & SW 846/6020 & \\
\hline Zinc & 0.0050 & 0.0010 & $\mathrm{mg} / \mathrm{L}$ & 2 & 19-Jun-2009 & 19-Jun-2009 & SW 846/6020 & B \\
\hline
\end{tabular}




\section{USACE ERDC-EP-C \\ 3909 Halls Ferry Road \\ Vicksburg, MS 39180-6199}

ERDC - ECB

Project: TVA Fly Ash Project

Reported:

$--,-$

Project Manager: Tony Bednar

03-Sep-2009

DRAFT: SLC MT N2 Hour 96 Rep B

9061701-08 (Water)

\begin{tabular}{|c|c|c|c|c|c|c|c|c|}
\hline & & orting & & & & & & \\
\hline Analyte & Result & Limit & Units & Dilution & Prepared & Analyzed & Method & Notes \\
\hline
\end{tabular}

ERDC- EL-EP-C (Environmental Chemistry Branch)

DRAFT: Miscellaneous Physical/Conventional Chemistry Parameters

\begin{tabular}{lrrrrrrr}
\hline Bromide & $\mathrm{ND}$ & 0.100 & $\mathrm{mg} / \mathrm{L}$ & 1 & $15-J u l-2009$ & $16-\mathrm{Jul}-2009$ & EPA 300.0 \\
Chloride & $\mathbf{3 . 7 9}$ & 1.00 & $\mathrm{mg} / \mathrm{L}$ & 1 & $15-J u l-2009$ & $16-J u l-2009$ & EPA 300.0 \\
Nitrate as N & $\mathbf{0 . 3 7 7}$ & 0.100 & $\mathrm{mg} / \mathrm{L}$ & 1 & $15-J u l-2009$ & $16-J u l-2009$ & EPA 300.0
\end{tabular}




\section{USACE ERDC-EP-C \\ 3909 Halls Ferry Road \\ Vicksburg, MS 39180-6199}

ERDC - ECB

$-\cdot$,
Project: TVA Fly Ash Project

Project Manager: Tony Bednar
Reported:

03-Sep-2009

DRAFT: SLC MT N2 Hour 96 Rep C

9061701-09 (Water)

\begin{tabular}{|c|c|c|c|c|c|c|c|c|}
\hline Analyte & Result & $\begin{array}{r}\text { porting } \\
\text { Limit }\end{array}$ & Units & Dilution & Prepared & Analyzed & Method & Notes \\
\hline
\end{tabular}

ERDC- EL-EP-C (Environmental Chemistry Branch)

DRAFT: Wet Chemistry

\begin{tabular}{llllllll}
\hline Hardness & $\mathbf{1 0 0}$ & 1.32 & $\begin{array}{c}\text { mg equil } \\
\text { CaCO3/ }\end{array}$ & 1 & 25-Jun-2009 & 25-Jun-2009
\end{tabular}

$\mathrm{L}$

DRAFT: Metals by EPA 6000/7000 Series Methods

\begin{tabular}{|c|c|c|c|c|c|c|c|c|}
\hline Mercury & ND & 0.000005 & $\mathrm{mg} / \mathrm{L}$ & 1 & 17-Jun-2009 & 01-Jul-2009 & EPA 7471A & $\mathrm{U}$ \\
\hline Strontium & 0.549 & 0.0200 & $\mathrm{mg} / \mathrm{L}$ & 1 & 25-Jun-2009 & 25-Jun-2009 & EPA 6010B & \\
\hline Aluminum & 0.110 & 0.200 & $\mathrm{mg} / \mathrm{L}$ & 1 & 25-Jun-2009 & 25-Jun-2009 & SW 846/6010 & $\mathrm{J}$ \\
\hline Calcium & 31.4 & 0.200 & $\mathrm{mg} / \mathrm{L}$ & 1 & 25-Jun-2009 & 25-Jun-2009 & SW 846/6010 & \\
\hline Iron & ND & 0.200 & $\mathrm{mg} / \mathrm{L}$ & 1 & 25-Jun-2009 & 25-Jun-2009 & SW 846/6010 & $\mathrm{U}$ \\
\hline Magnesium & 5.26 & 0.200 & $\mathrm{mg} / \mathrm{L}$ & 1 & 25-Jun-2009 & 25-Jun-2009 & SW 846/6010 & \\
\hline Potassium & 1.95 & 0.200 & $\mathrm{mg} / \mathrm{L}$ & 1 & 25-Jun-2009 & 25-Jun-2009 & SW 846/6010 & \\
\hline Sodium & 4.16 & 0.200 & $\mathrm{mg} / \mathrm{L}$ & 1 & 25-Jun-2009 & 25-Jun-2009 & SW 846/6010 & \\
\hline Antimony & 0.0090 & 0.0010 & $\mathrm{mg} / \mathrm{L}$ & 2 & 19-Jun-2009 & 19-Jun-2009 & SW 846/6020 & \\
\hline Arsenic & 0.0874 & 0.0010 & $\mathrm{mg} / \mathrm{L}$ & 2 & 19-Jun-2009 & 19-Jun-2009 & SW 846/6020 & \\
\hline Barium & 0.106 & 0.0010 & $\mathrm{mg} / \mathrm{L}$ & 2 & 19-Jun-2009 & 19-Jun-2009 & SW 846/6020 & \\
\hline Beryllium & ND & 0.0010 & $\mathrm{mg} / \mathrm{L}$ & 2 & 19-Jun-2009 & 19-Jun-2009 & SW 846/6020 & $\mathrm{U}$ \\
\hline Cadmium & 0.0003 & 0.0010 & $\mathrm{mg} / \mathrm{L}$ & 2 & 19-Jun-2009 & 19-Jun-2009 & SW 846/6020 & $\mathrm{J}$ \\
\hline Chromium & 0.0004 & 0.0010 & $\mathrm{mg} / \mathrm{L}$ & 2 & 19-Jun-2009 & 19-Jun-2009 & SW 846/6020 & $\mathrm{J}$ \\
\hline Cobalt & ND & 0.0010 & $\mathrm{mg} / \mathrm{L}$ & 2 & 19-Jun-2009 & 19-Jun-2009 & SW 846/6020 & $\mathrm{U}$ \\
\hline Copper & 0.0012 & 0.0010 & $\mathrm{mg} / \mathrm{L}$ & 2 & 19-Jun-2009 & 19-Jun-2009 & SW 846/6020 & \\
\hline Lead & ND & 0.0010 & $\mathrm{mg} / \mathrm{L}$ & 2 & 19-Jun-2009 & 19-Jun-2009 & SW 846/6020 & $\mathrm{U}$ \\
\hline Manganese & 0.0089 & 0.0010 & $\mathrm{mg} / \mathrm{L}$ & 2 & 19-Jun-2009 & 19-Jun-2009 & SW 846/6020 & \\
\hline Molybdenum & 0.0740 & 0.0010 & $\mathrm{mg} / \mathrm{L}$ & 2 & 19-Jun-2009 & 19-Jun-2009 & SW 846/6020 & \\
\hline Nickel & 0.0006 & 0.0010 & $\mathrm{mg} / \mathrm{L}$ & 2 & 19-Jun-2009 & 19-Jun-2009 & SW 846/6020 & $\mathrm{J}$ \\
\hline Selenium & 0.0097 & 0.0010 & $\mathrm{mg} / \mathrm{L}$ & 2 & 19-Jun-2009 & 19-Jun-2009 & SW 846/6020 & \\
\hline Silver & ND & 0.0010 & $\mathrm{mg} / \mathrm{L}$ & 2 & 19-Jun-2009 & 19-Jun-2009 & SW 846/6020 & $\mathrm{U}$ \\
\hline Thallium & 0.0008 & 0.0010 & $\mathrm{mg} / \mathrm{L}$ & 2 & 19-Jun-2009 & 19-Jun-2009 & SW 846/6020 & $\mathrm{J}$ \\
\hline Vanadium & 0.0527 & 0.0010 & $\mathrm{mg} / \mathrm{L}$ & 2 & 19-Jun-2009 & 19-Jun-2009 & SW 846/6020 & \\
\hline Zinc & 0.0048 & 0.0010 & $\mathrm{mg} / \mathrm{L}$ & 2 & 19-Jun-2009 & 19-Jun-2009 & SW 846/6020 & B \\
\hline
\end{tabular}




\section{USACE ERDC-EP-C \\ 3909 Halls Ferry Road \\ Vicksburg, MS 39180-6199}

ERDC - ECB

Project: TVA Fly Ash Project

Reported:

$--,-$

Project Manager: Tony Bednar

03-Sep-2009

DRAFT: SLC MT N2 Hour 96 Rep C

9061701-09 (Water)

\begin{tabular}{|c|c|c|c|c|c|c|c|c|}
\hline & & orting & & & & & & \\
\hline Analyte & Result & Limit & Units & Dilution & Prepared & Analyzed & Method & Notes \\
\hline
\end{tabular}

ERDC- EL-EP-C (Environmental Chemistry Branch)

DRAFT: Miscellaneous Physical/Conventional Chemistry Parameters

\begin{tabular}{lrrrrrrr}
\hline Bromide & $\mathrm{ND}$ & 0.100 & $\mathrm{mg} / \mathrm{L}$ & 1 & $15-J u l-2009$ & $16-\mathrm{Jul}-2009$ & EPA 300.0 \\
Chloride & $\mathbf{3 . 8 6}$ & 1.00 & $\mathrm{mg} / \mathrm{L}$ & 1 & $15-J u l-2009$ & $16-J u l-2009$ & EPA 300.0 \\
Nitrate as N & $\mathbf{0 . 3 7 4}$ & 0.100 & $\mathrm{mg} / \mathrm{L}$ & 1 & $15-J u l-2009$ & $16-J u l-2009$ & EPA 300.0
\end{tabular}




\section{USACE ERDC-EP-C \\ 3909 Halls Ferry Road \\ Vicksburg, MS 39180-6199}

ERDC - ECB

$--,-$
Project: TVA Fly Ash Project

Project Manager: Tony Bednar
Reported:

03-Sep-2009

DRAFT: SLC MT O2 Hour 96 Rep A

9061701-10 (Water)

\begin{tabular}{|c|c|c|c|c|c|c|c|c|}
\hline Analyte & Result & $\begin{array}{r}\text { porting } \\
\text { Limit }\end{array}$ & Units & Dilution & Prepared & Analyzed & Method & Notes \\
\hline
\end{tabular}

ERDC- EL-EP-C (Environmental Chemistry Branch)

DRAFT: Wet Chemistry

\begin{tabular}{llllllll}
\hline Hardness & $\mathbf{1 0 1}$ & 1.32 & $\begin{array}{c}\text { mg equil } \\
\text { CaCO3/ }\end{array}$ & 1 & 25-Jun-2009 & 25-Jun-2009
\end{tabular}

$\mathrm{L}$

DRAFT: Metals by EPA 6000/7000 Series Methods

\begin{tabular}{|c|c|c|c|c|c|c|c|c|}
\hline Mercury & ND & 0.000005 & $\mathrm{mg} / \mathrm{L}$ & 1 & 17-Jun-2009 & 01-Jul-2009 & EPA 7471A & $\mathrm{U}$ \\
\hline Strontium & 0.550 & 0.0200 & $\mathrm{mg} / \mathrm{L}$ & 1 & 25-Jun-2009 & 25-Jun-2009 & EPA 6010B & \\
\hline Aluminum & 0.0733 & 0.200 & $\mathrm{mg} / \mathrm{L}$ & 1 & 25-Jun-2009 & 25-Jun-2009 & SW 846/6010 & $\mathrm{J}$ \\
\hline Calcium & 31.6 & 0.200 & $\mathrm{mg} / \mathrm{L}$ & 1 & 25-Jun-2009 & 25-Jun-2009 & SW 846/6010 & \\
\hline Iron & ND & 0.200 & $\mathrm{mg} / \mathrm{L}$ & 1 & 25-Jun-2009 & 25-Jun-2009 & SW 846/6010 & $\mathrm{U}$ \\
\hline Magnesium & 5.28 & 0.200 & $\mathrm{mg} / \mathrm{L}$ & 1 & 25-Jun-2009 & 25-Jun-2009 & SW 846/6010 & \\
\hline Potassium & 2.03 & 0.200 & $\mathrm{mg} / \mathrm{L}$ & 1 & 25-Jun-2009 & 25-Jun-2009 & SW 846/6010 & \\
\hline Sodium & 4.33 & 0.200 & $\mathrm{mg} / \mathrm{L}$ & 1 & 25-Jun-2009 & 25-Jun-2009 & SW 846/6010 & \\
\hline Antimony & 0.0088 & 0.0010 & $\mathrm{mg} / \mathrm{L}$ & 2 & 19-Jun-2009 & 19-Jun-2009 & SW 846/6020 & \\
\hline Arsenic & 0.0800 & 0.0010 & $\mathrm{mg} / \mathrm{L}$ & 2 & 19-Jun-2009 & 19-Jun-2009 & SW 846/6020 & \\
\hline Barium & 0.109 & 0.0010 & $\mathrm{mg} / \mathrm{L}$ & 2 & 19-Jun-2009 & 19-Jun-2009 & SW 846/6020 & \\
\hline Beryllium & ND & 0.0010 & $\mathrm{mg} / \mathrm{L}$ & 2 & 19-Jun-2009 & 19-Jun-2009 & SW 846/6020 & $\mathrm{U}$ \\
\hline Cadmium & 0.0003 & 0.0010 & $\mathrm{mg} / \mathrm{L}$ & 2 & 19-Jun-2009 & 19-Jun-2009 & SW 846/6020 & $\mathrm{J}$ \\
\hline Chromium & 0.0004 & 0.0010 & $\mathrm{mg} / \mathrm{L}$ & 2 & 19-Jun-2009 & 19-Jun-2009 & SW 846/6020 & $\mathrm{J}$ \\
\hline Cobalt & ND & 0.0010 & $\mathrm{mg} / \mathrm{L}$ & 2 & 19-Jun-2009 & 19-Jun-2009 & SW 846/6020 & $\mathrm{U}$ \\
\hline Copper & 0.0012 & 0.0010 & $\mathrm{mg} / \mathrm{L}$ & 2 & 19-Jun-2009 & 19-Jun-2009 & SW 846/6020 & \\
\hline Lead & ND & 0.0010 & $\mathrm{mg} / \mathrm{L}$ & 2 & 19-Jun-2009 & 19-Jun-2009 & SW 846/6020 & $\mathrm{U}$ \\
\hline Manganese & 0.0114 & 0.0010 & $\mathrm{mg} / \mathrm{L}$ & 2 & 19-Jun-2009 & 19-Jun-2009 & SW 846/6020 & \\
\hline Molybdenum & 0.0725 & 0.0010 & $\mathrm{mg} / \mathrm{L}$ & 2 & 19-Jun-2009 & 19-Jun-2009 & SW 846/6020 & \\
\hline Nickel & 0.0006 & 0.0010 & $\mathrm{mg} / \mathrm{L}$ & 2 & 19-Jun-2009 & 19-Jun-2009 & SW 846/6020 & $\mathrm{J}$ \\
\hline Selenium & 0.0085 & 0.0010 & $\mathrm{mg} / \mathrm{L}$ & 2 & 19-Jun-2009 & 19-Jun-2009 & SW 846/6020 & \\
\hline Silver & ND & 0.0010 & $\mathrm{mg} / \mathrm{L}$ & 2 & 19-Jun-2009 & 19-Jun-2009 & SW 846/6020 & $\mathrm{U}$ \\
\hline Thallium & 0.0008 & 0.0010 & $\mathrm{mg} / \mathrm{L}$ & 2 & 19-Jun-2009 & 19-Jun-2009 & SW 846/6020 & $\mathrm{J}$ \\
\hline Vanadium & 0.0490 & 0.0010 & $\mathrm{mg} / \mathrm{L}$ & 2 & 19-Jun-2009 & 19-Jun-2009 & SW 846/6020 & \\
\hline Zinc & 0.0055 & 0.0010 & $\mathrm{mg} / \mathrm{L}$ & 2 & 19-Jun-2009 & 19-Jun-2009 & SW 846/6020 & B \\
\hline
\end{tabular}




\section{USACE ERDC-EP-C \\ 3909 Halls Ferry Road \\ Vicksburg, MS 39180-6199}

ERDC - ECB

Project: TVA Fly Ash Project

Reported:

Project Manager: Tony Bednar

03-Sep-2009

DRAFT: SLC MT O2 Hour 96 Rep A

9061701-10 (Water)

\begin{tabular}{|c|c|c|c|c|c|c|c|c|}
\hline & & orting & & & & & & \\
\hline Analyte & Result & Limit & Units & Dilution & Prepared & Analyzed & Method & Notes \\
\hline
\end{tabular}

ERDC- EL-EP-C (Environmental Chemistry Branch)

DRAFT: Miscellaneous Physical/Conventional Chemistry Parameters

\begin{tabular}{lrrrrrrr}
\hline Bromide & $\mathrm{ND}$ & 0.100 & $\mathrm{mg} / \mathrm{L}$ & 1 & $15-J u l-2009$ & $16-\mathrm{Jul}-2009$ & EPA 300.0 \\
Chloride & $\mathbf{4 . 0 8}$ & 1.00 & $\mathrm{mg} / \mathrm{L}$ & 1 & $15-J u l-2009$ & $16-J u l-2009$ & EPA 300.0 \\
Nitrate as N & $\mathbf{0 . 3 7 4}$ & 0.100 & $\mathrm{mg} / \mathrm{L}$ & 1 & $15-J u l-2009$ & $16-J u l-2009$ & EPA 300.0
\end{tabular}




\section{USACE ERDC-EP-C \\ 3909 Halls Ferry Road \\ Vicksburg, MS 39180-6199}

ERDC - ECB

$-\cdot$,
Project: TVA Fly Ash Project

Project Manager: Tony Bednar
Reported:

03-Sep-2009

DRAFT: SLC MT O2 Hour 96 Rep B

9061701-11 (Water)

\begin{tabular}{|c|c|c|c|c|c|c|c|c|}
\hline Analyte & Result & $\begin{array}{r}\text { porting } \\
\text { Limit }\end{array}$ & Units & Dilution & Prepared & Analyzed & Method & Notes \\
\hline
\end{tabular}

ERDC- EL-EP-C (Environmental Chemistry Branch)

DRAFT: Wet Chemistry

\begin{tabular}{llllllll}
\hline Hardness & $\mathbf{1 0 1}$ & 1.32 & $\begin{array}{c}\text { mg equil } \\
\text { CaCO3/ }\end{array}$ & 1 & 25-Jun-2009 & 25-Jun-2009
\end{tabular}

$\mathrm{L}$

DRAFT: Metals by EPA 6000/7000 Series Methods

\begin{tabular}{|c|c|c|c|c|c|c|c|c|}
\hline Mercury & ND & 0.000005 & $\mathrm{mg} / \mathrm{L}$ & 1 & 17-Jun-2009 & 01-Jul-2009 & EPA 7471A & $\mathrm{U}$ \\
\hline Strontium & 0.552 & 0.0200 & $\mathrm{mg} / \mathrm{L}$ & 1 & 25-Jun-2009 & 25-Jun-2009 & EPA 6010B & \\
\hline Aluminum & 0.0732 & 0.200 & $\mathrm{mg} / \mathrm{L}$ & 1 & 25-Jun-2009 & 25-Jun-2009 & SW 846/6010 & $\mathrm{J}$ \\
\hline Calcium & 31.6 & 0.200 & $\mathrm{mg} / \mathrm{L}$ & 1 & 25-Jun-2009 & 25-Jun-2009 & SW 846/6010 & \\
\hline Iron & ND & 0.200 & $\mathrm{mg} / \mathrm{L}$ & 1 & 25-Jun-2009 & 25-Jun-2009 & SW 846/6010 & $\mathrm{U}$ \\
\hline Magnesium & 5.34 & 0.200 & $\mathrm{mg} / \mathrm{L}$ & 1 & 25-Jun-2009 & 25-Jun-2009 & SW 846/6010 & \\
\hline Potassium & 1.98 & 0.200 & $\mathrm{mg} / \mathrm{L}$ & 1 & 25-Jun-2009 & 25-Jun-2009 & SW 846/6010 & \\
\hline Sodium & 4.29 & 0.200 & $\mathrm{mg} / \mathrm{L}$ & 1 & 25-Jun-2009 & 25-Jun-2009 & SW 846/6010 & \\
\hline Antimony & 0.0088 & 0.0010 & $\mathrm{mg} / \mathrm{L}$ & 2 & 19-Jun-2009 & 19-Jun-2009 & SW 846/6020 & \\
\hline Arsenic & 0.0834 & 0.0010 & $\mathrm{mg} / \mathrm{L}$ & 2 & 19-Jun-2009 & 19-Jun-2009 & SW 846/6020 & \\
\hline Barium & 0.110 & 0.0010 & $\mathrm{mg} / \mathrm{L}$ & 2 & 19-Jun-2009 & 19-Jun-2009 & SW 846/6020 & \\
\hline Beryllium & ND & 0.0010 & $\mathrm{mg} / \mathrm{L}$ & 2 & 19-Jun-2009 & 19-Jun-2009 & SW 846/6020 & $\mathrm{U}$ \\
\hline Cadmium & 0.0003 & 0.0010 & $\mathrm{mg} / \mathrm{L}$ & 2 & 19-Jun-2009 & 19-Jun-2009 & SW 846/6020 & $\mathrm{J}$ \\
\hline Chromium & 0.0004 & 0.0010 & $\mathrm{mg} / \mathrm{L}$ & 2 & 19-Jun-2009 & 19-Jun-2009 & SW 846/6020 & $\mathrm{J}$ \\
\hline Cobalt & ND & 0.0010 & $\mathrm{mg} / \mathrm{L}$ & 2 & 19-Jun-2009 & 19-Jun-2009 & SW 846/6020 & $\mathrm{U}$ \\
\hline Copper & 0.0012 & 0.0010 & $\mathrm{mg} / \mathrm{L}$ & 2 & 19-Jun-2009 & 19-Jun-2009 & SW 846/6020 & \\
\hline Lead & ND & 0.0010 & $\mathrm{mg} / \mathrm{L}$ & 2 & 19-Jun-2009 & 19-Jun-2009 & SW 846/6020 & $\mathrm{U}$ \\
\hline Manganese & 0.0102 & 0.0010 & $\mathrm{mg} / \mathrm{L}$ & 2 & 19-Jun-2009 & 19-Jun-2009 & SW 846/6020 & \\
\hline Molybdenum & 0.0720 & 0.0010 & $\mathrm{mg} / \mathrm{L}$ & 2 & 19-Jun-2009 & 19-Jun-2009 & SW 846/6020 & \\
\hline Nickel & 0.00064 & 0.0010 & $\mathrm{mg} / \mathrm{L}$ & 2 & 19-Jun-2009 & 19-Jun-2009 & SW 846/6020 & $\mathrm{J}$ \\
\hline Selenium & 0.0098 & 0.0010 & $\mathrm{mg} / \mathrm{L}$ & 2 & 19-Jun-2009 & 19-Jun-2009 & SW 846/6020 & \\
\hline Silver & ND & 0.0010 & $\mathrm{mg} / \mathrm{L}$ & 2 & 19-Jun-2009 & 19-Jun-2009 & SW 846/6020 & $\mathrm{U}$ \\
\hline Thallium & 0.0009 & 0.0010 & $\mathrm{mg} / \mathrm{L}$ & 2 & 19-Jun-2009 & 19-Jun-2009 & SW 846/6020 & $\mathrm{J}$ \\
\hline Vanadium & 0.0527 & 0.0010 & $\mathrm{mg} / \mathrm{L}$ & 2 & 19-Jun-2009 & 19-Jun-2009 & SW 846/6020 & \\
\hline Zinc & 0.0057 & 0.0010 & $\mathrm{mg} / \mathrm{L}$ & 2 & 19-Jun-2009 & 19-Jun-2009 & SW 846/6020 & B \\
\hline
\end{tabular}




\section{USACE ERDC-EP-C \\ 3909 Halls Ferry Road \\ Vicksburg, MS 39180-6199}

ERDC - ECB

Project: TVA Fly Ash Project

Reported:

Project Manager: Tony Bednar

03-Sep-2009

DRAFT: SLC MT O2 Hour 96 Rep B

9061701-11 (Water)

\begin{tabular}{|c|c|c|c|c|c|c|c|c|}
\hline & & orting & & & & & & \\
\hline Analyte & Result & Limit & Units & Dilution & Prepared & Analyzed & Method & Notes \\
\hline
\end{tabular}

ERDC- EL-EP-C (Environmental Chemistry Branch)

DRAFT: Miscellaneous Physical/Conventional Chemistry Parameters

\begin{tabular}{lrrrrrrr}
\hline Bromide & $\mathrm{ND}$ & 0.100 & $\mathrm{mg} / \mathrm{L}$ & 1 & $15-J u l-2009$ & $16-\mathrm{Jul}-2009$ & EPA 300.0 \\
Chloride & $\mathbf{3 . 9 7}$ & 1.00 & $\mathrm{mg} / \mathrm{L}$ & 1 & $15-J u l-2009$ & $16-J u l-2009$ & EPA 300.0 \\
Nitrate as N & $\mathbf{0 . 3 7 7}$ & 0.100 & $\mathrm{mg} / \mathrm{L}$ & 1 & $15-J u l-2009$ & $16-J u l-2009$ & EPA 300.0
\end{tabular}




\section{USACE ERDC-EP-C \\ 3909 Halls Ferry Road \\ Vicksburg, MS 39180-6199}

ERDC - ECB

$-\cdot$,
Project: TVA Fly Ash Project

Project Manager: Tony Bednar
Reported:

03-Sep-2009

DRAFT: SLC MT O2 Hour 96 Rep C

9061701-12 (Water)

\begin{tabular}{|c|c|c|c|c|c|c|c|c|}
\hline Analyte & Result & $\begin{array}{r}\text { porting } \\
\text { Limit }\end{array}$ & Units & Dilution & Prepared & Analyzed & Method & Notes \\
\hline
\end{tabular}

ERDC- EL-EP-C (Environmental Chemistry Branch)

DRAFT: Wet Chemistry

\begin{tabular}{llllllll}
\hline Hardness & $\mathbf{1 0 2}$ & 1.32 & $\begin{array}{c}\text { mg equil } \\
\text { CaCO3/ }\end{array}$ & 1 & 25-Jun-2009 & 25-Jun-2009
\end{tabular}

$\mathrm{L}$

DRAFT: Metals by EPA 6000/7000 Series Methods

\begin{tabular}{|c|c|c|c|c|c|c|c|c|}
\hline Mercury & ND & 0.000005 & $\mathrm{mg} / \mathrm{L}$ & 1 & 17-Jun-2009 & 01-Jul-2009 & EPA 7471A & $\mathrm{U}$ \\
\hline Strontium & 0.555 & 0.0200 & $\mathrm{mg} / \mathrm{L}$ & 1 & 25-Jun-2009 & 25-Jun-2009 & EPA 6010B & \\
\hline Aluminum & 0.0652 & 0.200 & $\mathrm{mg} / \mathrm{L}$ & 1 & 25-Jun-2009 & 25-Jun-2009 & SW 846/6010 & $\mathrm{J}$ \\
\hline Calcium & 31.9 & 0.200 & $\mathrm{mg} / \mathrm{L}$ & 1 & 25-Jun-2009 & 25-Jun-2009 & SW 846/6010 & \\
\hline Iron & ND & 0.200 & $\mathrm{mg} / \mathrm{L}$ & 1 & 25-Jun-2009 & 25-Jun-2009 & SW 846/6010 & $\mathrm{U}$ \\
\hline Magnesium & 5.32 & 0.200 & $\mathrm{mg} / \mathrm{L}$ & 1 & 25-Jun-2009 & 25-Jun-2009 & SW 846/6010 & \\
\hline Potassium & 2.00 & 0.200 & $\mathrm{mg} / \mathrm{L}$ & 1 & 25-Jun-2009 & 25-Jun-2009 & SW 846/6010 & \\
\hline Sodium & 4.23 & 0.200 & $\mathrm{mg} / \mathrm{L}$ & 1 & 25-Jun-2009 & 25-Jun-2009 & SW 846/6010 & \\
\hline Antimony & 0.0090 & 0.0010 & $\mathrm{mg} / \mathrm{L}$ & 2 & 19-Jun-2009 & 19-Jun-2009 & SW 846/6020 & \\
\hline Arsenic & 0.0824 & 0.0010 & $\mathrm{mg} / \mathrm{L}$ & 2 & 19-Jun-2009 & 19-Jun-2009 & SW 846/6020 & \\
\hline Barium & 0.111 & 0.0010 & $\mathrm{mg} / \mathrm{L}$ & 2 & 19-Jun-2009 & 19-Jun-2009 & SW 846/6020 & \\
\hline Beryllium & ND & 0.0010 & $\mathrm{mg} / \mathrm{L}$ & 2 & 19-Jun-2009 & 19-Jun-2009 & SW 846/6020 & $\mathrm{U}$ \\
\hline Cadmium & 0.0003 & 0.0010 & $\mathrm{mg} / \mathrm{L}$ & 2 & 19-Jun-2009 & 19-Jun-2009 & SW 846/6020 & $\mathrm{J}$ \\
\hline Chromium & 0.0005 & 0.0010 & $\mathrm{mg} / \mathrm{L}$ & 2 & 19-Jun-2009 & 19-Jun-2009 & SW 846/6020 & $\mathrm{J}$ \\
\hline Cobalt & ND & 0.0010 & $\mathrm{mg} / \mathrm{L}$ & 2 & 19-Jun-2009 & 19-Jun-2009 & SW 846/6020 & $\mathrm{U}$ \\
\hline Copper & 0.0016 & 0.0010 & $\mathrm{mg} / \mathrm{L}$ & 2 & 19-Jun-2009 & 19-Jun-2009 & SW 846/6020 & \\
\hline Lead & ND & 0.0010 & $\mathrm{mg} / \mathrm{L}$ & 2 & 19-Jun-2009 & 19-Jun-2009 & SW 846/6020 & $\mathrm{U}$ \\
\hline Manganese & 0.0105 & 0.0010 & $\mathrm{mg} / \mathrm{L}$ & 2 & 19-Jun-2009 & 19-Jun-2009 & SW 846/6020 & \\
\hline Molybdenum & 0.0727 & 0.0010 & $\mathrm{mg} / \mathrm{L}$ & 2 & 19-Jun-2009 & 19-Jun-2009 & SW 846/6020 & \\
\hline Nickel & 0.0008 & 0.0010 & $\mathrm{mg} / \mathrm{L}$ & 2 & 19-Jun-2009 & 19-Jun-2009 & SW 846/6020 & $\mathrm{J}$ \\
\hline Selenium & 0.0098 & 0.0010 & $\mathrm{mg} / \mathrm{L}$ & 2 & 19-Jun-2009 & 19-Jun-2009 & SW 846/6020 & \\
\hline Silver & ND & 0.0010 & $\mathrm{mg} / \mathrm{L}$ & 2 & 19-Jun-2009 & 19-Jun-2009 & SW 846/6020 & $\mathrm{U}$ \\
\hline Thallium & 0.0009 & 0.0010 & $\mathrm{mg} / \mathrm{L}$ & 2 & 19-Jun-2009 & 19-Jun-2009 & SW 846/6020 & $\mathrm{J}$ \\
\hline Vanadium & 0.0515 & 0.0010 & $\mathrm{mg} / \mathrm{L}$ & 2 & 19-Jun-2009 & 19-Jun-2009 & SW 846/6020 & \\
\hline Zinc & 0.0052 & 0.0010 & $\mathrm{mg} / \mathrm{L}$ & 2 & 19-Jun-2009 & 19-Jun-2009 & SW 846/6020 & B \\
\hline
\end{tabular}




\section{USACE ERDC-EP-C \\ 3909 Halls Ferry Road \\ Vicksburg, MS 39180-6199}

ERDC - ECB

Project: TVA Fly Ash Project

Reported:

$--,-$

Project Manager: Tony Bednar

03-Sep-2009

DRAFT: SLC MT O2 Hour 96 Rep C

9061701-12 (Water)

\begin{tabular}{|c|c|c|c|c|c|c|c|c|}
\hline & & orting & & & & & & \\
\hline Analyte & Result & Limit & Units & Dilution & Prepared & Analyzed & Method & Notes \\
\hline
\end{tabular}

ERDC- EL-EP-C (Environmental Chemistry Branch)

DRAFT: Miscellaneous Physical/Conventional Chemistry Parameters

\begin{tabular}{lrrrrrrr}
\hline Bromide & $\mathrm{ND}$ & 0.100 & $\mathrm{mg} / \mathrm{L}$ & 1 & $15-J u l-2009$ & $16-\mathrm{Jul}-2009$ & EPA 300.0 \\
Chloride & $\mathbf{3 . 9 3}$ & 1.00 & $\mathrm{mg} / \mathrm{L}$ & 1 & $15-J u l-2009$ & $16-J u l-2009$ & EPA 300.0 \\
Nitrate as N & $\mathbf{0 . 3 8 9}$ & 0.100 & $\mathrm{mg} / \mathrm{L}$ & 1 & $15-J u l-2009$ & $16-J u l-2009$ & EPA 300.0
\end{tabular}




\section{USACE ERDC-EP-C \\ 3909 Halls Ferry Road \\ Vicksburg, MS 39180-6199}

ERDC - ECB

$-\cdot$,
Project: TVA Fly Ash Project

Project Manager: Tony Bednar
Reported:

03-Sep-2009

DRAFT: EMR MT N2 Hour 96 Rep A - Total Metals

9061701-13 (Water)

\begin{tabular}{|c|c|c|c|c|c|c|c|c|}
\hline Analyte & Result & $\begin{array}{r}\text { porting } \\
\text { Limit }\end{array}$ & Units & Dilution & Prepared & Analyzed & Method & Notes \\
\hline
\end{tabular}

ERDC- EL-EP-C (Environmental Chemistry Branch)

DRAFT: Wet Chemistry

\begin{tabular}{llllllll}
\hline Hardness & $\mathbf{9 1 . 5}$ & 1.32 & $\begin{array}{c}\text { mg equil } \\
\text { CaCO3/ }\end{array}$ & 4 & 25-Jun-2009 & 25-Jun-2009
\end{tabular}

$\mathrm{L}$

DRAFT: Metals by EPA 6000/7000 Series Methods

\begin{tabular}{|c|c|c|c|c|c|c|c|c|}
\hline Mercury & 0.000177 & 0.000005 & $\mathrm{mg} / \mathrm{L}$ & 2 & 24-Jul-2009 & 24-Jul-2009 & EPA 7471A & \\
\hline Strontium & 0.707 & 0.0800 & $\mathrm{mg} / \mathrm{L}$ & 4 & 25-Jun-2009 & 25-Jun-2009 & EPA 6010B & \\
\hline Aluminum & 55.8 & 0.200 & $\mathrm{mg} / \mathrm{L}$ & 4 & 25-Jun-2009 & 25-Jun-2009 & SW 846/6010 & \\
\hline Calcium & 24.6 & 0.200 & $\mathrm{mg} / \mathrm{L}$ & 4 & 25-Jun-2009 & 25-Jun-2009 & SW 846/6010 & B \\
\hline Iron & 21.9 & 0.200 & $\mathrm{mg} / \mathrm{L}$ & 4 & 25-Jun-2009 & 25-Jun-2009 & SW 846/6010 & \\
\hline Magnesium & 7.33 & 0.200 & $\mathrm{mg} / \mathrm{L}$ & 4 & 25-Jun-2009 & 25-Jun-2009 & SW 846/6010 & \\
\hline Potassium & 11.4 & 0.200 & $\mathrm{mg} / \mathrm{L}$ & 4 & 25-Jun-2009 & 25-Jun-2009 & SW 846/6010 & B \\
\hline Sodium & 4.58 & 0.200 & $\mathrm{mg} / \mathrm{L}$ & 4 & 25-Jun-2009 & 25-Jun-2009 & SW 846/6010 & B \\
\hline Antimony & 0.0149 & 0.0020 & $\mathrm{mg} / \mathrm{L}$ & 4 & 19-Jun-2009 & 19-Jun-2009 & SW 846/6020 & B \\
\hline Arsenic & 0.182 & 0.0020 & $\mathrm{mg} / \mathrm{L}$ & 4 & 19-Jun-2009 & 19-Jun-2009 & SW 846/6020 & \\
\hline Barium & 1.02 & 0.0020 & $\mathrm{mg} / \mathrm{L}$ & 4 & 19-Jun-2009 & 19-Jun-2009 & SW 846/6020 & \\
\hline Beryllium & 0.0087 & 0.0020 & $\mathrm{mg} / \mathrm{L}$ & 4 & 19-Jun-2009 & 19-Jun-2009 & SW 846/6020 & \\
\hline Cadmium & 0.0013 & 0.0020 & $\mathrm{mg} / \mathrm{L}$ & 4 & 19-Jun-2009 & 19-Jun-2009 & SW 846/6020 & $\mathrm{J}$ \\
\hline Chromium & 0.0524 & 0.0020 & $\mathrm{mg} / \mathrm{L}$ & 4 & 19-Jun-2009 & 19-Jun-2009 & SW 846/6020 & \\
\hline Cobalt & 0.0358 & 0.0020 & $\mathrm{mg} / \mathrm{L}$ & 4 & 19-Jun-2009 & 19-Jun-2009 & SW 846/6020 & \\
\hline Copper & 0.120 & 0.0020 & $\mathrm{mg} / \mathrm{L}$ & 4 & 19-Jun-2009 & 19-Jun-2009 & SW 846/6020 & \\
\hline Lead & 0.0975 & 0.0020 & $\mathrm{mg} / \mathrm{L}$ & 4 & 19-Jun-2009 & 19-Jun-2009 & SW 846/6020 & \\
\hline Manganese & 0.124 & 0.0020 & $\mathrm{mg} / \mathrm{L}$ & 4 & 19-Jun-2009 & 19-Jun-2009 & SW 846/6020 & \\
\hline Molybdenum & 0.0095 & 0.0020 & $\mathrm{mg} / \mathrm{L}$ & 4 & 19-Jun-2009 & 19-Jun-2009 & SW 846/6020 & \\
\hline Nickel & 0.0701 & 0.0020 & $\mathrm{mg} / \mathrm{L}$ & 4 & 19-Jun-2009 & 19-Jun-2009 & SW 846/6020 & \\
\hline Selenium & 0.0098 & 0.0020 & $\mathrm{mg} / \mathrm{L}$ & 4 & 19-Jun-2009 & 19-Jun-2009 & SW 846/6020 & \\
\hline Silver & 0.0010 & 0.0020 & $\mathrm{mg} / \mathrm{L}$ & 4 & 19-Jun-2009 & 19-Jun-2009 & SW 846/6020 & $\mathrm{J}$ \\
\hline Thallium & 0.0050 & 0.0020 & $\mathrm{mg} / \mathrm{L}$ & 4 & 19-Jun-2009 & 19-Jun-2009 & SW 846/6020 & \\
\hline Vanadium & 0.184 & 0.0020 & $\mathrm{mg} / \mathrm{L}$ & 4 & 19-Jun-2009 & 19-Jun-2009 & SW 846/6020 & \\
\hline Zinc & 0.146 & 0.0020 & $\mathrm{mg} / \mathrm{L}$ & 4 & 19-Jun-2009 & 19-Jun-2009 & SW 846/6020 & B \\
\hline
\end{tabular}




\section{USACE ERDC-EP-C \\ 3909 Halls Ferry Road \\ Vicksburg, MS 39180-6199}

ERDC - ECB

$-\cdot$,
Project: TVA Fly Ash Project

Project Manager: Tony Bednar
Reported:

03-Sep-2009

DRAFT: EMR MT N2 Hour 96 Rep B - Total Metals

9061701-14 (Water)

\begin{tabular}{|c|c|c|c|c|c|c|c|c|}
\hline Analyte & Result & $\begin{array}{r}\text { porting } \\
\text { Limit }\end{array}$ & Units & Dilution & Prepared & Analyzed & Method & Notes \\
\hline
\end{tabular}

ERDC- EL-EP-C (Environmental Chemistry Branch)

DRAFT: Wet Chemistry

\begin{tabular}{llllllll}
\hline Hardness & $\mathbf{7 0 . 2}$ & 1.32 & $\begin{array}{c}\text { mg equil } \\
\text { CaCO3/ }\end{array}$ & 4 & 25-Jun-2009 & 25-Jun-2009
\end{tabular}

$\mathrm{L}$

DRAFT: Metals by EPA 6000/7000 Series Methods

\begin{tabular}{|c|c|c|c|c|c|c|c|c|}
\hline Mercury & 0.000163 & 0.000005 & $\mathrm{mg} / \mathrm{L}$ & 2 & 24-Jul-2009 & 24-Jul-2009 & EPA 7471A & \\
\hline Strontium & 0.477 & 0.0800 & $\mathrm{mg} / \mathrm{L}$ & 4 & 25-Jun-2009 & 25-Jun-2009 & EPA 6010B & \\
\hline Aluminum & 32.9 & 0.200 & $\mathrm{mg} / \mathrm{L}$ & 4 & 25-Jun-2009 & 25-Jun-2009 & SW 846/6010 & \\
\hline Calcium & 19.4 & 0.200 & $\mathrm{mg} / \mathrm{L}$ & 4 & 25-Jun-2009 & 25-Jun-2009 & SW 846/6010 & B \\
\hline Iron & 13.5 & 0.200 & $\mathrm{mg} / \mathrm{L}$ & 4 & 25-Jun-2009 & 25-Jun-2009 & SW 846/6010 & \\
\hline Magnesium & 5.28 & 0.200 & $\mathrm{mg} / \mathrm{L}$ & 4 & 25-Jun-2009 & 25-Jun-2009 & SW 846/6010 & \\
\hline Potassium & 7.50 & 0.200 & $\mathrm{mg} / \mathrm{L}$ & 4 & 25-Jun-2009 & 25-Jun-2009 & SW 846/6010 & B \\
\hline Sodium & 4.10 & 0.200 & $\mathrm{mg} / \mathrm{L}$ & 4 & 25-Jun-2009 & 25-Jun-2009 & SW 846/6010 & B \\
\hline Antimony & 0.0051 & 0.0020 & $\mathrm{mg} / \mathrm{L}$ & 4 & 19-Jun-2009 & 19-Jun-2009 & SW 846/6020 & B \\
\hline Arsenic & 0.140 & 0.0020 & $\mathrm{mg} / \mathrm{L}$ & 4 & 19-Jun-2009 & 19-Jun-2009 & SW 846/6020 & \\
\hline Barium & 0.661 & 0.0020 & $\mathrm{mg} / \mathrm{L}$ & 4 & 19-Jun-2009 & 19-Jun-2009 & SW 846/6020 & \\
\hline Beryllium & 0.0058 & 0.0020 & $\mathrm{mg} / \mathrm{L}$ & 4 & 19-Jun-2009 & 19-Jun-2009 & SW 846/6020 & \\
\hline Cadmium & 0.0018 & 0.0020 & $\mathrm{mg} / \mathrm{L}$ & 4 & 19-Jun-2009 & 19-Jun-2009 & SW 846/6020 & J \\
\hline Chromium & 0.0419 & 0.0020 & $\mathrm{mg} / \mathrm{L}$ & 4 & 19-Jun-2009 & 19-Jun-2009 & SW 846/6020 & \\
\hline Cobalt & 0.0252 & 0.0020 & $\mathrm{mg} / \mathrm{L}$ & 4 & 19-Jun-2009 & 19-Jun-2009 & SW 846/6020 & \\
\hline Copper & 0.0852 & 0.0020 & $\mathrm{mg} / \mathrm{L}$ & 4 & 19-Jun-2009 & 19-Jun-2009 & SW 846/6020 & \\
\hline Lead & 0.0619 & 0.0020 & $\mathrm{mg} / \mathrm{L}$ & 4 & 19-Jun-2009 & 19-Jun-2009 & SW 846/6020 & \\
\hline Manganese & 0.0962 & 0.0020 & $\mathrm{mg} / \mathrm{L}$ & 4 & 19-Jun-2009 & 19-Jun-2009 & SW 846/6020 & \\
\hline Molybdenum & 0.0071 & 0.0020 & $\mathrm{mg} / \mathrm{L}$ & 4 & 19-Jun-2009 & 19-Jun-2009 & SW 846/6020 & \\
\hline Nickel & 0.0488 & 0.0020 & $\mathrm{mg} / \mathrm{L}$ & 4 & 19-Jun-2009 & 19-Jun-2009 & SW 846/6020 & \\
\hline Selenium & 0.0088 & 0.0020 & $\mathrm{mg} / \mathrm{L}$ & 4 & 19-Jun-2009 & 19-Jun-2009 & SW 846/6020 & \\
\hline Silver & 0.0005 & 0.0020 & $\mathrm{mg} / \mathrm{L}$ & 4 & 19-Jun-2009 & 19-Jun-2009 & SW 846/6020 & J \\
\hline Thallium & 0.0034 & 0.0020 & $\mathrm{mg} / \mathrm{L}$ & 4 & 19-Jun-2009 & 19-Jun-2009 & SW 846/6020 & \\
\hline Vanadium & 0.144 & 0.0020 & $\mathrm{mg} / \mathrm{L}$ & 4 & 19-Jun-2009 & 19-Jun-2009 & SW 846/6020 & \\
\hline Zinc & 0.108 & 0.0020 & $\mathrm{mg} / \mathrm{L}$ & 4 & 19-Jun-2009 & 19-Jun-2009 & SW 846/6020 & B \\
\hline
\end{tabular}




\section{USACE ERDC-EP-C \\ 3909 Halls Ferry Road \\ Vicksburg, MS 39180-6199}

ERDC - ECB

$-\cdot$,
Project: TVA Fly Ash Project

Project Manager: Tony Bednar
Reported:

03-Sep-2009

DRAFT: EMR MT N2 Hour 96 Rep C - Total Metals

9061701-15 (Water)

\begin{tabular}{|c|c|c|c|c|c|c|c|c|}
\hline Analyte & Result & $\begin{array}{r}\text { porting } \\
\text { Limit }\end{array}$ & Units & Dilution & Prepared & Analyzed & Method & Notes \\
\hline
\end{tabular}

ERDC- EL-EP-C (Environmental Chemistry Branch)

DRAFT: Wet Chemistry

\begin{tabular}{llllllll}
\hline Hardness & $\mathbf{7 6 . 6}$ & 1.32 & $\begin{array}{c}\text { mg equil } \\
\text { CaCO3/ }\end{array}$ & 4 & 25-Jun-2009 & 25-Jun-2009
\end{tabular}

$\mathrm{L}$

DRAFT: Metals by EPA 6000/7000 Series Methods

\begin{tabular}{|c|c|c|c|c|c|c|c|c|}
\hline Mercury & 0.000176 & 0.000005 & $\mathrm{mg} / \mathrm{L}$ & 2 & 24-Jul-2009 & 24-Jul-2009 & EPA 7471A & \\
\hline Strontium & 0.569 & 0.0800 & $\mathrm{mg} / \mathrm{L}$ & 4 & 25-Jun-2009 & 25-Jun-2009 & EPA 6010B & \\
\hline Aluminum & 40.7 & 0.200 & $\mathrm{mg} / \mathrm{L}$ & 4 & 25-Jun-2009 & 25-Jun-2009 & SW 846/6010 & \\
\hline Calcium & 21.1 & 0.200 & $\mathrm{mg} / \mathrm{L}$ & 4 & 25-Jun-2009 & 25-Jun-2009 & SW 846/6010 & B \\
\hline Iron & 16.9 & 0.200 & $\mathrm{mg} / \mathrm{L}$ & 4 & 25-Jun-2009 & 25-Jun-2009 & SW 846/6010 & \\
\hline Magnesium & 5.82 & 0.200 & $\mathrm{mg} / \mathrm{L}$ & 4 & 25-Jun-2009 & 25-Jun-2009 & SW 846/6010 & \\
\hline Potassium & 8.79 & 0.200 & $\mathrm{mg} / \mathrm{L}$ & 4 & 25-Jun-2009 & 25-Jun-2009 & SW 846/6010 & B \\
\hline Sodium & 4.09 & 0.200 & $\mathrm{mg} / \mathrm{L}$ & 4 & 25-Jun-2009 & 25-Jun-2009 & SW 846/6010 & B \\
\hline Antimony & 0.0112 & 0.0020 & $\mathrm{mg} / \mathrm{L}$ & 4 & 19-Jun-2009 & 19-Jun-2009 & SW 846/6020 & B \\
\hline Arsenic & 0.164 & 0.0020 & $\mathrm{mg} / \mathrm{L}$ & 4 & 19-Jun-2009 & 19-Jun-2009 & SW 846/6020 & \\
\hline Barium & 0.824 & 0.0020 & $\mathrm{mg} / \mathrm{L}$ & 4 & 19-Jun-2009 & 19-Jun-2009 & SW 846/6020 & \\
\hline Beryllium & 0.0070 & 0.0020 & $\mathrm{mg} / \mathrm{L}$ & 4 & 19-Jun-2009 & 19-Jun-2009 & SW 846/6020 & \\
\hline Cadmium & 0.0011 & 0.0020 & $\mathrm{mg} / \mathrm{L}$ & 4 & 19-Jun-2009 & 19-Jun-2009 & SW 846/6020 & J \\
\hline Chromium & 0.0485 & 0.0020 & $\mathrm{mg} / \mathrm{L}$ & 4 & 19-Jun-2009 & 19-Jun-2009 & SW 846/6020 & \\
\hline Cobalt & 0.0315 & 0.0020 & $\mathrm{mg} / \mathrm{L}$ & 4 & 19-Jun-2009 & 19-Jun-2009 & SW 846/6020 & \\
\hline Copper & 0.103 & 0.0020 & $\mathrm{mg} / \mathrm{L}$ & 4 & 19-Jun-2009 & 19-Jun-2009 & SW 846/6020 & \\
\hline Lead & 0.0792 & 0.0020 & $\mathrm{mg} / \mathrm{L}$ & 4 & 19-Jun-2009 & 19-Jun-2009 & SW 846/6020 & \\
\hline Manganese & 0.111 & 0.0020 & $\mathrm{mg} / \mathrm{L}$ & 4 & 19-Jun-2009 & 19-Jun-2009 & SW 846/6020 & \\
\hline Molybdenum & 0.0082 & 0.0020 & $\mathrm{mg} / \mathrm{L}$ & 4 & 19-Jun-2009 & 19-Jun-2009 & SW 846/6020 & \\
\hline Nickel & 0.0617 & 0.0020 & $\mathrm{mg} / \mathrm{L}$ & 4 & 19-Jun-2009 & 19-Jun-2009 & SW 846/6020 & \\
\hline Selenium & 0.0093 & 0.0020 & $\mathrm{mg} / \mathrm{L}$ & 4 & 19-Jun-2009 & 19-Jun-2009 & SW 846/6020 & \\
\hline Silver & 0.0008 & 0.0020 & $\mathrm{mg} / \mathrm{L}$ & 4 & 19-Jun-2009 & 19-Jun-2009 & SW 846/6020 & J \\
\hline Thallium & 0.0041 & 0.0020 & $\mathrm{mg} / \mathrm{L}$ & 4 & 19-Jun-2009 & 19-Jun-2009 & SW 846/6020 & \\
\hline Vanadium & 0.170 & 0.0020 & $\mathrm{mg} / \mathrm{L}$ & 4 & 19-Jun-2009 & 19-Jun-2009 & SW 846/6020 & \\
\hline Zinc & 0.130 & 0.0020 & $\mathrm{mg} / \mathrm{L}$ & 4 & 19-Jun-2009 & 19-Jun-2009 & SW 846/6020 & B \\
\hline
\end{tabular}




\section{USACE ERDC-EP-C \\ 3909 Halls Ferry Road \\ Vicksburg, MS 39180-6199}

ERDC - ECB

$--,-$
Project: TVA Fly Ash Project

Project Manager: Tony Bednar
Reported:

03-Sep-2009

DRAFT: EMR MT O2 Hour 96 Rep A - Total Metals

9061701-16 (Water)

\begin{tabular}{|c|c|c|c|c|c|c|c|c|}
\hline Analyte & Result & $\begin{array}{r}\text { porting } \\
\text { Limit }\end{array}$ & Units & Dilution & Prepared & Analyzed & Method & Notes \\
\hline
\end{tabular}

ERDC- EL-EP-C (Environmental Chemistry Branch)

DRAFT: Wet Chemistry

\begin{tabular}{llllllll}
\hline Hardness & $\mathbf{6 7 . 6}$ & 1.32 & $\begin{array}{c}\mathrm{mg} \text { equil } \\
\text { CaCO3/ }\end{array}$ & 4 & 25-Jun-2009 & 25-Jun-2009
\end{tabular}

$\mathrm{L}$

DRAFT: Metals by EPA 6000/7000 Series Methods

\begin{tabular}{|c|c|c|c|c|c|c|c|c|}
\hline Mercury & 0.000127 & 0.000005 & $\mathrm{mg} / \mathrm{L}$ & 2 & 24-Jul-2009 & 24-Jul-2009 & EPA 7471A & \\
\hline Strontium & 0.462 & 0.0800 & $\mathrm{mg} / \mathrm{L}$ & 4 & 25-Jun-2009 & 25-Jun-2009 & EPA 6010B & \\
\hline Aluminum & 32.7 & 0.200 & $\mathrm{mg} / \mathrm{L}$ & 4 & 25-Jun-2009 & 25-Jun-2009 & SW 846/6010 & \\
\hline Calcium & 18.6 & 0.200 & $\mathrm{mg} / \mathrm{L}$ & 4 & 25-Jun-2009 & 25-Jun-2009 & SW 846/6010 & B \\
\hline Iron & 13.2 & 0.200 & $\mathrm{mg} / \mathrm{L}$ & 4 & 25-Jun-2009 & 25-Jun-2009 & SW 846/6010 & \\
\hline Magnesium & 5.15 & 0.200 & $\mathrm{mg} / \mathrm{L}$ & 4 & 25-Jun-2009 & 25-Jun-2009 & SW 846/6010 & \\
\hline Potassium & 7.32 & 0.200 & $\mathrm{mg} / \mathrm{L}$ & 4 & 25-Jun-2009 & 25-Jun-2009 & SW 846/6010 & B \\
\hline Sodium & 3.71 & 0.200 & $\mathrm{mg} / \mathrm{L}$ & 4 & 25-Jun-2009 & 25-Jun-2009 & SW 846/6010 & B \\
\hline Antimony & 0.0048 & 0.0020 & $\mathrm{mg} / \mathrm{L}$ & 4 & 19-Jun-2009 & 19-Jun-2009 & SW 846/6020 & B \\
\hline Arsenic & 0.130 & 0.0020 & $\mathrm{mg} / \mathrm{L}$ & 4 & 19-Jun-2009 & 19-Jun-2009 & SW 846/6020 & \\
\hline Barium & 0.668 & 0.0020 & $\mathrm{mg} / \mathrm{L}$ & 4 & 19-Jun-2009 & 19-Jun-2009 & SW 846/6020 & \\
\hline Beryllium & 0.0060 & 0.0020 & $\mathrm{mg} / \mathrm{L}$ & 4 & 19-Jun-2009 & 19-Jun-2009 & SW 846/6020 & \\
\hline Cadmium & 0.0010 & 0.0020 & $\mathrm{mg} / \mathrm{L}$ & 4 & 19-Jun-2009 & 19-Jun-2009 & SW 846/6020 & $\mathrm{J}$ \\
\hline Chromium & 0.0434 & 0.0020 & $\mathrm{mg} / \mathrm{L}$ & 4 & 19-Jun-2009 & 19-Jun-2009 & SW 846/6020 & \\
\hline Cobalt & 0.0256 & 0.0020 & $\mathrm{mg} / \mathrm{L}$ & 4 & 19-Jun-2009 & 19-Jun-2009 & SW 846/6020 & \\
\hline Copper & 0.0859 & 0.0020 & $\mathrm{mg} / \mathrm{L}$ & 4 & 19-Jun-2009 & 19-Jun-2009 & SW 846/6020 & \\
\hline Lead & 0.0623 & 0.0020 & $\mathrm{mg} / \mathrm{L}$ & 4 & 19-Jun-2009 & 19-Jun-2009 & SW 846/6020 & \\
\hline Manganese & 0.0926 & 0.0020 & $\mathrm{mg} / \mathrm{L}$ & 4 & 19-Jun-2009 & 19-Jun-2009 & SW 846/6020 & \\
\hline Molybdenum & 0.0067 & 0.0020 & $\mathrm{mg} / \mathrm{L}$ & 4 & 19-Jun-2009 & 19-Jun-2009 & SW 846/6020 & \\
\hline Nickel & 0.0501 & 0.0020 & $\mathrm{mg} / \mathrm{L}$ & 4 & 19-Jun-2009 & 19-Jun-2009 & SW 846/6020 & \\
\hline Selenium & 0.0074 & 0.0020 & $\mathrm{mg} / \mathrm{L}$ & 4 & 19-Jun-2009 & 19-Jun-2009 & SW 846/6020 & \\
\hline Silver & 0.0006 & 0.0020 & $\mathrm{mg} / \mathrm{L}$ & 4 & 19-Jun-2009 & 19-Jun-2009 & SW 846/6020 & $\mathrm{J}$ \\
\hline Thallium & 0.0034 & 0.0020 & $\mathrm{mg} / \mathrm{L}$ & 4 & 19-Jun-2009 & 19-Jun-2009 & SW 846/6020 & \\
\hline Vanadium & 0.143 & 0.0020 & $\mathrm{mg} / \mathrm{L}$ & 4 & 19-Jun-2009 & 19-Jun-2009 & SW 846/6020 & \\
\hline Zinc & 0.100 & 0.0020 & $\mathrm{mg} / \mathrm{L}$ & 4 & 19-Jun-2009 & 19-Jun-2009 & SW 846/6020 & $\mathrm{B}$ \\
\hline
\end{tabular}




\section{USACE ERDC-EP-C \\ 3909 Halls Ferry Road \\ Vicksburg, MS 39180-6199}

ERDC - ECB

$-\cdot$,
Project: TVA Fly Ash Project

Project Manager: Tony Bednar
Reported:

03-Sep-2009

DRAFT: EMR MT O2 Hour 96 Rep B - Total Metals

9061701-17 (Water)

\begin{tabular}{|c|c|c|c|c|c|c|c|c|}
\hline Analyte & Result & $\begin{array}{r}\text { porting } \\
\text { Limit }\end{array}$ & Units & Dilution & Prepared & Analyzed & Method & Notes \\
\hline
\end{tabular}

ERDC- EL-EP-C (Environmental Chemistry Branch)

DRAFT: Wet Chemistry

\begin{tabular}{llcccccc}
\hline Hardness & $\mathbf{6 6 . 8}$ & 1.32 & $\begin{array}{c}\text { mg equil } \\
\text { CaCO3/ }\end{array}$ & 4 & 25-Jun-2009 & 25-Jun-2009 & Hardness
\end{tabular}

$\mathrm{L}$

DRAFT: Metals by EPA 6000/7000 Series Methods

\begin{tabular}{|c|c|c|c|c|c|c|c|c|}
\hline Mercury & 0.000147 & 0.000005 & $\mathrm{mg} / \mathrm{L}$ & 2 & 24-Jul-2009 & 24-Jul-2009 & EPA 7471A & \\
\hline Strontium & 0.447 & 0.0800 & $\mathrm{mg} / \mathrm{L}$ & 4 & 25-Jun-2009 & 25-Jun-2009 & EPA 6010B & \\
\hline Aluminum & 30.1 & 0.200 & $\mathrm{mg} / \mathrm{L}$ & 4 & 25-Jun-2009 & 25-Jun-2009 & SW 846/6010 & \\
\hline Calcium & 18.6 & 0.200 & $\mathrm{mg} / \mathrm{L}$ & 4 & 25-Jun-2009 & 25-Jun-2009 & SW 846/6010 & B \\
\hline Iron & 12.4 & 0.200 & $\mathrm{mg} / \mathrm{L}$ & 4 & 25-Jun-2009 & 25-Jun-2009 & SW 846/6010 & \\
\hline Magnesium & 4.95 & 0.200 & $\mathrm{mg} / \mathrm{L}$ & 4 & 25-Jun-2009 & 25-Jun-2009 & SW 846/6010 & \\
\hline Potassium & 6.80 & 0.200 & $\mathrm{mg} / \mathrm{L}$ & 4 & 25-Jun-2009 & 25-Jun-2009 & SW 846/6010 & B \\
\hline Sodium & 3.65 & 0.200 & $\mathrm{mg} / \mathrm{L}$ & 4 & 25-Jun-2009 & 25-Jun-2009 & SW 846/6010 & B \\
\hline Antimony & 0.0042 & 0.0020 & $\mathrm{mg} / \mathrm{L}$ & 4 & 19-Jun-2009 & 19-Jun-2009 & SW 846/6020 & B \\
\hline Arsenic & 0.130 & 0.0020 & $\mathrm{mg} / \mathrm{L}$ & 4 & 19-Jun-2009 & 19-Jun-2009 & SW 846/6020 & \\
\hline Barium & 0.635 & 0.0020 & $\mathrm{mg} / \mathrm{L}$ & 4 & 19-Jun-2009 & 19-Jun-2009 & SW 846/6020 & \\
\hline Beryllium & 0.0054 & 0.0020 & $\mathrm{mg} / \mathrm{L}$ & 4 & 19-Jun-2009 & 19-Jun-2009 & SW 846/6020 & \\
\hline Cadmium & 0.0010 & 0.0020 & $\mathrm{mg} / \mathrm{L}$ & 4 & 19-Jun-2009 & 19-Jun-2009 & SW 846/6020 & J \\
\hline Chromium & 0.0411 & 0.0020 & $\mathrm{mg} / \mathrm{L}$ & 4 & 19-Jun-2009 & 19-Jun-2009 & SW 846/6020 & \\
\hline Cobalt & 0.0236 & 0.0020 & $\mathrm{mg} / \mathrm{L}$ & 4 & 19-Jun-2009 & 19-Jun-2009 & SW 846/6020 & \\
\hline Copper & 0.0790 & 0.0020 & $\mathrm{mg} / \mathrm{L}$ & 4 & 19-Jun-2009 & 19-Jun-2009 & SW 846/6020 & \\
\hline Lead & 0.0561 & 0.0020 & $\mathrm{mg} / \mathrm{L}$ & 4 & 19-Jun-2009 & 19-Jun-2009 & SW 846/6020 & \\
\hline Manganese & 0.0917 & 0.0020 & $\mathrm{mg} / \mathrm{L}$ & 4 & 19-Jun-2009 & 19-Jun-2009 & SW 846/6020 & \\
\hline Molybdenum & 0.0067 & 0.0020 & $\mathrm{mg} / \mathrm{L}$ & 4 & 19-Jun-2009 & 19-Jun-2009 & SW 846/6020 & \\
\hline Nickel & 0.0457 & 0.0020 & $\mathrm{mg} / \mathrm{L}$ & 4 & 19-Jun-2009 & 19-Jun-2009 & SW 846/6020 & \\
\hline Selenium & 0.0076 & 0.0020 & $\mathrm{mg} / \mathrm{L}$ & 4 & 19-Jun-2009 & 19-Jun-2009 & SW 846/6020 & \\
\hline Silver & 0.0005 & 0.0020 & $\mathrm{mg} / \mathrm{L}$ & 4 & 19-Jun-2009 & 19-Jun-2009 & SW 846/6020 & J \\
\hline Thallium & 0.0033 & 0.0020 & $\mathrm{mg} / \mathrm{L}$ & 4 & 19-Jun-2009 & 19-Jun-2009 & SW 846/6020 & \\
\hline Vanadium & 0.135 & 0.0020 & $\mathrm{mg} / \mathrm{L}$ & 4 & 19-Jun-2009 & 19-Jun-2009 & SW 846/6020 & \\
\hline Zinc & 0.0951 & 0.0020 & $\mathrm{mg} / \mathrm{L}$ & 4 & 19-Jun-2009 & 19-Jun-2009 & SW 846/6020 & B \\
\hline
\end{tabular}




\section{USACE ERDC-EP-C \\ 3909 Halls Ferry Road \\ Vicksburg, MS 39180-6199}

ERDC - ECB

$-\cdot$,
Project: TVA Fly Ash Project

Project Manager: Tony Bednar
Reported:

03-Sep-2009

DRAFT: EMR MT O2 Hour 96 Rep C - Total Metals

9061701-18 (Water)

\begin{tabular}{|c|c|c|c|c|c|c|c|c|}
\hline Analyte & Result & $\begin{array}{r}\text { porting } \\
\text { Limit }\end{array}$ & Units & Dilution & Prepared & Analyzed & Method & Notes \\
\hline
\end{tabular}

ERDC- EL-EP-C (Environmental Chemistry Branch)

DRAFT: Wet Chemistry

\begin{tabular}{llllllll}
\hline Hardness & $\mathbf{6 4 . 3}$ & 1.32 & $\begin{array}{c}\text { mg equil } \\
\text { CaCO3/ }\end{array}$ & 4 & 25-Jun-2009 & 25-Jun-2009
\end{tabular}

$\mathrm{L}$

DRAFT: Metals by EPA 6000/7000 Series Methods

\begin{tabular}{|c|c|c|c|c|c|c|c|c|}
\hline Mercury & 0.000132 & 0.000005 & $\mathrm{mg} / \mathrm{L}$ & 2 & 24-Jul-2009 & 24-Jul-2009 & EPA 7471A & \\
\hline Strontium & 0.418 & 0.0800 & $\mathrm{mg} / \mathrm{L}$ & 4 & 25-Jun-2009 & 25-Jun-2009 & EPA 6010B & \\
\hline Aluminum & 27.2 & 0.200 & $\mathrm{mg} / \mathrm{L}$ & 4 & 25-Jun-2009 & 25-Jun-2009 & SW 846/6010 & \\
\hline Calcium & 18.0 & 0.200 & $\mathrm{mg} / \mathrm{L}$ & 4 & 25-Jun-2009 & 25-Jun-2009 & SW 846/6010 & B \\
\hline Iron & 11.3 & 0.200 & $\mathrm{mg} / \mathrm{L}$ & 4 & 25-Jun-2009 & 25-Jun-2009 & SW 846/6010 & \\
\hline Magnesium & 4.73 & 0.200 & $\mathrm{mg} / \mathrm{L}$ & 4 & 25-Jun-2009 & 25-Jun-2009 & SW 846/6010 & \\
\hline Potassium & 6.43 & 0.200 & $\mathrm{mg} / \mathrm{L}$ & 4 & 25-Jun-2009 & 25-Jun-2009 & SW 846/6010 & B \\
\hline Sodium & 3.93 & 0.200 & $\mathrm{mg} / \mathrm{L}$ & 4 & 25-Jun-2009 & 25-Jun-2009 & SW 846/6010 & B \\
\hline Antimony & 0.0055 & 0.0020 & $\mathrm{mg} / \mathrm{L}$ & 4 & 19-Jun-2009 & 19-Jun-2009 & SW 846/6020 & B \\
\hline Arsenic & 0.120 & 0.0020 & $\mathrm{mg} / \mathrm{L}$ & 4 & 19-Jun-2009 & 19-Jun-2009 & SW 846/6020 & \\
\hline Barium & 0.580 & 0.0020 & $\mathrm{mg} / \mathrm{L}$ & 4 & 19-Jun-2009 & 19-Jun-2009 & SW 846/6020 & \\
\hline Beryllium & 0.0054 & 0.0020 & $\mathrm{mg} / \mathrm{L}$ & 4 & 19-Jun-2009 & 19-Jun-2009 & SW 846/6020 & \\
\hline Cadmium & 0.0008 & 0.0020 & $\mathrm{mg} / \mathrm{L}$ & 4 & 19-Jun-2009 & 19-Jun-2009 & SW 846/6020 & J \\
\hline Chromium & 0.0383 & 0.0020 & $\mathrm{mg} / \mathrm{L}$ & 4 & 19-Jun-2009 & 19-Jun-2009 & SW 846/6020 & \\
\hline Cobalt & 0.0222 & 0.0020 & $\mathrm{mg} / \mathrm{L}$ & 4 & 19-Jun-2009 & 19-Jun-2009 & SW 846/6020 & \\
\hline Copper & 0.0766 & 0.0020 & $\mathrm{mg} / \mathrm{L}$ & 4 & 19-Jun-2009 & 19-Jun-2009 & SW 846/6020 & \\
\hline Lead & 0.0551 & 0.0020 & $\mathrm{mg} / \mathrm{L}$ & 4 & 19-Jun-2009 & 19-Jun-2009 & SW 846/6020 & \\
\hline Manganese & 0.0866 & 0.0020 & $\mathrm{mg} / \mathrm{L}$ & 4 & 19-Jun-2009 & 19-Jun-2009 & SW 846/6020 & \\
\hline Molybdenum & 0.0061 & 0.0020 & $\mathrm{mg} / \mathrm{L}$ & 4 & 19-Jun-2009 & 19-Jun-2009 & SW 846/6020 & \\
\hline Nickel & 0.0431 & 0.0020 & $\mathrm{mg} / \mathrm{L}$ & 4 & 19-Jun-2009 & 19-Jun-2009 & SW 846/6020 & \\
\hline Selenium & 0.0072 & 0.0020 & $\mathrm{mg} / \mathrm{L}$ & 4 & 19-Jun-2009 & 19-Jun-2009 & SW 846/6020 & \\
\hline Silver & 0.0006 & 0.0020 & $\mathrm{mg} / \mathrm{L}$ & 4 & 19-Jun-2009 & 19-Jun-2009 & SW 846/6020 & J \\
\hline Thallium & 0.0031 & 0.0020 & $\mathrm{mg} / \mathrm{L}$ & 4 & 19-Jun-2009 & 19-Jun-2009 & SW 846/6020 & \\
\hline Vanadium & 0.132 & 0.0020 & $\mathrm{mg} / \mathrm{L}$ & 4 & 19-Jun-2009 & 19-Jun-2009 & SW 846/6020 & \\
\hline Zinc & 0.104 & 0.0020 & $\mathrm{mg} / \mathrm{L}$ & 4 & 19-Jun-2009 & 19-Jun-2009 & SW 846/6020 & B \\
\hline
\end{tabular}




\section{USACE ERDC-EP-C \\ 3909 Halls Ferry Road \\ Vicksburg, MS 39180-6199}

ERDC - ECB

$-\cdot$,
Project: TVA Fly Ash Project

Project Manager: Tony Bednar
Reported:

03-Sep-2009

DRAFT: SLC MT N2 Hour 96 Rep A - Total Metals

9061701-19 (Water)

\begin{tabular}{|c|c|c|c|c|c|c|c|c|}
\hline Analyte & Result & $\begin{array}{r}\text { porting } \\
\text { Limit }\end{array}$ & Units & Dilution & Prepared & Analyzed & Method & Notes \\
\hline
\end{tabular}

ERDC- EL-EP-C (Environmental Chemistry Branch)

DRAFT: Wet Chemistry

\begin{tabular}{llllllll}
\hline Hardness & $\mathbf{1 1 0}$ & 1.32 & $\begin{array}{c}\text { mg equil } \\
\text { CaCO3/ }\end{array}$ & 4 & 25-Jun-2009 & 25-Jun-2009
\end{tabular}

$\mathrm{L}$

DRAFT: Metals by EPA 6000/7000 Series Methods

\begin{tabular}{|c|c|c|c|c|c|c|c|c|}
\hline Mercury & 0.000078 & 0.000005 & $\mathrm{mg} / \mathrm{L}$ & 2 & 24-Jul-2009 & 24-Jul-2009 & EPA 7471A & \\
\hline Strontium & 0.673 & 0.0800 & $\mathrm{mg} / \mathrm{L}$ & 4 & 25-Jun-2009 & 25-Jun-2009 & EPA 6010B & \\
\hline Aluminum & 18.0 & 0.200 & $\mathrm{mg} / \mathrm{L}$ & 4 & 25-Jun-2009 & 25-Jun-2009 & SW 846/6010 & \\
\hline Calcium & 33.4 & 0.200 & $\mathrm{mg} / \mathrm{L}$ & 4 & 25-Jun-2009 & 25-Jun-2009 & SW 846/6010 & B \\
\hline Iron & 7.86 & 0.200 & $\mathrm{mg} / \mathrm{L}$ & 4 & 25-Jun-2009 & 25-Jun-2009 & SW 846/6010 & \\
\hline Magnesium & 6.48 & 0.200 & $\mathrm{mg} / \mathrm{L}$ & 4 & 25-Jun-2009 & 25-Jun-2009 & SW 846/6010 & \\
\hline Potassium & 5.35 & 0.200 & $\mathrm{mg} / \mathrm{L}$ & 4 & 25-Jun-2009 & 25-Jun-2009 & SW 846/6010 & B \\
\hline Sodium & 4.84 & 0.200 & $\mathrm{mg} / \mathrm{L}$ & 4 & 25-Jun-2009 & 25-Jun-2009 & SW 846/6010 & B \\
\hline Antimony & 0.0079 & 0.0020 & $\mathrm{mg} / \mathrm{L}$ & 4 & 19-Jun-2009 & 19-Jun-2009 & SW 846/6020 & B \\
\hline Arsenic & 0.131 & 0.0020 & $\mathrm{mg} / \mathrm{L}$ & 4 & 19-Jun-2009 & 19-Jun-2009 & SW 846/6020 & \\
\hline Barium & 0.363 & 0.0020 & $\mathrm{mg} / \mathrm{L}$ & 4 & 19-Jun-2009 & 19-Jun-2009 & SW 846/6020 & \\
\hline Beryllium & 0.0032 & 0.0020 & $\mathrm{mg} / \mathrm{L}$ & 4 & 19-Jun-2009 & 19-Jun-2009 & SW 846/6020 & \\
\hline Cadmium & 0.0008 & 0.0020 & $\mathrm{mg} / \mathrm{L}$ & 4 & 19-Jun-2009 & 19-Jun-2009 & SW 846/6020 & $\mathrm{J}$ \\
\hline Chromium & 0.0259 & 0.0020 & $\mathrm{mg} / \mathrm{L}$ & 4 & 19-Jun-2009 & 19-Jun-2009 & SW 846/6020 & \\
\hline Cobalt & 0.0147 & 0.0020 & $\mathrm{mg} / \mathrm{L}$ & 4 & 19-Jun-2009 & 19-Jun-2009 & SW 846/6020 & \\
\hline Copper & 0.0752 & 0.0020 & $\mathrm{mg} / \mathrm{L}$ & 4 & 19-Jun-2009 & 19-Jun-2009 & SW 846/6020 & \\
\hline Lead & 0.0345 & 0.0020 & $\mathrm{mg} / \mathrm{L}$ & 4 & 19-Jun-2009 & 19-Jun-2009 & SW 846/6020 & \\
\hline Manganese & 0.0750 & 0.0020 & $\mathrm{mg} / \mathrm{L}$ & 4 & 19-Jun-2009 & 19-Jun-2009 & SW 846/6020 & \\
\hline Molybdenum & 0.0747 & 0.0020 & $\mathrm{mg} / \mathrm{L}$ & 4 & 19-Jun-2009 & 19-Jun-2009 & SW 846/6020 & \\
\hline Nickel & 0.0432 & 0.0020 & $\mathrm{mg} / \mathrm{L}$ & 4 & 19-Jun-2009 & 19-Jun-2009 & SW 846/6020 & \\
\hline Selenium & 0.0095 & 0.0020 & $\mathrm{mg} / \mathrm{L}$ & 4 & 19-Jun-2009 & 19-Jun-2009 & SW 846/6020 & \\
\hline Silver & ND & 0.0020 & $\mathrm{mg} / \mathrm{L}$ & 4 & 19-Jun-2009 & 19-Jun-2009 & SW 846/6020 & $\mathrm{U}$ \\
\hline Thallium & 0.0023 & 0.0020 & $\mathrm{mg} / \mathrm{L}$ & 4 & 19-Jun-2009 & 19-Jun-2009 & SW 846/6020 & \\
\hline Vanadium & 0.129 & 0.0020 & $\mathrm{mg} / \mathrm{L}$ & 4 & 19-Jun-2009 & 19-Jun-2009 & SW 846/6020 & \\
\hline Zinc & 0.0808 & 0.0020 & $\mathrm{mg} / \mathrm{L}$ & 4 & 19-Jun-2009 & 19-Jun-2009 & SW 846/6020 & B \\
\hline
\end{tabular}




\section{USACE ERDC-EP-C \\ 3909 Halls Ferry Road \\ Vicksburg, MS 39180-6199}

ERDC - ECB

$-\cdot$,
Project: TVA Fly Ash Project

Project Manager: Tony Bednar
Reported:

03-Sep-2009

DRAFT: SLC MT N2 Hour 96 Rep B - Total Metals

9061701-20 (Water)

\begin{tabular}{|c|c|c|c|c|c|c|c|c|}
\hline Analyte & Result & $\begin{array}{r}\text { porting } \\
\text { Limit }\end{array}$ & Units & Dilution & Prepared & Analyzed & Method & Notes \\
\hline
\end{tabular}

ERDC- EL-EP-C (Environmental Chemistry Branch)

DRAFT: Wet Chemistry

\begin{tabular}{llllllll}
\hline Hardness & $\mathbf{1 1 2}$ & 1.32 & $\begin{array}{c}\text { mg equil } \\
\text { CaCO3/ }\end{array}$ & 4 & 25-Jun-2009 & 25-Jun-2009
\end{tabular}

$\mathrm{L}$

DRAFT: Metals by EPA 6000/7000 Series Methods

\begin{tabular}{|c|c|c|c|c|c|c|c|c|}
\hline Mercury & 0.000047 & 0.000005 & $\mathrm{mg} / \mathrm{L}$ & 2 & 24-Jul-2009 & 24-Jul-2009 & EPA 7471A & \\
\hline Strontium & 0.694 & 0.0800 & $\mathrm{mg} / \mathrm{L}$ & 4 & 25-Jun-2009 & 25-Jun-2009 & EPA 6010B & \\
\hline Aluminum & 20.0 & 0.200 & $\mathrm{mg} / \mathrm{L}$ & 4 & 25-Jun-2009 & 25-Jun-2009 & SW 846/6010 & \\
\hline Calcium & 33.9 & 0.200 & $\mathrm{mg} / \mathrm{L}$ & 4 & 25-Jun-2009 & 25-Jun-2009 & SW 846/6010 & B \\
\hline Iron & 8.68 & 0.200 & $\mathrm{mg} / \mathrm{L}$ & 4 & 25-Jun-2009 & 25-Jun-2009 & SW 846/6010 & \\
\hline Magnesium & 6.69 & 0.200 & $\mathrm{mg} / \mathrm{L}$ & 4 & 25-Jun-2009 & 25-Jun-2009 & SW 846/6010 & \\
\hline Potassium & 5.75 & 0.200 & $\mathrm{mg} / \mathrm{L}$ & 4 & 25-Jun-2009 & 25-Jun-2009 & SW 846/6010 & B \\
\hline Sodium & 4.84 & 0.200 & $\mathrm{mg} / \mathrm{L}$ & 4 & 25-Jun-2009 & 25-Jun-2009 & SW 846/6010 & B \\
\hline Antimony & 0.0083 & 0.0020 & $\mathrm{mg} / \mathrm{L}$ & 4 & 19-Jun-2009 & 19-Jun-2009 & SW 846/6020 & B \\
\hline Arsenic & 0.137 & 0.0020 & $\mathrm{mg} / \mathrm{L}$ & 4 & 19-Jun-2009 & 19-Jun-2009 & SW 846/6020 & \\
\hline Barium & 0.392 & 0.0020 & $\mathrm{mg} / \mathrm{L}$ & 4 & 19-Jun-2009 & 19-Jun-2009 & SW 846/6020 & \\
\hline Beryllium & 0.0035 & 0.0020 & $\mathrm{mg} / \mathrm{L}$ & 4 & 19-Jun-2009 & 19-Jun-2009 & SW 846/6020 & \\
\hline Cadmium & 0.0011 & 0.0020 & $\mathrm{mg} / \mathrm{L}$ & 4 & 19-Jun-2009 & 19-Jun-2009 & SW 846/6020 & $\mathrm{J}$ \\
\hline Chromium & 0.0263 & 0.0020 & $\mathrm{mg} / \mathrm{L}$ & 4 & 19-Jun-2009 & 19-Jun-2009 & SW 846/6020 & \\
\hline Cobalt & 0.0157 & 0.0020 & $\mathrm{mg} / \mathrm{L}$ & 4 & 19-Jun-2009 & 19-Jun-2009 & SW 846/6020 & \\
\hline Copper & 0.0549 & 0.0020 & $\mathrm{mg} / \mathrm{L}$ & 4 & 19-Jun-2009 & 19-Jun-2009 & SW 846/6020 & \\
\hline Lead & 0.0376 & 0.0020 & $\mathrm{mg} / \mathrm{L}$ & 4 & 19-Jun-2009 & 19-Jun-2009 & SW 846/6020 & \\
\hline Manganese & 0.0807 & 0.0020 & $\mathrm{mg} / \mathrm{L}$ & 4 & 19-Jun-2009 & 19-Jun-2009 & SW 846/6020 & \\
\hline Molybdenum & 0.0764 & 0.0020 & $\mathrm{mg} / \mathrm{L}$ & 4 & 19-Jun-2009 & 19-Jun-2009 & SW 846/6020 & \\
\hline Nickel & 0.0329 & 0.0020 & $\mathrm{mg} / \mathrm{L}$ & 4 & 19-Jun-2009 & 19-Jun-2009 & SW 846/6020 & \\
\hline Selenium & 0.0089 & 0.0020 & $\mathrm{mg} / \mathrm{L}$ & 4 & 19-Jun-2009 & 19-Jun-2009 & SW 846/6020 & \\
\hline Silver & ND & 0.0020 & $\mathrm{mg} / \mathrm{L}$ & 4 & 19-Jun-2009 & 19-Jun-2009 & SW 846/6020 & $\mathrm{U}$ \\
\hline Thallium & 0.0025 & 0.0020 & $\mathrm{mg} / \mathrm{L}$ & 4 & 19-Jun-2009 & 19-Jun-2009 & SW 846/6020 & \\
\hline Vanadium & 0.135 & 0.0020 & $\mathrm{mg} / \mathrm{L}$ & 4 & 19-Jun-2009 & 19-Jun-2009 & SW 846/6020 & \\
\hline Zinc & 0.0804 & 0.0020 & $\mathrm{mg} / \mathrm{L}$ & 4 & 19-Jun-2009 & 19-Jun-2009 & SW 846/6020 & B \\
\hline
\end{tabular}




\section{USACE ERDC-EP-C \\ 3909 Halls Ferry Road \\ Vicksburg, MS 39180-6199}

ERDC - ECB

$-\cdot$,
Project: TVA Fly Ash Project

Project Manager: Tony Bednar
Reported:

03-Sep-2009

DRAFT: SLC MT N2 Hour 96 Rep C - Total Metals

9061701-21 (Water)

\begin{tabular}{|c|c|c|c|c|c|c|c|c|}
\hline Analyte & Result & $\begin{array}{r}\text { porting } \\
\text { Limit }\end{array}$ & Units & Dilution & Prepared & Analyzed & Method & Notes \\
\hline
\end{tabular}

ERDC- EL-EP-C (Environmental Chemistry Branch)

DRAFT: Wet Chemistry

\begin{tabular}{llllllll}
\hline Hardness & $\mathbf{1 0 9}$ & 1.32 & $\begin{array}{c}\text { mg equil } \\
\text { CaCO3/ }\end{array}$ & 4 & 25-Jun-2009 & 25-Jun-2009
\end{tabular}

DRAFT: Metals by EPA 6000/7000 Series Methods

\begin{tabular}{|c|c|c|c|c|c|c|c|c|}
\hline Mercury & 0.000039 & 0.000005 & $\mathrm{mg} / \mathrm{L}$ & 2 & 24-Jul-2009 & 24-Jul-2009 & EPA 7471A & \\
\hline Strontium & 0.657 & 0.0800 & $\mathrm{mg} / \mathrm{L}$ & 4 & 25-Jun-2009 & 25-Jun-2009 & EPA 6010B & \\
\hline Aluminum & 15.6 & 0.200 & $\mathrm{mg} / \mathrm{L}$ & 4 & 25-Jun-2009 & 25-Jun-2009 & SW 846/6010 & \\
\hline Calcium & 33.1 & 0.200 & $\mathrm{mg} / \mathrm{L}$ & 4 & 25-Jun-2009 & 25-Jun-2009 & SW 846/6010 & B \\
\hline Iron & 6.87 & 0.200 & $\mathrm{mg} / \mathrm{L}$ & 4 & 25-Jun-2009 & 25-Jun-2009 & SW 846/6010 & \\
\hline Magnesium & 6.35 & 0.200 & $\mathrm{mg} / \mathrm{L}$ & 4 & 25-Jun-2009 & 25-Jun-2009 & SW 846/6010 & \\
\hline Potassium & 4.79 & 0.200 & $\mathrm{mg} / \mathrm{L}$ & 4 & 25-Jun-2009 & 25-Jun-2009 & SW 846/6010 & B \\
\hline Sodium & 4.65 & 0.200 & $\mathrm{mg} / \mathrm{L}$ & 4 & 25-Jun-2009 & 25-Jun-2009 & SW 846/6010 & B \\
\hline Antimony & 0.0082 & 0.0020 & $\mathrm{mg} / \mathrm{L}$ & 4 & 19-Jun-2009 & 19-Jun-2009 & SW 846/6020 & B \\
\hline Arsenic & 0.122 & 0.0020 & $\mathrm{mg} / \mathrm{L}$ & 4 & 19-Jun-2009 & 19-Jun-2009 & SW 846/6020 & \\
\hline Barium & 0.339 & 0.0020 & $\mathrm{mg} / \mathrm{L}$ & 4 & 19-Jun-2009 & 19-Jun-2009 & SW 846/6020 & \\
\hline Beryllium & 0.0028 & 0.0020 & $\mathrm{mg} / \mathrm{L}$ & 4 & 19-Jun-2009 & 19-Jun-2009 & SW 846/6020 & \\
\hline Cadmium & 0.0008 & 0.0020 & $\mathrm{mg} / \mathrm{L}$ & 4 & 19-Jun-2009 & 19-Jun-2009 & SW 846/6020 & J \\
\hline Chromium & 0.0217 & 0.0020 & $\mathrm{mg} / \mathrm{L}$ & 4 & 19-Jun-2009 & 19-Jun-2009 & SW 846/6020 & \\
\hline Cobalt & 0.0130 & 0.0020 & $\mathrm{mg} / \mathrm{L}$ & 4 & 19-Jun-2009 & 19-Jun-2009 & SW 846/6020 & \\
\hline Copper & 0.0460 & 0.0020 & $\mathrm{mg} / \mathrm{L}$ & 4 & 19-Jun-2009 & 19-Jun-2009 & SW 846/6020 & \\
\hline Lead & 0.0309 & 0.0020 & $\mathrm{mg} / \mathrm{L}$ & 4 & 19-Jun-2009 & 19-Jun-2009 & SW 846/6020 & \\
\hline Manganese & 0.0685 & 0.0020 & $\mathrm{mg} / \mathrm{L}$ & 4 & 19-Jun-2009 & 19-Jun-2009 & SW 846/6020 & \\
\hline Molybdenum & 0.0758 & 0.0020 & $\mathrm{mg} / \mathrm{L}$ & 4 & 19-Jun-2009 & 19-Jun-2009 & SW 846/6020 & \\
\hline Nickel & 0.0277 & 0.0020 & $\mathrm{mg} / \mathrm{L}$ & 4 & 19-Jun-2009 & 19-Jun-2009 & SW 846/6020 & \\
\hline Selenium & 0.0087 & 0.0020 & $\mathrm{mg} / \mathrm{L}$ & 4 & 19-Jun-2009 & 19-Jun-2009 & SW 846/6020 & \\
\hline Silver & ND & 0.0020 & $\mathrm{mg} / \mathrm{L}$ & 4 & 19-Jun-2009 & 19-Jun-2009 & SW 846/6020 & $\mathrm{U}$ \\
\hline Thallium & 0.0022 & 0.0020 & $\mathrm{mg} / \mathrm{L}$ & 4 & 19-Jun-2009 & 19-Jun-2009 & SW 846/6020 & \\
\hline Vanadium & 0.125 & 0.0020 & $\mathrm{mg} / \mathrm{L}$ & 4 & 19-Jun-2009 & 19-Jun-2009 & SW 846/6020 & \\
\hline Zinc & 0.0729 & 0.0020 & $\mathrm{mg} / \mathrm{L}$ & 4 & 19-Jun-2009 & 19-Jun-2009 & SW 846/6020 & B \\
\hline
\end{tabular}




\section{USACE ERDC-EP-C \\ 3909 Halls Ferry Road \\ Vicksburg, MS 39180-6199}

ERDC - ECB

$-\cdot$,
Project: TVA Fly Ash Project

Project Manager: Tony Bednar
Reported:

03-Sep-2009

DRAFT: SLC MT O2 Hour 96 Rep A - Total Metals

9061701-22 (Water)

\begin{tabular}{|c|c|c|c|c|c|c|c|c|}
\hline Analyte & Result & $\begin{array}{r}\text { porting } \\
\text { Limit }\end{array}$ & Units & Dilution & Prepared & Analyzed & Method & Notes \\
\hline
\end{tabular}

ERDC- EL-EP-C (Environmental Chemistry Branch)

DRAFT: Wet Chemistry

\begin{tabular}{llllllll}
\hline Hardness & $\mathbf{1 0 9}$ & 1.32 & $\begin{array}{c}\text { mg equil } \\
\text { CaCO3/ }\end{array}$ & 4 & 25-Jun-2009 & 25-Jun-2009
\end{tabular}

$\mathrm{L}$

DRAFT: Metals by EPA 6000/7000 Series Methods

\begin{tabular}{|c|c|c|c|c|c|c|c|c|}
\hline Mercury & 0.000188 & 0.000005 & $\mathrm{mg} / \mathrm{L}$ & 2 & 24-Jul-2009 & 24-Jul-2009 & EPA 7471A & \\
\hline Strontium & 0.662 & 0.0800 & $\mathrm{mg} / \mathrm{L}$ & 4 & 25-Jun-2009 & 25-Jun-2009 & EPA 6010B & \\
\hline Aluminum & 17.4 & 0.200 & $\mathrm{mg} / \mathrm{L}$ & 4 & 25-Jun-2009 & 25-Jun-2009 & SW 846/6010 & \\
\hline Calcium & 33.1 & 0.200 & $\mathrm{mg} / \mathrm{L}$ & 4 & 25-Jun-2009 & 25-Jun-2009 & SW 846/6010 & B \\
\hline Iron & 7.73 & 0.200 & $\mathrm{mg} / \mathrm{L}$ & 4 & 25-Jun-2009 & 25-Jun-2009 & SW 846/6010 & \\
\hline Magnesium & 6.43 & 0.200 & $\mathrm{mg} / \mathrm{L}$ & 4 & 25-Jun-2009 & 25-Jun-2009 & SW 846/6010 & \\
\hline Potassium & 5.29 & 0.200 & $\mathrm{mg} / \mathrm{L}$ & 4 & 25-Jun-2009 & 25-Jun-2009 & SW 846/6010 & B \\
\hline Sodium & 4.78 & 0.200 & $\mathrm{mg} / \mathrm{L}$ & 4 & 25-Jun-2009 & 25-Jun-2009 & SW 846/6010 & B \\
\hline Antimony & 0.0073 & 0.0020 & $\mathrm{mg} / \mathrm{L}$ & 4 & 19-Jun-2009 & 19-Jun-2009 & SW 846/6020 & B \\
\hline Arsenic & 0.125 & 0.0020 & $\mathrm{mg} / \mathrm{L}$ & 4 & 19-Jun-2009 & 19-Jun-2009 & SW 846/6020 & \\
\hline Barium & 0.342 & 0.0020 & $\mathrm{mg} / \mathrm{L}$ & 4 & 19-Jun-2009 & 19-Jun-2009 & SW 846/6020 & \\
\hline Beryllium & 0.0031 & 0.0020 & $\mathrm{mg} / \mathrm{L}$ & 4 & 19-Jun-2009 & 19-Jun-2009 & SW 846/6020 & \\
\hline Cadmium & 0.00081 & 0.0020 & $\mathrm{mg} / \mathrm{L}$ & 4 & 19-Jun-2009 & 19-Jun-2009 & SW 846/6020 & J \\
\hline Chromium & 0.0240 & 0.0020 & $\mathrm{mg} / \mathrm{L}$ & 4 & 19-Jun-2009 & 19-Jun-2009 & SW 846/6020 & \\
\hline Cobalt & 0.0139 & 0.0020 & $\mathrm{mg} / \mathrm{L}$ & 4 & 19-Jun-2009 & 19-Jun-2009 & SW 846/6020 & \\
\hline Copper & 0.0492 & 0.0020 & $\mathrm{mg} / \mathrm{L}$ & 4 & 19-Jun-2009 & 19-Jun-2009 & SW 846/6020 & \\
\hline Lead & 0.0325 & 0.0020 & $\mathrm{mg} / \mathrm{L}$ & 4 & 19-Jun-2009 & 19-Jun-2009 & SW 846/6020 & \\
\hline Manganese & 0.0768 & 0.0020 & $\mathrm{mg} / \mathrm{L}$ & 4 & 19-Jun-2009 & 19-Jun-2009 & SW 846/6020 & \\
\hline Molybdenum & 0.0753 & 0.0020 & $\mathrm{mg} / \mathrm{L}$ & 4 & 19-Jun-2009 & 19-Jun-2009 & SW 846/6020 & \\
\hline Nickel & 0.0301 & 0.0020 & $\mathrm{mg} / \mathrm{L}$ & 4 & 19-Jun-2009 & 19-Jun-2009 & SW 846/6020 & \\
\hline Selenium & 0.0088 & 0.0020 & $\mathrm{mg} / \mathrm{L}$ & 4 & 19-Jun-2009 & 19-Jun-2009 & SW 846/6020 & \\
\hline Silver & ND & 0.0020 & $\mathrm{mg} / \mathrm{L}$ & 4 & 19-Jun-2009 & 19-Jun-2009 & SW 846/6020 & $\mathrm{U}$ \\
\hline Thallium & 0.0023 & 0.0020 & $\mathrm{mg} / \mathrm{L}$ & 4 & 19-Jun-2009 & 19-Jun-2009 & SW 846/6020 & \\
\hline Vanadium & 0.126 & 0.0020 & $\mathrm{mg} / \mathrm{L}$ & 4 & 19-Jun-2009 & 19-Jun-2009 & SW 846/6020 & \\
\hline Zinc & 0.0850 & 0.0020 & $\mathrm{mg} / \mathrm{L}$ & 4 & 19-Jun-2009 & 19-Jun-2009 & SW 846/6020 & B \\
\hline
\end{tabular}




\section{USACE ERDC-EP-C \\ 3909 Halls Ferry Road \\ Vicksburg, MS 39180-6199}

ERDC - ECB

$-\cdot$,
Project: TVA Fly Ash Project

Project Manager: Tony Bednar
Reported:

03-Sep-2009

DRAFT: SLC MT O2 Hour 96 Rep B - Total Metals

9061701-23 (Water)

\begin{tabular}{|c|c|c|c|c|c|c|c|c|}
\hline Analyte & Result & $\begin{array}{r}\text { porting } \\
\text { Limit }\end{array}$ & Units & Dilution & Prepared & Analyzed & Method & Notes \\
\hline
\end{tabular}

ERDC- EL-EP-C (Environmental Chemistry Branch)

DRAFT: Wet Chemistry

\begin{tabular}{llllllll}
\hline Hardness & $\mathbf{1 1 1}$ & 1.32 & $\begin{array}{c}\text { mg equil } \\
\text { CaCO3/ }\end{array}$ & 4 & 25-Jun-2009 & 25-Jun-2009
\end{tabular}

$\mathrm{L}$

DRAFT: Metals by EPA 6000/7000 Series Methods

\begin{tabular}{|c|c|c|c|c|c|c|c|c|}
\hline Mercury & 0.000063 & 0.000005 & $\mathrm{mg} / \mathrm{L}$ & 2 & 24-Jul-2009 & 24-Jul-2009 & EPA 7471A & \\
\hline Strontium & 0.671 & 0.0800 & $\mathrm{mg} / \mathrm{L}$ & 4 & 25-Jun-2009 & 25-Jun-2009 & EPA 6010B & \\
\hline Aluminum & 17.2 & 0.200 & $\mathrm{mg} / \mathrm{L}$ & 4 & 25-Jun-2009 & 25-Jun-2009 & SW 846/6010 & \\
\hline Calcium & 33.7 & 0.200 & $\mathrm{mg} / \mathrm{L}$ & 4 & 25-Jun-2009 & 25-Jun-2009 & SW 846/6010 & B \\
\hline Iron & 7.50 & 0.200 & $\mathrm{mg} / \mathrm{L}$ & 4 & 25-Jun-2009 & 25-Jun-2009 & SW 846/6010 & \\
\hline Magnesium & 6.49 & 0.200 & $\mathrm{mg} / \mathrm{L}$ & 4 & 25-Jun-2009 & 25-Jun-2009 & SW 846/6010 & \\
\hline Potassium & 5.19 & 0.200 & $\mathrm{mg} / \mathrm{L}$ & 4 & 25-Jun-2009 & 25-Jun-2009 & SW 846/6010 & B \\
\hline Sodium & 4.79 & 0.200 & $\mathrm{mg} / \mathrm{L}$ & 4 & 25-Jun-2009 & 25-Jun-2009 & SW 846/6010 & B \\
\hline Antimony & 0.0083 & 0.0020 & $\mathrm{mg} / \mathrm{L}$ & 4 & 19-Jun-2009 & 19-Jun-2009 & SW 846/6020 & B \\
\hline Arsenic & 0.128 & 0.0020 & $\mathrm{mg} / \mathrm{L}$ & 4 & 19-Jun-2009 & 19-Jun-2009 & SW 846/6020 & \\
\hline Barium & 0.364 & 0.0020 & $\mathrm{mg} / \mathrm{L}$ & 4 & 19-Jun-2009 & 19-Jun-2009 & SW 846/6020 & \\
\hline Beryllium & 0.0030 & 0.0020 & $\mathrm{mg} / \mathrm{L}$ & 4 & 19-Jun-2009 & 19-Jun-2009 & SW 846/6020 & \\
\hline Cadmium & 0.0008 & 0.0020 & $\mathrm{mg} / \mathrm{L}$ & 4 & 19-Jun-2009 & 19-Jun-2009 & SW 846/6020 & J \\
\hline Chromium & 0.0262 & 0.0020 & $\mathrm{mg} / \mathrm{L}$ & 4 & 19-Jun-2009 & 19-Jun-2009 & SW 846/6020 & \\
\hline Cobalt & 0.0135 & 0.0020 & $\mathrm{mg} / \mathrm{L}$ & 4 & 19-Jun-2009 & 19-Jun-2009 & SW 846/6020 & \\
\hline Copper & 0.0485 & 0.0020 & $\mathrm{mg} / \mathrm{L}$ & 4 & 19-Jun-2009 & 19-Jun-2009 & SW 846/6020 & \\
\hline Lead & 0.0370 & 0.0020 & $\mathrm{mg} / \mathrm{L}$ & 4 & 19-Jun-2009 & 19-Jun-2009 & SW 846/6020 & \\
\hline Manganese & 0.0740 & 0.0020 & $\mathrm{mg} / \mathrm{L}$ & 4 & 19-Jun-2009 & 19-Jun-2009 & SW 846/6020 & \\
\hline Molybdenum & 0.0776 & 0.0020 & $\mathrm{mg} / \mathrm{L}$ & 4 & 19-Jun-2009 & 19-Jun-2009 & SW 846/6020 & \\
\hline Nickel & 0.0310 & 0.0020 & $\mathrm{mg} / \mathrm{L}$ & 4 & 19-Jun-2009 & 19-Jun-2009 & SW 846/6020 & \\
\hline Selenium & 0.0092 & 0.0020 & $\mathrm{mg} / \mathrm{L}$ & 4 & 19-Jun-2009 & 19-Jun-2009 & SW 846/6020 & \\
\hline Silver & ND & 0.0020 & $\mathrm{mg} / \mathrm{L}$ & 4 & 19-Jun-2009 & 19-Jun-2009 & SW 846/6020 & $\mathrm{U}$ \\
\hline Thallium & 0.0024 & 0.0020 & $\mathrm{mg} / \mathrm{L}$ & 4 & 19-Jun-2009 & 19-Jun-2009 & SW 846/6020 & \\
\hline Vanadium & 0.126 & 0.0020 & $\mathrm{mg} / \mathrm{L}$ & 4 & 19-Jun-2009 & 19-Jun-2009 & SW 846/6020 & \\
\hline Zinc & 0.0916 & 0.0020 & $\mathrm{mg} / \mathrm{L}$ & 4 & 19-Jun-2009 & 19-Jun-2009 & SW 846/6020 & B \\
\hline
\end{tabular}




\section{USACE ERDC-EP-C \\ 3909 Halls Ferry Road \\ Vicksburg, MS 39180-6199}

ERDC - ECB

$-\cdot$,
Project: TVA Fly Ash Project

Project Manager: Tony Bednar
Reported:

03-Sep-2009

DRAFT: SLC MT O2 Hour 96 Rep C - Total Metals

9061701-24 (Water)

\begin{tabular}{|c|c|c|c|c|c|c|c|c|}
\hline Analyte & Result & $\begin{array}{r}\text { porting } \\
\text { Limit }\end{array}$ & Units & Dilution & Prepared & Analyzed & Method & Notes \\
\hline
\end{tabular}

ERDC- EL-EP-C (Environmental Chemistry Branch)

DRAFT: Wet Chemistry

\begin{tabular}{llllllll}
\hline Hardness & $\mathbf{1 1 4}$ & 1.32 & $\begin{array}{c}\text { mg equil } \\
\text { CaCO3/ }\end{array}$ & 4 & 25-Jun-2009 & 25-Jun-2009
\end{tabular}

$\mathrm{L}$

DRAFT: Metals by EPA 6000/7000 Series Methods

\begin{tabular}{|c|c|c|c|c|c|c|c|c|}
\hline Mercury & 0.000098 & 0.000005 & $\mathrm{mg} / \mathrm{L}$ & 2 & 24-Jul-2009 & 24-Jul-2009 & EPA 7471A & \\
\hline Strontium & 0.704 & 0.0800 & $\mathrm{mg} / \mathrm{L}$ & 4 & 25-Jun-2009 & 25-Jun-2009 & EPA 6010B & \\
\hline Aluminum & 20.5 & 0.200 & $\mathrm{mg} / \mathrm{L}$ & 4 & 25-Jun-2009 & 25-Jun-2009 & SW 846/6010 & \\
\hline Calcium & 34.6 & 0.200 & $\mathrm{mg} / \mathrm{L}$ & 4 & 25-Jun-2009 & 25-Jun-2009 & SW 846/6010 & B \\
\hline Iron & 8.83 & 0.200 & $\mathrm{mg} / \mathrm{L}$ & 4 & 25-Jun-2009 & 25-Jun-2009 & SW 846/6010 & \\
\hline Magnesium & 6.77 & 0.200 & $\mathrm{mg} / \mathrm{L}$ & 4 & 25-Jun-2009 & 25-Jun-2009 & SW 846/6010 & \\
\hline Potassium & 5.90 & 0.200 & $\mathrm{mg} / \mathrm{L}$ & 4 & 25-Jun-2009 & 25-Jun-2009 & SW 846/6010 & B \\
\hline Sodium & 4.98 & 0.200 & $\mathrm{mg} / \mathrm{L}$ & 4 & 25-Jun-2009 & 25-Jun-2009 & SW 846/6010 & B \\
\hline Antimony & 0.0082 & 0.0020 & $\mathrm{mg} / \mathrm{L}$ & 4 & 19-Jun-2009 & 19-Jun-2009 & SW 846/6020 & B \\
\hline Arsenic & 0.136 & 0.0020 & $\mathrm{mg} / \mathrm{L}$ & 4 & 19-Jun-2009 & 19-Jun-2009 & SW 846/6020 & \\
\hline Barium & 0.394 & 0.0020 & $\mathrm{mg} / \mathrm{L}$ & 4 & 19-Jun-2009 & 19-Jun-2009 & SW 846/6020 & \\
\hline Beryllium & 0.0037 & 0.0020 & $\mathrm{mg} / \mathrm{L}$ & 4 & 19-Jun-2009 & 19-Jun-2009 & SW 846/6020 & \\
\hline Cadmium & 0.0009 & 0.0020 & $\mathrm{mg} / \mathrm{L}$ & 4 & 19-Jun-2009 & 19-Jun-2009 & SW 846/6020 & J \\
\hline Chromium & 0.0344 & 0.0020 & $\mathrm{mg} / \mathrm{L}$ & 4 & 19-Jun-2009 & 19-Jun-2009 & SW 846/6020 & \\
\hline Cobalt & 0.0159 & 0.0020 & $\mathrm{mg} / \mathrm{L}$ & 4 & 19-Jun-2009 & 19-Jun-2009 & SW 846/6020 & \\
\hline Copper & 0.0566 & 0.0020 & $\mathrm{mg} / \mathrm{L}$ & 4 & 19-Jun-2009 & 19-Jun-2009 & SW 846/6020 & \\
\hline Lead & 0.0379 & 0.0020 & $\mathrm{mg} / \mathrm{L}$ & 4 & 19-Jun-2009 & 19-Jun-2009 & SW 846/6020 & \\
\hline Manganese & 0.0933 & 0.0020 & $\mathrm{mg} / \mathrm{L}$ & 4 & 19-Jun-2009 & 19-Jun-2009 & SW 846/6020 & \\
\hline Molybdenum & 0.0786 & 0.0020 & $\mathrm{mg} / \mathrm{L}$ & 4 & 19-Jun-2009 & 19-Jun-2009 & SW 846/6020 & \\
\hline Nickel & 0.0365 & 0.0020 & $\mathrm{mg} / \mathrm{L}$ & 4 & 19-Jun-2009 & 19-Jun-2009 & SW 846/6020 & \\
\hline Selenium & 0.0098 & 0.0020 & $\mathrm{mg} / \mathrm{L}$ & 4 & 19-Jun-2009 & 19-Jun-2009 & SW 846/6020 & \\
\hline Silver & ND & 0.0020 & $\mathrm{mg} / \mathrm{L}$ & 4 & 19-Jun-2009 & 19-Jun-2009 & SW 846/6020 & $\mathrm{U}$ \\
\hline Thallium & 0.0025 & 0.0020 & $\mathrm{mg} / \mathrm{L}$ & 4 & 19-Jun-2009 & 19-Jun-2009 & SW 846/6020 & \\
\hline Vanadium & 0.140 & 0.0020 & $\mathrm{mg} / \mathrm{L}$ & 4 & 19-Jun-2009 & 19-Jun-2009 & SW 846/6020 & \\
\hline Zinc & 0.0907 & 0.0020 & $\mathrm{mg} / \mathrm{L}$ & 4 & 19-Jun-2009 & 19-Jun-2009 & SW 846/6020 & B \\
\hline
\end{tabular}




\section{USACE ERDC-EP-C \\ 3909 Halls Ferry Road \\ Vicksburg, MS 39180-6199}

ERDC - ECB

$-,-$
Project: TVA Fly Ash Project

Project Manager: Tony Bednar
Reported:

03-Sep-2009

DRAFT: EMR MT N2 Hour 240 Rep A

9062302-01 (Water)

\begin{tabular}{|c|c|c|c|c|c|c|c|c|}
\hline Analyte & Result & $\begin{array}{r}\text { porting } \\
\text { Limit }\end{array}$ & Units & Dilution & Prepared & Analyzed & Method & Notes \\
\hline
\end{tabular}

ERDC- EL-EP-C (Environmental Chemistry Branch)

DRAFT: Wet Chemistry

\begin{tabular}{llllllll}
\hline Hardness & $\mathbf{4 7 . 0}$ & 1.32 & $\begin{array}{c}\text { mg equil } \\
\text { CaCO3/ }\end{array}$ & 1 & 25-Jun-2009 & 25-Jun-2009
\end{tabular}

DRAFT: Metals by EPA 6000/7000 Series Methods

\begin{tabular}{|c|c|c|c|c|c|c|c|c|}
\hline Mercury & 0.000037 & 0.000005 & $\mathrm{mg} / \mathrm{L}$ & 1 & 17-Jun-2009 & 01-Jul-2009 & EPA 7471A & \\
\hline Strontium & 0.199 & 0.0200 & $\mathrm{mg} / \mathrm{L}$ & 1 & 25-Jun-2009 & 25-Jun-2009 & EPA 6010B & \\
\hline Aluminum & 0.123 & 0.200 & $\mathrm{mg} / \mathrm{L}$ & 1 & 25-Jun-2009 & 25-Jun-2009 & SW 846/6010 & $\mathrm{J}$ \\
\hline Calcium & 14.5 & 0.200 & $\mathrm{mg} / \mathrm{L}$ & 1 & 25-Jun-2009 & 25-Jun-2009 & SW 846/6010 & \\
\hline Iron & ND & 0.200 & $\mathrm{mg} / \mathrm{L}$ & 1 & 25-Jun-2009 & 25-Jun-2009 & SW 846/6010 & $\mathrm{U}$ \\
\hline Magnesium & 2.63 & 0.200 & $\mathrm{mg} / \mathrm{L}$ & 1 & 25-Jun-2009 & 25-Jun-2009 & SW 846/6010 & \\
\hline Potassium & 1.51 & 0.200 & $\mathrm{mg} / \mathrm{L}$ & 1 & 25-Jun-2009 & 25-Jun-2009 & SW 846/6010 & \\
\hline Sodium & 2.69 & 0.200 & $\mathrm{mg} / \mathrm{L}$ & 1 & 25-Jun-2009 & 25-Jun-2009 & SW 846/6010 & \\
\hline Antimony & 0.0022 & 0.0010 & $\mathrm{mg} / \mathrm{L}$ & 2 & 26-Jun-2009 & 26-Jun-2009 & SW 846/6020 & \\
\hline Arsenic & 0.0633 & 0.0010 & $\mathrm{mg} / \mathrm{L}$ & 2 & 26-Jun-2009 & 26-Jun-2009 & SW 846/6020 & \\
\hline Barium & 0.123 & 0.0010 & $\mathrm{mg} / \mathrm{L}$ & 2 & 26-Jun-2009 & 26-Jun-2009 & SW 846/6020 & \\
\hline Beryllium & ND & 0.0010 & $\mathrm{mg} / \mathrm{L}$ & 2 & 26-Jun-2009 & 26-Jun-2009 & SW 846/6020 & $\mathrm{U}$ \\
\hline Cadmium & ND & 0.0010 & $\mathrm{mg} / \mathrm{L}$ & 2 & 26-Jun-2009 & 26-Jun-2009 & SW 846/6020 & $\mathrm{U}$ \\
\hline Chromium & 0.0018 & 0.0010 & $\mathrm{mg} / \mathrm{L}$ & 2 & 26-Jun-2009 & 26-Jun-2009 & SW 846/6020 & \\
\hline Cobalt & ND & 0.0010 & $\mathrm{mg} / \mathrm{L}$ & 2 & 26-Jun-2009 & 26-Jun-2009 & SW 846/6020 & $\mathrm{U}$ \\
\hline Copper & 0.0011 & 0.0010 & $\mathrm{mg} / \mathrm{L}$ & 2 & 26-Jun-2009 & 26-Jun-2009 & SW 846/6020 & \\
\hline Lead & ND & 0.0010 & $\mathrm{mg} / \mathrm{L}$ & 2 & 26-Jun-2009 & 26-Jun-2009 & SW 846/6020 & $\mathrm{U}$ \\
\hline Manganese & 0.0005 & 0.0010 & $\mathrm{mg} / \mathrm{L}$ & 2 & 26-Jun-2009 & 26-Jun-2009 & SW 846/6020 & J \\
\hline Molybdenum & 0.0027 & 0.0010 & $\mathrm{mg} / \mathrm{L}$ & 2 & 26-Jun-2009 & 26-Jun-2009 & SW 846/6020 & \\
\hline Nickel & 0.0004 & 0.0010 & $\mathrm{mg} / \mathrm{L}$ & 2 & 26-Jun-2009 & 26-Jun-2009 & SW 846/6020 & $\mathrm{J}$ \\
\hline Selenium & 0.0064 & 0.0010 & $\mathrm{mg} / \mathrm{L}$ & 2 & 26-Jun-2009 & 26-Jun-2009 & SW 846/6020 & \\
\hline Silver & ND & 0.0010 & $\mathrm{mg} / \mathrm{L}$ & 2 & 26-Jun-2009 & 26-Jun-2009 & SW 846/6020 & $\mathrm{U}$ \\
\hline Thallium & 0.0006 & 0.0010 & $\mathrm{mg} / \mathrm{L}$ & 2 & 26-Jun-2009 & 26-Jun-2009 & SW 846/6020 & $\mathrm{J}$ \\
\hline Vanadium & 0.0387 & 0.0010 & $\mathrm{mg} / \mathrm{L}$ & 2 & 26-Jun-2009 & 26-Jun-2009 & SW 846/6020 & \\
\hline Zinc & 0.0041 & 0.0010 & $\mathrm{mg} / \mathrm{L}$ & 2 & 26-Jun-2009 & 26-Jun-2009 & SW 846/6020 & B \\
\hline
\end{tabular}




\section{USACE ERDC-EP-C \\ 3909 Halls Ferry Road \\ Vicksburg, MS 39180-6199}

ERDC - ECB

Project: TVA Fly Ash Project

Reported:

$--,-$

Project Manager: Tony Bednar

03-Sep-2009

DRAFT: EMR MT N2 Hour 240 Rep A

9062302-01 (Water)

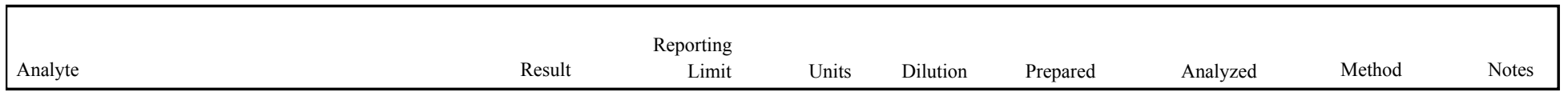

ERDC- EL-EP-C (Environmental Chemistry Branch)

DRAFT: Miscellaneous Physical/Conventional Chemistry Parameters

\begin{tabular}{llllrrrr}
\hline Total Dissolved Solids & $\mathbf{1 5 5}$ & 25.0 & mg/L & 1 & $25-J u n-2009$ & $25-J u n-2009$ & EPA 160.1 \\
Total Solids & $\mathbf{6 3 0}$ & 10.0 & mg/L & 1 & $25-J u n-2009$ & $25-J u n-2009$ & EPA 160.2 \\
Total Suspended Solids & $\mathbf{4 7 5}$ & 25.0 & mg/L & 1 & $25-J u n-2009$ & $25-J u n-2009$ & EPA 160.2
\end{tabular}




\section{USACE ERDC-EP-C \\ 3909 Halls Ferry Road \\ Vicksburg, MS 39180-6199}

ERDC - ECB

$-,-$
Project: TVA Fly Ash Project

Project Manager: Tony Bednar
Reported:

03-Sep-2009

DRAFT: EMR MT N2 Hour 240 Rep B

9062302-02 (Water)

\begin{tabular}{|c|c|c|c|c|c|c|c|c|}
\hline Analyte & Result & $\begin{array}{r}\text { porting } \\
\text { Limit }\end{array}$ & Units & Dilution & Prepared & Analyzed & Method & Notes \\
\hline
\end{tabular}

ERDC- EL-EP-C (Environmental Chemistry Branch)

DRAFT: Wet Chemistry

\begin{tabular}{llllllll}
\hline Hardness & $\mathbf{4 7 . 2}$ & 1.32 & $\begin{array}{c}\text { mg equil } \\
\text { CaCO3/ }\end{array}$ & 1 & 25-Jun-2009 & 25-Jun-2009
\end{tabular}

$\mathrm{L}$

DRAFT: Metals by EPA 6000/7000 Series Methods

\begin{tabular}{|c|c|c|c|c|c|c|c|c|}
\hline Mercury & ND & 0.000005 & $\mathrm{mg} / \mathrm{L}$ & 1 & 17-Jun-2009 & 01-Jul-2009 & EPA 7471A & $\mathrm{U}$ \\
\hline Strontium & 0.203 & 0.0200 & $\mathrm{mg} / \mathrm{L}$ & 1 & 25-Jun-2009 & 25-Jun-2009 & EPA 6010B & \\
\hline Aluminum & 0.130 & 0.200 & $\mathrm{mg} / \mathrm{L}$ & 1 & 25-Jun-2009 & 25-Jun-2009 & SW 846/6010 & $\mathrm{J}$ \\
\hline Calcium & 14.5 & 0.200 & $\mathrm{mg} / \mathrm{L}$ & 1 & 25-Jun-2009 & 25-Jun-2009 & SW 846/6010 & \\
\hline Iron & 0.0532 & 0.200 & $\mathrm{mg} / \mathrm{L}$ & 1 & 25-Jun-2009 & 25-Jun-2009 & SW 846/6010 & $\mathrm{J}$ \\
\hline Magnesium & 2.65 & 0.200 & $\mathrm{mg} / \mathrm{L}$ & 1 & 25-Jun-2009 & 25-Jun-2009 & SW 846/6010 & \\
\hline Potassium & 1.55 & 0.200 & $\mathrm{mg} / \mathrm{L}$ & 1 & 25-Jun-2009 & 25-Jun-2009 & SW 846/6010 & \\
\hline Sodium & 2.98 & 0.200 & $\mathrm{mg} / \mathrm{L}$ & 1 & 25-Jun-2009 & 25-Jun-2009 & SW 846/6010 & \\
\hline Antimony & 0.0026 & 0.0010 & $\mathrm{mg} / \mathrm{L}$ & 2 & 26-Jun-2009 & 26-Jun-2009 & SW 846/6020 & \\
\hline Arsenic & 0.0634 & 0.0010 & $\mathrm{mg} / \mathrm{L}$ & 2 & 26-Jun-2009 & 26-Jun-2009 & SW 846/6020 & \\
\hline Barium & 0.129 & 0.0010 & $\mathrm{mg} / \mathrm{L}$ & 2 & 26-Jun-2009 & 26-Jun-2009 & SW 846/6020 & \\
\hline Beryllium & ND & 0.0010 & $\mathrm{mg} / \mathrm{L}$ & 2 & 26-Jun-2009 & 26-Jun-2009 & SW 846/6020 & $\mathrm{U}$ \\
\hline Cadmium & ND & 0.0010 & $\mathrm{mg} / \mathrm{L}$ & 2 & 26-Jun-2009 & 26-Jun-2009 & SW 846/6020 & $\mathrm{U}$ \\
\hline Chromium & 0.0019 & 0.0010 & $\mathrm{mg} / \mathrm{L}$ & 2 & 26-Jun-2009 & 26-Jun-2009 & SW 846/6020 & \\
\hline Cobalt & ND & 0.0010 & $\mathrm{mg} / \mathrm{L}$ & 2 & 26-Jun-2009 & 26-Jun-2009 & SW 846/6020 & $\mathrm{U}$ \\
\hline Copper & 0.0010 & 0.0010 & $\mathrm{mg} / \mathrm{L}$ & 2 & 26-Jun-2009 & 26-Jun-2009 & SW 846/6020 & J \\
\hline Lead & ND & 0.0010 & $\mathrm{mg} / \mathrm{L}$ & 2 & 26-Jun-2009 & 26-Jun-2009 & SW 846/6020 & $\mathrm{U}$ \\
\hline Manganese & 0.00051 & 0.0010 & $\mathrm{mg} / \mathrm{L}$ & 2 & 26-Jun-2009 & 26-Jun-2009 & SW 846/6020 & J \\
\hline Molybdenum & 0.0029 & 0.0010 & $\mathrm{mg} / \mathrm{L}$ & 2 & 26-Jun-2009 & 26-Jun-2009 & SW 846/6020 & \\
\hline Nickel & 0.0004 & 0.0010 & $\mathrm{mg} / \mathrm{L}$ & 2 & 26-Jun-2009 & 26-Jun-2009 & SW 846/6020 & $\mathrm{J}$ \\
\hline Selenium & 0.0064 & 0.0010 & $\mathrm{mg} / \mathrm{L}$ & 2 & 26-Jun-2009 & 26-Jun-2009 & SW 846/6020 & \\
\hline Silver & ND & 0.0010 & $\mathrm{mg} / \mathrm{L}$ & 2 & 26-Jun-2009 & 26-Jun-2009 & SW 846/6020 & $\mathrm{U}$ \\
\hline Thallium & 0.0006 & 0.0010 & $\mathrm{mg} / \mathrm{L}$ & 2 & 26-Jun-2009 & 26-Jun-2009 & SW 846/6020 & $\mathrm{J}$ \\
\hline Vanadium & 0.0382 & 0.0010 & $\mathrm{mg} / \mathrm{L}$ & 2 & 26-Jun-2009 & 26-Jun-2009 & SW 846/6020 & \\
\hline Zinc & 0.0035 & 0.0010 & $\mathrm{mg} / \mathrm{L}$ & 2 & 26-Jun-2009 & 26-Jun-2009 & SW 846/6020 & $\mathrm{B}$ \\
\hline
\end{tabular}




\section{USACE ERDC-EP-C \\ 3909 Halls Ferry Road \\ Vicksburg, MS 39180-6199}

ERDC - ECB

Project: TVA Fly Ash Project

Reported:

$--,-$

Project Manager: Tony Bednar

03-Sep-2009

DRAFT: EMR MT N2 Hour 240 Rep B

9062302-02 (Water)

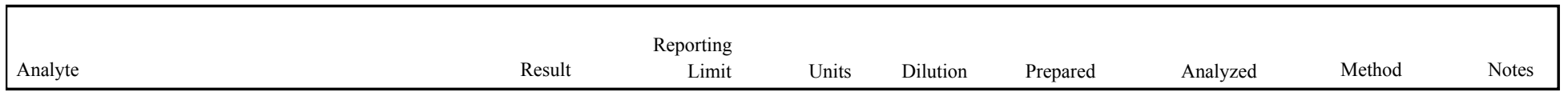

ERDC- EL-EP-C (Environmental Chemistry Branch)

DRAFT: Miscellaneous Physical/Conventional Chemistry Parameters

\begin{tabular}{|c|c|c|c|c|c|c|c|}
\hline Total Dissolved Solids & 165 & 25.0 & $\mathrm{mg} / \mathrm{L}$ & 1 & 25-Jun-2009 & 25-Jun-2009 & EPA 160.1 \\
\hline Total Solids & 560 & 10.0 & $\mathrm{mg} / \mathrm{L}$ & 1 & 25-Jun-2009 & 25-Jun-2009 & EPA 160.2 \\
\hline Total Suspended Solids & 395 & 25.0 & $\mathrm{mg} / \mathrm{L}$ & 1 & 25-Jun-2009 & 25-Jun-2009 & EPA 160.2 \\
\hline
\end{tabular}




\section{USACE ERDC-EP-C \\ 3909 Halls Ferry Road \\ Vicksburg, MS 39180-6199}

ERDC - ECB

$-,-$
Project: TVA Fly Ash Project

Project Manager: Tony Bednar
Reported:

03-Sep-2009

DRAFT: EMR MT N2 Hour 240 Rep C

9062302-03 (Water)

\begin{tabular}{|c|c|c|c|c|c|c|c|c|}
\hline Analyte & Result & $\begin{array}{r}\text { porting } \\
\text { Limit }\end{array}$ & Units & Dilution & Prepared & Analyzed & Method & Notes \\
\hline
\end{tabular}

ERDC- EL-EP-C (Environmental Chemistry Branch)

DRAFT: Wet Chemistry

\begin{tabular}{llcccccc}
\hline Hardness & $\mathbf{4 8 . 2}$ & 1.32 & $\begin{array}{c}\text { mg equil } \\
\text { CaCO3/ }\end{array}$ & 1 & 25-Jun-2009 & 25-Jun-2009
\end{tabular}

DRAFT: Metals by EPA 6000/7000 Series Methods

\begin{tabular}{|c|c|c|c|c|c|c|c|c|}
\hline Mercury & ND & 0.000005 & $\mathrm{mg} / \mathrm{L}$ & 1 & 17-Jun-2009 & 01-Jul-2009 & EPA 7471A & $\mathrm{U}$ \\
\hline Strontium & 0.208 & 0.0200 & $\mathrm{mg} / \mathrm{L}$ & 1 & 25-Jun-2009 & 25-Jun-2009 & EPA 6010B & \\
\hline Aluminum & 0.101 & 0.200 & $\mathrm{mg} / \mathrm{L}$ & 1 & 25-Jun-2009 & 25-Jun-2009 & SW 846/6010 & J \\
\hline Calcium & 14.9 & 0.200 & $\mathrm{mg} / \mathrm{L}$ & 1 & 25-Jun-2009 & 25-Jun-2009 & SW 846/6010 & \\
\hline Iron & ND & 0.200 & $\mathrm{mg} / \mathrm{L}$ & 1 & 25-Jun-2009 & 25-Jun-2009 & SW 846/6010 & $\mathrm{U}$ \\
\hline Magnesium & 2.70 & 0.200 & $\mathrm{mg} / \mathrm{L}$ & 1 & 25-Jun-2009 & 25-Jun-2009 & SW 846/6010 & \\
\hline Potassium & 1.66 & 0.200 & $\mathrm{mg} / \mathrm{L}$ & 1 & 25-Jun-2009 & 25-Jun-2009 & SW 846/6010 & \\
\hline Sodium & 3.04 & 0.200 & $\mathrm{mg} / \mathrm{L}$ & 1 & 25-Jun-2009 & 25-Jun-2009 & SW 846/6010 & \\
\hline Antimony & 0.0022 & 0.0010 & $\mathrm{mg} / \mathrm{L}$ & 2 & 26-Jun-2009 & 26-Jun-2009 & SW 846/6020 & \\
\hline Arsenic & 0.0657 & 0.0010 & $\mathrm{mg} / \mathrm{L}$ & 2 & 26-Jun-2009 & 26-Jun-2009 & SW 846/6020 & \\
\hline Barium & 0.127 & 0.0010 & $\mathrm{mg} / \mathrm{L}$ & 2 & 26-Jun-2009 & 26-Jun-2009 & SW 846/6020 & \\
\hline Beryllium & ND & 0.0010 & $\mathrm{mg} / \mathrm{L}$ & 2 & 26-Jun-2009 & 26-Jun-2009 & SW 846/6020 & $\mathrm{U}$ \\
\hline Cadmium & ND & 0.0010 & $\mathrm{mg} / \mathrm{L}$ & 2 & 26-Jun-2009 & 26-Jun-2009 & SW 846/6020 & $\mathrm{U}$ \\
\hline Chromium & 0.0019 & 0.0010 & $\mathrm{mg} / \mathrm{L}$ & 2 & 26-Jun-2009 & 26-Jun-2009 & SW 846/6020 & \\
\hline Cobalt & ND & 0.0010 & $\mathrm{mg} / \mathrm{L}$ & 2 & 26-Jun-2009 & 26-Jun-2009 & SW 846/6020 & $\mathrm{U}$ \\
\hline Copper & 0.0014 & 0.0010 & $\mathrm{mg} / \mathrm{L}$ & 2 & 26-Jun-2009 & 26-Jun-2009 & SW 846/6020 & \\
\hline Lead & ND & 0.0010 & $\mathrm{mg} / \mathrm{L}$ & 2 & 26-Jun-2009 & 26-Jun-2009 & SW 846/6020 & $\mathrm{U}$ \\
\hline Manganese & 0.0006 & 0.0010 & $\mathrm{mg} / \mathrm{L}$ & 2 & 26-Jun-2009 & 26-Jun-2009 & SW 846/6020 & $\mathrm{J}$ \\
\hline Molybdenum & 0.0029 & 0.0010 & $\mathrm{mg} / \mathrm{L}$ & 2 & 26-Jun-2009 & 26-Jun-2009 & SW 846/6020 & \\
\hline Nickel & 0.0005 & 0.0010 & $\mathrm{mg} / \mathrm{L}$ & 2 & 26-Jun-2009 & 26-Jun-2009 & SW 846/6020 & $\mathrm{J}$ \\
\hline Selenium & 0.0066 & 0.0010 & $\mathrm{mg} / \mathrm{L}$ & 2 & 26-Jun-2009 & 26-Jun-2009 & SW 846/6020 & \\
\hline Silver & ND & 0.0010 & $\mathrm{mg} / \mathrm{L}$ & 2 & 26-Jun-2009 & 26-Jun-2009 & SW 846/6020 & $\mathrm{U}$ \\
\hline Thallium & 0.0007 & 0.0010 & $\mathrm{mg} / \mathrm{L}$ & 2 & 26-Jun-2009 & 26-Jun-2009 & SW 846/6020 & $\mathrm{J}$ \\
\hline Vanadium & 0.0399 & 0.0010 & $\mathrm{mg} / \mathrm{L}$ & 2 & 26-Jun-2009 & 26-Jun-2009 & SW 846/6020 & \\
\hline Zinc & 0.0036 & 0.0010 & $\mathrm{mg} / \mathrm{L}$ & 2 & 26-Jun-2009 & 26-Jun-2009 & SW 846/6020 & B \\
\hline
\end{tabular}




\section{USACE ERDC-EP-C \\ 3909 Halls Ferry Road \\ Vicksburg, MS 39180-6199}

ERDC - ECB

Project: TVA Fly Ash Project

Reported:

$--,-$

Project Manager: Tony Bednar

03-Sep-2009

DRAFT: EMR MT N2 Hour 240 Rep C

9062302-03 (Water)

\begin{tabular}{|c|c|c|c|c|c|c|c|c|}
\hline & & orting & & & & & & \\
\hline Analyte & Result & Limit & Units & Dilution & Prepared & Analyzed & Method & Notes \\
\hline
\end{tabular}

ERDC- EL-EP-C (Environmental Chemistry Branch)

DRAFT: Miscellaneous Physical/Conventional Chemistry Parameters

\begin{tabular}{llllrrrr}
\hline Total Dissolved Solids & $\mathbf{2 2 5}$ & 25.0 & mg/L & 1 & $25-J u n-2009$ & $25-J u n-2009$ & EPA 160.1 \\
Total Solids & $\mathbf{6 3 0}$ & 10.0 & mg/L & 1 & $25-J u n-2009$ & $25-J u n-2009$ & EPA 160.2 \\
Total Suspended Solids & $\mathbf{4 0 5}$ & 25.0 & mg/L & 1 & $25-J u n-2009$ & $25-J u n-2009$ & EPA 160.2
\end{tabular}




\section{USACE ERDC-EP-C \\ 3909 Halls Ferry Road \\ Vicksburg, MS 39180-6199}

ERDC - ECB

$-\cdot$,
Project: TVA Fly Ash Project

Project Manager: Tony Bednar
Reported:

03-Sep-2009

DRAFT: EMR MT O2 Hour 240 Rep A

9062302-04 (Water)

\begin{tabular}{|c|c|c|c|c|c|c|c|c|}
\hline Analyte & Result & $\begin{array}{r}\text { porting } \\
\text { Limit }\end{array}$ & Units & Dilution & Prepared & Analyzed & Method & Notes \\
\hline
\end{tabular}

ERDC- EL-EP-C (Environmental Chemistry Branch)

DRAFT: Wet Chemistry

\begin{tabular}{lccccccc}
\hline Hardness & $\mathbf{4 4 . 2}$ & 1.32 & $\begin{array}{c}\text { mg equil } \\
\text { CaCO3/ }\end{array}$ & 1 & 25-Jun-2009 & 25-Jun-2009
\end{tabular}

$\mathrm{L}$

DRAFT: Metals by EPA 6000/7000 Series Methods

\begin{tabular}{|c|c|c|c|c|c|c|c|c|}
\hline Mercury & ND & 0.000005 & $\mathrm{mg} / \mathrm{L}$ & 1 & 17-Jun-2009 & 01-Jul-2009 & EPA 7471A & $\mathrm{U}$ \\
\hline Strontium & 0.182 & 0.0200 & $\mathrm{mg} / \mathrm{L}$ & 1 & 25-Jun-2009 & 25-Jun-2009 & EPA 6010B & \\
\hline Aluminum & 0.0944 & 0.200 & $\mathrm{mg} / \mathrm{L}$ & 1 & 25-Jun-2009 & 25-Jun-2009 & SW 846/6010 & J \\
\hline Calcium & 13.6 & 0.200 & $\mathrm{mg} / \mathrm{L}$ & 1 & 25-Jun-2009 & 25-Jun-2009 & SW 846/6010 & \\
\hline Iron & ND & 0.200 & $\mathrm{mg} / \mathrm{L}$ & 1 & 25-Jun-2009 & 25-Jun-2009 & SW 846/6010 & $\mathrm{U}$ \\
\hline Magnesium & 2.52 & 0.200 & $\mathrm{mg} / \mathrm{L}$ & 1 & 25-Jun-2009 & 25-Jun-2009 & SW 846/6010 & \\
\hline Potassium & 1.48 & 0.200 & $\mathrm{mg} / \mathrm{L}$ & 1 & 25-Jun-2009 & 25-Jun-2009 & SW 846/6010 & \\
\hline Sodium & 2.64 & 0.200 & $\mathrm{mg} / \mathrm{L}$ & 1 & 25-Jun-2009 & 25-Jun-2009 & SW 846/6010 & \\
\hline Antimony & 0.0017 & 0.0010 & $\mathrm{mg} / \mathrm{L}$ & 2 & 26-Jun-2009 & 26-Jun-2009 & SW 846/6020 & \\
\hline Arsenic & 0.0533 & 0.0010 & $\mathrm{mg} / \mathrm{L}$ & 2 & 26-Jun-2009 & 26-Jun-2009 & SW 846/6020 & \\
\hline Barium & 0.119 & 0.0010 & $\mathrm{mg} / \mathrm{L}$ & 2 & 26-Jun-2009 & 26-Jun-2009 & SW 846/6020 & \\
\hline Beryllium & ND & 0.0010 & $\mathrm{mg} / \mathrm{L}$ & 2 & 26-Jun-2009 & 26-Jun-2009 & SW 846/6020 & $\mathrm{U}$ \\
\hline Cadmium & ND & 0.0010 & $\mathrm{mg} / \mathrm{L}$ & 2 & 26-Jun-2009 & 26-Jun-2009 & SW 846/6020 & $\mathrm{U}$ \\
\hline Chromium & 0.0019 & 0.0010 & $\mathrm{mg} / \mathrm{L}$ & 2 & 26-Jun-2009 & 26-Jun-2009 & SW 846/6020 & \\
\hline Cobalt & ND & 0.0010 & $\mathrm{mg} / \mathrm{L}$ & 2 & 26-Jun-2009 & 26-Jun-2009 & SW 846/6020 & $\mathrm{U}$ \\
\hline Copper & 0.0010 & 0.0010 & $\mathrm{mg} / \mathrm{L}$ & 2 & 26-Jun-2009 & 26-Jun-2009 & SW 846/6020 & $\mathrm{J}$ \\
\hline Lead & ND & 0.0010 & $\mathrm{mg} / \mathrm{L}$ & 2 & 26-Jun-2009 & 26-Jun-2009 & SW 846/6020 & $\mathrm{U}$ \\
\hline Manganese & 0.0006 & 0.0010 & $\mathrm{mg} / \mathrm{L}$ & 2 & 26-Jun-2009 & 26-Jun-2009 & SW 846/6020 & J \\
\hline Molybdenum & 0.0025 & 0.0010 & $\mathrm{mg} / \mathrm{L}$ & 2 & 26-Jun-2009 & 26-Jun-2009 & SW 846/6020 & \\
\hline Nickel & 0.0004 & 0.0010 & $\mathrm{mg} / \mathrm{L}$ & 2 & 26-Jun-2009 & 26-Jun-2009 & SW 846/6020 & $\mathrm{J}$ \\
\hline Selenium & 0.0059 & 0.0010 & $\mathrm{mg} / \mathrm{L}$ & 2 & 26-Jun-2009 & 26-Jun-2009 & SW 846/6020 & \\
\hline Silver & ND & 0.0010 & $\mathrm{mg} / \mathrm{L}$ & 2 & 26-Jun-2009 & 26-Jun-2009 & SW 846/6020 & $\mathrm{U}$ \\
\hline Thallium & 0.0006 & 0.0010 & $\mathrm{mg} / \mathrm{L}$ & 2 & 26-Jun-2009 & 26-Jun-2009 & SW 846/6020 & $\mathrm{J}$ \\
\hline Vanadium & 0.0341 & 0.0010 & $\mathrm{mg} / \mathrm{L}$ & 2 & 26-Jun-2009 & 26-Jun-2009 & SW 846/6020 & \\
\hline Zinc & 0.0038 & 0.0010 & $\mathrm{mg} / \mathrm{L}$ & 2 & 26-Jun-2009 & 26-Jun-2009 & SW 846/6020 & B \\
\hline
\end{tabular}




\section{USACE ERDC-EP-C \\ 3909 Halls Ferry Road \\ Vicksburg, MS 39180-6199}

ERDC - ECB

Project: TVA Fly Ash Project

Reported:

$--,-$

Project Manager: Tony Bednar

03-Sep-2009

DRAFT: EMR MT O2 Hour 240 Rep A

9062302-04 (Water)

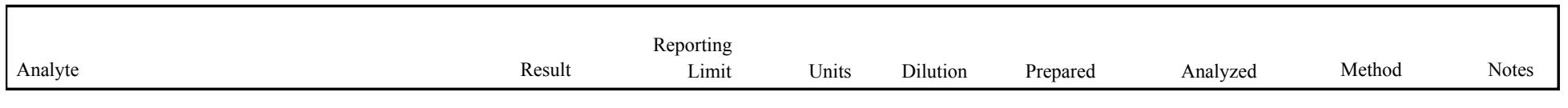

ERDC- EL-EP-C (Environmental Chemistry Branch)

DRAFT: Miscellaneous Physical/Conventional Chemistry Parameters

\begin{tabular}{|c|c|c|c|c|c|c|c|}
\hline Total Dissolved Solids & 140 & 25.0 & $\mathrm{mg} / \mathrm{L}$ & 1 & 25-Jun-2009 & 25-Jun-2009 & EPA 160.1 \\
\hline Total Solids & 700 & 10.0 & $\mathrm{mg} / \mathrm{L}$ & 1 & 25-Jun-2009 & 25-Jun-2009 & EPA 160.2 \\
\hline Total Suspended Solids & 560 & 25.0 & $\mathrm{mg} / \mathrm{L}$ & 1 & 25-Jun-2009 & 25-Jun-2009 & EPA 160.2 \\
\hline
\end{tabular}




\section{USACE ERDC-EP-C \\ 3909 Halls Ferry Road \\ Vicksburg, MS 39180-6199}

ERDC - ECB

$-,-$
Project: TVA Fly Ash Project

Project Manager: Tony Bednar
Reported:

03-Sep-2009

DRAFT: EMR MT O2 Hour 240 Rep B

9062302-05 (Water)

\begin{tabular}{|c|c|c|c|c|c|c|c|c|}
\hline Analyte & Result & $\begin{array}{r}\text { porting } \\
\text { Limit }\end{array}$ & Units & Dilution & Prepared & Analyzed & Method & Notes \\
\hline
\end{tabular}

ERDC- EL-EP-C (Environmental Chemistry Branch)

DRAFT: Wet Chemistry

\begin{tabular}{llllllll}
\hline Hardness & $\mathbf{4 4 . 2}$ & 1.32 & $\begin{array}{c}\text { mg equil } \\
\text { CaCO3/ }\end{array}$ & 1 & 25-Jun-2009 & 25-Jun-2009
\end{tabular}

$\mathrm{L}$

DRAFT: Metals by EPA 6000/7000 Series Methods

\begin{tabular}{|c|c|c|c|c|c|c|c|c|}
\hline Mercury & ND & 0.000005 & $\mathrm{mg} / \mathrm{L}$ & 1 & 17-Jun-2009 & 01-Jul-2009 & EPA 7471A & $\mathrm{U}$ \\
\hline Strontium & 0.184 & 0.0200 & $\mathrm{mg} / \mathrm{L}$ & 1 & 25-Jun-2009 & 25-Jun-2009 & EPA 6010B & \\
\hline Aluminum & 0.0891 & 0.200 & $\mathrm{mg} / \mathrm{L}$ & 1 & 25-Jun-2009 & 25-Jun-2009 & SW 846/6010 & J \\
\hline Calcium & 13.6 & 0.200 & $\mathrm{mg} / \mathrm{L}$ & 1 & 25-Jun-2009 & 25-Jun-2009 & SW 846/6010 & \\
\hline Iron & ND & 0.200 & $\mathrm{mg} / \mathrm{L}$ & 1 & 25-Jun-2009 & 25-Jun-2009 & SW 846/6010 & $\mathrm{U}$ \\
\hline Magnesium & 2.51 & 0.200 & $\mathrm{mg} / \mathrm{L}$ & 1 & 25-Jun-2009 & 25-Jun-2009 & SW 846/6010 & \\
\hline Potassium & 1.41 & 0.200 & $\mathrm{mg} / \mathrm{L}$ & 1 & 25-Jun-2009 & 25-Jun-2009 & SW 846/6010 & \\
\hline Sodium & 2.52 & 0.200 & $\mathrm{mg} / \mathrm{L}$ & 1 & 25-Jun-2009 & 25-Jun-2009 & SW 846/6010 & \\
\hline Antimony & 0.0016 & 0.0010 & $\mathrm{mg} / \mathrm{L}$ & 2 & 26-Jun-2009 & 26-Jun-2009 & SW 846/6020 & \\
\hline Arsenic & 0.0539 & 0.0010 & $\mathrm{mg} / \mathrm{L}$ & 2 & 26-Jun-2009 & 26-Jun-2009 & SW 846/6020 & \\
\hline Barium & 0.120 & 0.0010 & $\mathrm{mg} / \mathrm{L}$ & 2 & 26-Jun-2009 & 26-Jun-2009 & SW 846/6020 & \\
\hline Beryllium & ND & 0.0010 & $\mathrm{mg} / \mathrm{L}$ & 2 & 26-Jun-2009 & 26-Jun-2009 & SW 846/6020 & $\mathrm{U}$ \\
\hline Cadmium & ND & 0.0010 & $\mathrm{mg} / \mathrm{L}$ & 2 & 26-Jun-2009 & 26-Jun-2009 & SW 846/6020 & $\mathrm{U}$ \\
\hline Chromium & 0.0019 & 0.0010 & $\mathrm{mg} / \mathrm{L}$ & 2 & 26-Jun-2009 & 26-Jun-2009 & SW 846/6020 & \\
\hline Cobalt & ND & 0.0010 & $\mathrm{mg} / \mathrm{L}$ & 2 & 26-Jun-2009 & 26-Jun-2009 & SW 846/6020 & $\mathrm{U}$ \\
\hline Copper & 0.0007 & 0.0010 & $\mathrm{mg} / \mathrm{L}$ & 2 & 26-Jun-2009 & 26-Jun-2009 & SW 846/6020 & $\mathrm{J}$ \\
\hline Lead & ND & 0.0010 & $\mathrm{mg} / \mathrm{L}$ & 2 & 26-Jun-2009 & 26-Jun-2009 & SW 846/6020 & $\mathrm{U}$ \\
\hline Manganese & 0.0012 & 0.0010 & $\mathrm{mg} / \mathrm{L}$ & 2 & 26-Jun-2009 & 26-Jun-2009 & SW 846/6020 & \\
\hline Molybdenum & 0.0025 & 0.0010 & $\mathrm{mg} / \mathrm{L}$ & 2 & 26-Jun-2009 & 26-Jun-2009 & SW 846/6020 & \\
\hline Nickel & 0.0005 & 0.0010 & $\mathrm{mg} / \mathrm{L}$ & 2 & 26-Jun-2009 & 26-Jun-2009 & SW 846/6020 & $\mathrm{J}$ \\
\hline Selenium & 0.0056 & 0.0010 & $\mathrm{mg} / \mathrm{L}$ & 2 & 26-Jun-2009 & 26-Jun-2009 & SW 846/6020 & \\
\hline Silver & ND & 0.0010 & $\mathrm{mg} / \mathrm{L}$ & 2 & 26-Jun-2009 & 26-Jun-2009 & SW 846/6020 & $\mathrm{U}$ \\
\hline Thallium & 0.0005 & 0.0010 & $\mathrm{mg} / \mathrm{L}$ & 2 & 26-Jun-2009 & 26-Jun-2009 & SW 846/6020 & $\mathrm{J}$ \\
\hline Vanadium & 0.0341 & 0.0010 & $\mathrm{mg} / \mathrm{L}$ & 2 & 26-Jun-2009 & 26-Jun-2009 & SW 846/6020 & \\
\hline Zinc & 0.0033 & 0.0010 & $\mathrm{mg} / \mathrm{L}$ & 2 & 26-Jun-2009 & 26-Jun-2009 & SW 846/6020 & B \\
\hline
\end{tabular}




\section{USACE ERDC-EP-C \\ 3909 Halls Ferry Road \\ Vicksburg, MS 39180-6199}

ERDC - ECB

Project: TVA Fly Ash Project

Reported:

$--,-$

Project Manager: Tony Bednar

03-Sep-2009

DRAFT: EMR MT O2 Hour 240 Rep B

9062302-05 (Water)

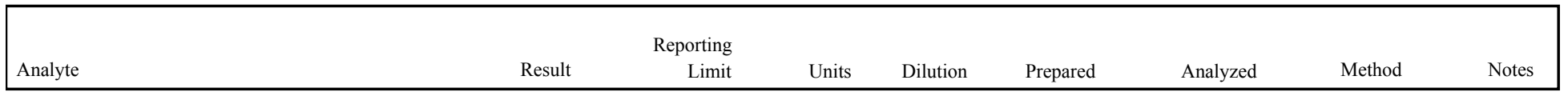

ERDC- EL-EP-C (Environmental Chemistry Branch)

DRAFT: Miscellaneous Physical/Conventional Chemistry Parameters

\begin{tabular}{llllrrrr}
\hline Total Dissolved Solids & $\mathbf{1 1 5}$ & 25.0 & mg/L & 1 & $25-J u n-2009$ & $25-J u n-2009$ & EPA 160.1 \\
Total Solids & $\mathbf{6 5 0}$ & 10.0 & mg/L & 1 & $25-J u n-2009$ & $25-J u n-2009$ & EPA 160.2 \\
Total Suspended Solids & $\mathbf{5 3 5}$ & 25.0 & mg/L & 1 & $25-J u n-2009$ & $25-J u n-2009$ & EPA 160.2
\end{tabular}




\section{USACE ERDC-EP-C \\ 3909 Halls Ferry Road \\ Vicksburg, MS 39180-6199}

ERDC - ECB

$-,-$
Project: TVA Fly Ash Project

Project Manager: Tony Bednar
Reported:

03-Sep-2009

DRAFT: EMR MT O2 Hour 240 Rep C

9062302-06 (Water)

\begin{tabular}{|c|c|c|c|c|c|c|c|c|}
\hline Analyte & Result & $\begin{array}{r}\text { porting } \\
\text { Limit }\end{array}$ & Units & Dilution & Prepared & Analyzed & Method & Notes \\
\hline
\end{tabular}

ERDC- EL-EP-C (Environmental Chemistry Branch)

DRAFT: Wet Chemistry

\begin{tabular}{llllllll}
\hline Hardness & $\mathbf{4 4 . 3}$ & 1.32 & $\begin{array}{c}\text { mg equil } \\
\text { CaCO3/ }\end{array}$ & 1 & 25-Jun-2009 & 25-Jun-2009
\end{tabular}

$\mathrm{L}$

DRAFT: Metals by EPA 6000/7000 Series Methods

\begin{tabular}{|c|c|c|c|c|c|c|c|c|}
\hline Mercury & ND & 0.000005 & $\mathrm{mg} / \mathrm{L}$ & 1 & 17-Jun-2009 & 01-Jul-2009 & EPA 7471A & $\mathrm{U}$ \\
\hline Strontium & 0.182 & 0.0200 & $\mathrm{mg} / \mathrm{L}$ & 1 & 25-Jun-2009 & 25-Jun-2009 & EPA 6010B & \\
\hline Aluminum & 0.0868 & 0.200 & $\mathrm{mg} / \mathrm{L}$ & 1 & 25-Jun-2009 & 25-Jun-2009 & SW 846/6010 & J \\
\hline Calcium & 13.6 & 0.200 & $\mathrm{mg} / \mathrm{L}$ & 1 & 25-Jun-2009 & 25-Jun-2009 & SW 846/6010 & \\
\hline Iron & ND & 0.200 & $\mathrm{mg} / \mathrm{L}$ & 1 & 25-Jun-2009 & 25-Jun-2009 & SW 846/6010 & $\mathrm{U}$ \\
\hline Magnesium & 2.52 & 0.200 & $\mathrm{mg} / \mathrm{L}$ & 1 & 25-Jun-2009 & 25-Jun-2009 & SW 846/6010 & \\
\hline Potassium & 1.43 & 0.200 & $\mathrm{mg} / \mathrm{L}$ & 1 & 25-Jun-2009 & 25-Jun-2009 & SW 846/6010 & \\
\hline Sodium & 2.57 & 0.200 & $\mathrm{mg} / \mathrm{L}$ & 1 & 25-Jun-2009 & 25-Jun-2009 & SW 846/6010 & \\
\hline Antimony & 0.0017 & 0.0010 & $\mathrm{mg} / \mathrm{L}$ & 2 & 26-Jun-2009 & 26-Jun-2009 & SW 846/6020 & \\
\hline Arsenic & 0.0518 & 0.0010 & $\mathrm{mg} / \mathrm{L}$ & 2 & 26-Jun-2009 & 26-Jun-2009 & SW 846/6020 & \\
\hline Barium & 0.126 & 0.0010 & $\mathrm{mg} / \mathrm{L}$ & 2 & 26-Jun-2009 & 26-Jun-2009 & SW 846/6020 & \\
\hline Beryllium & ND & 0.0010 & $\mathrm{mg} / \mathrm{L}$ & 2 & 26-Jun-2009 & 26-Jun-2009 & SW 846/6020 & $\mathrm{U}$ \\
\hline Cadmium & ND & 0.0010 & $\mathrm{mg} / \mathrm{L}$ & 2 & 26-Jun-2009 & 26-Jun-2009 & SW 846/6020 & $\mathrm{U}$ \\
\hline Chromium & 0.0017 & 0.0010 & $\mathrm{mg} / \mathrm{L}$ & 2 & 26-Jun-2009 & 26-Jun-2009 & SW 846/6020 & \\
\hline Cobalt & ND & 0.0010 & $\mathrm{mg} / \mathrm{L}$ & 2 & 26-Jun-2009 & 26-Jun-2009 & SW 846/6020 & $\mathrm{U}$ \\
\hline Copper & 0.0010 & 0.0010 & $\mathrm{mg} / \mathrm{L}$ & 2 & 26-Jun-2009 & 26-Jun-2009 & SW 846/6020 & \\
\hline Lead & ND & 0.0010 & $\mathrm{mg} / \mathrm{L}$ & 2 & 26-Jun-2009 & 26-Jun-2009 & SW 846/6020 & $\mathrm{U}$ \\
\hline Manganese & 0.0007 & 0.0010 & $\mathrm{mg} / \mathrm{L}$ & 2 & 26-Jun-2009 & 26-Jun-2009 & SW 846/6020 & J \\
\hline Molybdenum & 0.0023 & 0.0010 & $\mathrm{mg} / \mathrm{L}$ & 2 & 26-Jun-2009 & 26-Jun-2009 & SW 846/6020 & \\
\hline Nickel & 0.0004 & 0.0010 & $\mathrm{mg} / \mathrm{L}$ & 2 & 26-Jun-2009 & 26-Jun-2009 & SW 846/6020 & $\mathrm{J}$ \\
\hline Selenium & 0.0049 & 0.0010 & $\mathrm{mg} / \mathrm{L}$ & 2 & 26-Jun-2009 & 26-Jun-2009 & SW 846/6020 & \\
\hline Silver & ND & 0.0010 & $\mathrm{mg} / \mathrm{L}$ & 2 & 26-Jun-2009 & 26-Jun-2009 & SW 846/6020 & $\mathrm{U}$ \\
\hline Thallium & 0.0005 & 0.0010 & $\mathrm{mg} / \mathrm{L}$ & 2 & 26-Jun-2009 & 26-Jun-2009 & SW 846/6020 & $\mathrm{J}$ \\
\hline Vanadium & 0.0340 & 0.0010 & $\mathrm{mg} / \mathrm{L}$ & 2 & 26-Jun-2009 & 26-Jun-2009 & SW 846/6020 & \\
\hline Zinc & 0.0038 & 0.0010 & $\mathrm{mg} / \mathrm{L}$ & 2 & 26-Jun-2009 & 26-Jun-2009 & SW 846/6020 & B \\
\hline
\end{tabular}




\section{USACE ERDC-EP-C \\ 3909 Halls Ferry Road \\ Vicksburg, MS 39180-6199}

ERDC - ECB

Project: TVA Fly Ash Project

Reported:

$--,-$

Project Manager: Tony Bednar

03-Sep-2009

DRAFT: EMR MT O2 Hour 240 Rep C

9062302-06 (Water)

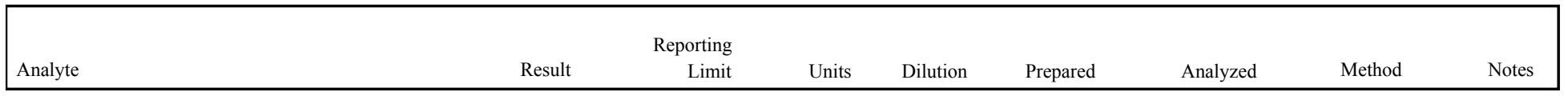

ERDC- EL-EP-C (Environmental Chemistry Branch)

DRAFT: Miscellaneous Physical/Conventional Chemistry Parameters

\begin{tabular}{llllrrrr}
\hline Total Dissolved Solids & $\mathbf{1 1 0}$ & 25.0 & mg/L & 1 & $25-J u n-2009$ & $25-J u n-2009$ & EPA 160.1 \\
Total Solids & $\mathbf{5 3 5}$ & 10.0 & mg/L & 1 & $25-J u n-2009$ & $25-J u n-2009$ & EPA 160.2 \\
Total Suspended Solids & $\mathbf{4 2 5}$ & 25.0 & mg/L & 1 & $25-J u n-2009$ & $25-J u n-2009$ & EPA 160.2
\end{tabular}




\section{USACE ERDC-EP-C \\ 3909 Halls Ferry Road \\ Vicksburg, MS 39180-6199}

ERDC - ECB

$-,-$
Project: TVA Fly Ash Project

Project Manager: Tony Bednar
Reported:

03-Sep-2009

DRAFT: SLC MT N2 Hour 240 Rep A

9062302-07 (Water)

\begin{tabular}{|c|c|c|c|c|c|c|c|c|}
\hline Analyte & Result & $\begin{array}{r}\text { porting } \\
\text { Limit }\end{array}$ & Units & Dilution & Prepared & Analyzed & Method & Notes \\
\hline
\end{tabular}

ERDC- EL-EP-C (Environmental Chemistry Branch)

DRAFT: Wet Chemistry

\begin{tabular}{llllllll}
\hline Hardness & $\mathbf{1 0 5}$ & 1.32 & $\begin{array}{c}\text { mg equil } \\
\text { CaCO3/ }\end{array}$ & 1 & 25-Jun-2009 & 25-Jun-2009
\end{tabular}

DRAFT: Metals by EPA 6000/7000 Series Methods

\begin{tabular}{|c|c|c|c|c|c|c|c|c|}
\hline Mercury & ND & 0.000005 & $\mathrm{mg} / \mathrm{L}$ & 1 & 17-Jun-2009 & 01-Jul-2009 & EPA 7471A & $\mathrm{U}$ \\
\hline Strontium & 0.572 & 0.0200 & $\mathrm{mg} / \mathrm{L}$ & 1 & 25-Jun-2009 & 25-Jun-2009 & EPA $6010 B$ & \\
\hline Aluminum & 0.170 & 0.200 & $\mathrm{mg} / \mathrm{L}$ & 1 & 25-Jun-2009 & 25-Jun-2009 & SW 846/6010 & $\mathrm{J}$ \\
\hline Calcium & 32.7 & 0.200 & $\mathrm{mg} / \mathrm{L}$ & 1 & 25-Jun-2009 & 25-Jun-2009 & SW 846/6010 & \\
\hline Iron & ND & 0.200 & $\mathrm{mg} / \mathrm{L}$ & 1 & 25-Jun-2009 & 25-Jun-2009 & SW 846/6010 & $\mathrm{U}$ \\
\hline Magnesium & 5.59 & 0.200 & $\mathrm{mg} / \mathrm{L}$ & 1 & 25-Jun-2009 & 25-Jun-2009 & SW 846/6010 & \\
\hline Potassium & 2.14 & 0.200 & $\mathrm{mg} / \mathrm{L}$ & 1 & 25-Jun-2009 & 25-Jun-2009 & SW 846/6010 & \\
\hline Sodium & 4.48 & 0.200 & $\mathrm{mg} / \mathrm{L}$ & 1 & 25-Jun-2009 & 25-Jun-2009 & SW 846/6010 & \\
\hline Antimony & 0.0096 & 0.0010 & $\mathrm{mg} / \mathrm{L}$ & 2 & 26-Jun-2009 & 26-Jun-2009 & SW 846/6020 & \\
\hline Arsenic & 0.0848 & 0.0010 & $\mathrm{mg} / \mathrm{L}$ & 2 & 26-Jun-2009 & 26-Jun-2009 & SW 846/6020 & \\
\hline Barium & 0.113 & 0.0010 & $\mathrm{mg} / \mathrm{L}$ & 2 & 26-Jun-2009 & 26-Jun-2009 & SW 846/6020 & \\
\hline Beryllium & ND & 0.0010 & $\mathrm{mg} / \mathrm{L}$ & 2 & 26-Jun-2009 & 26-Jun-2009 & SW 846/6020 & $\mathrm{U}$ \\
\hline Cadmium & 0.0003 & 0.0010 & $\mathrm{mg} / \mathrm{L}$ & 2 & 26-Jun-2009 & 26-Jun-2009 & SW 846/6020 & J \\
\hline Chromium & 0.0006 & 0.0010 & $\mathrm{mg} / \mathrm{L}$ & 2 & 26-Jun-2009 & 26-Jun-2009 & SW 846/6020 & J \\
\hline Cobalt & ND & 0.0010 & $\mathrm{mg} / \mathrm{L}$ & 2 & 26-Jun-2009 & 26-Jun-2009 & SW 846/6020 & $\mathrm{U}$ \\
\hline Copper & 0.0002 & 0.0010 & $\mathrm{mg} / \mathrm{L}$ & 2 & 26-Jun-2009 & 26-Jun-2009 & SW 846/6020 & J \\
\hline Lead & ND & 0.0010 & $\mathrm{mg} / \mathrm{L}$ & 2 & 26-Jun-2009 & 26-Jun-2009 & SW 846/6020 & $\mathrm{U}$ \\
\hline Manganese & 0.0005 & 0.0010 & $\mathrm{mg} / \mathrm{L}$ & 2 & 26-Jun-2009 & 26-Jun-2009 & SW 846/6020 & J \\
\hline Molybdenum & 0.0796 & 0.0010 & $\mathrm{mg} / \mathrm{L}$ & 2 & 26-Jun-2009 & 26-Jun-2009 & SW 846/6020 & \\
\hline Nickel & 0.0007 & 0.0010 & $\mathrm{mg} / \mathrm{L}$ & 2 & 26-Jun-2009 & 26-Jun-2009 & SW 846/6020 & $\mathrm{J}$ \\
\hline Selenium & 0.0121 & 0.0010 & $\mathrm{mg} / \mathrm{L}$ & 2 & 26-Jun-2009 & 26-Jun-2009 & SW 846/6020 & \\
\hline Silver & ND & 0.0010 & $\mathrm{mg} / \mathrm{L}$ & 2 & 26-Jun-2009 & 26-Jun-2009 & SW 846/6020 & $\mathrm{U}$ \\
\hline Thallium & 0.0006 & 0.0010 & $\mathrm{mg} / \mathrm{L}$ & 2 & 26-Jun-2009 & 26-Jun-2009 & SW 846/6020 & $\mathrm{J}$ \\
\hline Vanadium & 0.0540 & 0.0010 & $\mathrm{mg} / \mathrm{L}$ & 2 & 26-Jun-2009 & 26-Jun-2009 & SW 846/6020 & \\
\hline Zinc & 0.0030 & 0.0010 & $\mathrm{mg} / \mathrm{L}$ & 2 & 26-Jun-2009 & 26-Jun-2009 & SW 846/6020 & $\mathrm{B}$ \\
\hline
\end{tabular}




\section{USACE ERDC-EP-C \\ 3909 Halls Ferry Road \\ Vicksburg, MS 39180-6199}

ERDC - ECB

Project: TVA Fly Ash Project

Reported:

$--,-$

Project Manager: Tony Bednar

03-Sep-2009

DRAFT: SLC MT N2 Hour 240 Rep A

9062302-07 (Water)

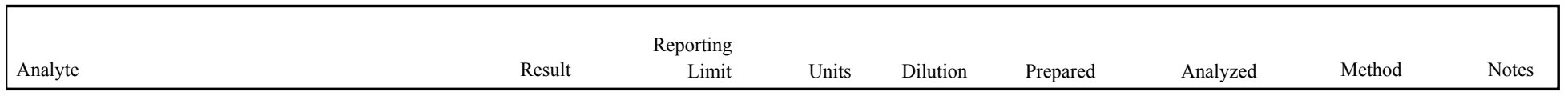

ERDC- EL-EP-C (Environmental Chemistry Branch)

DRAFT: Miscellaneous Physical/Conventional Chemistry Parameters

\begin{tabular}{llllrrrr}
\hline Total Dissolved Solids & $\mathbf{2 5 5}$ & 25.0 & mg/L & 1 & $25-J u n-2009$ & $25-J u n-2009$ & EPA 160.1 \\
Total Solids & $\mathbf{6 7 0}$ & 10.0 & mg/L & 1 & $25-J u n-2009$ & $25-J u n-2009$ & EPA 160.2 \\
Total Suspended Solids & $\mathbf{4 1 5}$ & 25.0 & mg/L & 1 & $25-J u n-2009$ & $25-J u n-2009$ & EPA 160.2
\end{tabular}




\section{USACE ERDC-EP-C \\ 3909 Halls Ferry Road \\ Vicksburg, MS 39180-6199}

ERDC - ECB

$-,-$
Project: TVA Fly Ash Project

Project Manager: Tony Bednar
Reported:

03-Sep-2009

DRAFT: SLC MT N2 Hour 240 Rep B

9062302-08 (Water)

\begin{tabular}{|c|c|c|c|c|c|c|c|c|}
\hline Analyte & Result & $\begin{array}{r}\text { porting } \\
\text { Limit }\end{array}$ & Units & Dilution & Prepared & Analyzed & Method & Notes \\
\hline
\end{tabular}

ERDC- EL-EP-C (Environmental Chemistry Branch)

DRAFT: Wet Chemistry

\begin{tabular}{llllllll}
\hline Hardness & $\mathbf{1 0 3}$ & 1.32 & $\begin{array}{c}\text { mg equil } \\
\text { CaCO3/ }\end{array}$ & 1 & 25-Jun-2009 & 25-Jun-2009
\end{tabular}

DRAFT: Metals by EPA 6000/7000 Series Methods

\begin{tabular}{|c|c|c|c|c|c|c|c|c|}
\hline Mercury & ND & 0.000005 & $\mathrm{mg} / \mathrm{L}$ & 1 & 17-Jun-2009 & 01-Jul-2009 & EPA 7471A & $\mathrm{U}$ \\
\hline Strontium & 0.569 & 0.0200 & $\mathrm{mg} / \mathrm{L}$ & 1 & 25-Jun-2009 & 25-Jun-2009 & EPA 6010B & \\
\hline Aluminum & 0.166 & 0.200 & $\mathrm{mg} / \mathrm{L}$ & 1 & 25-Jun-2009 & 25-Jun-2009 & SW 846/6010 & J \\
\hline Calcium & 32.3 & 0.200 & $\mathrm{mg} / \mathrm{L}$ & 1 & 25-Jun-2009 & 25-Jun-2009 & SW 846/6010 & \\
\hline Iron & ND & 0.200 & $\mathrm{mg} / \mathrm{L}$ & 1 & 25-Jun-2009 & 25-Jun-2009 & SW 846/6010 & $\mathrm{U}$ \\
\hline Magnesium & 5.38 & 0.200 & $\mathrm{mg} / \mathrm{L}$ & 1 & 25-Jun-2009 & 25-Jun-2009 & SW 846/6010 & \\
\hline Potassium & 1.93 & 0.200 & $\mathrm{mg} / \mathrm{L}$ & 1 & 25-Jun-2009 & 25-Jun-2009 & SW 846/6010 & \\
\hline Sodium & 4.26 & 0.200 & $\mathrm{mg} / \mathrm{L}$ & 1 & 25-Jun-2009 & 25-Jun-2009 & SW 846/6010 & \\
\hline Antimony & 0.0095 & 0.0010 & $\mathrm{mg} / \mathrm{L}$ & 2 & 26-Jun-2009 & 26-Jun-2009 & SW 846/6020 & \\
\hline Arsenic & 0.0850 & 0.0010 & $\mathrm{mg} / \mathrm{L}$ & 2 & 26-Jun-2009 & 26-Jun-2009 & SW 846/6020 & \\
\hline Barium & 0.109 & 0.0010 & $\mathrm{mg} / \mathrm{L}$ & 2 & 26-Jun-2009 & 26-Jun-2009 & SW 846/6020 & \\
\hline Beryllium & ND & 0.0010 & $\mathrm{mg} / \mathrm{L}$ & 2 & 26-Jun-2009 & 26-Jun-2009 & SW 846/6020 & $\mathrm{U}$ \\
\hline Cadmium & 0.0003 & 0.0010 & $\mathrm{mg} / \mathrm{L}$ & 2 & 26-Jun-2009 & 26-Jun-2009 & SW 846/6020 & $\mathrm{J}$ \\
\hline Chromium & 0.0006 & 0.0010 & $\mathrm{mg} / \mathrm{L}$ & 2 & 26-Jun-2009 & 26-Jun-2009 & SW 846/6020 & J \\
\hline Cobalt & ND & 0.0010 & $\mathrm{mg} / \mathrm{L}$ & 2 & 26-Jun-2009 & 26-Jun-2009 & SW 846/6020 & $\mathrm{U}$ \\
\hline Copper & ND & 0.0010 & $\mathrm{mg} / \mathrm{L}$ & 2 & 26-Jun-2009 & 26-Jun-2009 & SW 846/6020 & $\mathrm{U}$ \\
\hline Lead & ND & 0.0010 & $\mathrm{mg} / \mathrm{L}$ & 2 & 26-Jun-2009 & 26-Jun-2009 & SW 846/6020 & $\mathrm{U}$ \\
\hline Manganese & 0.0005 & 0.0010 & $\mathrm{mg} / \mathrm{L}$ & 2 & 26-Jun-2009 & 26-Jun-2009 & SW 846/6020 & $\mathrm{J}$ \\
\hline Molybdenum & 0.0790 & 0.0010 & $\mathrm{mg} / \mathrm{L}$ & 2 & 26-Jun-2009 & 26-Jun-2009 & SW 846/6020 & \\
\hline Nickel & 0.0040 & 0.0010 & $\mathrm{mg} / \mathrm{L}$ & 2 & 26-Jun-2009 & 26-Jun-2009 & SW 846/6020 & \\
\hline Selenium & 0.0130 & 0.0010 & $\mathrm{mg} / \mathrm{L}$ & 2 & 26-Jun-2009 & 26-Jun-2009 & SW 846/6020 & \\
\hline Silver & ND & 0.0010 & $\mathrm{mg} / \mathrm{L}$ & 2 & 26-Jun-2009 & 26-Jun-2009 & SW 846/6020 & $\mathrm{U}$ \\
\hline Thallium & 0.0005 & 0.0010 & $\mathrm{mg} / \mathrm{L}$ & 2 & 26-Jun-2009 & 26-Jun-2009 & SW 846/6020 & $\mathrm{J}$ \\
\hline Vanadium & 0.0534 & 0.0010 & $\mathrm{mg} / \mathrm{L}$ & 2 & 26-Jun-2009 & 26-Jun-2009 & SW 846/6020 & \\
\hline Zinc & 0.0033 & 0.0010 & $\mathrm{mg} / \mathrm{L}$ & 2 & 26-Jun-2009 & 26-Jun-2009 & SW 846/6020 & B \\
\hline
\end{tabular}




\section{USACE ERDC-EP-C \\ 3909 Halls Ferry Road \\ Vicksburg, MS 39180-6199}

ERDC - ECB

Project: TVA Fly Ash Project

Reported:

$--,-$

Project Manager: Tony Bednar

03-Sep-2009

DRAFT: SLC MT N2 Hour 240 Rep B

9062302-08 (Water)

\begin{tabular}{|c|c|c|c|c|c|c|c|c|}
\hline & & orting & & & & & & \\
\hline Analyte & Result & Limit & Units & Dilution & Prepared & Analyzed & Method & Notes \\
\hline
\end{tabular}

ERDC- EL-EP-C (Environmental Chemistry Branch)

DRAFT: Miscellaneous Physical/Conventional Chemistry Parameters

\begin{tabular}{llllrrrr}
\hline Total Dissolved Solids & $\mathbf{1 8 5}$ & 25.0 & mg/L & 1 & $25-J u n-2009$ & $25-J u n-2009$ & EPA 160.1 \\
Total Solids & $\mathbf{6 0 0}$ & 10.0 & mg/L & 1 & $25-J u n-2009$ & $25-J u n-2009$ & EPA 160.2 \\
Total Suspended Solids & $\mathbf{4 1 5}$ & 25.0 & mg/L & 1 & $25-J u n-2009$ & $25-J u n-2009$ & EPA 160.2
\end{tabular}




\section{USACE ERDC-EP-C \\ 3909 Halls Ferry Road \\ Vicksburg, MS 39180-6199}

ERDC - ECB

$-,-$
Project: TVA Fly Ash Project

Project Manager: Tony Bednar
Reported:

03-Sep-2009

DRAFT: SLC MT N2 Hour 240 Rep C

9062302-09 (Water)

\begin{tabular}{|c|c|c|c|c|c|c|c|c|}
\hline Analyte & Result & $\begin{array}{r}\text { porting } \\
\text { Limit }\end{array}$ & Units & Dilution & Prepared & Analyzed & Method & Notes \\
\hline
\end{tabular}

ERDC- EL-EP-C (Environmental Chemistry Branch)

DRAFT: Wet Chemistry

\begin{tabular}{lllllllll}
\hline Hardness & $\mathbf{1 0 4}$ & 1.32 & $\begin{array}{c}\text { mg equil } \\
\text { CaCO3/ }\end{array}$ & 1 & 25-Jun-2009 & 25-Jun-2009
\end{tabular}

DRAFT: Metals by EPA 6000/7000 Series Methods

\begin{tabular}{|c|c|c|c|c|c|c|c|c|}
\hline Mercury & ND & 0.000005 & $\mathrm{mg} / \mathrm{L}$ & 1 & 17-Jun-2009 & 02-Jul-2009 & EPA 7471A & $\mathrm{U}$ \\
\hline Strontium & 0.569 & 0.0200 & $\mathrm{mg} / \mathrm{L}$ & 1 & 25-Jun-2009 & 25-Jun-2009 & EPA $6010 B$ & \\
\hline Aluminum & 0.171 & 0.200 & $\mathrm{mg} / \mathrm{L}$ & 1 & 25-Jun-2009 & 25-Jun-2009 & SW 846/6010 & $\mathrm{J}$ \\
\hline Calcium & 32.5 & 0.200 & $\mathrm{mg} / \mathrm{L}$ & 1 & 25-Jun-2009 & 25-Jun-2009 & SW 846/6010 & \\
\hline Iron & ND & 0.200 & $\mathrm{mg} / \mathrm{L}$ & 1 & 25-Jun-2009 & 25-Jun-2009 & SW 846/6010 & $\mathrm{U}$ \\
\hline Magnesium & 5.46 & 0.200 & $\mathrm{mg} / \mathrm{L}$ & 1 & 25-Jun-2009 & 25-Jun-2009 & SW 846/6010 & \\
\hline Potassium & 1.99 & 0.200 & $\mathrm{mg} / \mathrm{L}$ & 1 & 25-Jun-2009 & 25-Jun-2009 & SW 846/6010 & \\
\hline Sodium & 4.30 & 0.200 & $\mathrm{mg} / \mathrm{L}$ & 1 & 25-Jun-2009 & 25-Jun-2009 & SW 846/6010 & \\
\hline Antimony & 0.0096 & 0.0010 & $\mathrm{mg} / \mathrm{L}$ & 2 & 26-Jun-2009 & 26-Jun-2009 & SW 846/6020 & \\
\hline Arsenic & 0.0850 & 0.0010 & $\mathrm{mg} / \mathrm{L}$ & 2 & 26-Jun-2009 & 26-Jun-2009 & SW 846/6020 & \\
\hline Barium & 0.110 & 0.0010 & $\mathrm{mg} / \mathrm{L}$ & 2 & 26-Jun-2009 & 26-Jun-2009 & SW 846/6020 & \\
\hline Beryllium & ND & 0.0010 & $\mathrm{mg} / \mathrm{L}$ & 2 & 26-Jun-2009 & 26-Jun-2009 & SW 846/6020 & $\mathrm{U}$ \\
\hline Cadmium & 0.0003 & 0.0010 & $\mathrm{mg} / \mathrm{L}$ & 2 & 26-Jun-2009 & 26-Jun-2009 & SW 846/6020 & J \\
\hline Chromium & 0.0006 & 0.0010 & $\mathrm{mg} / \mathrm{L}$ & 2 & 26-Jun-2009 & 26-Jun-2009 & SW 846/6020 & J \\
\hline Cobalt & ND & 0.0010 & $\mathrm{mg} / \mathrm{L}$ & 2 & 26-Jun-2009 & 26-Jun-2009 & SW 846/6020 & $\mathrm{U}$ \\
\hline Copper & 0.00025 & 0.0010 & $\mathrm{mg} / \mathrm{L}$ & 2 & 26-Jun-2009 & 26-Jun-2009 & SW 846/6020 & J \\
\hline Lead & ND & 0.0010 & $\mathrm{mg} / \mathrm{L}$ & 2 & 26-Jun-2009 & 26-Jun-2009 & SW 846/6020 & $\mathrm{U}$ \\
\hline Manganese & 0.0005 & 0.0010 & $\mathrm{mg} / \mathrm{L}$ & 2 & 26-Jun-2009 & 26-Jun-2009 & SW 846/6020 & J \\
\hline Molybdenum & 0.0801 & 0.0010 & $\mathrm{mg} / \mathrm{L}$ & 2 & 26-Jun-2009 & 26-Jun-2009 & SW 846/6020 & \\
\hline Nickel & 0.0006 & 0.0010 & $\mathrm{mg} / \mathrm{L}$ & 2 & 26-Jun-2009 & 26-Jun-2009 & SW 846/6020 & $\mathrm{J}$ \\
\hline Selenium & 0.0128 & 0.0010 & $\mathrm{mg} / \mathrm{L}$ & 2 & 26-Jun-2009 & 26-Jun-2009 & SW 846/6020 & \\
\hline Silver & ND & 0.0010 & $\mathrm{mg} / \mathrm{L}$ & 2 & 26-Jun-2009 & 26-Jun-2009 & SW 846/6020 & $\mathrm{U}$ \\
\hline Thallium & 0.0005 & 0.0010 & $\mathrm{mg} / \mathrm{L}$ & 2 & 26-Jun-2009 & 26-Jun-2009 & SW 846/6020 & $\mathrm{J}$ \\
\hline Vanadium & 0.0544 & 0.0010 & $\mathrm{mg} / \mathrm{L}$ & 2 & 26-Jun-2009 & 26-Jun-2009 & SW 846/6020 & \\
\hline Zinc & 0.0035 & 0.0010 & $\mathrm{mg} / \mathrm{L}$ & 2 & 26-Jun-2009 & 26-Jun-2009 & SW 846/6020 & $\mathrm{B}$ \\
\hline
\end{tabular}




\section{USACE ERDC-EP-C \\ 3909 Halls Ferry Road \\ Vicksburg, MS 39180-6199}

ERDC - ECB

Project: TVA Fly Ash Project

Reported:

$--,-$

Project Manager: Tony Bednar

03-Sep-2009

DRAFT: SLC MT N2 Hour 240 Rep C

9062302-09 (Water)

\begin{tabular}{|c|c|c|c|c|c|c|c|c|}
\hline & & orting & & & & & & \\
\hline Analyte & Result & Limit & Units & Dilution & Prepared & Analyzed & Method & Notes \\
\hline
\end{tabular}

ERDC- EL-EP-C (Environmental Chemistry Branch)

DRAFT: Miscellaneous Physical/Conventional Chemistry Parameters

\begin{tabular}{llllrrrr}
\hline Total Dissolved Solids & $\mathbf{2 3 0}$ & 25.0 & mg/L & 1 & $25-J u n-2009$ & $25-J u n-2009$ & EPA 160.1 \\
Total Solids & $\mathbf{6 4 0}$ & 10.0 & mg/L & 1 & $25-J u n-2009$ & $25-J u n-2009$ & EPA 160.2 \\
Total Suspended Solids & $\mathbf{4 1 0}$ & 25.0 & mg/L & 1 & $25-J u n-2009$ & $25-J u n-2009$ & EPA 160.2
\end{tabular}




\section{USACE ERDC-EP-C \\ 3909 Halls Ferry Road \\ Vicksburg, MS 39180-6199}

ERDC - ECB

$-,-$
Project: TVA Fly Ash Project

Project Manager: Tony Bednar
Reported:

03-Sep-2009

DRAFT: SLC MT O2 Hour 240 Rep A

9062302-10 (Water)

\begin{tabular}{|c|c|c|c|c|c|c|c|c|}
\hline Analyte & Result & $\begin{array}{r}\text { porting } \\
\text { Limit }\end{array}$ & Units & Dilution & Prepared & Analyzed & Method & Notes \\
\hline
\end{tabular}

ERDC- EL-EP-C (Environmental Chemistry Branch)

DRAFT: Wet Chemistry

\begin{tabular}{llllllll}
\hline Hardness & $\mathbf{1 0 4}$ & 1.32 & $\begin{array}{c}\text { mg equil } \\
\text { CaCO3/ }\end{array}$ & 1 & 25-Jun-2009 & 25-Jun-2009
\end{tabular}

$\mathrm{L}$

DRAFT: Metals by EPA 6000/7000 Series Methods

\begin{tabular}{|c|c|c|c|c|c|c|c|c|}
\hline Mercury & ND & 0.000005 & $\mathrm{mg} / \mathrm{L}$ & 1 & 17-Jun-2009 & 02-Jul-2009 & EPA 7471A & $\mathrm{U}$ \\
\hline Strontium & 0.575 & 0.0200 & $\mathrm{mg} / \mathrm{L}$ & 1 & 25-Jun-2009 & 25-Jun-2009 & EPA 6010B & \\
\hline Aluminum & 0.116 & 0.200 & $\mathrm{mg} / \mathrm{L}$ & 1 & 25-Jun-2009 & 25-Jun-2009 & SW 846/6010 & $\mathrm{J}$ \\
\hline Calcium & 32.7 & 0.200 & $\mathrm{mg} / \mathrm{L}$ & 1 & 25-Jun-2009 & 25-Jun-2009 & SW 846/6010 & \\
\hline Iron & ND & 0.200 & $\mathrm{mg} / \mathrm{L}$ & 1 & 25-Jun-2009 & 25-Jun-2009 & SW 846/6010 & $\mathrm{U}$ \\
\hline Magnesium & 5.35 & 0.200 & $\mathrm{mg} / \mathrm{L}$ & 1 & 25-Jun-2009 & 25-Jun-2009 & SW 846/6010 & \\
\hline Potassium & 2.04 & 0.200 & $\mathrm{mg} / \mathrm{L}$ & 1 & 25-Jun-2009 & 25-Jun-2009 & SW 846/6010 & \\
\hline Sodium & 4.48 & 0.200 & $\mathrm{mg} / \mathrm{L}$ & 1 & 25-Jun-2009 & 25-Jun-2009 & SW 846/6010 & \\
\hline Antimony & 0.0098 & 0.0010 & $\mathrm{mg} / \mathrm{L}$ & 2 & 26-Jun-2009 & 26-Jun-2009 & SW 846/6020 & \\
\hline Arsenic & 0.0798 & 0.0010 & $\mathrm{mg} / \mathrm{L}$ & 2 & 26-Jun-2009 & 26-Jun-2009 & SW 846/6020 & \\
\hline Barium & 0.117 & 0.0010 & $\mathrm{mg} / \mathrm{L}$ & 2 & 26-Jun-2009 & 26-Jun-2009 & SW 846/6020 & \\
\hline Beryllium & ND & 0.0010 & $\mathrm{mg} / \mathrm{L}$ & 2 & 26-Jun-2009 & 26-Jun-2009 & SW 846/6020 & $\mathrm{U}$ \\
\hline Cadmium & 0.0003 & 0.0010 & $\mathrm{mg} / \mathrm{L}$ & 2 & 26-Jun-2009 & 26-Jun-2009 & SW 846/6020 & J \\
\hline Chromium & 0.0007 & 0.0010 & $\mathrm{mg} / \mathrm{L}$ & 2 & 26-Jun-2009 & 26-Jun-2009 & SW 846/6020 & J \\
\hline Cobalt & ND & 0.0010 & $\mathrm{mg} / \mathrm{L}$ & 2 & 26-Jun-2009 & 26-Jun-2009 & SW 846/6020 & $\mathrm{U}$ \\
\hline Copper & 0.0003 & 0.0010 & $\mathrm{mg} / \mathrm{L}$ & 2 & 26-Jun-2009 & 26-Jun-2009 & SW 846/6020 & J \\
\hline Lead & ND & 0.0010 & $\mathrm{mg} / \mathrm{L}$ & 2 & 26-Jun-2009 & 26-Jun-2009 & SW 846/6020 & $\mathrm{U}$ \\
\hline Manganese & 0.00065 & 0.0010 & $\mathrm{mg} / \mathrm{L}$ & 2 & 26-Jun-2009 & 26-Jun-2009 & SW 846/6020 & J \\
\hline Molybdenum & 0.0787 & 0.0010 & $\mathrm{mg} / \mathrm{L}$ & 2 & 26-Jun-2009 & 26-Jun-2009 & SW 846/6020 & \\
\hline Nickel & 0.0006 & 0.0010 & $\mathrm{mg} / \mathrm{L}$ & 2 & 26-Jun-2009 & 26-Jun-2009 & SW 846/6020 & $\mathrm{J}$ \\
\hline Selenium & 0.0126 & 0.0010 & $\mathrm{mg} / \mathrm{L}$ & 2 & 26-Jun-2009 & 26-Jun-2009 & SW 846/6020 & \\
\hline Silver & ND & 0.0010 & $\mathrm{mg} / \mathrm{L}$ & 2 & 26-Jun-2009 & 26-Jun-2009 & SW 846/6020 & $\mathrm{U}$ \\
\hline Thallium & 0.0006 & 0.0010 & $\mathrm{mg} / \mathrm{L}$ & 2 & 26-Jun-2009 & 26-Jun-2009 & SW 846/6020 & $\mathrm{J}$ \\
\hline Vanadium & 0.0532 & 0.0010 & $\mathrm{mg} / \mathrm{L}$ & 2 & 26-Jun-2009 & 26-Jun-2009 & SW 846/6020 & \\
\hline Zinc & 0.0041 & 0.0010 & $\mathrm{mg} / \mathrm{L}$ & 2 & 26-Jun-2009 & 26-Jun-2009 & SW 846/6020 & $\mathrm{B}$ \\
\hline
\end{tabular}




\section{USACE ERDC-EP-C \\ 3909 Halls Ferry Road \\ Vicksburg, MS 39180-6199}

ERDC - ECB

Project: TVA Fly Ash Project

Reported:

$--,-$

Project Manager: Tony Bednar

03-Sep-2009

DRAFT: SLC MT O2 Hour 240 Rep A

9062302-10 (Water)

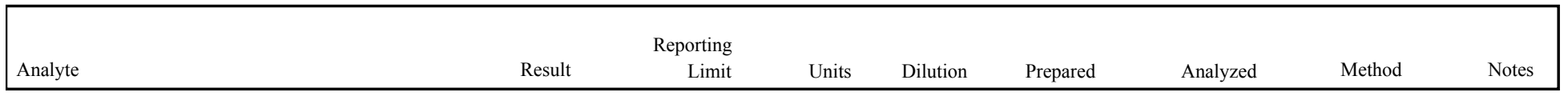

ERDC- EL-EP-C (Environmental Chemistry Branch)

DRAFT: Miscellaneous Physical/Conventional Chemistry Parameters

\begin{tabular}{llllrrrr}
\hline Total Dissolved Solids & $\mathbf{1 8 5}$ & 25.0 & mg/L & 1 & $25-J u n-2009$ & $25-J u n-2009$ & EPA 160.1 \\
Total Solids & $\mathbf{6 4 0}$ & 10.0 & mg/L & 1 & $25-J u n-2009$ & $25-J u n-2009$ & EPA 160.2 \\
Total Suspended Solids & $\mathbf{4 5 5}$ & 25.0 & mg/L & 1 & $25-J u n-2009$ & $25-J u n-2009$ & EPA 160.2
\end{tabular}




\section{USACE ERDC-EP-C \\ 3909 Halls Ferry Road \\ Vicksburg, MS 39180-6199}

ERDC - ECB

$-,-$
Project: TVA Fly Ash Project

Project Manager: Tony Bednar
Reported:

03-Sep-2009

DRAFT: SLC MT O2 Hour 240 Rep B

9062302-11 (Water)

\begin{tabular}{|c|c|c|c|c|c|c|c|c|}
\hline Analyte & Result & $\begin{array}{r}\text { porting } \\
\text { Limit }\end{array}$ & Units & Dilution & Prepared & Analyzed & Method & Notes \\
\hline
\end{tabular}

ERDC- EL-EP-C (Environmental Chemistry Branch)

DRAFT: Wet Chemistry

\begin{tabular}{llllllll}
\hline Hardness & $\mathbf{1 0 4}$ & 1.32 & $\begin{array}{c}\text { mg equil } \\
\text { CaCO3/ }\end{array}$ & 1 & 25-Jun-2009 & 25-Jun-2009
\end{tabular}

DRAFT: Metals by EPA 6000/7000 Series Methods

\begin{tabular}{|c|c|c|c|c|c|c|c|c|}
\hline Mercury & ND & 0.000005 & $\mathrm{mg} / \mathrm{L}$ & 1 & 17-Jun-2009 & 02-Jul-2009 & EPA 7471A & $\mathrm{U}$ \\
\hline Strontium & 0.574 & 0.0200 & $\mathrm{mg} / \mathrm{L}$ & 1 & 25-Jun-2009 & 25-Jun-2009 & EPA $6010 B$ & \\
\hline Aluminum & 0.124 & 0.200 & $\mathrm{mg} / \mathrm{L}$ & 1 & 25-Jun-2009 & 25-Jun-2009 & SW 846/6010 & $\mathrm{J}$ \\
\hline Calcium & 32.8 & 0.200 & $\mathrm{mg} / \mathrm{L}$ & 1 & 25-Jun-2009 & 25-Jun-2009 & SW 846/6010 & \\
\hline Iron & ND & 0.200 & $\mathrm{mg} / \mathrm{L}$ & 1 & 25-Jun-2009 & 25-Jun-2009 & SW 846/6010 & $\mathrm{U}$ \\
\hline Magnesium & 5.37 & 0.200 & $\mathrm{mg} / \mathrm{L}$ & 1 & 25-Jun-2009 & 25-Jun-2009 & SW 846/6010 & \\
\hline Potassium & 1.98 & 0.200 & $\mathrm{mg} / \mathrm{L}$ & 1 & 25-Jun-2009 & 25-Jun-2009 & SW 846/6010 & \\
\hline Sodium & 4.32 & 0.200 & $\mathrm{mg} / \mathrm{L}$ & 1 & 25-Jun-2009 & 25-Jun-2009 & SW 846/6010 & \\
\hline Antimony & 0.0094 & 0.0010 & $\mathrm{mg} / \mathrm{L}$ & 2 & 26-Jun-2009 & 26-Jun-2009 & SW 846/6020 & \\
\hline Arsenic & 0.0812 & 0.0010 & $\mathrm{mg} / \mathrm{L}$ & 2 & 26-Jun-2009 & 26-Jun-2009 & SW 846/6020 & \\
\hline Barium & 0.115 & 0.0010 & $\mathrm{mg} / \mathrm{L}$ & 2 & 26-Jun-2009 & 26-Jun-2009 & SW 846/6020 & \\
\hline Beryllium & ND & 0.0010 & $\mathrm{mg} / \mathrm{L}$ & 2 & 26-Jun-2009 & 26-Jun-2009 & SW 846/6020 & $\mathrm{U}$ \\
\hline Cadmium & 0.0003 & 0.0010 & $\mathrm{mg} / \mathrm{L}$ & 2 & 26-Jun-2009 & 26-Jun-2009 & SW 846/6020 & J \\
\hline Chromium & 0.0007 & 0.0010 & $\mathrm{mg} / \mathrm{L}$ & 2 & 26-Jun-2009 & 26-Jun-2009 & SW 846/6020 & J \\
\hline Cobalt & ND & 0.0010 & $\mathrm{mg} / \mathrm{L}$ & 2 & 26-Jun-2009 & 26-Jun-2009 & SW 846/6020 & $\mathrm{U}$ \\
\hline Copper & 0.0003 & 0.0010 & $\mathrm{mg} / \mathrm{L}$ & 2 & 26-Jun-2009 & 26-Jun-2009 & SW 846/6020 & J \\
\hline Lead & ND & 0.0010 & $\mathrm{mg} / \mathrm{L}$ & 2 & 26-Jun-2009 & 26-Jun-2009 & SW 846/6020 & $\mathrm{U}$ \\
\hline Manganese & 0.0005 & 0.0010 & $\mathrm{mg} / \mathrm{L}$ & 2 & 26-Jun-2009 & 26-Jun-2009 & SW 846/6020 & J \\
\hline Molybdenum & 0.0778 & 0.0010 & $\mathrm{mg} / \mathrm{L}$ & 2 & 26-Jun-2009 & 26-Jun-2009 & SW 846/6020 & \\
\hline Nickel & 0.0006 & 0.0010 & $\mathrm{mg} / \mathrm{L}$ & 2 & 26-Jun-2009 & 26-Jun-2009 & SW 846/6020 & $\mathrm{J}$ \\
\hline Selenium & 0.0128 & 0.0010 & $\mathrm{mg} / \mathrm{L}$ & 2 & 26-Jun-2009 & 26-Jun-2009 & SW 846/6020 & \\
\hline Silver & ND & 0.0010 & $\mathrm{mg} / \mathrm{L}$ & 2 & 26-Jun-2009 & 26-Jun-2009 & SW 846/6020 & $\mathrm{U}$ \\
\hline Thallium & 0.00059 & 0.0010 & $\mathrm{mg} / \mathrm{L}$ & 2 & 26-Jun-2009 & 26-Jun-2009 & SW 846/6020 & $\mathrm{J}$ \\
\hline Vanadium & 0.0529 & 0.0010 & $\mathrm{mg} / \mathrm{L}$ & 2 & 26-Jun-2009 & 26-Jun-2009 & SW 846/6020 & \\
\hline Zinc & 0.0033 & 0.0010 & $\mathrm{mg} / \mathrm{L}$ & 2 & 26-Jun-2009 & 26-Jun-2009 & SW 846/6020 & $\mathrm{B}$ \\
\hline
\end{tabular}




\section{USACE ERDC-EP-C \\ 3909 Halls Ferry Road \\ Vicksburg, MS 39180-6199}

ERDC - ECB

Project: TVA Fly Ash Project

Reported:

$--,-$

Project Manager: Tony Bednar

03-Sep-2009

DRAFT: SLC MT O2 Hour 240 Rep B

9062302-11 (Water)

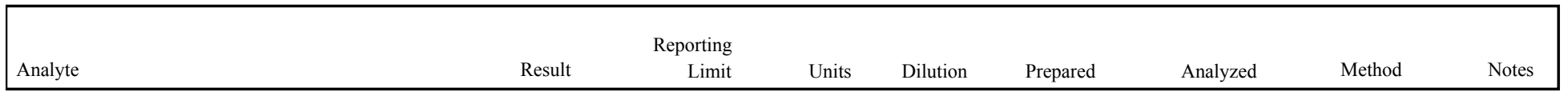

ERDC- EL-EP-C (Environmental Chemistry Branch)

DRAFT: Miscellaneous Physical/Conventional Chemistry Parameters

\begin{tabular}{llllrrrr}
\hline Total Dissolved Solids & $\mathbf{2 2 5}$ & 25.0 & mg/L & 1 & $25-J u n-2009$ & $25-J u n-2009$ & EPA 160.1 \\
Total Solids & $\mathbf{6 6 0}$ & 10.0 & mg/L & 1 & $25-J u n-2009$ & $25-J u n-2009$ & EPA 160.2 \\
Total Suspended Solids & $\mathbf{4 3 5}$ & 25.0 & mg/L & 1 & $25-J u n-2009$ & $25-J u n-2009$ & EPA 160.2
\end{tabular}




\section{USACE ERDC-EP-C \\ 3909 Halls Ferry Road \\ Vicksburg, MS 39180-6199}

ERDC - ECB

$-,-$
Project: TVA Fly Ash Project

Project Manager: Tony Bednar
Reported:

03-Sep-2009

DRAFT: SLC MT O2 Hour 240 Rep C

9062302-12 (Water)

\begin{tabular}{|c|c|c|c|c|c|c|c|c|}
\hline Analyte & Result & $\begin{array}{r}\text { porting } \\
\text { Limit }\end{array}$ & Units & Dilution & Prepared & Analyzed & Method & Notes \\
\hline
\end{tabular}

ERDC- EL-EP-C (Environmental Chemistry Branch)

DRAFT: Wet Chemistry

\begin{tabular}{llllllll}
\hline Hardness & $\mathbf{1 0 6}$ & 1.32 & $\begin{array}{c}\text { mg equil } \\
\text { CaCO3/ }\end{array}$ & 1 & 25-Jun-2009 & 25-Jun-2009
\end{tabular}

$\mathrm{L}$

DRAFT: Metals by EPA 6000/7000 Series Methods

\begin{tabular}{|c|c|c|c|c|c|c|c|c|}
\hline Mercury & ND & 0.000005 & $\mathrm{mg} / \mathrm{L}$ & 1 & 17-Jun-2009 & 02-Jul-2009 & EPA 7471A & $\mathrm{U}$ \\
\hline Strontium & 0.580 & 0.0200 & $\mathrm{mg} / \mathrm{L}$ & 1 & 25-Jun-2009 & 25-Jun-2009 & EPA 6010B & \\
\hline Aluminum & 0.123 & 0.200 & $\mathrm{mg} / \mathrm{L}$ & 1 & 25-Jun-2009 & 25-Jun-2009 & SW 846/6010 & $\mathrm{J}$ \\
\hline Calcium & 33.1 & 0.200 & $\mathrm{mg} / \mathrm{L}$ & 1 & 25-Jun-2009 & 25-Jun-2009 & SW 846/6010 & \\
\hline Iron & ND & 0.200 & $\mathrm{mg} / \mathrm{L}$ & 1 & 25-Jun-2009 & 25-Jun-2009 & SW 846/6010 & $\mathrm{U}$ \\
\hline Magnesium & 5.54 & 0.200 & $\mathrm{mg} / \mathrm{L}$ & 1 & 25-Jun-2009 & 25-Jun-2009 & SW 846/6010 & \\
\hline Potassium & 2.04 & 0.200 & $\mathrm{mg} / \mathrm{L}$ & 1 & 25-Jun-2009 & 25-Jun-2009 & SW 846/6010 & \\
\hline Sodium & 4.33 & 0.200 & $\mathrm{mg} / \mathrm{L}$ & 1 & 25-Jun-2009 & 25-Jun-2009 & SW 846/6010 & \\
\hline Antimony & 0.0098 & 0.0010 & $\mathrm{mg} / \mathrm{L}$ & 2 & 26-Jun-2009 & 26-Jun-2009 & SW 846/6020 & \\
\hline Arsenic & 0.0829 & 0.0010 & $\mathrm{mg} / \mathrm{L}$ & 2 & 26-Jun-2009 & 26-Jun-2009 & SW 846/6020 & \\
\hline Barium & 0.118 & 0.0010 & $\mathrm{mg} / \mathrm{L}$ & 2 & 26-Jun-2009 & 26-Jun-2009 & SW 846/6020 & \\
\hline Beryllium & ND & 0.0010 & $\mathrm{mg} / \mathrm{L}$ & 2 & 26-Jun-2009 & 26-Jun-2009 & SW 846/6020 & $\mathrm{U}$ \\
\hline Cadmium & 0.0004 & 0.0010 & $\mathrm{mg} / \mathrm{L}$ & 2 & 26-Jun-2009 & 26-Jun-2009 & SW 846/6020 & J \\
\hline Chromium & 0.0007 & 0.0010 & $\mathrm{mg} / \mathrm{L}$ & 2 & 26-Jun-2009 & 26-Jun-2009 & SW 846/6020 & J \\
\hline Cobalt & ND & 0.0010 & $\mathrm{mg} / \mathrm{L}$ & 2 & 26-Jun-2009 & 26-Jun-2009 & SW 846/6020 & $\mathrm{U}$ \\
\hline Copper & 0.0003 & 0.0010 & $\mathrm{mg} / \mathrm{L}$ & 2 & 26-Jun-2009 & 26-Jun-2009 & SW 846/6020 & J \\
\hline Lead & ND & 0.0010 & $\mathrm{mg} / \mathrm{L}$ & 2 & 26-Jun-2009 & 26-Jun-2009 & SW 846/6020 & $\mathrm{U}$ \\
\hline Manganese & 0.0005 & 0.0010 & $\mathrm{mg} / \mathrm{L}$ & 2 & 26-Jun-2009 & 26-Jun-2009 & SW 846/6020 & J \\
\hline Molybdenum & 0.0801 & 0.0010 & $\mathrm{mg} / \mathrm{L}$ & 2 & 26-Jun-2009 & 26-Jun-2009 & SW 846/6020 & \\
\hline Nickel & 0.0006 & 0.0010 & $\mathrm{mg} / \mathrm{L}$ & 2 & 26-Jun-2009 & 26-Jun-2009 & SW 846/6020 & $\mathrm{J}$ \\
\hline Selenium & 0.0128 & 0.0010 & $\mathrm{mg} / \mathrm{L}$ & 2 & 26-Jun-2009 & 26-Jun-2009 & SW 846/6020 & \\
\hline Silver & ND & 0.0010 & $\mathrm{mg} / \mathrm{L}$ & 2 & 26-Jun-2009 & 26-Jun-2009 & SW 846/6020 & $\mathrm{U}$ \\
\hline Thallium & 0.0006 & 0.0010 & $\mathrm{mg} / \mathrm{L}$ & 2 & 26-Jun-2009 & 26-Jun-2009 & SW 846/6020 & $\mathrm{J}$ \\
\hline Vanadium & 0.0520 & 0.0010 & $\mathrm{mg} / \mathrm{L}$ & 2 & 26-Jun-2009 & 26-Jun-2009 & SW 846/6020 & \\
\hline Zinc & 0.0036 & 0.0010 & $\mathrm{mg} / \mathrm{L}$ & 2 & 26-Jun-2009 & 26-Jun-2009 & SW 846/6020 & $\mathrm{B}$ \\
\hline
\end{tabular}




\section{USACE ERDC-EP-C \\ 3909 Halls Ferry Road \\ Vicksburg, MS 39180-6199}

ERDC - ECB

Project: TVA Fly Ash Project

Reported:

$--,-$

Project Manager: Tony Bednar

03-Sep-2009

DRAFT: SLC MT O2 Hour 240 Rep C

9062302-12 (Water)

\begin{tabular}{|lrrrrrrrr}
\hline & & & & & & & & \\
Analyte & Result & Limit & Units & Dilution & Prepared & Analyzed & Method & Notes \\
\hline
\end{tabular}

ERDC- EL-EP-C (Environmental Chemistry Branch)

DRAFT: Miscellaneous Physical/Conventional Chemistry Parameters

\begin{tabular}{lllllllr}
\hline Total Dissolved Solids & $\mathbf{2 2 5}$ & 25.0 & $\mathrm{mg} / \mathrm{L}$ & 1 & 25-Jun-2009 & 25-Jun-2009 & EPA 160.1 \\
Total Solids & $\mathbf{7 4 0}$ & 10.0 & $\mathrm{mg} / \mathrm{L}$ & 1 & 25-Jun-2009 & 25 -Jun-2009 & EPA 160.2 \\
Total Suspended Solids & $\mathbf{5 1 5}$ & 25.0 & $\mathrm{mg} / \mathrm{L}$ & 1 & 25-Jun-2009 & 25 -Jun-2009 & EPA 160.2
\end{tabular}




\section{USACE ERDC-EP-C \\ 3909 Halls Ferry Road \\ Vicksburg, MS 39180-6199}

ERDC - ECB

$-\cdot$,
Project: TVA Fly Ash Project

Project Manager: Tony Bednar
Reported:

03-Sep-2009

DRAFT: EMR MT N2 Hour 240 Rep A - Total Metals

9062302-13 (Water)

\begin{tabular}{|c|c|c|c|c|c|c|c|c|}
\hline Analyte & Result & $\begin{array}{r}\text { porting } \\
\text { Limit }\end{array}$ & Units & Dilution & Prepared & Analyzed & Method & Notes \\
\hline
\end{tabular}

ERDC- EL-EP-C (Environmental Chemistry Branch)

DRAFT: Wet Chemistry

\begin{tabular}{llllllll}
\hline Hardness & $\mathbf{6 6 . 5}$ & 1.32 & $\begin{array}{c}\text { mg equil } \\
\text { CaCO3/ }\end{array}$ & 4 & 25-Jun-2009 & 25-Jun-2009
\end{tabular}

$\mathrm{L}$

DRAFT: Metals by EPA 6000/7000 Series Methods

\begin{tabular}{|c|c|c|c|c|c|c|c|c|}
\hline Mercury & 0.000205 & 0.000005 & $\mathrm{mg} / \mathrm{L}$ & 2 & 24-Jul-2009 & 24-Jul-2009 & EPA 7471A & \\
\hline Strontium & 0.441 & 0.0800 & $\mathrm{mg} / \mathrm{L}$ & 4 & 25-Jun-2009 & 25-Jun-2009 & EPA 6010B & \\
\hline Aluminum & 22.1 & 0.200 & $\mathrm{mg} / \mathrm{L}$ & 4 & 25-Jun-2009 & 25-Jun-2009 & SW 846/6010 & \\
\hline Calcium & 19.2 & 0.200 & $\mathrm{mg} / \mathrm{L}$ & 4 & 25-Jun-2009 & 25-Jun-2009 & SW 846/6010 & B \\
\hline Iron & 8.97 & 0.200 & $\mathrm{mg} / \mathrm{L}$ & 4 & 25-Jun-2009 & 25-Jun-2009 & SW 846/6010 & \\
\hline Magnesium & 4.51 & 0.200 & $\mathrm{mg} / \mathrm{L}$ & 4 & 25-Jun-2009 & 25-Jun-2009 & SW 846/6010 & \\
\hline Potassium & 4.98 & 0.200 & $\mathrm{mg} / \mathrm{L}$ & 4 & 25-Jun-2009 & 25-Jun-2009 & SW 846/6010 & B \\
\hline Sodium & 3.11 & 0.200 & $\mathrm{mg} / \mathrm{L}$ & 4 & 25-Jun-2009 & 25-Jun-2009 & SW 846/6010 & B \\
\hline Antimony & 0.0069 & 0.0020 & $\mathrm{mg} / \mathrm{L}$ & 4 & 26-Jun-2009 & 26-Jun-2009 & SW 846/6020 & B \\
\hline Arsenic & 0.132 & 0.0020 & $\mathrm{mg} / \mathrm{L}$ & 4 & 26-Jun-2009 & 26-Jun-2009 & SW 846/6020 & \\
\hline Barium & 0.556 & 0.0020 & $\mathrm{mg} / \mathrm{L}$ & 4 & 26-Jun-2009 & 26-Jun-2009 & SW 846/6020 & \\
\hline Beryllium & 0.0033 & 0.0020 & $\mathrm{mg} / \mathrm{L}$ & 4 & 26-Jun-2009 & 26-Jun-2009 & SW 846/6020 & \\
\hline Cadmium & 0.0005 & 0.0020 & $\mathrm{mg} / \mathrm{L}$ & 4 & 26-Jun-2009 & 26-Jun-2009 & SW 846/6020 & J \\
\hline Chromium & 0.0245 & 0.0020 & $\mathrm{mg} / \mathrm{L}$ & 4 & 26-Jun-2009 & 26-Jun-2009 & SW 846/6020 & \\
\hline Cobalt & 0.0142 & 0.0020 & $\mathrm{mg} / \mathrm{L}$ & 4 & 26-Jun-2009 & 26-Jun-2009 & SW 846/6020 & \\
\hline Copper & 0.0516 & 0.0020 & $\mathrm{mg} / \mathrm{L}$ & 4 & 26-Jun-2009 & 26-Jun-2009 & SW 846/6020 & \\
\hline Lead & 0.0399 & 0.0020 & $\mathrm{mg} / \mathrm{L}$ & 4 & 26-Jun-2009 & 26-Jun-2009 & SW 846/6020 & \\
\hline Manganese & 0.0774 & 0.0020 & $\mathrm{mg} / \mathrm{L}$ & 4 & 26-Jun-2009 & 26-Jun-2009 & SW 846/6020 & \\
\hline Molybdenum & 0.0067 & 0.0020 & $\mathrm{mg} / \mathrm{L}$ & 4 & 26-Jun-2009 & 26-Jun-2009 & SW 846/6020 & \\
\hline Nickel & 0.0262 & 0.0020 & $\mathrm{mg} / \mathrm{L}$ & 4 & 26-Jun-2009 & 26-Jun-2009 & SW 846/6020 & \\
\hline Selenium & 0.0072 & 0.0020 & $\mathrm{mg} / \mathrm{L}$ & 4 & 26-Jun-2009 & 26-Jun-2009 & SW 846/6020 & \\
\hline Silver & 0.0004 & 0.0020 & $\mathrm{mg} / \mathrm{L}$ & 4 & 26-Jun-2009 & 26-Jun-2009 & SW 846/6020 & J \\
\hline Thallium & 0.0028 & 0.0020 & $\mathrm{mg} / \mathrm{L}$ & 4 & 26-Jun-2009 & 26-Jun-2009 & SW 846/6020 & \\
\hline Vanadium & 0.106 & 0.0020 & $\mathrm{mg} / \mathrm{L}$ & 4 & 26-Jun-2009 & 26-Jun-2009 & SW 846/6020 & \\
\hline Zinc & 0.0678 & 0.0020 & $\mathrm{mg} / \mathrm{L}$ & 4 & 26-Jun-2009 & 26-Jun-2009 & SW 846/6020 & B \\
\hline
\end{tabular}




\section{USACE ERDC-EP-C \\ 3909 Halls Ferry Road \\ Vicksburg, MS 39180-6199}

ERDC - ECB

$-,-$
Project: TVA Fly Ash Project

Project Manager: Tony Bednar
Reported:

03-Sep-2009

DRAFT: EMR MT N2 Hour 240 Rep B - Total Metals

9062302-14 (Water)

\begin{tabular}{|c|c|c|c|c|c|c|c|c|}
\hline Analyte & Result & $\begin{array}{r}\text { porting } \\
\text { Limit }\end{array}$ & Units & Dilution & Prepared & Analyzed & Method & Notes \\
\hline
\end{tabular}

ERDC- EL-EP-C (Environmental Chemistry Branch)

DRAFT: Wet Chemistry

\begin{tabular}{llllllll}
\hline Hardness & $\mathbf{6 4 . 6}$ & 1.32 & $\begin{array}{c}\text { mg equil } \\
\text { CaCO3/ }\end{array}$ & 4 & 25-Jun-2009 & 25-Jun-2009
\end{tabular}

$\mathrm{L}$

DRAFT: Metals by EPA 6000/7000 Series Methods

\begin{tabular}{|c|c|c|c|c|c|c|c|c|}
\hline Mercury & 0.00013 & 0.000005 & $\mathrm{mg} / \mathrm{L}$ & 1 & 24-Jul-2009 & 20-Aug-2009 & EPA 7471A & \\
\hline Strontium & 0.417 & 0.0800 & $\mathrm{mg} / \mathrm{L}$ & 4 & 25-Jun-2009 & 25-Jun-2009 & EPA 6010B & \\
\hline Aluminum & 19.8 & 0.200 & $\mathrm{mg} / \mathrm{L}$ & 4 & 25-Jun-2009 & 25-Jun-2009 & SW 846/6010 & \\
\hline Calcium & 18.7 & 0.200 & $\mathrm{mg} / \mathrm{L}$ & 4 & 25-Jun-2009 & 25-Jun-2009 & SW 846/6010 & B \\
\hline Iron & 8.03 & 0.200 & $\mathrm{mg} / \mathrm{L}$ & 4 & 25-Jun-2009 & 25-Jun-2009 & SW 846/6010 & \\
\hline Magnesium & 4.34 & 0.200 & $\mathrm{mg} / \mathrm{L}$ & 4 & 25-Jun-2009 & 25-Jun-2009 & SW 846/6010 & \\
\hline Potassium & 4.71 & 0.200 & $\mathrm{mg} / \mathrm{L}$ & 4 & 25-Jun-2009 & 25-Jun-2009 & SW 846/6010 & B \\
\hline Sodium & 3.36 & 0.200 & $\mathrm{mg} / \mathrm{L}$ & 4 & 25-Jun-2009 & 25-Jun-2009 & SW 846/6010 & B \\
\hline Antimony & 0.0052 & 0.0020 & $\mathrm{mg} / \mathrm{L}$ & 4 & 26-Jun-2009 & 26-Jun-2009 & SW 846/6020 & B \\
\hline Arsenic & 0.120 & 0.0020 & $\mathrm{mg} / \mathrm{L}$ & 4 & 26-Jun-2009 & 26-Jun-2009 & SW 846/6020 & \\
\hline Barium & 0.524 & 0.0020 & $\mathrm{mg} / \mathrm{L}$ & 4 & 26-Jun-2009 & 26-Jun-2009 & SW 846/6020 & \\
\hline Beryllium & 0.0029 & 0.0020 & $\mathrm{mg} / \mathrm{L}$ & 4 & 26-Jun-2009 & 26-Jun-2009 & SW 846/6020 & \\
\hline Cadmium & 0.0006 & 0.0020 & $\mathrm{mg} / \mathrm{L}$ & 4 & 26-Jun-2009 & 26-Jun-2009 & SW 846/6020 & $\mathrm{J}$ \\
\hline Chromium & 0.0238 & 0.0020 & $\mathrm{mg} / \mathrm{L}$ & 4 & 26-Jun-2009 & 26-Jun-2009 & SW 846/6020 & \\
\hline Cobalt & 0.0133 & 0.0020 & $\mathrm{mg} / \mathrm{L}$ & 4 & 26-Jun-2009 & 26-Jun-2009 & SW 846/6020 & \\
\hline Copper & 0.0474 & 0.0020 & $\mathrm{mg} / \mathrm{L}$ & 4 & 26-Jun-2009 & 26-Jun-2009 & SW 846/6020 & \\
\hline Lead & 0.0353 & 0.0020 & $\mathrm{mg} / \mathrm{L}$ & 4 & 26-Jun-2009 & 26-Jun-2009 & SW 846/6020 & \\
\hline Manganese & 0.0695 & 0.0020 & $\mathrm{mg} / \mathrm{L}$ & 4 & 26-Jun-2009 & 26-Jun-2009 & SW 846/6020 & \\
\hline Molybdenum & 0.0064 & 0.0020 & $\mathrm{mg} / \mathrm{L}$ & 4 & 26-Jun-2009 & 26-Jun-2009 & SW 846/6020 & \\
\hline Nickel & 0.0270 & 0.0020 & $\mathrm{mg} / \mathrm{L}$ & 4 & 26-Jun-2009 & 26-Jun-2009 & SW 846/6020 & \\
\hline Selenium & 0.0077 & 0.0020 & $\mathrm{mg} / \mathrm{L}$ & 4 & 26-Jun-2009 & 26-Jun-2009 & SW 846/6020 & \\
\hline Silver & ND & 0.0020 & $\mathrm{mg} / \mathrm{L}$ & 4 & 26-Jun-2009 & 26-Jun-2009 & SW 846/6020 & $\mathrm{U}$ \\
\hline Thallium & 0.0026 & 0.0020 & $\mathrm{mg} / \mathrm{L}$ & 4 & 26-Jun-2009 & 26-Jun-2009 & SW 846/6020 & \\
\hline Vanadium & 0.103 & 0.0020 & $\mathrm{mg} / \mathrm{L}$ & 4 & 26-Jun-2009 & 26-Jun-2009 & SW 846/6020 & \\
\hline Zinc & 0.0685 & 0.0020 & $\mathrm{mg} / \mathrm{L}$ & 4 & 26-Jun-2009 & 26-Jun-2009 & SW 846/6020 & B \\
\hline
\end{tabular}




\section{USACE ERDC-EP-C \\ 3909 Halls Ferry Road \\ Vicksburg, MS 39180-6199}

ERDC - ECB

$-,-$
Project: TVA Fly Ash Project

Project Manager: Tony Bednar
Reported:

03-Sep-2009

DRAFT: EMR MT N2 Hour 240 Rep C - Total Metals

9062302-15 (Water)

\begin{tabular}{|c|c|c|c|c|c|c|c|c|}
\hline Analyte & Result & $\begin{array}{r}\text { porting } \\
\text { Limit }\end{array}$ & Units & Dilution & Prepared & Analyzed & Method & Notes \\
\hline
\end{tabular}

ERDC- EL-EP-C (Environmental Chemistry Branch)

DRAFT: Wet Chemistry

\begin{tabular}{llllllll}
\hline Hardness & $\mathbf{6 9 . 6}$ & 1.32 & $\begin{array}{c}\text { mg equil } \\
\text { CaCO3/ }\end{array}$ & 4 & 25-Jun-2009 & 25-Jun-2009
\end{tabular}

$\mathrm{L}$

DRAFT: Metals by EPA 6000/7000 Series Methods

\begin{tabular}{|c|c|c|c|c|c|c|c|c|}
\hline Mercury & 0.000175 & 0.000005 & $\mathrm{mg} / \mathrm{L}$ & 1 & 24-Jul-2009 & 20-Aug-2009 & EPA 7471A & \\
\hline Strontium & 0.454 & 0.0800 & $\mathrm{mg} / \mathrm{L}$ & 4 & 25-Jun-2009 & 25-Jun-2009 & EPA 6010B & \\
\hline Aluminum & 22.5 & 0.200 & $\mathrm{mg} / \mathrm{L}$ & 4 & 25-Jun-2009 & 25-Jun-2009 & SW 846/6010 & \\
\hline Calcium & 20.2 & 0.200 & $\mathrm{mg} / \mathrm{L}$ & 4 & 25-Jun-2009 & 25-Jun-2009 & SW 846/6010 & B \\
\hline Iron & 9.19 & 0.200 & $\mathrm{mg} / \mathrm{L}$ & 4 & 25-Jun-2009 & 25-Jun-2009 & SW 846/6010 & \\
\hline Magnesium & 4.68 & 0.200 & $\mathrm{mg} / \mathrm{L}$ & 4 & 25-Jun-2009 & 25-Jun-2009 & SW 846/6010 & \\
\hline Potassium & 5.29 & 0.200 & $\mathrm{mg} / \mathrm{L}$ & 4 & 25-Jun-2009 & 25-Jun-2009 & SW 846/6010 & B \\
\hline Sodium & 3.52 & 0.200 & $\mathrm{mg} / \mathrm{L}$ & 4 & 25-Jun-2009 & 25-Jun-2009 & SW 846/6010 & B \\
\hline Antimony & 0.0053 & 0.0020 & $\mathrm{mg} / \mathrm{L}$ & 4 & 26-Jun-2009 & 26-Jun-2009 & SW 846/6020 & B \\
\hline Arsenic & 0.136 & 0.0020 & $\mathrm{mg} / \mathrm{L}$ & 4 & 26-Jun-2009 & 26-Jun-2009 & SW 846/6020 & \\
\hline Barium & 0.577 & 0.0020 & $\mathrm{mg} / \mathrm{L}$ & 4 & 26-Jun-2009 & 26-Jun-2009 & SW 846/6020 & \\
\hline Beryllium & 0.0036 & 0.0020 & $\mathrm{mg} / \mathrm{L}$ & 4 & 26-Jun-2009 & 26-Jun-2009 & SW 846/6020 & \\
\hline Cadmium & 0.00069 & 0.0020 & $\mathrm{mg} / \mathrm{L}$ & 4 & 26-Jun-2009 & 26-Jun-2009 & SW 846/6020 & J \\
\hline Chromium & 0.0253 & 0.0020 & $\mathrm{mg} / \mathrm{L}$ & 4 & 26-Jun-2009 & 26-Jun-2009 & SW 846/6020 & \\
\hline Cobalt & 0.0144 & 0.0020 & $\mathrm{mg} / \mathrm{L}$ & 4 & 26-Jun-2009 & 26-Jun-2009 & SW 846/6020 & \\
\hline Copper & 0.0605 & 0.0020 & $\mathrm{mg} / \mathrm{L}$ & 4 & 26-Jun-2009 & 26-Jun-2009 & SW 846/6020 & \\
\hline Lead & 0.0410 & 0.0020 & $\mathrm{mg} / \mathrm{L}$ & 4 & 26-Jun-2009 & 26-Jun-2009 & SW 846/6020 & \\
\hline Manganese & 0.0803 & 0.0020 & $\mathrm{mg} / \mathrm{L}$ & 4 & 26-Jun-2009 & 26-Jun-2009 & SW 846/6020 & \\
\hline Molybdenum & 0.0071 & 0.0020 & $\mathrm{mg} / \mathrm{L}$ & 4 & 26-Jun-2009 & 26-Jun-2009 & SW 846/6020 & \\
\hline Nickel & 0.0275 & 0.0020 & $\mathrm{mg} / \mathrm{L}$ & 4 & 26-Jun-2009 & 26-Jun-2009 & SW 846/6020 & \\
\hline Selenium & 0.0086 & 0.0020 & $\mathrm{mg} / \mathrm{L}$ & 4 & 26-Jun-2009 & 26-Jun-2009 & SW 846/6020 & \\
\hline Silver & 0.0004 & 0.0020 & $\mathrm{mg} / \mathrm{L}$ & 4 & 26-Jun-2009 & 26-Jun-2009 & SW 846/6020 & J \\
\hline Thallium & 0.0029 & 0.0020 & $\mathrm{mg} / \mathrm{L}$ & 4 & 26-Jun-2009 & 26-Jun-2009 & SW 846/6020 & \\
\hline Vanadium & 0.112 & 0.0020 & $\mathrm{mg} / \mathrm{L}$ & 4 & 26-Jun-2009 & 26-Jun-2009 & SW 846/6020 & \\
\hline Zinc & 0.0727 & 0.0020 & $\mathrm{mg} / \mathrm{L}$ & 4 & 26-Jun-2009 & 26-Jun-2009 & SW 846/6020 & B \\
\hline
\end{tabular}




\section{USACE ERDC-EP-C \\ 3909 Halls Ferry Road \\ Vicksburg, MS 39180-6199}

ERDC - ECB

$-\cdot$,
Project: TVA Fly Ash Project

Project Manager: Tony Bednar
Reported:

03-Sep-2009

DRAFT: EMR MT O2 Hour 240 Rep A - Total Metals

9062302-16 (Water)

\begin{tabular}{|c|c|c|c|c|c|c|c|c|}
\hline Analyte & Result & $\begin{array}{r}\text { porting } \\
\text { Limit }\end{array}$ & Units & Dilution & Prepared & Analyzed & Method & Notes \\
\hline
\end{tabular}

ERDC- EL-EP-C (Environmental Chemistry Branch)

DRAFT: Wet Chemistry

\begin{tabular}{llllllll}
\hline Hardness & $\mathbf{6 8 . 4}$ & 1.32 & $\begin{array}{c}\text { mg equil } \\
\text { CaCO3/ }\end{array}$ & 4 & 25-Jun-2009 & 25-Jun-2009
\end{tabular}

$\mathrm{L}$

DRAFT: Metals by EPA 6000/7000 Series Methods

\begin{tabular}{|c|c|c|c|c|c|c|c|c|}
\hline Mercury & 0.000155 & 0.000005 & $\mathrm{mg} / \mathrm{L}$ & 1 & 24-Jul-2009 & 20-Aug-2009 & EPA 7471A & \\
\hline Strontium & 0.465 & 0.0800 & $\mathrm{mg} / \mathrm{L}$ & 4 & 25-Jun-2009 & 25-Jun-2009 & EPA 6010B & \\
\hline Aluminum & 25.4 & 0.200 & $\mathrm{mg} / \mathrm{L}$ & 4 & 25-Jun-2009 & 25-Jun-2009 & SW 846/6010 & \\
\hline Calcium & 19.6 & 0.200 & $\mathrm{mg} / \mathrm{L}$ & 4 & 25-Jun-2009 & 25-Jun-2009 & SW 846/6010 & B \\
\hline Iron & 10.2 & 0.200 & $\mathrm{mg} / \mathrm{L}$ & 4 & 25-Jun-2009 & 25-Jun-2009 & SW 846/6010 & \\
\hline Magnesium & 4.76 & 0.200 & $\mathrm{mg} / \mathrm{L}$ & 4 & 25-Jun-2009 & 25-Jun-2009 & SW 846/6010 & \\
\hline Potassium & 5.53 & 0.200 & $\mathrm{mg} / \mathrm{L}$ & 4 & 25-Jun-2009 & 25-Jun-2009 & SW 846/6010 & B \\
\hline Sodium & 3.21 & 0.200 & $\mathrm{mg} / \mathrm{L}$ & 4 & 25-Jun-2009 & 25-Jun-2009 & SW 846/6010 & B \\
\hline Antimony & 0.0051 & 0.0020 & $\mathrm{mg} / \mathrm{L}$ & 4 & 26-Jun-2009 & 26-Jun-2009 & SW 846/6020 & B \\
\hline Arsenic & 0.129 & 0.0020 & $\mathrm{mg} / \mathrm{L}$ & 4 & 26-Jun-2009 & 26-Jun-2009 & SW 846/6020 & \\
\hline Barium & 0.608 & 0.0020 & $\mathrm{mg} / \mathrm{L}$ & 4 & 26-Jun-2009 & 26-Jun-2009 & SW 846/6020 & \\
\hline Beryllium & 0.0037 & 0.0020 & $\mathrm{mg} / \mathrm{L}$ & 4 & 26-Jun-2009 & 26-Jun-2009 & SW 846/6020 & \\
\hline Cadmium & 0.0006 & 0.0020 & $\mathrm{mg} / \mathrm{L}$ & 4 & 26-Jun-2009 & 26-Jun-2009 & SW 846/6020 & J \\
\hline Chromium & 0.0265 & 0.0020 & $\mathrm{mg} / \mathrm{L}$ & 4 & 26-Jun-2009 & 26-Jun-2009 & SW 846/6020 & \\
\hline Cobalt & 0.0156 & 0.0020 & $\mathrm{mg} / \mathrm{L}$ & 4 & 26-Jun-2009 & 26-Jun-2009 & SW 846/6020 & \\
\hline Copper & 0.0561 & 0.0020 & $\mathrm{mg} / \mathrm{L}$ & 4 & 26-Jun-2009 & 26-Jun-2009 & SW 846/6020 & \\
\hline Lead & 0.0448 & 0.0020 & $\mathrm{mg} / \mathrm{L}$ & 4 & 26-Jun-2009 & 26-Jun-2009 & SW 846/6020 & \\
\hline Manganese & 0.0790 & 0.0020 & $\mathrm{mg} / \mathrm{L}$ & 4 & 26-Jun-2009 & 26-Jun-2009 & SW 846/6020 & \\
\hline Molybdenum & 0.0070 & 0.0020 & $\mathrm{mg} / \mathrm{L}$ & 4 & 26-Jun-2009 & 26-Jun-2009 & SW 846/6020 & \\
\hline Nickel & 0.0291 & 0.0020 & $\mathrm{mg} / \mathrm{L}$ & 4 & 26-Jun-2009 & 26-Jun-2009 & SW 846/6020 & \\
\hline Selenium & 0.0070 & 0.0020 & $\mathrm{mg} / \mathrm{L}$ & 4 & 26-Jun-2009 & 26-Jun-2009 & SW 846/6020 & \\
\hline Silver & 0.0004 & 0.0020 & $\mathrm{mg} / \mathrm{L}$ & 4 & 26-Jun-2009 & 26-Jun-2009 & SW 846/6020 & J \\
\hline Thallium & 0.0030 & 0.0020 & $\mathrm{mg} / \mathrm{L}$ & 4 & 26-Jun-2009 & 26-Jun-2009 & SW 846/6020 & \\
\hline Vanadium & 0.110 & 0.0020 & $\mathrm{mg} / \mathrm{L}$ & 4 & 26-Jun-2009 & 26-Jun-2009 & SW 846/6020 & \\
\hline Zinc & 0.0732 & 0.0020 & $\mathrm{mg} / \mathrm{L}$ & 4 & 26-Jun-2009 & 26-Jun-2009 & SW 846/6020 & B \\
\hline
\end{tabular}




\section{USACE ERDC-EP-C \\ 3909 Halls Ferry Road \\ Vicksburg, MS 39180-6199}

ERDC - ECB

$-\cdot$,
Project: TVA Fly Ash Project

Project Manager: Tony Bednar
Reported:

03-Sep-2009

DRAFT: EMR MT O2 Hour 240 Rep B - Total Metals

9062302-17 (Water)

\begin{tabular}{|c|c|c|c|c|c|c|c|c|}
\hline Analyte & Result & $\begin{array}{r}\text { porting } \\
\text { Limit }\end{array}$ & Units & Dilution & Prepared & Analyzed & Method & Notes \\
\hline
\end{tabular}

ERDC- EL-EP-C (Environmental Chemistry Branch)

DRAFT: Wet Chemistry

\begin{tabular}{llllllll}
\hline Hardness & $\mathbf{6 9 . 0}$ & 1.32 & $\begin{array}{c}\text { mg equil } \\
\text { CaCO3/ }\end{array}$ & 4 & 25-Jun-2009 & 25-Jun-2009
\end{tabular}

$\mathrm{L}$

DRAFT: Metals by EPA 6000/7000 Series Methods

\begin{tabular}{|c|c|c|c|c|c|c|c|c|}
\hline Mercury & 0.000171 & 0.000005 & $\mathrm{mg} / \mathrm{L}$ & 1 & 24-Jul-2009 & 20-Aug-2009 & EPA 7471A & \\
\hline Strontium & 0.458 & 0.0800 & $\mathrm{mg} / \mathrm{L}$ & 4 & 25-Jun-2009 & 25-Jun-2009 & EPA 6010B & \\
\hline Aluminum & 25.1 & 0.200 & $\mathrm{mg} / \mathrm{L}$ & 4 & 25-Jun-2009 & 25-Jun-2009 & SW 846/6010 & \\
\hline Calcium & 19.7 & 0.200 & $\mathrm{mg} / \mathrm{L}$ & 4 & 25-Jun-2009 & 25-Jun-2009 & SW 846/6010 & B \\
\hline Iron & 10.1 & 0.200 & $\mathrm{mg} / \mathrm{L}$ & 4 & 25-Jun-2009 & 25-Jun-2009 & SW 846/6010 & \\
\hline Magnesium & 4.84 & 0.200 & $\mathrm{mg} / \mathrm{L}$ & 4 & 25-Jun-2009 & 25-Jun-2009 & SW 846/6010 & \\
\hline Potassium & 5.48 & 0.200 & $\mathrm{mg} / \mathrm{L}$ & 4 & 25-Jun-2009 & 25-Jun-2009 & SW 846/6010 & B \\
\hline Sodium & 3.09 & 0.200 & $\mathrm{mg} / \mathrm{L}$ & 4 & 25-Jun-2009 & 25-Jun-2009 & SW 846/6010 & B \\
\hline Antimony & 0.0049 & 0.0020 & $\mathrm{mg} / \mathrm{L}$ & 4 & 26-Jun-2009 & 26-Jun-2009 & SW 846/6020 & B \\
\hline Arsenic & 0.126 & 0.0020 & $\mathrm{mg} / \mathrm{L}$ & 4 & 26-Jun-2009 & 26-Jun-2009 & SW 846/6020 & \\
\hline Barium & 0.591 & 0.0020 & $\mathrm{mg} / \mathrm{L}$ & 4 & 26-Jun-2009 & 26-Jun-2009 & SW 846/6020 & \\
\hline Beryllium & 0.0035 & 0.0020 & $\mathrm{mg} / \mathrm{L}$ & 4 & 26-Jun-2009 & 26-Jun-2009 & SW 846/6020 & \\
\hline Cadmium & 0.0006 & 0.0020 & $\mathrm{mg} / \mathrm{L}$ & 4 & 26-Jun-2009 & 26-Jun-2009 & SW 846/6020 & J \\
\hline Chromium & 0.0270 & 0.0020 & $\mathrm{mg} / \mathrm{L}$ & 4 & 26-Jun-2009 & 26-Jun-2009 & SW 846/6020 & \\
\hline Cobalt & 0.0157 & 0.0020 & $\mathrm{mg} / \mathrm{L}$ & 4 & 26-Jun-2009 & 26-Jun-2009 & SW 846/6020 & \\
\hline Copper & 0.0547 & 0.0020 & $\mathrm{mg} / \mathrm{L}$ & 4 & 26-Jun-2009 & 26-Jun-2009 & SW 846/6020 & \\
\hline Lead & 0.0431 & 0.0020 & $\mathrm{mg} / \mathrm{L}$ & 4 & 26-Jun-2009 & 26-Jun-2009 & SW 846/6020 & \\
\hline Manganese & 0.0776 & 0.0020 & $\mathrm{mg} / \mathrm{L}$ & 4 & 26-Jun-2009 & 26-Jun-2009 & SW 846/6020 & \\
\hline Molybdenum & 0.0069 & 0.0020 & $\mathrm{mg} / \mathrm{L}$ & 4 & 26-Jun-2009 & 26-Jun-2009 & SW 846/6020 & \\
\hline Nickel & 0.0288 & 0.0020 & $\mathrm{mg} / \mathrm{L}$ & 4 & 26-Jun-2009 & 26-Jun-2009 & SW 846/6020 & \\
\hline Selenium & 0.0073 & 0.0020 & $\mathrm{mg} / \mathrm{L}$ & 4 & 26-Jun-2009 & 26-Jun-2009 & SW 846/6020 & \\
\hline Silver & 0.00041 & 0.0020 & $\mathrm{mg} / \mathrm{L}$ & 4 & 26-Jun-2009 & 26-Jun-2009 & SW 846/6020 & J \\
\hline Thallium & 0.0028 & 0.0020 & $\mathrm{mg} / \mathrm{L}$ & 4 & 26-Jun-2009 & 26-Jun-2009 & SW 846/6020 & \\
\hline Vanadium & 0.108 & 0.0020 & $\mathrm{mg} / \mathrm{L}$ & 4 & 26-Jun-2009 & 26-Jun-2009 & SW 846/6020 & \\
\hline Zinc & 0.0718 & 0.0020 & $\mathrm{mg} / \mathrm{L}$ & 4 & 26-Jun-2009 & 26-Jun-2009 & SW 846/6020 & B \\
\hline
\end{tabular}




\section{USACE ERDC-EP-C \\ 3909 Halls Ferry Road \\ Vicksburg, MS 39180-6199}

ERDC - ECB

$-\cdot$,
Project: TVA Fly Ash Project

Project Manager: Tony Bednar
Reported:

03-Sep-2009

DRAFT: EMR MT O2 Hour 240 Rep C - Total Metals

9062302-18 (Water)

\begin{tabular}{|c|c|c|c|c|c|c|c|c|}
\hline Analyte & Result & $\begin{array}{r}\text { porting } \\
\text { Limit }\end{array}$ & Units & Dilution & Prepared & Analyzed & Method & Notes \\
\hline
\end{tabular}

ERDC- EL-EP-C (Environmental Chemistry Branch)

DRAFT: Wet Chemistry

\begin{tabular}{llllllll}
\hline Hardness & $\mathbf{6 2 . 9}$ & 1.32 & $\begin{array}{c}\text { mg equil } \\
\text { CaCO3/ }\end{array}$ & 4 & 25-Jun-2009 & 25-Jun-2009
\end{tabular}

$\mathrm{L}$

DRAFT: Metals by EPA 6000/7000 Series Methods

\begin{tabular}{|c|c|c|c|c|c|c|c|c|}
\hline Mercury & 0.000167 & 0.000005 & $\mathrm{mg} / \mathrm{L}$ & 1 & 24-Jul-2009 & 20-Aug-2009 & EPA 7471A & \\
\hline Strontium & 0.402 & 0.0800 & $\mathrm{mg} / \mathrm{L}$ & 4 & 25-Jun-2009 & 25-Jun-2009 & EPA 6010B & \\
\hline Aluminum & 19.3 & 0.200 & $\mathrm{mg} / \mathrm{L}$ & 4 & 25-Jun-2009 & 25-Jun-2009 & SW 846/6010 & \\
\hline Calcium & 18.3 & 0.200 & $\mathrm{mg} / \mathrm{L}$ & 4 & 25-Jun-2009 & 25-Jun-2009 & SW 846/6010 & B \\
\hline Iron & 7.79 & 0.200 & $\mathrm{mg} / \mathrm{L}$ & 4 & 25-Jun-2009 & 25-Jun-2009 & SW 846/6010 & \\
\hline Magnesium & 4.21 & 0.200 & $\mathrm{mg} / \mathrm{L}$ & 4 & 25-Jun-2009 & 25-Jun-2009 & SW 846/6010 & \\
\hline Potassium & 4.52 & 0.200 & $\mathrm{mg} / \mathrm{L}$ & 4 & 25-Jun-2009 & 25-Jun-2009 & SW 846/6010 & B \\
\hline Sodium & 3.07 & 0.200 & $\mathrm{mg} / \mathrm{L}$ & 4 & 25-Jun-2009 & 25-Jun-2009 & SW 846/6010 & B \\
\hline Antimony & 0.0046 & 0.0020 & $\mathrm{mg} / \mathrm{L}$ & 4 & 26-Jun-2009 & 26-Jun-2009 & SW 846/6020 & B \\
\hline Arsenic & 0.112 & 0.0020 & $\mathrm{mg} / \mathrm{L}$ & 4 & 26-Jun-2009 & 26-Jun-2009 & SW 846/6020 & \\
\hline Barium & 0.509 & 0.0020 & $\mathrm{mg} / \mathrm{L}$ & 4 & 26-Jun-2009 & 26-Jun-2009 & SW 846/6020 & \\
\hline Beryllium & 0.0029 & 0.0020 & $\mathrm{mg} / \mathrm{L}$ & 4 & 26-Jun-2009 & 26-Jun-2009 & SW 846/6020 & \\
\hline Cadmium & 0.0005 & 0.0020 & $\mathrm{mg} / \mathrm{L}$ & 4 & 26-Jun-2009 & 26-Jun-2009 & SW 846/6020 & J \\
\hline Chromium & 0.0233 & 0.0020 & $\mathrm{mg} / \mathrm{L}$ & 4 & 26-Jun-2009 & 26-Jun-2009 & SW 846/6020 & \\
\hline Cobalt & 0.0131 & 0.0020 & $\mathrm{mg} / \mathrm{L}$ & 4 & 26-Jun-2009 & 26-Jun-2009 & SW 846/6020 & \\
\hline Copper & 0.0475 & 0.0020 & $\mathrm{mg} / \mathrm{L}$ & 4 & 26-Jun-2009 & 26-Jun-2009 & SW 846/6020 & \\
\hline Lead & 0.0361 & 0.0020 & $\mathrm{mg} / \mathrm{L}$ & 4 & 26-Jun-2009 & 26-Jun-2009 & SW 846/6020 & \\
\hline Manganese & 0.0684 & 0.0020 & $\mathrm{mg} / \mathrm{L}$ & 4 & 26-Jun-2009 & 26-Jun-2009 & SW 846/6020 & \\
\hline Molybdenum & 0.0059 & 0.0020 & $\mathrm{mg} / \mathrm{L}$ & 4 & 26-Jun-2009 & 26-Jun-2009 & SW 846/6020 & \\
\hline Nickel & 0.0252 & 0.0020 & $\mathrm{mg} / \mathrm{L}$ & 4 & 26-Jun-2009 & 26-Jun-2009 & SW 846/6020 & \\
\hline Selenium & 0.0065 & 0.0020 & $\mathrm{mg} / \mathrm{L}$ & 4 & 26-Jun-2009 & 26-Jun-2009 & SW 846/6020 & \\
\hline Silver & ND & 0.0020 & $\mathrm{mg} / \mathrm{L}$ & 4 & 26-Jun-2009 & 26-Jun-2009 & SW 846/6020 & $\mathrm{U}$ \\
\hline Thallium & 0.0026 & 0.0020 & $\mathrm{mg} / \mathrm{L}$ & 4 & 26-Jun-2009 & 26-Jun-2009 & SW 846/6020 & \\
\hline Vanadium & 0.0968 & 0.0020 & $\mathrm{mg} / \mathrm{L}$ & 4 & 26-Jun-2009 & 26-Jun-2009 & SW 846/6020 & \\
\hline Zinc & 0.0654 & 0.0020 & $\mathrm{mg} / \mathrm{L}$ & 4 & 26-Jun-2009 & 26-Jun-2009 & SW 846/6020 & B \\
\hline
\end{tabular}




\section{USACE ERDC-EP-C \\ 3909 Halls Ferry Road \\ Vicksburg, MS 39180-6199}

ERDC - ECB

$-\cdot$,
Project: TVA Fly Ash Project

Project Manager: Tony Bednar
Reported:

03-Sep-2009

DRAFT: SLC MT N2 Hour 240 Rep A - Total Metals

9062302-19 (Water)

\begin{tabular}{|c|c|c|c|c|c|c|c|c|}
\hline Analyte & Result & $\begin{array}{r}\text { porting } \\
\text { Limit }\end{array}$ & Units & Dilution & Prepared & Analyzed & Method & Notes \\
\hline
\end{tabular}

ERDC- EL-EP-C (Environmental Chemistry Branch)

DRAFT: Wet Chemistry

\begin{tabular}{llllllll}
\hline Hardness & $\mathbf{1 2 1}$ & 1.32 & $\begin{array}{c}\text { mg equil } \\
\text { CaCO3/ }\end{array}$ & 4 & 25-Jun-2009 & 25-Jun-2009
\end{tabular}

$\mathrm{L}$

DRAFT: Metals by EPA 6000/7000 Series Methods

\begin{tabular}{|c|c|c|c|c|c|c|c|c|}
\hline Mercury & 0.000158 & 0.000005 & $\mathrm{mg} / \mathrm{L}$ & 1 & 24-Jul-2009 & 20-Aug-2009 & EPA 7471A & \\
\hline Strontium & 0.777 & 0.0800 & $\mathrm{mg} / \mathrm{L}$ & 4 & 25-Jun-2009 & 25-Jun-2009 & EPA 6010B & \\
\hline Aluminum & 20.5 & 0.200 & $\mathrm{mg} / \mathrm{L}$ & 4 & 25-Jun-2009 & 25-Jun-2009 & SW 846/6010 & \\
\hline Calcium & 36.8 & 0.200 & $\mathrm{mg} / \mathrm{L}$ & 4 & 25-Jun-2009 & 25-Jun-2009 & SW 846/6010 & B \\
\hline Iron & 9.17 & 0.200 & $\mathrm{mg} / \mathrm{L}$ & 4 & 25-Jun-2009 & 25-Jun-2009 & SW 846/6010 & \\
\hline Magnesium & 7.15 & 0.200 & $\mathrm{mg} / \mathrm{L}$ & 4 & 25-Jun-2009 & 25-Jun-2009 & SW 846/6010 & \\
\hline Potassium & 5.57 & 0.200 & $\mathrm{mg} / \mathrm{L}$ & 4 & 25-Jun-2009 & 25-Jun-2009 & SW 846/6010 & B \\
\hline Sodium & 4.82 & 0.200 & $\mathrm{mg} / \mathrm{L}$ & 4 & 25-Jun-2009 & 25-Jun-2009 & SW 846/6010 & B \\
\hline Antimony & 0.0105 & 0.0020 & $\mathrm{mg} / \mathrm{L}$ & 4 & 26-Jun-2009 & 26-Jun-2009 & SW 846/6020 & B \\
\hline Arsenic & 0.156 & 0.0020 & $\mathrm{mg} / \mathrm{L}$ & 4 & 26-Jun-2009 & 26-Jun-2009 & SW 846/6020 & \\
\hline Barium & 0.448 & 0.0020 & $\mathrm{mg} / \mathrm{L}$ & 4 & 26-Jun-2009 & 26-Jun-2009 & SW 846/6020 & \\
\hline Beryllium & 0.0027 & 0.0020 & $\mathrm{mg} / \mathrm{L}$ & 4 & 26-Jun-2009 & 26-Jun-2009 & SW 846/6020 & \\
\hline Cadmium & 0.0009 & 0.0020 & $\mathrm{mg} / \mathrm{L}$ & 4 & 26-Jun-2009 & 26-Jun-2009 & SW 846/6020 & $\mathrm{J}$ \\
\hline Chromium & 0.0246 & 0.0020 & $\mathrm{mg} / \mathrm{L}$ & 4 & 26-Jun-2009 & 26-Jun-2009 & SW 846/6020 & \\
\hline Cobalt & 0.0131 & 0.0020 & $\mathrm{mg} / \mathrm{L}$ & 4 & 26-Jun-2009 & 26-Jun-2009 & SW 846/6020 & \\
\hline Copper & 0.0498 & 0.0020 & $\mathrm{mg} / \mathrm{L}$ & 4 & 26-Jun-2009 & 26-Jun-2009 & SW 846/6020 & \\
\hline Lead & 0.0365 & 0.0020 & $\mathrm{mg} / \mathrm{L}$ & 4 & 26-Jun-2009 & 26-Jun-2009 & SW 846/6020 & \\
\hline Manganese & 0.0915 & 0.0020 & $\mathrm{mg} / \mathrm{L}$ & 4 & 26-Jun-2009 & 26-Jun-2009 & SW 846/6020 & \\
\hline Molybdenum & 0.0852 & 0.0020 & $\mathrm{mg} / \mathrm{L}$ & 4 & 26-Jun-2009 & 26-Jun-2009 & SW 846/6020 & \\
\hline Nickel & 0.0267 & 0.0020 & $\mathrm{mg} / \mathrm{L}$ & 4 & 26-Jun-2009 & 26-Jun-2009 & SW 846/6020 & \\
\hline Selenium & 0.0115 & 0.0020 & $\mathrm{mg} / \mathrm{L}$ & 4 & 26-Jun-2009 & 26-Jun-2009 & SW 846/6020 & \\
\hline Silver & ND & 0.0020 & $\mathrm{mg} / \mathrm{L}$ & 4 & 26-Jun-2009 & 26-Jun-2009 & SW 846/6020 & $\mathrm{U}$ \\
\hline Thallium & 0.0028 & 0.0020 & $\mathrm{mg} / \mathrm{L}$ & 4 & 26-Jun-2009 & 26-Jun-2009 & SW 846/6020 & \\
\hline Vanadium & 0.129 & 0.0020 & $\mathrm{mg} / \mathrm{L}$ & 4 & 26-Jun-2009 & 26-Jun-2009 & SW 846/6020 & \\
\hline Zinc & 0.0685 & 0.0020 & $\mathrm{mg} / \mathrm{L}$ & 4 & 26-Jun-2009 & 26-Jun-2009 & SW 846/6020 & B \\
\hline
\end{tabular}




\section{USACE ERDC-EP-C \\ 3909 Halls Ferry Road \\ Vicksburg, MS 39180-6199}

ERDC - ECB

$-\cdot$,
Project: TVA Fly Ash Project

Project Manager: Tony Bednar
Reported:

03-Sep-2009

DRAFT: SLC MT N2 Hour 240 Rep B - Total Metals

9062302-20 (Water)

\begin{tabular}{|c|c|c|c|c|c|c|c|c|}
\hline Analyte & Result & $\begin{array}{r}\text { porting } \\
\text { Limit }\end{array}$ & Units & Dilution & Prepared & Analyzed & Method & Notes \\
\hline
\end{tabular}

ERDC- EL-EP-C (Environmental Chemistry Branch)

DRAFT: Wet Chemistry

\begin{tabular}{llllllll}
\hline Hardness & $\mathbf{1 1 8}$ & 1.32 & $\begin{array}{c}\text { mg equil } \\
\mathrm{CaCO} /\end{array}$ & 4 & 25-Jun-2009 & 25-Jun-2009
\end{tabular}

DRAFT: Metals by EPA 6000/7000 Series Methods

\begin{tabular}{|c|c|c|c|c|c|c|c|c|}
\hline Mercury & 0.000148 & 0.000005 & $\mathrm{mg} / \mathrm{L}$ & 1 & 24-Jul-2009 & 20-Aug-2009 & EPA 7471A & \\
\hline Strontium & 0.751 & 0.0800 & $\mathrm{mg} / \mathrm{L}$ & 4 & 25-Jun-2009 & 25-Jun-2009 & EPA 6010B & \\
\hline Aluminum & 17.8 & 0.200 & $\mathrm{mg} / \mathrm{L}$ & 4 & 25-Jun-2009 & 25-Jun-2009 & SW 846/6010 & \\
\hline Calcium & 36.2 & 0.200 & $\mathrm{mg} / \mathrm{L}$ & 4 & 25-Jun-2009 & 25-Jun-2009 & SW 846/6010 & B \\
\hline Iron & 7.98 & 0.200 & $\mathrm{mg} / \mathrm{L}$ & 4 & 25-Jun-2009 & 25-Jun-2009 & SW 846/6010 & \\
\hline Magnesium & 6.75 & 0.200 & $\mathrm{mg} / \mathrm{L}$ & 4 & 25-Jun-2009 & 25-Jun-2009 & SW 846/6010 & \\
\hline Potassium & 4.94 & 0.200 & $\mathrm{mg} / \mathrm{L}$ & 4 & 25-Jun-2009 & 25-Jun-2009 & SW 846/6010 & B \\
\hline Sodium & 4.59 & 0.200 & $\mathrm{mg} / \mathrm{L}$ & 4 & 25-Jun-2009 & 25-Jun-2009 & SW 846/6010 & B \\
\hline Antimony & 0.0102 & 0.0020 & $\mathrm{mg} / \mathrm{L}$ & 4 & 26-Jun-2009 & 26-Jun-2009 & SW 846/6020 & B \\
\hline Arsenic & 0.147 & 0.0020 & $\mathrm{mg} / \mathrm{L}$ & 4 & 26-Jun-2009 & 26-Jun-2009 & SW 846/6020 & \\
\hline Barium & 0.406 & 0.0020 & $\mathrm{mg} / \mathrm{L}$ & 4 & 26-Jun-2009 & 26-Jun-2009 & SW 846/6020 & \\
\hline Beryllium & 0.0026 & 0.0020 & $\mathrm{mg} / \mathrm{L}$ & 4 & 26-Jun-2009 & 26-Jun-2009 & SW 846/6020 & \\
\hline Cadmium & 0.0009 & 0.0020 & $\mathrm{mg} / \mathrm{L}$ & 4 & 26-Jun-2009 & 26-Jun-2009 & SW 846/6020 & J \\
\hline Chromium & 0.0220 & 0.0020 & $\mathrm{mg} / \mathrm{L}$ & 4 & 26-Jun-2009 & 26-Jun-2009 & SW 846/6020 & \\
\hline Cobalt & 0.0121 & 0.0020 & $\mathrm{mg} / \mathrm{L}$ & 4 & 26-Jun-2009 & 26-Jun-2009 & SW 846/6020 & \\
\hline Copper & 0.0455 & 0.0020 & $\mathrm{mg} / \mathrm{L}$ & 4 & 26-Jun-2009 & 26-Jun-2009 & SW 846/6020 & \\
\hline Lead & 0.0319 & 0.0020 & $\mathrm{mg} / \mathrm{L}$ & 4 & 26-Jun-2009 & 26-Jun-2009 & SW 846/6020 & \\
\hline Manganese & 0.0836 & 0.0020 & $\mathrm{mg} / \mathrm{L}$ & 4 & 26-Jun-2009 & 26-Jun-2009 & SW 846/6020 & \\
\hline Molybdenum & 0.0862 & 0.0020 & $\mathrm{mg} / \mathrm{L}$ & 4 & 26-Jun-2009 & 26-Jun-2009 & SW 846/6020 & \\
\hline Nickel & 0.0250 & 0.0020 & $\mathrm{mg} / \mathrm{L}$ & 4 & 26-Jun-2009 & 26-Jun-2009 & SW 846/6020 & \\
\hline Selenium & 0.0124 & 0.0020 & $\mathrm{mg} / \mathrm{L}$ & 4 & 26-Jun-2009 & 26-Jun-2009 & SW 846/6020 & \\
\hline Silver & ND & 0.0020 & $\mathrm{mg} / \mathrm{L}$ & 4 & 26-Jun-2009 & 26-Jun-2009 & SW 846/6020 & $\mathrm{U}$ \\
\hline Thallium & 0.0025 & 0.0020 & $\mathrm{mg} / \mathrm{L}$ & 4 & 26-Jun-2009 & 26-Jun-2009 & SW 846/6020 & \\
\hline Vanadium & 0.125 & 0.0020 & $\mathrm{mg} / \mathrm{L}$ & 4 & 26-Jun-2009 & 26-Jun-2009 & SW 846/6020 & \\
\hline Zinc & 0.0662 & 0.0020 & $\mathrm{mg} / \mathrm{L}$ & 4 & 26-Jun-2009 & 26-Jun-2009 & SW 846/6020 & B \\
\hline
\end{tabular}




\section{USACE ERDC-EP-C \\ 3909 Halls Ferry Road \\ Vicksburg, MS 39180-6199}

ERDC - ECB

$-\cdot$,
Project: TVA Fly Ash Project

Project Manager: Tony Bednar
Reported:

03-Sep-2009

DRAFT: SLC MT N2 Hour 240 Rep C - Total Metals

9062302-21 (Water)

\begin{tabular}{|c|c|c|c|c|c|c|c|c|}
\hline Analyte & Result & $\begin{array}{r}\text { porting } \\
\text { Limit }\end{array}$ & Units & Dilution & Prepared & Analyzed & Method & Notes \\
\hline
\end{tabular}

ERDC- EL-EP-C (Environmental Chemistry Branch)

DRAFT: Wet Chemistry

\begin{tabular}{llllllll}
\hline Hardness & $\mathbf{1 1 2}$ & 1.32 & $\begin{array}{c}\text { mg equil } \\
\text { CaCO3/ }\end{array}$ & 4 & 25-Jun-2009 & 25-Jun-2009
\end{tabular}

$\mathrm{L}$

DRAFT: Metals by EPA 6000/7000 Series Methods

\begin{tabular}{|c|c|c|c|c|c|c|c|c|}
\hline Mercury & 0.000118 & 0.000005 & $\mathrm{mg} / \mathrm{L}$ & 1 & 24-Jul-2009 & 20-Aug-2009 & EPA 7471A & \\
\hline Strontium & 0.709 & 0.0800 & $\mathrm{mg} / \mathrm{L}$ & 4 & 25-Jun-2009 & 25-Jun-2009 & EPA 6010B & \\
\hline Aluminum & 17.3 & 0.200 & $\mathrm{mg} / \mathrm{L}$ & 4 & 25-Jun-2009 & 25-Jun-2009 & SW 846/6010 & \\
\hline Calcium & 34.2 & 0.200 & $\mathrm{mg} / \mathrm{L}$ & 4 & 25-Jun-2009 & 25-Jun-2009 & SW 846/6010 & B \\
\hline Iron & 7.83 & 0.200 & $\mathrm{mg} / \mathrm{L}$ & 4 & 25-Jun-2009 & 25-Jun-2009 & SW 846/6010 & \\
\hline Magnesium & 6.48 & 0.200 & $\mathrm{mg} / \mathrm{L}$ & 4 & 25-Jun-2009 & 25-Jun-2009 & SW 846/6010 & \\
\hline Potassium & 4.78 & 0.200 & $\mathrm{mg} / \mathrm{L}$ & 4 & 25-Jun-2009 & 25-Jun-2009 & SW 846/6010 & B \\
\hline Sodium & 4.35 & 0.200 & $\mathrm{mg} / \mathrm{L}$ & 4 & 25-Jun-2009 & 25-Jun-2009 & SW 846/6010 & B \\
\hline Antimony & 0.0096 & 0.0020 & $\mathrm{mg} / \mathrm{L}$ & 4 & 26-Jun-2009 & 26-Jun-2009 & SW 846/6020 & B \\
\hline Arsenic & 0.142 & 0.0020 & $\mathrm{mg} / \mathrm{L}$ & 4 & 26-Jun-2009 & 26-Jun-2009 & SW 846/6020 & \\
\hline Barium & 0.399 & 0.0020 & $\mathrm{mg} / \mathrm{L}$ & 4 & 26-Jun-2009 & 26-Jun-2009 & SW 846/6020 & \\
\hline Beryllium & 0.0023 & 0.0020 & $\mathrm{mg} / \mathrm{L}$ & 4 & 26-Jun-2009 & 26-Jun-2009 & SW 846/6020 & \\
\hline Cadmium & 0.0008 & 0.0020 & $\mathrm{mg} / \mathrm{L}$ & 4 & 26-Jun-2009 & 26-Jun-2009 & SW 846/6020 & $\mathrm{J}$ \\
\hline Chromium & 0.0212 & 0.0020 & $\mathrm{mg} / \mathrm{L}$ & 4 & 26-Jun-2009 & 26-Jun-2009 & SW 846/6020 & \\
\hline Cobalt & 0.0119 & 0.0020 & $\mathrm{mg} / \mathrm{L}$ & 4 & 26-Jun-2009 & 26-Jun-2009 & SW 846/6020 & \\
\hline Copper & 0.0438 & 0.0020 & $\mathrm{mg} / \mathrm{L}$ & 4 & 26-Jun-2009 & 26-Jun-2009 & SW 846/6020 & \\
\hline Lead & 0.0310 & 0.0020 & $\mathrm{mg} / \mathrm{L}$ & 4 & 26-Jun-2009 & 26-Jun-2009 & SW 846/6020 & \\
\hline Manganese & 0.0798 & 0.0020 & $\mathrm{mg} / \mathrm{L}$ & 4 & 26-Jun-2009 & 26-Jun-2009 & SW 846/6020 & \\
\hline Molybdenum & 0.0800 & 0.0020 & $\mathrm{mg} / \mathrm{L}$ & 4 & 26-Jun-2009 & 26-Jun-2009 & SW 846/6020 & \\
\hline Nickel & 0.0238 & 0.0020 & $\mathrm{mg} / \mathrm{L}$ & 4 & 26-Jun-2009 & 26-Jun-2009 & SW 846/6020 & \\
\hline Selenium & 0.0110 & 0.0020 & $\mathrm{mg} / \mathrm{L}$ & 4 & 26-Jun-2009 & 26-Jun-2009 & SW 846/6020 & \\
\hline Silver & ND & 0.0020 & $\mathrm{mg} / \mathrm{L}$ & 4 & 26-Jun-2009 & 26-Jun-2009 & SW 846/6020 & $\mathrm{U}$ \\
\hline Thallium & 0.0024 & 0.0020 & $\mathrm{mg} / \mathrm{L}$ & 4 & 26-Jun-2009 & 26-Jun-2009 & SW 846/6020 & \\
\hline Vanadium & 0.120 & 0.0020 & $\mathrm{mg} / \mathrm{L}$ & 4 & 26-Jun-2009 & 26-Jun-2009 & SW 846/6020 & \\
\hline Zinc & 0.0669 & 0.0020 & $\mathrm{mg} / \mathrm{L}$ & 4 & 26-Jun-2009 & 26-Jun-2009 & SW 846/6020 & B \\
\hline
\end{tabular}




\section{USACE ERDC-EP-C \\ 3909 Halls Ferry Road \\ Vicksburg, MS 39180-6199}

ERDC - ECB

$-,-$
Project: TVA Fly Ash Project

Project Manager: Tony Bednar
Reported:

03-Sep-2009

DRAFT: SLC MT O2 Hour 240 Rep A - Total Metals

9062302-22 (Water)

\begin{tabular}{|c|c|c|c|c|c|c|c|c|}
\hline Analyte & Result & $\begin{array}{r}\text { porting } \\
\text { Limit }\end{array}$ & Units & Dilution & Prepared & Analyzed & Method & Notes \\
\hline
\end{tabular}

ERDC- EL-EP-C (Environmental Chemistry Branch)

DRAFT: Wet Chemistry

\begin{tabular}{llllllll}
\hline Hardness & $\mathbf{1 1 6}$ & 1.32 & $\begin{array}{c}\text { mg equil } \\
\text { CaCO3/ }\end{array}$ & 4 & 25-Jun-2009 & 25-Jun-2009
\end{tabular}

$\mathrm{L}$

DRAFT: Metals by EPA 6000/7000 Series Methods

\begin{tabular}{|c|c|c|c|c|c|c|c|c|}
\hline Mercury & 0.000182 & 0.000005 & $\mathrm{mg} / \mathrm{L}$ & 1 & 24-Jul-2009 & 20-Aug-2009 & EPA 7471A & \\
\hline Strontium & 0.735 & 0.0800 & $\mathrm{mg} / \mathrm{L}$ & 4 & 25-Jun-2009 & 25-Jun-2009 & EPA 6010B & \\
\hline Aluminum & 16.9 & 0.200 & $\mathrm{mg} / \mathrm{L}$ & 4 & 25-Jun-2009 & 25-Jun-2009 & SW 846/6010 & \\
\hline Calcium & 35.6 & 0.200 & $\mathrm{mg} / \mathrm{L}$ & 4 & 25-Jun-2009 & 25-Jun-2009 & SW 846/6010 & B \\
\hline Iron & 7.71 & 0.200 & $\mathrm{mg} / \mathrm{L}$ & 4 & 25-Jun-2009 & 25-Jun-2009 & SW 846/6010 & \\
\hline Magnesium & 6.59 & 0.200 & $\mathrm{mg} / \mathrm{L}$ & 4 & 25-Jun-2009 & 25-Jun-2009 & SW 846/6010 & \\
\hline Potassium & 4.85 & 0.200 & $\mathrm{mg} / \mathrm{L}$ & 4 & 25-Jun-2009 & 25-Jun-2009 & SW 846/6010 & B \\
\hline Sodium & 4.68 & 0.200 & $\mathrm{mg} / \mathrm{L}$ & 4 & 25-Jun-2009 & 25-Jun-2009 & SW 846/6010 & B \\
\hline Antimony & 0.0099 & 0.0020 & $\mathrm{mg} / \mathrm{L}$ & 4 & 26-Jun-2009 & 26-Jun-2009 & SW 846/6020 & B \\
\hline Arsenic & 0.145 & 0.0020 & $\mathrm{mg} / \mathrm{L}$ & 4 & 26-Jun-2009 & 26-Jun-2009 & SW 846/6020 & \\
\hline Barium & 0.406 & 0.0020 & $\mathrm{mg} / \mathrm{L}$ & 4 & 26-Jun-2009 & 26-Jun-2009 & SW 846/6020 & \\
\hline Beryllium & 0.0025 & 0.0020 & $\mathrm{mg} / \mathrm{L}$ & 4 & 26-Jun-2009 & 26-Jun-2009 & SW 846/6020 & \\
\hline Cadmium & 0.0009 & 0.0020 & $\mathrm{mg} / \mathrm{L}$ & 4 & 26-Jun-2009 & 26-Jun-2009 & SW 846/6020 & $\mathrm{J}$ \\
\hline Chromium & 0.0212 & 0.0020 & $\mathrm{mg} / \mathrm{L}$ & 4 & 26-Jun-2009 & 26-Jun-2009 & SW 846/6020 & \\
\hline Cobalt & 0.0114 & 0.0020 & $\mathrm{mg} / \mathrm{L}$ & 4 & 26-Jun-2009 & 26-Jun-2009 & SW 846/6020 & \\
\hline Copper & 0.0434 & 0.0020 & $\mathrm{mg} / \mathrm{L}$ & 4 & 26-Jun-2009 & 26-Jun-2009 & SW 846/6020 & \\
\hline Lead & 0.0297 & 0.0020 & $\mathrm{mg} / \mathrm{L}$ & 4 & 26-Jun-2009 & 26-Jun-2009 & SW 846/6020 & \\
\hline Manganese & 0.0851 & 0.0020 & $\mathrm{mg} / \mathrm{L}$ & 4 & 26-Jun-2009 & 26-Jun-2009 & SW 846/6020 & \\
\hline Molybdenum & 0.0839 & 0.0020 & $\mathrm{mg} / \mathrm{L}$ & 4 & 26-Jun-2009 & 26-Jun-2009 & SW 846/6020 & \\
\hline Nickel & 0.0232 & 0.0020 & $\mathrm{mg} / \mathrm{L}$ & 4 & 26-Jun-2009 & 26-Jun-2009 & SW 846/6020 & \\
\hline Selenium & 0.0111 & 0.0020 & $\mathrm{mg} / \mathrm{L}$ & 4 & 26-Jun-2009 & 26-Jun-2009 & SW 846/6020 & \\
\hline Silver & ND & 0.0020 & $\mathrm{mg} / \mathrm{L}$ & 4 & 26-Jun-2009 & 26-Jun-2009 & SW 846/6020 & $\mathrm{U}$ \\
\hline Thallium & 0.0026 & 0.0020 & $\mathrm{mg} / \mathrm{L}$ & 4 & 26-Jun-2009 & 26-Jun-2009 & SW 846/6020 & \\
\hline Vanadium & 0.119 & 0.0020 & $\mathrm{mg} / \mathrm{L}$ & 4 & 26-Jun-2009 & 26-Jun-2009 & SW 846/6020 & \\
\hline Zinc & 0.0638 & 0.0020 & $\mathrm{mg} / \mathrm{L}$ & 4 & 26-Jun-2009 & 26-Jun-2009 & SW 846/6020 & B \\
\hline
\end{tabular}




\section{USACE ERDC-EP-C \\ 3909 Halls Ferry Road \\ Vicksburg, MS 39180-6199}

ERDC - ECB

$-,-$
Project: TVA Fly Ash Project

Project Manager: Tony Bednar
Reported:

03-Sep-2009

DRAFT: SLC MT O2 Hour 240 Rep B - Total Metals

9062302-23 (Water)

\begin{tabular}{|c|c|c|c|c|c|c|c|c|}
\hline Analyte & Result & $\begin{array}{r}\text { porting } \\
\text { Limit }\end{array}$ & Units & Dilution & Prepared & Analyzed & Method & Notes \\
\hline
\end{tabular}

ERDC- EL-EP-C (Environmental Chemistry Branch)

DRAFT: Wet Chemistry

\begin{tabular}{llllllll}
\hline Hardness & 119 & 1.32 & $\begin{array}{c}\text { mg equil } \\
\text { CaCO3/ }\end{array}$ & 4 & 25-Jun-2009 & 25-Jun-2009
\end{tabular}

$\mathrm{L}$

DRAFT: Metals by EPA 6000/7000 Series Methods

\begin{tabular}{|c|c|c|c|c|c|c|c|c|}
\hline Mercury & 0.000189 & 0.000005 & $\mathrm{mg} / \mathrm{L}$ & 1 & 24-Jul-2009 & 20-Aug-2009 & EPA 7471A & \\
\hline Strontium & 0.759 & 0.0800 & $\mathrm{mg} / \mathrm{L}$ & 4 & 25-Jun-2009 & 25-Jun-2009 & EPA 6010B & \\
\hline Aluminum & 17.8 & 0.200 & $\mathrm{mg} / \mathrm{L}$ & 4 & 25-Jun-2009 & 25-Jun-2009 & SW 846/6010 & \\
\hline Calcium & 36.6 & 0.200 & $\mathrm{mg} / \mathrm{L}$ & 4 & 25-Jun-2009 & 25-Jun-2009 & SW 846/6010 & B \\
\hline Iron & 8.06 & 0.200 & $\mathrm{mg} / \mathrm{L}$ & 4 & 25-Jun-2009 & 25-Jun-2009 & SW 846/6010 & \\
\hline Magnesium & 6.77 & 0.200 & $\mathrm{mg} / \mathrm{L}$ & 4 & 25-Jun-2009 & 25-Jun-2009 & SW 846/6010 & \\
\hline Potassium & 4.99 & 0.200 & $\mathrm{mg} / \mathrm{L}$ & 4 & 25-Jun-2009 & 25-Jun-2009 & SW 846/6010 & B \\
\hline Sodium & 4.69 & 0.200 & $\mathrm{mg} / \mathrm{L}$ & 4 & 25-Jun-2009 & 25-Jun-2009 & SW 846/6010 & B \\
\hline Antimony & 0.0104 & 0.0020 & $\mathrm{mg} / \mathrm{L}$ & 4 & 26-Jun-2009 & 26-Jun-2009 & SW 846/6020 & B \\
\hline Arsenic & 0.149 & 0.0020 & $\mathrm{mg} / \mathrm{L}$ & 4 & 26-Jun-2009 & 26-Jun-2009 & SW 846/6020 & \\
\hline Barium & 0.429 & 0.0020 & $\mathrm{mg} / \mathrm{L}$ & 4 & 26-Jun-2009 & 26-Jun-2009 & SW 846/6020 & \\
\hline Beryllium & 0.0026 & 0.0020 & $\mathrm{mg} / \mathrm{L}$ & 4 & 26-Jun-2009 & 26-Jun-2009 & SW 846/6020 & \\
\hline Cadmium & 0.0009 & 0.0020 & $\mathrm{mg} / \mathrm{L}$ & 4 & 26-Jun-2009 & 26-Jun-2009 & SW 846/6020 & $\mathrm{J}$ \\
\hline Chromium & 0.0233 & 0.0020 & $\mathrm{mg} / \mathrm{L}$ & 4 & 26-Jun-2009 & 26-Jun-2009 & SW 846/6020 & \\
\hline Cobalt & 0.0124 & 0.0020 & $\mathrm{mg} / \mathrm{L}$ & 4 & 26-Jun-2009 & 26-Jun-2009 & SW 846/6020 & \\
\hline Copper & 0.0457 & 0.0020 & $\mathrm{mg} / \mathrm{L}$ & 4 & 26-Jun-2009 & 26-Jun-2009 & SW 846/6020 & \\
\hline Lead & 0.0321 & 0.0020 & $\mathrm{mg} / \mathrm{L}$ & 4 & 26-Jun-2009 & 26-Jun-2009 & SW 846/6020 & \\
\hline Manganese & 0.0875 & 0.0020 & $\mathrm{mg} / \mathrm{L}$ & 4 & 26-Jun-2009 & 26-Jun-2009 & SW 846/6020 & \\
\hline Molybdenum & 0.0837 & 0.0020 & $\mathrm{mg} / \mathrm{L}$ & 4 & 26-Jun-2009 & 26-Jun-2009 & SW 846/6020 & \\
\hline Nickel & 0.0253 & 0.0020 & $\mathrm{mg} / \mathrm{L}$ & 4 & 26-Jun-2009 & 26-Jun-2009 & SW 846/6020 & \\
\hline Selenium & 0.0118 & 0.0020 & $\mathrm{mg} / \mathrm{L}$ & 4 & 26-Jun-2009 & 26-Jun-2009 & SW 846/6020 & \\
\hline Silver & ND & 0.0020 & $\mathrm{mg} / \mathrm{L}$ & 4 & 26-Jun-2009 & 26-Jun-2009 & SW 846/6020 & $\mathrm{U}$ \\
\hline Thallium & 0.0027 & 0.0020 & $\mathrm{mg} / \mathrm{L}$ & 4 & 26-Jun-2009 & 26-Jun-2009 & SW 846/6020 & \\
\hline Vanadium & 0.127 & 0.0020 & $\mathrm{mg} / \mathrm{L}$ & 4 & 26-Jun-2009 & 26-Jun-2009 & SW 846/6020 & \\
\hline Zinc & 0.0660 & 0.0020 & $\mathrm{mg} / \mathrm{L}$ & 4 & 26-Jun-2009 & 26-Jun-2009 & SW 846/6020 & B \\
\hline
\end{tabular}




\section{USACE ERDC-EP-C \\ 3909 Halls Ferry Road \\ Vicksburg, MS 39180-6199}

ERDC - ECB

$-,-$
Project: TVA Fly Ash Project

Project Manager: Tony Bednar
Reported:

03-Sep-2009

DRAFT: SLC MT O2 Hour 240 Rep C - Total Metals

9062302-24 (Water)

\begin{tabular}{|c|c|c|c|c|c|c|c|c|}
\hline Analyte & Result & $\begin{array}{r}\text { porting } \\
\text { Limit }\end{array}$ & Units & Dilution & Prepared & Analyzed & Method & Notes \\
\hline
\end{tabular}

ERDC- EL-EP-C (Environmental Chemistry Branch)

DRAFT: Wet Chemistry

\begin{tabular}{llllllll}
\hline Hardness & $\mathbf{1 2 4}$ & 1.32 & $\begin{array}{c}\text { mg equil } \\
\text { CaCO3/ }\end{array}$ & 4 & 25-Jun-2009 & 25-Jun-2009
\end{tabular}

$\mathrm{L}$

DRAFT: Metals by EPA 6000/7000 Series Methods

\begin{tabular}{|c|c|c|c|c|c|c|c|c|}
\hline Mercury & 0.000264 & 0.000005 & $\mathrm{mg} / \mathrm{L}$ & 1 & 24-Jul-2009 & 20-Aug-2009 & EPA 7471A & \\
\hline Strontium & 0.814 & 0.0800 & $\mathrm{mg} / \mathrm{L}$ & 4 & 25-Jun-2009 & 25-Jun-2009 & EPA 6010B & \\
\hline Aluminum & 24.4 & 0.200 & $\mathrm{mg} / \mathrm{L}$ & 4 & 25-Jun-2009 & 25-Jun-2009 & SW 846/6010 & \\
\hline Calcium & 37.6 & 0.200 & $\mathrm{mg} / \mathrm{L}$ & 4 & 25-Jun-2009 & 25-Jun-2009 & SW 846/6010 & B \\
\hline Iron & 11.0 & 0.200 & $\mathrm{mg} / \mathrm{L}$ & 4 & 25-Jun-2009 & 25-Jun-2009 & SW 846/6010 & \\
\hline Magnesium & 7.33 & 0.200 & $\mathrm{mg} / \mathrm{L}$ & 4 & 25-Jun-2009 & 25-Jun-2009 & SW 846/6010 & \\
\hline Potassium & 6.13 & 0.200 & $\mathrm{mg} / \mathrm{L}$ & 4 & 25-Jun-2009 & 25-Jun-2009 & SW 846/6010 & B \\
\hline Sodium & 4.74 & 0.200 & $\mathrm{mg} / \mathrm{L}$ & 4 & 25-Jun-2009 & 25-Jun-2009 & SW 846/6010 & B \\
\hline Antimony & 0.0105 & 0.0020 & $\mathrm{mg} / \mathrm{L}$ & 4 & 26-Jun-2009 & 26-Jun-2009 & SW 846/6020 & B \\
\hline Arsenic & 0.171 & 0.0020 & $\mathrm{mg} / \mathrm{L}$ & 4 & 26-Jun-2009 & 26-Jun-2009 & SW 846/6020 & \\
\hline Barium & 0.512 & 0.0020 & $\mathrm{mg} / \mathrm{L}$ & 4 & 26-Jun-2009 & 26-Jun-2009 & SW 846/6020 & \\
\hline Beryllium & 0.0033 & 0.0020 & $\mathrm{mg} / \mathrm{L}$ & 4 & 26-Jun-2009 & 26-Jun-2009 & SW 846/6020 & \\
\hline Cadmium & 0.0011 & 0.0020 & $\mathrm{mg} / \mathrm{L}$ & 4 & 26-Jun-2009 & 26-Jun-2009 & SW 846/6020 & $\mathrm{J}$ \\
\hline Chromium & 0.0268 & 0.0020 & $\mathrm{mg} / \mathrm{L}$ & 4 & 26-Jun-2009 & 26-Jun-2009 & SW 846/6020 & \\
\hline Cobalt & 0.0154 & 0.0020 & $\mathrm{mg} / \mathrm{L}$ & 4 & 26-Jun-2009 & 26-Jun-2009 & SW 846/6020 & \\
\hline Copper & 0.0563 & 0.0020 & $\mathrm{mg} / \mathrm{L}$ & 4 & 26-Jun-2009 & 26-Jun-2009 & SW 846/6020 & \\
\hline Lead & 0.0415 & 0.0020 & $\mathrm{mg} / \mathrm{L}$ & 4 & 26-Jun-2009 & 26-Jun-2009 & SW 846/6020 & \\
\hline Manganese & 0.103 & 0.0020 & $\mathrm{mg} / \mathrm{L}$ & 4 & 26-Jun-2009 & 26-Jun-2009 & SW 846/6020 & \\
\hline Molybdenum & 0.0855 & 0.0020 & $\mathrm{mg} / \mathrm{L}$ & 4 & 26-Jun-2009 & 26-Jun-2009 & SW 846/6020 & \\
\hline Nickel & 0.0308 & 0.0020 & $\mathrm{mg} / \mathrm{L}$ & 4 & 26-Jun-2009 & 26-Jun-2009 & SW 846/6020 & \\
\hline Selenium & 0.0131 & 0.0020 & $\mathrm{mg} / \mathrm{L}$ & 4 & 26-Jun-2009 & 26-Jun-2009 & SW 846/6020 & \\
\hline Silver & ND & 0.0020 & $\mathrm{mg} / \mathrm{L}$ & 4 & 26-Jun-2009 & 26-Jun-2009 & SW 846/6020 & $\mathrm{U}$ \\
\hline Thallium & 0.0031 & 0.0020 & $\mathrm{mg} / \mathrm{L}$ & 4 & 26-Jun-2009 & 26-Jun-2009 & SW 846/6020 & \\
\hline Vanadium & 0.139 & 0.0020 & $\mathrm{mg} / \mathrm{L}$ & 4 & 26-Jun-2009 & 26-Jun-2009 & SW 846/6020 & \\
\hline Zinc & 0.0746 & 0.0020 & $\mathrm{mg} / \mathrm{L}$ & 4 & 26-Jun-2009 & 26-Jun-2009 & SW 846/6020 & B \\
\hline
\end{tabular}




\section{USACE ERDC-EP-C \\ 3909 Halls Ferry Road \\ Vicksburg, MS 39180-6199}

ERDC - ECB $--,-$
Reported: 03-Sep-2009

DRAFT: EMR - EL - AF - 100 - A

9071602-01 (Tissue)

\begin{tabular}{|c|c|c|c|c|c|c|c|c|}
\hline Analyte & Result & porting & Units & Dilution & Prenared & Analyzed & Method & Notes \\
\hline Analyte & Kesult & Limit & & & Prepared & & Metnod & \\
\hline
\end{tabular}

ERDC- EL-EP-C (Environmental Chemistry Branch)

DRAFT: Metals by EPA 6000/7000 Series Methods

\begin{tabular}{|c|c|c|c|c|c|c|c|c|}
\hline Mercury & 0.029 & 0.005 & $\mathrm{mg} / \mathrm{kg}$ & 1 & 22-Jul-2009 & 22-Jul-2009 & EPA 7471A & \\
\hline Aluminum & 1080 & 0.500 & $\mathrm{mg} / \mathrm{kg}$ & 2 & 17-Jul-2009 & 20-Jul-2009 & SW 846/6020 & \\
\hline Antimony & 0.141 & 0.500 & $\mathrm{mg} / \mathrm{kg}$ & 2 & 17-Jul-2009 & 20-Jul-2009 & SW 846/6020 & $\mathrm{J}$ \\
\hline Arsenic & 2.89 & 0.500 & $\mathrm{mg} / \mathrm{kg}$ & 2 & 17-Jul-2009 & 20-Jul-2009 & SW 846/6020 & \\
\hline Barium & 29.5 & 0.500 & $\mathrm{mg} / \mathrm{kg}$ & 2 & 17-Jul-2009 & 20-Jul-2009 & SW 846/6020 & \\
\hline Beryllium & 0.167 & 0.500 & $\mathrm{mg} / \mathrm{kg}$ & 2 & 17-Jul-2009 & 20-Jul-2009 & SW 846/6020 & $\mathrm{J}$ \\
\hline Cadmium & 0.393 & 0.500 & $\mathrm{mg} / \mathrm{kg}$ & 2 & 17-Jul-2009 & 20-Jul-2009 & SW 846/6020 & $\mathrm{J}$ \\
\hline Calcium & 4740 & 0.500 & $\mathrm{mg} / \mathrm{kg}$ & 2 & 17-Jul-2009 & 20-Jul-2009 & SW 846/6020 & \\
\hline Chromium & 16.5 & 0.500 & $\mathrm{mg} / \mathrm{kg}$ & 2 & 17-Jul-2009 & 20-Jul-2009 & SW 846/6020 & \\
\hline Cobalt & 1.09 & 0.500 & $\mathrm{mg} / \mathrm{kg}$ & 2 & 17-Jul-2009 & 20-Jul-2009 & SW 846/6020 & \\
\hline Copper & 4.87 & 0.500 & $\mathrm{mg} / \mathrm{kg}$ & 2 & 17-Jul-2009 & 20-Jul-2009 & SW 846/6020 & \\
\hline Iron & 584 & 0.500 & $\mathrm{mg} / \mathrm{kg}$ & 2 & 17-Jul-2009 & 20-Jul-2009 & SW 846/6020 & \\
\hline Lead & 1.83 & 0.500 & $\mathrm{mg} / \mathrm{kg}$ & 2 & 17-Jul-2009 & 20-Jul-2009 & SW 846/6020 & \\
\hline Magnesium & 360 & 0.500 & $\mathrm{mg} / \mathrm{kg}$ & 2 & 17-Jul-2009 & 20-Jul-2009 & SW 846/6020 & \\
\hline Manganese & 6.31 & 0.500 & $\mathrm{mg} / \mathrm{kg}$ & 2 & 17-Jul-2009 & 20-Jul-2009 & SW 846/6020 & \\
\hline Molybdenum & 0.458 & 0.500 & $\mathrm{mg} / \mathrm{kg}$ & 2 & 17-Jul-2009 & 20-Jul-2009 & SW 846/6020 & $\mathrm{J}$ \\
\hline Nickel & 9.52 & 0.500 & $\mathrm{mg} / \mathrm{kg}$ & 2 & 17-Jul-2009 & 20-Jul-2009 & SW 846/6020 & \\
\hline Potassium & 2650 & 0.500 & $\mathrm{mg} / \mathrm{kg}$ & 2 & 17-Jul-2009 & 20-Jul-2009 & SW 846/6020 & \\
\hline Selenium & 0.709 & 0.500 & $\mathrm{mg} / \mathrm{kg}$ & 2 & 17-Jul-2009 & 20-Jul-2009 & SW 846/6020 & \\
\hline Silver & 0.462 & 0.500 & $\mathrm{mg} / \mathrm{kg}$ & 2 & 17-Jul-2009 & 20-Jul-2009 & SW 846/6020 & $\mathrm{J}$ \\
\hline Sodium & 1010 & 0.500 & $\mathrm{mg} / \mathrm{kg}$ & 2 & 17-Jul-2009 & 20-Jul-2009 & SW 846/6020 & \\
\hline Thallium & 0.195 & 0.500 & $\mathrm{mg} / \mathrm{kg}$ & 2 & 17-Jul-2009 & 20-Jul-2009 & SW 846/6020 & $\mathrm{J}$ \\
\hline Vanadium & 4.12 & 0.500 & $\mathrm{mg} / \mathrm{kg}$ & 2 & 17-Jul-2009 & 20-Jul-2009 & SW 846/6020 & \\
\hline Zinc & 24.8 & 0.500 & $\mathrm{mg} / \mathrm{kg}$ & 2 & 17-Jul-2009 & 20-Jul-2009 & SW 846/6020 & B \\
\hline
\end{tabular}




\section{USACE ERDC-EP-C \\ 3909 Halls Ferry Road \\ Vicksburg, MS 39180-6199}

ERDC - ECB $--,-$

DRAFT: EMR - EL - AF - 100 - B

9071602-02 (Tissue)

\begin{tabular}{|c|c|c|c|c|c|c|c|c|}
\hline Analyte & Result & porting & Units & Dilution & Prenared & Analyzed & Method & Notes \\
\hline Analyte & Kesult & Limit & & & Prepared & & Metnod & \\
\hline
\end{tabular}

ERDC- EL-EP-C (Environmental Chemistry Branch)

DRAFT: Metals by EPA 6000/7000 Series Methods

\begin{tabular}{|c|c|c|c|c|c|c|c|c|}
\hline Mercury & 0.027 & 0.002 & $\mathrm{mg} / \mathrm{kg}$ & 1 & 22-Jul-2009 & 22-Jul-2009 & EPA 7471A & \\
\hline Aluminum & 1450 & 0.500 & $\mathrm{mg} / \mathrm{kg}$ & 2 & 17-Jul-2009 & 20-Jul-2009 & SW 846/6020 & \\
\hline Antimony & 0.435 & 0.500 & $\mathrm{mg} / \mathrm{kg}$ & 2 & 17-Jul-2009 & 20-Jul-2009 & SW 846/6020 & $\mathrm{J}$ \\
\hline Arsenic & 4.35 & 0.500 & $\mathrm{mg} / \mathrm{kg}$ & 2 & 17-Jul-2009 & 20-Jul-2009 & SW 846/6020 & \\
\hline Barium & 27.3 & 0.500 & $\mathrm{mg} / \mathrm{kg}$ & 2 & 17-Jul-2009 & 20-Jul-2009 & SW 846/6020 & \\
\hline Beryllium & 0.222 & 0.500 & $\mathrm{mg} / \mathrm{kg}$ & 2 & 17-Jul-2009 & 20-Jul-2009 & SW 846/6020 & $\mathrm{J}$ \\
\hline Cadmium & 0.532 & 0.500 & $\mathrm{mg} / \mathrm{kg}$ & 2 & 17-Jul-2009 & 20-Jul-2009 & SW 846/6020 & \\
\hline Calcium & 5210 & 0.500 & $\mathrm{mg} / \mathrm{kg}$ & 2 & 17-Jul-2009 & 20-Jul-2009 & SW 846/6020 & \\
\hline Chromium & 7.38 & 0.500 & $\mathrm{mg} / \mathrm{kg}$ & 2 & 17-Jul-2009 & 20-Jul-2009 & SW 846/6020 & \\
\hline Cobalt & 1.14 & 0.500 & $\mathrm{mg} / \mathrm{kg}$ & 2 & 17-Jul-2009 & 20-Jul-2009 & SW 846/6020 & \\
\hline Copper & 5.14 & 0.500 & $\mathrm{mg} / \mathrm{kg}$ & 2 & 17-Jul-2009 & 20-Jul-2009 & SW 846/6020 & \\
\hline Iron & 737 & 0.500 & $\mathrm{mg} / \mathrm{kg}$ & 2 & 17-Jul-2009 & 20-Jul-2009 & SW 846/6020 & \\
\hline Lead & 2.41 & 0.500 & $\mathrm{mg} / \mathrm{kg}$ & 2 & 17-Jul-2009 & 20-Jul-2009 & SW 846/6020 & \\
\hline Magnesium & 380 & 0.500 & $\mathrm{mg} / \mathrm{kg}$ & 2 & 17-Jul-2009 & 20-Jul-2009 & SW 846/6020 & \\
\hline Manganese & 7.28 & 0.500 & $\mathrm{mg} / \mathrm{kg}$ & 2 & 17-Jul-2009 & 20-Jul-2009 & SW 846/6020 & \\
\hline Molybdenum & 0.367 & 0.500 & $\mathrm{mg} / \mathrm{kg}$ & 2 & 17-Jul-2009 & 20-Jul-2009 & SW 846/6020 & J \\
\hline Nickel & 5.07 & 0.500 & $\mathrm{mg} / \mathrm{kg}$ & 2 & 17-Jul-2009 & 20-Jul-2009 & SW 846/6020 & \\
\hline Potassium & 2520 & 0.500 & $\mathrm{mg} / \mathrm{kg}$ & 2 & 17-Jul-2009 & 20-Jul-2009 & SW 846/6020 & \\
\hline Selenium & 0.639 & 0.500 & $\mathrm{mg} / \mathrm{kg}$ & 2 & 17-Jul-2009 & 20-Jul-2009 & SW 846/6020 & \\
\hline Silver & ND & 0.500 & $\mathrm{mg} / \mathrm{kg}$ & 2 & 17-Jul-2009 & 20-Jul-2009 & SW 846/6020 & $\mathrm{U}$ \\
\hline Sodium & 996 & 0.500 & $\mathrm{mg} / \mathrm{kg}$ & 2 & 17-Jul-2009 & 20-Jul-2009 & SW 846/6020 & \\
\hline Thallium & 0.270 & 0.500 & $\mathrm{mg} / \mathrm{kg}$ & 2 & 17-Jul-2009 & 20-Jul-2009 & SW 846/6020 & $\mathrm{J}$ \\
\hline Vanadium & 5.81 & 0.500 & $\mathrm{mg} / \mathrm{kg}$ & 2 & 17-Jul-2009 & 20-Jul-2009 & SW 846/6020 & \\
\hline Zinc & 29.1 & 0.500 & $\mathrm{mg} / \mathrm{kg}$ & 2 & 17-Jul-2009 & 20-Jul-2009 & SW 846/6020 & B \\
\hline
\end{tabular}




\section{USACE ERDC-EP-C \\ 3909 Halls Ferry Road \\ Vicksburg, MS 39180-6199}

ERDC - ECB $--,-$
Project: TVA Fly Ash Project

Project Manager: Tony Bednar
Reported:

03-Sep-2009

DRAFT: EMR - EL - AF - 100 - C

9071602-03 (Tissue)

\begin{tabular}{|c|c|c|c|c|c|c|c|c|}
\hline Analyte & Result & $\begin{array}{r}\text { porting } \\
\text { Limit }\end{array}$ & Units & Dilution & Prepared & Analyzed & Method & Notes \\
\hline
\end{tabular}

ERDC- EL-EP-C (Environmental Chemistry Branch)

DRAFT: Metals by EPA 6000/7000 Series Methods

\begin{tabular}{|c|c|c|c|c|c|c|c|c|}
\hline Mercury & 0.017 & 0.002 & $\mathrm{mg} / \mathrm{kg}$ & 1 & 22-Jul-2009 & 22-Jul-2009 & EPA 7471A & \\
\hline Aluminum & 756 & 0.500 & $\mathrm{mg} / \mathrm{kg}$ & 2 & 17-Jul-2009 & 20-Jul-2009 & SW 846/6020 & \\
\hline Antimony & 0.876 & 0.500 & $\mathrm{mg} / \mathrm{kg}$ & 2 & 17-Jul-2009 & 20-Jul-2009 & SW 846/6020 & \\
\hline Arsenic & 2.38 & 0.500 & $\mathrm{mg} / \mathrm{kg}$ & 2 & 17-Jul-2009 & 20-Jul-2009 & SW 846/6020 & \\
\hline Barium & 15.1 & 0.500 & $\mathrm{mg} / \mathrm{kg}$ & 2 & 17-Jul-2009 & 20-Jul-2009 & SW 846/6020 & \\
\hline Beryllium & 0.121 & 0.500 & $\mathrm{mg} / \mathrm{kg}$ & 2 & 17-Jul-2009 & 20-Jul-2009 & SW 846/6020 & $\mathrm{J}$ \\
\hline Cadmium & 0.324 & 0.500 & $\mathrm{mg} / \mathrm{kg}$ & 2 & 17-Jul-2009 & 20-Jul-2009 & SW 846/6020 & $\mathrm{J}$ \\
\hline Calcium & 4610 & 0.500 & $\mathrm{mg} / \mathrm{kg}$ & 2 & 17-Jul-2009 & 20-Jul-2009 & SW 846/6020 & \\
\hline Chromium & 6.71 & 0.500 & $\mathrm{mg} / \mathrm{kg}$ & 2 & 17-Jul-2009 & 20-Jul-2009 & SW 846/6020 & \\
\hline Cobalt & 0.685 & 0.500 & $\mathrm{mg} / \mathrm{kg}$ & 2 & 17-Jul-2009 & 20-Jul-2009 & SW 846/6020 & \\
\hline Copper & 3.22 & 0.500 & $\mathrm{mg} / \mathrm{kg}$ & 2 & 17-Jul-2009 & 20-Jul-2009 & SW 846/6020 & \\
\hline Iron & 393 & 0.500 & $\mathrm{mg} / \mathrm{kg}$ & 2 & 17-Jul-2009 & 20-Jul-2009 & SW 846/6020 & \\
\hline Lead & 1.11 & 0.500 & $\mathrm{mg} / \mathrm{kg}$ & 2 & 17-Jul-2009 & 20-Jul-2009 & SW 846/6020 & \\
\hline Magnesium & 311 & 0.500 & $\mathrm{mg} / \mathrm{kg}$ & 2 & 17-Jul-2009 & 20-Jul-2009 & SW 846/6020 & \\
\hline Manganese & 4.27 & 0.500 & $\mathrm{mg} / \mathrm{kg}$ & 2 & 17-Jul-2009 & 20-Jul-2009 & SW 846/6020 & \\
\hline Molybdenum & 0.265 & 0.500 & $\mathrm{mg} / \mathrm{kg}$ & 2 & 17-Jul-2009 & 20-Jul-2009 & SW 846/6020 & J \\
\hline Nickel & 4.24 & 0.500 & $\mathrm{mg} / \mathrm{kg}$ & 2 & 17-Jul-2009 & 20-Jul-2009 & SW 846/6020 & \\
\hline Potassium & 2370 & 0.500 & $\mathrm{mg} / \mathrm{kg}$ & 2 & 17-Jul-2009 & 20-Jul-2009 & SW 846/6020 & \\
\hline Selenium & 0.505 & 0.500 & $\mathrm{mg} / \mathrm{kg}$ & 2 & 17-Jul-2009 & 20-Jul-2009 & SW 846/6020 & \\
\hline Silver & ND & 0.500 & $\mathrm{mg} / \mathrm{kg}$ & 2 & 17-Jul-2009 & 20-Jul-2009 & SW 846/6020 & $\mathrm{U}$ \\
\hline Sodium & 946 & 0.500 & $\mathrm{mg} / \mathrm{kg}$ & 2 & 17-Jul-2009 & 20-Jul-2009 & SW 846/6020 & \\
\hline Thallium & 0.204 & 0.500 & $\mathrm{mg} / \mathrm{kg}$ & 2 & 17-Jul-2009 & 20-Jul-2009 & SW 846/6020 & J \\
\hline Vanadium & 3.19 & 0.500 & $\mathrm{mg} / \mathrm{kg}$ & 2 & 17-Jul-2009 & 20-Jul-2009 & SW 846/6020 & \\
\hline Zinc & 23.3 & 0.500 & $\mathrm{mg} / \mathrm{kg}$ & 2 & 17-Jul-2009 & 20-Jul-2009 & SW 846/6020 & B \\
\hline
\end{tabular}




\section{USACE ERDC-EP-C \\ 3909 Halls Ferry Road \\ Vicksburg, MS 39180-6199}

ERDC - ECB $--,-$

DRAFT: EMR - EL - AF - 100 - D

9071602-04 (Tissue)

\begin{tabular}{|c|c|c|c|c|c|c|c|c|}
\hline Analyte & Result & porting & Units & Dilution & Prenared & Analyzed & Method & Notes \\
\hline Analyte & Kesult & Limit & & & Prepared & & Metnod & \\
\hline
\end{tabular}

ERDC- EL-EP-C (Environmental Chemistry Branch)

DRAFT: Metals by EPA 6000/7000 Series Methods

\begin{tabular}{|c|c|c|c|c|c|c|c|c|}
\hline Mercury & 0.022 & 0.002 & $\mathrm{mg} / \mathrm{kg}$ & 1 & 22-Jul-2009 & 22-Jul-2009 & EPA 7471A & \\
\hline Aluminum & 914 & 0.500 & $\mathrm{mg} / \mathrm{kg}$ & 2 & 17-Jul-2009 & 20-Jul-2009 & SW 846/6020 & \\
\hline Antimony & 0.723 & 0.500 & $\mathrm{mg} / \mathrm{kg}$ & 2 & 17-Jul-2009 & 20-Jul-2009 & SW 846/6020 & \\
\hline Arsenic & 2.93 & 0.500 & $\mathrm{mg} / \mathrm{kg}$ & 2 & 17-Jul-2009 & 20-Jul-2009 & SW 846/6020 & \\
\hline Barium & 18.2 & 0.500 & $\mathrm{mg} / \mathrm{kg}$ & 2 & 17-Jul-2009 & 20-Jul-2009 & SW 846/6020 & \\
\hline Beryllium & 0.149 & 0.500 & $\mathrm{mg} / \mathrm{kg}$ & 2 & 17-Jul-2009 & 20-Jul-2009 & SW 846/6020 & J \\
\hline Cadmium & 0.408 & 0.500 & $\mathrm{mg} / \mathrm{kg}$ & 2 & 17-Jul-2009 & 20-Jul-2009 & SW 846/6020 & J \\
\hline Calcium & 5920 & 0.500 & $\mathrm{mg} / \mathrm{kg}$ & 2 & 17-Jul-2009 & 20-Jul-2009 & SW 846/6020 & \\
\hline Chromium & 7.55 & 0.500 & $\mathrm{mg} / \mathrm{kg}$ & 2 & 17-Jul-2009 & 20-Jul-2009 & SW 846/6020 & \\
\hline Cobalt & 0.789 & 0.500 & $\mathrm{mg} / \mathrm{kg}$ & 2 & 17-Jul-2009 & 20-Jul-2009 & SW 846/6020 & \\
\hline Copper & 4.32 & 0.500 & $\mathrm{mg} / \mathrm{kg}$ & 2 & 17-Jul-2009 & 20-Jul-2009 & SW 846/6020 & \\
\hline Iron & 492 & 0.500 & $\mathrm{mg} / \mathrm{kg}$ & 2 & 17-Jul-2009 & 20-Jul-2009 & SW 846/6020 & \\
\hline Lead & 1.41 & 0.500 & $\mathrm{mg} / \mathrm{kg}$ & 2 & 17-Jul-2009 & 20-Jul-2009 & SW 846/6020 & \\
\hline Magnesium & 369 & 0.500 & $\mathrm{mg} / \mathrm{kg}$ & 2 & 17-Jul-2009 & 20-Jul-2009 & SW 846/6020 & \\
\hline Manganese & 5.35 & 0.500 & $\mathrm{mg} / \mathrm{kg}$ & 2 & 17-Jul-2009 & 20-Jul-2009 & SW 846/6020 & \\
\hline Molybdenum & 0.300 & 0.500 & $\mathrm{mg} / \mathrm{kg}$ & 2 & 17-Jul-2009 & 20-Jul-2009 & SW 846/6020 & $\mathrm{J}$ \\
\hline Nickel & 4.72 & 0.500 & $\mathrm{mg} / \mathrm{kg}$ & 2 & 17-Jul-2009 & 20-Jul-2009 & SW 846/6020 & \\
\hline Potassium & 2800 & 0.500 & $\mathrm{mg} / \mathrm{kg}$ & 2 & 17-Jul-2009 & 20-Jul-2009 & SW 846/6020 & \\
\hline Selenium & 0.592 & 0.500 & $\mathrm{mg} / \mathrm{kg}$ & 2 & 17-Jul-2009 & 20-Jul-2009 & SW 846/6020 & \\
\hline Silver & ND & 0.500 & $\mathrm{mg} / \mathrm{kg}$ & 2 & 17-Jul-2009 & 20-Jul-2009 & SW 846/6020 & $\mathrm{U}$ \\
\hline Sodium & 1080 & 0.500 & $\mathrm{mg} / \mathrm{kg}$ & 2 & 17-Jul-2009 & 20-Jul-2009 & SW 846/6020 & \\
\hline Thallium & 0.213 & 0.500 & $\mathrm{mg} / \mathrm{kg}$ & 2 & 17-Jul-2009 & 20-Jul-2009 & SW 846/6020 & J \\
\hline Vanadium & 3.94 & 0.500 & $\mathrm{mg} / \mathrm{kg}$ & 2 & 17-Jul-2009 & 20-Jul-2009 & SW 846/6020 & \\
\hline Zinc & 32.7 & 0.500 & $\mathrm{mg} / \mathrm{kg}$ & 2 & 17-Jul-2009 & 20-Jul-2009 & SW 846/6020 & B \\
\hline
\end{tabular}




\section{USACE ERDC-EP-C \\ 3909 Halls Ferry Road \\ Vicksburg, MS 39180-6199}

ERDC - ECB $--,-$
Reported: 03-Sep-2009

DRAFT: EMR - EL - AF - 100 - E

9071602-05 (Tissue)

\begin{tabular}{|c|c|c|c|c|c|c|c|c|}
\hline Analyte & Result & $\begin{array}{l}\text { orting } \\
\text { Limit }\end{array}$ & Units & Dilution & Prepared & Analyzed & Method & Notes \\
\hline
\end{tabular}

ERDC- EL-EP-C (Environmental Chemistry Branch)

DRAFT: Metals by EPA 6000/7000 Series Methods

\begin{tabular}{|c|c|c|c|c|c|c|c|c|}
\hline Mercury & 0.020 & 0.002 & $\mathrm{mg} / \mathrm{kg}$ & 1 & 22-Jul-2009 & 22-Jul-2009 & EPA 7471A & \\
\hline Aluminum & 511 & 0.500 & $\mathrm{mg} / \mathrm{kg}$ & 2 & 17-Jul-2009 & 20-Jul-2009 & SW 846/6020 & \\
\hline Antimony & ND & 0.500 & $\mathrm{mg} / \mathrm{kg}$ & 2 & 17-Jul-2009 & 20-Jul-2009 & SW 846/6020 & $\mathrm{U}$ \\
\hline Arsenic & 1.64 & 0.500 & $\mathrm{mg} / \mathrm{kg}$ & 2 & 17-Jul-2009 & 20-Jul-2009 & SW 846/6020 & \\
\hline Barium & 11.1 & 0.500 & $\mathrm{mg} / \mathrm{kg}$ & 2 & 17-Jul-2009 & 20-Jul-2009 & SW 846/6020 & \\
\hline Beryllium & ND & 0.500 & $\mathrm{mg} / \mathrm{kg}$ & 2 & 17-Jul-2009 & 20-Jul-2009 & SW 846/6020 & $\mathrm{U}$ \\
\hline Cadmium & 0.291 & 0.500 & $\mathrm{mg} / \mathrm{kg}$ & 2 & 17-Jul-2009 & 20-Jul-2009 & SW 846/6020 & J \\
\hline Calcium & 5050 & 0.500 & $\mathrm{mg} / \mathrm{kg}$ & 2 & 17-Jul-2009 & 20-Jul-2009 & SW 846/6020 & \\
\hline Chromium & 2.44 & 0.500 & $\mathrm{mg} / \mathrm{kg}$ & 2 & 17-Jul-2009 & 20-Jul-2009 & SW 846/6020 & \\
\hline Cobalt & 0.421 & 0.500 & $\mathrm{mg} / \mathrm{kg}$ & 2 & 17-Jul-2009 & 20-Jul-2009 & SW 846/6020 & J \\
\hline Copper & 2.78 & 0.500 & $\mathrm{mg} / \mathrm{kg}$ & 2 & 17-Jul-2009 & 20-Jul-2009 & SW 846/6020 & \\
\hline Iron & 279 & 0.500 & $\mathrm{mg} / \mathrm{kg}$ & 2 & 17-Jul-2009 & 20-Jul-2009 & SW 846/6020 & \\
\hline Lead & 1.08 & 0.500 & $\mathrm{mg} / \mathrm{kg}$ & 2 & 17-Jul-2009 & 20-Jul-2009 & SW 846/6020 & \\
\hline Magnesium & 299 & 0.500 & $\mathrm{mg} / \mathrm{kg}$ & 2 & 17-Jul-2009 & 20-Jul-2009 & SW 846/6020 & \\
\hline Manganese & 2.87 & 0.500 & $\mathrm{mg} / \mathrm{kg}$ & 2 & 17-Jul-2009 & 20-Jul-2009 & SW 846/6020 & \\
\hline Molybdenum & 0.148 & 0.500 & $\mathrm{mg} / \mathrm{kg}$ & 2 & 17-Jul-2009 & 20-Jul-2009 & SW 846/6020 & $\mathrm{J}$ \\
\hline Nickel & 1.86 & 0.500 & $\mathrm{mg} / \mathrm{kg}$ & 2 & 17-Jul-2009 & 20-Jul-2009 & SW 846/6020 & \\
\hline Potassium & 2590 & 0.500 & $\mathrm{mg} / \mathrm{kg}$ & 2 & 17-Jul-2009 & 20-Jul-2009 & SW 846/6020 & \\
\hline Selenium & 0.465 & 0.500 & $\mathrm{mg} / \mathrm{kg}$ & 2 & 17-Jul-2009 & 20-Jul-2009 & SW 846/6020 & J \\
\hline Silver & $\mathrm{ND}$ & 0.500 & $\mathrm{mg} / \mathrm{kg}$ & 2 & 17-Jul-2009 & 20-Jul-2009 & SW 846/6020 & $\mathrm{U}$ \\
\hline Sodium & 938 & 0.500 & $\mathrm{mg} / \mathrm{kg}$ & 2 & 17-Jul-2009 & 20-Jul-2009 & SW 846/6020 & \\
\hline Thallium & 0.168 & 0.500 & $\mathrm{mg} / \mathrm{kg}$ & 2 & 17-Jul-2009 & 20-Jul-2009 & SW 846/6020 & $\mathrm{J}$ \\
\hline Vanadium & 2.35 & 0.500 & $\mathrm{mg} / \mathrm{kg}$ & 2 & 17-Jul-2009 & 20-Jul-2009 & SW 846/6020 & \\
\hline Zinc & 24.9 & 0.500 & $\mathrm{mg} / \mathrm{kg}$ & 2 & 17-Jul-2009 & 20-Jul-2009 & SW 846/6020 & B \\
\hline
\end{tabular}




\section{USACE ERDC-EP-C \\ 3909 Halls Ferry Road \\ Vicksburg, MS 39180-6199}

ERDC - ECB

$--,-$
Project: TVA Fly Ash Project

Project Manager: Tony Bednar
Reported:

03-Sep-2009

DRAFT: EMR - EL - AF - 50 - A

9071602-06 (Tissue)

\begin{tabular}{|c|c|c|c|c|c|c|c|c|}
\hline & & porting & & & & & & \\
\hline Analyte & Result & Limit & Units & Dilution & Prepared & Analyzed & Method & Notes \\
\hline
\end{tabular}

ERDC- EL-EP-C (Environmental Chemistry Branch)

DRAFT: Metals by EPA 6000/7000 Series Methods

\begin{tabular}{|c|c|c|c|c|c|c|c|c|}
\hline Mercury & 0.022 & 0.002 & $\mathrm{mg} / \mathrm{kg}$ & 1 & 22-Jul-2009 & 22-Jul-2009 & EPA 7471A & \\
\hline Aluminum & 1020 & 0.500 & $\mathrm{mg} / \mathrm{kg}$ & 2 & 17-Jul-2009 & 20-Jul-2009 & SW 846/6020 & \\
\hline Antimony & 0.123 & 0.500 & $\mathrm{mg} / \mathrm{kg}$ & 2 & 17-Jul-2009 & 20-Jul-2009 & SW 846/6020 & J \\
\hline Arsenic & 3.18 & 0.500 & $\mathrm{mg} / \mathrm{kg}$ & 2 & 17-Jul-2009 & 20-Jul-2009 & SW 846/6020 & \\
\hline Barium & 19.6 & 0.500 & $\mathrm{mg} / \mathrm{kg}$ & 2 & 17-Jul-2009 & 20-Jul-2009 & SW 846/6020 & \\
\hline Beryllium & 0.173 & 0.500 & $\mathrm{mg} / \mathrm{kg}$ & 2 & 17-Jul-2009 & 20-Jul-2009 & SW 846/6020 & $\mathrm{J}$ \\
\hline Cadmium & 0.353 & 0.500 & $\mathrm{mg} / \mathrm{kg}$ & 2 & 17-Jul-2009 & 20-Jul-2009 & SW 846/6020 & $\mathrm{J}$ \\
\hline Calcium & 5530 & 0.500 & $\mathrm{mg} / \mathrm{kg}$ & 2 & 17-Jul-2009 & 20-Jul-2009 & SW 846/6020 & \\
\hline Chromium & 4.83 & 0.500 & $\mathrm{mg} / \mathrm{kg}$ & 2 & 17-Jul-2009 & 20-Jul-2009 & SW 846/6020 & \\
\hline Cobalt & 0.845 & 0.500 & $\mathrm{mg} / \mathrm{kg}$ & 2 & 17-Jul-2009 & 20-Jul-2009 & SW 846/6020 & \\
\hline Copper & 4.41 & 0.500 & $\mathrm{mg} / \mathrm{kg}$ & 2 & 17-Jul-2009 & 20-Jul-2009 & SW 846/6020 & \\
\hline Iron & 551 & 0.500 & $\mathrm{mg} / \mathrm{kg}$ & 2 & 17-Jul-2009 & 20-Jul-2009 & SW 846/6020 & \\
\hline Lead & 1.58 & 0.500 & $\mathrm{mg} / \mathrm{kg}$ & 2 & 17-Jul-2009 & 20-Jul-2009 & SW 846/6020 & \\
\hline Magnesium & 344 & 0.500 & $\mathrm{mg} / \mathrm{kg}$ & 2 & 17-Jul-2009 & 20-Jul-2009 & SW 846/6020 & \\
\hline Manganese & 5.97 & 0.500 & $\mathrm{mg} / \mathrm{kg}$ & 2 & 17-Jul-2009 & 20-Jul-2009 & SW 846/6020 & \\
\hline Molybdenum & 0.278 & 0.500 & $\mathrm{mg} / \mathrm{kg}$ & 2 & 17-Jul-2009 & 20-Jul-2009 & SW 846/6020 & $\mathrm{J}$ \\
\hline Nickel & 3.51 & 0.500 & $\mathrm{mg} / \mathrm{kg}$ & 2 & 17-Jul-2009 & 20-Jul-2009 & SW 846/6020 & \\
\hline Potassium & 2730 & 0.500 & $\mathrm{mg} / \mathrm{kg}$ & 2 & 17-Jul-2009 & 20-Jul-2009 & SW 846/6020 & \\
\hline Selenium & 0.526 & 0.500 & $\mathrm{mg} / \mathrm{kg}$ & 2 & 17-Jul-2009 & 20-Jul-2009 & SW 846/6020 & \\
\hline Silver & 0.175 & 0.500 & $\mathrm{mg} / \mathrm{kg}$ & 2 & 17-Jul-2009 & 20-Jul-2009 & SW 846/6020 & J \\
\hline Sodium & 993 & 0.500 & $\mathrm{mg} / \mathrm{kg}$ & 2 & 17-Jul-2009 & 20-Jul-2009 & SW 846/6020 & \\
\hline Thallium & 0.170 & 0.500 & $\mathrm{mg} / \mathrm{kg}$ & 2 & 17-Jul-2009 & 20-Jul-2009 & SW 846/6020 & $\mathrm{J}$ \\
\hline Vanadium & 4.41 & 0.500 & $\mathrm{mg} / \mathrm{kg}$ & 2 & 17-Jul-2009 & 20-Jul-2009 & SW 846/6020 & \\
\hline Zinc & 26.7 & 0.500 & $\mathrm{mg} / \mathrm{kg}$ & 2 & 17-Jul-2009 & 20-Jul-2009 & SW 846/6020 & B \\
\hline
\end{tabular}




\section{USACE ERDC-EP-C \\ 3909 Halls Ferry Road \\ Vicksburg, MS 39180-6199}

ERDC - ECB

$--,-$
Project: TVA Fly Ash Project

Project Manager: Tony Bednar
Reported:

03-Sep-2009

DRAFT: EMR - EL - AF - 50 - B

9071602-07 (Tissue)

\begin{tabular}{|c|c|c|c|c|c|c|c|c|}
\hline Analyte & Result & $\begin{array}{r}\text { porting } \\
\text { Limit }\end{array}$ & Units & Dilution & Prepared & Analyzed & Method & Notes \\
\hline
\end{tabular}

ERDC- EL-EP-C (Environmental Chemistry Branch)

DRAFT: Metals by EPA 6000/7000 Series Methods

\begin{tabular}{|c|c|c|c|c|c|c|c|c|}
\hline Mercury & 0.020 & 0.004 & $\mathrm{mg} / \mathrm{kg}$ & 1 & 22-Jul-2009 & 22-Jul-2009 & EPA 7471A & \\
\hline Aluminum & 601 & 0.500 & $\mathrm{mg} / \mathrm{kg}$ & 2 & 17-Jul-2009 & 20-Jul-2009 & SW 846/6020 & \\
\hline Antimony & ND & 0.500 & $\mathrm{mg} / \mathrm{kg}$ & 2 & 17-Jul-2009 & 20-Jul-2009 & SW 846/6020 & $\mathrm{U}$ \\
\hline Arsenic & 1.83 & 0.500 & $\mathrm{mg} / \mathrm{kg}$ & 2 & 17-Jul-2009 & 20-Jul-2009 & SW 846/6020 & \\
\hline Barium & 11.5 & 0.500 & $\mathrm{mg} / \mathrm{kg}$ & 2 & 17-Jul-2009 & 20-Jul-2009 & SW 846/6020 & \\
\hline Beryllium & 0.104 & 0.500 & $\mathrm{mg} / \mathrm{kg}$ & 2 & 17-Jul-2009 & 20-Jul-2009 & SW 846/6020 & $\mathrm{J}$ \\
\hline Cadmium & 0.268 & 0.500 & $\mathrm{mg} / \mathrm{kg}$ & 2 & 17-Jul-2009 & 20-Jul-2009 & SW 846/6020 & $\mathrm{J}$ \\
\hline Calcium & 4990 & 0.500 & $\mathrm{mg} / \mathrm{kg}$ & 2 & 17-Jul-2009 & 20-Jul-2009 & SW 846/6020 & \\
\hline Chromium & 9.17 & 0.500 & $\mathrm{mg} / \mathrm{kg}$ & 2 & 17-Jul-2009 & 20-Jul-2009 & SW 846/6020 & \\
\hline Cobalt & 0.577 & 0.500 & $\mathrm{mg} / \mathrm{kg}$ & 2 & 17-Jul-2009 & 20-Jul-2009 & SW 846/6020 & \\
\hline Copper & 3.05 & 0.500 & $\mathrm{mg} / \mathrm{kg}$ & 2 & 17-Jul-2009 & 20-Jul-2009 & SW 846/6020 & \\
\hline Iron & 342 & 0.500 & $\mathrm{mg} / \mathrm{kg}$ & 2 & 17-Jul-2009 & 20-Jul-2009 & SW 846/6020 & \\
\hline Lead & 0.901 & 0.500 & $\mathrm{mg} / \mathrm{kg}$ & 2 & 17-Jul-2009 & 20-Jul-2009 & SW 846/6020 & \\
\hline Magnesium & 294 & 0.500 & $\mathrm{mg} / \mathrm{kg}$ & 2 & 17-Jul-2009 & 20-Jul-2009 & SW 846/6020 & \\
\hline Manganese & 4.04 & 0.500 & $\mathrm{mg} / \mathrm{kg}$ & 2 & 17-Jul-2009 & 20-Jul-2009 & SW 846/6020 & \\
\hline Molybdenum & 0.356 & 0.500 & $\mathrm{mg} / \mathrm{kg}$ & 2 & 17-Jul-2009 & 20-Jul-2009 & SW 846/6020 & $\mathrm{J}$ \\
\hline Nickel & 5.12 & 0.500 & $\mathrm{mg} / \mathrm{kg}$ & 2 & 17-Jul-2009 & 20-Jul-2009 & SW 846/6020 & \\
\hline Potassium & 2280 & 0.500 & $\mathrm{mg} / \mathrm{kg}$ & 2 & 17-Jul-2009 & 20-Jul-2009 & SW 846/6020 & \\
\hline Selenium & 0.444 & 0.500 & $\mathrm{mg} / \mathrm{kg}$ & 2 & 17-Jul-2009 & 20-Jul-2009 & SW 846/6020 & $\mathrm{J}$ \\
\hline Silver & ND & 0.500 & $\mathrm{mg} / \mathrm{kg}$ & 2 & 17-Jul-2009 & 20-Jul-2009 & SW 846/6020 & $\mathrm{U}$ \\
\hline Sodium & 939 & 0.500 & $\mathrm{mg} / \mathrm{kg}$ & 2 & 17-Jul-2009 & 20-Jul-2009 & SW 846/6020 & \\
\hline Thallium & 0.113 & 0.500 & $\mathrm{mg} / \mathrm{kg}$ & 2 & 17-Jul-2009 & 20-Jul-2009 & SW 846/6020 & $\mathrm{J}$ \\
\hline Vanadium & 2.69 & 0.500 & $\mathrm{mg} / \mathrm{kg}$ & 2 & 17-Jul-2009 & 20-Jul-2009 & SW 846/6020 & \\
\hline Zinc & 27.2 & 0.500 & $\mathrm{mg} / \mathrm{kg}$ & 2 & 17-Jul-2009 & 20-Jul-2009 & SW 846/6020 & B \\
\hline
\end{tabular}




\section{USACE ERDC-EP-C \\ 3909 Halls Ferry Road \\ Vicksburg, MS 39180-6199}

ERDC - ECB

Project: TVA Fly Ash Project

$-$

$\begin{array}{cc} & \text { Reported: } \\ \text { Project Manager: Tony Bednar } & 03-S e p-2009\end{array}$

DRAFT: EMR - EL - AF - 50 - C

9071602-08 (Tissue)

\begin{tabular}{|c|c|c|c|c|c|c|c|c|}
\hline & & porting & & & & & & \\
\hline Analyte & Result & Limit & Units & Dilution & Prepared & Analyzed & Method & Notes \\
\hline
\end{tabular}

ERDC- EL-EP-C (Environmental Chemistry Branch)

DRAFT: Metals by EPA 6000/7000 Series Methods

\begin{tabular}{|c|c|c|c|c|c|c|c|c|}
\hline Mercury & 0.023 & 0.002 & $\mathrm{mg} / \mathrm{kg}$ & 1 & 22-Jul-2009 & 22-Jul-2009 & EPA 7471A & \\
\hline Aluminum & 1210 & 0.500 & $\mathrm{mg} / \mathrm{kg}$ & 2 & 17-Jul-2009 & 20-Jul-2009 & SW 846/6020 & \\
\hline Antimony & 0.134 & 0.500 & $\mathrm{mg} / \mathrm{kg}$ & 2 & 17-Jul-2009 & 20-Jul-2009 & SW 846/6020 & $\mathrm{J}$ \\
\hline Arsenic & 3.69 & 0.500 & $\mathrm{mg} / \mathrm{kg}$ & 2 & 17-Jul-2009 & 20-Jul-2009 & SW 846/6020 & \\
\hline Barium & 23.5 & 0.500 & $\mathrm{mg} / \mathrm{kg}$ & 2 & 17-Jul-2009 & 20-Jul-2009 & SW 846/6020 & \\
\hline Beryllium & 0.205 & 0.500 & $\mathrm{mg} / \mathrm{kg}$ & 2 & 17-Jul-2009 & 20-Jul-2009 & SW 846/6020 & $\mathrm{J}$ \\
\hline Cadmium & 0.444 & 0.500 & $\mathrm{mg} / \mathrm{kg}$ & 2 & 17-Jul-2009 & 20-Jul-2009 & SW 846/6020 & $\mathrm{J}$ \\
\hline Calcium & 5950 & 0.500 & $\mathrm{mg} / \mathrm{kg}$ & 2 & 17-Jul-2009 & 20-Jul-2009 & SW 846/6020 & \\
\hline Chromium & 5.19 & 0.500 & $\mathrm{mg} / \mathrm{kg}$ & 2 & 17-Jul-2009 & 20-Jul-2009 & SW 846/6020 & \\
\hline Cobalt & 1.02 & 0.500 & $\mathrm{mg} / \mathrm{kg}$ & 2 & 17-Jul-2009 & 20-Jul-2009 & SW 846/6020 & \\
\hline Copper & 4.48 & 0.500 & $\mathrm{mg} / \mathrm{kg}$ & 2 & 17-Jul-2009 & 20-Jul-2009 & SW 846/6020 & \\
\hline Iron & 646 & 0.500 & $\mathrm{mg} / \mathrm{kg}$ & 2 & 17-Jul-2009 & 20-Jul-2009 & SW 846/6020 & \\
\hline Lead & 1.87 & 0.500 & $\mathrm{mg} / \mathrm{kg}$ & 2 & 17-Jul-2009 & 20-Jul-2009 & SW 846/6020 & \\
\hline Magnesium & 369 & 0.500 & $\mathrm{mg} / \mathrm{kg}$ & 2 & 17-Jul-2009 & 20-Jul-2009 & SW 846/6020 & \\
\hline Manganese & 7.14 & 0.500 & $\mathrm{mg} / \mathrm{kg}$ & 2 & 17-Jul-2009 & 20-Jul-2009 & SW 846/6020 & \\
\hline Molybdenum & 0.298 & 0.500 & $\mathrm{mg} / \mathrm{kg}$ & 2 & 17-Jul-2009 & 20-Jul-2009 & SW 846/6020 & $\mathrm{J}$ \\
\hline Nickel & 4.13 & 0.500 & $\mathrm{mg} / \mathrm{kg}$ & 2 & 17-Jul-2009 & 20-Jul-2009 & SW 846/6020 & \\
\hline Potassium & 2440 & 0.500 & $\mathrm{mg} / \mathrm{kg}$ & 2 & 17-Jul-2009 & 20-Jul-2009 & SW 846/6020 & \\
\hline Selenium & 0.566 & 0.500 & $\mathrm{mg} / \mathrm{kg}$ & 2 & 17-Jul-2009 & 20-Jul-2009 & SW 846/6020 & \\
\hline Silver & 0.104 & 0.500 & $\mathrm{mg} / \mathrm{kg}$ & 2 & 17-Jul-2009 & 20-Jul-2009 & SW 846/6020 & $\mathrm{J}$ \\
\hline Sodium & 953 & 0.500 & $\mathrm{mg} / \mathrm{kg}$ & 2 & 17-Jul-2009 & 20-Jul-2009 & SW 846/6020 & \\
\hline Thallium & 0.162 & 0.500 & $\mathrm{mg} / \mathrm{kg}$ & 2 & 17-Jul-2009 & 20-Jul-2009 & SW 846/6020 & $\mathrm{J}$ \\
\hline Vanadium & 4.93 & 0.500 & $\mathrm{mg} / \mathrm{kg}$ & 2 & 17-Jul-2009 & 20-Jul-2009 & SW 846/6020 & \\
\hline Zinc & 29.7 & 0.500 & $\mathrm{mg} / \mathrm{kg}$ & 2 & 17-Jul-2009 & 20-Jul-2009 & SW 846/6020 & B \\
\hline
\end{tabular}




\section{USACE ERDC-EP-C \\ 3909 Halls Ferry Road \\ Vicksburg, MS 39180-6199}

ERDC - ECB

Project: TVA Fly Ash Project

$-$

$\begin{array}{cc} & \text { Reported: } \\ \text { Project Manager: Tony Bednar } & 03-S e p-2009\end{array}$

DRAFT: EMR - EL - AF - 50 - D

9071602-09 (Tissue)

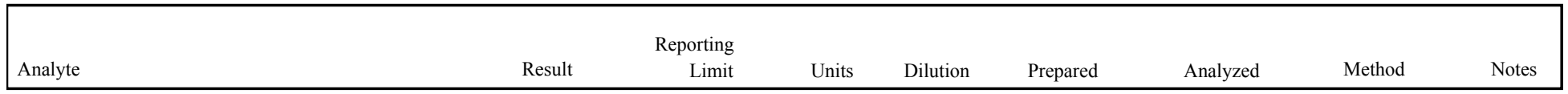

ERDC- EL-EP-C (Environmental Chemistry Branch)

DRAFT: Metals by EPA 6000/7000 Series Methods

\begin{tabular}{|c|c|c|c|c|c|c|c|c|}
\hline Mercury & 0.019 & 0.002 & $\mathrm{mg} / \mathrm{kg}$ & 1 & 22-Jul-2009 & 22-Jul-2009 & EPA 7471A & \\
\hline Aluminum & 798 & 0.500 & $\mathrm{mg} / \mathrm{kg}$ & 2 & 17-Jul-2009 & 20-Jul-2009 & SW 846/6020 & \\
\hline Antimony & ND & 0.500 & $\mathrm{mg} / \mathrm{kg}$ & 2 & 17-Jul-2009 & 20-Jul-2009 & SW 846/6020 & $\mathrm{U}$ \\
\hline Arsenic & 2.56 & 0.500 & $\mathrm{mg} / \mathrm{kg}$ & 2 & 17-Jul-2009 & 20-Jul-2009 & SW 846/6020 & \\
\hline Barium & 15.6 & 0.500 & $\mathrm{mg} / \mathrm{kg}$ & 2 & 17-Jul-2009 & 20-Jul-2009 & SW 846/6020 & \\
\hline Beryllium & 0.136 & 0.500 & $\mathrm{mg} / \mathrm{kg}$ & 2 & 17-Jul-2009 & 20-Jul-2009 & SW 846/6020 & $\mathrm{J}$ \\
\hline Cadmium & 0.339 & 0.500 & $\mathrm{mg} / \mathrm{kg}$ & 2 & 17-Jul-2009 & 20-Jul-2009 & SW 846/6020 & $\mathrm{J}$ \\
\hline Calcium & 5490 & 0.500 & $\mathrm{mg} / \mathrm{kg}$ & 2 & 17-Jul-2009 & 20-Jul-2009 & SW 846/6020 & \\
\hline Chromium & 3.97 & 0.500 & $\mathrm{mg} / \mathrm{kg}$ & 2 & 17-Jul-2009 & 20-Jul-2009 & SW 846/6020 & \\
\hline Cobalt & 0.665 & 0.500 & $\mathrm{mg} / \mathrm{kg}$ & 2 & 17-Jul-2009 & 20-Jul-2009 & SW 846/6020 & \\
\hline Copper & 3.30 & 0.500 & $\mathrm{mg} / \mathrm{kg}$ & 2 & 17-Jul-2009 & 20-Jul-2009 & SW 846/6020 & \\
\hline Iron & 433 & 0.500 & $\mathrm{mg} / \mathrm{kg}$ & 2 & 17-Jul-2009 & 20-Jul-2009 & SW 846/6020 & \\
\hline Lead & 1.22 & 0.500 & $\mathrm{mg} / \mathrm{kg}$ & 2 & 17-Jul-2009 & 20-Jul-2009 & SW 846/6020 & \\
\hline Magnesium & 328 & 0.500 & $\mathrm{mg} / \mathrm{kg}$ & 2 & 17-Jul-2009 & 20-Jul-2009 & SW 846/6020 & \\
\hline Manganese & 4.47 & 0.500 & $\mathrm{mg} / \mathrm{kg}$ & 2 & 17-Jul-2009 & 20-Jul-2009 & SW 846/6020 & \\
\hline Molybdenum & 0.215 & 0.500 & $\mathrm{mg} / \mathrm{kg}$ & 2 & 17-Jul-2009 & 20-Jul-2009 & SW 846/6020 & $\mathrm{J}$ \\
\hline Nickel & 2.90 & 0.500 & $\mathrm{mg} / \mathrm{kg}$ & 2 & 17-Jul-2009 & 20-Jul-2009 & SW 846/6020 & \\
\hline Potassium & 2590 & 0.500 & $\mathrm{mg} / \mathrm{kg}$ & 2 & 17-Jul-2009 & 20-Jul-2009 & SW 846/6020 & \\
\hline Selenium & 0.475 & 0.500 & $\mathrm{mg} / \mathrm{kg}$ & 2 & 17-Jul-2009 & 20-Jul-2009 & SW 846/6020 & J \\
\hline Silver & ND & 0.500 & $\mathrm{mg} / \mathrm{kg}$ & 2 & 17-Jul-2009 & 20-Jul-2009 & SW 846/6020 & $\mathrm{U}$ \\
\hline Sodium & 982 & 0.500 & $\mathrm{mg} / \mathrm{kg}$ & 2 & 17-Jul-2009 & 20-Jul-2009 & SW 846/6020 & \\
\hline Thallium & 0.131 & 0.500 & $\mathrm{mg} / \mathrm{kg}$ & 2 & 17-Jul-2009 & 20-Jul-2009 & SW 846/6020 & $\mathrm{J}$ \\
\hline Vanadium & 3.54 & 0.500 & $\mathrm{mg} / \mathrm{kg}$ & 2 & 17-Jul-2009 & 20-Jul-2009 & SW 846/6020 & \\
\hline Zinc & 26.9 & 0.500 & $\mathrm{mg} / \mathrm{kg}$ & 2 & 17-Jul-2009 & 20-Jul-2009 & SW 846/6020 & B \\
\hline
\end{tabular}




\section{USACE ERDC-EP-C \\ 3909 Halls Ferry Road \\ Vicksburg, MS 39180-6199}

ERDC - ECB

Project: TVA Fly Ash Project

$-$

$\begin{array}{cc} & \text { Reported: } \\ \text { Project Manager: Tony Bednar } & 03-S e p-2009\end{array}$

DRAFT: EMR - EL - AF - 50 - E

9071602-10 (Tissue)

\begin{tabular}{|c|c|c|c|c|c|c|c|c|}
\hline & & porting & & & & & & \\
\hline Analyte & Result & Limit & Units & Dilution & Prepared & Analyzed & Method & Notes \\
\hline
\end{tabular}

ERDC- EL-EP-C (Environmental Chemistry Branch)

DRAFT: Metals by EPA 6000/7000 Series Methods

\begin{tabular}{|c|c|c|c|c|c|c|c|c|}
\hline Mercury & 0.029 & 0.003 & $\mathrm{mg} / \mathrm{kg}$ & 1 & 22-Jul-2009 & 22-Jul-2009 & EPA 7471A & \\
\hline Aluminum & 1150 & 0.500 & $\mathrm{mg} / \mathrm{kg}$ & 2 & 17-Jul-2009 & 20-Jul-2009 & SW 846/6020 & \\
\hline Antimony & 0.125 & 0.500 & $\mathrm{mg} / \mathrm{kg}$ & 2 & 17-Jul-2009 & 20-Jul-2009 & SW 846/6020 & J \\
\hline Arsenic & 3.50 & 0.500 & $\mathrm{mg} / \mathrm{kg}$ & 2 & 17-Jul-2009 & 20-Jul-2009 & SW 846/6020 & \\
\hline Barium & 22.5 & 0.500 & $\mathrm{mg} / \mathrm{kg}$ & 2 & 17-Jul-2009 & 20-Jul-2009 & SW 846/6020 & \\
\hline Beryllium & 0.196 & 0.500 & $\mathrm{mg} / \mathrm{kg}$ & 2 & 17-Jul-2009 & 20-Jul-2009 & SW 846/6020 & J \\
\hline Cadmium & 0.436 & 0.500 & $\mathrm{mg} / \mathrm{kg}$ & 2 & 17-Jul-2009 & 20-Jul-2009 & SW 846/6020 & $\mathrm{J}$ \\
\hline Calcium & 5870 & 0.500 & $\mathrm{mg} / \mathrm{kg}$ & 2 & 17-Jul-2009 & 20-Jul-2009 & SW 846/6020 & \\
\hline Chromium & 4.08 & 0.500 & $\mathrm{mg} / \mathrm{kg}$ & 2 & 17-Jul-2009 & 20-Jul-2009 & SW 846/6020 & \\
\hline Cobalt & 0.961 & 0.500 & $\mathrm{mg} / \mathrm{kg}$ & 2 & 17-Jul-2009 & 20-Jul-2009 & SW 846/6020 & \\
\hline Copper & 5.20 & 0.500 & $\mathrm{mg} / \mathrm{kg}$ & 2 & 17-Jul-2009 & 20-Jul-2009 & SW 846/6020 & \\
\hline Iron & 616 & 0.500 & $\mathrm{mg} / \mathrm{kg}$ & 2 & 17-Jul-2009 & 20-Jul-2009 & SW 846/6020 & \\
\hline Lead & 1.80 & 0.500 & $\mathrm{mg} / \mathrm{kg}$ & 2 & 17-Jul-2009 & 20-Jul-2009 & SW 846/6020 & \\
\hline Magnesium & 355 & 0.500 & $\mathrm{mg} / \mathrm{kg}$ & 2 & 17-Jul-2009 & 20-Jul-2009 & SW 846/6020 & \\
\hline Manganese & 6.50 & 0.500 & $\mathrm{mg} / \mathrm{kg}$ & 2 & 17-Jul-2009 & 20-Jul-2009 & SW 846/6020 & \\
\hline Molybdenum & 0.262 & 0.500 & $\mathrm{mg} / \mathrm{kg}$ & 2 & 17-Jul-2009 & 20-Jul-2009 & SW 846/6020 & $\mathrm{J}$ \\
\hline Nickel & 3.49 & 0.500 & $\mathrm{mg} / \mathrm{kg}$ & 2 & 17-Jul-2009 & 20-Jul-2009 & SW 846/6020 & \\
\hline Potassium & 2450 & 0.500 & $\mathrm{mg} / \mathrm{kg}$ & 2 & 17-Jul-2009 & 20-Jul-2009 & SW 846/6020 & \\
\hline Selenium & 0.535 & 0.500 & $\mathrm{mg} / \mathrm{kg}$ & 2 & 17-Jul-2009 & 20-Jul-2009 & SW 846/6020 & \\
\hline Silver & 0.104 & 0.500 & $\mathrm{mg} / \mathrm{kg}$ & 2 & 17-Jul-2009 & 20-Jul-2009 & SW 846/6020 & $\mathrm{J}$ \\
\hline Sodium & 992 & 0.500 & $\mathrm{mg} / \mathrm{kg}$ & 2 & 17-Jul-2009 & 20-Jul-2009 & SW 846/6020 & \\
\hline Thallium & 0.172 & 0.500 & $\mathrm{mg} / \mathrm{kg}$ & 2 & 17-Jul-2009 & 20-Jul-2009 & SW 846/6020 & $\mathrm{J}$ \\
\hline Vanadium & 4.71 & 0.500 & $\mathrm{mg} / \mathrm{kg}$ & 2 & 17-Jul-2009 & 20-Jul-2009 & SW 846/6020 & \\
\hline Zinc & 28.6 & 0.500 & $\mathrm{mg} / \mathrm{kg}$ & 2 & 17-Jul-2009 & 20-Jul-2009 & SW 846/6020 & B \\
\hline
\end{tabular}




\section{USACE ERDC-EP-C \\ 3909 Halls Ferry Road \\ Vicksburg, MS 39180-6199}

ERDC - ECB

Project: TVA Fly Ash Project

-

$\begin{array}{cc} & \text { Reported: } \\ \text { Project Manager: Tony Bednar } & 03-S e p-2009\end{array}$

DRAFT: EMR - EL - AF - 10 - A

9071602-11 (Tissue)

\begin{tabular}{|c|c|c|c|c|c|c|c|c|}
\hline & & porting & & & & & & \\
\hline Analyte & Result & Limit & Units & Dilution & Prepared & Analyzed & Method & Notes \\
\hline
\end{tabular}

ERDC- EL-EP-C (Environmental Chemistry Branch)

DRAFT: Metals by EPA 6000/7000 Series Methods

\begin{tabular}{|c|c|c|c|c|c|c|c|c|}
\hline Mercury & 0.020 & 0.002 & $\mathrm{mg} / \mathrm{kg}$ & 1 & 22-Jul-2009 & 22-Jul-2009 & EPA 7471A & \\
\hline Aluminum & 236 & 0.500 & $\mathrm{mg} / \mathrm{kg}$ & 2 & 17-Jul-2009 & 20-Jul-2009 & SW 846/6020 & \\
\hline Antimony & $\mathrm{ND}$ & 0.500 & $\mathrm{mg} / \mathrm{kg}$ & 2 & 17-Jul-2009 & 20-Jul-2009 & SW 846/6020 & U \\
\hline Arsenic & 0.902 & 0.500 & $\mathrm{mg} / \mathrm{kg}$ & 2 & 17-Jul-2009 & 20-Jul-2009 & SW 846/6020 & \\
\hline Barium & 5.61 & 0.500 & $\mathrm{mg} / \mathrm{kg}$ & 2 & 17-Jul-2009 & 20-Jul-2009 & SW 846/6020 & \\
\hline Beryllium & ND & 0.500 & $\mathrm{mg} / \mathrm{kg}$ & 2 & 17-Jul-2009 & 20-Jul-2009 & SW 846/6020 & U \\
\hline Cadmium & 0.115 & 0.500 & $\mathrm{mg} / \mathrm{kg}$ & 2 & 17-Jul-2009 & 20-Jul-2009 & SW 846/6020 & J \\
\hline Calcium & 6270 & 0.500 & $\mathrm{mg} / \mathrm{kg}$ & 2 & 17-Jul-2009 & 20-Jul-2009 & SW 846/6020 & \\
\hline Chromium & 6.00 & 0.500 & $\mathrm{mg} / \mathrm{kg}$ & 2 & 17-Jul-2009 & 20-Jul-2009 & SW 846/6020 & \\
\hline Cobalt & 0.251 & 0.500 & $\mathrm{mg} / \mathrm{kg}$ & 2 & 17-Jul-2009 & 20-Jul-2009 & SW 846/6020 & J \\
\hline Copper & 1.75 & 0.500 & $\mathrm{mg} / \mathrm{kg}$ & 2 & 17-Jul-2009 & 20-Jul-2009 & SW 846/6020 & \\
\hline Iron & 160 & 0.500 & $\mathrm{mg} / \mathrm{kg}$ & 2 & 17-Jul-2009 & 20-Jul-2009 & SW 846/6020 & \\
\hline Lead & 0.462 & 0.500 & $\mathrm{mg} / \mathrm{kg}$ & 2 & 17-Jul-2009 & 20-Jul-2009 & SW 846/6020 & $\mathrm{J}$ \\
\hline Magnesium & 302 & 0.500 & $\mathrm{mg} / \mathrm{kg}$ & 2 & 17-Jul-2009 & 20-Jul-2009 & SW 846/6020 & \\
\hline Manganese & 2.55 & 0.500 & $\mathrm{mg} / \mathrm{kg}$ & 2 & 17-Jul-2009 & 20-Jul-2009 & SW 846/6020 & \\
\hline Molybdenum & 0.199 & 0.500 & $\mathrm{mg} / \mathrm{kg}$ & 2 & 17-Jul-2009 & 20-Jul-2009 & SW 846/6020 & $\mathrm{J}$ \\
\hline Nickel & 3.20 & 0.500 & $\mathrm{mg} / \mathrm{kg}$ & 2 & 17-Jul-2009 & 20-Jul-2009 & SW 846/6020 & \\
\hline Potassium & 2500 & 0.500 & $\mathrm{mg} / \mathrm{kg}$ & 2 & 17-Jul-2009 & 20-Jul-2009 & SW 846/6020 & \\
\hline Selenium & 0.358 & 0.500 & $\mathrm{mg} / \mathrm{kg}$ & 2 & 17-Jul-2009 & 20-Jul-2009 & SW 846/6020 & $\mathrm{J}$ \\
\hline Silver & ND & 0.500 & $\mathrm{mg} / \mathrm{kg}$ & 2 & 17-Jul-2009 & 20-Jul-2009 & SW 846/6020 & $\mathrm{U}$ \\
\hline Sodium & 965 & 0.500 & $\mathrm{mg} / \mathrm{kg}$ & 2 & 17-Jul-2009 & 20-Jul-2009 & SW 846/6020 & \\
\hline Thallium & ND & 0.500 & $\mathrm{mg} / \mathrm{kg}$ & 2 & 17-Jul-2009 & 20-Jul-2009 & SW 846/6020 & $\mathrm{U}$ \\
\hline Vanadium & 0.907 & 0.500 & $\mathrm{mg} / \mathrm{kg}$ & 2 & 17-Jul-2009 & 20-Jul-2009 & SW 846/6020 & \\
\hline Zinc & 26.5 & 0.500 & $\mathrm{mg} / \mathrm{kg}$ & 2 & 17-Jul-2009 & 20-Jul-2009 & SW 846/6020 & B \\
\hline
\end{tabular}




\section{USACE ERDC-EP-C \\ 3909 Halls Ferry Road \\ Vicksburg, MS 39180-6199}

ERDC - ECB

Project: TVA Fly Ash Project

$-$

$\begin{array}{cc} & \text { Reported: } \\ \text { Project Manager: Tony Bednar } & 03-S e p-2009\end{array}$

DRAFT: EMR - EL - AF - 10 - B

9071602-12 (Tissue)

\begin{tabular}{|c|c|c|c|c|c|c|c|c|}
\hline & & porting & & & & & & \\
\hline Analyte & Result & Limit & Units & Dilution & Prepared & Analyzed & Method & Notes \\
\hline
\end{tabular}

ERDC- EL-EP-C (Environmental Chemistry Branch)

DRAFT: Metals by EPA 6000/7000 Series Methods

\begin{tabular}{|c|c|c|c|c|c|c|c|c|}
\hline Mercury & 0.016 & 0.002 & $\mathrm{mg} / \mathrm{kg}$ & 1 & 22-Jul-2009 & 22-Jul-2009 & EPA 7471A & \\
\hline Aluminum & 475 & 0.500 & $\mathrm{mg} / \mathrm{kg}$ & 2 & 17-Jul-2009 & 20-Jul-2009 & SW 846/6020 & \\
\hline Antimony & ND & 0.500 & $\mathrm{mg} / \mathrm{kg}$ & 2 & 17-Jul-2009 & 20-Jul-2009 & SW 846/6020 & $\mathrm{U}$ \\
\hline Arsenic & 1.32 & 0.500 & $\mathrm{mg} / \mathrm{kg}$ & 2 & 17-Jul-2009 & 20-Jul-2009 & SW 846/6020 & \\
\hline Barium & 9.27 & 0.500 & $\mathrm{mg} / \mathrm{kg}$ & 2 & 17-Jul-2009 & 20-Jul-2009 & SW 846/6020 & \\
\hline Beryllium & ND & 0.500 & $\mathrm{mg} / \mathrm{kg}$ & 2 & 17-Jul-2009 & 20-Jul-2009 & SW 846/6020 & $\mathrm{U}$ \\
\hline Cadmium & 0.127 & 0.500 & $\mathrm{mg} / \mathrm{kg}$ & 2 & 17-Jul-2009 & 20-Jul-2009 & SW 846/6020 & $\mathrm{J}$ \\
\hline Calcium & 5990 & 0.500 & $\mathrm{mg} / \mathrm{kg}$ & 2 & 17-Jul-2009 & 20-Jul-2009 & SW 846/6020 & \\
\hline Chromium & 3.85 & 0.500 & $\mathrm{mg} / \mathrm{kg}$ & 2 & 17-Jul-2009 & 20-Jul-2009 & SW 846/6020 & \\
\hline Cobalt & 0.399 & 0.500 & $\mathrm{mg} / \mathrm{kg}$ & 2 & 17-Jul-2009 & 20-Jul-2009 & SW 846/6020 & $\mathrm{J}$ \\
\hline Copper & 2.10 & 0.500 & $\mathrm{mg} / \mathrm{kg}$ & 2 & 17-Jul-2009 & 20-Jul-2009 & SW 846/6020 & \\
\hline Iron & 277 & 0.500 & $\mathrm{mg} / \mathrm{kg}$ & 2 & 17-Jul-2009 & 20-Jul-2009 & SW 846/6020 & \\
\hline Lead & 1.03 & 0.500 & $\mathrm{mg} / \mathrm{kg}$ & 2 & 17-Jul-2009 & 20-Jul-2009 & SW 846/6020 & \\
\hline Magnesium & 306 & 0.500 & $\mathrm{mg} / \mathrm{kg}$ & 2 & 17-Jul-2009 & 20-Jul-2009 & SW 846/6020 & \\
\hline Manganese & 3.41 & 0.500 & $\mathrm{mg} / \mathrm{kg}$ & 2 & 17-Jul-2009 & 20-Jul-2009 & SW 846/6020 & \\
\hline Molybdenum & 0.176 & 0.500 & $\mathrm{mg} / \mathrm{kg}$ & 2 & 17-Jul-2009 & 20-Jul-2009 & SW 846/6020 & $\mathrm{J}$ \\
\hline Nickel & 2.41 & 0.500 & $\mathrm{mg} / \mathrm{kg}$ & 2 & 17-Jul-2009 & 20-Jul-2009 & SW 846/6020 & \\
\hline Potassium & 2470 & 0.500 & $\mathrm{mg} / \mathrm{kg}$ & 2 & 17-Jul-2009 & 20-Jul-2009 & SW 846/6020 & \\
\hline Selenium & 0.360 & 0.500 & $\mathrm{mg} / \mathrm{kg}$ & 2 & 17-Jul-2009 & 20-Jul-2009 & SW 846/6020 & J \\
\hline Silver & ND & 0.500 & $\mathrm{mg} / \mathrm{kg}$ & 2 & 17-Jul-2009 & 20-Jul-2009 & SW 846/6020 & $\mathrm{U}$ \\
\hline Sodium & 1010 & 0.500 & $\mathrm{mg} / \mathrm{kg}$ & 2 & 17-Jul-2009 & 20-Jul-2009 & SW 846/6020 & \\
\hline Thallium & ND & 0.500 & $\mathrm{mg} / \mathrm{kg}$ & 2 & 17-Jul-2009 & 20-Jul-2009 & SW 846/6020 & $\mathrm{U}$ \\
\hline Vanadium & 1.77 & 0.500 & $\mathrm{mg} / \mathrm{kg}$ & 2 & 17-Jul-2009 & 20-Jul-2009 & SW 846/6020 & \\
\hline Zinc & 27.8 & 0.500 & $\mathrm{mg} / \mathrm{kg}$ & 2 & 17-Jul-2009 & 20-Jul-2009 & SW 846/6020 & B \\
\hline
\end{tabular}




\section{USACE ERDC-EP-C \\ 3909 Halls Ferry Road \\ Vicksburg, MS 39180-6199}

ERDC - ECB

Project: TVA Fly Ash Project

$-$

$\begin{array}{cc} & \text { Reported: } \\ \text { Project Manager: Tony Bednar } & 03-S e p-2009\end{array}$

DRAFT: EMR - EL - AF - 10 - C

9071602-13 (Tissue)

\begin{tabular}{|c|c|c|c|c|c|c|c|c|}
\hline & & porting & & & & & & \\
\hline Analyte & Result & Limit & Units & Dilution & Prepared & Analyzed & Method & Notes \\
\hline
\end{tabular}

ERDC- EL-EP-C (Environmental Chemistry Branch)

DRAFT: Metals by EPA 6000/7000 Series Methods

\begin{tabular}{|c|c|c|c|c|c|c|c|c|}
\hline Mercury & 0.015 & 0.002 & $\mathrm{mg} / \mathrm{kg}$ & 1 & 22-Jul-2009 & 22-Jul-2009 & EPA 7471A & \\
\hline Aluminum & 339 & 0.500 & $\mathrm{mg} / \mathrm{kg}$ & 2 & 17-Jul-2009 & 20-Jul-2009 & SW 846/6020 & \\
\hline Antimony & ND & 0.500 & $\mathrm{mg} / \mathrm{kg}$ & 2 & 17-Jul-2009 & 20-Jul-2009 & SW 846/6020 & $\mathrm{U}$ \\
\hline Arsenic & 1.07 & 0.500 & $\mathrm{mg} / \mathrm{kg}$ & 2 & 17-Jul-2009 & 20-Jul-2009 & SW 846/6020 & \\
\hline Barium & 7.08 & 0.500 & $\mathrm{mg} / \mathrm{kg}$ & 2 & 17-Jul-2009 & 20-Jul-2009 & SW 846/6020 & \\
\hline Beryllium & ND & 0.500 & $\mathrm{mg} / \mathrm{kg}$ & 2 & 17-Jul-2009 & 20-Jul-2009 & SW 846/6020 & $\mathrm{U}$ \\
\hline Cadmium & 0.112 & 0.500 & $\mathrm{mg} / \mathrm{kg}$ & 2 & 17-Jul-2009 & 20-Jul-2009 & SW 846/6020 & $\mathrm{J}$ \\
\hline Calcium & 5140 & 0.500 & $\mathrm{mg} / \mathrm{kg}$ & 2 & 17-Jul-2009 & 20-Jul-2009 & SW 846/6020 & \\
\hline Chromium & 10.4 & 0.500 & $\mathrm{mg} / \mathrm{kg}$ & 2 & 17-Jul-2009 & 20-Jul-2009 & SW 846/6020 & \\
\hline Cobalt & 0.368 & 0.500 & $\mathrm{mg} / \mathrm{kg}$ & 2 & 17-Jul-2009 & 20-Jul-2009 & SW 846/6020 & $\mathrm{J}$ \\
\hline Copper & 2.62 & 0.500 & $\mathrm{mg} / \mathrm{kg}$ & 2 & 17-Jul-2009 & 20-Jul-2009 & SW 846/6020 & \\
\hline Iron & 255 & 0.500 & $\mathrm{mg} / \mathrm{kg}$ & 2 & 17-Jul-2009 & 20-Jul-2009 & SW 846/6020 & \\
\hline Lead & 0.620 & 0.500 & $\mathrm{mg} / \mathrm{kg}$ & 2 & 17-Jul-2009 & 20-Jul-2009 & SW 846/6020 & \\
\hline Magnesium & 268 & 0.500 & $\mathrm{mg} / \mathrm{kg}$ & 2 & 17-Jul-2009 & 20-Jul-2009 & SW 846/6020 & \\
\hline Manganese & 3.54 & 0.500 & $\mathrm{mg} / \mathrm{kg}$ & 2 & 17-Jul-2009 & 20-Jul-2009 & SW 846/6020 & \\
\hline Molybdenum & 0.588 & 0.500 & $\mathrm{mg} / \mathrm{kg}$ & 2 & 17-Jul-2009 & 20-Jul-2009 & SW 846/6020 & \\
\hline Nickel & 4.93 & 0.500 & $\mathrm{mg} / \mathrm{kg}$ & 2 & 17-Jul-2009 & 20-Jul-2009 & SW 846/6020 & \\
\hline Potassium & 2110 & 0.500 & $\mathrm{mg} / \mathrm{kg}$ & 2 & 17-Jul-2009 & 20-Jul-2009 & SW 846/6020 & \\
\hline Selenium & 0.303 & 0.500 & $\mathrm{mg} / \mathrm{kg}$ & 2 & 17-Jul-2009 & 20-Jul-2009 & SW 846/6020 & J \\
\hline Silver & ND & 0.500 & $\mathrm{mg} / \mathrm{kg}$ & 2 & 17-Jul-2009 & 20-Jul-2009 & SW 846/6020 & $\mathrm{U}$ \\
\hline Sodium & 882 & 0.500 & $\mathrm{mg} / \mathrm{kg}$ & 2 & 17-Jul-2009 & 20-Jul-2009 & SW 846/6020 & \\
\hline Thallium & ND & 0.500 & $\mathrm{mg} / \mathrm{kg}$ & 2 & 17-Jul-2009 & 20-Jul-2009 & SW 846/6020 & $\mathrm{U}$ \\
\hline Vanadium & 1.43 & 0.500 & $\mathrm{mg} / \mathrm{kg}$ & 2 & 17-Jul-2009 & 20-Jul-2009 & SW 846/6020 & \\
\hline Zinc & 25.5 & 0.500 & $\mathrm{mg} / \mathrm{kg}$ & 2 & 17-Jul-2009 & 20-Jul-2009 & SW 846/6020 & B \\
\hline
\end{tabular}




\section{USACE ERDC-EP-C \\ 3909 Halls Ferry Road \\ Vicksburg, MS 39180-6199}

ERDC - ECB

Project: TVA Fly Ash Project

$-$

$\begin{array}{cc} & \text { Reported: } \\ \text { Project Manager: Tony Bednar } & 03-S e p-2009\end{array}$

DRAFT: EMR - EL - AF - 10 - D

9071602-14 (Tissue)

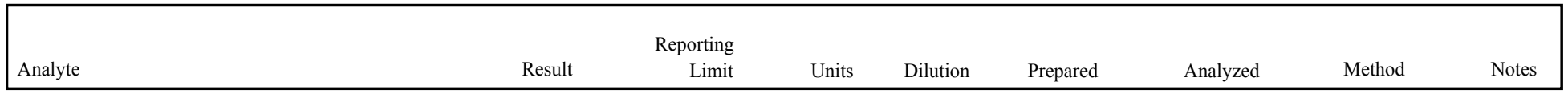

ERDC- EL-EP-C (Environmental Chemistry Branch)

DRAFT: Metals by EPA 6000/7000 Series Methods

\begin{tabular}{|c|c|c|c|c|c|c|c|c|}
\hline Mercury & 0.018 & 0.002 & $\mathrm{mg} / \mathrm{kg}$ & 1 & 22-Jul-2009 & 22-Jul-2009 & EPA 7471A & \\
\hline Aluminum & 511 & 0.500 & $\mathrm{mg} / \mathrm{kg}$ & 2 & 17-Jul-2009 & 20-Jul-2009 & SW 846/6020 & \\
\hline Antimony & 0.540 & 0.500 & $\mathrm{mg} / \mathrm{kg}$ & 2 & 17-Jul-2009 & 20-Jul-2009 & SW 846/6020 & \\
\hline Arsenic & 1.42 & 0.500 & $\mathrm{mg} / \mathrm{kg}$ & 2 & 17-Jul-2009 & 20-Jul-2009 & SW 846/6020 & \\
\hline Barium & 10.1 & 0.500 & $\mathrm{mg} / \mathrm{kg}$ & 2 & 17-Jul-2009 & 20-Jul-2009 & SW 846/6020 & \\
\hline Beryllium & ND & 0.500 & $\mathrm{mg} / \mathrm{kg}$ & 2 & 17-Jul-2009 & 20-Jul-2009 & SW 846/6020 & $\mathrm{U}$ \\
\hline Cadmium & 0.146 & 0.500 & $\mathrm{mg} / \mathrm{kg}$ & 2 & 17-Jul-2009 & 20-Jul-2009 & SW 846/6020 & $\mathrm{J}$ \\
\hline Calcium & $\mathbf{5 5 5 0}$ & 0.500 & $\mathrm{mg} / \mathrm{kg}$ & 2 & 17-Jul-2009 & 20-Jul-2009 & SW 846/6020 & \\
\hline Chromium & 2.62 & 0.500 & $\mathrm{mg} / \mathrm{kg}$ & 2 & 17-Jul-2009 & 20-Jul-2009 & SW 846/6020 & \\
\hline Cobalt & 0.436 & 0.500 & $\mathrm{mg} / \mathrm{kg}$ & 2 & 17-Jul-2009 & 20-Jul-2009 & SW 846/6020 & $\mathrm{J}$ \\
\hline Copper & 2.62 & 0.500 & $\mathrm{mg} / \mathrm{kg}$ & 2 & 17-Jul-2009 & 20-Jul-2009 & SW 846/6020 & \\
\hline Iron & 289 & 0.500 & $\mathrm{mg} / \mathrm{kg}$ & 2 & 17-Jul-2009 & 20-Jul-2009 & SW 846/6020 & \\
\hline Lead & 1.20 & 0.500 & $\mathrm{mg} / \mathrm{kg}$ & 2 & 17-Jul-2009 & 20-Jul-2009 & SW 846/6020 & \\
\hline Magnesium & 286 & 0.500 & $\mathrm{mg} / \mathrm{kg}$ & 2 & 17-Jul-2009 & 20-Jul-2009 & SW 846/6020 & \\
\hline Manganese & 3.75 & 0.500 & $\mathrm{mg} / \mathrm{kg}$ & 2 & 17-Jul-2009 & 20-Jul-2009 & SW 846/6020 & \\
\hline Molybdenum & 0.153 & 0.500 & $\mathrm{mg} / \mathrm{kg}$ & 2 & 17-Jul-2009 & 20-Jul-2009 & SW 846/6020 & $\mathrm{J}$ \\
\hline Nickel & 1.92 & 0.500 & $\mathrm{mg} / \mathrm{kg}$ & 2 & 17-Jul-2009 & 20-Jul-2009 & SW 846/6020 & \\
\hline Potassium & 2370 & 0.500 & $\mathrm{mg} / \mathrm{kg}$ & 2 & 17-Jul-2009 & 20-Jul-2009 & SW 846/6020 & \\
\hline Selenium & 0.369 & 0.500 & $\mathrm{mg} / \mathrm{kg}$ & 2 & 17-Jul-2009 & 20-Jul-2009 & SW 846/6020 & $\mathrm{J}$ \\
\hline Silver & ND & 0.500 & $\mathrm{mg} / \mathrm{kg}$ & 2 & 17-Jul-2009 & 20-Jul-2009 & SW 846/6020 & $\mathrm{U}$ \\
\hline Sodium & 937 & 0.500 & $\mathrm{mg} / \mathrm{kg}$ & 2 & 17-Jul-2009 & 20-Jul-2009 & SW 846/6020 & \\
\hline Thallium & ND & 0.500 & $\mathrm{mg} / \mathrm{kg}$ & 2 & 17-Jul-2009 & 20-Jul-2009 & SW 846/6020 & $\mathrm{U}$ \\
\hline Vanadium & 1.92 & 0.500 & $\mathrm{mg} / \mathrm{kg}$ & 2 & 17-Jul-2009 & 20-Jul-2009 & SW 846/6020 & \\
\hline Zinc & 28.0 & 0.500 & $\mathrm{mg} / \mathrm{kg}$ & 2 & 17-Jul-2009 & 20-Jul-2009 & SW 846/6020 & B \\
\hline
\end{tabular}




\section{USACE ERDC-EP-C \\ 3909 Halls Ferry Road \\ Vicksburg, MS 39180-6199}

ERDC - ECB

Project: TVA Fly Ash Project

$-$

$\begin{array}{cc} & \text { Reported: } \\ \text { Project Manager: Tony Bednar } & 03-S e p-2009\end{array}$

DRAFT: EMR - EL - AF - 10 - E

9071602-15 (Tissue)

\begin{tabular}{|c|c|c|c|c|c|c|c|c|}
\hline & & porting & & & & & & \\
\hline Analyte & Result & Limit & Units & Dilution & Prepared & Analyzed & Method & Notes \\
\hline
\end{tabular}

ERDC- EL-EP-C (Environmental Chemistry Branch)

DRAFT: Metals by EPA 6000/7000 Series Methods

\begin{tabular}{|c|c|c|c|c|c|c|c|c|}
\hline Mercury & 0.018 & 0.002 & $\mathrm{mg} / \mathrm{kg}$ & 1 & 22-Jul-2009 & 22-Jul-2009 & EPA 7471A & \\
\hline Aluminum & 442 & 0.500 & $\mathrm{mg} / \mathrm{kg}$ & 2 & 17-Jul-2009 & 20-Jul-2009 & SW 846/6020 & \\
\hline Antimony & ND & 0.500 & $\mathrm{mg} / \mathrm{kg}$ & 2 & 17-Jul-2009 & 20-Jul-2009 & SW 846/6020 & $\mathrm{U}$ \\
\hline Arsenic & 1.36 & 0.500 & $\mathrm{mg} / \mathrm{kg}$ & 2 & 17-Jul-2009 & 20-Jul-2009 & SW 846/6020 & \\
\hline Barium & 8.96 & 0.500 & $\mathrm{mg} / \mathrm{kg}$ & 2 & 17-Jul-2009 & 20-Jul-2009 & SW 846/6020 & \\
\hline Beryllium & ND & 0.500 & $\mathrm{mg} / \mathrm{kg}$ & 2 & 17-Jul-2009 & 20-Jul-2009 & SW 846/6020 & $\mathrm{U}$ \\
\hline Cadmium & 0.130 & 0.500 & $\mathrm{mg} / \mathrm{kg}$ & 2 & 17-Jul-2009 & 20-Jul-2009 & SW 846/6020 & $\mathrm{J}$ \\
\hline Calcium & 5880 & 0.500 & $\mathrm{mg} / \mathrm{kg}$ & 2 & 17-Jul-2009 & 20-Jul-2009 & SW 846/6020 & \\
\hline Chromium & 4.63 & 0.500 & $\mathrm{mg} / \mathrm{kg}$ & 2 & 17-Jul-2009 & 20-Jul-2009 & SW 846/6020 & \\
\hline Cobalt & 0.402 & 0.500 & $\mathrm{mg} / \mathrm{kg}$ & 2 & 17-Jul-2009 & 20-Jul-2009 & SW 846/6020 & $\mathrm{J}$ \\
\hline Copper & 2.13 & 0.500 & $\mathrm{mg} / \mathrm{kg}$ & 2 & 17-Jul-2009 & 20-Jul-2009 & SW 846/6020 & \\
\hline Iron & 262 & 0.500 & $\mathrm{mg} / \mathrm{kg}$ & 2 & 17-Jul-2009 & 20-Jul-2009 & SW 846/6020 & \\
\hline Lead & 0.755 & 0.500 & $\mathrm{mg} / \mathrm{kg}$ & 2 & 17-Jul-2009 & 20-Jul-2009 & SW 846/6020 & \\
\hline Magnesium & 305 & 0.500 & $\mathrm{mg} / \mathrm{kg}$ & 2 & 17-Jul-2009 & 20-Jul-2009 & SW 846/6020 & \\
\hline Manganese & 3.80 & 0.500 & $\mathrm{mg} / \mathrm{kg}$ & 2 & 17-Jul-2009 & 20-Jul-2009 & SW 846/6020 & \\
\hline Molybdenum & 0.173 & 0.500 & $\mathrm{mg} / \mathrm{kg}$ & 2 & 17-Jul-2009 & 20-Jul-2009 & SW 846/6020 & $\mathrm{J}$ \\
\hline Nickel & 2.91 & 0.500 & $\mathrm{mg} / \mathrm{kg}$ & 2 & 17-Jul-2009 & 20-Jul-2009 & SW 846/6020 & \\
\hline Potassium & 2420 & 0.500 & $\mathrm{mg} / \mathrm{kg}$ & 2 & 17-Jul-2009 & 20-Jul-2009 & SW 846/6020 & \\
\hline Selenium & 0.353 & 0.500 & $\mathrm{mg} / \mathrm{kg}$ & 2 & 17-Jul-2009 & 20-Jul-2009 & SW 846/6020 & J \\
\hline Silver & ND & 0.500 & $\mathrm{mg} / \mathrm{kg}$ & 2 & 17-Jul-2009 & 20-Jul-2009 & SW 846/6020 & $\mathrm{U}$ \\
\hline Sodium & 933 & 0.500 & $\mathrm{mg} / \mathrm{kg}$ & 2 & 17-Jul-2009 & 20-Jul-2009 & SW 846/6020 & \\
\hline Thallium & ND & 0.500 & $\mathrm{mg} / \mathrm{kg}$ & 2 & 17-Jul-2009 & 20-Jul-2009 & SW 846/6020 & $\mathrm{U}$ \\
\hline Vanadium & 1.69 & 0.500 & $\mathrm{mg} / \mathrm{kg}$ & 2 & 17-Jul-2009 & 20-Jul-2009 & SW 846/6020 & \\
\hline Zinc & 27.8 & 0.500 & $\mathrm{mg} / \mathrm{kg}$ & 2 & 17-Jul-2009 & 20-Jul-2009 & SW 846/6020 & B \\
\hline
\end{tabular}




\section{USACE ERDC-EP-C \\ 3909 Halls Ferry Road \\ Vicksburg, MS 39180-6199}

ERDC - ECB

Project: TVA Fly Ash Project

$-$

Reported:

Project Manager: Tony Bednar

03-Sep-2009

DRAFT: EMR - EL - AF - 0 - A

9071602-16 (Tissue)

\begin{tabular}{|c|c|c|c|c|c|c|c|c|}
\hline Analyte & Result & $\begin{array}{r}\text { porting } \\
\text { Limit }\end{array}$ & Units & Dilution & Prepared & Analyzed & Method & Notes \\
\hline
\end{tabular}

ERDC- EL-EP-C (Environmental Chemistry Branch)

DRAFT: Metals by EPA 6000/7000 Series Methods

\begin{tabular}{|c|c|c|c|c|c|c|c|c|}
\hline Mercury & 0.014 & 0.002 & $\mathrm{mg} / \mathrm{kg}$ & 1 & 22-Jul-2009 & 22-Jul-2009 & EPA 7471A & \\
\hline Aluminum & 33.9 & 0.500 & $\mathrm{mg} / \mathrm{kg}$ & 2 & 17-Jul-2009 & 20-Jul-2009 & SW 846/6020 & \\
\hline Antimony & ND & 0.500 & $\mathrm{mg} / \mathrm{kg}$ & 2 & 17-Jul-2009 & 20-Jul-2009 & SW 846/6020 & $\mathrm{U}$ \\
\hline Arsenic & 0.360 & 0.500 & $\mathrm{mg} / \mathrm{kg}$ & 2 & 17-Jul-2009 & 20-Jul-2009 & SW 846/6020 & $\mathrm{J}$ \\
\hline Barium & 2.62 & 0.500 & $\mathrm{mg} / \mathrm{kg}$ & 2 & 17-Jul-2009 & 20-Jul-2009 & SW 846/6020 & \\
\hline Beryllium & $\mathrm{ND}$ & 0.500 & $\mathrm{mg} / \mathrm{kg}$ & 2 & 17-Jul-2009 & 20-Jul-2009 & SW 846/6020 & $\mathrm{U}$ \\
\hline Cadmium & ND & 0.500 & $\mathrm{mg} / \mathrm{kg}$ & 2 & 17-Jul-2009 & 20-Jul-2009 & SW 846/6020 & $\mathrm{U}$ \\
\hline Calcium & 5920 & 0.500 & $\mathrm{mg} / \mathrm{kg}$ & 2 & 17-Jul-2009 & 20-Jul-2009 & SW 846/6020 & \\
\hline Chromium & 1.92 & 0.500 & $\mathrm{mg} / \mathrm{kg}$ & 2 & 17-Jul-2009 & 20-Jul-2009 & SW 846/6020 & \\
\hline Cobalt & ND & 0.500 & $\mathrm{mg} / \mathrm{kg}$ & 2 & 17-Jul-2009 & 20-Jul-2009 & SW 846/6020 & $\mathrm{U}$ \\
\hline Copper & 1.02 & 0.500 & $\mathrm{mg} / \mathrm{kg}$ & 2 & 17-Jul-2009 & 20-Jul-2009 & SW 846/6020 & \\
\hline Iron & 71.6 & 0.500 & $\mathrm{mg} / \mathrm{kg}$ & 2 & 17-Jul-2009 & 20-Jul-2009 & SW 846/6020 & \\
\hline Lead & 0.510 & 0.500 & $\mathrm{mg} / \mathrm{kg}$ & 2 & 17-Jul-2009 & 20-Jul-2009 & SW 846/6020 & \\
\hline Magnesium & 261 & 0.500 & $\mathrm{mg} / \mathrm{kg}$ & 2 & 17-Jul-2009 & 20-Jul-2009 & SW 846/6020 & \\
\hline Manganese & 3.52 & 0.500 & $\mathrm{mg} / \mathrm{kg}$ & 2 & 17-Jul-2009 & 20-Jul-2009 & SW 846/6020 & \\
\hline Molybdenum & ND & 0.500 & $\mathrm{mg} / \mathrm{kg}$ & 2 & 17-Jul-2009 & 20-Jul-2009 & SW 846/6020 & $\mathrm{U}$ \\
\hline Nickel & 1.13 & 0.500 & $\mathrm{mg} / \mathrm{kg}$ & 2 & 17-Jul-2009 & 20-Jul-2009 & SW 846/6020 & \\
\hline Potassium & 2310 & 0.500 & $\mathrm{mg} / \mathrm{kg}$ & 2 & 17-Jul-2009 & 20-Jul-2009 & SW 846/6020 & \\
\hline Selenium & 0.252 & 0.500 & $\mathrm{mg} / \mathrm{kg}$ & 2 & 17-Jul-2009 & 20-Jul-2009 & SW 846/6020 & $\mathrm{J}$ \\
\hline Silver & ND & 0.500 & $\mathrm{mg} / \mathrm{kg}$ & 2 & 17-Jul-2009 & 20-Jul-2009 & SW 846/6020 & $\mathrm{U}$ \\
\hline Sodium & 913 & 0.500 & $\mathrm{mg} / \mathrm{kg}$ & 2 & 17-Jul-2009 & 20-Jul-2009 & SW 846/6020 & \\
\hline Thallium & ND & 0.500 & $\mathrm{mg} / \mathrm{kg}$ & 2 & 17-Jul-2009 & 20-Jul-2009 & SW 846/6020 & $\mathrm{U}$ \\
\hline Vanadium & 0.115 & 0.500 & $\mathrm{mg} / \mathrm{kg}$ & 2 & 17-Jul-2009 & 20-Jul-2009 & SW 846/6020 & $\mathrm{J}$ \\
\hline Zinc & 27.7 & 0.500 & $\mathrm{mg} / \mathrm{kg}$ & 2 & 17-Jul-2009 & 20-Jul-2009 & SW 846/6020 & B \\
\hline
\end{tabular}




\section{USACE ERDC-EP-C \\ 3909 Halls Ferry Road \\ Vicksburg, MS 39180-6199}

ERDC - ECB

Project: TVA Fly Ash Project

$-$

Project Manager: Tony Bednar

Reported:

$--,-$

03-Sep-2009

DRAFT: EMR - EL - AF - 0 - B

9071602-17 (Tissue)

\begin{tabular}{|c|c|c|c|c|c|c|c|c|}
\hline & & porting & & & & & & \\
\hline Analyte & Result & Limit & Units & Dilution & Prepared & Analyzed & Method & Notes \\
\hline
\end{tabular}

ERDC- EL-EP-C (Environmental Chemistry Branch)

DRAFT: Metals by EPA 6000/7000 Series Methods

\begin{tabular}{|c|c|c|c|c|c|c|c|c|}
\hline Mercury & 0.014 & 0.002 & $\mathrm{mg} / \mathrm{kg}$ & 1 & 22-Jul-2009 & 22-Jul-2009 & EPA 7471A & \\
\hline Aluminum & 11.8 & 0.500 & $\mathrm{mg} / \mathrm{kg}$ & 2 & 17-Jul-2009 & 20-Jul-2009 & SW 846/6020 & \\
\hline Antimony & ND & 0.500 & $\mathrm{mg} / \mathrm{kg}$ & 2 & 17-Jul-2009 & 20-Jul-2009 & SW 846/6020 & $\mathrm{U}$ \\
\hline Arsenic & 0.379 & 0.500 & $\mathrm{mg} / \mathrm{kg}$ & 2 & 17-Jul-2009 & 20-Jul-2009 & SW 846/6020 & $\mathrm{J}$ \\
\hline Barium & 1.73 & 0.500 & $\mathrm{mg} / \mathrm{kg}$ & 2 & 17-Jul-2009 & 20-Jul-2009 & SW 846/6020 & \\
\hline Beryllium & ND & 0.500 & $\mathrm{mg} / \mathrm{kg}$ & 2 & 17-Jul-2009 & 20-Jul-2009 & SW 846/6020 & $\mathrm{U}$ \\
\hline Calcium & 4520 & 0.500 & $\mathrm{mg} / \mathrm{kg}$ & 2 & 17-Jul-2009 & 20-Jul-2009 & SW 846/6020 & \\
\hline Chromium & 3.01 & 0.500 & $\mathrm{mg} / \mathrm{kg}$ & 2 & 17-Jul-2009 & 20-Jul-2009 & SW 846/6020 & \\
\hline Cobalt & ND & 0.500 & $\mathrm{mg} / \mathrm{kg}$ & 2 & 17-Jul-2009 & 20-Jul-2009 & SW 846/6020 & $\mathrm{U}$ \\
\hline Copper & 1.13 & 0.500 & $\mathrm{mg} / \mathrm{kg}$ & 2 & 17-Jul-2009 & 20-Jul-2009 & SW 846/6020 & \\
\hline Iron & 43.0 & 0.500 & $\mathrm{mg} / \mathrm{kg}$ & 2 & 17-Jul-2009 & 20-Jul-2009 & SW $846 / 6020$ & \\
\hline Lead & ND & 0.500 & $\mathrm{mg} / \mathrm{kg}$ & 2 & 17-Jul-2009 & 20-Jul-2009 & SW 846/6020 & $\mathrm{U}$ \\
\hline Magnesium & 247 & 0.500 & $\mathrm{mg} / \mathrm{kg}$ & 2 & 17-Jul-2009 & 20-Jul-2009 & SW 846/6020 & \\
\hline Manganese & 1.88 & 0.500 & $\mathrm{mg} / \mathrm{kg}$ & 2 & 17-Jul-2009 & 20-Jul-2009 & SW 846/6020 & \\
\hline Molybdenum & $\mathrm{ND}$ & 0.500 & $\mathrm{mg} / \mathrm{kg}$ & 2 & 17-Jul-2009 & 20-Jul-2009 & SW 846/6020 & $\mathrm{U}$ \\
\hline Nickel & 1.50 & 0.500 & $\mathrm{mg} / \mathrm{kg}$ & 2 & 17-Jul-2009 & 20-Jul-2009 & SW 846/6020 & \\
\hline Potassium & 2340 & 0.500 & $\mathrm{mg} / \mathrm{kg}$ & 2 & 17-Jul-2009 & 20-Jul-2009 & SW 846/6020 & \\
\hline Selenium & 0.282 & 0.500 & $\mathrm{mg} / \mathrm{kg}$ & 2 & 17-Jul-2009 & 20-Jul-2009 & SW 846/6020 & $\mathrm{J}$ \\
\hline Silver & ND & 0.500 & $\mathrm{mg} / \mathrm{kg}$ & 2 & 17-Jul-2009 & 20-Jul-2009 & SW 846/6020 & $\mathrm{U}$ \\
\hline Sodium & 872 & 0.500 & $\mathrm{mg} / \mathrm{kg}$ & 2 & 17-Jul-2009 & 20-Jul-2009 & SW 846/6020 & \\
\hline Thallium & ND & 0.500 & $\mathrm{mg} / \mathrm{kg}$ & 2 & 17-Jul-2009 & 20-Jul-2009 & SW 846/6020 & $\mathrm{U}$ \\
\hline Vanadium & ND & 0.500 & $\mathrm{mg} / \mathrm{kg}$ & 2 & 17-Jul-2009 & 20-Jul-2009 & SW 846/6020 & $\mathrm{U}$ \\
\hline Zinc & 25.6 & 0.500 & $\mathrm{mg} / \mathrm{kg}$ & 2 & 17-Jul-2009 & 20-Jul-2009 & SW 846/6020 & B \\
\hline
\end{tabular}




\section{USACE ERDC-EP-C \\ 3909 Halls Ferry Road \\ Vicksburg, MS 39180-6199}

ERDC - ECB

Project: TVA Fly Ash Project

$-$

Project Manager: Tony Bednar

Reported:

$--,-$

03-Sep-2009

DRAFT: EMR - EL - AF - 0 - C

9071602-18 (Tissue)

\begin{tabular}{|c|c|c|c|c|c|c|c|c|}
\hline Analyte & Result & $\begin{array}{r}\text { porting } \\
\text { Limit }\end{array}$ & Units & Dilution & Prepared & Analyzed & Method & Notes \\
\hline
\end{tabular}

ERDC- EL-EP-C (Environmental Chemistry Branch)

DRAFT: Metals by EPA 6000/7000 Series Methods

\begin{tabular}{|c|c|c|c|c|c|c|c|c|}
\hline Mercury & 0.017 & 0.002 & $\mathrm{mg} / \mathrm{kg}$ & 1 & 22-Jul-2009 & 22-Jul-2009 & EPA 7471A & \\
\hline Aluminum & 22.1 & 0.500 & $\mathrm{mg} / \mathrm{kg}$ & 2 & 17-Jul-2009 & 20-Jul-2009 & SW 846/6020 & \\
\hline Antimony & ND & 0.500 & $\mathrm{mg} / \mathrm{kg}$ & 2 & 17-Jul-2009 & 20-Jul-2009 & SW 846/6020 & $\mathrm{U}$ \\
\hline Arsenic & 0.342 & 0.500 & $\mathrm{mg} / \mathrm{kg}$ & 2 & 17-Jul-2009 & 20-Jul-2009 & SW 846/6020 & $\mathrm{J}$ \\
\hline Barium & 2.01 & 0.500 & $\mathrm{mg} / \mathrm{kg}$ & 2 & 17-Jul-2009 & 20-Jul-2009 & SW 846/6020 & \\
\hline Beryllium & ND & 0.500 & $\mathrm{mg} / \mathrm{kg}$ & 2 & 17-Jul-2009 & 20-Jul-2009 & SW 846/6020 & $\mathrm{U}$ \\
\hline Cadmium & ND & 0.500 & $\mathrm{mg} / \mathrm{kg}$ & 2 & 17-Jul-2009 & 20-Jul-2009 & SW 846/6020 & $\mathrm{U}$ \\
\hline Calcium & 5270 & 0.500 & $\mathrm{mg} / \mathrm{kg}$ & 2 & 17-Jul-2009 & 20-Jul-2009 & SW 846/6020 & \\
\hline Chromium & 1.38 & 0.500 & $\mathrm{mg} / \mathrm{kg}$ & 2 & 17-Jul-2009 & 20-Jul-2009 & SW 846/6020 & \\
\hline Cobalt & ND & 0.500 & $\mathrm{mg} / \mathrm{kg}$ & 2 & 17-Jul-2009 & 20-Jul-2009 & SW 846/6020 & $\mathrm{U}$ \\
\hline Copper & 1.27 & 0.500 & $\mathrm{mg} / \mathrm{kg}$ & 2 & 17-Jul-2009 & 20-Jul-2009 & SW 846/6020 & \\
\hline Iron & 62.5 & 0.500 & $\mathrm{mg} / \mathrm{kg}$ & 2 & 17-Jul-2009 & 20-Jul-2009 & SW 846/6020 & \\
\hline Lead & 0.278 & 0.500 & $\mathrm{mg} / \mathrm{kg}$ & 2 & 17-Jul-2009 & 20-Jul-2009 & SW 846/6020 & J \\
\hline Magnesium & 261 & 0.500 & $\mathrm{mg} / \mathrm{kg}$ & 2 & 17-Jul-2009 & 20-Jul-2009 & SW 846/6020 & \\
\hline Manganese & 2.94 & 0.500 & $\mathrm{mg} / \mathrm{kg}$ & 2 & 17-Jul-2009 & 20-Jul-2009 & SW 846/6020 & \\
\hline Molybdenum & ND & 0.500 & $\mathrm{mg} / \mathrm{kg}$ & 2 & 17-Jul-2009 & 20-Jul-2009 & SW 846/6020 & $\mathrm{U}$ \\
\hline Nickel & 0.600 & 0.500 & $\mathrm{mg} / \mathrm{kg}$ & 2 & 17-Jul-2009 & 20-Jul-2009 & SW 846/6020 & \\
\hline Potassium & 2350 & 0.500 & $\mathrm{mg} / \mathrm{kg}$ & 2 & 17-Jul-2009 & 20-Jul-2009 & SW 846/6020 & \\
\hline Selenium & 0.267 & 0.500 & $\mathrm{mg} / \mathrm{kg}$ & 2 & 17-Jul-2009 & 20-Jul-2009 & SW 846/6020 & J \\
\hline Silver & 0.647 & 0.500 & $\mathrm{mg} / \mathrm{kg}$ & 2 & 17-Jul-2009 & 20-Jul-2009 & SW 846/6020 & \\
\hline Sodium & 880 & 0.500 & $\mathrm{mg} / \mathrm{kg}$ & 2 & 17-Jul-2009 & 20-Jul-2009 & SW 846/6020 & \\
\hline Thallium & ND & 0.500 & $\mathrm{mg} / \mathrm{kg}$ & 2 & 17-Jul-2009 & 20-Jul-2009 & SW 846/6020 & $\mathrm{U}$ \\
\hline Vanadium & ND & 0.500 & $\mathrm{mg} / \mathrm{kg}$ & 2 & 17-Jul-2009 & 20-Jul-2009 & SW 846/6020 & $\mathrm{U}$ \\
\hline Zinc & 28.1 & 0.500 & $\mathrm{mg} / \mathrm{kg}$ & 2 & 17-Jul-2009 & 20-Jul-2009 & SW 846/6020 & B \\
\hline
\end{tabular}




\section{USACE ERDC-EP-C \\ 3909 Halls Ferry Road \\ Vicksburg, MS 39180-6199}

ERDC - ECB

Project: TVA Fly Ash Project

$-$

Reported:

Project Manager: Tony Bednar

03-Sep-2009

DRAFT: EMR - EL - AF - 0 - D

9071602-19 (Tissue)

\begin{tabular}{|c|c|c|c|c|c|c|c|c|}
\hline Analyte & Result & $\begin{array}{r}\text { porting } \\
\text { Limit }\end{array}$ & Units & Dilution & Prepared & Analyzed & Method & Notes \\
\hline
\end{tabular}

ERDC- EL-EP-C (Environmental Chemistry Branch)

DRAFT: Metals by EPA 6000/7000 Series Methods

\begin{tabular}{|c|c|c|c|c|c|c|c|c|}
\hline Mercury & 0.020 & 0.003 & $\mathrm{mg} / \mathrm{kg}$ & 1 & 22-Jul-2009 & 22-Jul-2009 & EPA $7471 \mathrm{~A}$ & \\
\hline Aluminum & 27.0 & 0.500 & $\mathrm{mg} / \mathrm{kg}$ & 2 & 17-Jul-2009 & 20-Jul-2009 & SW 846/6020 & \\
\hline Antimony & ND & 0.500 & $\mathrm{mg} / \mathrm{kg}$ & 2 & 17-Jul-2009 & 20-Jul-2009 & SW 846/6020 & $\mathrm{U}$ \\
\hline Arsenic & 0.328 & 0.500 & $\mathrm{mg} / \mathrm{kg}$ & 2 & 17-Jul-2009 & 20-Jul-2009 & SW 846/6020 & $\mathrm{J}$ \\
\hline Barium & 2.64 & 0.500 & $\mathrm{mg} / \mathrm{kg}$ & 2 & 17-Jul-2009 & 20-Jul-2009 & SW 846/6020 & \\
\hline Beryllium & ND & 0.500 & $\mathrm{mg} / \mathrm{kg}$ & 2 & 17-Jul-2009 & 20-Jul-2009 & SW 846/6020 & $\mathrm{U}$ \\
\hline Calcium & 5230 & 0.500 & $\mathrm{mg} / \mathrm{kg}$ & 2 & 17-Jul-2009 & 20-Jul-2009 & SW $846 / 6020$ & \\
\hline Chromium & 2.18 & 0.500 & $\mathrm{mg} / \mathrm{kg}$ & 2 & 17-Jul-2009 & 20-Jul-2009 & SW 846/6020 & \\
\hline Cobalt & ND & 0.500 & $\mathrm{mg} / \mathrm{kg}$ & 2 & 17-Jul-2009 & 20-Jul-2009 & SW 846/6020 & $\mathrm{U}$ \\
\hline Copper & 1.31 & 0.500 & $\mathrm{mg} / \mathrm{kg}$ & 2 & 17-Jul-2009 & 20-Jul-2009 & SW 846/6020 & \\
\hline Iron & 68.4 & 0.500 & $\mathrm{mg} / \mathrm{kg}$ & 2 & 17-Jul-2009 & 20-Jul-2009 & SW 846/6020 & \\
\hline Lead & 0.524 & 0.500 & $\mathrm{mg} / \mathrm{kg}$ & 2 & 17-Jul-2009 & 20-Jul-2009 & SW 846/6020 & \\
\hline Magnesium & 262 & 0.500 & $\mathrm{mg} / \mathrm{kg}$ & 2 & 17-Jul-2009 & 20-Jul-2009 & SW 846/6020 & \\
\hline Manganese & 3.38 & 0.500 & $\mathrm{mg} / \mathrm{kg}$ & 2 & 17-Jul-2009 & 20-Jul-2009 & SW 846/6020 & \\
\hline Molybdenum & 0.101 & 0.500 & $\mathrm{mg} / \mathrm{kg}$ & 2 & 17-Jul-2009 & 20-Jul-2009 & SW 846/6020 & $\mathrm{J}$ \\
\hline Nickel & 1.07 & 0.500 & $\mathrm{mg} / \mathrm{kg}$ & 2 & 17-Jul-2009 & 20-Jul-2009 & SW 846/6020 & \\
\hline Potassium & 2230 & 0.500 & $\mathrm{mg} / \mathrm{kg}$ & 2 & 17-Jul-2009 & 20-Jul-2009 & SW 846/6020 & \\
\hline Selenium & 0.229 & 0.500 & $\mathrm{mg} / \mathrm{kg}$ & 2 & 17-Jul-2009 & 20-Jul-2009 & SW 846/6020 & $\mathrm{J}$ \\
\hline Silver & ND & 0.500 & $\mathrm{mg} / \mathrm{kg}$ & 2 & 17-Jul-2009 & 20-Jul-2009 & SW 846/6020 & $\mathrm{U}$ \\
\hline Sodium & 931 & 0.500 & $\mathrm{mg} / \mathrm{kg}$ & 2 & 17-Jul-2009 & 20-Jul-2009 & SW 846/6020 & \\
\hline Thallium & $\mathrm{ND}$ & 0.500 & $\mathrm{mg} / \mathrm{kg}$ & 2 & 17-Jul-2009 & 20-Jul-2009 & SW 846/6020 & $\mathrm{U}$ \\
\hline Vanadium & 0.112 & 0.500 & $\mathrm{mg} / \mathrm{kg}$ & 2 & 17-Jul-2009 & 20-Jul-2009 & SW 846/6020 & $\mathrm{J}$ \\
\hline Zinc & 24.9 & 0.500 & $\mathrm{mg} / \mathrm{kg}$ & 2 & 17-Jul-2009 & 20-Jul-2009 & SW 846/6020 & B \\
\hline
\end{tabular}




\section{USACE ERDC-EP-C \\ 3909 Halls Ferry Road \\ Vicksburg, MS 39180-6199}

ERDC - ECB

Project: TVA Fly Ash Project

$-$

$\begin{array}{cc} & \text { Reported: } \\ \text { Project Manager: Tony Bednar } & \text { 03-Sep-2009 }\end{array}$

DRAFT: EMR - EL - AF - 0 - E

9071602-20 (Tissue)

\begin{tabular}{|c|c|c|c|c|c|c|c|c|}
\hline & & porting & & & & & & \\
\hline Analyte & Result & Limit & Units & Dilution & Prepared & Analyzed & Method & Notes \\
\hline
\end{tabular}

ERDC- EL-EP-C (Environmental Chemistry Branch)

DRAFT: Metals by EPA 6000/7000 Series Methods

\begin{tabular}{|c|c|c|c|c|c|c|c|c|}
\hline Mercury & 0.019 & 0.003 & $\mathrm{mg} / \mathrm{kg}$ & 1 & 22-Jul-2009 & 22-Jul-2009 & EPA 7471A & \\
\hline Aluminum & 33.0 & 0.500 & $\mathrm{mg} / \mathrm{kg}$ & 2 & 17-Jul-2009 & 20-Jul-2009 & SW 846/6020 & \\
\hline Antimony & 0.169 & 0.500 & $\mathrm{mg} / \mathrm{kg}$ & 2 & 17-Jul-2009 & 20-Jul-2009 & SW 846/6020 & J \\
\hline Arsenic & 0.425 & 0.500 & $\mathrm{mg} / \mathrm{kg}$ & 2 & 17-Jul-2009 & 20-Jul-2009 & SW 846/6020 & J \\
\hline Barium & 3.01 & 0.500 & $\mathrm{mg} / \mathrm{kg}$ & 2 & 17-Jul-2009 & 20-Jul-2009 & SW 846/6020 & \\
\hline Beryllium & ND & 0.500 & $\mathrm{mg} / \mathrm{kg}$ & 2 & 17-Jul-2009 & 20-Jul-2009 & SW 846/6020 & $\mathrm{U}$ \\
\hline Cadmium & ND & 0.500 & $\mathrm{mg} / \mathrm{kg}$ & 2 & 17-Jul-2009 & 20-Jul-2009 & SW 846/6020 & $\mathrm{U}$ \\
\hline Calcium & 6780 & 0.500 & $\mathrm{mg} / \mathrm{kg}$ & 2 & 17-Jul-2009 & 20-Jul-2009 & SW 846/6020 & \\
\hline Chromium & 9.15 & 0.500 & $\mathrm{mg} / \mathrm{kg}$ & 2 & 17-Jul-2009 & 20-Jul-2009 & SW 846/6020 & \\
\hline Cobalt & 0.121 & 0.500 & $\mathrm{mg} / \mathrm{kg}$ & 2 & 17-Jul-2009 & 20-Jul-2009 & SW 846/6020 & J \\
\hline Copper & 1.77 & 0.500 & $\mathrm{mg} / \mathrm{kg}$ & 2 & 17-Jul-2009 & 20-Jul-2009 & SW 846/6020 & \\
\hline Iron & 110 & 0.500 & $\mathrm{mg} / \mathrm{kg}$ & 2 & 17-Jul-2009 & 20-Jul-2009 & SW 846/6020 & \\
\hline Lead & 0.436 & 0.500 & $\mathrm{mg} / \mathrm{kg}$ & 2 & 17-Jul-2009 & 20-Jul-2009 & SW 846/6020 & J \\
\hline Magnesium & 299 & 0.500 & $\mathrm{mg} / \mathrm{kg}$ & 2 & 17-Jul-2009 & 20-Jul-2009 & SW 846/6020 & \\
\hline Manganese & 5.32 & 0.500 & $\mathrm{mg} / \mathrm{kg}$ & 2 & 17-Jul-2009 & 20-Jul-2009 & SW 846/6020 & \\
\hline Molybdenum & 0.215 & 0.500 & $\mathrm{mg} / \mathrm{kg}$ & 2 & 17-Jul-2009 & 20-Jul-2009 & SW 846/6020 & $\mathrm{J}$ \\
\hline Nickel & 4.25 & 0.500 & $\mathrm{mg} / \mathrm{kg}$ & 2 & 17-Jul-2009 & 20-Jul-2009 & SW 846/6020 & \\
\hline Potassium & 2290 & 0.500 & $\mathrm{mg} / \mathrm{kg}$ & 2 & 17-Jul-2009 & 20-Jul-2009 & SW 846/6020 & \\
\hline Selenium & 0.301 & 0.500 & $\mathrm{mg} / \mathrm{kg}$ & 2 & 17-Jul-2009 & 20-Jul-2009 & SW 846/6020 & J \\
\hline Silver & ND & 0.500 & $\mathrm{mg} / \mathrm{kg}$ & 2 & 17-Jul-2009 & 20-Jul-2009 & SW 846/6020 & $\mathrm{U}$ \\
\hline Sodium & 991 & 0.500 & $\mathrm{mg} / \mathrm{kg}$ & 2 & 17-Jul-2009 & 20-Jul-2009 & SW 846/6020 & \\
\hline Thallium & ND & 0.500 & $\mathrm{mg} / \mathrm{kg}$ & 2 & 17-Jul-2009 & 20-Jul-2009 & SW 846/6020 & $\mathrm{U}$ \\
\hline Vanadium & 0.179 & 0.500 & $\mathrm{mg} / \mathrm{kg}$ & 2 & 17-Jul-2009 & 20-Jul-2009 & SW 846/6020 & J \\
\hline Zinc & 33.6 & 0.500 & $\mathrm{mg} / \mathrm{kg}$ & 2 & 17-Jul-2009 & 20-Jul-2009 & SW 846/6020 & B \\
\hline
\end{tabular}




\section{USACE ERDC-EP-C \\ 3909 Halls Ferry Road \\ Vicksburg, MS 39180-6199}

ERDC - ECB

Project: TVA Fly Ash Project

-

Project Manager: Tony Bednar

Reported:

$--,-$

Project Manager: Tony Bednar

03-Sep-2009

DRAFT: Control - A

9071602-21 (Tissue)

\begin{tabular}{|lrrrrrrrr}
\hline & & Reporting & & & & & & \\
Analyte & Result & Limit & Units & Dilution & Prepared & Analyzed & Method & Notes \\
\hline
\end{tabular}

ERDC- EL-EP-C (Environmental Chemistry Branch)

DRAFT: Metals by EPA 6000/7000 Series Methods

\begin{tabular}{|c|c|c|c|c|c|c|c|c|}
\hline Mercury & 0.013 & 0.001 & $\mathrm{mg} / \mathrm{kg}$ & 1 & 22-Jul-2009 & 22-Jul-2009 & EPA 7471A & \\
\hline Aluminum & 8.11 & 0.500 & $\mathrm{mg} / \mathrm{kg}$ & 2 & 17-Jul-2009 & 20-Jul-2009 & SW 846/6020 & \\
\hline Antimony & ND & 0.500 & $\mathrm{mg} / \mathrm{kg}$ & 2 & 17-Jul-2009 & 20-Jul-2009 & SW 846/6020 & $\mathrm{U}$ \\
\hline Arsenic & 0.152 & 0.500 & $\mathrm{mg} / \mathrm{kg}$ & 2 & 17-Jul-2009 & 20-Jul-2009 & SW 846/6020 & $\mathrm{J}$ \\
\hline Barium & 1.83 & 0.500 & $\mathrm{mg} / \mathrm{kg}$ & 2 & 17-Jul-2009 & 20-Jul-2009 & SW 846/6020 & \\
\hline Beryllium & ND & 0.500 & $\mathrm{mg} / \mathrm{kg}$ & 2 & 17-Jul-2009 & 20-Jul-2009 & SW 846/6020 & $\mathrm{U}$ \\
\hline Cadmium & ND & 0.500 & $\mathrm{mg} / \mathrm{kg}$ & 2 & 17-Jul-2009 & 20-Jul-2009 & SW 846/6020 & $\mathrm{U}$ \\
\hline Calcium & 6000 & 0.500 & $\mathrm{mg} / \mathrm{kg}$ & 2 & 17-Jul-2009 & 20-Jul-2009 & SW 846/6020 & \\
\hline Chromium & 2.06 & 0.500 & $\mathrm{mg} / \mathrm{kg}$ & 2 & 17-Jul-2009 & 20-Jul-2009 & SW 846/6020 & \\
\hline Cobalt & ND & 0.500 & $\mathrm{mg} / \mathrm{kg}$ & 2 & 17-Jul-2009 & 20-Jul-2009 & SW 846/6020 & $\mathrm{U}$ \\
\hline Copper & 0.979 & 0.500 & $\mathrm{mg} / \mathrm{kg}$ & 2 & 17-Jul-2009 & 20-Jul-2009 & SW 846/6020 & \\
\hline Iron & 37.2 & 0.500 & $\mathrm{mg} / \mathrm{kg}$ & 2 & 17-Jul-2009 & 20-Jul-2009 & SW 846/6020 & \\
\hline Lead & 0.192 & 0.500 & $\mathrm{mg} / \mathrm{kg}$ & 2 & 17-Jul-2009 & 20-Jul-2009 & SW 846/6020 & $\mathrm{J}$ \\
\hline Magnesium & 283 & 0.500 & $\mathrm{mg} / \mathrm{kg}$ & 2 & 17-Jul-2009 & 20-Jul-2009 & SW 846/6020 & \\
\hline Manganese & 1.15 & 0.500 & $\mathrm{mg} / \mathrm{kg}$ & 2 & 17-Jul-2009 & 20-Jul-2009 & SW 846/6020 & \\
\hline Molybdenum & ND & 0.500 & $\mathrm{mg} / \mathrm{kg}$ & 2 & 17-Jul-2009 & 20-Jul-2009 & SW 846/6020 & $\mathrm{U}$ \\
\hline Nickel & 1.03 & 0.500 & $\mathrm{mg} / \mathrm{kg}$ & 2 & 17-Jul-2009 & 20-Jul-2009 & SW 846/6020 & \\
\hline Potassium & 2400 & 0.500 & $\mathrm{mg} / \mathrm{kg}$ & 2 & 17-Jul-2009 & 20-Jul-2009 & SW 846/6020 & \\
\hline Selenium & 0.260 & 0.500 & $\mathrm{mg} / \mathrm{kg}$ & 2 & 17-Jul-2009 & 20-Jul-2009 & SW 846/6020 & $\mathrm{J}$ \\
\hline Silver & ND & 0.500 & $\mathrm{mg} / \mathrm{kg}$ & 2 & 17-Jul-2009 & 20-Jul-2009 & SW 846/6020 & $\mathrm{U}$ \\
\hline Sodium & 924 & 0.500 & $\mathrm{mg} / \mathrm{kg}$ & 2 & 17-Jul-2009 & 20-Jul-2009 & SW 846/6020 & \\
\hline Thallium & ND & 0.500 & $\mathrm{mg} / \mathrm{kg}$ & 2 & 17-Jul-2009 & 20-Jul-2009 & SW 846/6020 & $\mathrm{U}$ \\
\hline Vanadium & ND & 0.500 & $\mathrm{mg} / \mathrm{kg}$ & 2 & 17-Jul-2009 & 20-Jul-2009 & SW 846/6020 & $\mathrm{U}$ \\
\hline Zinc & 27.8 & 0.500 & $\mathrm{mg} / \mathrm{kg}$ & 2 & 17-Jul-2009 & 20-Jul-2009 & SW 846/6020 & B \\
\hline
\end{tabular}




\section{USACE ERDC-EP-C \\ 3909 Halls Ferry Road \\ Vicksburg, MS 39180-6199}

ERDC - ECB

Project: TVA Fly Ash Project

-

Project Manager: Tony Bednar

Reported:

$--,-$

Project Manager: Tony Bednar

03-Sep-2009

DRAFT: Control - B

9071602-22 (Tissue)

\begin{tabular}{|c|c|c|c|c|c|c|c|c|}
\hline & & porting & & & & & & \\
\hline Analyte & Result & Limit & Units & Dilution & Prepared & Analyzed & Method & Notes \\
\hline
\end{tabular}

ERDC- EL-EP-C (Environmental Chemistry Branch)

DRAFT: Metals by EPA 6000/7000 Series Methods

\begin{tabular}{|c|c|c|c|c|c|c|c|c|}
\hline Mercury & 0.017 & 0.002 & $\mathrm{mg} / \mathrm{kg}$ & 1 & 22-Jul-2009 & 22-Jul-2009 & EPA 7471A & \\
\hline Aluminum & 11.4 & 0.500 & $\mathrm{mg} / \mathrm{kg}$ & 2 & 17-Jul-2009 & 20-Jul-2009 & SW 846/6020 & \\
\hline Antimony & ND & 0.500 & $\mathrm{mg} / \mathrm{kg}$ & 2 & 17-Jul-2009 & 20-Jul-2009 & SW 846/6020 & $\mathrm{U}$ \\
\hline Arsenic & 0.114 & 0.500 & $\mathrm{mg} / \mathrm{kg}$ & 2 & 17-Jul-2009 & 20-Jul-2009 & SW 846/6020 & J \\
\hline Barium & 1.93 & 0.500 & $\mathrm{mg} / \mathrm{kg}$ & 2 & 17-Jul-2009 & 20-Jul-2009 & SW 846/6020 & \\
\hline Beryllium & ND & 0.500 & $\mathrm{mg} / \mathrm{kg}$ & 2 & 17-Jul-2009 & 20-Jul-2009 & SW 846/6020 & $\mathrm{U}$ \\
\hline Cadmium & ND & 0.500 & $\mathrm{mg} / \mathrm{kg}$ & 2 & 17-Jul-2009 & 20-Jul-2009 & SW 846/6020 & $\mathrm{U}$ \\
\hline Calcium & 5920 & 0.500 & $\mathrm{mg} / \mathrm{kg}$ & 2 & 17-Jul-2009 & 20-Jul-2009 & SW 846/6020 & \\
\hline Chromium & 1.41 & 0.500 & $\mathrm{mg} / \mathrm{kg}$ & 2 & 17-Jul-2009 & 20-Jul-2009 & SW 846/6020 & \\
\hline Cobalt & ND & 0.500 & $\mathrm{mg} / \mathrm{kg}$ & 2 & 17-Jul-2009 & 20-Jul-2009 & SW 846/6020 & $\mathrm{U}$ \\
\hline Copper & 1.04 & 0.500 & $\mathrm{mg} / \mathrm{kg}$ & 2 & 17-Jul-2009 & 20-Jul-2009 & SW 846/6020 & \\
\hline Iron & 34.0 & 0.500 & $\mathrm{mg} / \mathrm{kg}$ & 2 & 17-Jul-2009 & 20-Jul-2009 & SW 846/6020 & \\
\hline Lead & 0.248 & 0.500 & $\mathrm{mg} / \mathrm{kg}$ & 2 & 17-Jul-2009 & 20-Jul-2009 & SW 846/6020 & $\mathrm{J}$ \\
\hline Magnesium & 263 & 0.500 & $\mathrm{mg} / \mathrm{kg}$ & 2 & 17-Jul-2009 & 20-Jul-2009 & SW 846/6020 & \\
\hline Manganese & 1.36 & 0.500 & $\mathrm{mg} / \mathrm{kg}$ & 2 & 17-Jul-2009 & 20-Jul-2009 & SW 846/6020 & \\
\hline Molybdenum & ND & 0.500 & $\mathrm{mg} / \mathrm{kg}$ & 2 & 17-Jul-2009 & 20-Jul-2009 & SW 846/6020 & $\mathrm{U}$ \\
\hline Nickel & 0.651 & 0.500 & $\mathrm{mg} / \mathrm{kg}$ & 2 & 17-Jul-2009 & 20-Jul-2009 & SW 846/6020 & \\
\hline Potassium & 2240 & 0.500 & $\mathrm{mg} / \mathrm{kg}$ & 2 & 17-Jul-2009 & 20-Jul-2009 & SW 846/6020 & \\
\hline Selenium & 0.244 & 0.500 & $\mathrm{mg} / \mathrm{kg}$ & 2 & 17-Jul-2009 & 20-Jul-2009 & SW 846/6020 & $\mathrm{J}$ \\
\hline Silver & ND & 0.500 & $\mathrm{mg} / \mathrm{kg}$ & 2 & 17-Jul-2009 & 20-Jul-2009 & SW 846/6020 & $\mathrm{U}$ \\
\hline Sodium & 1010 & 0.500 & $\mathrm{mg} / \mathrm{kg}$ & 2 & 17-Jul-2009 & 20-Jul-2009 & SW 846/6020 & \\
\hline Thallium & ND & 0.500 & $\mathrm{mg} / \mathrm{kg}$ & 2 & 17-Jul-2009 & 20-Jul-2009 & SW 846/6020 & $\mathrm{U}$ \\
\hline Vanadium & 0.120 & 0.500 & $\mathrm{mg} / \mathrm{kg}$ & 2 & 17-Jul-2009 & 20-Jul-2009 & SW 846/6020 & $\mathrm{J}$ \\
\hline Zinc & 25.4 & 0.500 & $\mathrm{mg} / \mathrm{kg}$ & 2 & 17-Jul-2009 & 20-Jul-2009 & SW 846/6020 & B \\
\hline
\end{tabular}




\section{USACE ERDC-EP-C \\ 3909 Halls Ferry Road \\ Vicksburg, MS 39180-6199}

ERDC - ECB

Project: TVA Fly Ash Project

-

Project Manager: Tony Bednar

Reported:

$--,-$

Project Manager: Tony Bednar

03-Sep-2009

DRAFT: Control - C

9071602-23 (Tissue)

\begin{tabular}{|c|c|c|c|c|c|c|c|c|}
\hline & & porting & & & & & & \\
\hline Analyte & Result & Limit & Units & Dilution & Prepared & Analyzed & Method & Notes \\
\hline
\end{tabular}

ERDC- EL-EP-C (Environmental Chemistry Branch)

DRAFT: Metals by EPA 6000/7000 Series Methods

\begin{tabular}{|c|c|c|c|c|c|c|c|c|}
\hline Mercury & 0.015 & 0.002 & $\mathrm{mg} / \mathrm{kg}$ & 1 & 22-Jul-2009 & 22-Jul-2009 & EPA 7471A & \\
\hline Aluminum & 15.6 & 0.500 & $\mathrm{mg} / \mathrm{kg}$ & 2 & 17-Jul-2009 & 20-Jul-2009 & SW 846/6020 & \\
\hline Antimony & ND & 0.500 & $\mathrm{mg} / \mathrm{kg}$ & 2 & 17-Jul-2009 & 20-Jul-2009 & SW 846/6020 & $\mathrm{U}$ \\
\hline Arsenic & 0.101 & 0.500 & $\mathrm{mg} / \mathrm{kg}$ & 2 & 17-Jul-2009 & 20-Jul-2009 & SW 846/6020 & $\mathrm{J}$ \\
\hline Barium & 2.00 & 0.500 & $\mathrm{mg} / \mathrm{kg}$ & 2 & 17-Jul-2009 & 20-Jul-2009 & SW 846/6020 & \\
\hline Beryllium & ND & 0.500 & $\mathrm{mg} / \mathrm{kg}$ & 2 & 17-Jul-2009 & 20-Jul-2009 & SW 846/6020 & $\mathrm{U}$ \\
\hline Cadmium & ND & 0.500 & $\mathrm{mg} / \mathrm{kg}$ & 2 & 17-Jul-2009 & 20-Jul-2009 & SW 846/6020 & $\mathrm{U}$ \\
\hline Calcium & 5680 & 0.500 & $\mathrm{mg} / \mathrm{kg}$ & 2 & 17-Jul-2009 & 20-Jul-2009 & SW 846/6020 & \\
\hline Chromium & 3.70 & 0.500 & $\mathrm{mg} / \mathrm{kg}$ & 2 & 17-Jul-2009 & 20-Jul-2009 & SW 846/6020 & \\
\hline Cobalt & ND & 0.500 & $\mathrm{mg} / \mathrm{kg}$ & 2 & 17-Jul-2009 & 20-Jul-2009 & SW 846/6020 & $\mathrm{U}$ \\
\hline Copper & 1.21 & 0.500 & $\mathrm{mg} / \mathrm{kg}$ & 2 & 17-Jul-2009 & 20-Jul-2009 & SW 846/6020 & \\
\hline Iron & 39.8 & 0.500 & $\mathrm{mg} / \mathrm{kg}$ & 2 & 17-Jul-2009 & 20-Jul-2009 & SW 846/6020 & \\
\hline Lead & 0.324 & 0.500 & $\mathrm{mg} / \mathrm{kg}$ & 2 & 17-Jul-2009 & 20-Jul-2009 & SW 846/6020 & $\mathrm{J}$ \\
\hline Magnesium & 262 & 0.500 & $\mathrm{mg} / \mathrm{kg}$ & 2 & 17-Jul-2009 & 20-Jul-2009 & SW 846/6020 & \\
\hline Manganese & 1.52 & 0.500 & $\mathrm{mg} / \mathrm{kg}$ & 2 & 17-Jul-2009 & 20-Jul-2009 & SW 846/6020 & \\
\hline Molybdenum & 0.100 & 0.500 & $\mathrm{mg} / \mathrm{kg}$ & 2 & 17-Jul-2009 & 20-Jul-2009 & SW 846/6020 & $\mathrm{J}$ \\
\hline Nickel & 1.78 & 0.500 & $\mathrm{mg} / \mathrm{kg}$ & 2 & 17-Jul-2009 & 20-Jul-2009 & SW 846/6020 & \\
\hline Potassium & 2220 & 0.500 & $\mathrm{mg} / \mathrm{kg}$ & 2 & 17-Jul-2009 & 20-Jul-2009 & SW 846/6020 & \\
\hline Selenium & 0.248 & 0.500 & $\mathrm{mg} / \mathrm{kg}$ & 2 & 17-Jul-2009 & 20-Jul-2009 & SW 846/6020 & $\mathrm{J}$ \\
\hline Silver & ND & 0.500 & $\mathrm{mg} / \mathrm{kg}$ & 2 & 17-Jul-2009 & 20-Jul-2009 & SW 846/6020 & $\mathrm{U}$ \\
\hline Sodium & 962 & 0.500 & $\mathrm{mg} / \mathrm{kg}$ & 2 & 17-Jul-2009 & 20-Jul-2009 & SW 846/6020 & \\
\hline Thallium & ND & 0.500 & $\mathrm{mg} / \mathrm{kg}$ & 2 & 17-Jul-2009 & 20-Jul-2009 & SW 846/6020 & $\mathrm{U}$ \\
\hline Vanadium & ND & 0.500 & $\mathrm{mg} / \mathrm{kg}$ & 2 & 17-Jul-2009 & 20-Jul-2009 & SW 846/6020 & $\mathrm{U}$ \\
\hline Zinc & 25.3 & 0.500 & $\mathrm{mg} / \mathrm{kg}$ & 2 & 17-Jul-2009 & 20-Jul-2009 & SW 846/6020 & B \\
\hline
\end{tabular}




\section{USACE ERDC-EP-C \\ 3909 Halls Ferry Road \\ Vicksburg, MS 39180-6199}

ERDC - ECB

Project: TVA Fly Ash Project

-

Project Manager: Tony Bednar

Reported:

$--,-$

Project Manager: Tony Bednar

03-Sep-2009

DRAFT: Control - D

9071602-24 (Tissue)

\begin{tabular}{|c|c|c|c|c|c|c|c|c|}
\hline & & porting & & & & & & \\
\hline Analyte & Result & Limit & Units & Dilution & Prepared & Analyzed & Method & Notes \\
\hline
\end{tabular}

ERDC- EL-EP-C (Environmental Chemistry Branch)

DRAFT: Metals by EPA 6000/7000 Series Methods

\begin{tabular}{|c|c|c|c|c|c|c|c|c|}
\hline Mercury & 0.016 & 0.002 & $\mathrm{mg} / \mathrm{kg}$ & 1 & 22-Jul-2009 & 22-Jul-2009 & EPA 7471A & \\
\hline Aluminum & 13.4 & 0.500 & $\mathrm{mg} / \mathrm{kg}$ & 2 & 17-Jul-2009 & 20-Jul-2009 & SW 846/6020 & \\
\hline Antimony & ND & 0.500 & $\mathrm{mg} / \mathrm{kg}$ & 2 & 17-Jul-2009 & 20-Jul-2009 & SW 846/6020 & $\mathrm{U}$ \\
\hline Arsenic & 0.110 & 0.500 & $\mathrm{mg} / \mathrm{kg}$ & 2 & 17-Jul-2009 & 20-Jul-2009 & SW 846/6020 & J \\
\hline Barium & 1.84 & 0.500 & $\mathrm{mg} / \mathrm{kg}$ & 2 & 17-Jul-2009 & 20-Jul-2009 & SW 846/6020 & \\
\hline Beryllium & ND & 0.500 & $\mathrm{mg} / \mathrm{kg}$ & 2 & 17-Jul-2009 & 20-Jul-2009 & SW 846/6020 & $\mathrm{U}$ \\
\hline Cadmium & ND & 0.500 & $\mathrm{mg} / \mathrm{kg}$ & 2 & 17-Jul-2009 & 20-Jul-2009 & SW 846/6020 & $\mathrm{U}$ \\
\hline Calcium & 5710 & 0.500 & $\mathrm{mg} / \mathrm{kg}$ & 2 & 17-Jul-2009 & 20-Jul-2009 & SW 846/6020 & \\
\hline Chromium & 1.35 & 0.500 & $\mathrm{mg} / \mathrm{kg}$ & 2 & 17-Jul-2009 & 20-Jul-2009 & SW 846/6020 & \\
\hline Cobalt & ND & 0.500 & $\mathrm{mg} / \mathrm{kg}$ & 2 & 17-Jul-2009 & 20-Jul-2009 & SW 846/6020 & $\mathrm{U}$ \\
\hline Copper & 0.914 & 0.500 & $\mathrm{mg} / \mathrm{kg}$ & 2 & 17-Jul-2009 & 20-Jul-2009 & SW 846/6020 & \\
\hline Iron & 34.2 & 0.500 & $\mathrm{mg} / \mathrm{kg}$ & 2 & 17-Jul-2009 & 20-Jul-2009 & SW 846/6020 & \\
\hline Lead & 0.221 & 0.500 & $\mathrm{mg} / \mathrm{kg}$ & 2 & 17-Jul-2009 & 20-Jul-2009 & SW 846/6020 & $\mathrm{J}$ \\
\hline Magnesium & 252 & 0.500 & $\mathrm{mg} / \mathrm{kg}$ & 2 & 17-Jul-2009 & 20-Jul-2009 & SW 846/6020 & \\
\hline Manganese & 1.18 & 0.500 & $\mathrm{mg} / \mathrm{kg}$ & 2 & 17-Jul-2009 & 20-Jul-2009 & SW 846/6020 & \\
\hline Molybdenum & ND & 0.500 & $\mathrm{mg} / \mathrm{kg}$ & 2 & 17-Jul-2009 & 20-Jul-2009 & SW 846/6020 & $\mathrm{U}$ \\
\hline Nickel & 0.504 & 0.500 & $\mathrm{mg} / \mathrm{kg}$ & 2 & 17-Jul-2009 & 20-Jul-2009 & SW 846/6020 & \\
\hline Potassium & 2130 & 0.500 & $\mathrm{mg} / \mathrm{kg}$ & 2 & 17-Jul-2009 & 20-Jul-2009 & SW 846/6020 & \\
\hline Selenium & 0.241 & 0.500 & $\mathrm{mg} / \mathrm{kg}$ & 2 & 17-Jul-2009 & 20-Jul-2009 & SW 846/6020 & $\mathrm{J}$ \\
\hline Silver & ND & 0.500 & $\mathrm{mg} / \mathrm{kg}$ & 2 & 17-Jul-2009 & 20-Jul-2009 & SW 846/6020 & $\mathrm{U}$ \\
\hline Sodium & 887 & 0.500 & $\mathrm{mg} / \mathrm{kg}$ & 2 & 17-Jul-2009 & 20-Jul-2009 & SW 846/6020 & \\
\hline Thallium & ND & 0.500 & $\mathrm{mg} / \mathrm{kg}$ & 2 & 17-Jul-2009 & 20-Jul-2009 & SW 846/6020 & $\mathrm{U}$ \\
\hline Vanadium & ND & 0.500 & $\mathrm{mg} / \mathrm{kg}$ & 2 & 17-Jul-2009 & 20-Jul-2009 & SW 846/6020 & $\mathrm{U}$ \\
\hline Zinc & 22.3 & 0.500 & $\mathrm{mg} / \mathrm{kg}$ & 2 & 17-Jul-2009 & 20-Jul-2009 & SW 846/6020 & B \\
\hline
\end{tabular}




\section{USACE ERDC-EP-C \\ 3909 Halls Ferry Road \\ Vicksburg, MS 39180-6199}

ERDC - ECB

Project: TVA Fly Ash Project

$-$

$--,-$

Project Manager: Tony Bednar

Reported:

03-Sep-2009

DRAFT: Control - E

9071602-25 (Tissue)

\begin{tabular}{|c|c|c|c|c|c|c|c|c|}
\hline & & porting & & & & & & \\
\hline Analyte & Result & Limit & Units & Dilution & Prepared & Analyzed & Method & Notes \\
\hline
\end{tabular}

ERDC- EL-EP-C (Environmental Chemistry Branch)

DRAFT: Metals by EPA 6000/7000 Series Methods

\begin{tabular}{|c|c|c|c|c|c|c|c|c|}
\hline Mercury & 0.015 & 0.002 & $\mathrm{mg} / \mathrm{kg}$ & 1 & 22-Jul-2009 & 22-Jul-2009 & EPA 7471A & \\
\hline Aluminum & 7.01 & 0.500 & $\mathrm{mg} / \mathrm{kg}$ & 2 & 17-Jul-2009 & 20-Jul-2009 & SW 846/6020 & \\
\hline Antimony & 0.812 & 0.500 & $\mathrm{mg} / \mathrm{kg}$ & 2 & 17-Jul-2009 & 20-Jul-2009 & SW 846/6020 & \\
\hline Arsenic & 0.130 & 0.500 & $\mathrm{mg} / \mathrm{kg}$ & 2 & 17-Jul-2009 & 20-Jul-2009 & SW 846/6020 & $\mathrm{J}$ \\
\hline Barium & 1.55 & 0.500 & $\mathrm{mg} / \mathrm{kg}$ & 2 & 17-Jul-2009 & 20-Jul-2009 & SW 846/6020 & \\
\hline Beryllium & ND & 0.500 & $\mathrm{mg} / \mathrm{kg}$ & 2 & 17-Jul-2009 & 20-Jul-2009 & SW 846/6020 & $\mathrm{U}$ \\
\hline Cadmium & ND & 0.500 & $\mathrm{mg} / \mathrm{kg}$ & 2 & 17-Jul-2009 & 20-Jul-2009 & SW 846/6020 & $\mathrm{U}$ \\
\hline Calcium & 5740 & 0.500 & $\mathrm{mg} / \mathrm{kg}$ & 2 & 17-Jul-2009 & 20-Jul-2009 & SW 846/6020 & \\
\hline Chromium & 0.797 & 0.500 & $\mathrm{mg} / \mathrm{kg}$ & 2 & 17-Jul-2009 & 20-Jul-2009 & SW 846/6020 & \\
\hline Cobalt & ND & 0.500 & $\mathrm{mg} / \mathrm{kg}$ & 2 & 17-Jul-2009 & 20-Jul-2009 & SW 846/6020 & $\mathrm{U}$ \\
\hline Copper & 1.22 & 0.500 & $\mathrm{mg} / \mathrm{kg}$ & 2 & 17-Jul-2009 & 20-Jul-2009 & SW 846/6020 & \\
\hline Iron & 31.9 & 0.500 & $\mathrm{mg} / \mathrm{kg}$ & 2 & 17-Jul-2009 & 20-Jul-2009 & SW 846/6020 & \\
\hline Lead & ND & 0.500 & $\mathrm{mg} / \mathrm{kg}$ & 2 & 17-Jul-2009 & 20-Jul-2009 & SW 846/6020 & $\mathrm{U}$ \\
\hline Magnesium & 241 & 0.500 & $\mathrm{mg} / \mathrm{kg}$ & 2 & 17-Jul-2009 & 20-Jul-2009 & SW 846/6020 & \\
\hline Manganese & 0.805 & 0.500 & $\mathrm{mg} / \mathrm{kg}$ & 2 & 17-Jul-2009 & 20-Jul-2009 & SW 846/6020 & \\
\hline Molybdenum & ND & 0.500 & $\mathrm{mg} / \mathrm{kg}$ & 2 & 17-Jul-2009 & 20-Jul-2009 & SW 846/6020 & $\mathrm{U}$ \\
\hline Nickel & 0.338 & 0.500 & $\mathrm{mg} / \mathrm{kg}$ & 2 & 17-Jul-2009 & 20-Jul-2009 & SW 846/6020 & $\mathrm{J}$ \\
\hline Potassium & 2020 & 0.500 & $\mathrm{mg} / \mathrm{kg}$ & 2 & 17-Jul-2009 & 20-Jul-2009 & SW 846/6020 & \\
\hline Selenium & 0.220 & 0.500 & $\mathrm{mg} / \mathrm{kg}$ & 2 & 17-Jul-2009 & 20-Jul-2009 & SW 846/6020 & $\mathrm{J}$ \\
\hline Silver & ND & 0.500 & $\mathrm{mg} / \mathrm{kg}$ & 2 & 17-Jul-2009 & 20-Jul-2009 & SW 846/6020 & $\mathrm{U}$ \\
\hline Sodium & 874 & 0.500 & $\mathrm{mg} / \mathrm{kg}$ & 2 & 17-Jul-2009 & 20-Jul-2009 & SW 846/6020 & \\
\hline Thallium & ND & 0.500 & $\mathrm{mg} / \mathrm{kg}$ & 2 & 17-Jul-2009 & 20-Jul-2009 & SW 846/6020 & $\mathrm{U}$ \\
\hline Vanadium & ND & 0.500 & $\mathrm{mg} / \mathrm{kg}$ & 2 & 17-Jul-2009 & 20-Jul-2009 & SW 846/6020 & $\mathrm{U}$ \\
\hline Zinc & 23.9 & 0.500 & $\mathrm{mg} / \mathrm{kg}$ & 2 & 17-Jul-2009 & 20-Jul-2009 & SW 846/6020 & B \\
\hline
\end{tabular}




\section{USACE ERDC-EP-C \\ 3909 Halls Ferry Road \\ Vicksburg, MS 39180-6199}

ERDC - ECB

Project: TVA Fly Ash Project

$-$

$--,-$

Project Manager: Tony Bednar

Reported:

03-Sep-2009

\section{DRAFT: Larvae Food}

9071602-26 (Solid)

\begin{tabular}{|c|c|c|c|c|c|c|c|c|}
\hline & & porting & & & & & & \\
\hline Analyte & Result & Limit & Units & Dilution & Prepared & Analyzed & Method & Notes \\
\hline
\end{tabular}

ERDC- EL-EP-C (Environmental Chemistry Branch)

DRAFT: Metals by EPA 6000/7000 Series Methods

\begin{tabular}{|c|c|c|c|c|c|c|c|c|}
\hline Aluminum & 23.7 & 0.500 & $\mathrm{mg} / \mathrm{kg}$ & 2 & 17-Jul-2009 & 20-Jul-2009 & SW 846/6020 & \\
\hline Antimony & ND & 0.500 & $\mathrm{mg} / \mathrm{kg}$ & 2 & 17-Jul-2009 & 20-Jul-2009 & SW 846/6020 & $\mathrm{U}$ \\
\hline Arsenic & 14.3 & 0.500 & $\mathrm{mg} / \mathrm{kg}$ & 2 & 17-Jul-2009 & 20-Jul-2009 & SW 846/6020 & \\
\hline Barium & 2.21 & 0.500 & $\mathrm{mg} / \mathrm{kg}$ & 2 & 17-Jul-2009 & 20-Jul-2009 & SW 846/6020 & \\
\hline Beryllium & ND & 0.500 & $\mathrm{mg} / \mathrm{kg}$ & 2 & 17-Jul-2009 & 20-Jul-2009 & SW 846/6020 & $\mathrm{U}$ \\
\hline Cadmium & ND & 0.500 & $\mathrm{mg} / \mathrm{kg}$ & 2 & 17-Jul-2009 & 20-Jul-2009 & SW 846/6020 & $\mathrm{U}$ \\
\hline Calcium & 1470 & 0.500 & $\mathrm{mg} / \mathrm{kg}$ & 2 & 17-Jul-2009 & 20-Jul-2009 & SW 846/6020 & \\
\hline Chromium & 0.237 & 0.500 & $\mathrm{mg} / \mathrm{kg}$ & 2 & 17-Jul-2009 & 20-Jul-2009 & SW 846/6020 & $\mathrm{J}$ \\
\hline Cobalt & 0.280 & 0.500 & $\mathrm{mg} / \mathrm{kg}$ & 2 & 17-Jul-2009 & 20-Jul-2009 & SW 846/6020 & $\mathrm{J}$ \\
\hline Copper & 8.68 & 0.500 & $\mathrm{mg} / \mathrm{kg}$ & 2 & 17-Jul-2009 & 20-Jul-2009 & SW 846/6020 & \\
\hline Iron & 600 & 0.500 & $\mathrm{mg} / \mathrm{kg}$ & 2 & 17-Jul-2009 & 20-Jul-2009 & SW 846/6020 & \\
\hline Lead & 0.478 & 0.500 & $\mathrm{mg} / \mathrm{kg}$ & 2 & 17-Jul-2009 & 20-Jul-2009 & SW 846/6020 & $\mathrm{J}$ \\
\hline Magnesium & 2280 & 0.500 & $\mathrm{mg} / \mathrm{kg}$ & 2 & 17-Jul-2009 & 20-Jul-2009 & SW 846/6020 & \\
\hline Manganese & 7.33 & 0.500 & $\mathrm{mg} / \mathrm{kg}$ & 2 & 17-Jul-2009 & 20-Jul-2009 & SW 846/6020 & \\
\hline Molybdenum & 0.467 & 0.500 & $\mathrm{mg} / \mathrm{kg}$ & 2 & 17-Jul-2009 & 20-Jul-2009 & SW 846/6020 & $\mathrm{J}$ \\
\hline Nickel & 0.570 & 0.500 & $\mathrm{mg} / \mathrm{kg}$ & 2 & 17-Jul-2009 & 20-Jul-2009 & SW 846/6020 & \\
\hline Potassium & 4850 & 0.500 & $\mathrm{mg} / \mathrm{kg}$ & 2 & 17-Jul-2009 & 20-Jul-2009 & SW 846/6020 & \\
\hline Selenium & 2.15 & 0.500 & $\mathrm{mg} / \mathrm{kg}$ & 2 & 17-Jul-2009 & 20-Jul-2009 & SW 846/6020 & \\
\hline Silver & ND & 0.500 & $\mathrm{mg} / \mathrm{kg}$ & 2 & 17-Jul-2009 & 20-Jul-2009 & SW 846/6020 & $\mathrm{U}$ \\
\hline Sodium & 2950 & 0.500 & $\mathrm{mg} / \mathrm{kg}$ & 2 & 17-Jul-2009 & 20-Jul-2009 & SW 846/6020 & \\
\hline Thallium & ND & 0.500 & $\mathrm{mg} / \mathrm{kg}$ & 2 & 17-Jul-2009 & 20-Jul-2009 & SW 846/6020 & $\mathrm{U}$ \\
\hline Vanadium & 0.133 & 0.500 & $\mathrm{mg} / \mathrm{kg}$ & 2 & 17-Jul-2009 & 20-Jul-2009 & SW 846/6020 & $\mathrm{J}$ \\
\hline Zinc & 52.9 & 0.500 & $\mathrm{mg} / \mathrm{kg}$ & 2 & 17-Jul-2009 & 20-Jul-2009 & SW 846/6020 & B \\
\hline
\end{tabular}




\section{USACE ERDC-EP-C \\ 3909 Halls Ferry Road \\ Vicksburg, MS 39180-6199}

ERDC - ECB

Project: TVA Fly Ash Project

$-$

$--,-$

Project Manager: Tony Bednar

Reported:

03-Sep-2009

DRAFT: Adult Food

9071602-27 (Solid)

\begin{tabular}{|c|c|c|c|c|c|c|c|c|}
\hline & & porting & & & & & & \\
\hline Analyte & Result & Limit & Units & Dilution & Prepared & Analyzed & Method & Notes \\
\hline
\end{tabular}

ERDC- EL-EP-C (Environmental Chemistry Branch)

DRAFT: Metals by EPA 6000/7000 Series Methods

\begin{tabular}{|c|c|c|c|c|c|c|c|c|}
\hline Aluminum & 32.3 & 0.500 & $\mathrm{mg} / \mathrm{kg}$ & 2 & 17-Jul-2009 & 20-Jul-2009 & SW 846/6020 & \\
\hline Antimony & ND & 0.500 & $\mathrm{mg} / \mathrm{kg}$ & 2 & 17-Jul-2009 & 20-Jul-2009 & SW 846/6020 & $\mathrm{U}$ \\
\hline Arsenic & 1.70 & 0.500 & $\mathrm{mg} / \mathrm{kg}$ & 2 & 17-Jul-2009 & 20-Jul-2009 & SW 846/6020 & \\
\hline Barium & 3.17 & 0.500 & $\mathrm{mg} / \mathrm{kg}$ & 2 & 17-Jul-2009 & 20-Jul-2009 & SW 846/6020 & \\
\hline Beryllium & ND & 0.500 & $\mathrm{mg} / \mathrm{kg}$ & 2 & 17-Jul-2009 & 20-Jul-2009 & SW 846/6020 & $\mathrm{U}$ \\
\hline Cadmium & 0.101 & 0.500 & $\mathrm{mg} / \mathrm{kg}$ & 2 & 17-Jul-2009 & 20-Jul-2009 & SW 846/6020 & $\mathrm{J}$ \\
\hline Calcium & 12300 & 0.500 & $\mathrm{mg} / \mathrm{kg}$ & 2 & 17-Jul-2009 & 20-Jul-2009 & SW 846/6020 & \\
\hline Chromium & 5.50 & 0.500 & $\mathrm{mg} / \mathrm{kg}$ & 2 & 17-Jul-2009 & 20-Jul-2009 & SW 846/6020 & \\
\hline Cobalt & 0.195 & 0.500 & $\mathrm{mg} / \mathrm{kg}$ & 2 & 17-Jul-2009 & 20-Jul-2009 & SW 846/6020 & $\mathrm{J}$ \\
\hline Copper & 16.9 & 0.500 & $\mathrm{mg} / \mathrm{kg}$ & 2 & 17-Jul-2009 & 20-Jul-2009 & SW 846/6020 & \\
\hline Iron & 199 & 0.500 & $\mathrm{mg} / \mathrm{kg}$ & 2 & 17-Jul-2009 & 20-Jul-2009 & SW 846/6020 & \\
\hline Lead & 0.523 & 0.500 & $\mathrm{mg} / \mathrm{kg}$ & 2 & 17-Jul-2009 & 20-Jul-2009 & SW 846/6020 & \\
\hline Magnesium & 1630 & 0.500 & $\mathrm{mg} / \mathrm{kg}$ & 2 & 17-Jul-2009 & 20-Jul-2009 & SW 846/6020 & \\
\hline Manganese & 42.6 & 0.500 & $\mathrm{mg} / \mathrm{kg}$ & 2 & 17-Jul-2009 & 20-Jul-2009 & SW 846/6020 & \\
\hline Molybdenum & 0.446 & 0.500 & $\mathrm{mg} / \mathrm{kg}$ & 2 & 17-Jul-2009 & 20-Jul-2009 & SW 846/6020 & J \\
\hline Nickel & 1.07 & 0.500 & $\mathrm{mg} / \mathrm{kg}$ & 2 & 17-Jul-2009 & 20-Jul-2009 & SW 846/6020 & \\
\hline Potassium & 7810 & 0.500 & $\mathrm{mg} / \mathrm{kg}$ & 2 & 17-Jul-2009 & 20-Jul-2009 & SW 846/6020 & \\
\hline Selenium & 1.30 & 0.500 & $\mathrm{mg} / \mathrm{kg}$ & 2 & 17-Jul-2009 & 20-Jul-2009 & SW 846/6020 & \\
\hline Silver & ND & 0.500 & $\mathrm{mg} / \mathrm{kg}$ & 2 & 17-Jul-2009 & 20-Jul-2009 & SW 846/6020 & $\mathrm{U}$ \\
\hline Sodium & 5590 & 0.500 & $\mathrm{mg} / \mathrm{kg}$ & 2 & 17-Jul-2009 & 20-Jul-2009 & SW 846/6020 & \\
\hline Thallium & ND & 0.500 & $\mathrm{mg} / \mathrm{kg}$ & 2 & 17-Jul-2009 & 20-Jul-2009 & SW 846/6020 & $\mathrm{U}$ \\
\hline Vanadium & 0.357 & 0.500 & $\mathrm{mg} / \mathrm{kg}$ & 2 & 17-Jul-2009 & 20-Jul-2009 & SW 846/6020 & J \\
\hline Zinc & 93.5 & 0.500 & $\mathrm{mg} / \mathrm{kg}$ & 2 & 17-Jul-2009 & 20-Jul-2009 & SW 846/6020 & B \\
\hline
\end{tabular}




\section{USACE ERDC-EP-C \\ 3909 Halls Ferry Road \\ Vicksburg, MS 39180-6199}

ERDC - ECB

Project: TVA Fly Ash Project

Reported:

Project Manager: Tony Bednar

03-Sep-2009

DRAFT: Fish Tissue Dechlor 1

9082706-01 (Tissue)

\begin{tabular}{|c|c|c|c|c|c|c|c|c|}
\hline Analyte & Result & $\begin{array}{r}\text { porting } \\
\text { Limit }\end{array}$ & Units & Dilution & Prepared & Analyzed & Method & Notes \\
\hline
\end{tabular}

ERDC- EL-EP-C (Environmental Chemistry Branch)

DRAFT: Metals by EPA 6000/7000 Series Methods

\begin{tabular}{|c|c|c|c|c|c|c|c|c|}
\hline Mercury & 0.007 & 0.003 & $\mathrm{mg} / \mathrm{kg}$ & 1 & 03-Sep-2009 & 03-Sep-2009 & EPA 7471A & \\
\hline Antimony & ND & 0.400 & $\mathrm{mg} / \mathrm{kg}$ & 2 & 28-Aug-2009 & 29-Aug-2009 & SW 846/6020 & $\mathrm{U}$ \\
\hline Arsenic & 0.101 & 0.400 & $\mathrm{mg} / \mathrm{kg}$ & 2 & 28-Aug-2009 & 29-Aug-2009 & SW 846/6020 & $\mathrm{J}$ \\
\hline Beryllium & ND & 0.400 & $\mathrm{mg} / \mathrm{kg}$ & 2 & 28-Aug-2009 & 29-Aug-2009 & SW 846/6020 & $\mathrm{U}$ \\
\hline Cadmium & ND & 0.400 & $\mathrm{mg} / \mathrm{kg}$ & 2 & 28-Aug-2009 & 29-Aug-2009 & SW 846/6020 & $\mathrm{U}$ \\
\hline Chromium & 6.45 & 0.400 & $\mathrm{mg} / \mathrm{kg}$ & 2 & 28-Aug-2009 & 29-Aug-2009 & SW 846/6020 & \\
\hline Cobalt & 0.104 & 0.400 & $\mathrm{mg} / \mathrm{kg}$ & 2 & 28-Aug-2009 & 29-Aug-2009 & SW 846/6020 & $\mathrm{J}$ \\
\hline Copper & 1.62 & 0.400 & $\mathrm{mg} / \mathrm{kg}$ & 2 & 28-Aug-2009 & 29-Aug-2009 & SW 846/6020 & \\
\hline Lead & 1.52 & 0.400 & $\mathrm{mg} / \mathrm{kg}$ & 2 & 28-Aug-2009 & 29-Aug-2009 & SW 846/6020 & B \\
\hline Manganese & 1.51 & 0.400 & $\mathrm{mg} / \mathrm{kg}$ & 2 & 28-Aug-2009 & 29-Aug-2009 & SW 846/6020 & \\
\hline Molybdenum & 0.124 & 0.400 & $\mathrm{mg} / \mathrm{kg}$ & 2 & 28-Aug-2009 & 29-Aug-2009 & SW 846/6020 & $\mathrm{J}$ \\
\hline Nickel & 4.68 & 0.400 & $\mathrm{mg} / \mathrm{kg}$ & 2 & 28-Aug-2009 & 29-Aug-2009 & SW 846/6020 & \\
\hline Silver & 0.193 & 0.400 & $\mathrm{mg} / \mathrm{kg}$ & 2 & 28-Aug-2009 & 29-Aug-2009 & SW 846/6020 & $\mathrm{J}$ \\
\hline Thallium & ND & 0.400 & $\mathrm{mg} / \mathrm{kg}$ & 2 & 28-Aug-2009 & 29-Aug-2009 & SW 846/6020 & $\mathrm{U}$ \\
\hline Vanadium & 0.094 & 0.400 & $\mathrm{mg} / \mathrm{kg}$ & 2 & 28-Aug-2009 & 29-Aug-2009 & SW 846/6020 & $\mathrm{J}$ \\
\hline Zinc & 19.0 & 0.400 & $\mathrm{mg} / \mathrm{kg}$ & 2 & 28-Aug-2009 & 29-Aug-2009 & SW 846/6020 & \\
\hline
\end{tabular}




\section{USACE ERDC-EP-C \\ 3909 Halls Ferry Road \\ Vicksburg, MS 39180-6199}

ERDC - ECB

Project: TVA Fly Ash Project

Reported:

Project Manager: Tony Bednar

03-Sep-2009

DRAFT: Fish Tissue Dechlor 2

9082706-02 (Tissue)

\begin{tabular}{|c|c|c|c|c|c|c|c|c|}
\hline Analyte & Result & $\begin{array}{r}\text { porting } \\
\text { Limit }\end{array}$ & Units & Dilution & Prepared & Analyzed & Method & Notes \\
\hline
\end{tabular}

ERDC- EL-EP-C (Environmental Chemistry Branch)

DRAFT: Metals by EPA 6000/7000 Series Methods

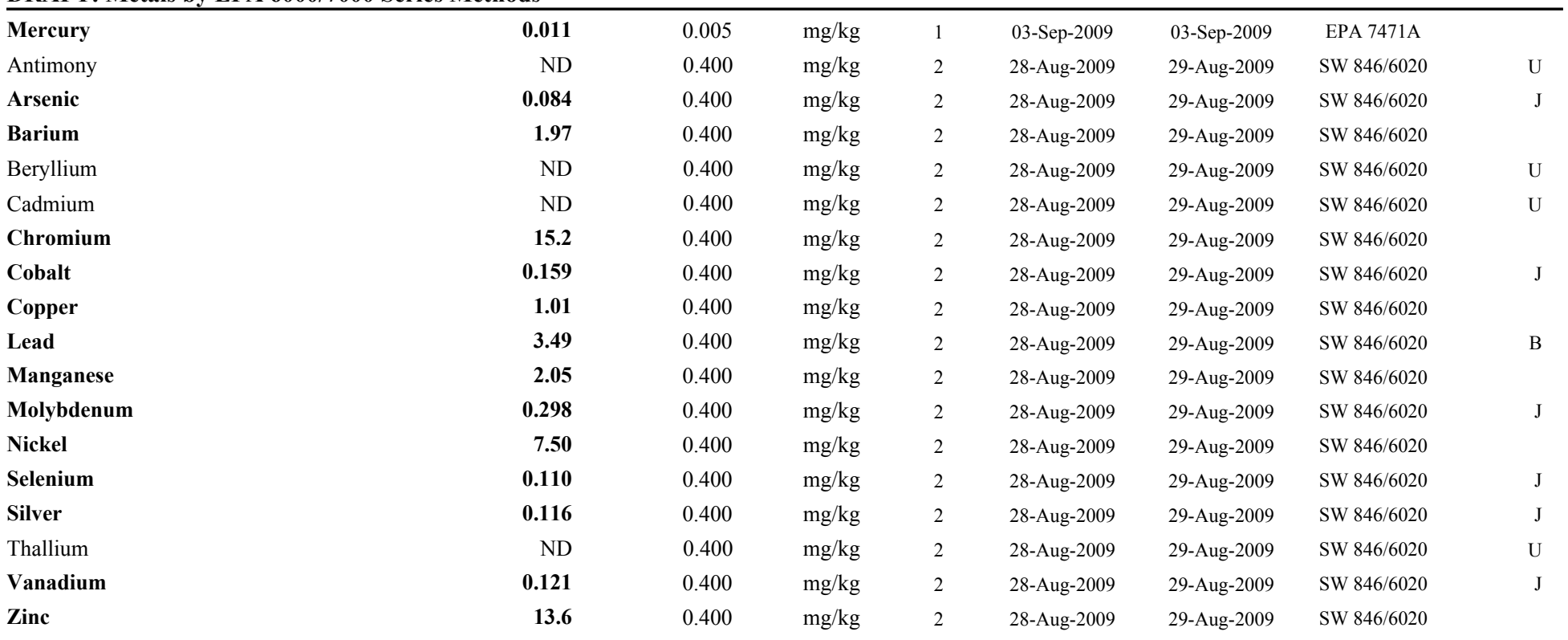




\section{USACE ERDC-EP-C \\ 3909 Halls Ferry Road \\ Vicksburg, MS 39180-6199}

ERDC - ECB

Project: TVA Fly Ash Project

Reported:

Project Manager: Tony Bednar

03-Sep-2009

DRAFT: Fish Tissue Dechlor 3

9082706-03 (Tissue)

\begin{tabular}{|c|c|c|c|c|c|c|c|c|}
\hline Analyte & Result & $\begin{array}{r}\text { porting } \\
\text { Limit }\end{array}$ & Units & Dilution & Prepared & Analyzed & Method & Notes \\
\hline
\end{tabular}

ERDC- EL-EP-C (Environmental Chemistry Branch)

DRAFT: Metals by EPA 6000/7000 Series Methods

\begin{tabular}{|c|c|c|c|c|c|c|c|c|}
\hline Mercury & 0.007 & 0.004 & $\mathrm{mg} / \mathrm{kg}$ & 1 & 03-Sep-2009 & 03-Sep-2009 & EPA 7471A & \\
\hline Antimony & ND & 0.400 & $\mathrm{mg} / \mathrm{kg}$ & 2 & 28-Aug-2009 & 29-Aug-2009 & SW 846/6020 & $\mathrm{U}$ \\
\hline Arsenic & ND & 0.400 & $\mathrm{mg} / \mathrm{kg}$ & 2 & 28-Aug-2009 & 29-Aug-2009 & SW 846/6020 & $\mathrm{U}$ \\
\hline Beryllium & ND & 0.400 & $\mathrm{mg} / \mathrm{kg}$ & 2 & 28-Aug-2009 & 29-Aug-2009 & SW 846/6020 & $\mathrm{U}$ \\
\hline Cadmium & ND & 0.400 & $\mathrm{mg} / \mathrm{kg}$ & 2 & 28-Aug-2009 & 29-Aug-2009 & SW 846/6020 & $\mathrm{U}$ \\
\hline Cobalt & ND & 0.400 & $\mathrm{mg} / \mathrm{kg}$ & 2 & 28-Aug-2009 & 29-Aug-2009 & SW 846/6020 & $\mathrm{U}$ \\
\hline Copper & 0.663 & 0.400 & $\mathrm{mg} / \mathrm{kg}$ & 2 & 28-Aug-2009 & 29-Aug-2009 & SW 846/6020 & \\
\hline Lead & 0.588 & 0.400 & $\mathrm{mg} / \mathrm{kg}$ & 2 & 28-Aug-2009 & 29-Aug-2009 & SW 846/6020 & B \\
\hline Manganese & 0.742 & 0.400 & $\mathrm{mg} / \mathrm{kg}$ & 2 & 28-Aug-2009 & 29-Aug-2009 & SW 846/6020 & \\
\hline Molybdenum & ND & 0.400 & $\mathrm{mg} / \mathrm{kg}$ & 2 & 28-Aug-2009 & 29-Aug-2009 & SW 846/6020 & $\mathrm{U}$ \\
\hline Nickel & 1.20 & 0.400 & $\mathrm{mg} / \mathrm{kg}$ & 2 & 28-Aug-2009 & 29-Aug-2009 & SW 846/6020 & \\
\hline Silver & ND & 0.400 & $\mathrm{mg} / \mathrm{kg}$ & 2 & 28-Aug-2009 & 29-Aug-2009 & SW 846/6020 & $\mathrm{U}$ \\
\hline Thallium & ND & 0.400 & $\mathrm{mg} / \mathrm{kg}$ & 2 & 28-Aug-2009 & 29-Aug-2009 & SW 846/6020 & $\mathrm{U}$ \\
\hline Vanadium & ND & 0.400 & $\mathrm{mg} / \mathrm{kg}$ & 2 & 28-Aug-2009 & 29-Aug-2009 & SW 846/6020 & $\mathrm{U}$ \\
\hline Zinc & 22.5 & 0.400 & $\mathrm{mg} / \mathrm{kg}$ & 2 & 28-Aug-2009 & 29-Aug-2009 & SW 846/6020 & \\
\hline
\end{tabular}




\section{USACE ERDC-EP-C \\ 3909 Halls Ferry Road \\ Vicksburg, MS 39180-6199}

ERDC - ECB

Project: TVA Fly Ash Project

Reported:

Project Manager: Tony Bednar

03-Sep-2009

DRAFT: Fish Tissue Dechlor 4

9082706-04 (Tissue)

\begin{tabular}{|c|c|c|c|c|c|c|c|c|}
\hline Analyte & Result & $\begin{array}{r}\text { porting } \\
\text { Limit }\end{array}$ & Units & Dilution & Prepared & Analyzed & Method & Notes \\
\hline
\end{tabular}

ERDC- EL-EP-C (Environmental Chemistry Branch)

DRAFT: Metals by EPA 6000/7000 Series Methods

\begin{tabular}{|c|c|c|c|c|c|c|c|c|}
\hline Mercury & 0.009 & 0.003 & $\mathrm{mg} / \mathrm{kg}$ & 1 & 03-Sep-2009 & 03-Sep-2009 & EPA 7471A & \\
\hline Antimony & 0.084 & 0.400 & $\mathrm{mg} / \mathrm{kg}$ & 2 & 28-Aug-2009 & 29-Aug-2009 & SW 846/6020 & $\mathrm{J}, \mathrm{B}$ \\
\hline Arsenic & ND & 0.400 & $\mathrm{mg} / \mathrm{kg}$ & 2 & 28-Aug-2009 & 29-Aug-2009 & SW 846/6020 & $\mathrm{U}$ \\
\hline Barium & 1.61 & 0.400 & $\mathrm{mg} / \mathrm{kg}$ & 2 & 28-Aug-2009 & 29-Aug-2009 & SW 846/6020 & \\
\hline Beryllium & ND & 0.400 & $\mathrm{mg} / \mathrm{kg}$ & 2 & 28-Aug-2009 & 29-Aug-2009 & SW 846/6020 & $\mathrm{U}$ \\
\hline Cadmium & ND & 0.400 & $\mathrm{mg} / \mathrm{kg}$ & 2 & 28-Aug-2009 & 29-Aug-2009 & SW 846/6020 & $\mathrm{U}$ \\
\hline Cobalt & ND & 0.400 & $\mathrm{mg} / \mathrm{kg}$ & 2 & 28-Aug-2009 & 29-Aug-2009 & SW 846/6020 & $\mathrm{U}$ \\
\hline Copper & 0.806 & 0.400 & $\mathrm{mg} / \mathrm{kg}$ & 2 & 28-Aug-2009 & 29-Aug-2009 & SW 846/6020 & \\
\hline Lead & 0.590 & 0.400 & $\mathrm{mg} / \mathrm{kg}$ & 2 & 28-Aug-2009 & 29-Aug-2009 & SW 846/6020 & $\mathrm{B}$ \\
\hline Manganese & 1.02 & 0.400 & $\mathrm{mg} / \mathrm{kg}$ & 2 & 28-Aug-2009 & 29-Aug-2009 & SW 846/6020 & \\
\hline Molybdenum & 0.083 & 0.400 & $\mathrm{mg} / \mathrm{kg}$ & 2 & 28-Aug-2009 & 29-Aug-2009 & SW 846/6020 & $\mathrm{J}$ \\
\hline Nickel & 2.29 & 0.400 & $\mathrm{mg} / \mathrm{kg}$ & 2 & 28-Aug-2009 & 29-Aug-2009 & SW 846/6020 & \\
\hline Silver & ND & 0.400 & $\mathrm{mg} / \mathrm{kg}$ & 2 & 28-Aug-2009 & 29-Aug-2009 & SW 846/6020 & $\mathrm{U}$ \\
\hline Thallium & ND & 0.400 & $\mathrm{mg} / \mathrm{kg}$ & 2 & 28-Aug-2009 & 29-Aug-2009 & SW 846/6020 & $\mathrm{U}$ \\
\hline Vanadium & 0.098 & 0.400 & $\mathrm{mg} / \mathrm{kg}$ & 2 & 28-Aug-2009 & 29-Aug-2009 & SW 846/6020 & $\mathrm{J}$ \\
\hline Zinc & 17.8 & 0.400 & $\mathrm{mg} / \mathrm{kg}$ & 2 & 28-Aug-2009 & 29-Aug-2009 & SW 846/6020 & \\
\hline
\end{tabular}




\section{USACE ERDC-EP-C \\ 3909 Halls Ferry Road \\ Vicksburg, MS 39180-6199}

ERDC - ECB

Project: TVA Fly Ash Project

Reported:

Project Manager: Tony Bednar

03-Sep-2009

DRAFT: Fish Tissue Dechlor 5

9082706-05 (Tissue)

\begin{tabular}{|c|c|c|c|c|c|c|c|c|}
\hline Analyte & Result & $\begin{array}{r}\text { porting } \\
\text { Limit }\end{array}$ & Units & Dilution & Prepared & Analyzed & Method & Notes \\
\hline
\end{tabular}

ERDC- EL-EP-C (Environmental Chemistry Branch)

DRAFT: Metals by EPA 6000/7000 Series Methods

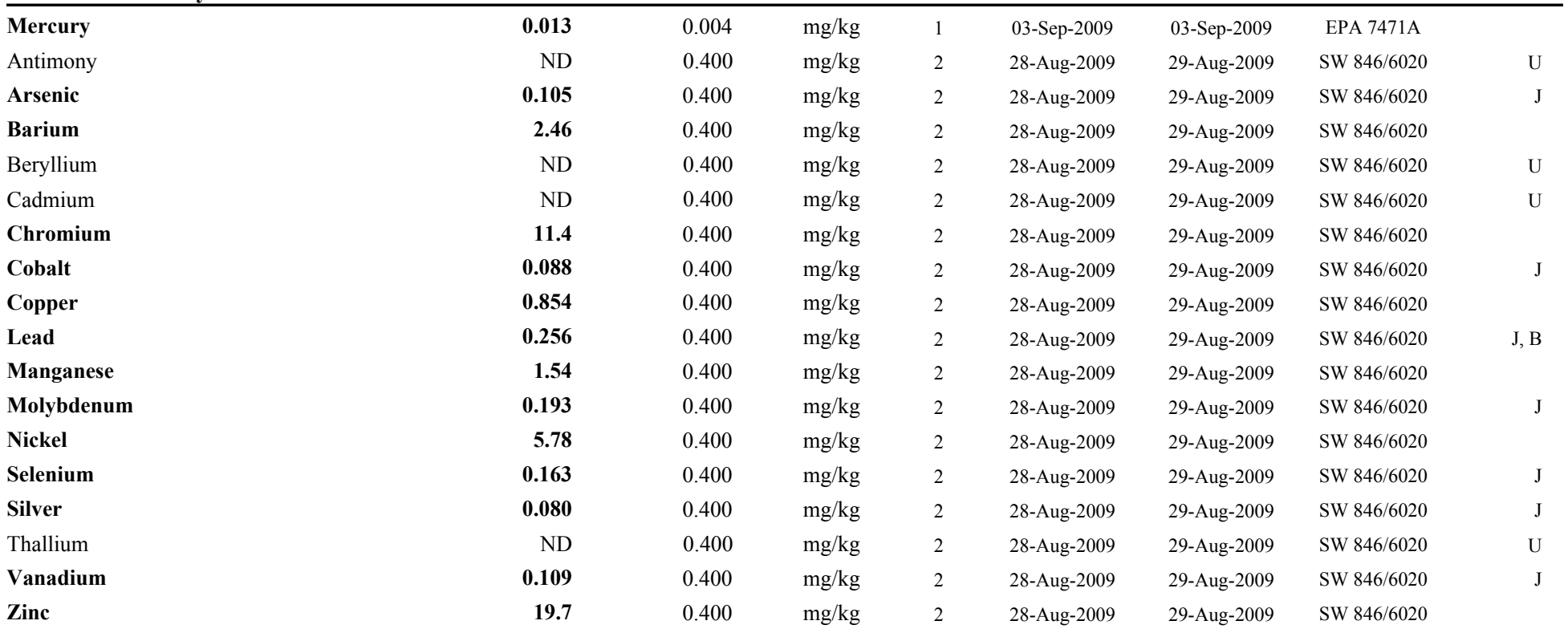




\section{USACE ERDC-EP-C \\ 3909 Halls Ferry Road \\ Vicksburg, MS 39180-6199}

ERDC - ECB

$-,-$
Project: TVA Fly Ash Project

Project Manager: Tony Bednar
Reported:

03-Sep-2009

DRAFT: Fish Tissue EMR - WA 1

9082706-06 (Tissue)

\begin{tabular}{|c|c|c|c|c|c|c|c|c|}
\hline & & porting & & & & & & \\
\hline Analyte & Result & Limit & Units & Dilution & Prepared & Analyzed & Method & Notes \\
\hline
\end{tabular}

ERDC- EL-EP-C (Environmental Chemistry Branch)

DRAFT: Metals by EPA 6000/7000 Series Methods

\begin{tabular}{|c|c|c|c|c|c|c|c|c|}
\hline Mercury & 0.011 & 0.003 & $\mathrm{mg} / \mathrm{kg}$ & 1 & 03-Sep-2009 & 03-Sep-2009 & EPA 7471A & \\
\hline Antimony & 0.426 & 0.400 & $\mathrm{mg} / \mathrm{kg}$ & 2 & 28-Aug-2009 & 29-Aug-2009 & SW 846/6020 & $\mathrm{B}$ \\
\hline Arsenic & 0.184 & 0.400 & $\mathrm{mg} / \mathrm{kg}$ & 2 & 28-Aug-2009 & 29-Aug-2009 & SW 846/6020 & $\mathrm{J}$ \\
\hline Barium & 4.15 & 0.400 & $\mathrm{mg} / \mathrm{kg}$ & 2 & 28-Aug-2009 & 29-Aug-2009 & SW 846/6020 & \\
\hline Beryllium & ND & 0.400 & $\mathrm{mg} / \mathrm{kg}$ & 2 & 28-Aug-2009 & 29-Aug-2009 & SW 846/6020 & $\mathrm{U}$ \\
\hline Cadmium & ND & 0.400 & $\mathrm{mg} / \mathrm{kg}$ & 2 & 28-Aug-2009 & 29-Aug-2009 & SW 846/6020 & $\mathrm{U}$ \\
\hline Cobalt & 0.136 & 0.400 & $\mathrm{mg} / \mathrm{kg}$ & 2 & 28-Aug-2009 & 29-Aug-2009 & SW 846/6020 & $\mathrm{J}$ \\
\hline Copper & 2.13 & 0.400 & $\mathrm{mg} / \mathrm{kg}$ & 2 & 28-Aug-2009 & 29-Aug-2009 & SW 846/6020 & \\
\hline Lead & 0.414 & 0.400 & $\mathrm{mg} / \mathrm{kg}$ & 2 & 28-Aug-2009 & 29-Aug-2009 & SW 846/6020 & B \\
\hline Manganese & 2.00 & 0.400 & $\mathrm{mg} / \mathrm{kg}$ & 2 & 28-Aug-2009 & 29-Aug-2009 & SW 846/6020 & \\
\hline Molybdenum & 0.203 & 0.400 & $\mathrm{mg} / \mathrm{kg}$ & 2 & 28-Aug-2009 & 29-Aug-2009 & SW 846/6020 & $\mathrm{J}$ \\
\hline Nickel & 5.05 & 0.400 & $\mathrm{mg} / \mathrm{kg}$ & 2 & 28-Aug-2009 & 29-Aug-2009 & SW 846/6020 & \\
\hline Selenium & 0.229 & 0.400 & $\mathrm{mg} / \mathrm{kg}$ & 2 & 28-Aug-2009 & 29-Aug-2009 & SW 846/6020 & $\mathrm{J}$ \\
\hline Silver & 0.156 & 0.400 & $\mathrm{mg} / \mathrm{kg}$ & 2 & 28-Aug-2009 & 29-Aug-2009 & SW 846/6020 & $\mathrm{J}$ \\
\hline Thallium & ND & 0.400 & $\mathrm{mg} / \mathrm{kg}$ & 2 & 28-Aug-2009 & 29-Aug-2009 & SW 846/6020 & $\mathrm{U}$ \\
\hline Vanadium & 0.259 & 0.400 & $\mathrm{mg} / \mathrm{kg}$ & 2 & 28-Aug-2009 & 29-Aug-2009 & SW 846/6020 & $\mathrm{J}$ \\
\hline Zinc & 39.0 & 0.400 & $\mathrm{mg} / \mathrm{kg}$ & 2 & 28-Aug-2009 & 29-Aug-2009 & SW 846/6020 & \\
\hline
\end{tabular}




\section{USACE ERDC-EP-C \\ 3909 Halls Ferry Road \\ Vicksburg, MS 39180-6199}

ERDC - ECB

Project: TVA Fly Ash Project

Reported:

Project Manager: Tony Bednar

03-Sep-2009

DRAFT: Fish Tissue EMR - WA 2

9082706-07 (Tissue)

\begin{tabular}{|c|c|c|c|c|c|c|c|c|}
\hline Analyte & Result & $\begin{array}{r}\text { porting } \\
\text { Limit }\end{array}$ & Units & Dilution & Prepared & Analyzed & Method & Notes \\
\hline
\end{tabular}

ERDC- EL-EP-C (Environmental Chemistry Branch)

DRAFT: Metals by EPA 6000/7000 Series Methods

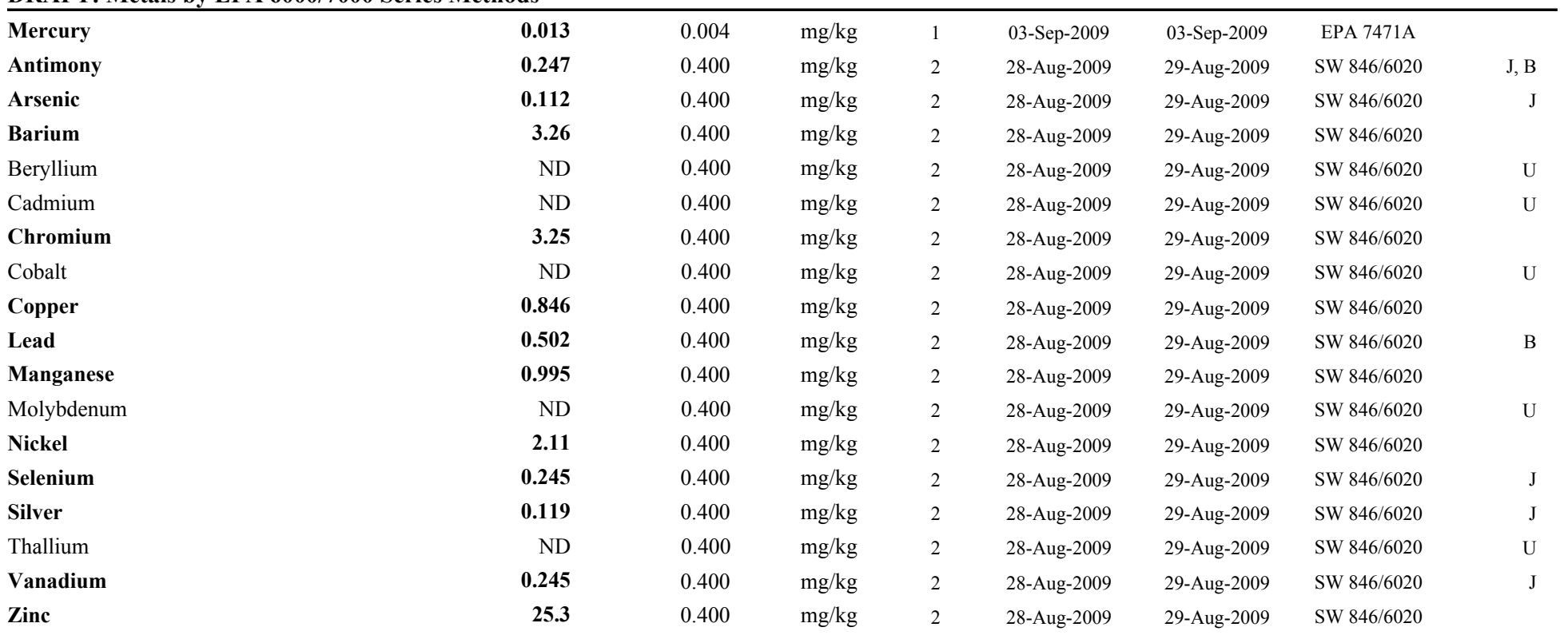




\section{USACE ERDC-EP-C \\ 3909 Halls Ferry Road \\ Vicksburg, MS 39180-6199}

ERDC - ECB

Project: TVA Fly Ash Project

Reported:

Project Manager: Tony Bednar

03-Sep-2009

DRAFT: Fish Tissue EMR - WA 3

9082706-08 (Tissue)

\begin{tabular}{|c|c|c|c|c|c|c|c|c|}
\hline Analyte & Result & $\begin{array}{r}\text { porting } \\
\text { Limit }\end{array}$ & Units & Dilution & Prepared & Analyzed & Method & Notes \\
\hline
\end{tabular}

ERDC- EL-EP-C (Environmental Chemistry Branch)

DRAFT: Metals by EPA 6000/7000 Series Methods

\begin{tabular}{|c|c|c|c|c|c|c|c|c|}
\hline Mercury & 0.010 & 0.004 & $\mathrm{mg} / \mathrm{kg}$ & 1 & 03-Sep-2009 & 03-Sep-2009 & EPA 7471A & \\
\hline Antimony & 0.205 & 0.400 & $\mathrm{mg} / \mathrm{kg}$ & 2 & 28-Aug-2009 & 29-Aug-2009 & SW 846/6020 & $\mathrm{J}, \mathrm{B}$ \\
\hline Arsenic & 0.083 & 0.400 & $\mathrm{mg} / \mathrm{kg}$ & 2 & 28-Aug-2009 & 29-Aug-2009 & SW 846/6020 & $\mathrm{J}$ \\
\hline Barium & 1.96 & 0.400 & $\mathrm{mg} / \mathrm{kg}$ & 2 & 28-Aug-2009 & 29-Aug-2009 & SW 846/6020 & \\
\hline Beryllium & ND & 0.400 & $\mathrm{mg} / \mathrm{kg}$ & 2 & 28-Aug-2009 & 29-Aug-2009 & SW 846/6020 & $\mathrm{U}$ \\
\hline Cadmium & ND & 0.400 & $\mathrm{mg} / \mathrm{kg}$ & 2 & 28-Aug-2009 & 29-Aug-2009 & SW 846/6020 & $\mathrm{U}$ \\
\hline Cobalt & ND & 0.400 & $\mathrm{mg} / \mathrm{kg}$ & 2 & 28-Aug-2009 & 29-Aug-2009 & SW 846/6020 & $\mathrm{U}$ \\
\hline Copper & 0.722 & 0.400 & $\mathrm{mg} / \mathrm{kg}$ & 2 & 28-Aug-2009 & 29-Aug-2009 & SW 846/6020 & \\
\hline Lead & 0.480 & 0.400 & $\mathrm{mg} / \mathrm{kg}$ & 2 & 28-Aug-2009 & 29-Aug-2009 & SW 846/6020 & B \\
\hline Manganese & 1.17 & 0.400 & $\mathrm{mg} / \mathrm{kg}$ & 2 & 28-Aug-2009 & 29-Aug-2009 & SW 846/6020 & \\
\hline Molybdenum & 0.110 & 0.400 & $\mathrm{mg} / \mathrm{kg}$ & 2 & 28-Aug-2009 & 29-Aug-2009 & SW 846/6020 & $\mathrm{J}$ \\
\hline Nickel & 3.66 & 0.400 & $\mathrm{mg} / \mathrm{kg}$ & 2 & 28-Aug-2009 & 29-Aug-2009 & SW 846/6020 & \\
\hline Selenium & 0.108 & 0.400 & $\mathrm{mg} / \mathrm{kg}$ & 2 & 28-Aug-2009 & 29-Aug-2009 & SW 846/6020 & $\mathrm{J}$ \\
\hline Silver & 0.238 & 0.400 & $\mathrm{mg} / \mathrm{kg}$ & 2 & 28-Aug-2009 & 29-Aug-2009 & SW 846/6020 & $\mathrm{J}$ \\
\hline Thallium & ND & 0.400 & $\mathrm{mg} / \mathrm{kg}$ & 2 & 28-Aug-2009 & 29-Aug-2009 & SW 846/6020 & $\mathrm{U}$ \\
\hline Vanadium & 0.096 & 0.400 & $\mathrm{mg} / \mathrm{kg}$ & 2 & 28-Aug-2009 & 29-Aug-2009 & SW 846/6020 & $\mathrm{J}$ \\
\hline Zinc & 16.0 & 0.400 & $\mathrm{mg} / \mathrm{kg}$ & 2 & 28-Aug-2009 & 29-Aug-2009 & SW 846/6020 & \\
\hline
\end{tabular}




\section{USACE ERDC-EP-C \\ 3909 Halls Ferry Road \\ Vicksburg, MS 39180-6199}

ERDC - ECB

Project: TVA Fly Ash Project

Reported:

Project Manager: Tony Bednar

03-Sep-2009

DRAFT: Fish Tissue EMR - WA 4

9082706-09 (Tissue)

\begin{tabular}{|c|c|c|c|c|c|c|c|c|}
\hline Analyte & Result & $\begin{array}{r}\text { porting } \\
\text { Limit }\end{array}$ & Units & Dilution & Prepared & Analyzed & Method & Notes \\
\hline
\end{tabular}

ERDC- EL-EP-C (Environmental Chemistry Branch)

DRAFT: Metals by EPA 6000/7000 Series Methods

\begin{tabular}{|c|c|c|c|c|c|c|c|c|}
\hline Mercury & 0.006 & 0.003 & $\mathrm{mg} / \mathrm{kg}$ & 1 & 03-Sep-2009 & 03-Sep-2009 & EPA 7471A & \\
\hline Antimony & ND & 0.400 & $\mathrm{mg} / \mathrm{kg}$ & 2 & 28-Aug-2009 & 29-Aug-2009 & SW 846/6020 & $\mathrm{U}$ \\
\hline Arsenic & 0.099 & 0.400 & $\mathrm{mg} / \mathrm{kg}$ & 2 & 28-Aug-2009 & 29-Aug-2009 & SW 846/6020 & $\mathrm{J}$ \\
\hline Barium & 4.02 & 0.400 & $\mathrm{mg} / \mathrm{kg}$ & 2 & 28-Aug-2009 & 29-Aug-2009 & SW 846/6020 & \\
\hline Beryllium & ND & 0.400 & $\mathrm{mg} / \mathrm{kg}$ & 2 & 28-Aug-2009 & 29-Aug-2009 & SW 846/6020 & $\mathrm{U}$ \\
\hline Cadmium & ND & 0.400 & $\mathrm{mg} / \mathrm{kg}$ & 2 & 28-Aug-2009 & 29-Aug-2009 & SW 846/6020 & $\mathrm{U}$ \\
\hline Cobalt & 0.097 & 0.400 & $\mathrm{mg} / \mathrm{kg}$ & 2 & 28-Aug-2009 & 29-Aug-2009 & SW 846/6020 & $\mathrm{J}$ \\
\hline Copper & 1.33 & 0.400 & $\mathrm{mg} / \mathrm{kg}$ & 2 & 28-Aug-2009 & 29-Aug-2009 & SW 846/6020 & \\
\hline Lead & 0.392 & 0.400 & $\mathrm{mg} / \mathrm{kg}$ & 2 & 28-Aug-2009 & 29-Aug-2009 & SW 846/6020 & $\mathrm{J}, \mathrm{B}$ \\
\hline Manganese & 1.70 & 0.400 & $\mathrm{mg} / \mathrm{kg}$ & 2 & 28-Aug-2009 & 29-Aug-2009 & SW 846/6020 & \\
\hline Molybdenum & 0.156 & 0.400 & $\mathrm{mg} / \mathrm{kg}$ & 2 & 28-Aug-2009 & 29-Aug-2009 & SW 846/6020 & $\mathrm{J}$ \\
\hline Nickel & 4.78 & 0.400 & $\mathrm{mg} / \mathrm{kg}$ & 2 & 28-Aug-2009 & 29-Aug-2009 & SW 846/6020 & \\
\hline Silver & ND & 0.400 & $\mathrm{mg} / \mathrm{kg}$ & 2 & 28-Aug-2009 & 29-Aug-2009 & SW 846/6020 & U \\
\hline Thallium & ND & 0.400 & $\mathrm{mg} / \mathrm{kg}$ & 2 & 28-Aug-2009 & 29-Aug-2009 & SW 846/6020 & $\mathrm{U}$ \\
\hline Vanadium & 0.123 & 0.400 & $\mathrm{mg} / \mathrm{kg}$ & 2 & 28-Aug-2009 & 29-Aug-2009 & SW 846/6020 & J \\
\hline Zinc & 19.6 & 0.400 & $\mathrm{mg} / \mathrm{kg}$ & 2 & 28-Aug-2009 & 29-Aug-2009 & SW 846/6020 & \\
\hline
\end{tabular}




\section{USACE ERDC-EP-C \\ 3909 Halls Ferry Road \\ Vicksburg, MS 39180-6199}

ERDC - ECB

Project: TVA Fly Ash Project

Reported:

Project Manager: Tony Bednar

03-Sep-2009

DRAFT: Fish Tissue EMR - WA 5

9082706-10 (Tissue)

\begin{tabular}{|c|c|c|c|c|c|c|c|c|}
\hline Analyte & Result & $\begin{array}{r}\text { porting } \\
\text { Limit }\end{array}$ & Units & Dilution & Prepared & Analyzed & Method & Notes \\
\hline
\end{tabular}

ERDC- EL-EP-C (Environmental Chemistry Branch)

DRAFT: Metals by EPA 6000/7000 Series Methods

\begin{tabular}{|c|c|c|c|c|c|c|c|c|}
\hline Mercury & 0.015 & 0.006 & $\mathrm{mg} / \mathrm{kg}$ & 1 & 03-Sep-2009 & 03-Sep-2009 & EPA 7471A & \\
\hline Antimony & ND & 0.400 & $\mathrm{mg} / \mathrm{kg}$ & 2 & 28-Aug-2009 & 29-Aug-2009 & SW 846/6020 & $\mathrm{U}$ \\
\hline Arsenic & 0.119 & 0.400 & $\mathrm{mg} / \mathrm{kg}$ & 2 & 28-Aug-2009 & 29-Aug-2009 & SW $846 / 6020$ & $\mathrm{~J}$ \\
\hline Barium & 3.31 & 0.400 & $\mathrm{mg} / \mathrm{kg}$ & 2 & 28-Aug-2009 & 29-Aug-2009 & SW 846/6020 & \\
\hline Beryllium & ND & 0.400 & $\mathrm{mg} / \mathrm{kg}$ & 2 & 28-Aug-2009 & 29-Aug-2009 & SW 846/6020 & $\mathrm{U}$ \\
\hline Cadmium & ND & 0.400 & $\mathrm{mg} / \mathrm{kg}$ & 2 & 28-Aug-2009 & 29-Aug-2009 & SW 846/6020 & $\mathrm{U}$ \\
\hline Cobalt & 0.092 & 0.400 & $\mathrm{mg} / \mathrm{kg}$ & 2 & 28-Aug-2009 & 29-Aug-2009 & SW 846/6020 & $\mathrm{J}$ \\
\hline Copper & 0.994 & 0.400 & $\mathrm{mg} / \mathrm{kg}$ & 2 & 28-Aug-2009 & 29-Aug-2009 & SW 846/6020 & \\
\hline Lead & 0.414 & 0.400 & $\mathrm{mg} / \mathrm{kg}$ & 2 & 28-Aug-2009 & 29-Aug-2009 & SW 846/6020 & B \\
\hline Manganese & 2.11 & 0.400 & $\mathrm{mg} / \mathrm{kg}$ & 2 & 28-Aug-2009 & 29-Aug-2009 & SW 846/6020 & \\
\hline Molybdenum & 0.194 & 0.400 & $\mathrm{mg} / \mathrm{kg}$ & 2 & 28-Aug-2009 & 29-Aug-2009 & SW 846/6020 & $\mathrm{J}$ \\
\hline Nickel & 6.22 & 0.400 & $\mathrm{mg} / \mathrm{kg}$ & 2 & 28-Aug-2009 & 29-Aug-2009 & SW 846/6020 & \\
\hline Silver & ND & 0.400 & $\mathrm{mg} / \mathrm{kg}$ & 2 & 28-Aug-2009 & 29-Aug-2009 & SW $846 / 6020$ & $\mathrm{U}$ \\
\hline Thallium & ND & 0.400 & $\mathrm{mg} / \mathrm{kg}$ & 2 & 28-Aug-2009 & 29-Aug-2009 & SW $846 / 6020$ & $\mathrm{U}$ \\
\hline Vanadium & 0.160 & 0.400 & $\mathrm{mg} / \mathrm{kg}$ & 2 & 28-Aug-2009 & 29-Aug-2009 & SW 846/6020 & $\mathrm{J}$ \\
\hline Zinc & 26.1 & 0.400 & $\mathrm{mg} / \mathrm{kg}$ & 2 & 28-Aug-2009 & 29-Aug-2009 & SW 846/6020 & \\
\hline
\end{tabular}




\section{USACE ERDC-EP-C \\ 3909 Halls Ferry Road \\ Vicksburg, MS 39180-6199}

ERDC - ECB

$-,-$
Project: TVA Fly Ash Project

Project Manager: Tony Bednar
Reported:

03-Sep-2009

DRAFT: Fish Tissue EMR - EL 1

9082706-11 (Tissue)

\begin{tabular}{|c|c|c|c|c|c|c|c|c|}
\hline & & porting & & & & & & \\
\hline Analyte & Result & Limit & Units & Dilution & Prepared & Analyzed & Method & Notes \\
\hline
\end{tabular}

ERDC- EL-EP-C (Environmental Chemistry Branch)

DRAFT: Metals by EPA 6000/7000 Series Methods

\begin{tabular}{|c|c|c|c|c|c|c|c|c|}
\hline Mercury & 0.013 & 0.005 & $\mathrm{mg} / \mathrm{kg}$ & 1 & 03-Sep-2009 & 03-Sep-2009 & EPA 7471A & \\
\hline Antimony & ND & 0.400 & $\mathrm{mg} / \mathrm{kg}$ & 2 & 28-Aug-2009 & 29-Aug-2009 & SW 846/6020 & $\mathrm{U}$ \\
\hline Arsenic & 0.493 & 0.400 & $\mathrm{mg} / \mathrm{kg}$ & 2 & 28-Aug-2009 & 29-Aug-2009 & SW 846/6020 & \\
\hline Barium & 4.18 & 0.400 & $\mathrm{mg} / \mathrm{kg}$ & 2 & 28-Aug-2009 & 29-Aug-2009 & SW 846/6020 & \\
\hline Beryllium & ND & 0.400 & $\mathrm{mg} / \mathrm{kg}$ & 2 & 28-Aug-2009 & 29-Aug-2009 & SW 846/6020 & $\mathrm{U}$ \\
\hline Cadmium & ND & 0.400 & $\mathrm{mg} / \mathrm{kg}$ & 2 & 28-Aug-2009 & 29-Aug-2009 & SW 846/6020 & $\mathrm{U}$ \\
\hline Cobalt & ND & 0.400 & $\mathrm{mg} / \mathrm{kg}$ & 2 & 28-Aug-2009 & 29-Aug-2009 & SW 846/6020 & $\mathrm{U}$ \\
\hline Copper & 0.992 & 0.400 & $\mathrm{mg} / \mathrm{kg}$ & 2 & 28-Aug-2009 & 29-Aug-2009 & SW $846 / 6020$ & \\
\hline Lead & 0.356 & 0.400 & $\mathrm{mg} / \mathrm{kg}$ & 2 & 28-Aug-2009 & 29-Aug-2009 & SW 846/6020 & $\mathrm{J}, \mathrm{B}$ \\
\hline Manganese & 1.21 & 0.400 & $\mathrm{mg} / \mathrm{kg}$ & 2 & 28-Aug-2009 & 29-Aug-2009 & SW 846/6020 & \\
\hline Molybdenum & ND & 0.400 & $\mathrm{mg} / \mathrm{kg}$ & 2 & 28-Aug-2009 & 29-Aug-2009 & SW $846 / 6020$ & $\mathrm{U}$ \\
\hline Nickel & 1.65 & 0.400 & $\mathrm{mg} / \mathrm{kg}$ & 2 & 28-Aug-2009 & 29-Aug-2009 & SW $846 / 6020$ & \\
\hline Silver & ND & 0.400 & $\mathrm{mg} / \mathrm{kg}$ & 2 & 28-Aug-2009 & 29-Aug-2009 & SW 846/6020 & $\mathrm{U}$ \\
\hline Thallium & ND & 0.400 & $\mathrm{mg} / \mathrm{kg}$ & 2 & 28-Aug-2009 & 29-Aug-2009 & SW 846/6020 & $\mathrm{U}$ \\
\hline Vanadium & 0.669 & 0.400 & $\mathrm{mg} / \mathrm{kg}$ & 2 & 28-Aug-2009 & 29-Aug-2009 & SW 846/6020 & \\
\hline Zinc & 28.6 & 0.400 & $\mathrm{mg} / \mathrm{kg}$ & 2 & 28-Aug-2009 & 29-Aug-2009 & SW 846/6020 & \\
\hline
\end{tabular}




\section{USACE ERDC-EP-C \\ 3909 Halls Ferry Road \\ Vicksburg, MS 39180-6199}

ERDC - ECB

Project: TVA Fly Ash Project

Reported:

Project Manager: Tony Bednar

03-Sep-2009

DRAFT: Fish Tissue EMR - EL 2

9082706-12 (Tissue)

\begin{tabular}{|c|c|c|c|c|c|c|c|c|}
\hline Analyte & Result & $\begin{array}{r}\text { porting } \\
\text { Limit }\end{array}$ & Units & Dilution & Prepared & Analyzed & Method & Notes \\
\hline
\end{tabular}

ERDC- EL-EP-C (Environmental Chemistry Branch)

DRAFT: Metals by EPA 6000/7000 Series Methods

\begin{tabular}{|c|c|c|c|c|c|c|c|c|}
\hline Mercury & 0.013 & 0.005 & $\mathrm{mg} / \mathrm{kg}$ & 1 & 03-Sep-2009 & 03-Sep-2009 & EPA 7471A & \\
\hline Antimony & ND & 0.400 & $\mathrm{mg} / \mathrm{kg}$ & 2 & 28-Aug-2009 & 29-Aug-2009 & SW 846/6020 & $\mathrm{U}$ \\
\hline Arsenic & 0.470 & 0.400 & $\mathrm{mg} / \mathrm{kg}$ & 2 & 28-Aug-2009 & 29-Aug-2009 & SW $846 / 6020$ & \\
\hline Barium & 4.03 & 0.400 & $\mathrm{mg} / \mathrm{kg}$ & 2 & 28-Aug-2009 & 29-Aug-2009 & SW 846/6020 & \\
\hline Beryllium & ND & 0.400 & $\mathrm{mg} / \mathrm{kg}$ & 2 & 28-Aug-2009 & 29-Aug-2009 & SW 846/6020 & $\mathrm{U}$ \\
\hline Cadmium & ND & 0.400 & $\mathrm{mg} / \mathrm{kg}$ & 2 & 28-Aug-2009 & 29-Aug-2009 & SW 846/6020 & $\mathrm{U}$ \\
\hline Cobalt & $\mathrm{ND}$ & 0.400 & $\mathrm{mg} / \mathrm{kg}$ & 2 & 28-Aug-2009 & 29-Aug-2009 & SW 846/6020 & $\mathrm{U}$ \\
\hline Copper & 0.847 & 0.400 & $\mathrm{mg} / \mathrm{kg}$ & 2 & 28-Aug-2009 & 29-Aug-2009 & SW 846/6020 & \\
\hline Lead & 0.305 & 0.400 & $\mathrm{mg} / \mathrm{kg}$ & 2 & 28-Aug-2009 & 29-Aug-2009 & SW 846/6020 & $\mathrm{J}, \mathrm{B}$ \\
\hline Manganese & 1.12 & 0.400 & $\mathrm{mg} / \mathrm{kg}$ & 2 & 28-Aug-2009 & 29-Aug-2009 & SW 846/6020 & \\
\hline Molybdenum & ND & 0.400 & $\mathrm{mg} / \mathrm{kg}$ & 2 & 28-Aug-2009 & 29-Aug-2009 & SW 846/6020 & $\mathrm{U}$ \\
\hline Nickel & 1.72 & 0.400 & $\mathrm{mg} / \mathrm{kg}$ & 2 & 28-Aug-2009 & 29-Aug-2009 & SW $846 / 6020$ & \\
\hline Selenium & 0.335 & 0.400 & $\mathrm{mg} / \mathrm{kg}$ & 2 & 28-Aug-2009 & 29-Aug-2009 & SW 846/6020 & $\mathrm{J}$ \\
\hline Silver & ND & 0.400 & $\mathrm{mg} / \mathrm{kg}$ & 2 & 28-Aug-2009 & 29-Aug-2009 & SW 846/6020 & $\mathrm{U}$ \\
\hline Thallium & ND & 0.400 & $\mathrm{mg} / \mathrm{kg}$ & 2 & 28-Aug-2009 & 29-Aug-2009 & SW $846 / 6020$ & $\mathrm{U}$ \\
\hline Vanadium & 0.386 & 0.400 & $\mathrm{mg} / \mathrm{kg}$ & 2 & 28-Aug-2009 & 29-Aug-2009 & SW $846 / 6020$ & $\mathrm{~J}$ \\
\hline Zinc & 28.8 & 0.400 & $\mathrm{mg} / \mathrm{kg}$ & 2 & 28-Aug-2009 & 29-Aug-2009 & SW 846/6020 & \\
\hline
\end{tabular}




\section{USACE ERDC-EP-C \\ 3909 Halls Ferry Road \\ Vicksburg, MS 39180-6199}

ERDC - ECB

Project: TVA Fly Ash Project

Reported:

Project Manager: Tony Bednar

03-Sep-2009

DRAFT: Fish Tissue EMR - EL 3

9082706-13 (Tissue)

\begin{tabular}{|c|c|c|c|c|c|c|c|c|}
\hline Analyte & Result & $\begin{array}{r}\text { porting } \\
\text { Limit }\end{array}$ & Units & Dilution & Prepared & Analyzed & Method & Notes \\
\hline
\end{tabular}

ERDC- EL-EP-C (Environmental Chemistry Branch)

DRAFT: Metals by EPA 6000/7000 Series Methods

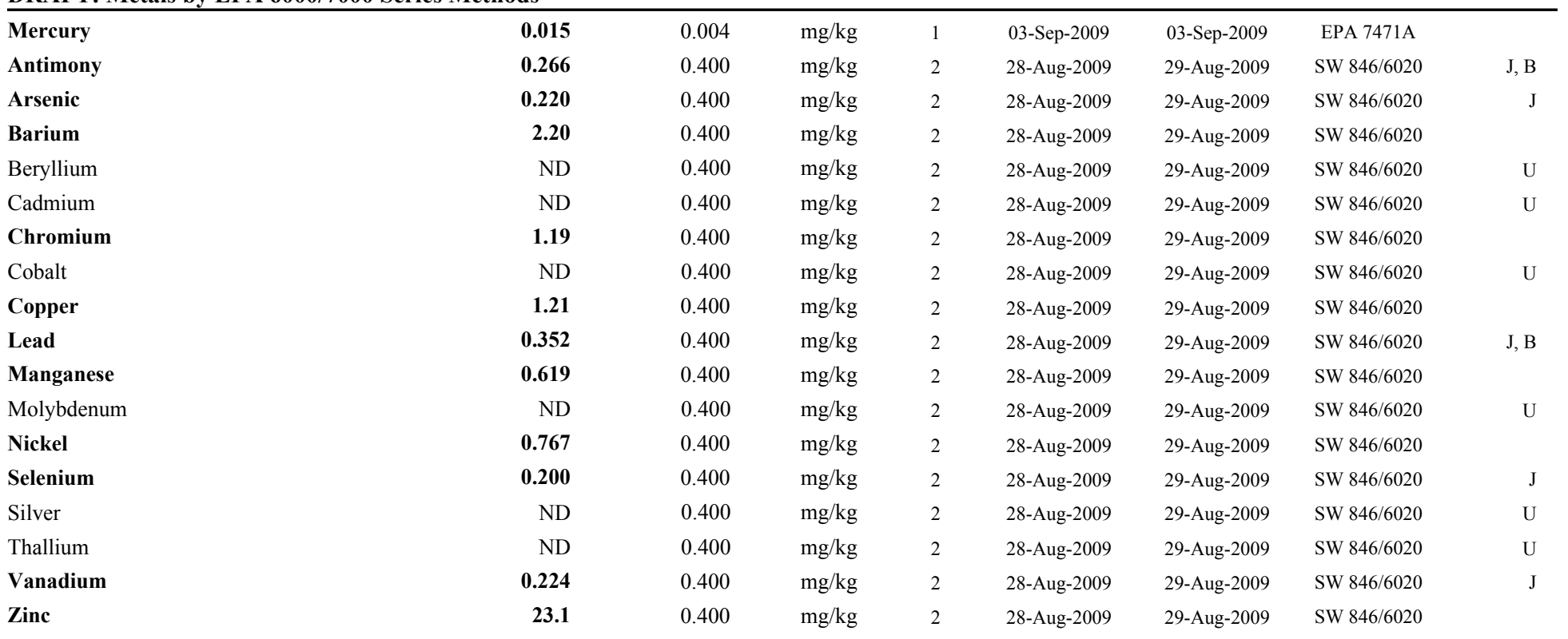




\section{USACE ERDC-EP-C \\ 3909 Halls Ferry Road \\ Vicksburg, MS 39180-6199}

ERDC - ECB

Project: TVA Fly Ash Project

Reported:

Project Manager: Tony Bednar

03-Sep-2009

DRAFT: Fish Tissue EMR - EL 4

9082706-14 (Tissue)

\begin{tabular}{|c|c|c|c|c|c|c|c|c|}
\hline Analyte & Result & $\begin{array}{r}\text { porting } \\
\text { Limit }\end{array}$ & Units & Dilution & Prepared & Analyzed & Method & Notes \\
\hline
\end{tabular}

ERDC- EL-EP-C (Environmental Chemistry Branch)

DRAFT: Metals by EPA 6000/7000 Series Methods

\begin{tabular}{|c|c|c|c|c|c|c|c|c|}
\hline Mercury & 0.009 & 0.004 & $\mathrm{mg} / \mathrm{kg}$ & 1 & 03-Sep-2009 & 03-Sep-2009 & EPA 7471A & \\
\hline Antimony & ND & 0.400 & $\mathrm{mg} / \mathrm{kg}$ & 2 & 28-Aug-2009 & 29-Aug-2009 & SW 846/6020 & $\mathrm{U}$ \\
\hline Arsenic & 0.199 & 0.400 & $\mathrm{mg} / \mathrm{kg}$ & 2 & 28-Aug-2009 & 29-Aug-2009 & SW $846 / 6020$ & $\mathrm{~J}$ \\
\hline Barium & 2.06 & 0.400 & $\mathrm{mg} / \mathrm{kg}$ & 2 & 28-Aug-2009 & 29-Aug-2009 & SW 846/6020 & \\
\hline Beryllium & ND & 0.400 & $\mathrm{mg} / \mathrm{kg}$ & 2 & 28-Aug-2009 & 29-Aug-2009 & SW 846/6020 & $\mathrm{U}$ \\
\hline Cadmium & ND & 0.400 & $\mathrm{mg} / \mathrm{kg}$ & 2 & 28-Aug-2009 & 29-Aug-2009 & SW 846/6020 & $\mathrm{U}$ \\
\hline Cobalt & ND & 0.400 & $\mathrm{mg} / \mathrm{kg}$ & 2 & 28-Aug-2009 & 29-Aug-2009 & SW 846/6020 & $\mathrm{U}$ \\
\hline Copper & 0.787 & 0.400 & $\mathrm{mg} / \mathrm{kg}$ & 2 & 28-Aug-2009 & 29-Aug-2009 & SW 846/6020 & \\
\hline Lead & 0.350 & 0.400 & $\mathrm{mg} / \mathrm{kg}$ & 2 & 28-Aug-2009 & 29-Aug-2009 & SW 846/6020 & $\mathrm{J}, \mathrm{B}$ \\
\hline Manganese & 0.848 & 0.400 & $\mathrm{mg} / \mathrm{kg}$ & 2 & 28-Aug-2009 & 29-Aug-2009 & SW 846/6020 & \\
\hline Molybdenum & ND & 0.400 & $\mathrm{mg} / \mathrm{kg}$ & 2 & 28-Aug-2009 & 29-Aug-2009 & SW 846/6020 & $\mathrm{U}$ \\
\hline Nickel & 2.09 & 0.400 & $\mathrm{mg} / \mathrm{kg}$ & 2 & 28-Aug-2009 & 29-Aug-2009 & SW $846 / 6020$ & \\
\hline Selenium & 0.171 & 0.400 & $\mathrm{mg} / \mathrm{kg}$ & 2 & 28-Aug-2009 & 29-Aug-2009 & SW 846/6020 & $\mathrm{J}$ \\
\hline Silver & ND & 0.400 & $\mathrm{mg} / \mathrm{kg}$ & 2 & 28-Aug-2009 & 29-Aug-2009 & SW 846/6020 & $\mathrm{U}$ \\
\hline Thallium & ND & 0.400 & $\mathrm{mg} / \mathrm{kg}$ & 2 & 28-Aug-2009 & 29-Aug-2009 & SW $846 / 6020$ & $\mathrm{U}$ \\
\hline Vanadium & 0.358 & 0.400 & $\mathrm{mg} / \mathrm{kg}$ & 2 & 28-Aug-2009 & 29-Aug-2009 & SW 846/6020 & $\mathrm{J}$ \\
\hline Zinc & 20.1 & 0.400 & $\mathrm{mg} / \mathrm{kg}$ & 2 & 28-Aug-2009 & 29-Aug-2009 & SW 846/6020 & \\
\hline
\end{tabular}




\section{USACE ERDC-EP-C \\ 3909 Halls Ferry Road \\ Vicksburg, MS 39180-6199}

ERDC - ECB

Project: TVA Fly Ash Project

Reported:

Project Manager: Tony Bednar

03-Sep-2009

DRAFT: Fish Tissue EMR - EL 5

9082706-15 (Tissue)

\begin{tabular}{|c|c|c|c|c|c|c|c|c|}
\hline Analyte & Result & $\begin{array}{r}\text { porting } \\
\text { Limit }\end{array}$ & Units & Dilution & Prepared & Analyzed & Method & Notes \\
\hline
\end{tabular}

ERDC- EL-EP-C (Environmental Chemistry Branch)

DRAFT: Metals by EPA 6000/7000 Series Methods

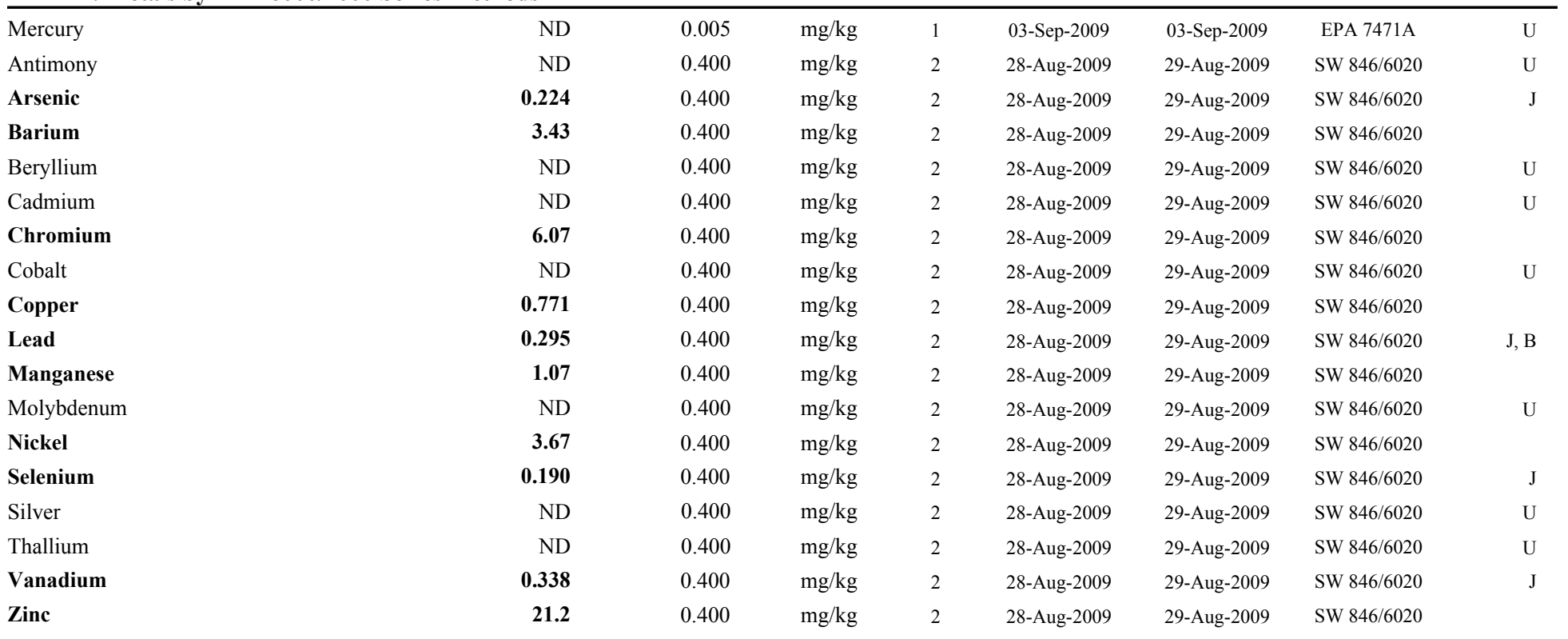




\section{USACE ERDC-EP-C \\ 3909 Halls Ferry Road \\ Vicksburg, MS 39180-6199}

ERDC - ECB

Project: TVA Fly Ash Project

Reported:

Project Manager: Tony Bednar

03-Sep-2009

DRAFT: Fish Gut Dechlor 1

9082706-16 (Tissue)

\begin{tabular}{|c|c|c|c|c|c|c|c|c|}
\hline Analyte & Result & $\begin{array}{r}\text { porting } \\
\text { Limit }\end{array}$ & Units & Dilution & Prepared & Analyzed & Method & Notes \\
\hline
\end{tabular}

ERDC- EL-EP-C (Environmental Chemistry Branch)

DRAFT: Metals by EPA 6000/7000 Series Methods

\begin{tabular}{|c|c|c|c|c|c|c|c|c|}
\hline Antimony & ND & 40.0 & $\mathrm{mg} / \mathrm{kg}$ & 2 & 28-Aug-2009 & 29-Aug-2009 & SW 846/6020 & $\mathrm{U}$ \\
\hline Arsenic & ND & 40.0 & $\mathrm{mg} / \mathrm{kg}$ & 2 & 28-Aug-2009 & 29-Aug-2009 & SW 846/6020 & $\mathrm{U}$ \\
\hline Barium & 1060 & 40.0 & $\mathrm{mg} / \mathrm{kg}$ & 2 & 28-Aug-2009 & 29-Aug-2009 & SW 846/6020 & \\
\hline Beryllium & ND & 40.0 & $\mathrm{mg} / \mathrm{kg}$ & 2 & 28-Aug-2009 & 29-Aug-2009 & SW 846/6020 & $\mathrm{U}$ \\
\hline Cadmium & ND & 40.0 & $\mathrm{mg} / \mathrm{kg}$ & 2 & 28-Aug-2009 & 29-Aug-2009 & SW 846/6020 & $\mathrm{U}$ \\
\hline Chromium & 1020 & 40.0 & $\mathrm{mg} / \mathrm{kg}$ & 2 & 28-Aug-2009 & 29-Aug-2009 & SW 846/6020 & \\
\hline Copper & 460 & 40.0 & $\mathrm{mg} / \mathrm{kg}$ & 2 & 28-Aug-2009 & 29-Aug-2009 & SW 846/6020 & \\
\hline Lead & 169 & 40.0 & $\mathrm{mg} / \mathrm{kg}$ & 2 & 28-Aug-2009 & 29-Aug-2009 & SW 846/6020 & B \\
\hline Manganese & 3090 & 40.0 & $\mathrm{mg} / \mathrm{kg}$ & 2 & 28-Aug-2009 & 29-Aug-2009 & SW 846/6020 & \\
\hline Molybdenum & ND & 40.0 & $\mathrm{mg} / \mathrm{kg}$ & 2 & 28-Aug-2009 & 29-Aug-2009 & SW 846/6020 & $\mathrm{U}$ \\
\hline Nickel & 66.3 & 40.0 & $\mathrm{mg} / \mathrm{kg}$ & 2 & 28-Aug-2009 & 29-Aug-2009 & SW 846/6020 & \\
\hline Selenium & 13.3 & 40.0 & $\mathrm{mg} / \mathrm{kg}$ & 2 & 28-Aug-2009 & 29-Aug-2009 & SW 846/6020 & $\mathrm{J}$ \\
\hline Thallium & ND & 40.0 & $\mathrm{mg} / \mathrm{kg}$ & 2 & 28-Aug-2009 & 29-Aug-2009 & SW 846/6020 & $\mathrm{U}$ \\
\hline Vanadium & 28.0 & 40.0 & $\mathrm{mg} / \mathrm{kg}$ & 2 & 28-Aug-2009 & 29-Aug-2009 & SW 846/6020 & $\mathrm{J}$ \\
\hline Zinc & 2370 & 40.0 & $\mathrm{mg} / \mathrm{kg}$ & 2 & 28-Aug-2009 & 29-Aug-2009 & SW 846/6020 & \\
\hline
\end{tabular}




\section{USACE ERDC-EP-C \\ 3909 Halls Ferry Road \\ Vicksburg, MS 39180-6199}

ERDC - ECB

Project: TVA Fly Ash Project

Reported:

Project Manager: Tony Bednar

03-Sep-2009

DRAFT: Fish Gut Dechlor 2

9082706-17 (Tissue)

\begin{tabular}{|c|c|c|c|c|c|c|c|c|}
\hline Analyte & Result & $\begin{array}{r}\text { porting } \\
\text { Limit }\end{array}$ & Units & Dilution & Prepared & Analyzed & Method & Notes \\
\hline
\end{tabular}

ERDC- EL-EP-C (Environmental Chemistry Branch)

DRAFT: Metals by EPA 6000/7000 Series Methods

\begin{tabular}{|c|c|c|c|c|c|c|c|c|}
\hline Antimony & 27.3 & 40.0 & $\mathrm{mg} / \mathrm{kg}$ & 2 & 28-Aug-2009 & 29-Aug-2009 & SW 846/6020 & $\mathrm{J}, \mathrm{B}$ \\
\hline Arsenic & ND & 40.0 & $\mathrm{mg} / \mathrm{kg}$ & 2 & 28-Aug-2009 & 29-Aug-2009 & SW 846/6020 & $\mathrm{U}$ \\
\hline Barium & 299 & 40.0 & $\mathrm{mg} / \mathrm{kg}$ & 2 & 28-Aug-2009 & 29-Aug-2009 & SW 846/6020 & \\
\hline Beryllium & ND & 40.0 & $\mathrm{mg} / \mathrm{kg}$ & 2 & 28-Aug-2009 & 29-Aug-2009 & SW 846/6020 & $\mathrm{U}$ \\
\hline Cadmium & ND & 40.0 & $\mathrm{mg} / \mathrm{kg}$ & 2 & 28-Aug-2009 & 29-Aug-2009 & SW 846/6020 & $\mathrm{U}$ \\
\hline Chromium & 224 & 40.0 & $\mathrm{mg} / \mathrm{kg}$ & 2 & 28-Aug-2009 & 29-Aug-2009 & SW 846/6020 & \\
\hline Copper & 167 & 40.0 & $\mathrm{mg} / \mathrm{kg}$ & 2 & 28-Aug-2009 & 29-Aug-2009 & SW 846/6020 & \\
\hline Lead & 172 & 40.0 & $\mathrm{mg} / \mathrm{kg}$ & 2 & 28-Aug-2009 & 29-Aug-2009 & SW 846/6020 & B \\
\hline Manganese & 727 & 40.0 & $\mathrm{mg} / \mathrm{kg}$ & 2 & 28-Aug-2009 & 29-Aug-2009 & SW 846/6020 & \\
\hline Molybdenum & ND & 40.0 & $\mathrm{mg} / \mathrm{kg}$ & 2 & 28-Aug-2009 & 29-Aug-2009 & SW 846/6020 & $\mathrm{U}$ \\
\hline Nickel & 66.1 & 40.0 & $\mathrm{mg} / \mathrm{kg}$ & 2 & 28-Aug-2009 & 29-Aug-2009 & SW 846/6020 & \\
\hline Selenium & ND & 40.0 & $\mathrm{mg} / \mathrm{kg}$ & 2 & 28-Aug-2009 & 29-Aug-2009 & SW 846/6020 & U \\
\hline Thallium & ND & 40.0 & $\mathrm{mg} / \mathrm{kg}$ & 2 & 28-Aug-2009 & 29-Aug-2009 & SW 846/6020 & $\mathrm{U}$ \\
\hline Vanadium & 9.40 & 40.0 & $\mathrm{mg} / \mathrm{kg}$ & 2 & 28-Aug-2009 & 29-Aug-2009 & SW 846/6020 & $\mathrm{J}$ \\
\hline Zinc & 1080 & 40.0 & $\mathrm{mg} / \mathrm{kg}$ & 2 & 28-Aug-2009 & 29-Aug-2009 & SW $846 / 6020$ & \\
\hline
\end{tabular}




\section{USACE ERDC-EP-C \\ 3909 Halls Ferry Road \\ Vicksburg, MS 39180-6199}

ERDC - ECB

Project: TVA Fly Ash Project

Reported:

Project Manager: Tony Bednar

03-Sep-2009

DRAFT: Fish Gut Dechlor 3

9082706-18 (Tissue)

\begin{tabular}{|c|c|c|c|c|c|c|c|c|}
\hline Analyte & Result & $\begin{array}{r}\text { porting } \\
\text { Limit }\end{array}$ & Units & Dilution & Prepared & Analyzed & Method & Notes \\
\hline
\end{tabular}

ERDC- EL-EP-C (Environmental Chemistry Branch)

DRAFT: Metals by EPA 6000/7000 Series Methods

\begin{tabular}{|c|c|c|c|c|c|c|c|c|}
\hline Antimony & ND & 40.0 & $\mathrm{mg} / \mathrm{kg}$ & 2 & 28-Aug-2009 & 29-Aug-2009 & SW 846/6020 & $\mathrm{U}$ \\
\hline Arsenic & ND & 40.0 & $\mathrm{mg} / \mathrm{kg}$ & 2 & 28-Aug-2009 & 29-Aug-2009 & SW 846/6020 & $\mathrm{U}$ \\
\hline Barium & 210 & 40.0 & $\mathrm{mg} / \mathrm{kg}$ & 2 & 28-Aug-2009 & 29-Aug-2009 & SW 846/6020 & \\
\hline Beryllium & ND & 40.0 & $\mathrm{mg} / \mathrm{kg}$ & 2 & 28-Aug-2009 & 29-Aug-2009 & SW 846/6020 & $\mathrm{U}$ \\
\hline Cadmium & ND & 40.0 & $\mathrm{mg} / \mathrm{kg}$ & 2 & 28-Aug-2009 & 29-Aug-2009 & SW 846/6020 & $\mathrm{U}$ \\
\hline Chromium & 184 & 40.0 & $\mathrm{mg} / \mathrm{kg}$ & 2 & 28-Aug-2009 & 29-Aug-2009 & SW 846/6020 & \\
\hline Copper & 107 & 40.0 & $\mathrm{mg} / \mathrm{kg}$ & 2 & 28-Aug-2009 & 29-Aug-2009 & SW 846/6020 & \\
\hline Lead & 77.5 & 40.0 & $\mathrm{mg} / \mathrm{kg}$ & 2 & 28-Aug-2009 & 29-Aug-2009 & SW 846/6020 & B \\
\hline Manganese & 586 & 40.0 & $\mathrm{mg} / \mathrm{kg}$ & 2 & 28-Aug-2009 & 29-Aug-2009 & SW 846/6020 & \\
\hline Molybdenum & ND & 40.0 & $\mathrm{mg} / \mathrm{kg}$ & 2 & 28-Aug-2009 & 29-Aug-2009 & SW 846/6020 & $\mathrm{U}$ \\
\hline Nickel & 25.1 & 40.0 & $\mathrm{mg} / \mathrm{kg}$ & 2 & 28-Aug-2009 & 29-Aug-2009 & SW 846/6020 & $\mathrm{J}$ \\
\hline Selenium & ND & 40.0 & $\mathrm{mg} / \mathrm{kg}$ & 2 & 28-Aug-2009 & 29-Aug-2009 & SW 846/6020 & $\mathrm{U}$ \\
\hline Silver & ND & 40.0 & $\mathrm{mg} / \mathrm{kg}$ & 2 & 28-Aug-2009 & 29-Aug-2009 & SW 846/6020 & $\mathrm{U}$ \\
\hline Thallium & ND & 40.0 & $\mathrm{mg} / \mathrm{kg}$ & 2 & 28-Aug-2009 & 29-Aug-2009 & SW 846/6020 & $\mathrm{U}$ \\
\hline Vanadium & ND & 40.0 & $\mathrm{mg} / \mathrm{kg}$ & 2 & 28-Aug-2009 & 29-Aug-2009 & SW 846/6020 & $\mathrm{U}$ \\
\hline Zinc & 700 & 40.0 & $\mathrm{mg} / \mathrm{kg}$ & 2 & 28-Aug-2009 & 29-Aug-2009 & SW 846/6020 & \\
\hline
\end{tabular}




\section{USACE ERDC-EP-C \\ 3909 Halls Ferry Road \\ Vicksburg, MS 39180-6199}

ERDC - ECB

Project: TVA Fly Ash Project

Reported:

Project Manager: Tony Bednar

03-Sep-2009

DRAFT: Fish Gut Dechlor 4

9082706-19 (Tissue)

\begin{tabular}{|c|c|c|c|c|c|c|c|c|}
\hline Analyte & Result & $\begin{array}{r}\text { porting } \\
\text { Limit }\end{array}$ & Units & Dilution & Prepared & Analyzed & Method & Notes \\
\hline
\end{tabular}

ERDC- EL-EP-C (Environmental Chemistry Branch)

DRAFT: Metals by EPA 6000/7000 Series Methods

\begin{tabular}{|c|c|c|c|c|c|c|c|c|}
\hline Antimony & ND & 40.0 & $\mathrm{mg} / \mathrm{kg}$ & 2 & 28-Aug-2009 & 29-Aug-2009 & SW 846/6020 & $\mathrm{U}$ \\
\hline Arsenic & ND & 40.0 & $\mathrm{mg} / \mathrm{kg}$ & 2 & 28-Aug-2009 & 29-Aug-2009 & SW 846/6020 & U \\
\hline Barium & 358 & 40.0 & $\mathrm{mg} / \mathrm{kg}$ & 2 & 28-Aug-2009 & 29-Aug-2009 & SW 846/6020 & \\
\hline Beryllium & ND & 40.0 & $\mathrm{mg} / \mathrm{kg}$ & 2 & 28-Aug-2009 & 29-Aug-2009 & SW 846/6020 & $\mathrm{U}$ \\
\hline Cadmium & ND & 40.0 & $\mathrm{mg} / \mathrm{kg}$ & 2 & 28-Aug-2009 & 29-Aug-2009 & SW 846/6020 & $\mathrm{U}$ \\
\hline Chromium & 272 & 40.0 & $\mathrm{mg} / \mathrm{kg}$ & 2 & 28-Aug-2009 & 29-Aug-2009 & SW 846/6020 & \\
\hline Copper & 566 & 40.0 & $\mathrm{mg} / \mathrm{kg}$ & 2 & 28-Aug-2009 & 29-Aug-2009 & SW 846/6020 & \\
\hline Lead & 96.0 & 40.0 & $\mathrm{mg} / \mathrm{kg}$ & 2 & 28-Aug-2009 & 29-Aug-2009 & SW 846/6020 & B \\
\hline Manganese & 987 & 40.0 & $\mathrm{mg} / \mathrm{kg}$ & 2 & 28-Aug-2009 & 29-Aug-2009 & SW 846/6020 & \\
\hline Molybdenum & ND & 40.0 & $\mathrm{mg} / \mathrm{kg}$ & 2 & 28-Aug-2009 & 29-Aug-2009 & SW 846/6020 & $\mathrm{U}$ \\
\hline Nickel & 53.6 & 40.0 & $\mathrm{mg} / \mathrm{kg}$ & 2 & 28-Aug-2009 & 29-Aug-2009 & SW 846/6020 & \\
\hline Selenium & ND & 40.0 & $\mathrm{mg} / \mathrm{kg}$ & 2 & 28-Aug-2009 & 29-Aug-2009 & SW 846/6020 & $\mathrm{U}$ \\
\hline Silver & ND & 40.0 & $\mathrm{mg} / \mathrm{kg}$ & 2 & 28-Aug-2009 & 29-Aug-2009 & SW 846/6020 & $\mathrm{U}$ \\
\hline Thallium & ND & 40.0 & $\mathrm{mg} / \mathrm{kg}$ & 2 & 28-Aug-2009 & 29-Aug-2009 & SW 846/6020 & $\mathrm{U}$ \\
\hline Vanadium & 14.2 & 40.0 & $\mathrm{mg} / \mathrm{kg}$ & 2 & 28-Aug-2009 & 29-Aug-2009 & SW 846/6020 & $\mathrm{J}$ \\
\hline Zinc & 1170 & 40.0 & $\mathrm{mg} / \mathrm{kg}$ & 2 & 28-Aug-2009 & 29-Aug-2009 & SW 846/6020 & \\
\hline
\end{tabular}




\section{USACE ERDC-EP-C \\ 3909 Halls Ferry Road \\ Vicksburg, MS 39180-6199}

ERDC - ECB

Project: TVA Fly Ash Project

Reported:

Project Manager: Tony Bednar

03-Sep-2009

DRAFT: Fish Gut Dechlor 5

9082706-20 (Tissue)

\begin{tabular}{|c|c|c|c|c|c|c|c|c|}
\hline Analyte & Result & $\begin{array}{r}\text { porting } \\
\text { Limit }\end{array}$ & Units & Dilution & Prepared & Analyzed & Method & Notes \\
\hline
\end{tabular}

ERDC- EL-EP-C (Environmental Chemistry Branch)

DRAFT: Metals by EPA 6000/7000 Series Methods

\begin{tabular}{|c|c|c|c|c|c|c|c|c|}
\hline Antimony & ND & 40.0 & $\mathrm{mg} / \mathrm{kg}$ & 2 & 28-Aug-2009 & 29-Aug-2009 & SW 846/6020 & $\mathrm{U}$ \\
\hline Arsenic & ND & 40.0 & $\mathrm{mg} / \mathrm{kg}$ & 2 & 28-Aug-2009 & 29-Aug-2009 & SW 846/6020 & $\mathrm{U}$ \\
\hline Barium & 110 & 40.0 & $\mathrm{mg} / \mathrm{kg}$ & 2 & 28-Aug-2009 & 29-Aug-2009 & SW 846/6020 & \\
\hline Beryllium & ND & 40.0 & $\mathrm{mg} / \mathrm{kg}$ & 2 & 28-Aug-2009 & 29-Aug-2009 & SW 846/6020 & $\mathrm{U}$ \\
\hline Cadmium & ND & 40.0 & $\mathrm{mg} / \mathrm{kg}$ & 2 & 28-Aug-2009 & 29-Aug-2009 & SW 846/6020 & $\mathrm{U}$ \\
\hline Chromium & 195 & 40.0 & $\mathrm{mg} / \mathrm{kg}$ & 2 & 28-Aug-2009 & 29-Aug-2009 & SW 846/6020 & \\
\hline Copper & 99.3 & 40.0 & $\mathrm{mg} / \mathrm{kg}$ & 2 & 28-Aug-2009 & 29-Aug-2009 & SW 846/6020 & \\
\hline Lead & 86.2 & 40.0 & $\mathrm{mg} / \mathrm{kg}$ & 2 & 28-Aug-2009 & 29-Aug-2009 & SW 846/6020 & B \\
\hline Manganese & 199 & 40.0 & $\mathrm{mg} / \mathrm{kg}$ & 2 & 28-Aug-2009 & 29-Aug-2009 & SW 846/6020 & \\
\hline Molybdenum & ND & 40.0 & $\mathrm{mg} / \mathrm{kg}$ & 2 & 28-Aug-2009 & 29-Aug-2009 & SW 846/6020 & $\mathrm{U}$ \\
\hline Nickel & 57.0 & 40.0 & $\mathrm{mg} / \mathrm{kg}$ & 2 & 28-Aug-2009 & 29-Aug-2009 & SW 846/6020 & \\
\hline Selenium & ND & 40.0 & $\mathrm{mg} / \mathrm{kg}$ & 2 & 28-Aug-2009 & 29-Aug-2009 & SW 846/6020 & $\mathrm{U}$ \\
\hline Thallium & $\mathrm{ND}$ & 40.0 & $\mathrm{mg} / \mathrm{kg}$ & 2 & 28-Aug-2009 & 29-Aug-2009 & SW 846/6020 & $\mathrm{U}$ \\
\hline Vanadium & ND & 40.0 & $\mathrm{mg} / \mathrm{kg}$ & 2 & 28-Aug-2009 & 29-Aug-2009 & SW 846/6020 & $\mathrm{U}$ \\
\hline Zinc & 551 & 40.0 & $\mathrm{mg} / \mathrm{kg}$ & 2 & 28-Aug-2009 & 29-Aug-2009 & SW 846/6020 & \\
\hline
\end{tabular}




\section{USACE ERDC-EP-C \\ 3909 Halls Ferry Road \\ Vicksburg, MS 39180-6199}

ERDC - ECB

Project: TVA Fly Ash Project

Reported:

Project Manager: Tony Bednar

03-Sep-2009

DRAFT: Fish Gut EMR - WA 1

9082706-21 (Tissue)

\begin{tabular}{|c|c|c|c|c|c|c|c|c|}
\hline & & porting & & & & & & \\
\hline Analyte & Result & Limit & Units & Dilution & Prepared & Analyzed & Method & Notes \\
\hline
\end{tabular}

ERDC- EL-EP-C (Environmental Chemistry Branch)

DRAFT: Metals by EPA 6000/7000 Series Methods

\begin{tabular}{|c|c|c|c|c|c|c|c|c|}
\hline Antimony & ND & 40.0 & $\mathrm{mg} / \mathrm{kg}$ & 2 & 28-Aug-2009 & 29-Aug-2009 & SW 846/6020 & $\mathrm{U}$ \\
\hline Arsenic & ND & 40.0 & $\mathrm{mg} / \mathrm{kg}$ & 2 & 28-Aug-2009 & 29-Aug-2009 & SW 846/6020 & $\mathrm{U}$ \\
\hline Barium & 348 & 40.0 & $\mathrm{mg} / \mathrm{kg}$ & 2 & 28-Aug-2009 & 29-Aug-2009 & SW 846/6020 & \\
\hline Beryllium & ND & 40.0 & $\mathrm{mg} / \mathrm{kg}$ & 2 & 28-Aug-2009 & 29-Aug-2009 & SW 846/6020 & $\mathrm{U}$ \\
\hline Cadmium & ND & 40.0 & $\mathrm{mg} / \mathrm{kg}$ & 2 & 28-Aug-2009 & 29-Aug-2009 & SW 846/6020 & $\mathrm{U}$ \\
\hline Chromium & 94.1 & 40.0 & $\mathrm{mg} / \mathrm{kg}$ & 2 & 28-Aug-2009 & 29-Aug-2009 & SW 846/6020 & \\
\hline Copper & 135 & 40.0 & $\mathrm{mg} / \mathrm{kg}$ & 2 & 28-Aug-2009 & 29-Aug-2009 & SW 846/6020 & \\
\hline Lead & 90.7 & 40.0 & $\mathrm{mg} / \mathrm{kg}$ & 2 & 28-Aug-2009 & 29-Aug-2009 & SW 846/6020 & B \\
\hline Manganese & 488 & 40.0 & $\mathrm{mg} / \mathrm{kg}$ & 2 & 28-Aug-2009 & 29-Aug-2009 & SW 846/6020 & \\
\hline Molybdenum & ND & 40.0 & $\mathrm{mg} / \mathrm{kg}$ & 2 & 28-Aug-2009 & 29-Aug-2009 & SW 846/6020 & $\mathrm{U}$ \\
\hline Nickel & 31.3 & 40.0 & $\mathrm{mg} / \mathrm{kg}$ & 2 & 28-Aug-2009 & 29-Aug-2009 & SW 846/6020 & $\mathrm{J}$ \\
\hline Selenium & ND & 40.0 & $\mathrm{mg} / \mathrm{kg}$ & 2 & 28-Aug-2009 & 29-Aug-2009 & SW 846/6020 & $\mathrm{U}$ \\
\hline Thallium & ND & 40.0 & $\mathrm{mg} / \mathrm{kg}$ & 2 & 28-Aug-2009 & 29-Aug-2009 & SW 846/6020 & $\mathrm{U}$ \\
\hline Vanadium & 12.1 & 40.0 & $\mathrm{mg} / \mathrm{kg}$ & 2 & 28-Aug-2009 & 29-Aug-2009 & SW 846/6020 & $\mathrm{J}$ \\
\hline Zinc & 1490 & 40.0 & $\mathrm{mg} / \mathrm{kg}$ & 2 & 28-Aug-2009 & 29-Aug-2009 & SW 846/6020 & \\
\hline
\end{tabular}




\section{USACE ERDC-EP-C \\ 3909 Halls Ferry Road \\ Vicksburg, MS 39180-6199}

ERDC - ECB

Project: TVA Fly Ash Project

Reported:

Project Manager: Tony Bednar

03-Sep-2009

DRAFT: Fish Gut EMR - WA 2

9082706-22 (Tissue)

\begin{tabular}{|c|c|c|c|c|c|c|c|c|}
\hline Analyte & Result & $\begin{array}{r}\text { porting } \\
\text { Limit }\end{array}$ & Units & Dilution & Prepared & Analyzed & Method & Notes \\
\hline
\end{tabular}

ERDC- EL-EP-C (Environmental Chemistry Branch)

DRAFT: Metals by EPA 6000/7000 Series Methods

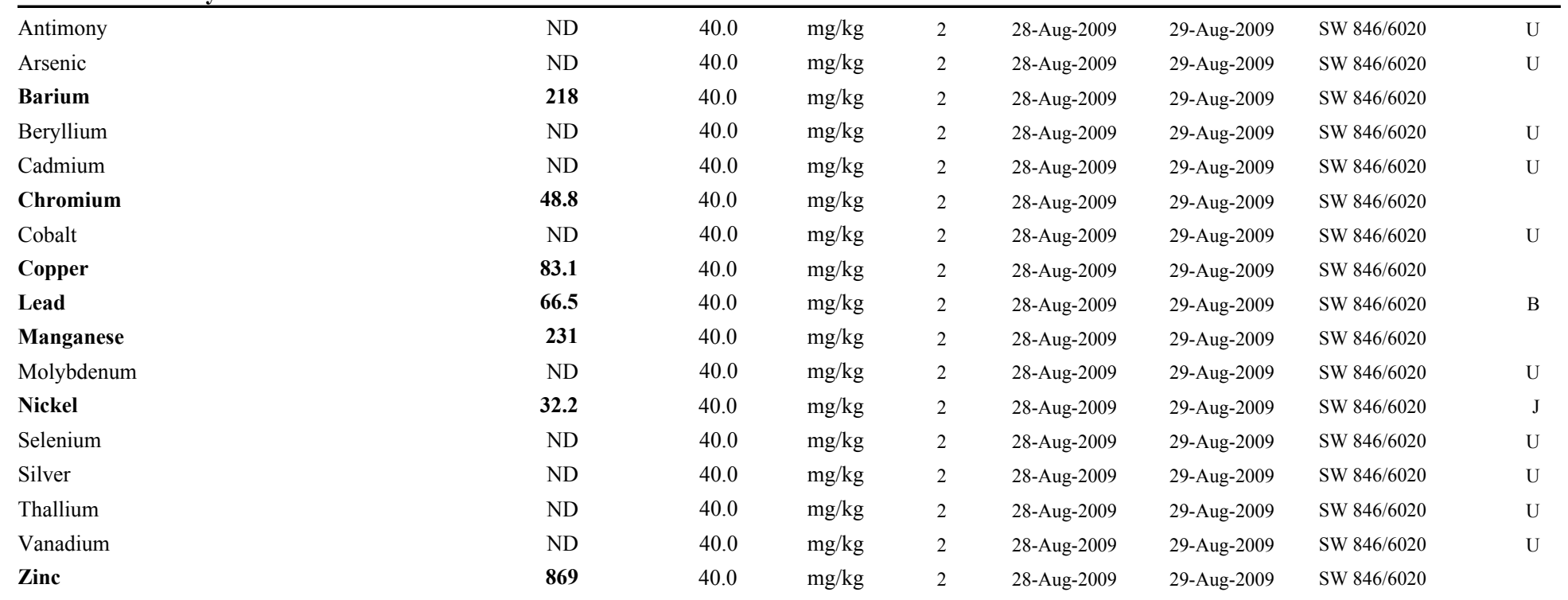




\section{USACE ERDC-EP-C \\ 3909 Halls Ferry Road \\ Vicksburg, MS 39180-6199}

ERDC - ECB

Project: TVA Fly Ash Project

Reported:

Project Manager: Tony Bednar

03-Sep-2009

DRAFT: Fish Gut EMR - WA 3

9082706-23 (Tissue)

\begin{tabular}{|c|c|c|c|c|c|c|c|c|}
\hline Analyte & Result & $\begin{array}{r}\text { porting } \\
\text { Limit }\end{array}$ & Units & Dilution & Prepared & Analyzed & Method & Notes \\
\hline
\end{tabular}

ERDC- EL-EP-C (Environmental Chemistry Branch)

DRAFT: Metals by EPA 6000/7000 Series Methods

\begin{tabular}{|c|c|c|c|c|c|c|c|c|}
\hline Antimony & ND & 40.0 & $\mathrm{mg} / \mathrm{kg}$ & 2 & 28-Aug-2009 & 29-Aug-2009 & SW 846/6020 & $\mathrm{U}$ \\
\hline Arsenic & ND & 40.0 & $\mathrm{mg} / \mathrm{kg}$ & 2 & 28-Aug-2009 & 29-Aug-2009 & SW 846/6020 & $\mathrm{U}$ \\
\hline Barium & 481 & 40.0 & $\mathrm{mg} / \mathrm{kg}$ & 2 & 28-Aug-2009 & 29-Aug-2009 & SW 846/6020 & \\
\hline Beryllium & ND & 40.0 & $\mathrm{mg} / \mathrm{kg}$ & 2 & 28-Aug-2009 & 29-Aug-2009 & SW 846/6020 & $\mathrm{U}$ \\
\hline Cadmium & ND & 40.0 & $\mathrm{mg} / \mathrm{kg}$ & 2 & 28-Aug-2009 & 29-Aug-2009 & SW 846/6020 & $\mathrm{U}$ \\
\hline Chromium & 189 & 40.0 & $\mathrm{mg} / \mathrm{kg}$ & 2 & 28-Aug-2009 & 29-Aug-2009 & SW 846/6020 & \\
\hline Copper & 181 & 40.0 & $\mathrm{mg} / \mathrm{kg}$ & 2 & 28-Aug-2009 & 29-Aug-2009 & SW 846/6020 & \\
\hline Lead & 123 & 40.0 & $\mathrm{mg} / \mathrm{kg}$ & 2 & 28-Aug-2009 & 29-Aug-2009 & SW 846/6020 & B \\
\hline Manganese & 786 & 40.0 & $\mathrm{mg} / \mathrm{kg}$ & 2 & 28-Aug-2009 & 29-Aug-2009 & SW 846/6020 & \\
\hline Molybdenum & ND & 40.0 & $\mathrm{mg} / \mathrm{kg}$ & 2 & 28-Aug-2009 & 29-Aug-2009 & SW 846/6020 & $\mathrm{U}$ \\
\hline Nickel & 70.8 & 40.0 & $\mathrm{mg} / \mathrm{kg}$ & 2 & 28-Aug-2009 & 29-Aug-2009 & SW 846/6020 & \\
\hline Selenium & ND & 40.0 & $\mathrm{mg} / \mathrm{kg}$ & 2 & 28-Aug-2009 & 29-Aug-2009 & SW 846/6020 & $\mathrm{U}$ \\
\hline Thallium & ND & 40.0 & $\mathrm{mg} / \mathrm{kg}$ & 2 & 28-Aug-2009 & 29-Aug-2009 & SW 846/6020 & $\mathrm{U}$ \\
\hline Vanadium & 20.1 & 40.0 & $\mathrm{mg} / \mathrm{kg}$ & 2 & 28-Aug-2009 & 29-Aug-2009 & SW 846/6020 & $\mathrm{J}$ \\
\hline Zinc & 1670 & 40.0 & $\mathrm{mg} / \mathrm{kg}$ & 2 & 28-Aug-2009 & 29-Aug-2009 & SW 846/6020 & \\
\hline
\end{tabular}




\section{USACE ERDC-EP-C \\ 3909 Halls Ferry Road \\ Vicksburg, MS 39180-6199}

ERDC - ECB

Project: TVA Fly Ash Project

Reported:

Project Manager: Tony Bednar

03-Sep-2009

DRAFT: Fish Gut EMR - WA 4

9082706-24 (Tissue)

\begin{tabular}{|c|c|c|c|c|c|c|c|c|}
\hline & & porting & & & & & & \\
\hline Analyte & Result & Limit & Units & Dilution & Prepared & Analyzed & Method & Notes \\
\hline
\end{tabular}

ERDC- EL-EP-C (Environmental Chemistry Branch)

DRAFT: Metals by EPA 6000/7000 Series Methods

\begin{tabular}{|c|c|c|c|c|c|c|c|c|}
\hline Antimony & 25.0 & 40.0 & $\mathrm{mg} / \mathrm{kg}$ & 2 & 28-Aug-2009 & 29-Aug-2009 & SW 846/6020 & $\mathrm{J}, \mathrm{B}$ \\
\hline Arsenic & ND & 40.0 & $\mathrm{mg} / \mathrm{kg}$ & 2 & 28-Aug-2009 & 29-Aug-2009 & SW 846/6020 & $\mathrm{U}$ \\
\hline Barium & 517 & 40.0 & $\mathrm{mg} / \mathrm{kg}$ & 2 & 28-Aug-2009 & 29-Aug-2009 & SW 846/6020 & \\
\hline Beryllium & ND & 40.0 & $\mathrm{mg} / \mathrm{kg}$ & 2 & 28-Aug-2009 & 29-Aug-2009 & SW 846/6020 & $\mathrm{U}$ \\
\hline Cadmium & ND & 40.0 & $\mathrm{mg} / \mathrm{kg}$ & 2 & 28-Aug-2009 & 29-Aug-2009 & SW 846/6020 & $\mathrm{U}$ \\
\hline Chromium & 168 & 40.0 & $\mathrm{mg} / \mathrm{kg}$ & 2 & 28-Aug-2009 & 29-Aug-2009 & SW 846/6020 & \\
\hline Copper & 160 & 40.0 & $\mathrm{mg} / \mathrm{kg}$ & 2 & 28-Aug-2009 & 29-Aug-2009 & SW 846/6020 & \\
\hline Lead & 112 & 40.0 & $\mathrm{mg} / \mathrm{kg}$ & 2 & 28-Aug-2009 & 29-Aug-2009 & SW 846/6020 & B \\
\hline Manganese & 728 & 40.0 & $\mathrm{mg} / \mathrm{kg}$ & 2 & 28-Aug-2009 & 29-Aug-2009 & SW 846/6020 & \\
\hline Molybdenum & ND & 40.0 & $\mathrm{mg} / \mathrm{kg}$ & 2 & 28-Aug-2009 & 29-Aug-2009 & SW 846/6020 & $\mathrm{U}$ \\
\hline Nickel & 45.9 & 40.0 & $\mathrm{mg} / \mathrm{kg}$ & 2 & 28-Aug-2009 & 29-Aug-2009 & SW 846/6020 & \\
\hline Selenium & ND & 40.0 & $\mathrm{mg} / \mathrm{kg}$ & 2 & 28-Aug-2009 & 29-Aug-2009 & SW 846/6020 & $\mathrm{U}$ \\
\hline Thallium & ND & 40.0 & $\mathrm{mg} / \mathrm{kg}$ & 2 & 28-Aug-2009 & 29-Aug-2009 & SW 846/6020 & U \\
\hline Vanadium & 18.2 & 40.0 & $\mathrm{mg} / \mathrm{kg}$ & 2 & 28-Aug-2009 & 29-Aug-2009 & SW 846/6020 & $\mathrm{J}$ \\
\hline Zinc & 1620 & 40.0 & $\mathrm{mg} / \mathrm{kg}$ & 2 & 28-Aug-2009 & 29-Aug-2009 & SW $846 / 6020$ & \\
\hline
\end{tabular}




\section{USACE ERDC-EP-C \\ 3909 Halls Ferry Road \\ Vicksburg, MS 39180-6199}

ERDC - ECB

Project: TVA Fly Ash Project

Reported:

Project Manager: Tony Bednar

03-Sep-2009

DRAFT: Fish Gut EMR - WA 5

9082706-25 (Tissue)

\begin{tabular}{|c|c|c|c|c|c|c|c|c|}
\hline Analyte & Result & $\begin{array}{r}\text { porting } \\
\text { Limit }\end{array}$ & Units & Dilution & Prepared & Analyzed & Method & Notes \\
\hline
\end{tabular}

ERDC- EL-EP-C (Environmental Chemistry Branch)

DRAFT: Metals by EPA 6000/7000 Series Methods

\begin{tabular}{|c|c|c|c|c|c|c|c|c|}
\hline Antimony & ND & 40.0 & $\mathrm{mg} / \mathrm{kg}$ & 2 & 28-Aug-2009 & 29-Aug-2009 & SW 846/6020 & $\mathrm{U}$ \\
\hline Arsenic & ND & 40.0 & $\mathrm{mg} / \mathrm{kg}$ & 2 & 28-Aug-2009 & 29-Aug-2009 & SW 846/6020 & $\mathrm{U}$ \\
\hline Barium & 351 & 40.0 & $\mathrm{mg} / \mathrm{kg}$ & 2 & 28-Aug-2009 & 29-Aug-2009 & SW 846/6020 & \\
\hline Beryllium & ND & 40.0 & $\mathrm{mg} / \mathrm{kg}$ & 2 & 28-Aug-2009 & 29-Aug-2009 & SW 846/6020 & $\mathrm{U}$ \\
\hline Cadmium & ND & 40.0 & $\mathrm{mg} / \mathrm{kg}$ & 2 & 28-Aug-2009 & 29-Aug-2009 & SW 846/6020 & $\mathrm{U}$ \\
\hline Chromium & 96.4 & 40.0 & $\mathrm{mg} / \mathrm{kg}$ & 2 & 28-Aug-2009 & 29-Aug-2009 & SW 846/6020 & \\
\hline Copper & 109 & 40.0 & $\mathrm{mg} / \mathrm{kg}$ & 2 & 28-Aug-2009 & 29-Aug-2009 & SW 846/6020 & \\
\hline Lead & 100 & 40.0 & $\mathrm{mg} / \mathrm{kg}$ & 2 & 28-Aug-2009 & 29-Aug-2009 & SW 846/6020 & B \\
\hline Manganese & 479 & 40.0 & $\mathrm{mg} / \mathrm{kg}$ & 2 & 28-Aug-2009 & 29-Aug-2009 & SW 846/6020 & \\
\hline Molybdenum & $\mathrm{ND}$ & 40.0 & $\mathrm{mg} / \mathrm{kg}$ & 2 & 28-Aug-2009 & 29-Aug-2009 & SW 846/6020 & $\mathrm{U}$ \\
\hline Nickel & 32.3 & 40.0 & $\mathrm{mg} / \mathrm{kg}$ & 2 & 28-Aug-2009 & 29-Aug-2009 & SW 846/6020 & $\mathrm{J}$ \\
\hline Selenium & ND & 40.0 & $\mathrm{mg} / \mathrm{kg}$ & 2 & 28-Aug-2009 & 29-Aug-2009 & SW 846/6020 & $\mathrm{U}$ \\
\hline Silver & ND & 40.0 & $\mathrm{mg} / \mathrm{kg}$ & 2 & 28-Aug-2009 & 29-Aug-2009 & SW 846/6020 & $\mathrm{U}$ \\
\hline Thallium & ND & 40.0 & $\mathrm{mg} / \mathrm{kg}$ & 2 & 28-Aug-2009 & 29-Aug-2009 & SW 846/6020 & $\mathrm{U}$ \\
\hline Vanadium & 14.4 & 40.0 & $\mathrm{mg} / \mathrm{kg}$ & 2 & 28-Aug-2009 & 29-Aug-2009 & SW 846/6020 & $\mathrm{J}$ \\
\hline Zinc & 1280 & 40.0 & $\mathrm{mg} / \mathrm{kg}$ & 2 & 28-Aug-2009 & 29-Aug-2009 & SW $846 / 6020$ & \\
\hline
\end{tabular}




\section{USACE ERDC-EP-C \\ 3909 Halls Ferry Road \\ Vicksburg, MS 39180-6199}

ERDC - ECB

Project: TVA Fly Ash Project

Reported:

Project Manager: Tony Bednar

03-Sep-2009

DRAFT: Fish Gut EMR - EL 1

9082706-26 (Tissue)

\begin{tabular}{|c|c|c|c|c|c|c|c|c|}
\hline & & porting & & & & & & \\
\hline Analyte & Result & Limit & Units & Dilution & Prepared & Analyzed & Method & Notes \\
\hline
\end{tabular}

ERDC- EL-EP-C (Environmental Chemistry Branch)

DRAFT: Metals by EPA 6000/7000 Series Methods

\begin{tabular}{|c|c|c|c|c|c|c|c|c|}
\hline Antimony & ND & 40.0 & $\mathrm{mg} / \mathrm{kg}$ & 2 & 28-Aug-2009 & 29-Aug-2009 & SW 846/6020 & $\mathrm{U}$ \\
\hline Arsenic & 183 & 40.0 & $\mathrm{mg} / \mathrm{kg}$ & 2 & 28-Aug-2009 & 29-Aug-2009 & SW 846/6020 & \\
\hline Barium & 1280 & 40.0 & $\mathrm{mg} / \mathrm{kg}$ & 2 & 28-Aug-2009 & 29-Aug-2009 & SW 846/6020 & \\
\hline Cadmium & ND & 40.0 & $\mathrm{mg} / \mathrm{kg}$ & 2 & 28-Aug-2009 & 29-Aug-2009 & SW 846/6020 & $\mathrm{U}$ \\
\hline Chromium & 138 & 40.0 & $\mathrm{mg} / \mathrm{kg}$ & 2 & 28-Aug-2009 & 29-Aug-2009 & SW 846/6020 & \\
\hline Copper & 242 & 40.0 & $\mathrm{mg} / \mathrm{kg}$ & 2 & 28-Aug-2009 & 29-Aug-2009 & SW 846/6020 & \\
\hline Lead & 114 & 40.0 & $\mathrm{mg} / \mathrm{kg}$ & 2 & 28-Aug-2009 & 29-Aug-2009 & SW 846/6020 & B \\
\hline Manganese & 317 & 40.0 & $\mathrm{mg} / \mathrm{kg}$ & 2 & 28-Aug-2009 & 29-Aug-2009 & SW 846/6020 & \\
\hline Molybdenum & 11.2 & 40.0 & $\mathrm{mg} / \mathrm{kg}$ & 2 & 28-Aug-2009 & 29-Aug-2009 & SW 846/6020 & $\mathrm{J}$ \\
\hline Nickel & 118 & 40.0 & $\mathrm{mg} / \mathrm{kg}$ & 2 & 28-Aug-2009 & 29-Aug-2009 & SW 846/6020 & \\
\hline Selenium & ND & 40.0 & $\mathrm{mg} / \mathrm{kg}$ & 2 & 28-Aug-2009 & 29-Aug-2009 & SW 846/6020 & $\mathrm{U}$ \\
\hline Thallium & ND & 40.0 & $\mathrm{mg} / \mathrm{kg}$ & 2 & 28-Aug-2009 & 29-Aug-2009 & SW 846/6020 & $\mathrm{U}$ \\
\hline Vanadium & 286 & 40.0 & $\mathrm{mg} / \mathrm{kg}$ & 2 & 28-Aug-2009 & 29-Aug-2009 & SW 846/6020 & \\
\hline Zinc & 382 & 40.0 & $\mathrm{mg} / \mathrm{kg}$ & 2 & 28-Aug-2009 & 29-Aug-2009 & SW 846/6020 & \\
\hline
\end{tabular}




\section{USACE ERDC-EP-C \\ 3909 Halls Ferry Road \\ Vicksburg, MS 39180-6199}

ERDC - ECB

Project: TVA Fly Ash Project

Reported:

Project Manager: Tony Bednar

03-Sep-2009

DRAFT: Fish Gut EMR - EL 2

9082706-27 (Tissue)

\begin{tabular}{|c|c|c|c|c|c|c|c|c|}
\hline & & porting & & & & & & \\
\hline Analyte & Result & Limit & Units & Dilution & Prepared & Analyzed & Method & Notes \\
\hline
\end{tabular}

ERDC- EL-EP-C (Environmental Chemistry Branch)

DRAFT: Metals by EPA 6000/7000 Series Methods

\begin{tabular}{|c|c|c|c|c|c|c|c|c|}
\hline Antimony & 46.1 & 40.0 & $\mathrm{mg} / \mathrm{kg}$ & 2 & 28-Aug-2009 & 29-Aug-2009 & SW 846/6020 & B \\
\hline Arsenic & 1390 & 40.0 & $\mathrm{mg} / \mathrm{kg}$ & 2 & 28-Aug-2009 & 29-Aug-2009 & SW 846/6020 & \\
\hline Barium & 11100 & 40.0 & $\mathrm{mg} / \mathrm{kg}$ & 2 & 28-Aug-2009 & 29-Aug-2009 & SW 846/6020 & \\
\hline Beryllium & 93.6 & 40.0 & $\mathrm{mg} / \mathrm{kg}$ & 2 & 28-Aug-2009 & 29-Aug-2009 & SW 846/6020 & \\
\hline Cadmium & 60.2 & 40.0 & $\mathrm{mg} / \mathrm{kg}$ & 2 & 28-Aug-2009 & 29-Aug-2009 & SW 846/6020 & \\
\hline Chromium & 1120 & 40.0 & $\mathrm{mg} / \mathrm{kg}$ & 2 & 28-Aug-2009 & 29-Aug-2009 & SW 846/6020 & \\
\hline Copper & 2280 & 40.0 & $\mathrm{mg} / \mathrm{kg}$ & 2 & 28-Aug-2009 & 29-Aug-2009 & SW 846/6020 & \\
\hline Lead & 970 & 40.0 & $\mathrm{mg} / \mathrm{kg}$ & 2 & 28-Aug-2009 & 29-Aug-2009 & SW 846/6020 & B \\
\hline Manganese & 2710 & 40.0 & $\mathrm{mg} / \mathrm{kg}$ & 2 & 28-Aug-2009 & 29-Aug-2009 & SW 846/6020 & \\
\hline Molybdenum & 94.1 & 40.0 & $\mathrm{mg} / \mathrm{kg}$ & 2 & 28-Aug-2009 & 29-Aug-2009 & SW 846/6020 & \\
\hline Nickel & 1010 & 40.0 & $\mathrm{mg} / \mathrm{kg}$ & 2 & 28-Aug-2009 & 29-Aug-2009 & SW 846/6020 & \\
\hline Selenium & 36.0 & 40.0 & $\mathrm{mg} / \mathrm{kg}$ & 2 & 28-Aug-2009 & 29-Aug-2009 & SW 846/6020 & $\mathrm{J}$ \\
\hline Thallium & 38.4 & 40.0 & $\mathrm{mg} / \mathrm{kg}$ & 2 & 28-Aug-2009 & 29-Aug-2009 & SW 846/6020 & $\mathrm{J}$ \\
\hline Vanadium & 2360 & 40.0 & $\mathrm{mg} / \mathrm{kg}$ & 2 & 28-Aug-2009 & 29-Aug-2009 & SW 846/6020 & \\
\hline Zinc & 3570 & 40.0 & $\mathrm{mg} / \mathrm{kg}$ & 2 & 28-Aug-2009 & 29-Aug-2009 & SW 846/6020 & \\
\hline
\end{tabular}




\section{USACE ERDC-EP-C \\ 3909 Halls Ferry Road \\ Vicksburg, MS 39180-6199}

ERDC - ECB

Project: TVA Fly Ash Project

Reported:

Project Manager: Tony Bednar

03-Sep-2009

DRAFT: Fish Gut EMR - EL 3

9082706-28 (Tissue)

\begin{tabular}{|c|c|c|c|c|c|c|c|c|}
\hline & & porting & & & & & & \\
\hline Analyte & Result & Limit & Units & Dilution & Prepared & Analyzed & Method & Notes \\
\hline
\end{tabular}

ERDC- EL-EP-C (Environmental Chemistry Branch)

DRAFT: Metals by EPA 6000/7000 Series Methods

\begin{tabular}{|c|c|c|c|c|c|c|c|c|}
\hline Antimony & 23.6 & 40.0 & $\mathrm{mg} / \mathrm{kg}$ & 2 & 28-Aug-2009 & 29-Aug-2009 & SW 846/6020 & $\mathrm{J}, \mathrm{B}$ \\
\hline Arsenic & 611 & 40.0 & $\mathrm{mg} / \mathrm{kg}$ & 2 & 28-Aug-2009 & 29-Aug-2009 & SW 846/6020 & \\
\hline Barium & 6140 & 40.0 & $\mathrm{mg} / \mathrm{kg}$ & 2 & 28-Aug-2009 & 29-Aug-2009 & SW 846/6020 & \\
\hline Cadmium & 34.4 & 40.0 & $\mathrm{mg} / \mathrm{kg}$ & 2 & 28-Aug-2009 & 29-Aug-2009 & SW 846/6020 & $\mathrm{J}$ \\
\hline Chromium & 611 & 40.0 & $\mathrm{mg} / \mathrm{kg}$ & 2 & 28-Aug-2009 & 29-Aug-2009 & SW 846/6020 & \\
\hline Cobalt & 252 & 40.0 & $\mathrm{mg} / \mathrm{kg}$ & 2 & 28-Aug-2009 & 29-Aug-2009 & SW 846/6020 & \\
\hline Copper & 1170 & 40.0 & $\mathrm{mg} / \mathrm{kg}$ & 2 & 28-Aug-2009 & 29-Aug-2009 & SW 846/6020 & \\
\hline Lead & 550 & 40.0 & $\mathrm{mg} / \mathrm{kg}$ & 2 & 28-Aug-2009 & 29-Aug-2009 & SW 846/6020 & B \\
\hline Manganese & 1480 & 40.0 & $\mathrm{mg} / \mathrm{kg}$ & 2 & 28-Aug-2009 & 29-Aug-2009 & SW 846/6020 & \\
\hline Molybdenum & 50.9 & 40.0 & $\mathrm{mg} / \mathrm{kg}$ & 2 & 28-Aug-2009 & 29-Aug-2009 & SW 846/6020 & \\
\hline Nickel & 560 & 40.0 & $\mathrm{mg} / \mathrm{kg}$ & 2 & 28-Aug-2009 & 29-Aug-2009 & SW 846/6020 & \\
\hline Selenium & 12.2 & 40.0 & $\mathrm{mg} / \mathrm{kg}$ & 2 & 28-Aug-2009 & 29-Aug-2009 & SW 846/6020 & $\mathrm{J}$ \\
\hline Thallium & 19.4 & 40.0 & $\mathrm{mg} / \mathrm{kg}$ & 2 & 28-Aug-2009 & 29-Aug-2009 & SW 846/6020 & $\mathrm{J}$ \\
\hline Vanadium & 1300 & 40.0 & $\mathrm{mg} / \mathrm{kg}$ & 2 & 28-Aug-2009 & 29-Aug-2009 & SW 846/6020 & \\
\hline Zinc & 2440 & 40.0 & $\mathrm{mg} / \mathrm{kg}$ & 2 & 28-Aug-2009 & 29-Aug-2009 & SW 846/6020 & \\
\hline
\end{tabular}




\section{USACE ERDC-EP-C \\ 3909 Halls Ferry Road \\ Vicksburg, MS 39180-6199}

ERDC - ECB

Project: TVA Fly Ash Project

Reported:

Project Manager: Tony Bednar

03-Sep-2009

DRAFT: Fish Gut EMR - EL 4

9082706-29 (Tissue)

\begin{tabular}{|c|c|c|c|c|c|c|c|c|}
\hline & & porting & & & & & & \\
\hline Analyte & Result & Limit & Units & Dilution & Prepared & Analyzed & Method & Notes \\
\hline
\end{tabular}

ERDC- EL-EP-C (Environmental Chemistry Branch)

DRAFT: Metals by EPA 6000/7000 Series Methods

\begin{tabular}{|c|c|c|c|c|c|c|c|c|}
\hline Antimony & 25.6 & 40.0 & $\mathrm{mg} / \mathrm{kg}$ & 2 & 28-Aug-2009 & 29-Aug-2009 & SW 846/6020 & $\mathrm{J}, \mathrm{B}$ \\
\hline Arsenic & 734 & 40.0 & $\mathrm{mg} / \mathrm{kg}$ & 2 & 28-Aug-2009 & 29-Aug-2009 & SW 846/6020 & \\
\hline Barium & 6380 & 40.0 & $\mathrm{mg} / \mathrm{kg}$ & 2 & 28-Aug-2009 & 29-Aug-2009 & SW 846/6020 & \\
\hline Cadmium & 32.0 & 40.0 & $\mathrm{mg} / \mathrm{kg}$ & 2 & 28-Aug-2009 & 29-Aug-2009 & SW 846/6020 & $\mathrm{J}$ \\
\hline Chromium & 625 & 40.0 & $\mathrm{mg} / \mathrm{kg}$ & 2 & 28-Aug-2009 & 29-Aug-2009 & SW 846/6020 & \\
\hline Cobalt & 261 & 40.0 & $\mathrm{mg} / \mathrm{kg}$ & 2 & 28-Aug-2009 & 29-Aug-2009 & SW 846/6020 & \\
\hline Copper & 1180 & 40.0 & $\mathrm{mg} / \mathrm{kg}$ & 2 & 28-Aug-2009 & 29-Aug-2009 & SW 846/6020 & \\
\hline Lead & 1600 & 40.0 & $\mathrm{mg} / \mathrm{kg}$ & 2 & 28-Aug-2009 & 29-Aug-2009 & SW 846/6020 & B \\
\hline Manganese & 1520 & 40.0 & $\mathrm{mg} / \mathrm{kg}$ & 2 & 28-Aug-2009 & 29-Aug-2009 & SW 846/6020 & \\
\hline Molybdenum & 52.5 & 40.0 & $\mathrm{mg} / \mathrm{kg}$ & 2 & 28-Aug-2009 & 29-Aug-2009 & SW 846/6020 & \\
\hline Nickel & 580 & 40.0 & $\mathrm{mg} / \mathrm{kg}$ & 2 & 28-Aug-2009 & 29-Aug-2009 & SW 846/6020 & \\
\hline Selenium & ND & 40.0 & $\mathrm{mg} / \mathrm{kg}$ & 2 & 28-Aug-2009 & 29-Aug-2009 & SW 846/6020 & $\mathrm{U}$ \\
\hline Thallium & 20.2 & 40.0 & $\mathrm{mg} / \mathrm{kg}$ & 2 & 28-Aug-2009 & 29-Aug-2009 & SW 846/6020 & $\mathrm{J}$ \\
\hline Vanadium & 1340 & 40.0 & $\mathrm{mg} / \mathrm{kg}$ & 2 & 28-Aug-2009 & 29-Aug-2009 & SW 846/6020 & \\
\hline Zinc & 2460 & 40.0 & $\mathrm{mg} / \mathrm{kg}$ & 2 & 28-Aug-2009 & 29-Aug-2009 & SW 846/6020 & \\
\hline
\end{tabular}




\section{USACE ERDC-EP-C \\ 3909 Halls Ferry Road \\ Vicksburg, MS 39180-6199}

ERDC - ECB

Project: TVA Fly Ash Project

Reported:

Project Manager: Tony Bednar

03-Sep-2009

DRAFT: Fish Gut EMR - EL 5

9082706-30 (Tissue)

\begin{tabular}{|c|c|c|c|c|c|c|c|c|}
\hline & & porting & & & & & & \\
\hline Analyte & Result & Limit & Units & Dilution & Prepared & Analyzed & Method & Notes \\
\hline
\end{tabular}

ERDC- EL-EP-C (Environmental Chemistry Branch)

DRAFT: Metals by EPA 6000/7000 Series Methods

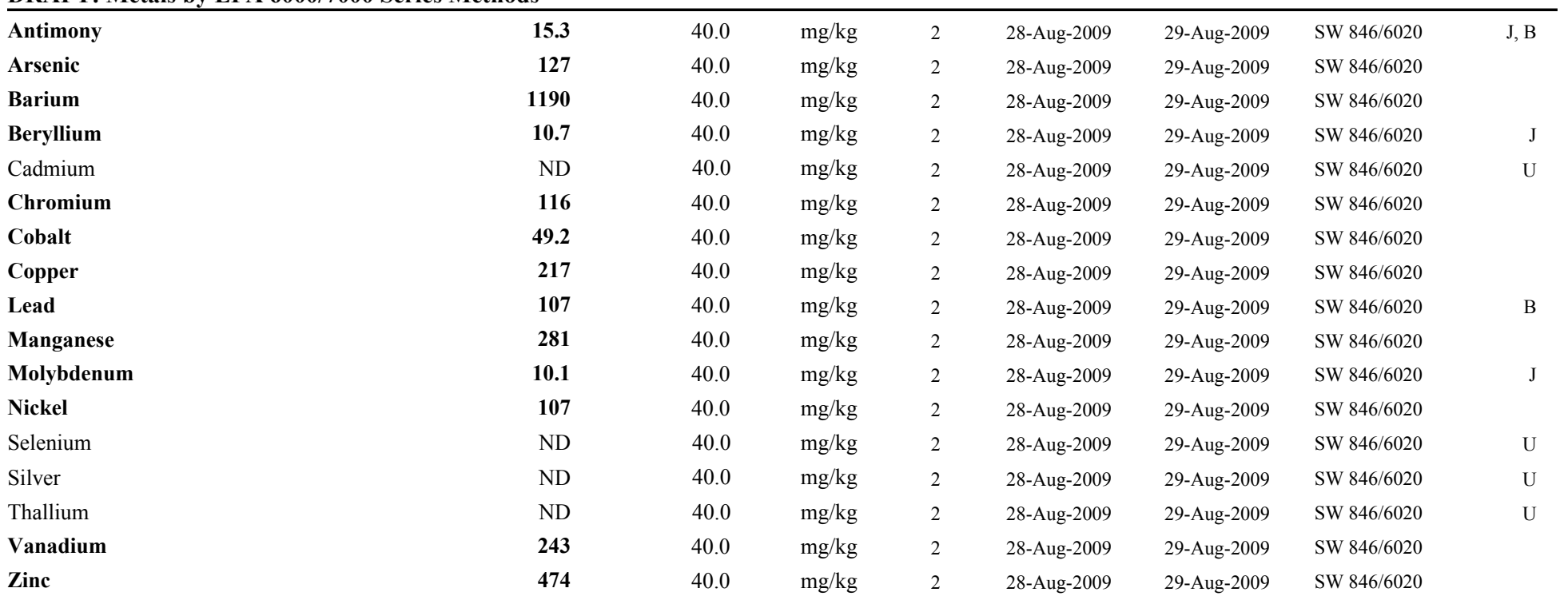




\section{USACE ERDC-EP-C \\ 3909 Halls Ferry Road \\ Vicksburg, MS 39180-6199}

ERDC - ECB

Project: TVA Fly Ash Project

Reported:

Project Manager: Tony Bednar

03-Sep-2009

DRAFT: Metals by EPA 6000/7000 Series Methods - Quality Control

ERDC- EL-EP-C (Environmental Chemistry Branch)

\begin{tabular}{|lrrrrrrrrrr}
\hline & & Reporting & & Spike & Source & & & \%REC & & RPD \\
Analyte & Result & Limit & Units & Level & Result & $\%$ REC & Limits & RPD & Limit & Notes \\
\hline
\end{tabular}

Batch B906047 - Default Prep Metals

\begin{tabular}{|c|c|c|c|c|c|}
\hline \multirow{2}{*}{$\begin{array}{l}\text { Blank (B906047-BLK1) } \\
\text { Antimony }\end{array}$} & \multicolumn{5}{|c|}{ Prepared \& Analyzed: 16-Jun-2009 } \\
\hline & 0.0006 & 0.0010 & $\mathrm{mg} / \mathrm{L}$ & & $\overline{\mathrm{J}}$ \\
\hline Arsenic & ND & 0.0010 & $\mathrm{mg} / \mathrm{L}$ & & $\mathrm{U}$ \\
\hline Barium & ND & 0.0010 & $\mathrm{mg} / \mathrm{L}$ & & $\mathrm{U}$ \\
\hline Beryllium & ND & 0.0010 & $\mathrm{mg} / \mathrm{L}$ & & $\mathrm{U}$ \\
\hline Cadmium & ND & 0.0010 & $\mathrm{mg} / \mathrm{L}$ & & $\mathrm{U}$ \\
\hline Chromium & ND & 0.0010 & $\mathrm{mg} / \mathrm{L}$ & & $\mathrm{U}$ \\
\hline Cobalt & ND & 0.0010 & $\mathrm{mg} / \mathrm{L}$ & & $\mathrm{U}$ \\
\hline Copper & 0.0004 & 0.0010 & $\mathrm{mg} / \mathrm{L}$ & & $\mathrm{J}$ \\
\hline Lead & ND & 0.0010 & $\mathrm{mg} / \mathrm{L}$ & & $\mathrm{U}$ \\
\hline Manganese & ND & 0.0010 & $\mathrm{mg} / \mathrm{L}$ & & $\mathrm{U}$ \\
\hline Molybdenum & ND & 0.0010 & $\mathrm{mg} / \mathrm{L}$ & & $\mathrm{U}$ \\
\hline Nickel & ND & 0.0010 & $\mathrm{mg} / \mathrm{L}$ & & $\mathrm{U}$ \\
\hline Selenium & ND & 0.0010 & $\mathrm{mg} / \mathrm{L}$ & & $\mathrm{U}$ \\
\hline Silver & 0.0063 & 0.0010 & $\mathrm{mg} / \mathrm{L}$ & & \\
\hline Thallium & ND & 0.0010 & $\mathrm{mg} / \mathrm{L}$ & & $\mathrm{U}$ \\
\hline Vanadium & ND & 0.0010 & $\mathrm{mg} / \mathrm{L}$ & & $\mathrm{U}$ \\
\hline Zinc & 0.0012 & 0.0010 & $\mathrm{mg} / \mathrm{L}$ & & \\
\hline Blank (B906047-BLK2) & \multicolumn{5}{|c|}{ Prepared: 16-Jun-2009 Analyzed: 30-Jun-2009 } \\
\hline Antimony & ND & 0.0010 & $\mathrm{mg} / \mathrm{L}$ & & $\mathrm{U}$ \\
\hline Arsenic & ND & 0.0010 & $\mathrm{mg} / \mathrm{L}$ & & U \\
\hline Barium & ND & 0.0010 & $\mathrm{mg} / \mathrm{L}$ & & $\mathrm{U}$ \\
\hline Beryllium & ND & 0.0010 & $\mathrm{mg} / \mathrm{L}$ & & $\mathrm{U}$ \\
\hline Cadmium & ND & 0.0010 & $\mathrm{mg} / \mathrm{L}$ & & U \\
\hline Chromium & ND & 0.0010 & $\mathrm{mg} / \mathrm{L}$ & & U \\
\hline Cobalt & ND & 0.0010 & $\mathrm{mg} / \mathrm{L}$ & & U \\
\hline Copper & ND & 0.0010 & $\mathrm{mg} / \mathrm{L}$ & & $\mathrm{U}$ \\
\hline Lead & ND & 0.0010 & $\mathrm{mg} / \mathrm{L}$ & & $\mathrm{U}$ \\
\hline Manganese & ND & 0.0010 & $\mathrm{mg} / \mathrm{L}$ & & $\mathrm{U}$ \\
\hline Molybdenum & ND & 0.0010 & $\mathrm{mg} / \mathrm{L}$ & & $\mathrm{U}$ \\
\hline Nickel & ND & 0.0010 & $\mathrm{mg} / \mathrm{L}$ & & $\mathrm{U}$ \\
\hline Selenium & ND & 0.0010 & $\mathrm{mg} / \mathrm{L}$ & & $\mathrm{U}$ \\
\hline Silver & ND & 0.0010 & $\mathrm{mg} / \mathrm{L}$ & & $\mathrm{U}$ \\
\hline Thallium & ND & 0.0010 & $\mathrm{mg} / \mathrm{L}$ & & $\mathrm{U}$ \\
\hline Vanadium & ND & 0.0010 & $\mathrm{mg} / \mathrm{L}$ & & $\mathrm{U}$ \\
\hline Zinc & ND & 0.0010 & $\mathrm{mg} / \mathrm{L}$ & & $\mathrm{U}$ \\
\hline
\end{tabular}




\section{USACE ERDC-EP-C \\ 3909 Halls Ferry Road \\ Vicksburg, MS 39180-6199}

ERDC - ECB
Project: TVA Fly Ash Project

Project Manager: Tony Bednar
Reported:

03-Sep-2009

DRAFT: Metals by EPA 6000/7000 Series Methods - Quality Control

ERDC- EL-EP-C (Environmental Chemistry Branch)

\begin{tabular}{|c|c|c|c|c|c|c|c|c|c|c|}
\hline & & eporting & & Spike & Source & & $\%$ REC & & RPD & \\
\hline Analyte & Result & Limit & Units & Level & Result & $\%$ REC & Limits & RPD & Limit & Notes \\
\hline
\end{tabular}

Batch B906047 - Default Prep Metals

\begin{tabular}{|c|c|c|c|c|c|c|c|c|c|}
\hline \multirow{2}{*}{$\frac{\text { LCS (B906047-BS1) }}{\text { Antimony }}$} & \multicolumn{9}{|c|}{ Prepared \& Analyzed: 16-Jun-2009 } \\
\hline & 0.0246 & 0.0010 & $\mathrm{mg} / \mathrm{L}$ & 0.02500 & 98.5 & $80-120$ & & & \\
\hline Arsenic & 0.0249 & 0.0010 & $\mathrm{mg} / \mathrm{L}$ & 0.02500 & 99.6 & $80-120$ & & & \\
\hline Beryllium & 0.0247 & 0.0010 & $\mathrm{mg} / \mathrm{L}$ & 0.02500 & 98.9 & $80-120$ & & & \\
\hline Cadmium & 0.0246 & 0.0010 & $\mathrm{mg} / \mathrm{L}$ & 0.02500 & 98.3 & $80-120$ & & & \\
\hline Chromium & 0.0262 & 0.0010 & $\mathrm{mg} / \mathrm{L}$ & 0.02500 & 105 & $80-120$ & & & \\
\hline Cobalt & 0.0274 & 0.0010 & $\mathrm{mg} / \mathrm{L}$ & 0.02500 & 110 & $80-120$ & & & \\
\hline Copper & 0.0259 & 0.0010 & $\mathrm{mg} / \mathrm{L}$ & 0.02500 & 104 & $80-120$ & & & \\
\hline Lead & 0.0268 & 0.0010 & $\mathrm{mg} / \mathrm{L}$ & 0.02500 & 107 & $80-120$ & & & \\
\hline Manganese & 0.0262 & 0.0010 & $\mathrm{mg} / \mathrm{L}$ & 0.02500 & 105 & $80-120$ & & & \\
\hline Molybdenum & 0.0253 & 0.0010 & $\mathrm{mg} / \mathrm{L}$ & 0.02500 & 101 & $80-120$ & & & \\
\hline Nickel & 0.0260 & 0.0010 & $\mathrm{mg} / \mathrm{L}$ & 0.02500 & 104 & $80-120$ & & & \\
\hline Selenium & 0.0239 & 0.0010 & $\mathrm{mg} / \mathrm{L}$ & 0.02500 & 95.6 & $80-120$ & & & \\
\hline Thallium & 0.0248 & 0.0010 & $\mathrm{mg} / \mathrm{L}$ & 0.02500 & 99.4 & $80-120$ & & & \\
\hline Vanadium & 0.0266 & 0.0010 & $\mathrm{mg} / \mathrm{L}$ & 0.02500 & 107 & $80-120$ & & & \\
\hline Zinc & 0.0250 & 0.0010 & $\mathrm{mg} / \mathrm{L}$ & 0.02500 & 100 & $80-120$ & & & B \\
\hline Duplicate (B906047-DUP1) & \multicolumn{3}{|c|}{ Source: $9061502-40$} & \multicolumn{3}{|c|}{ Prepared \& Analyzed: 16-Jun-2009 } & & & \\
\hline Antimony & 0.0005 & 0.0010 & $\mathrm{mg} / \mathrm{L}$ & & & & 4.14 & 20 & $\mathrm{~J}$ \\
\hline Arsenic & 0.0348 & 0.0010 & $\mathrm{mg} / \mathrm{L}$ & & & & 2.99 & 20 & \\
\hline Barium & 0.0831 & 0.0010 & $\mathrm{mg} / \mathrm{L}$ & & & & 5.54 & 20 & \\
\hline Beryllium & ND & 0.0010 & $\mathrm{mg} / \mathrm{L}$ & & & & & 20 & $\mathrm{U}$ \\
\hline Cadmium & ND & 0.0010 & $\mathrm{mg} / \mathrm{L}$ & & & & & 20 & $\mathrm{U}$ \\
\hline Chromium & ND & 0.0010 & $\mathrm{mg} / \mathrm{L}$ & & & & & 20 & $\mathrm{U}$ \\
\hline Cobalt & ND & 0.0010 & $\mathrm{mg} / \mathrm{L}$ & & & & & 20 & U \\
\hline Copper & 0.0015 & 0.0010 & $\mathrm{mg} / \mathrm{L}$ & & & & 5.19 & 20 & \\
\hline Molybdenum & 0.0017 & 0.0010 & $\mathrm{mg} / \mathrm{L}$ & & & & 5.01 & 20 & \\
\hline Nickel & 0.0006 & 0.0010 & $\mathrm{mg} / \mathrm{L}$ & & & & 4.41 & 20 & $\mathrm{~J}$ \\
\hline Selenium & 0.0013 & 0.0010 & $\mathrm{mg} / \mathrm{L}$ & & & & 3.22 & 20 & \\
\hline Silver & ND & 0.0010 & $\mathrm{mg} / \mathrm{L}$ & & & & & 20 & $\mathrm{U}$ \\
\hline Thallium & 0.0003 & 0.0010 & $\mathrm{mg} / \mathrm{L}$ & & & & 19.2 & 20 & $\mathrm{~J}$ \\
\hline Vanadium & 0.0235 & 0.0010 & $\mathrm{mg} / \mathrm{L}$ & & & & 0.126 & 20 & \\
\hline Zinc & 0.0055 & 0.0010 & $\mathrm{mg} / \mathrm{L}$ & & & & 8.81 & 20 & B \\
\hline
\end{tabular}




\section{USACE ERDC-EP-C \\ 3909 Halls Ferry Road \\ Vicksburg, MS 39180-6199}

ERDC - ECB

Project: TVA Fly Ash Project

Reported:

Project Manager: Tony Bednar

03-Sep-2009

DRAFT: Metals by EPA 6000/7000 Series Methods - Quality Control

ERDC- EL-EP-C (Environmental Chemistry Branch)

\begin{tabular}{|lcccccccccccc}
\hline & & Reporting & & Spike & Source & & $\%$ REC & & RPD \\
Analyte & Result & Limit & Units & Level & Result & $\%$ REC & Limits & RPD & Limit & Notes \\
\hline
\end{tabular}

Batch B906047 - Default Prep Metals

\begin{tabular}{|c|c|c|c|c|c|c|c|c|}
\hline \multirow{2}{*}{$\begin{array}{l}\text { Matrix Spike (B906047-MS1) } \\
\text { Antimony }\end{array}$} & \multicolumn{3}{|c|}{ Source: $9061502-40$} & \multicolumn{5}{|c|}{ Prepared \& Analyzed: 16-Jun-2009 } \\
\hline & 0.0399 & 0.0010 & $\mathrm{mg} / \mathrm{L}$ & 0.04000 & 0.0006 & 98.4 & $80-120$ & \\
\hline Arsenic & 0.0764 & 0.0010 & $\mathrm{mg} / \mathrm{L}$ & 0.04000 & 0.0359 & 101 & $80-120$ & \\
\hline Barium & 0.124 & 0.0010 & $\mathrm{mg} / \mathrm{L}$ & 0.04000 & 0.0879 & 89.3 & $80-120$ & \\
\hline Beryllium & 0.0439 & 0.0010 & $\mathrm{mg} / \mathrm{L}$ & 0.04000 & ND & 110 & $80-120$ & \\
\hline Cadmium & 0.0396 & 0.0010 & $\mathrm{mg} / \mathrm{L}$ & 0.04000 & ND & 98.9 & $80-120$ & \\
\hline Chromium & 0.0398 & 0.0010 & $\mathrm{mg} / \mathrm{L}$ & 0.04000 & ND & 99.5 & $80-120$ & \\
\hline Cobalt & 0.0404 & 0.0010 & $\mathrm{mg} / \mathrm{L}$ & 0.04000 & ND & 101 & $80-120$ & \\
\hline Copper & 0.0406 & 0.0010 & $\mathrm{mg} / \mathrm{L}$ & 0.04000 & 0.0014 & 98.0 & $80-120$ & \\
\hline Lead & 0.0405 & 0.0010 & $\mathrm{mg} / \mathrm{L}$ & 0.04000 & ND & 101 & $80-120$ & \\
\hline Manganese & 0.0411 & 0.0010 & $\mathrm{mg} / \mathrm{L}$ & 0.04000 & 0.0033 & 94.4 & $80-120$ & \\
\hline Molybdenum & 0.0410 & 0.0010 & $\mathrm{mg} / \mathrm{L}$ & 0.04000 & 0.0018 & 97.9 & $80-120$ & \\
\hline Nickel & 0.0402 & 0.0010 & $\mathrm{mg} / \mathrm{L}$ & 0.04000 & 0.0006 & 99.0 & $80-120$ & \\
\hline Selenium & 0.0429 & 0.0010 & $\mathrm{mg} / \mathrm{L}$ & 0.04000 & 0.0013 & 104 & $80-120$ & \\
\hline Silver & 0.0369 & 0.0010 & $\mathrm{mg} / \mathrm{L}$ & 0.04000 & ND & 92.2 & $80-120$ & $\mathrm{~B}$ \\
\hline Thallium & 0.0371 & 0.0010 & $\mathrm{mg} / \mathrm{L}$ & 0.04000 & 0.0004 & 91.9 & $80-120$ & \\
\hline Vanadium & 0.0645 & 0.0010 & $\mathrm{mg} / \mathrm{L}$ & 0.04000 & 0.0235 & 102 & $80-120$ & \\
\hline Zinc & 0.0489 & 0.0010 & $\mathrm{mg} / \mathrm{L}$ & 0.04000 & 0.0060 & 107 & $80-120$ & B \\
\hline \multicolumn{9}{|l|}{ Batch B906052 - EPA 1631} \\
\hline Blank (B906052-BLK1) & \multicolumn{8}{|c|}{ Prepared: 17-Jun-2009 Analyzed: 01-Jul-2009 } \\
\hline Mercury & ND & 0.000005 & $\mathrm{mg} / \mathrm{L}$ & & & & & $\overline{\mathrm{U}}$ \\
\hline Blank (B906052-BLK2) & \multicolumn{8}{|c|}{ Prepared: 17-Jun-2009 Analyzed: 01-Jul-2009 } \\
\hline Mercury & ND & 0.000005 & $\mathrm{mg} / \mathrm{L}$ & & & & & $\overline{\mathrm{U}}$ \\
\hline Blank (B906052-BLK3) & \multicolumn{8}{|c|}{ Prepared: 17-Jun-2009 Analyzed: 01-Jul-2009 } \\
\hline Mercury & ND & 0.000005 & $\mathrm{mg} / \mathrm{L}$ & & & & & $\overline{\mathrm{U}}$ \\
\hline
\end{tabular}




\section{USACE ERDC-EP-C \\ 3909 Halls Ferry Road \\ Vicksburg, MS 39180-6199}

ERDC - ECB

Project: TVA Fly Ash Project

$-\cdot,-$

Project Manager: Tony Bednar

Reported:

03-Sep-2009

DRAFT: Metals by EPA 6000/7000 Series Methods - Quality Control

ERDC- EL-EP-C (Environmental Chemistry Branch)

\begin{tabular}{|lcccccccccccc}
\hline & & Reporting & & Spike & Source & & & $\%$ REC & & RPD \\
Analyte & Result & Limit & Units & Level & Result & $\%$ REC & Limits & RPD & Limit & Notes & \\
\hline
\end{tabular}

Batch B906052 - EPA 1631

\begin{tabular}{|c|c|c|c|c|c|c|c|c|}
\hline \multirow{2}{*}{$\begin{array}{l}\text { Blank (B906052-BLK4) } \\
\text { Mercury }\end{array}$} & \multicolumn{8}{|c|}{ Prepared: 17-Jun-2009 Analyzed: 21-Aug-2009 } \\
\hline & ND & 0.000005 & $\mathrm{mg} / \mathrm{L}$ & & & & & $\overline{\mathrm{U}}$ \\
\hline LCS (B906052-BS1) & \multicolumn{8}{|c|}{ Prepared: 17-Jun-2009 Analyzed: 01-Jul-2009 } \\
\hline Mercury & 0.000183 & 0.000005 & $\mathrm{mg} / \mathrm{L}$ & $2.000 \mathrm{E}-4$ & 91.4 & $75-125$ & & \\
\hline LCS (B906052-BS2) & \multicolumn{8}{|c|}{ Prepared: 17-Jun-2009 Analyzed: 01-Jul-2009 } \\
\hline Mercury & 0.000178 & 0.000005 & $\mathrm{mg} / \mathrm{L}$ & $2.000 \mathrm{E}-4$ & 88.9 & $75-125$ & & \\
\hline LCS (B906052-BS3) & \multicolumn{8}{|c|}{ Prepared: 17-Jun-2009 Analyzed: 01-Jul-2009 } \\
\hline Mercury & 0.000185 & 0.000005 & $\mathrm{mg} / \mathrm{L}$ & $2.000 \mathrm{E}-4$ & 92.6 & $75-125$ & & \\
\hline LCS (B906052-BS4) & \multicolumn{8}{|c|}{ Prepared: 17-Jun-2009 Analyzed: 01-Jul-2009 } \\
\hline Mercury & 0.000178 & 0.000005 & $\mathrm{mg} / \mathrm{L}$ & $2.000 \mathrm{E}-4$ & 89.1 & $75-125$ & & \\
\hline LCS (B906052-BS6) & \multicolumn{8}{|c|}{ Prepared: 17-Jun-2009 Analyzed: 02-Jul-2009 } \\
\hline Mercury & 0.000196 & 0.000005 & $\mathrm{mg} / \mathrm{L}$ & $2.000 \mathrm{E}-4$ & 98.0 & $75-125$ & & \\
\hline Duplicate (B906052-DUP1) & \multicolumn{3}{|c|}{ Source: 9061002-01 } & \multicolumn{5}{|c|}{ Prepared: 17-Jun-2009 Analyzed: 01-Jul-2009 } \\
\hline Mercury & 0.000371 & 0.000005 & $\mathrm{mg} / \mathrm{L}$ & & & 0.329 & 25 & \\
\hline Duplicate (B906052-DUP2) & \multicolumn{3}{|c|}{ Source: $9061502-40$} & \multicolumn{5}{|c|}{ Prepared: 17-Jun-2009 Analyzed: 21-Aug-2009 } \\
\hline Mercury & ND & 0.000005 & $\mathrm{mg} / \mathrm{L}$ & & & & 25 & $\overline{\mathrm{U}}$ \\
\hline Duplicate (B906052-DUP3) & \multicolumn{3}{|c|}{ Source: 9061503-04 } & \multicolumn{5}{|c|}{ Prepared: 17-Jun-2009 Analyzed: 21-Aug-2009 } \\
\hline Mercury & ND & 0.000005 & $\mathrm{mg} / \mathrm{L}$ & & & & 25 & $\mathrm{U}$ \\
\hline Duplicate (B906052-DUP4) & \multicolumn{3}{|c|}{ Source: 9061504-01 } & \multicolumn{4}{|c|}{ Prepared: 17-Jun-2009 Analyzed: 01-Jul-2009 } & \\
\hline Mercury & 0.000366 & 0.000005 & $\mathrm{mg} / \mathrm{L}$ & & & 6.64 & 25 & \\
\hline
\end{tabular}




\section{USACE ERDC-EP-C \\ 3909 Halls Ferry Road \\ Vicksburg, MS 39180-6199}

ERDC - ECB

$-,-$
Project: TVA Fly Ash Project

Reported:

03-Sep-2009

\section{DRAFT: Metals by EPA 6000/7000 Series Methods - Quality Control}

\section{ERDC- EL-EP-C (Environmental Chemistry Branch)}

\begin{tabular}{|lccccccccccc}
\hline & & Reporting & & Spike & Source & & & $\%$ REC & & RPD \\
Analyte & Result & Limit & Units & Level & Result & \%REC & Limits & RPD & Limit & Notes & \\
\hline
\end{tabular}

Batch B906052 - EPA 1631

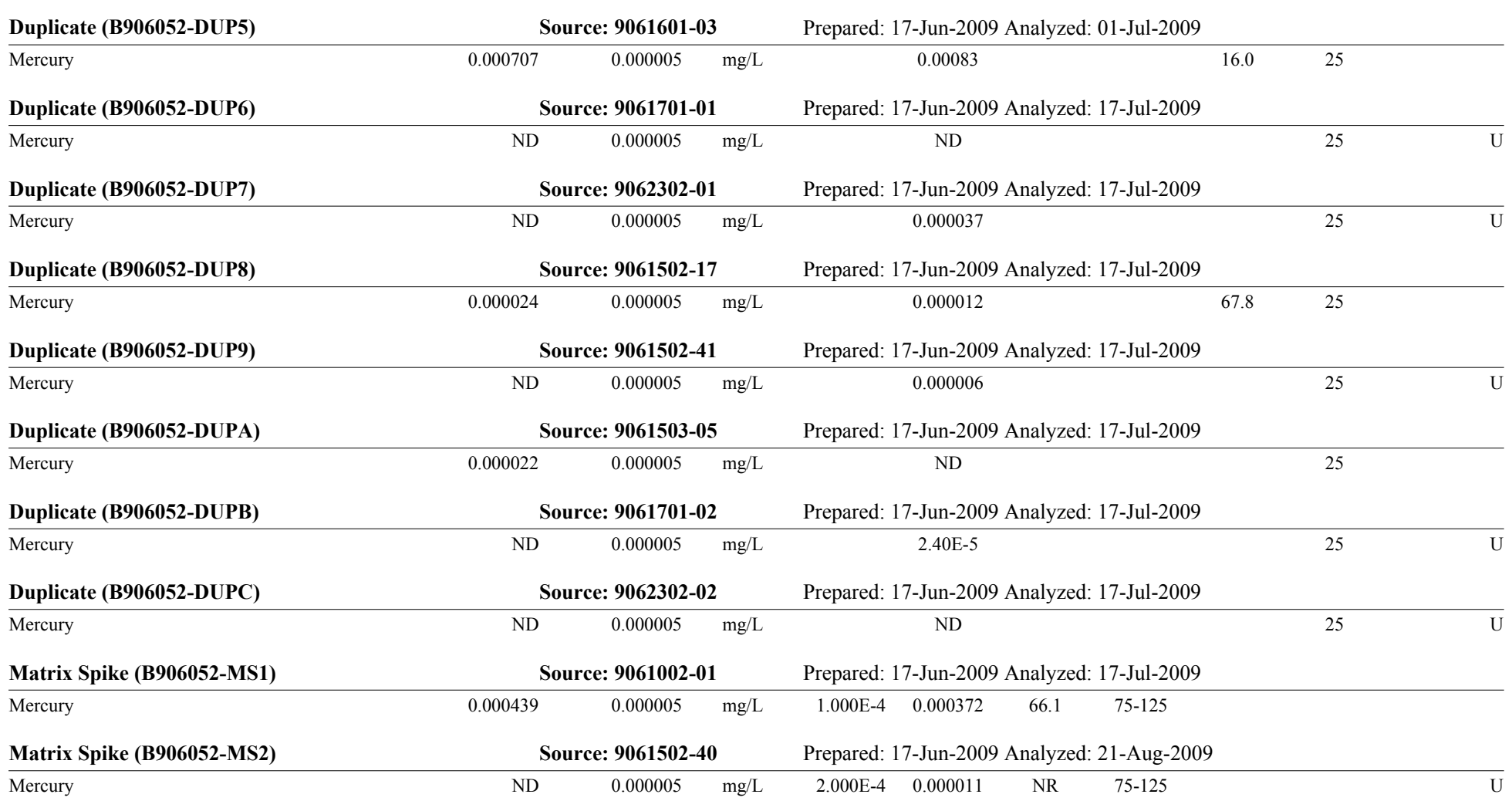




\section{USACE ERDC-EP-C \\ 3909 Halls Ferry Road \\ Vicksburg, MS 39180-6199}

ERDC - ECB

$-\cdot,-$
Project: TVA Fly Ash Project

Reported:

03-Sep-2009

DRAFT: Metals by EPA 6000/7000 Series Methods - Quality Control

ERDC- EL-EP-C (Environmental Chemistry Branch)

\begin{tabular}{|lccccccccccc}
\hline & & Reporting & & Spike & Source & & & \%REC & & RPD \\
Analyte & Result & Limit & Units & Level & Result & \%REC & Limits & RPD & Limit & Notes \\
\hline
\end{tabular}

Batch B906052 - EPA 1631

Matrix Spike (B906052-MS3)

Mercury

Source: 9061503-04

Prepared: 17-Jun-2009 Analyzed: 21-Aug-2009

ND $\quad 0.000005 \quad \mathrm{mg} / \mathrm{L}$

$4.000 \mathrm{E}-4 \quad \mathrm{ND}$

$75-125$

Matrix Spike (B906052-MS4)

Mercury

Source: 9061504-01

Prepared: 17-Jun-2009 Analyzed: 01-Jul-2009

Matrix Spike (B906052-MS5)

Mercury

$\begin{array}{lllllll}0.000549 & 0.000005 & \mathrm{mg} / \mathrm{L} & 2.000 \mathrm{E}-4 & 0.000391 & 78.9 & 75-125\end{array}$

Matrix Spike (B906052-MS6)

Source: $9061601-03$

Prepared: 17-Jun-2009 Analyzed: 01-Jul-2009

Mercury

$\begin{array}{lllllll}0.000945 & 0.000005 & \mathrm{mg} / \mathrm{L} & 1.000 \mathrm{E}-4 & 0.00083 & 114 & 75-125\end{array}$

Matrix Spike (B906052-MS7)

Source: 9061701-01 Prepared: 17-Jun-2009 Analyzed: 21-Aug-2009

Mercury

Source: 9062302-01 Prepared: 17-Jun-2009 Analyzed: 21-Aug-2009

$\begin{array}{lllllll}\mathrm{ND} & 0.000005 & \mathrm{mg} / \mathrm{L} & 1.000 \mathrm{E}-4 & 0.000037 & \mathrm{NR} & 75-125\end{array}$

Matrix Spike Dup (B906052-MSD1)

Source: 9061002-01 Prepared: 17-Jun-2009 Analyzed: 01-Jul-2009

Mercury

$\begin{array}{lllllllll}0.000437 & 0.000005 & \mathrm{mg} / \mathrm{L} & 1.000 \mathrm{E}-4 & 0.000372 & 65.1 & 75-125 & 1.61 & 25\end{array}$

Matrix Spike Dup (B906052-MSD2)

Source: 9061502-40

Prepared: 17-Jun-2009 Analyzed: 21-Aug-2009

Mercury

ND $\quad 0.000005 \quad \mathrm{mg}$

Source: 9061503-04

Prepared: 17-Jun-2009 Analyzed: 21-Aug-2009

Matrix Spike Dup (B906052-MSD3)

Mercury

4.000E-4 ND

$75-125$

25

Matrix Spike Dup (B906052-MSD4)

Mercury

Source: 9061504-01

Prepared: 17-Jun-2009 Analyzed: 01-Jul-2009

Matrix Spike Dup (B906052-MSD6)

$\begin{array}{lllllllll}0.00056 & 0.000005 & \mathrm{mg} / \mathrm{L} & 2.000 \mathrm{E}-4 & 0.000391 & 84.3 & 75-125 & 6.64 & 25\end{array}$

Mercury

Source: 9061701-01

Prepared: 17-Jun-2009 Analyzed: 01-Jul-2009

$\begin{array}{lllllll}0.000338 & 0.000005 & \mathrm{mg} / \mathrm{L} & 2.000 \mathrm{E}-4 & \mathrm{ND} & 169 & 75-125\end{array}$

25 


\section{USACE ERDC-EP-C \\ 3909 Halls Ferry Road \\ Vicksburg, MS 39180-6199}

ERDC - ECB

Project: TVA Fly Ash Project

Reported:

Project Manager: Tony Bednar

03-Sep-2009

DRAFT: Metals by EPA 6000/7000 Series Methods - Quality Control

ERDC- EL-EP-C (Environmental Chemistry Branch)

\begin{tabular}{|c|c|c|c|c|c|c|c|c|c|c|}
\hline & & eporting & & Spike & Source & & $\%$ REC & & RPD & \\
\hline Analyte & Result & Limit & Units & Level & Result & $\%$ REC & Limits & RPD & Limit & Notes \\
\hline
\end{tabular}

Batch B906052 - EPA 1631

\begin{tabular}{llllllll}
\hline Matrix Spike Dup (B906052-MSD7) & \multicolumn{3}{c}{ Source: 9062302-01 } & \multicolumn{2}{c}{ Prepared: 17-Jun-2009 Analyzed: 01-Jul-2009 } \\
\hline Mercury & 0.000327 & 0.000005 & $\mathrm{mg} / \mathrm{L}$ & $1.000 \mathrm{E}-4$ & 0.000037 & 290 & $75-125$
\end{tabular}

Batch B906092 - Default Prep Metals

\begin{tabular}{|c|c|c|c|c|c|}
\hline \multirow{2}{*}{$\begin{array}{l}\text { Blank (B906092-BLK1) } \\
\text { Antimony }\end{array}$} & \multicolumn{5}{|c|}{ Prepared \& Analyzed: 17-Jun-2009 } \\
\hline & 0.0019 & 0.0020 & $\mathrm{mg} / \mathrm{L}$ & & $\overline{\mathrm{J}}$ \\
\hline Arsenic & ND & 0.0020 & $\mathrm{mg} / \mathrm{L}$ & & $\mathrm{U}$ \\
\hline Barium & 0.0028 & 0.0020 & $\mathrm{mg} / \mathrm{L}$ & & \\
\hline Beryllium & ND & 0.0020 & $\mathrm{mg} / \mathrm{L}$ & & $\mathrm{U}$ \\
\hline Cadmium & ND & 0.0020 & $\mathrm{mg} / \mathrm{L}$ & & $\mathrm{U}$ \\
\hline Chromium & 0.0004 & 0.0020 & $\mathrm{mg} / \mathrm{L}$ & & $\mathrm{J}$ \\
\hline Cobalt & ND & 0.0020 & $\mathrm{mg} / \mathrm{L}$ & & $\mathrm{U}$ \\
\hline Copper & 0.0029 & 0.0020 & $\mathrm{mg} / \mathrm{L}$ & & \\
\hline Lead & 0.0005 & 0.0020 & $\mathrm{mg} / \mathrm{L}$ & & $\mathrm{J}$ \\
\hline Manganese & 0.0013 & 0.0020 & $\mathrm{mg} / \mathrm{L}$ & & $\mathrm{J}$ \\
\hline Molybdenum & $\mathrm{ND}$ & 0.0020 & $\mathrm{mg} / \mathrm{L}$ & & U \\
\hline Nickel & 0.0010 & 0.0020 & $\mathrm{mg} / \mathrm{L}$ & & $\mathrm{J}$ \\
\hline Selenium & ND & 0.0020 & $\mathrm{mg} / \mathrm{L}$ & & U \\
\hline Silver & 0.0126 & 0.0020 & $\mathrm{mg} / \mathrm{L}$ & & \\
\hline Thallium & ND & 0.0020 & $\mathrm{mg} / \mathrm{L}$ & & U \\
\hline Vanadium & ND & 0.0020 & $\mathrm{mg} / \mathrm{L}$ & & $\mathrm{U}$ \\
\hline Zinc & 0.0165 & 0.0020 & $\mathrm{mg} / \mathrm{L}$ & & \\
\hline Blank (B906092-BLK2) & \multicolumn{5}{|c|}{ Prepared \& Analyzed: 17-Jun-2009 } \\
\hline Antimony & 0.0009 & 0.0020 & $\mathrm{mg} / \mathrm{L}$ & & $\mathrm{J}$ \\
\hline Arsenic & ND & 0.0020 & $\mathrm{mg} / \mathrm{L}$ & & $\mathrm{U}$ \\
\hline Barium & 0.0025 & 0.0020 & $\mathrm{mg} / \mathrm{L}$ & & \\
\hline Beryllium & ND & 0.0020 & $\mathrm{mg} / \mathrm{L}$ & & $\mathrm{U}$ \\
\hline Cadmium & ND & 0.0020 & $\mathrm{mg} / \mathrm{L}$ & & U \\
\hline Chromium & 0.0012 & 0.0020 & $\mathrm{mg} / \mathrm{L}$ & & $\mathrm{J}$ \\
\hline Cobalt & ND & 0.0020 & $\mathrm{mg} / \mathrm{L}$ & & U \\
\hline Copper & 0.0021 & 0.0020 & $\mathrm{mg} / \mathrm{L}$ & & \\
\hline Lead & 0.0006 & 0.0020 & $\mathrm{mg} / \mathrm{L}$ & & $\mathrm{J}$ \\
\hline Manganese & 0.0012 & 0.0020 & $\mathrm{mg} / \mathrm{L}$ & & $\mathrm{J}$ \\
\hline Molybdenum & ND & 0.0020 & $\mathrm{mg} / \mathrm{L}$ & & U \\
\hline Nickel & 0.0010 & 0.0020 & $\mathrm{mg} / \mathrm{L}$ & & $\mathrm{J}$ \\
\hline Selenium & ND & 0.0020 & $\mathrm{mg} / \mathrm{L}$ & & U \\
\hline Silver & 0.0038 & 0.0020 & $\mathrm{mg} / \mathrm{L}$ & & \\
\hline Thallium & ND & 0.0020 & $\mathrm{mg} / \mathrm{L}$ & & $\mathrm{U}$ \\
\hline Vanadium & ND & 0.0020 & $\mathrm{mg} / \mathrm{L}$ & & U \\
\hline Zinc & 0.0233 & 0.0020 & $\mathrm{mg} / \mathrm{L}$ & & \\
\hline
\end{tabular}




\section{USACE ERDC-EP-C \\ 3909 Halls Ferry Road \\ Vicksburg, MS 39180-6199}

ERDC - ECB
Project: TVA Fly Ash Project

Project Manager: Tony Bednar
Reported:

03-Sep-2009

DRAFT: Metals by EPA 6000/7000 Series Methods - Quality Control

ERDC- EL-EP-C (Environmental Chemistry Branch)

\begin{tabular}{|c|c|c|c|c|c|c|c|c|c|c|}
\hline & & Reporting & & Spike & Source & & $\%$ REC & & RPD & \\
\hline Analyte & Result & Limit & Units & Level & Result & $\%$ REC & Limits & RPD & Limit & Notes \\
\hline
\end{tabular}

Batch B906092 - Default Prep Metals

\begin{tabular}{|c|c|c|c|c|c|c|c|}
\hline \multirow{2}{*}{$\frac{\text { LCS (B906092-BS1) }}{\text { Antimony }}$} & \multicolumn{7}{|c|}{ Prepared \& Analyzed: 17-Jun-2009 } \\
\hline & 5.79 & 0.0020 & $\mathrm{mg} / \mathrm{L}$ & 4.800 & 121 & $80-120$ & \\
\hline Arsenic & 2.02 & 0.0020 & $\mathrm{mg} / \mathrm{L}$ & 2.400 & 84.2 & $80-120$ & \\
\hline Beryllium & 1.12 & 0.0020 & $\mathrm{mg} / \mathrm{L}$ & 1.200 & 92.9 & $80-120$ & \\
\hline Cadmium & 1.05 & 0.0020 & $\mathrm{mg} / \mathrm{L}$ & 1.200 & 87.9 & $80-120$ & \\
\hline Chromium & 5.87 & 0.0020 & $\mathrm{mg} / \mathrm{L}$ & 4.800 & 122 & $80-120$ & \\
\hline Cobalt & 6.10 & 0.0020 & $\mathrm{mg} / \mathrm{L}$ & 4.800 & 127 & $80-120$ & \\
\hline Copper & 5.73 & 0.0020 & $\mathrm{mg} / \mathrm{L}$ & 4.800 & 119 & $80-120$ & B \\
\hline Lead & 3.46 & 0.0020 & $\mathrm{mg} / \mathrm{L}$ & 2.400 & 144 & $80-120$ & \\
\hline Manganese & 5.27 & 0.0020 & $\mathrm{mg} / \mathrm{L}$ & 4.800 & 110 & $80-120$ & \\
\hline Molybdenum & ND & 0.0020 & $\mathrm{mg} / \mathrm{L}$ & & & $80-120$ & $\mathrm{U}$ \\
\hline Nickel & 4.65 & 0.0020 & $\mathrm{mg} / \mathrm{L}$ & 4.800 & 97.0 & $80-120$ & \\
\hline Selenium & 1.08 & 0.0020 & $\mathrm{mg} / \mathrm{L}$ & 1.200 & 89.8 & $80-120$ & \\
\hline Thallium & 1.81 & 0.0020 & $\mathrm{mg} / \mathrm{L}$ & 1.200 & 151 & $80-120$ & \\
\hline Vanadium & 5.70 & 0.0020 & $\mathrm{mg} / \mathrm{L}$ & 4.800 & 119 & $80-120$ & \\
\hline Zinc & 11.8 & 0.0020 & $\mathrm{mg} / \mathrm{L}$ & 12.00 & 98.4 & $80-120$ & B \\
\hline LCS (B906092-BS2) & \multicolumn{7}{|c|}{ Prepared \& Analyzed: 17-Jun-2009 } \\
\hline Antimony & 5.78 & 0.0020 & $\mathrm{mg} / \mathrm{L}$ & 4.800 & 120 & $80-120$ & \\
\hline Arsenic & 2.07 & 0.0020 & $\mathrm{mg} / \mathrm{L}$ & 2.400 & 86.0 & $80-120$ & \\
\hline Barium & 16.2 & 0.0020 & $\mathrm{mg} / \mathrm{L}$ & 12.00 & 135 & $80-120$ & B \\
\hline Beryllium & 1.07 & 0.0020 & $\mathrm{mg} / \mathrm{L}$ & 1.200 & 89.3 & $80-120$ & \\
\hline Cadmium & 1.07 & 0.0020 & $\mathrm{mg} / \mathrm{L}$ & 1.200 & 88.8 & $80-120$ & \\
\hline Chromium & 5.87 & 0.0020 & $\mathrm{mg} / \mathrm{L}$ & 4.800 & 122 & $80-120$ & \\
\hline Cobalt & 5.80 & 0.0020 & $\mathrm{mg} / \mathrm{L}$ & 4.800 & 121 & $80-120$ & \\
\hline Copper & 5.60 & 0.0020 & $\mathrm{mg} / \mathrm{L}$ & 4.800 & 117 & $80-120$ & B \\
\hline Molybdenum & ND & 0.0020 & $\mathrm{mg} / \mathrm{L}$ & & & $80-120$ & $\mathrm{U}$ \\
\hline Nickel & 5.76 & 0.0020 & $\mathrm{mg} / \mathrm{L}$ & 4.800 & 120 & $80-120$ & \\
\hline Selenium & 1.11 & 0.0020 & $\mathrm{mg} / \mathrm{L}$ & 1.200 & 92.6 & $80-120$ & \\
\hline Silver & 1.47 & 0.0020 & $\mathrm{mg} / \mathrm{L}$ & 1.200 & 123 & $80-120$ & B \\
\hline Thallium & 1.88 & 0.0020 & $\mathrm{mg} / \mathrm{L}$ & 1.200 & 157 & $80-120$ & \\
\hline Vanadium & 5.73 & 0.0020 & $\mathrm{mg} / \mathrm{L}$ & 4.800 & 119 & $80-120$ & \\
\hline Zinc & 11.4 & 0.0020 & $\mathrm{mg} / \mathrm{L}$ & 12.00 & 95.3 & $80-120$ & B \\
\hline
\end{tabular}




\section{USACE ERDC-EP-C \\ 3909 Halls Ferry Road \\ Vicksburg, MS 39180-6199}

ERDC - ECB
Project: TVA Fly Ash Project

Project Manager: Tony Bednar
Reported:

03-Sep-2009

DRAFT: Metals by EPA 6000/7000 Series Methods - Quality Control

ERDC- EL-EP-C (Environmental Chemistry Branch)

\begin{tabular}{|c|c|c|c|c|c|c|c|c|c|c|}
\hline & & eporting & & Spike & Source & & $\%$ REC & & RPD & \\
\hline Analyte & Result & Limit & Units & Level & Result & $\%$ REC & Limits & RPD & Limit & Notes \\
\hline
\end{tabular}

Batch B906092 - Default Prep Metals

\begin{tabular}{|c|c|c|c|c|c|c|c|c|c|c|}
\hline \multirow{2}{*}{$\begin{array}{l}\text { Duplicate (B906092-DUP1) } \\
\text { Antimony }\end{array}$} & \multicolumn{3}{|c|}{ Source: $9061502-76$} & \multicolumn{7}{|c|}{ Prepared \& Analyzed: 17-Jun-2009 } \\
\hline & 0.0126 & 0.0020 & $\mathrm{mg} / \mathrm{L}$ & & 0.0041 & & & 102 & 20 & \\
\hline Arsenic & 0.107 & 0.0020 & $\mathrm{mg} / \mathrm{L}$ & & 0.106 & & & 1.55 & 20 & \\
\hline Barium & 0.512 & 0.0020 & $\mathrm{mg} / \mathrm{L}$ & & 0.513 & & & 0.257 & 20 & B \\
\hline Beryllium & 0.0044 & 0.0020 & $\mathrm{mg} / \mathrm{L}$ & & 0.0042 & & & 5.26 & 20 & \\
\hline Cadmium & 0.0008 & 0.0020 & $\mathrm{mg} / \mathrm{L}$ & & 0.0010 & & & 27.0 & 20 & $\mathrm{~J}$ \\
\hline Chromium & 0.0339 & 0.0020 & $\mathrm{mg} / \mathrm{L}$ & & 0.0338 & & & 0.377 & 20 & \\
\hline Cobalt & 0.0183 & 0.0020 & $\mathrm{mg} / \mathrm{L}$ & & 0.0188 & & & 2.79 & 20 & \\
\hline Copper & 0.0681 & 0.0020 & $\mathrm{mg} / \mathrm{L}$ & & 0.0675 & & & 0.909 & 20 & B \\
\hline Lead & 0.0381 & 0.0020 & $\mathrm{mg} / \mathrm{L}$ & & 0.0380 & & & 0.269 & 20 & \\
\hline Manganese & 0.0980 & 0.0020 & $\mathrm{mg} / \mathrm{L}$ & & 0.104 & & & 6.33 & 20 & \\
\hline Molybdenum & 0.0057 & 0.0020 & $\mathrm{mg} / \mathrm{L}$ & & 0.0055 & & & 2.14 & 20 & \\
\hline Nickel & 0.0381 & 0.0020 & $\mathrm{mg} / \mathrm{L}$ & & 0.0377 & & & 1.01 & 20 & \\
\hline Selenium & 0.0050 & 0.0020 & $\mathrm{mg} / \mathrm{L}$ & & 0.0044 & & & 12.1 & 20 & \\
\hline Silver & ND & 0.0020 & $\mathrm{mg} / \mathrm{L}$ & & ND & & & & 20 & $\mathrm{U}$ \\
\hline Thallium & 0.0026 & 0.0020 & $\mathrm{mg} / \mathrm{L}$ & & 0.0025 & & & 3.55 & 20 & \\
\hline Vanadium & 0.105 & 0.0020 & $\mathrm{mg} / \mathrm{L}$ & & 0.110 & & & 4.41 & 20 & \\
\hline Zinc & 0.0810 & 0.0020 & $\mathrm{mg} / \mathrm{L}$ & & 0.0917 & & & 12.5 & 20 & B \\
\hline Matrix Spike (B906092-MS1) & \multicolumn{3}{|c|}{ Source: $9061502-76$} & \multicolumn{4}{|c|}{ Prepared \& Analyzed: 17-Jun-2009 } & & & \\
\hline Antimony & 5.79 & 0.0020 & $\mathrm{mg} / \mathrm{L}$ & 4.800 & 0.0041 & 121 & $80-120$ & & & \\
\hline Arsenic & 2.23 & 0.0020 & $\mathrm{mg} / \mathrm{L}$ & 2.400 & 0.106 & 88.7 & $80-120$ & & & \\
\hline Barium & 16.5 & 0.0020 & $\mathrm{mg} / \mathrm{L}$ & 12.00 & 0.513 & 133 & $80-120$ & & & B \\
\hline Beryllium & 0.955 & 0.0020 & $\mathrm{mg} / \mathrm{L}$ & 1.200 & 0.0042 & 79.2 & $80-120$ & & & \\
\hline Cadmium & 1.03 & 0.0020 & $\mathrm{mg} / \mathrm{L}$ & 1.200 & 0.0010 & 86.0 & $80-120$ & & & \\
\hline Chromium & 5.25 & 0.0020 & $\mathrm{mg} / \mathrm{L}$ & 4.800 & 0.0338 & 109 & $80-120$ & & & \\
\hline Cobalt & 5.32 & 0.0020 & $\mathrm{mg} / \mathrm{L}$ & 4.800 & 0.0188 & 110 & $80-120$ & & & \\
\hline Copper & 5.32 & 0.0020 & $\mathrm{mg} / \mathrm{L}$ & 4.800 & 0.0675 & 109 & $80-120$ & & & B \\
\hline Lead & 3.45 & 0.0020 & $\mathrm{mg} / \mathrm{L}$ & 2.400 & 0.0380 & 142 & $80-120$ & & & \\
\hline Manganese & 4.78 & 0.0020 & $\mathrm{mg} / \mathrm{L}$ & 4.800 & 0.104 & 97.4 & $80-120$ & & & \\
\hline Molybdenum & 0.0057 & 0.0020 & $\mathrm{mg} / \mathrm{L}$ & & 0.0055 & & $80-120$ & & & \\
\hline Nickel & 5.35 & 0.0020 & $\mathrm{mg} / \mathrm{L}$ & 4.800 & 0.0377 & 111 & $80-120$ & & & \\
\hline Selenium & 1.09 & 0.0020 & $\mathrm{mg} / \mathrm{L}$ & 1.200 & 0.0044 & 90.5 & $80-120$ & & & \\
\hline Silver & 1.53 & 0.0020 & $\mathrm{mg} / \mathrm{L}$ & 1.200 & ND & 127 & $80-120$ & & & B \\
\hline Thallium & 1.79 & 0.0020 & $\mathrm{mg} / \mathrm{L}$ & 1.200 & 0.0025 & 149 & $80-120$ & & & \\
\hline Vanadium & 5.29 & 0.0020 & $\mathrm{mg} / \mathrm{L}$ & 4.800 & 0.110 & 108 & $80-120$ & & & \\
\hline Zinc & 10.9 & 0.0020 & $\mathrm{mg} / \mathrm{L}$ & 12.00 & 0.0917 & 90.3 & $80-120$ & & & B \\
\hline
\end{tabular}




\section{USACE ERDC-EP-C \\ 3909 Halls Ferry Road \\ Vicksburg, MS 39180-6199}

ERDC - ECB

Project: TVA Fly Ash Project

Reported:

Project Manager: Tony Bednar

03-Sep-2009

DRAFT: Metals by EPA 6000/7000 Series Methods - Quality Control

ERDC- EL-EP-C (Environmental Chemistry Branch)

\begin{tabular}{|lcccccccccccc}
\hline & & Reporting & & Spike & Source & & $\%$ REC & & RPD \\
Analyte & Result & Limit & Units & Level & Result & $\%$ REC & Limits & RPD & Limit & Notes \\
\hline
\end{tabular}

Batch B906093 - Default Prep Metals

\begin{tabular}{|c|c|c|c|c|}
\hline Blank (B906093-BLK1) & & & & Prepared \& Analyzed: 19-Jun-2009 \\
\hline Antimony & 0.0005 & 0.0010 & $\mathrm{mg} / \mathrm{L}$ & $\overline{\mathrm{J}}$ \\
\hline Arsenic & ND & 0.0010 & $\mathrm{mg} / \mathrm{L}$ & $\mathrm{U}$ \\
\hline Barium & ND & 0.0010 & $\mathrm{mg} / \mathrm{L}$ & $\mathrm{U}$ \\
\hline Beryllium & $\mathrm{ND}$ & 0.0010 & $\mathrm{mg} / \mathrm{L}$ & $\mathrm{U}$ \\
\hline Cadmium & ND & 0.0010 & $\mathrm{mg} / \mathrm{L}$ & $\mathrm{U}$ \\
\hline Chromium & ND & 0.0010 & $\mathrm{mg} / \mathrm{L}$ & $\mathrm{U}$ \\
\hline Cobalt & ND & 0.0010 & $\mathrm{mg} / \mathrm{L}$ & $\mathrm{U}$ \\
\hline Copper & ND & 0.0010 & $\mathrm{mg} / \mathrm{L}$ & $\mathrm{U}$ \\
\hline Lead & ND & 0.0010 & $\mathrm{mg} / \mathrm{L}$ & $\mathrm{U}$ \\
\hline Manganese & ND & 0.0010 & $\mathrm{mg} / \mathrm{L}$ & $\mathrm{U}$ \\
\hline Molybdenum & ND & 0.0010 & $\mathrm{mg} / \mathrm{L}$ & $\mathrm{U}$ \\
\hline Nickel & ND & 0.0010 & $\mathrm{mg} / \mathrm{L}$ & $\mathrm{U}$ \\
\hline Selenium & ND & 0.0010 & $\mathrm{mg} / \mathrm{L}$ & $\mathrm{U}$ \\
\hline Silver & 0.0038 & 0.0010 & $\mathrm{mg} / \mathrm{L}$ & \\
\hline Thallium & ND & 0.0010 & $\mathrm{mg} / \mathrm{L}$ & $\mathrm{U}$ \\
\hline Vanadium & ND & 0.0010 & $\mathrm{mg} / \mathrm{L}$ & $\mathrm{U}$ \\
\hline Zinc & ND & 0.0010 & $\mathrm{mg} / \mathrm{L}$ & $\mathrm{U}$ \\
\hline Blank (B906093-BLK2) & & & & Prepared: 19-Jun-2009 Analyzed: 26-Jun-2009 \\
\hline Antimony & 0.0006 & 0.0010 & $\mathrm{mg} / \mathrm{L}$ & $\mathrm{J}$ \\
\hline Arsenic & ND & 0.0010 & $\mathrm{mg} / \mathrm{L}$ & $\mathrm{U}$ \\
\hline Barium & ND & 0.0010 & $\mathrm{mg} / \mathrm{L}$ & $\mathrm{U}$ \\
\hline Beryllium & ND & 0.0010 & $\mathrm{mg} / \mathrm{L}$ & $\mathrm{U}$ \\
\hline Cadmium & ND & 0.0010 & $\mathrm{mg} / \mathrm{L}$ & $\mathrm{U}$ \\
\hline Chromium & ND & 0.0010 & $\mathrm{mg} / \mathrm{L}$ & $\mathrm{U}$ \\
\hline Cobalt & ND & 0.0010 & $\mathrm{mg} / \mathrm{L}$ & $\mathrm{U}$ \\
\hline Copper & ND & 0.0010 & $\mathrm{mg} / \mathrm{L}$ & $\mathrm{U}$ \\
\hline Lead & ND & 0.0010 & $\mathrm{mg} / \mathrm{L}$ & $\mathrm{U}$ \\
\hline Manganese & ND & 0.0010 & $\mathrm{mg} / \mathrm{L}$ & $\mathrm{U}$ \\
\hline Molybdenum & ND & 0.0010 & $\mathrm{mg} / \mathrm{L}$ & $\mathrm{U}$ \\
\hline Nickel & ND & 0.0010 & $\mathrm{mg} / \mathrm{L}$ & $\mathrm{U}$ \\
\hline Selenium & ND & 0.0010 & $\mathrm{mg} / \mathrm{L}$ & $\mathrm{U}$ \\
\hline Silver & ND & 0.0010 & $\mathrm{mg} / \mathrm{L}$ & $\mathrm{U}$ \\
\hline Thallium & ND & 0.0010 & $\mathrm{mg} / \mathrm{L}$ & $\mathrm{U}$ \\
\hline Vanadium & ND & 0.0010 & $\mathrm{mg} / \mathrm{L}$ & $\mathrm{U}$ \\
\hline Zinc & 0.0014 & 0.0010 & $\mathrm{mg} / \mathrm{L}$ & \\
\hline
\end{tabular}




\section{USACE ERDC-EP-C \\ 3909 Halls Ferry Road \\ Vicksburg, MS 39180-6199}

ERDC - ECB

$-,-$
Project: TVA Fly Ash Project

Project Manager: Tony Bednar
Reported:

03-Sep-2009

\section{DRAFT: Metals by EPA 6000/7000 Series Methods - Quality Control}

ERDC- EL-EP-C (Environmental Chemistry Branch)

\begin{tabular}{|c|c|c|c|c|c|c|c|c|c|c|}
\hline & & porting & & Spike & Source & & $\%$ REC & & RPD & \\
\hline Analyte & Result & Limit & Units & Level & Result & $\%$ REC & Limits & RPD & Limit & Notes \\
\hline
\end{tabular}

\section{Batch B906093 - Default Prep Metals}

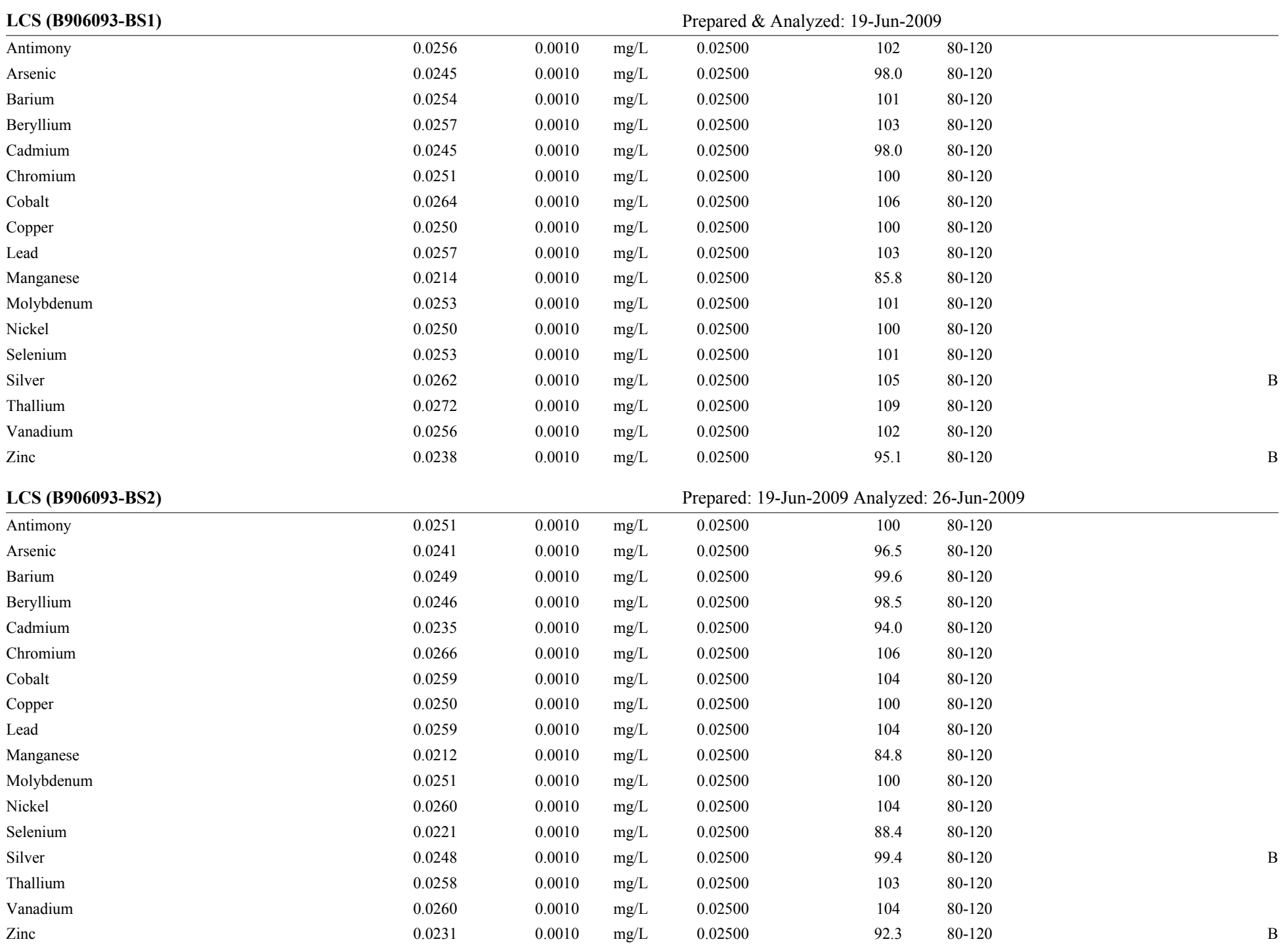




\section{USACE ERDC-EP-C \\ 3909 Halls Ferry Road \\ Vicksburg, MS 39180-6199}

ERDC - ECB
Project: TVA Fly Ash Project

Project Manager: Tony Bednar
Reported:

03-Sep-2009

DRAFT: Metals by EPA 6000/7000 Series Methods - Quality Control

ERDC- EL-EP-C (Environmental Chemistry Branch)

\begin{tabular}{|lcccccccccccc}
\hline & & Reporting & & Spike & Source & & $\%$ REC & & RPD \\
Analyte & Result & Limit & Units & Level & Result & $\%$ REC & Limits & RPD & Limit & Notes \\
\hline
\end{tabular}

Batch B906093 - Default Prep Metals

\begin{tabular}{|c|c|c|c|c|c|c|c|}
\hline \multirow{2}{*}{$\begin{array}{l}\text { Duplicate (B906093-DUP1) } \\
\text { Antimony }\end{array}$} & \multicolumn{3}{|c|}{ Source: 9061701-01 } & \multicolumn{4}{|c|}{ Prepared \& Analyzed: 19-Jun-2009 } \\
\hline & 0.0016 & 0.0010 & $\mathrm{mg} / \mathrm{L}$ & 0.0017 & 3.86 & 20 & \\
\hline Arsenic & 0.0507 & 0.0010 & $\mathrm{mg} / \mathrm{L}$ & 0.0508 & 0.147 & 20 & \\
\hline Barium & 0.113 & 0.0010 & $\mathrm{mg} / \mathrm{L}$ & 0.109 & 3.09 & 20 & \\
\hline Beryllium & ND & 0.0010 & $\mathrm{mg} / \mathrm{L}$ & ND & & 20 & $\mathrm{U}$ \\
\hline Cadmium & ND & 0.0010 & $\mathrm{mg} / \mathrm{L}$ & ND & & 20 & $\mathrm{U}$ \\
\hline Chromium & 0.0013 & 0.0010 & $\mathrm{mg} / \mathrm{L}$ & 0.0012 & 4.28 & 20 & \\
\hline Cobalt & ND & 0.0010 & $\mathrm{mg} / \mathrm{L}$ & ND & & 20 & $\mathrm{U}$ \\
\hline Copper & 0.0024 & 0.0010 & $\mathrm{mg} / \mathrm{L}$ & 0.0025 & 3.77 & 20 & \\
\hline Lead & ND & 0.0010 & $\mathrm{mg} / \mathrm{L}$ & ND & & 20 & $\mathrm{U}$ \\
\hline Manganese & 0.0012 & 0.0010 & $\mathrm{mg} / \mathrm{L}$ & 0.0012 & 2.11 & 20 & \\
\hline Molybdenum & 0.0021 & 0.0010 & $\mathrm{mg} / \mathrm{L}$ & 0.0022 & 2.44 & 20 & \\
\hline Nickel & 0.0006 & 0.0010 & $\mathrm{mg} / \mathrm{L}$ & 0.0008 & 26.6 & 20 & $\mathrm{~J}$ \\
\hline Selenium & 0.0033 & 0.0010 & $\mathrm{mg} / \mathrm{L}$ & 0.0034 & 2.42 & 20 & \\
\hline Silver & 0.0006 & 0.0010 & $\mathrm{mg} / \mathrm{L}$ & 0.0008 & 29.4 & 20 & $\mathrm{~J}, \mathrm{~B}$ \\
\hline Thallium & 0.0006 & 0.0010 & $\mathrm{mg} / \mathrm{L}$ & 0.0005 & 2.43 & 20 & $\mathrm{~J}$ \\
\hline Vanadium & 0.0296 & 0.0010 & $\mathrm{mg} / \mathrm{L}$ & 0.0298 & 0.602 & 20 & \\
\hline Zinc & 0.0056 & 0.0010 & $\mathrm{mg} / \mathrm{L}$ & 0.0058 & 2.62 & 20 & B \\
\hline Duplicate (B906093-DUP2) & \multicolumn{3}{|c|}{ Source: 9062302-01 } & \multicolumn{3}{|c|}{ Prepared: 19-Jun-2009 Analyzed: 26-Jun-2009 } & \\
\hline Antimony & 0.0020 & 0.0010 & $\mathrm{mg} / \mathrm{L}$ & 0.0022 & 6.10 & 20 & \\
\hline Arsenic & 0.0640 & 0.0010 & $\mathrm{mg} / \mathrm{L}$ & 0.0633 & 1.09 & 20 & \\
\hline Barium & 0.125 & 0.0010 & $\mathrm{mg} / \mathrm{L}$ & 0.123 & 1.75 & 20 & \\
\hline Beryllium & $\mathrm{ND}$ & 0.0010 & $\mathrm{mg} / \mathrm{L}$ & ND & & 20 & $\mathrm{U}$ \\
\hline Cadmium & $\mathrm{ND}$ & 0.0010 & $\mathrm{mg} / \mathrm{L}$ & ND & & 20 & $\mathrm{U}$ \\
\hline Chromium & 0.0017 & 0.0010 & $\mathrm{mg} / \mathrm{L}$ & 0.0018 & 5.97 & 20 & \\
\hline Cobalt & ND & 0.0010 & $\mathrm{mg} / \mathrm{L}$ & ND & & 20 & $\mathrm{U}$ \\
\hline Copper & 0.0009 & 0.0010 & $\mathrm{mg} / \mathrm{L}$ & 0.0011 & 14.8 & 20 & $\mathrm{~J}$ \\
\hline Lead & ND & 0.0010 & $\mathrm{mg} / \mathrm{L}$ & ND & & 20 & $\mathrm{U}$ \\
\hline Manganese & 0.00042 & 0.0010 & $\mathrm{mg} / \mathrm{L}$ & 0.0005 & 20.0 & 20 & $\mathrm{~J}$ \\
\hline Molybdenum & 0.0027 & 0.0010 & $\mathrm{mg} / \mathrm{L}$ & 0.0027 & 0.877 & 20 & \\
\hline Nickel & 0.0004 & 0.0010 & $\mathrm{mg} / \mathrm{L}$ & 0.0004 & 5.39 & 20 & $\mathrm{~J}$ \\
\hline Selenium & 0.0065 & 0.0010 & $\mathrm{mg} / \mathrm{L}$ & 0.0064 & 1.73 & 20 & \\
\hline Silver & ND & 0.0010 & $\mathrm{mg} / \mathrm{L}$ & ND & & 20 & $\mathrm{U}$ \\
\hline Thallium & 0.0006 & 0.0010 & $\mathrm{mg} / \mathrm{L}$ & 0.0006 & 0.244 & 20 & $\mathrm{~J}$ \\
\hline Vanadium & 0.0384 & 0.0010 & $\mathrm{mg} / \mathrm{L}$ & 0.0387 & 0.865 & 20 & \\
\hline Zinc & 0.0032 & 0.0010 & $\mathrm{mg} / \mathrm{L}$ & 0.0041 & 22.1 & 20 & B \\
\hline
\end{tabular}




\section{USACE ERDC-EP-C \\ 3909 Halls Ferry Road \\ Vicksburg, MS 39180-6199}

ERDC - ECB

$-\cdot$,
Project: TVA Fly Ash Project

Reported:

Project Manager: Tony Bednar
03-Sep-2009

\section{DRAFT: Metals by EPA 6000/7000 Series Methods - Quality Control}

ERDC- EL-EP-C (Environmental Chemistry Branch)

\begin{tabular}{|c|c|c|c|c|c|c|c|c|c|c|}
\hline & & porting & & Spike & Source & & $\%$ REC & & RPD & \\
\hline Analyte & Result & Limit & Units & Level & Result & $\%$ REC & Limits & RPD & Limit & Notes \\
\hline
\end{tabular}

\section{Batch B906093 - Default Prep Metals}

\begin{tabular}{|c|c|c|c|c|c|c|c|c|}
\hline \multirow{2}{*}{$\begin{array}{l}\text { Matrix Spike (B906093-MS1) } \\
\text { Antimony }\end{array}$} & \multicolumn{3}{|c|}{ Source: $9061701-01$} & \multicolumn{5}{|c|}{ Prepared \& Analyzed: 19-Jun-2009 } \\
\hline & 0.0427 & 0.0010 & $\mathrm{mg} / \mathrm{L}$ & 0.04000 & 0.0017 & 103 & $80-120$ & \\
\hline Arsenic & 0.0887 & 0.0010 & $\mathrm{mg} / \mathrm{L}$ & 0.04000 & 0.0508 & 94.9 & $80-120$ & \\
\hline Beryllium & 0.0427 & 0.0010 & $\mathrm{mg} / \mathrm{L}$ & 0.04000 & ND & 107 & $80-120$ & \\
\hline Cadmium & 0.0423 & 0.0010 & $\mathrm{mg} / \mathrm{L}$ & 0.04000 & ND & 106 & $80-120$ & \\
\hline Chromium & 0.0425 & 0.0010 & $\mathrm{mg} / \mathrm{L}$ & 0.04000 & 0.0012 & 103 & $80-120$ & \\
\hline Cobalt & 0.0411 & 0.0010 & $\mathrm{mg} / \mathrm{L}$ & 0.04000 & ND & 103 & $80-120$ & \\
\hline Copper & 0.0416 & 0.0010 & $\mathrm{mg} / \mathrm{L}$ & 0.04000 & 0.0025 & 97.9 & $80-120$ & \\
\hline Lead & 0.0403 & 0.0010 & $\mathrm{mg} / \mathrm{L}$ & 0.04000 & ND & 101 & $80-120$ & \\
\hline Manganese & 0.0335 & 0.0010 & $\mathrm{mg} / \mathrm{L}$ & 0.04000 & 0.0012 & 80.8 & $80-120$ & \\
\hline Molybdenum & 0.0417 & 0.0010 & $\mathrm{mg} / \mathrm{L}$ & 0.04000 & 0.0022 & 98.8 & $80-120$ & \\
\hline Nickel & 0.0402 & 0.0010 & $\mathrm{mg} / \mathrm{L}$ & 0.04000 & 0.0008 & 98.6 & $80-120$ & \\
\hline Selenium & 0.0460 & 0.0010 & $\mathrm{mg} / \mathrm{L}$ & 0.04000 & 0.0034 & 107 & $80-120$ & \\
\hline Thallium & 0.0436 & 0.0010 & $\mathrm{mg} / \mathrm{L}$ & 0.04000 & 0.0005 & 108 & $80-120$ & \\
\hline Vanadium & 0.0677 & 0.0010 & $\mathrm{mg} / \mathrm{L}$ & 0.04000 & 0.0298 & 94.9 & $80-120$ & \\
\hline Zinc & 0.0484 & 0.0010 & $\mathrm{mg} / \mathrm{L}$ & 0.04000 & 0.0058 & 107 & $80-120$ & B \\
\hline Matrix Spike (B906093-MS2) & \multicolumn{3}{|c|}{ Source: $9062302-01$} & \multicolumn{4}{|c|}{ Prepared: 19-Jun-2009 Analyzed: 26-Jun-2009 } & \\
\hline Antimony & 0.0417 & 0.0010 & $\mathrm{mg} / \mathrm{L}$ & 0.04000 & 0.0022 & 98.7 & $80-120$ & \\
\hline Arsenic & 0.105 & 0.0010 & $\mathrm{mg} / \mathrm{L}$ & 0.04000 & 0.0633 & 105 & $80-120$ & \\
\hline Barium & 0.158 & 0.0010 & $\mathrm{mg} / \mathrm{L}$ & 0.04000 & 0.123 & 87.8 & $80-120$ & \\
\hline Beryllium & 0.0420 & 0.0010 & $\mathrm{mg} / \mathrm{L}$ & 0.04000 & ND & 105 & $80-120$ & \\
\hline Cadmium & 0.0394 & 0.0010 & $\mathrm{mg} / \mathrm{L}$ & 0.04000 & ND & 98.4 & $80-120$ & \\
\hline Chromium & 0.0466 & 0.0010 & $\mathrm{mg} / \mathrm{L}$ & 0.04000 & 0.0018 & 112 & $80-120$ & \\
\hline Cobalt & 0.0407 & 0.0010 & $\mathrm{mg} / \mathrm{L}$ & 0.04000 & ND & 102 & $80-120$ & \\
\hline Copper & 0.0416 & 0.0010 & $\mathrm{mg} / \mathrm{L}$ & 0.04000 & 0.0011 & 101 & $80-120$ & \\
\hline Molybdenum & 0.0434 & 0.0010 & $\mathrm{mg} / \mathrm{L}$ & 0.04000 & 0.0027 & 102 & $80-120$ & \\
\hline Nickel & 0.0435 & 0.0010 & $\mathrm{mg} / \mathrm{L}$ & 0.04000 & 0.0004 & 108 & $80-120$ & \\
\hline Selenium & 0.0488 & 0.0010 & $\mathrm{mg} / \mathrm{L}$ & 0.04000 & 0.0064 & 106 & $80-120$ & \\
\hline Silver & 0.0406 & 0.0010 & $\mathrm{mg} / \mathrm{L}$ & 0.04000 & ND & 101 & $80-120$ & B \\
\hline Thallium & 0.0407 & 0.0010 & $\mathrm{mg} / \mathrm{L}$ & 0.04000 & 0.0006 & 100 & $80-120$ & \\
\hline Vanadium & 0.0776 & 0.0010 & $\mathrm{mg} / \mathrm{L}$ & 0.04000 & 0.0387 & 97.3 & $80-120$ & \\
\hline Zinc & 0.0452 & 0.0010 & $\mathrm{mg} / \mathrm{L}$ & 0.04000 & 0.0041 & 103 & $80-120$ & B \\
\hline
\end{tabular}




\section{USACE ERDC-EP-C \\ 3909 Halls Ferry Road \\ Vicksburg, MS 39180-6199}

ERDC - ECB

Project: TVA Fly Ash Project

Reported:

Project Manager: Tony Bednar

03-Sep-2009

DRAFT: Metals by EPA 6000/7000 Series Methods - Quality Control

ERDC- EL-EP-C (Environmental Chemistry Branch)

\begin{tabular}{|c|c|c|c|c|c|c|c|c|c|c|}
\hline & & eporting & & Spike & Source & & $\%$ REC & & RPD & \\
\hline Analyte & Result & Limit & Units & Level & Result & $\%$ REC & Limits & RPD & Limit & Notes \\
\hline
\end{tabular}

Batch B906094 - Default Prep Metals

\begin{tabular}{|c|c|c|c|c|c|}
\hline Blank (B906094-BLK1) & & & & Prepared \& Analyzed: 19-Jun-2009 & \\
\hline Antimony & 0.0047 & 0.0020 & $\mathrm{mg} / \mathrm{L}$ & & \\
\hline Arsenic & ND & 0.0020 & $\mathrm{mg} / \mathrm{L}$ & & $\mathrm{U}$ \\
\hline Barium & 0.00063 & 0.0020 & $\mathrm{mg} / \mathrm{L}$ & & $\mathrm{J}$ \\
\hline Beryllium & $\mathrm{ND}$ & 0.0020 & $\mathrm{mg} / \mathrm{L}$ & & $\mathrm{U}$ \\
\hline Cadmium & ND & 0.0020 & $\mathrm{mg} / \mathrm{L}$ & & $\mathrm{U}$ \\
\hline Chromium & 0.0007 & 0.0020 & $\mathrm{mg} / \mathrm{L}$ & & $\mathrm{J}$ \\
\hline Cobalt & ND & 0.0020 & $\mathrm{mg} / \mathrm{L}$ & & $\mathrm{U}$ \\
\hline Copper & 0.0005 & 0.0020 & $\mathrm{mg} / \mathrm{L}$ & & $\mathrm{J}$ \\
\hline Lead & 0.0005 & 0.0020 & $\mathrm{mg} / \mathrm{L}$ & & $\mathrm{J}$ \\
\hline Manganese & ND & 0.0020 & $\mathrm{mg} / \mathrm{L}$ & & $\mathrm{U}$ \\
\hline Molybdenum & ND & 0.0020 & $\mathrm{mg} / \mathrm{L}$ & & $\mathrm{U}$ \\
\hline Nickel & ND & 0.0020 & $\mathrm{mg} / \mathrm{L}$ & & $\mathrm{U}$ \\
\hline Selenium & ND & 0.0020 & $\mathrm{mg} / \mathrm{L}$ & & $\mathrm{U}$ \\
\hline Silver & ND & 0.0020 & $\mathrm{mg} / \mathrm{L}$ & & $\mathrm{U}$ \\
\hline Thallium & ND & 0.0020 & $\mathrm{mg} / \mathrm{L}$ & & U \\
\hline Vanadium & ND & 0.0020 & $\mathrm{mg} / \mathrm{L}$ & & $\mathrm{U}$ \\
\hline Zinc & 0.0061 & 0.0020 & $\mathrm{mg} / \mathrm{L}$ & & \\
\hline Blank (B906094-BLK2) & & & & Prepared \& Analyzed: 26-Jun-2009 & \\
\hline Antimony & 0.0007 & 0.0020 & $\mathrm{mg} / \mathrm{L}$ & & $\mathrm{J}$ \\
\hline Arsenic & ND & 0.0020 & $\mathrm{mg} / \mathrm{L}$ & & $\mathrm{U}$ \\
\hline Barium & ND & 0.0020 & $\mathrm{mg} / \mathrm{L}$ & & $\mathrm{U}$ \\
\hline Beryllium & ND & 0.0020 & $\mathrm{mg} / \mathrm{L}$ & & $\mathrm{U}$ \\
\hline Cadmium & ND & 0.0020 & $\mathrm{mg} / \mathrm{L}$ & & $\mathrm{U}$ \\
\hline Chromium & ND & 0.0020 & $\mathrm{mg} / \mathrm{L}$ & & $\mathrm{U}$ \\
\hline Cobalt & ND & 0.0020 & $\mathrm{mg} / \mathrm{L}$ & & $\mathrm{U}$ \\
\hline Copper & ND & 0.0020 & $\mathrm{mg} / \mathrm{L}$ & & $\mathrm{U}$ \\
\hline Lead & ND & 0.0020 & $\mathrm{mg} / \mathrm{L}$ & & $\mathrm{U}$ \\
\hline Manganese & ND & 0.0020 & $\mathrm{mg} / \mathrm{L}$ & & $\mathrm{U}$ \\
\hline Molybdenum & ND & 0.0020 & $\mathrm{mg} / \mathrm{L}$ & & $\mathrm{U}$ \\
\hline Nickel & ND & 0.0020 & $\mathrm{mg} / \mathrm{L}$ & & $\mathrm{U}$ \\
\hline Selenium & ND & 0.0020 & $\mathrm{mg} / \mathrm{L}$ & & $\mathrm{U}$ \\
\hline Silver & ND & 0.0020 & $\mathrm{mg} / \mathrm{L}$ & & $\mathrm{U}$ \\
\hline Thallium & ND & 0.0020 & $\mathrm{mg} / \mathrm{L}$ & & $\mathrm{U}$ \\
\hline Vanadium & ND & 0.0020 & $\mathrm{mg} / \mathrm{L}$ & & U \\
\hline Zinc & 0.0301 & 0.0020 & $\mathrm{mg} / \mathrm{L}$ & & \\
\hline
\end{tabular}




\section{USACE ERDC-EP-C \\ 3909 Halls Ferry Road \\ Vicksburg, MS 39180-6199}

ERDC - ECB
Project: TVA Fly Ash Project

Project Manager: Tony Bednar
Reported:

03-Sep-2009

DRAFT: Metals by EPA 6000/7000 Series Methods - Quality Control

ERDC- EL-EP-C (Environmental Chemistry Branch)

\begin{tabular}{|c|c|c|c|c|c|c|c|c|c|c|}
\hline & & Reporting & & Spike & Source & & $\%$ REC & & RPD & \\
\hline Analyte & Result & Limit & Units & Level & Result & $\%$ REC & Limits & RPD & Limit & Notes \\
\hline
\end{tabular}

Batch B906094 - Default Prep Metals

\begin{tabular}{|c|c|c|c|c|c|c|c|}
\hline \multirow{2}{*}{$\frac{\text { LCS (B906094-BS1) }}{\text { Antimony }}$} & \multicolumn{7}{|c|}{ Prepared \& Analyzed: 19-Jun-2009 } \\
\hline & 1.22 & 0.0020 & $\mathrm{mg} / \mathrm{L}$ & 1.200 & 102 & $80-120$ & $\mathrm{~B}$ \\
\hline Arsenic & 0.606 & 0.0020 & $\mathrm{mg} / \mathrm{L}$ & 0.6000 & 101 & $80-120$ & \\
\hline Beryllium & 0.321 & 0.0020 & $\mathrm{mg} / \mathrm{L}$ & 0.3000 & 107 & $80-120$ & \\
\hline Cadmium & 0.308 & 0.0020 & $\mathrm{mg} / \mathrm{L}$ & 0.3000 & 103 & $80-120$ & \\
\hline Chromium & 1.28 & 0.0020 & $\mathrm{mg} / \mathrm{L}$ & 1.200 & 106 & $80-120$ & \\
\hline Cobalt & 1.26 & 0.0020 & $\mathrm{mg} / \mathrm{L}$ & 1.200 & 105 & $80-120$ & \\
\hline Copper & 1.23 & 0.0020 & $\mathrm{mg} / \mathrm{L}$ & 1.200 & 103 & $80-120$ & \\
\hline Lead & 0.613 & 0.0020 & $\mathrm{mg} / \mathrm{L}$ & 0.6000 & 102 & $80-120$ & \\
\hline Manganese & 1.22 & 0.0020 & $\mathrm{mg} / \mathrm{L}$ & 1.200 & 101 & $80-120$ & \\
\hline Molybdenum & ND & 0.0020 & $\mathrm{mg} / \mathrm{L}$ & & & $80-120$ & $\mathrm{U}$ \\
\hline Nickel & 1.25 & 0.0020 & $\mathrm{mg} / \mathrm{L}$ & 1.200 & 104 & $80-120$ & \\
\hline Selenium & 0.302 & 0.0020 & $\mathrm{mg} / \mathrm{L}$ & 0.3000 & 101 & $80-120$ & \\
\hline Thallium & 0.329 & 0.0020 & $\mathrm{mg} / \mathrm{L}$ & 0.3000 & 110 & $80-120$ & \\
\hline Vanadium & 1.36 & 0.0020 & $\mathrm{mg} / \mathrm{L}$ & 1.200 & 113 & $80-120$ & \\
\hline Zinc & 3.09 & 0.0020 & $\mathrm{mg} / \mathrm{L}$ & 3.000 & 103 & $80-120$ & B \\
\hline LCS (B906094-BS2) & \multicolumn{7}{|c|}{ Prepared \& Analyzed: 26-Jun-2009 } \\
\hline Antimony & 4.99 & 0.0020 & $\mathrm{mg} / \mathrm{L}$ & 4.800 & 104 & $80-120$ & B \\
\hline Arsenic & 2.44 & 0.0020 & $\mathrm{mg} / \mathrm{L}$ & 2.400 & 102 & $80-120$ & \\
\hline Barium & 12.2 & 0.0020 & $\mathrm{mg} / \mathrm{L}$ & 12.00 & 102 & $80-120$ & \\
\hline Beryllium & 1.19 & 0.0020 & $\mathrm{mg} / \mathrm{L}$ & 1.200 & 99.5 & $80-120$ & \\
\hline Cadmium & 1.19 & 0.0020 & $\mathrm{mg} / \mathrm{L}$ & 1.200 & 99.2 & $80-120$ & \\
\hline Chromium & 4.74 & 0.0020 & $\mathrm{mg} / \mathrm{L}$ & 4.800 & 98.7 & $80-120$ & \\
\hline Cobalt & 4.82 & 0.0020 & $\mathrm{mg} / \mathrm{L}$ & 4.800 & 100 & $80-120$ & \\
\hline Copper & 4.73 & 0.0020 & $\mathrm{mg} / \mathrm{L}$ & 4.800 & 98.4 & $80-120$ & \\
\hline Molybdenum & 0.0006 & 0.0020 & $\mathrm{mg} / \mathrm{L}$ & & & $80-120$ & $\mathrm{~J}$ \\
\hline Nickel & 4.76 & 0.0020 & $\mathrm{mg} / \mathrm{L}$ & 4.800 & 99.2 & $80-120$ & \\
\hline Selenium & 1.16 & 0.0020 & $\mathrm{mg} / \mathrm{L}$ & 1.200 & 96.9 & $80-120$ & \\
\hline Silver & 1.27 & 0.0020 & $\mathrm{mg} / \mathrm{L}$ & 1.200 & 106 & $80-120$ & \\
\hline Thallium & 1.31 & 0.0020 & $\mathrm{mg} / \mathrm{L}$ & 1.200 & 109 & $80-120$ & \\
\hline Vanadium & 5.66 & 0.0020 & $\mathrm{mg} / \mathrm{L}$ & 4.800 & 118 & $80-120$ & \\
\hline Zinc & 11.0 & 0.0020 & $\mathrm{mg} / \mathrm{L}$ & 12.00 & 91.6 & $80-120$ & B \\
\hline
\end{tabular}




\section{USACE ERDC-EP-C \\ 3909 Halls Ferry Road \\ Vicksburg, MS 39180-6199}

ERDC - ECB
Project: TVA Fly Ash Project

Project Manager: Tony Bednar
Reported:

03-Sep-2009

DRAFT: Metals by EPA 6000/7000 Series Methods - Quality Control

ERDC- EL-EP-C (Environmental Chemistry Branch)

\begin{tabular}{|c|c|c|c|c|c|c|c|c|c|c|}
\hline & & eporting & & Spike & Source & & $\%$ REC & & RPD & \\
\hline Analyte & Result & Limit & Units & Level & Result & $\%$ REC & Limits & RPD & Limit & Notes \\
\hline
\end{tabular}

Batch B906094 - Default Prep Metals

\begin{tabular}{|c|c|c|c|c|c|c|c|}
\hline Duplicate (B906094-DUP1) & \multicolumn{3}{|c|}{ Source: 9061701-13 } & \multicolumn{4}{|l|}{ Prepared \& Analyzed: 19-Jun-2009 } \\
\hline Antimony & 0.0063 & 0.0020 & $\mathrm{mg} / \mathrm{L}$ & 0.0149 & 81.5 & 20 & $\bar{B}$ \\
\hline Arsenic & 0.190 & 0.0020 & $\mathrm{mg} / \mathrm{L}$ & 0.182 & 4.46 & 20 & \\
\hline Barium & 1.03 & 0.0020 & $\mathrm{mg} / \mathrm{L}$ & 1.02 & 0.362 & 20 & \\
\hline Beryllium & 0.0081 & 0.0020 & $\mathrm{mg} / \mathrm{L}$ & 0.0087 & 6.66 & 20 & \\
\hline Cadmium & 0.0014 & 0.0020 & $\mathrm{mg} / \mathrm{L}$ & 0.0013 & 1.35 & 20 & $\mathrm{~J}$ \\
\hline Chromium & 0.0525 & 0.0020 & $\mathrm{mg} / \mathrm{L}$ & 0.0524 & 0.133 & 20 & \\
\hline Cobalt & 0.0351 & 0.0020 & $\mathrm{mg} / \mathrm{L}$ & 0.0358 & 1.83 & 20 & \\
\hline Copper & 0.117 & 0.0020 & $\mathrm{mg} / \mathrm{L}$ & 0.120 & 1.90 & 20 & \\
\hline Lead & 0.0998 & 0.0020 & $\mathrm{mg} / \mathrm{L}$ & 0.0975 & 2.28 & 20 & \\
\hline Manganese & 0.127 & 0.0020 & $\mathrm{mg} / \mathrm{L}$ & 0.124 & 2.19 & 20 & \\
\hline Molybdenum & 0.0096 & 0.0020 & $\mathrm{mg} / \mathrm{L}$ & 0.0095 & 1.02 & 20 & \\
\hline Nickel & 0.0678 & 0.0020 & $\mathrm{mg} / \mathrm{L}$ & 0.0701 & 3.37 & 20 & \\
\hline Selenium & 0.0101 & 0.0020 & $\mathrm{mg} / \mathrm{L}$ & 0.0098 & 3.66 & 20 & \\
\hline Silver & 0.0008 & 0.0020 & $\mathrm{mg} / \mathrm{L}$ & 0.0010 & 18.3 & 20 & $\mathrm{~J}$ \\
\hline Thallium & 0.0050 & 0.0020 & $\mathrm{mg} / \mathrm{L}$ & 0.0050 & 0.210 & 20 & \\
\hline Vanadium & 0.189 & 0.0020 & $\mathrm{mg} / \mathrm{L}$ & 0.184 & 2.36 & 20 & \\
\hline Zinc & 0.152 & 0.0020 & $\mathrm{mg} / \mathrm{L}$ & 0.146 & 3.81 & 20 & B \\
\hline Duplicate (B906094-DUP2) & \multicolumn{3}{|c|}{ Source: $9062302-13$} & Prepared \& Analyzed: 26-Jun-2009 & & & \\
\hline Antimony & 0.0057 & 0.0020 & $\mathrm{mg} / \mathrm{L}$ & 0.0069 & 18.7 & 20 & B \\
\hline Arsenic & 0.128 & 0.0020 & $\mathrm{mg} / \mathrm{L}$ & 0.132 & 3.43 & 20 & \\
\hline Barium & 0.548 & 0.0020 & $\mathrm{mg} / \mathrm{L}$ & 0.556 & 1.38 & 20 & \\
\hline Beryllium & 0.0033 & 0.0020 & $\mathrm{mg} / \mathrm{L}$ & 0.0033 & 2.50 & 20 & \\
\hline Cadmium & 0.0006 & 0.0020 & $\mathrm{mg} / \mathrm{L}$ & 0.0005 & 9.29 & 20 & $\mathrm{~J}$ \\
\hline Chromium & 0.0258 & 0.0020 & $\mathrm{mg} / \mathrm{L}$ & 0.0245 & 4.89 & 20 & \\
\hline Cobalt & 0.0142 & 0.0020 & $\mathrm{mg} / \mathrm{L}$ & 0.0142 & 0.318 & 20 & \\
\hline Copper & 0.0520 & 0.0020 & $\mathrm{mg} / \mathrm{L}$ & 0.0516 & 0.759 & 20 & \\
\hline Lead & 0.0393 & 0.0020 & $\mathrm{mg} / \mathrm{L}$ & 0.0399 & 1.54 & 20 & \\
\hline Manganese & 0.0794 & 0.0020 & $\mathrm{mg} / \mathrm{L}$ & 0.0774 & 2.54 & 20 & \\
\hline Molybdenum & 0.0066 & 0.0020 & $\mathrm{mg} / \mathrm{L}$ & 0.0067 & 1.89 & 20 & \\
\hline Nickel & 0.0274 & 0.0020 & $\mathrm{mg} / \mathrm{L}$ & 0.0262 & 4.70 & 20 & \\
\hline Selenium & 0.0070 & 0.0020 & $\mathrm{mg} / \mathrm{L}$ & 0.0072 & 1.82 & 20 & \\
\hline Silver & ND & 0.0020 & $\mathrm{mg} / \mathrm{L}$ & 0.0004 & & 20 & $\mathrm{U}$ \\
\hline Thallium & 0.0027 & 0.0020 & $\mathrm{mg} / \mathrm{L}$ & 0.0028 & 4.18 & 20 & \\
\hline Vanadium & 0.104 & 0.0020 & $\mathrm{mg} / \mathrm{L}$ & 0.106 & 1.89 & 20 & \\
\hline Zinc & 0.0681 & 0.0020 & $\mathrm{mg} / \mathrm{L}$ & 0.0678 & 0.340 & 20 & B \\
\hline
\end{tabular}




\section{USACE ERDC-EP-C \\ 3909 Halls Ferry Road \\ Vicksburg, MS 39180-6199}

ERDC - ECB

$-,-$
Project: TVA Fly Ash Project

Project Manager: Tony Bednar
Reported:

03-Sep-2009

\section{DRAFT: Metals by EPA 6000/7000 Series Methods - Quality Control}

ERDC- EL-EP-C (Environmental Chemistry Branch)

\begin{tabular}{|lcccccccccccc}
\hline & & Reporting & & Spike & Source & & $\%$ REC & & RPD \\
Analyte & Result & Limit & Units & Level & Result & $\%$ REC & Limits & RPD & Limit & Notes \\
\hline
\end{tabular}

Batch B906094 - Default Prep Metals

\begin{tabular}{lccccccc}
\hline Matrix Spike (B906094-MS1) & \multicolumn{3}{c}{ Source: } & $\mathbf{9 0 6 1 7 0 1 - 1 3}$ & \multicolumn{2}{c}{ Prepared \& Analyzed: 19-Jun-2009 } \\
\hline Antimony & 4.05 & 0.0020 & $\mathrm{mg} / \mathrm{L}$ & 4.800 & 0.0149 & 84.0 & $80-120$ \\
Arsenic & 2.71 & 0.0020 & $\mathrm{mg} / \mathrm{L}$ & 2.400 & 0.182 & 105 & $80-120$ \\
Barium & 13.3 & 0.0020 & $\mathrm{mg} / \mathrm{L}$ & 12.00 & 1.02 & 102 & $80-120$ \\
Beryllium & 1.27 & 0.0020 & $\mathrm{mg} / \mathrm{L}$ & 1.200 & 0.0087 & 105 & $80-120$ \\
Cadmium & 1.25 & 0.0020 & $\mathrm{mg} / \mathrm{L}$ & 1.200 & 0.0013 & 104 & $80-120$ \\
Chromium & 5.02 & 0.0020 & $\mathrm{mg} / \mathrm{L}$ & 4.800 & 0.0524 & 104 & $80-120$ \\
Cobalt & 5.02 & 0.0020 & $\mathrm{mg} / \mathrm{L}$ & 4.800 & 0.0358 & 104 & $80-120$ \\
Copper & 4.98 & 0.0020 & $\mathrm{mg} / \mathrm{L}$ & 4.800 & 0.120 & 101 & $80-120$ \\
Lead & 2.67 & 0.0020 & $\mathrm{mg} / \mathrm{L}$ & 2.400 & 0.0975 & 107 & $80-120$ \\
Manganese & 4.99 & 0.0020 & $\mathrm{mg} / \mathrm{L}$ & 4.800 & 0.124 & 101 & $80-120$ \\
Molybdenum & 0.0087 & 0.0020 & $\mathrm{mg} / \mathrm{L}$ & & 0.0095 & & $80-120$ \\
Nickel & 5.05 & 0.0020 & $\mathrm{mg} / \mathrm{L}$ & 4.800 & 0.0701 & 104 & $80-120$ \\
Selenium & 1.21 & 0.0020 & $\mathrm{mg} / \mathrm{L}$ & 1.200 & 0.0098 & 100 & $80-120$ \\
Silver & 0.329 & 0.0020 & $\mathrm{mg} / \mathrm{L}$ & 1.200 & 0.0010 & 27.3 & $80-120$ \\
Thallium & 1.32 & 0.0020 & $\mathrm{mg} / \mathrm{L}$ & 1.200 & 0.0050 & 110 & $80-120$ \\
Vanadium & 6.55 & 0.0020 & $\mathrm{mg} / \mathrm{L}$ & 4.800 & 0.184 & 133 & $80-120$ \\
Zinc & 12.2 & 0.0020 & $\mathrm{mg} / \mathrm{L}$ & 12.00 & 0.146 & 101 & $80-120$
\end{tabular}

Batch B906096 - EPA 3050B

Blank (B906096-BLK1)

Antimony

Arsenic

Barium

Beryllium

Cadmium

Chromium

Cobalt

Copper

Lead

Manganese

Molybdenum

Nickel

Selenium

Silver

Thallium

Vanadium

Zinc
Prepared: 25-Jun-2009 Analyzed: 26-Jun-2009

$\begin{array}{rll}0.254 & 0.0500 & \mathrm{mg} / \mathrm{kg} \\ \mathrm{ND} & 0.0500 & \mathrm{mg} / \mathrm{kg} \\ 0.126 & 0.0500 & \mathrm{mg} / \mathrm{kg} \\ \mathrm{ND} & 0.0500 & \mathrm{mg} / \mathrm{kg} \\ \mathrm{ND} & 0.0500 & \mathrm{mg} / \mathrm{kg} \\ 0.200 & 0.0500 & \mathrm{mg} / \mathrm{kg} \\ \mathrm{ND} & 0.0500 & \mathrm{mg} / \mathrm{kg} \\ \mathrm{ND} & 0.0500 & \mathrm{mg} / \mathrm{kg} \\ 0.0333 & 0.0500 & \mathrm{mg} / \mathrm{kg} \\ 0.0727 & 0.0500 & \mathrm{mg} / \mathrm{kg} \\ 0.0306 & 0.0500 & \mathrm{mg} / \mathrm{kg} \\ 0.0456 & 0.0500 & \mathrm{mg} / \mathrm{kg} \\ \mathrm{ND} & 0.0500 & \mathrm{mg} / \mathrm{kg} \\ \mathrm{ND} & 0.0500 & \mathrm{mg} / \mathrm{kg} \\ \mathrm{ND} & 0.0500 & \mathrm{mg} / \mathrm{kg} \\ 0.0117 & 0.0500 & \mathrm{mg} / \mathrm{kg} \\ 1.96 & 0.0500 & \mathrm{mg} / \mathrm{kg}\end{array}$




\section{USACE ERDC-EP-C \\ 3909 Halls Ferry Road \\ Vicksburg, MS 39180-6199}

ERDC - ECB
Project: TVA Fly Ash Project

Project Manager: Tony Bednar
Reported:

03-Sep-2009

\section{DRAFT: Metals by EPA 6000/7000 Series Methods - Quality Control}

\section{ERDC- EL-EP-C (Environmental Chemistry Branch)}

\begin{tabular}{|c|c|c|c|c|c|c|c|c|c|c|}
\hline & & porting & & Spike & Source & & $\%$ REC & & RPD & \\
\hline Analyte & Result & Limit & Units & Level & Result & $\%$ REC & Limits & RPD & Limit & Notes \\
\hline
\end{tabular}

\section{Batch B906096 - EPA 3050B}

\section{LCS (B906096-BS1)}

Antimony

Arsenic

Barium

Beryllium

Cadmium

Chromium

Cobalt

Copper

Lead

Manganese

Molybdenum

Nickel

Selenium

Silver

Thallium

Vanadium

Zinc

B

(1)




\section{USACE ERDC-EP-C \\ 3909 Halls Ferry Road \\ Vicksburg, MS 39180-6199}

ERDC - ECB

Project: TVA Fly Ash Project

Reported:

Project Manager: Tony Bednar

03-Sep-2009

DRAFT: Metals by EPA 6000/7000 Series Methods - Quality Control

ERDC- EL-EP-C (Environmental Chemistry Branch)

\begin{tabular}{|c|c|c|c|c|c|c|c|c|c|c|}
\hline & & porting & & Spike & Source & & $\%$ REC & & RPD & \\
\hline Analyte & Result & Limit & Units & Level & Result & $\%$ REC & Limits & RPD & Limit & Notes \\
\hline
\end{tabular}

Batch B906096 - EPA 3050B

\begin{tabular}{|c|c|c|c|c|c|c|c|c|}
\hline \multirow{2}{*}{$\begin{array}{l}\text { Matrix Spike (B906096-MS1) } \\
\text { Antimony }\end{array}$} & \multicolumn{3}{|c|}{ Source: 9061502-01 } & \multicolumn{4}{|c|}{ Prepared: 25-Jun-2009 Analyzed: 26-Jun-2009 } & \multirow[b]{2}{*}{ B } \\
\hline & 5.79 & 0.0500 & $\mathrm{mg} / \mathrm{kg}$ & 100.1 & 0.233 & 5.55 & $80-120$ & \\
\hline Arsenic & 101 & 0.0500 & $\mathrm{mg} / \mathrm{kg}$ & 50.05 & 65.8 & 71.0 & $80-120$ & \\
\hline Barium & 879 & 0.0500 & $\mathrm{mg} / \mathrm{kg}$ & 250.3 & 655 & 89.7 & $80-120$ & B \\
\hline Beryllium & 34.3 & 0.0500 & $\mathrm{mg} / \mathrm{kg}$ & 25.03 & 7.15 & 108 & $80-120$ & \\
\hline Cadmium & 23.2 & 0.0500 & $\mathrm{mg} / \mathrm{kg}$ & 25.03 & 0.576 & 90.2 & $80-120$ & \\
\hline Chromium & 156 & 0.0500 & $\mathrm{mg} / \mathrm{kg}$ & 100.1 & 48.7 & 107 & $80-120$ & B \\
\hline Cobalt & 130 & 0.0500 & $\mathrm{mg} / \mathrm{kg}$ & 100.1 & 25.6 & 104 & $80-120$ & \\
\hline Copper & 180 & 0.0500 & $\mathrm{mg} / \mathrm{kg}$ & 100.1 & 84.1 & 95.6 & $80-120$ & \\
\hline Lead & 83.2 & 0.0500 & $\mathrm{mg} / \mathrm{kg}$ & 50.05 & 36.4 & 93.4 & $80-120$ & \\
\hline Manganese & 276 & 0.0500 & $\mathrm{mg} / \mathrm{kg}$ & 100.1 & 153 & 123 & $80-120$ & B \\
\hline Molybdenum & 4.00 & 0.0500 & $\mathrm{mg} / \mathrm{kg}$ & & 4.04 & & $80-120$ & \\
\hline Nickel & 155 & 0.0500 & $\mathrm{mg} / \mathrm{kg}$ & 100.1 & 52.6 & 103 & $80-120$ & \\
\hline Selenium & 28.8 & 0.0500 & $\mathrm{mg} / \mathrm{kg}$ & 25.03 & 6.97 & 87.3 & $80-120$ & \\
\hline Silver & 24.7 & 0.0500 & $\mathrm{mg} / \mathrm{kg}$ & 25.03 & 0.426 & 97.1 & $80-120$ & \\
\hline Thallium & 26.7 & 0.0500 & $\mathrm{mg} / \mathrm{kg}$ & 25.03 & 2.19 & 98.1 & $80-120$ & \\
\hline Vanadium & 321 & 0.0500 & $\mathrm{mg} / \mathrm{kg}$ & 100.1 & 133 & 187 & $80-120$ & \\
\hline Zinc & 303 & 0.0500 & $\mathrm{mg} / \mathrm{kg}$ & 250.3 & 77.4 & 90.3 & $80-120$ & B \\
\hline \multicolumn{9}{|c|}{ Batch B907001 - Default Prep Metals } \\
\hline Blank (B907001-BLK1) & \multicolumn{7}{|c|}{ Prepared \& Analyzed: 25-Jun-2009 } & \\
\hline Aluminum & ND & 0.200 & $\mathrm{mg} / \mathrm{L}$ & & & & & $\mathrm{U}$ \\
\hline Calcium & ND & 0.200 & $\mathrm{mg} / \mathrm{L}$ & & & & & $\mathrm{U}$ \\
\hline Iron & ND & 0.200 & $\mathrm{mg} / \mathrm{L}$ & & & & & $\mathrm{U}$ \\
\hline Magnesium & ND & 0.200 & $\mathrm{mg} / \mathrm{L}$ & & & & & $\mathrm{U}$ \\
\hline Potassium & 0.0690 & 0.200 & $\mathrm{mg} / \mathrm{L}$ & & & & & $\mathrm{J}$ \\
\hline Sodium & ND & 0.200 & $\mathrm{mg} / \mathrm{L}$ & & & & & $\mathrm{U}$ \\
\hline
\end{tabular}




\section{USACE ERDC-EP-C \\ 3909 Halls Ferry Road \\ Vicksburg, MS 39180-6199}

ERDC - ECB $-$

$-,-$
Project: TVA Fly Ash Project

Reported:

Project Manager: Tony Bednar
03-Sep-2009

DRAFT: Metals by EPA 6000/7000 Series Methods - Quality Control

ERDC- EL-EP-C (Environmental Chemistry Branch)

\begin{tabular}{|lcccccccccccc}
\hline & & Reporting & & Spike & Source & & $\%$ REC & & RPD \\
Analyte & Result & Limit & Units & Level & Result & $\%$ REC & Limits & RPD & Limit & Notes \\
\hline
\end{tabular}

Batch B907001 - Default Prep Metals

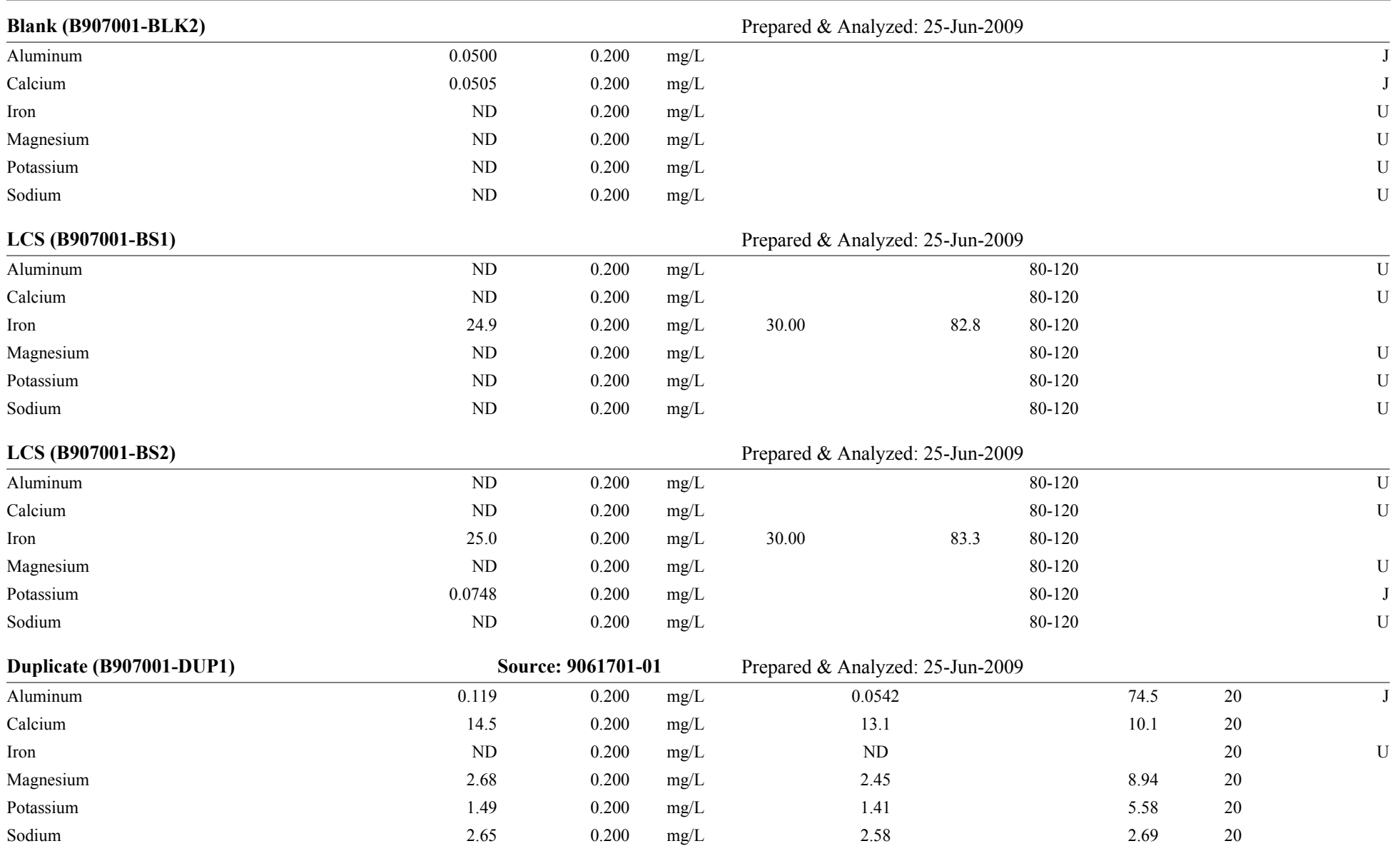




\section{USACE ERDC-EP-C \\ 3909 Halls Ferry Road \\ Vicksburg, MS 39180-6199}

ERDC - ECB $-$

$-\cdot$,
Project: TVA Fly Ash Project

Reported:

Project Manager: Tony Bednar
03-Sep-2009

DRAFT: Metals by EPA 6000/7000 Series Methods - Quality Control

ERDC- EL-EP-C (Environmental Chemistry Branch)

\begin{tabular}{|lcccccccccccc}
\hline & & Reporting & & Spike & Source & & \%REC & & RPD \\
Analyte & Result & Limit & Units & Level & Result & $\%$ REC & Limits & RPD & Limit & Notes & \\
\hline
\end{tabular}

Batch B907001 - Default Prep Metals

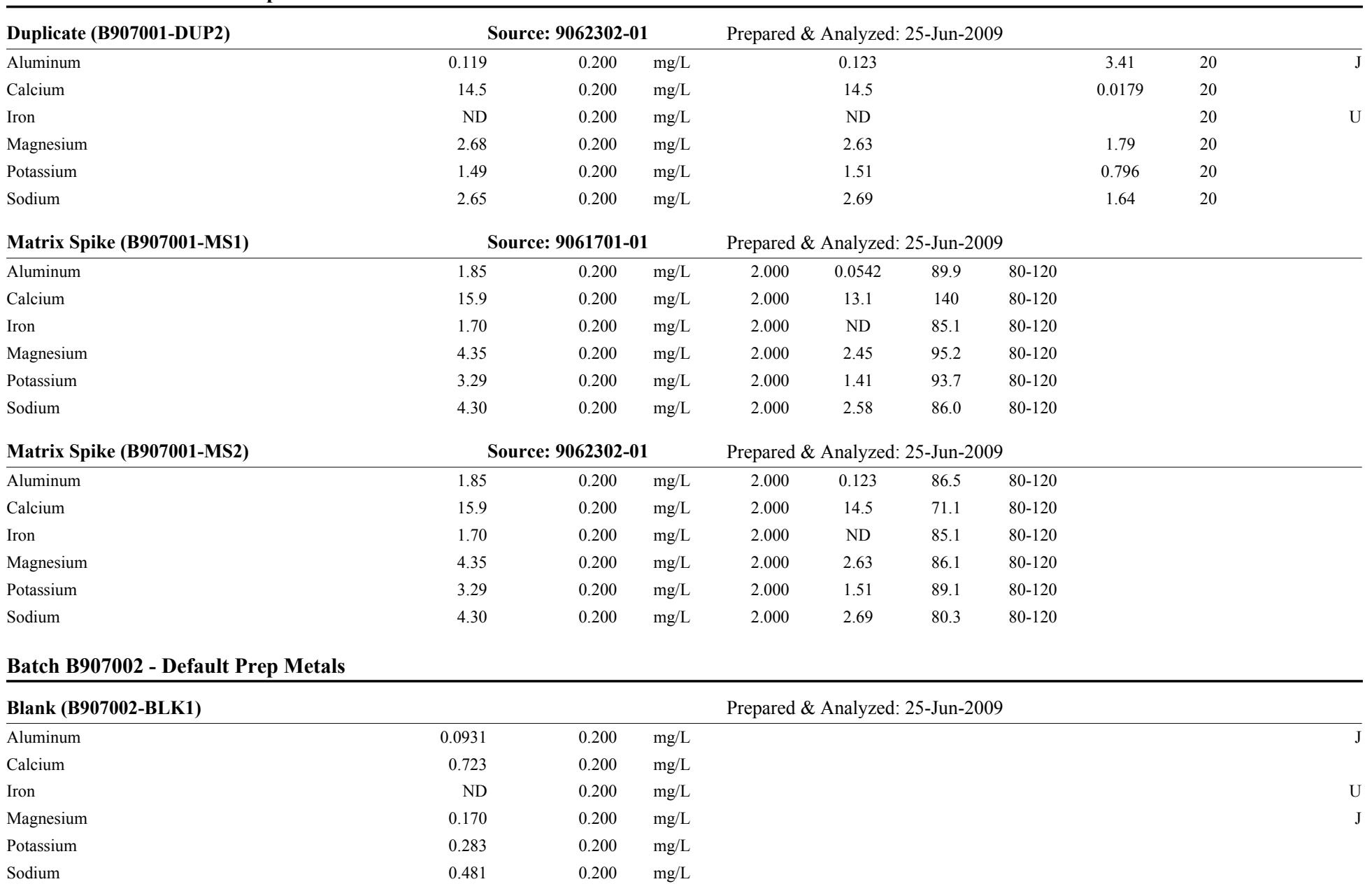




\section{USACE ERDC-EP-C \\ 3909 Halls Ferry Road \\ Vicksburg, MS 39180-6199}

ERDC - ECB $-$

$-,-$
Project: TVA Fly Ash Project

Reported:

Project Manager: Tony Bednar
03-Sep-2009

DRAFT: Metals by EPA 6000/7000 Series Methods - Quality Control

ERDC- EL-EP-C (Environmental Chemistry Branch)

\begin{tabular}{|lcccccccccccc}
\hline & & Reporting & & Spike & Source & & $\%$ REC & & RPD \\
Analyte & Result & Limit & Units & Level & Result & $\%$ REC & Limits & RPD & Limit & Notes \\
\hline
\end{tabular}

Batch B907002 - Default Prep Metals

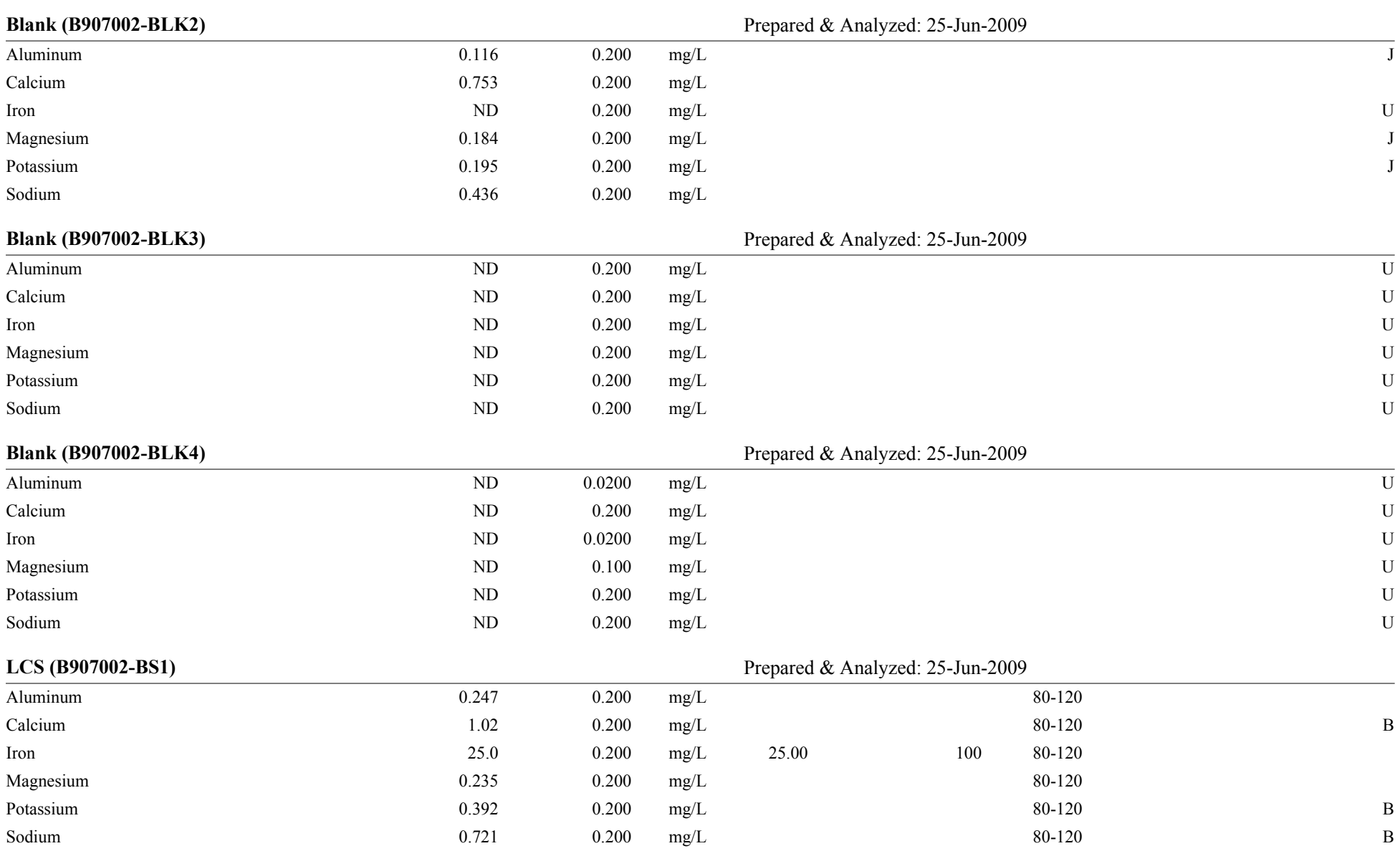




\section{USACE ERDC-EP-C \\ 3909 Halls Ferry Road \\ Vicksburg, MS 39180-6199}

ERDC - ECB $-$

$--,-$
Project: TVA Fly Ash Project

Reported:

Project Manager: Tony Bednar
03-Sep-2009

DRAFT: Metals by EPA 6000/7000 Series Methods - Quality Control

ERDC- EL-EP-C (Environmental Chemistry Branch)

\begin{tabular}{|c|c|c|c|c|c|c|c|c|c|c|}
\hline & & Reporting & & Spike & Source & & $\%$ REC & & RPD & \\
\hline Analyte & Result & Limit & Units & Level & Result & $\%$ REC & Limits & RPD & Limit & Notes \\
\hline
\end{tabular}

Batch B907002 - Default Prep Metals

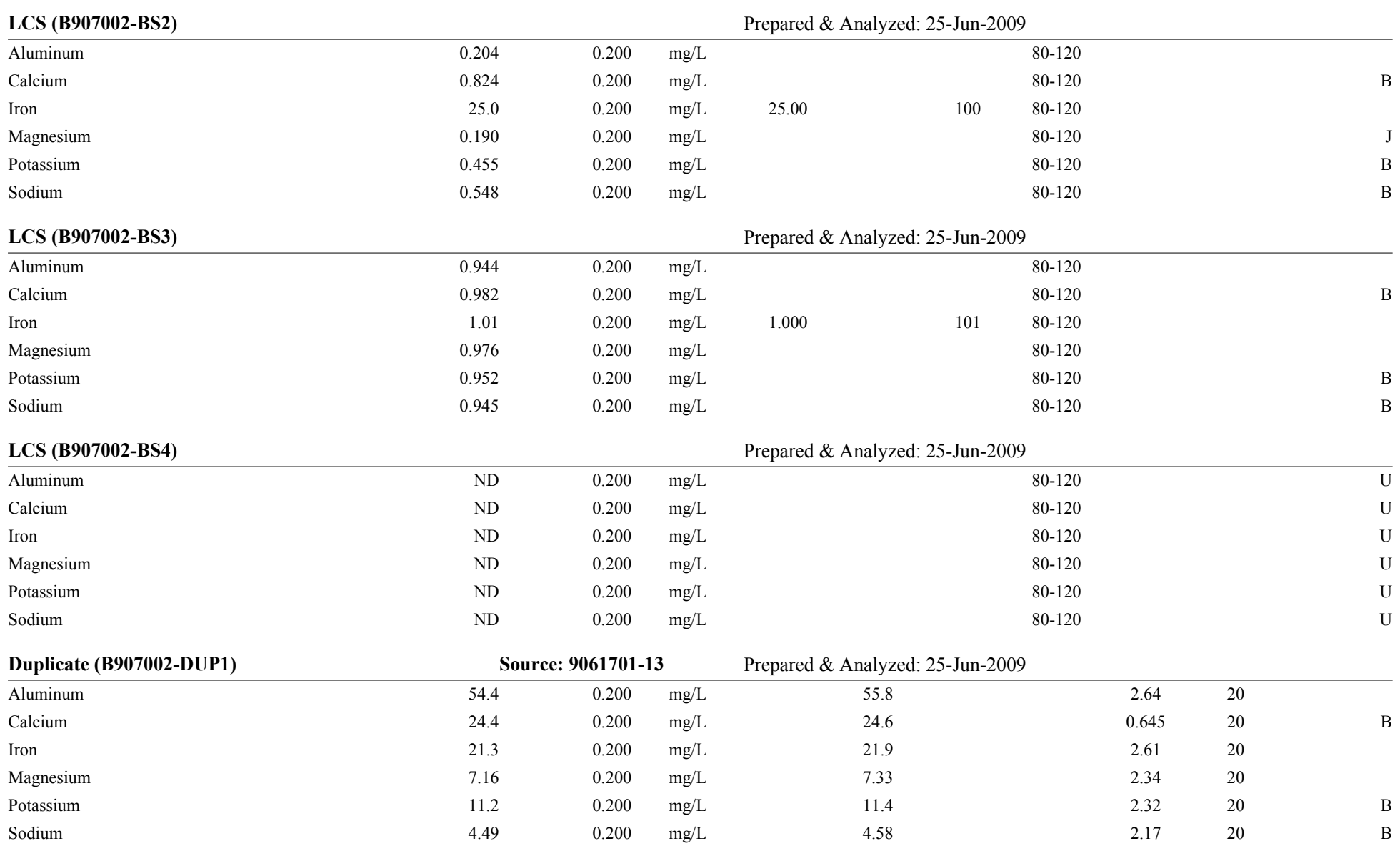




\section{USACE ERDC-EP-C \\ 3909 Halls Ferry Road \\ Vicksburg, MS 39180-6199}

ERDC - ECB $-$
Project: TVA Fly Ash Project

Project Manager: Tony Bednar
Reported:

03-Sep-2009

DRAFT: Metals by EPA 6000/7000 Series Methods - Quality Control

ERDC- EL-EP-C (Environmental Chemistry Branch)

\begin{tabular}{|lcccccccccccc}
\hline & & Reporting & & Spike & Source & & $\%$ REC & & RPD \\
Analyte & Result & Limit & Units & Level & Result & $\%$ REC & Limits & RPD & Limit & Notes \\
\hline
\end{tabular}

Batch B907002 - Default Prep Metals

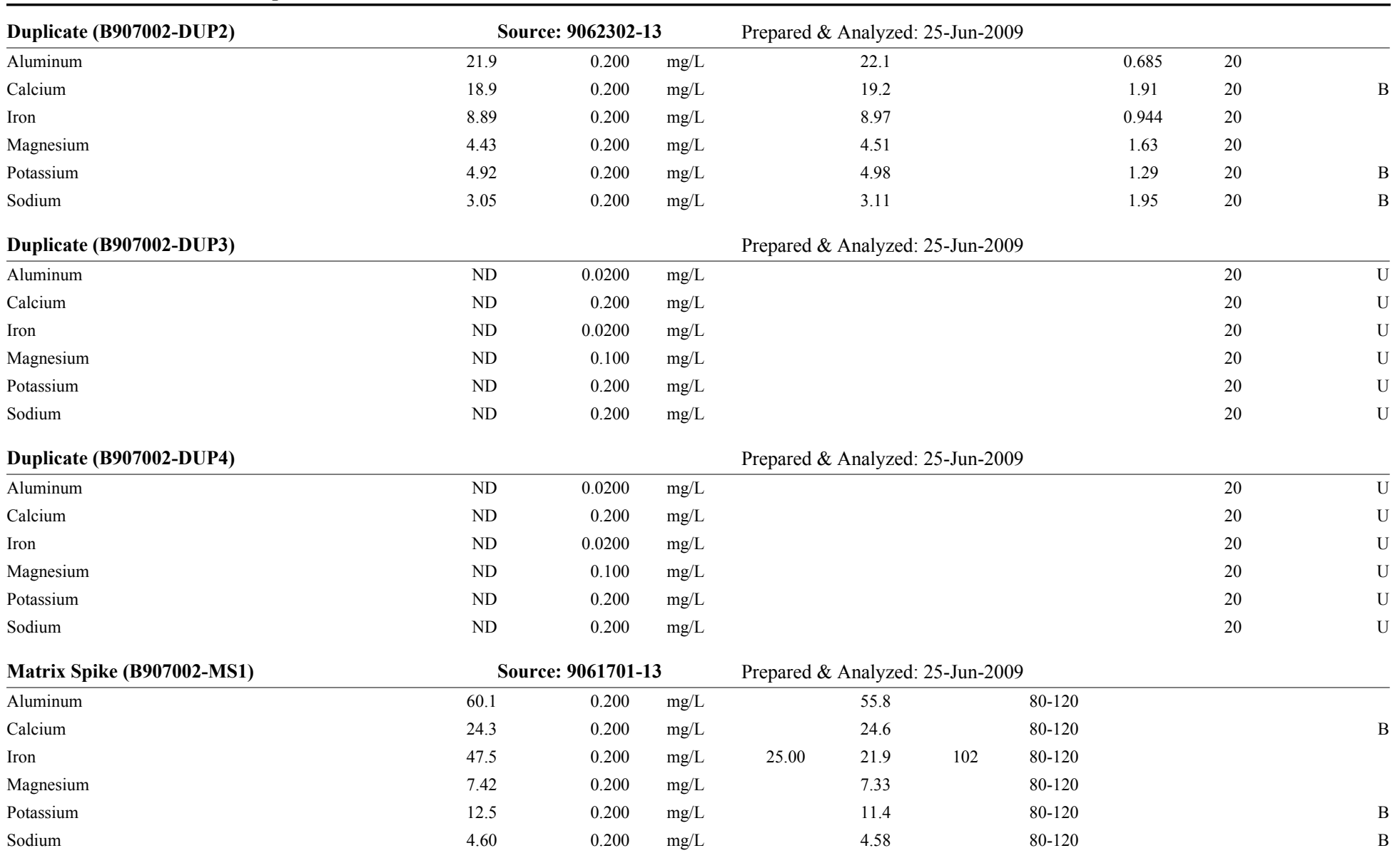




\section{USACE ERDC-EP-C \\ 3909 Halls Ferry Road \\ Vicksburg, MS 39180-6199}

ERDC - ECB

Project: TVA Fly Ash Project

Reported:

Project Manager: Tony Bednar

03-Sep-2009

DRAFT: Metals by EPA 6000/7000 Series Methods - Quality Control

ERDC- EL-EP-C (Environmental Chemistry Branch)

\begin{tabular}{|c|c|c|c|c|c|c|c|c|c|c|}
\hline & & porting & & Spike & Source & & $\%$ REC & & RPD & \\
\hline Analyte & Result & Limit & Units & Level & Result & $\%$ REC & Limits & RPD & Limit & Notes \\
\hline
\end{tabular}

Batch B907002 - Default Prep Metals

\begin{tabular}{|c|c|c|c|c|c|c|c|c|}
\hline \multirow{2}{*}{$\begin{array}{l}\text { Matrix Spike (B907002-MS2) } \\
\text { Aluminum }\end{array}$} & \multicolumn{3}{|c|}{ Source: $9062302-13$} & \multicolumn{5}{|c|}{ Prepared \& Analyzed: 25-Jun-2009 } \\
\hline & 21.7 & 0.200 & $\mathrm{mg} / \mathrm{L}$ & & 22.1 & & $80-120$ & \\
\hline Calcium & 18.9 & 0.200 & $\mathrm{mg} / \mathrm{L}$ & & 19.2 & & $80-120$ & B \\
\hline Iron & 8.83 & 0.200 & $\mathrm{mg} / \mathrm{L}$ & 25.00 & 8.97 & NR & $80-120$ & \\
\hline Magnesium & 4.36 & 0.200 & $\mathrm{mg} / \mathrm{L}$ & & 4.51 & & $80-120$ & \\
\hline Potassium & 4.90 & 0.200 & $\mathrm{mg} / \mathrm{L}$ & & 4.98 & & $80-120$ & B \\
\hline Sodium & 3.02 & 0.200 & $\mathrm{mg} / \mathrm{L}$ & & 3.11 & & $80-120$ & B \\
\hline
\end{tabular}

Sodium

Prepared \& Analyzed: 25-Jun-2009

Matrix Spike (B907002-MS3)

Aluminum

ND $\quad 0.0200 \quad \mathrm{mg} / \mathrm{L}$

Calcium

Iron

Magnesium

Potassium

ND $\quad 0.200 \quad \mathrm{mg} / \mathrm{L}$

$80-120$

80-120

ND $\quad 0.0200 \quad \mathrm{mg} / \mathrm{L} \quad 80-120$

ND $\quad 0.100 \quad \mathrm{mg} / \mathrm{L} \quad 80-120$

Sodium

ND $\quad 0.200 \quad \mathrm{mg} / \mathrm{L}$

$80-120$

ND $\quad 0.200 \quad \mathrm{mg} / \mathrm{L}$

Matrix Spike (B907002-MS4)

Aluminum

Calcium

Iron

Magnesium

Potassium

ND $\quad$ Prepared \& Analyzed: 25-Jun-2009

Sodium

$\begin{array}{lrl}\text { ND } & 0.0200 & \mathrm{mg} / \mathrm{L} \\ \text { ND } & 0.200 & \mathrm{mg} / \mathrm{L} \\ \text { ND } & 0.0200 & \mathrm{mg} / \mathrm{L} \\ \text { ND } & 0.100 & \mathrm{mg} / \mathrm{L} \\ \text { ND } & 0.200 & \mathrm{mg} / \mathrm{L} \\ \text { ND } & 0.200 & \mathrm{mg} / \mathrm{L}\end{array}$

$80-120$

$80-120$

$80-120$

$80-120$

$80-120$

$80-120$

Batch B907003 - EPA 3050B

\begin{tabular}{llllll}
\hline Blank (B907003-BLK1) & & & & Prepared \& Analyzed: 25-Jun-2009 \\
\hline Aluminum & 9.31 & 2.00 & $\mathrm{mg} / \mathrm{kg}$ & \\
Calcium & 12.8 & 2.00 & $\mathrm{mg} / \mathrm{kg}$ & \\
Iron & 1.79 & 2.00 & $\mathrm{mg} / \mathrm{kg}$ & \\
Magnesium & 2.69 & 2.00 & $\mathrm{mg} / \mathrm{kg}$ & \\
Potassium & 16.5 & 2.00 & $\mathrm{mg} / \mathrm{kg}$ & \\
Sodium & 23.0 & 2.00 & $\mathrm{mg} / \mathrm{kg}$ & $\mathrm{J}$
\end{tabular}




\section{USACE ERDC-EP-C \\ 3909 Halls Ferry Road \\ Vicksburg, MS 39180-6199}

ERDC - ECB

Project: TVA Fly Ash Project

Reported:

Project Manager: Tony Bednar

03-Sep-2009

DRAFT: Metals by EPA 6000/7000 Series Methods - Quality Control

ERDC- EL-EP-C (Environmental Chemistry Branch)

\begin{tabular}{|lrrrrrrrrrrr}
\hline & & Reporting & & Spike & Source & & & $\%$ REC & & RPD \\
Analyte & Result & Limit & Units & Level & Result & $\%$ REC & Limits & RPD & Limit & Notes & \\
\hline
\end{tabular}

Batch B907003 - EPA 3050B

\begin{tabular}{|c|c|c|c|c|c|c|c|c|c|c|}
\hline \multirow{2}{*}{$\frac{\text { LCS (B907003-BS1) }}{\text { Aluminum }}$} & \multicolumn{10}{|c|}{ Prepared \& Analyzed: 25-Jun-2009 } \\
\hline & 11.3 & 2.00 & $\mathrm{mg} / \mathrm{kg}$ & & & & $80-120$ & & & B \\
\hline Calcium & 12.5 & 2.00 & $\mathrm{mg} / \mathrm{kg}$ & & & & $80-120$ & & & B \\
\hline Magnesium & 2.33 & 2.00 & $\mathrm{mg} / \mathrm{kg}$ & & & & $80-120$ & & & B \\
\hline Potassium & 21.8 & 2.00 & $\mathrm{mg} / \mathrm{kg}$ & & & & $80-120$ & & & B \\
\hline Sodium & 23.1 & 2.00 & $\mathrm{mg} / \mathrm{kg}$ & & & & $80-120$ & & & B \\
\hline Duplicate (B907003-DUP1) & \multicolumn{3}{|c|}{ Source: $9061502-01$} & \multicolumn{7}{|c|}{ Prepared \& Analyzed: 25-Jun-2009 } \\
\hline Aluminum & 31000 & 2.00 & $\mathrm{mg} / \mathrm{kg}$ & & 4890 & & & 145 & 20 & B \\
\hline Calcium & 9140 & 2.00 & $\mathrm{mg} / \mathrm{kg}$ & & 2410 & & & 117 & 20 & B \\
\hline Iron & 18000 & 2.00 & $\mathrm{mg} / \mathrm{kg}$ & & 10400 & & & 52.9 & 20 & \\
\hline Magnesium & 2810 & 2.00 & $\mathrm{mg} / \mathrm{kg}$ & & 559 & & & 134 & 20 & B \\
\hline Potassium & 4740 & 2.00 & $\mathrm{mg} / \mathrm{kg}$ & & 625 & & & 153 & 20 & B \\
\hline Matrix Spike (B907003-MS1) & \multicolumn{3}{|c|}{ Source: $9061502-01$} & \multicolumn{7}{|c|}{ Prepared \& Analyzed: 25-Jun-2009 } \\
\hline Aluminum & 32000 & 2.00 & $\mathrm{mg} / \mathrm{kg}$ & & 4890 & & $80-120$ & & & B \\
\hline Calcium & 9320 & 2.00 & $\mathrm{mg} / \mathrm{kg}$ & & 2410 & & $80-120$ & & & $\mathrm{~B}$ \\
\hline Iron & 18400 & 2.00 & $\mathrm{mg} / \mathrm{kg}$ & 1001 & 10400 & 797 & $80-120$ & & & \\
\hline Magnesium & 2880 & 2.00 & $\mathrm{mg} / \mathrm{kg}$ & & 559 & & $80-120$ & & & B \\
\hline Potassium & 4960 & 2.00 & $\mathrm{mg} / \mathrm{kg}$ & & 625 & & $80-120$ & & & B \\
\hline Sodium & 747 & 2.00 & $\mathrm{mg} / \mathrm{kg}$ & & 65.2 & & $80-120$ & & & B \\
\hline
\end{tabular}

Batch B907024 - Default Prep Metals

\begin{tabular}{lllll}
\hline Blank (B907024-BLK1) & & & & Prepared \& Analyzed: 25-Jun-2009 \\
\hline Strontium & ND & 0.0200 & $\mathrm{mg} / \mathrm{L}$ &
\end{tabular}




\section{USACE ERDC-EP-C \\ 3909 Halls Ferry Road \\ Vicksburg, MS 39180-6199}

ERDC - ECB

Project: TVA Fly Ash Project

Project Manager: Tony Bednar

Reported:

03-Sep-2009

DRAFT: Metals by EPA 6000/7000 Series Methods - Quality Control

ERDC- EL-EP-C (Environmental Chemistry Branch)

\begin{tabular}{|lccccccccccc}
\hline & & Reporting & & Spike & Source & & & \%REC & & RPD \\
Analyte & Result & Limit & Units & Level & Result & $\%$ REC & Limits & RPD & Limit & Notes \\
\hline
\end{tabular}

Batch B907024 - Default Prep Metals

\begin{tabular}{|c|c|c|c|c|c|c|}
\hline \multirow{2}{*}{$\begin{array}{l}\text { Blank (B907024-BLK2) } \\
\text { Strontium }\end{array}$} & \multicolumn{6}{|c|}{ Prepared \& Analyzed: 25-Jun-2009 } \\
\hline & ND & 0.0200 & $\mathrm{mg} / \mathrm{L}$ & & & $\overline{\mathrm{U}}$ \\
\hline Blank (B907024-BLK3) & \multicolumn{6}{|c|}{ Prepared \& Analyzed: 25-Jun-2009 } \\
\hline Strontium & ND & 0.0200 & $\mathrm{mg} / \mathrm{L}$ & & & $\overline{\mathrm{U}}$ \\
\hline Blank (B907024-BLK4) & \multicolumn{6}{|c|}{ Prepared: 25-Jun-2009 Analyzed: 16-Jul-2009 } \\
\hline Strontium & ND & 0.0200 & $\mathrm{mg} / \mathrm{L}$ & & & $\overline{\mathrm{U}}$ \\
\hline Blank (B907024-BLK5) & \multicolumn{6}{|c|}{ Prepared: 25-Jun-2009 Analyzed: 16-Jul-2009 } \\
\hline Strontium & ND & 0.0200 & $\mathrm{mg} / \mathrm{L}$ & & & $\overline{\mathrm{U}}$ \\
\hline Blank (B907024-BLK6) & \multicolumn{6}{|c|}{ Prepared: 25-Jun-2009 Analyzed: 16-Jul-2009 } \\
\hline Strontium & ND & 0.0200 & $\mathrm{mg} / \mathrm{L}$ & & & $\overline{\mathrm{U}}$ \\
\hline Blank (B907024-BLK7) & \multicolumn{6}{|c|}{ Prepared: 25-Jun-2009 Analyzed: 16-Jul-2009 } \\
\hline Strontium & ND & 0.0200 & $\mathrm{mg} / \mathrm{L}$ & & & $\overline{\mathrm{U}}$ \\
\hline Duplicate (B907024-DUP1) & \multicolumn{3}{|c|}{ Source: $9061502-40$} & \multicolumn{3}{|l|}{ Prepared \& Analyzed: 25-Jun-2009 } \\
\hline Strontium & 0.197 & 0.0200 & $\mathrm{mg} / \mathrm{L}$ & 0.135 & 20 & \\
\hline \multicolumn{7}{|l|}{ Batch B907025 - EPA 3050B } \\
\hline Blank (B907025-BLK1) & & & & Prepared \& Analyzed: 25-Jun-2009 & & \\
\hline Strontium & ND & 4.00 & $\mathrm{mg} / \mathrm{kg}$ & & & $\overline{\mathrm{U}}$ \\
\hline Duplicate (B907025-DUP1) & \multicolumn{3}{|c|}{ Source: 9061502-01 } & Prepared \& Analyzed: 25-Jun-2009 & & \\
\hline Strontium & 89.2 & 4.00 & $\mathrm{mg} / \mathrm{kg}$ & 87.4 & 20 & \\
\hline
\end{tabular}




\section{USACE ERDC-EP-C \\ 3909 Halls Ferry Road \\ Vicksburg, MS 39180-6199}

ERDC - ECB

Project: TVA Fly Ash Project

Reported:

Project Manager: Tony Bednar

03-Sep-2009

DRAFT: Metals by EPA 6000/7000 Series Methods - Quality Control

ERDC- EL-EP-C (Environmental Chemistry Branch)

\begin{tabular}{|c|c|c|c|c|c|c|c|c|c|c|}
\hline & & Reporting & & Spike & Source & & $\%$ REC & & RPD & \\
\hline Analyte & Result & Limit & Units & Level & Result & $\%$ REC & Limits & RPD & Limit & Notes \\
\hline
\end{tabular}

Batch B907026 - Default Prep Metals

\begin{tabular}{|c|c|c|c|c|c|}
\hline \multirow{2}{*}{$\begin{array}{l}\text { Blank (B907026-BLK1) } \\
\text { Strontium }\end{array}$} & \multicolumn{5}{|c|}{ Prepared \& Analyzed: 25-Jun-2009 } \\
\hline & ND & 0.0800 & $\mathrm{mg} / \mathrm{L}$ & & $\overline{\mathrm{U}}$ \\
\hline Blank (B907026-BLK2) & \multicolumn{5}{|c|}{ Prepared \& Analyzed: 25-Jun-2009 } \\
\hline Strontium & ND & 0.0800 & $\mathrm{mg} / \mathrm{L}$ & & $\mathrm{U}$ \\
\hline Blank (B907026-BLK3) & \multicolumn{5}{|c|}{ Prepared \& Analyzed: 25-Jun-2009 } \\
\hline Strontium & ND & 0.0800 & $\mathrm{mg} / \mathrm{L}$ & & $\overline{\mathrm{U}}$ \\
\hline Blank (B907026-BLK4) & \multicolumn{5}{|c|}{ Prepared \& Analyzed: 25-Jun-2009 } \\
\hline Strontium & ND & 0.0800 & $\mathrm{mg} / \mathrm{L}$ & & $\overline{\mathrm{U}}$ \\
\hline Duplicate (B907026-DUP1) & \multicolumn{3}{|c|}{ Source: $9061502-76$} & \multicolumn{2}{|l|}{ Prepared \& Analyzed: 25-Jun-2009 } \\
\hline Strontium & 0.362 & 0.0800 & $\mathrm{mg} / \mathrm{L}$ & 0.800 & 20 \\
\hline \multicolumn{6}{|c|}{ Batch B907033 - EPA 3050B } \\
\hline Blank (B907033-BLK1) & \multicolumn{5}{|c|}{ Prepared: 17-Jul-2009 Analyzed: 20-Jul-2009 } \\
\hline Antimony & ND & 0.500 & $\mathrm{mg} / \mathrm{kg}$ & & $\overline{\mathrm{U}}$ \\
\hline Arsenic & ND & 0.500 & $\mathrm{mg} / \mathrm{kg}$ & & $\mathrm{U}$ \\
\hline Barium & ND & 0.500 & $\mathrm{mg} / \mathrm{kg}$ & & $\mathrm{U}$ \\
\hline Beryllium & ND & 0.500 & $\mathrm{mg} / \mathrm{kg}$ & & $\mathrm{U}$ \\
\hline Cadmium & ND & 0.500 & $\mathrm{mg} / \mathrm{kg}$ & & $\mathrm{U}$ \\
\hline Chromium & ND & 0.500 & $\mathrm{mg} / \mathrm{kg}$ & & $\mathrm{U}$ \\
\hline Cobalt & ND & 0.500 & $\mathrm{mg} / \mathrm{kg}$ & & $\mathrm{U}$ \\
\hline Copper & ND & 0.500 & $\mathrm{mg} / \mathrm{kg}$ & & $\mathrm{U}$ \\
\hline Lead & ND & 0.500 & $\mathrm{mg} / \mathrm{kg}$ & & $\mathrm{U}$ \\
\hline Manganese & ND & 0.500 & $\mathrm{mg} / \mathrm{kg}$ & & $\mathrm{U}$ \\
\hline Molybdenum & ND & 0.500 & $\mathrm{mg} / \mathrm{kg}$ & & $\mathrm{U}$ \\
\hline Nickel & ND & 0.500 & $\mathrm{mg} / \mathrm{kg}$ & & $\mathrm{U}$ \\
\hline Selenium & ND & 0.500 & $\mathrm{mg} / \mathrm{kg}$ & & $\mathrm{U}$ \\
\hline Silver & ND & 0.500 & $\mathrm{mg} / \mathrm{kg}$ & & $\mathrm{U}$ \\
\hline Thallium & ND & 0.500 & $\mathrm{mg} / \mathrm{kg}$ & & $\mathrm{U}$ \\
\hline Vanadium & ND & 0.500 & $\mathrm{mg} / \mathrm{kg}$ & & $\mathrm{U}$ \\
\hline Zinc & 0.153 & 0.500 & $\mathrm{mg} / \mathrm{kg}$ & & $\mathrm{J}$ \\
\hline
\end{tabular}




\section{USACE ERDC-EP-C \\ 3909 Halls Ferry Road \\ Vicksburg, MS 39180-6199}

ERDC - ECB

$-,-$
Project: TVA Fly Ash Project

Reported:

Project Manager: Tony Bednar
03-Sep-2009

\section{DRAFT: Metals by EPA 6000/7000 Series Methods - Quality Control}

\section{ERDC- EL-EP-C (Environmental Chemistry Branch)}

\begin{tabular}{|lcccccccccccc}
\hline & & Reporting & & Spike & Source & & & \%REC & & RPD \\
Analyte & Result & Limit & Units & Level & Result & \%REC & Limits & RPD & Limit & Notes & \\
\hline
\end{tabular}

\section{Batch B907033 - EPA 3050B}

Blank (B907033-BLK2)

Antimony

Arsenic

Barium

Beryllium

Cadmium

Chromium

Cobalt

Copper

Lead

Manganese

Molybdenum

Nickel

Selenium

Silver

Thallium

Vanadium

Zinc

LCS (B907033-BS1)

Antimony

Arsenic

Barium

Beryllium

Cadmium

Chromium

Cobalt

Copper

Lead

Manganese

Molybdenum

Nickel

Selenium

Silver

Thallium

Vanadium

Zinc
Prepared: 17-Jul-2009 Analyzed: 20-Jul-2009

$\begin{array}{rll}0.485 & 0.500 & \mathrm{mg} / \mathrm{kg} \\ \mathrm{ND} & 0.500 & \mathrm{mg} / \mathrm{kg} \\ 0.113 & 0.500 & \mathrm{mg} / \mathrm{kg} \\ \mathrm{ND} & 0.500 & \mathrm{mg} / \mathrm{kg} \\ \mathrm{ND} & 0.500 & \mathrm{mg} / \mathrm{kg} \\ \mathrm{ND} & 0.500 & \mathrm{mg} / \mathrm{kg} \\ \mathrm{ND} & 0.500 & \mathrm{mg} / \mathrm{kg} \\ \mathrm{ND} & 0.500 & \mathrm{mg} / \mathrm{kg} \\ 0.217 & 0.500 & \mathrm{mg} / \mathrm{kg} \\ \mathrm{ND} & 0.500 & \mathrm{mg} / \mathrm{kg} \\ \mathrm{ND} & 0.500 & \mathrm{mg} / \mathrm{kg} \\ \mathrm{ND} & 0.500 & \mathrm{mg} / \mathrm{kg} \\ \mathrm{ND} & 0.500 & \mathrm{mg} / \mathrm{kg} \\ \mathrm{ND} & 0.500 & \mathrm{mg} / \mathrm{kg} \\ \mathrm{ND} & 0.500 & \mathrm{mg} / \mathrm{kg} \\ \mathrm{ND} & 0.500 & \mathrm{mg} / \mathrm{kg} \\ 0.514 & 0.500 & \mathrm{mg} / \mathrm{kg}\end{array}$

Prepared: 17-Jul-2009 Analyzed: 20-Jul-2009

$\begin{array}{lllll}0.500 & \mathrm{mg} / \mathrm{kg} & 2.500 & 101 & 80-120 \\ 0.500 & \mathrm{mg} / \mathrm{kg} & 2.500 & 101 & 80-120 \\ 0.500 & \mathrm{mg} / \mathrm{kg} & 2.500 & 103 & 80-120 \\ 0.500 & \mathrm{mg} / \mathrm{kg} & 2.500 & 102 & 80-120 \\ 0.500 & \mathrm{mg} / \mathrm{kg} & 2.500 & 105 & 80-120 \\ 0.500 & \mathrm{mg} / \mathrm{kg} & 2.500 & 101 & 80-120 \\ 0.500 & \mathrm{mg} / \mathrm{kg} & 2.500 & 102 & 80-120 \\ 0.500 & \mathrm{mg} / \mathrm{kg} & 2.500 & 101 & 80-120 \\ 0.500 & \mathrm{mg} / \mathrm{kg} & 2.500 & 102 & 80-120 \\ 0.500 & \mathrm{mg} / \mathrm{kg} & 2.500 & 97.0 & 80-120 \\ 0.500 & \mathrm{mg} / \mathrm{kg} & 2.500 & 100 & 80-120 \\ 0.500 & \mathrm{mg} / \mathrm{kg} & 2.500 & 102 & 80-120 \\ 0.500 & \mathrm{mg} / \mathrm{kg} & 2.500 & 102 & 80-120 \\ 0.500 & \mathrm{mg} / \mathrm{kg} & 2.500 & 102 & 80-120 \\ 0.500 & \mathrm{mg} / \mathrm{kg} & 2.500 & 100 & 80-120 \\ 0.500 & \mathrm{mg} / \mathrm{kg} & 2.500 & 100 & 80-120 \\ 0.500 & \mathrm{mg} / \mathrm{kg} & 2.500 & 103 & 80-120\end{array}$




\section{USACE ERDC-EP-C \\ 3909 Halls Ferry Road \\ Vicksburg, MS 39180-6199}

ERDC - ECB
Project: TVA Fly Ash Project

Project Manager: Tony Bednar
Reported:

03-Sep-2009

\section{DRAFT: Metals by EPA 6000/7000 Series Methods - Quality Control}

\section{ERDC- EL-EP-C (Environmental Chemistry Branch)}

\begin{tabular}{|lcccccccccccc}
\hline & & Reporting & & Spike & Source & & & \%REC & & RPD \\
Analyte & Result & Limit & Units & Level & Result & \%REC & Limits & RPD & Limit & Notes & \\
\hline
\end{tabular}

\section{Batch B907033 - EPA 3050B}

\begin{tabular}{|c|c|c|c|c|c|c|c|c|c|}
\hline \multirow{2}{*}{$\frac{\text { LCS (B907033-BS2) }}{\text { Antimony }}$} & \multicolumn{9}{|c|}{ Prepared: 17-Jul-2009 Analyzed: 20-Jul-2009 } \\
\hline & 96.3 & 0.500 & $\mathrm{mg} / \mathrm{kg}$ & 100.0 & 96.3 & $80-120$ & & & \\
\hline Arsenic & 45.5 & 0.500 & $\mathrm{mg} / \mathrm{kg}$ & 50.00 & 91.0 & $80-120$ & & & \\
\hline Barium & 196 & 0.500 & $\mathrm{mg} / \mathrm{kg}$ & 200.0 & 97.9 & $80-120$ & & & \\
\hline Beryllium & 45.2 & 0.500 & $\mathrm{mg} / \mathrm{kg}$ & 50.00 & 90.3 & $80-120$ & & & \\
\hline Cadmium & 47.2 & 0.500 & $\mathrm{mg} / \mathrm{kg}$ & 50.00 & 94.4 & $80-120$ & & & \\
\hline Chromium & 97.5 & 0.500 & $\mathrm{mg} / \mathrm{kg}$ & 100.0 & 97.5 & $80-120$ & & & \\
\hline Copper & 95.5 & 0.500 & $\mathrm{mg} / \mathrm{kg}$ & 100.0 & 95.5 & $80-120$ & & & \\
\hline Lead & 99.5 & 0.500 & $\mathrm{mg} / \mathrm{kg}$ & 100.0 & 99.5 & $80-120$ & & & \\
\hline Manganese & 239 & 0.500 & $\mathrm{mg} / \mathrm{kg}$ & 250.0 & 95.7 & $80-120$ & & & \\
\hline Molybdenum & 49.7 & 0.500 & $\mathrm{mg} / \mathrm{kg}$ & 50.00 & 99.4 & $80-120$ & & & \\
\hline Nickel & 96.6 & 0.500 & $\mathrm{mg} / \mathrm{kg}$ & 100.0 & 96.6 & $80-120$ & & & \\
\hline Selenium & 42.9 & 0.500 & $\mathrm{mg} / \mathrm{kg}$ & 50.00 & 85.9 & $80-120$ & & & \\
\hline Thallium & 48.7 & 0.500 & $\mathrm{mg} / \mathrm{kg}$ & 50.00 & 97.4 & $80-120$ & & & \\
\hline Vanadium & 96.9 & 0.500 & $\mathrm{mg} / \mathrm{kg}$ & 100.0 & 96.9 & $80-120$ & & & \\
\hline Zinc & 168 & 0.500 & $\mathrm{mg} / \mathrm{kg}$ & 200.0 & 84.0 & $80-120$ & & & B \\
\hline Duplicate (B907033-DUP1) & \multicolumn{3}{|c|}{ Source: $9071602-01$} & \multicolumn{5}{|c|}{ Prepared: 17-Jul-2009 Analyzed: 20-Jul-2009 } & \\
\hline Antimony & 0.146 & 0.500 & $\mathrm{mg} / \mathrm{kg}$ & & & & 3.30 & 20 & $\mathrm{~J}$ \\
\hline Arsenic & 2.83 & 0.500 & $\mathrm{mg} / \mathrm{kg}$ & & & & 2.00 & 20 & \\
\hline Barium & 19.6 & 0.500 & $\mathrm{mg} / \mathrm{kg}$ & & & & 40.4 & 20 & \\
\hline Beryllium & 0.172 & 0.500 & $\mathrm{mg} / \mathrm{kg}$ & & & & 2.99 & 20 & $\mathrm{~J}$ \\
\hline Cadmium & 0.374 & 0.500 & $\mathrm{mg} / \mathrm{kg}$ & & & & 4.86 & 20 & $\mathrm{~J}$ \\
\hline Chromium & 18.4 & 0.500 & $\mathrm{mg} / \mathrm{kg}$ & & & & 10.6 & 20 & \\
\hline Cobalt & 1.23 & 0.500 & $\mathrm{mg} / \mathrm{kg}$ & & & & 11.5 & 20 & \\
\hline Copper & 5.10 & 0.500 & $\mathrm{mg} / \mathrm{kg}$ & & & & 4.63 & 20 & \\
\hline Lead & 1.57 & 0.500 & $\mathrm{mg} / \mathrm{kg}$ & & & & 15.3 & 20 & \\
\hline Molybdenum & 0.489 & 0.500 & $\mathrm{mg} / \mathrm{kg}$ & & & & 6.58 & 20 & $\mathrm{~J}$ \\
\hline Nickel & 10.4 & 0.500 & $\mathrm{mg} / \mathrm{kg}$ & & & & 8.43 & 20 & \\
\hline Selenium & 0.592 & 0.500 & $\mathrm{mg} / \mathrm{kg}$ & & & & 18.0 & 20 & \\
\hline Silver & 0.512 & 0.500 & $\mathrm{mg} / \mathrm{kg}$ & & & & 10.3 & 20 & \\
\hline Thallium & 0.189 & 0.500 & $\mathrm{mg} / \mathrm{kg}$ & & & & 2.97 & 20 & $\mathrm{~J}$ \\
\hline Vanadium & 4.22 & 0.500 & $\mathrm{mg} / \mathrm{kg}$ & & & & 2.45 & 20 & \\
\hline Zinc & 25.4 & 0.500 & $\mathrm{mg} / \mathrm{kg}$ & & & & 2.07 & 20 & B \\
\hline
\end{tabular}




\section{USACE ERDC-EP-C \\ 3909 Halls Ferry Road \\ Vicksburg, MS 39180-6199}

ERDC - ECB

Project: TVA Fly Ash Project

Reported:

Project Manager: Tony Bednar

03-Sep-2009

DRAFT: Metals by EPA 6000/7000 Series Methods - Quality Control

ERDC- EL-EP-C (Environmental Chemistry Branch)

\begin{tabular}{|c|c|c|c|c|c|c|c|c|c|c|}
\hline & & eporting & & Spike & Source & & $\%$ REC & & RPD & \\
\hline Analyte & Result & Limit & Units & Level & Result & $\%$ REC & Limits & RPD & Limit & Notes \\
\hline
\end{tabular}

Batch B907033 - EPA 3050B

\begin{tabular}{|c|c|c|c|c|c|c|c|c|}
\hline \multirow{2}{*}{$\begin{array}{l}\text { Matrix Spike (B907033-MS1) } \\
\text { Antimony }\end{array}$} & \multicolumn{3}{|c|}{ Source: 9071602-01 } & \multicolumn{4}{|c|}{ Prepared: 17-Jul-2009 Analyzed: 20-Jul-2009 } & \\
\hline & 254 & 0.500 & $\mathrm{mg} / \mathrm{kg}$ & 259.1 & 0.141 & 98.1 & $80-120$ & \\
\hline Arsenic & 129 & 0.500 & $\mathrm{mg} / \mathrm{kg}$ & 129.5 & 2.89 & 97.5 & $80-120$ & \\
\hline Barium & 560 & 0.500 & $\mathrm{mg} / \mathrm{kg}$ & 518.1 & 29.5 & 102 & $80-120$ & \\
\hline Beryllium & 129 & 0.500 & $\mathrm{mg} / \mathrm{kg}$ & 129.5 & 0.167 & 99.3 & $80-120$ & \\
\hline Cadmium & 130 & 0.500 & $\mathrm{mg} / \mathrm{kg}$ & 129.5 & 0.393 & 99.9 & $80-120$ & \\
\hline Chromium & 297 & 0.500 & $\mathrm{mg} / \mathrm{kg}$ & 259.1 & 16.5 & 108 & $80-120$ & \\
\hline Cobalt & 274 & 0.500 & $\mathrm{mg} / \mathrm{kg}$ & 259.1 & 1.09 & 106 & $80-120$ & \\
\hline Copper & 267 & 0.500 & $\mathrm{mg} / \mathrm{kg}$ & 259.1 & 4.87 & 101 & $80-120$ & \\
\hline Lead & 272 & 0.500 & $\mathrm{mg} / \mathrm{kg}$ & 259.1 & 1.83 & 104 & $80-120$ & \\
\hline Manganese & 662 & 0.500 & $\mathrm{mg} / \mathrm{kg}$ & 647.7 & 6.31 & 101 & $80-120$ & \\
\hline Molybdenum & 137 & 0.500 & $\mathrm{mg} / \mathrm{kg}$ & 129.5 & 0.458 & 105 & $80-120$ & \\
\hline Nickel & 279 & 0.500 & $\mathrm{mg} / \mathrm{kg}$ & 259.1 & 9.52 & 104 & $80-120$ & \\
\hline Selenium & 116 & 0.500 & $\mathrm{mg} / \mathrm{kg}$ & 129.5 & 0.709 & 89.3 & $80-120$ & \\
\hline Silver & 130 & 0.500 & $\mathrm{mg} / \mathrm{kg}$ & 129.5 & 0.462 & 100 & $80-120$ & \\
\hline Thallium & 131 & 0.500 & $\mathrm{mg} / \mathrm{kg}$ & 129.5 & 0.195 & 101 & $80-120$ & \\
\hline Vanadium & 275 & 0.500 & $\mathrm{mg} / \mathrm{kg}$ & 259.1 & 4.12 & 104 & $80-120$ & \\
\hline Zinc & 495 & 0.500 & $\mathrm{mg} / \mathrm{kg}$ & 518.1 & 24.8 & 90.8 & $80-120$ & B \\
\hline \multicolumn{9}{|c|}{ Batch B907036 - EPA 7471A Prep } \\
\hline Blank (B907036-BLK1) & \multicolumn{8}{|c|}{ Prepared: 06-Jul-2009 Analyzed: 22-Jul-2009 } \\
\hline Mercury & ND & 0.00400 & $\mathrm{mg} / \mathrm{kg}$ & & & & & \\
\hline Blank (B907036-BLK2) & \multicolumn{8}{|c|}{ Prepared: 06-Jul-2009 Analyzed: 22-Jul-2009 } \\
\hline Mercury & ND & 0.00400 & $\mathrm{mg} / \mathrm{kg}$ & & & & & \\
\hline LCS (B907036-BS1) & \multicolumn{8}{|c|}{ Prepared: 06-Jul-2009 Analyzed: 22-Jul-2009 } \\
\hline Mercury & 0609 & 0.00400 & $\mathrm{mg} / \mathrm{kg}$ & 0.06000 & & 101 & $75-125$ & \\
\hline
\end{tabular}




\section{USACE ERDC-EP-C \\ 3909 Halls Ferry Road \\ Vicksburg, MS 39180-6199}

ERDC - ECB

Project: TVA Fly Ash Project

Reported:

Project Manager: Tony Bednar

03-Sep-2009

DRAFT: Metals by EPA 6000/7000 Series Methods - Quality Control

ERDC- EL-EP-C (Environmental Chemistry Branch)

\begin{tabular}{|lcccccccccccc}
\hline & & Reporting & & Spike & Source & & & $\%$ REC & & RPD \\
Analyte & Result & Limit & Units & Level & Result & $\%$ REC & Limits & RPD & Limit & Notes & \\
\hline
\end{tabular}

Batch B907036 - EPA 7471A Prep

LCS (B907036-BS2)

Mercury

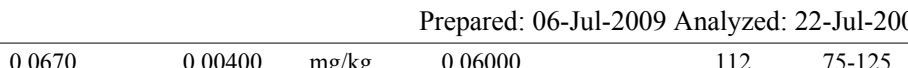

Duplicate (B907036-DUP1)

Source: 9061502-05 Prepared: 06-Jul-2009 Analyzed: 22-Jul-2009

Mercury

$0.0132 \quad 0.00395 \quad \mathrm{mg} / \mathrm{kg}$

Matrix Spike (B907036-MS1)

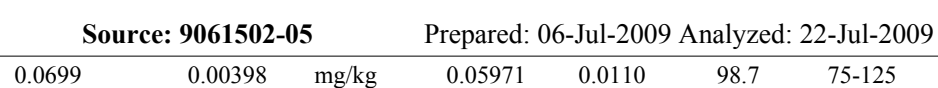

Mercury

Batch B907044 - EPA 7471A Prep

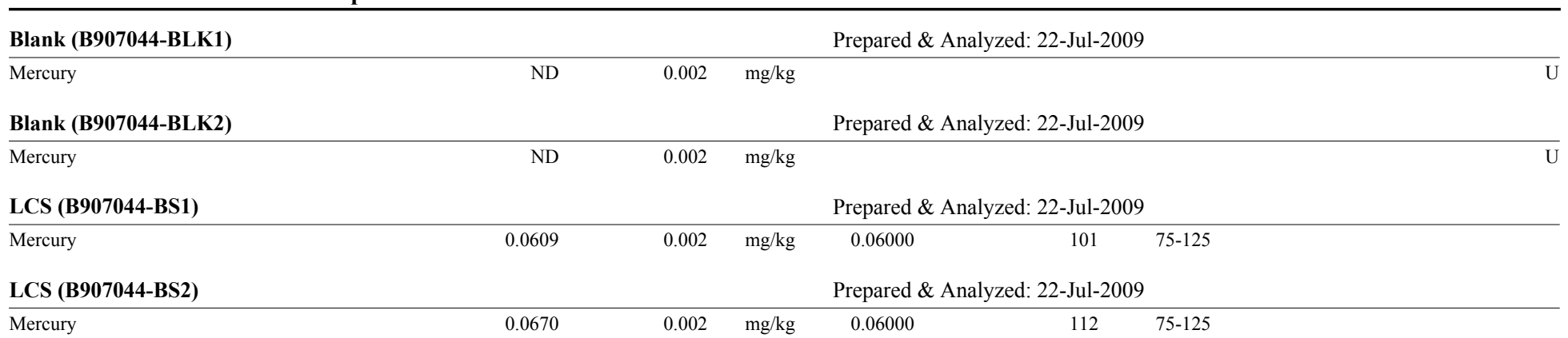

Batch B907052 - EPA 7470A Prep

Blank (B907052-BLK1)

Mercury

ND $\quad 0.000005 \quad \mathrm{mg} / \mathrm{L}$

Prepared \& Analyzed: 24-Jul-2009 


\section{USACE ERDC-EP-C \\ 3909 Halls Ferry Road \\ Vicksburg, MS 39180-6199}

ERDC - ECB

$-,-$
Project: TVA Fly Ash Project

Reported:

03-Sep-2009

DRAFT: Metals by EPA 6000/7000 Series Methods - Quality Control

ERDC- EL-EP-C (Environmental Chemistry Branch)

\begin{tabular}{|lcccccccccccc}
\hline & & Reporting & & Spike & Source & & \%REC & & RPD \\
Analyte & Result & Limit & Units & Level & Result & $\%$ REC & Limits & RPD & Limit & Notes & \\
\hline
\end{tabular}

Batch B907052 - EPA 7470A Prep

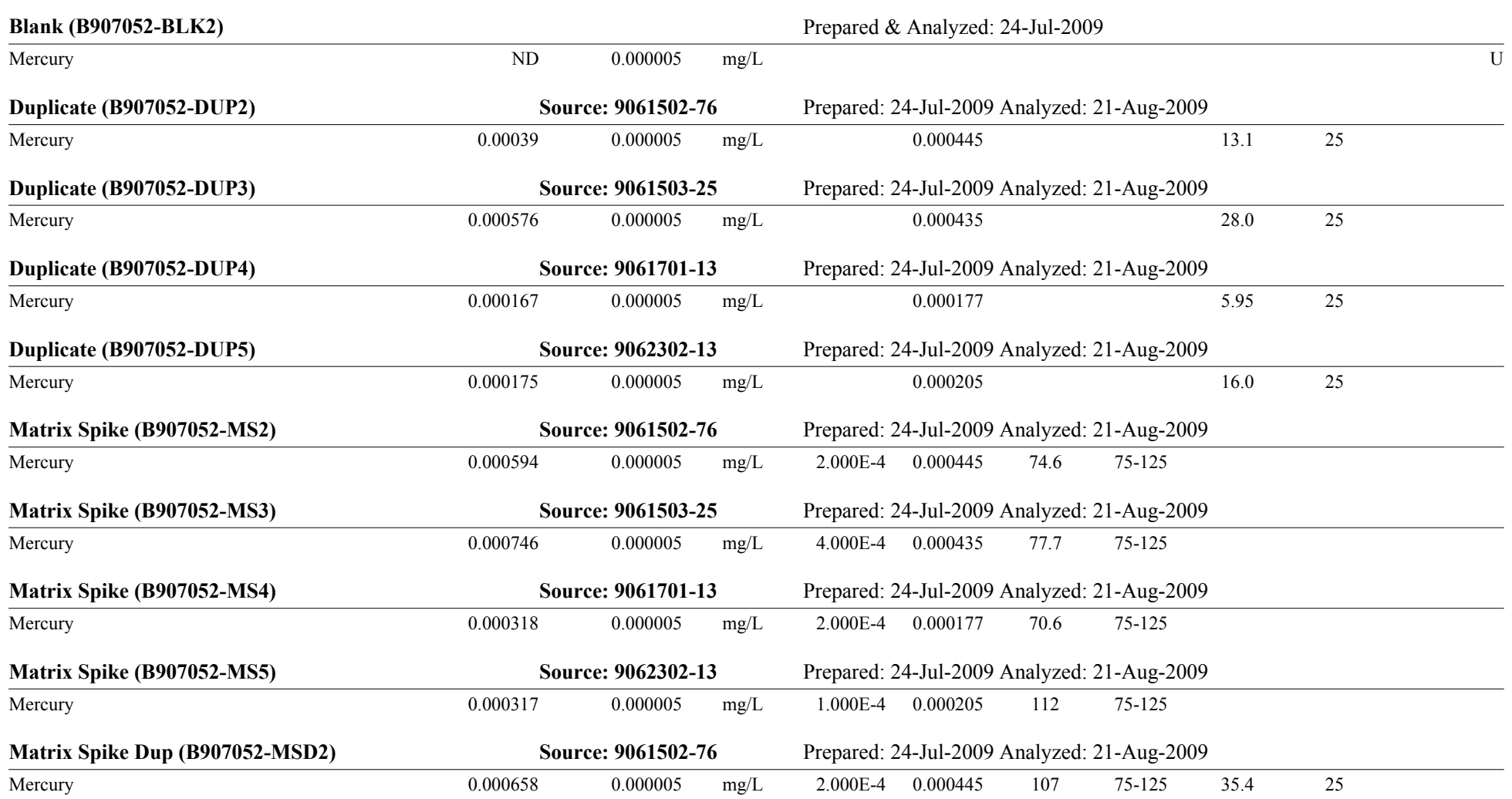




\section{USACE ERDC-EP-C \\ 3909 Halls Ferry Road \\ Vicksburg, MS 39180-6199}

ERDC - ECB

Project: TVA Fly Ash Project

Reported:

Project Manager: Tony Bednar

03-Sep-2009

DRAFT: Metals by EPA 6000/7000 Series Methods - Quality Control

ERDC- EL-EP-C (Environmental Chemistry Branch)

\begin{tabular}{|c|c|c|c|c|c|c|c|c|c|c|}
\hline & & eporting & & Spike & Source & & $\%$ REC & & RPD & \\
\hline Analyte & Result & Limit & Units & Level & Result & $\%$ REC & Limits & RPD & Limit & Notes \\
\hline
\end{tabular}

Batch B907052 - EPA 7470A Prep

\begin{tabular}{|c|c|c|c|c|c|c|c|c|c|}
\hline \multirow{2}{*}{$\begin{array}{l}\text { Matrix Spike Dup (B907052-MSD3) } \\
\text { Mercury }\end{array}$} & \multicolumn{3}{|c|}{ Source: $9061503-25$} & \multicolumn{6}{|c|}{ Prepared: 24-Jul-2009 Analyzed: 21-Aug-2009 } \\
\hline & 0.000829 & 0.000005 & $\mathrm{mg} / \mathrm{L}$ & $4.000 \mathrm{E}-4$ & 0.000435 & 98.5 & $75-125$ & 23.6 & 25 \\
\hline Matrix Spike Dup (B907052-MSD4) & \multicolumn{3}{|c|}{ Source: $9061701-13$} & \multicolumn{6}{|c|}{ Prepared: 24-Jul-2009 Analyzed: 21-Aug-2009 } \\
\hline Mercury & 0.000338 & 0.000005 & $\mathrm{mg} / \mathrm{L}$ & $2.000 \mathrm{E}-4$ & 0.000177 & 80.8 & $75-125$ & 13.5 & 25 \\
\hline Matrix Spike Dup (B907052-MSD5) & \multicolumn{3}{|c|}{ Source: $9062302-13$} & \multicolumn{6}{|c|}{ Prepared: 24-Jul-2009 Analyzed: 21-Aug-2009 } \\
\hline Mercury & 0.000327 & 0.000005 & $\mathrm{mg} / \mathrm{L}$ & $1.000 \mathrm{E}-4$ & 0.000205 & 122 & $75-125$ & 8.04 & 25 \\
\hline
\end{tabular}

Batch B908051 - EPA 3050B

\begin{tabular}{|c|c|c|c|c|}
\hline \multirow{2}{*}{$\frac{\text { Blank (B908051-BLK1) }}{\text { Antimony }}$} & \multicolumn{4}{|c|}{ Prepared: 28-Aug-2009 Analyzed: 29-Aug-2009 } \\
\hline & ND & 0.400 & $\mathrm{mg} / \mathrm{kg}$ & $\overline{\mathrm{U}}$ \\
\hline Arsenic & ND & 0.400 & $\mathrm{mg} / \mathrm{kg}$ & $\mathrm{U}$ \\
\hline Beryllium & ND & 0.400 & $\mathrm{mg} / \mathrm{kg}$ & $\mathrm{U}$ \\
\hline Cadmium & ND & 0.400 & $\mathrm{mg} / \mathrm{kg}$ & $\mathrm{U}$ \\
\hline Chromium & ND & 0.400 & $\mathrm{mg} / \mathrm{kg}$ & $\mathrm{U}$ \\
\hline Cobalt & ND & 0.400 & $\mathrm{mg} / \mathrm{kg}$ & $\mathrm{U}$ \\
\hline Copper & ND & 0.400 & $\mathrm{mg} / \mathrm{kg}$ & $\mathrm{U}$ \\
\hline Lead & 1.22 & 0.400 & $\mathrm{mg} / \mathrm{kg}$ & \\
\hline Manganese & ND & 0.400 & $\mathrm{mg} / \mathrm{kg}$ & $\mathrm{U}$ \\
\hline Molybdenum & ND & 0.400 & $\mathrm{mg} / \mathrm{kg}$ & $\mathrm{U}$ \\
\hline Nickel & ND & 0.400 & $\mathrm{mg} / \mathrm{kg}$ & $\mathrm{U}$ \\
\hline Selenium & ND & 0.400 & $\mathrm{mg} / \mathrm{kg}$ & $\mathrm{U}$ \\
\hline Thallium & ND & 0.400 & $\mathrm{mg} / \mathrm{kg}$ & $\mathrm{U}$ \\
\hline Vanadium & ND & 0.400 & $\mathrm{mg} / \mathrm{kg}$ & U \\
\hline Zinc & 0.348 & 0.400 & $\mathrm{mg} / \mathrm{kg}$ & J \\
\hline
\end{tabular}




\section{USACE ERDC-EP-C \\ 3909 Halls Ferry Road \\ Vicksburg, MS 39180-6199}

ERDC - ECB

$-,-$
Project: TVA Fly Ash Project

Project Manager: Tony Bednar
Reported:

03-Sep-2009

DRAFT: Metals by EPA 6000/7000 Series Methods - Quality Control

ERDC- EL-EP-C (Environmental Chemistry Branch)

\begin{tabular}{|c|c|c|c|c|c|c|c|c|c|c|}
\hline & & eporting & & Spike & Source & & $\%$ REC & & RPD & \\
\hline Analyte & Result & Limit & Units & Level & Result & $\%$ REC & Limits & RPD & Limit & Notes \\
\hline
\end{tabular}

Batch B908051 - EPA 3050B

\begin{tabular}{|c|c|c|c|c|c|c|c|}
\hline \multirow{2}{*}{$\frac{\text { Blank (B908051-BLK2) }}{\text { Antimony }}$} & \multicolumn{7}{|c|}{ Prepared: 28-Aug-2009 Analyzed: 29-Aug-2009 } \\
\hline & 0.498 & 0.400 & $\mathrm{mg} / \mathrm{kg}$ & & & & \\
\hline Arsenic & ND & 0.400 & $\mathrm{mg} / \mathrm{kg}$ & & & & $\mathrm{U}$ \\
\hline Beryllium & ND & 0.400 & $\mathrm{mg} / \mathrm{kg}$ & & & & $\mathrm{U}$ \\
\hline Cadmium & ND & 0.400 & $\mathrm{mg} / \mathrm{kg}$ & & & & $\mathrm{U}$ \\
\hline Chromium & ND & 0.400 & $\mathrm{mg} / \mathrm{kg}$ & & & & $\mathrm{U}$ \\
\hline Cobalt & ND & 0.400 & $\mathrm{mg} / \mathrm{kg}$ & & & & $\mathrm{U}$ \\
\hline Copper & ND & 0.400 & $\mathrm{mg} / \mathrm{kg}$ & & & & $\mathrm{U}$ \\
\hline Lead & ND & 0.400 & $\mathrm{mg} / \mathrm{kg}$ & & & & $\mathrm{U}$ \\
\hline Manganese & ND & 0.400 & $\mathrm{mg} / \mathrm{kg}$ & & & & $\mathrm{U}$ \\
\hline Molybdenum & ND & 0.400 & $\mathrm{mg} / \mathrm{kg}$ & & & & $\mathrm{U}$ \\
\hline Nickel & ND & 0.400 & $\mathrm{mg} / \mathrm{kg}$ & & & & $\mathrm{U}$ \\
\hline Selenium & ND & 0.400 & $\mathrm{mg} / \mathrm{kg}$ & & & & $\mathrm{U}$ \\
\hline Thallium & ND & 0.400 & $\mathrm{mg} / \mathrm{kg}$ & & & & $\mathrm{U}$ \\
\hline Vanadium & ND & 0.400 & $\mathrm{mg} / \mathrm{kg}$ & & & & $\mathrm{U}$ \\
\hline Zinc & 0.365 & 0.400 & $\mathrm{mg} / \mathrm{kg}$ & & & & $\mathrm{J}$ \\
\hline LCS (B908051-BS1) & \multicolumn{7}{|c|}{ Prepared: 28-Aug-2009 Analyzed: 29-Aug-2009 } \\
\hline Antimony & 93.0 & 0.400 & $\mathrm{mg} / \mathrm{kg}$ & 100.0 & 93.0 & $80-120$ & B \\
\hline Arsenic & 46.3 & 0.400 & $\mathrm{mg} / \mathrm{kg}$ & 50.00 & 92.6 & $80-120$ & \\
\hline Barium & 194 & 0.400 & $\mathrm{mg} / \mathrm{kg}$ & 200.0 & 97.1 & $80-120$ & \\
\hline Beryllium & 48.2 & 0.400 & $\mathrm{mg} / \mathrm{kg}$ & 50.00 & 96.4 & $80-120$ & \\
\hline Cadmium & 46.6 & 0.400 & $\mathrm{mg} / \mathrm{kg}$ & 50.00 & 93.1 & $80-120$ & \\
\hline Chromium & 100 & 0.400 & $\mathrm{mg} / \mathrm{kg}$ & 100.0 & 100 & $80-120$ & \\
\hline Cobalt & 102 & 0.400 & $\mathrm{mg} / \mathrm{kg}$ & 100.0 & 102 & $80-120$ & \\
\hline Copper & 97.4 & 0.400 & $\mathrm{mg} / \mathrm{kg}$ & 100.0 & 97.4 & $80-120$ & \\
\hline Molybdenum & 48.0 & 0.400 & $\mathrm{mg} / \mathrm{kg}$ & 50.00 & 95.9 & $80-120$ & \\
\hline Nickel & 100 & 0.400 & $\mathrm{mg} / \mathrm{kg}$ & 100.0 & 100 & $80-120$ & \\
\hline Selenium & 42.8 & 0.400 & $\mathrm{mg} / \mathrm{kg}$ & 50.00 & 85.6 & $80-120$ & \\
\hline Silver & 46.9 & 0.400 & $\mathrm{mg} / \mathrm{kg}$ & 50.00 & 93.7 & $80-120$ & \\
\hline Thallium & 47.8 & 0.400 & $\mathrm{mg} / \mathrm{kg}$ & 50.00 & 95.6 & $80-120$ & \\
\hline Vanadium & 99.7 & 0.400 & $\mathrm{mg} / \mathrm{kg}$ & 100.0 & 99.7 & $80-120$ & \\
\hline Zinc & 176 & 0.400 & $\mathrm{mg} / \mathrm{kg}$ & 200.0 & 88.2 & $80-120$ & \\
\hline
\end{tabular}




\section{USACE ERDC-EP-C \\ 3909 Halls Ferry Road \\ Vicksburg, MS 39180-6199}

ERDC - ECB
Project: TVA Fly Ash Project

Project Manager: Tony Bednar
Reported:

03-Sep-2009

\section{DRAFT: Metals by EPA 6000/7000 Series Methods - Quality Control}

\section{ERDC- EL-EP-C (Environmental Chemistry Branch)}

\begin{tabular}{|lcccccccccccc}
\hline & & Reporting & & Spike & Source & & & \%REC & & RPD \\
Analyte & Result & Limit & Units & Level & Result & \%REC & Limits & RPD & Limit & Notes & \\
\hline
\end{tabular}

\section{Batch B908051 - EPA 3050B}

\begin{tabular}{|c|c|c|c|c|c|c|c|c|c|}
\hline \multirow{2}{*}{$\frac{\text { LCS (B908051-BS2) }}{\text { Antimony }}$} & \multicolumn{9}{|c|}{ Prepared: 28-Aug-2009 Analyzed: 29-Aug-2009 } \\
\hline & 95.7 & 0.400 & $\mathrm{mg} / \mathrm{kg}$ & 100.0 & 95.7 & \multicolumn{3}{|l|}{$80-120$} & \multirow[t]{2}{*}{ B } \\
\hline Arsenic & 46.8 & 0.400 & $\mathrm{mg} / \mathrm{kg}$ & 50.00 & 93.6 & \multicolumn{3}{|l|}{$80-120$} & \\
\hline Barium & 197 & 0.400 & $\mathrm{mg} / \mathrm{kg}$ & 200.0 & 98.3 & \multicolumn{3}{|l|}{$80-120$} & \\
\hline Beryllium & 47.3 & 0.400 & $\mathrm{mg} / \mathrm{kg}$ & 50.00 & 94.5 & \multicolumn{3}{|l|}{$80-120$} & \\
\hline Cadmium & 46.4 & 0.400 & $\mathrm{mg} / \mathrm{kg}$ & 50.00 & 92.8 & \multicolumn{3}{|l|}{$80-120$} & \\
\hline Chromium & 101 & 0.400 & $\mathrm{mg} / \mathrm{kg}$ & 100.0 & 101 & \multicolumn{3}{|l|}{$80-120$} & \\
\hline Copper & 96.5 & 0.400 & $\mathrm{mg} / \mathrm{kg}$ & 100.0 & 96.5 & \multicolumn{3}{|l|}{$80-120$} & \\
\hline Lead & 97.5 & 0.400 & $\mathrm{mg} / \mathrm{kg}$ & 100.0 & 97.5 & \multicolumn{3}{|l|}{$80-120$} & B \\
\hline Manganese & 235 & 0.400 & $\mathrm{mg} / \mathrm{kg}$ & 250.0 & 94.2 & \multicolumn{3}{|l|}{$80-120$} & \\
\hline Molybdenum & 48.6 & 0.400 & $\mathrm{mg} / \mathrm{kg}$ & 50.00 & 97.1 & \multicolumn{3}{|l|}{$80-120$} & \\
\hline Nickel & 97.8 & 0.400 & $\mathrm{mg} / \mathrm{kg}$ & 100.0 & 97.8 & \multicolumn{3}{|l|}{$80-120$} & \\
\hline Selenium & 43.1 & 0.400 & $\mathrm{mg} / \mathrm{kg}$ & 50.00 & 86.1 & \multicolumn{3}{|l|}{$80-120$} & \\
\hline Thallium & 48.3 & 0.400 & $\mathrm{mg} / \mathrm{kg}$ & 50.00 & 96.7 & \multicolumn{3}{|l|}{$80-120$} & \\
\hline Vanadium & 99.5 & 0.400 & $\mathrm{mg} / \mathrm{kg}$ & 100.0 & 99.5 & \multicolumn{3}{|l|}{$80-120$} & \\
\hline Zinc & 176 & 0.400 & $\mathrm{mg} / \mathrm{kg}$ & 200.0 & 88.2 & \multicolumn{3}{|l|}{$80-120$} & \\
\hline Duplicate (B908051-DUP1) & \multicolumn{3}{|c|}{ Source: $9082706-03$} & \multicolumn{5}{|c|}{ Prepared: 28-Aug-2009 Analyzed: 29-Aug-2009 } & \\
\hline Antimony & 0.721 & 0.400 & $\mathrm{mg} / \mathrm{kg}$ & & & & & 20 & B \\
\hline Arsenic & 0.113 & 0.400 & $\mathrm{mg} / \mathrm{kg}$ & & & & & 20 & $\mathrm{~J}$ \\
\hline Barium & 2.62 & 0.400 & $\mathrm{mg} / \mathrm{kg}$ & & & & 35.0 & 20 & \\
\hline Beryllium & ND & 0.400 & $\mathrm{mg} / \mathrm{kg}$ & & & & & 20 & $\mathrm{U}$ \\
\hline Cadmium & ND & 0.400 & $\mathrm{mg} / \mathrm{kg}$ & & & & & 20 & $\mathrm{U}$ \\
\hline Chromium & 2.01 & 0.400 & $\mathrm{mg} / \mathrm{kg}$ & & & & 11.6 & 20 & \\
\hline Cobalt & ND & 0.400 & $\mathrm{mg} / \mathrm{kg}$ & & & & & 20 & $\mathrm{U}$ \\
\hline Copper & 0.878 & 0.400 & $\mathrm{mg} / \mathrm{kg}$ & & & & 27.9 & 20 & \\
\hline Lead & 0.397 & 0.400 & $\mathrm{mg} / \mathrm{kg}$ & & & & 38.9 & 20 & $\mathrm{~J}, \mathrm{~B}$ \\
\hline Molybdenum & 0.096 & 0.400 & $\mathrm{mg} / \mathrm{kg}$ & & & & & 20 & $\mathrm{~J}$ \\
\hline Nickel & 1.51 & 0.400 & $\mathrm{mg} / \mathrm{kg}$ & & & & 22.9 & 20 & \\
\hline Selenium & 0.182 & 0.400 & $\mathrm{mg} / \mathrm{kg}$ & & & & 70.3 & 20 & $\mathrm{~J}$ \\
\hline Silver & 0.143 & 0.400 & $\mathrm{mg} / \mathrm{kg}$ & & & & & 20 & $\mathrm{~J}$ \\
\hline Thallium & ND & 0.400 & $\mathrm{mg} / \mathrm{kg}$ & & & & & 20 & $\mathrm{U}$ \\
\hline Vanadium & 0.081 & 0.400 & $\mathrm{mg} / \mathrm{kg}$ & & & & & 20 & $\mathrm{~J}$ \\
\hline Zinc & 31.5 & 0.400 & $\mathrm{mg} / \mathrm{kg}$ & & & & 33.1 & 20 & \\
\hline
\end{tabular}




\section{USACE ERDC-EP-C \\ 3909 Halls Ferry Road \\ Vicksburg, MS 39180-6199}

ERDC - ECB

Project: TVA Fly Ash Project

Reported:

Project Manager: Tony Bednar

03-Sep-2009

DRAFT: Metals by EPA 6000/7000 Series Methods - Quality Control

ERDC- EL-EP-C (Environmental Chemistry Branch)

\begin{tabular}{|lrrrrrrrrrrr}
\hline & & Reporting & & Spike & Source & & & $\%$ REC & & RPD \\
Analyte & Result & Limit & Units & Level & Result & $\%$ REC & Limits & RPD & Limit & Notes & \\
\hline
\end{tabular}

Batch B908051 - EPA 3050B

\begin{tabular}{|c|c|c|c|c|c|c|c|c|}
\hline \multirow{2}{*}{$\begin{array}{l}\text { Matrix Spike (B908051-MS1) } \\
\text { Antimony }\end{array}$} & \multicolumn{3}{|c|}{ Source: 9082706-03 } & \multicolumn{4}{|c|}{ Prepared: 28-Aug-2009 Analyzed: 29-Aug-2009 } & \multirow[b]{2}{*}{$\mathrm{B}$} \\
\hline & 172 & 0.400 & $\mathrm{mg} / \mathrm{kg}$ & 170.2 & ND & 101 & $80-120$ & \\
\hline Arsenic & 87.7 & 0.400 & $\mathrm{mg} / \mathrm{kg}$ & 85.09 & ND & 103 & $80-120$ & \\
\hline Barium & 360 & 0.400 & $\mathrm{mg} / \mathrm{kg}$ & 340.4 & 1.84 & 105 & $80-120$ & \\
\hline Beryllium & 85.0 & 0.400 & $\mathrm{mg} / \mathrm{kg}$ & 85.09 & ND & 99.9 & $80-120$ & \\
\hline Cadmium & 84.2 & 0.400 & $\mathrm{mg} / \mathrm{kg}$ & 85.09 & ND & 98.9 & $80-120$ & \\
\hline Chromium & 189 & 0.400 & $\mathrm{mg} / \mathrm{kg}$ & 170.2 & 1.79 & 110 & $80-120$ & \\
\hline Cobalt & 184 & 0.400 & $\mathrm{mg} / \mathrm{kg}$ & 170.2 & ND & 108 & $80-120$ & \\
\hline Copper & 180 & 0.400 & $\mathrm{mg} / \mathrm{kg}$ & 170.2 & 0.663 & 105 & $80-120$ & \\
\hline Lead & 175 & 0.400 & $\mathrm{mg} / \mathrm{kg}$ & 170.2 & 0.588 & 102 & $80-120$ & B \\
\hline Manganese & 435 & 0.400 & $\mathrm{mg} / \mathrm{kg}$ & 425.5 & 0.742 & 102 & $80-120$ & \\
\hline Molybdenum & 90.5 & 0.400 & $\mathrm{mg} / \mathrm{kg}$ & 85.09 & $\mathrm{ND}$ & 106 & $80-120$ & \\
\hline Nickel & 181 & 0.400 & $\mathrm{mg} / \mathrm{kg}$ & 170.2 & 1.20 & 106 & $80-120$ & \\
\hline Selenium & 79.7 & 0.400 & $\mathrm{mg} / \mathrm{kg}$ & 85.09 & 0.087 & 93.6 & $80-120$ & \\
\hline Silver & 84.7 & 0.400 & $\mathrm{mg} / \mathrm{kg}$ & 85.09 & ND & 99.6 & $80-120$ & \\
\hline Thallium & 87.5 & 0.400 & $\mathrm{mg} / \mathrm{kg}$ & 85.09 & ND & 103 & $80-120$ & \\
\hline Vanadium & 184 & 0.400 & $\mathrm{mg} / \mathrm{kg}$ & 170.2 & $\mathrm{ND}$ & 108 & $80-120$ & \\
\hline Zinc & 368 & 0.400 & $\mathrm{mg} / \mathrm{kg}$ & 340.4 & 22.5 & 102 & $80-120$ & \\
\hline \multicolumn{9}{|c|}{ Batch B909005 - EPA 7471A Prep } \\
\hline Blank (B909005-BLK1) & \multicolumn{7}{|c|}{ Prepared \& Analyzed: 03-Sep-2009 } & \\
\hline Mercury & ND & 0.002 & $\mathrm{mg} / \mathrm{kg}$ & & & & & $\overline{\mathrm{U}}$ \\
\hline Blank (B909005-BLK2) & \multicolumn{7}{|c|}{ Prepared \& Analyzed: 03-Sep-2009 } & \\
\hline Mercury & ND & 0.002 & $\mathrm{mg} / \mathrm{kg}$ & & & & & $\mathrm{U}$ \\
\hline LCS (B909005-BS1) & \multicolumn{7}{|c|}{ Prepared \& Analyzed: 03-Sep-2009 } & \\
\hline Mercury & 0.0691 & 0.002 & $\mathrm{mg} / \mathrm{kg}$ & 0.06000 & & 115 & $75-125$ & \\
\hline
\end{tabular}




\section{USACE ERDC-EP-C \\ 3909 Halls Ferry Road \\ Vicksburg, MS 39180-6199}

ERDC - ECB

Project: TVA Fly Ash Project

Reported:

Project Manager: Tony Bednar

03-Sep-2009

DRAFT: Metals by EPA 6000/7000 Series Methods - Quality Control

ERDC- EL-EP-C (Environmental Chemistry Branch)

\begin{tabular}{|c|c|c|c|c|c|c|c|c|c|c|}
\hline & & eporting & & Spike & Source & & $\%$ REC & & RPD & \\
\hline Analyte & Result & Limit & Units & Level & Result & $\%$ REC & Limits & RPD & Limit & Notes \\
\hline
\end{tabular}

Batch B909005 - EPA 7471A Prep

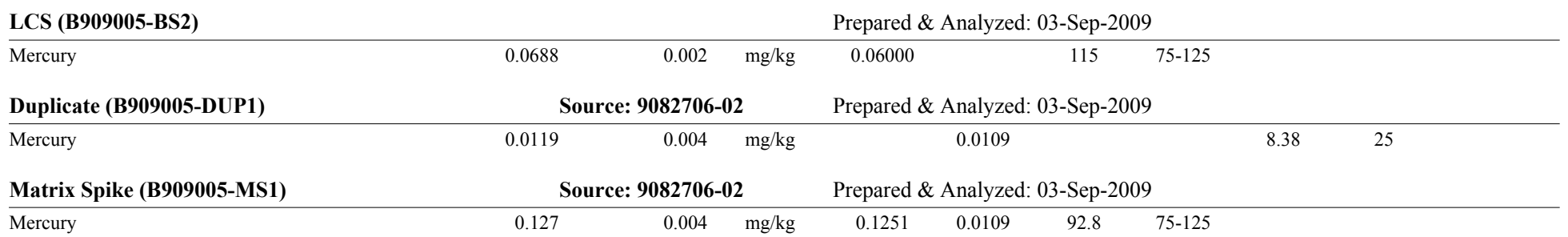




\section{USACE ERDC-EP-C \\ 3909 Halls Ferry Road \\ Vicksburg, MS 39180-6199}

ERDC - ECB

Project: TVA Fly Ash Project

$-\cdot,-$

Project Manager: Tony Bednar

Reported:

03-Sep-2009

DRAFT: Miscellaneous Physical/Conventional Chemistry Parameters - Quality Control

ERDC- EL-EP-C (Environmental Chemistry Branch)

\begin{tabular}{|lccccccccccc}
\hline & & Reporting & & Spike & Source & & & $\%$ REC & & RPD \\
Analyte & Result & Limit & Units & Level & Result & \%REC & Limits & RPD & Limit & Notes & \\
\hline
\end{tabular}

Batch B906084 - TS/TSS/TDS Prep

Blank (B906084-BLK1)

Total Solids

Prepared \& Analyzed: 25-Jun-2009

Duplicate (B906084-DUP1)

$10.0 \quad \mathrm{mg} / \mathrm{L}$

Total Solids

Source: 9062302-04

Prepared \& Analyzed: 25-Jun-2009

$695 \quad 10.0 \quad \mathrm{mg} / \mathrm{L}$

700

0.717

30

Batch B906085 - TS/TSS/TDS Prep

Blank (B906085-BLK1)

Total Suspended Solids

ND $25.0 \quad \mathrm{mg} / \mathrm{L}$

Prepared \& Analyzed: 25-Jun-2009

Duplicate (B906085-DUP1)

Source: 9062302-09 Prepared \& Analyzed: 25-Jun-2009

Total Suspended Solids

$425 \quad 25.0 \quad \mathrm{mg} / \mathrm{L}$

3.59

20

Batch B906086 - TS/TSS/TDS Prep

Blank (B906086-BLK1)

Total Dissolved Solids

Duplicate (B906086-DUP1)

Total Dissolved Solids

Batch B907020 - Default Prep GenChem

Blank (B907020-BLK2)

Chloride

Sulfate

Bromide

Nitrate as $\mathrm{N}$

SS/TDS Prep

Project: TVA Fly Ash Project
Reported:
Project Manager: Tony Bednar




\section{USACE ERDC-EP-C \\ 3909 Halls Ferry Road \\ Vicksburg, MS 39180-6199}

ERDC - ECB

$--,-$
Project: TVA Fly Ash Project

Reported:

Project Manager: Tony Bednar
03-Sep-2009

DRAFT: Miscellaneous Physical/Conventional Chemistry Parameters - Quality Control

ERDC- EL-EP-C (Environmental Chemistry Branch)

\begin{tabular}{|lcccccccccccc}
\hline & & Reporting & & Spike & Source & & $\%$ REC & & RPD \\
Analyte & Result & Limit & Units & Level & Result & $\%$ REC & Limits & RPD & Limit & Notes \\
\hline
\end{tabular}

Batch B907020 - Default Prep GenChem

\begin{tabular}{llclccc}
\hline Duplicate (B907020-DUP1) & \multicolumn{7}{c}{ Source: 9061502-24 } & Prepared \& Analyzed: 14-Jul-2009 & \\
\hline Chloride & 2.34 & 1.00 & $\mathrm{mg} / \mathrm{L}$ & 2.36 & 2.851 & 20 \\
Sulfate & 12.2 & 0.100 & $\mathrm{mg} / \mathrm{L}$ & 12.5 & 2.19 \\
Bromide & $\mathrm{ND}$ & 0.100 & $\mathrm{mg} / \mathrm{L}$ & $\mathrm{ND}$ & 20 \\
Nitrate as N & 0.390 & 0.100 & $\mathrm{mg} / \mathrm{L}$ & 0.400 & 20 \\
\end{tabular}

Duplicate (B907020-DUP2)

Chloride

Sulfate

Source: 9061502-41

Prepared \& Analyzed: 14-Jul-2009

\begin{tabular}{rrrccc}
\multicolumn{7}{c}{ Source: 9061502-41 } & Prepared \& Analyzed: 14-Jul-2009 \\
\hline 2.60 & 1.00 & $\mathrm{mg} / \mathrm{L}$ & 2.60 & 0.00 & 20 \\
13.3 & 0.100 & $\mathrm{mg} / \mathrm{L}$ & 13.2 & 0.452 & 20 \\
ND & 0.100 & $\mathrm{mg} / \mathrm{L}$ & $\mathrm{ND}$ & & 20 \\
0.390 & 0.100 & $\mathrm{mg} / \mathrm{L}$ & 0.396 & 1.53 & 20
\end{tabular}

Nitrate as $\mathrm{N}$

0.390

$0.100 \quad \mathrm{mg} / \mathrm{L}$

0.396

Batch B907021 - Default Prep GenChem

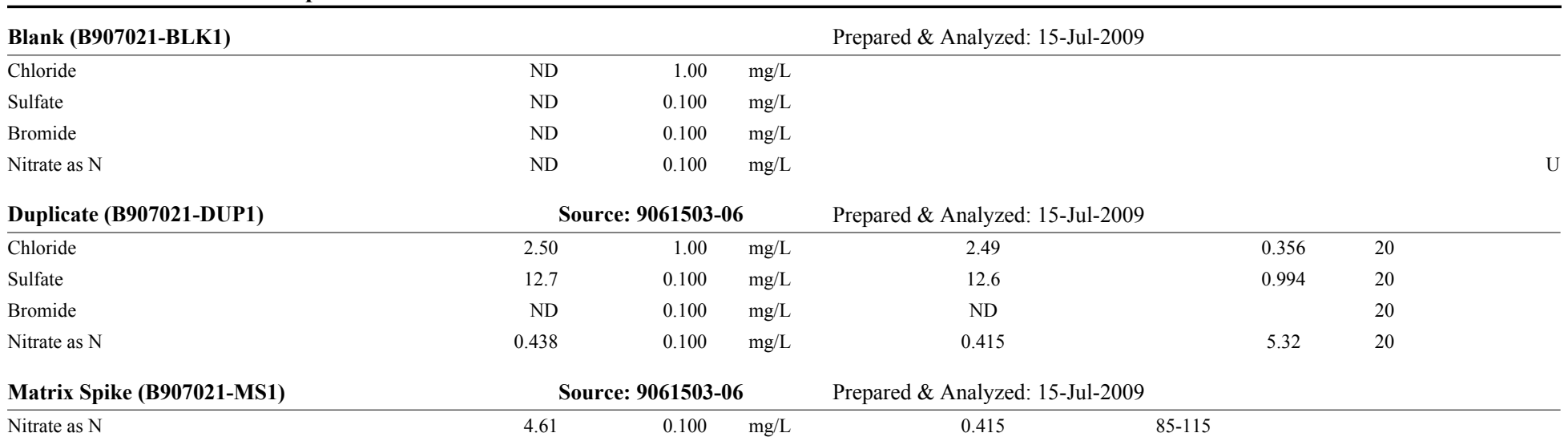




\section{USACE ERDC-EP-C \\ 3909 Halls Ferry Road \\ Vicksburg, MS 39180-6199}

ERDC - ECB

Project: TVA Fly Ash Project

Reported:

Project Manager: Tony Bednar

03-Sep-2009

DRAFT: Miscellaneous Physical/Conventional Chemistry Parameters - Quality Control

ERDC- EL-EP-C (Environmental Chemistry Branch)

\begin{tabular}{|c|c|c|c|c|c|c|c|c|c|c|}
\hline & & eporting & & Spike & Source & & $\%$ REC & & RPD & \\
\hline Analyte & Result & Limit & Units & Level & Result & $\%$ REC & Limits & RPD & Limit & Notes \\
\hline
\end{tabular}

Batch B907021 - Default Prep GenChem

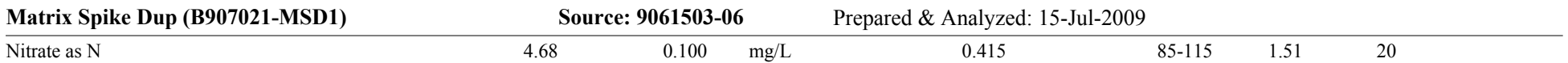

Batch B907022 - Default Prep GenChem

\begin{tabular}{lccrrr} 
Duplicate (B907022-DUP1) & \multicolumn{2}{c}{ Source: 9061701-03 } & \multicolumn{2}{c}{ Prepared: 15-Jul-2009 Analyzed: 16-Jul-2009 } \\
\hline Chloride & 2.68 & 1.00 & $\mathrm{mg} / \mathrm{L}$ & 2.68 & 12.9 \\
Sulfate & 12.9 & 0.100 & $\mathrm{mg} / \mathrm{L}$ & 0.201 & 0.102 \\
Bromide & $\mathrm{ND}$ & 0.100 & $\mathrm{mg} / \mathrm{L}$ & 20 & $\mathrm{ND}$ \\
Nitrate as N & 0.390 & 0.100 & $\mathrm{mg} / \mathrm{L}$ & 0.393
\end{tabular}




\section{USACE ERDC-EP-C \\ 3909 Halls Ferry Road \\ Vicksburg, MS 39180-6199}

ERDC - ECB

Project: TVA Fly Ash Project

Reported:

Project Manager: Tony Bednar

03-Sep-2009

\section{Notes and Definitions}

U Analyte included in the analysis, but not detected

J Detected but below the Reporting Limit; therefore, result is an estimated concentration (CLP J-Flag).

B Analyte is found in the associated blank as well as in the sample (CLP B-flag).

DET Analyte DETECTED

ND Analyte NOT DETECTED at or above the reporting limit

NR Not Reported

dry Sample results reported on a dry weight basis

RPD Relative Percent Difference 


\section{USACE ERDC-EP-C \\ 3909 Halls Ferry Road \\ Vicksburg, MS 39180-6199}

03 September 2009

Tony Bednar

ERDC - ECB

$-,--$

RE: TVA Fly Ash Project

Enclosed are the results of analyses for samples received by the laboratory on 27-Aug-2009. The samples associated with this report will be held for 90 days from the date of this report. The raw data associated with this report will be held for 5 years from the date of this report. If you need us to hold onto the samples or the data longer then these specified times, you will need to notify us in writing at least 30 days before the expiration dates. If you have any questions concerning this report, please feel free to contact me.

Sincerely,

Patty Tuminello

Project Coordinator 


\section{USACE ERDC-EP-C \\ 3909 Halls Ferry Road \\ Vicksburg, MS 39180-6199}

ERDC - ECB

Project: TVA Fly Ash Project

Reported:

$--,-$

Project Manager: Tony Bednar

03-Sep-2009

WORK ORDER SUMMARY

\begin{tabular}{|c|c|c|c|c|}
\hline Sample ID & Laboratory ID & Matrix & Date Sampled & Date of Work Order \\
\hline Fish Tissue Dechlor 1 & $9082706-01$ & Tissue & 26-Aug-2009 & 27-Aug-2009 \\
\hline Fish Tissue Dechlor 2 & $9082706-02$ & Tissue & 26-Aug-2009 & 27-Aug-2009 \\
\hline Fish Tissue Dechlor 3 & $9082706-03$ & Tissue & 26-Aug-2009 & 27-Aug-2009 \\
\hline Fish Tissue Dechlor 4 & 9082706-04 & Tissue & 26-Aug-2009 & 27-Aug-2009 \\
\hline Fish Tissue Dechlor 5 & $9082706-05$ & Tissue & 26-Aug-2009 & 27-Aug-2009 \\
\hline Fish Tissue EMR - WA 1 & $9082706-06$ & Tissue & 26-Aug-2009 & 27-Aug-2009 \\
\hline Fish Tissue EMR - WA 2 & $9082706-07$ & Tissue & 26-Aug-2009 & 27-Aug-2009 \\
\hline Fish Tissue EMR - WA 3 & $9082706-08$ & Tissue & 26-Aug-2009 & 27-Aug-2009 \\
\hline Fish Tissue EMR - WA 4 & $9082706-09$ & Tissue & 26-Aug-2009 & 27-Aug-2009 \\
\hline Fish Tissue EMR - WA 5 & $9082706-10$ & Tissue & 26-Aug-2009 & 27-Aug-2009 \\
\hline Fish Tissue EMR - EL 1 & $9082706-11$ & Tissue & 26-Aug-2009 & 27-Aug-2009 \\
\hline Fish Tissue EMR - EL 2 & $9082706-12$ & Tissue & 26-Aug-2009 & 27-Aug-2009 \\
\hline Fish Tissue EMR - EL 3 & $9082706-13$ & Tissue & 26-Aug-2009 & 27-Aug-2009 \\
\hline Fish Tissue EMR - EL 4 & $9082706-14$ & Tissue & 26-Aug-2009 & 27-Aug-2009 \\
\hline Fish Tissue EMR - EL 5 & $9082706-15$ & Tissue & 26-Aug-2009 & 27-Aug-2009 \\
\hline Fish Gut Dechlor 1 & $9082706-16$ & Tissue & 26-Aug-2009 & 27-Aug-2009 \\
\hline Fish Gut Dechlor 2 & $9082706-17$ & Tissue & 26-Aug-2009 & 27-Aug-2009 \\
\hline Fish Gut Dechlor 3 & $9082706-18$ & Tissue & 26-Aug-2009 & 27-Aug-2009 \\
\hline Fish Gut Dechlor 4 & $9082706-19$ & Tissue & 26-Aug-2009 & 27-Aug-2009 \\
\hline Fish Gut Dechlor 5 & $9082706-20$ & Tissue & 26-Aug-2009 & 27-Aug-2009 \\
\hline Fish Gut EMR - WA 1 & $9082706-21$ & Tissue & 26-Aug-2009 & 27-Aug-2009 \\
\hline Fish Gut EMR - WA 2 & $9082706-22$ & Tissue & 26-Aug-2009 & 27-Aug-2009 \\
\hline Fish Gut EMR - WA 3 & $9082706-23$ & Tissue & 26-Aug-2009 & 27-Aug-2009 \\
\hline Fish Gut EMR - WA 4 & $9082706-24$ & Tissue & 26-Aug-2009 & 27-Aug-2009 \\
\hline Fish Gut EMR - WA 5 & $9082706-25$ & Tissue & 26-Aug-2009 & 27-Aug-2009 \\
\hline Fish Gut EMR - EL 1 & $9082706-26$ & Tissue & 26-Aug-2009 & 27-Aug-2009 \\
\hline Fish Gut EMR - EL 2 & $9082706-27$ & Tissue & 26-Aug-2009 & 27-Aug-2009 \\
\hline Fish Gut EMR - EL 3 & $9082706-28$ & Tissue & 26-Aug-2009 & 27-Aug-2009 \\
\hline Fish Gut EMR - EL 4 & $9082706-29$ & Tissue & 26-Aug-2009 & 27-Aug-2009 \\
\hline Fish Gut EMR - EL 5 & $9082706-30$ & Tissue & 26-Aug-2009 & 27-Aug-2009 \\
\hline
\end{tabular}




\section{USACE ERDC-EP-C \\ 3909 Halls Ferry Road \\ Vicksburg, MS 39180-6199}

ERDC - ECB

Project: TVA Fly Ash Project

Reported:

Project Manager: Tony Bednar

03-Sep-2009

\section{Fish Tissue Dechlor 1}

9082706-01 (Tissue)

\begin{tabular}{|c|c|c|c|c|c|c|c|c|}
\hline & & porting & & & & & & \\
\hline Analyte & Result & Limit & Units & Dilution & Prepared & Analyzed & Method & Notes \\
\hline
\end{tabular}

ERDC- EL-EP-C (Environmental Chemistry Branch)

Metals by EPA 6000/7000 Series Methods

\begin{tabular}{|c|c|c|c|c|c|c|c|c|}
\hline Mercury & 0.007 & 0.003 & $\mathrm{mg} / \mathrm{kg}$ & 1 & 03-Sep-2009 & 03-Sep-2009 & EPA 7471A & \\
\hline Antimony & ND & 0.400 & $\mathrm{mg} / \mathrm{kg}$ & 2 & 28-Aug-2009 & 29-Aug-2009 & SW 846/6020 & $\mathrm{U}$ \\
\hline Arsenic & 0.101 & 0.400 & $\mathrm{mg} / \mathrm{kg}$ & 2 & 28-Aug-2009 & 29-Aug-2009 & SW 846/6020 & $\mathrm{J}$ \\
\hline Barium & 2.28 & 0.400 & $\mathrm{mg} / \mathrm{kg}$ & 2 & 28-Aug-2009 & 29-Aug-2009 & SW 846/6020 & \\
\hline Beryllium & ND & 0.400 & $\mathrm{mg} / \mathrm{kg}$ & 2 & 28-Aug-2009 & 29-Aug-2009 & SW 846/6020 & $\mathrm{U}$ \\
\hline Cadmium & ND & 0.400 & $\mathrm{mg} / \mathrm{kg}$ & 2 & 28-Aug-2009 & 29-Aug-2009 & SW 846/6020 & $\mathrm{U}$ \\
\hline Cobalt & 0.104 & 0.400 & $\mathrm{mg} / \mathrm{kg}$ & 2 & 28-Aug-2009 & 29-Aug-2009 & SW 846/6020 & $\mathrm{J}$ \\
\hline Copper & 1.62 & 0.400 & $\mathrm{mg} / \mathrm{kg}$ & 2 & 28-Aug-2009 & 29-Aug-2009 & SW 846/6020 & \\
\hline Lead & 1.52 & 0.400 & $\mathrm{mg} / \mathrm{kg}$ & 2 & 28-Aug-2009 & 29-Aug-2009 & SW $846 / 6020$ & B \\
\hline Manganese & 1.51 & 0.400 & $\mathrm{mg} / \mathrm{kg}$ & 2 & 28-Aug-2009 & 29-Aug-2009 & SW 846/6020 & \\
\hline Molybdenum & 0.124 & 0.400 & $\mathrm{mg} / \mathrm{kg}$ & 2 & 28-Aug-2009 & 29-Aug-2009 & SW 846/6020 & $\mathrm{J}$ \\
\hline Nickel & 4.68 & 0.400 & $\mathrm{mg} / \mathrm{kg}$ & 2 & 28-Aug-2009 & 29-Aug-2009 & SW 846/6020 & \\
\hline Silver & 0.193 & 0.400 & $\mathrm{mg} / \mathrm{kg}$ & 2 & 28-Aug-2009 & 29-Aug-2009 & SW $846 / 6020$ & $\mathrm{~J}$ \\
\hline Thallium & ND & 0.400 & $\mathrm{mg} / \mathrm{kg}$ & 2 & 28-Aug-2009 & 29-Aug-2009 & SW 846/6020 & $\mathrm{U}$ \\
\hline Vanadium & 0.094 & 0.400 & $\mathrm{mg} / \mathrm{kg}$ & 2 & 28-Aug-2009 & 29-Aug-2009 & SW 846/6020 & $\mathrm{J}$ \\
\hline Zinc & 19.0 & 0.400 & $\mathrm{mg} / \mathrm{kg}$ & 2 & 28-Aug-2009 & 29-Aug-2009 & SW 846/6020 & \\
\hline
\end{tabular}




\section{USACE ERDC-EP-C \\ 3909 Halls Ferry Road \\ Vicksburg, MS 39180-6199}

ERDC - ECB

Project: TVA Fly Ash Project

Reported:

Project Manager: Tony Bednar

03-Sep-2009

\section{Fish Tissue Dechlor 2}

9082706-02 (Tissue)

\begin{tabular}{|c|c|c|c|c|c|c|c|c|}
\hline Analyte & Result & $\begin{array}{r}\text { porting } \\
\text { Limit }\end{array}$ & Units & Dilution & Prepared & Analyzed & Method & Notes \\
\hline
\end{tabular}

ERDC- EL-EP-C (Environmental Chemistry Branch)

Metals by EPA 6000/7000 Series Methods

\begin{tabular}{|c|c|c|c|c|c|c|c|c|}
\hline Mercury & 0.011 & 0.005 & $\mathrm{mg} / \mathrm{kg}$ & 1 & 03-Sep-2009 & 03-Sep-2009 & EPA 7471A & \\
\hline Antimony & ND & 0.400 & $\mathrm{mg} / \mathrm{kg}$ & 2 & 28-Aug-2009 & 29-Aug-2009 & SW 846/6020 & $\mathrm{U}$ \\
\hline Arsenic & 0.084 & 0.400 & $\mathrm{mg} / \mathrm{kg}$ & 2 & 28-Aug-2009 & 29-Aug-2009 & SW 846/6020 & $\mathrm{J}$ \\
\hline Barium & 1.97 & 0.400 & $\mathrm{mg} / \mathrm{kg}$ & 2 & 28-Aug-2009 & 29-Aug-2009 & SW 846/6020 & \\
\hline Beryllium & ND & 0.400 & $\mathrm{mg} / \mathrm{kg}$ & 2 & 28-Aug-2009 & 29-Aug-2009 & SW 846/6020 & $\mathrm{U}$ \\
\hline Cadmium & ND & 0.400 & $\mathrm{mg} / \mathrm{kg}$ & 2 & 28-Aug-2009 & 29-Aug-2009 & SW 846/6020 & $\mathrm{U}$ \\
\hline Chromium & 15.2 & 0.400 & $\mathrm{mg} / \mathrm{kg}$ & 2 & 28-Aug-2009 & 29-Aug-2009 & SW 846/6020 & \\
\hline Cobalt & 0.159 & 0.400 & $\mathrm{mg} / \mathrm{kg}$ & 2 & 28-Aug-2009 & 29-Aug-2009 & SW 846/6020 & J \\
\hline Copper & 1.01 & 0.400 & $\mathrm{mg} / \mathrm{kg}$ & 2 & 28-Aug-2009 & 29-Aug-2009 & SW 846/6020 & \\
\hline Lead & 3.49 & 0.400 & $\mathrm{mg} / \mathrm{kg}$ & 2 & 28-Aug-2009 & 29-Aug-2009 & SW 846/6020 & B \\
\hline Manganese & 2.05 & 0.400 & $\mathrm{mg} / \mathrm{kg}$ & 2 & 28-Aug-2009 & 29-Aug-2009 & SW 846/6020 & \\
\hline Molybdenum & 0.298 & 0.400 & $\mathrm{mg} / \mathrm{kg}$ & 2 & 28-Aug-2009 & 29-Aug-2009 & SW 846/6020 & J \\
\hline Nickel & 7.50 & 0.400 & $\mathrm{mg} / \mathrm{kg}$ & 2 & 28-Aug-2009 & 29-Aug-2009 & SW 846/6020 & \\
\hline Selenium & 0.110 & 0.400 & $\mathrm{mg} / \mathrm{kg}$ & 2 & 28-Aug-2009 & 29-Aug-2009 & SW 846/6020 & $\mathrm{J}$ \\
\hline Silver & 0.116 & 0.400 & $\mathrm{mg} / \mathrm{kg}$ & 2 & 28-Aug-2009 & 29-Aug-2009 & SW 846/6020 & $\mathrm{J}$ \\
\hline Thallium & ND & 0.400 & $\mathrm{mg} / \mathrm{kg}$ & 2 & 28-Aug-2009 & 29-Aug-2009 & SW 846/6020 & $\mathrm{U}$ \\
\hline Vanadium & 0.121 & 0.400 & $\mathrm{mg} / \mathrm{kg}$ & 2 & 28-Aug-2009 & 29-Aug-2009 & SW 846/6020 & J \\
\hline Zinc & 13.6 & 0.400 & $\mathrm{mg} / \mathrm{kg}$ & 2 & 28-Aug-2009 & 29-Aug-2009 & SW 846/6020 & \\
\hline
\end{tabular}




\section{USACE ERDC-EP-C \\ 3909 Halls Ferry Road \\ Vicksburg, MS 39180-6199}

ERDC - ECB

Project: TVA Fly Ash Project

Reported:

Project Manager: Tony Bednar

03-Sep-2009

\section{Fish Tissue Dechlor 3}

9082706-03 (Tissue)

\begin{tabular}{|c|c|c|c|c|c|c|c|c|}
\hline Analyte & Result & $\begin{array}{r}\text { porting } \\
\text { Limit }\end{array}$ & Units & Dilution & Prepared & Analyzed & Method & Notes \\
\hline
\end{tabular}

ERDC- EL-EP-C (Environmental Chemistry Branch)

Metals by EPA 6000/7000 Series Methods

\begin{tabular}{|c|c|c|c|c|c|c|c|c|}
\hline Mercury & 0.007 & 0.004 & $\mathrm{mg} / \mathrm{kg}$ & 1 & 03-Sep-2009 & 03-Sep-2009 & EPA 7471A & \\
\hline Antimony & ND & 0.400 & $\mathrm{mg} / \mathrm{kg}$ & 2 & 28-Aug-2009 & 29-Aug-2009 & SW 846/6020 & $\mathrm{U}$ \\
\hline Arsenic & ND & 0.400 & $\mathrm{mg} / \mathrm{kg}$ & 2 & 28-Aug-2009 & 29-Aug-2009 & SW 846/6020 & $\mathrm{U}$ \\
\hline Barium & 1.84 & 0.400 & $\mathrm{mg} / \mathrm{kg}$ & 2 & 28-Aug-2009 & 29-Aug-2009 & SW 846/6020 & \\
\hline Beryllium & ND & 0.400 & $\mathrm{mg} / \mathrm{kg}$ & 2 & 28-Aug-2009 & 29-Aug-2009 & SW 846/6020 & $\mathrm{U}$ \\
\hline Cadmium & ND & 0.400 & $\mathrm{mg} / \mathrm{kg}$ & 2 & 28-Aug-2009 & 29-Aug-2009 & SW 846/6020 & $\mathrm{U}$ \\
\hline Chromium & 1.79 & 0.400 & $\mathrm{mg} / \mathrm{kg}$ & 2 & 28-Aug-2009 & 29-Aug-2009 & SW 846/6020 & \\
\hline Cobalt & ND & 0.400 & $\mathrm{mg} / \mathrm{kg}$ & 2 & 28-Aug-2009 & 29-Aug-2009 & SW 846/6020 & $\mathrm{U}$ \\
\hline Copper & 0.663 & 0.400 & $\mathrm{mg} / \mathrm{kg}$ & 2 & 28-Aug-2009 & 29-Aug-2009 & SW 846/6020 & \\
\hline Lead & 0.588 & 0.400 & $\mathrm{mg} / \mathrm{kg}$ & 2 & 28-Aug-2009 & 29-Aug-2009 & SW 846/6020 & B \\
\hline Manganese & 0.742 & 0.400 & $\mathrm{mg} / \mathrm{kg}$ & 2 & 28-Aug-2009 & 29-Aug-2009 & SW 846/6020 & \\
\hline Molybdenum & ND & 0.400 & $\mathrm{mg} / \mathrm{kg}$ & 2 & 28-Aug-2009 & 29-Aug-2009 & SW 846/6020 & $\mathrm{U}$ \\
\hline Nickel & 1.20 & 0.400 & $\mathrm{mg} / \mathrm{kg}$ & 2 & 28-Aug-2009 & 29-Aug-2009 & SW 846/6020 & \\
\hline Selenium & 0.087 & 0.400 & $\mathrm{mg} / \mathrm{kg}$ & 2 & 28-Aug-2009 & 29-Aug-2009 & SW 846/6020 & $\mathrm{J}$ \\
\hline Silver & ND & 0.400 & $\mathrm{mg} / \mathrm{kg}$ & 2 & 28-Aug-2009 & 29-Aug-2009 & SW 846/6020 & $\mathrm{U}$ \\
\hline Thallium & ND & 0.400 & $\mathrm{mg} / \mathrm{kg}$ & 2 & 28-Aug-2009 & 29-Aug-2009 & SW 846/6020 & $\mathrm{U}$ \\
\hline Vanadium & ND & 0.400 & $\mathrm{mg} / \mathrm{kg}$ & 2 & 28-Aug-2009 & 29-Aug-2009 & SW 846/6020 & $\mathrm{U}$ \\
\hline Zinc & 22.5 & 0.400 & $\mathrm{mg} / \mathrm{kg}$ & 2 & 28-Aug-2009 & 29-Aug-2009 & SW 846/6020 & \\
\hline
\end{tabular}




\section{USACE ERDC-EP-C \\ 3909 Halls Ferry Road \\ Vicksburg, MS 39180-6199}

ERDC - ECB

Project: TVA Fly Ash Project

Reported:

Project Manager: Tony Bednar

03-Sep-2009

\section{Fish Tissue Dechlor 4}

9082706-04 (Tissue)

\begin{tabular}{|c|c|c|c|c|c|c|c|c|}
\hline & & porting & & & & & & \\
\hline Analyte & Result & Limit & Units & Dilution & Prepared & Analyzed & Method & Notes \\
\hline
\end{tabular}

ERDC- EL-EP-C (Environmental Chemistry Branch)

Metals by EPA 6000/7000 Series Methods

\begin{tabular}{|c|c|c|c|c|c|c|c|c|}
\hline Mercury & 0.009 & 0.003 & $\mathrm{mg} / \mathrm{kg}$ & 1 & 03-Sep-2009 & 03-Sep-2009 & EPA 7471A & \\
\hline Antimony & 0.084 & 0.400 & $\mathrm{mg} / \mathrm{kg}$ & 2 & 28-Aug-2009 & 29-Aug-2009 & SW 846/6020 & $\mathrm{J}, \mathrm{B}$ \\
\hline Arsenic & ND & 0.400 & $\mathrm{mg} / \mathrm{kg}$ & 2 & 28-Aug-2009 & 29-Aug-2009 & SW 846/6020 & $\mathrm{U}$ \\
\hline Barium & 1.61 & 0.400 & $\mathrm{mg} / \mathrm{kg}$ & 2 & 28-Aug-2009 & 29-Aug-2009 & SW 846/6020 & \\
\hline Beryllium & ND & 0.400 & $\mathrm{mg} / \mathrm{kg}$ & 2 & 28-Aug-2009 & 29-Aug-2009 & SW 846/6020 & $\mathrm{U}$ \\
\hline Cadmium & ND & 0.400 & $\mathrm{mg} / \mathrm{kg}$ & 2 & 28-Aug-2009 & 29-Aug-2009 & SW 846/6020 & $\mathrm{U}$ \\
\hline Chromium & 4.04 & 0.400 & $\mathrm{mg} / \mathrm{kg}$ & 2 & 28-Aug-2009 & 29-Aug-2009 & SW 846/6020 & \\
\hline Cobalt & ND & 0.400 & $\mathrm{mg} / \mathrm{kg}$ & 2 & 28-Aug-2009 & 29-Aug-2009 & SW 846/6020 & $\mathrm{U}$ \\
\hline Copper & 0.806 & 0.400 & $\mathrm{mg} / \mathrm{kg}$ & 2 & 28-Aug-2009 & 29-Aug-2009 & SW 846/6020 & \\
\hline Lead & 0.590 & 0.400 & $\mathrm{mg} / \mathrm{kg}$ & 2 & 28-Aug-2009 & 29-Aug-2009 & SW 846/6020 & B \\
\hline Manganese & 1.02 & 0.400 & $\mathrm{mg} / \mathrm{kg}$ & 2 & 28-Aug-2009 & 29-Aug-2009 & SW 846/6020 & \\
\hline Molybdenum & 0.083 & 0.400 & $\mathrm{mg} / \mathrm{kg}$ & 2 & 28-Aug-2009 & 29-Aug-2009 & SW 846/6020 & $\mathrm{J}$ \\
\hline Nickel & 2.29 & 0.400 & $\mathrm{mg} / \mathrm{kg}$ & 2 & 28-Aug-2009 & 29-Aug-2009 & SW 846/6020 & \\
\hline Selenium & 0.112 & 0.400 & $\mathrm{mg} / \mathrm{kg}$ & 2 & 28-Aug-2009 & 29-Aug-2009 & SW 846/6020 & $\mathrm{J}$ \\
\hline Silver & ND & 0.400 & $\mathrm{mg} / \mathrm{kg}$ & 2 & 28-Aug-2009 & 29-Aug-2009 & SW 846/6020 & $\mathrm{U}$ \\
\hline Thallium & ND & 0.400 & $\mathrm{mg} / \mathrm{kg}$ & 2 & 28-Aug-2009 & 29-Aug-2009 & SW 846/6020 & $\mathrm{U}$ \\
\hline Vanadium & 0.098 & 0.400 & $\mathrm{mg} / \mathrm{kg}$ & 2 & 28-Aug-2009 & 29-Aug-2009 & SW 846/6020 & $\mathrm{J}$ \\
\hline Zinc & 17.8 & 0.400 & $\mathrm{mg} / \mathrm{kg}$ & 2 & 28-Aug-2009 & 29-Aug-2009 & SW 846/6020 & \\
\hline
\end{tabular}




\section{USACE ERDC-EP-C \\ 3909 Halls Ferry Road \\ Vicksburg, MS 39180-6199}

ERDC - ECB

Project: TVA Fly Ash Project

Reported:

Project Manager: Tony Bednar

03-Sep-2009

\section{Fish Tissue Dechlor 5}

9082706-05 (Tissue)

\begin{tabular}{|c|c|c|c|c|c|c|c|c|}
\hline Analyte & Result & $\begin{array}{r}\text { porting } \\
\text { Limit }\end{array}$ & Units & Dilution & Prepared & Analyzed & Method & Notes \\
\hline
\end{tabular}

ERDC- EL-EP-C (Environmental Chemistry Branch)

Metals by EPA 6000/7000 Series Methods

\begin{tabular}{|c|c|c|c|c|c|c|c|c|}
\hline Mercury & 0.013 & 0.004 & $\mathrm{mg} / \mathrm{kg}$ & 1 & 03-Sep-2009 & 03-Sep-2009 & EPA 7471A & \\
\hline Antimony & ND & 0.400 & $\mathrm{mg} / \mathrm{kg}$ & 2 & 28-Aug-2009 & 29-Aug-2009 & SW 846/6020 & $\mathrm{U}$ \\
\hline Arsenic & 0.105 & 0.400 & $\mathrm{mg} / \mathrm{kg}$ & 2 & 28-Aug-2009 & 29-Aug-2009 & SW 846/6020 & $\mathrm{J}$ \\
\hline Barium & 2.46 & 0.400 & $\mathrm{mg} / \mathrm{kg}$ & 2 & 28-Aug-2009 & 29-Aug-2009 & SW 846/6020 & \\
\hline Beryllium & ND & 0.400 & $\mathrm{mg} / \mathrm{kg}$ & 2 & 28-Aug-2009 & 29-Aug-2009 & SW 846/6020 & $\mathrm{U}$ \\
\hline Cadmium & ND & 0.400 & $\mathrm{mg} / \mathrm{kg}$ & 2 & 28-Aug-2009 & 29-Aug-2009 & SW 846/6020 & $\mathrm{U}$ \\
\hline Chromium & 11.4 & 0.400 & $\mathrm{mg} / \mathrm{kg}$ & 2 & 28-Aug-2009 & 29-Aug-2009 & SW 846/6020 & \\
\hline Cobalt & 0.088 & 0.400 & $\mathrm{mg} / \mathrm{kg}$ & 2 & 28-Aug-2009 & 29-Aug-2009 & SW 846/6020 & $\mathrm{J}$ \\
\hline Copper & 0.854 & 0.400 & $\mathrm{mg} / \mathrm{kg}$ & 2 & 28-Aug-2009 & 29-Aug-2009 & SW 846/6020 & \\
\hline Lead & 0.256 & 0.400 & $\mathrm{mg} / \mathrm{kg}$ & 2 & 28-Aug-2009 & 29-Aug-2009 & SW 846/6020 & $\mathrm{J}, \mathrm{B}$ \\
\hline Manganese & 1.54 & 0.400 & $\mathrm{mg} / \mathrm{kg}$ & 2 & 28-Aug-2009 & 29-Aug-2009 & SW 846/6020 & \\
\hline Molybdenum & 0.193 & 0.400 & $\mathrm{mg} / \mathrm{kg}$ & 2 & 28-Aug-2009 & 29-Aug-2009 & SW 846/6020 & $\mathrm{J}$ \\
\hline Nickel & 5.78 & 0.400 & $\mathrm{mg} / \mathrm{kg}$ & 2 & 28-Aug-2009 & 29-Aug-2009 & SW 846/6020 & \\
\hline Selenium & 0.163 & 0.400 & $\mathrm{mg} / \mathrm{kg}$ & 2 & 28-Aug-2009 & 29-Aug-2009 & SW 846/6020 & $\mathrm{J}$ \\
\hline Silver & 0.080 & 0.400 & $\mathrm{mg} / \mathrm{kg}$ & 2 & 28-Aug-2009 & 29-Aug-2009 & SW 846/6020 & $\mathrm{J}$ \\
\hline Thallium & ND & 0.400 & $\mathrm{mg} / \mathrm{kg}$ & 2 & 28-Aug-2009 & 29-Aug-2009 & SW 846/6020 & $\mathrm{U}$ \\
\hline Vanadium & 0.109 & 0.400 & $\mathrm{mg} / \mathrm{kg}$ & 2 & 28-Aug-2009 & 29-Aug-2009 & SW 846/6020 & J \\
\hline Zinc & 19.7 & 0.400 & $\mathrm{mg} / \mathrm{kg}$ & 2 & 28-Aug-2009 & 29-Aug-2009 & SW 846/6020 & \\
\hline
\end{tabular}




\section{USACE ERDC-EP-C \\ 3909 Halls Ferry Road \\ Vicksburg, MS 39180-6199}

ERDC - ECB

Project: TVA Fly Ash Project

Reported:

Project Manager: Tony Bednar

03-Sep-2009

Fish Tissue EMR - WA 1

9082706-06 (Tissue)

\begin{tabular}{|c|c|c|c|c|c|c|c|c|}
\hline Analyte & Result & porting & Units & Dilution & Prenared & Analyzed & Method & Notes \\
\hline Analyte & Kesult & Limit & & & Prepared & & Metnod & \\
\hline
\end{tabular}

ERDC- EL-EP-C (Environmental Chemistry Branch)

Metals by EPA 6000/7000 Series Methods

\begin{tabular}{|c|c|c|c|c|c|c|c|c|}
\hline Mercury & 0.011 & 0.003 & $\mathrm{mg} / \mathrm{kg}$ & 1 & 03-Sep-2009 & 03-Sep-2009 & EPA 7471A & \\
\hline Antimony & 0.426 & 0.400 & $\mathrm{mg} / \mathrm{kg}$ & 2 & 28-Aug-2009 & 29-Aug-2009 & SW 846/6020 & B \\
\hline Arsenic & 0.184 & 0.400 & $\mathrm{mg} / \mathrm{kg}$ & 2 & 28-Aug-2009 & 29-Aug-2009 & SW 846/6020 & $\mathrm{J}$ \\
\hline Barium & 4.15 & 0.400 & $\mathrm{mg} / \mathrm{kg}$ & 2 & 28-Aug-2009 & 29-Aug-2009 & SW 846/6020 & \\
\hline Beryllium & ND & 0.400 & $\mathrm{mg} / \mathrm{kg}$ & 2 & 28-Aug-2009 & 29-Aug-2009 & SW 846/6020 & $\mathrm{U}$ \\
\hline Cadmium & ND & 0.400 & $\mathrm{mg} / \mathrm{kg}$ & 2 & 28-Aug-2009 & 29-Aug-2009 & SW 846/6020 & $\mathrm{U}$ \\
\hline Chromium & 9.76 & 0.400 & $\mathrm{mg} / \mathrm{kg}$ & 2 & 28-Aug-2009 & 29-Aug-2009 & SW 846/6020 & \\
\hline Cobalt & 0.136 & 0.400 & $\mathrm{mg} / \mathrm{kg}$ & 2 & 28-Aug-2009 & 29-Aug-2009 & SW 846/6020 & $\mathrm{J}$ \\
\hline Copper & 2.13 & 0.400 & $\mathrm{mg} / \mathrm{kg}$ & 2 & 28-Aug-2009 & 29-Aug-2009 & SW 846/6020 & \\
\hline Lead & 0.414 & 0.400 & $\mathrm{mg} / \mathrm{kg}$ & 2 & 28-Aug-2009 & 29-Aug-2009 & SW 846/6020 & B \\
\hline Manganese & 2.00 & 0.400 & $\mathrm{mg} / \mathrm{kg}$ & 2 & 28-Aug-2009 & 29-Aug-2009 & SW 846/6020 & \\
\hline Molybdenum & 0.203 & 0.400 & $\mathrm{mg} / \mathrm{kg}$ & 2 & 28-Aug-2009 & 29-Aug-2009 & SW 846/6020 & $\mathrm{J}$ \\
\hline Nickel & 5.05 & 0.400 & $\mathrm{mg} / \mathrm{kg}$ & 2 & 28-Aug-2009 & 29-Aug-2009 & SW 846/6020 & \\
\hline Selenium & 0.229 & 0.400 & $\mathrm{mg} / \mathrm{kg}$ & 2 & 28-Aug-2009 & 29-Aug-2009 & SW 846/6020 & $\mathrm{J}$ \\
\hline Silver & 0.156 & 0.400 & $\mathrm{mg} / \mathrm{kg}$ & 2 & 28-Aug-2009 & 29-Aug-2009 & SW 846/6020 & $\mathrm{J}$ \\
\hline Thallium & ND & 0.400 & $\mathrm{mg} / \mathrm{kg}$ & 2 & 28-Aug-2009 & 29-Aug-2009 & SW 846/6020 & $\mathrm{U}$ \\
\hline Vanadium & 0.259 & 0.400 & $\mathrm{mg} / \mathrm{kg}$ & 2 & 28-Aug-2009 & 29-Aug-2009 & SW 846/6020 & J \\
\hline Zinc & 39.0 & 0.400 & $\mathrm{mg} / \mathrm{kg}$ & 2 & 28-Aug-2009 & 29-Aug-2009 & SW 846/6020 & \\
\hline
\end{tabular}




\section{USACE ERDC-EP-C \\ 3909 Halls Ferry Road \\ Vicksburg, MS 39180-6199}

ERDC - ECB

Project: TVA Fly Ash Project

Reported:

Project Manager: Tony Bednar

03-Sep-2009

Fish Tissue EMR - WA 2

9082706-07 (Tissue)

\begin{tabular}{|c|c|c|c|c|c|c|c|c|}
\hline Analyte & Result & porting & Units & Dilution & Prenared & Analyzed & Method & Notes \\
\hline Analyte & Kesult & Limit & & & Prepared & & Metnod & \\
\hline
\end{tabular}

ERDC- EL-EP-C (Environmental Chemistry Branch)

Metals by EPA 6000/7000 Series Methods

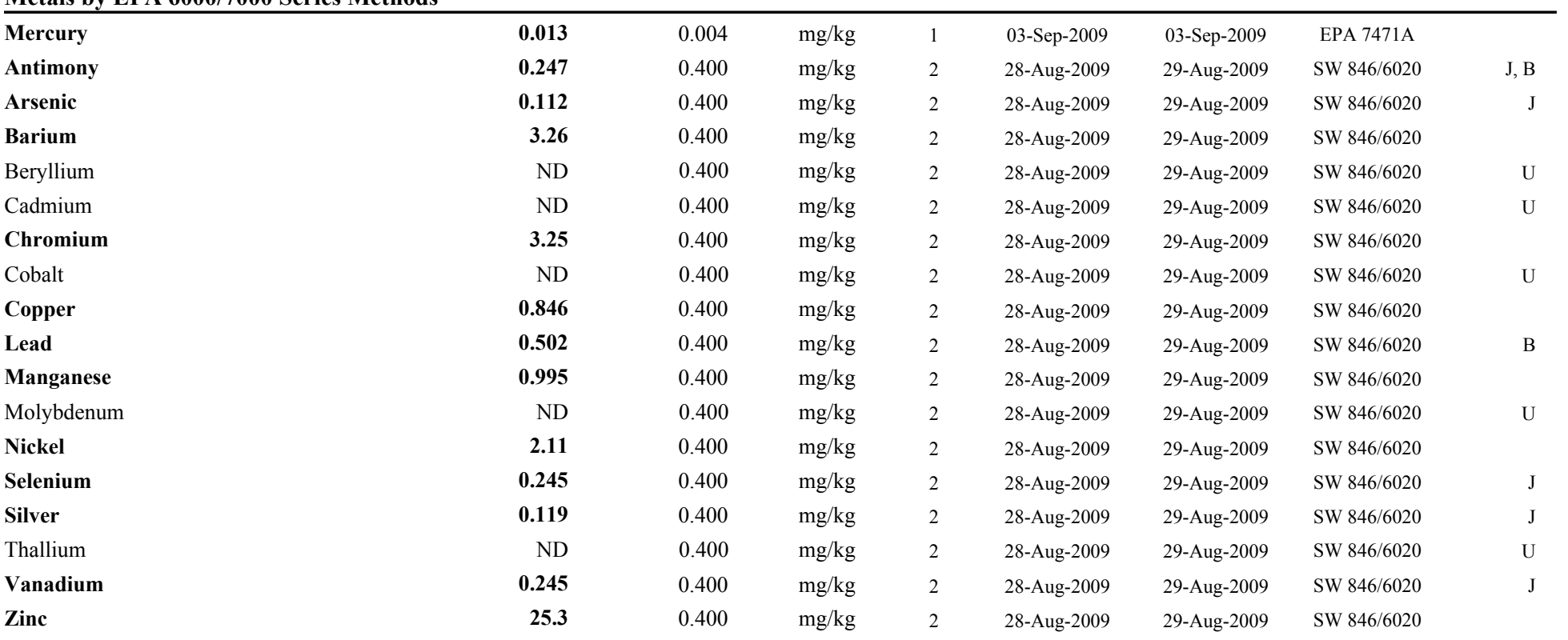




\section{USACE ERDC-EP-C \\ 3909 Halls Ferry Road \\ Vicksburg, MS 39180-6199}

ERDC - ECB

Project: TVA Fly Ash Project

Reported:

Project Manager: Tony Bednar

03-Sep-2009

Fish Tissue EMR - WA 3

9082706-08 (Tissue)

\begin{tabular}{|c|c|c|c|c|c|c|c|c|}
\hline Analyte & Result & porting & Units & Dilution & Prenared & Analyzed & Method & Notes \\
\hline Analyte & Kesult & Limit & & & Prepared & & Metnod & \\
\hline
\end{tabular}

ERDC- EL-EP-C (Environmental Chemistry Branch)

Metals by EPA 6000/7000 Series Methods

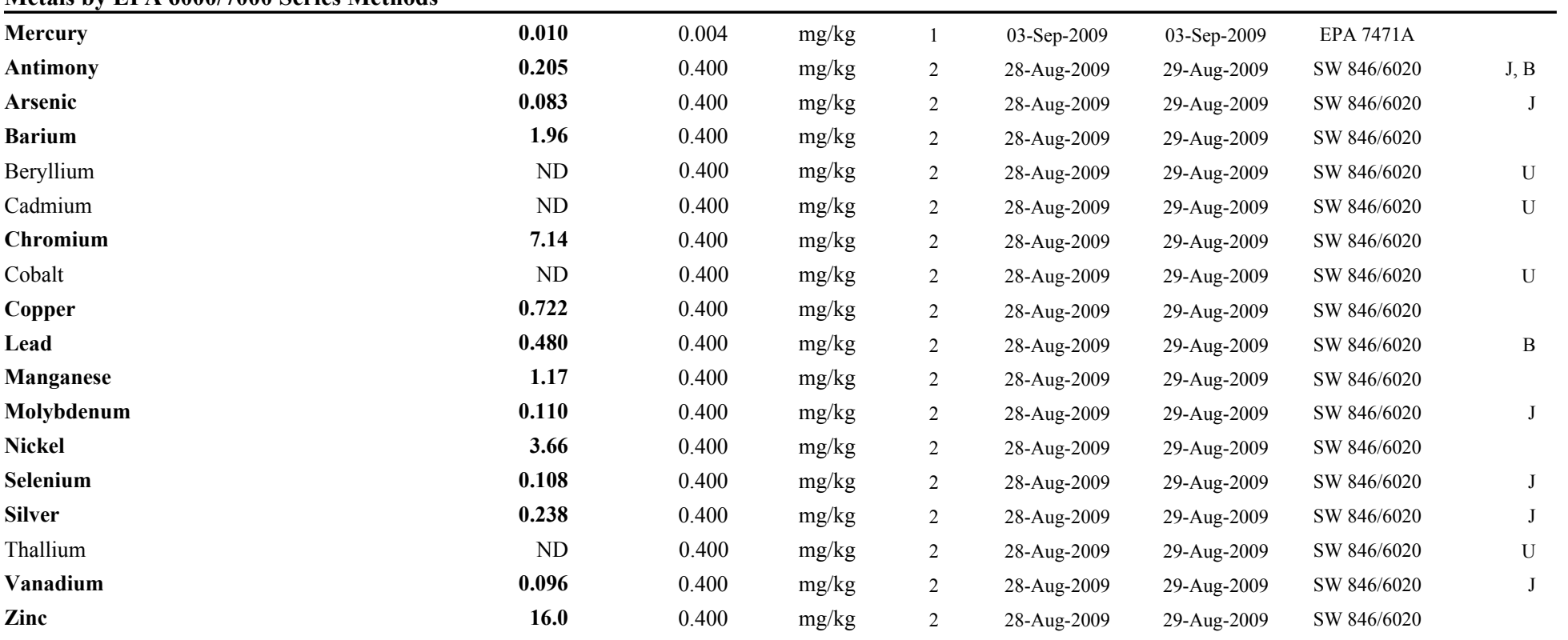




\section{USACE ERDC-EP-C \\ 3909 Halls Ferry Road \\ Vicksburg, MS 39180-6199}

ERDC - ECB

Project: TVA Fly Ash Project

Reported:

Project Manager: Tony Bednar

03-Sep-2009

Fish Tissue EMR - WA 4

9082706-09 (Tissue)

\begin{tabular}{|c|c|c|c|c|c|c|c|c|}
\hline Analyte & Result & $\begin{array}{r}\text { porting } \\
\text { Limit }\end{array}$ & Units & Dilution & Prepared & Analyzed & Method & Notes \\
\hline
\end{tabular}

ERDC- EL-EP-C (Environmental Chemistry Branch)

Metals by EPA 6000/7000 Series Methods

\begin{tabular}{|c|c|c|c|c|c|c|c|c|}
\hline Mercury & 0.006 & 0.003 & $\mathrm{mg} / \mathrm{kg}$ & 1 & 03-Sep-2009 & 03-Sep-2009 & EPA 7471A & \\
\hline Antimony & ND & 0.400 & $\mathrm{mg} / \mathrm{kg}$ & 2 & 28-Aug-2009 & 29-Aug-2009 & SW 846/6020 & $\mathrm{U}$ \\
\hline Arsenic & 0.099 & 0.400 & $\mathrm{mg} / \mathrm{kg}$ & 2 & 28-Aug-2009 & 29-Aug-2009 & SW 846/6020 & $\mathrm{J}$ \\
\hline Barium & 4.02 & 0.400 & $\mathrm{mg} / \mathrm{kg}$ & 2 & 28-Aug-2009 & 29-Aug-2009 & SW 846/6020 & \\
\hline Beryllium & ND & 0.400 & $\mathrm{mg} / \mathrm{kg}$ & 2 & 28-Aug-2009 & 29-Aug-2009 & SW 846/6020 & $\mathrm{U}$ \\
\hline Cadmium & ND & 0.400 & $\mathrm{mg} / \mathrm{kg}$ & 2 & 28-Aug-2009 & 29-Aug-2009 & SW 846/6020 & $\mathrm{U}$ \\
\hline Chromium & 8.94 & 0.400 & $\mathrm{mg} / \mathrm{kg}$ & 2 & 28-Aug-2009 & 29-Aug-2009 & SW 846/6020 & \\
\hline Cobalt & 0.097 & 0.400 & $\mathrm{mg} / \mathrm{kg}$ & 2 & 28-Aug-2009 & 29-Aug-2009 & SW 846/6020 & $\mathrm{J}$ \\
\hline Copper & 1.33 & 0.400 & $\mathrm{mg} / \mathrm{kg}$ & 2 & 28-Aug-2009 & 29-Aug-2009 & SW 846/6020 & \\
\hline Lead & 0.392 & 0.400 & $\mathrm{mg} / \mathrm{kg}$ & 2 & 28-Aug-2009 & 29-Aug-2009 & SW 846/6020 & $\mathrm{J}, \mathrm{B}$ \\
\hline Manganese & 1.70 & 0.400 & $\mathrm{mg} / \mathrm{kg}$ & 2 & 28-Aug-2009 & 29-Aug-2009 & SW 846/6020 & \\
\hline Molybdenum & 0.156 & 0.400 & $\mathrm{mg} / \mathrm{kg}$ & 2 & 28-Aug-2009 & 29-Aug-2009 & SW 846/6020 & $\mathrm{J}$ \\
\hline Nickel & 4.78 & 0.400 & $\mathrm{mg} / \mathrm{kg}$ & 2 & 28-Aug-2009 & 29-Aug-2009 & SW 846/6020 & \\
\hline Selenium & 0.208 & 0.400 & $\mathrm{mg} / \mathrm{kg}$ & 2 & 28-Aug-2009 & 29-Aug-2009 & SW 846/6020 & $\mathrm{J}$ \\
\hline Silver & ND & 0.400 & $\mathrm{mg} / \mathrm{kg}$ & 2 & 28-Aug-2009 & 29-Aug-2009 & SW 846/6020 & $\mathrm{U}$ \\
\hline Thallium & ND & 0.400 & $\mathrm{mg} / \mathrm{kg}$ & 2 & 28-Aug-2009 & 29-Aug-2009 & SW 846/6020 & $\mathrm{U}$ \\
\hline Vanadium & 0.123 & 0.400 & $\mathrm{mg} / \mathrm{kg}$ & 2 & 28-Aug-2009 & 29-Aug-2009 & SW 846/6020 & $\mathrm{J}$ \\
\hline Zinc & 19.6 & 0.400 & $\mathrm{mg} / \mathrm{kg}$ & 2 & 28-Aug-2009 & 29-Aug-2009 & SW 846/6020 & \\
\hline
\end{tabular}




\section{USACE ERDC-EP-C \\ 3909 Halls Ferry Road \\ Vicksburg, MS 39180-6199}

ERDC - ECB

Project: TVA Fly Ash Project

Reported:

Project Manager: Tony Bednar

03-Sep-2009

Fish Tissue EMR - WA 5

9082706-10 (Tissue)

\begin{tabular}{|c|c|c|c|c|c|c|c|c|}
\hline Analyte & Result & porting & Units & Dilution & Prenared & Analyzed & Method & Notes \\
\hline Analyte & Kesult & Limit & & & Prepared & & Metnod & \\
\hline
\end{tabular}

ERDC- EL-EP-C (Environmental Chemistry Branch)

Metals by EPA 6000/7000 Series Methods

\begin{tabular}{|c|c|c|c|c|c|c|c|c|}
\hline Mercury & 0.015 & 0.006 & $\mathrm{mg} / \mathrm{kg}$ & 1 & 03-Sep-2009 & 03-Sep-2009 & EPA 7471A & \\
\hline Antimony & ND & 0.400 & $\mathrm{mg} / \mathrm{kg}$ & 2 & 28-Aug-2009 & 29-Aug-2009 & SW 846/6020 & $\mathrm{U}$ \\
\hline Arsenic & 0.119 & 0.400 & $\mathrm{mg} / \mathrm{kg}$ & 2 & 28-Aug-2009 & 29-Aug-2009 & SW 846/6020 & $\mathrm{J}$ \\
\hline Barium & 3.31 & 0.400 & $\mathrm{mg} / \mathrm{kg}$ & 2 & 28-Aug-2009 & 29-Aug-2009 & SW 846/6020 & \\
\hline Beryllium & ND & 0.400 & $\mathrm{mg} / \mathrm{kg}$ & 2 & 28-Aug-2009 & 29-Aug-2009 & SW 846/6020 & $\mathrm{U}$ \\
\hline Cadmium & ND & 0.400 & $\mathrm{mg} / \mathrm{kg}$ & 2 & 28-Aug-2009 & 29-Aug-2009 & SW 846/6020 & $\mathrm{U}$ \\
\hline Chromium & 12.6 & 0.400 & $\mathrm{mg} / \mathrm{kg}$ & 2 & 28-Aug-2009 & 29-Aug-2009 & SW 846/6020 & \\
\hline Cobalt & 0.092 & 0.400 & $\mathrm{mg} / \mathrm{kg}$ & 2 & 28-Aug-2009 & 29-Aug-2009 & SW 846/6020 & $\mathrm{J}$ \\
\hline Copper & 0.994 & 0.400 & $\mathrm{mg} / \mathrm{kg}$ & 2 & 28-Aug-2009 & 29-Aug-2009 & SW 846/6020 & \\
\hline Lead & 0.414 & 0.400 & $\mathrm{mg} / \mathrm{kg}$ & 2 & 28-Aug-2009 & 29-Aug-2009 & SW 846/6020 & B \\
\hline Manganese & 2.11 & 0.400 & $\mathrm{mg} / \mathrm{kg}$ & 2 & 28-Aug-2009 & 29-Aug-2009 & SW 846/6020 & \\
\hline Molybdenum & 0.194 & 0.400 & $\mathrm{mg} / \mathrm{kg}$ & 2 & 28-Aug-2009 & 29-Aug-2009 & SW 846/6020 & $\mathrm{J}$ \\
\hline Nickel & 6.22 & 0.400 & $\mathrm{mg} / \mathrm{kg}$ & 2 & 28-Aug-2009 & 29-Aug-2009 & SW 846/6020 & \\
\hline Selenium & 0.240 & 0.400 & $\mathrm{mg} / \mathrm{kg}$ & 2 & 28-Aug-2009 & 29-Aug-2009 & SW 846/6020 & $\mathrm{J}$ \\
\hline Silver & ND & 0.400 & $\mathrm{mg} / \mathrm{kg}$ & 2 & 28-Aug-2009 & 29-Aug-2009 & SW 846/6020 & $\mathrm{U}$ \\
\hline Thallium & ND & 0.400 & $\mathrm{mg} / \mathrm{kg}$ & 2 & 28-Aug-2009 & 29-Aug-2009 & SW 846/6020 & $\mathrm{U}$ \\
\hline Vanadium & 0.160 & 0.400 & $\mathrm{mg} / \mathrm{kg}$ & 2 & 28-Aug-2009 & 29-Aug-2009 & SW 846/6020 & $\mathrm{J}$ \\
\hline Zinc & 26.1 & 0.400 & $\mathrm{mg} / \mathrm{kg}$ & 2 & 28-Aug-2009 & 29-Aug-2009 & SW 846/6020 & \\
\hline
\end{tabular}




\section{USACE ERDC-EP-C \\ 3909 Halls Ferry Road \\ Vicksburg, MS 39180-6199}

ERDC - ECB

Project: TVA Fly Ash Project

Reported:

Project Manager: Tony Bednar

03-Sep-2009

Fish Tissue EMR - EL 1

9082706-11 (Tissue)

\begin{tabular}{|c|c|c|c|c|c|c|c|c|}
\hline Analyte & Result & $\begin{array}{r}\text { porting } \\
\text { Limit }\end{array}$ & Units & Dilution & Prepared & Analyzed & Method & Notes \\
\hline
\end{tabular}

ERDC- EL-EP-C (Environmental Chemistry Branch)

Metals by EPA 6000/7000 Series Methods

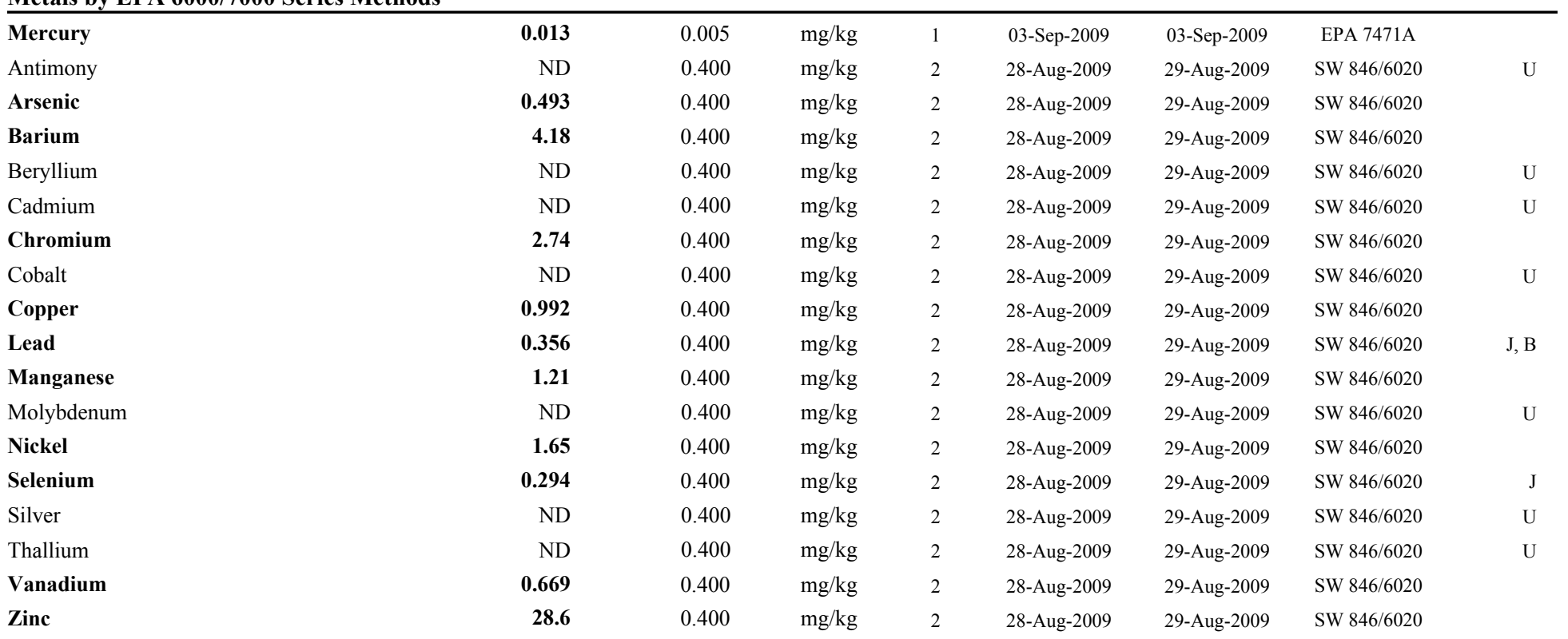




\section{USACE ERDC-EP-C \\ 3909 Halls Ferry Road \\ Vicksburg, MS 39180-6199}

ERDC - ECB

Project: TVA Fly Ash Project

Reported:

Project Manager: Tony Bednar

03-Sep-2009

Fish Tissue EMR - EL 2

9082706-12 (Tissue)

\begin{tabular}{|c|c|c|c|c|c|c|c|c|}
\hline & & porting & & & & & & \\
\hline Analyte & Result & Limit & Units & Dilution & Prepared & Analyzed & Method & Notes \\
\hline
\end{tabular}

ERDC- EL-EP-C (Environmental Chemistry Branch)

Metals by EPA 6000/7000 Series Methods

\begin{tabular}{|c|c|c|c|c|c|c|c|c|}
\hline Mercury & 0.013 & 0.005 & $\mathrm{mg} / \mathrm{kg}$ & 1 & 03-Sep-2009 & 03-Sep-2009 & EPA 7471A & \\
\hline Antimony & ND & 0.400 & $\mathrm{mg} / \mathrm{kg}$ & 2 & 28-Aug-2009 & 29-Aug-2009 & SW 846/6020 & $\mathrm{U}$ \\
\hline Arsenic & 0.470 & 0.400 & $\mathrm{mg} / \mathrm{kg}$ & 2 & 28-Aug-2009 & 29-Aug-2009 & SW 846/6020 & \\
\hline Barium & 4.03 & 0.400 & $\mathrm{mg} / \mathrm{kg}$ & 2 & 28-Aug-2009 & 29-Aug-2009 & SW 846/6020 & \\
\hline Beryllium & ND & 0.400 & $\mathrm{mg} / \mathrm{kg}$ & 2 & 28-Aug-2009 & 29-Aug-2009 & SW 846/6020 & $\mathrm{U}$ \\
\hline Cadmium & ND & 0.400 & $\mathrm{mg} / \mathrm{kg}$ & 2 & 28-Aug-2009 & 29-Aug-2009 & SW 846/6020 & $\mathrm{U}$ \\
\hline Chromium & 2.97 & 0.400 & $\mathrm{mg} / \mathrm{kg}$ & 2 & 28-Aug-2009 & 29-Aug-2009 & SW 846/6020 & \\
\hline Cobalt & ND & 0.400 & $\mathrm{mg} / \mathrm{kg}$ & 2 & 28-Aug-2009 & 29-Aug-2009 & SW 846/6020 & $\mathrm{U}$ \\
\hline Copper & 0.847 & 0.400 & $\mathrm{mg} / \mathrm{kg}$ & 2 & 28-Aug-2009 & 29-Aug-2009 & SW 846/6020 & \\
\hline Lead & 0.305 & 0.400 & $\mathrm{mg} / \mathrm{kg}$ & 2 & 28-Aug-2009 & 29-Aug-2009 & SW 846/6020 & $\mathrm{J}, \mathrm{B}$ \\
\hline Manganese & 1.12 & 0.400 & $\mathrm{mg} / \mathrm{kg}$ & 2 & 28-Aug-2009 & 29-Aug-2009 & SW 846/6020 & \\
\hline Molybdenum & ND & 0.400 & $\mathrm{mg} / \mathrm{kg}$ & 2 & 28-Aug-2009 & 29-Aug-2009 & SW 846/6020 & $\mathrm{U}$ \\
\hline Nickel & 1.72 & 0.400 & $\mathrm{mg} / \mathrm{kg}$ & 2 & 28-Aug-2009 & 29-Aug-2009 & SW 846/6020 & \\
\hline Selenium & 0.335 & 0.400 & $\mathrm{mg} / \mathrm{kg}$ & 2 & 28-Aug-2009 & 29-Aug-2009 & SW 846/6020 & $\mathrm{J}$ \\
\hline Silver & ND & 0.400 & $\mathrm{mg} / \mathrm{kg}$ & 2 & 28-Aug-2009 & 29-Aug-2009 & SW 846/6020 & $\mathrm{U}$ \\
\hline Thallium & ND & 0.400 & $\mathrm{mg} / \mathrm{kg}$ & 2 & 28-Aug-2009 & 29-Aug-2009 & SW 846/6020 & $\mathrm{U}$ \\
\hline Vanadium & 0.386 & 0.400 & $\mathrm{mg} / \mathrm{kg}$ & 2 & 28-Aug-2009 & 29-Aug-2009 & SW 846/6020 & $\mathrm{J}$ \\
\hline Zinc & 28.8 & 0.400 & $\mathrm{mg} / \mathrm{kg}$ & 2 & 28-Aug-2009 & 29-Aug-2009 & SW 846/6020 & \\
\hline
\end{tabular}




\section{USACE ERDC-EP-C \\ 3909 Halls Ferry Road \\ Vicksburg, MS 39180-6199}

ERDC - ECB

Project: TVA Fly Ash Project

Reported:

Project Manager: Tony Bednar

03-Sep-2009

Fish Tissue EMR - EL 3

9082706-13 (Tissue)

\begin{tabular}{|c|c|c|c|c|c|c|c|c|}
\hline Analyte & Result & porting & Units & Dilution & Prenared & Analyzed & Method & Notes \\
\hline Analyte & Kesult & Limit & & & Prepared & & Metnod & \\
\hline
\end{tabular}

ERDC- EL-EP-C (Environmental Chemistry Branch)

Metals by EPA 6000/7000 Series Methods

\begin{tabular}{|c|c|c|c|c|c|c|c|c|}
\hline Mercury & 0.015 & 0.004 & $\mathrm{mg} / \mathrm{kg}$ & 1 & 03-Sep-2009 & 03-Sep-2009 & EPA 7471A & \\
\hline Antimony & 0.266 & 0.400 & $\mathrm{mg} / \mathrm{kg}$ & 2 & 28-Aug-2009 & 29-Aug-2009 & SW 846/6020 & $\mathrm{J}, \mathrm{B}$ \\
\hline Arsenic & 0.220 & 0.400 & $\mathrm{mg} / \mathrm{kg}$ & 2 & 28-Aug-2009 & 29-Aug-2009 & SW 846/6020 & $\mathrm{J}$ \\
\hline Barium & 2.20 & 0.400 & $\mathrm{mg} / \mathrm{kg}$ & 2 & 28-Aug-2009 & 29-Aug-2009 & SW 846/6020 & \\
\hline Beryllium & ND & 0.400 & $\mathrm{mg} / \mathrm{kg}$ & 2 & 28-Aug-2009 & 29-Aug-2009 & SW 846/6020 & $\mathrm{U}$ \\
\hline Cadmium & ND & 0.400 & $\mathrm{mg} / \mathrm{kg}$ & 2 & 28-Aug-2009 & 29-Aug-2009 & SW 846/6020 & $\mathrm{U}$ \\
\hline Chromium & 1.19 & 0.400 & $\mathrm{mg} / \mathrm{kg}$ & 2 & 28-Aug-2009 & 29-Aug-2009 & SW 846/6020 & \\
\hline Cobalt & ND & 0.400 & $\mathrm{mg} / \mathrm{kg}$ & 2 & 28-Aug-2009 & 29-Aug-2009 & SW 846/6020 & $\mathrm{U}$ \\
\hline Copper & 1.21 & 0.400 & $\mathrm{mg} / \mathrm{kg}$ & 2 & 28-Aug-2009 & 29-Aug-2009 & SW 846/6020 & \\
\hline Lead & 0.352 & 0.400 & $\mathrm{mg} / \mathrm{kg}$ & 2 & 28-Aug-2009 & 29-Aug-2009 & SW 846/6020 & $\mathrm{J}, \mathrm{B}$ \\
\hline Manganese & 0.619 & 0.400 & $\mathrm{mg} / \mathrm{kg}$ & 2 & 28-Aug-2009 & 29-Aug-2009 & SW 846/6020 & \\
\hline Molybdenum & ND & 0.400 & $\mathrm{mg} / \mathrm{kg}$ & 2 & 28-Aug-2009 & 29-Aug-2009 & SW 846/6020 & $\mathrm{U}$ \\
\hline Nickel & 0.767 & 0.400 & $\mathrm{mg} / \mathrm{kg}$ & 2 & 28-Aug-2009 & 29-Aug-2009 & SW 846/6020 & \\
\hline Selenium & 0.200 & 0.400 & $\mathrm{mg} / \mathrm{kg}$ & 2 & 28-Aug-2009 & 29-Aug-2009 & SW 846/6020 & $\mathrm{J}$ \\
\hline Silver & ND & 0.400 & $\mathrm{mg} / \mathrm{kg}$ & 2 & 28-Aug-2009 & 29-Aug-2009 & SW 846/6020 & $\mathrm{U}$ \\
\hline Thallium & ND & 0.400 & $\mathrm{mg} / \mathrm{kg}$ & 2 & 28-Aug-2009 & 29-Aug-2009 & SW 846/6020 & $\mathrm{U}$ \\
\hline Vanadium & 0.224 & 0.400 & $\mathrm{mg} / \mathrm{kg}$ & 2 & 28-Aug-2009 & 29-Aug-2009 & SW 846/6020 & $\mathrm{J}$ \\
\hline Zinc & 23.1 & 0.400 & $\mathrm{mg} / \mathrm{kg}$ & 2 & 28-Aug-2009 & 29-Aug-2009 & SW 846/6020 & \\
\hline
\end{tabular}




\section{USACE ERDC-EP-C \\ 3909 Halls Ferry Road \\ Vicksburg, MS 39180-6199}

ERDC - ECB

Project: TVA Fly Ash Project

Reported:

Project Manager: Tony Bednar

03-Sep-2009

Fish Tissue EMR - EL 4

9082706-14 (Tissue)

\begin{tabular}{|c|c|c|c|c|c|c|c|c|}
\hline & & porting & & & & & & \\
\hline Analyte & Result & Limit & Units & Dilution & Prepared & Analyzed & Method & Notes \\
\hline
\end{tabular}

ERDC- EL-EP-C (Environmental Chemistry Branch)

Metals by EPA 6000/7000 Series Methods

\begin{tabular}{|c|c|c|c|c|c|c|c|c|}
\hline Mercury & 0.009 & 0.004 & $\mathrm{mg} / \mathrm{kg}$ & 1 & 03-Sep-2009 & 03-Sep-2009 & EPA 7471A & \\
\hline Antimony & ND & 0.400 & $\mathrm{mg} / \mathrm{kg}$ & 2 & 28-Aug-2009 & 29-Aug-2009 & SW 846/6020 & $\mathrm{U}$ \\
\hline Arsenic & 0.199 & 0.400 & $\mathrm{mg} / \mathrm{kg}$ & 2 & 28-Aug-2009 & 29-Aug-2009 & SW 846/6020 & $\mathrm{J}$ \\
\hline Barium & 2.06 & 0.400 & $\mathrm{mg} / \mathrm{kg}$ & 2 & 28-Aug-2009 & 29-Aug-2009 & SW 846/6020 & \\
\hline Beryllium & ND & 0.400 & $\mathrm{mg} / \mathrm{kg}$ & 2 & 28-Aug-2009 & 29-Aug-2009 & SW 846/6020 & $\mathrm{U}$ \\
\hline Cadmium & ND & 0.400 & $\mathrm{mg} / \mathrm{kg}$ & 2 & 28-Aug-2009 & 29-Aug-2009 & SW 846/6020 & $\mathrm{U}$ \\
\hline Chromium & 3.99 & 0.400 & $\mathrm{mg} / \mathrm{kg}$ & 2 & 28-Aug-2009 & 29-Aug-2009 & SW 846/6020 & \\
\hline Cobalt & ND & 0.400 & $\mathrm{mg} / \mathrm{kg}$ & 2 & 28-Aug-2009 & 29-Aug-2009 & SW 846/6020 & $\mathrm{U}$ \\
\hline Copper & 0.787 & 0.400 & $\mathrm{mg} / \mathrm{kg}$ & 2 & 28-Aug-2009 & 29-Aug-2009 & SW 846/6020 & \\
\hline Lead & 0.350 & 0.400 & $\mathrm{mg} / \mathrm{kg}$ & 2 & 28-Aug-2009 & 29-Aug-2009 & SW 846/6020 & $\mathrm{J}, \mathrm{B}$ \\
\hline Manganese & 0.848 & 0.400 & $\mathrm{mg} / \mathrm{kg}$ & 2 & 28-Aug-2009 & 29-Aug-2009 & SW 846/6020 & \\
\hline Molybdenum & ND & 0.400 & $\mathrm{mg} / \mathrm{kg}$ & 2 & 28-Aug-2009 & 29-Aug-2009 & SW 846/6020 & $\mathrm{U}$ \\
\hline Nickel & 2.09 & 0.400 & $\mathrm{mg} / \mathrm{kg}$ & 2 & 28-Aug-2009 & 29-Aug-2009 & SW 846/6020 & \\
\hline Selenium & 0.171 & 0.400 & $\mathrm{mg} / \mathrm{kg}$ & 2 & 28-Aug-2009 & 29-Aug-2009 & SW 846/6020 & J \\
\hline Silver & ND & 0.400 & $\mathrm{mg} / \mathrm{kg}$ & 2 & 28-Aug-2009 & 29-Aug-2009 & SW 846/6020 & $\mathrm{U}$ \\
\hline Thallium & ND & 0.400 & $\mathrm{mg} / \mathrm{kg}$ & 2 & 28-Aug-2009 & 29-Aug-2009 & SW 846/6020 & $\mathrm{U}$ \\
\hline Vanadium & 0.358 & 0.400 & $\mathrm{mg} / \mathrm{kg}$ & 2 & 28-Aug-2009 & 29-Aug-2009 & SW 846/6020 & $\mathrm{J}$ \\
\hline Zinc & 20.1 & 0.400 & $\mathrm{mg} / \mathrm{kg}$ & 2 & 28-Aug-2009 & 29-Aug-2009 & SW 846/6020 & \\
\hline
\end{tabular}




\section{USACE ERDC-EP-C \\ 3909 Halls Ferry Road \\ Vicksburg, MS 39180-6199}

ERDC - ECB

Project: TVA Fly Ash Project

Reported:

Project Manager: Tony Bednar

03-Sep-2009

Fish Tissue EMR - EL 5

9082706-15 (Tissue)

\begin{tabular}{|c|c|c|c|c|c|c|c|c|}
\hline & & porting & & & & & & \\
\hline Analyte & Result & Limit & Units & Dilution & Prepared & Analyzed & Method & Notes \\
\hline
\end{tabular}

ERDC- EL-EP-C (Environmental Chemistry Branch)

Metals by EPA 6000/7000 Series Methods

\begin{tabular}{|c|c|c|c|c|c|c|c|c|}
\hline Mercury & ND & 0.005 & $\mathrm{mg} / \mathrm{kg}$ & 1 & 03-Sep-2009 & 03-Sep-2009 & EPA 7471A & $\mathrm{U}$ \\
\hline Antimony & ND & 0.400 & $\mathrm{mg} / \mathrm{kg}$ & 2 & 28-Aug-2009 & 29-Aug-2009 & SW 846/6020 & $\mathrm{U}$ \\
\hline Arsenic & 0.224 & 0.400 & $\mathrm{mg} / \mathrm{kg}$ & 2 & 28-Aug-2009 & 29-Aug-2009 & SW 846/6020 & $\mathrm{J}$ \\
\hline Barium & 3.43 & 0.400 & $\mathrm{mg} / \mathrm{kg}$ & 2 & 28-Aug-2009 & 29-Aug-2009 & SW 846/6020 & \\
\hline Beryllium & ND & 0.400 & $\mathrm{mg} / \mathrm{kg}$ & 2 & 28-Aug-2009 & 29-Aug-2009 & SW 846/6020 & $\mathrm{U}$ \\
\hline Cadmium & ND & 0.400 & $\mathrm{mg} / \mathrm{kg}$ & 2 & 28-Aug-2009 & 29-Aug-2009 & SW 846/6020 & $\mathrm{U}$ \\
\hline Cobalt & ND & 0.400 & $\mathrm{mg} / \mathrm{kg}$ & 2 & 28-Aug-2009 & 29-Aug-2009 & SW 846/6020 & $\mathrm{U}$ \\
\hline Copper & 0.771 & 0.400 & $\mathrm{mg} / \mathrm{kg}$ & 2 & 28-Aug-2009 & 29-Aug-2009 & SW 846/6020 & \\
\hline Lead & 0.295 & 0.400 & $\mathrm{mg} / \mathrm{kg}$ & 2 & 28-Aug-2009 & 29-Aug-2009 & SW 846/6020 & $\mathrm{J}, \mathrm{B}$ \\
\hline Manganese & 1.07 & 0.400 & $\mathrm{mg} / \mathrm{kg}$ & 2 & 28-Aug-2009 & 29-Aug-2009 & SW 846/6020 & \\
\hline Molybdenum & ND & 0.400 & $\mathrm{mg} / \mathrm{kg}$ & 2 & 28-Aug-2009 & 29-Aug-2009 & SW 846/6020 & $\mathrm{U}$ \\
\hline Nickel & 3.67 & 0.400 & $\mathrm{mg} / \mathrm{kg}$ & 2 & 28-Aug-2009 & 29-Aug-2009 & SW 846/6020 & \\
\hline Selenium & 0.190 & 0.400 & $\mathrm{mg} / \mathrm{kg}$ & 2 & 28-Aug-2009 & 29-Aug-2009 & SW 846/6020 & $\mathrm{J}$ \\
\hline Silver & ND & 0.400 & $\mathrm{mg} / \mathrm{kg}$ & 2 & 28-Aug-2009 & 29-Aug-2009 & SW 846/6020 & $\mathrm{U}$ \\
\hline Thallium & ND & 0.400 & $\mathrm{mg} / \mathrm{kg}$ & 2 & 28-Aug-2009 & 29-Aug-2009 & SW 846/6020 & $\mathrm{U}$ \\
\hline Vanadium & 0.338 & 0.400 & $\mathrm{mg} / \mathrm{kg}$ & 2 & 28-Aug-2009 & 29-Aug-2009 & SW 846/6020 & $\mathrm{J}$ \\
\hline Zinc & 21.2 & 0.400 & $\mathrm{mg} / \mathrm{kg}$ & 2 & 28-Aug-2009 & 29-Aug-2009 & SW 846/6020 & \\
\hline
\end{tabular}




\section{USACE ERDC-EP-C \\ 3909 Halls Ferry Road \\ Vicksburg, MS 39180-6199}

ERDC - ECB

Project: TVA Fly Ash Project

Reported:

Project Manager: Tony Bednar

03-Sep-2009

\section{Fish Gut Dechlor 1}

9082706-16 (Tissue)

\begin{tabular}{|c|c|c|c|c|c|c|c|c|}
\hline & & porting & & & & & & \\
\hline Analyte & Result & Limit & Units & Dilution & Prepared & Analyzed & Method & Notes \\
\hline
\end{tabular}

ERDC- EL-EP-C (Environmental Chemistry Branch)

Metals by EPA 6000/7000 Series Methods

\begin{tabular}{|c|c|c|c|c|c|c|c|c|}
\hline Antimony & ND & 40.0 & $\mathrm{mg} / \mathrm{kg}$ & 2 & 28-Aug-2009 & 29-Aug-2009 & SW 846/6020 & $\mathrm{U}$ \\
\hline Arsenic & ND & 40.0 & $\mathrm{mg} / \mathrm{kg}$ & 2 & 28-Aug-2009 & 29-Aug-2009 & SW 846/6020 & $\mathrm{U}$ \\
\hline Barium & 1060 & 40.0 & $\mathrm{mg} / \mathrm{kg}$ & 2 & 28-Aug-2009 & 29-Aug-2009 & SW 846/6020 & \\
\hline Beryllium & ND & 40.0 & $\mathrm{mg} / \mathrm{kg}$ & 2 & 28-Aug-2009 & 29-Aug-2009 & SW 846/6020 & $\mathrm{U}$ \\
\hline Cadmium & ND & 40.0 & $\mathrm{mg} / \mathrm{kg}$ & 2 & 28-Aug-2009 & 29-Aug-2009 & SW 846/6020 & $\mathrm{U}$ \\
\hline Chromium & 1020 & 40.0 & $\mathrm{mg} / \mathrm{kg}$ & 2 & 28-Aug-2009 & 29-Aug-2009 & SW 846/6020 & \\
\hline Cobalt & 17.4 & 40.0 & $\mathrm{mg} / \mathrm{kg}$ & 2 & 28-Aug-2009 & 29-Aug-2009 & SW 846/6020 & $\mathrm{J}$ \\
\hline Copper & 460 & 40.0 & $\mathrm{mg} / \mathrm{kg}$ & 2 & 28-Aug-2009 & 29-Aug-2009 & SW 846/6020 & \\
\hline Lead & 169 & 40.0 & $\mathrm{mg} / \mathrm{kg}$ & 2 & 28-Aug-2009 & 29-Aug-2009 & SW 846/6020 & B \\
\hline Manganese & 3090 & 40.0 & $\mathrm{mg} / \mathrm{kg}$ & 2 & 28-Aug-2009 & 29-Aug-2009 & SW 846/6020 & \\
\hline Molybdenum & ND & 40.0 & $\mathrm{mg} / \mathrm{kg}$ & 2 & 28-Aug-2009 & 29-Aug-2009 & SW 846/6020 & $\mathrm{U}$ \\
\hline Nickel & 66.3 & 40.0 & $\mathrm{mg} / \mathrm{kg}$ & 2 & 28-Aug-2009 & 29-Aug-2009 & SW 846/6020 & \\
\hline Selenium & 13.3 & 40.0 & $\mathrm{mg} / \mathrm{kg}$ & 2 & 28-Aug-2009 & 29-Aug-2009 & SW 846/6020 & $\mathrm{J}$ \\
\hline Silver & ND & 40.0 & $\mathrm{mg} / \mathrm{kg}$ & 2 & 28-Aug-2009 & 29-Aug-2009 & SW 846/6020 & $\mathrm{U}$ \\
\hline Thallium & ND & 40.0 & $\mathrm{mg} / \mathrm{kg}$ & 2 & 28-Aug-2009 & 29-Aug-2009 & SW 846/6020 & $\mathrm{U}$ \\
\hline Vanadium & 28.0 & 40.0 & $\mathrm{mg} / \mathrm{kg}$ & 2 & 28-Aug-2009 & 29-Aug-2009 & SW 846/6020 & $\mathrm{J}$ \\
\hline Zinc & 2370 & 40.0 & $\mathrm{mg} / \mathrm{kg}$ & 2 & 28-Aug-2009 & 29-Aug-2009 & SW 846/6020 & \\
\hline
\end{tabular}




\section{USACE ERDC-EP-C \\ 3909 Halls Ferry Road \\ Vicksburg, MS 39180-6199}

ERDC - ECB

Project: TVA Fly Ash Project

Reported:

Project Manager: Tony Bednar

03-Sep-2009

Fish Gut Dechlor 2

9082706-17 (Tissue)

\begin{tabular}{|c|c|c|c|c|c|c|c|c|}
\hline Analyte & Result & porting & Units & Dilution & Prenared & Analyzed & Method & Notes \\
\hline Analyte & Kesult & Limit & & & Prepared & & Metnod & \\
\hline
\end{tabular}

ERDC- EL-EP-C (Environmental Chemistry Branch)

Metals by EPA 6000/7000 Series Methods

\begin{tabular}{|c|c|c|c|c|c|c|c|c|}
\hline Antimony & 27.3 & 40.0 & $\mathrm{mg} / \mathrm{kg}$ & 2 & 28-Aug-2009 & 29-Aug-2009 & SW 846/6020 & $\mathrm{J}, \mathrm{B}$ \\
\hline Arsenic & ND & 40.0 & $\mathrm{mg} / \mathrm{kg}$ & 2 & 28-Aug-2009 & 29-Aug-2009 & SW 846/6020 & $\mathrm{U}$ \\
\hline Barium & 299 & 40.0 & $\mathrm{mg} / \mathrm{kg}$ & 2 & 28-Aug-2009 & 29-Aug-2009 & SW 846/6020 & \\
\hline Beryllium & ND & 40.0 & $\mathrm{mg} / \mathrm{kg}$ & 2 & 28-Aug-2009 & 29-Aug-2009 & SW 846/6020 & $\mathrm{U}$ \\
\hline Cadmium & ND & 40.0 & $\mathrm{mg} / \mathrm{kg}$ & 2 & 28-Aug-2009 & 29-Aug-2009 & SW 846/6020 & $\mathrm{U}$ \\
\hline Chromium & 224 & 40.0 & $\mathrm{mg} / \mathrm{kg}$ & 2 & 28-Aug-2009 & 29-Aug-2009 & SW 846/6020 & \\
\hline Copper & 167 & 40.0 & $\mathrm{mg} / \mathrm{kg}$ & 2 & 28-Aug-2009 & 29-Aug-2009 & SW 846/6020 & \\
\hline Lead & 172 & 40.0 & $\mathrm{mg} / \mathrm{kg}$ & 2 & 28-Aug-2009 & 29-Aug-2009 & SW 846/6020 & B \\
\hline Manganese & 727 & 40.0 & $\mathrm{mg} / \mathrm{kg}$ & 2 & 28-Aug-2009 & 29-Aug-2009 & SW 846/6020 & \\
\hline Molybdenum & ND & 40.0 & $\mathrm{mg} / \mathrm{kg}$ & 2 & 28-Aug-2009 & 29-Aug-2009 & SW 846/6020 & $\mathrm{U}$ \\
\hline Nickel & 66.1 & 40.0 & $\mathrm{mg} / \mathrm{kg}$ & 2 & 28-Aug-2009 & 29-Aug-2009 & SW 846/6020 & \\
\hline Selenium & ND & 40.0 & $\mathrm{mg} / \mathrm{kg}$ & 2 & 28-Aug-2009 & 29-Aug-2009 & SW 846/6020 & U \\
\hline Silver & ND & 40.0 & $\mathrm{mg} / \mathrm{kg}$ & 2 & 28-Aug-2009 & 29-Aug-2009 & SW 846/6020 & $\mathrm{U}$ \\
\hline Thallium & ND & 40.0 & $\mathrm{mg} / \mathrm{kg}$ & 2 & 28-Aug-2009 & 29-Aug-2009 & SW 846/6020 & $\mathrm{U}$ \\
\hline Vanadium & 9.40 & 40.0 & $\mathrm{mg} / \mathrm{kg}$ & 2 & 28-Aug-2009 & 29-Aug-2009 & SW 846/6020 & $\mathrm{J}$ \\
\hline Zinc & 1080 & 40.0 & $\mathrm{mg} / \mathrm{kg}$ & 2 & 28-Aug-2009 & 29-Aug-2009 & SW $846 / 6020$ & \\
\hline
\end{tabular}




\section{USACE ERDC-EP-C \\ 3909 Halls Ferry Road \\ Vicksburg, MS 39180-6199}

ERDC - ECB

Project: TVA Fly Ash Project

Reported:

Project Manager: Tony Bednar

03-Sep-2009

Fish Gut Dechlor 3

9082706-18 (Tissue)

\begin{tabular}{|c|c|c|c|c|c|c|c|c|}
\hline & & porting & & & & & & \\
\hline Analyte & Result & Limit & Units & Dilution & Prepared & Analyzed & Method & Notes \\
\hline
\end{tabular}

ERDC- EL-EP-C (Environmental Chemistry Branch)

Metals by EPA 6000/7000 Series Methods

\begin{tabular}{|c|c|c|c|c|c|c|c|c|}
\hline Antimony & ND & 40.0 & $\mathrm{mg} / \mathrm{kg}$ & 2 & 28-Aug-2009 & 29-Aug-2009 & SW 846/6020 & $\mathrm{U}$ \\
\hline Arsenic & ND & 40.0 & $\mathrm{mg} / \mathrm{kg}$ & 2 & 28-Aug-2009 & 29-Aug-2009 & SW 846/6020 & $\mathrm{U}$ \\
\hline Barium & 210 & 40.0 & $\mathrm{mg} / \mathrm{kg}$ & 2 & 28-Aug-2009 & 29-Aug-2009 & SW 846/6020 & \\
\hline Beryllium & ND & 40.0 & $\mathrm{mg} / \mathrm{kg}$ & 2 & 28-Aug-2009 & 29-Aug-2009 & SW 846/6020 & $\mathrm{U}$ \\
\hline Cadmium & ND & 40.0 & $\mathrm{mg} / \mathrm{kg}$ & 2 & 28-Aug-2009 & 29-Aug-2009 & SW 846/6020 & $\mathrm{U}$ \\
\hline Chromium & 184 & 40.0 & $\mathrm{mg} / \mathrm{kg}$ & 2 & 28-Aug-2009 & 29-Aug-2009 & SW 846/6020 & \\
\hline Cobalt & ND & 40.0 & $\mathrm{mg} / \mathrm{kg}$ & 2 & 28-Aug-2009 & 29-Aug-2009 & SW 846/6020 & $\mathrm{U}$ \\
\hline Copper & 107 & 40.0 & $\mathrm{mg} / \mathrm{kg}$ & 2 & 28-Aug-2009 & 29-Aug-2009 & SW 846/6020 & \\
\hline Lead & 77.5 & 40.0 & $\mathrm{mg} / \mathrm{kg}$ & 2 & 28-Aug-2009 & 29-Aug-2009 & SW 846/6020 & B \\
\hline Manganese & 586 & 40.0 & $\mathrm{mg} / \mathrm{kg}$ & 2 & 28-Aug-2009 & 29-Aug-2009 & SW 846/6020 & \\
\hline Molybdenum & ND & 40.0 & $\mathrm{mg} / \mathrm{kg}$ & 2 & 28-Aug-2009 & 29-Aug-2009 & SW 846/6020 & $\mathrm{U}$ \\
\hline Nickel & 25.1 & 40.0 & $\mathrm{mg} / \mathrm{kg}$ & 2 & 28-Aug-2009 & 29-Aug-2009 & SW 846/6020 & $\mathrm{J}$ \\
\hline Selenium & ND & 40.0 & $\mathrm{mg} / \mathrm{kg}$ & 2 & 28-Aug-2009 & 29-Aug-2009 & SW 846/6020 & $\mathrm{U}$ \\
\hline Silver & ND & 40.0 & $\mathrm{mg} / \mathrm{kg}$ & 2 & 28-Aug-2009 & 29-Aug-2009 & SW 846/6020 & $\mathrm{U}$ \\
\hline Thallium & ND & 40.0 & $\mathrm{mg} / \mathrm{kg}$ & 2 & 28-Aug-2009 & 29-Aug-2009 & SW 846/6020 & $\mathrm{U}$ \\
\hline Vanadium & ND & 40.0 & $\mathrm{mg} / \mathrm{kg}$ & 2 & 28-Aug-2009 & 29-Aug-2009 & SW $846 / 6020$ & $\mathrm{U}$ \\
\hline Zinc & 700 & 40.0 & $\mathrm{mg} / \mathrm{kg}$ & 2 & 28-Aug-2009 & 29-Aug-2009 & SW 846/6020 & \\
\hline
\end{tabular}




\section{USACE ERDC-EP-C \\ 3909 Halls Ferry Road \\ Vicksburg, MS 39180-6199}

ERDC - ECB

Project: TVA Fly Ash Project

Reported:

Project Manager: Tony Bednar

03-Sep-2009

\section{Fish Gut Dechlor 4}

9082706-19 (Tissue)

\begin{tabular}{|c|c|c|c|c|c|c|c|c|}
\hline Analyte & Result & $\begin{array}{r}\text { porting } \\
\text { Limit }\end{array}$ & Units & Dilution & Prepared & Analyzed & Method & Notes \\
\hline
\end{tabular}

ERDC- EL-EP-C (Environmental Chemistry Branch)

Metals by EPA 6000/7000 Series Methods

\begin{tabular}{|c|c|c|c|c|c|c|c|c|}
\hline Antimony & ND & 40.0 & $\mathrm{mg} / \mathrm{kg}$ & 2 & 28-Aug-2009 & 29-Aug-2009 & SW 846/6020 & $\mathrm{U}$ \\
\hline Arsenic & ND & 40.0 & $\mathrm{mg} / \mathrm{kg}$ & 2 & 28-Aug-2009 & 29-Aug-2009 & SW 846/6020 & $\mathrm{U}$ \\
\hline Barium & 358 & 40.0 & $\mathrm{mg} / \mathrm{kg}$ & 2 & 28-Aug-2009 & 29-Aug-2009 & SW 846/6020 & \\
\hline Beryllium & ND & 40.0 & $\mathrm{mg} / \mathrm{kg}$ & 2 & 28-Aug-2009 & 29-Aug-2009 & SW 846/6020 & $\mathrm{U}$ \\
\hline Cadmium & ND & 40.0 & $\mathrm{mg} / \mathrm{kg}$ & 2 & 28-Aug-2009 & 29-Aug-2009 & SW 846/6020 & $\mathrm{U}$ \\
\hline Chromium & 272 & 40.0 & $\mathrm{mg} / \mathrm{kg}$ & 2 & 28-Aug-2009 & 29-Aug-2009 & SW 846/6020 & \\
\hline Cobalt & ND & 40.0 & $\mathrm{mg} / \mathrm{kg}$ & 2 & 28-Aug-2009 & 29-Aug-2009 & SW 846/6020 & $\mathrm{U}$ \\
\hline Copper & 566 & 40.0 & $\mathrm{mg} / \mathrm{kg}$ & 2 & 28-Aug-2009 & 29-Aug-2009 & SW 846/6020 & \\
\hline Lead & 96.0 & 40.0 & $\mathrm{mg} / \mathrm{kg}$ & 2 & 28-Aug-2009 & 29-Aug-2009 & SW 846/6020 & B \\
\hline Manganese & 987 & 40.0 & $\mathrm{mg} / \mathrm{kg}$ & 2 & 28-Aug-2009 & 29-Aug-2009 & SW 846/6020 & \\
\hline Molybdenum & ND & 40.0 & $\mathrm{mg} / \mathrm{kg}$ & 2 & 28-Aug-2009 & 29-Aug-2009 & SW 846/6020 & $\mathrm{U}$ \\
\hline Nickel & 53.6 & 40.0 & $\mathrm{mg} / \mathrm{kg}$ & 2 & 28-Aug-2009 & 29-Aug-2009 & SW 846/6020 & \\
\hline Selenium & ND & 40.0 & $\mathrm{mg} / \mathrm{kg}$ & 2 & 28-Aug-2009 & 29-Aug-2009 & SW 846/6020 & $\mathrm{U}$ \\
\hline Silver & ND & 40.0 & $\mathrm{mg} / \mathrm{kg}$ & 2 & 28-Aug-2009 & 29-Aug-2009 & SW 846/6020 & $\mathrm{U}$ \\
\hline Thallium & ND & 40.0 & $\mathrm{mg} / \mathrm{kg}$ & 2 & 28-Aug-2009 & 29-Aug-2009 & SW 846/6020 & $\mathrm{U}$ \\
\hline Vanadium & 14.2 & 40.0 & $\mathrm{mg} / \mathrm{kg}$ & 2 & 28-Aug-2009 & 29-Aug-2009 & SW 846/6020 & $\mathrm{J}$ \\
\hline Zinc & 1170 & 40.0 & $\mathrm{mg} / \mathrm{kg}$ & 2 & 28-Aug-2009 & 29-Aug-2009 & SW 846/6020 & \\
\hline
\end{tabular}




\section{USACE ERDC-EP-C \\ 3909 Halls Ferry Road \\ Vicksburg, MS 39180-6199}

ERDC - ECB

Project: TVA Fly Ash Project

Reported:

Project Manager: Tony Bednar

03-Sep-2009

Fish Gut Dechlor 5

9082706-20 (Tissue)

\begin{tabular}{|c|c|c|c|c|c|c|c|c|}
\hline & & porting & & & & & & \\
\hline Analyte & Result & Limit & Units & Dilution & Prepared & Analyzed & Method & Notes \\
\hline
\end{tabular}

ERDC- EL-EP-C (Environmental Chemistry Branch)

Metals by EPA 6000/7000 Series Methods

\begin{tabular}{|c|c|c|c|c|c|c|c|c|}
\hline Antimony & ND & 40.0 & $\mathrm{mg} / \mathrm{kg}$ & 2 & 28-Aug-2009 & 29-Aug-2009 & SW 846/6020 & $\mathrm{U}$ \\
\hline Arsenic & ND & 40.0 & $\mathrm{mg} / \mathrm{kg}$ & 2 & 28-Aug-2009 & 29-Aug-2009 & SW 846/6020 & $\mathrm{U}$ \\
\hline Barium & 110 & 40.0 & $\mathrm{mg} / \mathrm{kg}$ & 2 & 28-Aug-2009 & 29-Aug-2009 & SW 846/6020 & \\
\hline Beryllium & ND & 40.0 & $\mathrm{mg} / \mathrm{kg}$ & 2 & 28-Aug-2009 & 29-Aug-2009 & SW 846/6020 & $\mathrm{U}$ \\
\hline Cadmium & ND & 40.0 & $\mathrm{mg} / \mathrm{kg}$ & 2 & 28-Aug-2009 & 29-Aug-2009 & SW 846/6020 & $\mathrm{U}$ \\
\hline Chromium & 195 & 40.0 & $\mathrm{mg} / \mathrm{kg}$ & 2 & 28-Aug-2009 & 29-Aug-2009 & SW 846/6020 & \\
\hline Copper & 99.3 & 40.0 & $\mathrm{mg} / \mathrm{kg}$ & 2 & 28-Aug-2009 & 29-Aug-2009 & SW 846/6020 & \\
\hline Lead & 86.2 & 40.0 & $\mathrm{mg} / \mathrm{kg}$ & 2 & 28-Aug-2009 & 29-Aug-2009 & SW 846/6020 & B \\
\hline Manganese & 199 & 40.0 & $\mathrm{mg} / \mathrm{kg}$ & 2 & 28-Aug-2009 & 29-Aug-2009 & SW 846/6020 & \\
\hline Molybdenum & $\mathrm{ND}$ & 40.0 & $\mathrm{mg} / \mathrm{kg}$ & 2 & 28-Aug-2009 & 29-Aug-2009 & SW 846/6020 & $\mathrm{U}$ \\
\hline Nickel & 57.0 & 40.0 & $\mathrm{mg} / \mathrm{kg}$ & 2 & 28-Aug-2009 & 29-Aug-2009 & SW 846/6020 & \\
\hline Selenium & ND & 40.0 & $\mathrm{mg} / \mathrm{kg}$ & 2 & 28-Aug-2009 & 29-Aug-2009 & SW 846/6020 & $\mathrm{U}$ \\
\hline Silver & ND & 40.0 & $\mathrm{mg} / \mathrm{kg}$ & 2 & 28-Aug-2009 & 29-Aug-2009 & SW 846/6020 & $\mathrm{U}$ \\
\hline Thallium & ND & 40.0 & $\mathrm{mg} / \mathrm{kg}$ & 2 & 28-Aug-2009 & 29-Aug-2009 & SW 846/6020 & $\mathrm{U}$ \\
\hline Vanadium & ND & 40.0 & $\mathrm{mg} / \mathrm{kg}$ & 2 & 28-Aug-2009 & 29-Aug-2009 & SW 846/6020 & $\mathrm{U}$ \\
\hline Zinc & 551 & 40.0 & $\mathrm{mg} / \mathrm{kg}$ & 2 & 28-Aug-2009 & 29-Aug-2009 & SW 846/6020 & \\
\hline
\end{tabular}




\section{USACE ERDC-EP-C \\ 3909 Halls Ferry Road \\ Vicksburg, MS 39180-6199}

ERDC - ECB

Project: TVA Fly Ash Project

Reported:

Project Manager: Tony Bednar

03-Sep-2009

Fish Gut EMR - WA 1

9082706-21 (Tissue)

\begin{tabular}{|c|c|c|c|c|c|c|c|c|}
\hline Analyte & Result & porting & Units & Dilution & Prenared & Analyzed & Method & Notes \\
\hline Analyte & Kesult & Limit & & & Prepared & & Metnod & \\
\hline
\end{tabular}

ERDC- EL-EP-C (Environmental Chemistry Branch)

Metals by EPA 6000/7000 Series Methods

\begin{tabular}{|c|c|c|c|c|c|c|c|c|}
\hline Antimony & ND & 40.0 & $\mathrm{mg} / \mathrm{kg}$ & 2 & 28-Aug-2009 & 29-Aug-2009 & SW 846/6020 & $\mathrm{U}$ \\
\hline Arsenic & ND & 40.0 & $\mathrm{mg} / \mathrm{kg}$ & 2 & 28-Aug-2009 & 29-Aug-2009 & SW 846/6020 & $\mathrm{U}$ \\
\hline Barium & 348 & 40.0 & $\mathrm{mg} / \mathrm{kg}$ & 2 & 28-Aug-2009 & 29-Aug-2009 & SW 846/6020 & \\
\hline Beryllium & ND & 40.0 & $\mathrm{mg} / \mathrm{kg}$ & 2 & 28-Aug-2009 & 29-Aug-2009 & SW 846/6020 & $\mathrm{U}$ \\
\hline Cadmium & ND & 40.0 & $\mathrm{mg} / \mathrm{kg}$ & 2 & 28-Aug-2009 & 29-Aug-2009 & SW 846/6020 & $\mathrm{U}$ \\
\hline Chromium & 94.1 & 40.0 & $\mathrm{mg} / \mathrm{kg}$ & 2 & 28-Aug-2009 & 29-Aug-2009 & SW 846/6020 & \\
\hline Cobalt & ND & 40.0 & $\mathrm{mg} / \mathrm{kg}$ & 2 & 28-Aug-2009 & 29-Aug-2009 & SW 846/6020 & $\mathrm{U}$ \\
\hline Copper & 135 & 40.0 & $\mathrm{mg} / \mathrm{kg}$ & 2 & 28-Aug-2009 & 29-Aug-2009 & SW 846/6020 & \\
\hline Lead & 90.7 & 40.0 & $\mathrm{mg} / \mathrm{kg}$ & 2 & 28-Aug-2009 & 29-Aug-2009 & SW 846/6020 & B \\
\hline Manganese & 488 & 40.0 & $\mathrm{mg} / \mathrm{kg}$ & 2 & 28-Aug-2009 & 29-Aug-2009 & SW 846/6020 & \\
\hline Molybdenum & ND & 40.0 & $\mathrm{mg} / \mathrm{kg}$ & 2 & 28-Aug-2009 & 29-Aug-2009 & SW 846/6020 & $\mathrm{U}$ \\
\hline Nickel & 31.3 & 40.0 & $\mathrm{mg} / \mathrm{kg}$ & 2 & 28-Aug-2009 & 29-Aug-2009 & SW 846/6020 & $\mathrm{J}$ \\
\hline Selenium & $\mathrm{ND}$ & 40.0 & $\mathrm{mg} / \mathrm{kg}$ & 2 & 28-Aug-2009 & 29-Aug-2009 & SW 846/6020 & $\mathrm{U}$ \\
\hline Silver & 16.9 & 40.0 & $\mathrm{mg} / \mathrm{kg}$ & 2 & 28-Aug-2009 & 29-Aug-2009 & SW 846/6020 & $\mathrm{J}$ \\
\hline Thallium & ND & 40.0 & $\mathrm{mg} / \mathrm{kg}$ & 2 & 28-Aug-2009 & 29-Aug-2009 & SW 846/6020 & $\mathrm{U}$ \\
\hline Vanadium & 12.1 & 40.0 & $\mathrm{mg} / \mathrm{kg}$ & 2 & 28-Aug-2009 & 29-Aug-2009 & SW 846/6020 & $\mathrm{J}$ \\
\hline Zinc & 1490 & 40.0 & $\mathrm{mg} / \mathrm{kg}$ & 2 & 28-Aug-2009 & 29-Aug-2009 & SW 846/6020 & \\
\hline
\end{tabular}




\section{USACE ERDC-EP-C \\ 3909 Halls Ferry Road \\ Vicksburg, MS 39180-6199}

ERDC - ECB

Project: TVA Fly Ash Project

Reported:

Project Manager: Tony Bednar

03-Sep-2009

Fish Gut EMR - WA 2

9082706-22 (Tissue)

\begin{tabular}{|c|c|c|c|c|c|c|c|c|}
\hline Analyte & Result & porting & Units & Dilution & Prenared & Analyzed & Method & Notes \\
\hline Analyte & Kesult & Limit & & & Prepared & & Metnod & \\
\hline
\end{tabular}

ERDC- EL-EP-C (Environmental Chemistry Branch)

Metals by EPA 6000/7000 Series Methods

\begin{tabular}{|c|c|c|c|c|c|c|c|c|}
\hline Antimony & ND & 40.0 & $\mathrm{mg} / \mathrm{kg}$ & 2 & 28-Aug-2009 & 29-Aug-2009 & SW 846/6020 & $\mathrm{U}$ \\
\hline Arsenic & ND & 40.0 & $\mathrm{mg} / \mathrm{kg}$ & 2 & 28-Aug-2009 & 29-Aug-2009 & SW 846/6020 & $\mathrm{U}$ \\
\hline Barium & 218 & 40.0 & $\mathrm{mg} / \mathrm{kg}$ & 2 & 28-Aug-2009 & 29-Aug-2009 & SW 846/6020 & \\
\hline Beryllium & ND & 40.0 & $\mathrm{mg} / \mathrm{kg}$ & 2 & 28-Aug-2009 & 29-Aug-2009 & SW 846/6020 & $\mathrm{U}$ \\
\hline Cadmium & ND & 40.0 & $\mathrm{mg} / \mathrm{kg}$ & 2 & 28-Aug-2009 & 29-Aug-2009 & SW 846/6020 & $\mathrm{U}$ \\
\hline Chromium & 48.8 & 40.0 & $\mathrm{mg} / \mathrm{kg}$ & 2 & 28-Aug-2009 & 29-Aug-2009 & SW 846/6020 & \\
\hline Cobalt & ND & 40.0 & $\mathrm{mg} / \mathrm{kg}$ & 2 & 28-Aug-2009 & 29-Aug-2009 & SW 846/6020 & $\mathrm{U}$ \\
\hline Copper & 83.1 & 40.0 & $\mathrm{mg} / \mathrm{kg}$ & 2 & 28-Aug-2009 & 29-Aug-2009 & SW 846/6020 & \\
\hline Lead & 66.5 & 40.0 & $\mathrm{mg} / \mathrm{kg}$ & 2 & 28-Aug-2009 & 29-Aug-2009 & SW 846/6020 & B \\
\hline Manganese & 231 & 40.0 & $\mathrm{mg} / \mathrm{kg}$ & 2 & 28-Aug-2009 & 29-Aug-2009 & SW 846/6020 & \\
\hline Molybdenum & ND & 40.0 & $\mathrm{mg} / \mathrm{kg}$ & 2 & 28-Aug-2009 & 29-Aug-2009 & SW 846/6020 & $\mathrm{U}$ \\
\hline Nickel & 32.2 & 40.0 & $\mathrm{mg} / \mathrm{kg}$ & 2 & 28-Aug-2009 & 29-Aug-2009 & SW 846/6020 & $\mathrm{J}$ \\
\hline Selenium & ND & 40.0 & $\mathrm{mg} / \mathrm{kg}$ & 2 & 28-Aug-2009 & 29-Aug-2009 & SW 846/6020 & $\mathrm{U}$ \\
\hline Silver & ND & 40.0 & $\mathrm{mg} / \mathrm{kg}$ & 2 & 28-Aug-2009 & 29-Aug-2009 & SW 846/6020 & $\mathrm{U}$ \\
\hline Thallium & ND & 40.0 & $\mathrm{mg} / \mathrm{kg}$ & 2 & 28-Aug-2009 & 29-Aug-2009 & SW 846/6020 & $\mathrm{U}$ \\
\hline Vanadium & ND & 40.0 & $\mathrm{mg} / \mathrm{kg}$ & 2 & 28-Aug-2009 & 29-Aug-2009 & SW $846 / 6020$ & $\mathrm{U}$ \\
\hline Zinc & 869 & 40.0 & $\mathrm{mg} / \mathrm{kg}$ & 2 & 28-Aug-2009 & 29-Aug-2009 & SW 846/6020 & \\
\hline
\end{tabular}




\section{USACE ERDC-EP-C \\ 3909 Halls Ferry Road \\ Vicksburg, MS 39180-6199}

ERDC - ECB

Project: TVA Fly Ash Project

Reported:

Project Manager: Tony Bednar

03-Sep-2009

Fish Gut EMR - WA 3

9082706-23 (Tissue)

\begin{tabular}{|c|c|c|c|c|c|c|c|c|}
\hline Analyte & Result & porting & Units & Dilution & Prenared & Analyzed & Method & Notes \\
\hline Analyte & Kesult & Limit & & & Prepared & & Metnod & \\
\hline
\end{tabular}

ERDC- EL-EP-C (Environmental Chemistry Branch)

Metals by EPA 6000/7000 Series Methods

\begin{tabular}{|c|c|c|c|c|c|c|c|c|}
\hline Antimony & ND & 40.0 & $\mathrm{mg} / \mathrm{kg}$ & 2 & 28-Aug-2009 & 29-Aug-2009 & SW 846/6020 & $\mathrm{U}$ \\
\hline Arsenic & ND & 40.0 & $\mathrm{mg} / \mathrm{kg}$ & 2 & 28-Aug-2009 & 29-Aug-2009 & SW 846/6020 & $\mathrm{U}$ \\
\hline Barium & 481 & 40.0 & $\mathrm{mg} / \mathrm{kg}$ & 2 & 28-Aug-2009 & 29-Aug-2009 & SW 846/6020 & \\
\hline Beryllium & ND & 40.0 & $\mathrm{mg} / \mathrm{kg}$ & 2 & 28-Aug-2009 & 29-Aug-2009 & SW 846/6020 & $\mathrm{U}$ \\
\hline Cadmium & ND & 40.0 & $\mathrm{mg} / \mathrm{kg}$ & 2 & 28-Aug-2009 & 29-Aug-2009 & SW 846/6020 & $\mathrm{U}$ \\
\hline Chromium & 189 & 40.0 & $\mathrm{mg} / \mathrm{kg}$ & 2 & 28-Aug-2009 & 29-Aug-2009 & SW 846/6020 & \\
\hline Cobalt & ND & 40.0 & $\mathrm{mg} / \mathrm{kg}$ & 2 & 28-Aug-2009 & 29-Aug-2009 & SW 846/6020 & $\mathrm{U}$ \\
\hline Copper & 181 & 40.0 & $\mathrm{mg} / \mathrm{kg}$ & 2 & 28-Aug-2009 & 29-Aug-2009 & SW 846/6020 & \\
\hline Lead & 123 & 40.0 & $\mathrm{mg} / \mathrm{kg}$ & 2 & 28-Aug-2009 & 29-Aug-2009 & SW 846/6020 & B \\
\hline Manganese & 786 & 40.0 & $\mathrm{mg} / \mathrm{kg}$ & 2 & 28-Aug-2009 & 29-Aug-2009 & SW 846/6020 & \\
\hline Molybdenum & ND & 40.0 & $\mathrm{mg} / \mathrm{kg}$ & 2 & 28-Aug-2009 & 29-Aug-2009 & SW 846/6020 & $\mathrm{U}$ \\
\hline Nickel & 70.8 & 40.0 & $\mathrm{mg} / \mathrm{kg}$ & 2 & 28-Aug-2009 & 29-Aug-2009 & SW 846/6020 & \\
\hline Selenium & ND & 40.0 & $\mathrm{mg} / \mathrm{kg}$ & 2 & 28-Aug-2009 & 29-Aug-2009 & SW 846/6020 & $\mathrm{U}$ \\
\hline Silver & 10.6 & 40.0 & $\mathrm{mg} / \mathrm{kg}$ & 2 & 28-Aug-2009 & 29-Aug-2009 & SW 846/6020 & $\mathrm{J}$ \\
\hline Thallium & ND & 40.0 & $\mathrm{mg} / \mathrm{kg}$ & 2 & 28-Aug-2009 & 29-Aug-2009 & SW 846/6020 & $\mathrm{U}$ \\
\hline Vanadium & 20.1 & 40.0 & $\mathrm{mg} / \mathrm{kg}$ & 2 & 28-Aug-2009 & 29-Aug-2009 & SW 846/6020 & $\mathrm{J}$ \\
\hline Zinc & 1670 & 40.0 & $\mathrm{mg} / \mathrm{kg}$ & 2 & 28-Aug-2009 & 29-Aug-2009 & SW 846/6020 & \\
\hline
\end{tabular}




\section{USACE ERDC-EP-C \\ 3909 Halls Ferry Road \\ Vicksburg, MS 39180-6199}

ERDC - ECB

Project: TVA Fly Ash Project

Reported:

Project Manager: Tony Bednar

03-Sep-2009

Fish Gut EMR - WA 4

9082706-24 (Tissue)

\begin{tabular}{|c|c|c|c|c|c|c|c|c|}
\hline Analyte & Result & porting & Units & Dilution & Prenared & Analyzed & Method & Notes \\
\hline Analyte & Kesult & Limit & & & Prepared & & Metnod & \\
\hline
\end{tabular}

ERDC- EL-EP-C (Environmental Chemistry Branch)

Metals by EPA 6000/7000 Series Methods

\begin{tabular}{|c|c|c|c|c|c|c|c|c|}
\hline Antimony & 25.0 & 40.0 & $\mathrm{mg} / \mathrm{kg}$ & 2 & 28-Aug-2009 & 29-Aug-2009 & SW 846/6020 & $\mathrm{J}, \mathrm{B}$ \\
\hline Arsenic & $\mathrm{ND}$ & 40.0 & $\mathrm{mg} / \mathrm{kg}$ & 2 & 28-Aug-2009 & 29-Aug-2009 & SW 846/6020 & $\mathrm{U}$ \\
\hline Barium & 517 & 40.0 & $\mathrm{mg} / \mathrm{kg}$ & 2 & 28-Aug-2009 & 29-Aug-2009 & SW $846 / 6020$ & \\
\hline Beryllium & ND & 40.0 & $\mathrm{mg} / \mathrm{kg}$ & 2 & 28-Aug-2009 & 29-Aug-2009 & SW 846/6020 & $\mathrm{U}$ \\
\hline Cadmium & ND & 40.0 & $\mathrm{mg} / \mathrm{kg}$ & 2 & 28-Aug-2009 & 29-Aug-2009 & SW 846/6020 & $\mathrm{U}$ \\
\hline Chromium & 168 & 40.0 & $\mathrm{mg} / \mathrm{kg}$ & 2 & 28-Aug-2009 & 29-Aug-2009 & SW 846/6020 & \\
\hline Copper & 160 & 40.0 & $\mathrm{mg} / \mathrm{kg}$ & 2 & 28-Aug-2009 & 29-Aug-2009 & SW 846/6020 & \\
\hline Lead & 112 & 40.0 & $\mathrm{mg} / \mathrm{kg}$ & 2 & 28-Aug-2009 & 29-Aug-2009 & SW 846/6020 & B \\
\hline Manganese & 728 & 40.0 & $\mathrm{mg} / \mathrm{kg}$ & 2 & 28-Aug-2009 & 29-Aug-2009 & SW 846/6020 & \\
\hline Molybdenum & $\mathrm{ND}$ & 40.0 & $\mathrm{mg} / \mathrm{kg}$ & 2 & 28-Aug-2009 & 29-Aug-2009 & SW 846/6020 & $\mathrm{U}$ \\
\hline Nickel & 45.9 & 40.0 & $\mathrm{mg} / \mathrm{kg}$ & 2 & 28-Aug-2009 & 29-Aug-2009 & SW $846 / 6020$ & \\
\hline Selenium & $\mathrm{ND}$ & 40.0 & $\mathrm{mg} / \mathrm{kg}$ & 2 & 28-Aug-2009 & 29-Aug-2009 & SW $846 / 6020$ & $\mathrm{U}$ \\
\hline Thallium & ND & 40.0 & $\mathrm{mg} / \mathrm{kg}$ & 2 & 28-Aug-2009 & 29-Aug-2009 & SW 846/6020 & $\mathrm{U}$ \\
\hline Vanadium & 18.2 & 40.0 & $\mathrm{mg} / \mathrm{kg}$ & 2 & 28-Aug-2009 & 29-Aug-2009 & SW 846/6020 & $\mathrm{J}$ \\
\hline Zinc & 1620 & 40.0 & $\mathrm{mg} / \mathrm{kg}$ & 2 & 28-Aug-2009 & 29-Aug-2009 & SW $846 / 6020$ & \\
\hline
\end{tabular}




\section{USACE ERDC-EP-C \\ 3909 Halls Ferry Road \\ Vicksburg, MS 39180-6199}

ERDC - ECB

Project: TVA Fly Ash Project

Reported:

Project Manager: Tony Bednar

03-Sep-2009

Fish Gut EMR - WA 5

9082706-25 (Tissue)

\begin{tabular}{|c|c|c|c|c|c|c|c|c|}
\hline Analyte & Result & porting & Units & Dilution & Prenared & Analyzed & Method & Notes \\
\hline Analyte & Kesult & Limit & & & Prepared & & Metnod & \\
\hline
\end{tabular}

ERDC- EL-EP-C (Environmental Chemistry Branch)

Metals by EPA 6000/7000 Series Methods

\begin{tabular}{|c|c|c|c|c|c|c|c|c|}
\hline Antimony & ND & 40.0 & $\mathrm{mg} / \mathrm{kg}$ & 2 & 28-Aug-2009 & 29-Aug-2009 & SW 846/6020 & $\mathrm{U}$ \\
\hline Arsenic & ND & 40.0 & $\mathrm{mg} / \mathrm{kg}$ & 2 & 28-Aug-2009 & 29-Aug-2009 & SW 846/6020 & $\mathrm{U}$ \\
\hline Barium & 351 & 40.0 & $\mathrm{mg} / \mathrm{kg}$ & 2 & 28-Aug-2009 & 29-Aug-2009 & SW 846/6020 & \\
\hline Beryllium & ND & 40.0 & $\mathrm{mg} / \mathrm{kg}$ & 2 & 28-Aug-2009 & 29-Aug-2009 & SW 846/6020 & $\mathrm{U}$ \\
\hline Cadmium & ND & 40.0 & $\mathrm{mg} / \mathrm{kg}$ & 2 & 28-Aug-2009 & 29-Aug-2009 & SW 846/6020 & $\mathrm{U}$ \\
\hline Chromium & 96.4 & 40.0 & $\mathrm{mg} / \mathrm{kg}$ & 2 & 28-Aug-2009 & 29-Aug-2009 & SW 846/6020 & \\
\hline Cobalt & ND & 40.0 & $\mathrm{mg} / \mathrm{kg}$ & 2 & 28-Aug-2009 & 29-Aug-2009 & SW 846/6020 & $\mathrm{U}$ \\
\hline Copper & 109 & 40.0 & $\mathrm{mg} / \mathrm{kg}$ & 2 & 28-Aug-2009 & 29-Aug-2009 & SW 846/6020 & \\
\hline Lead & 100 & 40.0 & $\mathrm{mg} / \mathrm{kg}$ & 2 & 28-Aug-2009 & 29-Aug-2009 & SW 846/6020 & B \\
\hline Manganese & 479 & 40.0 & $\mathrm{mg} / \mathrm{kg}$ & 2 & 28-Aug-2009 & 29-Aug-2009 & SW 846/6020 & \\
\hline Molybdenum & ND & 40.0 & $\mathrm{mg} / \mathrm{kg}$ & 2 & 28-Aug-2009 & 29-Aug-2009 & SW 846/6020 & $\mathrm{U}$ \\
\hline Nickel & 32.3 & 40.0 & $\mathrm{mg} / \mathrm{kg}$ & 2 & 28-Aug-2009 & 29-Aug-2009 & SW 846/6020 & $\mathrm{J}$ \\
\hline Selenium & ND & 40.0 & $\mathrm{mg} / \mathrm{kg}$ & 2 & 28-Aug-2009 & 29-Aug-2009 & SW 846/6020 & $\mathrm{U}$ \\
\hline Silver & ND & 40.0 & $\mathrm{mg} / \mathrm{kg}$ & 2 & 28-Aug-2009 & 29-Aug-2009 & SW 846/6020 & $\mathrm{U}$ \\
\hline Thallium & ND & 40.0 & $\mathrm{mg} / \mathrm{kg}$ & 2 & 28-Aug-2009 & 29-Aug-2009 & SW 846/6020 & $\mathrm{U}$ \\
\hline Vanadium & 14.4 & 40.0 & $\mathrm{mg} / \mathrm{kg}$ & 2 & 28-Aug-2009 & 29-Aug-2009 & SW 846/6020 & $\mathrm{J}$ \\
\hline Zinc & 1280 & 40.0 & $\mathrm{mg} / \mathrm{kg}$ & 2 & 28-Aug-2009 & 29-Aug-2009 & SW 846/6020 & \\
\hline
\end{tabular}




\section{USACE ERDC-EP-C \\ 3909 Halls Ferry Road \\ Vicksburg, MS 39180-6199}

ERDC - ECB

Project: TVA Fly Ash Project

Reported:

Project Manager: Tony Bednar

03-Sep-2009

Fish Gut EMR - EL 1

9082706-26 (Tissue)

\begin{tabular}{|c|c|c|c|c|c|c|c|c|}
\hline & & porting & & & & & & \\
\hline Analyte & Result & Limit & Units & Dilution & Prepared & Analyzed & Method & Notes \\
\hline
\end{tabular}

ERDC- EL-EP-C (Environmental Chemistry Branch)

Metals by EPA 6000/7000 Series Methods

\begin{tabular}{|c|c|c|c|c|c|c|c|c|}
\hline Antimony & ND & 40.0 & $\mathrm{mg} / \mathrm{kg}$ & 2 & 28-Aug-2009 & 29-Aug-2009 & SW 846/6020 & $\mathrm{U}$ \\
\hline Arsenic & 183 & 40.0 & $\mathrm{mg} / \mathrm{kg}$ & 2 & 28-Aug-2009 & 29-Aug-2009 & SW 846/6020 & \\
\hline Barium & 1280 & 40.0 & $\mathrm{mg} / \mathrm{kg}$ & 2 & 28-Aug-2009 & 29-Aug-2009 & SW 846/6020 & \\
\hline Beryllium & 10.9 & 40.0 & $\mathrm{mg} / \mathrm{kg}$ & 2 & 28-Aug-2009 & 29-Aug-2009 & SW 846/6020 & $\mathrm{J}$ \\
\hline Cadmium & ND & 40.0 & $\mathrm{mg} / \mathrm{kg}$ & 2 & 28-Aug-2009 & 29-Aug-2009 & SW 846/6020 & $\mathrm{U}$ \\
\hline Chromium & 138 & 40.0 & $\mathrm{mg} / \mathrm{kg}$ & 2 & 28-Aug-2009 & 29-Aug-2009 & SW 846/6020 & \\
\hline Cobalt & 55.1 & 40.0 & $\mathrm{mg} / \mathrm{kg}$ & 2 & 28-Aug-2009 & 29-Aug-2009 & SW 846/6020 & \\
\hline Copper & 242 & 40.0 & $\mathrm{mg} / \mathrm{kg}$ & 2 & 28-Aug-2009 & 29-Aug-2009 & SW 846/6020 & \\
\hline Lead & 114 & 40.0 & $\mathrm{mg} / \mathrm{kg}$ & 2 & 28-Aug-2009 & 29-Aug-2009 & SW 846/6020 & B \\
\hline Manganese & 317 & 40.0 & $\mathrm{mg} / \mathrm{kg}$ & 2 & 28-Aug-2009 & 29-Aug-2009 & SW 846/6020 & \\
\hline Molybdenum & 11.2 & 40.0 & $\mathrm{mg} / \mathrm{kg}$ & 2 & 28-Aug-2009 & 29-Aug-2009 & SW 846/6020 & $\mathrm{J}$ \\
\hline Nickel & 118 & 40.0 & $\mathrm{mg} / \mathrm{kg}$ & 2 & 28-Aug-2009 & 29-Aug-2009 & SW 846/6020 & \\
\hline Selenium & ND & 40.0 & $\mathrm{mg} / \mathrm{kg}$ & 2 & 28-Aug-2009 & 29-Aug-2009 & SW 846/6020 & $\mathrm{U}$ \\
\hline Silver & ND & 40.0 & $\mathrm{mg} / \mathrm{kg}$ & 2 & 28-Aug-2009 & 29-Aug-2009 & SW 846/6020 & $\mathrm{U}$ \\
\hline Thallium & ND & 40.0 & $\mathrm{mg} / \mathrm{kg}$ & 2 & 28-Aug-2009 & 29-Aug-2009 & SW 846/6020 & $\mathrm{U}$ \\
\hline Vanadium & 286 & 40.0 & $\mathrm{mg} / \mathrm{kg}$ & 2 & 28-Aug-2009 & 29-Aug-2009 & SW 846/6020 & \\
\hline Zinc & 382 & 40.0 & $\mathrm{mg} / \mathrm{kg}$ & 2 & 28-Aug-2009 & 29-Aug-2009 & SW 846/6020 & \\
\hline
\end{tabular}




\section{USACE ERDC-EP-C \\ 3909 Halls Ferry Road \\ Vicksburg, MS 39180-6199}

ERDC - ECB

Project: TVA Fly Ash Project

Reported:

Project Manager: Tony Bednar

03-Sep-2009

Fish Gut EMR - EL 2

9082706-27 (Tissue)

\begin{tabular}{|c|c|c|c|c|c|c|c|c|}
\hline & & porting & & & & & & \\
\hline Analyte & Result & Limit & Units & Dilution & Prepared & Analyzed & Method & Notes \\
\hline
\end{tabular}

ERDC- EL-EP-C (Environmental Chemistry Branch)

Metals by EPA 6000/7000 Series Methods

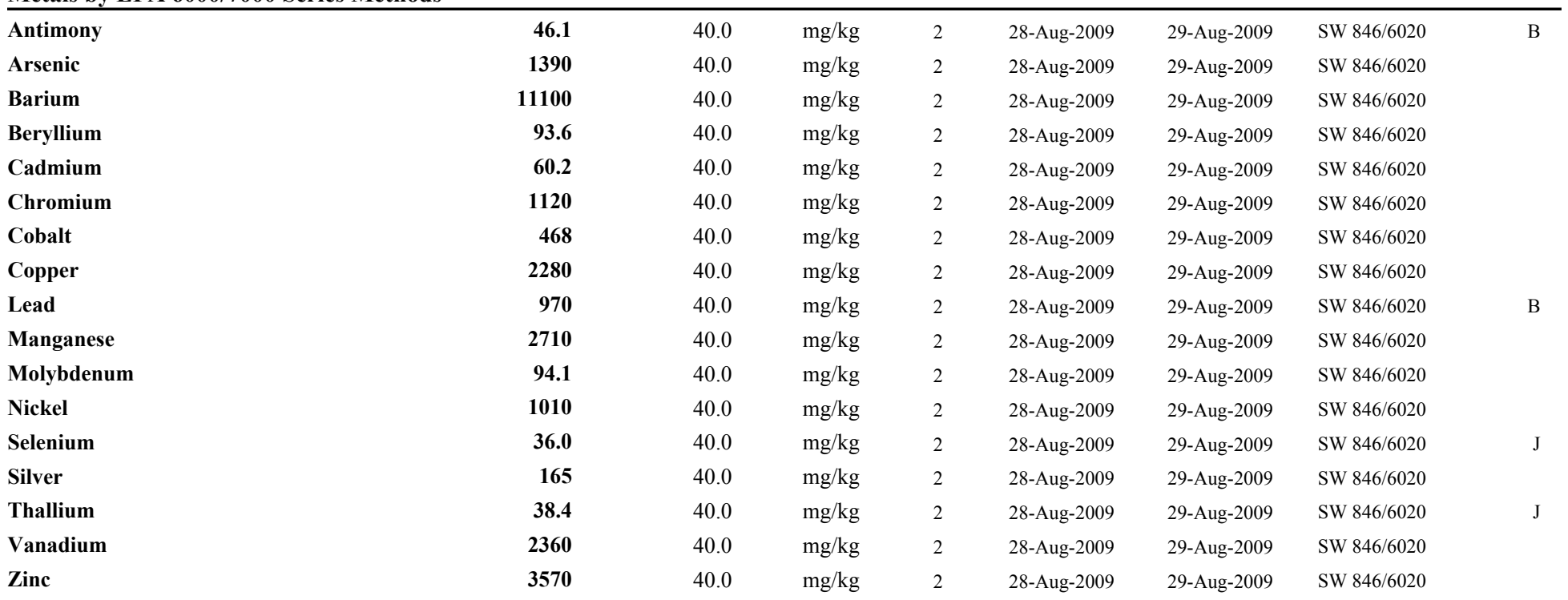




\section{USACE ERDC-EP-C \\ 3909 Halls Ferry Road \\ Vicksburg, MS 39180-6199}

ERDC - ECB

Project: TVA Fly Ash Project

Reported:

Project Manager: Tony Bednar

03-Sep-2009

Fish Gut EMR - EL 3

9082706-28 (Tissue)

\begin{tabular}{|c|c|c|c|c|c|c|c|c|}
\hline Analyte & Result & porting & Units & Dilution & Prenared & Analyzed & Method & Notes \\
\hline Analyte & Kesult & Limit & & & Prepared & & Metnod & \\
\hline
\end{tabular}

ERDC- EL-EP-C (Environmental Chemistry Branch)

Metals by EPA 6000/7000 Series Methods

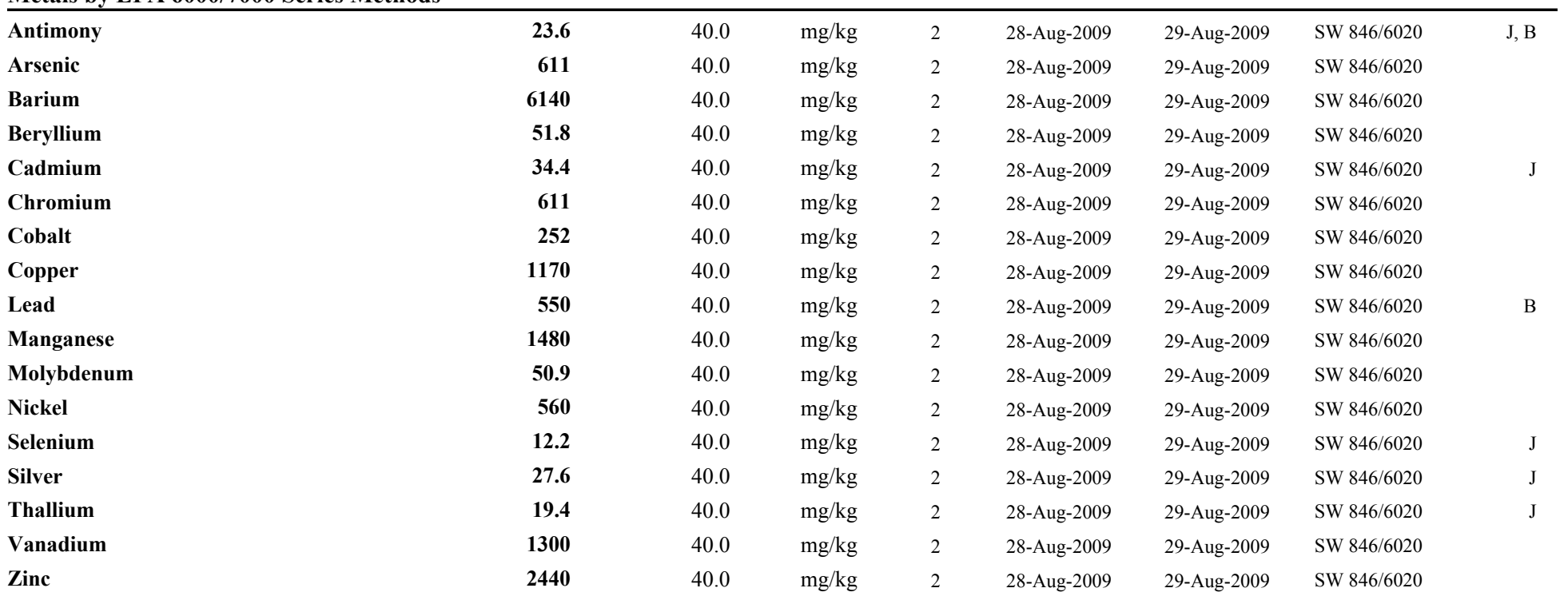




\section{USACE ERDC-EP-C \\ 3909 Halls Ferry Road \\ Vicksburg, MS 39180-6199}

ERDC - ECB

Project: TVA Fly Ash Project

Reported:

Project Manager: Tony Bednar

03-Sep-2009

Fish Gut EMR - EL 4

9082706-29 (Tissue)

\begin{tabular}{|c|c|c|c|c|c|c|c|c|}
\hline & & porting & & & & & & \\
\hline Analyte & Result & Limit & Units & Dilution & Prepared & Analyzed & Method & Notes \\
\hline
\end{tabular}

ERDC- EL-EP-C (Environmental Chemistry Branch)

Metals by EPA 6000/7000 Series Methods

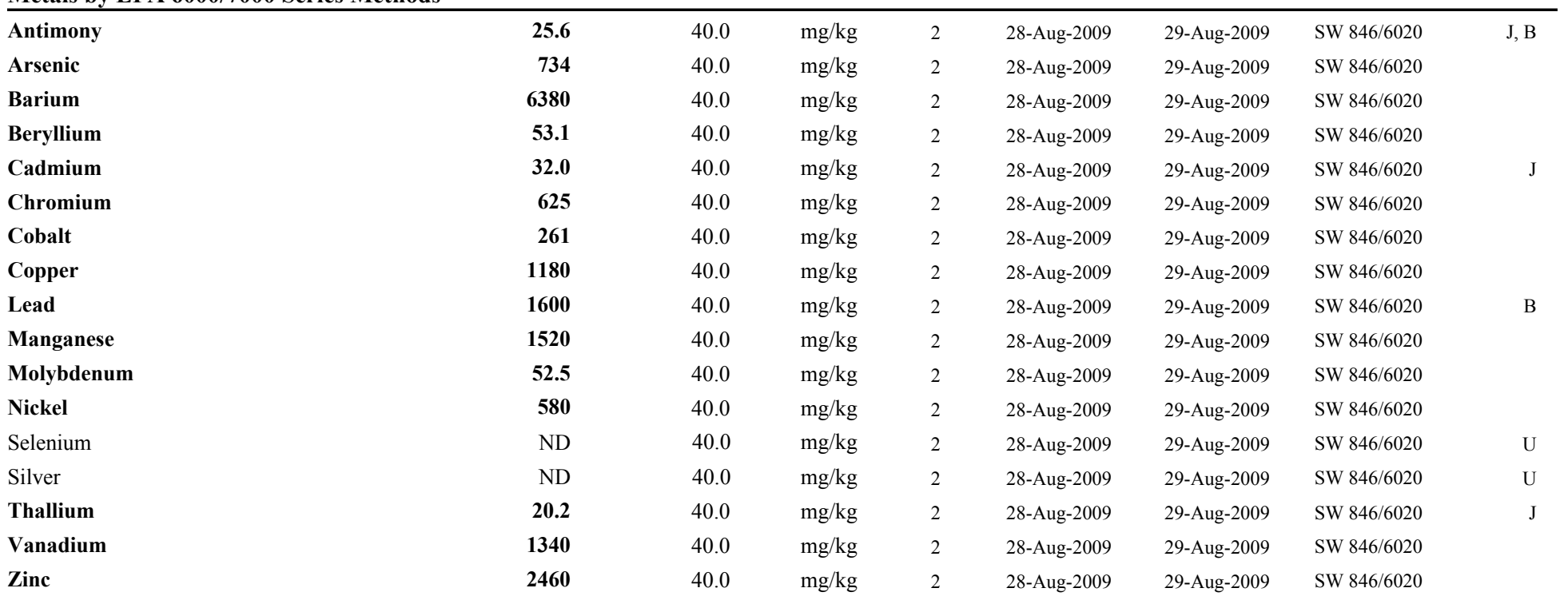




\section{USACE ERDC-EP-C \\ 3909 Halls Ferry Road \\ Vicksburg, MS 39180-6199}

ERDC - ECB

Project: TVA Fly Ash Project

Reported:

Project Manager: Tony Bednar

03-Sep-2009

Fish Gut EMR - EL 5

9082706-30 (Tissue)

\begin{tabular}{|c|c|c|c|c|c|c|c|c|}
\hline & & porting & & & & & & \\
\hline Analyte & Result & Limit & Units & Dilution & Prepared & Analyzed & Method & Notes \\
\hline
\end{tabular}

ERDC- EL-EP-C (Environmental Chemistry Branch)

Metals by EPA 6000/7000 Series Methods

\begin{tabular}{|c|c|c|c|c|c|c|c|c|}
\hline Antimony & 15.3 & 40.0 & $\mathrm{mg} / \mathrm{kg}$ & 2 & 28-Aug-2009 & 29-Aug-2009 & SW 846/6020 & $\mathrm{J}, \mathrm{B}$ \\
\hline Arsenic & 127 & 40.0 & $\mathrm{mg} / \mathrm{kg}$ & 2 & 28-Aug-2009 & 29-Aug-2009 & SW 846/6020 & \\
\hline Barium & 1190 & 40.0 & $\mathrm{mg} / \mathrm{kg}$ & 2 & 28-Aug-2009 & 29-Aug-2009 & SW 846/6020 & \\
\hline Beryllium & 10.7 & 40.0 & $\mathrm{mg} / \mathrm{kg}$ & 2 & 28-Aug-2009 & 29-Aug-2009 & SW 846/6020 & $\mathrm{J}$ \\
\hline Cadmium & ND & 40.0 & $\mathrm{mg} / \mathrm{kg}$ & 2 & 28-Aug-2009 & 29-Aug-2009 & SW 846/6020 & $\mathrm{U}$ \\
\hline Chromium & 116 & 40.0 & $\mathrm{mg} / \mathrm{kg}$ & 2 & 28-Aug-2009 & 29-Aug-2009 & SW 846/6020 & \\
\hline Cobalt & 49.2 & 40.0 & $\mathrm{mg} / \mathrm{kg}$ & 2 & 28-Aug-2009 & 29-Aug-2009 & SW 846/6020 & \\
\hline Copper & 217 & 40.0 & $\mathrm{mg} / \mathrm{kg}$ & 2 & 28-Aug-2009 & 29-Aug-2009 & SW 846/6020 & \\
\hline Lead & 107 & 40.0 & $\mathrm{mg} / \mathrm{kg}$ & 2 & 28-Aug-2009 & 29-Aug-2009 & SW 846/6020 & B \\
\hline Manganese & 281 & 40.0 & $\mathrm{mg} / \mathrm{kg}$ & 2 & 28-Aug-2009 & 29-Aug-2009 & SW 846/6020 & \\
\hline Molybdenum & 10.1 & 40.0 & $\mathrm{mg} / \mathrm{kg}$ & 2 & 28-Aug-2009 & 29-Aug-2009 & SW $846 / 6020$ & $\mathrm{~J}$ \\
\hline Nickel & 107 & 40.0 & $\mathrm{mg} / \mathrm{kg}$ & 2 & 28-Aug-2009 & 29-Aug-2009 & SW 846/6020 & \\
\hline Selenium & $\mathrm{ND}$ & 40.0 & $\mathrm{mg} / \mathrm{kg}$ & 2 & 28-Aug-2009 & 29-Aug-2009 & SW 846/6020 & $\mathrm{U}$ \\
\hline Silver & ND & 40.0 & $\mathrm{mg} / \mathrm{kg}$ & 2 & 28-Aug-2009 & 29-Aug-2009 & SW $846 / 6020$ & $\mathrm{U}$ \\
\hline Thallium & ND & 40.0 & $\mathrm{mg} / \mathrm{kg}$ & 2 & 28-Aug-2009 & 29-Aug-2009 & SW 846/6020 & $\mathrm{U}$ \\
\hline Vanadium & 243 & 40.0 & $\mathrm{mg} / \mathrm{kg}$ & 2 & 28-Aug-2009 & 29-Aug-2009 & SW 846/6020 & \\
\hline Zinc & 474 & 40.0 & $\mathrm{mg} / \mathrm{kg}$ & 2 & 28-Aug-2009 & 29-Aug-2009 & SW 846/6020 & \\
\hline
\end{tabular}




\section{USACE ERDC-EP-C \\ 3909 Halls Ferry Road \\ Vicksburg, MS 39180-6199}

ERDC - ECB

$-\cdot,-$
Project: TVA Fly Ash Project

Reported:

Project Manager: Tony Bednar
03-Sep-2009

Metals by EPA 6000/7000 Series Methods - Quality Control

ERDC- EL-EP-C (Environmental Chemistry Branch)

\begin{tabular}{|c|c|c|c|c|c|c|c|c|c|c|}
\hline & & eporting & & Spike & Source & & $\%$ REC & & RPD & \\
\hline Analyte & Result & Limit & Units & Level & Result & $\%$ REC & Limits & RPD & Limit & Notes \\
\hline
\end{tabular}

Batch B908051 - EPA 3050B

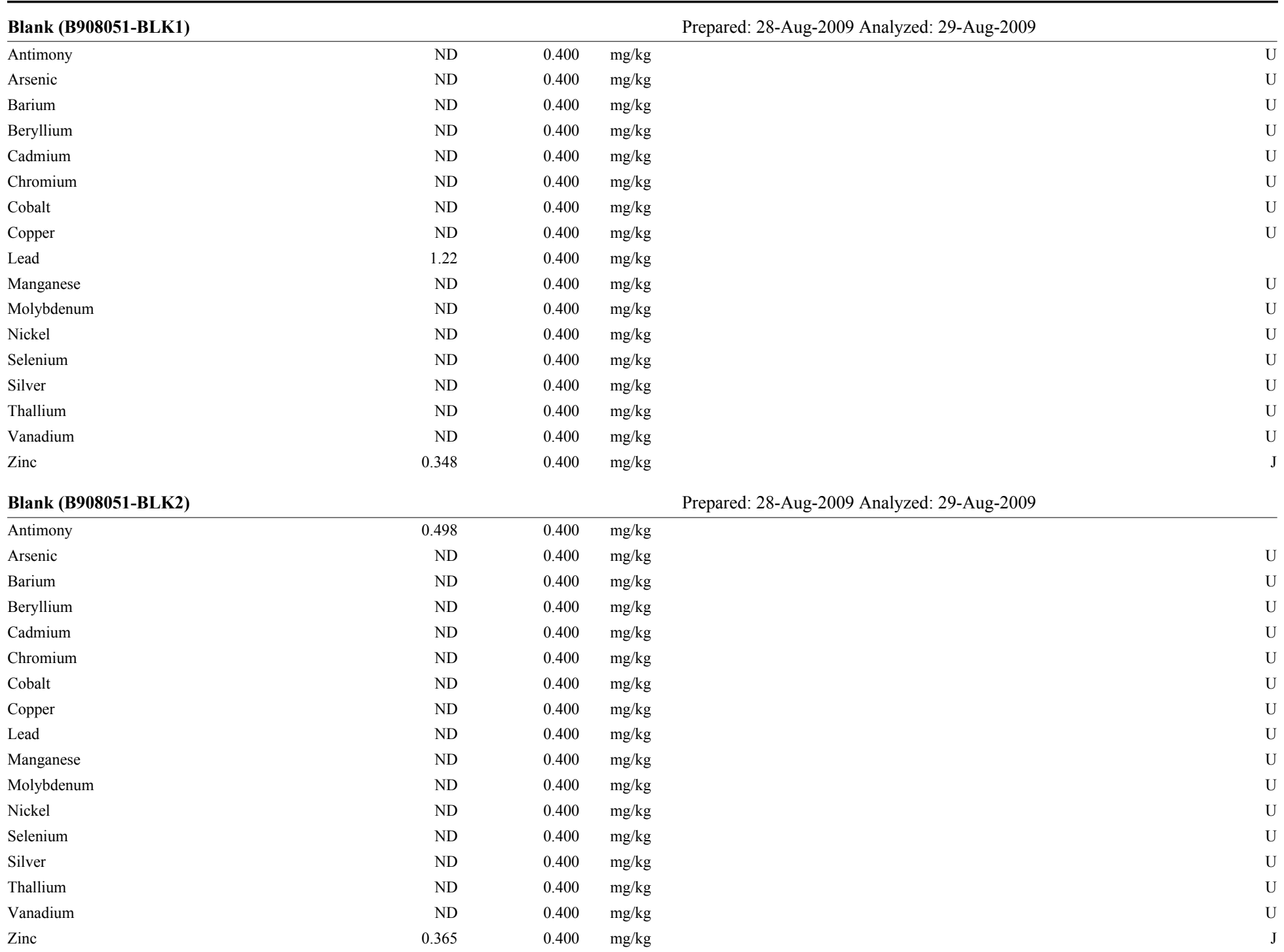




\section{USACE ERDC-EP-C \\ 3909 Halls Ferry Road \\ Vicksburg, MS 39180-6199}

ERDC - ECB

$-\cdot,-$
Project: TVA Fly Ash Project

Project Manager: Tony Bednar
Reported:

03-Sep-2009

Metals by EPA 6000/7000 Series Methods - Quality Control

\section{ERDC- EL-EP-C (Environmental Chemistry Branch)}

\begin{tabular}{|c|c|c|c|c|c|c|c|c|c|c|}
\hline & & porting & & Spike & Source & & $\%$ REC & & RPD & \\
\hline Analyte & Result & Limit & Units & Level & Result & $\%$ REC & Limits & RPD & Limit & Notes \\
\hline
\end{tabular}

\section{Batch B908051 - EPA 3050B}

\section{LCS (B908051-BS1)}

Antimony

Arsenic

Barium

Beryllium

Cadmium

Chromium

Cobalt

Copper

Lead

Manganese

Molybdenum

Nickel

Selenium

Silver

Thallium

Vanadium

Zinc
Prepared: 28-Aug-2009 Analyzed: 29-Aug-2009

$\begin{array}{lllll}0.400 & \mathrm{mg} / \mathrm{kg} & 100.0 & 93.0 & 80-120 \\ 0.400 & \mathrm{mg} / \mathrm{kg} & 50.00 & 92.6 & 80-120 \\ 0.400 & \mathrm{mg} / \mathrm{kg} & 200.0 & 97.1 & 80-120 \\ 0.400 & \mathrm{mg} / \mathrm{kg} & 50.00 & 96.4 & 80-120 \\ 0.400 & \mathrm{mg} / \mathrm{kg} & 50.00 & 93.1 & 80-120 \\ 0.400 & \mathrm{mg} / \mathrm{kg} & 100.0 & 100 & 80-120 \\ 0.400 & \mathrm{mg} / \mathrm{kg} & 100.0 & 102 & 80-120 \\ 0.400 & \mathrm{mg} / \mathrm{kg} & 100.0 & 97.4 & 80-120 \\ 0.400 & \mathrm{mg} / \mathrm{kg} & 100.0 & 99.3 & 80-120 \\ 0.400 & \mathrm{mg} / \mathrm{kg} & 250.0 & 93.2 & 80-120 \\ 0.400 & \mathrm{mg} / \mathrm{kg} & 50.00 & 95.9 & 80-120 \\ 0.400 & \mathrm{mg} / \mathrm{kg} & 100.0 & 100 & 80-120 \\ 0.400 & \mathrm{mg} / \mathrm{kg} & 50.00 & 85.6 & 80-120 \\ 0.400 & \mathrm{mg} / \mathrm{kg} & 50.00 & 93.7 & 80-120 \\ 0.400 & \mathrm{mg} / \mathrm{kg} & 50.00 & 95.6 & 80-120 \\ 0.400 & \mathrm{mg} / \mathrm{kg} & 100.0 & 99.7 & 80-120 \\ 0.400 & \mathrm{mg} / \mathrm{kg} & 200.0 & 88.2 & 80-120\end{array}$

Prepared: 28-Aug-2009 Analyzed: 29-Aug-2009

$\begin{array}{llllll}0.400 & \mathrm{mg} / \mathrm{kg} & 100.0 & 95.7 & 80-120 & \text { B } \\ 0.400 & \mathrm{mg} / \mathrm{kg} & 50.00 & 93.6 & 80-120 & 98.3 \\ 0.400 & \mathrm{mg} / \mathrm{kg} & 200.0 & 94-120 & 80-120 \\ 0.400 & \mathrm{mg} / \mathrm{kg} & 50.00 & 92.8 & 80-120 \\ 0.400 & \mathrm{mg} / \mathrm{kg} & 50.00 & 101 & 80-120 \\ 0.400 & \mathrm{mg} / \mathrm{kg} & 100.0 & 100 & 80-120 \\ 0.400 & \mathrm{mg} / \mathrm{kg} & 100.0 & 96.5 & 80-120 \\ 0.400 & \mathrm{mg} / \mathrm{kg} & 100.0 & 97.5 & 80-120 \\ 0.400 & \mathrm{mg} / \mathrm{kg} & 100.0 & 94.2 & 80-120 \\ 0.400 & \mathrm{mg} / \mathrm{kg} & 250.0 & 97.1 & 80-120 \\ 0.400 & \mathrm{mg} / \mathrm{kg} & 50.00 & 97.8 & 80-120 \\ 0.400 & \mathrm{mg} / \mathrm{kg} & 100.0 & 86.1 & 80-120 \\ 0.400 & \mathrm{mg} / \mathrm{kg} & 50.00 & 93.4 & 80-120 \\ 0.400 & \mathrm{mg} / \mathrm{kg} & 50.00 & 96.7 & 80-120 \\ 0.400 & \mathrm{mg} / \mathrm{kg} & 50.00 & 99.5 & 80-120 \\ 0.400 & \mathrm{mg} / \mathrm{kg} & 100.0 & 88.2 & 80-120 \\ 0.400 & \mathrm{mg} / \mathrm{kg} & 200.0 & & \end{array}$

B

LCS (B908051-BS2)

Antimony

Arsenic

Barium

Beryllium

Cadmium

Chromium

Cobalt

Copper

Lead

Manganese

Molybdenum

Nickel

Selenium

Silver

Thallium

Vanadium

Zinc

$\begin{array}{rll}93.0 & 0.400 & \mathrm{mg} / \mathrm{k} \\ 46.3 & 0.400 & \mathrm{mg} / \mathrm{k} \\ 194 & 0.400 & \mathrm{mg} / \mathrm{k} \\ 48.2 & 0.400 & \mathrm{mg} / \mathrm{k} \\ 46.6 & 0.400 & \mathrm{mg} / \mathrm{k} \\ 100 & 0.400 & \mathrm{mg} / \mathrm{k} \\ 102 & 0.400 & \mathrm{mg} / \mathrm{k} \\ 97.4 & 0.400 & \mathrm{mg} / \mathrm{k} \\ 99.3 & 0.400 & \mathrm{mg} / \mathrm{k} \\ 233 & 0.400 & \mathrm{mg} / \mathrm{k} \\ 48.0 & 0.400 & \mathrm{mg} / \mathrm{k} \\ 100 & 0.400 & \mathrm{mg} / \mathrm{k} \\ 42.8 & 0.400 & \mathrm{mg} / \mathrm{k} \\ 46.9 & 0.400 & \mathrm{mg} / \mathrm{k} \\ 47.8 & 0.400 & \mathrm{mg} / \mathrm{k} \\ 99.7 & 0.400 & \mathrm{mg} / \mathrm{k} \\ 176 & 0.400 & \mathrm{mg} / \mathrm{k}\end{array}$

95.7

46.8

197

47.3

46.4

101

100

96.5

97.5

235

48.6

97.8

43.1

46.7

48.3

99.5

176

.7
.8
.3
.4
101
100
.5
35
.6
.8
.1
176




\section{USACE ERDC-EP-C \\ 3909 Halls Ferry Road \\ Vicksburg, MS 39180-6199}

ERDC - ECB
Project: TVA Fly Ash Project

Reported:

Project Manager: Tony Bednar
03-Sep-2009

Metals by EPA 6000/7000 Series Methods - Quality Control

\section{ERDC- EL-EP-C (Environmental Chemistry Branch)}

\begin{tabular}{|lcccccccccccc}
\hline & & Reporting & & Spike & Source & & & \%REC & & RPD \\
Analyte & Result & Limit & Units & Level & Result & \%REC & Limits & RPD & Limit & Notes & \\
\hline
\end{tabular}

\section{Batch B908051 - EPA 3050B}

\begin{tabular}{|c|c|c|c|c|c|c|c|c|c|c|}
\hline \multirow{2}{*}{$\begin{array}{l}\text { Duplicate (B908051-DUP1) } \\
\text { Antimony }\end{array}$} & \multicolumn{3}{|c|}{ Source: $9082706-03$} & \multicolumn{7}{|c|}{ Prepared: 28-Aug-2009 Analyzed: 29-Aug-2009 } \\
\hline & 0.721 & 0.400 & $\mathrm{mg} / \mathrm{kg}$ & & ND & & & & 20 & $\bar{B}$ \\
\hline Arsenic & 0.113 & 0.400 & $\mathrm{mg} / \mathrm{kg}$ & & ND & & & & 20 & $\mathrm{~J}$ \\
\hline Beryllium & ND & 0.400 & $\mathrm{mg} / \mathrm{kg}$ & & ND & & & & 20 & $\mathrm{U}$ \\
\hline Cadmium & ND & 0.400 & $\mathrm{mg} / \mathrm{kg}$ & & ND & & & & 20 & $\mathrm{U}$ \\
\hline Chromium & 2.01 & 0.400 & $\mathrm{mg} / \mathrm{kg}$ & & 1.79 & & & 11.6 & 20 & \\
\hline Cobalt & ND & 0.400 & $\mathrm{mg} / \mathrm{kg}$ & & ND & & & & 20 & $\mathrm{U}$ \\
\hline Copper & 0.878 & 0.400 & $\mathrm{mg} / \mathrm{kg}$ & & 0.663 & & & 27.9 & 20 & \\
\hline Lead & 0.397 & 0.400 & $\mathrm{mg} / \mathrm{kg}$ & & 0.588 & & & 38.9 & 20 & $\mathrm{~J}, \mathrm{~B}$ \\
\hline Manganese & 0.970 & 0.400 & $\mathrm{mg} / \mathrm{kg}$ & & 0.742 & & & 26.6 & 20 & \\
\hline Molybdenum & 0.096 & 0.400 & $\mathrm{mg} / \mathrm{kg}$ & & ND & & & & 20 & $\mathrm{~J}$ \\
\hline Nickel & 1.51 & 0.400 & $\mathrm{mg} / \mathrm{kg}$ & & 1.20 & & & 22.9 & 20 & \\
\hline Selenium & 0.182 & 0.400 & $\mathrm{mg} / \mathrm{kg}$ & & 0.087 & & & 70.3 & 20 & $\mathrm{~J}$ \\
\hline Thallium & ND & 0.400 & $\mathrm{mg} / \mathrm{kg}$ & & ND & & & & 20 & $\mathrm{U}$ \\
\hline Vanadium & 0.081 & 0.400 & $\mathrm{mg} / \mathrm{kg}$ & & ND & & & & 20 & $\mathrm{~J}$ \\
\hline Zinc & 31.5 & 0.400 & $\mathrm{mg} / \mathrm{kg}$ & & 22.5 & & & 33.1 & 20 & \\
\hline Matrix Spike (B908051-MS1) & \multicolumn{3}{|c|}{ Source: $9082706-03$} & \multicolumn{7}{|c|}{ Prepared: 28-Aug-2009 Analyzed: 29-Aug-2009 } \\
\hline Antimony & 172 & 0.400 & $\mathrm{mg} / \mathrm{kg}$ & 170.2 & ND & 101 & $80-120$ & & & B \\
\hline Arsenic & 87.7 & 0.400 & $\mathrm{mg} / \mathrm{kg}$ & 85.09 & ND & 103 & $80-120$ & & & \\
\hline Barium & 360 & 0.400 & $\mathrm{mg} / \mathrm{kg}$ & 340.4 & 1.84 & 105 & $80-120$ & & & \\
\hline Beryllium & 85.0 & 0.400 & $\mathrm{mg} / \mathrm{kg}$ & 85.09 & ND & 99.9 & $80-120$ & & & \\
\hline Cadmium & 84.2 & 0.400 & $\mathrm{mg} / \mathrm{kg}$ & 85.09 & ND & 98.9 & $80-120$ & & & \\
\hline Chromium & 189 & 0.400 & $\mathrm{mg} / \mathrm{kg}$ & 170.2 & 1.79 & 110 & $80-120$ & & & \\
\hline Cobalt & 184 & 0.400 & $\mathrm{mg} / \mathrm{kg}$ & 170.2 & ND & 108 & $80-120$ & & & \\
\hline Copper & 180 & 0.400 & $\mathrm{mg} / \mathrm{kg}$ & 170.2 & 0.663 & 105 & $80-120$ & & & \\
\hline Molybdenum & 90.5 & 0.400 & $\mathrm{mg} / \mathrm{kg}$ & 85.09 & ND & 106 & $80-120$ & & & \\
\hline Nickel & 181 & 0.400 & $\mathrm{mg} / \mathrm{kg}$ & 170.2 & 1.20 & 106 & $80-120$ & & & \\
\hline Selenium & 79.7 & 0.400 & $\mathrm{mg} / \mathrm{kg}$ & 85.09 & 0.087 & 93.6 & $80-120$ & & & \\
\hline Silver & 84.7 & 0.400 & $\mathrm{mg} / \mathrm{kg}$ & 85.09 & ND & 99.6 & $80-120$ & & & \\
\hline Thallium & 87.5 & 0.400 & $\mathrm{mg} / \mathrm{kg}$ & 85.09 & ND & 103 & $80-120$ & & & \\
\hline Vanadium & 184 & 0.400 & $\mathrm{mg} / \mathrm{kg}$ & 170.2 & ND & 108 & $80-120$ & & & \\
\hline Zinc & 368 & 0.400 & $\mathrm{mg} / \mathrm{kg}$ & 340.4 & 22.5 & 102 & $80-120$ & & & \\
\hline
\end{tabular}




\section{USACE ERDC-EP-C \\ 3909 Halls Ferry Road \\ Vicksburg, MS 39180-6199}

ERDC - ECB

Project: TVA Fly Ash Project

Reported:

Project Manager: Tony Bednar

03-Sep-2009

Metals by EPA 6000/7000 Series Methods - Quality Control

ERDC- EL-EP-C (Environmental Chemistry Branch)

\begin{tabular}{|c|c|c|c|c|c|c|c|c|c|c|}
\hline & & eporting & & Spike & Source & & $\%$ REC & & RPD & \\
\hline Analyte & Result & Limit & Units & Level & Result & $\%$ REC & Limits & RPD & Limit & Notes \\
\hline
\end{tabular}

Batch B909005 - EPA 7471A Prep

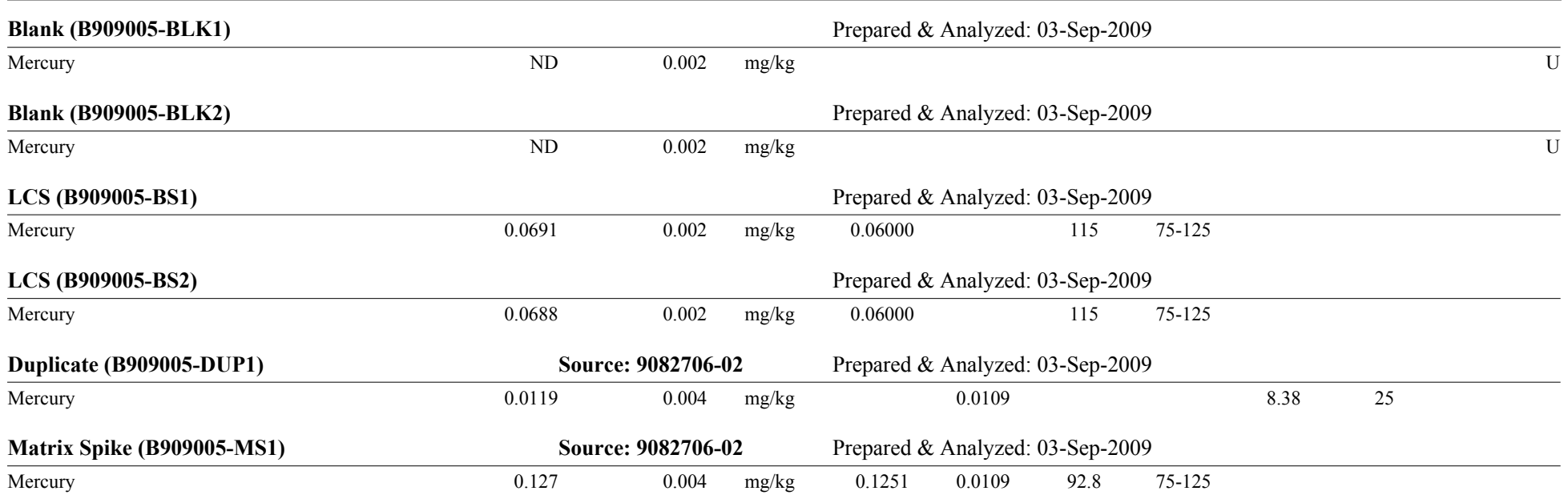




\section{USACE ERDC-EP-C \\ 3909 Halls Ferry Road \\ Vicksburg, MS 39180-6199}

ERDC - ECB

Project: TVA Fly Ash Project

Reported:

Project Manager: Tony Bednar

03-Sep-2009

\section{Notes and Definitions}

U Analyte included in the analysis, but not detected

J Detected but below the Reporting Limit; therefore, result is an estimated concentration (CLP J-Flag).

B Analyte is found in the associated blank as well as in the sample (CLP B-flag).

DET Analyte DETECTED

ND Analyte NOT DETECTED at or above the reporting limit

NR Not Reported

dry Sample results reported on a dry weight basis

RPD Relative Percent Difference 


\section{Appendix E: TOC data, Grain Size Analysis, and Speciation Data Sheets}




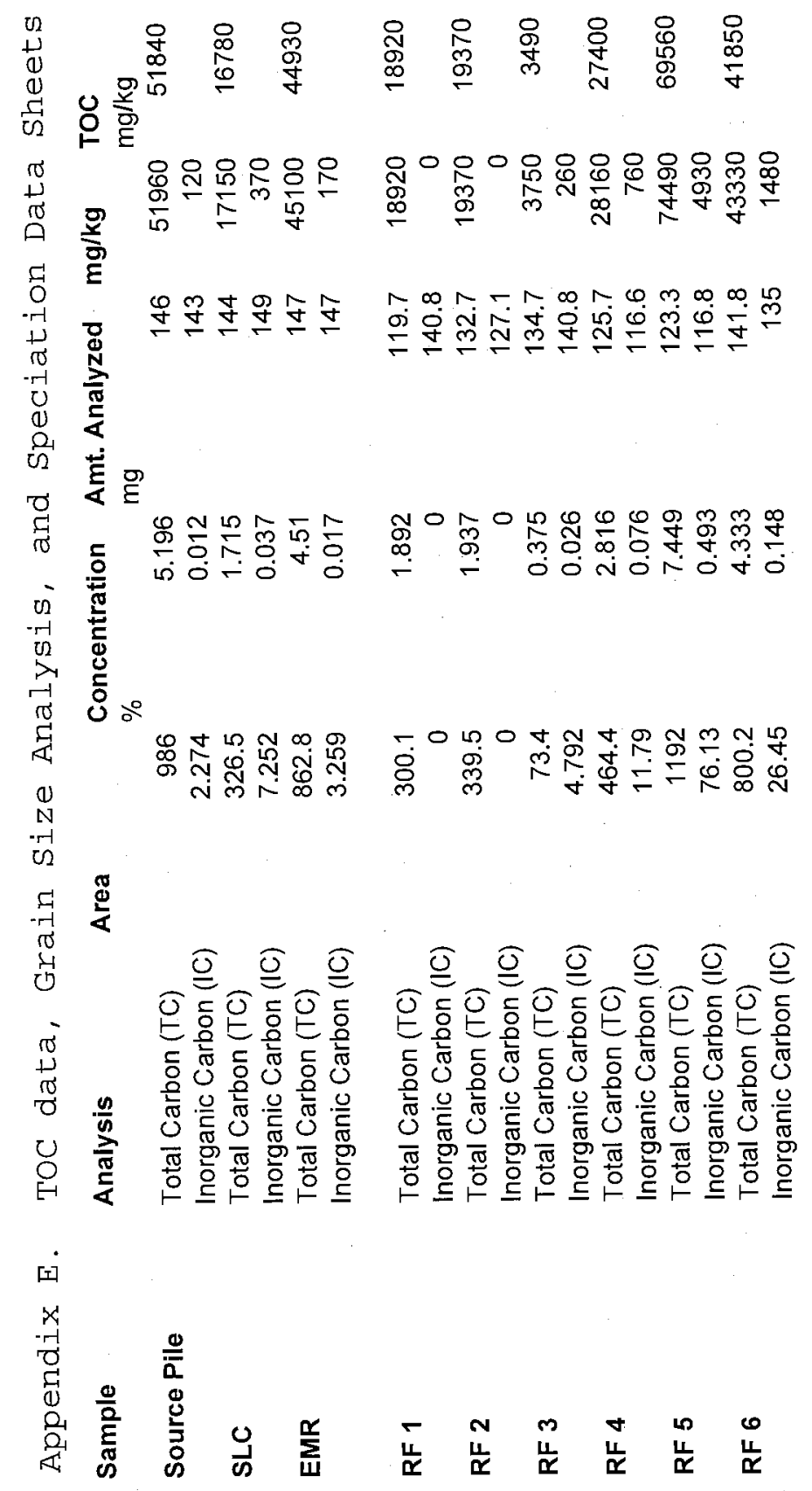




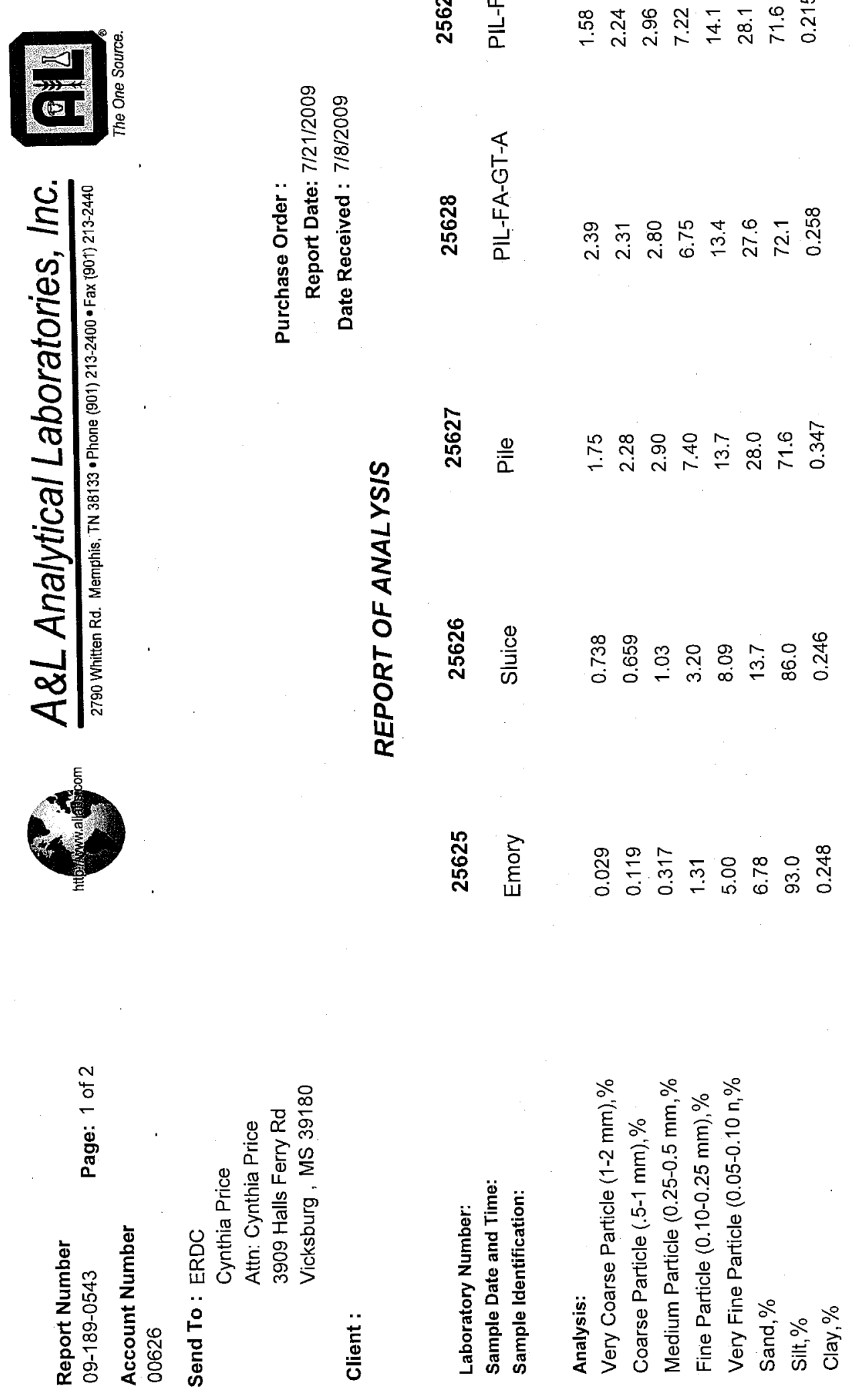




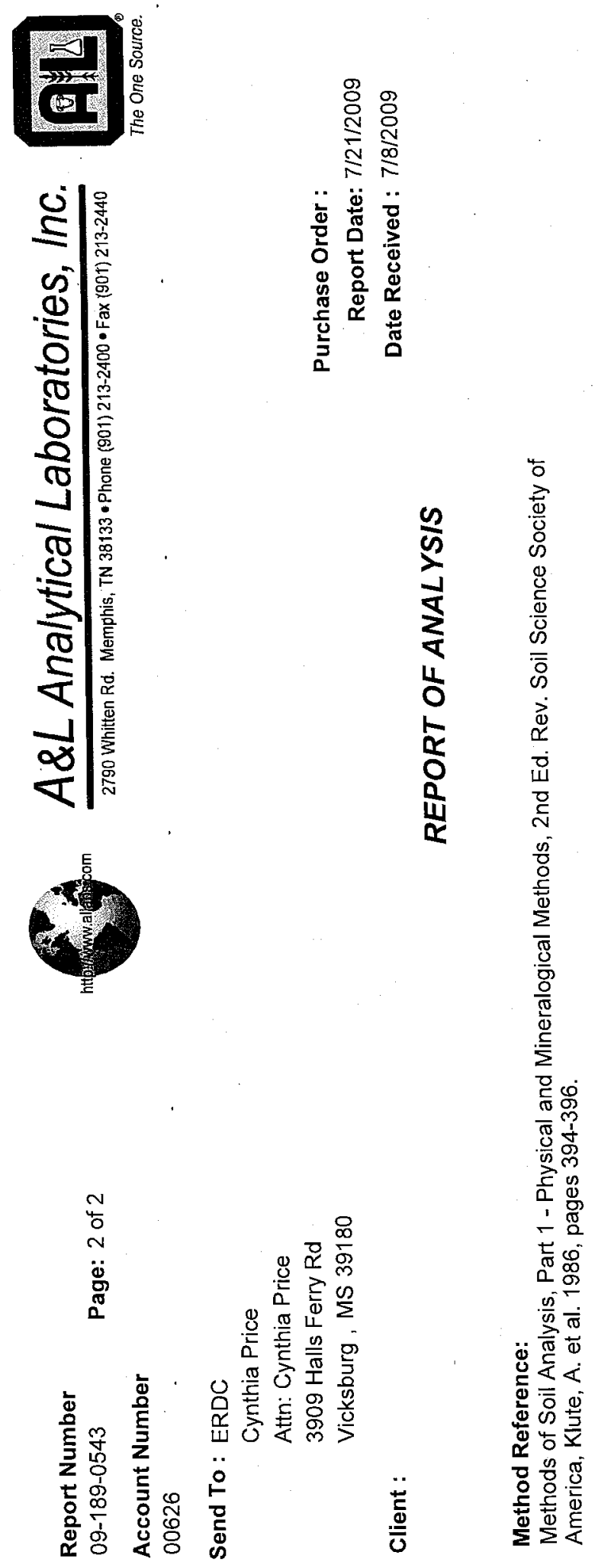




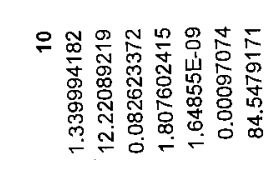

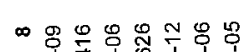

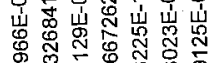

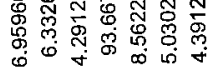

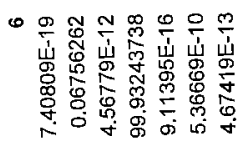

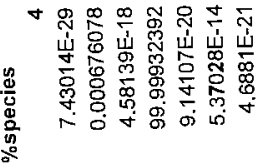

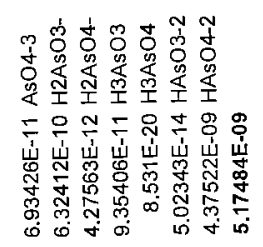

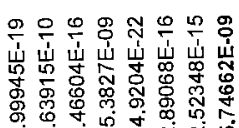

ำ ำํํำำำ

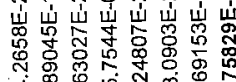

+ $\begin{gathered}\infty \\ \text { 过 }\end{gathered}$

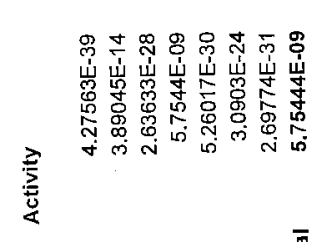

홈

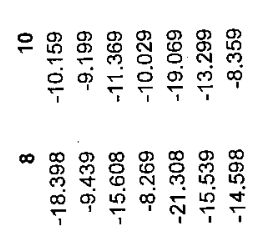
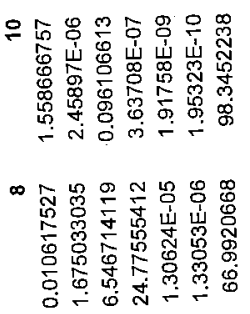

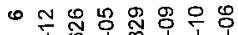

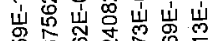

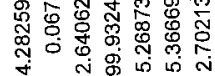

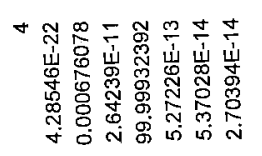

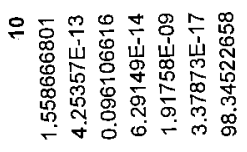

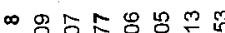

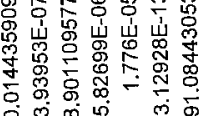

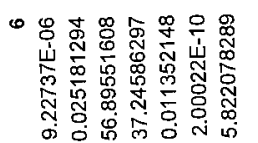

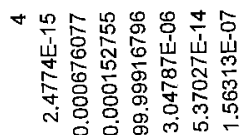

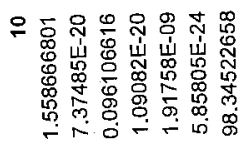

$\infty$ 훈

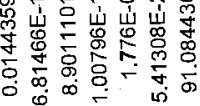

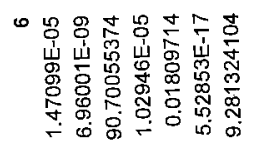

용 녕 要

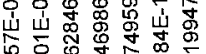

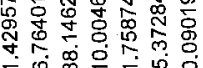
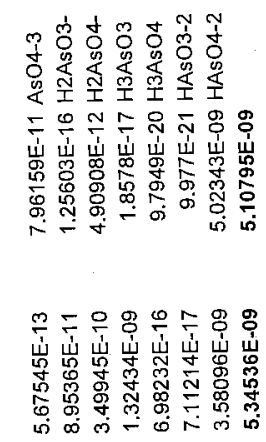

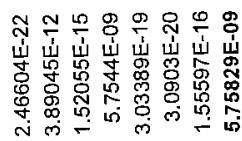

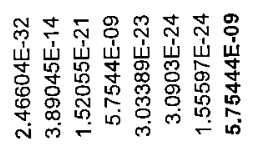

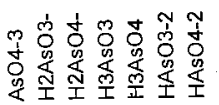

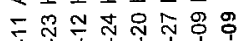

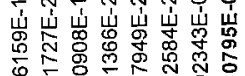

过

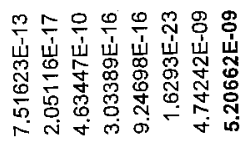

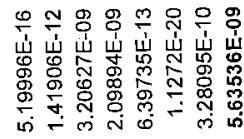

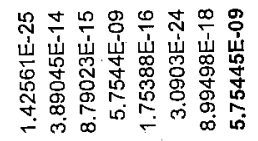

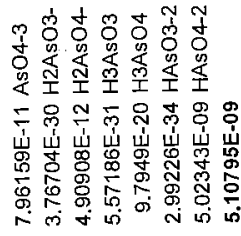

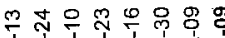

崩岕岕岕岩岩嵌

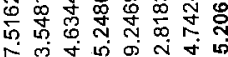

음용은ㅊㅇㅇㅇㅇ

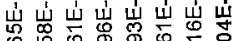

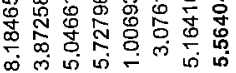

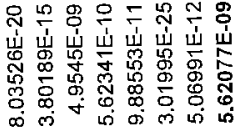

퐁

ฐ্

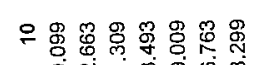

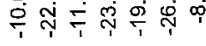

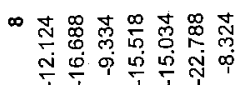

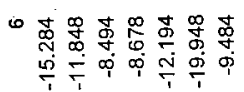

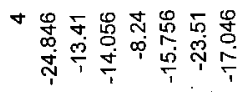

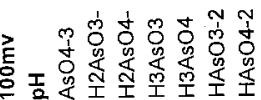

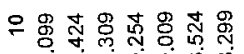

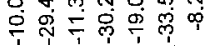

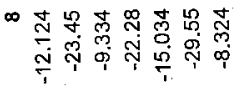

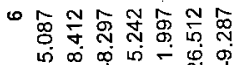
फ़

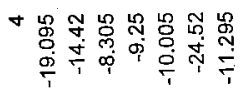




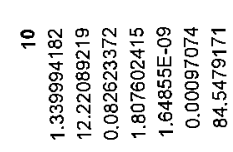

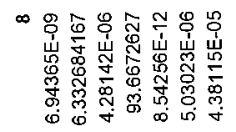

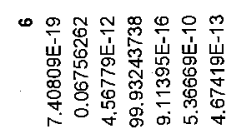

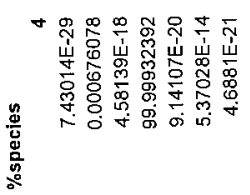
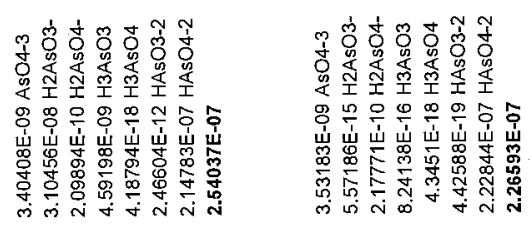

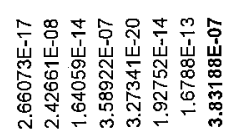

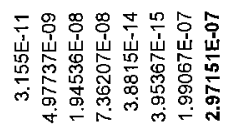

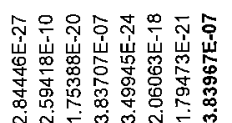

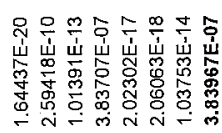

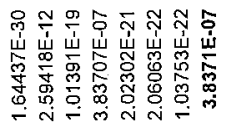

密

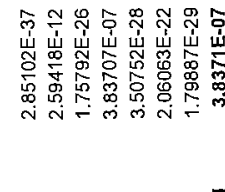

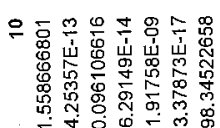

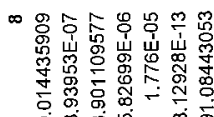

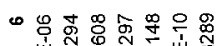

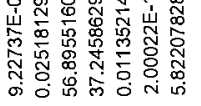

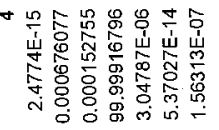

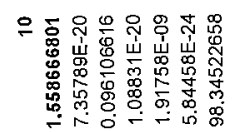

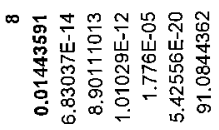

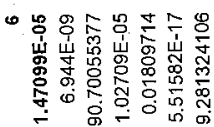

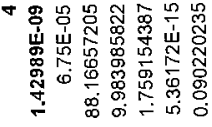

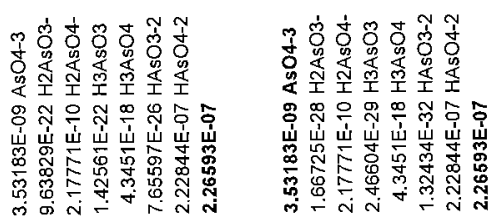

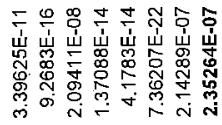

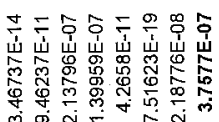

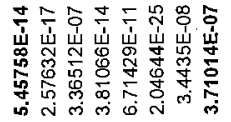

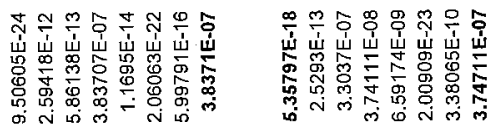

ฐ্

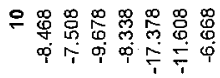

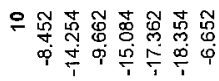

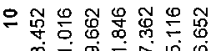

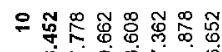

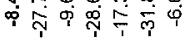

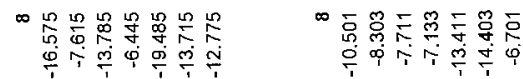

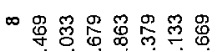

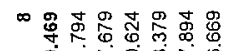

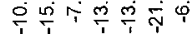

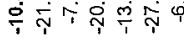

••

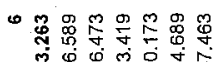

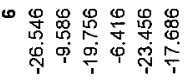

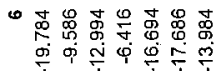

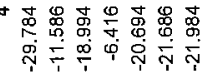

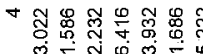

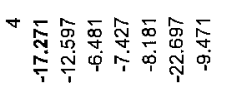

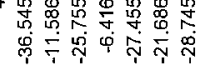
ํํำ ํㅜㄴ 


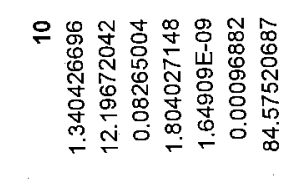

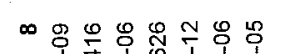

岁志岕造出岕岕

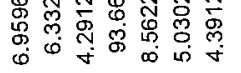

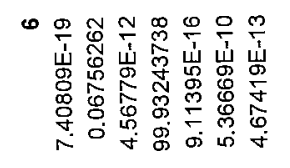

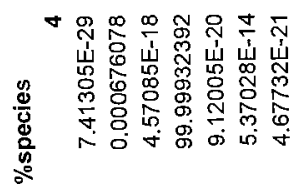

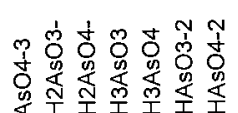

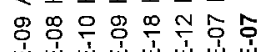

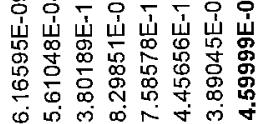

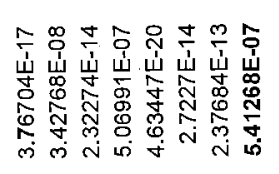

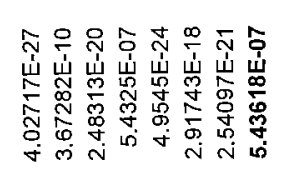

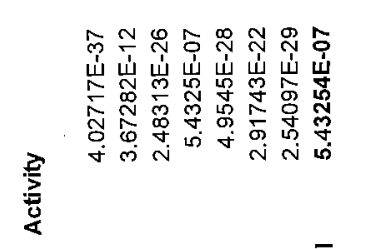

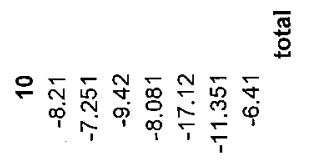

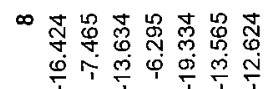

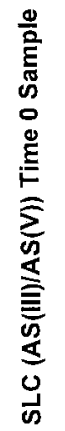

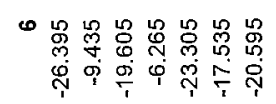

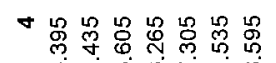

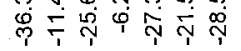

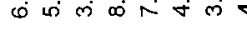

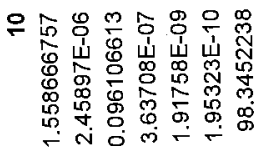

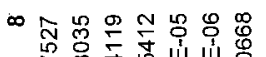

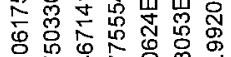

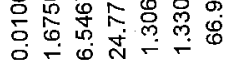

- 뚱ㅇㅇㅇ용ㅇㅇ웅

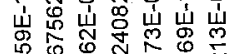

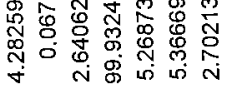

ช พิ

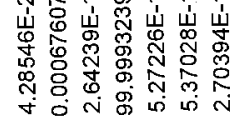

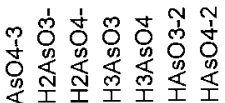

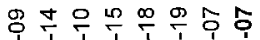

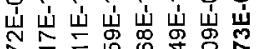

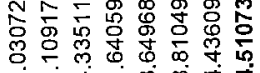

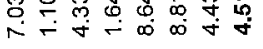

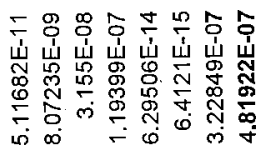

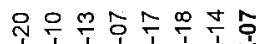

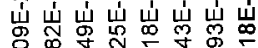

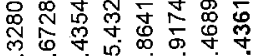

نั

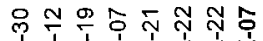

岁岕岁岁岕岕岁岁

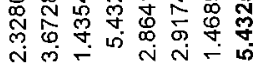

N然

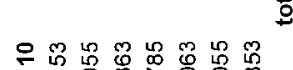

舟

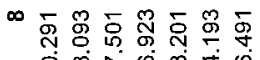

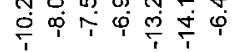

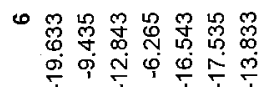

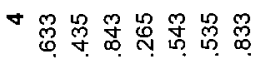

ণัণ

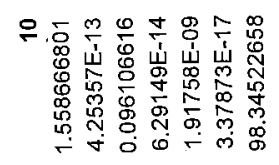

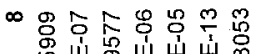

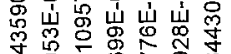

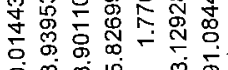

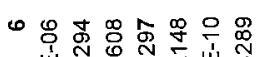

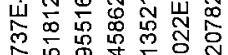

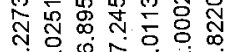

๙

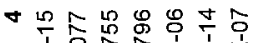

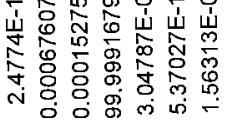

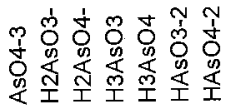

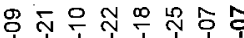

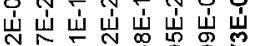

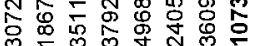

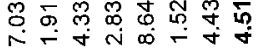

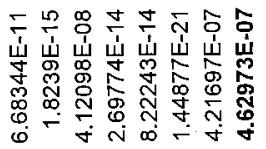

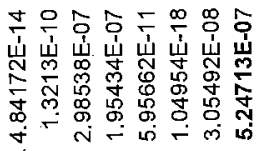

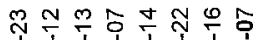

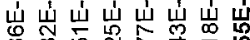

品点岕

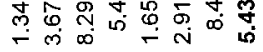

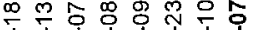

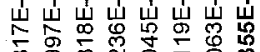

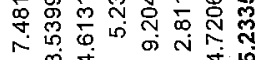

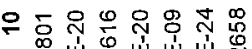

峞芯岕㟧 苜

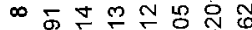

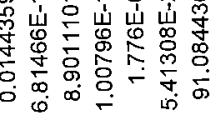

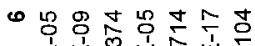

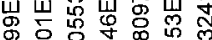

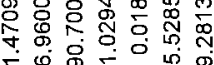

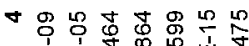

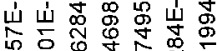

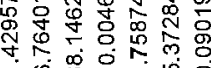

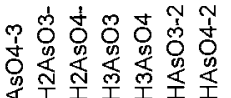

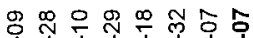

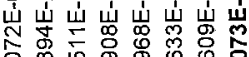

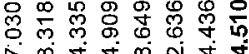

テ ำธัง

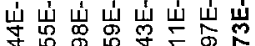

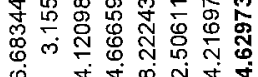

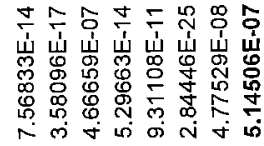

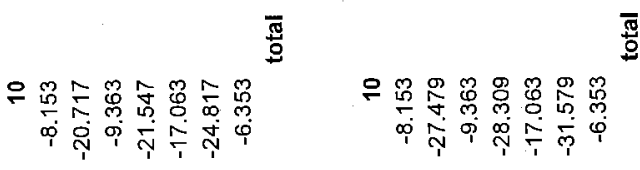

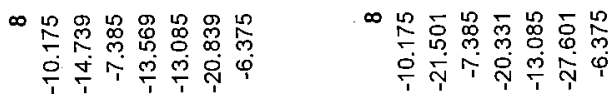

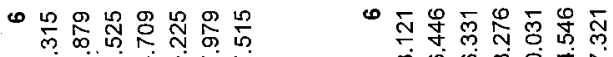

宁品

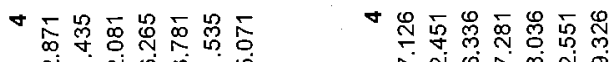

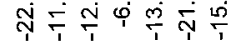

동ㅎㅎㅇㅇㅇ 之嵌㟧岩崖 ठे लं ले

홍ㅎㅇㅇㅇ

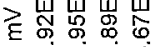
वलंखें

동ㅇㅇ 》虫出岁㟧 员 ले ले

동ㅎㅇㅇ

ไ岕岕岕岕 है ले ले ले

$\checkmark 0 \infty$ 음

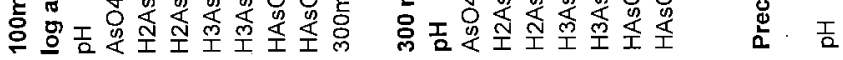




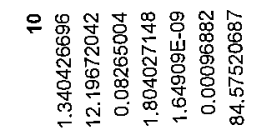

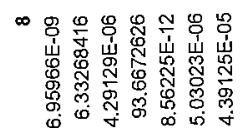

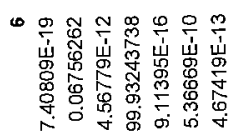

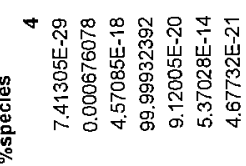

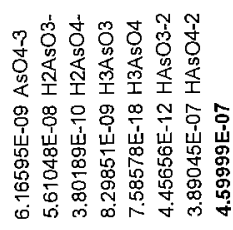

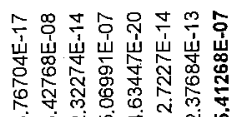

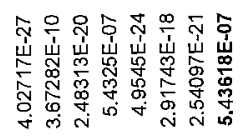

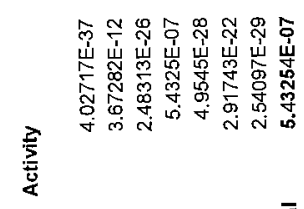

ฐ্

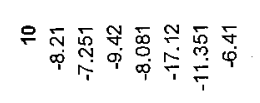

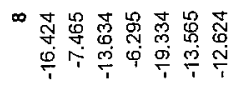

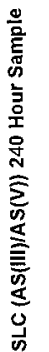

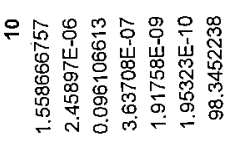

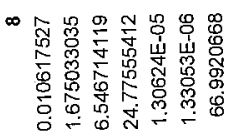

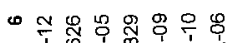

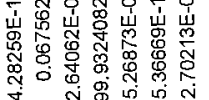

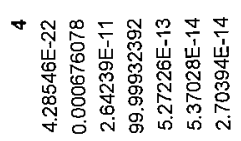

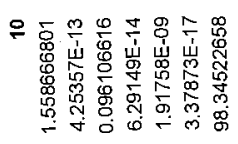

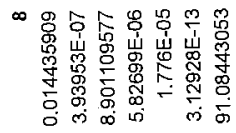

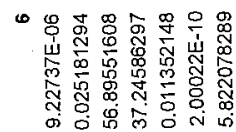

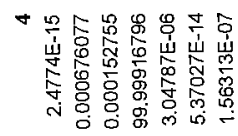

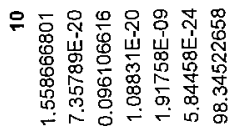

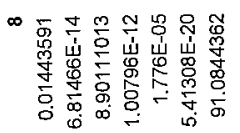

-

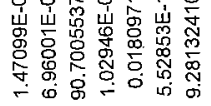

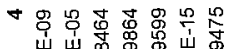

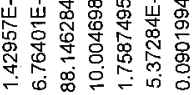

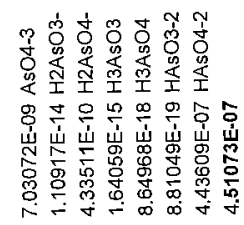

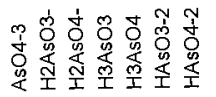

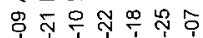

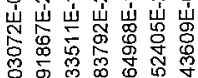
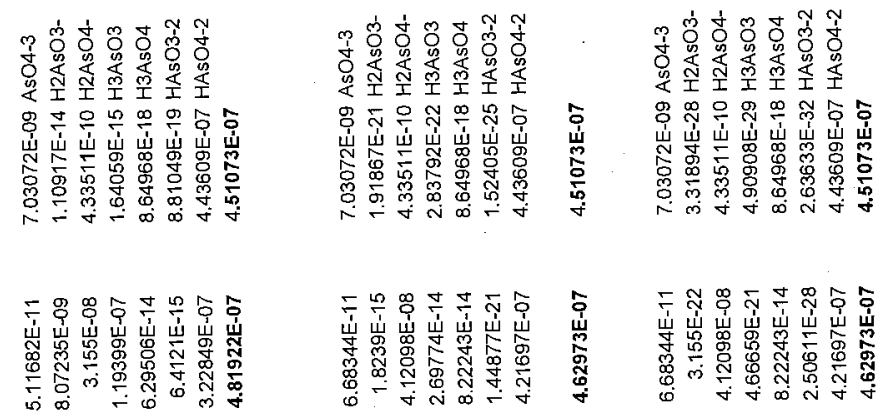

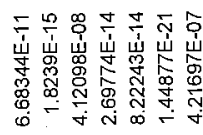

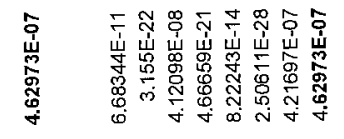

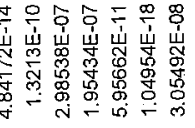

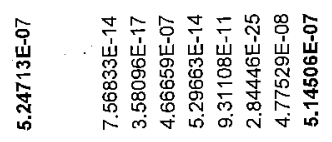

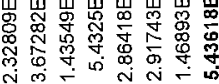
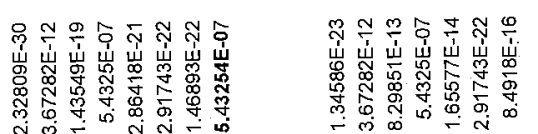

产

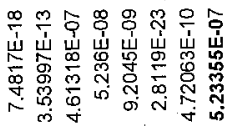

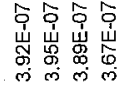

芒

홍

离

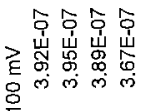

동ㅇㅇㅇ

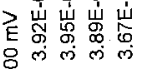

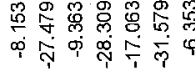

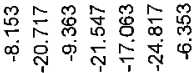

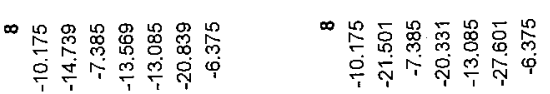

8888

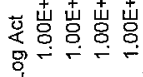

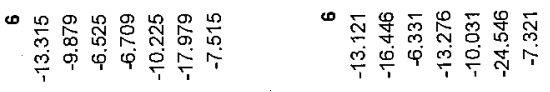

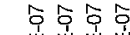

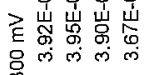

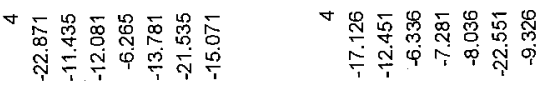

+ 0 \%

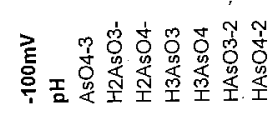

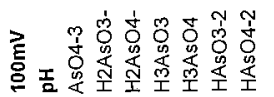

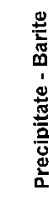




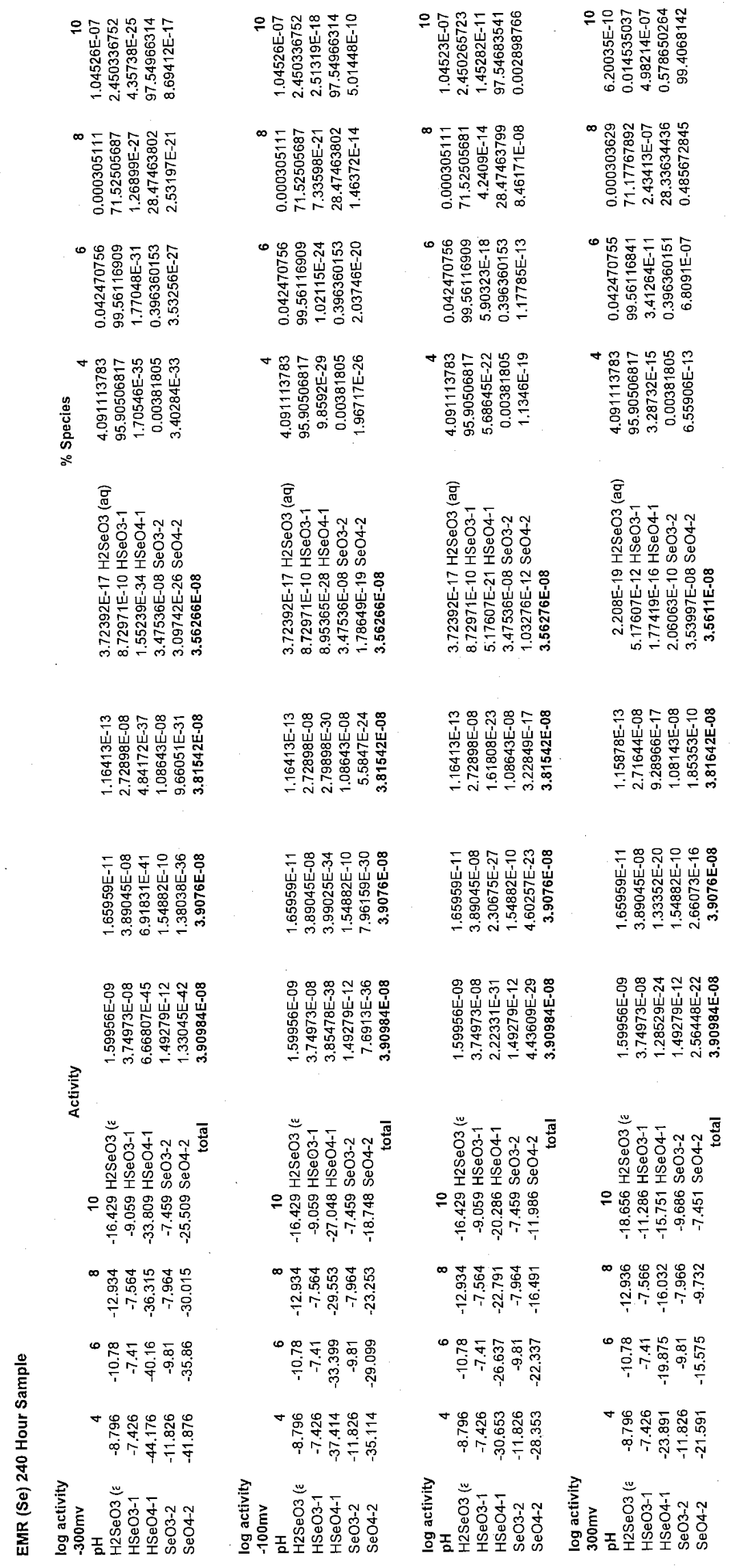



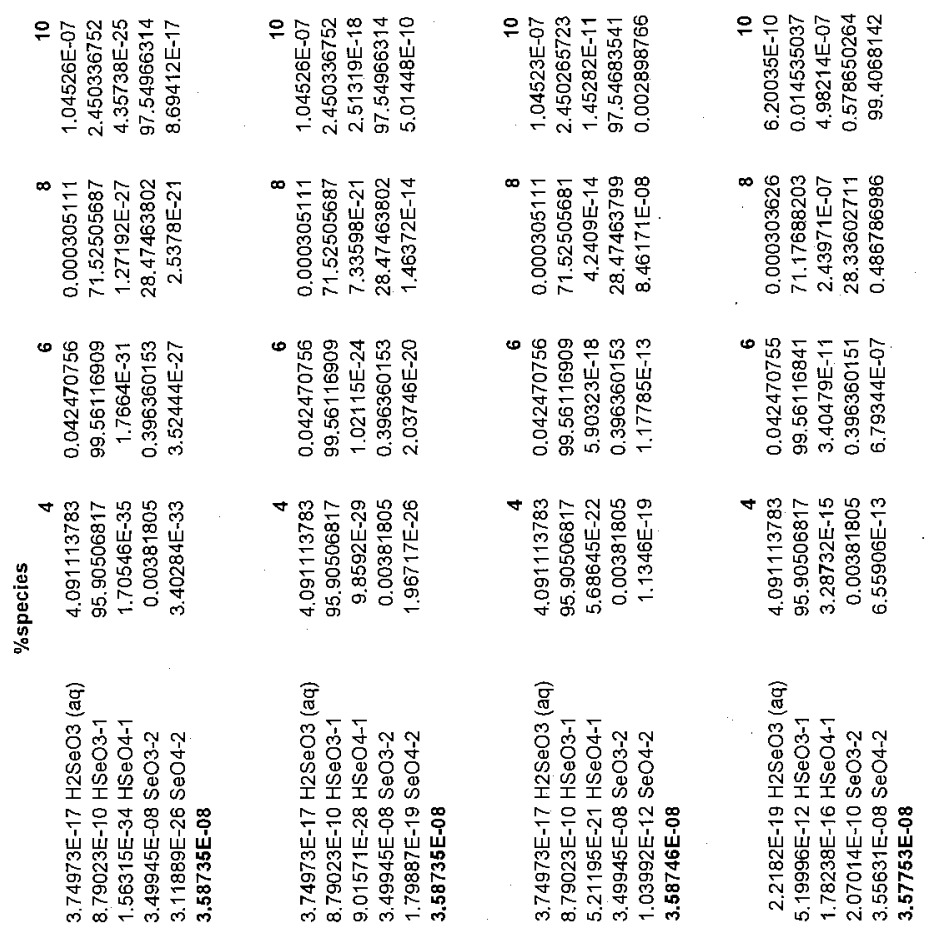

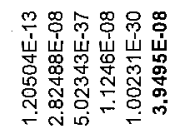

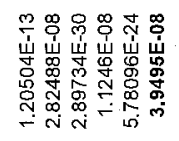

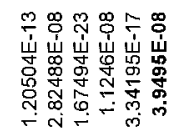

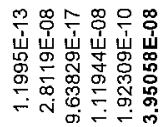

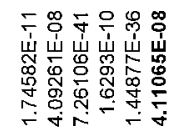
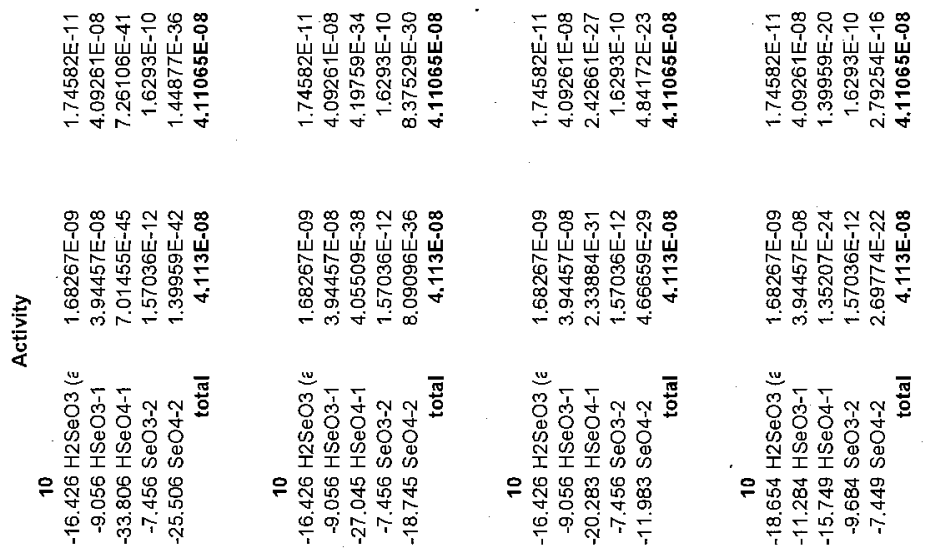

"艹

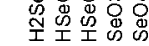

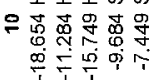

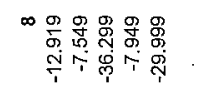

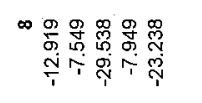

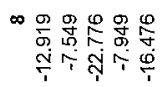

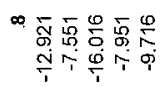

$\begin{array}{rl}0 & 0 \\ 0 \\ 0 \\ 0\end{array}$

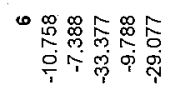

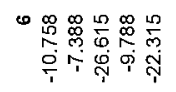

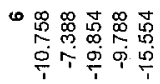

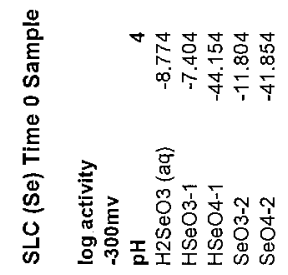

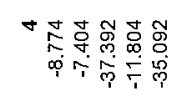

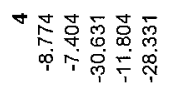

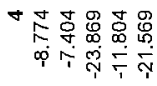



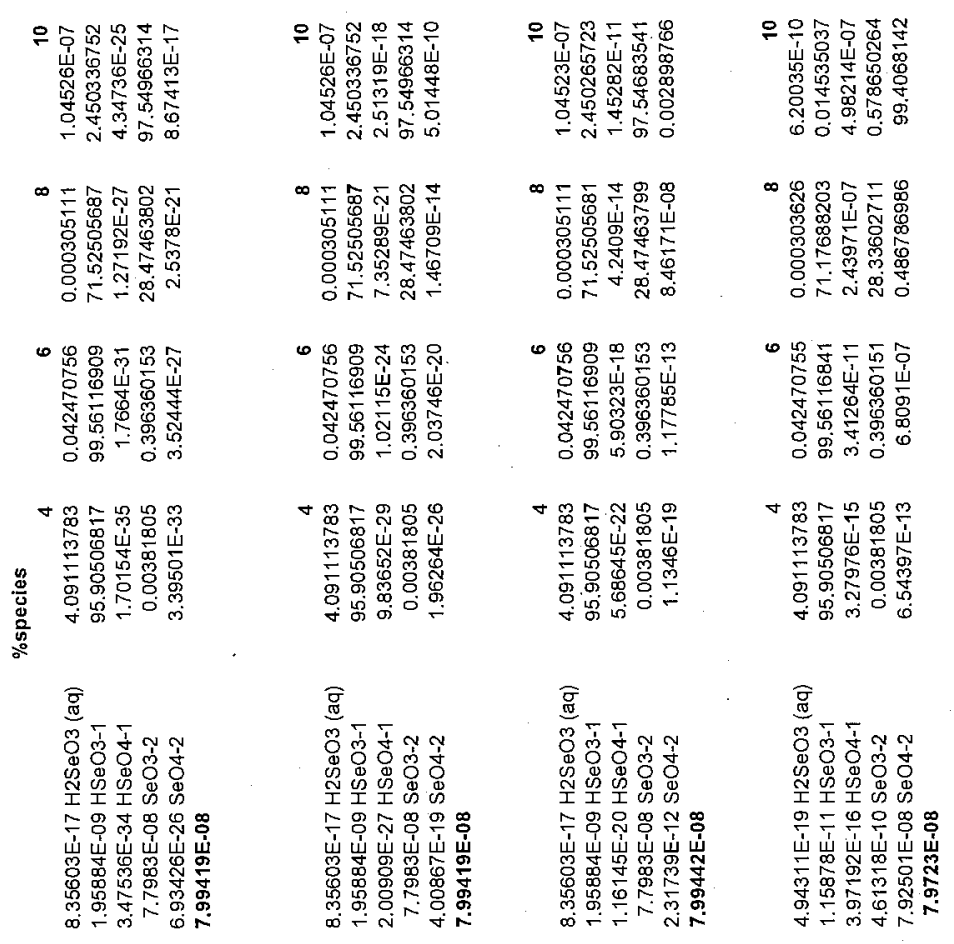

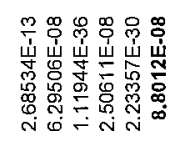
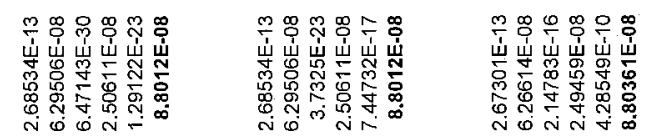

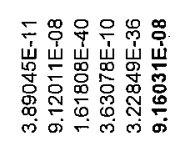

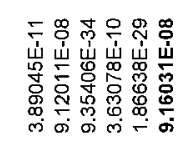

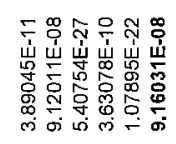
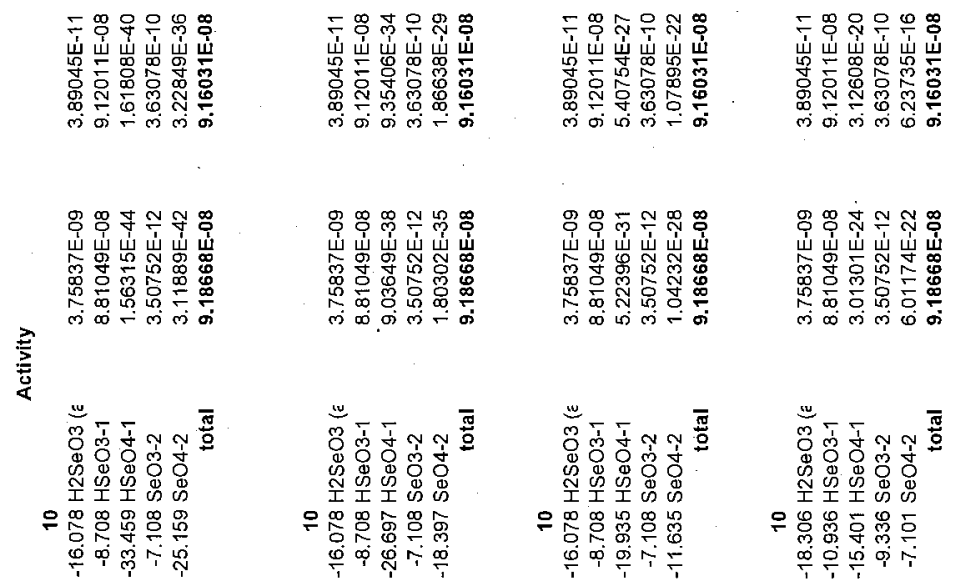

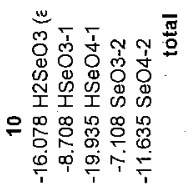

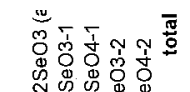

N

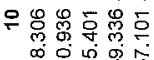

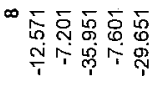

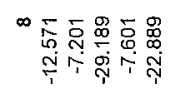

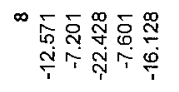

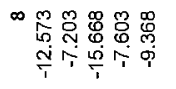

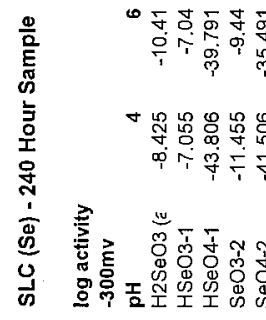

$$
\begin{aligned}
& \text { • }
\end{aligned}
$$

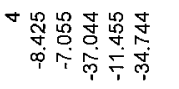

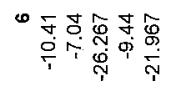

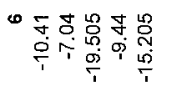

•

จ 


\section{Appendix F: Elutriate Bioassay Laboratory Data Sheets}




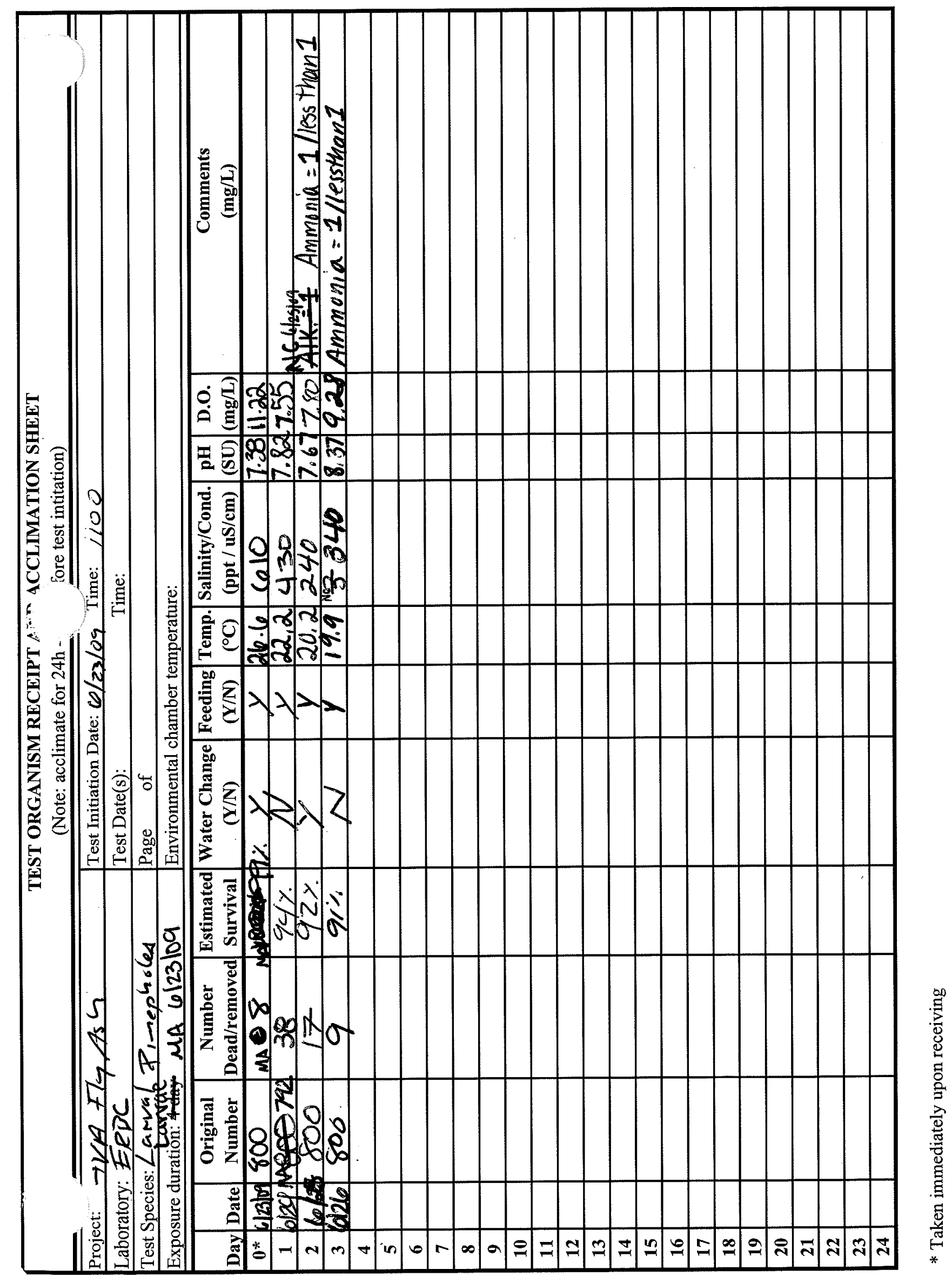




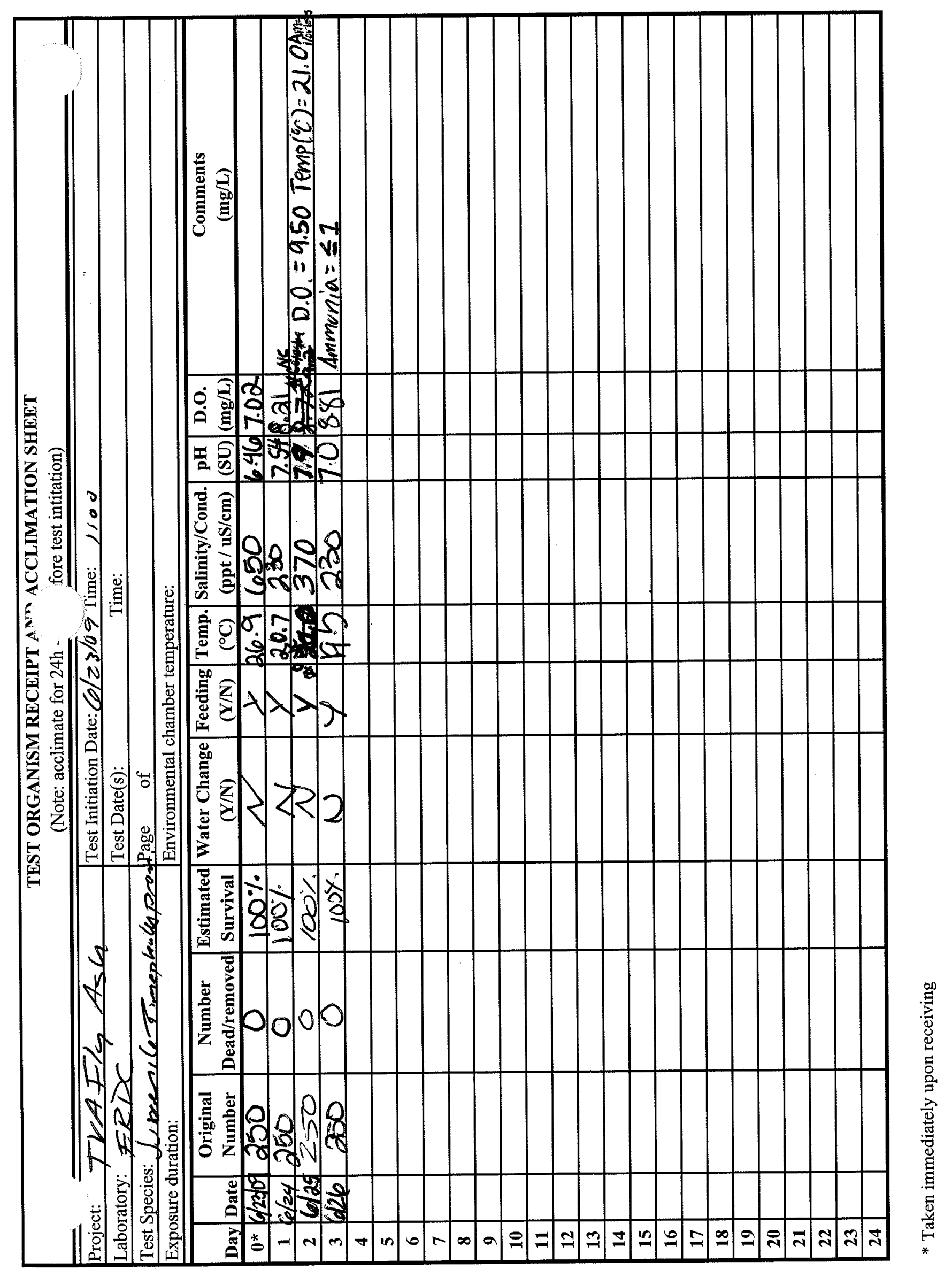




\section{$300 \mathrm{ML}$}

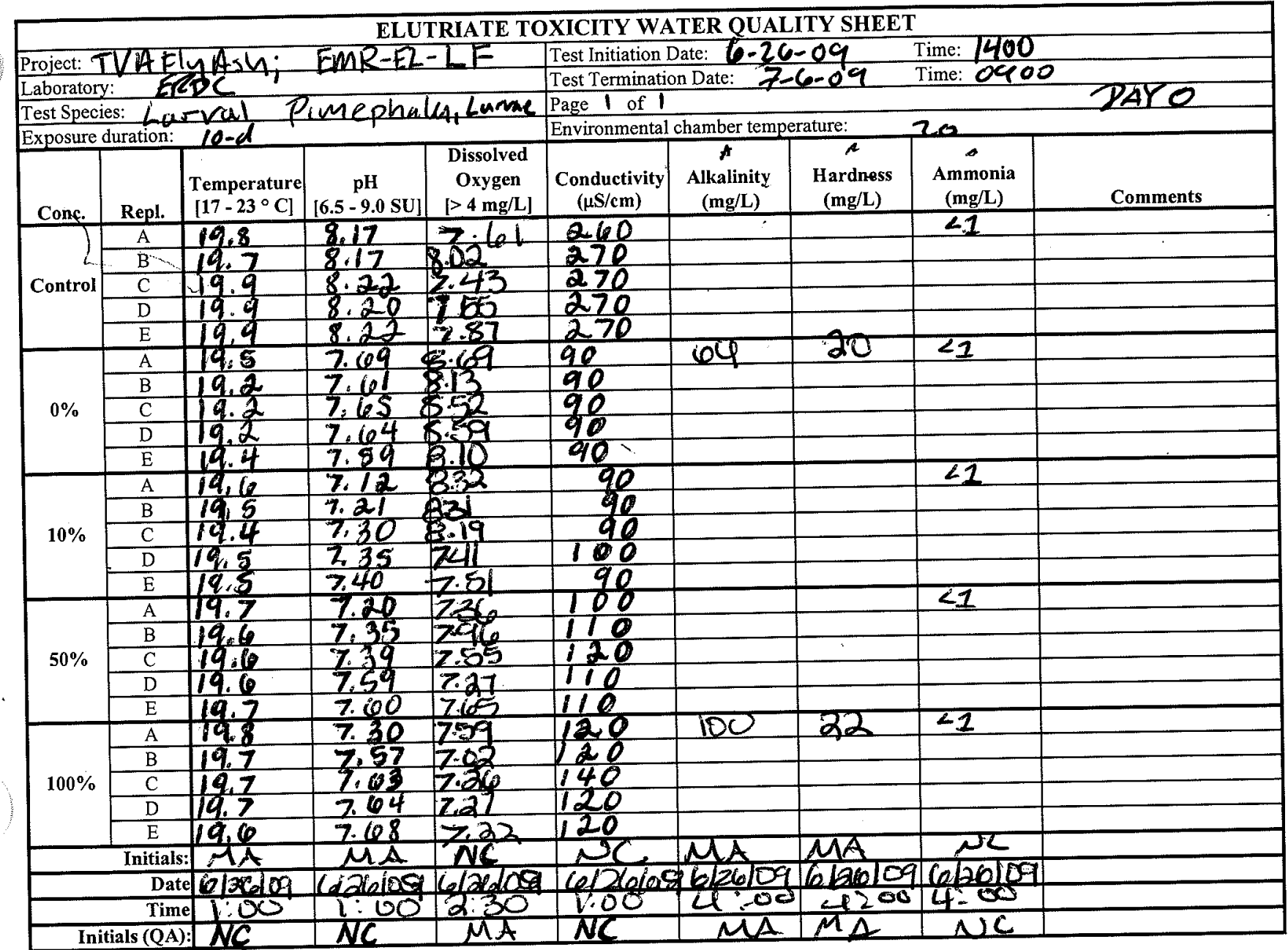

Reviewed by

7-14-09

Comment 


\section{$300 \mathrm{~mL}$}

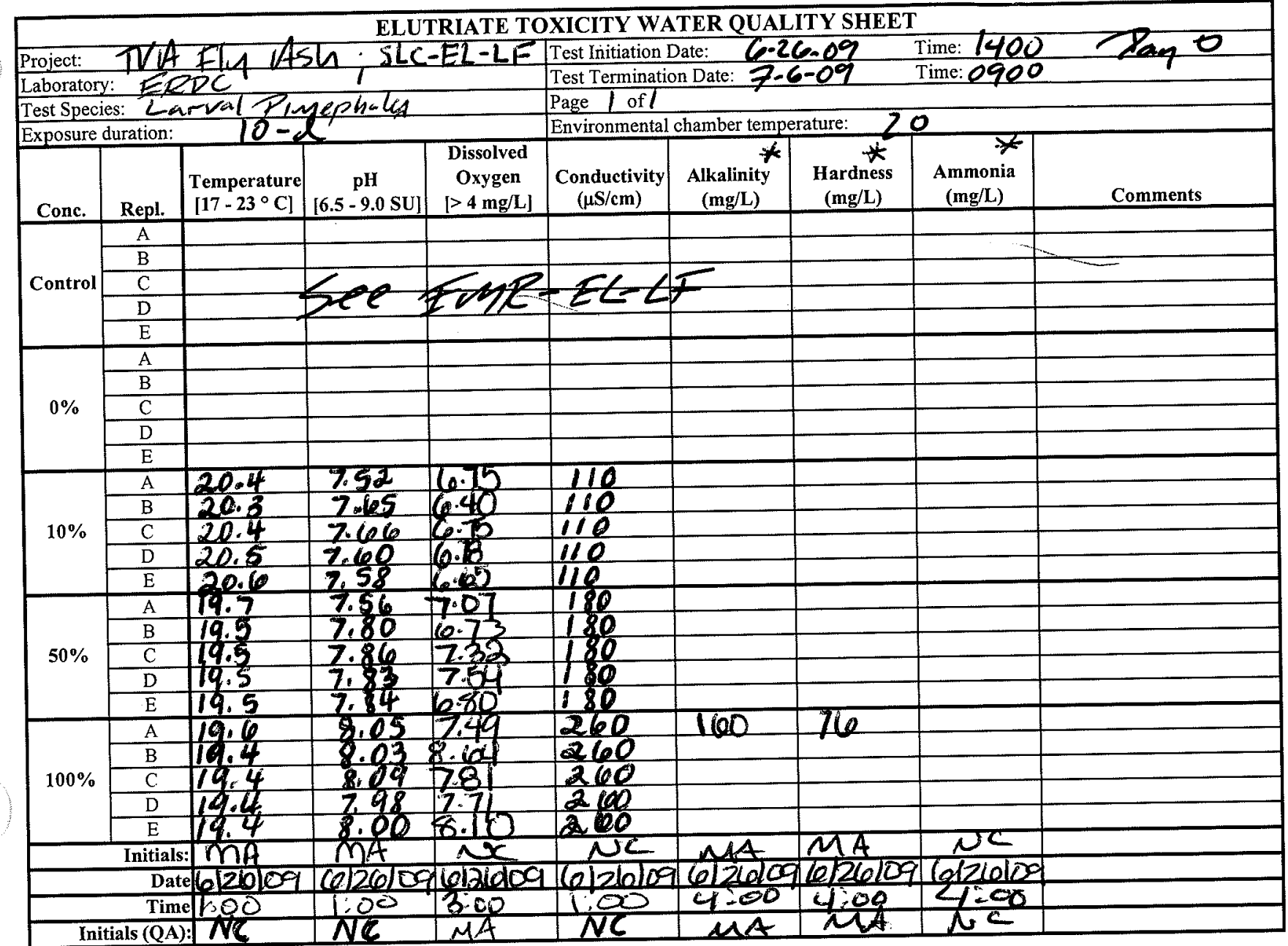

Reviewed by AK Date 7-14-09 comment 
4-L JAR

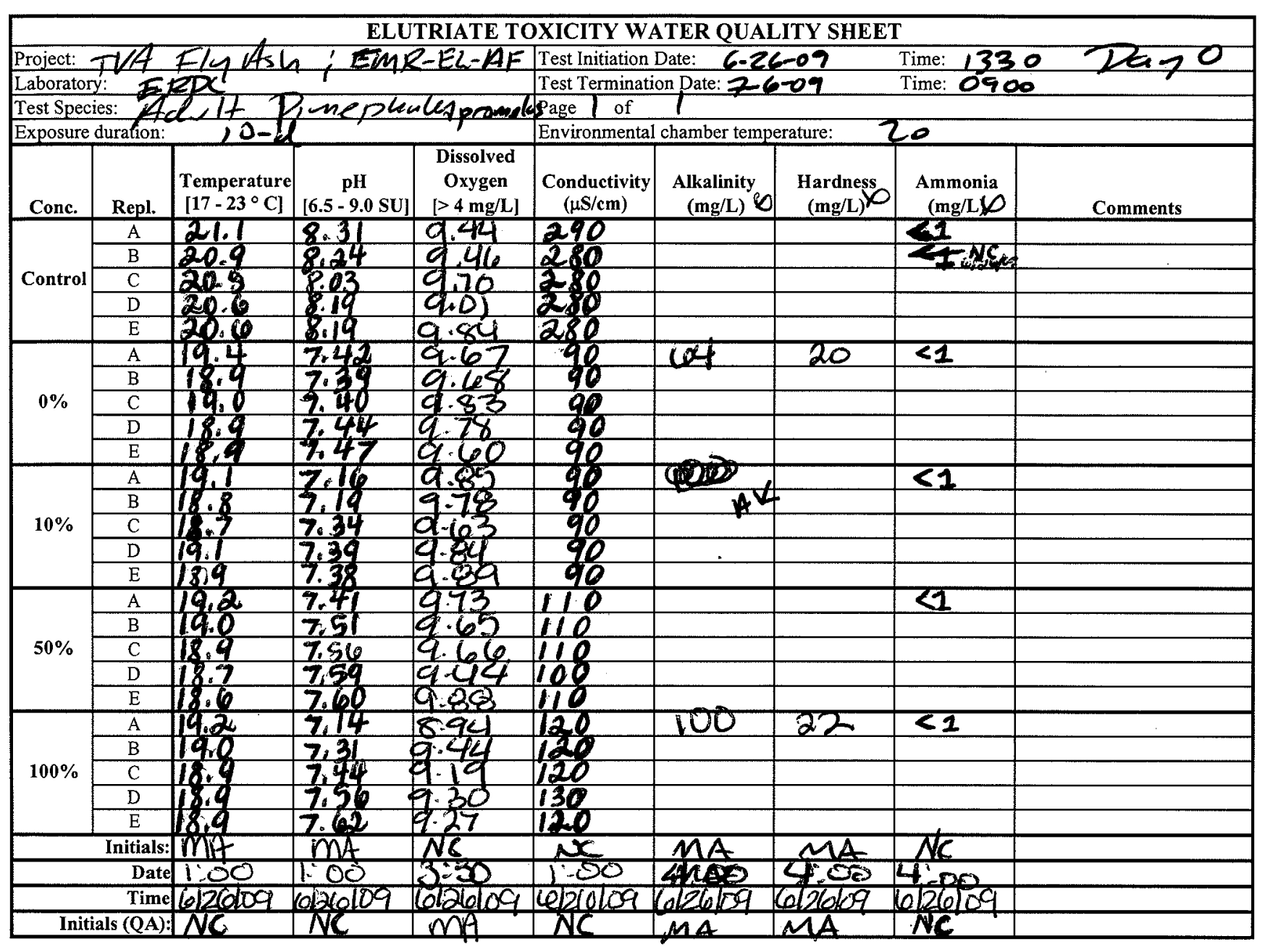

Reviewed by 4 K

Date 7-14-09

$\frac{\text { comment crossed out value }}{\text { not needed }}$ 


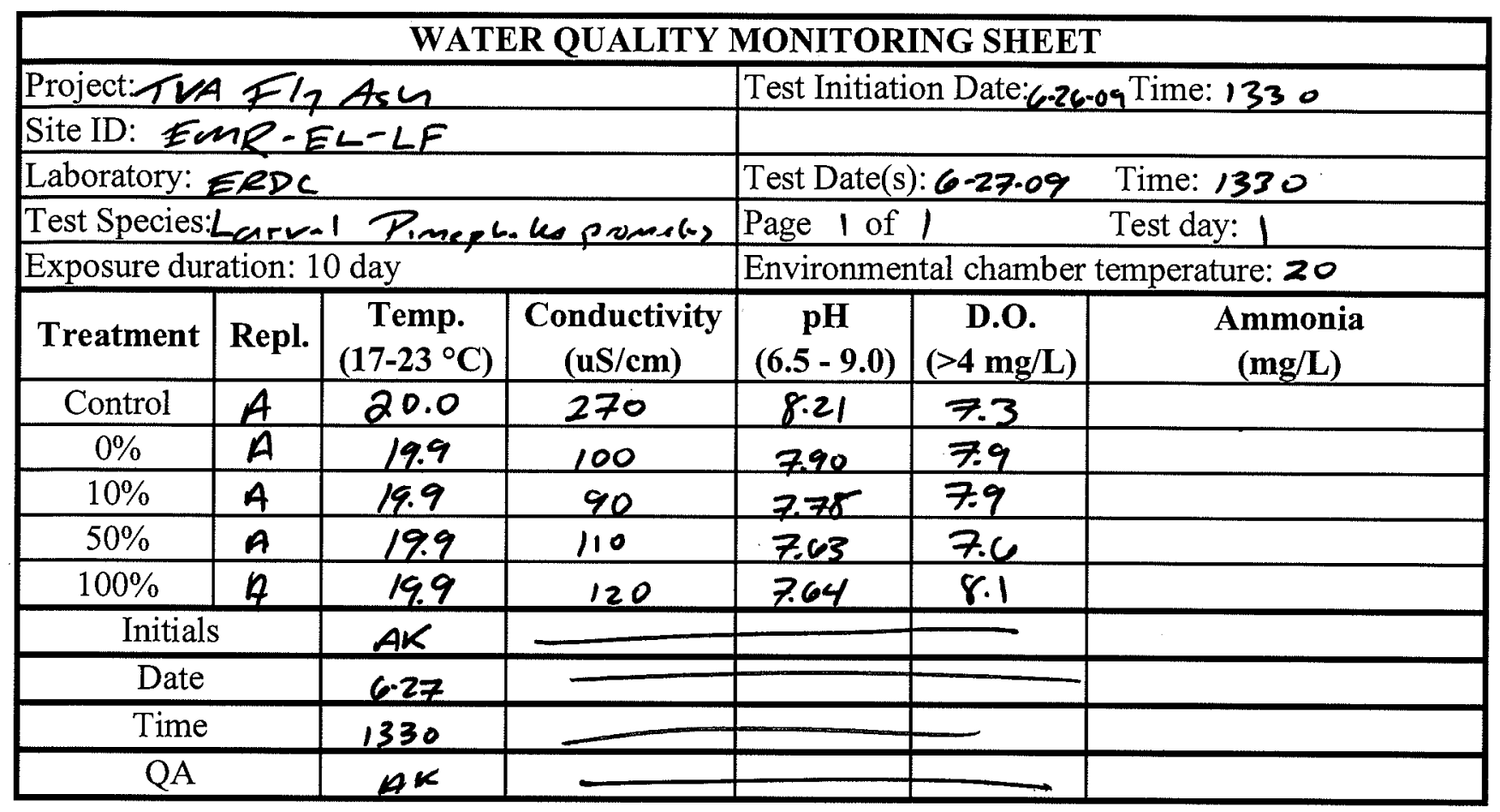

AK $7-14-09$ 


\begin{tabular}{|c|c|c|c|c|c|c|}
\hline \multicolumn{7}{|c|}{ WATER QUALITY MONITORING SHEET } \\
\hline \multicolumn{4}{|c|}{ Project: TVA FlAAsh } & \multicolumn{3}{|c|}{ Test Initiation Date:Gr6-og Time: $/ 400$} \\
\hline \multicolumn{4}{|c|}{ Site ID: $\quad S L C-E L-\angle F$} & & & \\
\hline \multicolumn{4}{|c|}{ Laboratory: ERPC } & \multicolumn{3}{|c|}{ Test Date(s): $6-27-09$ Time: 1330} \\
\hline \multirow{2}{*}{\multicolumn{4}{|c|}{$\begin{array}{l}\text { Test Species: Larval Pimeq h. Ces pemeles } \\
\text { Exposure duration: } 10 \text { day }\end{array}$}} & Page 1 of & 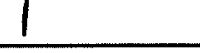 & lay: 1 \\
\hline & & & & \multicolumn{3}{|c|}{ Environmental chamber temperature: $\mathrm{Zo}$} \\
\hline Treatment & Repl. & $\begin{array}{c}\text { Temp. } \\
\left(17-23^{\circ} \mathrm{C}\right)\end{array}$ & $\begin{array}{c}\text { Conductivity } \\
\text { (uS/cm) }\end{array}$ & $\begin{array}{c}\mathrm{pH} \\
(6.5-9.0) \\
\end{array}$ & $\begin{array}{c}\text { D.O. } \\
(>4 \mathrm{mg} / \mathrm{L})\end{array}$ & $\begin{array}{c}\text { Ammonia } \\
(\mathrm{mg} / \mathrm{L})\end{array}$ \\
\hline Control & $\mathcal{A}$ & 20.0 & 270 & 8.21 & 7.3 & \\
\hline $0 \%$ & $A$ & 19.9 & 100 & 7.90 & 7.9 & \\
\hline $10 \%$ & $\bar{A}$ & 199 & 110 & $7-52$ & 7.9 & \\
\hline $50 \%$ & A & 20.1 & 180 & 7.68 & 8.1 & \\
\hline $100 \%$ & A & 2.1 & 260 & 7.99 & 8.2 & \\
\hline \multicolumn{2}{|c|}{ Initials } & Ak & & & & \\
\hline \multicolumn{2}{|l|}{ Date } & 6.27 & & & $=$ & \\
\hline \multicolumn{2}{|l|}{ Time } & 1330 & - & & $=$ & \\
\hline \multicolumn{2}{|l|}{ QA } & AK & & & $=$ & \\
\hline
\end{tabular}

$A K \quad 7-14-09$ 


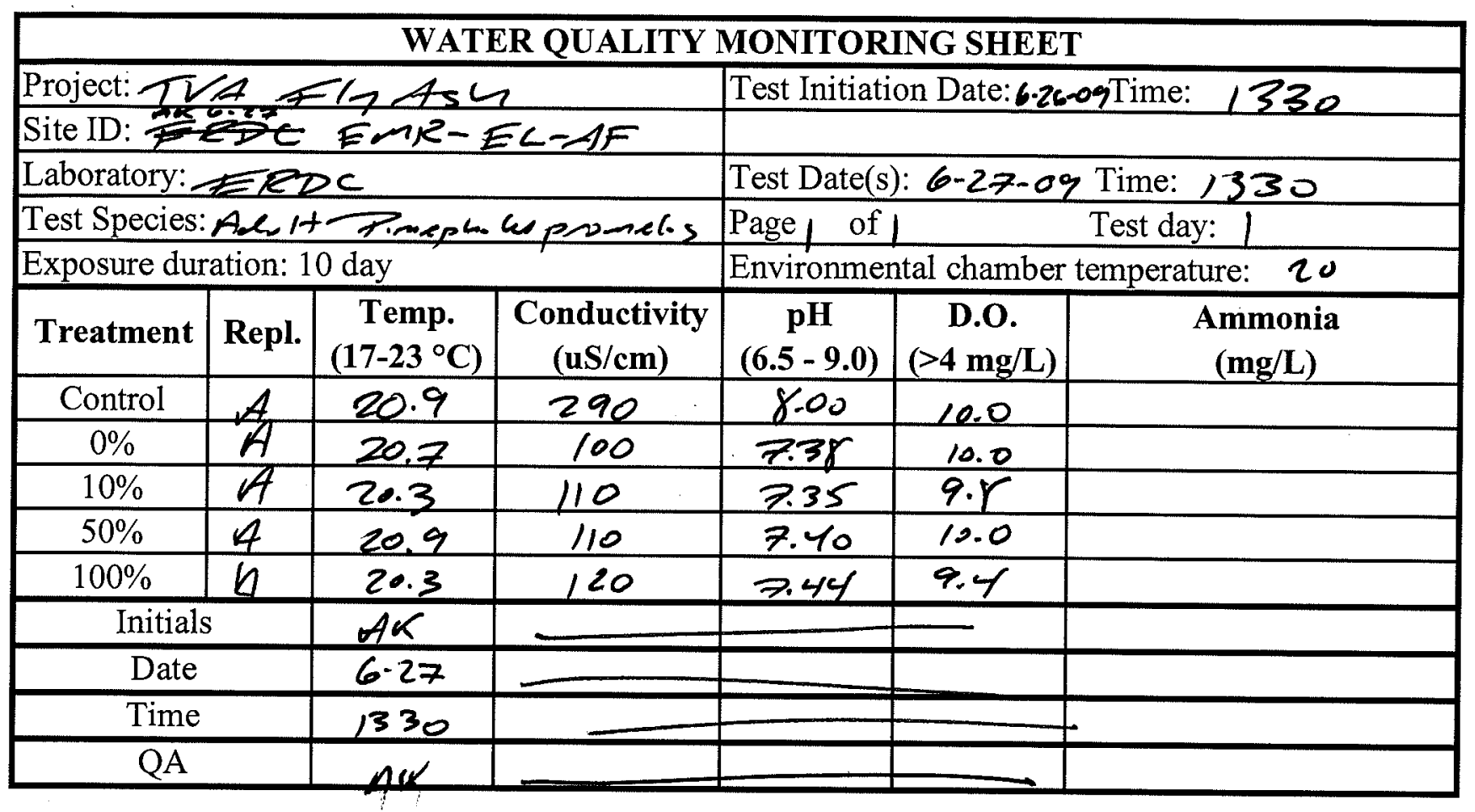

Ax 7-1409 


\section{WATER QUALITY MONITORING SHEET}

\begin{tabular}{|c|c|c|c|c|c|c|}
\hline Project: & 47 & $17 A_{5}=$ & & Test Initiati & n Date: 626 & osTime: 1330 \\
\hline Site ID: $F$ & $M R-$ & EL- LF & & & & \\
\hline Laboratory: & $=2 D C$ & & & Test Date(s & $6-28-09$ & Time: 1300 \\
\hline Test Species: & Larr & 1 Pinep & Copremplos & Page / of & & Test day: 2 \\
\hline Exposure dur & ation: 1 & 0 day & & Environmer & tal chamber & temperature: 20 \\
\hline Treatment & Repl. & $\begin{array}{c}\text { Temp. } \\
\left(17-23^{\circ} \mathrm{C}\right)\end{array}$ & $\begin{array}{l}\text { Conductivity } \\
\text { (uS/cm) }\end{array}$ & $\begin{array}{c}\text { pH } \\
(6.5-9.0)\end{array}$ & $\begin{array}{c}\text { D.O. } \\
(>4 \mathrm{mg} / \mathrm{L})\end{array}$ & $\begin{array}{c}\text { Ammonia } \\
(\mathrm{mg} / \mathrm{L})\end{array}$ \\
\hline Control & $B$ & 80.0 & 270 & 8.10 & r. & \\
\hline $0 \%$ & $\beta$ & 20.0 & 90 & 7.57 & 7.2 & \\
\hline $10 \%$ & $B$ & 20.0 & 90 & 7.54 & 7.6 & \\
\hline $50 \%$ & $B$ & 20.0 & 110 & $7.5 \gamma$ & 8.3 & \\
\hline $100 \%$ & $B$ & 19.9 & 120 & 7.61 & 8.2 & \\
\hline Initial & & $A K$ & AK & & & \\
\hline Date & & $6-2 \gamma$ & $6-28$ & & & \\
\hline Time & & 1300 & 1300 & & - & \\
\hline QA & & $C C K$ & & & & \\
\hline
\end{tabular}

fik 7-k-09 


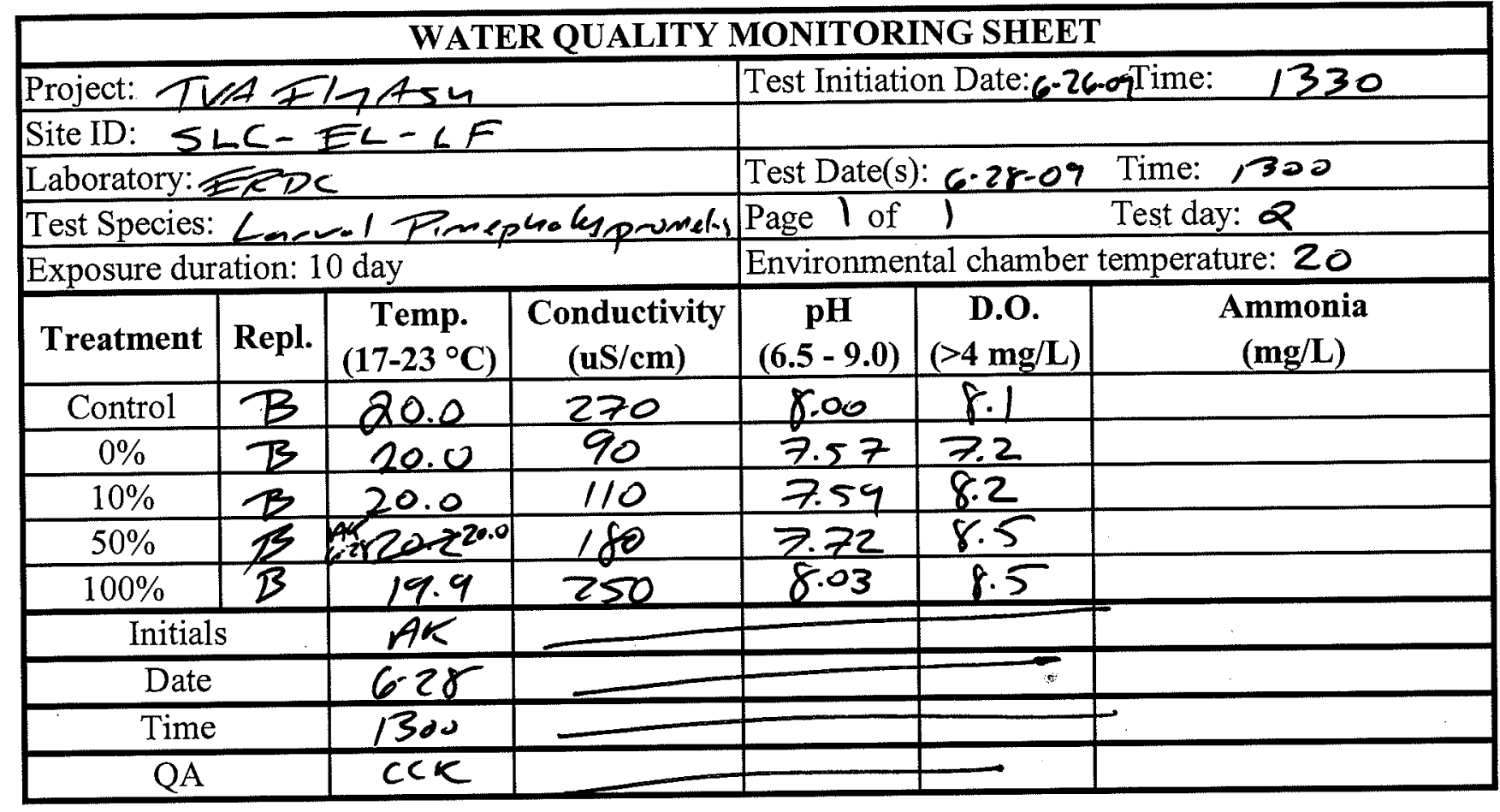

AK 7-14-09 


\begin{tabular}{|c|c|c|c|c|c|c|}
\hline \multicolumn{7}{|c|}{ WATER QUALITY MONITORING SHEET } \\
\hline \multicolumn{4}{|c|}{ Project: T化 $F / \neg A=4$} & \multicolumn{3}{|c|}{ Test Initiation Date: 6.2607 Time: 1330} \\
\hline \multicolumn{4}{|c|}{ Site ID: $M M R-E L-A F$} & \multicolumn{3}{|c|}{ (2) } \\
\hline \multirow{2}{*}{\multicolumn{4}{|c|}{$\begin{array}{l}\text { Laboratory: } \\
\text { Test Species: } A P P C\end{array}$}} & \multicolumn{3}{|c|}{ Test Date(s): $6-2 \gamma-09$ Time: $\mathbf{1 3 \Delta 0}$} \\
\hline \multirow{2}{*}{\multicolumn{4}{|c|}{$\begin{array}{l}\text { Test Species: } A \text { l, } 1 \text { P.meph les promers } \\
\text { Exposure duration: } 10 \text { day }\end{array}$}} & \multirow{2}{*}{\multicolumn{3}{|c|}{\begin{tabular}{|l|c|} 
Page 1 of & Test day: 2 \\
Environmental chamber temperature: 20
\end{tabular}}} \\
\hline & & & & & & \\
\hline Treatment & Repl. & $\begin{array}{c}\text { Temp. } \\
\left(17-23^{\circ} \mathrm{C}\right)\end{array}$ & $\begin{array}{c}\text { Conductivity } \\
\text { (uS/cm) }\end{array}$ & $\begin{array}{c}\mathrm{pH} \\
(6.5-9.0) \\
\end{array}$ & $\begin{array}{c}\text { D.O. } \\
(>4 \mathrm{mg} / \mathrm{L})\end{array}$ & $\begin{array}{c}\text { Ammonia } \\
(\mathrm{mg} / \mathrm{L})\end{array}$ \\
\hline Control & $B$ & 20.8 & 280 & 8.00 & 9.2 & \\
\hline $0 \%$ & 3 & 20.7 & 100 & 7.41 & $4.2 *$ & \\
\hline $10 \%$ & $B$ & 20.1 & 100 & 7.42 & 9.5 & \\
\hline $50 \%$ & $B$ & 20.8 & 110 & 7.44 & 9.7 & \\
\hline $100 \%$ & 13 & 20.3 & 130 & 751 & 9.3 & \\
\hline \multicolumn{2}{|l|}{ Initials } & $\overline{A K}$ & $\overline{-}$ & & & \\
\hline \multicolumn{2}{|l|}{ Date } & $6 \cdot 28$ & - & & $\overline{-}$ & \\
\hline \multicolumn{2}{|l|}{ Time } & 1300 & - & & & \\
\hline \multicolumn{2}{|l|}{$\overline{\mathrm{QA}}$} & CCK & & & & \\
\hline
\end{tabular}

* Corrective action initiatal. Acraton Tatored

AK. 7-14-09 


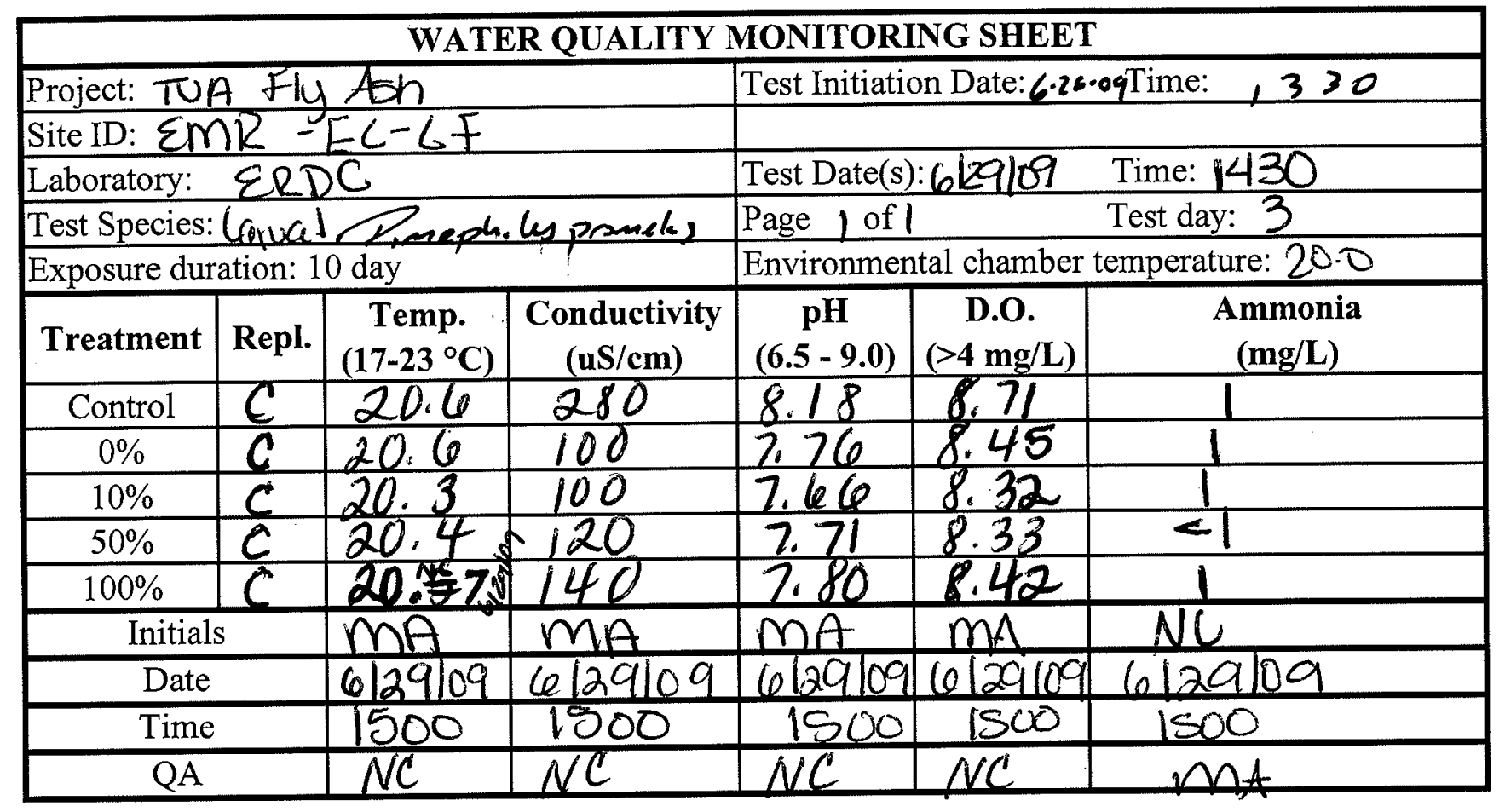

AK 7-14-09 cross shold be c/29109 


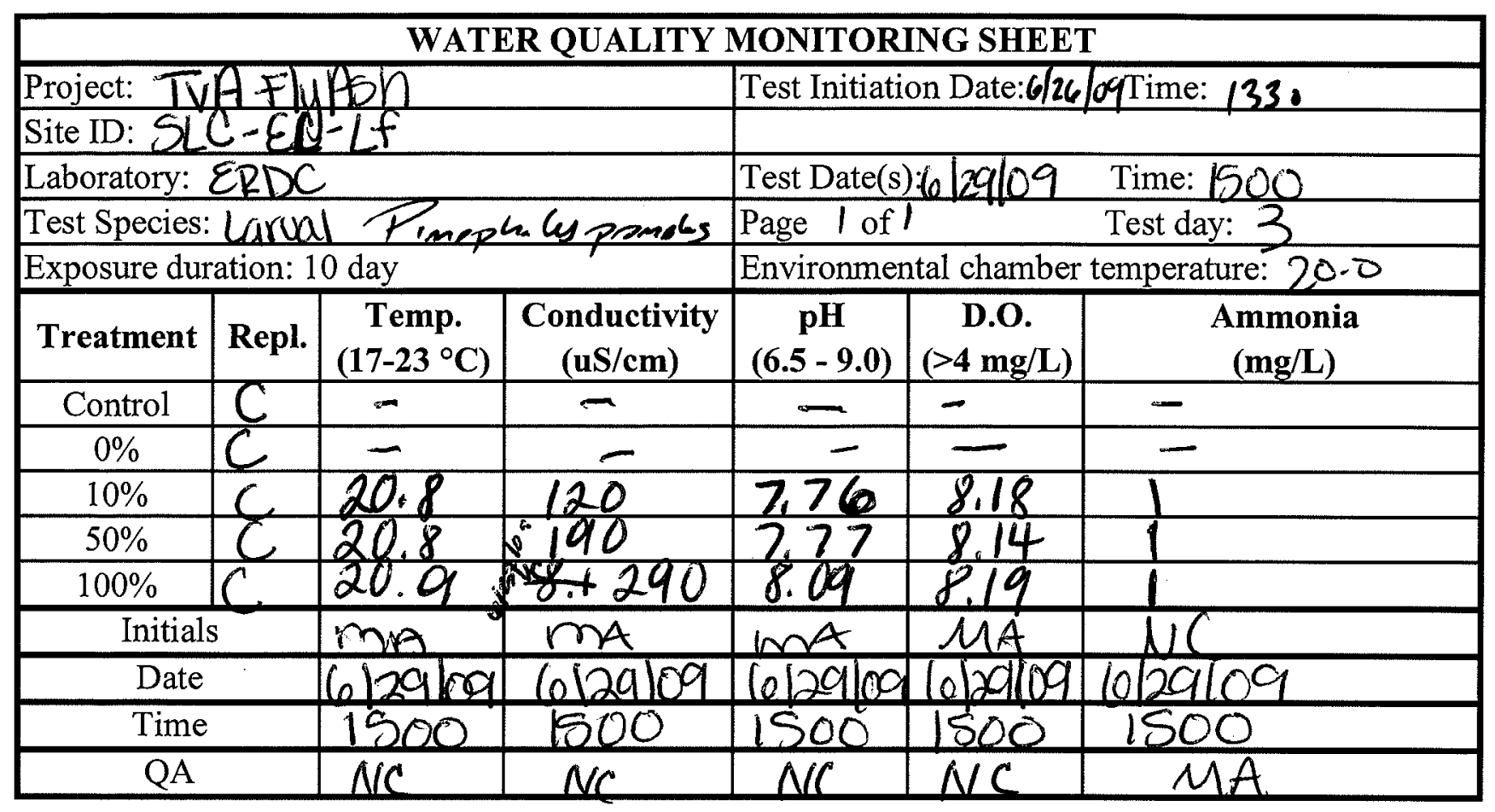

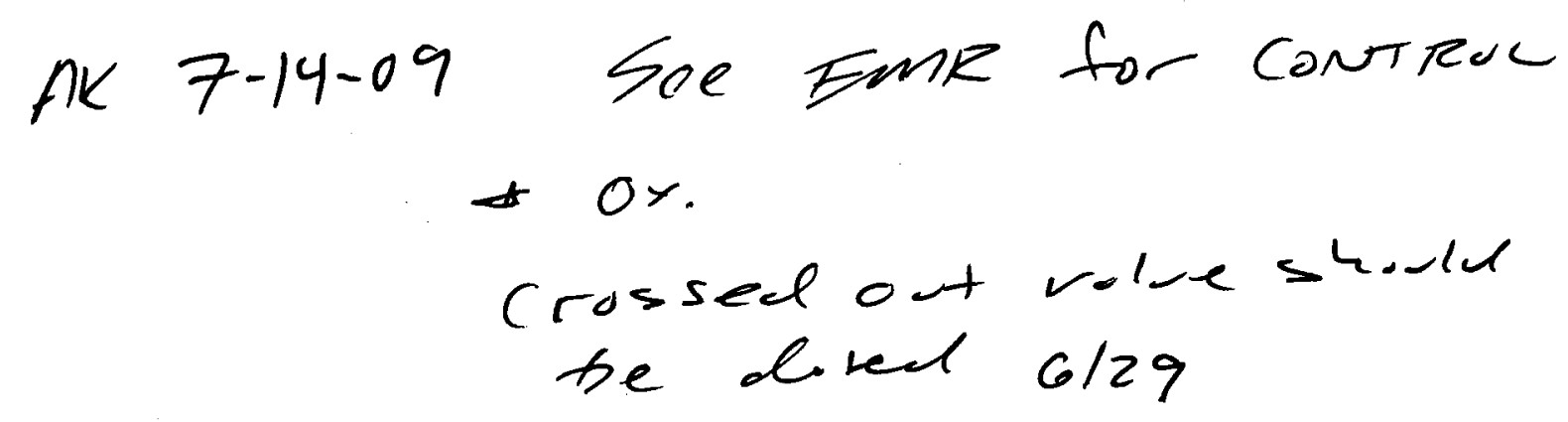




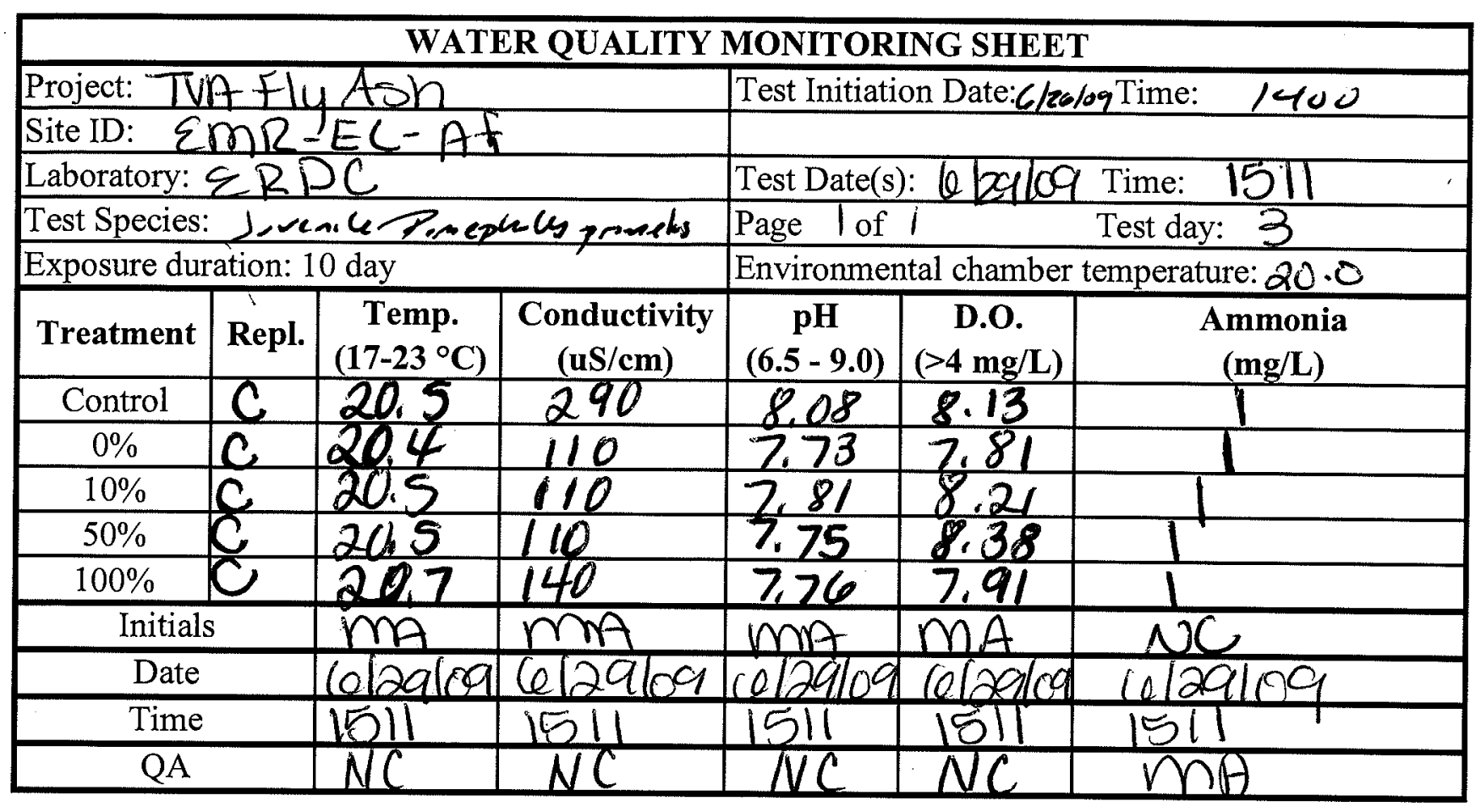

$4 k 7-14-09$ 


\begin{tabular}{|c|c|c|c|c|c|c|}
\hline \multicolumn{7}{|c|}{ WATER QUALITY MONITORING SHEET } \\
\hline & \multicolumn{3}{|c|}{ Test Initiation Date: $6 / 2 \mathrm{a} / \mathrm{og}$ Time: $/$ fou } \\
\hline & & & & \multirow{2}{*}{\multicolumn{3}{|c|}{ Test Date(s): 0130109 Time: $9: 00$}} \\
\hline \multicolumn{4}{|c|}{$\begin{array}{l}\text { Site ID: } 2 M R-E L-L f \\
\text { Laboratory: } E R D C\end{array}$} & & & \\
\hline \multicolumn{4}{|c|}{$\begin{array}{l}\text { Laboratory: ERDC } \\
\text { Test Species: Larual Pimephales }\end{array}$} & \multicolumn{3}{|c|}{ Page 1 of $1 \quad$ Test day: 4} \\
\hline \multicolumn{4}{|c|}{ Exposure duration: 10 day } & \multicolumn{3}{|c|}{ Environmental chamber temperature: $20-1$} \\
\hline Treatment & Repl. & $\begin{array}{c}\text { Temp. } \\
\left(17-23^{\circ} \mathrm{C}\right)\end{array}$ & $\begin{array}{c}\text { Conductivity } \\
\text { (uS/cm) }\end{array}$ & $\begin{array}{c}\mathrm{pH} \\
(6.5-9.0)\end{array}$ & $\begin{array}{c}\text { D.O. } \\
(>4 \mathrm{mg} / \mathrm{L})\end{array}$ & $\begin{array}{c}\text { Ammonia } \\
(\mathrm{mg} / \mathrm{L})\end{array}$ \\
\hline Control & $\bar{D}$ & 20.1 & 280 & 8.010 & 7.93 & $\leqslant$ \\
\hline $0 \%$ & $D$ & 20.1 & 90 & 7.93 & 8.17 & $\angle$ \\
\hline $10 \%$ & $D$ & 20.1 & 90 & 7.76 & 8.26 & 41 \\
\hline $50 \%$ & $\bar{D}$ & 20.1 & 110 & 770 & 8.30 & $<1$ \\
\hline $100 \%$ & $D$ & 20.1 & 130 & 7.56 & 6.64 & $<$ \\
\hline \multicolumn{2}{|l|}{ Initials } & NC & $\mathrm{NC}$ & Ne & $N C$ & $N C$ \\
\hline \multicolumn{2}{|l|}{ Date } & 6130109 & 6130109 & 10130109 & 613000 & 6130109 \\
\hline \multicolumn{2}{|l|}{ Time } & $9: 00$ & $9: 00$ & $9: 00$ & $9: 00$ & 9.00 \\
\hline \multicolumn{2}{|l|}{$\overline{\mathrm{QA}}$} & $m A$ & $m A$ & $m$ & $M A$ & $M A$ \\
\hline
\end{tabular}

$$
\text { AK } 7-14-09
$$




\begin{tabular}{|c|c|c|c|c|c|c|}
\hline \multicolumn{7}{|c|}{ WATER QUALITY MONITORING SHEET } \\
\hline \multirow{2}{*}{\multicolumn{4}{|c|}{ Project: TVA FlyPSh }} & \multicolumn{3}{|c|}{ Test Initiation Date: 6 /26/oyTime: $/ 400$} \\
\hline \multicolumn{6}{|c|}{ Site ID: $S L C-\varepsilon L-L f$} & \\
\hline \multicolumn{4}{|c|}{ Laboratory: ER } & \multicolumn{3}{|c|}{ Test Date(s): Ce 30109 Time: $10: 00$} \\
\hline \multirow{2}{*}{\multicolumn{4}{|c|}{$\begin{array}{l}\text { Test Species: Larual Pimedhales prome Kes } \\
\text { Exposure duration: } 10 \text { day }\end{array}$}} & Page ) of & 1 & Test day: 4 \\
\hline & & & & \multicolumn{3}{|c|}{ Environmental chamber temperature: 20.} \\
\hline Treatment & Repl. & \begin{tabular}{|c|} 
Temp. \\
$\left(17-23^{\circ} \mathrm{C}\right)$
\end{tabular} & $\begin{array}{c}\text { Conductivity } \\
(\mathrm{uS} / \mathrm{cm})\end{array}$ & $\begin{array}{c}\mathrm{pH} \\
(6.5-9.0)\end{array}$ & \begin{tabular}{|c|} 
D.O. \\
$(>4 \mathrm{mg} / \mathrm{L})$
\end{tabular} & $\begin{array}{c}\text { Ammonia } \\
(\mathbf{m g} / \mathbf{L})\end{array}$ \\
\hline Control & & & & & $\measuredangle$ & \\
\hline $0 \%$ & & & & & E & $=$ \\
\hline $10 \%$ & P & 201 & 110 & 7.87 & 8.24 & $\angle 1$ \\
\hline $50 \%$ & $D$ & $20 \cdot 0$ & 160 & 7.76 & 8.42 & $<$ \\
\hline $100 \%$ & $D$ & 20.0 & 200 & 8.00 & 8.35 & $\leq 1$ \\
\hline \multicolumn{2}{|l|}{ Initials } & N - & & & & ב \\
\hline \multicolumn{2}{|l|}{ Date } & 6130109 & & & & $=$ \\
\hline \multicolumn{2}{|l|}{ Time } & $10: 00-$ & & & & {[} \\
\hline \multicolumn{2}{|l|}{ QA } & $m a$ & $m A$ & MA & MA & MA \\
\hline
\end{tabular}

Ak $7-1-1-09$ 


\begin{tabular}{|c|c|c|c|c|c|c|}
\hline \multicolumn{7}{|c|}{ WATER QUALITY MONITORING SHEET } \\
\hline \multicolumn{4}{|c|}{ Project: TVA Flyltsh } & \multirow{2}{*}{\multicolumn{3}{|c|}{ Test Initiation Date: ${ }_{6} / 29$ log Time: $/ 400$}} \\
\hline \multicolumn{4}{|c|}{ Site ID: EMR-EL-TAF } & & & \\
\hline \multicolumn{4}{|c|}{ Laboratory: ERDC } & \multicolumn{3}{|c|}{ Test Date(s): Cel30 lo9 Time: 10.00} \\
\hline \multicolumn{4}{|c|}{ Test Species: Al Pimephalespromolas } & \multicolumn{3}{|c|}{ Page $\mid$ of $\mid$ Test day: 4} \\
\hline \multicolumn{4}{|c|}{ Exposure duration: 10 day } & \multicolumn{3}{|c|}{ Environmental chamber temperature: 20.1} \\
\hline Treatment & Repl. & \begin{tabular}{|c|} 
Temp. \\
$\left(17-23^{\circ} \mathrm{C}\right)$ \\
\end{tabular} & $\begin{array}{c}\text { Conductivity } \\
\text { (uS/cm) }\end{array}$ & $\begin{array}{c}\mathrm{pH} \\
(6.5-9.0) \\
\end{array}$ & $\begin{array}{c}\text { D.O. } \\
(>4 \mathrm{mg} / \mathrm{L})\end{array}$ & $\begin{array}{c}\text { Ammonia } \\
(\mathrm{mg} / \mathrm{L})\end{array}$ \\
\hline Control & D & 20.2 & 280 & 8.04 & 8.41 & 1 \\
\hline $0 \%$ & D & 20.1 & 100 & 7.75 & 8.56 & 1 \\
\hline $10 \%$ & $D$ & 20.0 & 100 & 7.74 & 8.60 & 1 \\
\hline $50 \%$ & D & 20,4 & 100 & 7.71 & 8.54 & 1 \\
\hline $100 \%$ & D & 20,2 & 130 & 7.73 & 8.52 & $\frac{1}{1}$ \\
\hline \multicolumn{2}{|c|}{ Initials } & $M A$ & & & & \\
\hline \multicolumn{2}{|l|}{ Date } & 6130104 & & & & - \\
\hline \multicolumn{2}{|l|}{ Time } & $10: 00$ & & & & \\
\hline QA & & NC & $\overline{N C}$ & NC & $N C$ & $\overline{N C}$ \\
\hline
\end{tabular}

AK 7-14-09 


\begin{tabular}{|c|c|c|c|c|c|c|}
\hline \multicolumn{7}{|c|}{ WATER QUALITY MONITORING SHEET } \\
\hline \multicolumn{4}{|c|}{ Project: TVA FlyAsh } & \multicolumn{3}{|c|}{ Test Initiation Date:6-26.09 Time: 1330} \\
\hline \multicolumn{7}{|c|}{ Site ID: $E M R-E L-L F$} \\
\hline \multicolumn{4}{|c|}{ Laboratory: ERDC } & \multicolumn{3}{|c|}{ Test Date(s): 71109 Time: $8: 30$} \\
\hline \multicolumn{4}{|c|}{ Test Species:Carval Pimephales F } & \multirow{2}{*}{\multicolumn{3}{|c|}{$\begin{array}{ll}\text { Page } 1 \text { of } 1 & \text { Test day: } 5 \\
\text { Environmental chamber temperature: } 20 \cdot 0\end{array}$}} \\
\hline \multicolumn{4}{|c|}{ Exposure duration: 10 day } & & & \\
\hline Treatment & Repl. & \begin{tabular}{|c|} 
Temp. \\
$\left(17-23^{\circ} \mathrm{C}\right)$
\end{tabular} & $\begin{array}{c}\text { Conductivity } \\
\text { (uS/cm) }\end{array}$ & $\begin{array}{c}\text { pH } \\
(6.5-9.0)\end{array}$ & $\begin{array}{c}\text { D.O. } \\
(>4 \mathrm{mg} / \mathrm{L})\end{array}$ & $\begin{array}{c}\text { Ammonia } \\
(\mathrm{mg} / \mathrm{L})\end{array}$ \\
\hline Control & $E$ & 20.3 & 280 & 8.14 & 8.60 & $<1$ \\
\hline $0 \%$ & E & 20.2 & 100 & 8.19 & 8.99 & $<1$ \\
\hline $10 \%$ & E & 20,1 & 100 & 8.02 & 8.73 & $\angle 1$ \\
\hline $50 \%$ & $E$ & 20.1 & 110 & 7.94 & 8.49 & $\angle 1$ \\
\hline $100 \%$ & $E$ & 20.2 & 120 & 7.95 & 8.42 & $\leq 1$ \\
\hline \multicolumn{2}{|c|}{ Initials } & $M A$ & & & & \\
\hline \multicolumn{2}{|c|}{ Date } & $71109=$ & & & & \\
\hline \multicolumn{2}{|c|}{ Time } & $8: 30=$ & & & & \\
\hline \multicolumn{2}{|l|}{$\overline{Q A}$} & $N C$ & $N C$ & $N C$ & NC & \\
\hline
\end{tabular}

$A K \quad 7.11-09$ 


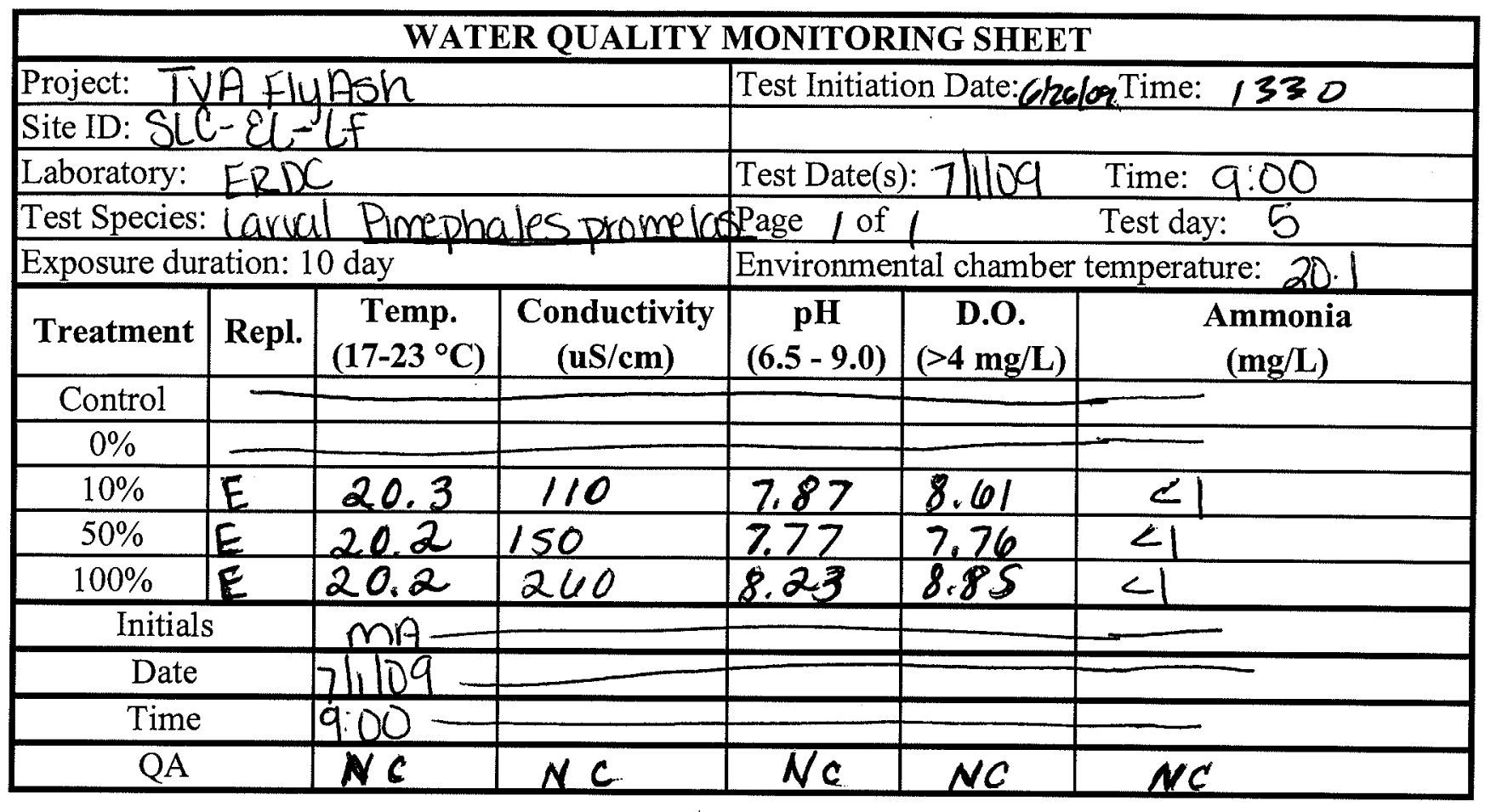

Ak 7-14-09 


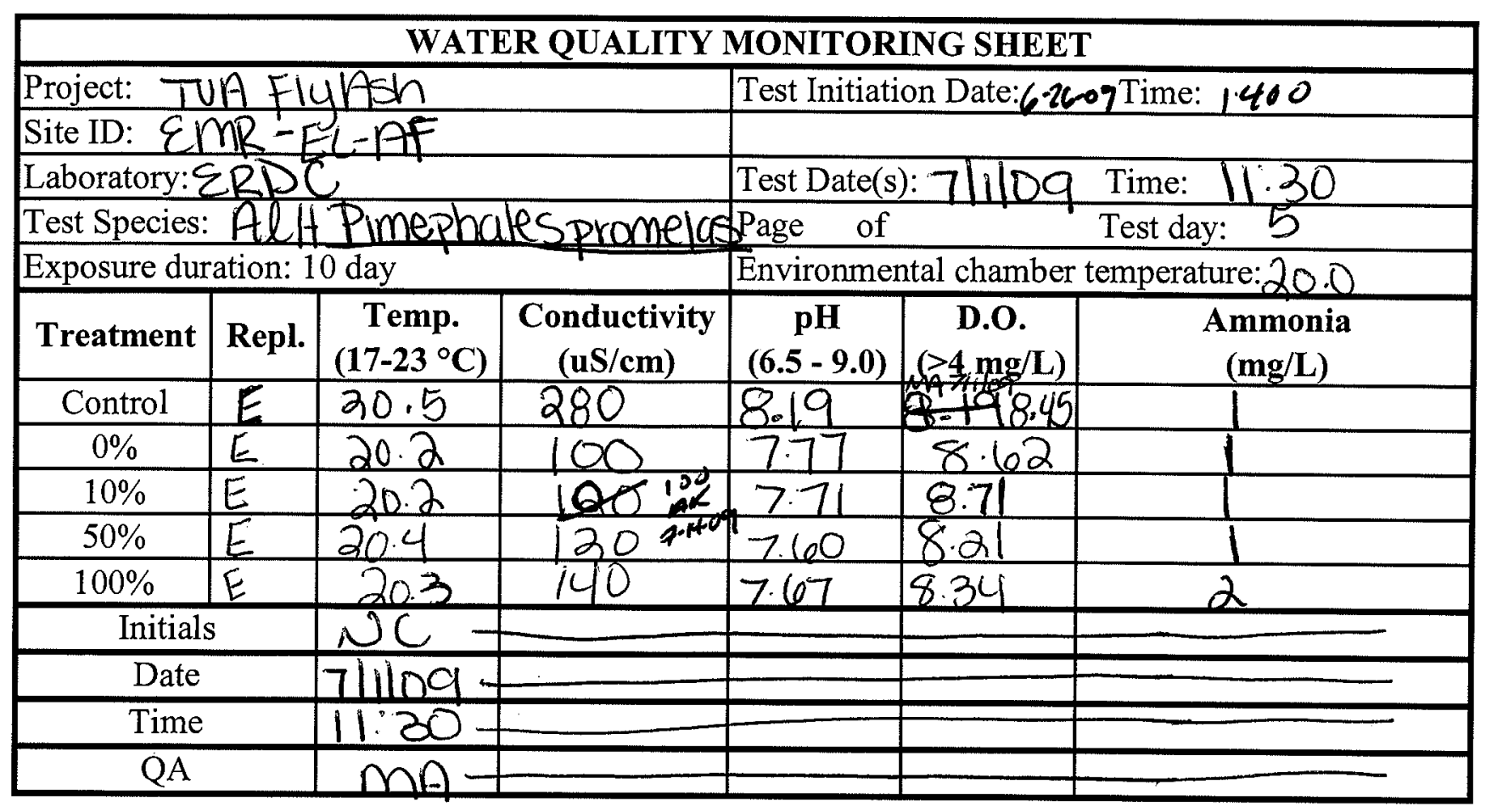

$1 k \quad 7-14-09$ 


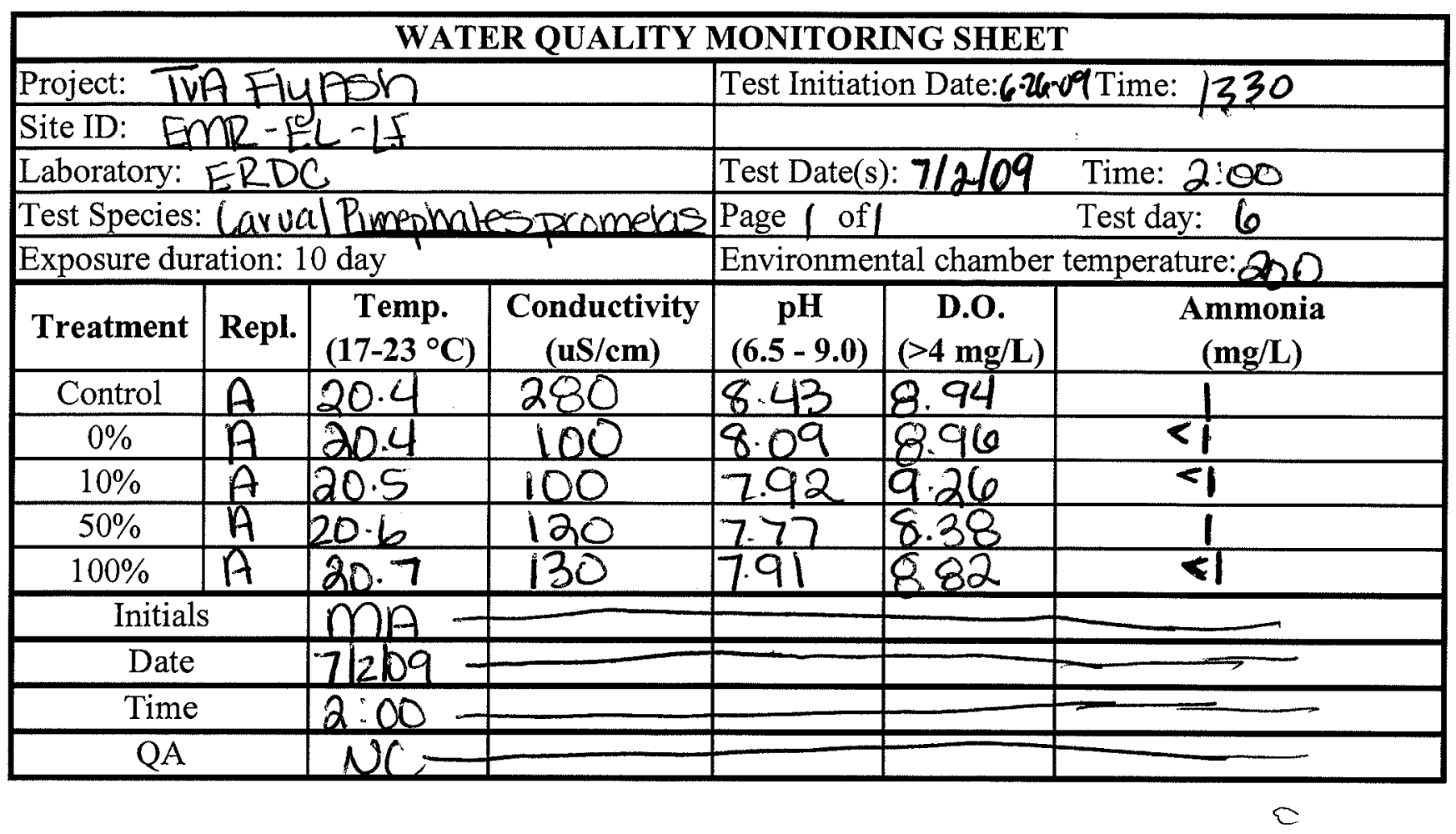

AK 7-14-09 


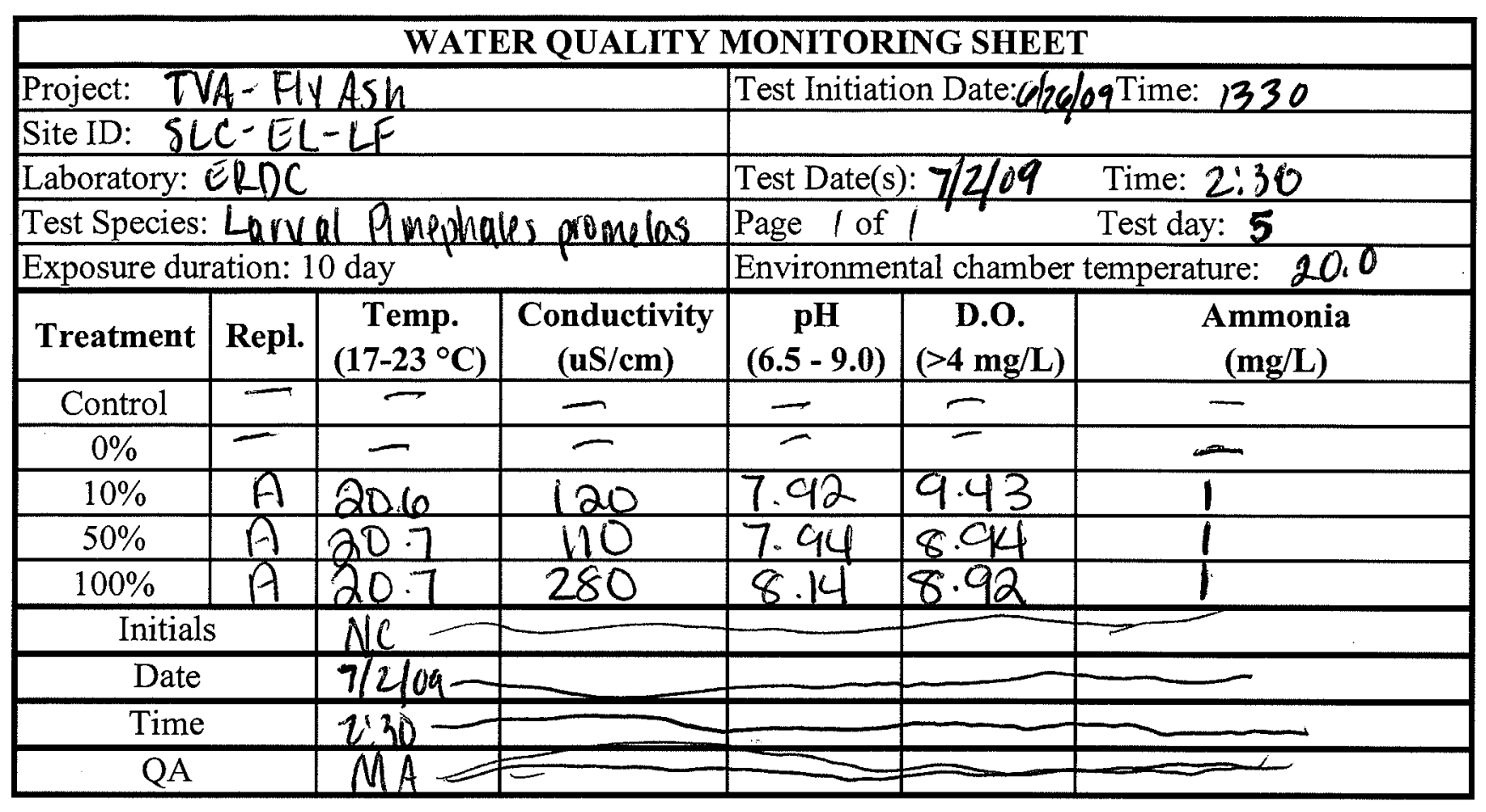

A) $\quad 7-14-09$ 


\begin{tabular}{|c|c|c|c|c|c|c|}
\hline \multicolumn{7}{|c|}{ WATER QUALITY MONITORING SHEET } \\
\hline \multicolumn{4}{|c|}{ Project: TUA fluAsh } & \multicolumn{3}{|c|}{ Test Initiation Date:6.26-09Time: 1400} \\
\hline \multicolumn{7}{|c|}{ Site ID:EMR-LL-Af } \\
\hline \multicolumn{4}{|c|}{ Laboratory: $\sum R P C$} & \multicolumn{3}{|c|}{ Test Date(s): $2 / 2 / 09$ Time: 3.00} \\
\hline \multicolumn{4}{|c|}{ Test Species:Adult Pumephales promelas } & \multicolumn{3}{|c|}{ Page 1 of $\quad$ Test day:S } \\
\hline \multicolumn{4}{|c|}{ Exposure duration: 10 day } & \multicolumn{3}{|c|}{ Environmental chamber temperature: $20 \mathrm{CO}$} \\
\hline Treatment & Repl. & $\begin{array}{c}\text { Temp. } \\
\left(17-23^{\circ} \mathrm{C}\right)\end{array}$ & $\begin{array}{c}\text { Conductivity } \\
\text { (uS/cm) }\end{array}$ & $\begin{array}{c}\mathrm{pH} \\
(6.5-9.0)\end{array}$ & $\begin{array}{c}\text { D.O. } \\
(>4 \mathrm{mg} / \mathrm{L})\end{array}$ & $\begin{array}{c}\text { Ammonia } \\
(\mathrm{mg} / \mathrm{L})\end{array}$ \\
\hline Control & $A$ & 21.1 & 300 & 8.22 & 9.31 & 2 \\
\hline $0 \%$ & A & 20.8 & 120 & 7.87 & 8.85 & 2 \\
\hline$\pi 2^{5}=50 \%$ & $A$ & 20.9 & 140 & 7.87 & 899 & \\
\hline $120 \% 0 \% 0 \%$ & $A$ & 20,3 & 110 & 7,87 & 8.95 & 2 \\
\hline $100 \%$ & $A$ & 20,3 & 140 & 7.86 & 8.86 & 2 \\
\hline \multicolumn{2}{|c|}{ Initials } & NA & & & & \\
\hline \multicolumn{2}{|l|}{ Date } & $712109=$ & & & & 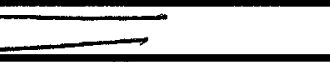 \\
\hline \multicolumn{2}{|l|}{ Time } & $3: 00$ & & & & \\
\hline \multicolumn{2}{|l|}{$\overline{\mathrm{QA}}$} & $N C$ & & & & \\
\hline
\end{tabular}

$$
\begin{aligned}
& M K \Rightarrow-14-09 \\
& \text { Note: All wlees } \\
& \text { switched. - blent } \\
& \text { fo- Sov \& 10\% }
\end{aligned}
$$




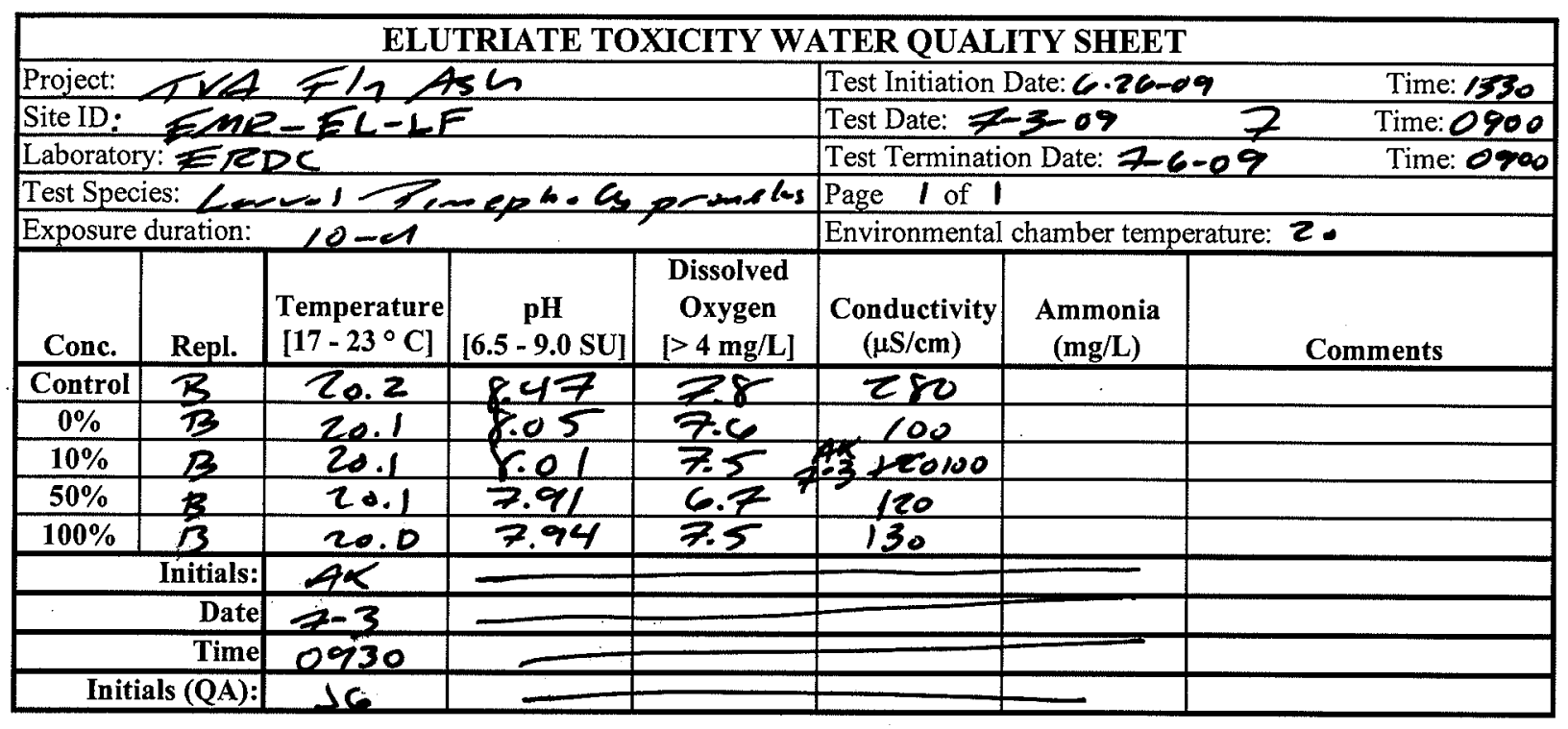

Reviewed by

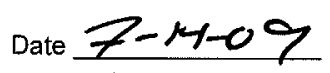

Comment 


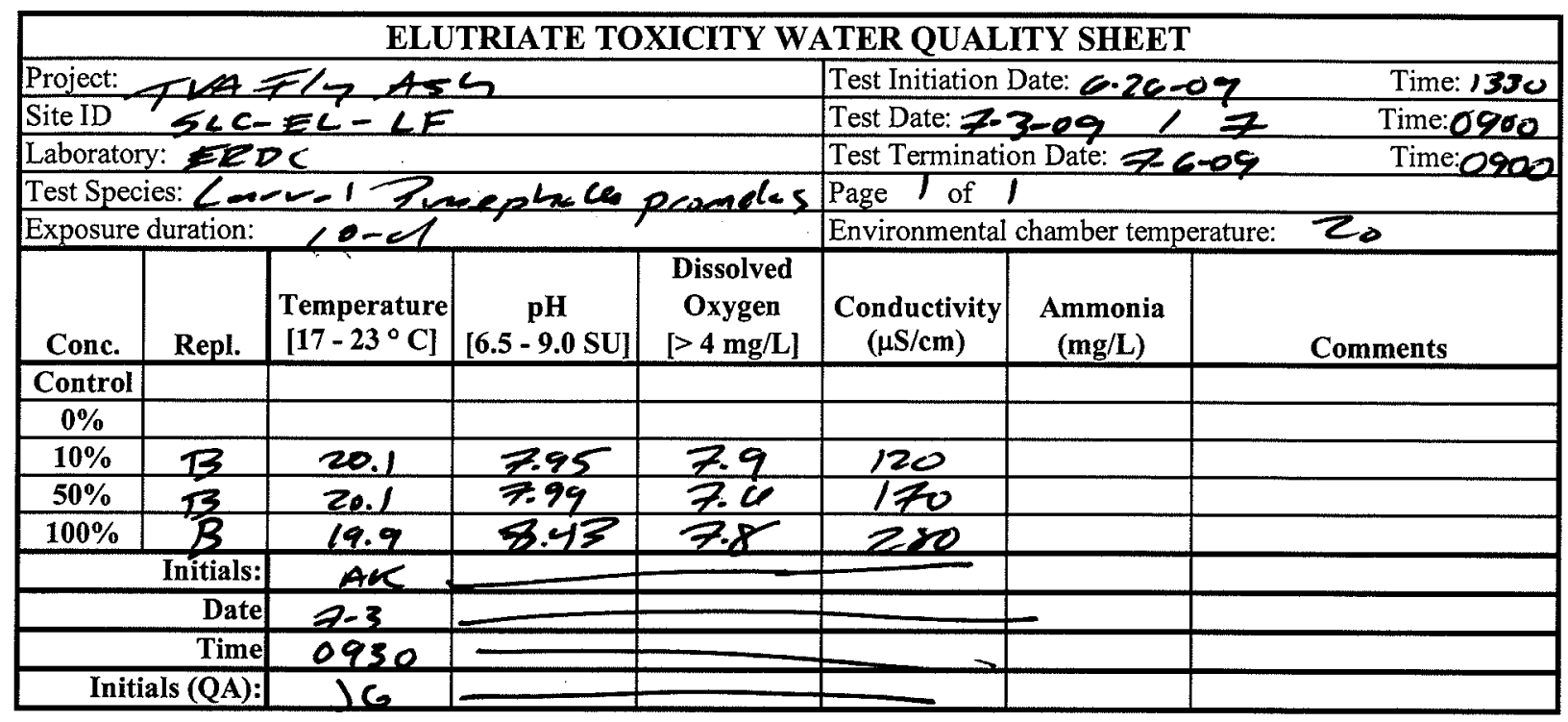

Reviewed by $2 / 2$

Date $7-11-09$ Comment 


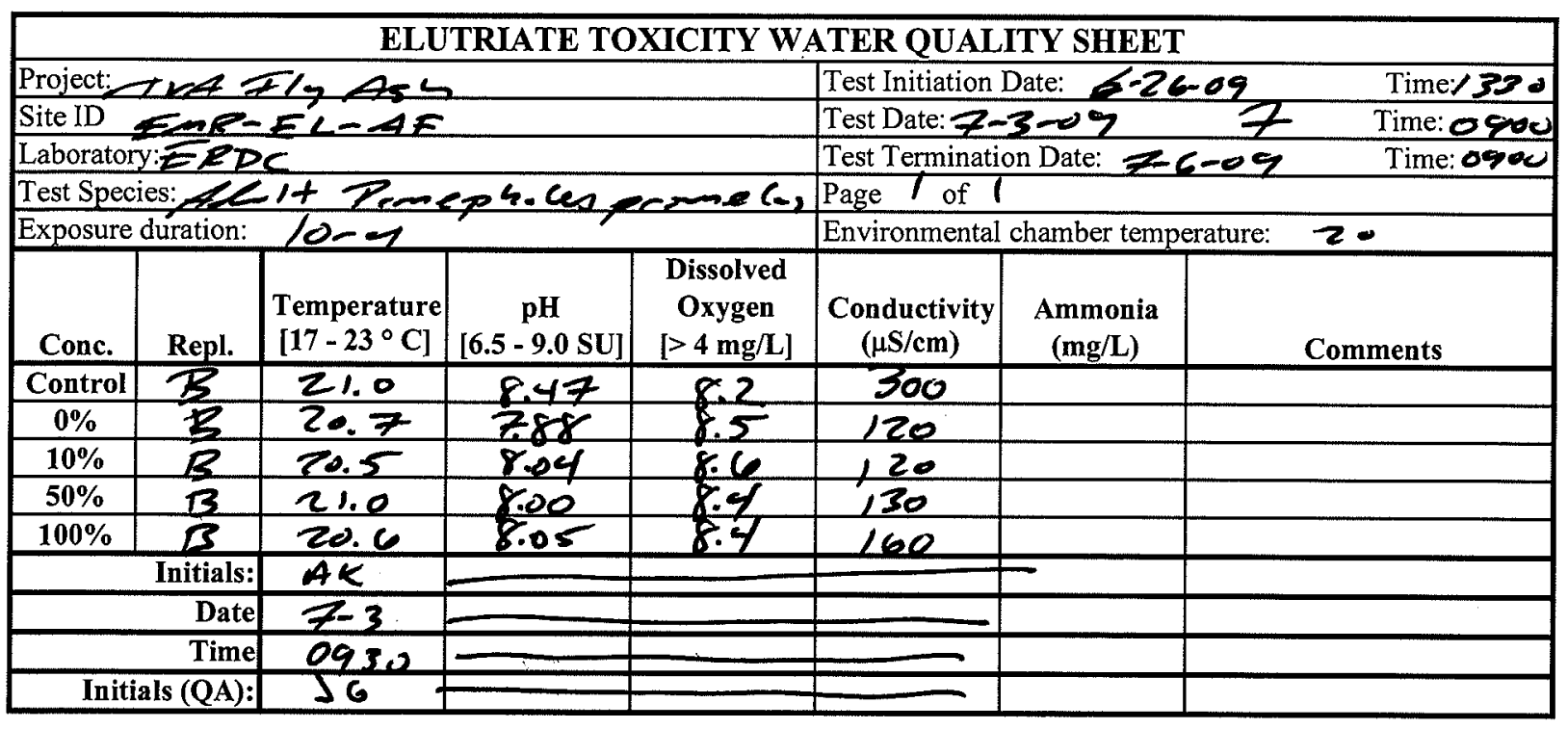

Reviewed by

Date $z-14-09$ Comment 


\begin{tabular}{|c|c|c|c|c|c|c|c|}
\hline \multicolumn{8}{|c|}{ ELUTRIATE TOXICITY WATER QUALITY SHEET } \\
\hline \multicolumn{5}{|c|}{ Project Tha $\% / 7$ As } & \multicolumn{3}{|c|}{ Test Initiation Date: $6-26-09 \quad$ Time:/330 } \\
\hline \multicolumn{5}{|c|}{ Site ID : $\leq M E-E C-\angle F$} & \multicolumn{3}{|c|}{ Test Date: $7-4-09 \quad \& \quad$ Time:1200 } \\
\hline \multicolumn{5}{|c|}{ Laboratory: $\approx \geq$} & \multicolumn{3}{|c|}{ Test Termination Date: $\geq 6-09$} \\
\hline \multicolumn{5}{|c|}{ 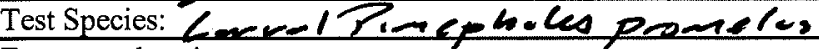 } & \multicolumn{3}{|c|}{ Page 1 of $\quad 1$} \\
\hline \multicolumn{2}{|c|}{ Exposure duration: } & \multicolumn{3}{|c|}{$10-2$} & \multicolumn{3}{|c|}{ Environmental chamber temperature: 20} \\
\hline Conc. & Repl. & $\begin{array}{c}\text { Temperature } \\
{\left[17-23^{\circ} \mathrm{C}\right]}\end{array}$ & $\begin{array}{c}\text { pH } \\
{[6.5-9.0 \mathrm{SU}]}\end{array}$ & $\begin{array}{c}\text { Dissolved } \\
\text { Oxygen } \\
{[>4 \mathrm{mg} / \mathrm{L}]}\end{array}$ & $\begin{array}{c}\text { Conductivity } \\
(\mu \mathrm{S} / \mathrm{cm})\end{array}$ & $\begin{array}{c}\text { Ammonia } \\
(\mathrm{mg} / \mathrm{L})\end{array}$ & Comments \\
\hline \begin{tabular}{|c|} 
Control \\
\end{tabular} & $C$ & 20,1 & 8.36 & C.9. & 260 & & \\
\hline $0 \%$ & C & 20.1 & 2.74 & 6.8 & 100 & & \\
\hline $10 \%$ & $\mathbf{C}$ & 20.1 & 786 & $\geq 0$ & 90 & & \\
\hline $50 \%$ & $C$ & 20.1 & 7.42 & 7.1 & 110 & & \\
\hline $100 \%$ & $C$ & 20.1 & 7.94 & 7.4 & 130 & & \\
\hline \multicolumn{2}{|r|}{ Initials: } & $2 k$ & & & & & \\
\hline \multicolumn{2}{|r|}{ Date } & 74 & & & & & \\
\hline \multicolumn{2}{|r|}{ Time } & 1200 & & & & & \\
\hline \multicolumn{2}{|c|}{ Initials (QA): } & Ak & & & & & \\
\hline
\end{tabular}

Reviewed by

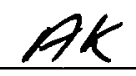




\begin{tabular}{|c|c|c|c|c|c|c|c|c|}
\hline \multicolumn{9}{|c|}{ ELUTRIATE TOXICITY WATER QUALITY SHEET } \\
\hline \multicolumn{5}{|c|}{ Project: TrA $Z / Z A=4$} & \multicolumn{4}{|c|}{ Test Initiation Date: $6.26 .09 \quad$ Time: $\boldsymbol{B} 30$} \\
\hline \multicolumn{5}{|c|}{ Site ID : $5 c c-1 c-L F$} & \multirow{2}{*}{\multicolumn{4}{|c|}{ Test Date: $7-4-09 \quad 8 \quad$ Time: 1700}} \\
\hline \multirow{2}{*}{\multicolumn{5}{|c|}{$\begin{array}{l}\text { Laboratory: EDPC } \\
\text { Test Species: Lerel Peneph as promeles }\end{array}$}} & & & & \\
\hline & & & & & \multicolumn{4}{|c|}{$\begin{array}{l}\text { Test Termination Date: } z-6-09 \text { Time: } 0900 \\
\text { Page l of }\end{array}$} \\
\hline \multicolumn{5}{|c|}{ Exposure duration: $10-d$} & \multicolumn{4}{|c|}{ Environmental chamber temperature: $>0$} \\
\hline Conc. & Repl. & $\begin{array}{c}\text { Temperature } \\
{\left[17-23^{\circ} \mathrm{C}\right]}\end{array}$ & {$\left[\begin{array}{c}\mathrm{pH} \\
{[6.5-9.0 \mathrm{Su}]}\end{array}\right]$} & $\begin{array}{c}\text { Dissolved } \\
\text { Oxygen } \\
{[>4 \mathrm{mg} / \mathrm{L}]}\end{array}$ & $\begin{array}{c}\text { Conductivity } \\
(\mu \mathrm{S} / \mathrm{cm})\end{array}$ & $\begin{array}{c}\text { Ammonia } \\
(\mathbf{m g} / \mathbf{L})\end{array}$ & & Comments \\
\hline \multicolumn{9}{|l|}{ Control } \\
\hline \multicolumn{9}{|l|}{$\mathbf{0 \%}$} \\
\hline $10 \%$ & C & 20.2 & 7.85 & 7.3 & 110 & & & \\
\hline $\mathbf{5 0 \%}$ & C & Zo.1 & 8.05 & 7.6 & 180 & & & \\
\hline $100 \%$ & C & 20.0 & 8.28 & 7.2 & 260 & & & \\
\hline & Initials: & ARK & & & & & & \\
\hline & Date & 7.4 & & & - & & & \\
\hline & Time & 1200 & & & - & & & \\
\hline & As (QA): & AK & & & & & & \\
\hline
\end{tabular}

Reviewed by

Date $Z-14-09$ Comment 


\begin{tabular}{|c|c|c|c|c|c|c|c|}
\hline \multicolumn{8}{|c|}{ ELUTRIATE TOXICITY WATER QUALITY SHEET } \\
\hline \multicolumn{5}{|c|}{ Project: Tw< Fl $\rightarrow$ \& 4} & \multicolumn{3}{|c|}{ Test Initiation Date: $\mathbf{E - 2 0 . 0 4}$ Timel 330} \\
\hline \multicolumn{5}{|c|}{ Site ID: $E M 2-E C-\alpha E$} & \multicolumn{3}{|c|}{ Test Date: $Z$ ZC/ } \\
\hline \multicolumn{5}{|c|}{ Laboratory: $=2 x$} & \multicolumn{3}{|c|}{ 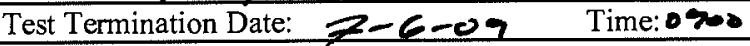 } \\
\hline \multicolumn{5}{|c|}{ 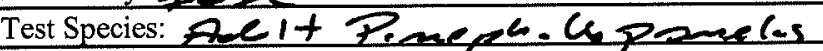 } & \multirow{2}{*}{\multicolumn{3}{|c|}{$\begin{array}{l}\text { Page lof } 1 \\
\text { Environmental chamber temperature: } \mathbf{2} 0\end{array}$}} \\
\hline \multicolumn{5}{|c|}{ Exposure duration: $/ \omega-7$} & & & \\
\hline Conc. & Repl. & $\begin{array}{l}\text { Temperature } \\
{\left[17-23^{\circ} \mathrm{C}\right]}\end{array}$ & $\begin{array}{c}\mathrm{pH} \\
{[6.5-9.0 \mathrm{SU}]}\end{array}$ & $\begin{array}{c}\text { Dissolved } \\
\text { Oxygen } \\
{[>4 \mathrm{mg} / \mathrm{L}]}\end{array}$ & $\begin{array}{c}\text { Conductivity } \\
(\mu \mathrm{S} / \mathrm{cm})\end{array}$ & $\begin{array}{c}\text { Ammonia } \\
(\mathrm{mg} / \mathrm{L})\end{array}$ & Comments \\
\hline Control & $c$ & 20.6 & 8.26 & 96 & 280 & & \\
\hline $0 \%$ & $C$ & $2 \cdot 5$ & 7.71 & 9.1 & 100 & & \\
\hline $10 \%$ & C & 2.4 & & 8.9 & 100 & & \\
\hline $\mathbf{5 0 \%}$ & $\bar{S}$ & 20.9 & 7.73 & 8.6 & 120 & & \\
\hline $100 \%$ & & 23.4 & 794 & 91 & 130 & & \\
\hline & Initials: & $A K$ & & & 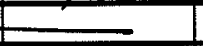 & & \\
\hline & Date & $=2.4$ & & & $=$ & & \\
\hline & Time & 120 & & & & & \\
\hline Initi & als (QA): & $A K$ & & & & & \\
\hline
\end{tabular}

Reviewed by

Ak

Date $7-14-09$

Comment 


\begin{tabular}{|c|c|c|c|c|c|c|c|}
\hline \multicolumn{8}{|c|}{ ELUTRIATE TOXICITY WATER QUALITY SHEET } \\
\hline \multicolumn{5}{|c|}{ Project: TuPE/AASh } & \multicolumn{3}{|c|}{ Test Initiation Date: $6.26-09 \quad$ Time: 1330} \\
\hline \multicolumn{5}{|c|}{ Site ID : $=M E-E L-\angle F$} & \multicolumn{3}{|c|}{ Test Date/Day: 7 Seq 9 Time: 1130} \\
\hline \multicolumn{5}{|c|}{ Laboratory: } & \multicolumn{3}{|c|}{ Test Termination Date: $>-6-09 \quad$ Time: 0900} \\
\hline \multicolumn{5}{|c|}{ Test Species: Lewen I Pimeph.ap permels } & \multicolumn{3}{|c|}{ Page / of $/$} \\
\hline \multicolumn{5}{|c|}{ Exposure duration: $10-1$} & \multicolumn{3}{|c|}{ Environmental chamber temperature: $\mathbf{2 0}$} \\
\hline Conc. & Repl. & $\begin{array}{l}\text { Temperature } \\
{\left[17-23^{\circ} \mathrm{C}\right]}\end{array}$ & $\begin{array}{c}\mathrm{pH} \\
{[6.5-9.0 \mathrm{SU}]}\end{array}$ & $\begin{array}{c}\text { Dissolved } \\
\text { Oxygen } \\
{[>4 \mathrm{mg} / \mathrm{L}]}\end{array}$ & $\begin{array}{c}\text { Conductivity } \\
(\mu \mathrm{S} / \mathrm{cm})\end{array}$ & $\begin{array}{c}\text { Ammonia } \\
(\mathrm{mg} / \mathrm{L})\end{array}$ & Comments \\
\hline Control & $D$ & 20.3 & 8.17 & 6.9 & 270 & $\angle 1$ & \\
\hline $0 \%$ & $\mathcal{D}$ & 20.1 & $\frac{0}{7.61}$ & 6.9 & 100 & $<1$ & \\
\hline $10 \%$ & $P$ & 20.1 & 7.60 & 6.6 & 100 & $\leq 1$ & \\
\hline $50 \%$ & $D$ & 20.1 & 7.46 & 6.4 & 110 & $\leq 1$ & \\
\hline $100 \%$ & $D$ & 20.0 & 7.73 & 6.7 & 130 & 41 & \\
\hline \multicolumn{2}{|r|}{ Initials: } & $A K$ & & & & & \\
\hline \multicolumn{2}{|r|}{ Date } & $7-5$ & & & & & \\
\hline \multicolumn{2}{|r|}{ Time } & 1130 & & & & & \\
\hline Initi & Is (QA): & $A K$ & & & & & \\
\hline
\end{tabular}

Reviewed by

Date $7-14-09$

Comment 


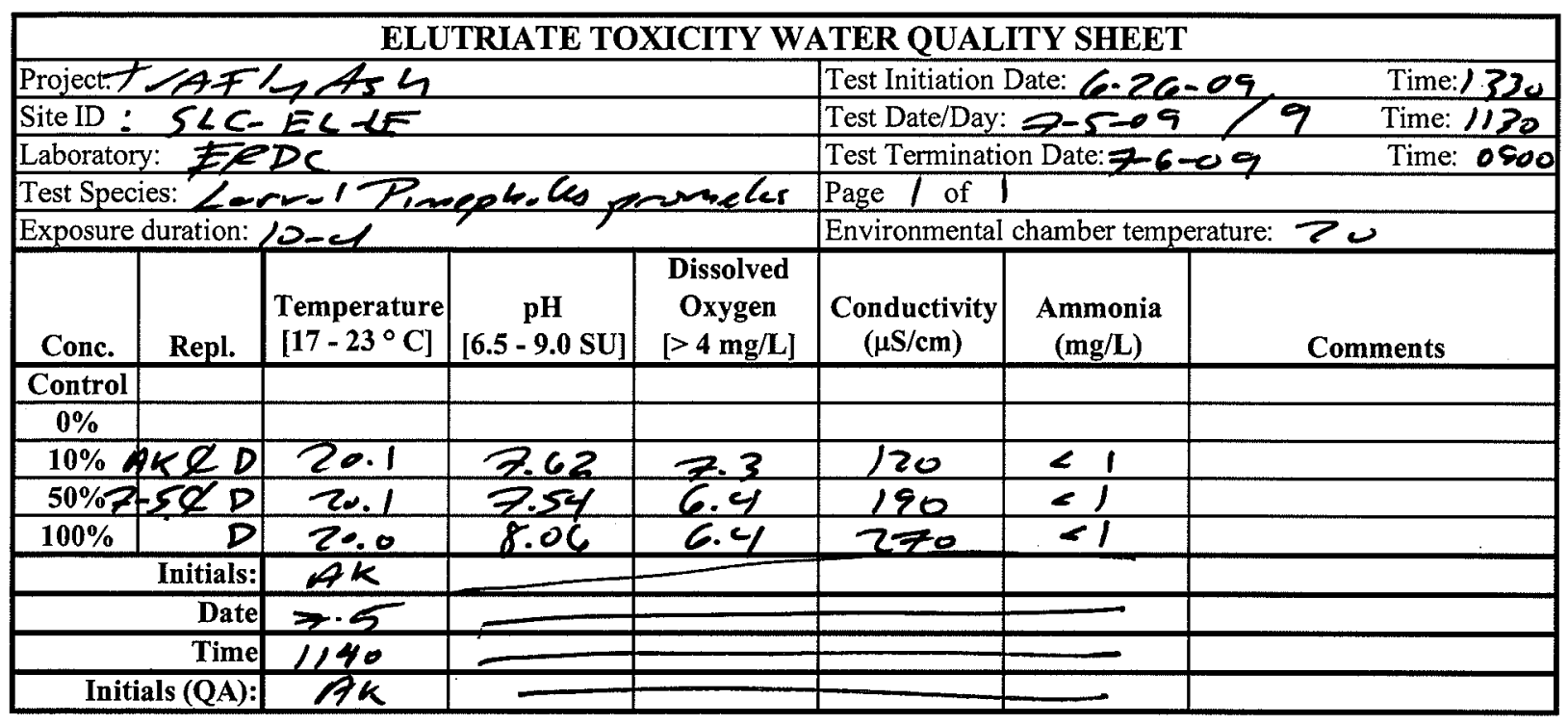

Reviewed by 


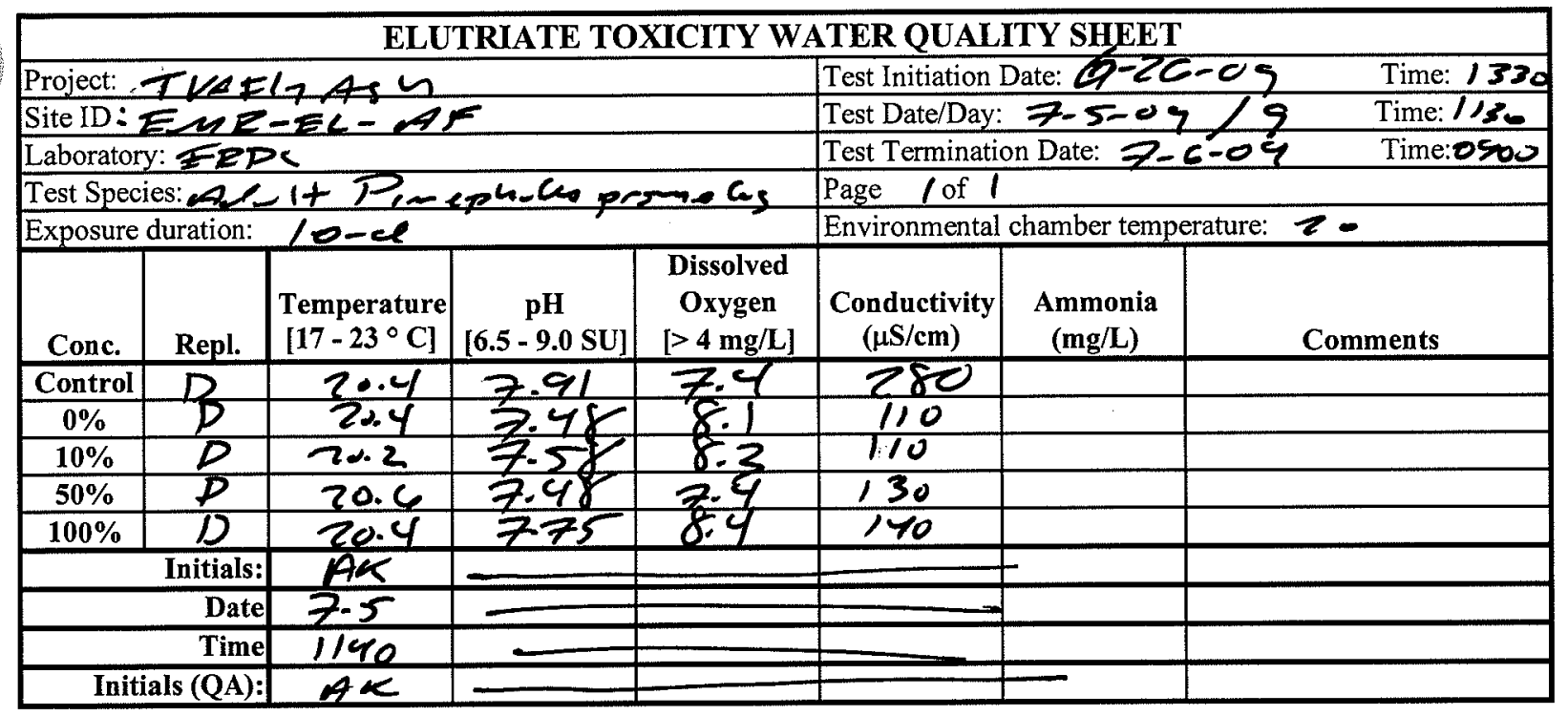

Reviewed by AK

Date $721 \% 0 \%$

Comment 


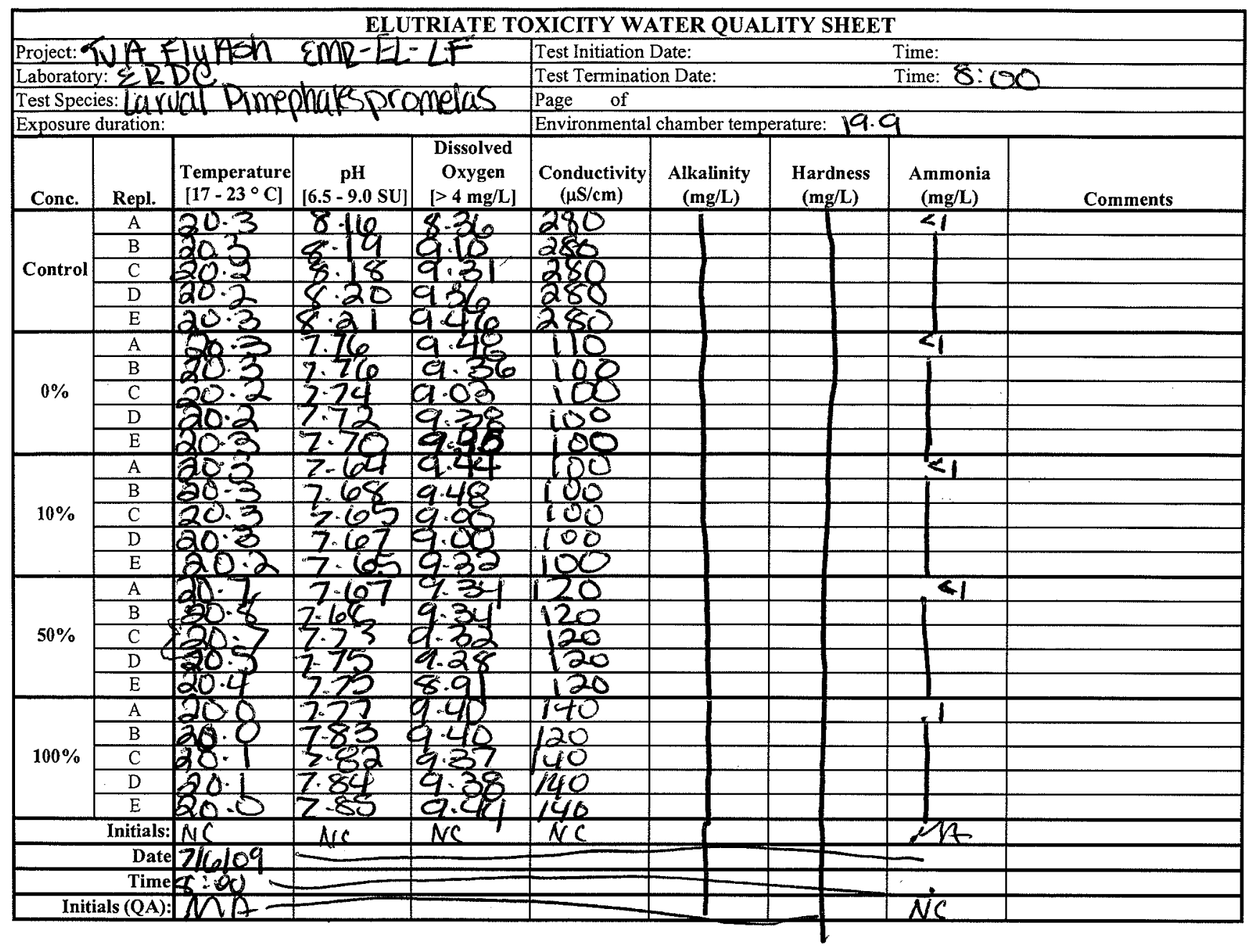

Reviewed by

Date

Comment 


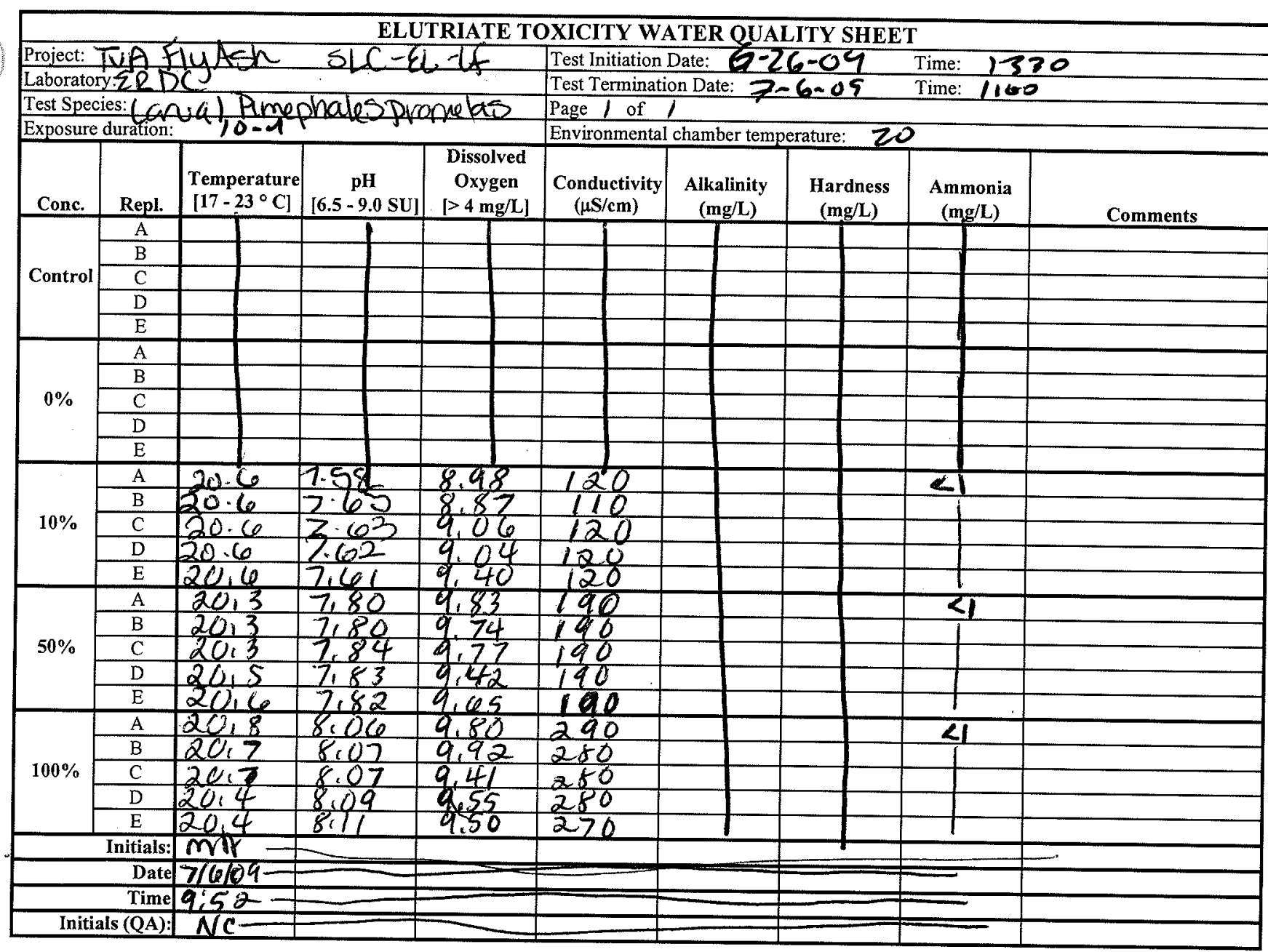

Reviewed by $4 k$ Date 7.14 .07 Comment 


\begin{tabular}{|c|c|c|c|c|c|c|c|c|c|}
\hline \multicolumn{10}{|c|}{ ELUTRIATE TOXICITY WATER QUALITY SHEET } \\
\hline \multirow{2}{*}{\multicolumn{5}{|c|}{$\begin{array}{l}\text { Project: TVA FlVA } \\
\text { Laboratory: } \angle R D C\end{array}$}} & \multicolumn{5}{|c|}{ Test Initiation Date: $6.26-09$ Time: } \\
\hline & & & & & \multicolumn{3}{|c|}{ Test Termination Date: $7-6-07$} & \multicolumn{2}{|c|}{ Time: 1100} \\
\hline \multicolumn{5}{|c|}{ Test Species: Adult Pimepholes promeles } & \multicolumn{5}{|c|}{ Page | of | } \\
\hline \multicolumn{5}{|c|}{ Exposure duration: $\quad 1,2-\mathrm{Cl}$} & \multicolumn{5}{|c|}{ Environmental chamber temperature: 20} \\
\hline Conc. & Repl. & $\begin{array}{c}\text { Temperature } \\
{\left[17-23^{\circ} \mathrm{C}\right]} \\
\end{array}$ & \begin{tabular}{|c|}
$\mathrm{pH}$ \\
{$[6.5-9.0 \mathrm{SU}]$} \\
\end{tabular} & $\begin{array}{c}\text { Dissolved } \\
\text { Oxygen } \\
{[>4 \mathrm{mg} / \mathrm{L}]}\end{array}$ & $\begin{array}{c}\begin{array}{c}\text { Conductivity } \\
(\mu \mathrm{S} / \mathrm{cm})\end{array} \\
\end{array}$ & $\begin{array}{c}\text { Alkalinity } \\
\text { (mg/L) }\end{array}$ & $\begin{array}{c}\text { Hardness } \\
(\mathrm{mg} / \mathrm{L})\end{array}$ & $\begin{array}{c}\text { Ammonia } \\
(\mathrm{mg} / \mathrm{L})\end{array}$ & Comments \\
\hline \multirow{4}{*}{ Control } & $\mathrm{A}$ & 20.8 & 96 & 9,04 & 300 & $=$ & & 9 & \\
\hline & $\mathrm{B}$ & 20 & & 27 & 70 & & & & \\
\hline & $\frac{C}{D}$ & 20. & & 9.20 & 9 & & & & \\
\hline & $\frac{\mathrm{D}}{\mathrm{E}}$ & $\frac{20}{20}$ & & $\frac{9.08}{9.36}$ & $\frac{290}{290}$ & & & & \\
\hline \multirow{5}{*}{$0 \%$} & $\frac{\mathrm{L}}{\mathrm{A}}$ & 20,5 & $\frac{11}{72}$ & $\frac{4,30}{4,37}$ & $\frac{290}{110}$ & & & & \\
\hline & $\mathrm{B}$ & 20,5 & & 9.63 & 0 & & & & \\
\hline & C & 20. & 66 & 9,48 & 0 & & & & \\
\hline & $\mathrm{D}$ & 20 & 62 & 9 & & & & & \\
\hline & E & 20 & 64 & 4,42 & 0 & & & & \\
\hline \multirow{4}{*}{$10 \%$} & $\frac{\mathrm{A}}{\mathrm{B}}$ & 20,2 & 100 & 27 & 110 & & & 1 & \\
\hline & $\frac{\mathrm{B}}{\mathrm{C}}$ & $\frac{20.4}{20.3}$ & 62 & 9.52 & 110 & & & & \\
\hline & $\frac{\mathrm{C}}{\mathrm{D}}$ & $\frac{20,3}{20,2}$ & 56 & 9.38 & 120 & & & & \\
\hline & $\frac{\mathrm{D}}{\mathrm{E}}$ & $\frac{20,2}{9}$ & 7.66 & 9,24 & 110 & & & & \\
\hline \multirow{5}{*}{$50 \%$} & $\frac{E}{A}$ & $\frac{2}{2}$ & $\frac{7.65}{7.66}$ & $\frac{9.37}{9.08}$ & 1.20 & A & & & \\
\hline & $\frac{\mathrm{A}}{\mathrm{B}}$ & $\frac{2}{2}$ & $\frac{7.68}{7.59}$ & 9.08 & BO4 & & & 1 & \\
\hline & $\mathrm{C}$ & 20y & $\frac{11}{7} 60$ & 9,57 & 40 & & & 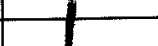 & \\
\hline & $\mathrm{D}$ & 20.5 & 7.62 & 9,19 & 130 & & & & \\
\hline & $\bar{E}$ & 20,5 & 7.64 & 9,22 & 1460 & & & & \\
\hline \multirow{5}{*}{$100 \%$} & $\mathrm{~A}$ & 2015 & 2,59 & 9,33 & 150 & & & 2 & \\
\hline & $\mathrm{B}$ & 20.5 & 7.71 & 9123 & & & & & \\
\hline & $\mathrm{C}$ & 20.4 & 174 & 9.08 & 140 & & & & \\
\hline & $\mathrm{D}$ & 20,4 & 7,71 & 9.27 & 150 & & & & \\
\hline & $\mathrm{E}$ & 20,4 & 7.75 & 9,48 & 150 & & 1 & & \\
\hline \multirow{2}{*}{\multicolumn{10}{|c|}{ Initials: $\mathrm{MT}=$}} \\
\hline & Date & $7 / 6109$ & & & & & & & \\
\hline & Time & $11: \infty$ & & & & & & & $=$ \\
\hline & QA): & $N C$ & & - & 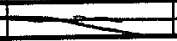 & & & 1 & \\
\hline
\end{tabular}

Reviewed by $A K$

Date $7.14-09$ comment

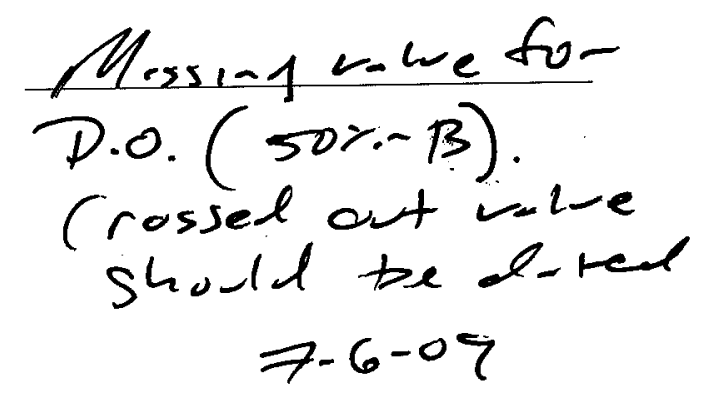




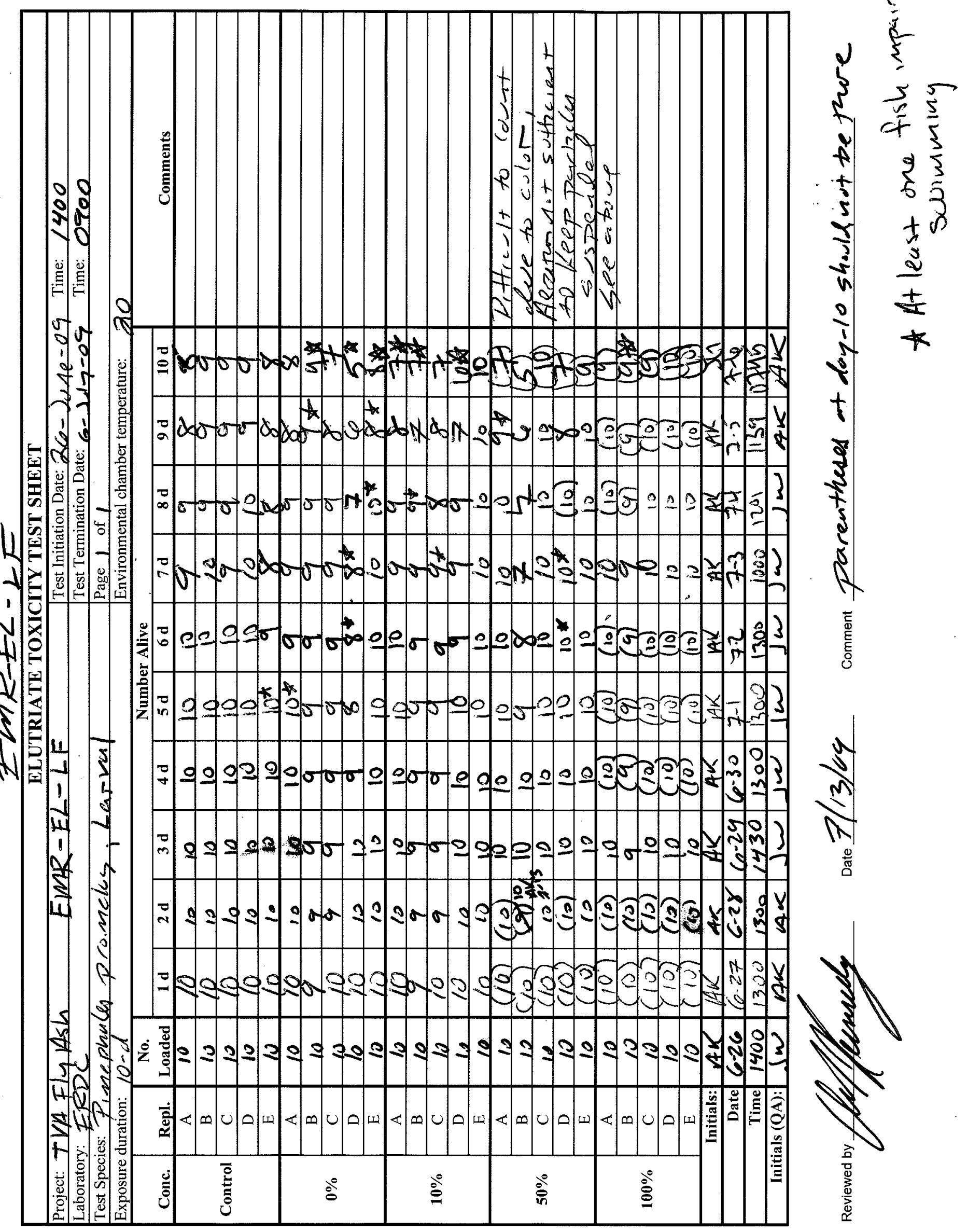




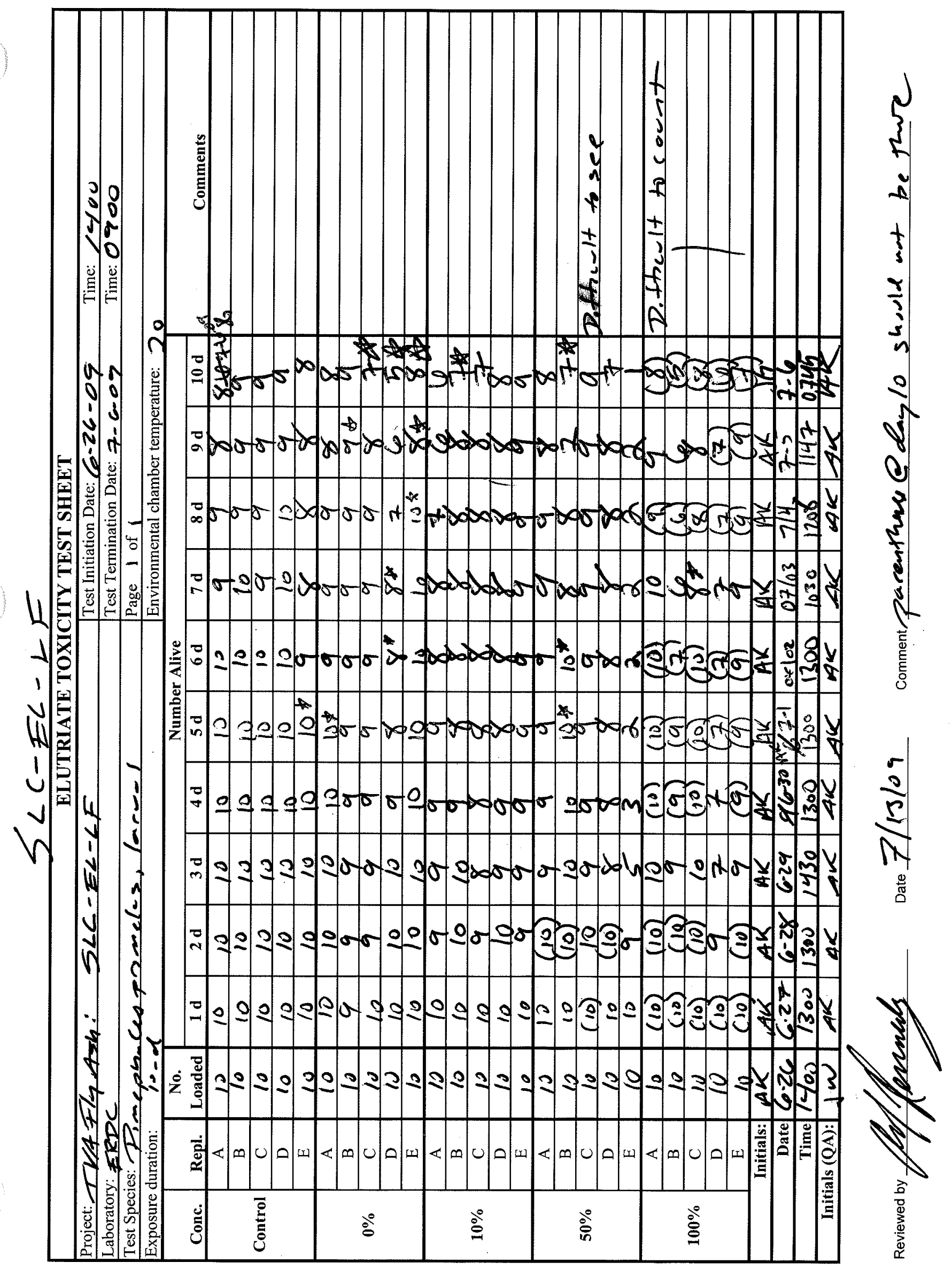




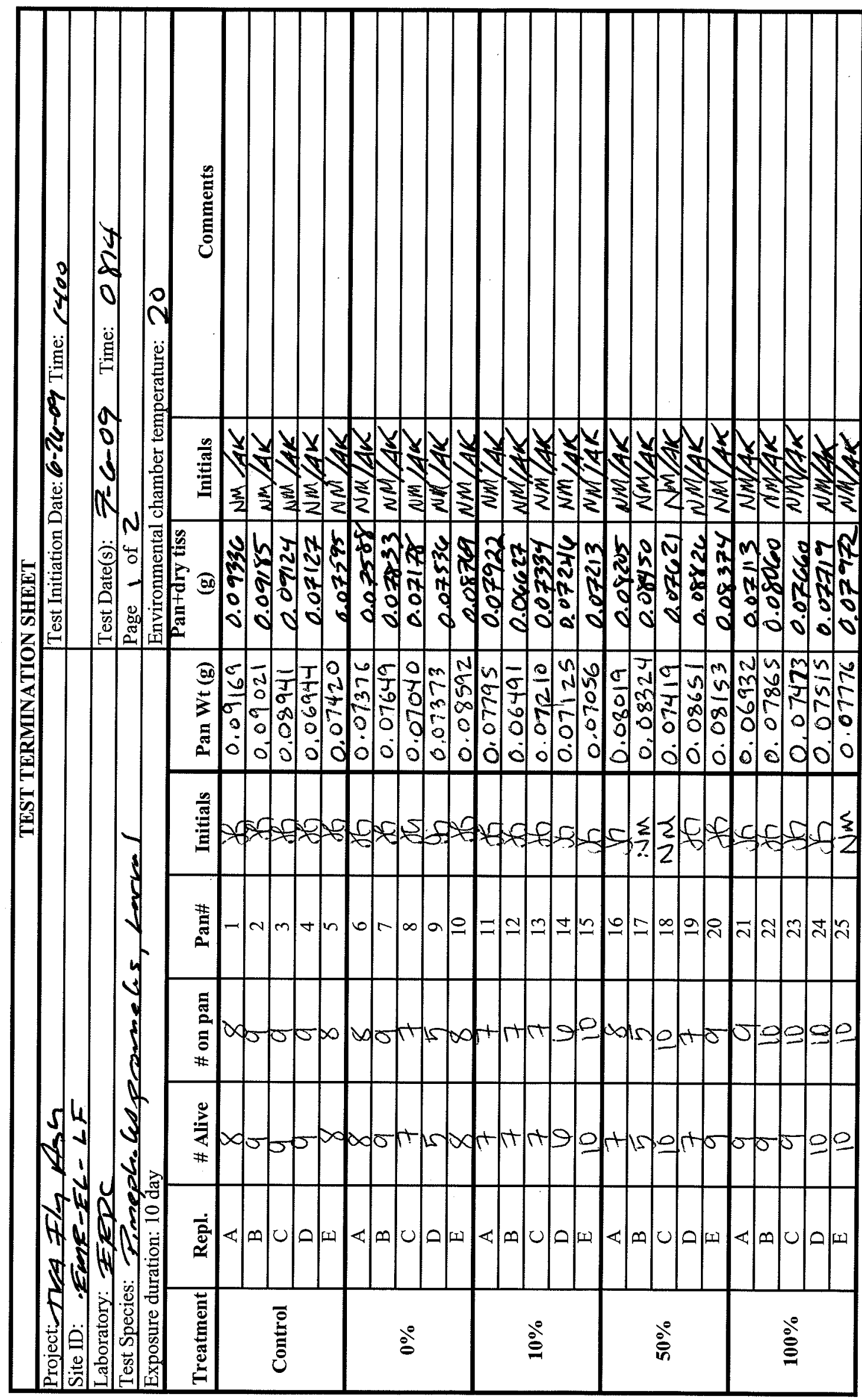

$\frac{5}{9}$

$\frac{v}{q}$ 


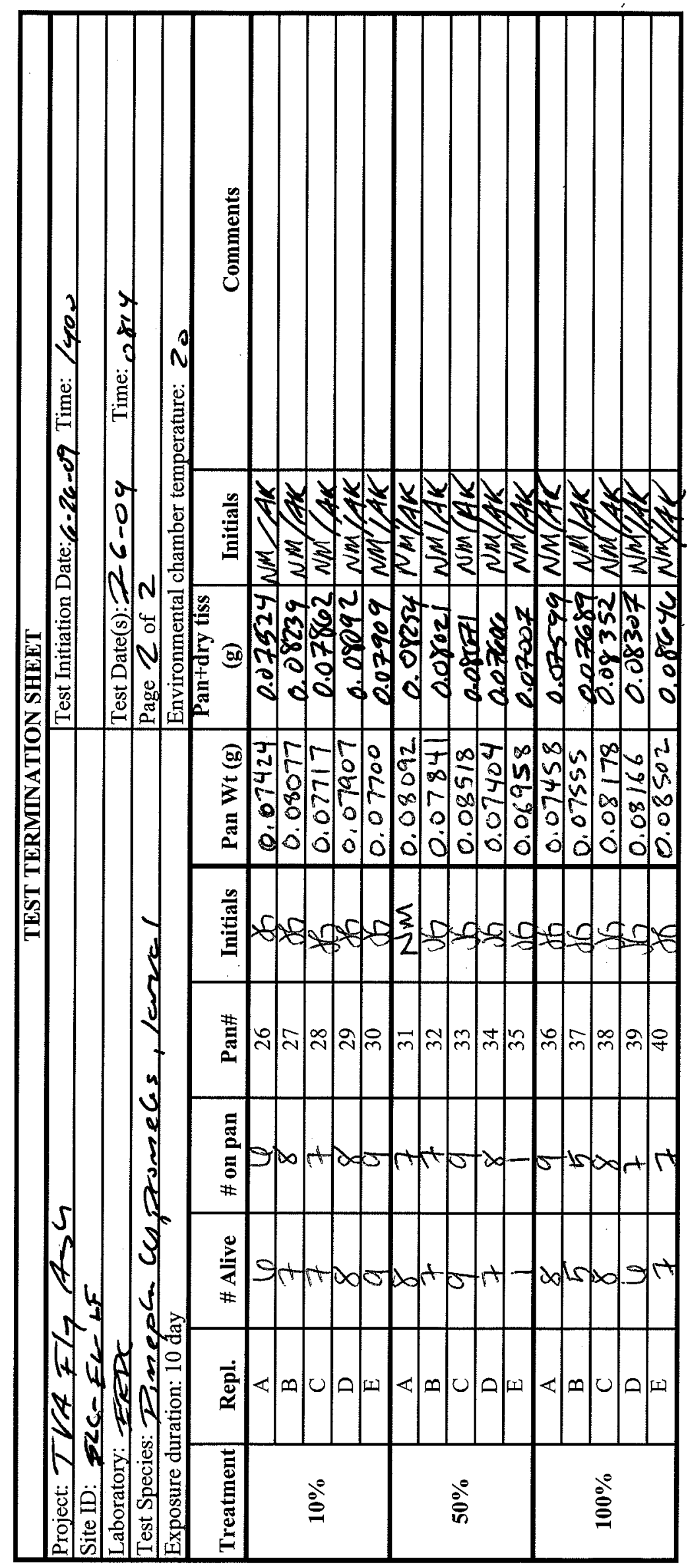




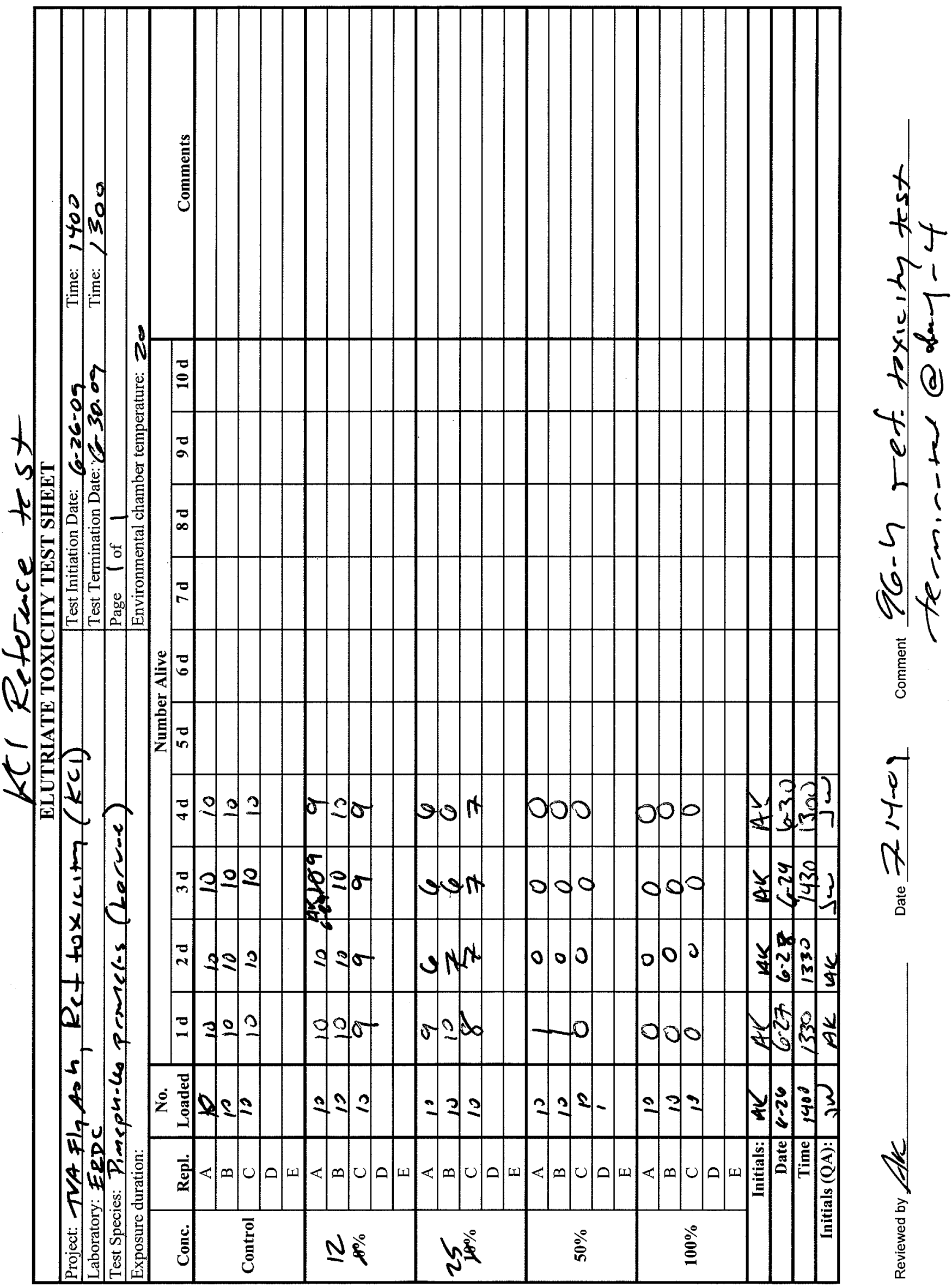




\section{KCI Peference toxish tost}

\begin{tabular}{|c|c|c|c|c|c|c|c|c|c|}
\hline \multicolumn{10}{|c|}{ ELUTRIATE TOXICITY WATER QUALITY SHEET } \\
\hline \multirow{3}{*}{\multicolumn{5}{|c|}{ 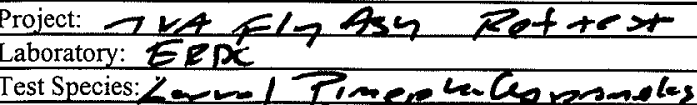 }} & \multirow{2}{*}{\multicolumn{3}{|c|}{$\begin{array}{l}\text { Test Initiation Date: } \quad 6.26-04 \\
\text { Test Termination Date: } 6.30-07 \\
\end{array}$}} & \multirow{2}{*}{\multicolumn{2}{|c|}{ Time: /Yoo }} \\
\hline & & & & & & & & & \\
\hline & & & & & \multirow{2}{*}{\multicolumn{5}{|c|}{\begin{tabular}{|l|} 
Page 1 of 1 \\
Environmental chamber temperature:
\end{tabular}}} \\
\hline \multicolumn{5}{|c|}{ 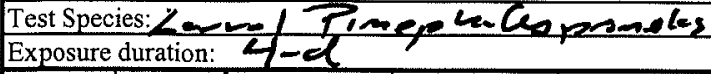 } & & & & & \\
\hline Conc. & Repl. & \begin{tabular}{|l} 
Temperature \\
{$\left[17-23^{\circ} \mathrm{C}\right]$}
\end{tabular} & $\begin{array}{c}\mathrm{pH} \\
{[6.5-9.0 \mathrm{SU}]} \\
\end{array}$ & $\begin{array}{c}\text { Dissolved } \\
\text { Oxygen } \\
{[>4 \mathrm{mg} / \mathrm{L}]} \\
\end{array}$ & $\begin{array}{c}\begin{array}{c}\text { Conductivity } \\
(\mu \mathrm{S} / \mathrm{cm})\end{array} \\
\end{array}$ & $\begin{array}{c}\text { Alkalinity } \\
(\mathrm{mg} / \mathrm{L})\end{array}$ & $\begin{array}{c}\text { Hardness } \\
(\mathrm{mg} / \mathrm{L})\end{array}$ & $\begin{array}{c}\text { Ammonia } \\
(\mathrm{mg} / \mathrm{L})\end{array}$ & Comments \\
\hline \multirow{4}{*}{ Control } & $\mathrm{A}$ & & & & & & & & \\
\hline & $\mathrm{B}$ & & & & & & & E & \\
\hline & $\frac{C}{D}$ & & & & & & & & \\
\hline & $\mathrm{E}$ & & & & & & 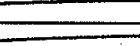 & $\sqrt{7}$ & $E$ \\
\hline \multirow{4}{*}{$12 \%$} & $\bar{A}$ & 20.8 & 8.00 & 8.35 & वा० & & $=$ & 21 & \\
\hline & $B$ & 20.1 & 8.02 & & 900 & & $=$ & $<1$ & \\
\hline & $\mathrm{C}$ & 20.9 & 8.03 & 32 & 880 & & $=$ & $<1$ & \\
\hline & $\frac{D}{F}$ & & & & & & & $\Longleftarrow$ & \\
\hline \multirow{5}{*}{$25 \%$} & $\frac{1}{4}$ & & & & & & & & \\
\hline & $\frac{A}{D}$ & 20.6 & & & 1490 & & $=$ & $<1$ & \\
\hline & $\frac{B}{C}$ & $\frac{20}{n a}$ & & 41 & 1530 & $=$ & $\longrightarrow$ & $<1$ & \\
\hline & $\frac{C}{D}$ & 00.8 & 8.02 & 8.39 & 1440 & & - & $<1$ & \\
\hline & $E$ & & & & & & & & \\
\hline \multirow{5}{*}{$\mathbf{5 0} \%$} & $\bar{A}$ & $20 \cdot 8$ & 8.25 & 8.88 & ouex & 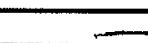 & - & टा & \\
\hline & $\mathrm{B}$ & 20.8 & 8.27 & 8.65 & Ger & $\equiv$ & $\underline{E}$ & 21 & \\
\hline & $\mathrm{C}$ & 20.9 & 8.28 & 8.72 & over & $=$ & $=$ & $<1$ & \\
\hline & D & & & & & & & & $=$ \\
\hline & $E$ & & & & & & & & 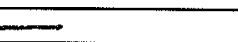 \\
\hline \multirow{5}{*}{$100 \%$} & $\mathrm{~A}$ & 20.9 & $8 \cdot 20$ & 8.82 & over & $=$ & $=$ & 21 & \\
\hline & $\mathrm{B}$ & 20.9 & 8.20 & 8.83 & ouer & & $\overline{-}$ & $\mathrm{Ci}$ & \\
\hline & $\mathrm{C}$ & 21.0 & 8.23 & 8.47 & over & & & 4 & \\
\hline & $\mathrm{D}$ & & & & & & & & \\
\hline & E & & & & & & & $=$ & \\
\hline \multirow{2}{*}{\multicolumn{2}{|c|}{ Initials: }} & $m A=$ & & & & & & $=$ & \\
\hline \multirow{2}{*}{\multicolumn{10}{|c|}{ Time 12200}} \\
\hline & & & & & & & & & \\
\hline Initi & als $(\mathrm{QA}):$ & $N C$ & $\overline{N C}$ & $N C$ & $N C$ & $x$ & 715 & NG & \\
\hline
\end{tabular}

Reviewed by $A k$ Date 7.14-09 Comment outweter chands 


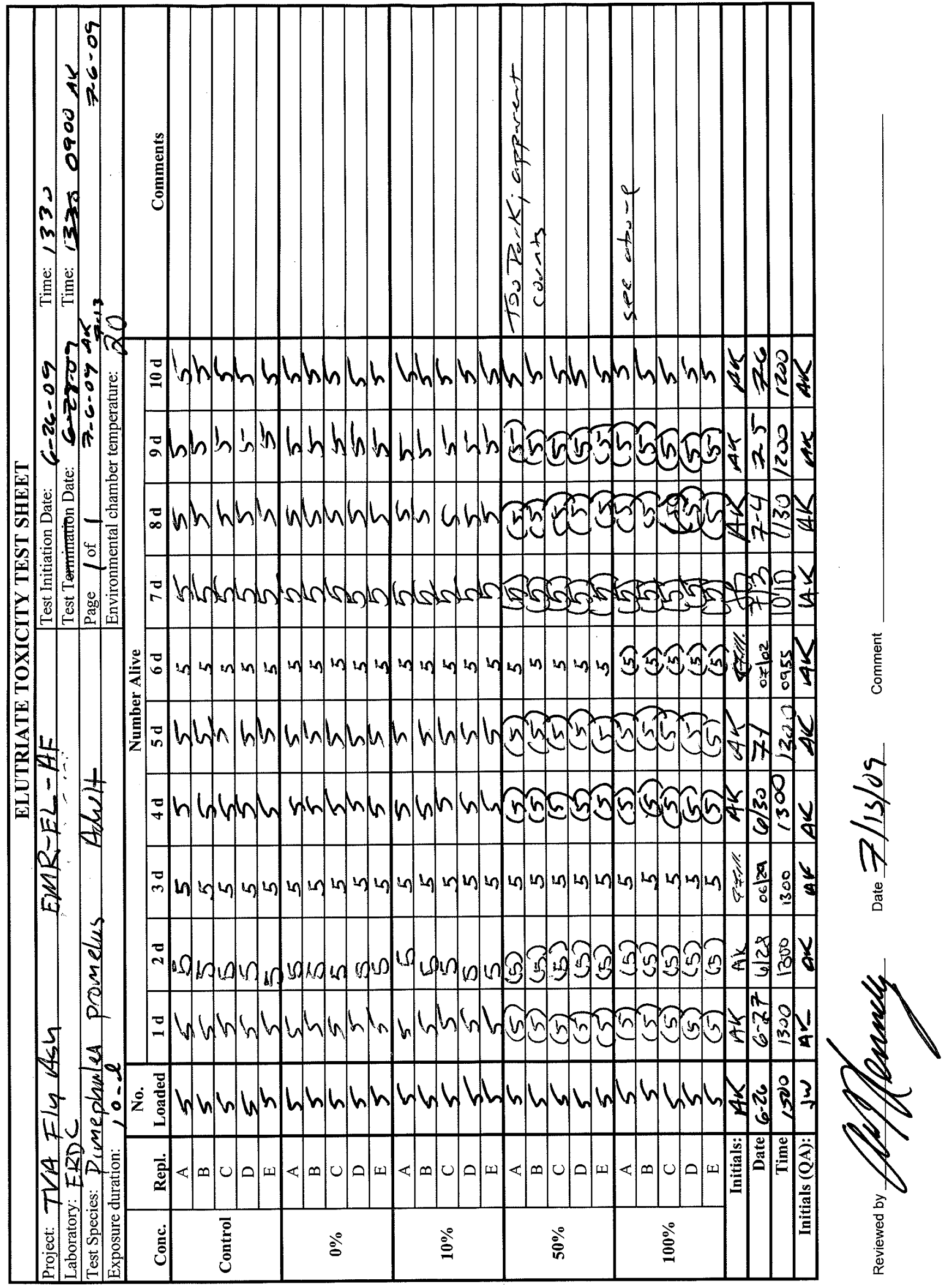




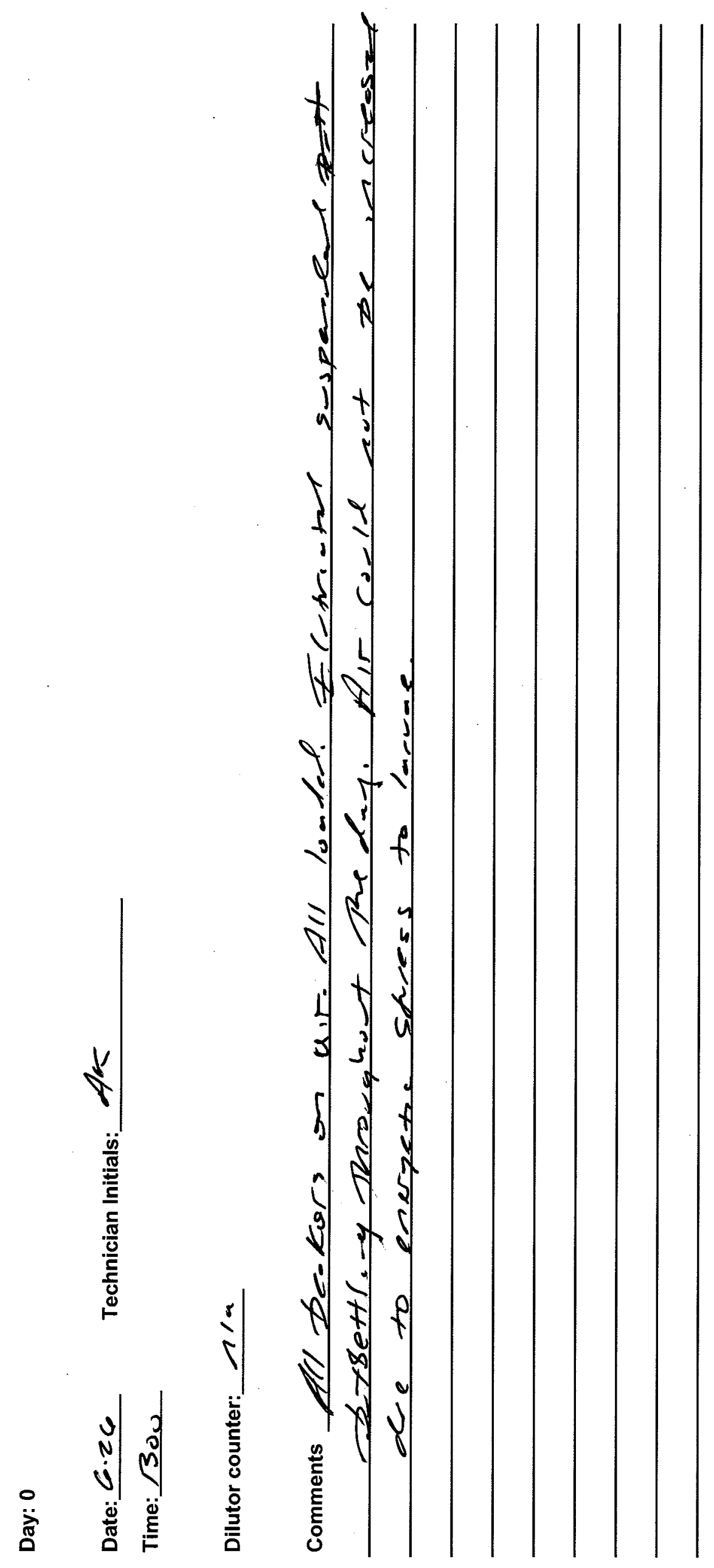




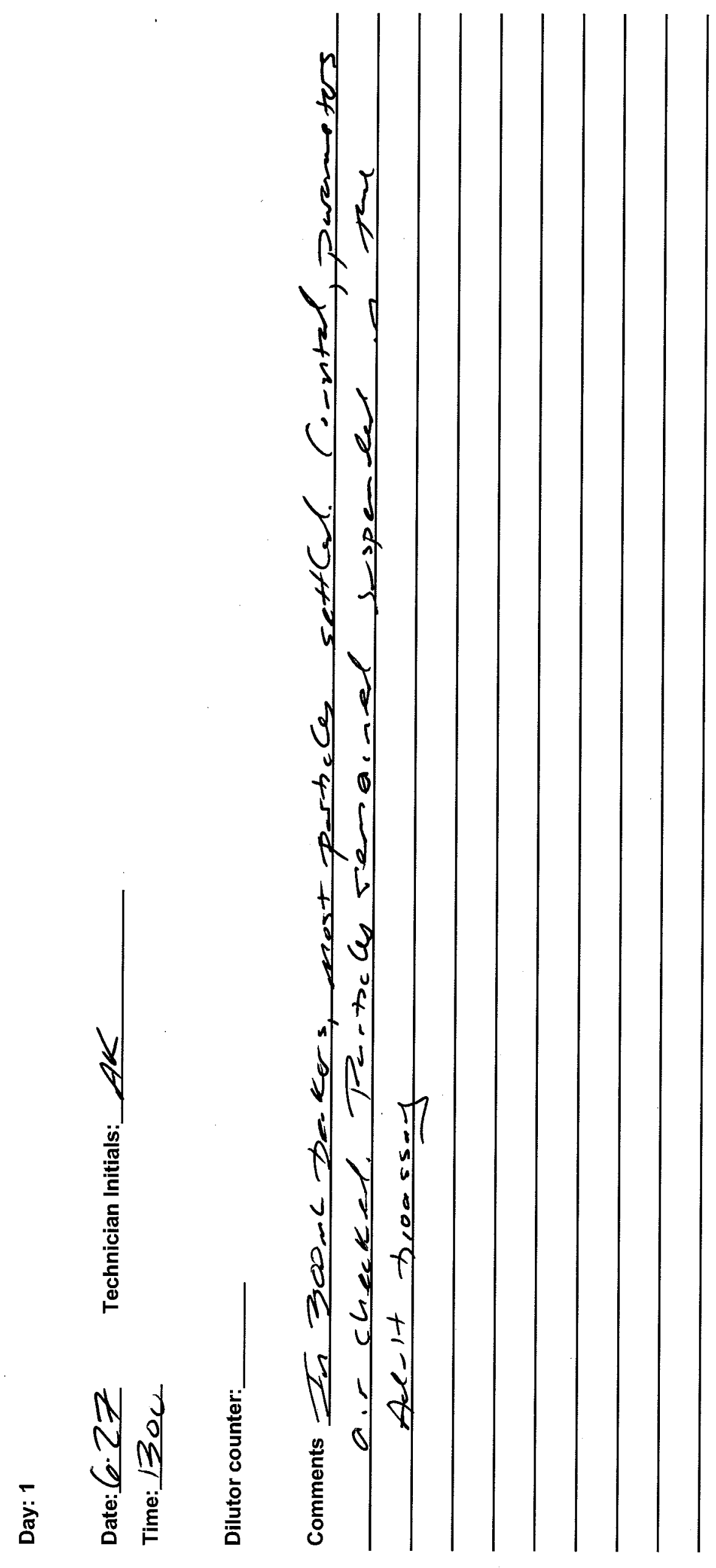




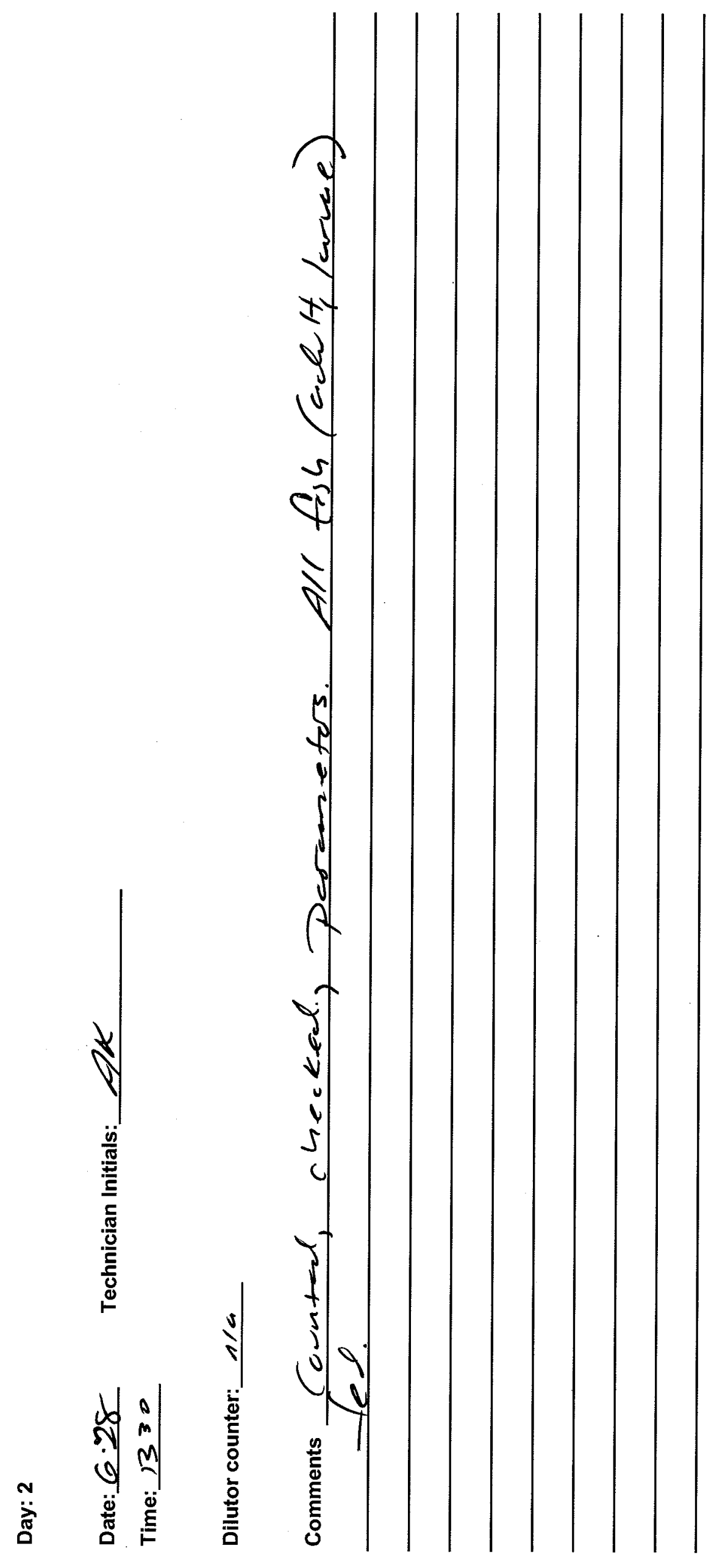




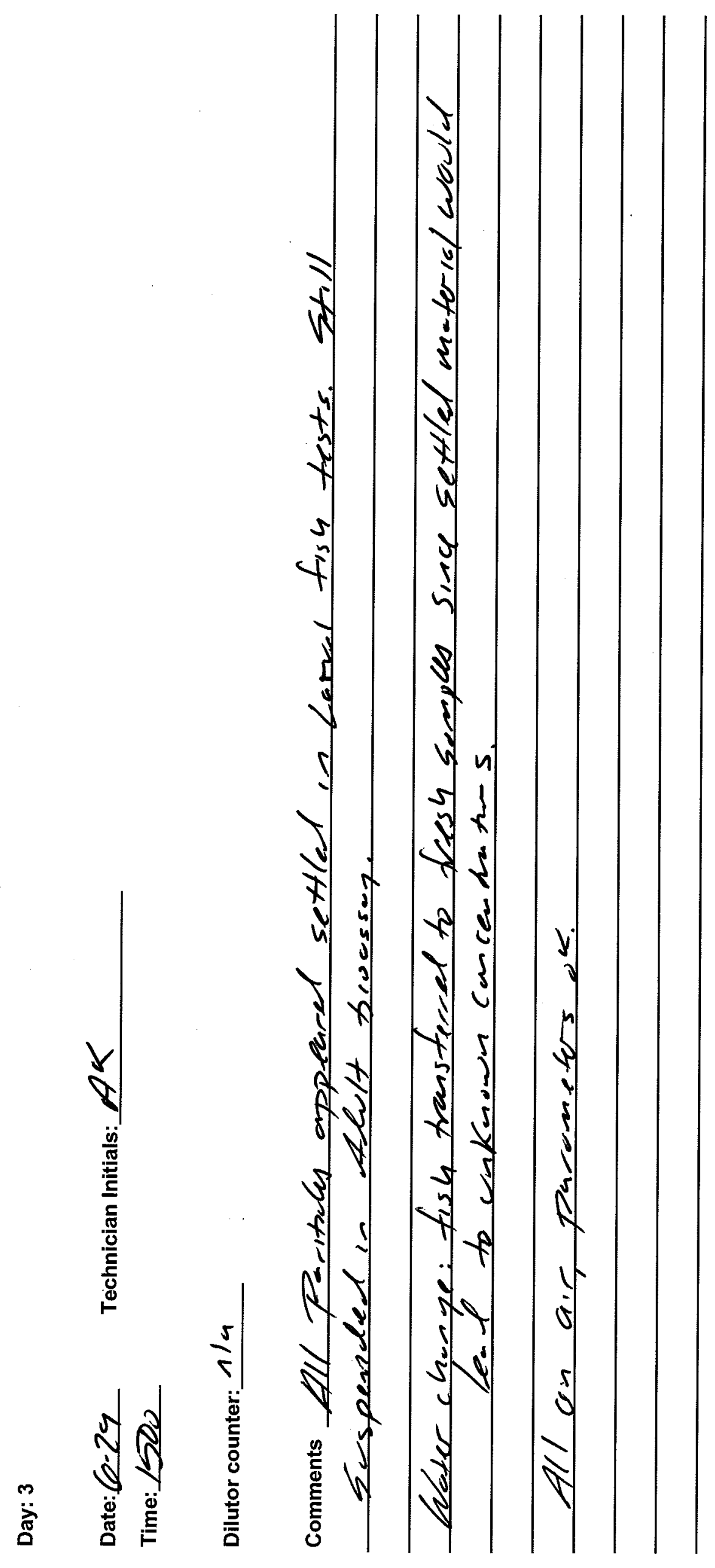




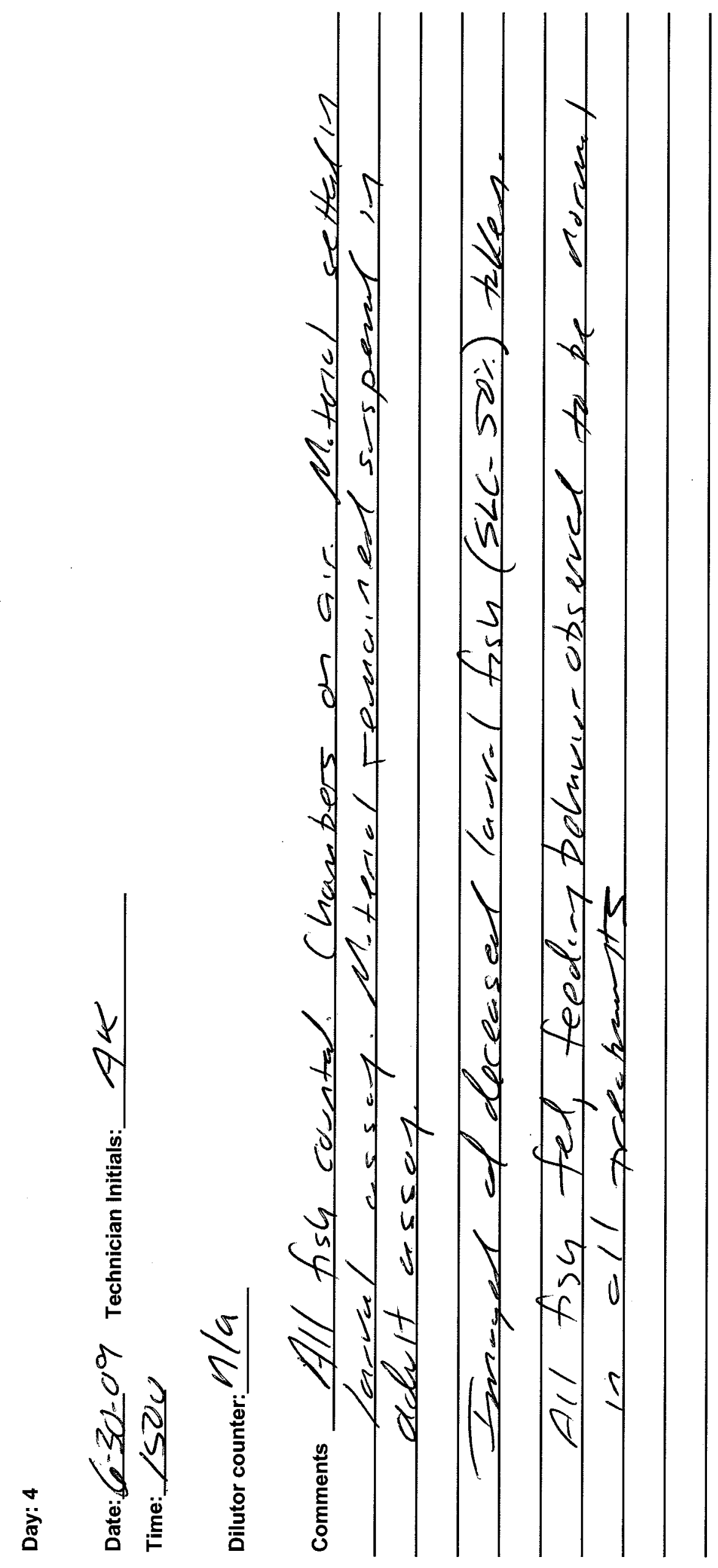




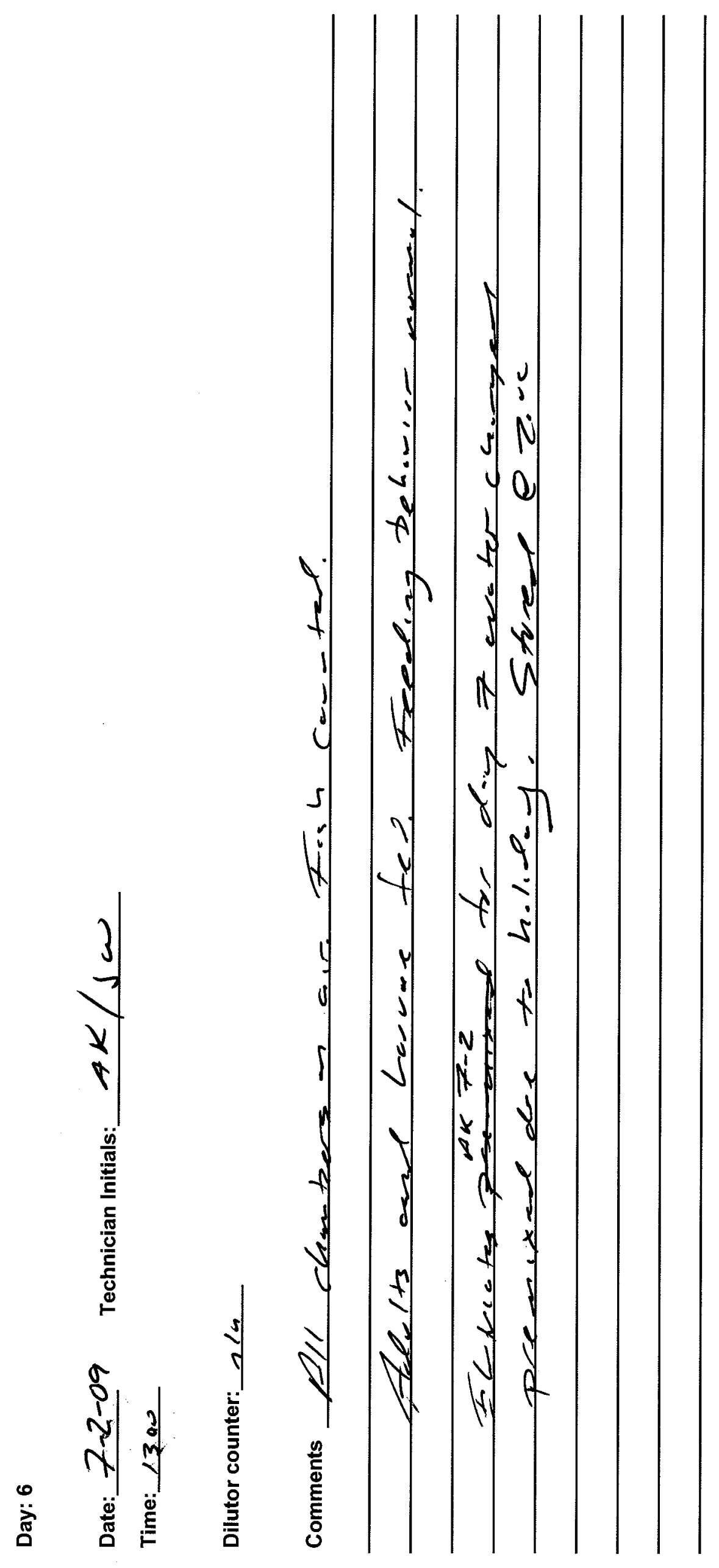




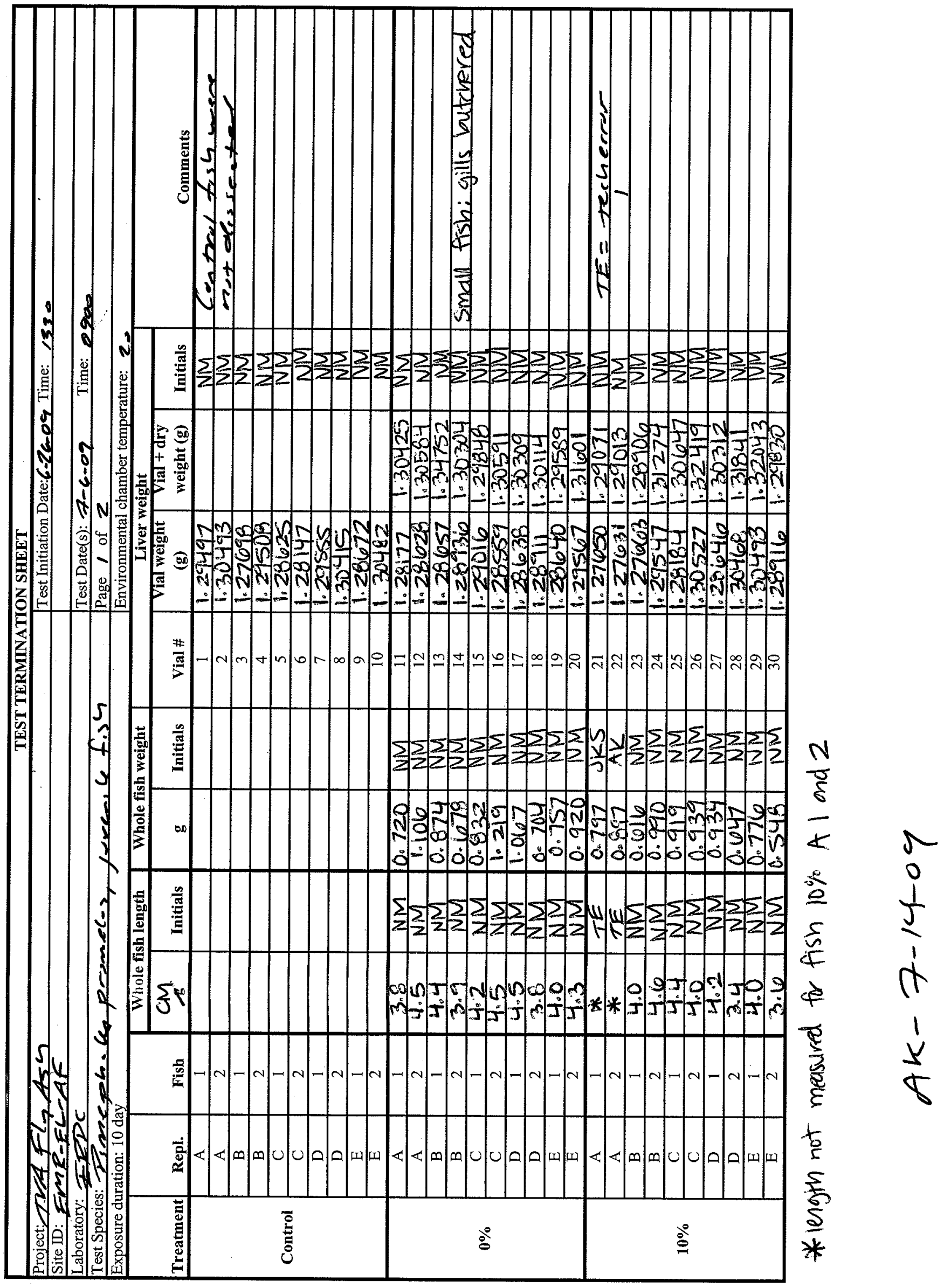



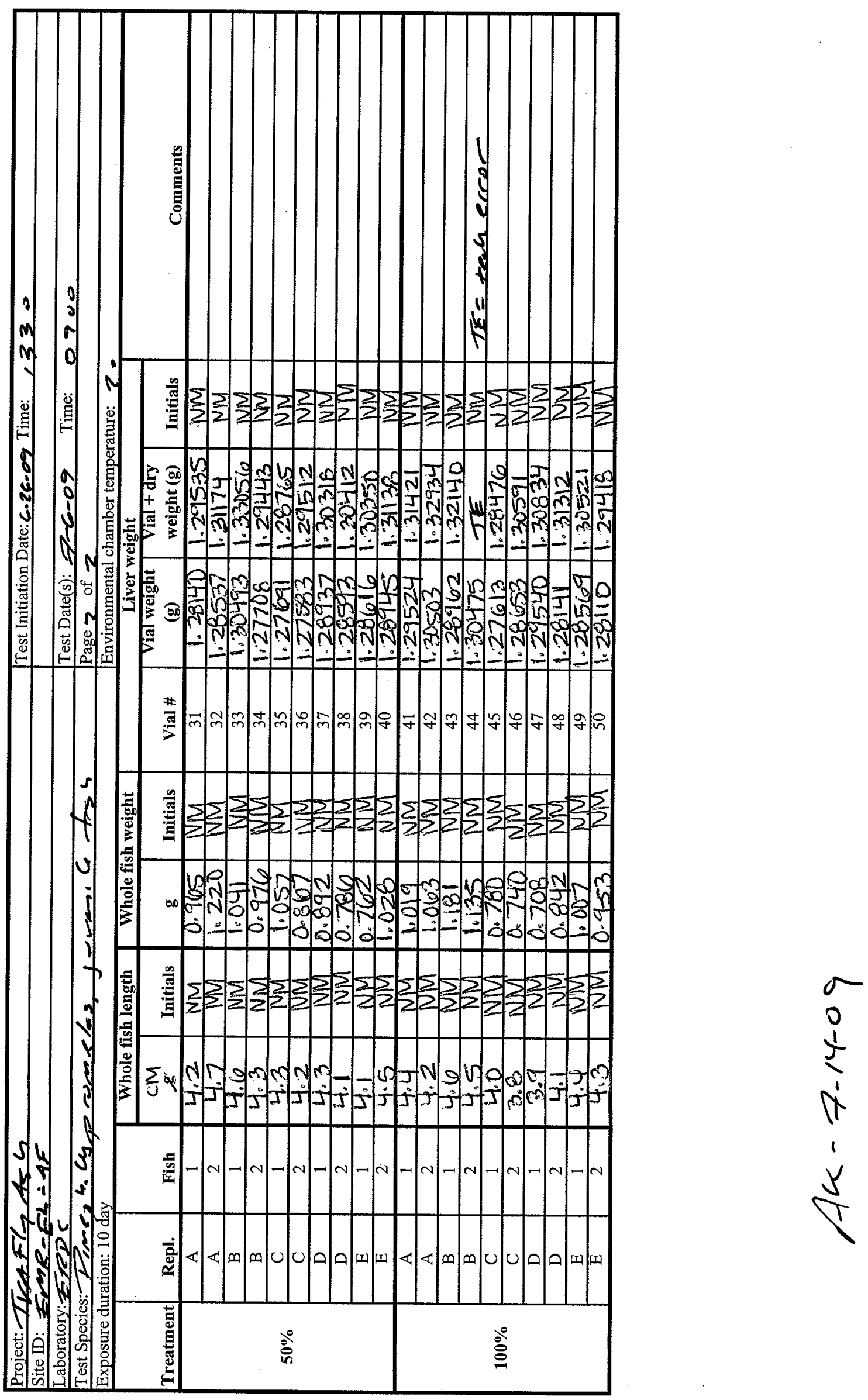


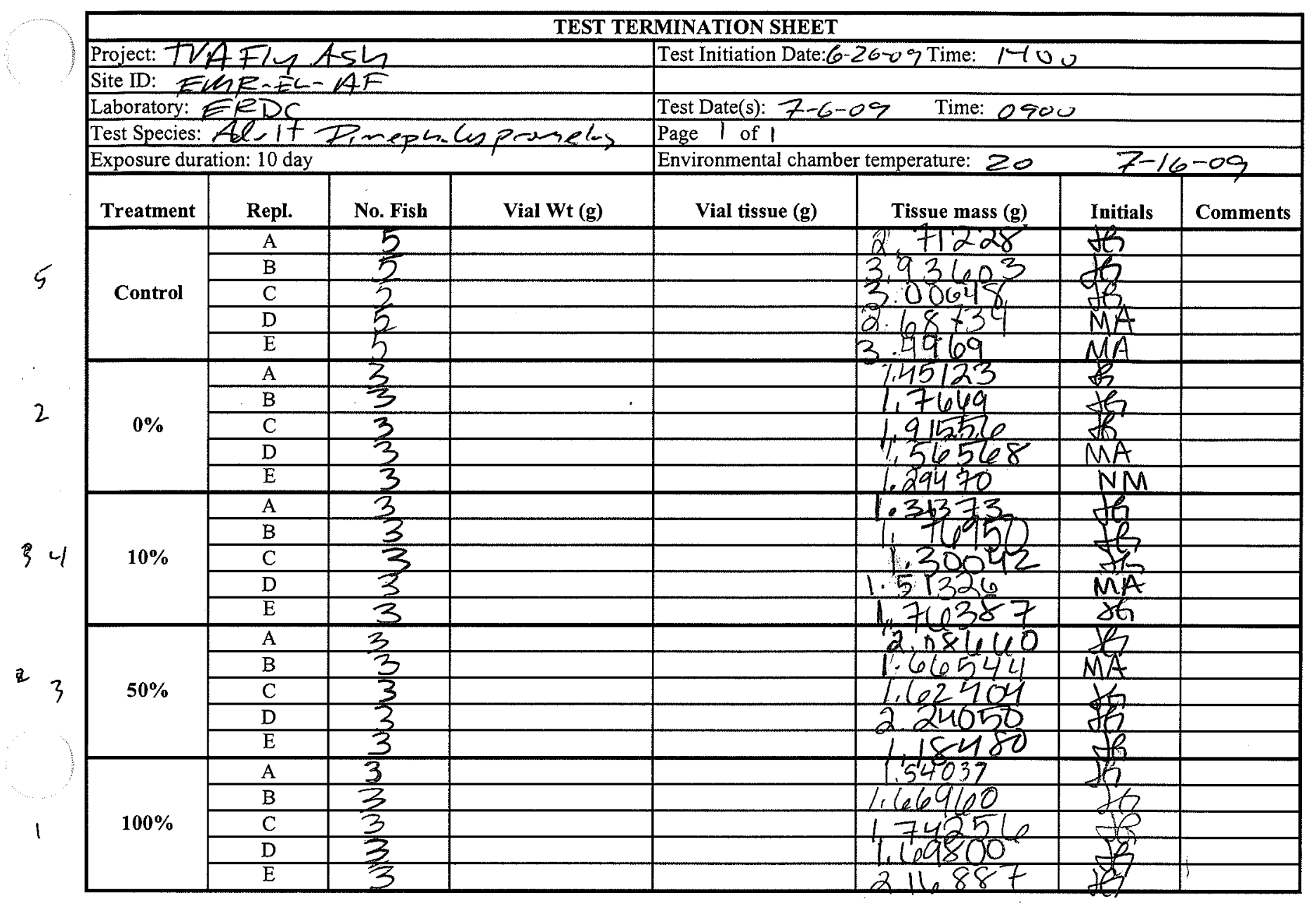




\begin{tabular}{|c|c|c|c|c|c|}
\hline \multicolumn{6}{|c|}{ Acute Fish Test-96 Hr Survival } \\
\hline Start Date: & $6 / 26 / 2009$ & & Test ID: & Sample ID: & \\
\hline End Date: & $6 / 30 / 2009$ & & Lab ID: & Sample Type: & \\
\hline Sample Date: & & & Protocol: EPAA 91-EPA Acute & Test Species: & PP-Pimephales promelas \\
\hline Comments: & & & & & \\
\hline Conc-gm/L & 1 & 2 & 3 & & \\
\hline 0 & 1.0000 & 1.0000 & 1.0000 & & \\
\hline 0.34 & 0.9000 & 1.0000 & 0.9000 & & \\
\hline 0.68 & 0.6000 & 0.6000 & 0.7000 & & \\
\hline 1.35 & 0.0000 & 0.0000 & 0.0000 & & \\
\hline 2.7 & 0.0000 & 0.0000 & 0.0000 & & \\
\hline
\end{tabular}

\begin{tabular}{|c|c|c|c|c|c|c|c|c|c|}
\hline \multirow[b]{2}{*}{ Conc-gm/L } & \multirow[b]{2}{*}{ Mean } & \multirow[b]{2}{*}{ N-Mean } & \multicolumn{5}{|c|}{ Transform: Arcsin Square Root } & \multirow{2}{*}{$\begin{array}{c}\text { Number } \\
\text { Resp }\end{array}$} & \multirow{2}{*}{$\begin{array}{c}\text { Total } \\
\text { Number }\end{array}$} \\
\hline & & & Mean & Min & Max & CV\% & $\mathbf{N}$ & & \\
\hline 0 & 1.0000 & 1.0000 & 1.4120 & 1.4120 & 1.4120 & 0.000 & 3 & 0 & 30 \\
\hline 0.34 & 0.9333 & 0.9333 & 1.3034 & 1.2490 & 1.4120 & 7.219 & 3 & 2 & 30 \\
\hline 0.68 & 0.6333 & 0.6333 & 0.9211 & 0.8861 & 0.9912 & 6.586 & 3 & 11 & 30 \\
\hline 1.35 & 0.0000 & 0.0000 & 0.1588 & 0.1588 & 0.1588 & 0.000 & 3 & 30 & 30 \\
\hline 2.7 & 0.0000 & 0.0000 & 0.1588 & 0.1588 & 0.1588 & 0.000 & 3 & 30 & 30 \\
\hline
\end{tabular}

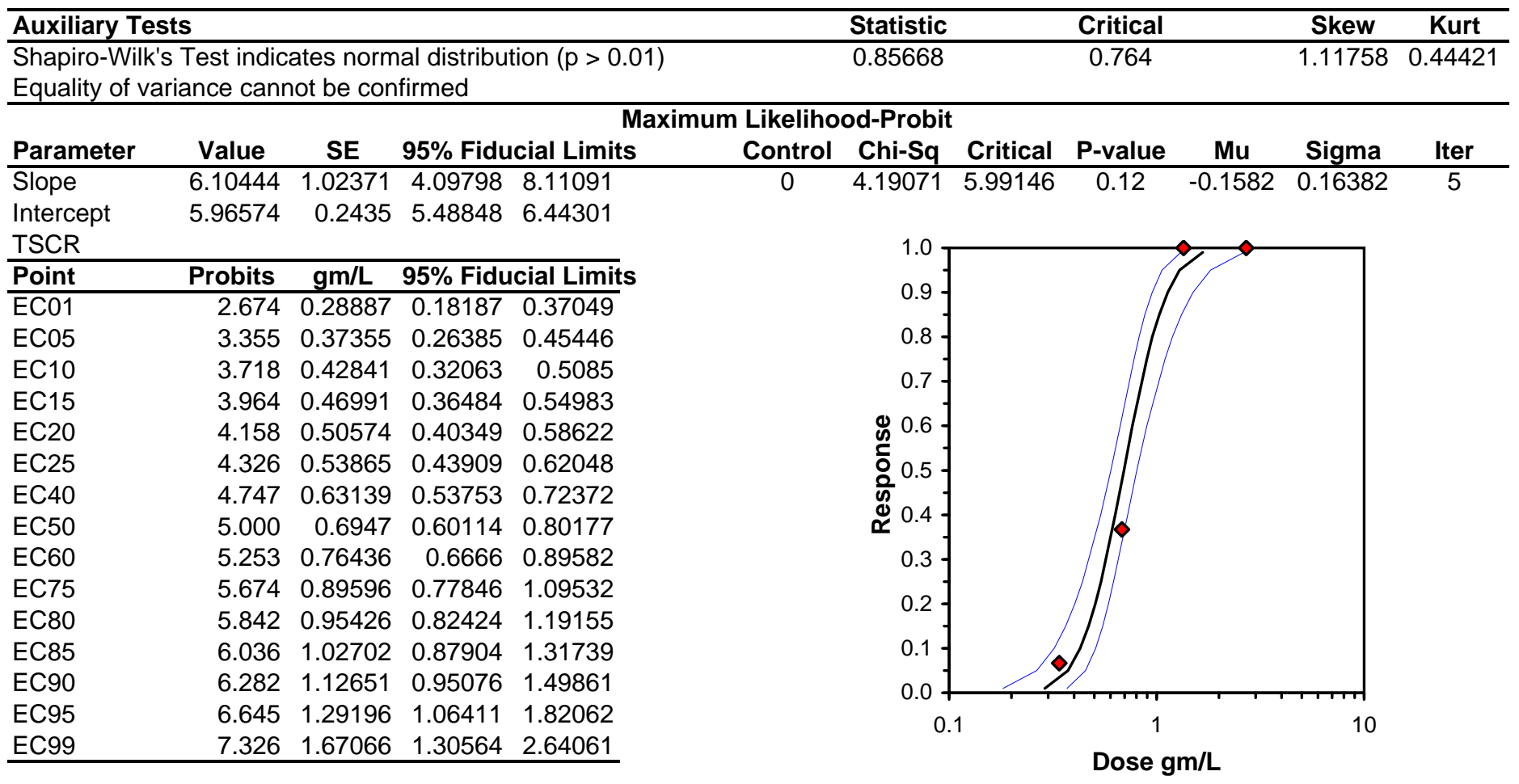




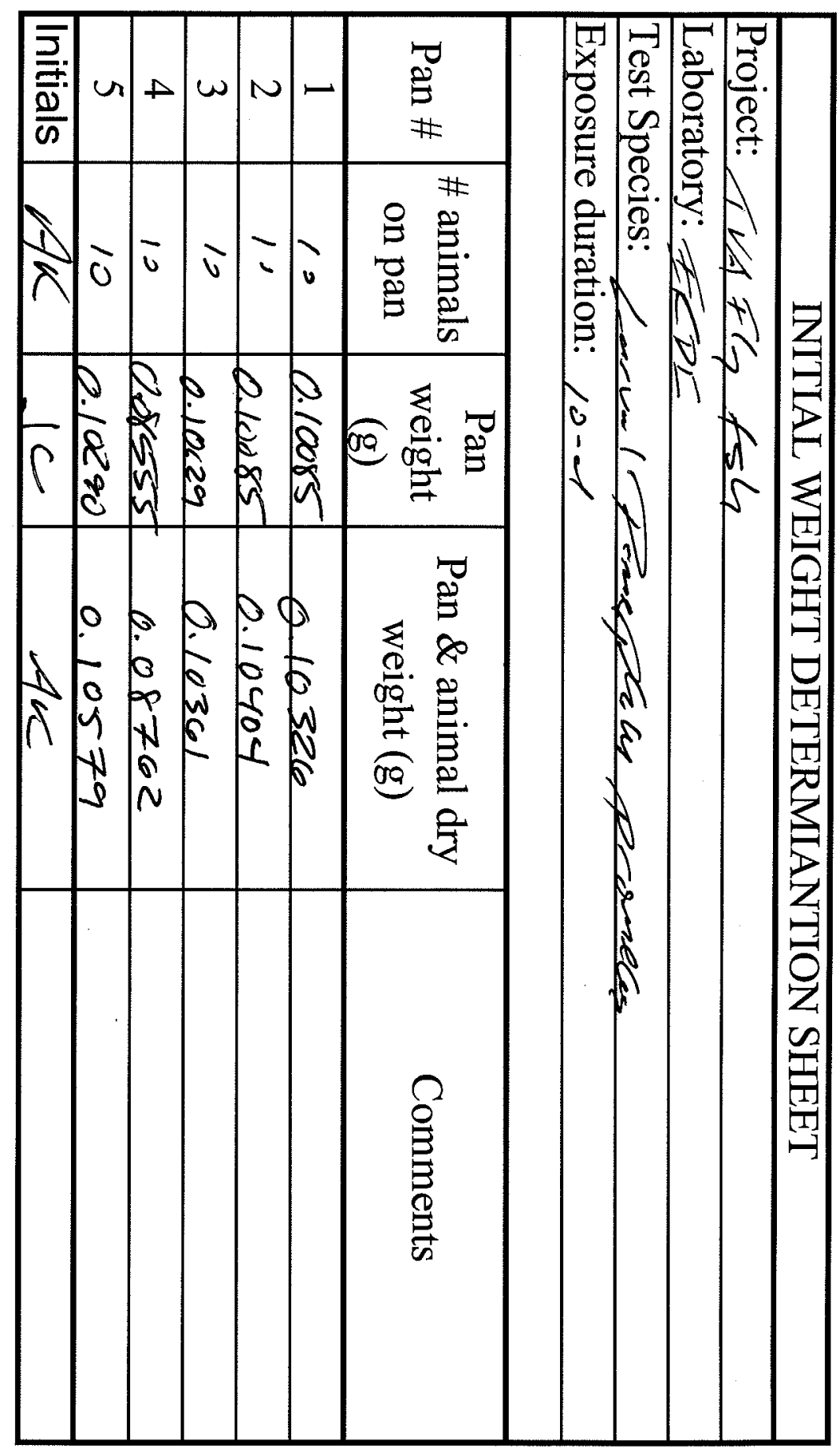




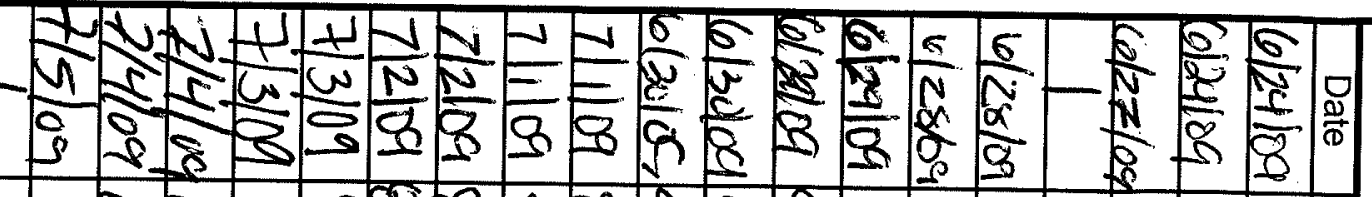

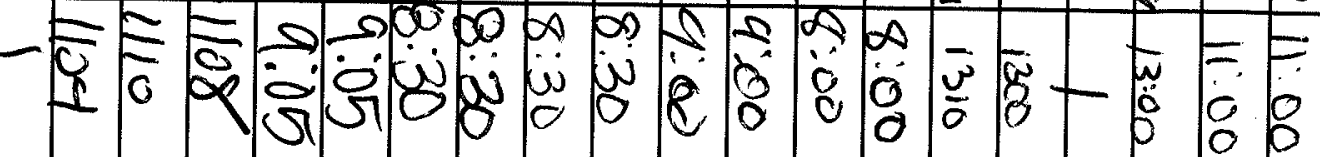

\section{0}

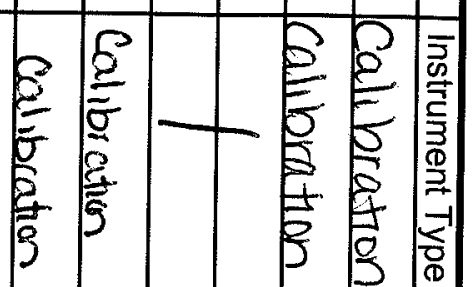

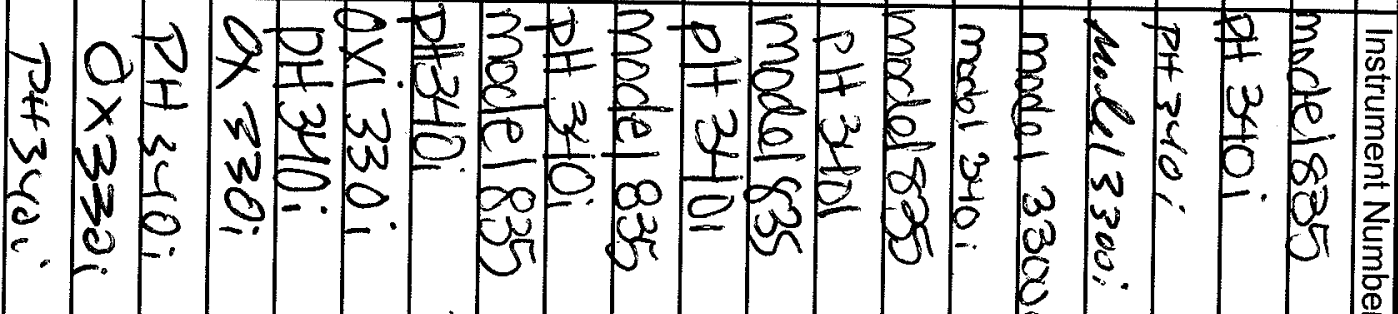

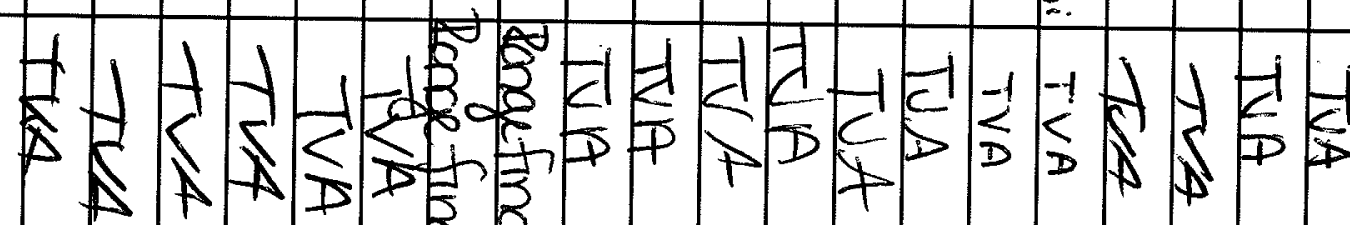

$$
\frac{5}{2}
$$

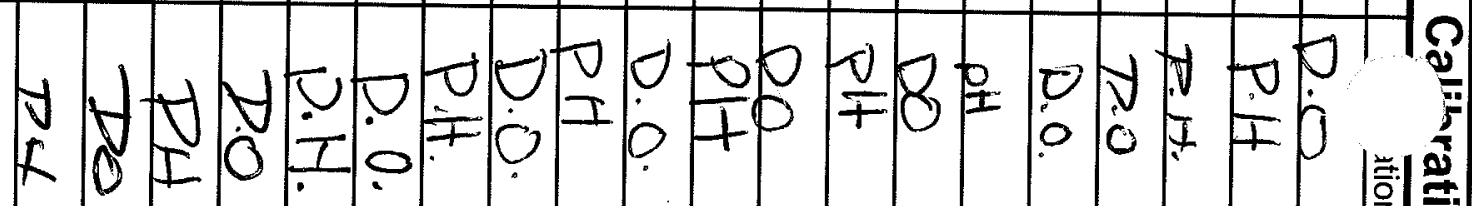

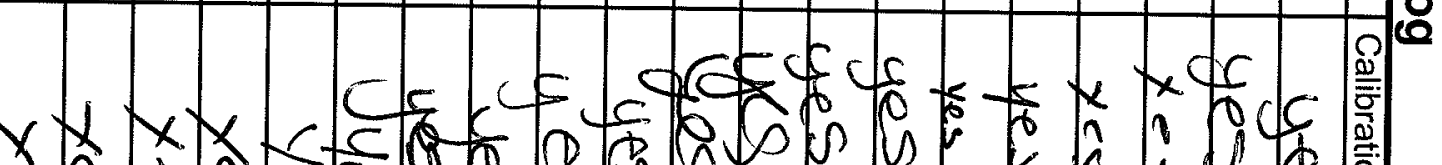

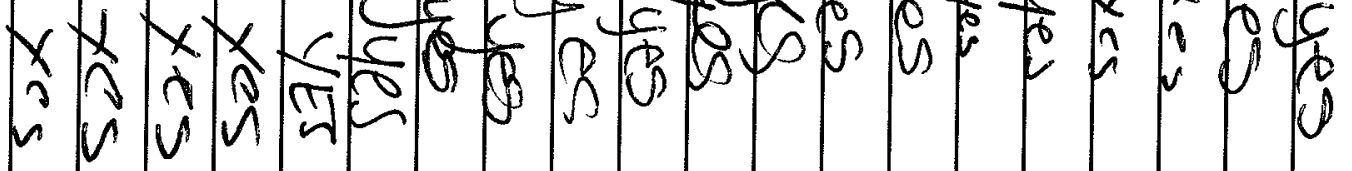

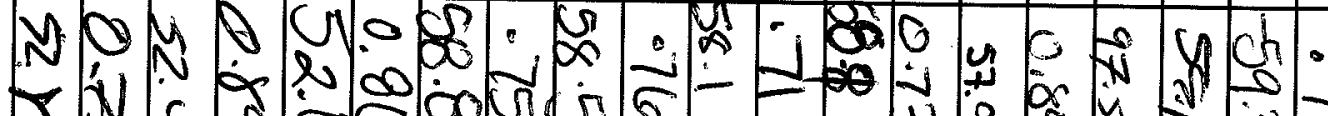

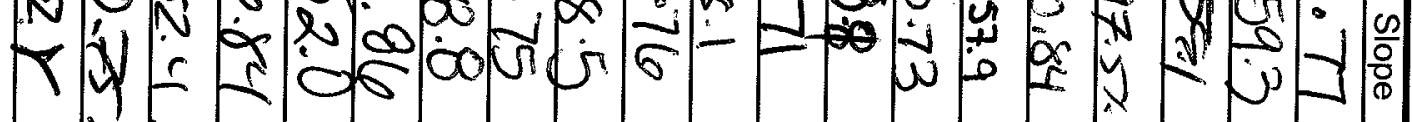

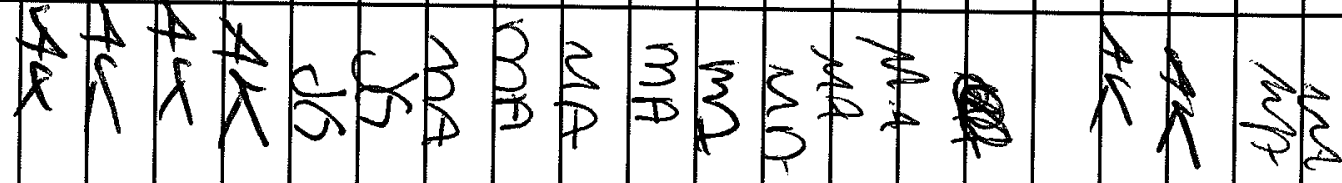




\section{Appendix G: Elutriate Bioassay Water Quality Parameters}




\section{Appendix G. Elutriate Bioassay Water Quality Parameters}

Summary of water quality parameters measured during the 10 days the larval and juvenile Pimephales promelas exposures. Means and one standard deviation from the mean are provided. Numbers in parentheses represent the minimum and maximum values.

\begin{tabular}{|c|c|c|c|c|c|c|c|c|}
\hline Site ID & Concentration & $\begin{array}{c}\text { Temperature } \\
\left({ }^{\circ} \mathrm{C}\right)\end{array}$ & $\begin{array}{c}\mathrm{pH} \\
(\mathrm{SU})\end{array}$ & $\begin{array}{l}\text { Conductivity } \\
(\mu \mathrm{S} / \mathrm{cm})\end{array}$ & $\begin{array}{c}\text { Dissolved } \\
\text { Oxygen } \\
\text { (mg/L) }\end{array}$ & $\begin{array}{c}\text { Ammonia } \\
(\mathrm{mg} / \mathrm{L})\end{array}$ & $\begin{array}{l}\text { Alkalinity } \\
\text { (mg/L) }\end{array}$ & $\begin{array}{c}\text { Hardness } \\
(\mathrm{mg} / \mathrm{L})\end{array}$ \\
\hline Control & NA & $\begin{array}{c}20.1 \pm 0.2 \\
(19.7-20.6)\end{array}$ & $\begin{array}{c}8.12 \pm 0.14 \\
(7.91-8.47)\end{array}$ & $\begin{array}{c}274 \pm 7 \\
(260-280)\end{array}$ & $\begin{array}{c}8.2 \pm 0.8 \\
(6.9-9.5) \\
\end{array}$ & $(<1-1)$ & 110 & 100 \\
\hline REF-WA-LF & $0 \%$ & $\begin{array}{c}20.0 \pm 0.4 \\
(19.2-20.6)\end{array}$ & $\begin{array}{c}7.79 \pm 0.20 \\
(7.57-8.21)\end{array}$ & $\begin{array}{c}97 \pm 6 \\
(90-110) \\
\end{array}$ & $\begin{array}{c}8.4 \pm 0.9 \\
(6.8-9.5)\end{array}$ & $(<1-1)$ & 64 & 20 \\
\hline \multirow{3}{*}{ EMR-EL-LF } & $10 \%$ & $\begin{array}{c}20.0 \pm 0.3 \\
(19.4-20.5)\end{array}$ & $\begin{array}{c}7.62 \pm 0.26 \\
(7.12-8.02)\end{array}$ & $\begin{array}{c}96 \pm 5 \\
(90-100)\end{array}$ & $\begin{array}{c}8.3 \pm 0.8 \\
(6.6-9.5)\end{array}$ & $(<1-1)$ & & \\
\hline & $50 \%$ & $\begin{array}{c}20.1 \pm 0.4 \\
(19.6-20.8)\end{array}$ & $\begin{array}{c}7.63 \pm 0.20 \\
(7.20-7.94)\end{array}$ & $\begin{array}{c}114 \pm 6 \\
(100-120)\end{array}$ & $\begin{array}{c}8.1 \pm 0.9 \\
(6.4-9.3)\end{array}$ & $(<1-1)$ & & \\
\hline & $100 \%$ & $\begin{array}{c}20.0 \pm 0.3 \\
(19.6-20.7)\end{array}$ & $\begin{array}{c}7.74 \pm 0.17 \\
(7.30-7.95)\end{array}$ & $\begin{array}{c}129 \pm 9 \\
(120-140)\end{array}$ & $\begin{array}{c}8.1 \pm 1.0 \\
(6.6-9.4)\end{array}$ & $(<1-1)$ & 100 & 22 \\
\hline \multirow{3}{*}{ SLC-EL-LF } & $10 \%$ & $\begin{array}{c}20.4 \pm 0.3 \\
(20.0-20.6)\end{array}$ & $\begin{array}{c}7.69 \pm 0.14 \\
(7.52-7.95)\end{array}$ & $\begin{array}{c}114 \pm 5 \\
(110-120)\end{array}$ & $\begin{array}{c}7.7 \pm 1.0 \\
(6.0-9.4)\end{array}$ & $(<1-1)$ & & \\
\hline & $50 \%$ & $\begin{array}{c}20.1 \pm 0.4 \\
(19.5-20.8)\end{array}$ & $\begin{array}{c}7.79 \pm 0.12 \\
(7.54-8.05)\end{array}$ & $\begin{array}{c}177 \pm 19 \\
(110-190)\end{array}$ & $\begin{array}{c}8.2 \pm 1.1 \\
(6.4-9.8)\end{array}$ & $(<1-1)$ & & \\
\hline & $100 \%$ & $\begin{array}{c}20.1 \pm 0.5 \\
(19.4-21.0)\end{array}$ & $\begin{array}{c}8.09 \pm 0.12 \\
(7.91-8.43)\end{array}$ & $\begin{array}{c}269 \pm 12 \\
(250-290)\end{array}$ & $\begin{array}{c}8.4 \pm 0.9 \\
(6.4-9.9)\end{array}$ & $(<1-1)$ & 160 & 76 \\
\hline
\end{tabular}




\begin{tabular}{|c|c|c|c|c|c|c|c|c|}
\hline Site ID & Concentration & $\begin{array}{c}\text { Temperature } \\
\left({ }^{\circ} \mathrm{C}\right)\end{array}$ & $\begin{array}{c}\mathrm{pH} \\
(\mathrm{SU})\end{array}$ & $\begin{array}{l}\text { Conductivity } \\
\qquad(\mu \mathrm{S} / \mathrm{cm})\end{array}$ & $\begin{array}{c}\text { Dissolved } \\
\text { Oxygen } \\
(\mathrm{mg} / \mathrm{L})\end{array}$ & $\begin{array}{l}\text { Ammonia } \\
(\mathrm{mg} / \mathrm{L})\end{array}$ & $\begin{array}{l}\text { Alkalinity } \\
(\mathrm{mg} / \mathrm{L})\end{array}$ & $\begin{array}{c}\text { Hardness } \\
(\mathrm{mg} / \mathrm{L})\end{array}$ \\
\hline Control & NA & $\begin{array}{c}20.6 \pm 0.3 \\
(20.2-21.1) \\
\end{array}$ & $\begin{array}{c}8.12 \pm 0.14 \\
(7.91-8.47)\end{array}$ & $\begin{array}{c}287 \pm 7 \\
(280-300) \\
\end{array}$ & $\begin{array}{c}9.0 \pm 0.7 \\
(7.4-10.0)\end{array}$ & $(<1-2)$ & 110 & 100 \\
\hline REF-WA-AF & $0 \%$ & $\begin{array}{c}20.1 \pm 0.7 \\
(18.9-20.8)\end{array}$ & $\begin{array}{c}7.61 \pm 0.17 \\
(7.38-7.88)\end{array}$ & $\begin{array}{c}104 \pm 11 \\
(90-120)\end{array}$ & $\begin{array}{c}8.9 \pm 1.3 \\
(4.2-10.0)\end{array}$ & $(<1-2)$ & 64 & 20 \\
\hline \multirow{3}{*}{ EMR-EL-AF } & $10 \%$ & $\begin{array}{c}19.9 \pm 0.6 \\
(18.7-20.5)\end{array}$ & $\begin{array}{c}7.57 \pm 0.23 \\
(7.16-8.04)\end{array}$ & $\begin{array}{c}104 \pm 11 \\
(90-120)\end{array}$ & $\begin{array}{c}9.2 \pm 0.6 \\
(8.2-9.9)\end{array}$ & $(<1-2)$ & & \\
\hline & $50 \%$ & $\begin{array}{c}20.2 \pm 0.8 \\
(18.6-21.0)\end{array}$ & $\begin{array}{c}7.62 \pm 0.15 \\
(7.40-8.00)\end{array}$ & $\begin{array}{c}120 \pm 13 \\
(100-140)\end{array}$ & $\begin{array}{c}9.1 \pm 0.7 \\
(7.4-10.0)\end{array}$ & $(<1-1)$ & & \\
\hline & $100 \%$ & $\begin{array}{c}20.0 \pm 0.7 \\
(18.9-20.7)\end{array}$ & $\begin{array}{c}7.65 \pm 0.22 \\
(7.14-8.05)\end{array}$ & $\begin{array}{c}136 \pm 13 \\
(120-160)\end{array}$ & $\begin{array}{c}9.0 \pm 0.5 \\
(7.9-9.5)\end{array}$ & $(<1-2)$ & 100 & 22 \\
\hline
\end{tabular}




\section{Appendix H: Histological Examination of Fish Gills}


Appendix H. Histological examination of fish gills

\title{
Evaluation of Metals Release from Oxidation of Fly Ash during Dredging of the Emory River, TN
}

\section{Gill Histology}

\author{
August 12, 2009 \\ Prepared by: \\ University of Mississippi \\ School of Pharmacy \\ Environmental Toxicology Research Program \\ Box 1848, 200 Faser Hall \\ University, MS 38677 \\ Technical Point of Contact: \\ Dr. Jeffery A. Steevens \\ Tel: 601-634-4199; Email: Jeffery.A.Steevens@us.army.mil \\ Dr. Kristine Willett \\ Tel: 662-915-6691; Email: kwillett@olemiss.edu
}

Technical Team Members

Ms. Cammi Thornton and Ms. Adrienne Wells at UM

Mr. Alan Kennedy, Dr. Jacob Stanley, Ms. Jessica Coleman, Ms. Jennifer Goss, Mr.

Jamma Williams at ERDC 


\section{INTRODUCTION}

Herein we report specifically on the gill histopathology resulting following 10 day exposures of juvenile fathead minnows (Pimephales promelas) to zero or $100 \%$ elutriate from the Emory River (EMR-EL).

\section{METHODS}

\subsection{Exposure}

For full description of fish testing methods please refer to section 6. Fish were anesthetized (Tricaine methanesulfonate, MS-222), and gills were carefully removed using micro-dissection scissors assisted by dissecting microscopes. Gills were imaged and immediately placed into $10 \mathrm{ml}$ Bouin's Solution (Sigma HT10132-1L) in $20 \mathrm{ml}$ glass scintillation vials. Specimens were shipped overnight to the Department of Pharmacology at the University of Mississippi (Oxford, MS, USA) for gill histopathology.

\subsection{Gill Histopathology}

After fixation in Bouin's solution for $\sim 24$ hrs at $4^{\circ} \mathrm{C}$, gills were rinsed in 1X PBS 2 times for $2 \mathrm{hr}$ and dehydrated in a gradient of 70, 80, and 90\% ethanol for $3 \mathrm{hr}$ each and 95 and $100 \%$ ethanol for $1.5 \mathrm{hr}$ each. Gills were then cycled through Clearify (American Master Tech Scientific Inc., Lodi, CA) rinses for $2 \mathrm{hr}$ and $3 \mathrm{hr}$ followed by paraffin embedding at $55^{\circ} \mathrm{C}$ for $50 \mathrm{~min}$ and another cycle for $3 \mathrm{hr}$ (Leica TP1020). Gills were removed from cassettes and positioned into a well of a 6-well plate for further block embedding. Embedded gills were stored at $-20^{\circ} \mathrm{C}$.

Efforts were made to position the gills to facilitate sectioning oriented cross sectionally to the filaments and transverse to the gill. Five $\mu \mathrm{m}$ sections were prepared with an Olympus Cut 4055. Three adjacent slide sets (A-C) were generated to allow for additional toxicity testing or replicate staining if necessary.

To determine morphological changes in the gill filaments or lamellae, hematoxylin and eosin (H\&E) staining was performed on the slides (set A). Tissues were dewaxed with Clearify and then rehydrated through a series of decreasing ethanol rinses (100 I, 100 II, 95, 90, 80 and 70\%). Tissues were then stained with Harris's hematoxylin and eosin Y. Sections were visualized and photographed with an Olympus BX40 and Optronics camera at 630x magnification.

Filament width was determined by measuring across the filament between 2 adjacent lamellae. When possible, filament width was measured 1 to 5 times per filament on 5 different filaments per fish ( 6 - 25 measurements per gill; $\mathrm{n}=5-7$ fish/treatment). Qualitative observations of other gill pathologies were noted as described by Bernet et al., 1999 and Costa et al., 2009. 


\section{RESULTS}

Gills from 10 individuals exposed to either 0 or 100\% Emory River elutriate were fixed and sectioned for histopathological analysis. Once sectioning was complete, a number of the fish were either not positioned properly to visualize cross sections of filaments and lamellae or had been damaged during processing (Table 1).

Figure 1 shows an overview of a representative gill section and subsequent magnifications of that section. The diagram in panel C shows how measurement of filament width was determined. When possible, this was measured up to five times per filament. However, because the widths naturally changed across the filament and in some sections the entire length of a filament was not in the plane of the section, it was determined a more consistent measurement was found at the base of the filament near where it attached to the gill arch. Base filament widths were measured for 2-5 filaments per fish ( $\mathrm{n}=7$ and 5 , for control and $100 \%$, respectively). There was a relatively high interindividual variability in filament width and the fish with the highest and lowest average widths were both in the control group (Figure 2A). When averaged for all the fish within treatment there was not a statistically significant ( $p>0.05$, Student's T-test) increase in filament width in the $100 \%$ elutriate-treated fish.

An increase in gill filament (padding) width has previously been reported in zebrafish exposed to silver nitrate, copper sulfate, and nanocopper for 24 or $48 \mathrm{hr}$ (Griffitt et al., 2009) and yellow perch chronically exposed to metals in the wild (Levesque et al., 2003). Decreased blood-water exchange is the pathophysiology presumed to be associated with increased epithelial width. In contrast, juvenile pike from a reference lake had significantly thicker gill filaments compared to those exposed to Key Lake uranium milling effluent (Kelly and Janz, 2009).

Both Bernet et al., 1999 and Costa et al., 2009 have utilized a weighted indices approach to assess gill histological biomarkers of environmental effect. The weighted index includes an importance factor $(\mathrm{w})$ of 1,2 or 3 on a scale from minimal, moderate to marked pathological importance (Bernet et al., 1999). Sections were investigated for signs of epithelial lifting ( $w=1)$, desquamation (1), lamellae deformation (1), lamellae fusion (1), chloride cell hypertrophy (2) and epithelial hyperplasia (2). Single incidences of pathologies were not considered positive, rather an abnormality had to be persistent across filaments to be considered positive. Figure 3 shows some examples of these lesions and Table 1 lists the lesions noted per sample. As shown a high number of gills could not be definitively classified with regard to lesions because the gill filaments/lamellae were not in focus across the $5 \mu \mathrm{m}$ sections. Gill is a particularly hard organ to section because they are curved, and thus, it is very difficult to get the arches to lay flat (especially on a small fish like fathead minnows) as shown in Figure 1A. In future studies, we would recommend dissecting the two halves of the gill apart so they can be embedded separately and potentially yield higher $\mathrm{n}$ numbers for histopathology and statistical evaluation. In addition, thinner sections $(3 \mu \mathrm{m})$ may yield clearer photos. Lesions noted in fish from this study included fused and deformed lamellae, 
desquamation, and epithelial hyperplasia. In summary, higher sample numbers will be needed to be able to statistically compare histological indices between treatment fish.

Table 1. Sample numbers, analyses conducted and pathologies noted.

\begin{tabular}{|c|c|c|c|c|}
\hline $\begin{array}{c}\text { ERDC ID } \\
0 \% \text { Controls }\end{array}$ & UM ID & $\begin{array}{c}\text { Filament } \\
\text { Width Measured }\end{array}$ & $\begin{array}{l}\text { Pathology } \\
\text { Scored }\end{array}$ & $\begin{array}{l}\text { Pathology } \\
\text { Noted }\end{array}$ \\
\hline EMR-EL-AF-A-0-1 & $1 \mathrm{~A}$ & Yes & Yes & Lamellar Fusion \\
\hline EMR-EL-AF-A-0-2 & 1B & No & No & \\
\hline EMR-EL-AF-B-0-1 & $2 A$ & Yes & Yes & None - normal \\
\hline EMR-EL-AF-B-0-2 & 2B & No & No & \\
\hline EMR-EL-AF-C-0-1 & $3 A$ & Yes & No & \\
\hline EMR-EL-AF-C-0-2 & 3B & Yes & No & \\
\hline EMR-EL-AF-D-0-1 & $4 \mathrm{~A}$ & No & No & \\
\hline EMR-EL-AF-D-0-2 & 4B & Yes & Yes & \\
\hline EMR-EL-AF-E-0-1 & $5 A$ & Yes & No & \\
\hline EMR-EL-AF-E-0-2 & $5 B$ & Yes & Yes & \\
\hline \multicolumn{5}{|l|}{$100 \%$ Elutriate } \\
\hline EMR-EL-AF-A-100-1 & $16 \mathrm{~A}$ & Yes & Yes & \\
\hline EMR-EL-AF-A-100-2 & 16B & Yes & Yes & Desquamation \\
\hline EMR-EL-AF-B-100-1 & $17 \mathrm{~A}$ & Yes & Yes & $\begin{array}{l}\text { Desquamation, Deformed } \\
\text { and Fused Lamellae }\end{array}$ \\
\hline EMR-EL-AF-B-100-2 & 17B & No & No & \\
\hline EMR-EL-AF-C-100-1 & $18 \mathrm{~A}$ & No & No & \\
\hline EMR-EL-AF-C-100-2 & 18B & Yes & Yes & \\
\hline EMR-EL-AF-D-100-1 & $19 A$ & Yes & Yes & Epithelial Hyperplasia \\
\hline EMR-EL-AF-D-100-2 & $19 B$ & No & No & \\
\hline EMR-EL-AF-E-100-1 & $20 \mathrm{~A}$ & No & No & \\
\hline EMR-EL-AF-E-100-2 & 20B & No & No & \\
\hline
\end{tabular}

Samples that were not measured or scored yielded sections that were not in the proper orientation for accurate histological evaluation of gill filaments or lamellae. If samples were analyzed for pathology, but there is nothing noted in the last column then no conclusive lesions were identified but also they could not be conclusively determined as normal. 

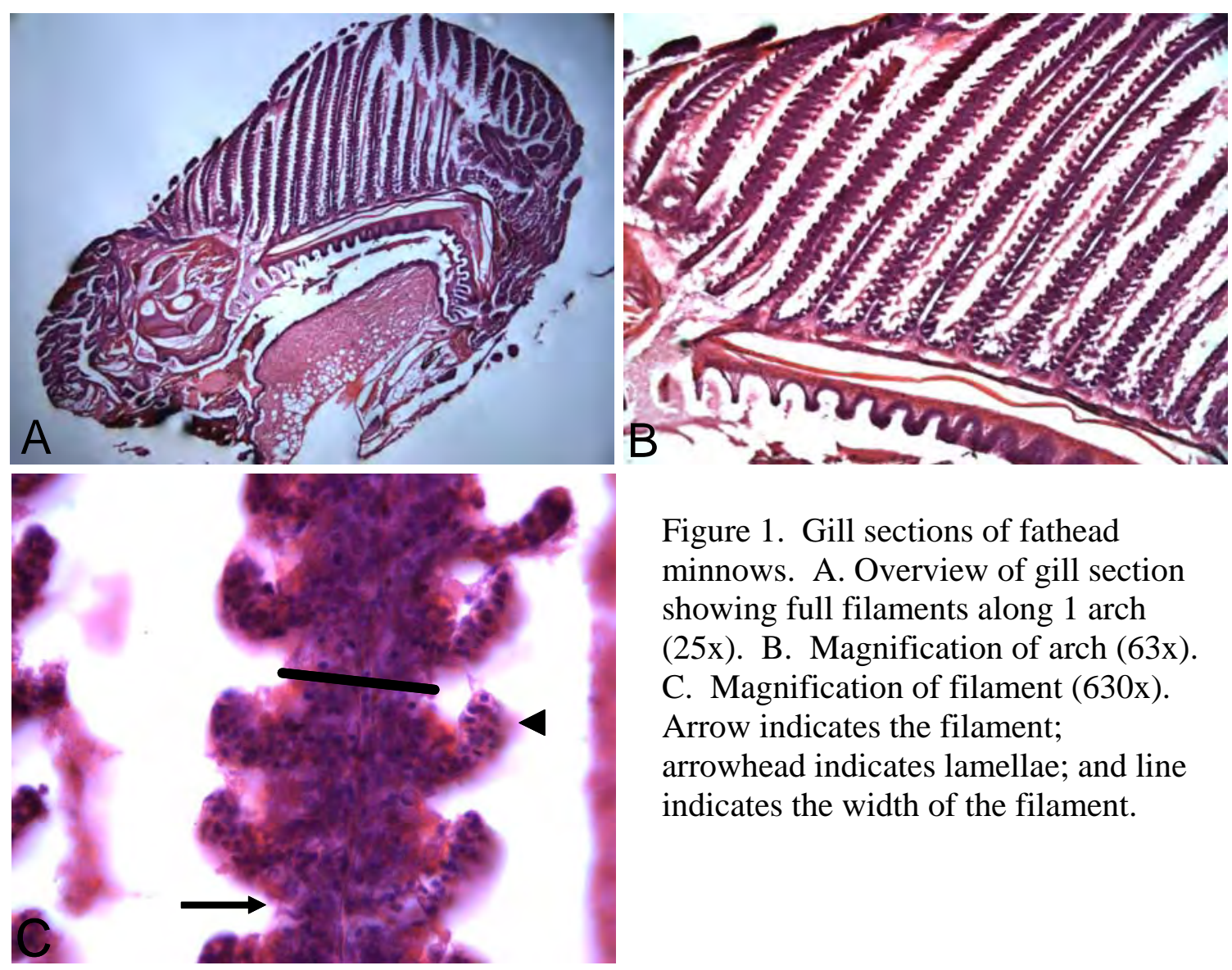

Figure 1. Gill sections of fathead minnows. A. Overview of gill section showing full filaments along 1 arch (25x). B. Magnification of arch (63x). C. Magnification of filament (630x). Arrow indicates the filament; arrowhead indicates lamellae; and line indicates the width of the filament.

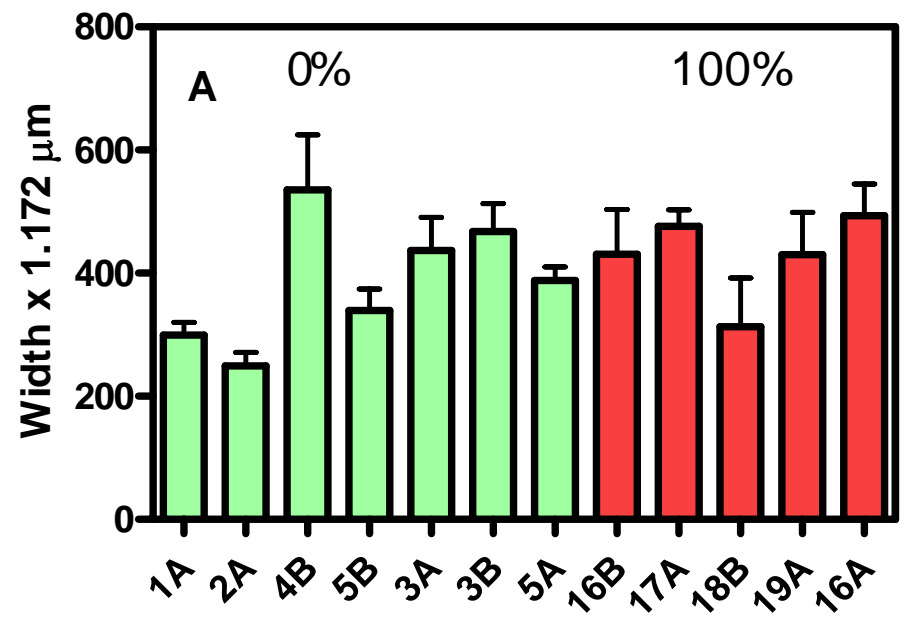

Fish Sample Number

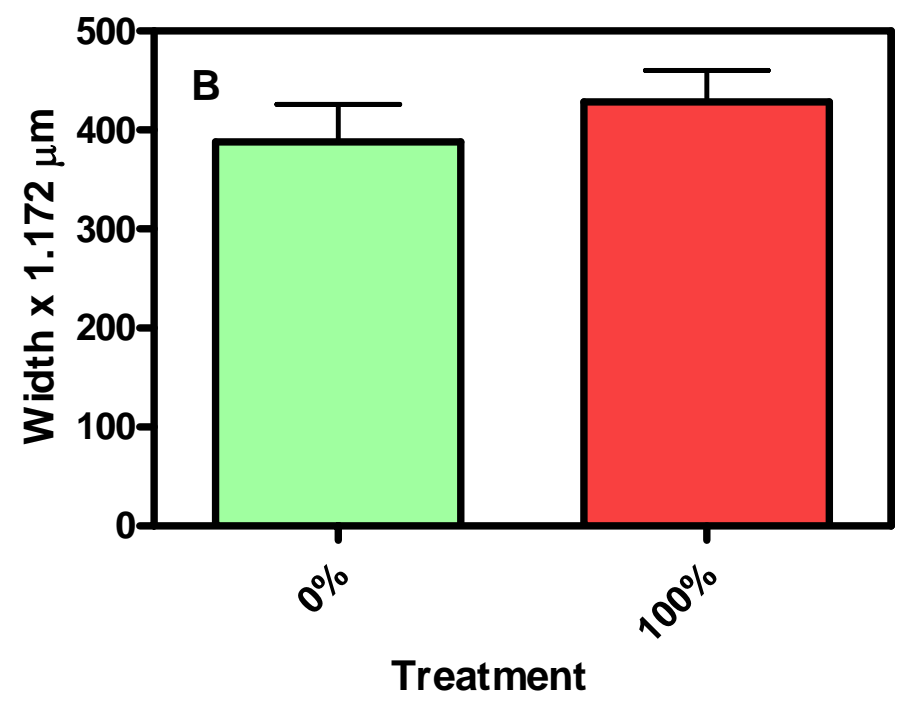

Figure 2. Gill filament widths measured at the base of the filament near the arch. A) Average widths \pm S.E. per fish ( $n=2-5$ filaments per fish), B) Average widths for all fish per treatment, $p$ $>0.05$, Student's T-test, $\mathrm{n}=5-7$. 

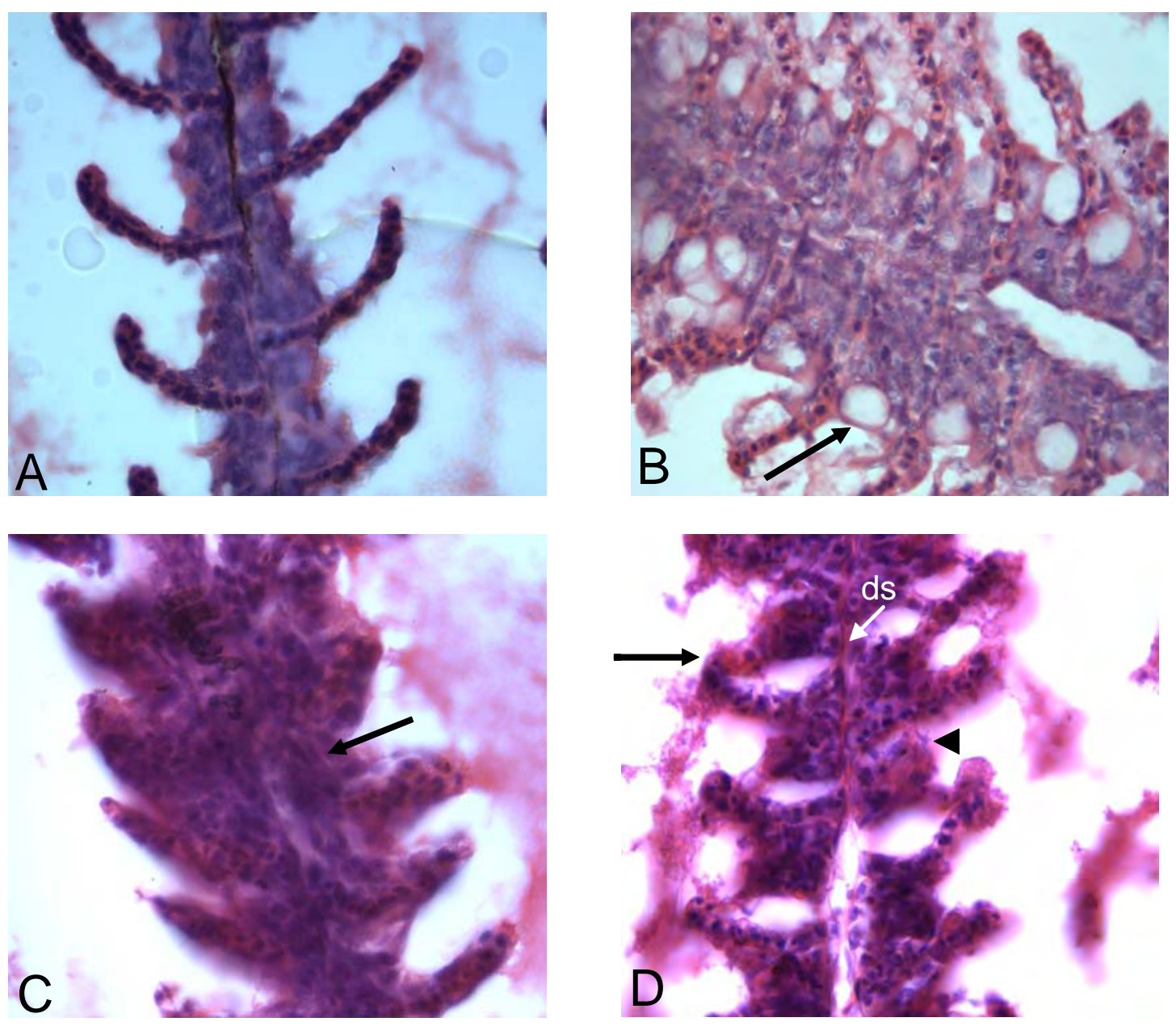

Figure 3. Gills sections of treated fish. A. Control fish showing no gross signs of toxicity. B. Control fish with potential goblet cell hyperplasia (arrow). C. Treated fish with epithelial hyperplasia (arrow). D. Treated fish with toxicities including desquamation (ds), deformed lamellae (arrow head) and lamellar fusion 


\section{Reference List}

Bernet, D., Schmidt, H., Burkhardt-Holm, P., and Wahli, T. (1999). Histopathology in fish: proposal for a protocol to assess aquatic pollution. J. Fish Dis. 22, 25-34.

Costa, P. M., Diniz, M. S., Caeiro, S., Lobo, J., Martins, M., Ferreira, A. M., Caetano, M., Vale, C., DelValls, T. A., and Costa, M. H. (2009). Histological biomarkers in liver and gills of juvenile Solea senegalensis exposed to contaminated estuarine sediments: a weighted indices approach. Aquat. Toxicol. 92(3), 202-212.

Griffitt, R. J., Hyndman, K., Denslow, N. D., and Barber, D. S. (2009). Comparison of molecular and histological changes in zebrafish gills exposed to metallic nanoparticles. Toxicol. Sci. 107(2), 404-415.

Kelly, J. M., and Janz, D. M. (2009). Assessment of oxidative stress and histopathology in juvenile northern pike (Esox lucius) inhabiting lakes downstream of a uranium mill. Aquat. Toxicol. 92(4), 240-249.

Levesque, H. M., Dorval, J., Hontela, A., Van Der Kraak, G. J., and Campbell, P. G. (2003). Hormonal, morphological, and physiological responses of yellow perch (Perca flavescens) to chronic environmental metal exposures. J Toxicol. Environ. Health A 66(7), 657-676. 


\section{Appendix I: Juvenile Mussel Toxicity}


Appendix I. Potential fly ash toxicity to juvenile mussels.

Evaluation of Elutriate Toxicity of Fly Ash Released during Dredging of the Emory River, TN, to Juvenile Mussels (fatmucket, Lampsilis siliquoidea and rainbow mussels, Villosa iris)

\section{Mussel Toxicity}

September 4, 2009

Prepared by:

Ning Wang, Chris D. Ivey, and Chris G. Ingersoll

U.S. Geological Survey

Columbia Environmental Research Center (CERC)

Columbia, MO, 65201

Technical Point of Contact:

Dr. Jeffery A. Steevens

Tel: 601-634-4199; Email: Jeffery.A.Steevens@usace.army.mil

Dr. Ning Wang

Tel: 573-441-2946 (work); Email: nwang@usgs.gov 


\section{INTRODUCTION}

Fly ash was released to the Emory River, TN from the Tennessee Valley Authority Kingston Fossil Plant (USEPA 2009, TVA 2009). The environmental cleanup of this spill will most likely result in the removal of over 5 million cubic yards of fly ash from the river and surrounding areas in connection with recovery and remediation efforts. The impact of this release on the river ecosystem is currently unknown. The objective of this study was to evaluate the potential toxicity of resuspended fly ash to juvenile freshwater mussels in 10-d static-renewal elutriate toxicity test following the method following general procedures outlined in ASTM (2009a) for conducting water toxicity tests with juvenile mussels.

\section{METHODS}

\subsection{Test 1 with newly transformed juvenile mussels.}

Fly ash was collected from two sites (Emory River and Sluice channel). Elutriates were prepared by U.S. Army Engineer Research and Development Center (ERDC) to represent water surrounding the dredge and released from the fly ash storage/dewatering facility. Two 8-L 100\% elutriate samples (EMR-EL-MS and SLC-EL-MS) and 20-L upstream reference water (EMRWA-MS, as dilution water for two elutriate tests) were delivered to U.S. Geological Survey, Columbia Environmental Research Center (CERC) on June 25, 2009 and kept at $4^{\circ} \mathrm{C}$. These water samples were splits of the same water samples used to conduct the elutriate toxicity tests with fathead minnows (Pimephales promelas) by the ERDC. Newly transformed (1-week-old) fatmucket (Lampsilis siliquoidea) were obtained from the laboratory cultures at Missouri State University in Springfield, MO. Juvenile mussels $(0.35 \pm 0.03 \mathrm{~mm}$ shell length, $n=20)$ were acclimated to CERC well water (hardness $280 \mathrm{mg} / \mathrm{L}$ as $\mathrm{CaCO}_{3}, \mathrm{pH}$ 8.0) and test temperature $\left(20^{\circ} \mathrm{C}\right)$ for $48 \mathrm{~h}$ before starting tests. During the acclimation period, the mussels were fed a nonviable algal mixture (Wang et al. 2007) twice daily.

Toxicity test conditions are summarized in Table 1 . Three elutriate concentrations (100, 50, 10\% elutriate) were prepared by diluting the $100 \%$ elutriate with reference site water ( $0 \%$ elutriate). At the beginning of the test, ten mussels were transferred impartially into each of five replicate 300-ml glass beakers containing about $200 \mathrm{ml}$ of water or elutriate. The beakers were placed in a water bath at $20 \pm 1^{\circ} \mathrm{C}$. Test duration was $10 \mathrm{~d}$. Mussels were fed $2 \mathrm{ml}$ of the non-viable algal mixture once daily. About 75\% water was renewed on Days 3 and 7 . Water quality characteristics ( $\mathrm{pH}$, dissolved oxygen, hardness, alkalinity, conductivity, ammonia) were measured from each treatment at the beginning and end of tests following standard methods (APHA 2005). Dissolved oxygen was also measured before each water renewal. About $40 \mathrm{ml}$ of water samples for potential chemical analysis were collected from each treatment at the beginning of tests, and was shipped at about $4^{\circ} \mathrm{C}$ in a cooler overnight to ERDC. Survival of mussels (defined as foot movement during a five-minute observation period) was determined at the end of the tests. The acceptability criterion for a toxicity test was $\geq 80 \%$ control survival (ASTM 2009a).

A subset of test organisms used in toxicity tests was evaluated by conducting an acute staticrenewal toxicity test with a reference toxicant (reagent-grade $\mathrm{NaCl}$ ) following standard test 
methods (ASTM 2009). Mussels will be acclimated to ASTM hard water (hardness 160-180 $\mathrm{mg} / \mathrm{L}$ as $\mathrm{CaCO}_{3}$; ASTM 2009b) for $48 \mathrm{~h}$ before the start of the reference test. The test conditions for reference toxicant test are summarized in Table 2.

The statistical difference in mean survival among four elutriate concentrations $(0,10,50$, and $100 \%$ elutriate) were determined by analysis of variance (ANOVA) with mean comparison made by one-tailed Dunnett's test (USEPA 2002) with TOXSTAT ${ }^{\circledR}$ software (WEST 1996). The level of statistical significance was set at $p \leq 0.05$. Median effective concentrations (EC50s) for survival were calculated using a Probit model (USEPA 1993). If the data did not meet the requirements of the Probit model, either a Spearman-Karber or trimmed Spearman-Karber method was used (USEPA 1993).

\subsection{Test 2 with 5-month-old juvenile mussels.}

Test 2 was conducted because Test 1 conducted with newly transformed fatmucket juveniles failed due to high mortality in well water control (100\% mortality) and site water control (70\% mortality) at the end of 10-d tests. Survival of the newly transformed fatmucket juvenile mussels was only $85 \%$ at Day 4 in the reference toxicant test ( $\geq 90 \%$ survival is the acceptability requirement for a 4-d acute test; ASTM 2009a). The ERDC recreated the two new preparations of the elutriate samples that were sent to the CERC on July 28, 2009 for retesting. The test conditions were similar to those described for Test 1, with following exceptions.

(1) Because no young juvenile mussels were available at that time, about 5-month-old rainbow mussels (Villosa iris) were used in Test 2. The juveniles were obtained from the laboratory cultures at CERC (the batch of mussels was originally obtained from the laboratory cultures at Virginia Department of Game and Inland Fisheries, Marion, VA).

(2) The mussels had a large range of size (1.9 to $5.7 \mathrm{~mm}$, mean $3.7 \pm 0.9 \mathrm{~mm}, \mathrm{n}=43)$. These older juvenile mussels were primarily filter feeders (similar to adult mussels) using their gills to remove suspended particles from the water column. Specifically, older mussels tend to exhibit less frequent food movement compared to younger mussels. In contrast, newly transformed juveniles are primarily foot feeding (obtaining food using cilia lining the surface of the foot; ASTM 2009a). Hence, the older mussels tend to move their foot out of shell less often than the newly transformed juveniles. Therefore, heartbeat was used to determine the survival if no foot movement was observed (ASTM 2009a).

(3) Juvenile mussels were acclimated to the reference site water (the dilution water) for $48 \mathrm{~h}$ before starting tests. A subset of mussels was also acclimated in the CERC well water for organisms evaluated in the CERC well water control treatment.

(4) Mussels were fed $2 \mathrm{ml}$ of the non-viable algal mixture twice daily (4 ml once daily at weekend).

(5) Test organisms in each test beaker were transferred to a clean beaker containing newly prepared control or elutriate water on Day 7.

(6) Because of limited number of juvenile mussel available for testing, each treatment included four replicates in elutriate tests and 2 replicates in $\mathrm{NaCl}$ reference toxicant treatment. 


\section{RESULTS}

\subsection{Test 1 with newly transformed juvenile mussels.}

The water quality characteristics were consistent during the exposures (Table 3). Concentrations of dissolved oxygen were higher than $5.8 \mathrm{mg} / \mathrm{L}$ and concentrations of total ammonia were low during the 10 day test (Table 3). The 10-d control survival was low: $0 \%$ in well water control and $30 \%$ in site water ( $0 \%$ elutriate, Table 3$)$. The 96 -h survival in the $\mathrm{NaCl}$ reference toxicant test was $85 \%$ (Table 4 ), below the test acceptability criterion of $\geq 90 \%$ control survival (ASTM 2009a).

\subsection{Test 2 with 5-month-old juvenile mussels.}

The water quality characteristics were consistent during the exposure concentrations (Table 3). Concentrations of dissolved oxygen were higher than $5.9 \mathrm{mg} / \mathrm{L}$ and concentrations of ammonia were low during the 10 day test (Table 3). The survival based on heartbeat was $\geq 95 \%$ in well water control, reference site waters (Table 3), and in the ASTM control water in the reference toxicant test (Table 4). The 96-h LC50 for $\mathrm{NaCl}$ was $3.5 \mathrm{~g} / \mathrm{L}$, which was slightly higher than 96h EC50s for $\mathrm{NaCl}$ obtained from the tests with newly transformed juvenile mussels or up to 2month-old mussels (EC50 <3.0 g/L based on foot movement, unpublished data). The 10-d survival based on foot movement or heartbeat was not significantly different between site water ( $0 \%$ elutriate) and any elutriate concentrations for the two elutriate samples, except for $100 \%$ elutriate from the sample collected in Sluice channel, where significantly lower percentage of the mussels were observed with foot movement compared to mussels in reference site water (Table 3).

\section{Discussion}

Test 1 with newly transformed juvenile mussels failed due to poor survival in the control water or in the reference site water at the end of the 10-d test. The 96-h reference toxicant test also failed due to low control survival in ASTM hard water. In addition, the same batch of juvenile mussels held in a mussel culture system at CERC died off at the same period of the elutriate tests. These results indicate that a bad batch of newly transformed fatmucket juveniles was used in Test 1 . These data are therefore not considered valid for assessing the toxicity for the fly ash material.

Test 2 with 5-month-old rainbow mussels were successfully completed with acceptable control survival. Concentrations of up to $100 \%$ elutriate from two samples did not affect the mussel survival (heartbeat) in the 10-d exposure. Foot movement is not a reliable indicator for control survival of old mussels because older mussels less frequently move their foot out of their shell compared to younger mussel. However, the low percentage of surviving mussels with foot movement in the $100 \%$ elutriate from Sluice channel indicate that exposure to the $100 \%$ of this elutriate adversely affected mussel behavior. Older juvenile mussels, like adult mussels, are able to close their shells to avoid exposures (ASTM 2009a). Because of large size and variation in size of the rainbow mussels and short test period, potential effect of exposure to elutriates on the growth of mussels was not determined in this test. 
The USGS CERC laboratory conducted 28-d whole-sediment toxicity tests with fly ash collected from Emory River with the amphipod (Hyalella azteca) and two mussel species (about 3-monthold rainbow mussel or wavy-rayed lampmussels (Lampsilis fasciola)). Methods used to conduct these whole-sediment toxicity tests were in basic accordance with methods outlined in ASTM (2009a,c). Both species of mussels exhibited 100\% survival (foot movement) after 28-d exposures to the fly ash sample. In contrast 28-d survival of amphipods in the fly ash sample was significantly lower than the amphipod exposed to a control sediment. Analyses of the physical and chemical characteristics of the ash sample and analyses of growth of amphipods and mussels at the end of the 28-d exposures are ongoing. 
Table 1. Summary of conditions for fly ash elutriate toxicity tests with juvenile mussels (Fatmucket, Lampsilis siliquoidea; rainbow mussel, Villosa iris) in basic accordance with ASTM (2009a)

\begin{tabular}{|c|c|}
\hline Species & Fatmucket (FM) or rainbow mussel (RM) \\
\hline Test Type & Static renewal \\
\hline Test Duration & $10 \mathrm{~d}$ \\
\hline Temperature & Mean: $20^{\circ} \mathrm{C}$ \\
\hline Light Quality & Ambient laboratory \\
\hline Light Intensity & $10-20 \mathrm{uE} / \mathrm{m} 2 / \mathrm{s}$ \\
\hline Photoperiod & 16L:8D \\
\hline Test Chamber Size & $300 \mathrm{ml}$ beakers \\
\hline Test Solution Volume & $200 \mathrm{~mL}$ \\
\hline $\begin{array}{l}\text { Renewal of Overlying } \\
\text { water }\end{array}$ & $\begin{array}{l}\text { About } 75 \% \text { water renewal on Days } 3 \text { and } 7 \text { in FM test; } 75 \% \\
\text { renewal on day } 3 \text { and } 100 \% \text { water renewal on Day } 7 \text { (with } \\
\text { replacement of exposure beakers) in RM test }\end{array}$ \\
\hline Age of Test Organisms & $\begin{array}{l}\text { Newly transformed (1-week-old) juvenile FM; about 5- } \\
\text { month-old RM }\end{array}$ \\
\hline $\begin{array}{l}\text { No. Organisms per } \\
\text { Chamber }\end{array}$ & 10 \\
\hline No. Replicates & 5 for $\mathrm{FM}, 4$ for RM \\
\hline Feeding Regime & $\begin{array}{l}\text { FM: } 2 \mathrm{ml} \text { of algal mixture once daily; } \\
\text { RM: } 2 \mathrm{ml} \text { of algal mixture twice daily }\end{array}$ \\
\hline Test Chamber Cleaning & $\begin{array}{l}\text { Transfer mussel to clean beakers containing exposure water } \\
\text { on Day } 7\end{array}$ \\
\hline Test Solution Aeration & None \\
\hline Dilution Water & Reference site water \\
\hline Test Concentrations & $\begin{array}{l}100 \%, 50 \%, 10 \%, 0 \% \text { (dilutions prepared with site water) } \\
\text { and CERC well water }\end{array}$ \\
\hline Endpoint & Survival \\
\hline Sampling and hold time & $1 \mathrm{~d}$ for FM test, $3 \mathrm{~d}$ for RM test \\
\hline Water quality & $\begin{array}{l}\text { pH, dissolved oxygen, hardness, alkalinity, conductivity, } \\
\text { ammonia were measured at the beginning and end of tests } \\
\text { Dissolved oxygen was measured on Days } 3 \text { and } 7\end{array}$ \\
\hline $\begin{array}{l}\text { Test Acceptability } \\
\text { Criterion }\end{array}$ & $\geq 80 \%$ survival in control \\
\hline
\end{tabular}


Table 2. Summary of conditions for conducting reference toxicant test with juvenile mussels (fatmucket, Lampsilis siliquoidea; rainbow mussel, Villosa iris) in basic accordance with ASTM (2009a)

\begin{tabular}{ll}
\hline Test species: & Fatmucket (FM) or rainbow mussel (RM) \\
Test chemical: & Sodium chloride (reagent-grade NaCl) \\
Test type: & Static renewal \\
Test Duration: & $96 \mathrm{~h}$ \\
Temperature: & $20^{\circ} \mathrm{C}$ \\
Light quality: & Ambient laboratory light \\
Light intensity: & 200 lux \\
Photoperiod: & $16 \mathrm{~L}: 8 \mathrm{D}$ \\
Test chamber size: & $50 \mathrm{ml}$ \\
Test solution volume: & $30 \mathrm{ml}$ \\
Renewal of solution: & After $48 \mathrm{~h}$ \\
Age of test organism: & About 1-week-old FM, about 5-month-old RM \\
Organisms/beaker: & 5 \\
Replicate number: & 4 in FM test, 2 in RM test (limited RM available) \\
Feeding: & No feeding \\
Chamber cleaning: & None \\
Aeration: & None \\
Dilution water: & Reconstituted ASTM hard water (160-180 mg/L as CaCO 3 ; ASTM \\
& $2009 b)$ \\
Dilution factor: & 0.5 \\
Test concentration: & $0,0.5,1,2,4$, and 8 g NaCl/L \\
Chemical residues: & Salinity and conductivity at each NaCl concentration measured at \\
& the beginning and the end of test \\
Water quality: & Dissolved orxgen, pH, hardness, and alkalinity were determined at \\
& the control, medium, and high concentrations of chemicals at the \\
beginning and the end of test \\
Endpoint: & Survival (foot movement within 5 min in FM test, foot movement \\
& and heartbeat in RM test) \\
Test acceptability criterion: & $\geq 90 \%$ control survival \\
\hline
\end{tabular}


Table 3. Water quality characteristics (test day 0 and 10) and survival of newly transformed fatmucket (Lampsilis siliquoidea) and 5-month-old rainbow mussel (Villosa iris) in 10-d static-renewal tests with fly ash elutriates diluted by reference site water (0\%).

\begin{tabular}{|c|c|c|c|c|c|c|c|c|c|c|}
\hline \multirow[t]{2}{*}{ Test } & \multirow{2}{*}{$\begin{array}{l}\text { Species } \\
\text { (age) }\end{array}$} & \multirow[t]{2}{*}{ Site ID } & \multirow[t]{2}{*}{ Concentration } & \multirow[t]{2}{*}{$\mathrm{pH}$} & \multirow{2}{*}{$\begin{array}{c}\text { Conductivity } \\
(\mu \mathrm{S} / \mathrm{cm})\end{array}$} & Alkalinity & Hardness & \multirow{2}{*}{$\begin{array}{l}\text { Ammonia } \\
\text { (mg TN/L) }\end{array}$} & \multicolumn{2}{|c|}{ Survival $(\%, S D, n=4)$} \\
\hline & & & & & & \multicolumn{2}{|c|}{$\left(\mathrm{mg} / \mathrm{L}\right.$ as $\left.\mathrm{CaCO}_{3}\right)$} & & (foot movement) & (heartbeat) \\
\hline \multirow[t]{9}{*}{1} & Fatmucket & CERC well water & Control & $8.0 / 8.6$ & $597 / 664$ & $136 / 256$ & $300 / 292$ & $0.08 / 0.11$ & $0(0)$ & $\mathrm{ND}^{\mathrm{a}}$ \\
\hline & & EMR-EL-MS (2) & $0 \%$ & $7.7 / 7.7$ & $99 / 123$ & $28 / 38$ & $22 / 36$ & $0.06 / 0.32$ & $30(27)$ & ND \\
\hline & & & $10 \%$ & $7.8 / 7.9$ & $93 / 124$ & $30 / 34$ & $24 / 38$ & $0.07 / 0.05$ & $40(27)$ & ND \\
\hline & & & $50 \%$ & $7.8 / 7.8$ & $101 / 140$ & $36 / 40$ & $34 / 44$ & $0.20 / 0.14$ & $28(4.5)$ & ND \\
\hline & & & $100 \%$ & $7.9 / 7.9$ & $115 / 162$ & $40 / 50$ & $40 / 54$ & $0.03 / 0.17$ & $38(8.4)$ & ND \\
\hline & & SLC-EL-MS (2) & $0 \%$ & 7.7/7.7 & $99 / 123$ & $28 / 38$ & $22 / 36$ & $0.06 / 0.32$ & $30(27)$ & ND \\
\hline & & & $10 \%$ & $7.7 / 7.9$ & $102 / 150$ & $40 / 38$ & $30 / 50$ & $0.06 / 0.05$ & $42(15)$ & ND \\
\hline & & & $50 \%$ & $7.9 / 8.0$ & $173 / 208$ & $40 / 54$ & $66 / 98$ & $0.03 / 0.15$ & $74(15)$ & ND \\
\hline & & & $100 \%$ & $8.0 / 8.0$ & $243 / 276$ & $70 / 72$ & $102 / 108$ & $0.04 / 0.05$ & $86(13)$ & ND \\
\hline \multirow[t]{9}{*}{2} & $\begin{array}{c}\text { Rainbow musse } \\
\text { (5 month old) }\end{array}$ & I CERC well water & Control & 8.6/8.6 & $625 / 637$ & $260 / 260$ & $300 / 280$ & $0.11 / 0.18$ & 73 (19) & $95(10)$ \\
\hline & & EMR-EL-MS (2) & $0 \%$ & $7.2 / 8.0$ & $84 / 107$ & $22 / 40$ & $40 / 32$ & $0.05 / 0.19$ & $88(15)$ & $100(0)$ \\
\hline & & & $10 \%$ & $7.3 / 7.6$ & $120 / 115$ & $36 / 40$ & $40 / 34$ & $0.08 / 0.19$ & $78(21)$ & $100(0)$ \\
\hline & & & $50 \%$ & $7.6 / 7.6$ & $119 / 125$ & $36 / 46$ & $48 / 36$ & $0.06 / 0.16$ & 85 (19) & $95(10)$ \\
\hline & & & $100 \%$ & $7.5 / 8.0$ & $151 / 178$ & $48 / 54$ & $60 / 66$ & $0.04 / 0.22$ & $83(10)$ & $90(0)$ \\
\hline & & SLC-EL-MS (2) & $0 \%$ & $7.2 / 8.0$ & $84 / 107$ & $22 / 40$ & $40 / 32$ & $0.05 / 0.19$ & $88(15)$ & $100(0)$ \\
\hline & & & $10 \%$ & $7.4 / 7.7$ & $120 / 128$ & $28 / 40$ & $50 / 40$ & $0.05 / 0.19$ & $68(10)$ & $98(5.0)$ \\
\hline & & & $50 \%$ & $7.4 / 7.9$ & $167 / 177$ & $44 / 40$ & $70 / 60$ & $0.06 / 0.15$ & $70(20)$ & $90(14)$ \\
\hline & & & $100 \%$ & 7.6/8.1 & $254 / 289$ & $68 / 70$ & $110 / 102$ & $0.05 / 0.20$ & $35(17)^{*}$ & $98(5.0)$ \\
\hline
\end{tabular}

\footnotetext{
* Significant reduction relative to the site water $(0 \%)$.
}

${ }^{a}$ Not determined. 
Table 4. Water quality characteristics and survival in 96-h reference toxicant ( $\mathrm{NaCl})$ tests with newly transformed fatmucket (Lampsilis siliquoidea) and 5-month-old rainbow mussel (Villosa iris)

\begin{tabular}{|c|c|c|c|c|c|c|c|c|c|}
\hline Test & $\begin{array}{c}\text { Species } \\
\text { (age) }\end{array}$ & $\begin{array}{c}\text { Nominal } \\
\mathrm{NaCL}(\mathrm{g} / \mathrm{L}) \\
\end{array}$ & $\begin{array}{c}\text { Measured } \\
\text { salinity (g/L) }\end{array}$ & $\begin{array}{c}\text { Dissolved oxygen } \\
(\mathrm{mg} / \mathrm{L})\end{array}$ & $\mathrm{pH}$ & $\begin{array}{l}\text { Conductivity } \\
(\mu \mathrm{S} / \mathrm{cm})\end{array}$ & $\begin{array}{l}\text { Alkalinity } \\
\text { (mg/L as }\end{array}$ & $\begin{array}{l}\text { Hardness } \\
\left.\mathrm{CaCO}_{3}\right) \\
\end{array}$ & $\begin{array}{c}\text { Survival }^{\mathrm{a}} \\
(\%) \\
\end{array}$ \\
\hline \multirow[t]{6}{*}{1} & Fatmucket & 0 & $0.1 / 0.1$ & $8.4 / 7.3$ & $8.6 / 8.5$ & $632 / 704$ & $130 / 148$ & $186 / 194$ & 85 \\
\hline & (1 week old) & 0.5 & $0.6 / 0.6$ & & & $1493 / 1588$ & & & 85 \\
\hline & & 1 & $1.1 / 1.2$ & & & $2360 / 2500$ & & & 70 \\
\hline & & 2 & $2.1 / 2.2$ & $8.4 / 7.3$ & $8.6 / 8.5$ & $4060 / 4290$ & $130 / 148$ & $186 / 194$ & 85 \\
\hline & & 4 & $4.0 / 4.2$ & & & $7290 / 7720$ & & & 5 \\
\hline & & 8 & $8.1 / 8.7$ & $8.4 / 7.3$ & $8.6 / 8.4$ & $13970 / 14890$ & $130 / 148$ & $186 / 194$ & 0 \\
\hline \multirow[t]{6}{*}{2} & Rainbow mussel & 0 & $0.1 / 0.1$ & $8.0 / 7.5$ & $8.8 / 8.7$ & $618 / 741$ & $140 / 142$ & $180 / 188$ & 100 \\
\hline & (5 month old) & 0.5 & $0.6 / 0.7$ & & & $1429 / 1666$ & & & 100 \\
\hline & & 1 & $1.1 / 1.2$ & & & $2222 / 2600$ & & & 100 \\
\hline & & 2 & $2.1 / 2.2$ & $8.0 / 7.4$ & /8.6 & $3790 / 4420$ & $140 / 148$ & $/ 188$ & 100 \\
\hline & & 4 & $4.1 / 4.4$ & & & $6840 / 8040$ & & & 30 \\
\hline & & 8 & $8.1 / 8.8$ & $8.0 / 7.1$ & 18.5 & $12970 / 15120$ & $140 / 148$ & $/ 188$ & 0 \\
\hline
\end{tabular}

a Survival was based on foot movement of newly transformed mussels in Test 1 and based on foot movement or heartbeat of 5month-old mussels in Test 2. 


\section{References List:}

American Public Health Association (APHA). 2005. Standard Methods for the Examination of Water and Waster Water, 21st ed. American Public Health Association, Washington, DC.

American Society for Testing and Materials (ASTM). 2009a. Standard guide for conducting laboratory toxicity tests with freshwater mussels (ASTM E2455-06). Annual Book of ASTM Standards Volume 11.06. West Conshohocken, PA.

ASTM 2009b. Standard guide for conducting acute toxicity tests on test materials with fishes, macroinvertebrates, and amphibians (ASTM E729 92007)). Annual Book of ASTM Standards Volume 11.06. West Conshohocken, PA.

ASTM 2009c. Standard test method for measuring the toxicity of sediment-associated contaminants with freshwater invertebrates (ASTM E1706-05). Annual Book of ASTM Standards Volume 11.06. West Conshohocken, PA.

Tennessee Valley Authority (TVA). 2009. Kingston Recovery Update. Accessed on the web at: http://www.tva.gov/kingston/index.htm

USEPA. 1993. Methods for measuring the acute toxicity of effluents and receiving waters to freshwater and marine organisms, fourth edition. EPA/600/4-90/027F. Washington, DC.

U.S. Environmental Protection Agency (USEPA). 2002. Short-term methods for estimating the chronic toxicity of effluents and receiving waters to freshwater organisms, 4th ed. EPA-821-R02-013. Washington, DC.

U.S. Environmental Protection Agency (USEPA). 2009. EPA's Response to the TVA Kingston Fossil Plant Fly Ash Release. Region 4, Southeast. Accessed on the web at: http://www.epa.gov/region4/kingston/index.html

Wang N, Ingersoll CG, Greer IE, Hardesty DK, Ivey CD, Kunz JL, Brumbaugh WG, Dwyer FJ, Roberts AD, Augspurger T, Kane CM, Neves RJ, Barnhart MC. 2007. Chronic toxicity of copper and ammonia to juvenile freshwater mussels (Unionidae). Environ Toxicol Chem 26:2048-2056.

Western EcoSystems Technology. 1996. TOXSTAT ${ }^{\circledR}$ 3.5. Cheyenne, WY, USA. 


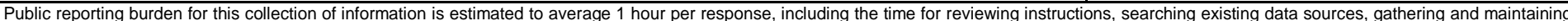

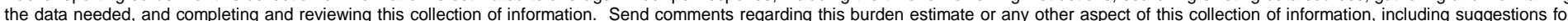

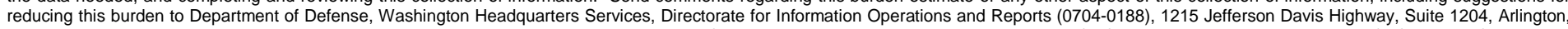

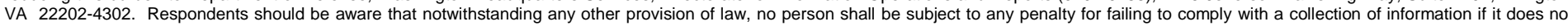
display a currently valid OMB control number. PLEASE DO NOT RETURN YOUR FORM TO THE ABOVE ADDRESS.
1. REPORT DATE (DD-MM-YYYY)
2. REPORT TYPE
3. DATES COVERED (From - To)
August 2011
Final report

\section{TITLE AND SUBTITLE}

Evaluation of Metals Release from Oxidation of Fly Ash during Dredging of the Emory

River, TN

\section{5a. CONTRACT NUMBER}

5b. GRANT NUMBER

5c. PROGRAM ELEMENT NUMBER

6. AUTHOR(S)

Jeffery A. Steevens, Anthony J. Bednar, Mark A. Chappell, Alan J. Kennedy, Jennifer M.

Seiter, Jacob K. Stanley, and Daniel E. Averett

\section{5d. PROJECT NUMBER}

5e. TASK NUMBER

5f. WORK UNIT NUMBER

7. PERFORMING ORGANIZATION NAME(S) AND ADDRESS(ES)

8. PERFORMING ORGANIZATION REPORT NUMBER

Environmental Laboratory

U.S. Army Engineer Research and Development Center

ERDC/EL TR-11-9

3909 Halls Ferry Road

Vicksburg, MS 39180-6199

9. SPONSORING I MONITORING AGENCY NAME(S) AND ADDRESS(ES)

10. SPONSOR/MONITOR'S ACRONYM(S)

Tennessee Department of Environmental Conservation

401 Church St., Nashville, TN 37243

11. SPONSOR/MONITOR'S REPORT NUMBER(S)

\section{DISTRIBUTION / AVAILABILITY STATEMENT}

Approved for public release; distribution is unlimited.

\section{SUPPLEMENTARY NOTES}

\section{ABSTRACT}

This report describes a study examining the effects of Kingston Fossil Plant fly ash on geochemistry, water quality, and aquatic biota relevant to the Emory River in Tennessee. It focuses on the processes specific to the changes in the fly ash chemistry and potential releases as a result of a spill that occurred on December 22, 2008 and the subsequent dredging operations to remove the fly ash from the Emory River. Fly ash from three sites including the original pile, fly ash spilled in the Emory River, and dredged fly ash from the sluice channel were evaluated to assess the potential for metal releases. Fly ash from the Emory River and sluice channel were used to prepare relevant suspensions (e.g., elutriates) to represent extreme conditions for the release, dissolution, and metal speciation changes that might occur during dredging. Advanced chemical assessment techniques were used to quantify the chemical concentrations and speciation in this system. Because multiple metals were present, biological studies were also conducted to assess the potential for toxic effects and uptake in aquatic organisms. Chemistry results from this study were compared to measurements from ongoing monitoring at the site.

\begin{tabular}{|ll}
\hline 15. SUBJECT TERMS & Emory River, TN \\
Arsenic & Fly ash \\
Barium & Selenium \\
\hline
\end{tabular}

16. SECURITY CLASSIFICATION OF:

Fly ash

16. SECURITY CLASSIFICATION OF:

\section{a. REPORT}

UNCLASSIFIED

b. ABSTRACT
UNCLASSIFIED

c. THIS PAGE

UNCLASSIFIED

17. LIMITATION
OF ABSTRACT
UNLIMITED

18. NUMBER OF PAGES

624
Tennessee Valley Authority

19a. NAME OF RESPONSIBLE PERSON

19b. TELEPHONE NUMBER (include area code) 